NUREG/CR-5913

ORNL/TM-12156/V2

Vol. 2

\title{
Irradiation Effects on
}

Fracture Toughness of

Two High-Copper Submerged-Arc

Welds, HSSI Series 5

Appendices $\mathrm{E}$ and $\mathrm{F}$

Prepared by

R. K. Nanstad, F. M. Haggag, D. E. McCabe,

S. K. Iskander, K. O. Bowman, B. H. Menke

Oak Ridge National Laboratory

Prepared for

U.S. Nuclear Regulatory Commission 


\section{AVAILABILITY NOTICE}

Availability of Reference Msiarials Cited in NRC Publications

Most documents cited in NRC publications will be avallable from one of the following sources:

1. The NRC Public Document Room, 2120 L Street, NW., Lower Level, Washington, DC 20555

2. The Superintendent of Documents, U.S. Government Printing Office, P.O. Box 37082, Washington, DC 20013-7082

3. The National Technical Information Service, Springfield, VA 22161

Although the listing that follows represents the majority of documents cited in NRC publications, it is not Intended to be exhaustive.

Referenced documents available for inspection and copying for a fee from the NRC Public Document Room include NRC correspondence and internal NRC memoranda; NRC bulletins, circulars, information notices, inspection and Investigation notices; licensee event reports; vendor reports and correspondence; Commission papers; and applicant and licensee documents and correspondence.

The following documents in the NUREG series are available for purchase from the GPO Sales Program: formal NRC staff and contractor reports, NRC-sponsored conference proceedings, international agreement reports, grant publications, and NRC booklets and brochures. Also available are regulatory guides, NRC regulations in the Code of Federal Regulations, and Nuclear Regulatory Commission Issuances.

Documents available from the National Technical Information Service include NUREG-serles reports and technical reports prepared by other Federal agencies and reports prepared by the Atomic Energy Commission, forerunner agency to the Nuclear Regulatory Commission.

Documents avallable from public and special technical libraries include all open literature items, such as books, Journal articles, and transactions. Federal Register notices, Federal and State legislation, and congressional reports can usually be obtained from these libraries.

Documents such as theses, dissertations, foreign reports and translations, and non-NRC conference proceedings are available for purchase from the organization sponsoring the publication cited.

Single copies of NRC draft reports are available free, to the extent of supply, upon written request to the Office of Administration, Distribution and Mail Services Section, U.S. Nuclear Regulatory Commission, Washington, DC 20555.

Copies of industry codes and standards used in a substantive manner in the NRC regulatory process are maintained at the NRC Library, 7920 Norfolk Avenue, Bethesda, Maryland, for use by the public. Codes and standards are usually copyrighted and may be purchased from the originating organization or, if they are American National Standards, from the American National Standards Institute, 1430 Broadway, New York, NY 10018.

\section{DISCLAIMER NOTICE}

This report was prepared as an account of work sponsored by an agency of the United States Government. Neither the United States Government nor any agency thereof, or any of their employees, makes any warranty, expressed or implied, or assumes any legal liability of responsibility for any third party's use, or the results of such use, of any information, apparatus, product or process disclosed in this report, or represents that its use by such third party wculd not infringe privately owned rights. 


\section{DISCLAIMER}

This report was prepared as an account of work sponsored by an agency of the United States Government. Neither the United States Government nor any agency Thereof, nor any of their employees, makes any warranty, express or implied, or assumes any legal liability or responsibility for the accuracy, completeness, or usefulness of any information, apparatus, product, or process disclosed, or represents that its use would not infringe privately owned rights. Reference herein to any specific commercial product, process, or service by trade name, trademark, manufacturer, or otherwise does not necessarily constitute or imply its endorsement, recommendation, or favoring by the United States Government or any agency thereof. The views and opinions of authors expressed herein do not necessarily state or reflect those of the United States Government or any agency thereof. 


\section{DISCLAIMER}

Portions of this document may be illegible in electronic image products. Images are produced from the best available original document. 


\title{
Irradiation Effects on
}

\section{Fracture Toughness of}

\section{Two High-Copper Submerged-Arc Welds, HSSI Series 5}

\author{
Appendices $\mathrm{E}$ and $\mathrm{F}$
}

Manuscript Completed: August 1992

Date Published: October 1992

Prepared by

R. K. Nanstad, F. M. Haggag, D. E. McCabe,

S. K. Iskander, K. O. Bowman, B. H. Menke*

Oak Ridge National Laboratory

Managed by Martin Marietta Energy Systems, Inc.

Oak Ridge National Laboratory

Oak Ridge, TN 37831-6285

\section{Prepared for}

Division of Engineering

Office of Nuclear Regulatory Research

U.S. Nuclear Regulatory Commission

Washington, DC 20555

NRC FIN L1098

\section{DISCLAIMER}

\section{Under Contract No. DE-AC05-840R21400}

*Materials Engineering Associates, Inc. 9700B Martin Luther King, Jr. Highway

Lanham, MD 20706 



\section{Abstract}

The Fifth Irradiation Series in the Heavy-Section Steel Irradiation (HSSI) Program was aimed at obtaining a statistically significant fracture toughness data base on two weldments with highcopper contents to determine the shift and shape of the $K_{1 c}$ curve as a consequence of irradiation. The program included irradiated Charpy V-notch impact, tensile, and drop-weight specimens in addition to compact fracture toughness specimens. Compact specimens with thicknesses of 25.4, 50.8, and $101.6 \mathrm{~mm}$ [1T C (T), 2T C (T), and 4T C (T), respectively] were irradiated. Additionally, unirradiated $6 \mathrm{~T} \mathrm{C}(\mathrm{T})$ and $8 \mathrm{~T} \mathrm{C}(\mathrm{T})$ specimens with the same $\mathrm{K}_{\mathrm{lc}}$ measuring capacity as the irradiated specimens were tested. The materials for this irradiation series were two weldments fabricated from special heats of weld wire with copper added to the melt. One lot of Linde 0124 flux was used for all the welds. Copper levels for the two welds are 0.23 and $0.31 \mathrm{wt} \%$, while the nickel contents for both welds are $0.60 \mathrm{wt} \%$.

Twelve capsules of specimens were irradiated in the poolside facility of the Oak Ridge Research Reactor at a nominal temperature of $288^{\circ} \mathrm{C}$ and an average fluence of about $1.5 \times 10^{19}$ neutrons $/ \mathrm{cm}^{2}$ $(>1 \mathrm{MeV})$. The Charpy $41-\mathrm{J}$ temperature shifts are about the same as the corresponding drop-weight nil-ductility transition temperature shifts. The
Charpy data were analyzed by various curve-fitting techniques, as well as a Weibull-based parametric analysis, to determine standard errors and associated temperature intervals. The irradiated welds exhibited substantial numbers of cleavage pop-ins that were judged significant and included in the data base for analysis. Mean curve fits using two-parameter (with fixed intercept) nonlinear and linearized exponential regression analyses revealed that the fracture toughness shifts (at $100 \mathrm{MPa} \cdot \sqrt{\mathrm{m}}$ ) exceeded the Charpy $41-\mathrm{J}$ shifts for both welds. The same observation applies to the shifts of curves fit as lower boundaries to the fracture toughness data. The data were also analyzed to examine effects of precleavage ductile tearing, small-scale yielding limits, and the $\boldsymbol{\beta}_{1 \mathrm{c}}$ adjustment. Those analyses provided essentially the same results as with the full data sets. Analyses of curve shape changes using the linearized model indicate irradiation-induced decreases in the slopes of the fracture toughness curves, especially for the higher copper weld. Three-parameter Weibull analyses were performed to investigate development of lower bound curves to the data, including the use of a variable $\mathrm{K}_{\min }$ parameter. The results were used to compare various procedures for shifting the $\mathrm{K}_{\mathrm{lc}}$ curve to establish a suitable bounding curve for irradiated material. 



\section{Contents}

Abstract

Previous Reports in Series

ix

Appendix E. Load-Displacement Curves and Photographs of the Fracture Toughness Specimens from the $72 \mathrm{~W}$ Weld $(0.23 \mathrm{wt} \% \mathrm{Cu})$

Appendix F. Load-Displacement Curves and Photographs of the Fracture Toughness Specimens from the $73 \mathrm{~W}$ Weld $(0.31 \mathrm{wt} \% \mathrm{Cu})$ 


\section{Acknowledgments}

The authors acknowledge Julia L. Bishop for preparation of the draft manuscript, Marcia Hodges for the final report, Kathy Spence for editing, and David J. Alexander and Roger E. Stoller for their helpful reviews.

Program management support from William R. Corwin and financial support from the U.S. Nuclear Regulatory Commission, Alfred Taboada and Ed Hackett, former and current technical monitors, respectively, are much appreciated. We also acknowledge Reynold Berggren and John McGowan for their many contributions earlier in the program and Daryl Downing for valuable consultations on statistical analyses. Finally, the dedication to quality shown by Ken Thoms and his group in capsule design, fabrication, and operation, and Frank Kam and his group for dosimetry, are much appreciated. 


\section{Previous Reports In Series}

The work reported here was performed at Oak Ridge National Laboratory (ORNL) under the Heavy-Section Steel Irradiation (HSSI) Program, W. R. Corwin, Program Manager. The program is sponsored by the Office of Nuclear Regulatory Research of the U.S. Nuclear Regulatory Commission (NRC). The former and current technical monitors for the NRC are A. Taboada and E. Hackett, respectively.

This report is designated HSSI Report 7. It is published in two volumes. Volume 1 contains the main body with Appendices A, B, C, and D. Volume 2 contains only Appendices $E$ and $F$, which provide detailed load-displacement traces, fracture surfaces, etcs. In recognition of its large size, Vol. 2 will be distributed only to those in the RF distribution requesting a copy from the HSSI Program Office. Reports in this series are listed below:

1. F. M. Haggag, W. R. Corwin, and R. K. Nanstad, Martin Marietta Energy Systems, Inc., Oak Ridge Natl. Lab., Oak Ridge, Tenn., Irradiation Effects on Strength and Toughness of Three-Wire SeriesArc Stainless Steel Weld Overlay Cladding, NUREG/CR-5511 (ORNL/TM-11439), February 1990.

2. L. F. Miller, C. A. Baldwin, F. W. Stallman, and F. B. K. Kam, Martin Marietta Energy Systems, Inc., Oak Ridge Natl. Lab., Oak Ridge, Tenn., Neutron Exposure Parameters for the Metallurgical Test Specimens in the Sixth Heavy-Section Steel Irradiation Series, NUREG/CR-5409 (ORNL/TM-11267), March 1990.

3. S. K. Iskander, W. R. Conwin, and R. K. Nanstad, Martin Marietta Energy Systems, Inc., Oak Ridge Natl. Lab., Oak Ridge, Tenn., Results of Crack-Arrest Tests on Two Irradiated High-Copper Welds,
NUREG/CR-5584 (ORNL/TM-11575), December 1990.

4. R. K. Nanstad and R. G. Berggren, Martin Marietta Energy Systems, Inc., Oak Ridge Natl. Lab., Irradiation Effects on Charpy Impact and Tensile Properties of Low Upper-Shelf Welds, HSSI Series 2 and 3, USNRC Report NUREG/CR-5696 (ORNL/TM-11804), August 1991.

5. R. E. Stoller, Martin Marietta Energy Systems, Inc., Oak Ridge Natl. Lab., Modeling the Influence of Irradiation Temperature and Displacement Rate on Radiation-Induced Hardening in Ferritic Steels, USNRC Report NUREG/CR-5859 (ORNL/TM-12073), August 1992.

6. R. K. Nanstad, D. E. McCabe, and R. L. Swain, Martin Marietta Energy Systems, Inc., Oak Ridge Natl. Lab., Chemical Composition and $R T_{N D T}$ Determinations For Midland Weld WF-70, USNRC Report NUREG/CR-5914 (ORNL/TM-12157), to be published.

7. This report.

The HSSI Program includes both follow-on and the direct continuation of work that was performed under the Heavy-Section Steel Technology (HSST) Program. The HSST reports related to irradiation effects in pressure vessel materials and those containing unirradiated properties of materials used in HSSI and HSST irradiation programs are tabulated below as a convenience to the reader.

C. E. Childress, Union Carbide Corp. Nuclear Div., Oak Ridge Natl. Lab., Oak Ridge, Tenn., Fabrication History of the First Two 12-in.-Thick A-533 Grade B, Class 1 Steel Plates of the Heavy Section Steel Technology Program, ORNL-4313, February 1969.

T. R. Mager and F. O. Thomas, Westinghouse Electric Corporation, PWR Systems Division, Pittsburgh, Pa., Evaluation by Linear Elastic Fracture Mechanics of Radiation Damage to 
Pressure Vessel Steels, WCAP-7328 (Rev.), October 1969.

P. N. Randall, TRW Systems Group, Redondo Beach, Calif., Gross Strain Measure of Fracture Toughness of Steels, HSSTP-TR-3, Nov. 1, 1969.

L. W. Loechel, Martin Marietta Corporation, Denver, Colo., The Effect of Testing Variables on the Transition Temperature in Steel, MCR-69-189, Nov. 20, 1969.

W. O. Shabbits, W. H. Pryle, and E. T. Wessel, Westinghouse Electric Corporation, PWR Systems Division, Pittsburgh, Pa., Heavy-Section Fracture Toughness Properties of A533 Grade B Class 1 Steel Plate and Submerged Arc Weldment, WCAP-7414, December 1969.

C. E. Childress, Union Carbide Corp. Nuclear Div., Oak Ridge Natl. Lab., Oak Ridge, Tenn., Fabrication History of the Third and Fourth ASTM A-533 Steel Plates of the Heavy Section Steel Technology Program, ORNL-4313-2, February 1970.

P. B. Crosley and E. J. Ripling, Materials Research Laboratory, Inc., Glenwood, III., Crack Arrest Fracture Toughness of A533 Grade B Class 1 Pressure Vessel Steel, HSSTP-TR-8, March 1970.

F. J. Loss, Naval Research Laboratory, Washington, D.C., Dynamic Tear Test Investigations of the Fracture Toughness of Thick-Section Steel, NRL-7056, May 14, 1970.

T. R. Mager, Westinghouse Electric Corporation, PWR Systems Div., Pittsburgh, Pa., Post-Irradiation Testing of $2 T$ Compact Tension Specimens, WCAP-7561, August 1970.

F. J. Witt and R. G. Berggren, Union Carbide Corp. Nuclear Div., Oak Ridge Natl. Lab., Oak Ridge, Tenn., Size Effects and Energy Disposition in Impact Specimen Testing of ASTM A533 Grade B Steel, ORNL/TM-3030, August 1970.
D. A. Canonico, Union Carbide Corp. Nuclear Div., Oak Ridge Natl. Lab., Oak Ridge, Tenn., Transition Temperature Considerations for Thick-Wall Nuclear Pressure Vesse/s, ORNL/TM-3114, October 1970.

T. R. Mager, Westinghouse Electric Corporation, PWR Systems Div., Pittsburgh, Pa., Fracture Toughness Characterization Study of A533, Grade B, Class 1 Steel, WCAP-7578, October 1970.

W. O. Shabbits, Westinghouse Electric Corp., PWR Systems Div., Pittsburgh, Pa., Dynamic Fracture Toughness Properties of Heavy Section A533 Grade B Class 1 Steel Plate, WCAP-7623, December 1970.

C. E. Childress, Union Carbide Corp. Nuclear Div., Oak Ridge Natl. Lab., Oak Ridge, Tenn., Fabrication Procedures and Acceptance Data for ASTM A-533 Welds and a 10-in.-Thick ASTM A-543 Plate of the Heavy Section Steel Technology Program, ORNL-TM-4313-3, January 1971.

D. A. Canonico and R. G. Berggren, Union Carbide Corp. Nuclear Div., Oak Ridge Natl. Lab., Oak Ridge, Tenn., Tensile and Impact Properties of Thick-Section Plate and Weldments, ORNL/TM-3211, January 1971.

C. W. Hunter and J. A. Williams, Hanford Eng. Dev. Lab., Richland, Wash., Fracture and Tensile Behavior of Neutron-Irradiated A533-B Pressure Vessel Steel, HEDL-TME-71-76, February 6, 1971.

C. E. Childress, Union Carbide Corp. Nuclear Div., Oak Ridge Natl. Lab., Oak Ridge, Tenn., Manual for ASTM A533 Grade B Class 1 Steel (HSST Plate 03) Provided to the International Atomic Energy Agency, ORNL/TM-3193, March 1971.

P. N. Randall, TRW Systems Group, Redondo Beach, Calif., Gross Strain Crack Tolerance of A533-B Steel, HSSTP-TR-14, May 1, 1971.

C. L. Segaser, Union Carbide Corp. Nuclear Div., Oak Ridge Natl. Lab., Oak Ridge, Tenn., Feasibility 
Study, Irradiation of Heavy-Section Steel Specimens in the South Test Facility of the Oak Ridge Research Reactor, ORNL/TM-3234, May 1971.

H. T. Corten and R. H. Sailors, University of Illinois, Urbana, III., Relationship Between Material Fracture Toughness Using Fracture Mechanics and Transition Temperature Tests, T\&AM Report 346, August 1, 1971.

L. A. James and J. A. Williams, Hanford Eng. Dev. Lab., Richland, Wash., Heavy Section Steel Technology Program Technical Report No. 21, The Effect of Temperature and Neutron Irradiation Upon the Fatigue-Crack Propagation Behavior of ASTM A533 Grade B, Class 1 Steel, HEDL-TME 72-132, September 1972.

P. B. Crosley and E. J. Ripling, Materials Research Laboratory, Inc., Glenwood, III., Crack Arrest in an Increasing K-Field, HSSTP-TR-27, January 1973.

W. J. Stelzman and R. G. Berggren, Union Carbide Corp. Nuclear Div., Oak Ridge Natl. Lab., Oak Ridge, Tenn., Radiation Strengthening and Embrittlement in Heavy-Section Steel Plates and Welds, ORNL-4871, June 1973.

J. M. Steichen and J. A. Williams, Hanford Eng. Dev. Lab., Richland, Wash., High Strain Rate Tensile Properties of Irradiated ASTM A533 Grade B Class 1 Pressure Vessel Steel, HEDL-TME 73-74, July 1973.

J. A. Williams, Hanford Eng. Dev. Lab., Richland, Wash., The Irradiation and Temperature Dependence of Tensile and Fracture Properties of ASTM A533, Grade B, Class 1 Steel Plate and Weldment, HEDL-TME 73-75, August 1973.

J. A. Williams, Hanford Eng. Dev. Lab., Richland, Wash., Some Comments Related to the Effect of Rate on the Fracture Toughness of Irradiated ASTM A553-B Steel Based on Yield Strength Behavior, HEDL-SA 797, December 1974.

J. A. Williams, Hanford Eng. Dev. Lab., Richland, Wash., The Irradiated Fracture Toughness of ASTM A533, Grade B, Class 1 Steel Measured with a
Four-Inch-Thick Compact Tension Specimen, HEDL-TME 75-10, January 1975.

J. G. Merkle, G. D. Whitman, and R. H. Bryan, Union Carbide Corp. Nuclear Div., Oak Ridge Natl. Lab., Oak Ridge, Tenn., An Evaluation of the HSST Program Intermediate Pressure Vessel Tests in Terms of Light-Water-Reactor Pressure Vessel Safety, ORNL/TM-5090, November 1975.

J. A. Davidson, L. J. Ceschini, R. P. Shogan, and G. V. Rao, Westinghouse Electric Corporation, Pittsburgh, Pa., The Irradiated Dynamic Fracture Toughness of ASTM A533, Grade B, Class 1 Steel Plate and Submerged Arc Weldment, WCAP-8775, October 1976.

J. A. Williams, Hanford Eng. Dev. Lab., Richland, Wash., Tensile Properties of Irradiated and Unirradiated Welds of A533 Steel Plate and A508 Forgings, NUREG/CR-1158

(ORNL/SUB-79/50917/2), July 1979.

J. A. Williams, Hanford Eng. Dev. Lab., Richland, Wash., The Ductile Fracture Toughness of Heavy Section Steel Plate, NUREG/CR-0859, September 1979.

K. W. Carlson and J. A. Williams, Hanford Eng. Dev. Lab., Richland, Wash., The Effect of Crack Length and Side Grooves on the Ductile Fracture Toughness Properties of ASTM A533 Steel, NUREG/CR-1171 (ORNL/SUB-79/50917/3), October 1979.

G. A. Clarke, Westinghouse Electric Corp., Pittsburgh, Pa., An Evaluation of the Unloading Compliance Procedure for J-Integral Testing in the Hot Cell, Final Report, NUREG/CR-1070 (ORNL/Sub-7394/1), October 1979.

P. B. Crosley and E. J. Ripling, Materials Research Laboratory, Inc., Glenwood, III., Development of a Standard Test for Measuring $K_{/ a}$ with a Modified Compact Specimen, NUREG/CR-2294 (ORNL/SUB-81/7755/1), August 1981.

H. A. Domian, Babcock and Wilcox Company, Alliance, Ohio, Vessel V-8 Repair and Preparation 
of Low Upper-Shelf Weldment, NUREG/CR-2676 (ORNL/Sub/81-85813/1), June 1982.

R. D. Cheverton, S. K. Iskander, and D. G. Ball, Union Carbide Corp. Nuclear Div., Oak Ridge Natl. Lab., Oak Ridge, Tenn., PWR Pressure Vessel Integrity During Overcooling Accidents: $A$ Parametric Analysis, NUREG/CR-2895 (ORNL/TM-7931), February 1983.

J. G. Merkle, Union Carbide Corp. Nuclear Div., Oak Ridge Natl. Lab., Oak Ridge, Tenn., An Examination of the Size Effects and Data Scatter Observed in Small Specimen Cleavage Fracture Toughness Testing, NUREG/CR-3672 (ORNL/TM-9088), April 1984.

W. R. Corwin, Martin Marietta Energy Systems, Inc., Oak Ridge Natl. Lab., Oak Ridge, Tenn., Assessment of Radiation Effects Relating to Reactor Pressure Vessel Cladding, NUREG/CR-3671 (ORNL-6047), July 1984.

W. R. Conwin, R. G. Berggren, and R. K. Nanstad, Martin Marietta Energy Systems, Inc., Oak Ridge Natl. Lab., Oak Ridge, Tenn., Charpy Toughness and Tensile Properties of a Neutron Irradiated Stainless Steel Submerged-Arc Weld Cladding Overlay, NUREG/CR-3927 (ORNL/TM-9709), September 1984.

J. J. McGowan, Martin Marietta Energy Systems, Inc., Oak Ridge Natl. Lab., Oak Ridge, Tenn., Tensile Properties of Irradiated Nuclear Grade Pressure Vessel Plate and Welds for the Fourth HSST Irradiation Series, NUREG/CR-3978 (ORNL/TM-9516), January 1985.

J. J. McGowan, Martin Marietta Energy Systems, Inc., Oak Ridge Natl. Lab., Oak Ridge, Tenn., Tensile Properties of Irradiated Nuclear Grade Pressure Vessel Welds for the Third HSST Irradiation Series, NUREG/CR-4086 (ORNL/TM-9477), March 1985.

W. R. Corwin, G. C. Robinson, R. K. Nanstad, J. G. Merkle, R. G. Berggren, G. M. Goodwin, R. L. Swain, and T. D. Owings, Martin Marietta Energy Systems, Inc., Oak Ridge Natl. Lab.,
Oak Ridge, Tenn., Effects of Stainless Steel Weld Overlay Cladding on the Structural Integrity of Flawed Steel Plates in Bending, Series 1, NUREG/CR-4015 (ORNL/TM-9390), April 1985.

W. J. Stelzman, R. G. Berggren, and T. N. Jones, Martin Marietta Energy Systems, Inc., Oak Ridge Natl. Lab., Oak Ridge, Tenn., ORNL Characterization of Heavy-Section Steel Technology Program Plates 01, 02, and 03, NUREG/CR-4092 (ORNL/TM-9491), April 1985.

G. D. Whitman, Martin Marietta Energy Systems, Inc., Oak Ridge Natl. Lab., Oak Ridge, Tenn., Historical Summary of the Heavy-Section Steel Technology Program and Some Related Activities in Light-Water Reactor Pressure Vessel Safety Research, NUREG/CR-4489 (ORNL-6259), March 1986.

R. H. Bryan, B. R. Bass, S. E. Bolt, J. W. Bryson, J. G. Merkle, R. K. Nanstad, and G. C. Robinson, Martin Marietta Energy Systems, Inc., Oak Ridge Natl. Lab., Oak Ridge, Tenn., Test of 6-in.-Thick Pressure Vesse/s. Series 3: Intermediate Test Vessel V-8A - Tearing Behavior of Low Upper-Shelf Material, NUREG-CR-4760 (ORNL-6187), May 1987.

D. B. Barker, R. Chona, W. L. Fourney, and G. R. Irwin, University of Maryland, College Park, Md., A Report on the Round Robin Program Conducted to Evaluate the Proposed ASTM Standard Test Method for Determining the Plane Strain Crack Arrest Fracture Toughness, $K_{\text {la }}$, of Ferritic Materials, NUREG/CR-4966 (ORNL/Sub/79-7778/4), January 1988.

L. F. Miller, C. A. Baldwin, F. W. Stallman, and F. B. K. Kam, Martin Marietta Energy Systems, Inc., Oak Ridge Natl. Lab., Oak Ridge, Tenn., Neutron Exposure Parameters for the Metallurgical Test Specimens in the Fifth Heavy-Section Steel Technology Irradiation Series Capsules, NUREG/CR-5019 (ORNL/TM-10582), March 1988.

J. J. McGowan, R. K. Nanstad, and K. R. Thoms, Martin Marietta Energy Systems, Inc., Oak Ridge Natl. Lab., Oak Ridge, Tenn., Characterization of Irradiated Current-Practice Welds and A533 
Grade B Class 1 Plate for Nuclear Pressure Vessel Service, NUREG/CR-4880 (ORNL-6484/N1 and V2), July 1988.

R. D. Cheverton, W. E. Pennell, G. C. Robinson, and R. K. Nanstad, Martin Marietta Energy Systems, Oak Ridge Natl. Lab., Oak Ridge, Tenn., Impact of Radiation Embrittlement on Integrity of Pressure Vessel Supports for Two PWR Plants, NUREG/CR-5320 (ORNL/TM-10966), February 1989.
J. G. Merkle, Martin Marietta Energy Systems, Inc., Oak Ridge Natl. Lab., Oak Ridge, Tenn., An Overview of the Low-Upper-Shelf Toughness Safety Margin Issue, NUREG/CR-5552 (ORNL/TM-11314), August 1990.

R. D. Cheverton, T. L. Dickson, J. G. Merkle, and R. K. Nanstad, Martin Marietta Energy Systems, Inc., Oak Ridge Natl. Lab., Oak Ridge, Tenn., Review of Reactor Pressure Vessel Evaluation Report for Yankee Rowe Nuclear Power Station (YAEC No. 1735), NUREG/CR-5799 (ORNL/TM-11982), March 1992. 
Appendix E

Load-Displacement Curves and Photographs of the Fracture

Toughness Specimens From the $72 \mathrm{~W}$ Weld $(0.23 \mathrm{wt} \% \mathrm{Cu})$ 

$8 / 22 / 85$
$72 \omega-135$
$-150^{\circ} \mathrm{C}$
$I T-C T$
$0 \% 5 G$
$8 / 22 / 85$

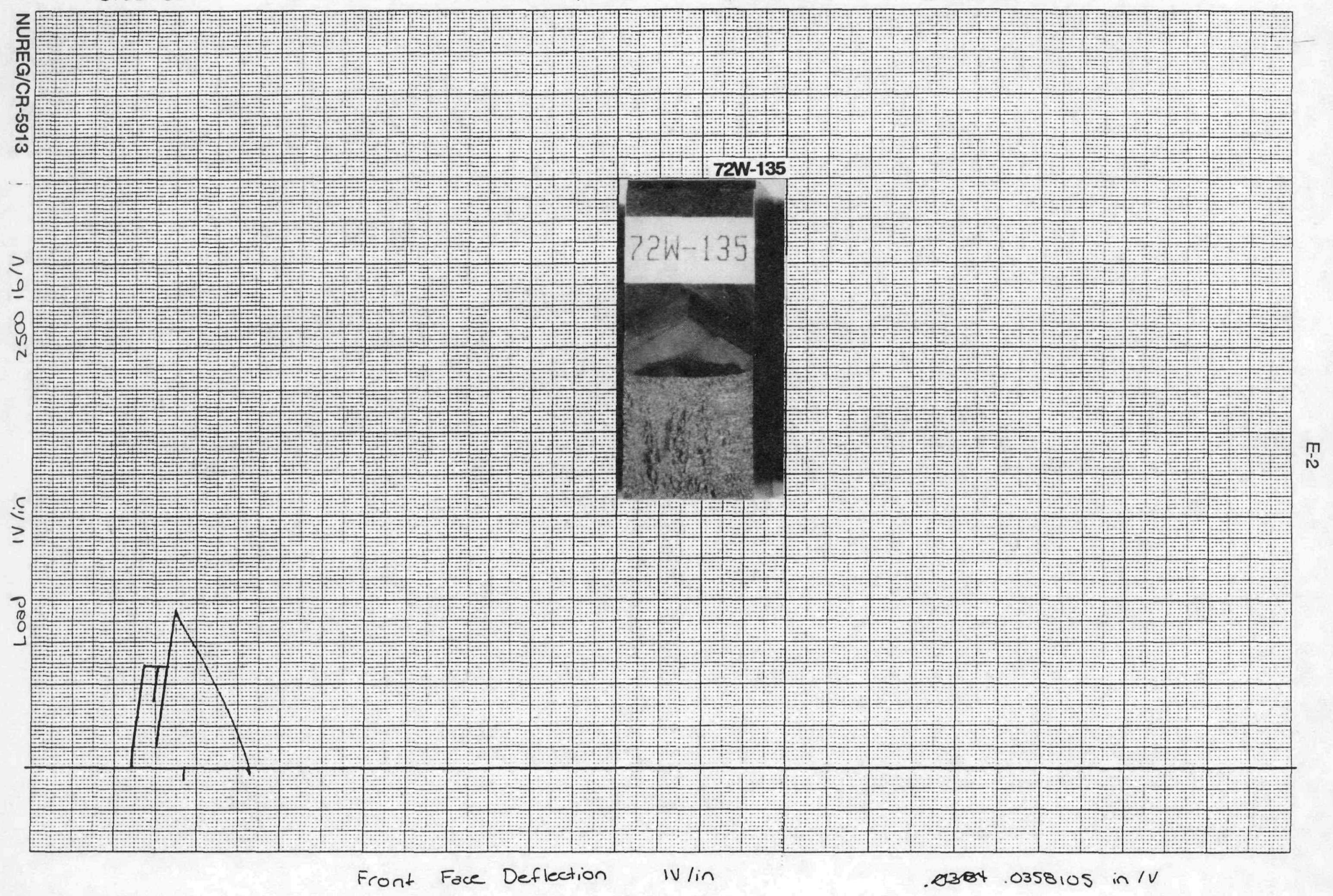




\section{$8 / 21 / 85 \mathrm{MRP} / \mathrm{MJL} \quad 72 \mathrm{~W}-138 \quad-150^{\circ} \mathrm{C} \quad 17-\mathrm{CT} \quad 0 \% 56$}

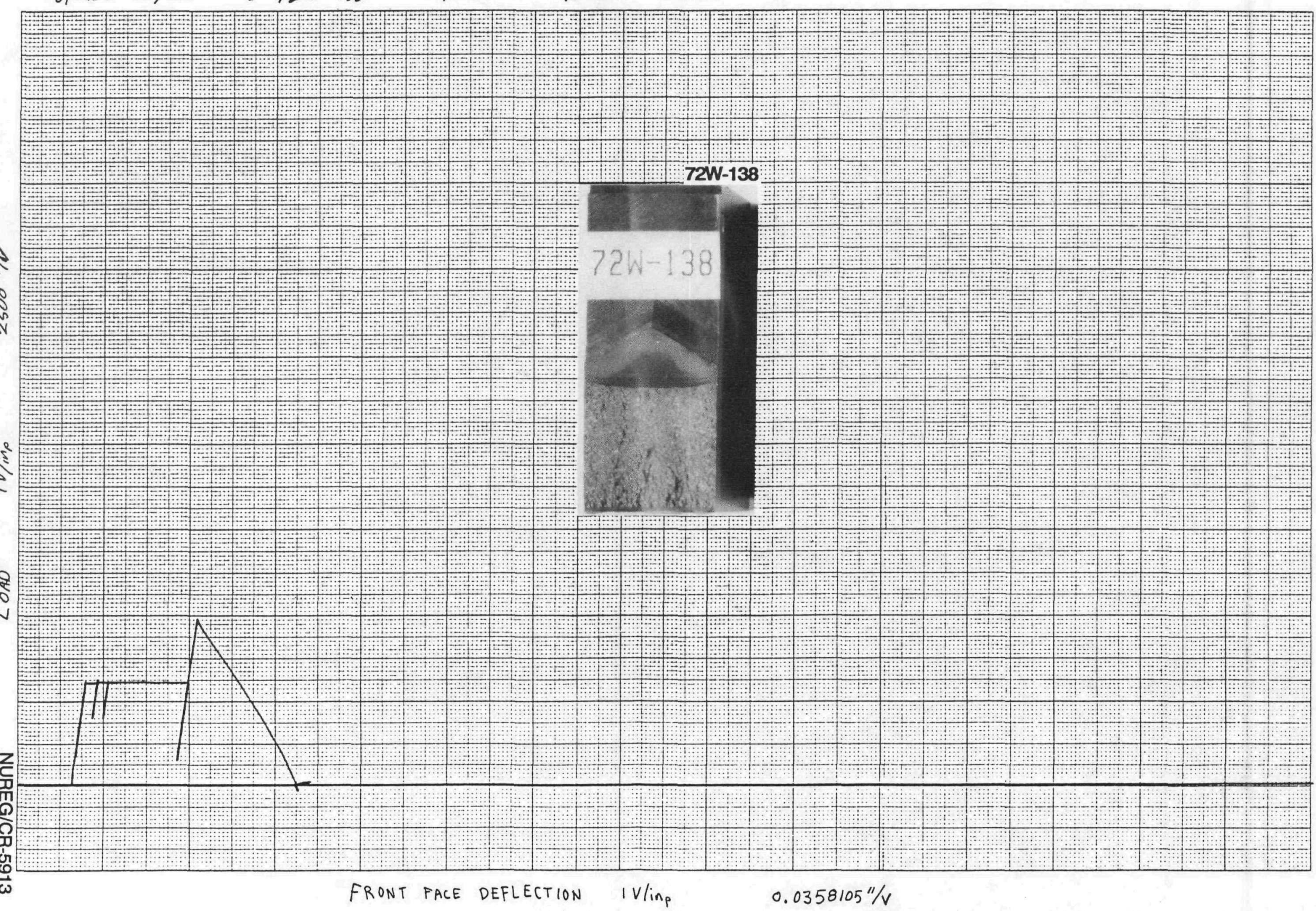


SPECIMEN ID $=72 W 113$

MATERIAL TYPE = MCUWELD

TEST TEMPERATURE $=-150$ DEG C

THICKNESS $=25.4 \mathrm{~mm}$

SIDE GROOUES $=0 \%$

INITIAL CRACK LENGTH $=26.9 \mathrm{~mm}$

$a / W) i=.529$

MEASURED DUETILE DELTA-A $=.05 \mathrm{~mm}$

$Y$ IELD STRENGTH $=733 \mathrm{MPa}$

FLOW STRENGTH $=775 \mathrm{MPa}$

ESTIMATED YOUNG'S MODULUS $=216 \mathrm{GPa}$

SPECIMEN CLEAUED AT KIc $=41.7$ MPa SQR $[\mathrm{m}]$ (UALID ASTM E399)

UALUES AT MAXIMUM LOAD

-

J-INTEGRAL $=8 \mathrm{k} \cdot \mathrm{J} / \mathrm{m}^{\wedge} 2$

$K J c=41.6 \mathrm{MPa} S Q R[\mathrm{~m}]$

K-beta-c $=41.2 \mathrm{MPa} \operatorname{SQR}[\mathrm{m}]$

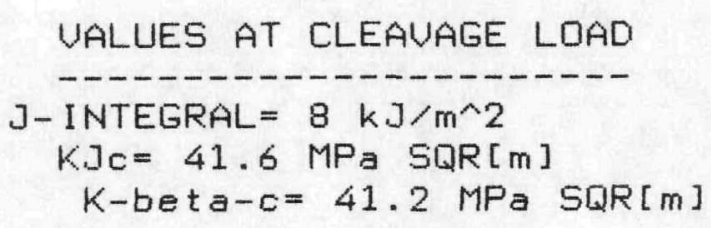


E-5

ORNL-DWG 92-11495

72W113-150 DEGREES C

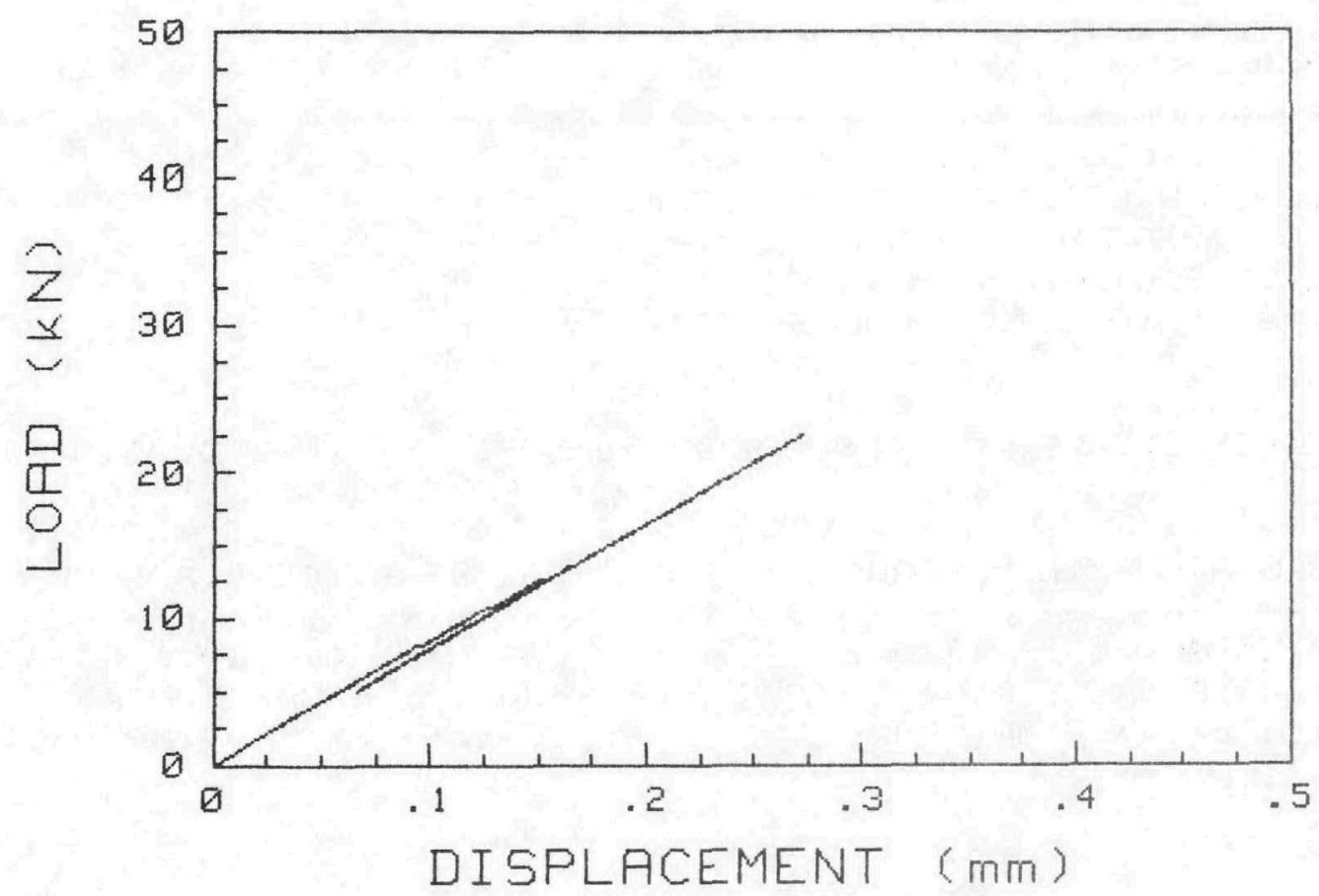

$72 W-113$

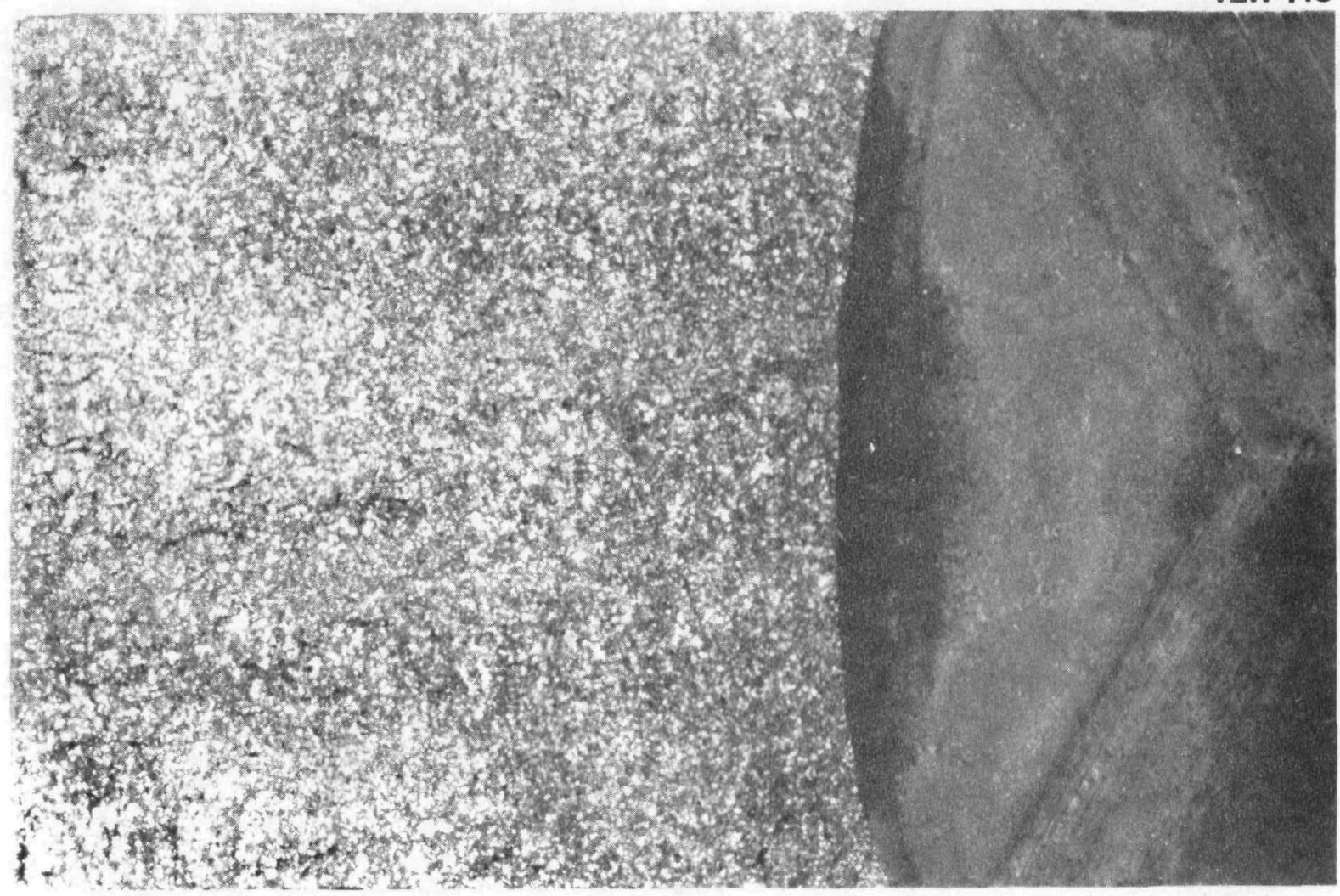

NUREG/CR-5913 


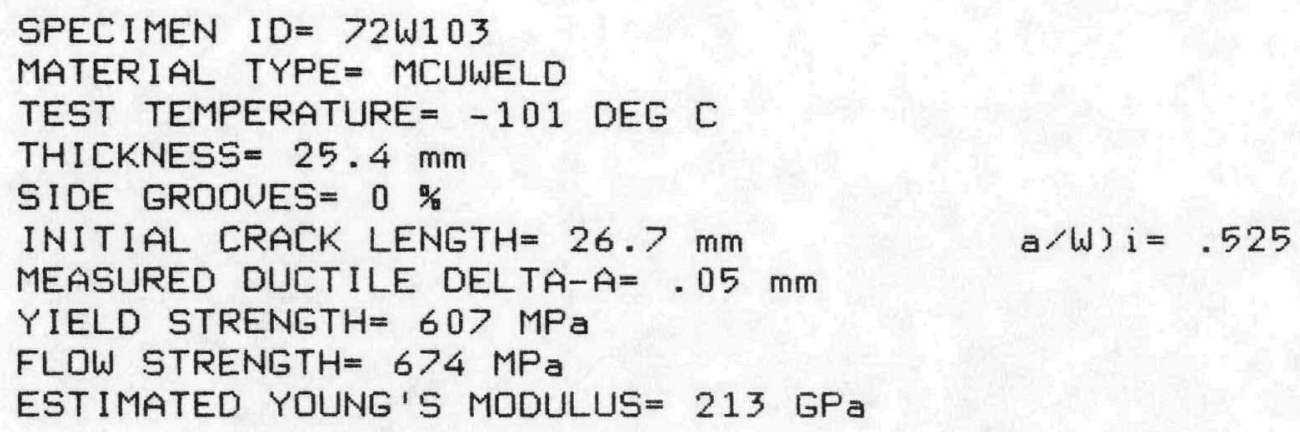

VALUES AT MAXIMUM LOAD

$J$-INTEGRAL $=10.3 \mathrm{~kJ} / \mathrm{m}^{\wedge} 2$

$K J c=46.8 \mathrm{MPa} S Q R[\mathrm{~m}]$

$K$-beta-c $=45.3 \mathrm{MPa} \operatorname{SQR}[\mathrm{m}]$
UALUES AT CLEAUAGE LOAD

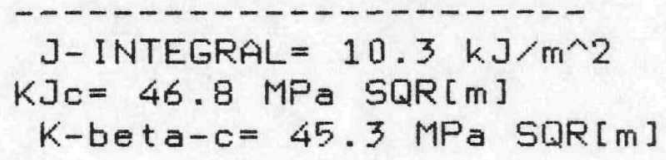


E-7

ORNL-DWG 92-11496

72W103-101 DEGREES C

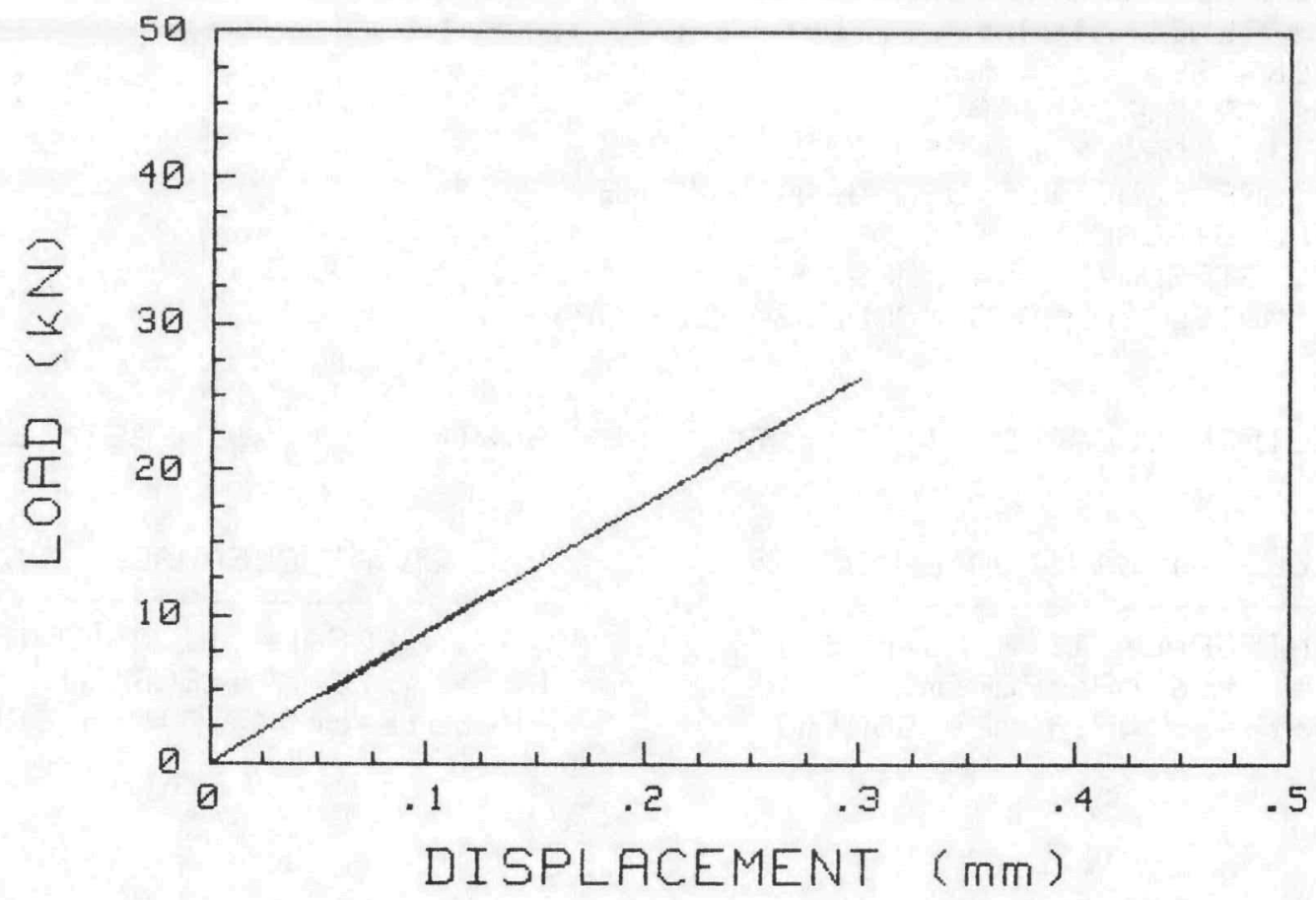

$72 W-103$

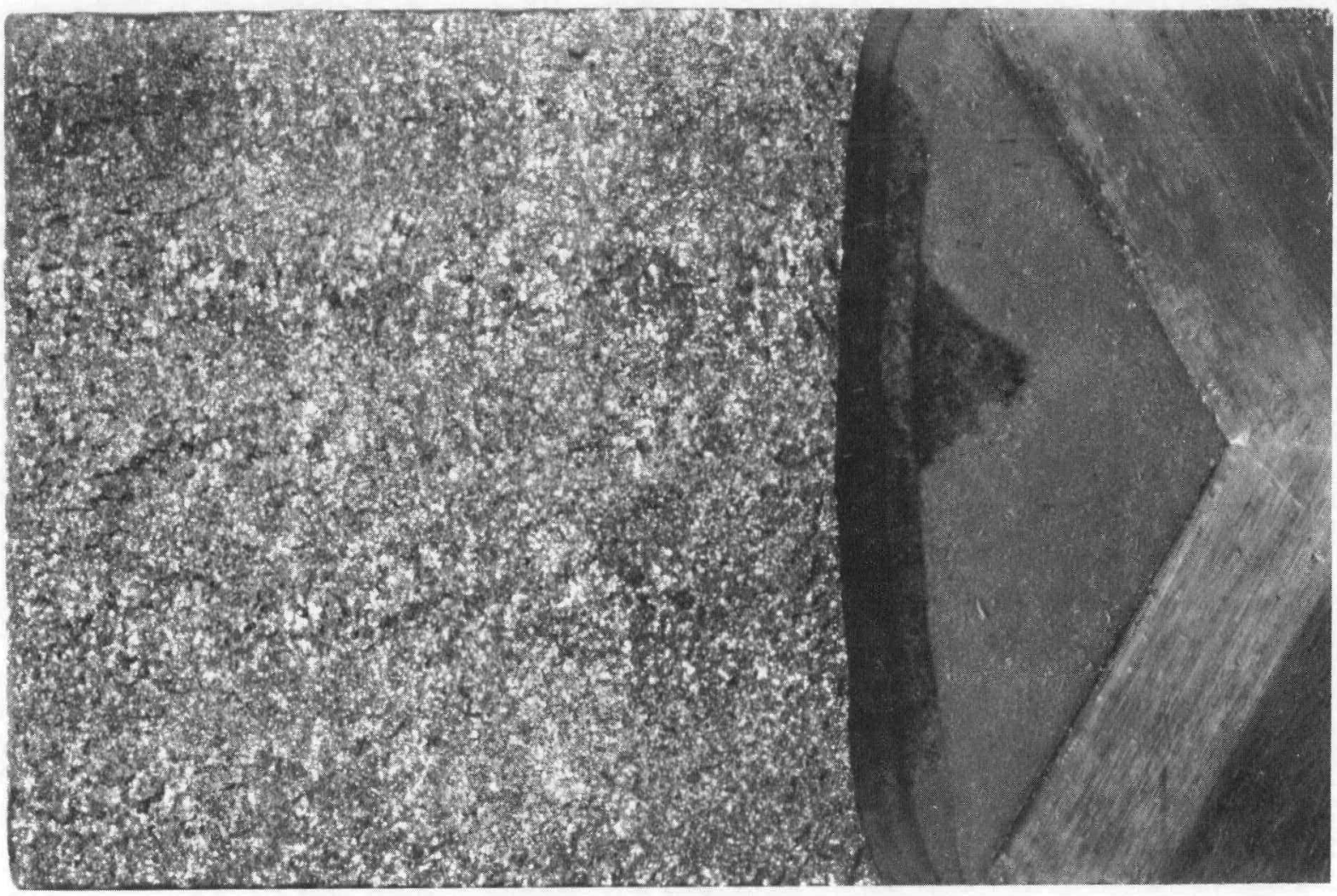

NUREG/CR-5913 
SPECIMEN ID $=72 W 168$

MATERIAL TYPE $=$ MCUWELD

TEST TEMPERATURE $=-80$ DEG C

THICKNESS $=25.4 \mathrm{~mm}$

SIDE GROOUES $=0 \%$

INITIAL CRACK LENGTH $=27.6 \mathrm{~mm}$

$a / W) i=.544$

MEASURED DUCTILE DELTA-A $=.05 \mathrm{~mm}$

YIELD STRENGTH $=576 \mathrm{MPa}$

FLOW STRENGTH $=645 \mathrm{MPa}$

EST IMATED YOUNG'S MODULUS $=212 \mathrm{GPa}$

SPECIMEN CLEAUED AT KIc=50.1 MPa SQR $[\mathrm{m}]$ (UALID ASTM E399)

UALUES AT MAXIMUM LOAD

J-INTEGRAL $=11.6 \mathrm{~kJ} / \mathrm{m}^{\wedge} 2$

$\mathrm{KJc}=49.6 \mathrm{MPa} \operatorname{SQR}[\mathrm{m}]$

$K$-beta-c $=47.3 \mathrm{MPa} S Q R[\mathrm{~m}]$
UALUES AT CLEAUAGE LDAD

J-INTEGRAL $=11.6 \mathrm{~kJ} / \mathrm{m}^{\wedge} 2$

$K J c=49.6 \mathrm{MPa} \operatorname{SQR}[\mathrm{m}]$

K-beta-c $=47.3 \mathrm{MPa} \operatorname{SQR}[\mathrm{m}]$ 
E-9

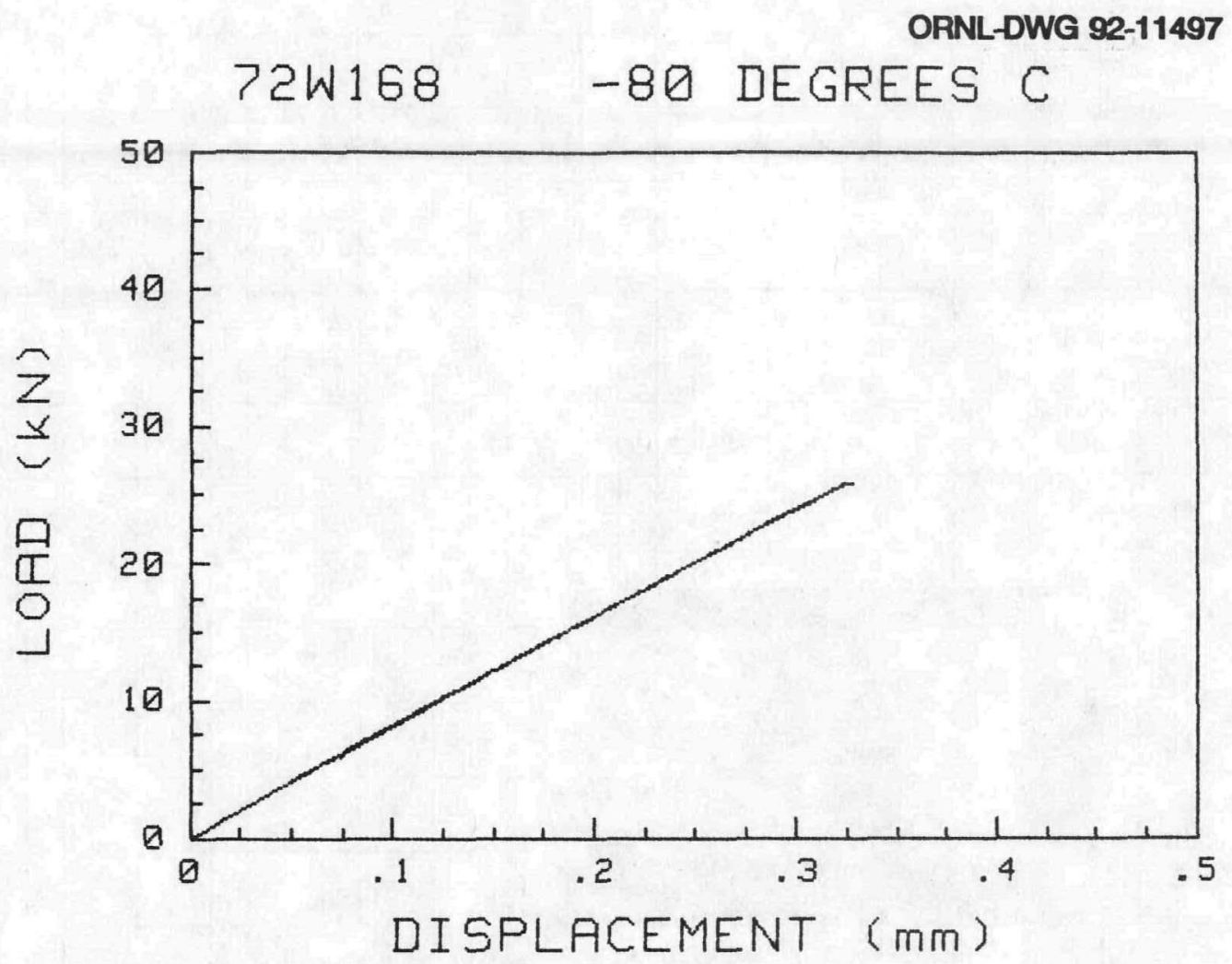

$72 W-168$

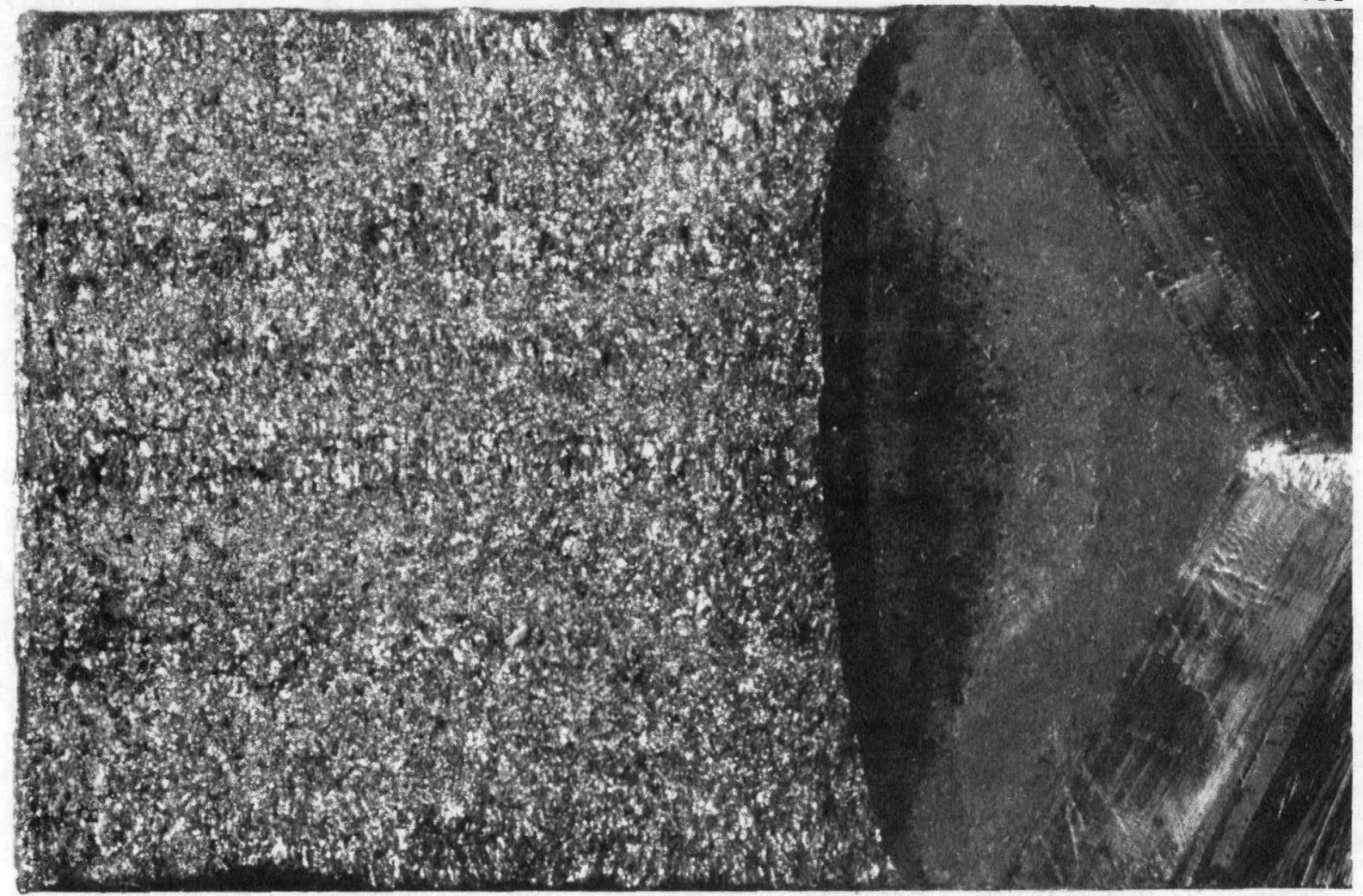

NUREG/CR-5913 
LAF $9 / 5 / 85$

$72 \omega-140$

IT-CT O\%S6 $-80^{\circ} \mathrm{C}$
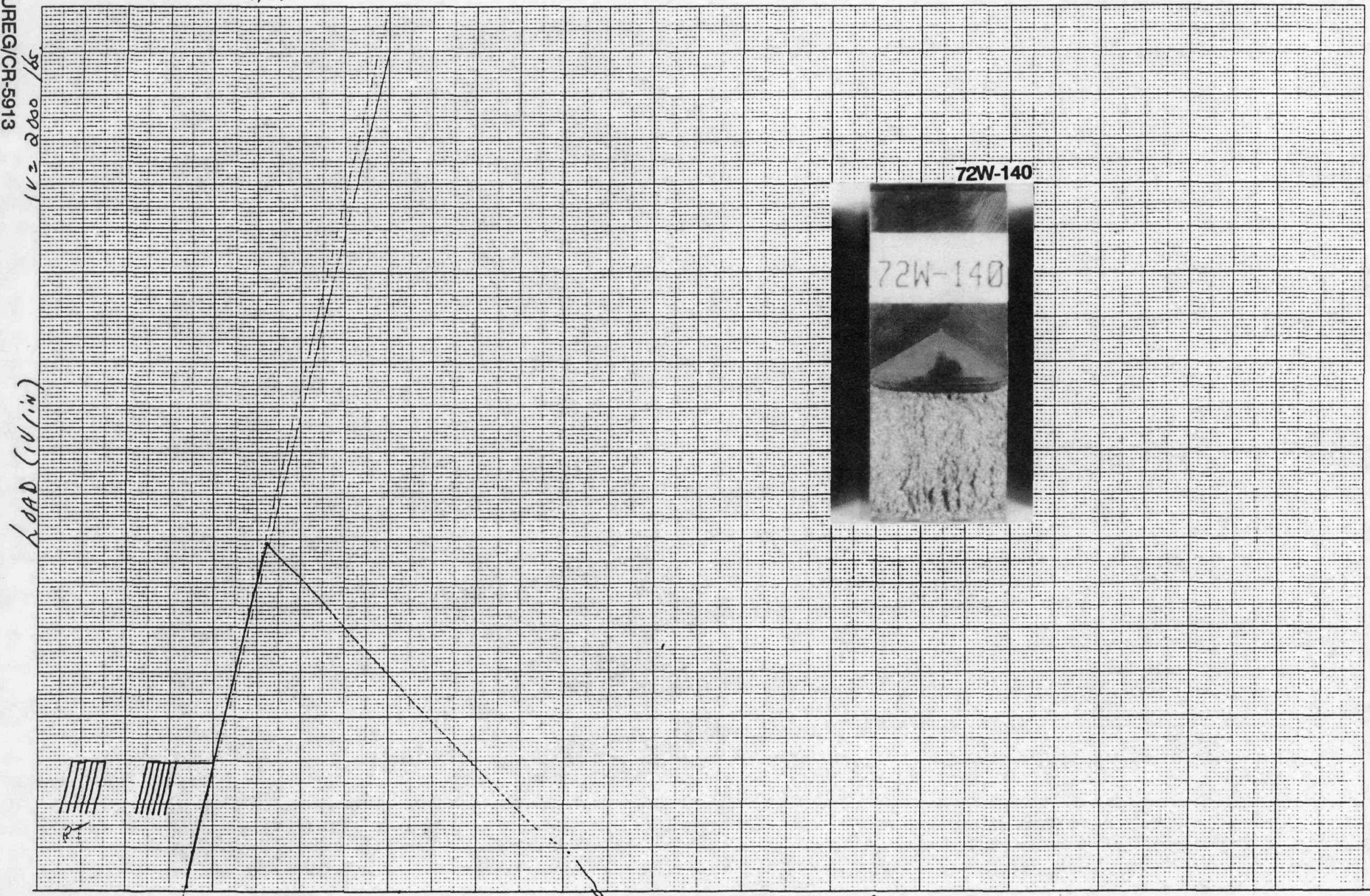
SPECIMEN ID $=72 W 164$

MATERIAL TYPE $=$ MCUWELD

TEST TEMPERATURE $=-80$ DEG C

THICKNESS $=25.4 \mathrm{~mm}$

SIDE GROOUES $=0 \%$

INITIAL CRACK LENGTH $=27.8 \mathrm{~mm}$

MEASURED DUCTILE DELTA-A $=.05 \mathrm{~mm}$

$a / W) i=.548$

YIELD STRENGTH $=576 \mathrm{MPa}$

FLOW STRENGTH $=645 \mathrm{MPa}$

ESTIMATED YOUNG'S MODULUS $=212 \mathrm{GPa}$

SPECIMEN CLEAUED AT KQ $=66 \mathrm{MPa}$ SQR [m] (NOT VALID KIc)

UALUES AT MAXIMUM LDAD

J-INTEGRAL $=21 \mathrm{~kJ} / \mathrm{m}^{\wedge} 2$

$K J c=66.7 \mathrm{MPa} \operatorname{SQR}[\mathrm{m}]$

$\mathrm{K}-$ beta-c $=59.7 \mathrm{MPa} \operatorname{SQR}[\mathrm{m}]$

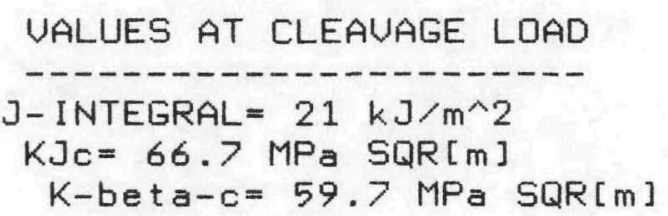


E-12

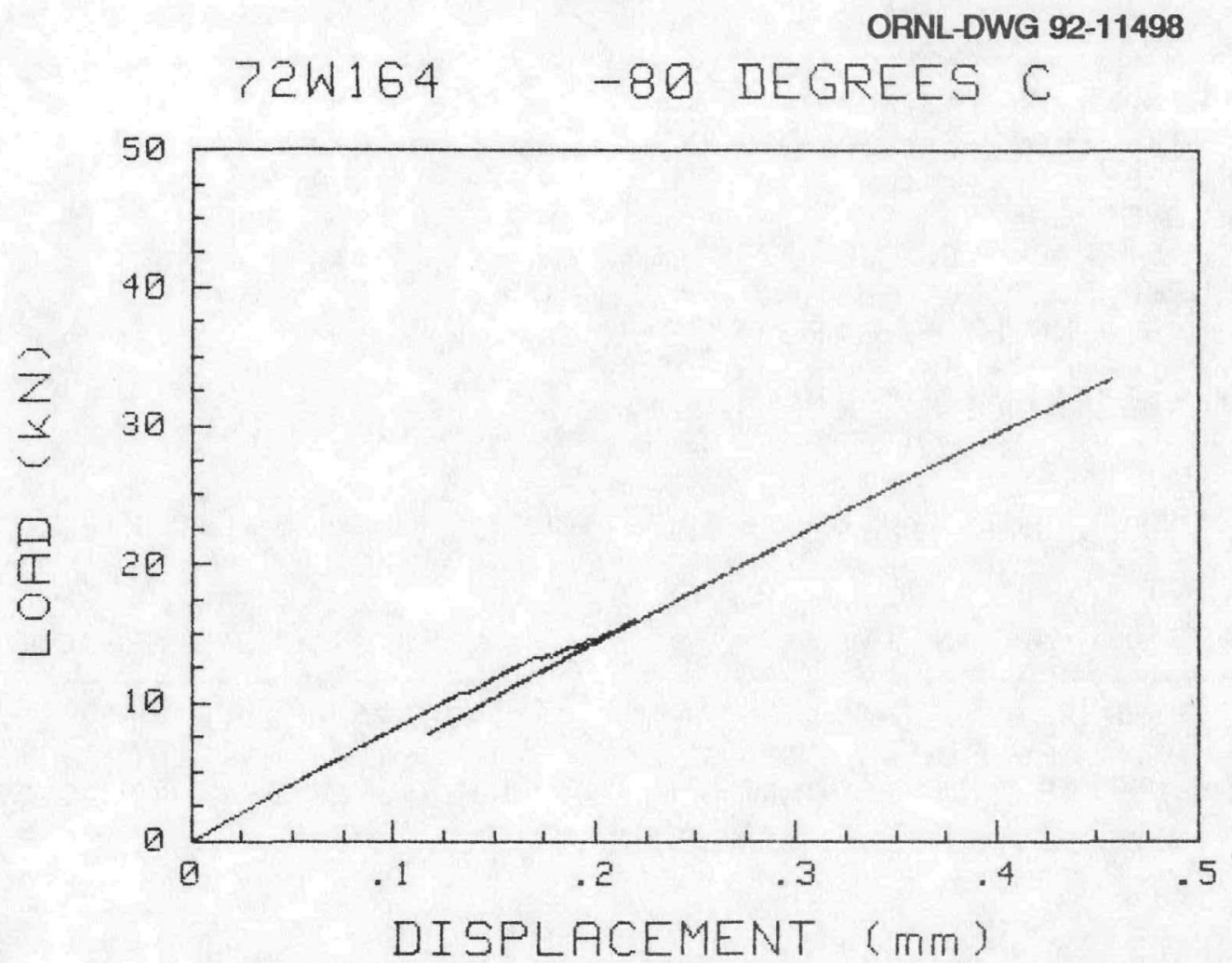

72W-164

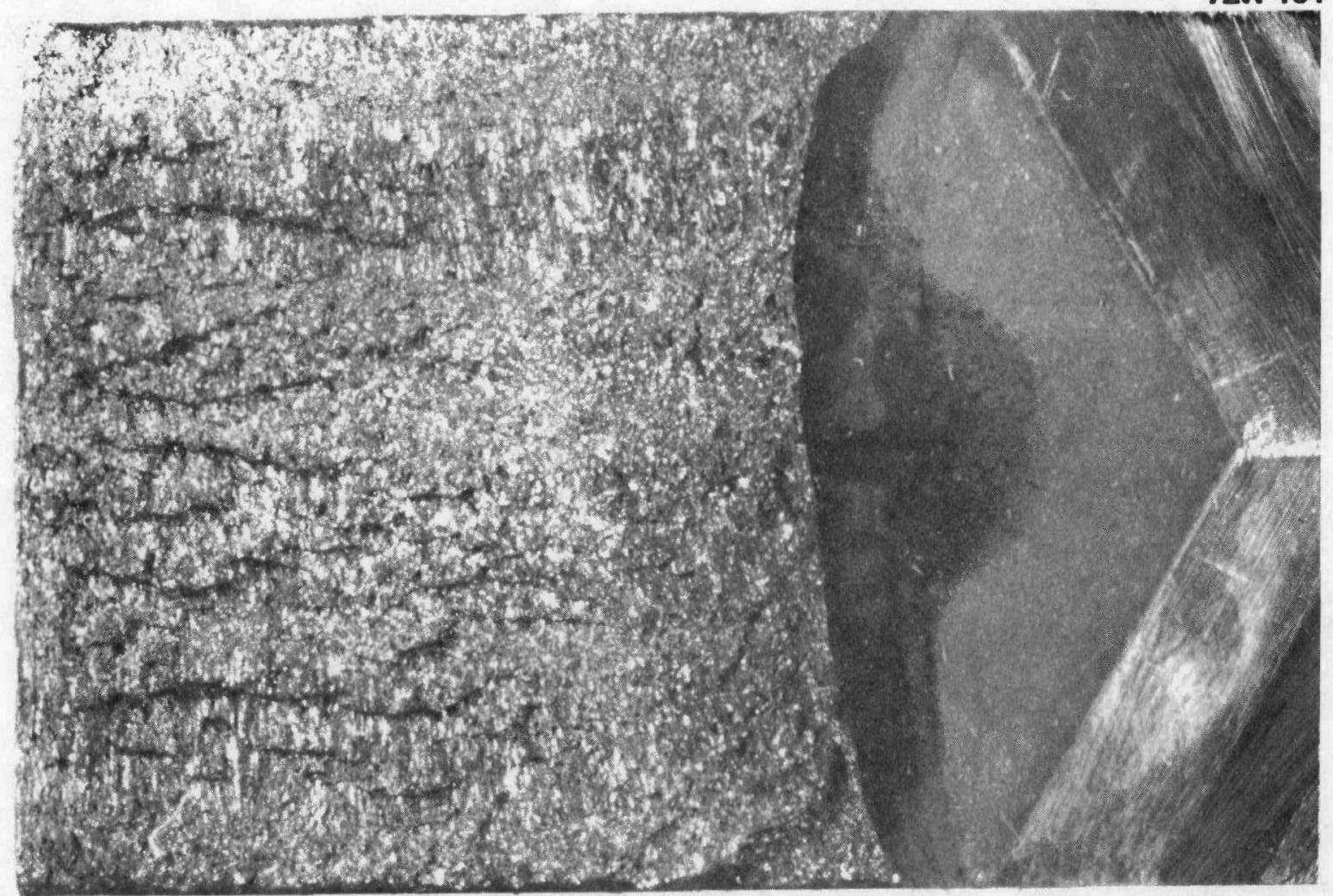


SPECIMEN ID $=72 W 121$

MATERIAL TYPE = MEUWELD

TEST TEMPERATURE $=-80$ DEG C

THICKNESS $=25.4 \mathrm{~mm}$

SIDE GRDOUES = $0 \%$

INITIAL CRACK LENGTH $=26.7 \mathrm{~mm}$

$a / w) i=.528$

MEASURED DUCTILE DELTA-A $=.05 \mathrm{~mm}$

$Y$ IELD STRENGTH $=576 \mathrm{MPa}$

FLOW STRENGTH $=645 \mathrm{MPa}$

ESTIMATED YOUNG'S MODULUS $=212$ GPa

SPECIMEN CLEAUED AT KQ= $88.9 \mathrm{MPa} S Q R[\mathrm{~m}]$ (NOT VALID KIC)

UALUES AT MAXIMUM LOAD

J-INTEGRAL $=40.5 \mathrm{~kJ} / \mathrm{m}^{\wedge} 2$

$K J c=92.6 \mathrm{MPa} S Q R[\mathrm{~m}]$

$K-b e t a-c=73.6 \mathrm{MPa} S Q R[\mathrm{~m}]$
WALUES AT CLEAUAGE LDAD

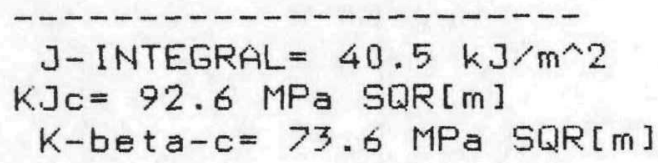




\section{$E-14$}
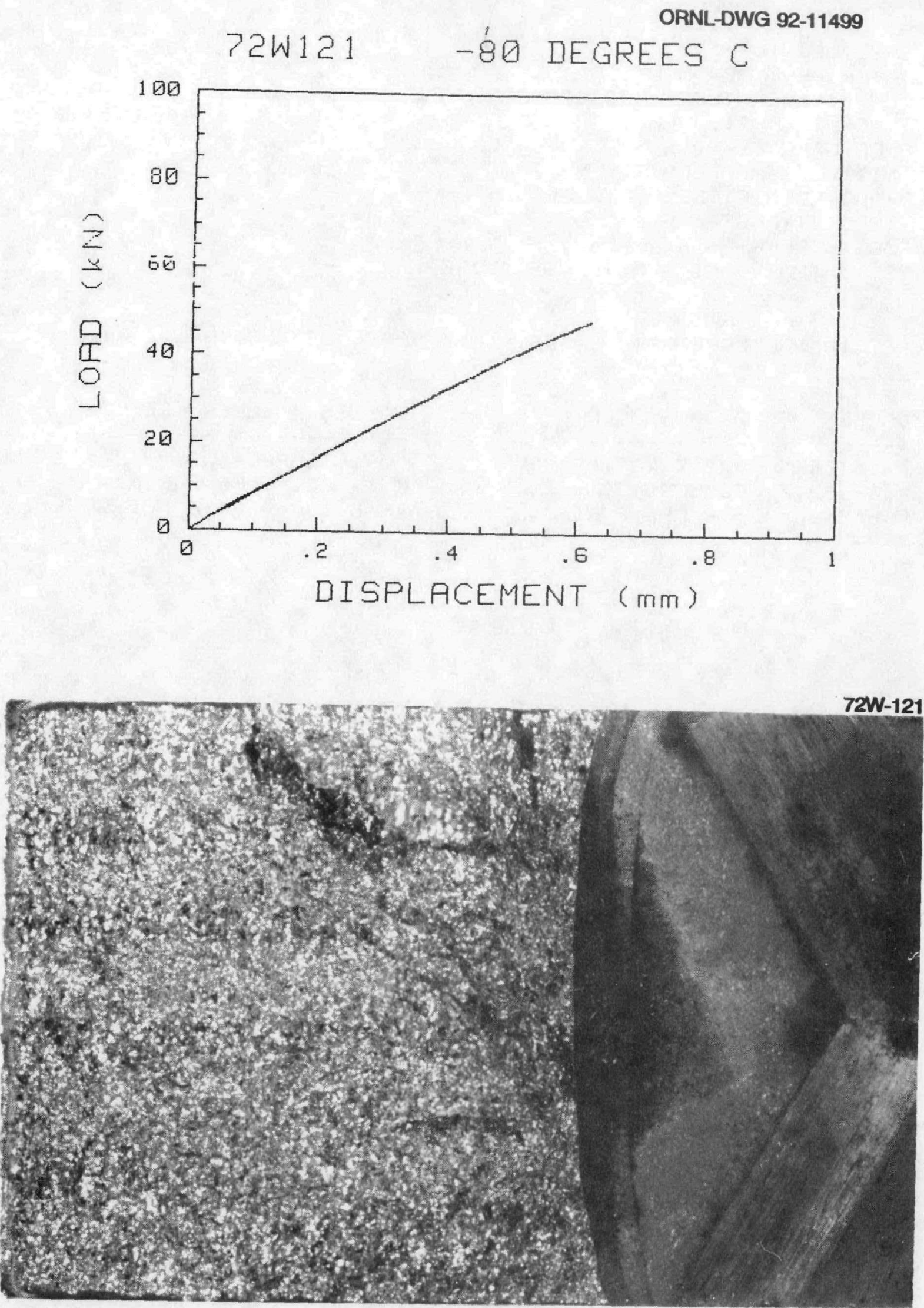
SPEC IMEN ID $=72 \mathrm{~W} 127$

MATERIAL TYPE = MCUWELD

TEST TEMPERATURE $=-80$ DEG C

THICKNESS $=25.4 \mathrm{~mm}$

SIDE GROOUES $=0 \%$

INITIAL CRACK LENGTH $=27 \mathrm{~mm}$

MEASURED DUCTILE DELTA-A $=.05 \mathrm{~mm}$

$a(w) i=.532$

$Y$ IELD STRENGTH $=576 \mathrm{MPa}$

FLOW STRENGTH $=645 \mathrm{MPa}$

ESTIMATED YOUNG'S MODULUS= 212 GPa

SPECIMEN CLEAUED AT KQ $=102.4 \mathrm{MPa} S Q R[\mathrm{~m}]$ (NDT UALID KIc)

UALUES AT MAXIMUM LOAD

J-INTEGRAL $=52 \mathrm{~kJ} / \mathrm{m}^{\wedge} 2$

$\mathrm{KJ} c=105 \mathrm{MPa}$ SQR $[\mathrm{m}]$

$K$-beta-c $=79 \mathrm{MPa} \operatorname{SQR}[\mathrm{m}]$
UALUES AT CLEAUAGE LDAD

J-INTEGRAL $=52 \mathrm{~kJ} / \mathrm{m}^{\wedge} 2$

$\mathrm{KJ} c=105 \mathrm{MPa} \operatorname{SQR}[\mathrm{m}]$

$K$-beta-c $=79 \mathrm{MPa} \operatorname{SQR}[\mathrm{m}]$ 
E-16

ORNL-DWG 92-11500

72W127 -80 DEGREES C

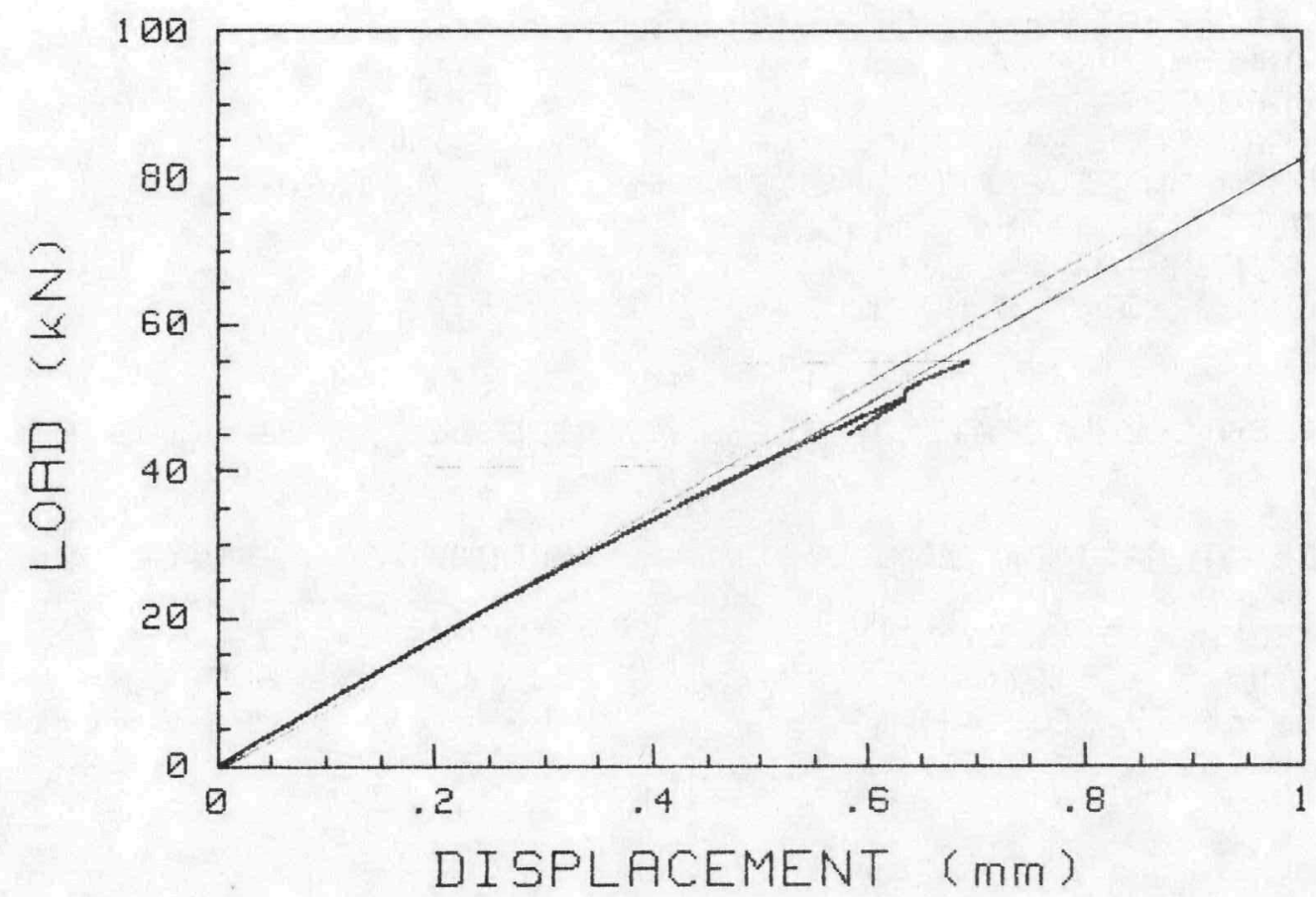

$72 W-127$

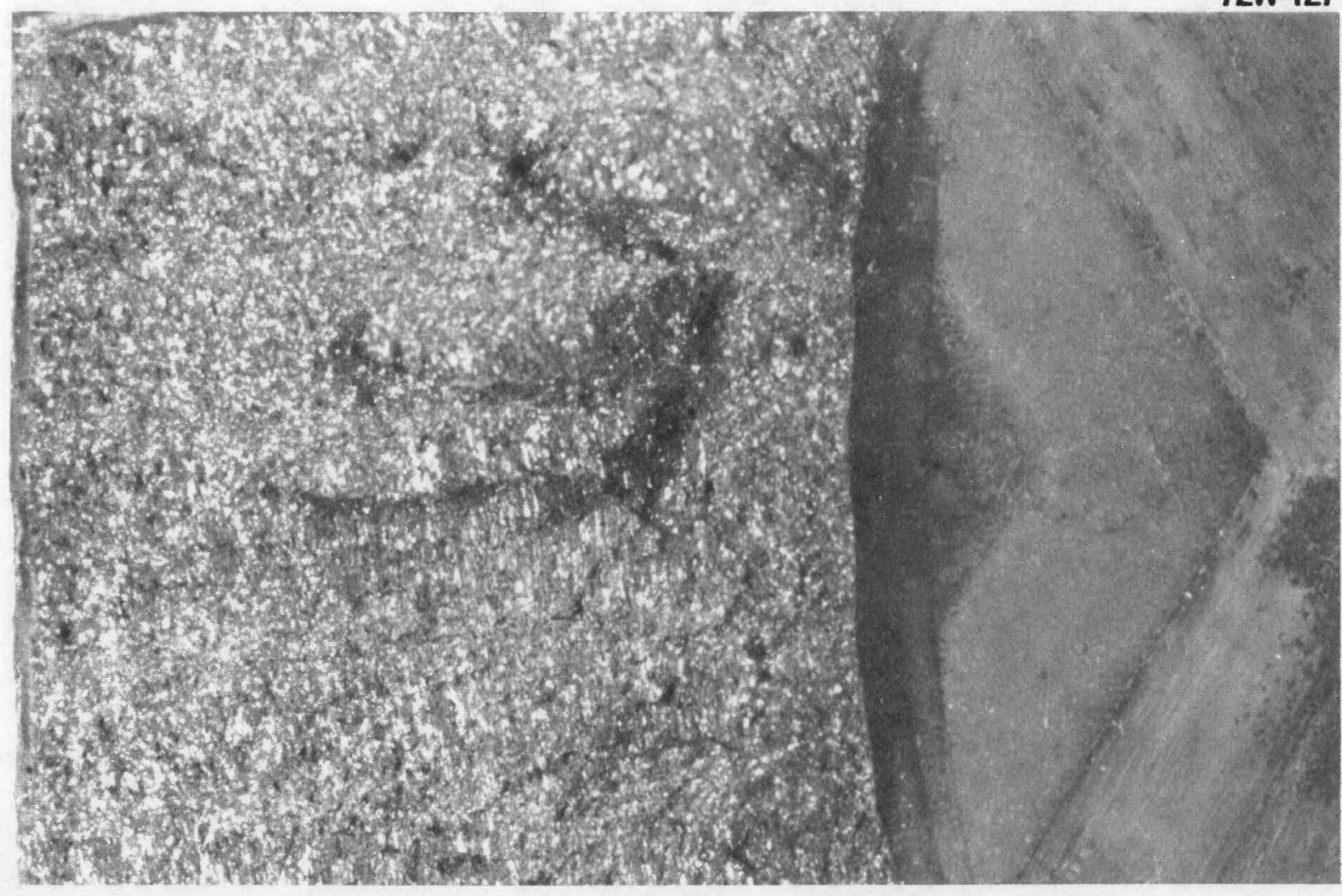




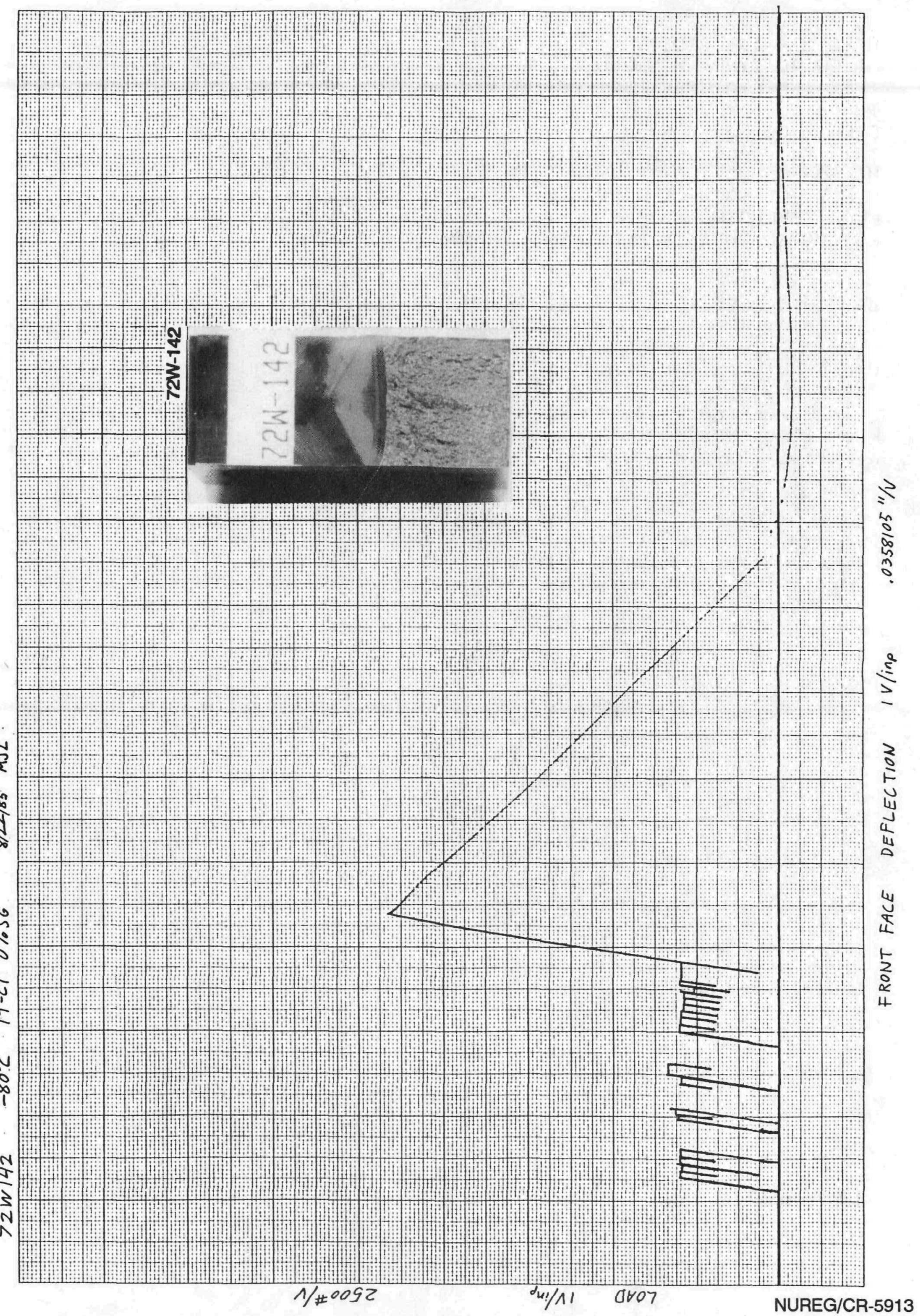


SPEC IMEN ID $=72 W 105$

MATERIAL TYPE = MCUWELD

TEST TEMPERATURE $=-59$ DEG $C$

THICKNESS $=25.4 \mathrm{~mm}$

SIDE GROOUES $=0 \%$

INITIAL CRACK LENGTH $=26.8 \mathrm{~mm}$

$a / W\rangle i=.528$

MEASURED DUCTILE DELTA-A $=.06 \mathrm{~mm}$

YIELD STRENGTH $=554 \mathrm{MPa}$

FLOW STRENGTH $=623 \mathrm{MPa}$

ESTIMATED YOUNG'S MODULUS $=211 \mathrm{GPa}$

SPECIMEN CLEAUED AT KQ= $108.6 \mathrm{MPa}$ SQR $[\mathrm{m}]$ (NOT UALID KIc)

UALUES AT MAXIMUM LOAD

J-INTEGRAL $=68.7 \mathrm{~kJ} / \mathrm{m}^{\wedge} 2$

$K \cdot] c=120.3 \mathrm{MPa} S Q R[\mathrm{~m}]$

$K$-bet $a-c=83.1 \mathrm{MPa} \operatorname{SQR}[\mathrm{m}]$

\author{
UALUES AT CLEAUAGE LOAD \\ J-INTEGRAL $=69.1 \mathrm{~kJ} / \mathrm{m}^{\wedge} 2$ \\ $\mathrm{KJc}=120.7 \mathrm{MPa} \operatorname{SBR}[\mathrm{m}]$ \\ $K$-beta-c $=83.2 \mathrm{MPa} \operatorname{SQR}[\mathrm{m}]$
}




\section{E-19}

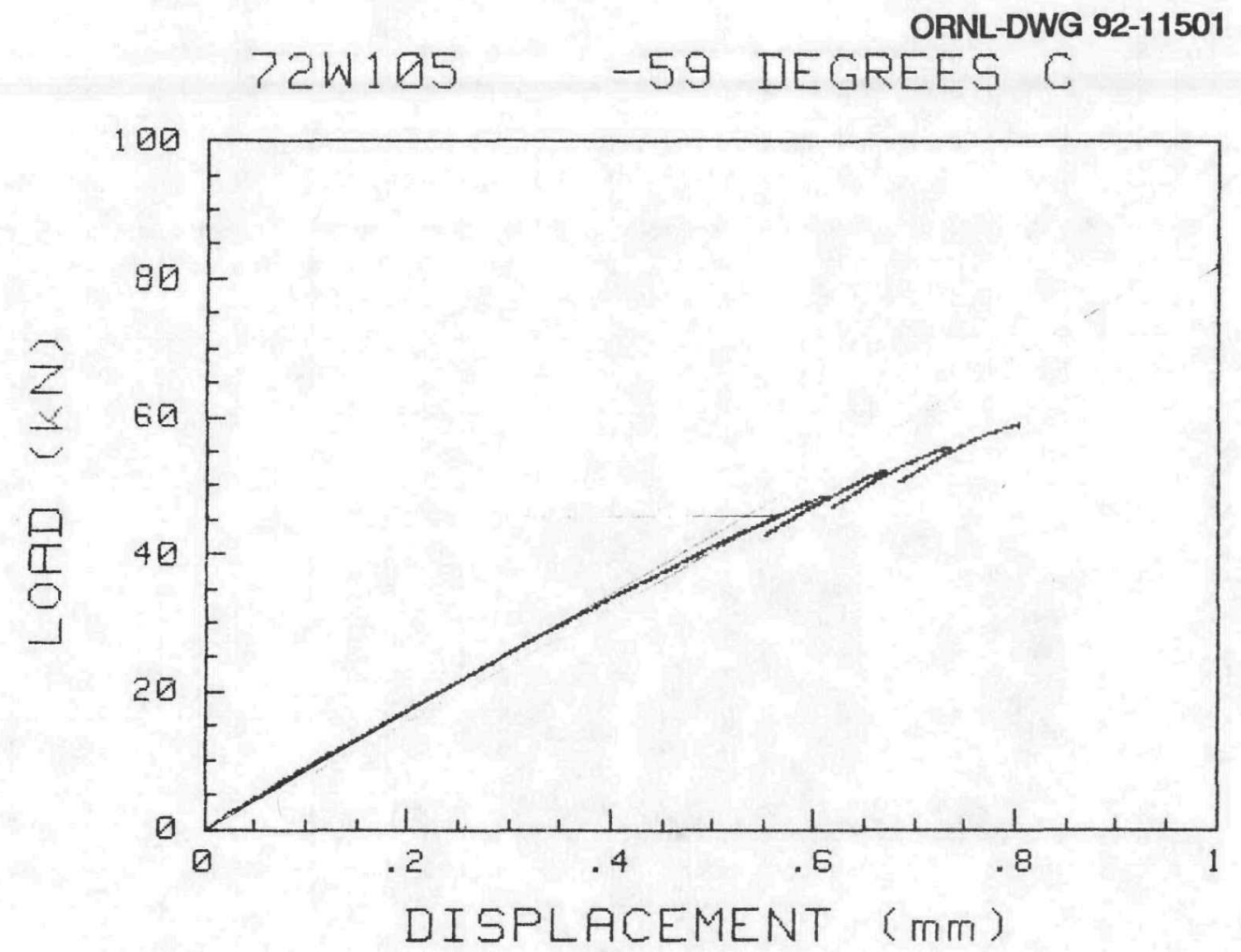

72W-105

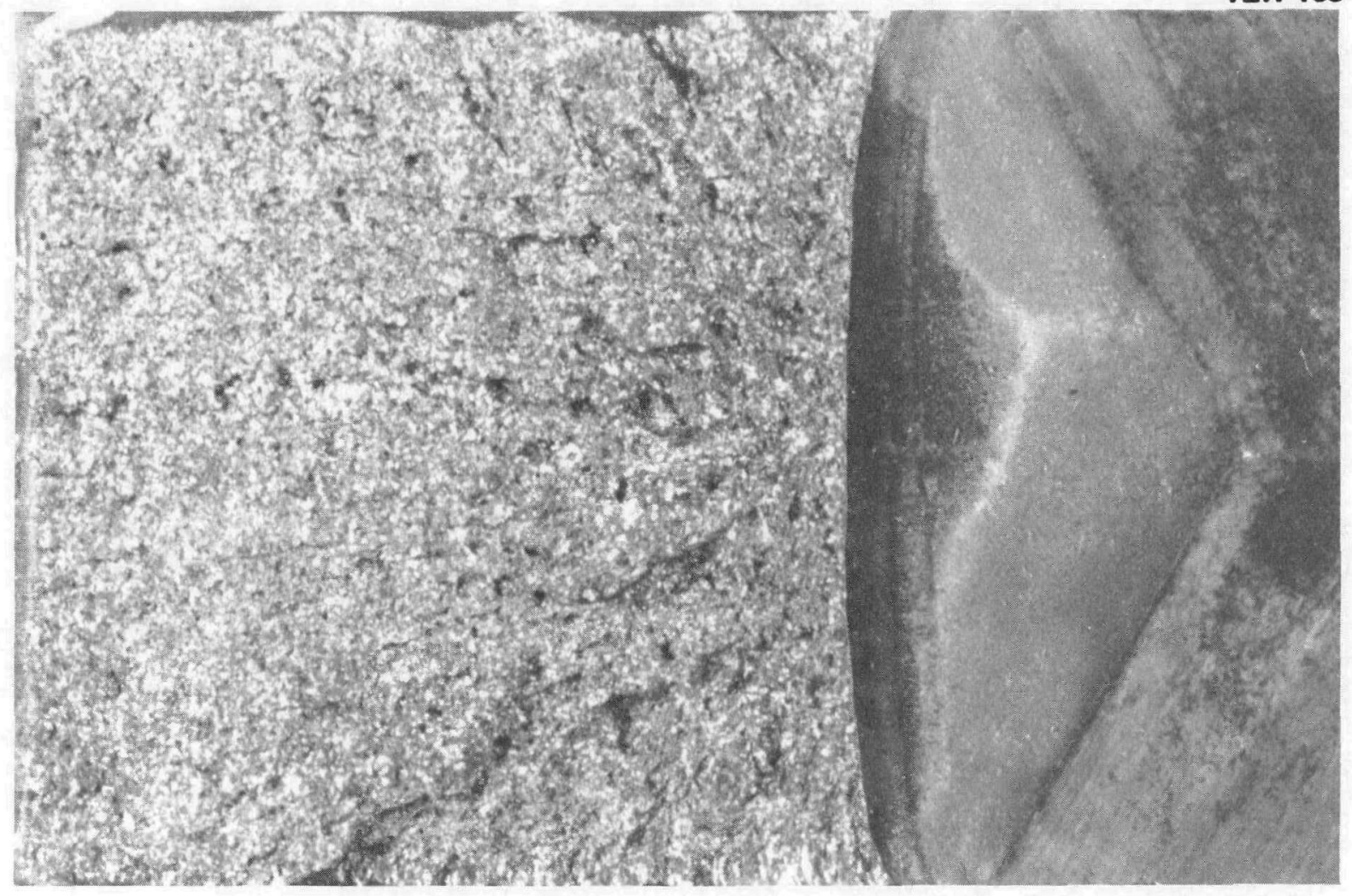




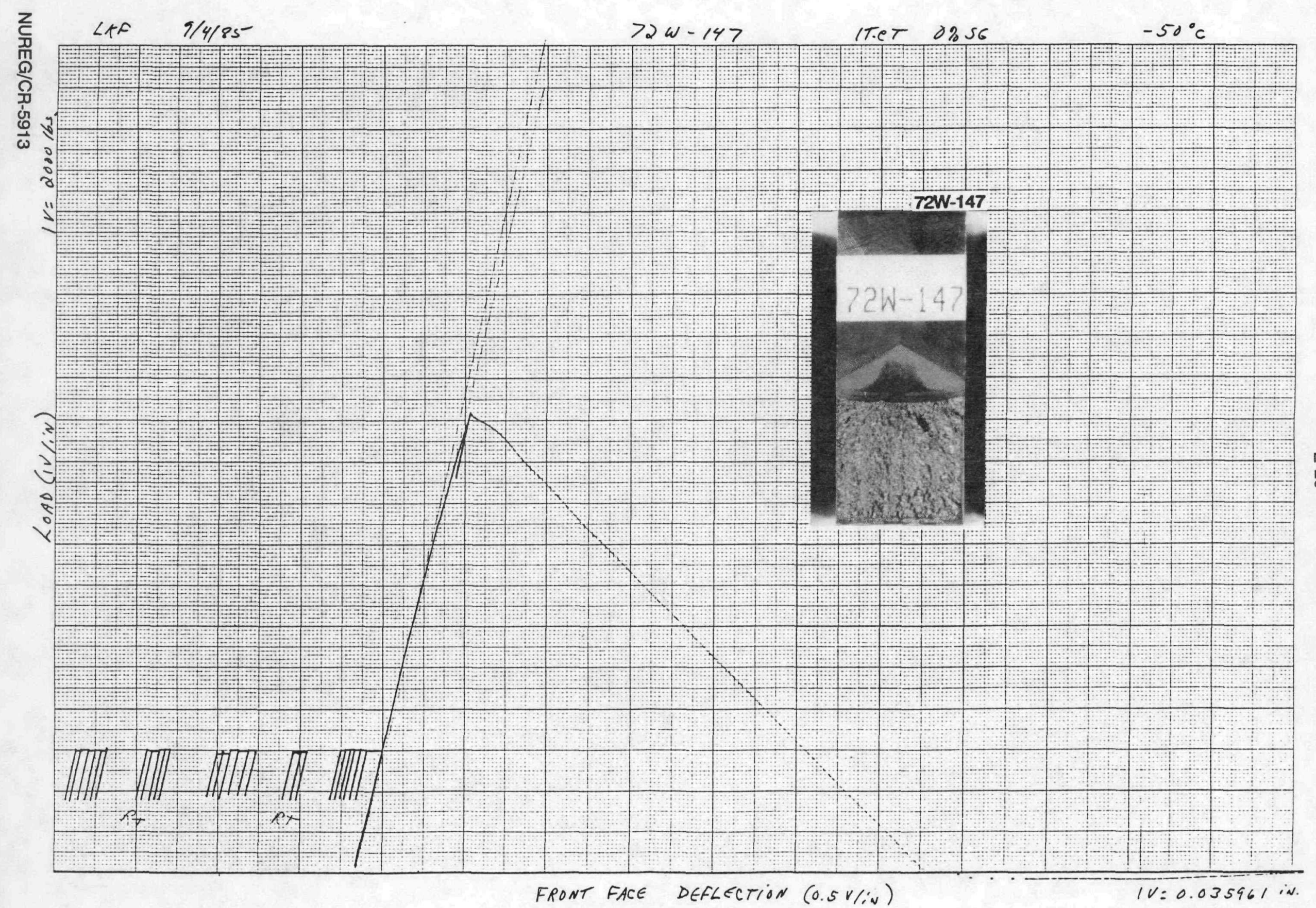




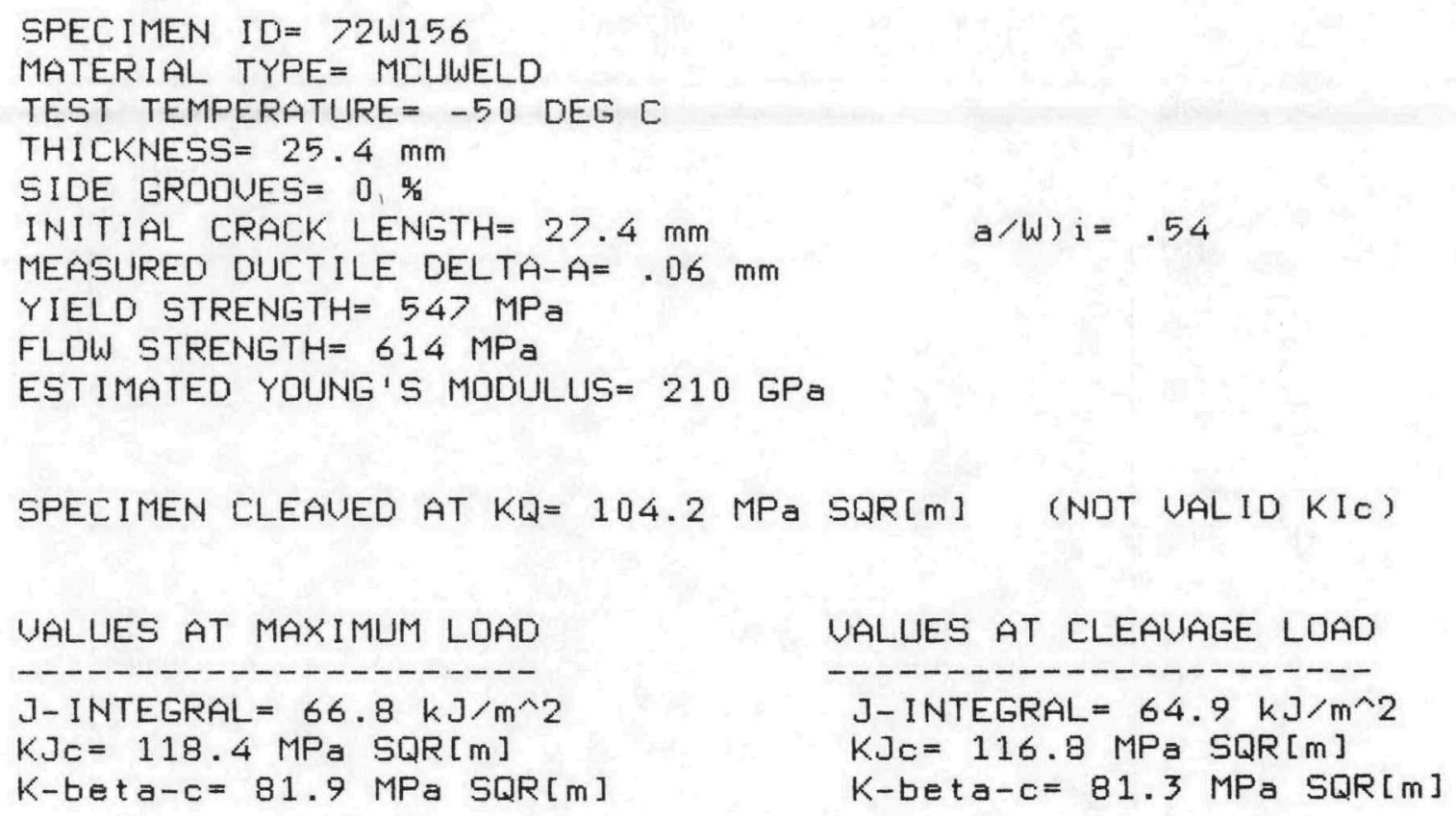




\section{E-22}

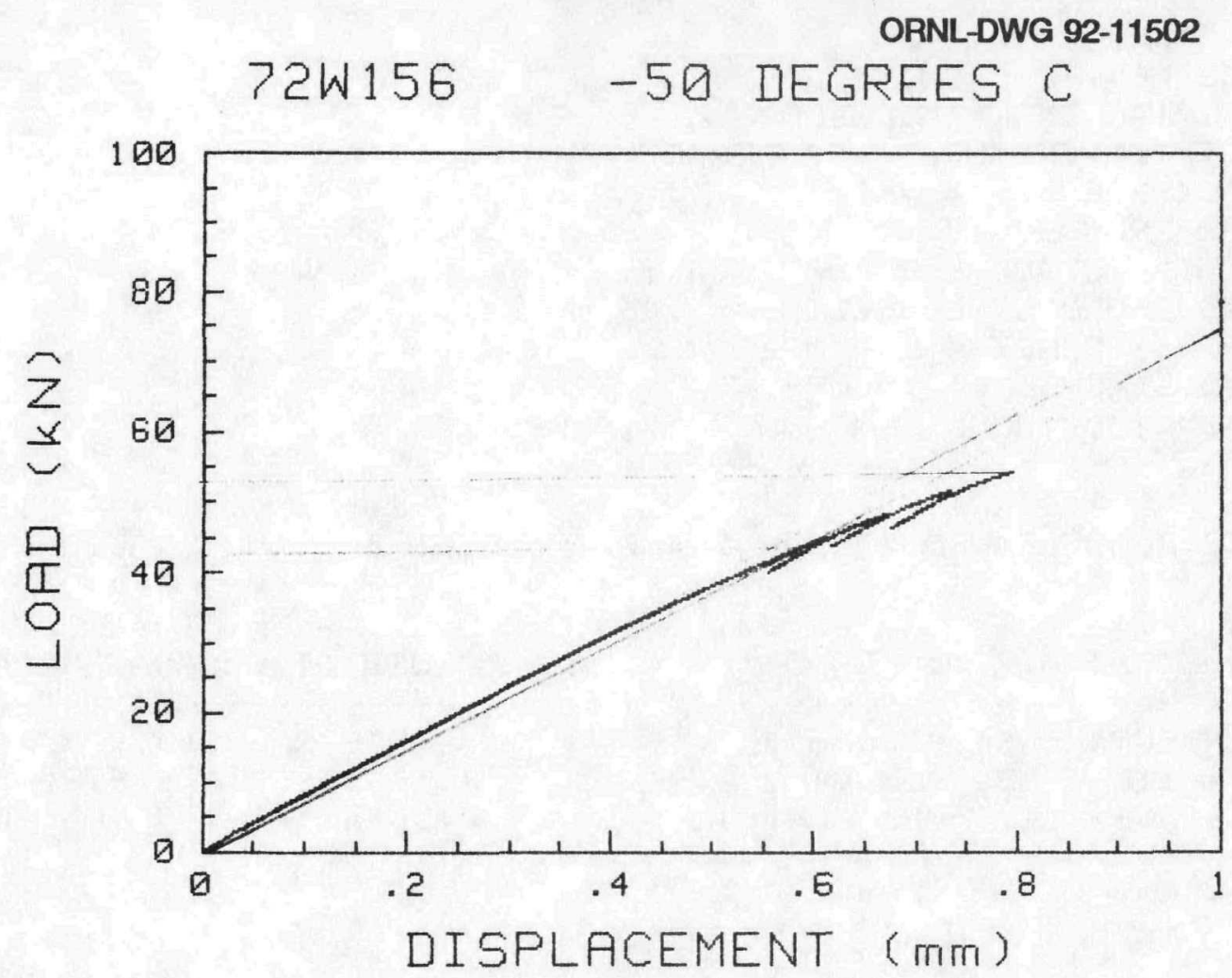

72W-156

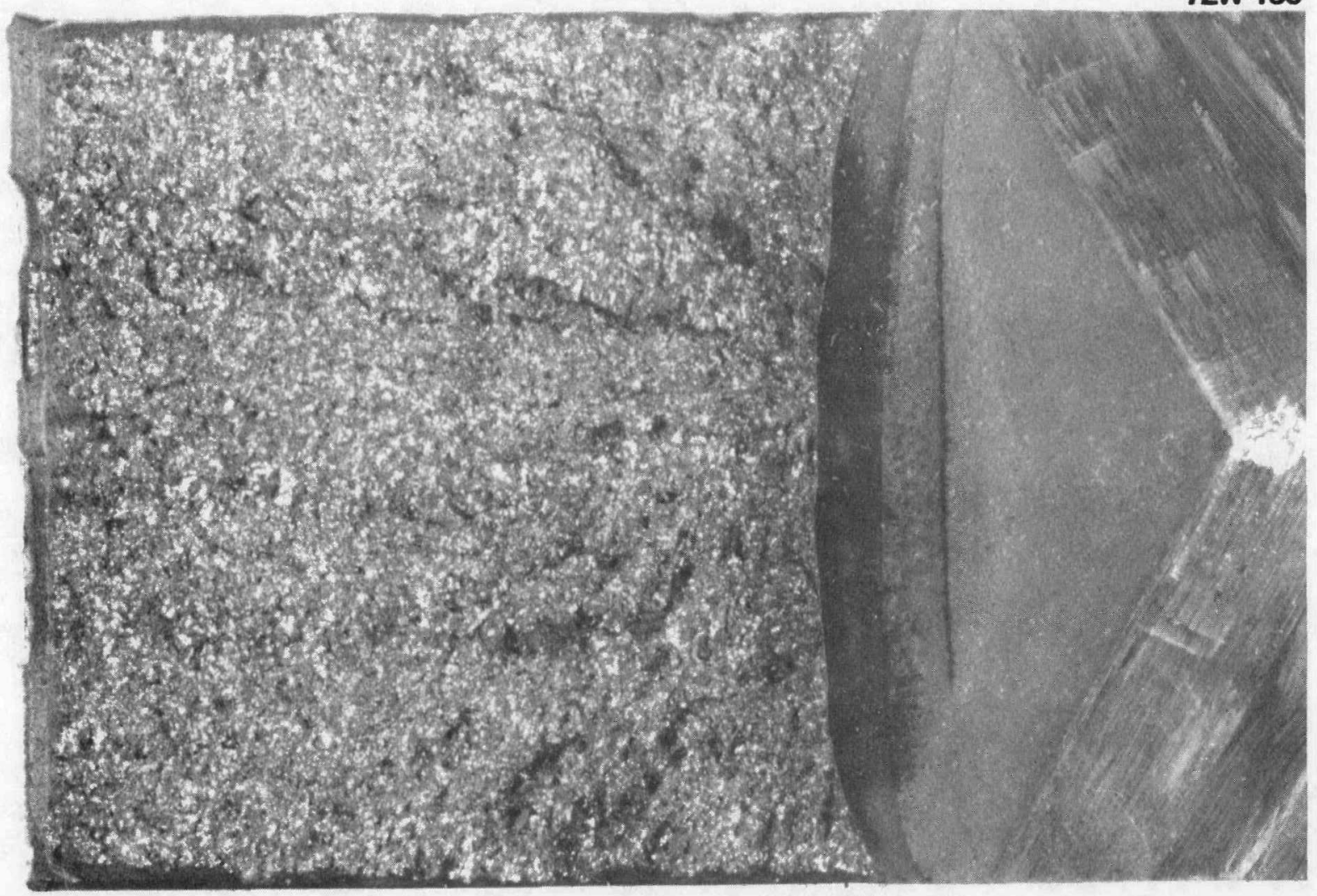

NUREG/CR-5913 
SPECIMEN ID $=72 W 154$

MATERIAL TYPE = MCUWELD

TEST TEMPERATURE $=-50$ DEG C

THILKNESS $=25.4 \mathrm{~mm}$

SIDE GROOUES $=0 \%$

INITIAL CRACK LENGTH $=26.7 \mathrm{~mm}$

$a / W) i=.526$

MEASURED DUCTILE DELTA-A $=.07 \mathrm{~mm}$

$Y$ IELD STRENGTH $=547 \mathrm{MPa}$

FLOW STRENGTH $=614 \mathrm{MPa}$

ESTIMATED YOUNG'S MODULUS $=210 \mathrm{GPa}$

SPECIMEN CLEAUED AT KQ= $106 \mathrm{MPa}$ SQR [m] (NDT WALID KIc)

UALUES AT MAXIMUM LOAD

J-INTEGRAL $=67.3 \mathrm{~kJ} / \mathrm{m}^{\wedge} 2$

$\mathrm{KJc}=118.9 \mathrm{MPa} \operatorname{SQR}[\mathrm{m}]$

K-bet $a-c=82.1 \mathrm{MPa} \operatorname{SQR}[\mathrm{m}]$
UALUES at CLEaUAGE LOAD

J-INTEGRAL $=67.1 \mathrm{~kJ} / \mathrm{m}^{\wedge} 2$

$\mathrm{KJ} c=118.7 \mathrm{MPa} \operatorname{SQR}[\mathrm{m}]$

$\mathrm{K}$-beta-c $=82 \mathrm{MPa} \operatorname{SQR}[\mathrm{m}]$ 
E-24

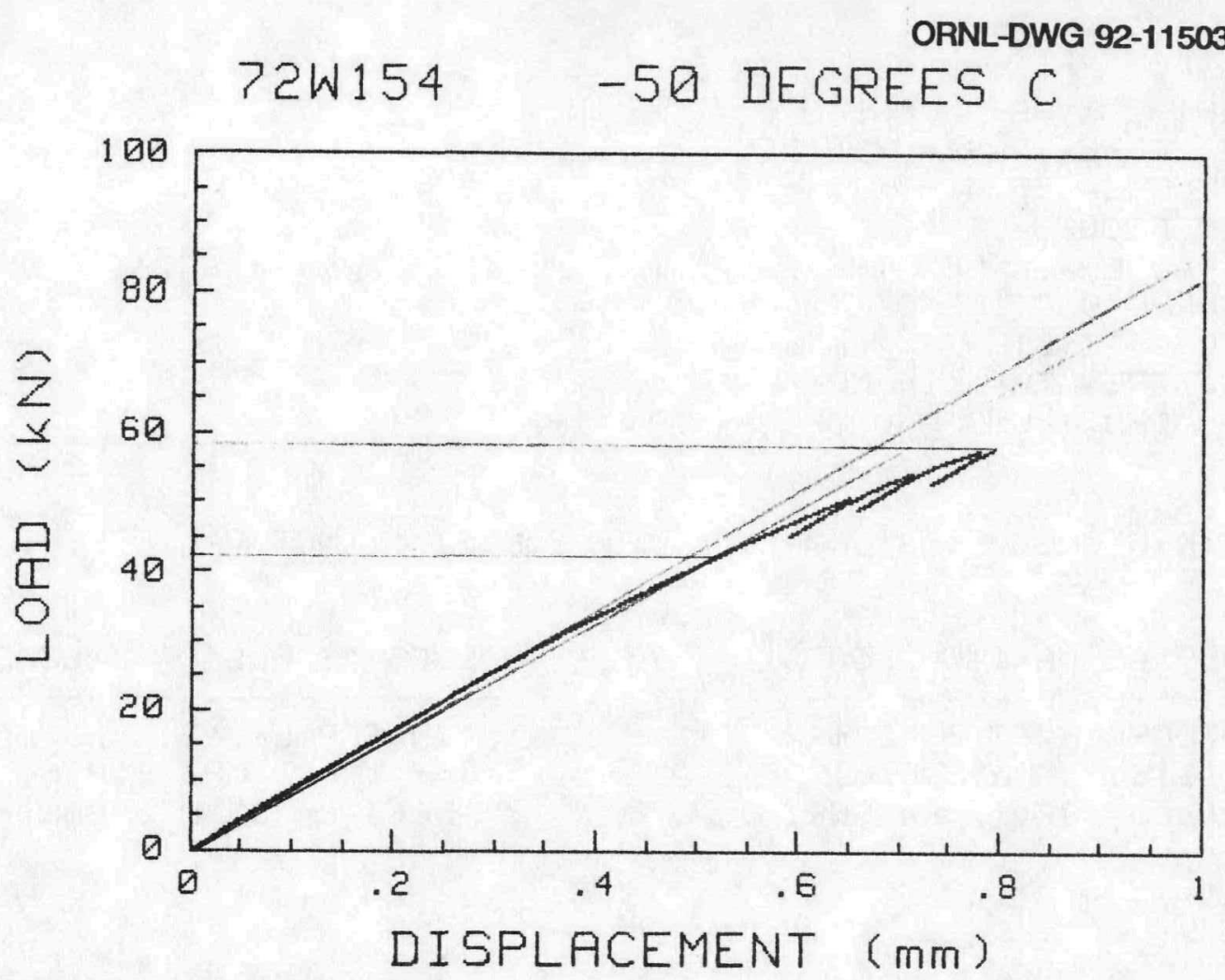

$72 W-154$

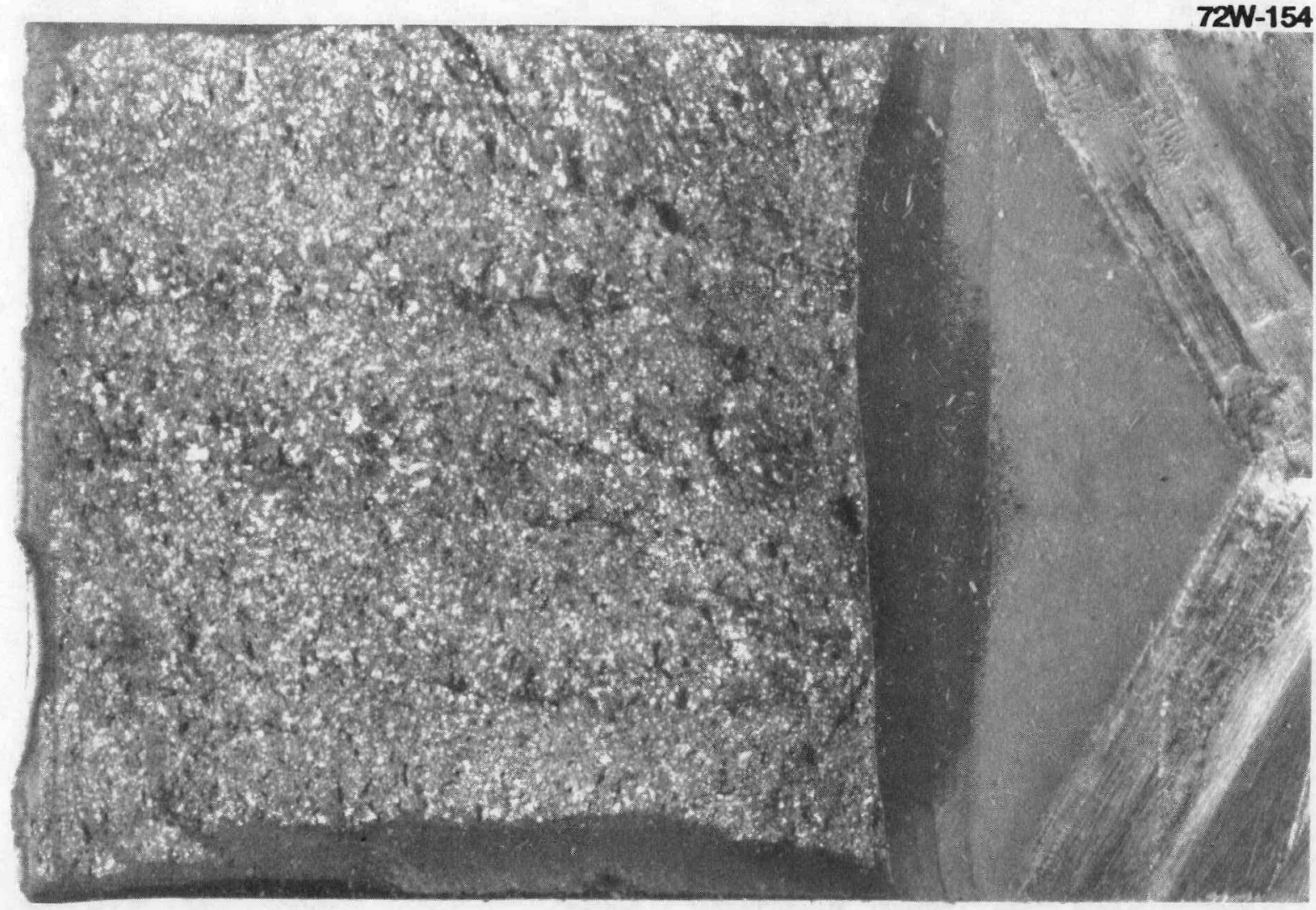

NUREG/CR-5913 
SPECIMEN ID $=72 W 157$

MATERIAL TYPE $=$ MCUWELD

TEST TEMPERATURE $=-50$ DEG $C$

THICKNESS $=25.4 \mathrm{~mm}$

SIDE GROOUES = $0 \%$

INITIAL CRACK LENGTH $=26.9 \mathrm{~mm}$

$a / w) i=.53$

MEASURED DUCTILE DELTA-A $=.06 \mathrm{~mm}$

YIELD STRENGTH $=547 \mathrm{MPa}$

FLOW STRENGTH $=614 \mathrm{MPa}$

ESTIMATED YOUNG'S MODULUS $=210 \mathrm{GPa}$

SPECIMEN CLEAUED AT KQ= $107.4 \mathrm{MPa}$ SQR $[\mathrm{m}]$ (NDT UALID KIc)

UALUES AT MAXIMUM LOAD

$J$-INTEGRAL $=68.2 \mathrm{~kJ} / \mathrm{m}^{\wedge} 2$

$\mathrm{KJ} c=119.7 \mathrm{MPa} S Q R[\mathrm{~m}]$

K-beta-c $=82.3 \mathrm{MPa} \operatorname{SQR}[\mathrm{m}]$
UALUES AT CLEaUAGE LDAD

J-INTEGRAL $=68.2 \mathrm{~kJ} / \mathrm{m}^{\wedge} 2$

$K J c=119.7 \mathrm{MPa} S Q R[\mathrm{~m}]$

$\mathrm{K}$-beta-c=82.3 MPa SQR $[\mathrm{m}]$ 
E-26
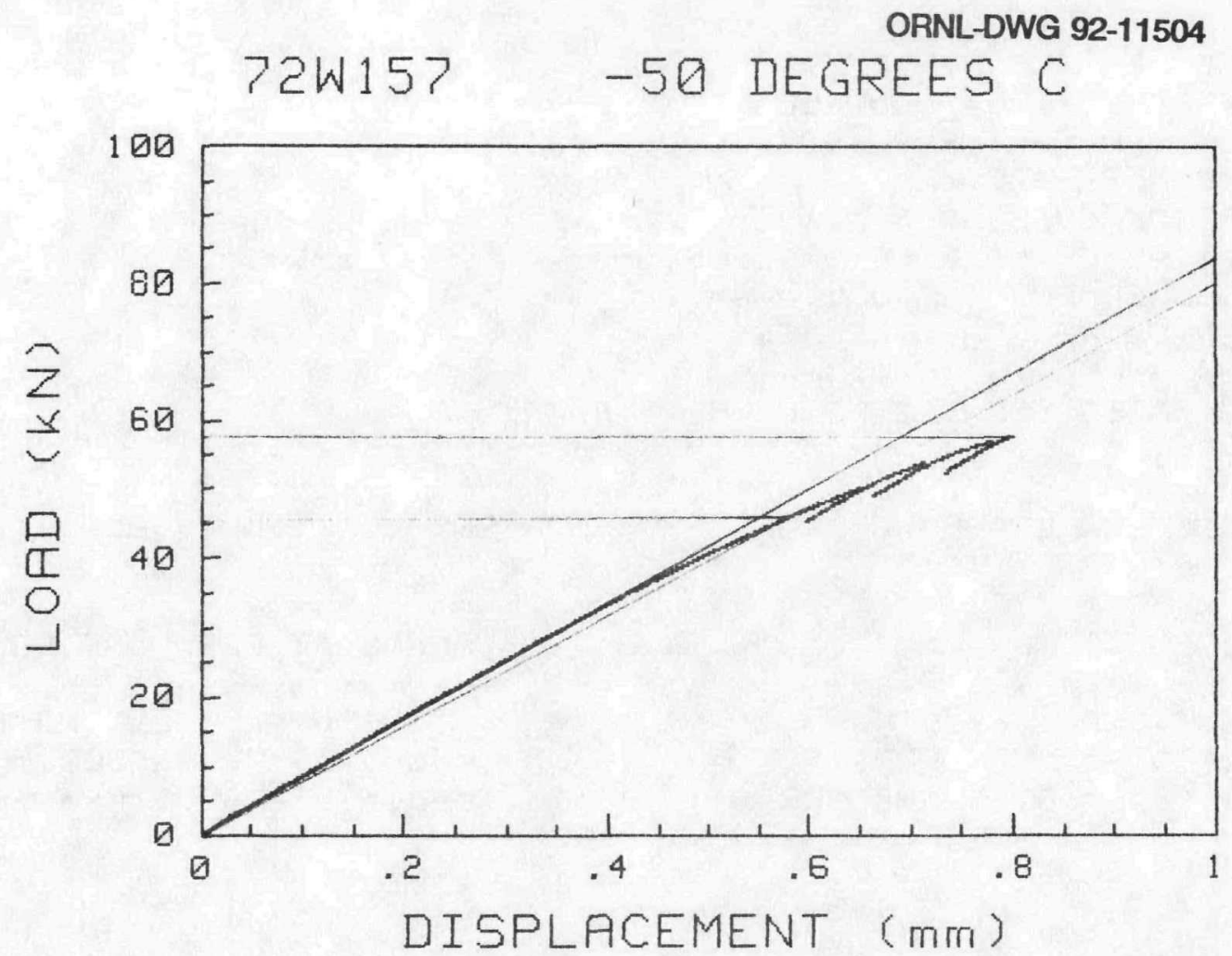

72W-157

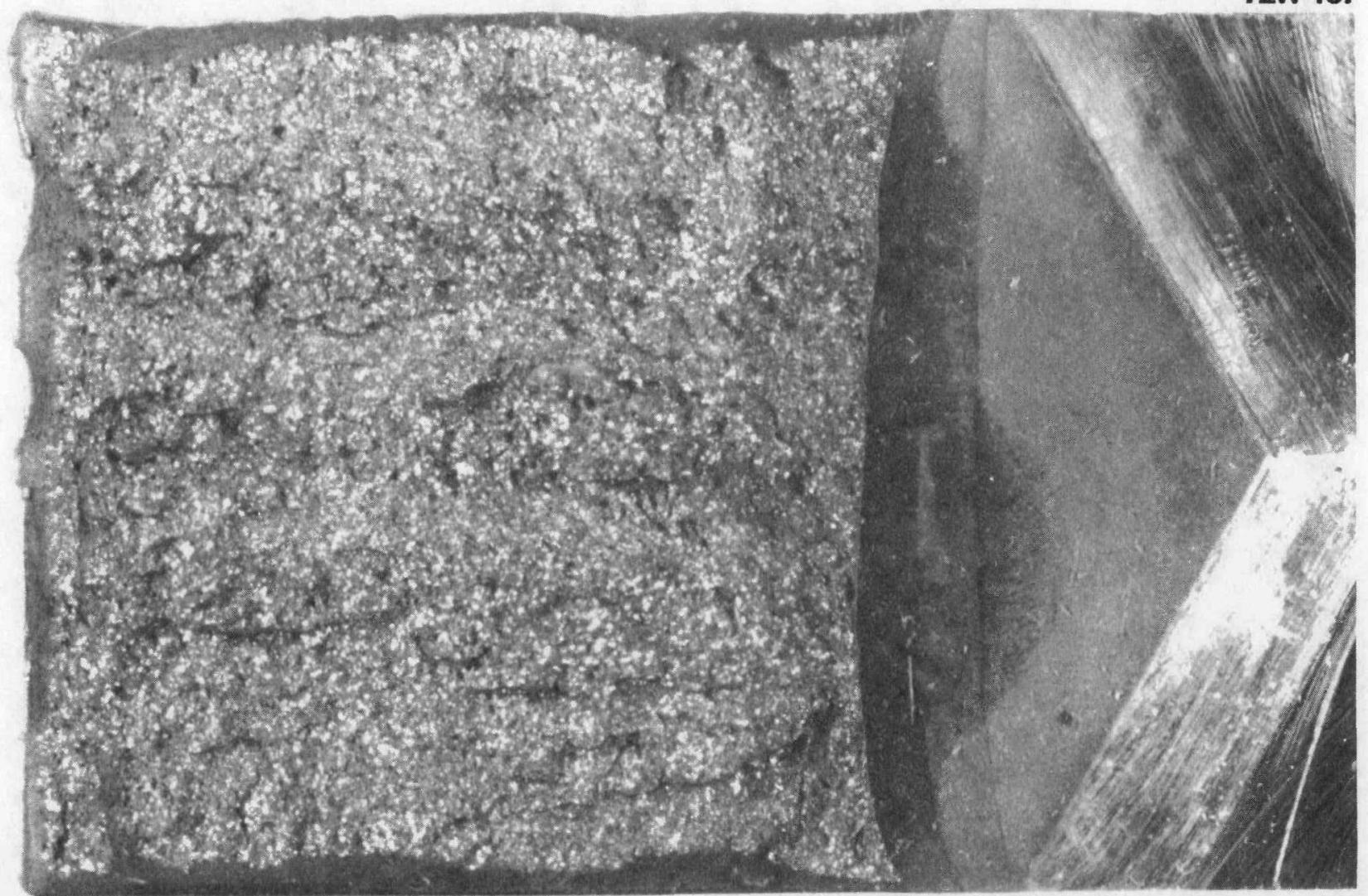




\section{Aug $23,1985 \quad 72-\omega-148 \quad-50^{\circ} \mathrm{C} \quad$ O\% SG $\quad$ IT-CT MRP/MJL}

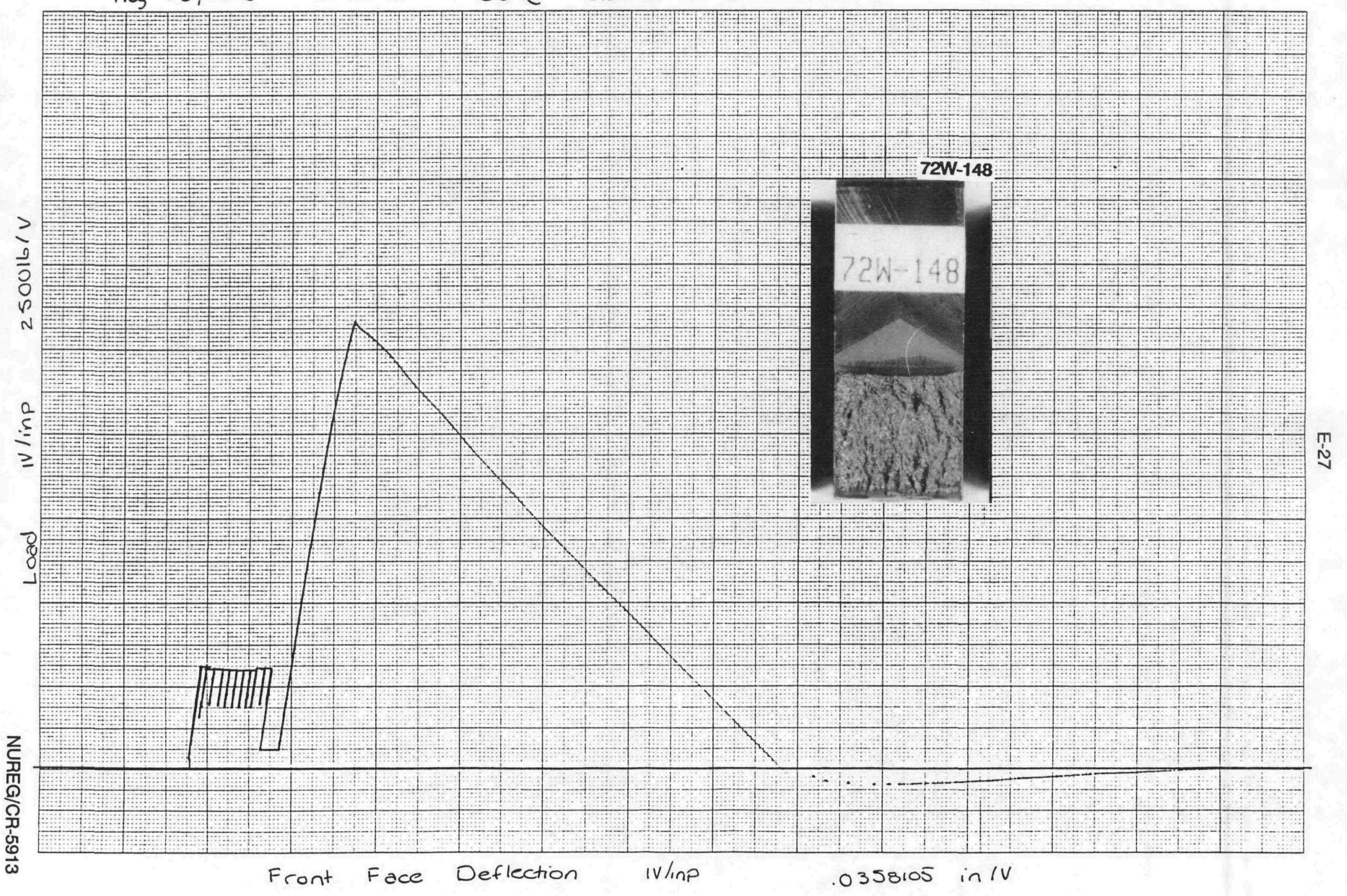


SPECIMEN ID $=72 W 153$

MATERIAL TYPE = MCUWELD

TEST TEMPERATURE $=-50$ DEG $\mathrm{C}$

THICKNESS $=25.3 \mathrm{~mm}$

SIDE GROOUES = $0 \%$

INITIAL CRACK LENGTH $=30.1 \mathrm{~mm}$

MEASURED DUCTILE DELTA-A $=.07 \mathrm{~mm}$

$a / W) \mathrm{i}=.592$

$Y I E L D$ STRENGTH $=547 \mathrm{MPa}$

FLDW STRENGTH $=614 \mathrm{MPa}$

ESTIMATED 'YOUNG'S MODULUS= $210 \mathrm{GPa}$

SPECIMEN CLEAUEO AT KQ= $110.6 \mathrm{MPa}$ SQR $[\mathrm{m}]$ (NDT VALID KIc)

UALUES AT MAXIMUM LOAD

J-INTEGRAL $=73.8 \mathrm{~kJ} / \mathrm{m}^{\wedge} 2$

$\mathrm{KJ} c=124.5 \mathrm{MPa} \operatorname{SQR}[\mathrm{m}]$

K-beta-c $=83.9 \mathrm{MPa} \operatorname{SQR}[\mathrm{m}]$
UALUES AT CLEAUAGE LOAD

J-INTEGRAL $=74.9 \mathrm{~kJ} / \mathrm{m}^{\wedge} 2$

$\mathrm{K} \cdot \mathrm{Jc}=125.4 \mathrm{MPa} \operatorname{SQR}[\mathrm{m}]$

$K$-beta-c $=84.2 \mathrm{MPa} \operatorname{SQR}[\mathrm{m}]$ 


\section{E-29}

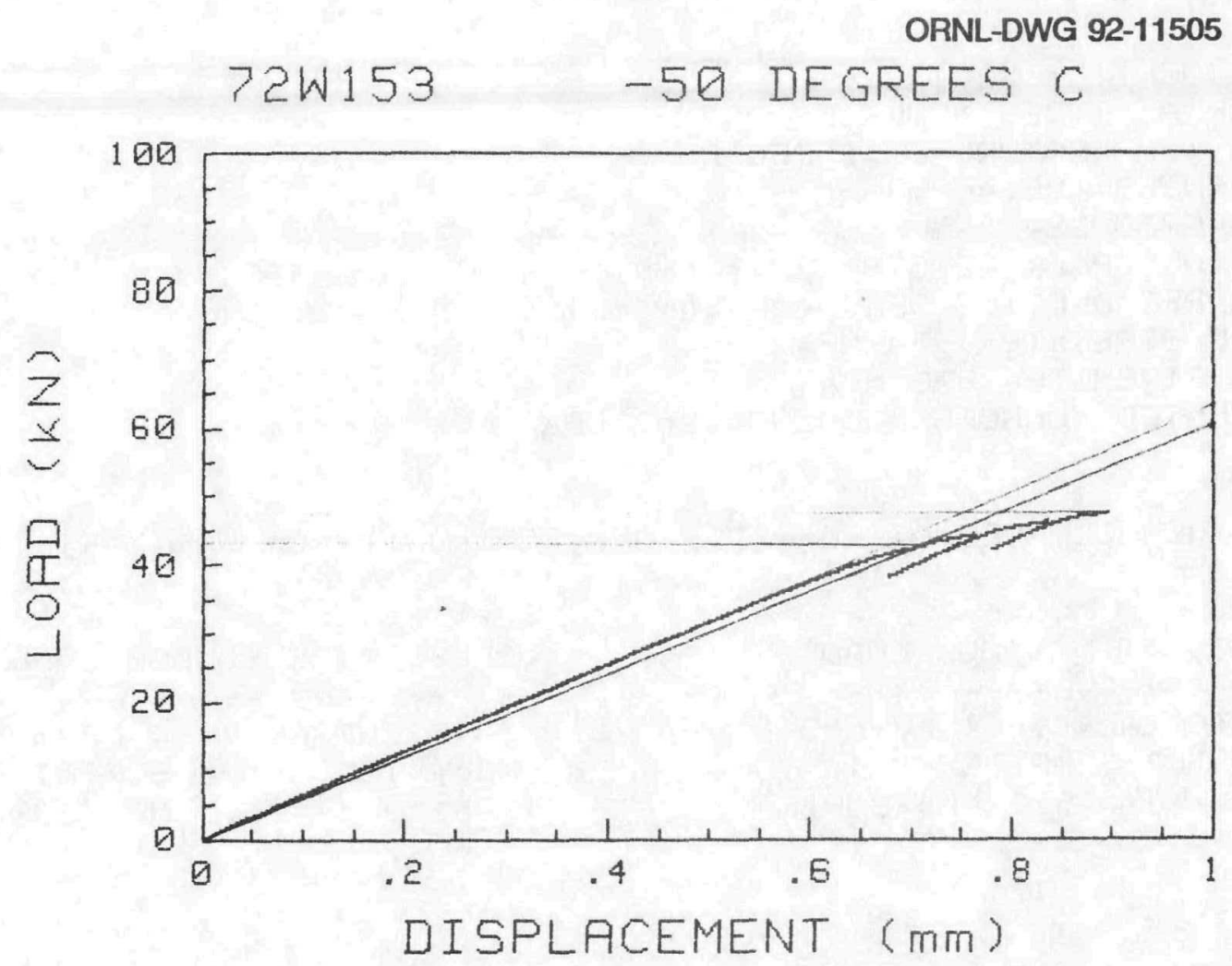

$72 W-153$

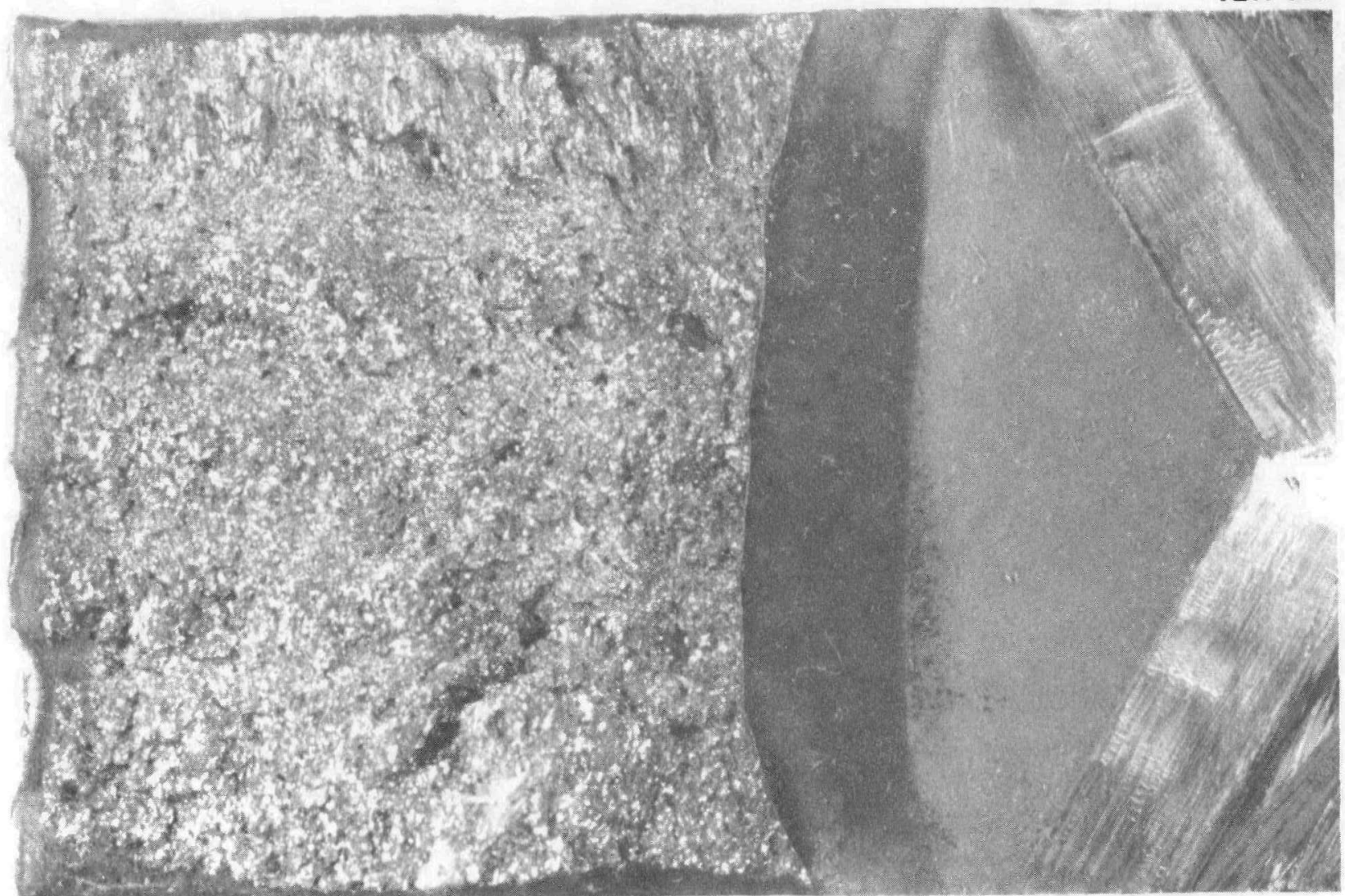


SPECIMEN ID $=72 W 162$

MATERIAL TYPE $=$ MCUWELD

TEST TEMPERATURE $=-30$ DEG C

THICKNESS $=25.4 \mathrm{~mm}$

SIDE GROOUES $=0 \%$

INITIAL CRACK LENGTH $=27.3 \mathrm{~mm}$

$a / w) i=.537$

MEASURED DUCTILE DELTA-A $=.05 \mathrm{~mm}$

$Y$ IELD STRENGTH $=534 \mathrm{MPa}$

FLOW STRENGTH $=598 \mathrm{MPa}$

ESTIMATED YOUNG'S MODULUS $=209 \mathrm{GPa}$

SPECIMEN CLEAUEO AT KQ $=97.5 \mathrm{MPa}$ SQR $[\mathrm{m}]$ (NOT UALID KIc)

UALUES AT MAXIMUM LOAD

$J-I N T E G R A L=50.1 \mathrm{~kJ} / \mathrm{m}^{\wedge} 2$

$\mathrm{KJc}=102.3 \mathrm{MPa} \operatorname{SQR}[\mathrm{m}]$

K-beta-c $=75.2 \mathrm{MPa}$ SQR $[\mathrm{m}]$
VALUES AT CLEAUAGE LDAD

J-INTEGRAL $=50.4 \mathrm{~kJ} / \mathrm{m}^{\wedge} 2$

$\mathrm{KJ} c=102.6 \mathrm{MPa} \operatorname{SQR}[\mathrm{m}]$

K-beta-c $=75.3 \mathrm{MPa} \operatorname{SQR}[\mathrm{m}]$ 
E-31

ORNL-DWG 92-11506

72W162 -30 DEGREES C

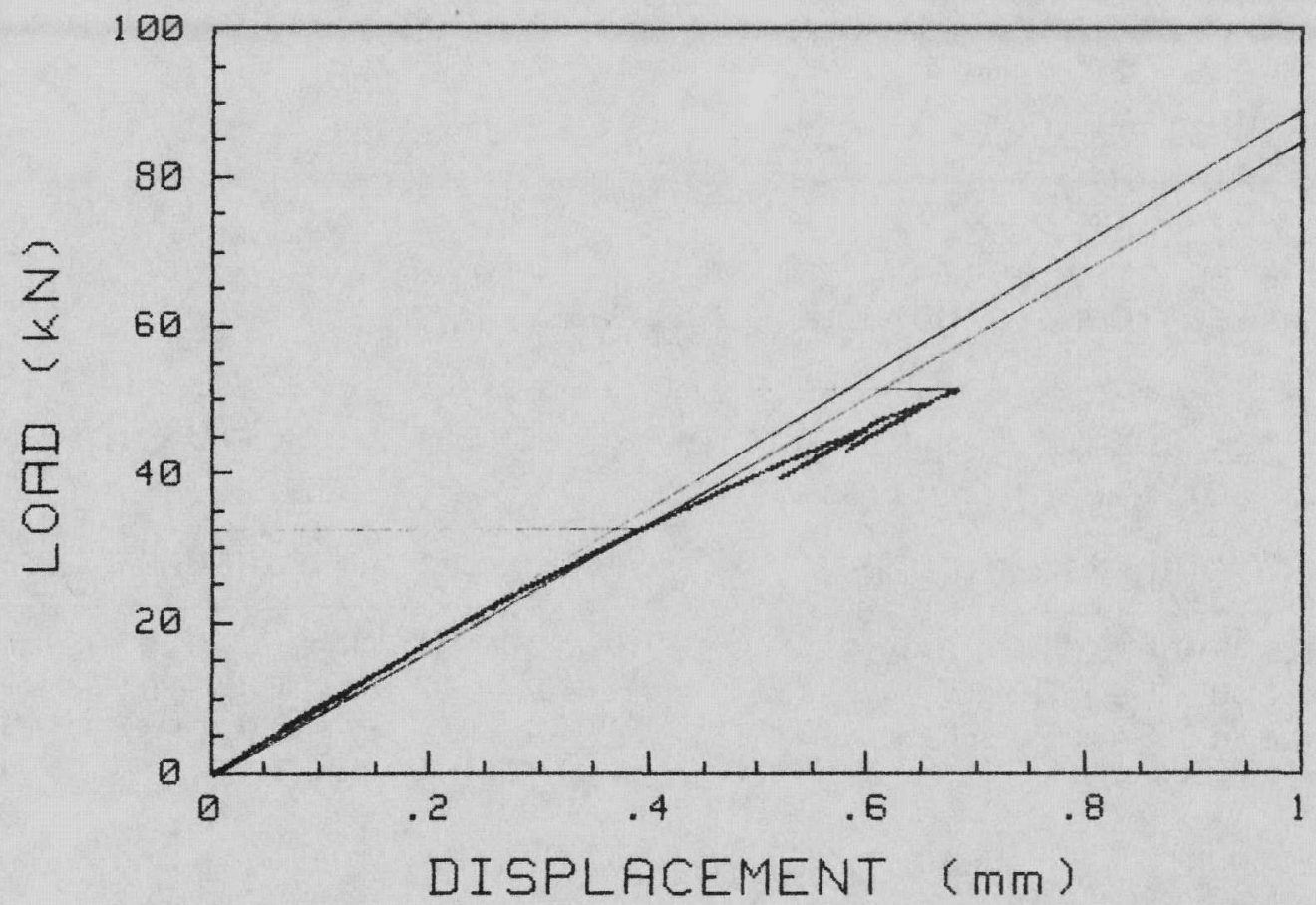

$72 W-162$

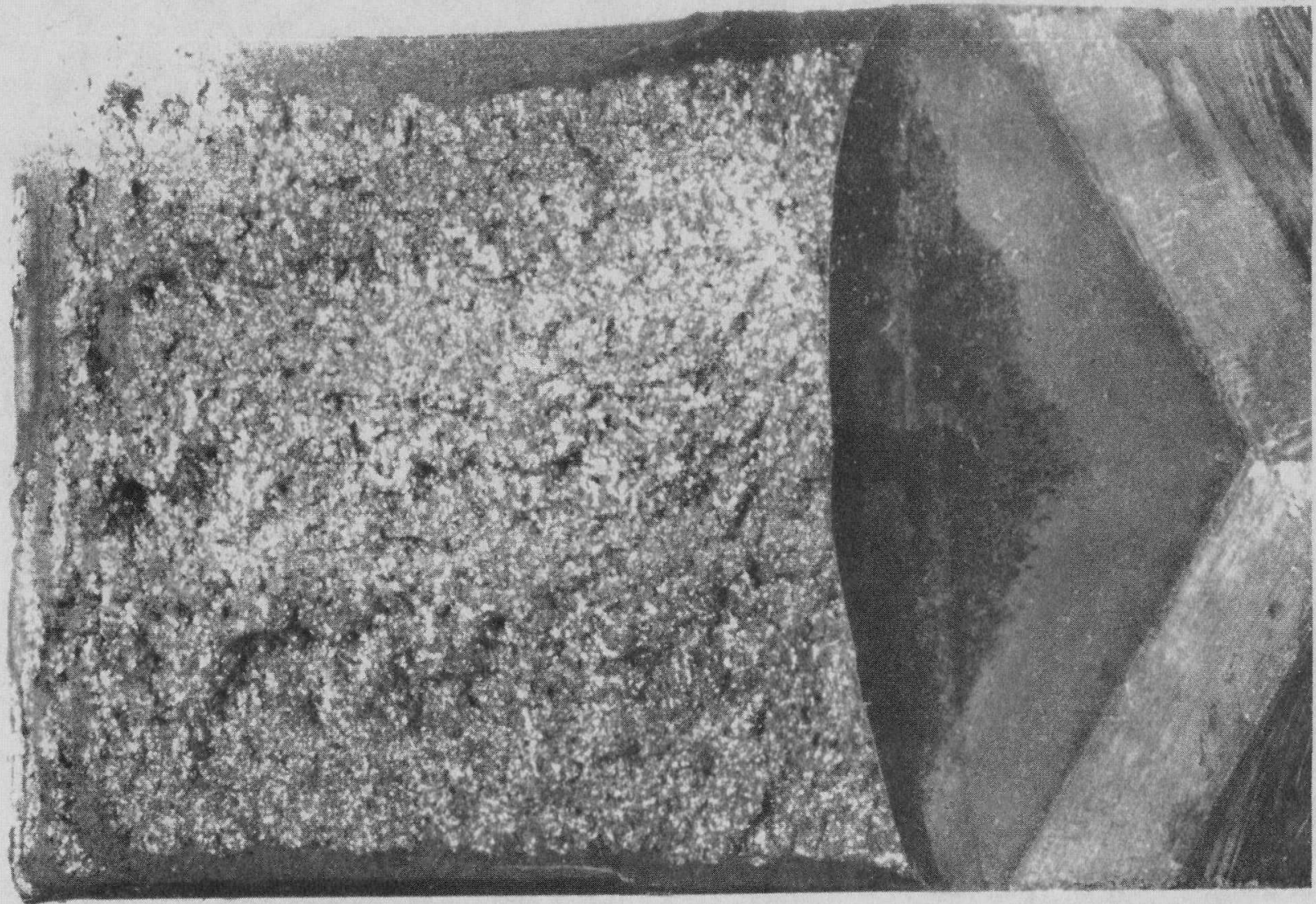

NUREG/CR-5913 
SPECIMEN $I D=72 W 158$

MATERIAL TYPE = MCUWELD

TEST TEMPERATURE $=-30$ DEG $C$

THICKNESS $=25.4 \mathrm{~mm}$

SIDE GROOUES = $0 \%$

INITIAL CRACK LENGTH $=26.8 \mathrm{~mm}$

MEASURED DUCTILE DELTA-A $=.08 \mathrm{~mm}$

$a / W)_{i}=.528$

YIELD STRENGTH $=534 \mathrm{MPa}$

FLOW STRENGTH $=598 \mathrm{MPa}$

ESTIMATED YOUNG'S MODULUS $=209 \mathrm{GPa}$

SPECIMEN CLEAUED AT KQ $=111.2 \mathrm{MPa}$ SQR $[\mathrm{m}]$ (NOT UALID $K I \mathrm{c}$ )

UALUES AT MAXIMUM LDAD

J-INTEGRAL $=78.4 \mathrm{~kJ} / \mathrm{m}^{\wedge} 2$

$\mathrm{KJc}=128 \mathrm{MPa} S Q R[\mathrm{~m}]$

$K$-beta-c $=84 \mathrm{MPa} \operatorname{SQR}[\mathrm{m}]$
UALUES AT CLEAUAGE LOAD

J-INTEGRAL $=78.2 \mathrm{~kJ} / \mathrm{m}^{\wedge} 2$

$K J c=127.8 \mathrm{MPa} \operatorname{SQR}[\mathrm{m}]$

$K$-bet $a-c=83.9 \mathrm{MPa} S Q R[\mathrm{~m}]$ 
E-33

ORNL-DWG 92-11507

\section{W158 -30 DEGREES C}

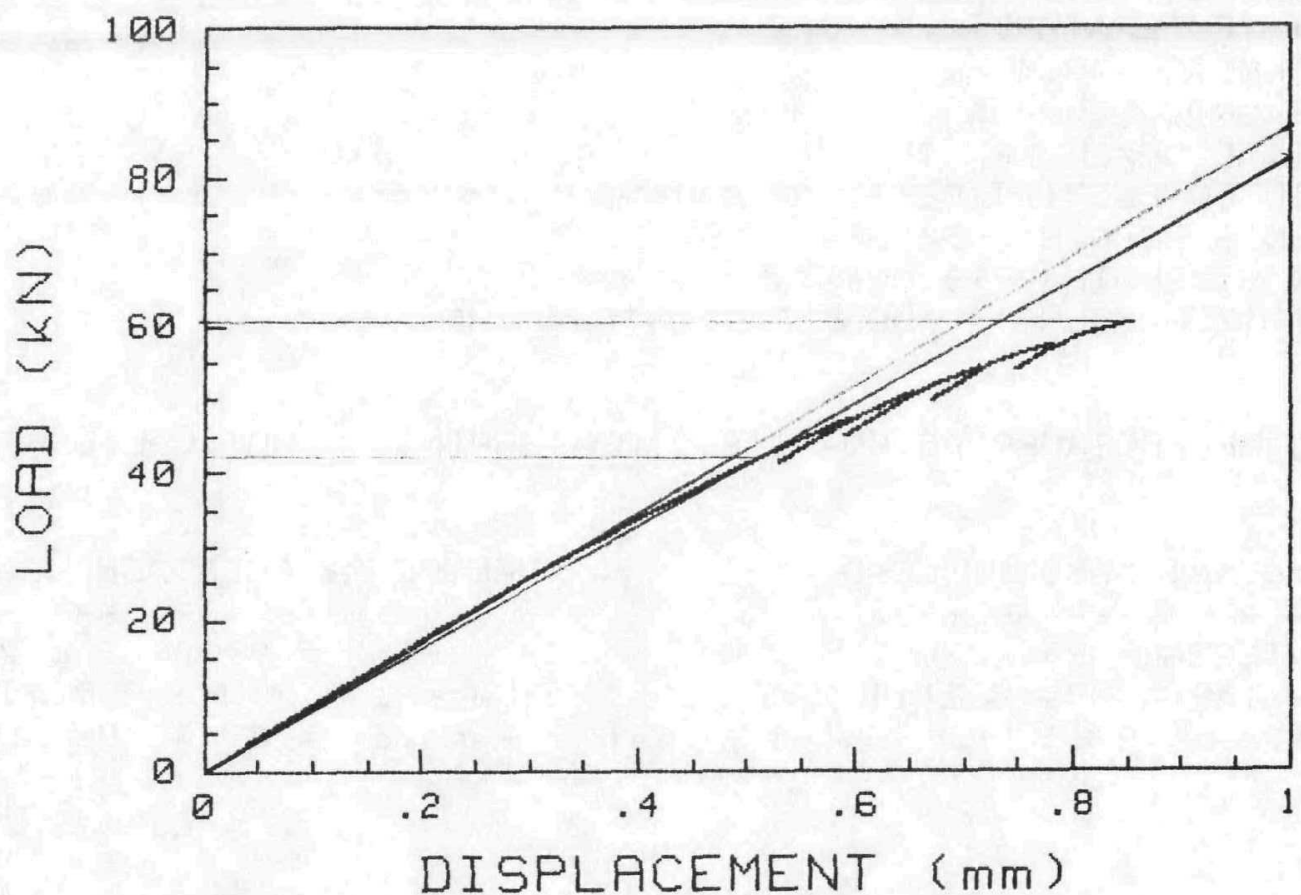

$72 W-158$

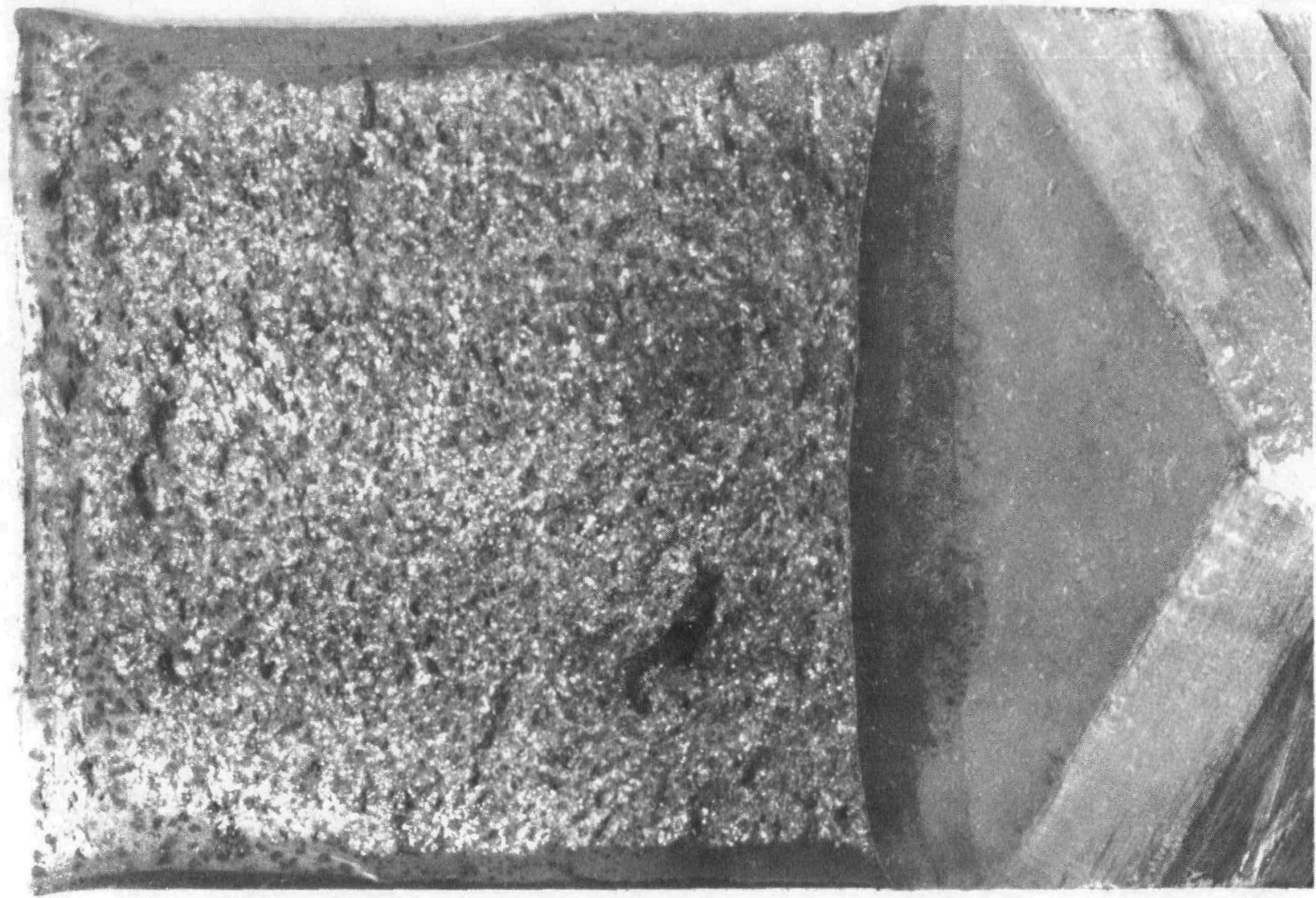

NUREG/CR-5913 
SPECIMEN ID $=72 W 131$

MATERIAL TYPE = MCUWELD

TEST TEMPERATURE $=-30$ DEG C

THICKNESS $=25.4 \mathrm{~mm}$

SIDE GROOUES $=0 \%$

INITIAL CRACK LENGTH $=26.9 \mathrm{~mm}$

$a / W\rangle i=.53$

MEASURED DUCT ILE DELTA-A $=.11 \mathrm{~mm}$

YIELD STRENGTH $=534 \mathrm{MPa}$

FLOW STRENGTH $=598 \mathrm{MPa}$

ESTIMATED YOUNG'S MODULUS $=209 \mathrm{GPa}$

SPECIMEN CLEAUED AT $K Q=118.2 \mathrm{MPa} S \mathrm{SQR}[\mathrm{m}]$ (NOT UALID $K I c$ )

UALUES AT MAXIMUM LDAD

J-INTEGRAL $=93 \mathrm{~kJ} / \mathrm{m}^{\wedge} 2$

$\mathrm{K}.]_{\mathrm{c}}=139.4 \mathrm{MPa} \operatorname{SQR}[\mathrm{m}]$

K-beta-c $=87.3 \mathrm{MPa}$ SQR $[\mathrm{m}]$
UALUES AT CLEAUAGE LDAD

$J-I N T E G R A L=93.5 \mathrm{~kJ} / \mathrm{m}^{\wedge} 2$

$K \cdot{ }_{c}=139.7 \mathrm{MPa} \operatorname{SQR}[\mathrm{m}]$

$K$-beta-c $=87.4 \mathrm{MPa} S Q R[\mathrm{~m}]$ 


\section{E-35}

ORNL-DWG 92-11508
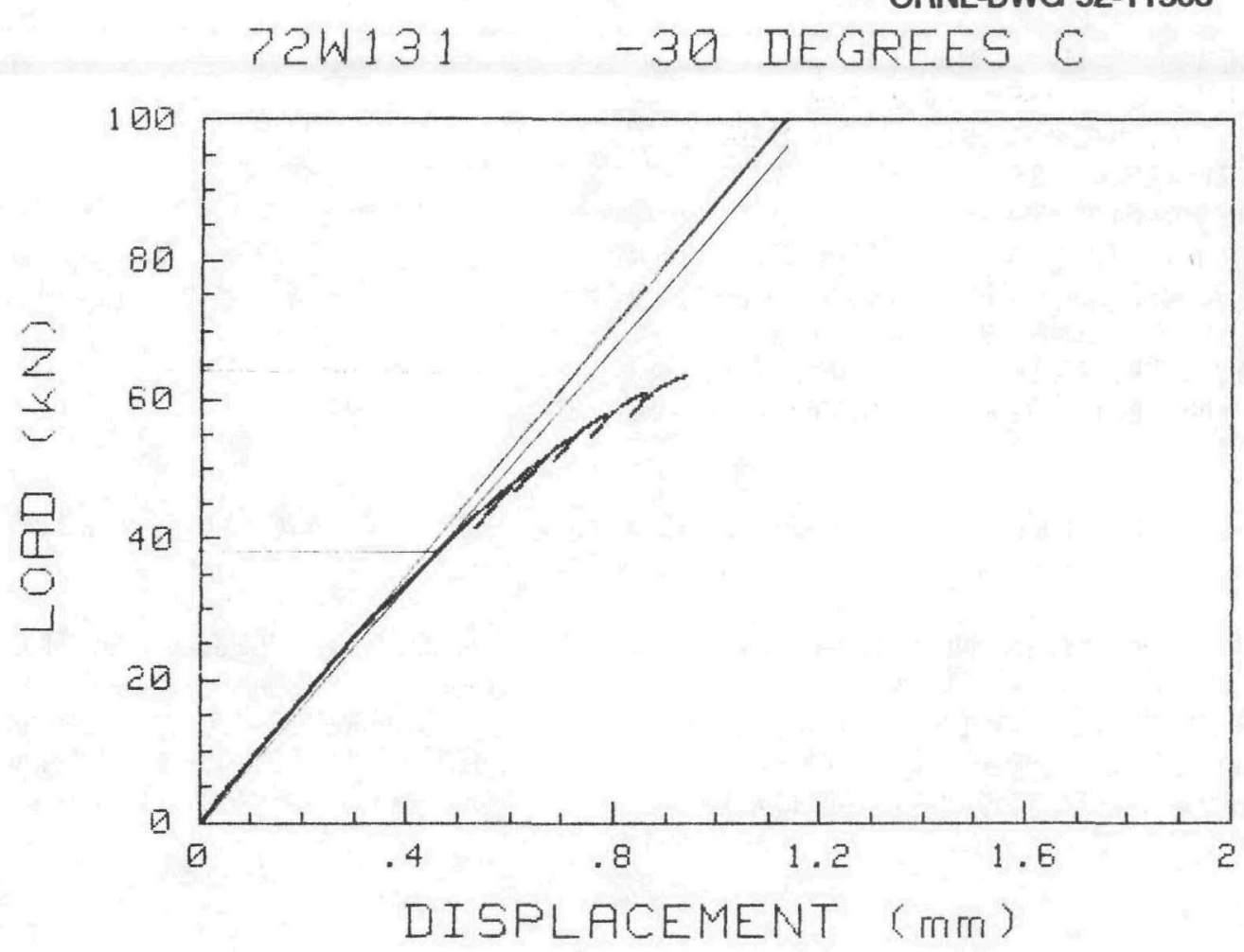

72W-131

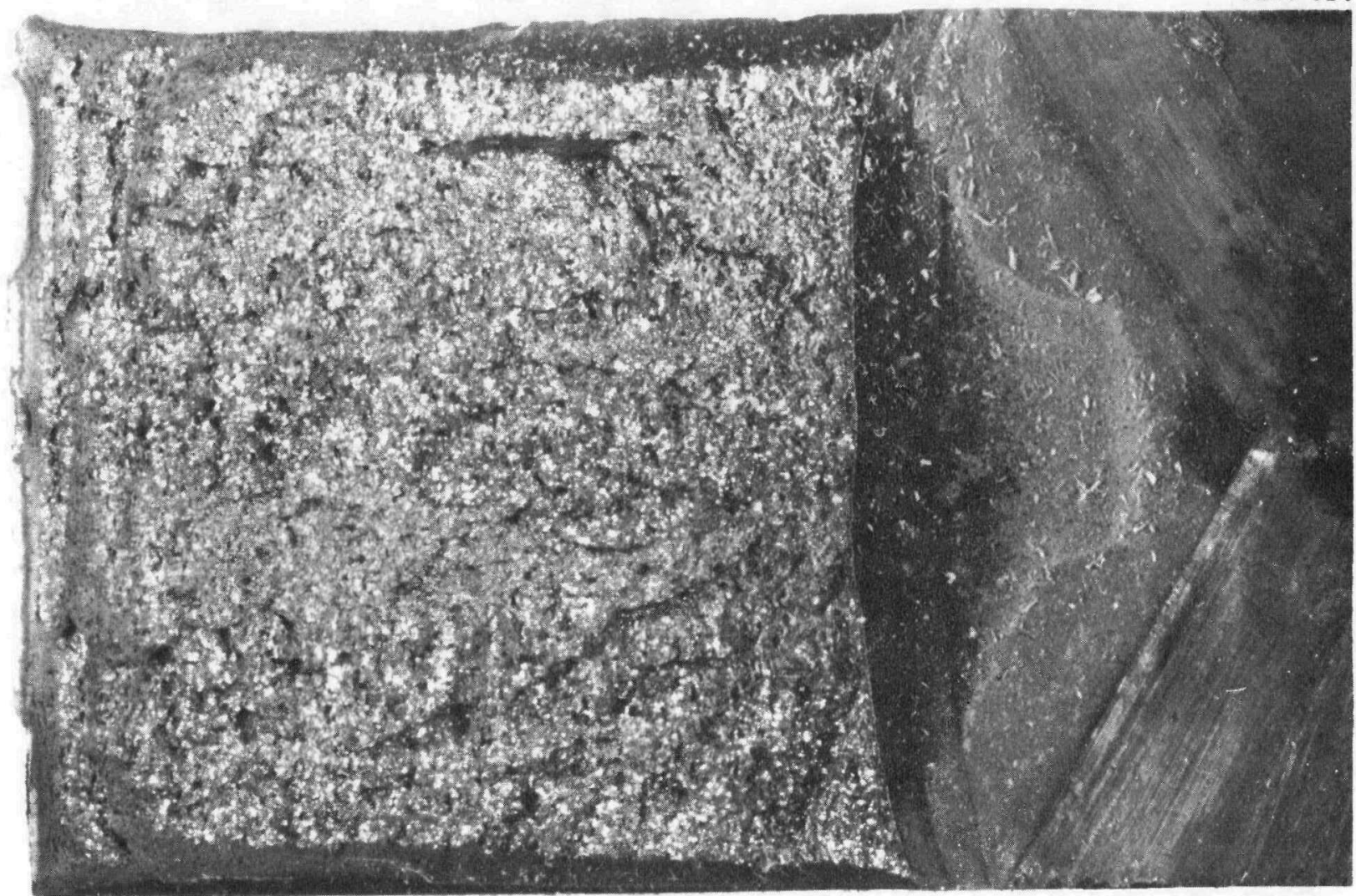


SPECIMEN ID $=72 W 133$

MATERIAL TYPE $=$ MCUWELD

TEST TEMPERATURE $=-30$ DEG C

THICKNESS $=25.4 \mathrm{~mm}$

SIDE GROOUES $=0 \%$

INITIAL CRACK LENGTH $=27.2 \mathrm{~mm}$

MEASURED DUCT ILE DELTA-A $=.25 \mathrm{~mm}$

$a / W) i=.536$

$Y$ IELD STRENGTH $=534 \mathrm{MPa}$

FLOW STRENGTH $=598 \mathrm{MPa}$

ESTIMATED YOUNG'S MODULUS $=209 \mathrm{GPa}$

SPECIMEN CLEAUED AT KQ= $125.4 \mathrm{MPa} S Q R[\mathrm{~m}]$ (NDT VALID $K I \mathrm{c}$ )

UALUES AT MAXIMUM LOAD

- - - - - - - - - - - - - -

$J$-INTEGRAL $=141.1 \mathrm{~kJ} / \mathrm{m}^{\wedge} 2$

$\mathrm{KJ} c=171.7 \mathrm{MPa} \operatorname{SQR}[\mathrm{m}]$

K-beta-c $=95.6$ MPa SQR $[\mathrm{m}]$
UALUES AT CLEAUAGE LDAD

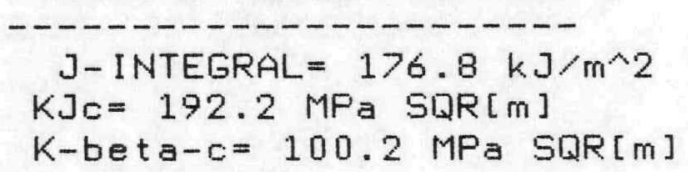




\section{E-37}

72W133 -30 DEGREES C

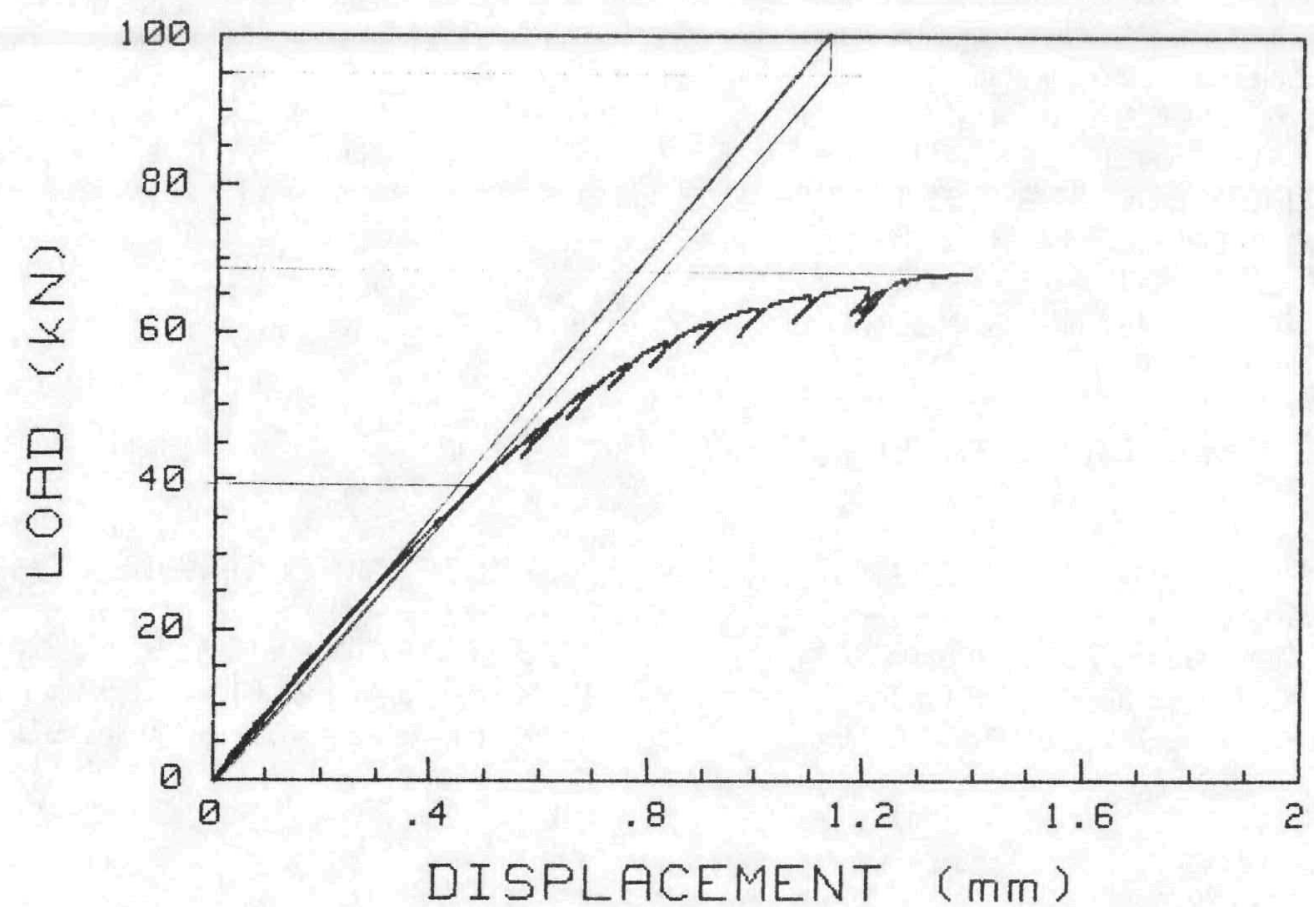

72W-133

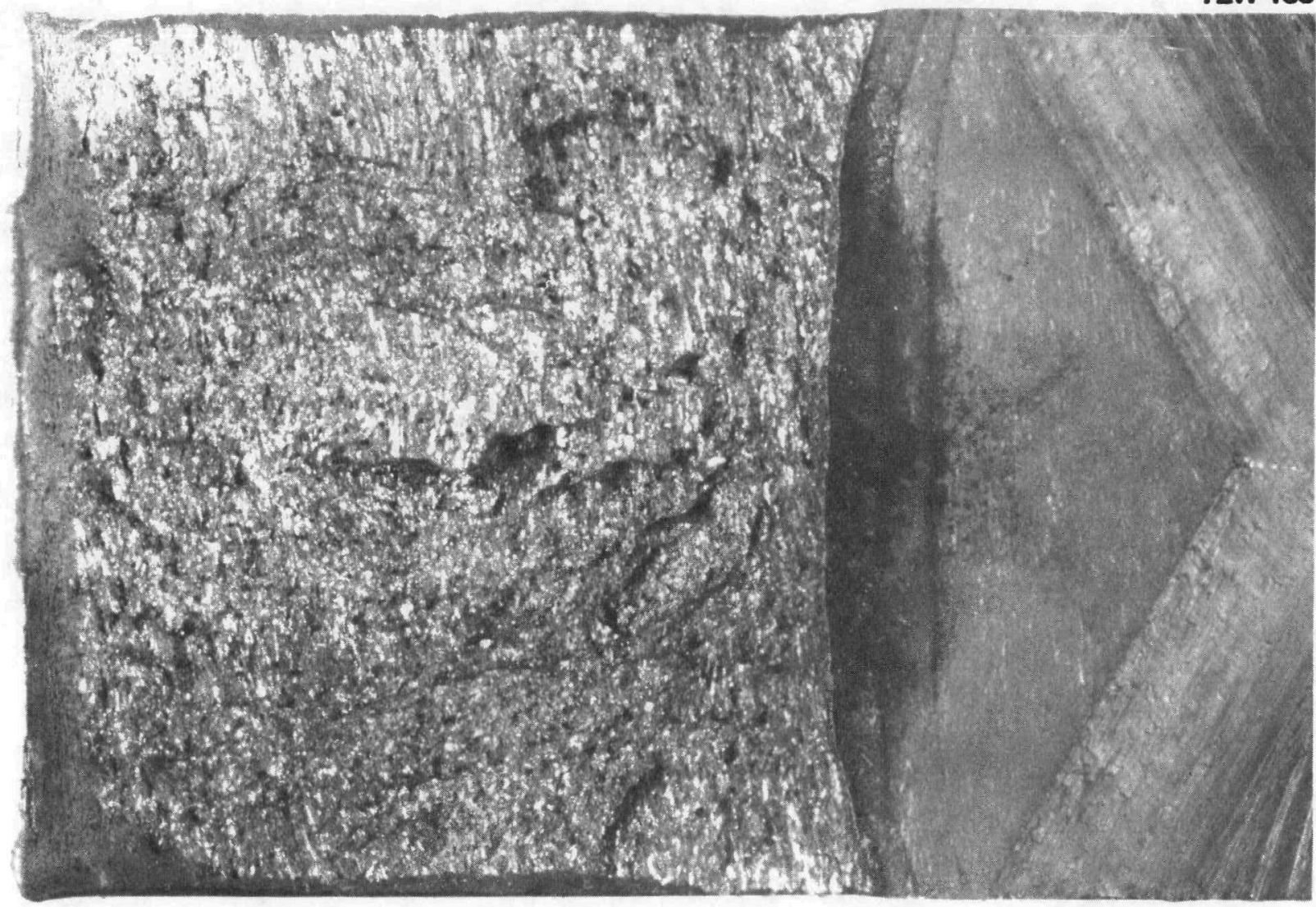


SPECIMEN ID $=72 W 109$

MATERIAL TYPE = MCUWELD

TEST TEMPERATURE $=-26$ DEG $C$

THICKNESS $=25.4 \mathrm{~mm}$

SIDE GROOUES = $0 \%$

INITIAL CRACK LENGTH $=26.7 \mathrm{~mm}$

$a / w) i=.526$

MEASURED DUCTILE DELTA-A $=.11 \mathrm{~mm}$

$Y$ IELD STRENGTH $=532 \mathrm{MPa}$

FLOW STRENGTH $=595 \mathrm{MPa}$

EST IMATED YOUNG'S MODULUS $=209 \mathrm{GPa}$

SPECIMEN CLEAUED AT KQ= $119.8 \mathrm{MPa} S Q R[\mathrm{~m}]$ (NDT UALID $K I \mathrm{c}$ )

UALUES AT MAXIMUM LDAD

$\mathrm{J}$-INTEGRAL $=96.3 \mathrm{~kJ} / \mathrm{m}^{\wedge} 2$

$\mathrm{K} \cdot \mathrm{J} c=141.8 \mathrm{MPa} \operatorname{SQR}[\mathrm{m}]$

K-beta-c $=87.8 \mathrm{MPa} \operatorname{SQR}[\mathrm{m}]$
UALUES AT CLEAUAGE LOAD

J-INTEGRAL $=96.9 \mathrm{~kJ} / \mathrm{m}^{\wedge} 2$

$\mathrm{KJc}=142.2 \mathrm{MPa} \operatorname{SQR}[\mathrm{m}]$

$K$-beta-c $=87.9 \mathrm{MPa} S Q R[\mathrm{~m}]$ 
E-39

ORNL-DWG 92-11510

72W109 -26 DEGREES C

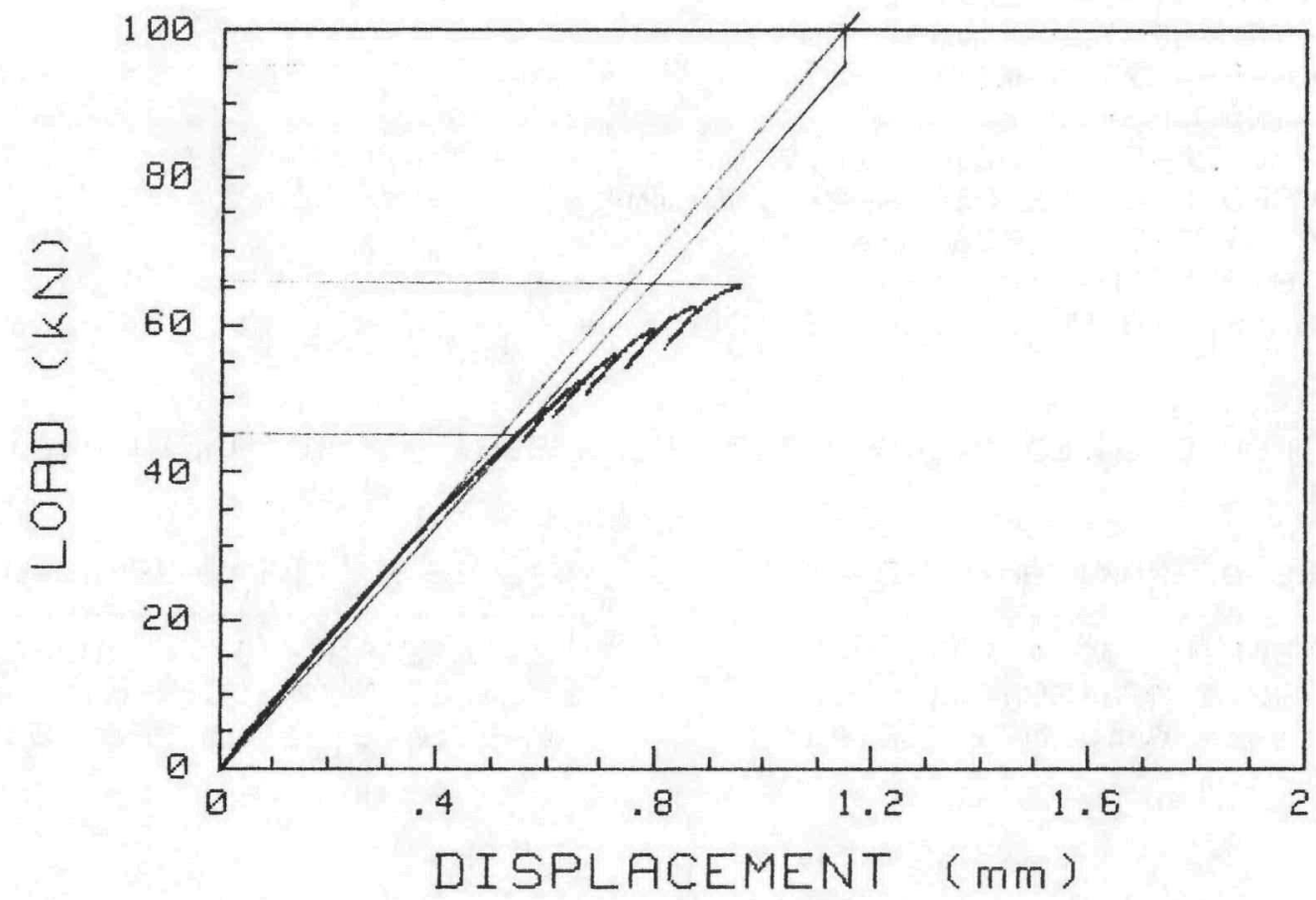

72W-109

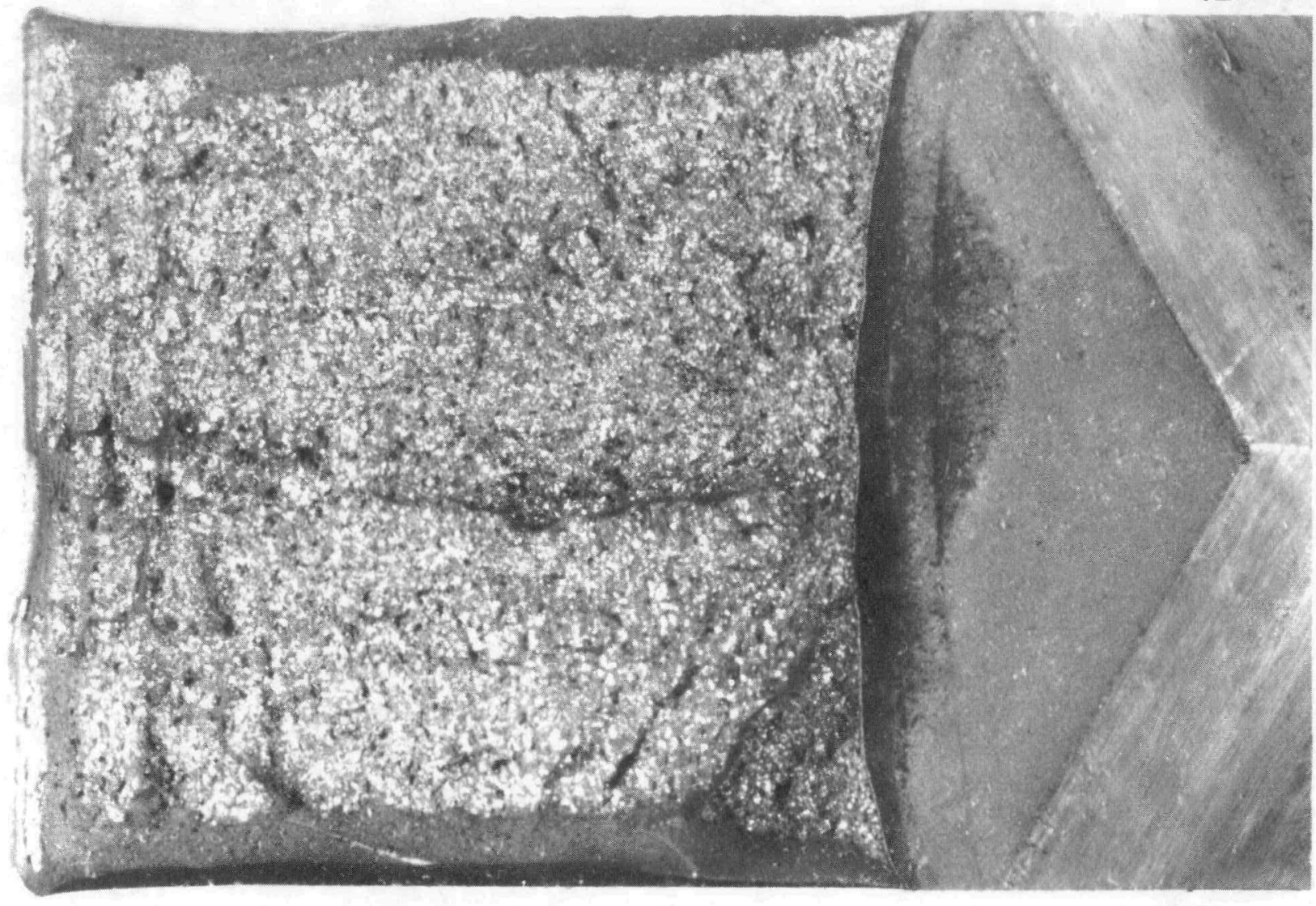


SPECIMEN ID $=72 W 161$

MATERIAL TYPE $=$ MCUWELD

TEST TEMPERATURE $=-15$ DEG $C$

THICKNESS $=25.4 \mathrm{~mm}$

SIDE GROOUES $=0 \%$

INITIAL CRACK LENGTH $=26.9 \mathrm{~mm}$

$a / W\rangle i=.53$

MEASURED DUCTILE DELTA-A $=.05 \mathrm{~mm}$

$Y I E L D$ STRENGTH $=526 \mathrm{MPa}$

FLOW STRENGTH $=586 \mathrm{MPa}$

ESTIMATED YOUNG'S MODULUS $=208 \mathrm{GPa}$

SPECIMEN CLEAUED AT KQ= $81.7 \mathrm{MPa}$ SQR $[\mathrm{m}]$ (NOT WALID KIC)

UALUES AT MAXIMUM LOAD

-

J-INTEGRAL $=32.4 \mathrm{~kJ} / \mathrm{m}^{\wedge} 2$

$\mathrm{KJ} c=82.1 \mathrm{MPa} \operatorname{SQR}[\mathrm{m}]$

$K$-beta-c $=66.1 \mathrm{MPa}$ SQR $[\mathrm{m}]$
UALUES AT CLEAUAGE LDAD

J-INTEGRAL $=32.4 \mathrm{~kJ} / \mathrm{m}^{\wedge} 2$

$K J c=82.1 \mathrm{MPa} \operatorname{SQR}[\mathrm{m}]$

K-beta-c $=66.1 \mathrm{MPa} \operatorname{SQR}[\mathrm{m}]$ 
E-41

ORNL-DWG 92-11511

72W161-15 DEGREES C

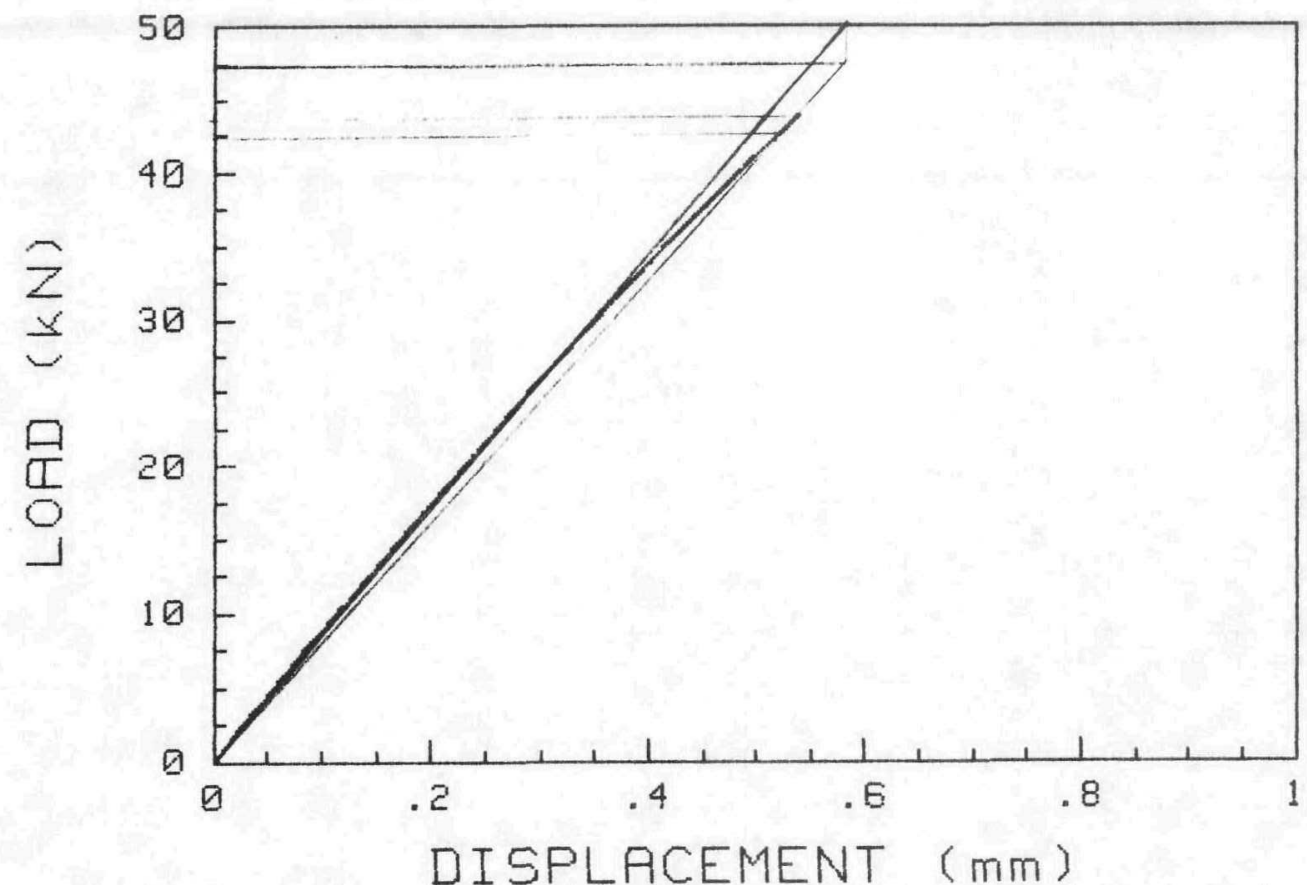

$72 W-161$

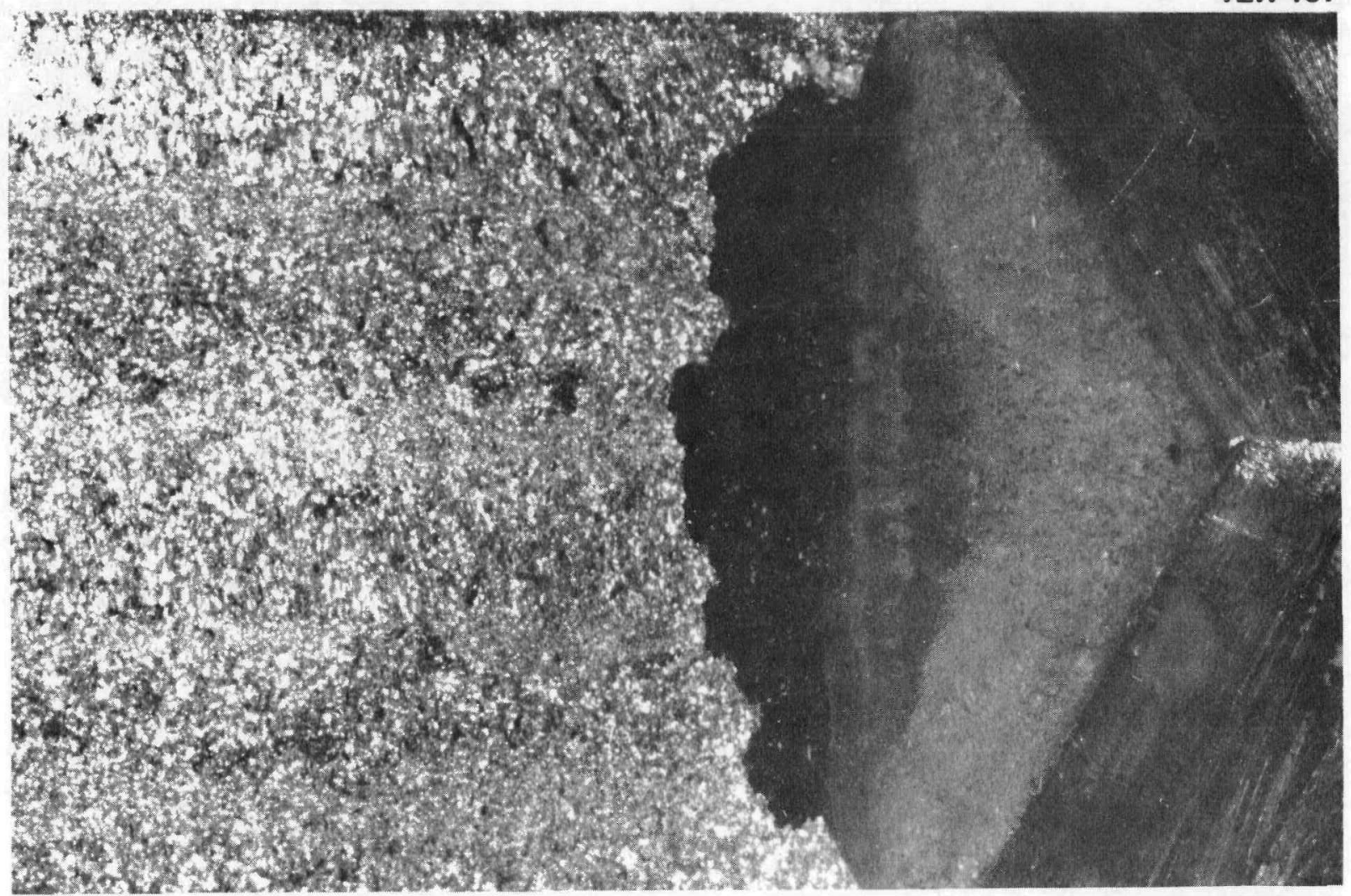

NUREG/CR-5913 


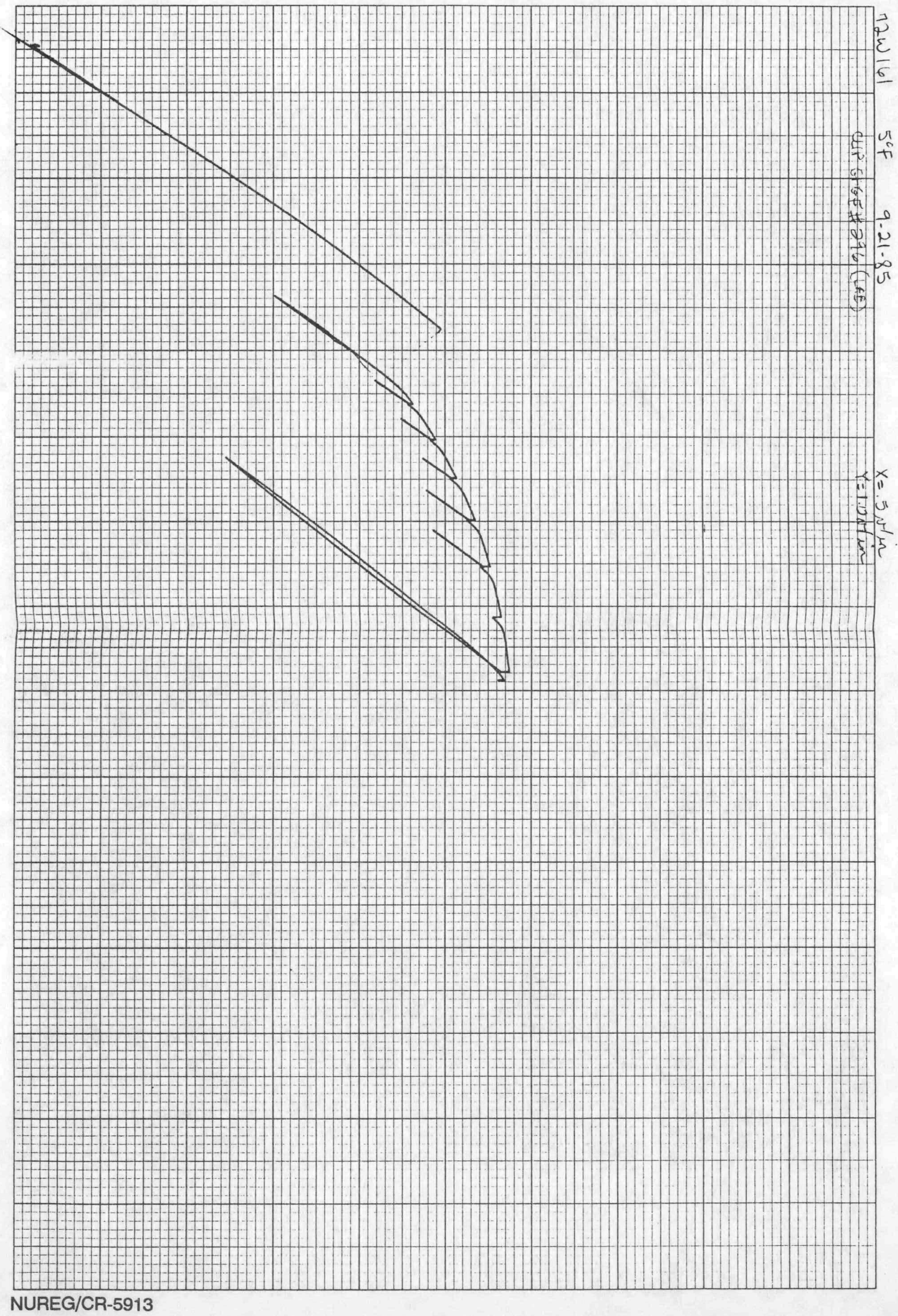


SPECIMEN ID $=72 W 169$

MATERIAL TYPE $=$ MCULIELD

TEST TEMPERATURE $=-15$ DEG $\quad \mathrm{C}$

THICKNESS $=25.4 \mathrm{~mm}$

SIDE GROOUES $=0 \%$

INITIAL CRACK LENGTH $=27.1 \mathrm{~mm}$

$\mathrm{a} /$ (w) $\mathrm{i}=.535$

MEASURED DUCTILE DELTA-A $=.05 \mathrm{~mm}$

$Y$ IELD STRENGTH $=526 \mathrm{MPa}$

FLOW STRENGTH $=586 \mathrm{MPa}$

ESTIMATED YOUNG'S MODULUS $=208 \mathrm{GPa}$

SPECIMEN CLEAUED AT KQ=84 MPa SQR $[\mathrm{m}]$ (NDT VALID KIC)

VALUES AT MAXIMUM LOAD

J-INTEGRAL $=35.8 \mathrm{~kJ} / \mathrm{m}^{\wedge} 2$

$\mathrm{KJ} c=86.3 \mathrm{MPa} \operatorname{SQR}[\mathrm{m}]$

$K$-bet $a-c=68.1 \mathrm{MPa} \operatorname{SQR}[\mathrm{m}]$
UALUES at CLEAUAGE LDAD

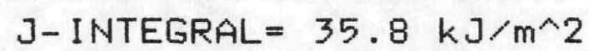

$\mathrm{KJ} c=86.3 \mathrm{MPa} \operatorname{SQR}[\mathrm{m}]$

$K$-beta-c $=68.1 \mathrm{MPa} S Q R[\mathrm{~m}]$ 
E-44

ORNL-DWG $92-11512$

72W 169

-15 DEGREES C

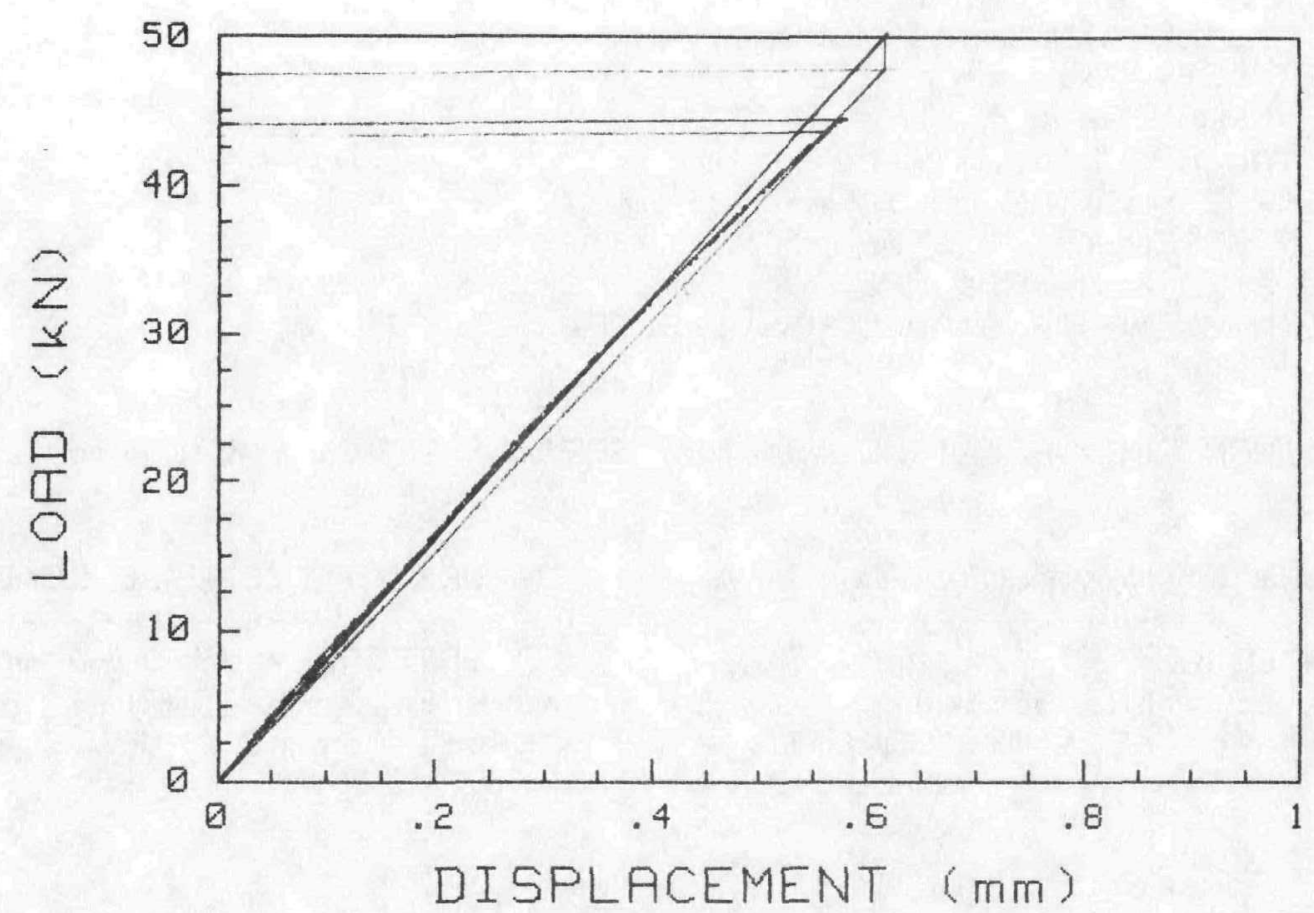

$72 W-169$

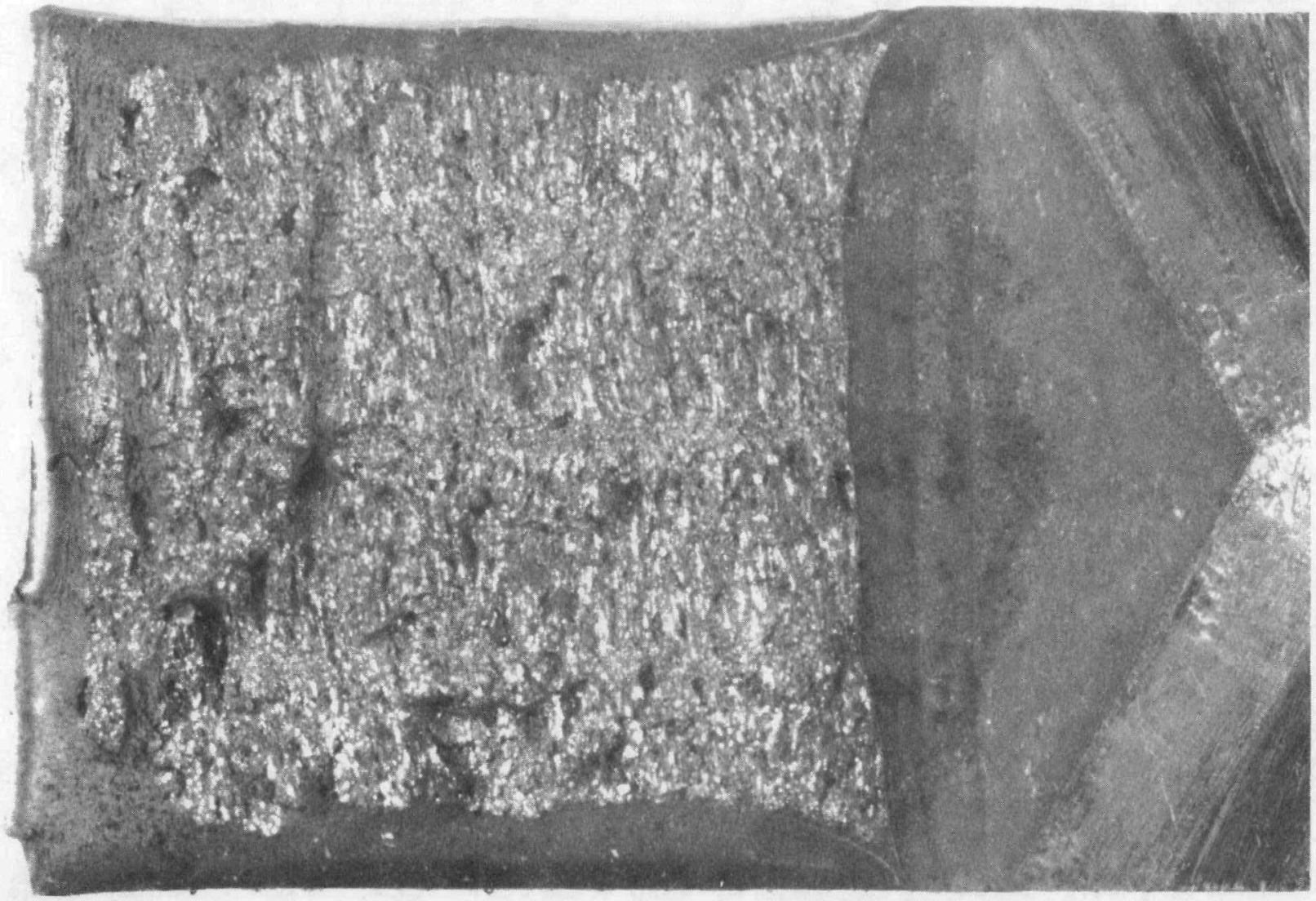

NUREG/CR-5913 
SPECIMEN $I D=72 W 1>0$

MATERIAL TYPE = MCUWELD

TEST TEMPERATURE $=-15$ DEG $C$

THICKNESS $=25.4 \mathrm{~mm}$

SIDE GROOUES $=0 \%$

INITIAL CRACK LENGTH $=27.1 \mathrm{~mm}$

MEASURED DUCTILE DELTA-A $=.14 \mathrm{~mm}$

$a / w) i=.534$

YIELD STRENGTH $=526 \mathrm{MPa}$

FLOW STRENGTH $=586 \mathrm{MPa}$

ESTIMATED YOUNG'S MODULUS $=208 \mathrm{GPa}$

SPECIMEN CLEAUED AT KQ $=118.5 \mathrm{MPa}$ SQR $[\mathrm{m}]$ (NOT WALID KIc)

VALUES AT MAXIMUM LOAD

-

J-INTEGRAL $=109.7 \mathrm{~kJ} / \mathrm{m}^{\wedge} 2$

$\mathrm{KJ} \mathrm{c}=151 \mathrm{MPa} \operatorname{SQR}[\mathrm{m}]$

K-beta-c $=89.7 \mathrm{MPa} \operatorname{SQR}[\mathrm{m}]$

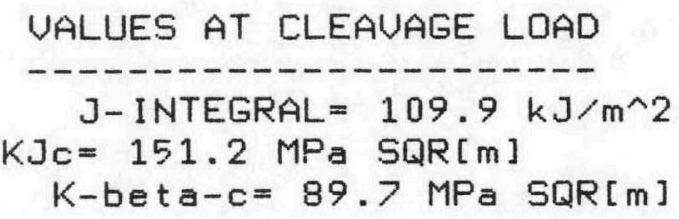




\section{E-46}

72W170 -15 DEGREES C

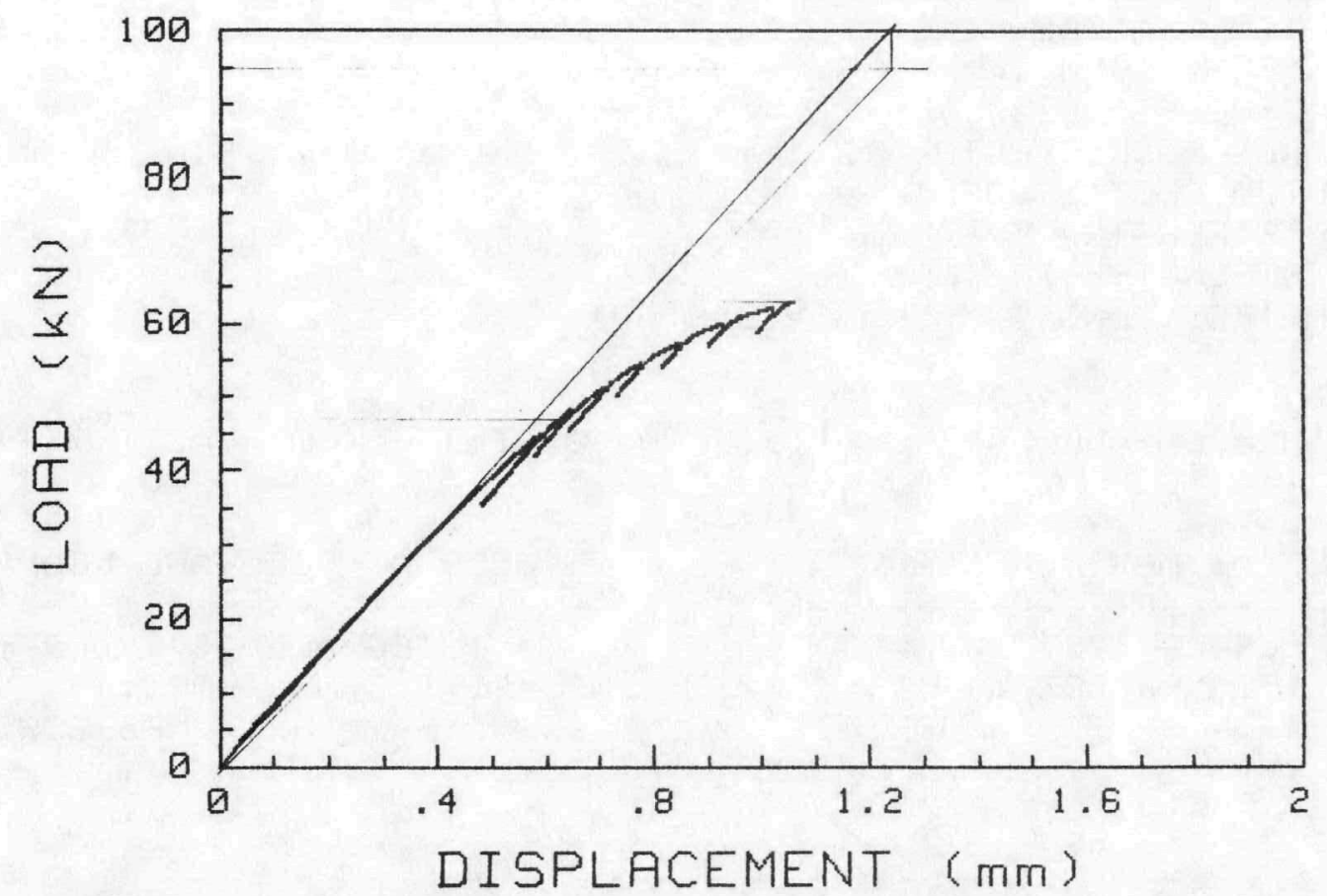

$72 W-170$

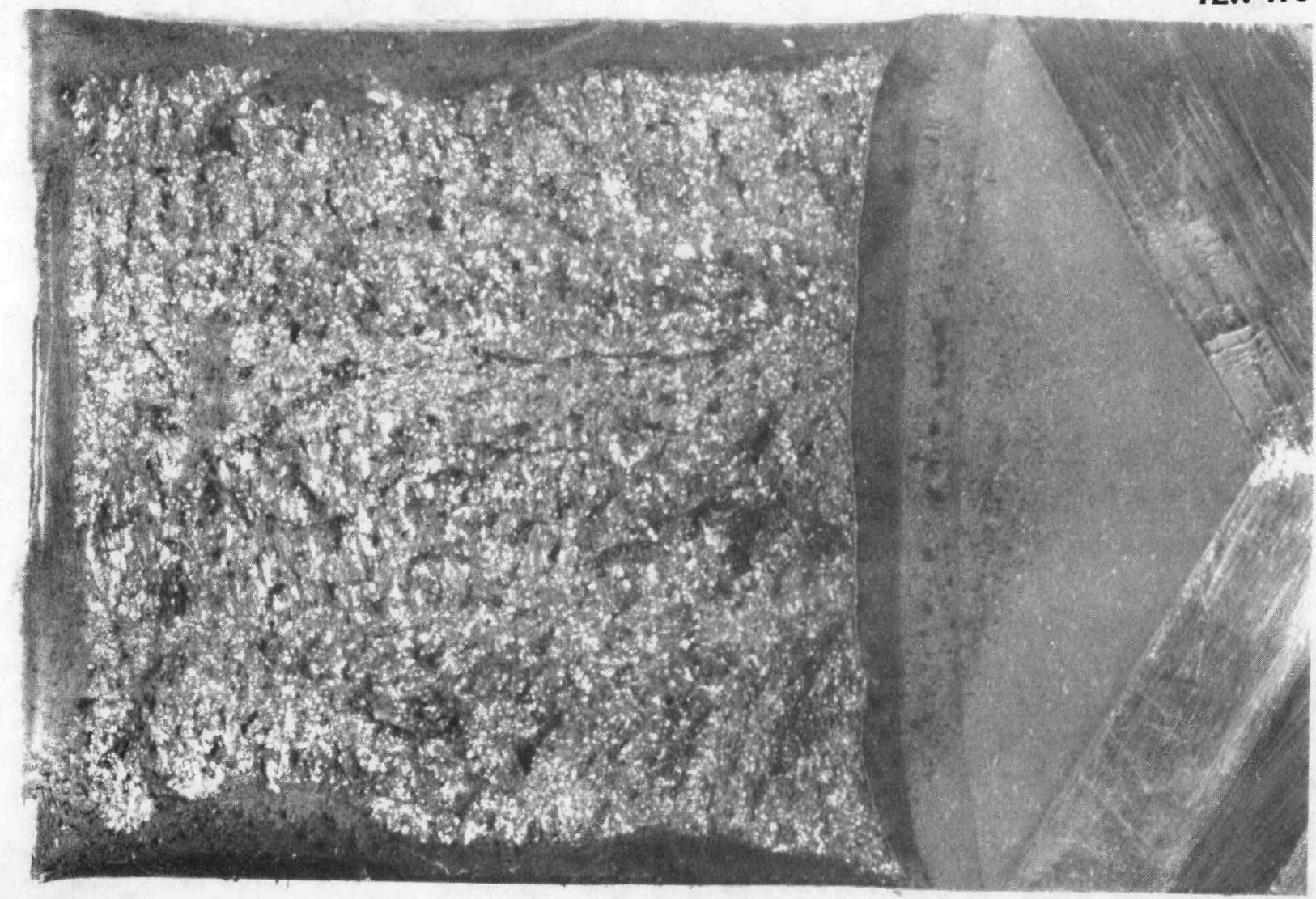


SPECIMEN ID $=72 W 166$

MATERIAL TYPE = MCUWELD

TEST TEMPERATURE $=-15$ DEG $\quad \mathrm{C}$

THICKNESS $=25.4 \mathrm{~mm}$

SIDE GROOUES $=0 \%$

INITIAL CRACK LENGTH $=26.6 \mathrm{~mm}$

MEASURED DUCTILE DELTA-A $=.15 \mathrm{~mm}$

$a / w) i=.525$

$Y$ IELD STRENGTH $=526 \mathrm{MPa}$

FLOW STRENGTH $=586 \mathrm{MPa}$

ESTIMATED YOUNG'S MDDULUS $=208 \mathrm{GPa}$

SPECIMEN CLEAUED AT KQ= $123.8 \mathrm{MPa} S Q R[\mathrm{~m}]$ (NOT UALID KIC)

UALUES AT MAXIMUM LDAD

J-INTEGRAL $=139.1 \mathrm{~kJ} / \mathrm{m}^{\wedge} 2$

$\mathrm{KJ} c=170.1 \mathrm{MPa} \operatorname{SQR}[\mathrm{m}]$

$K$-bet $a-c=94.3 \mathrm{MPa} \operatorname{SQR}[\mathrm{m}]$
UALUES at CLEAUAGE LDAD

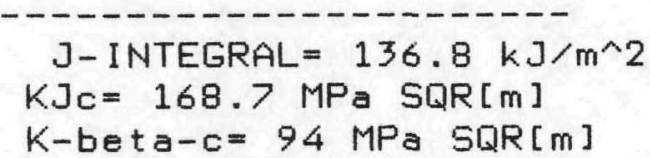




\section{E-48}

ORNL-DWG 94-11514

72W166 -15 DEGREES C

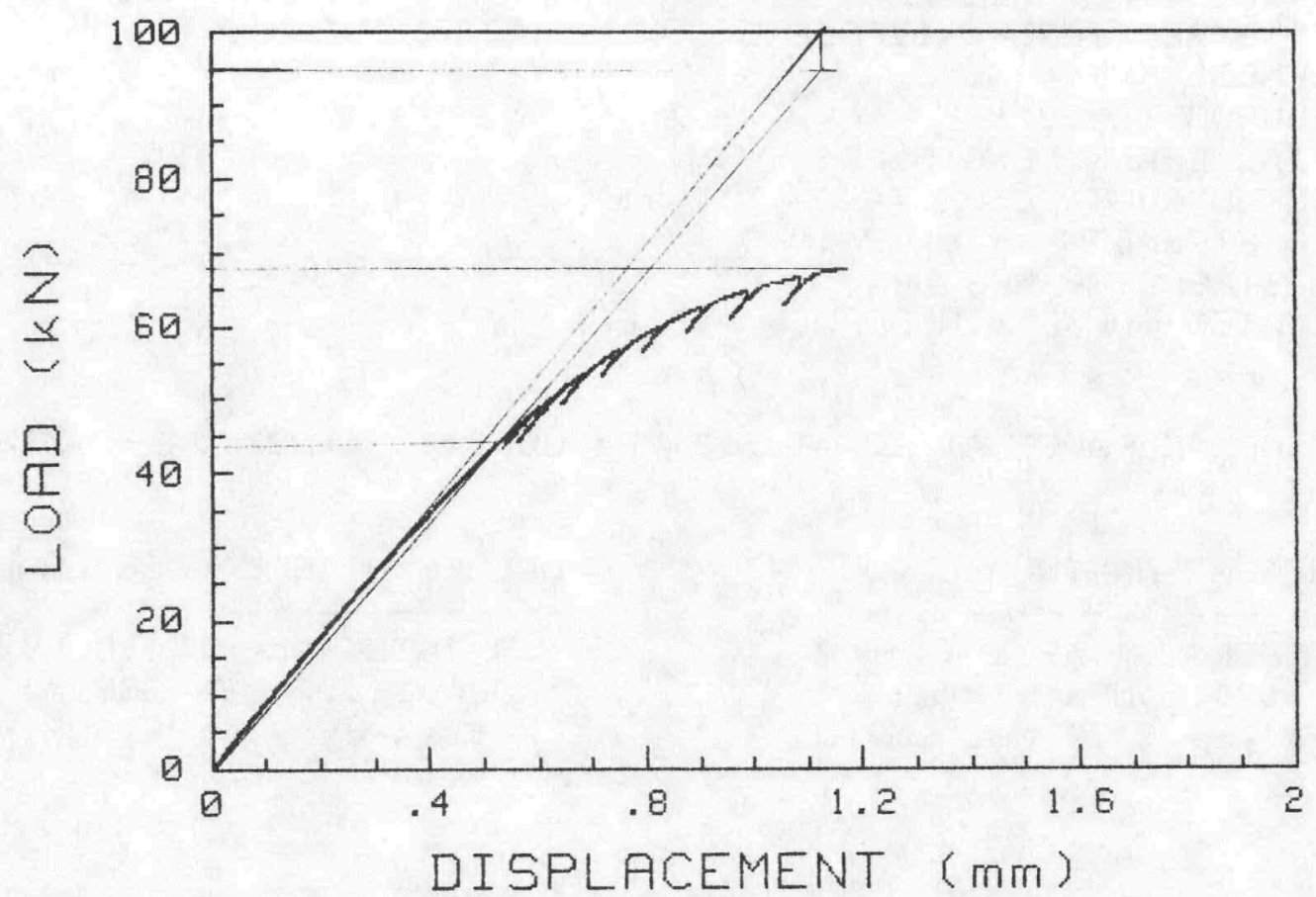

72W-166

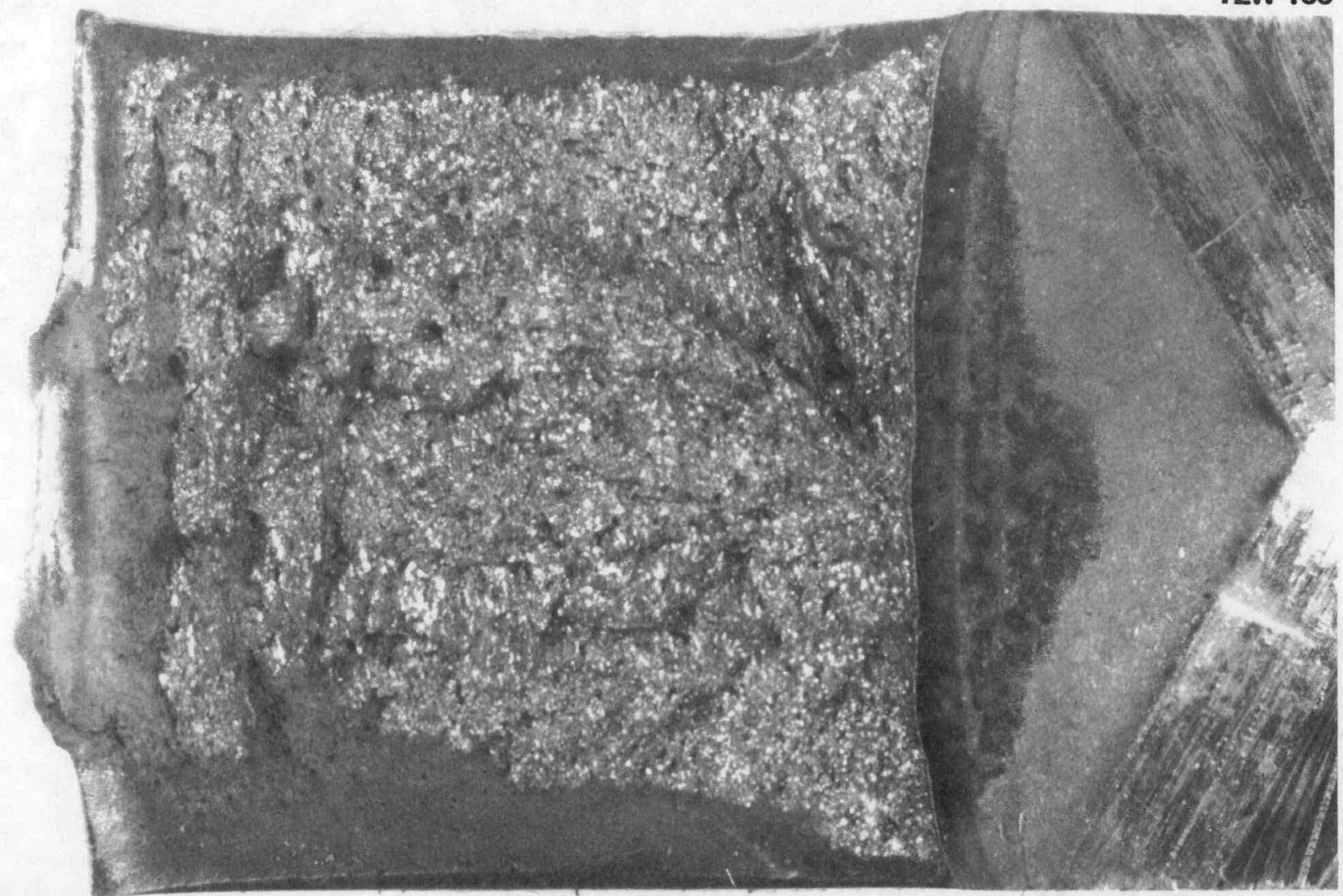


Specimen Crackmouth Deflection (in.)

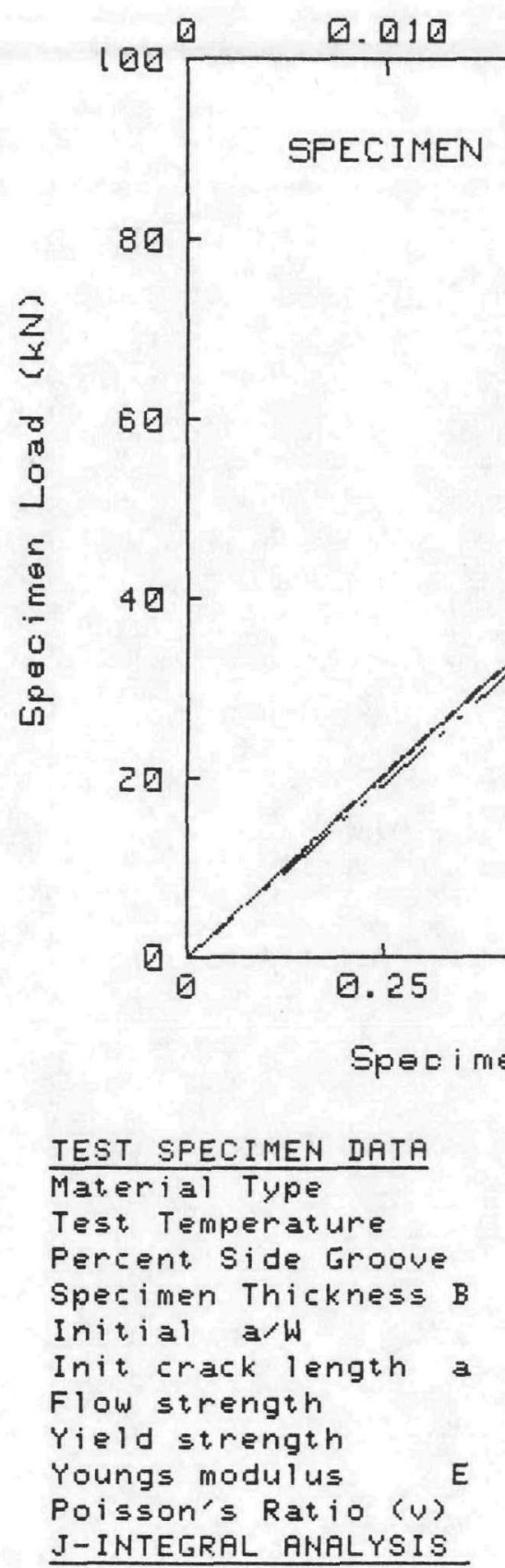

$\mathrm{Je}$

$K j c=\left\langle E J c\left\langle\left(1-w^{2}\right)\right\rangle 1 / 2\right.$

Kc (Beta Corrected)

ASTM E399-81 ANALYSIS

Modified Kic Equation;

Maximum Load $P=60.61 \mathrm{kN}$

$5 \%$ Secant offset $P q=43.35 \mathrm{kN}$

$\mathrm{Kq}$

Validity

$K$ at. Maximum Load
ㅁ. 040 口. 050

Cleavage

R curve $\frac{\text { FRILURE TYPE } A}{\text { (0.15mm exclusion line }}$ 5 (Insufficient for analysis)
20

15

0
0
0
2
2
7
0
0
0

10

要

DATA CORRECTIONS USED FOR J-INTEGRAL DATA

Merkle-Corten correction

Correction to J for crack extension (Deformation-J)

OFFSET LOAD $=0 \mathrm{kM}$ OFFSET DEFLECTION $=.007 \mathrm{~mm}$

Fitted elastic load range $=10 \mathrm{kN}$ TO $25 \mathrm{kN}$

Elastic slope $=8.133 E+01 \mathrm{kN} / \mathrm{mm}$ 


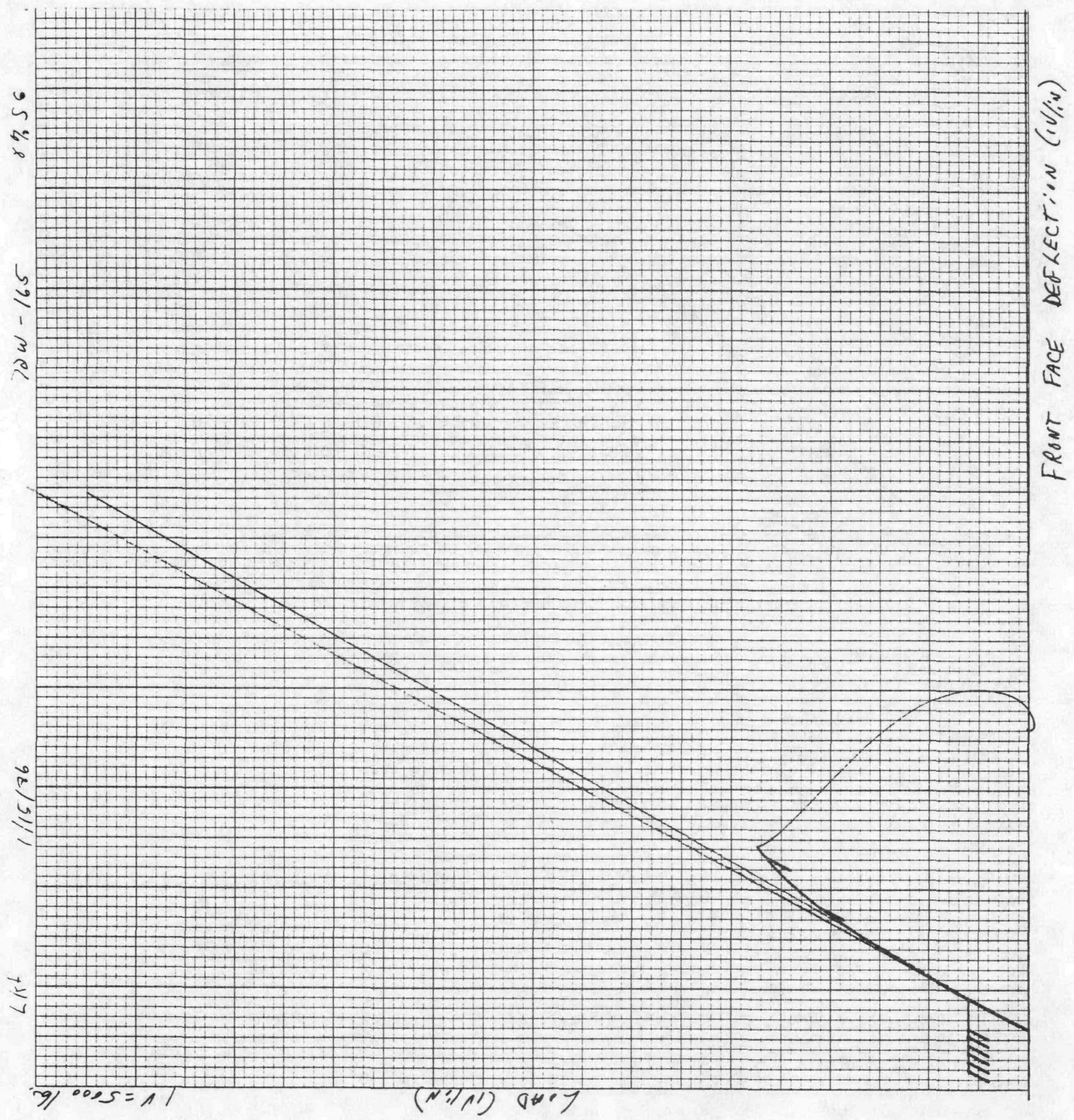

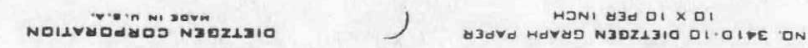


SPECIMEN ID $=72 W 151$

MATERIAL TYPE = MCU WELD

TEST TEMPERATURE $=0$ DEG $C$

THICKNESS $=25.4 \mathrm{~mm}$

SIDE GROOUES = $0 \%$

INITIAL CRACK LENGTH $=26.6 \mathrm{~mm}$

MEASURED DUCTILE DELTA-A $=.13 \mathrm{~mm}$

$a / W) i=.525$

YIELD STRENGTH $=517 \mathrm{MPa}$

FLOW STRENGTH $=575 \mathrm{MPa}$

ESTIMATED YOUNG'S MODULUS $=207 \mathrm{GPa}$

SPECIMEN CLEAUED AT KQ= $121.3 \mathrm{MPa} S Q R[\mathrm{~m}]$ (NOT WALID $K I C$ )

UALUES AT MAXIMUM LDAD

J-INTEGRAL $=124.3 \mathrm{~kJ} / \mathrm{m}^{\wedge} 2$

$K J c=160.5 \mathrm{MPa} S Q R[\mathrm{~m}]$

$K$-bet $a-c=91.2 \mathrm{MPa} \operatorname{SQR}[\mathrm{m}]$
UALUES at CLEaUAGE LDAD

J-INTEGRAL $=124 \mathrm{~kJ} / \mathrm{m}^{\wedge} 2$

$\mathrm{KJ} c=160.3 \mathrm{MPa} \operatorname{SQR}[\mathrm{m}]$

K-beta-c $=91.2 \mathrm{MPa} \operatorname{SQR}[\mathrm{m}]$ 


\section{E-52}

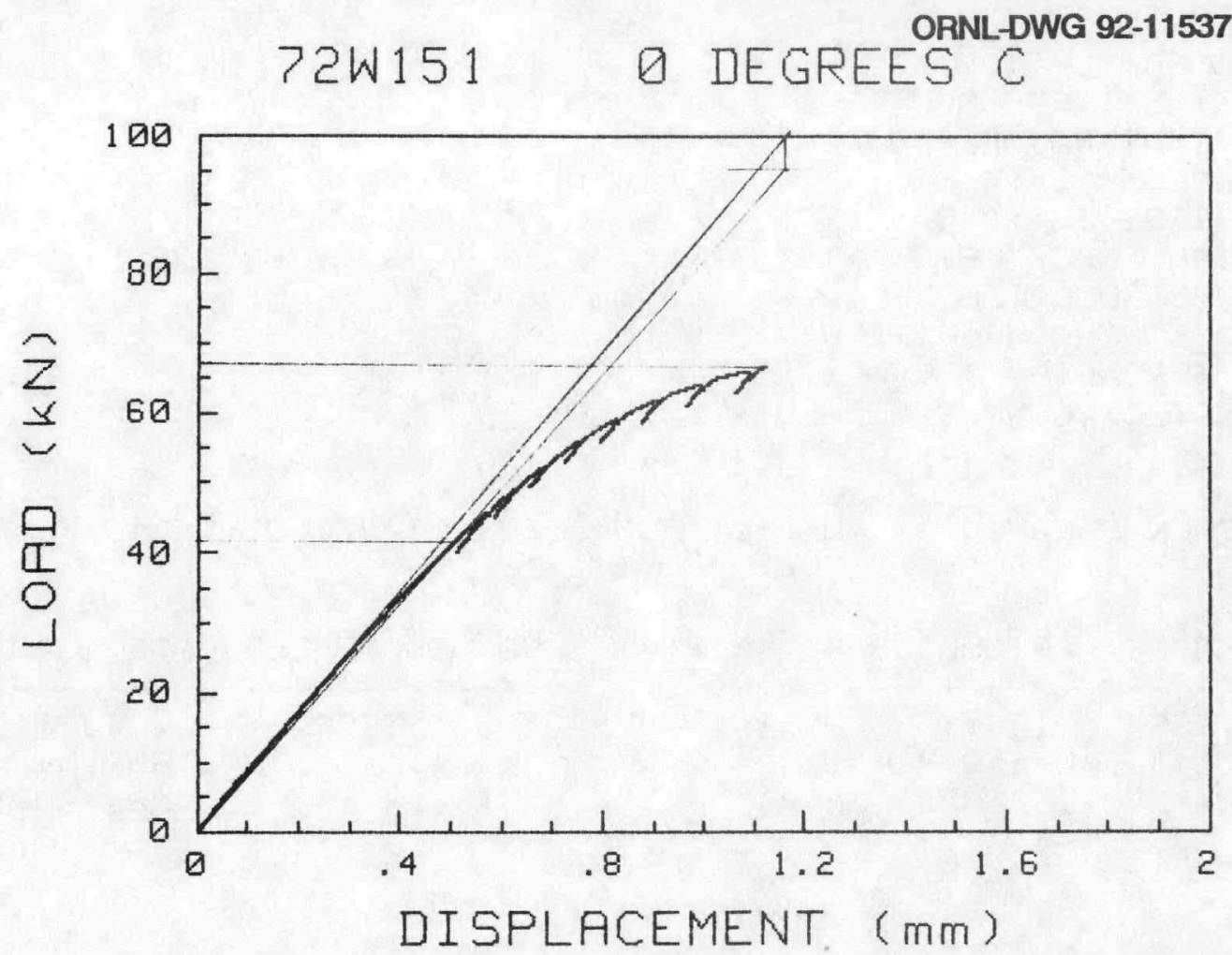

72W-151

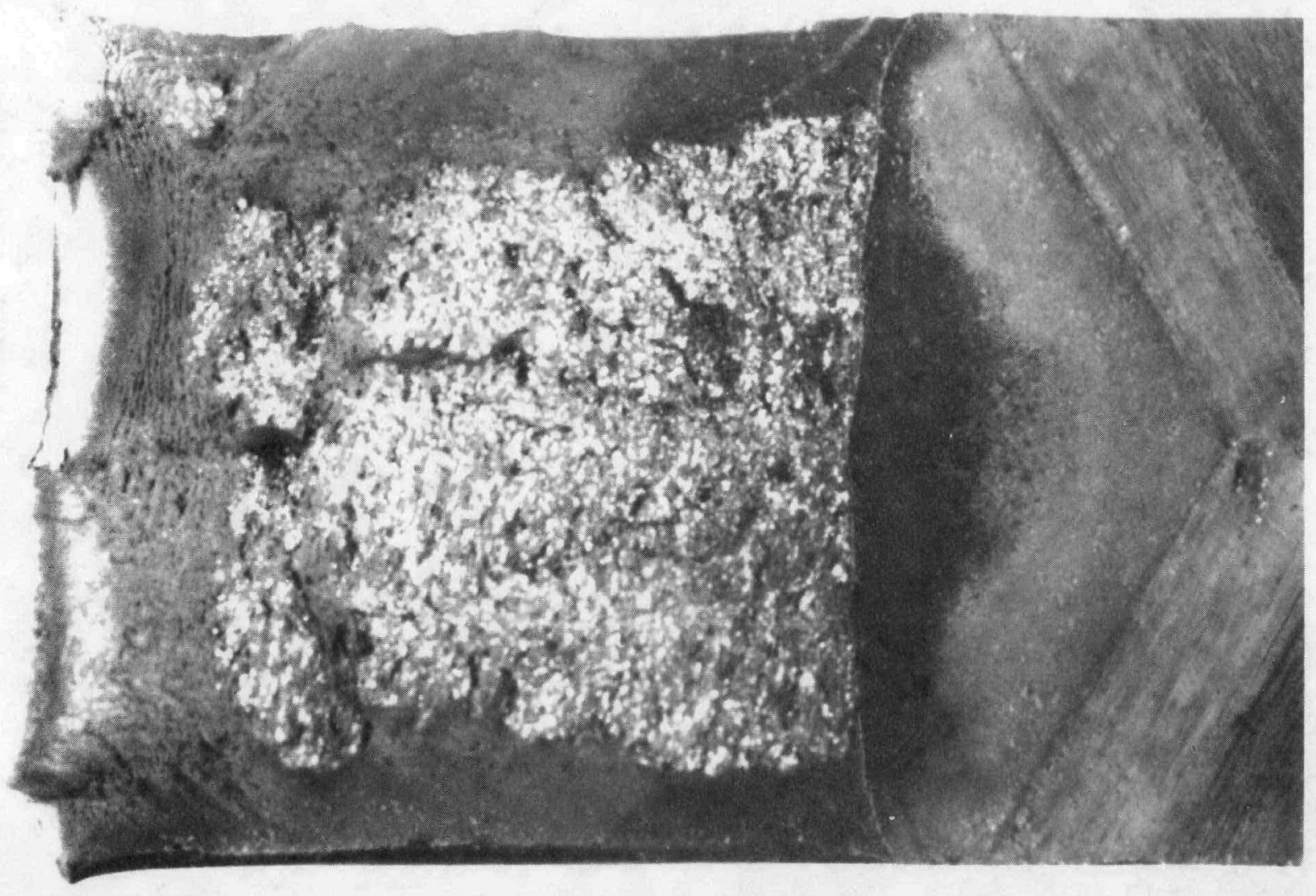

NUREG/CR-5913 
SPECIMEN ID $=72 W 128$

MATERIAL TYPE = MCU WELD

TEST TEMPERATURE $=0$ DEG C

THICKNESS $=25.4 \mathrm{~mm}$

SIDE GRDOUES $=0 \%$

INITIAL CRACK LENGTH $=26.9 \mathrm{~mm}$

$a / W\rangle i=.529$

MEASURED DUCTILE DELTA-A $=.34 \mathrm{~mm}$

$Y$ IELD STRENGTH $=517 \mathrm{MPa}$

FLOW STRENGTH $=575 \mathrm{MPa}$

ESTIMATED YOUNG'S MODLLUS $=207 \mathrm{GPa}$

SPECIMEN CLEAUED AT KQ= $127.5 \mathrm{MPa} S Q R[\mathrm{~m}]$ (NOT WALID KIc)

UALUES AT MAXIMUM LDAD

J-INTEGRAL $=209.2 \mathrm{~kJ} / \mathrm{m}^{\wedge} 2$

$K J c=208.2 \mathrm{MPa} \operatorname{SQR}[\mathrm{m}]$

$K$-beta-c $=101.4 \mathrm{MPa} \operatorname{SQR}[\mathrm{m}]$
WALUES AT CLEAUAGE LDAD

J-INTEGRAL $=209.8 \mathrm{~kJ} / \mathrm{m}^{\wedge} 2$

$K J c=208.5 \mathrm{MPa} \operatorname{SQR}[\mathrm{m}]$

$K$-beta-c= $101.5 \mathrm{MPa} \operatorname{SQR}[\mathrm{m}]$ 
E-54

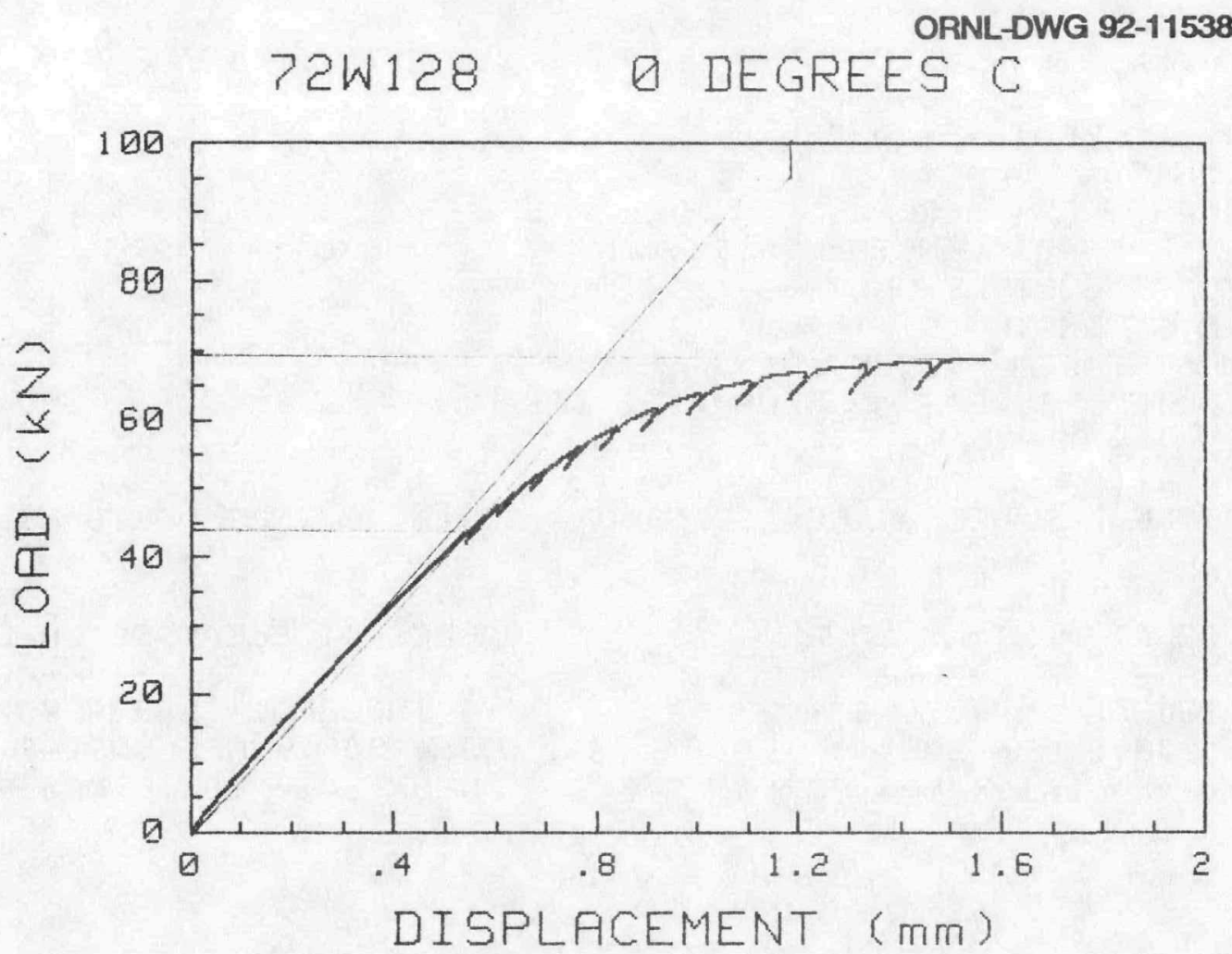

72W-128

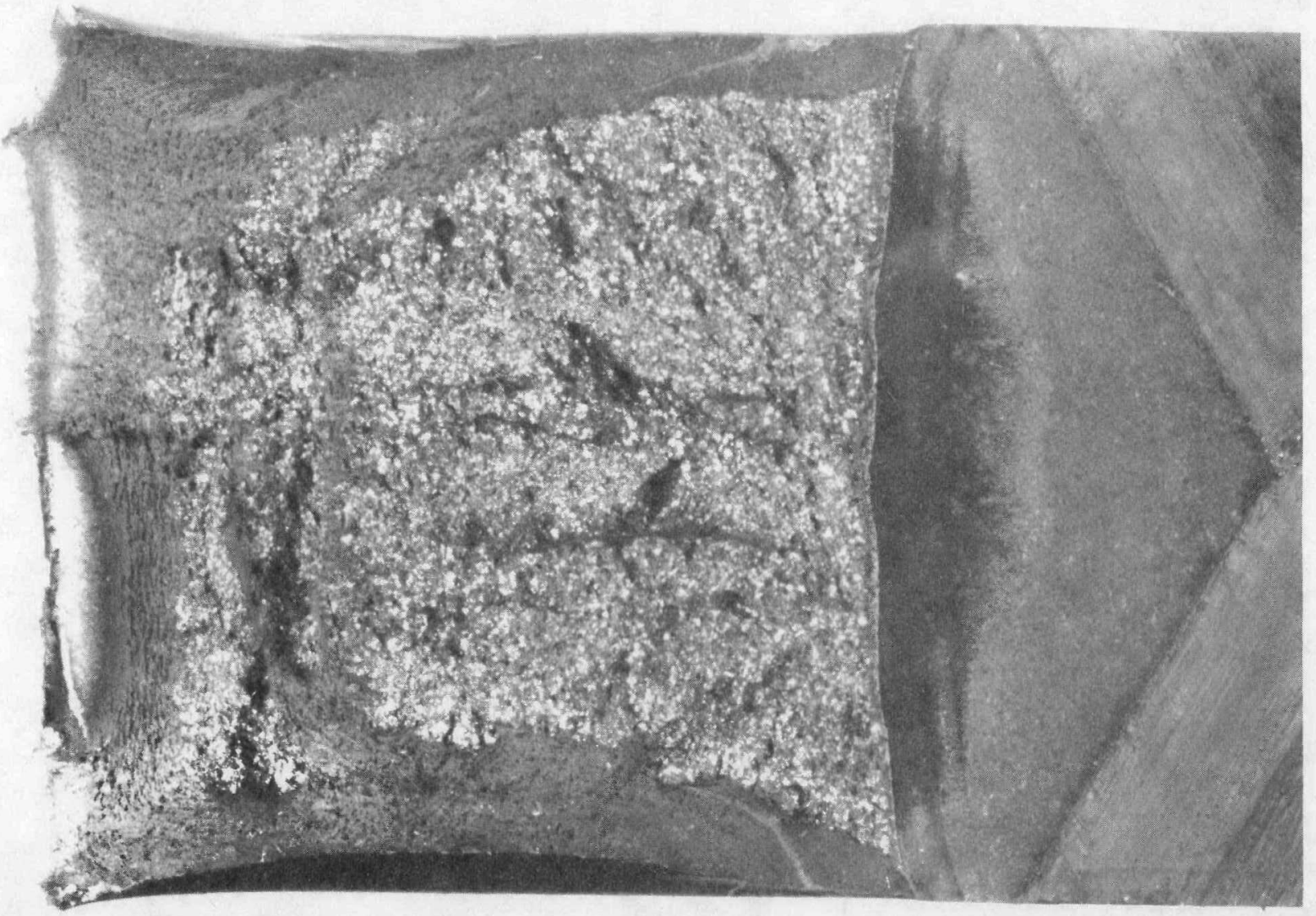


Specimen Crackmouth Deflectian (in.)

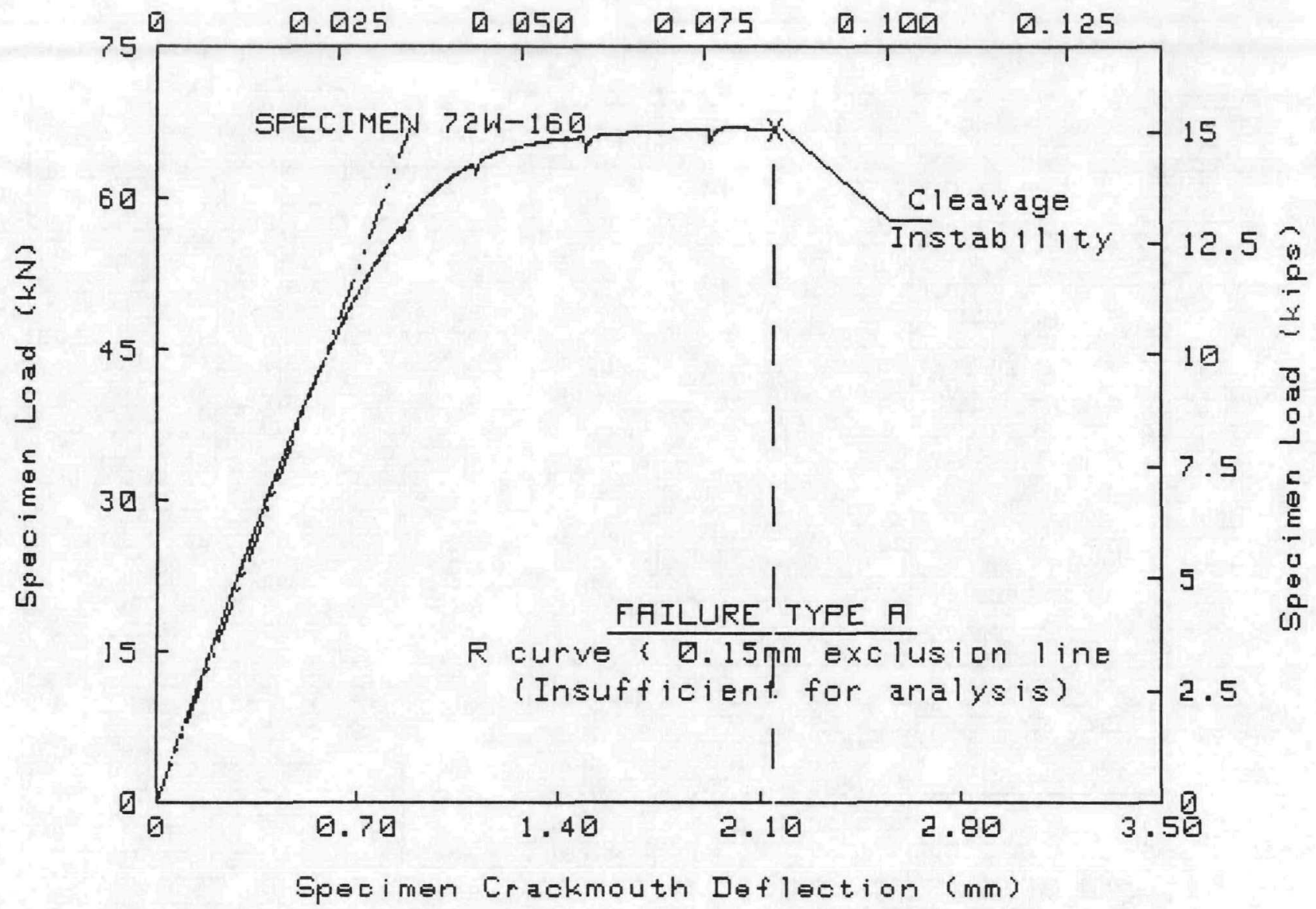

TEST SFECIMEN DATA

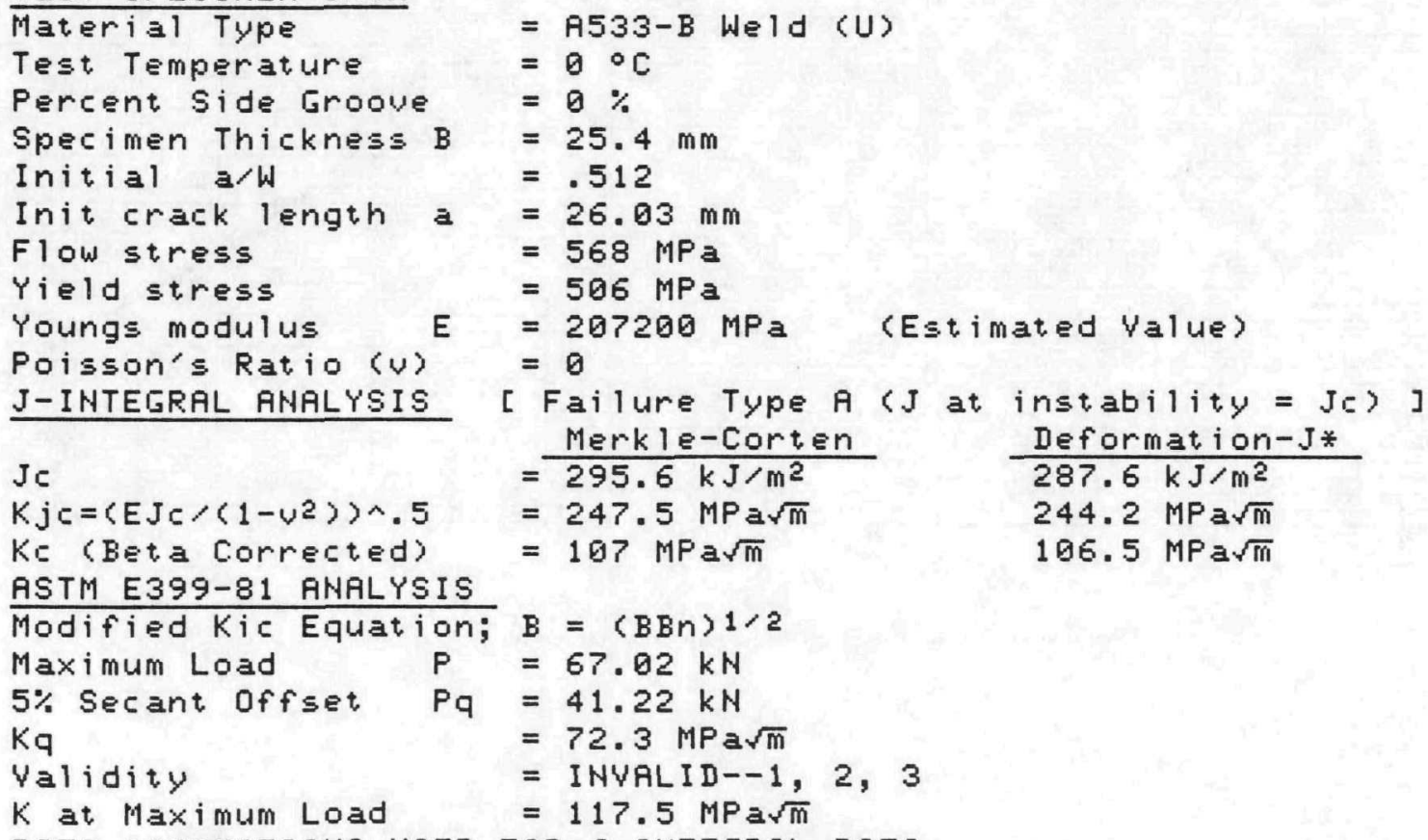

IATA CORRECTIONS USED FOR J-INTEGRAL DATA

Merkle-Corten correction

Correction to J for crack extension (Deformation-J)

OFFSET LOAD $=\square \mathrm{kN}$ DFFSET DEFLECTION $=.015 \mathrm{~mm}$

Fitted elastic load range $=5 \mathrm{kN}$ TO $25 \mathrm{kN}$

Elastic slope $=8.007 E+01 \mathrm{kN} / \mathrm{mm}$ 


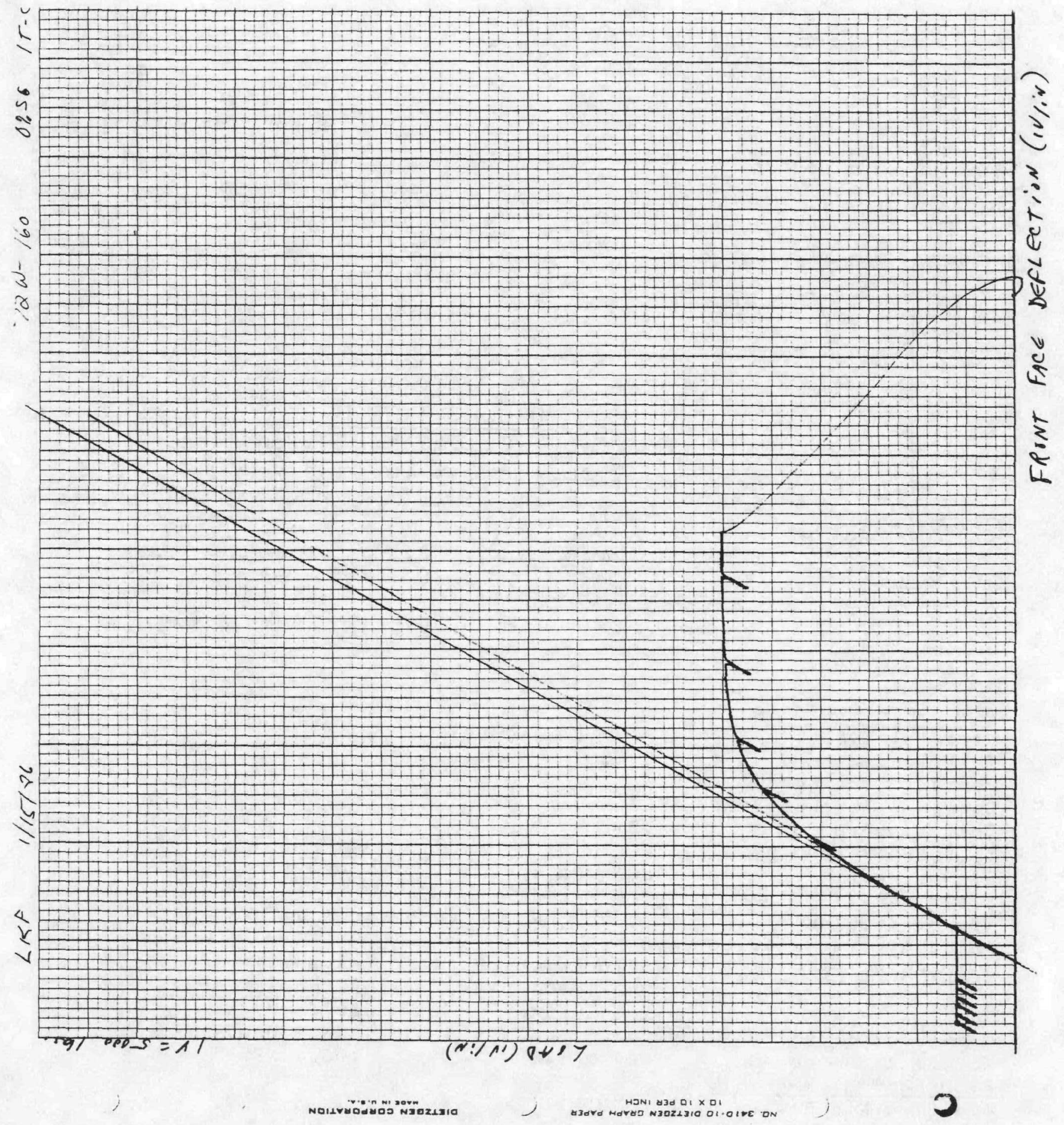


Spec imen Loadline Deflection (in.)

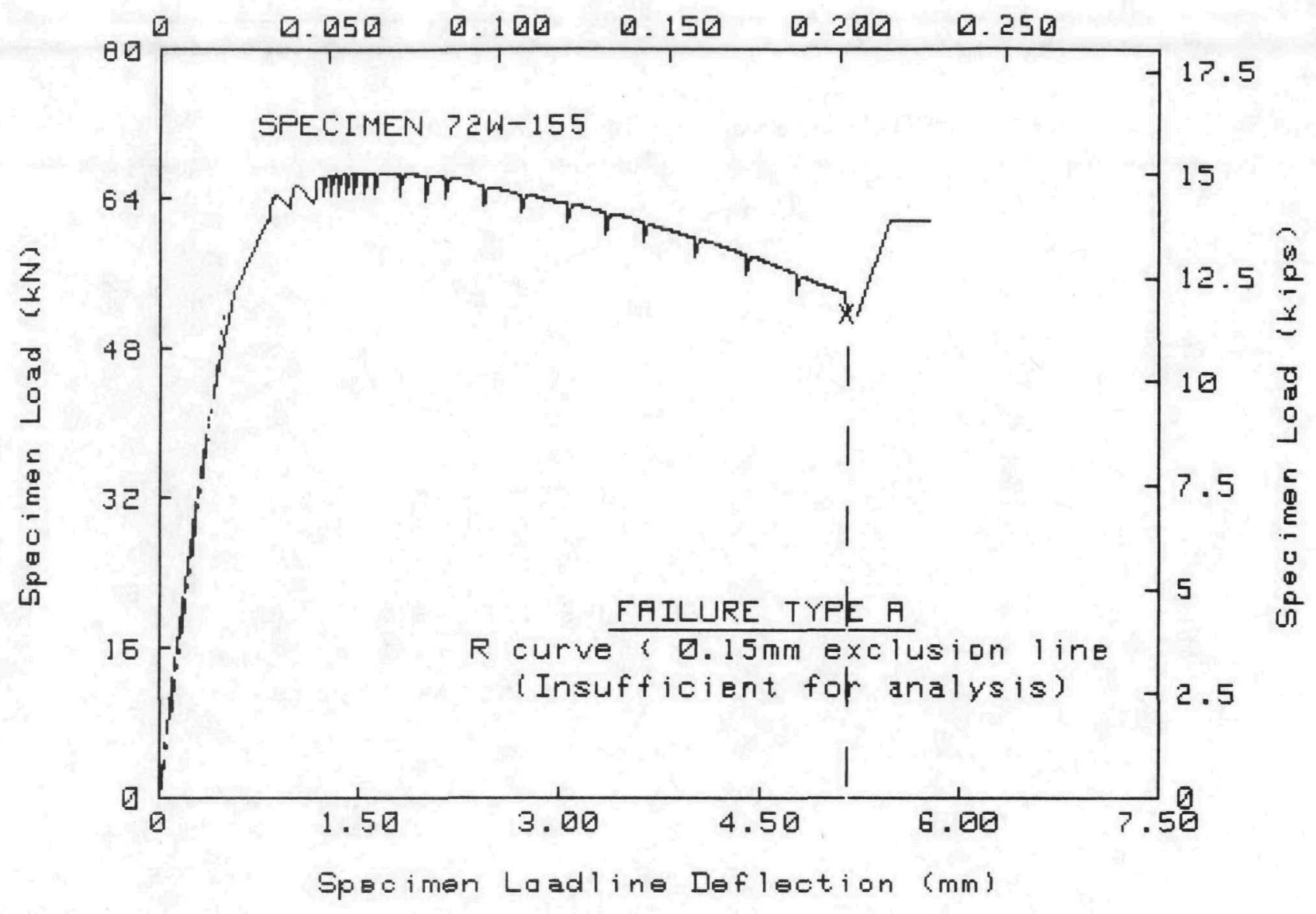

TEST SPECIMEN DATA

Material Type
Test Temperature
Percent Side Groove
Specimen Thickness B
Initial a/W
Init crack length a
Flow stress
Yield stress
Youngs modulus
Poisson's Ratio (U)
J-INTEGRAL RNALYSIS

$=A 533-B$ Weld (U)

$=0{ }^{\circ} \mathrm{C}$

$=0 \%$

$=25.4 \mathrm{~mm}$

$=.521$

$=26.47 \mathrm{~mm}$

$=568 \mathrm{MPa}$

$=506 \mathrm{MPa}$

= 207200 MPa (Estimated Value)

J-INTEGRAL ANALYSIS

$\mathrm{Jc}$

$=0$

$K j c=\left(E J c /\left(1-v^{2}\right)\right\rangle \wedge .5$

Ke (Beta Corrected)

ASTM E399-81 ANALYSIS

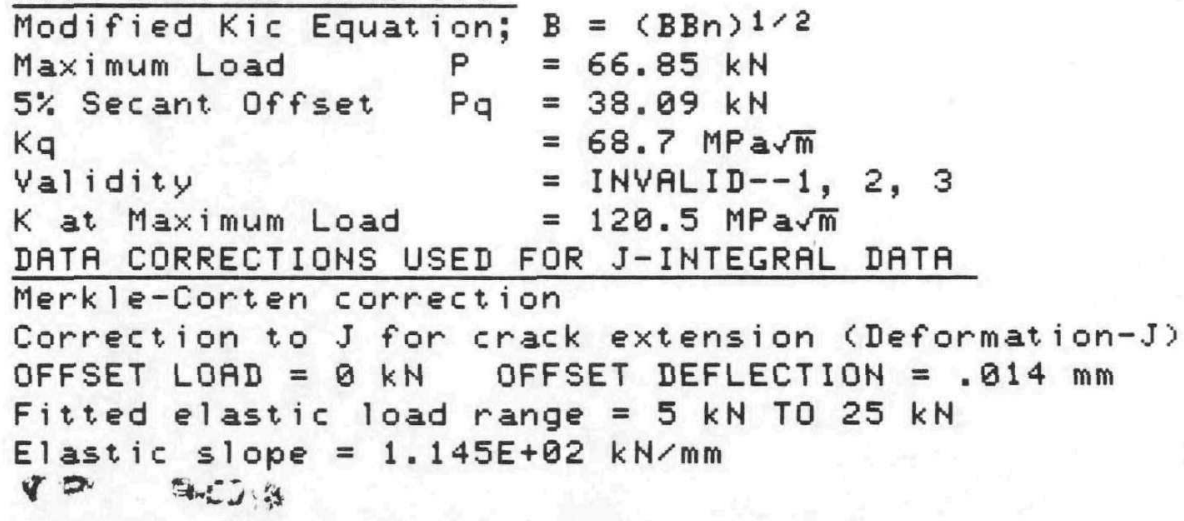

$\begin{array}{lll}\text { Merkle-Corten } & & \text { Deformation-J* } \\ = & & 1123.1 \mathrm{~kJ} / \mathrm{m}^{2} \\ =1367.4 \mathrm{~kJ} / \mathrm{m}^{2} & & 482.4 \mathrm{MPa} \sqrt{\mathrm{m}} \\ =135.9 \mathrm{MPa} \sqrt{\mathrm{m}} & 136.5 \mathrm{MPa} \sqrt{\mathrm{m}}\end{array}$


Specimen Crackmauth Deflection (in.)

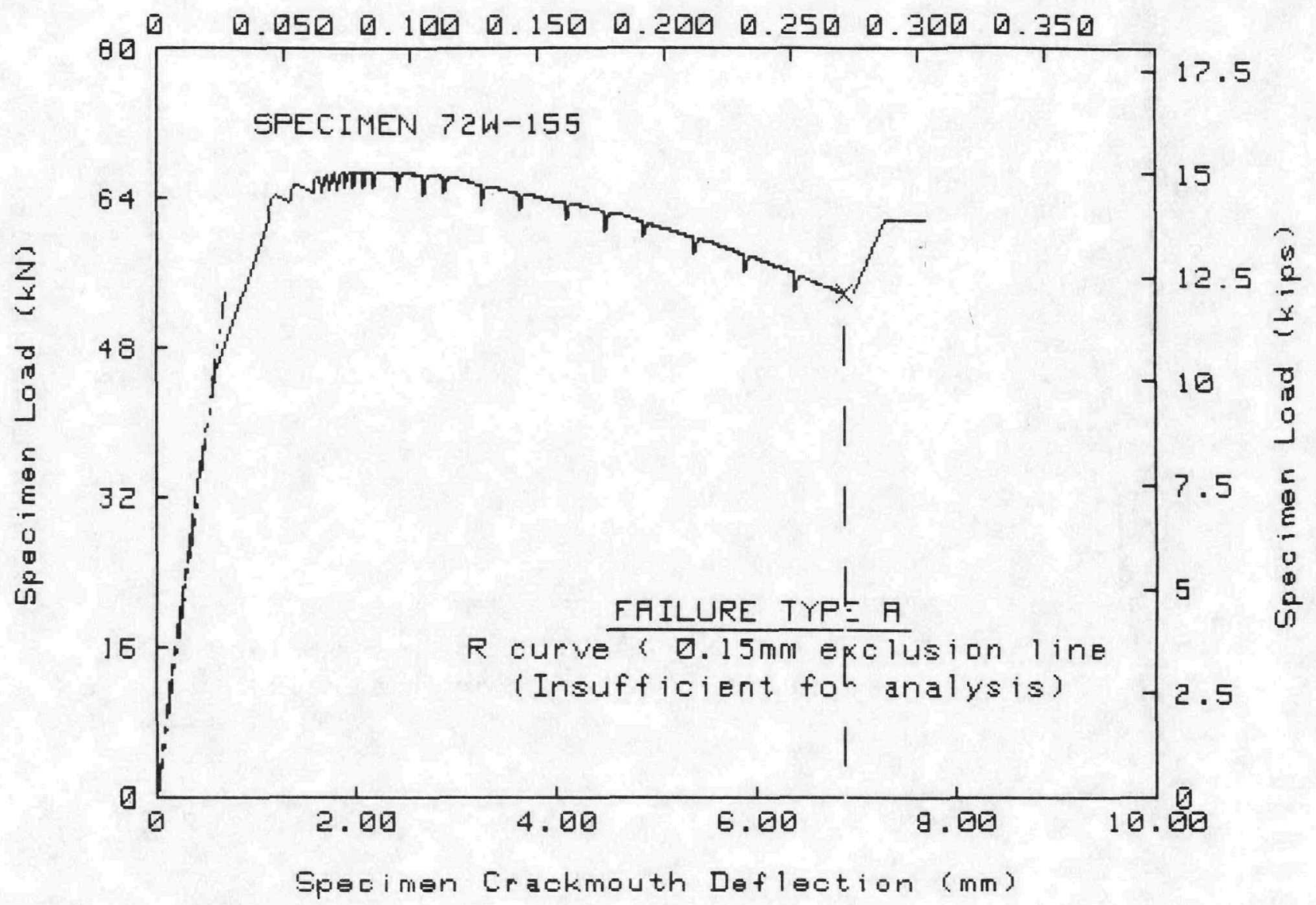

TEST SFECIMEN IATA

Material Type
Test Temperature
Percent Side Groove
Specimen Thickness B
Initial a/W
Init crack length a
Flow stress
Yield stress
Youngs modulus (U)
Poisson's Ratio (UST
J-INTEGRAL ANALYSIS

Je

$K j c=\left(E J c<\left(1-v^{2}\right)\right) \wedge .5$

$=A 533-B$ Weld (U)

$=0 \circ \mathrm{C}$

$=0 \%$

$=25.4 \mathrm{~mm}$

$=.521$

$=26.47 \mathrm{~mm}$

$=568 \mathrm{MPa}$

$=506 \mathrm{MPa}$

= $207200 \mathrm{MPa}$ (Estimated Value)

$$
=0
$$

[ Failure Type $A$ ( $J$ at instability = JE) ]

$$
\begin{aligned}
& =\frac{\text { Merkle-Corten }}{1082.7 \mathrm{~kJ} / \mathrm{m}^{2}} \\
& =473.7 \mathrm{MPa} \sqrt{\mathrm{m}} \\
& =135.6 \mathrm{MPa} \sqrt{\mathrm{m}}
\end{aligned}
$$

$\frac{\text { Deformat ion }-\mathrm{J} *}{1103.2 \mathrm{~kJ} / \mathrm{m}^{2}}$

$478.2 \mathrm{MPa} \sqrt{\mathrm{m}}$

$136.1 \mathrm{MPa} \cdot \overline{\mathrm{m}}$

ASTM E399-81 ANALYSIS

Modified Kic Equation;

Modified Kic Equation; $B=\langle$ B Bn>1/2
Maximum Load

$5 \%$ secant offset $P Q=39.59 \mathrm{kN}$

$\mathrm{Kq}$

Validity

$K$ at Maximum Load

$=66.85 \mathrm{kN}$
$=39.59 \mathrm{kN}$

$=71.4 \mathrm{MPa} \sqrt{\mathrm{m}}$

= INVALID--1, 2, 3

$=120.5 \mathrm{MPa} \sqrt{\mathrm{m}}$

DATA CORRECTIONS USED FOR J-INTEGRAL DATA

Merkle-Corten correction

Correction to $J$ for crack extension (Deformation-J)

OFFSET LOAD $=0 \mathrm{kN}$ OFFSET DEFLECTION $=.021 \mathrm{~mm}$

Fitted elastic load range $=5 \mathrm{kN}$ TO $25 \mathrm{kN}$

Elastic slope $=8.202 E+01 \mathrm{kN} / \mathrm{mm}$ 


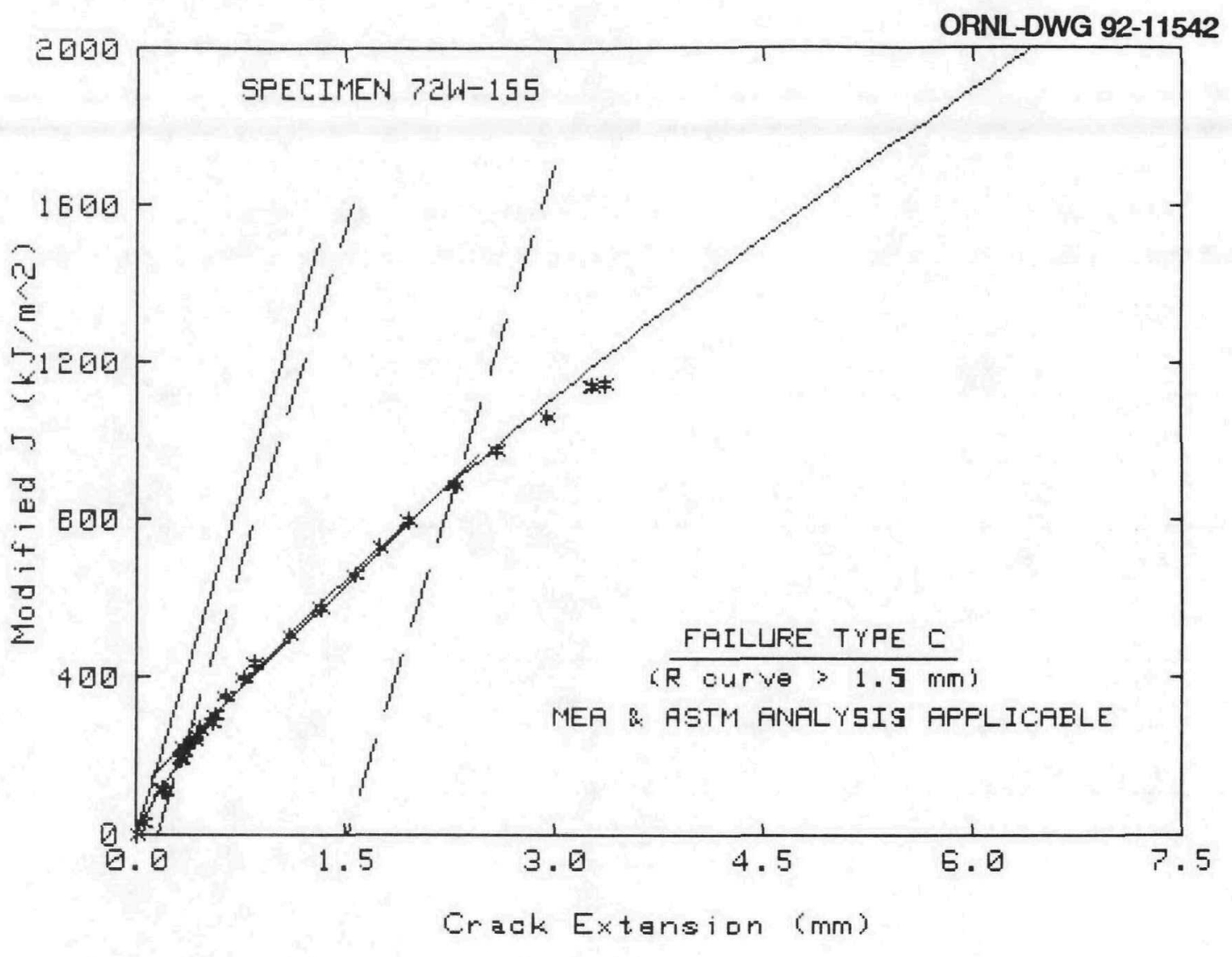

TEST SPECIMEN DATA

\begin{tabular}{|c|c|c|}
\hline aterial Typ & & $=$ HSST $5 \mathrm{th}$ WEL \\
\hline Test Tempera & ure & $=0 \mathrm{C}$ \\
\hline ercent Side & Groove & $=0 \%$ \\
\hline pecimen Thi & iess & $=25.4 \mathrm{~mm}$ \\
\hline nit erack 1 & $n$ & $=26.47 \mathrm{~mm}$ \\
\hline inal erack & en & $=3 \mathrm{~m}, 24 \mathrm{~mm}$ \\
\hline low stress & & $=569 \mathrm{MPa}$ \\
\hline$g=$ modul & & $=20>00 \mathrm{MPa}$ \\
\hline ER LAW IA & $J=$ & $=c(-1 a a)$ \\
\hline $\mathrm{J}(\mathrm{E} \mathrm{J} / \mathrm{T}=8.8)$ & & $=1458.1 \mathrm{~kJ}$ \\
\hline Jic & & $=195.6 \mathrm{~kJ} / \mathrm{m}^{\wedge} 3$ \\
\hline & & $.3 \mathrm{MPa}$ \\
\hline & $\mathrm{H}$ & .7779 \\
\hline$i c i e n t$ & $\mathrm{c}$ & $471.6 \mathrm{~kJ} /$ \\
\hline T (average) & & $=220$ \\
\hline
\end{tabular}

LEAST SQUARE LINEAR LINE (ASTM) J $=M$ (DElta a) + E

Jic

$\mathrm{K}, \mathrm{jc}$

$=161.6 \mathrm{~kJ} / \mathrm{m}^{\wedge}$
$=183 \mathrm{MPa} \backslash \mathrm{m}$

Slope $M \quad=347354.4 \mathrm{~kJ} / \mathrm{m}^{\wedge} 3$

Interiept B $\quad=112.8 \mathrm{~kJ} / \mathrm{m}^{\wedge} 2$

$T$ (ASTM) $\quad=218$

Validity (Jic) = VALID

Validity (R-curve) = INVALID--1, 3 (.5 vs.36)

$\mathrm{J}$ maximum allowed $=699 \mathrm{~kJ} / \mathrm{m} 22$ (Jmax=Bnet $* \mathrm{Flow} s t r e s s / 20$ )

Delta a max. allowed $=2.43 \mathrm{~mm}$ (Delta a max $=0.1$ *bo)

Final Delta a $\quad=3.26 \mathrm{~mm}$

Poisson's Ratio $u\rangle=0$

NUREG/CR-5913 


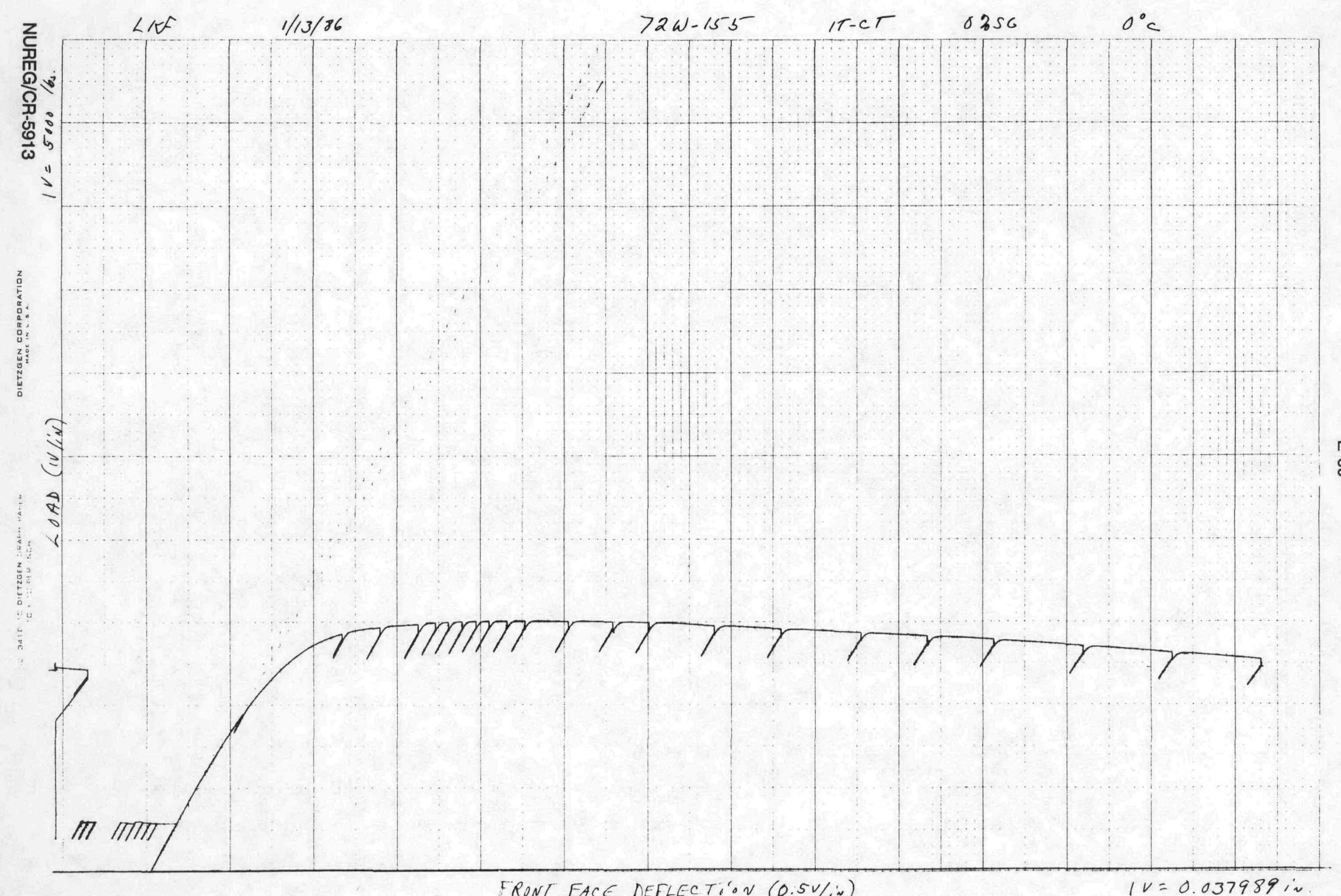


Specimen Crackmouth Deflection (in.)

ORNL-DWG 92-11543

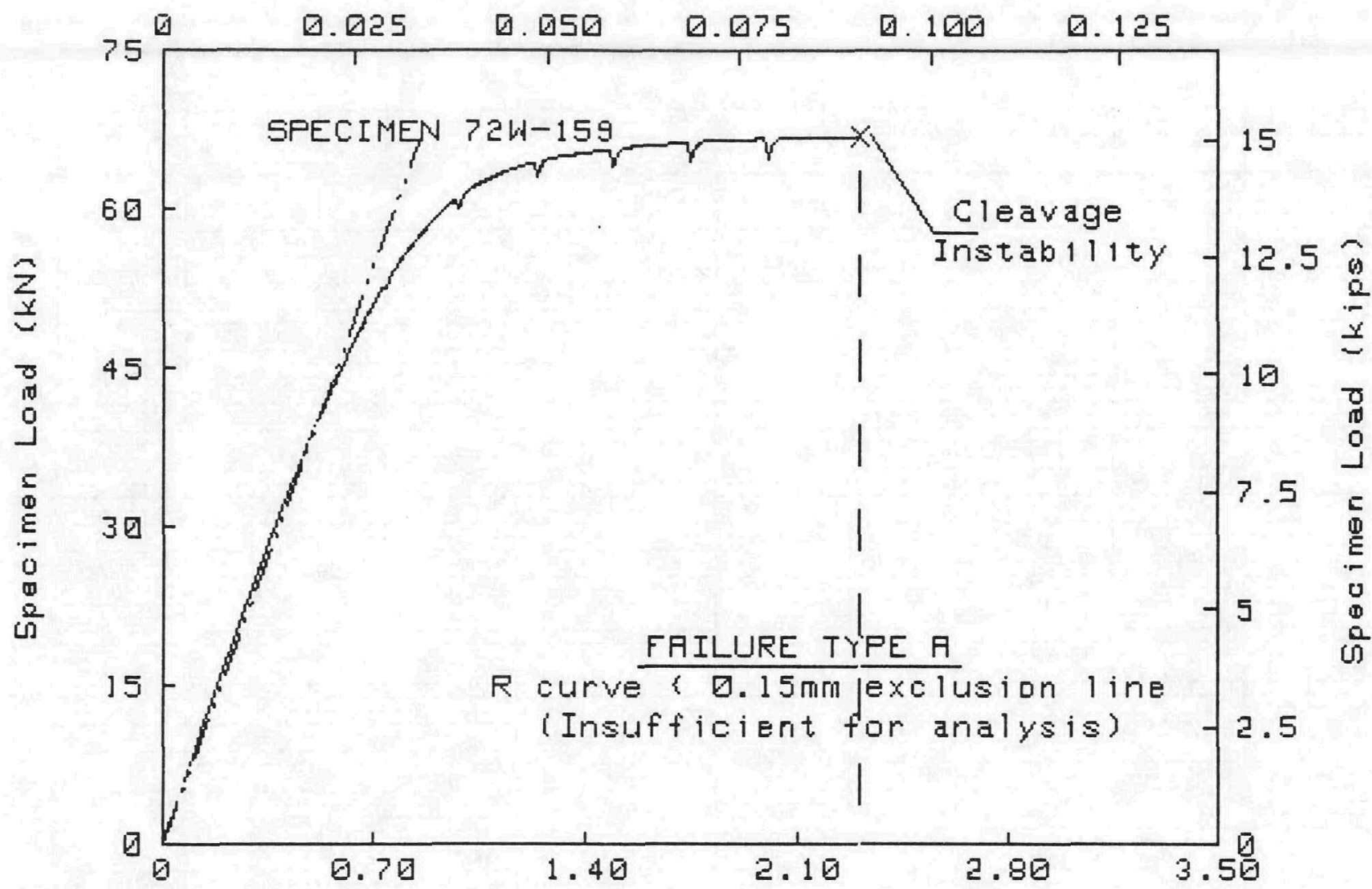

Specimen Crackmouth Defleotion ( $m m$ )

TEST SFECIMEN DATA

Material Type

$=A 533-B$ We $d d$ (U)

Test Temperature

$=0{ }^{\circ} \mathrm{C}$

Percent Side Groove $=0 \%$

Specimen Thickness B

Initial a/w

$=25.4 \mathrm{~mm}$

Init crack length a

Flow stress

Yield stress

$=.52$

$=26.39 \mathrm{~mm}$

$=568 \mathrm{MPa}$

$=506 \mathrm{MPa}$

Youngs modulus E

$=207200 \mathrm{MPa}$

Poisson's Ratio (u)

$=0$

J-INTEGRAL ANALYSIS

Je

[ Failure Type $A$ ( $J$ at instability = JC) ]

$$
\begin{aligned}
& =\frac{\text { Merkle-Corten }}{327.8 \mathrm{~kJ} / \mathrm{m}^{2}} \\
& =260.6 \mathrm{MPa} \sqrt{\mathrm{m}}
\end{aligned}
$$

Deformat ion- $J *$

$=109.2 \mathrm{MPa} \sqrt{\mathrm{m}}$

(Estimated Value〉

Ke 〈Beta Corrected)

ASTM E399-81 ANALYSIS

Modified Kic Equation; $B=(B B n) 1 / 2$

Maximum Load $P=66.99 \mathrm{kN}$

$5 \%$ Secant offset $\mathrm{Pq}=39.82 \mathrm{kN}$

$\mathrm{Kq}$

Validity

$=71.4 \mathrm{MPa} \sqrt{\mathrm{m}}$

$K$ at Maximum Load

$=$ INVALID $--1,2,3$

$=120.2 \mathrm{MPa} \sqrt{\mathrm{m}}$

DATA CORRECTIONS USED FOR J-INTEGRAL IATA

Merkle-Corten correction

Correction to J for crack extension (Deformation-J)

OFFSET LOAD $=9 \mathrm{kN}$ OFFSET DEFLECTION $=.006 \mathrm{~mm}$

Fitted elastic load range $=5 \mathrm{kN} \mathrm{TO} 25 \mathrm{kN}$

Elastic slope $=8.257 E+01 \mathrm{kN} / \mathrm{mm}$ 


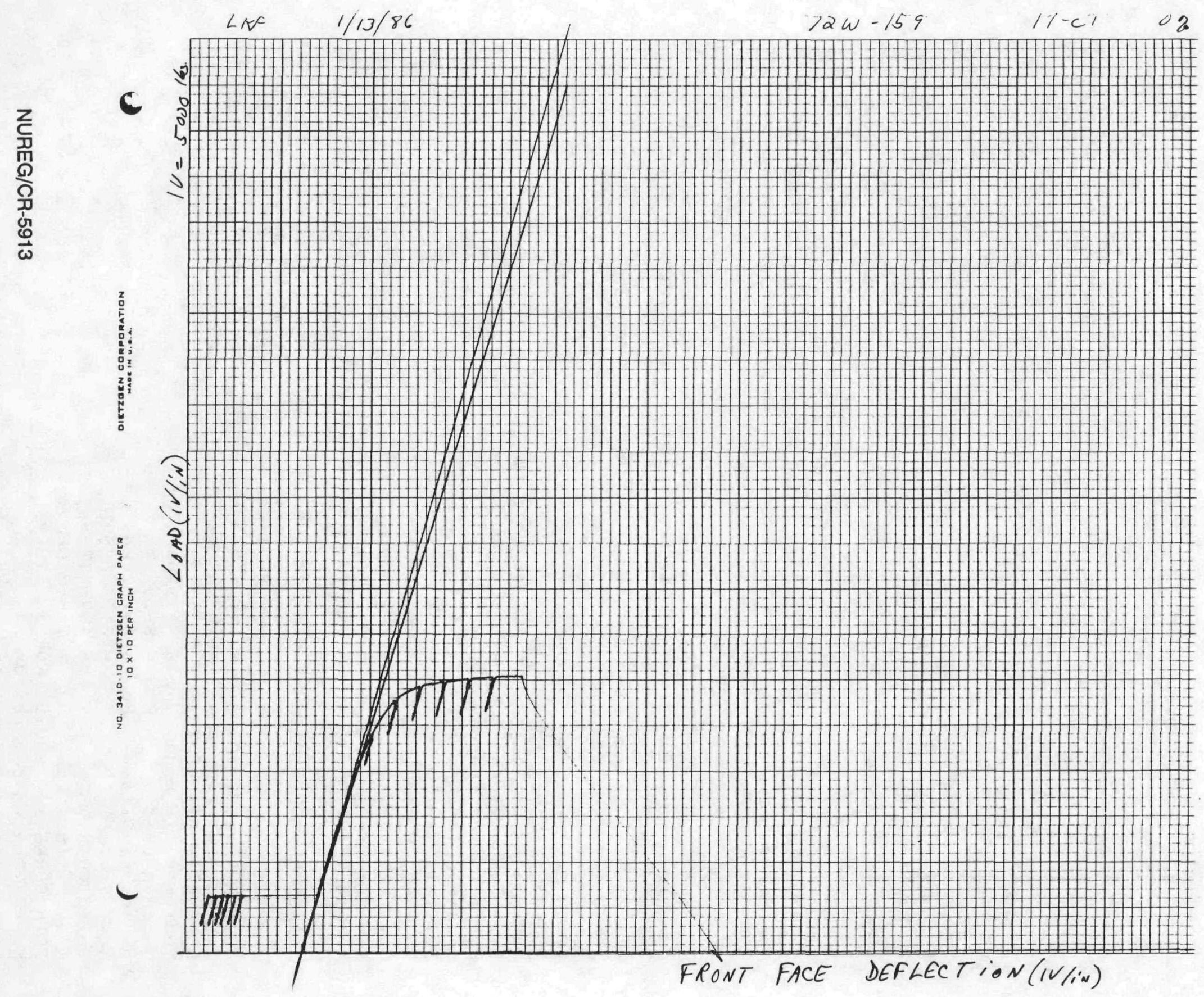


SPECIMEN ID = 72W111

TEST TEMPERATURE $=23$ DEG L

THICKNESS $=25.4 \mathrm{~mm}$

SIDE GRODUES $=0 \%$

INITIAL CRACK LENGTH $=26.4 \mathrm{~mm}$

MEASIJRED DUCTILE DELTA-A $=.92 \mathrm{~mm}$

$a / W\rangle i=.52$

YIELD STRENGTH $=501 \mathrm{MPa}$

FLOW STRENGTH $=556 \mathrm{MPa}$

ESTIMATED YOUNG'S MODULUS $=206$ GPa

SPECIMEN CLEAUED AT KQ= $121 \mathrm{MPa} S D R[\mathrm{~m}]$ (NOT UALID KIc)

UALUES AT MAXIMUM LOAD

WALUES at CLEAUAGE LDAD

J-INTEGRAL $=271.1 \mathrm{~kJ} / \mathrm{m}^{\wedge} 2$

$K \cdot] c=236.2 \mathrm{MPa} S Q R[\mathrm{~m}]$

$K$-bet $a-c=104.5 \mathrm{MPa} \operatorname{SQR}[\mathrm{m}]$

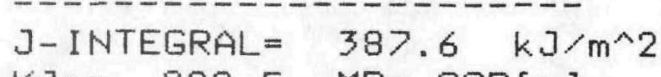

$K J c=282.5 \mathrm{MPa} S Q R[\mathrm{~m}]$

$K$-beta-c= $111.8 \mathrm{MPa}$ SQR $[\mathrm{m}]$

POWER LAW PRDCEDURE

$\mathrm{JIC}=162.4 \mathrm{k} \cdot \mathrm{J} / \mathrm{m}^{\wedge} 2$

$K-J I c=182.9 \quad M P a \operatorname{SGR}[\mathrm{m}]$

POLER $=.7588$

CDEFFICIENT $=408.9 \mathrm{~kJ} / \mathrm{m}^{\wedge} 2$

$T$ (AUERAGE) $=201$

JI-R CURUE CALCULATIONS

了 $($ 回了 $/ T=8.8)=11>5.9 \mathrm{~kJ} / \mathrm{m}^{\wedge} 2$

POWER $=\quad .7342$
$J-M A X$ ALLOWED $=676.2 \mathrm{~kJ} / \mathrm{m}^{\wedge} 2$

RCURUE INUALID, 2
ASTM LINEAR PRDCEDURE

-

$J I_{c}=87.9 \mathrm{~kJ} / \mathrm{m}^{\wedge} 2$

$K-J I C=134.5 \mathrm{MPa} S Q R[\mathrm{~m}]$

$S L D P E=357781 \mathrm{~kJ} / \mathrm{m}^{\wedge} 3$

INTERCEPT $=59.6 \mathrm{~kJ} / \mathrm{m}^{\wedge} 2$

$T(A S T M)=239$

UALID, E813
COEFFICIENT $=402.2 \mathrm{~kJ} / \mathrm{m}^{\wedge} 2$

DELTA-A MAX ALLOWED $=2.43 \mathrm{~mm}$

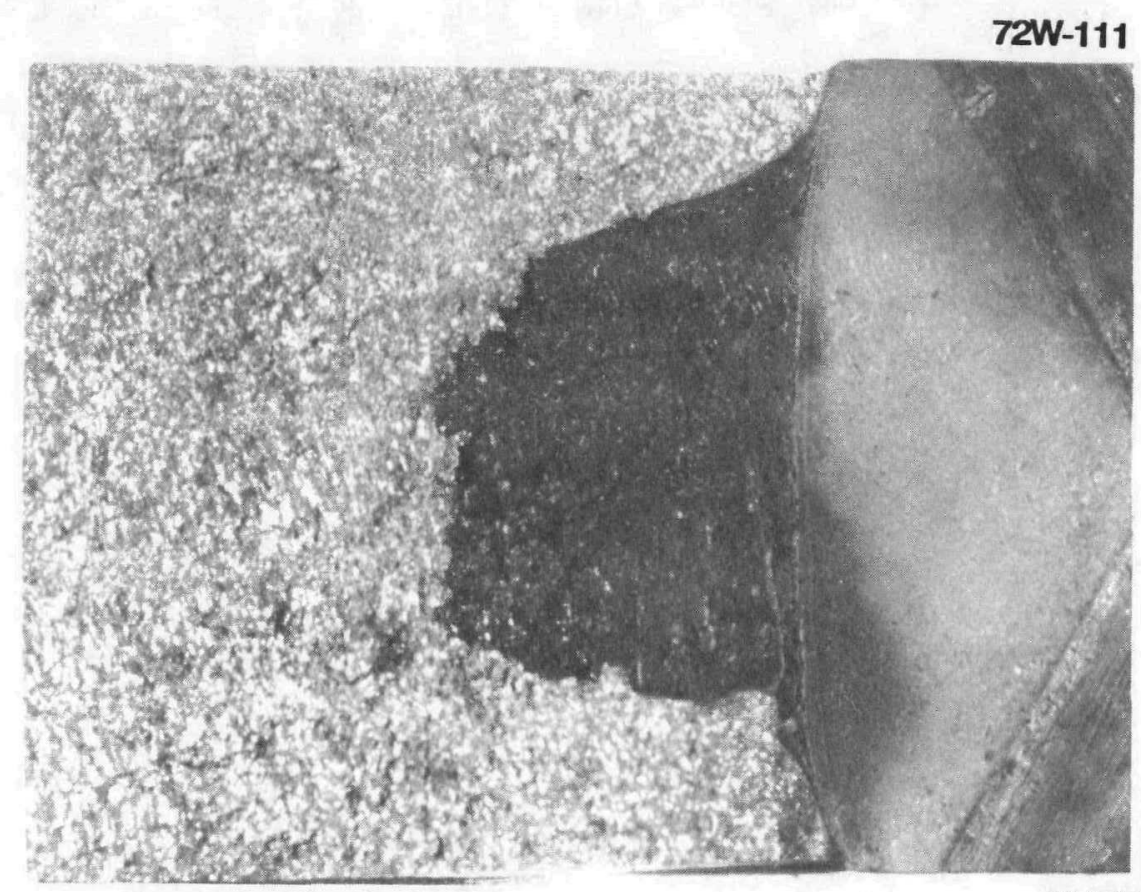

NUREG/CR-5913 


\section{W111 23 DEGREES C}

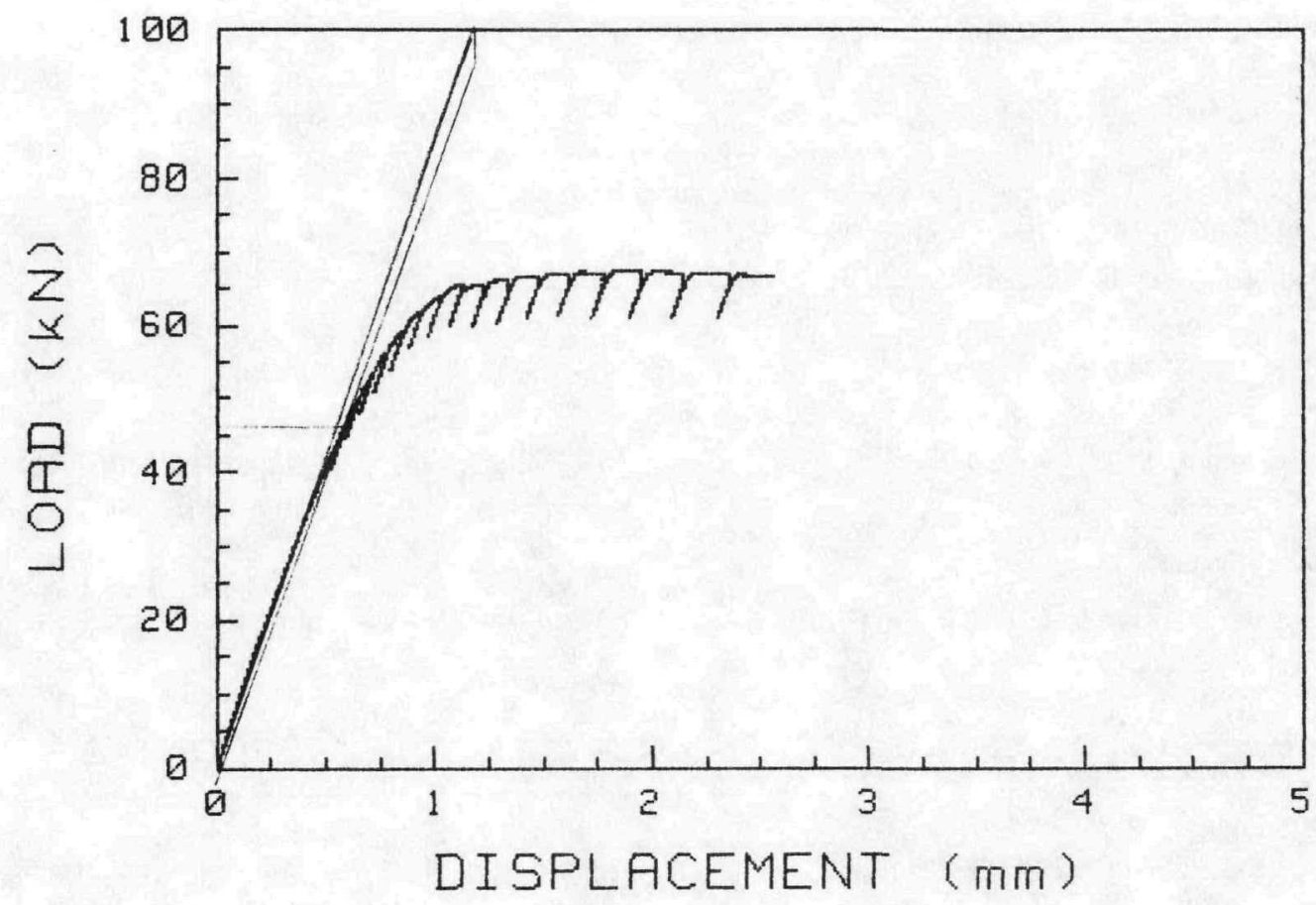

ORNL-DWG 92-11545

72W111 23 DEGREES C

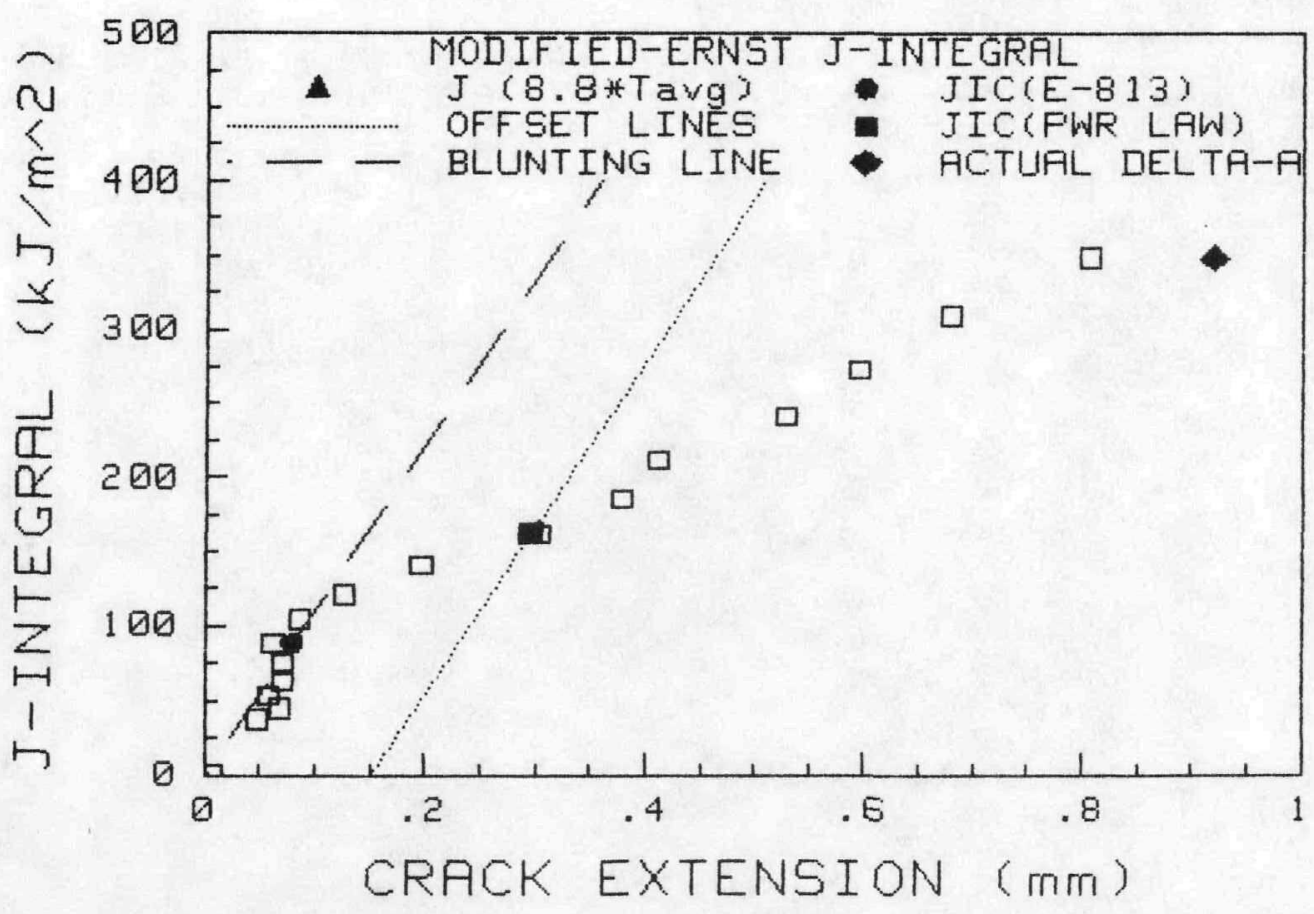


SPECIMEN ID = 72W101

TEST TEMPERATURE $=50$ DEE $C$

THICKNESS $=25.4 \mathrm{~mm}$

SIDE GRDOUES $=20 \%$

INITIAL CRACK LENGTH $=30.9 \mathrm{~mm} \quad \mathrm{a} / \mathrm{W}) \mathrm{i}=.609$

MEASURED DUCTILE DELTA-A $=5.52 \mathrm{~mm}$

$Y$ IELD STRENGTH $=472 \mathrm{MPa}$

FLOW STRENGTH $=527 \mathrm{MPa}$

ESTIMATED YOUNG'S MDDULUS $=204$ GPa

SPECIMEN DID NOT CLEAUE, J-MAX LOAD $=241.7 \mathrm{~kJ} / \mathrm{m}^{\wedge} 2$

$K J-M A X \quad L D A D=222.2 \mathrm{MPa} \operatorname{SQR}[\mathrm{m}]$

POWER LAW PROCEDURE

$\mathrm{J} \mathrm{Ic}=165.2 \mathrm{~kJ} / \mathrm{m}^{\wedge} 2$

$K-J I c=183.7 \mathrm{MPa}$ SQR $[\mathrm{m}]$

POWER $=.6141$

COEFFICIENT $=341.4 \mathrm{~kJ} / \mathrm{m}^{\wedge} 2$

$T(A \cup E R A G E)=151$

JI-R CURUE CALCULATIONS

了 $($ 回了 $/ T=8.8)=793.5 \mathrm{~kJ} / \mathrm{m}^{\wedge} 2$

POWER $=.6128$

$J-M A X$ ALLDWED $=523.3 \mathrm{~kJ} / \mathrm{m}^{\wedge} 2$

UAL ID R-CURUE
ASTM LINEAR PROCEDURE

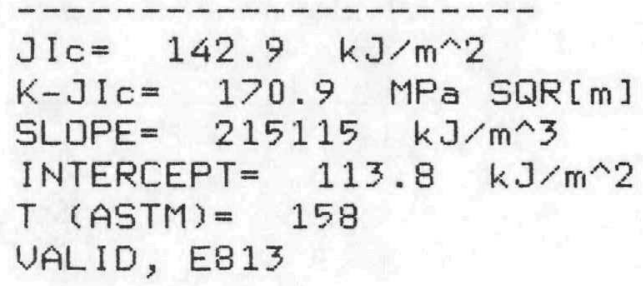

COEFFICIENT $=341.3 \mathrm{~kJ} / \mathrm{m}^{\wedge} 2$

DELTA-A MAX ALLOWED $=1.98 \mathrm{~mm}$

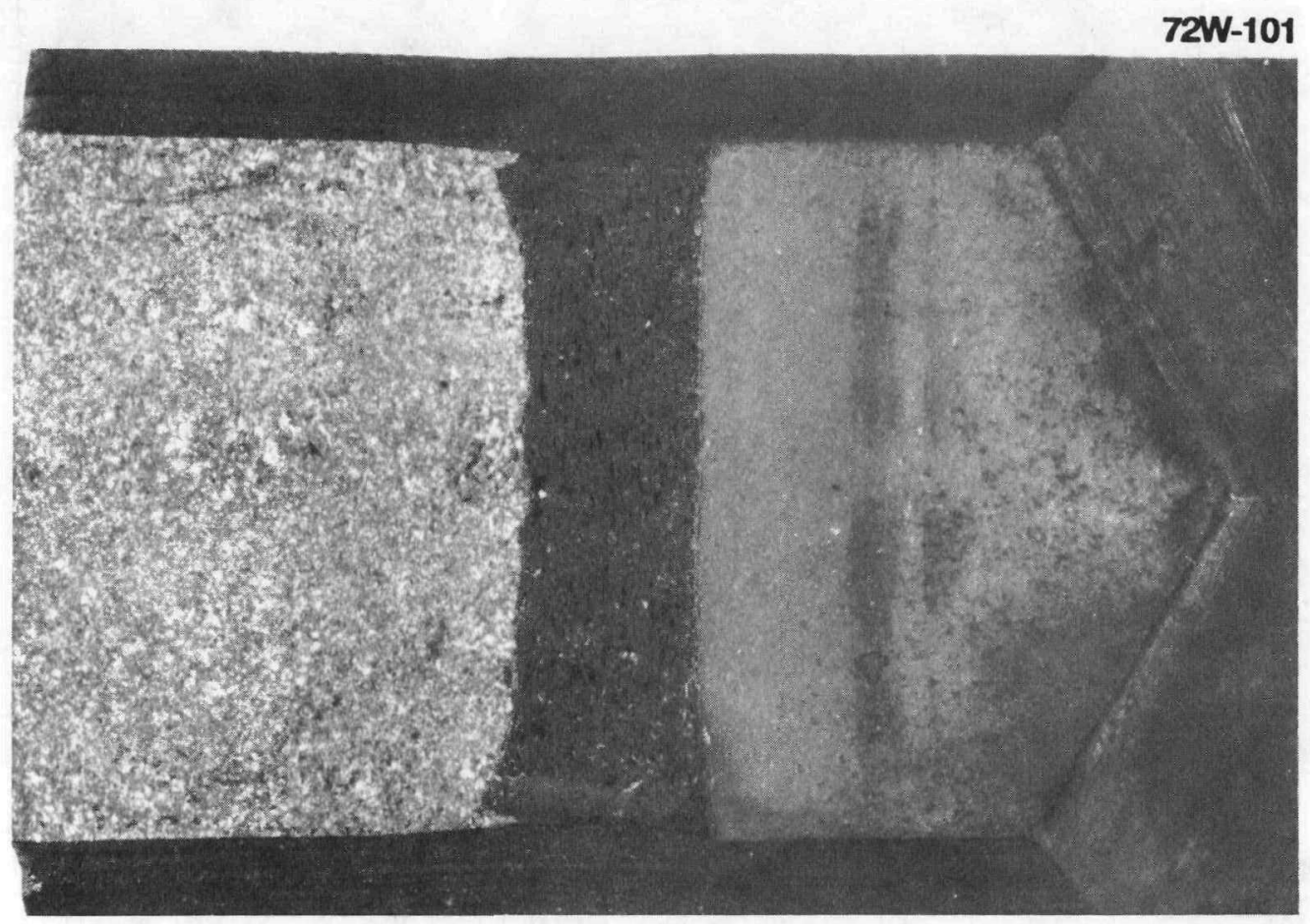



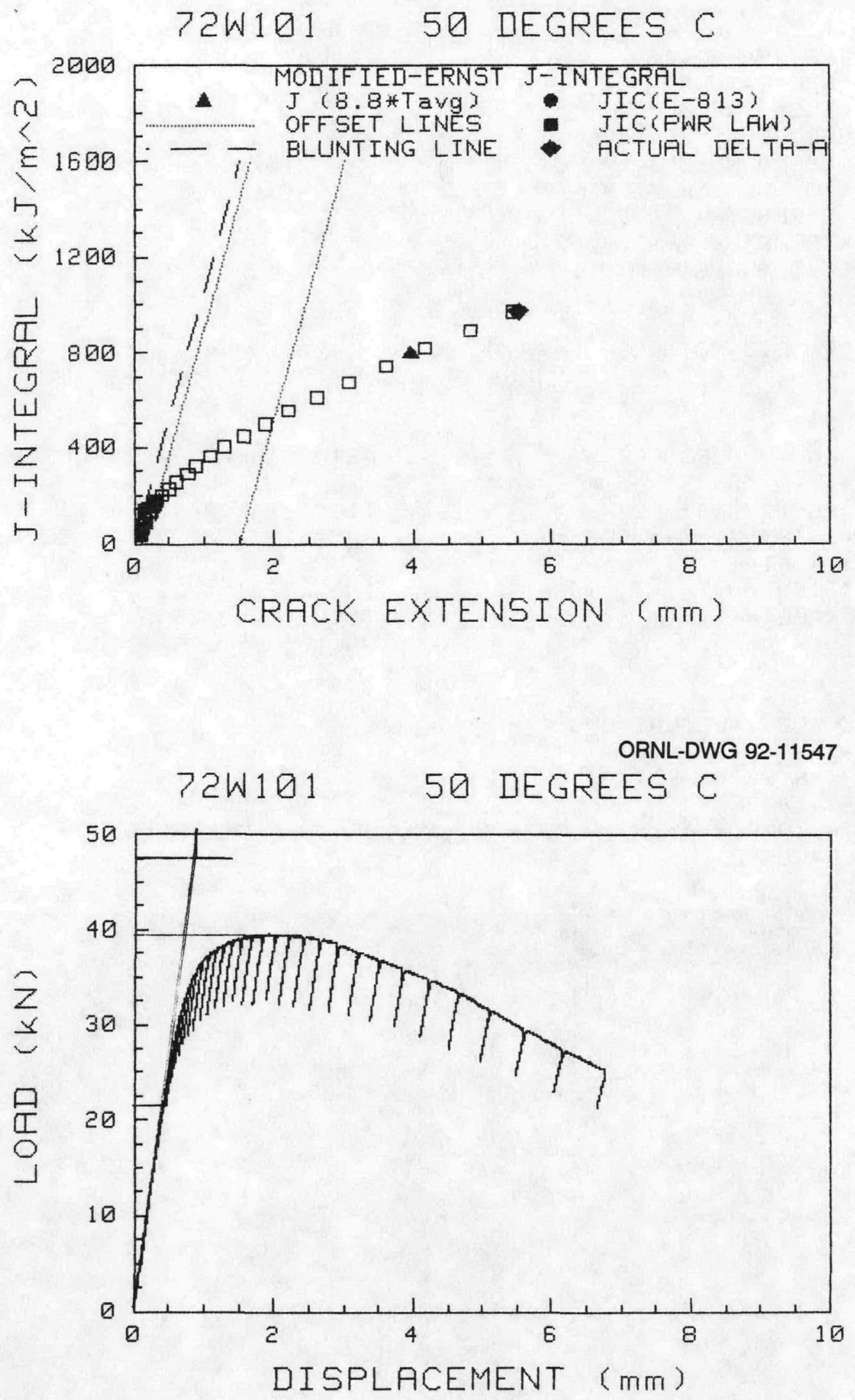

NUREG/CR-5913 


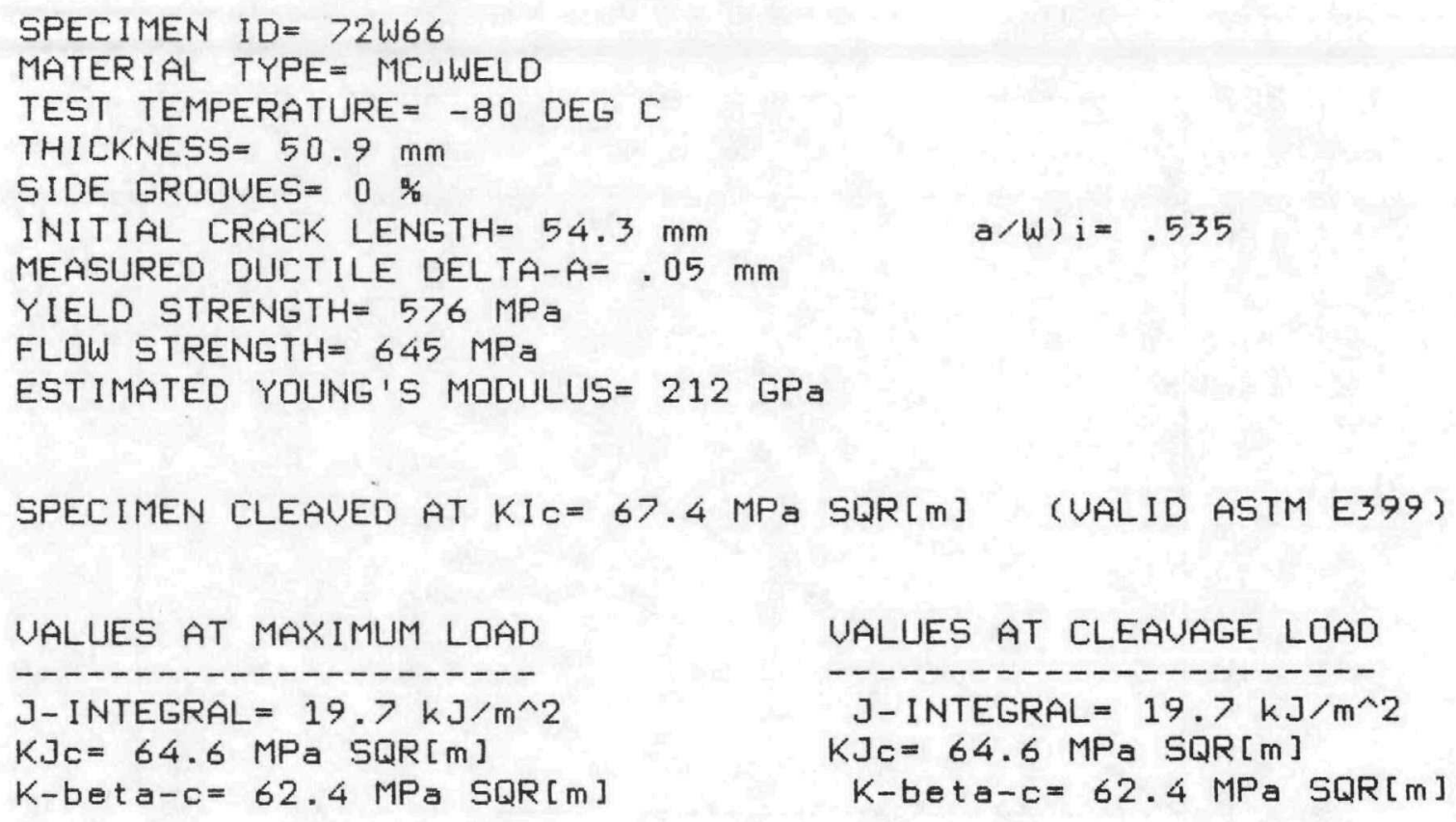




\section{E-68}
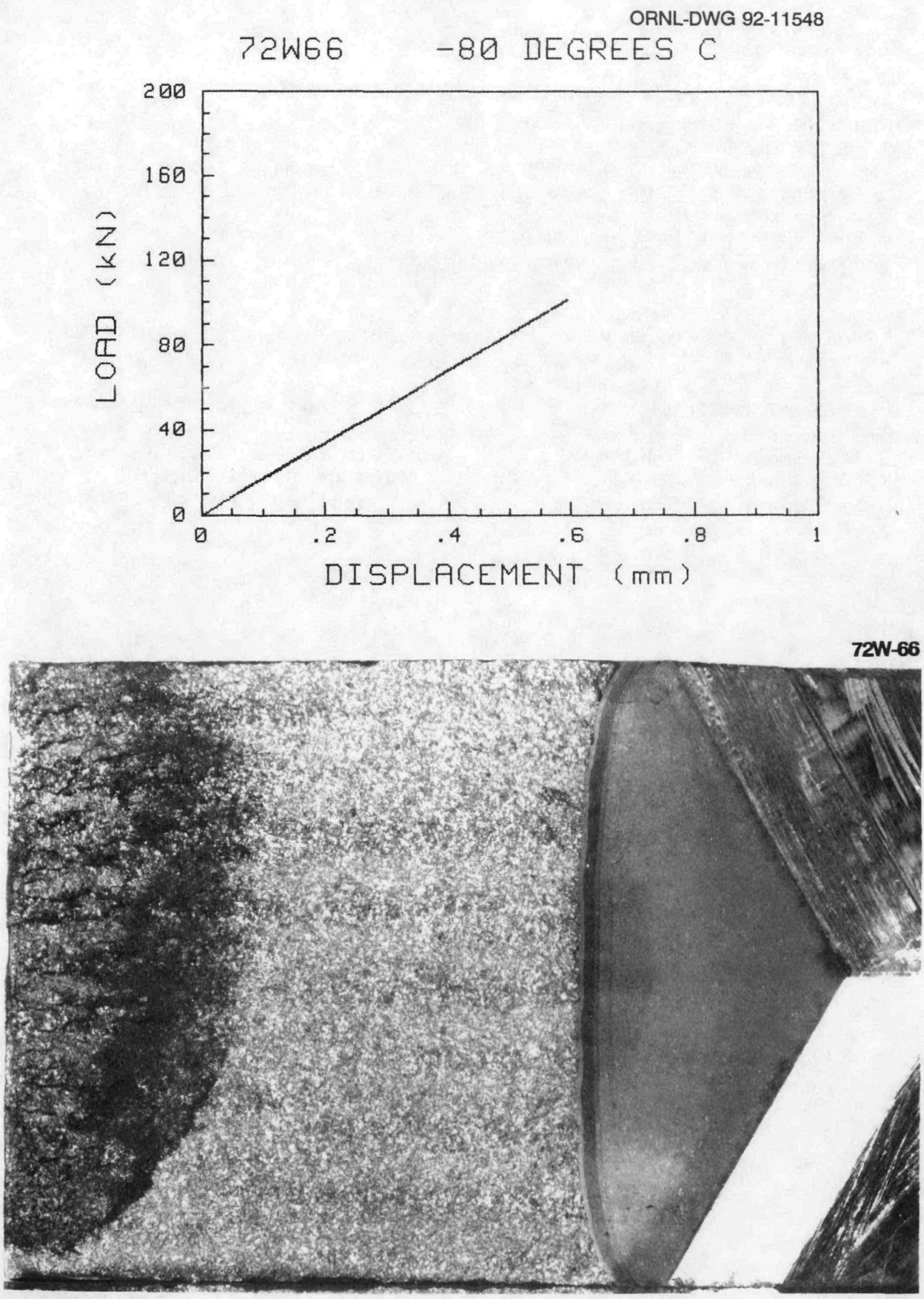

NUREG/CR-5913 


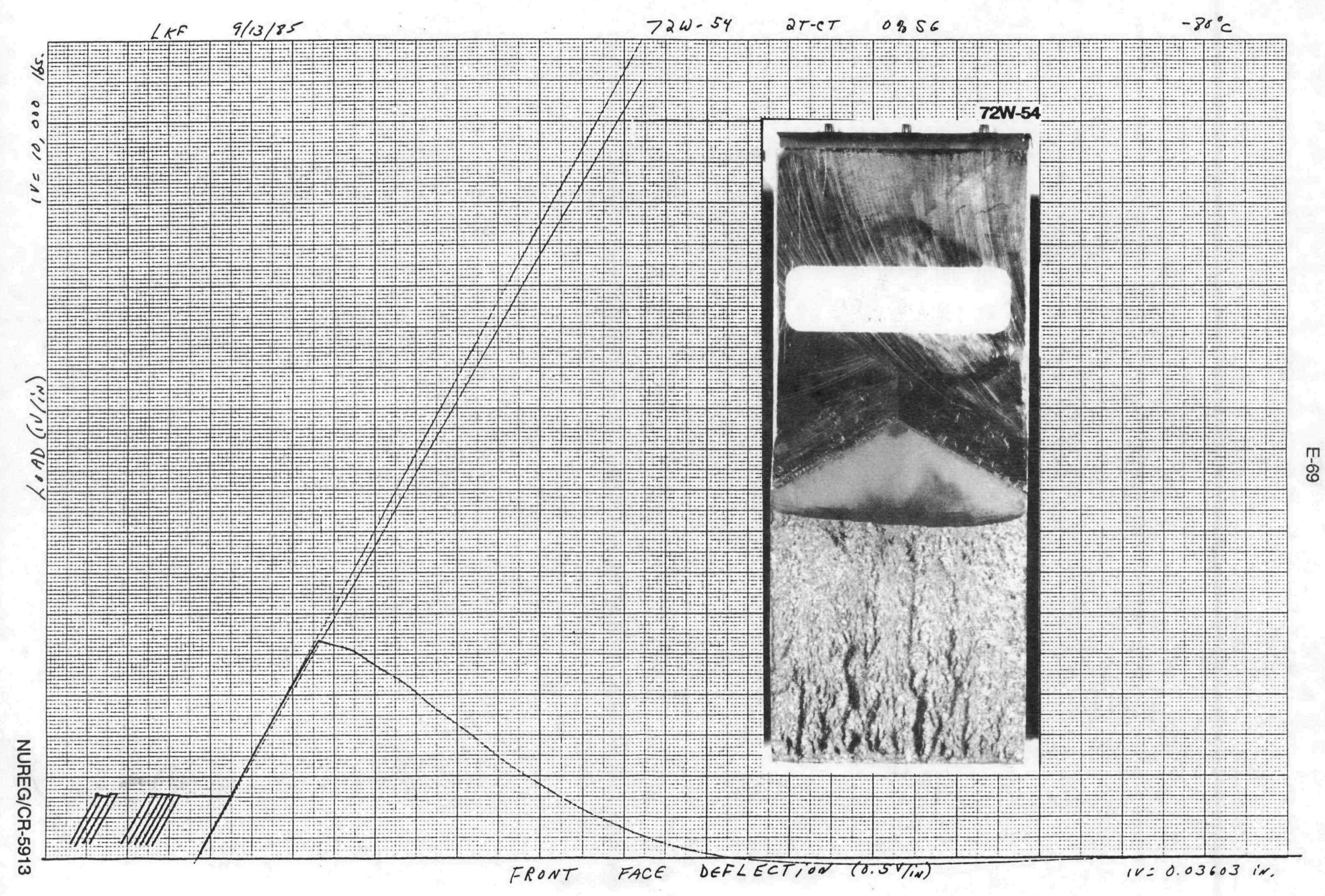


SPECIMEN ID $=72 W>3$

MATERIAL TYPE $=$ MCUWELD

TEST TEMPERATURE $=-80$ DEG $C$

THICKNESS $=50.9 \mathrm{~mm}$

SIDE GROOUES = $0 \%$

INITIAL CRACK LENGTH $=53.3 \mathrm{~mm}$

$a / w) i=.524$

MEASURED DUCTILE DELTA-A $=.05 \mathrm{~mm}$

YIELD STRENGTH $=576 \mathrm{MPa}$

FLOW STRENGTH $=645 \mathrm{MPa}$

ESTIMATED YOUNG'S MODULUS $=212 \mathrm{GPa}$

SPECIMEN CLEAUED AT KQ= $91 \mathrm{MPa}$ SQR $[\mathrm{m}]$ (NOT UALID KIC)

UALUES AT MAXIMUM LOAD

$J-I N T E G R A L=39.5 \mathrm{~kJ} / \mathrm{m}^{\wedge} 2$

$\mathrm{KJ} c=91.4 \mathrm{MPa} \operatorname{SQR}[\mathrm{m}]$

$K-b e t a-c=82.5 \mathrm{MPa} \operatorname{SQR}[\mathrm{m}]$
UALUES AT CLEAUAGE LOAD

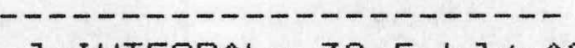

J-INTEGRAL $=39.5 \mathrm{~kJ} / \mathrm{m}^{\wedge} 2$

$\mathrm{KJ} c=91.4 \mathrm{MPa} \operatorname{SQR}[\mathrm{m}]$

$K$-beta-c $=82.5 \mathrm{MPa} \operatorname{SQR}[\mathrm{m}]$ 
E-71

ORNL-DWG 92-11549

72W73 -80 DEGREES C

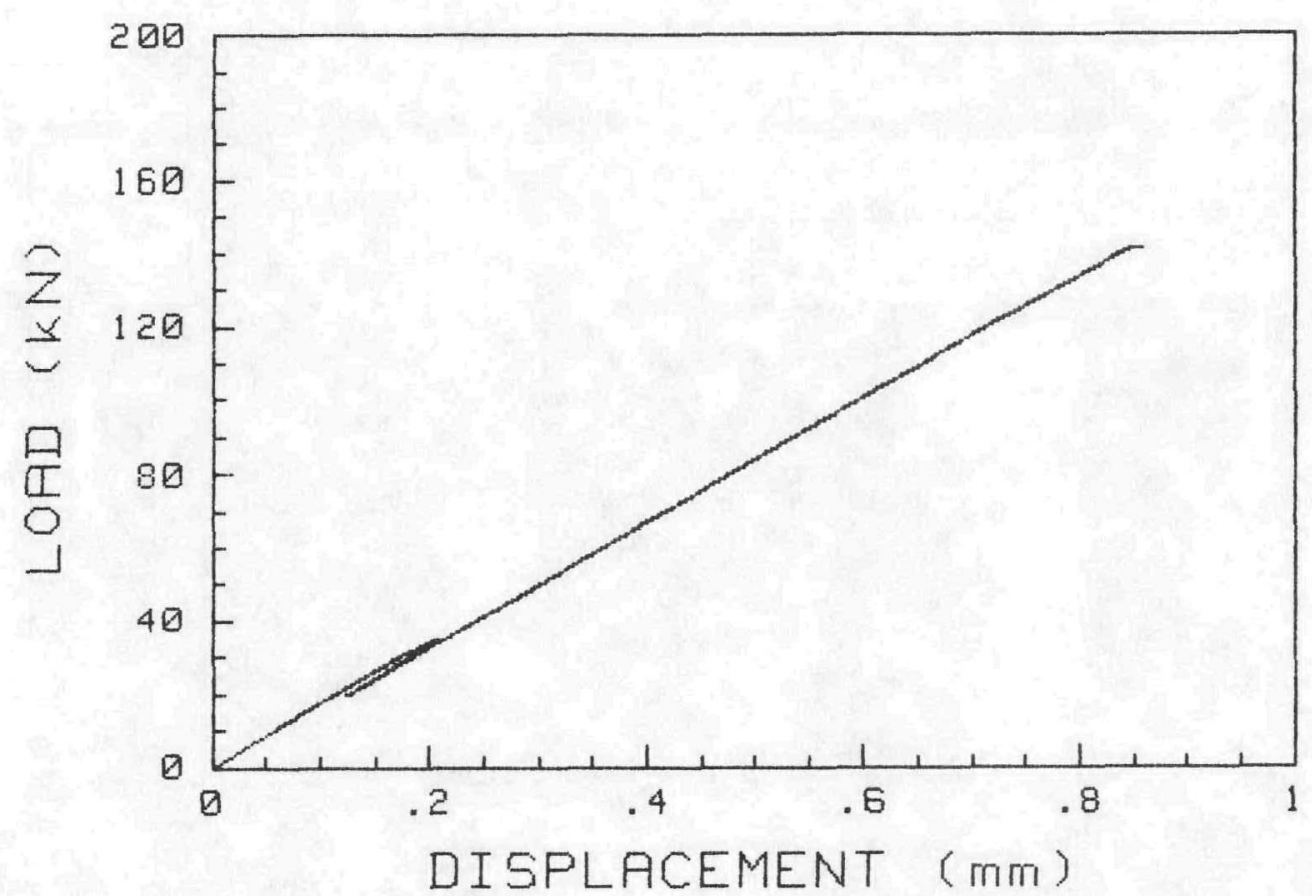

72W-73

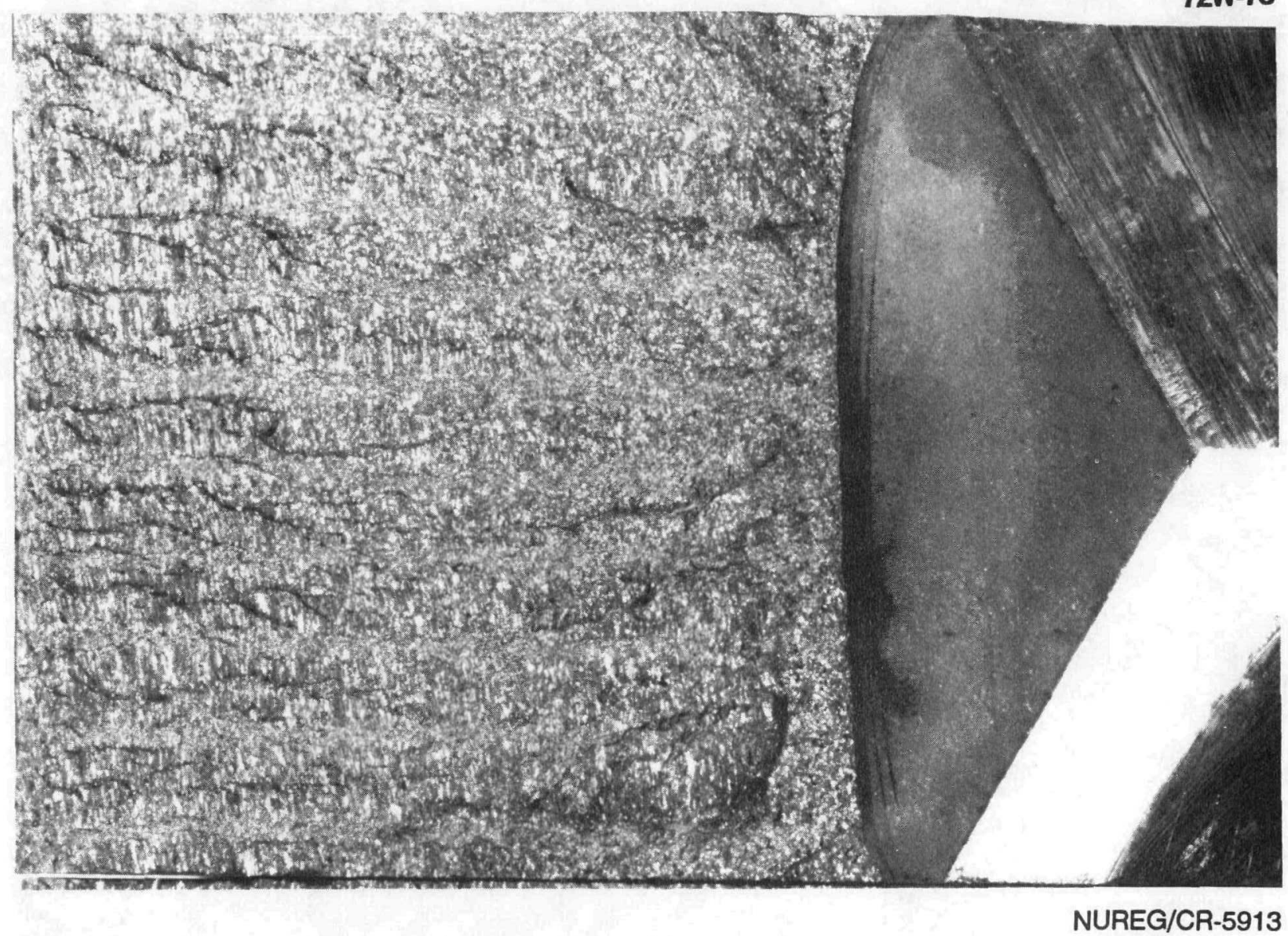




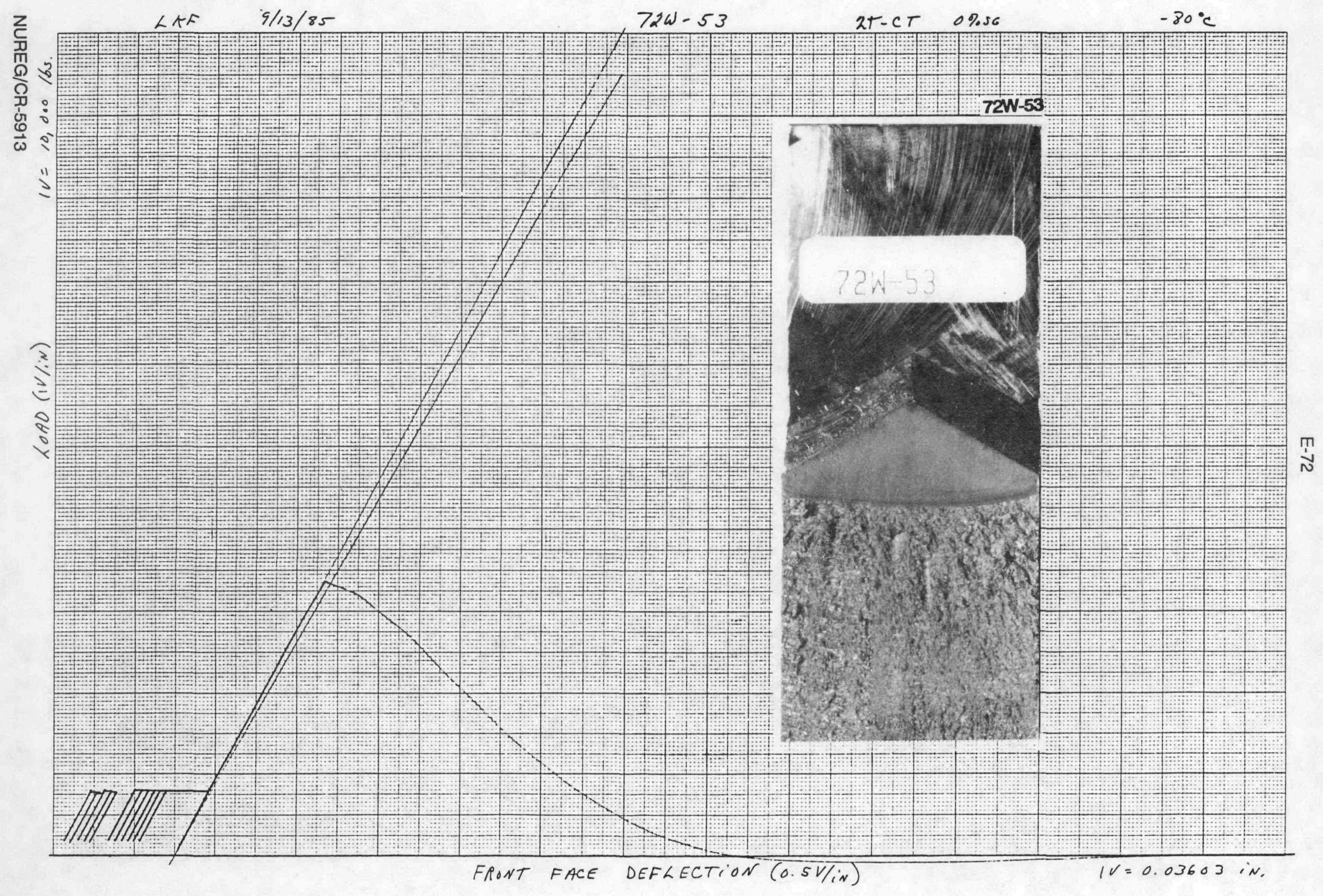


SPECIMEN ID $=72 W 72$

MATERIAL TYPE $=$ MCUWELD

TEST TEMPERATURE $=-50$ DEG C

THICKNESS $=50.9 \mathrm{~mm}$

SIDE GROOUES $=0 \%$

INITIAL CRACK LENGTH $=53.8 \mathrm{~mm}$

MEASURED DUCTILE DELTA-A $=.05 \mathrm{~mm}$

$a / W) \mathrm{i}=.53$

YIELD STRENGTH $=547 \mathrm{MPa}$

FLOW STRENGTH $=614 \mathrm{MPa}$

ESTIMATED YOUNG'S MODULUS $=210 \mathrm{GPa}$

SPECIMEN CLEAUED AT $K I c=71.1 \mathrm{MPa}$ SQR $[\mathrm{m}]$ (UALID ASTM E399)

VALUES AT MAXIMUM LDAD

J-INTEGRAL $=23 \mathrm{~kJ} / \mathrm{m}^{\wedge} 2$

$\mathrm{K}] \mathrm{c}=69.6 \mathrm{MPa} \operatorname{SQR}[\mathrm{m}]$

$K$-beta-c $=65.9 \mathrm{MPa} \operatorname{SQR}[\mathrm{m}]$
UALUES at CLEaUage LoAd

$J-I N T E G R A L=23 \mathrm{~kJ} / \mathrm{m}^{\wedge} 2$

$\mathrm{KJ} c=69.6 \mathrm{MPa} S Q R[\mathrm{~m}]$

$K$-bet $a-c=65.9 \mathrm{MPa} \operatorname{SQR}[\mathrm{m}]$ 


\section{E-74}

ORNL-DWG 92-11550

72W72 -50 DEGREES C

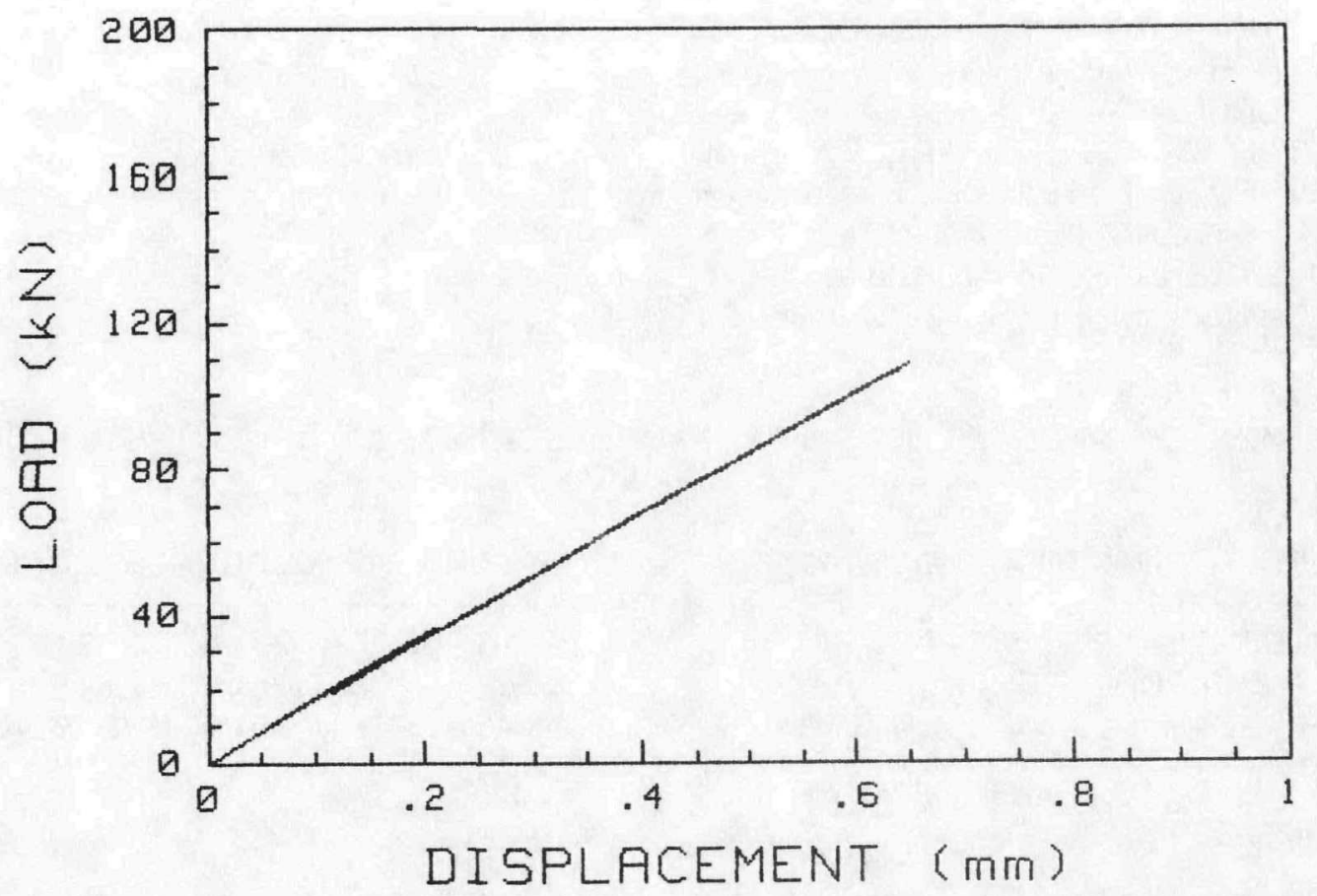

$72 W-72$

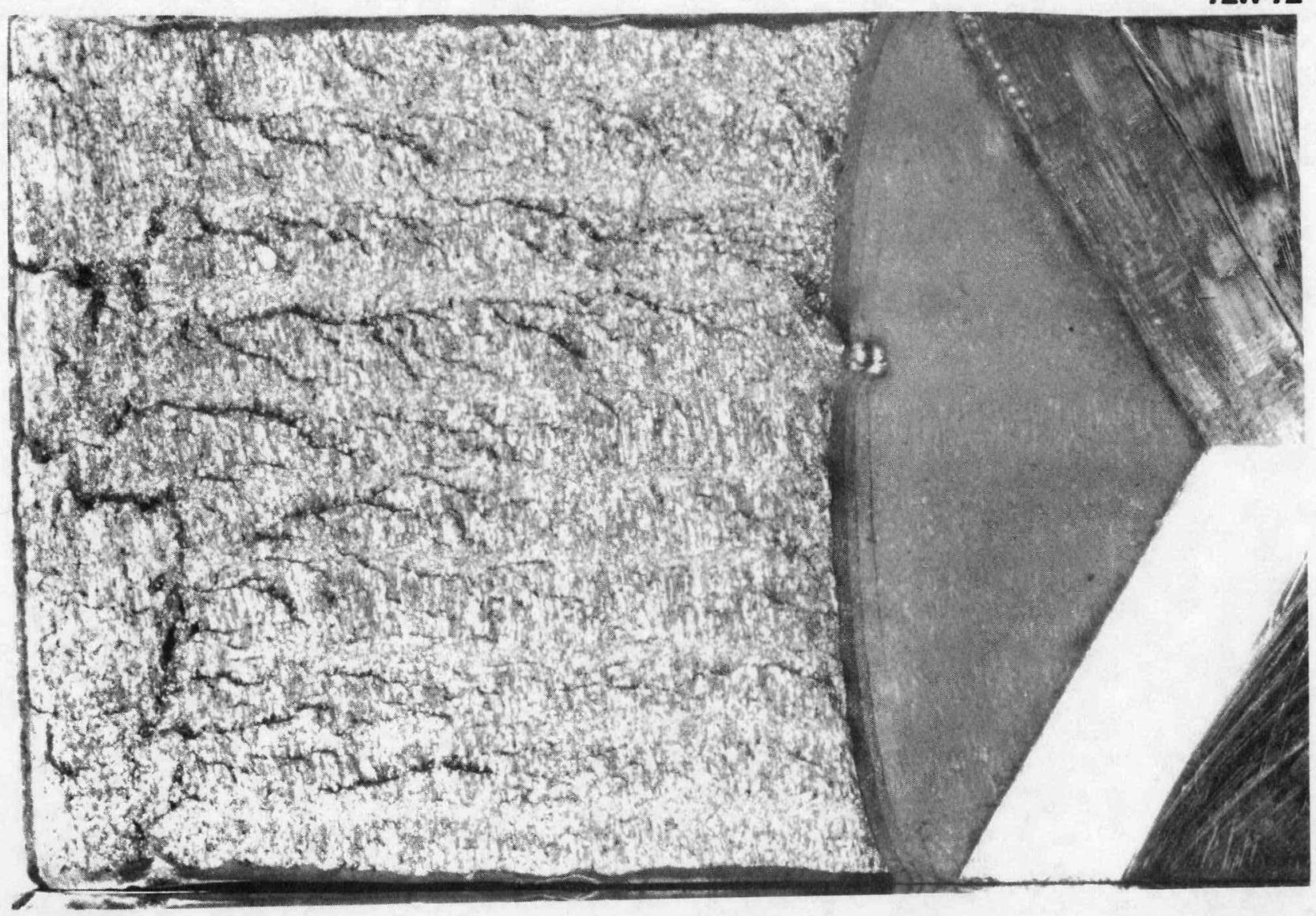




\section{E-75}

SPECIMEN ID $=72 W 86$

MATERIAL TYPE = MCUWELD

TEST TEMPERATURE $=-50$ DEG $C$

THICKNESS $=50.8 \mathrm{~mm}$

SIDE GROOUES $=0 \%$

INITIAL CRACK LENGTH $=53.4 \mathrm{~mm}$

MEASURED DUCTILE DELTA-A $=.05 \mathrm{~mm}$

$a /$ (J) $i=.526$

$Y$ IELD STRENGTH $=547 \mathrm{MPa}$

FLDW STRENGTH $=614 \mathrm{MPa}$

ESTIMATED YOUNG'S MODULUS $=210 \mathrm{GPa}$

SPECIMEN CLEAUED AT KQ $=84.8 \mathrm{MPa}$ SQR $[\mathrm{m}]$ (NOT VALID $K I \mathrm{c}$ )

UALUES AT MAXIMUM LOAD

J-INTEGRAL $=33.8 \mathrm{~kJ} / \mathrm{m}^{\wedge} 2$

$\mathrm{KJC}=84.3 \mathrm{MPa} \operatorname{SQR}[\mathrm{m}]$

$K$-bet $a-c=76.7 \mathrm{MPa} S Q R[\mathrm{~m}]$
Ualues at cLeavage LoAd

$J$-INTEGRAL $=33.8 \mathrm{~kJ} / \mathrm{m}^{\wedge} 2$

$K J c=84.3 \mathrm{MPa} \operatorname{SQR}[\mathrm{m}]$

$K-$ bet $a-c=76.7 \mathrm{MPa} S Q R[\mathrm{~m}]$ 


\section{E-76}

ORNL-DWG 92-11551

\section{W86 -50 DEGREES C}

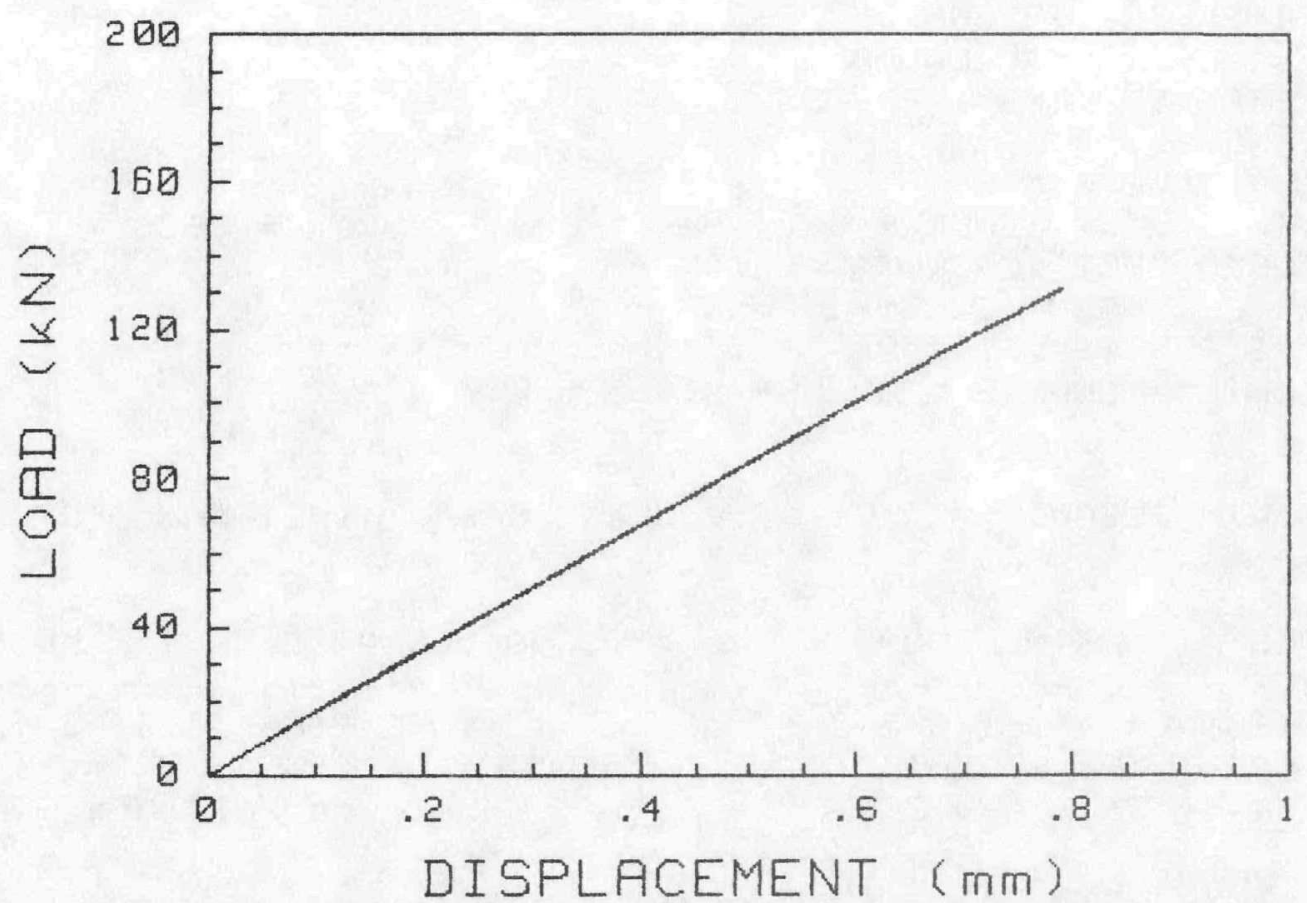

$72 W-86$

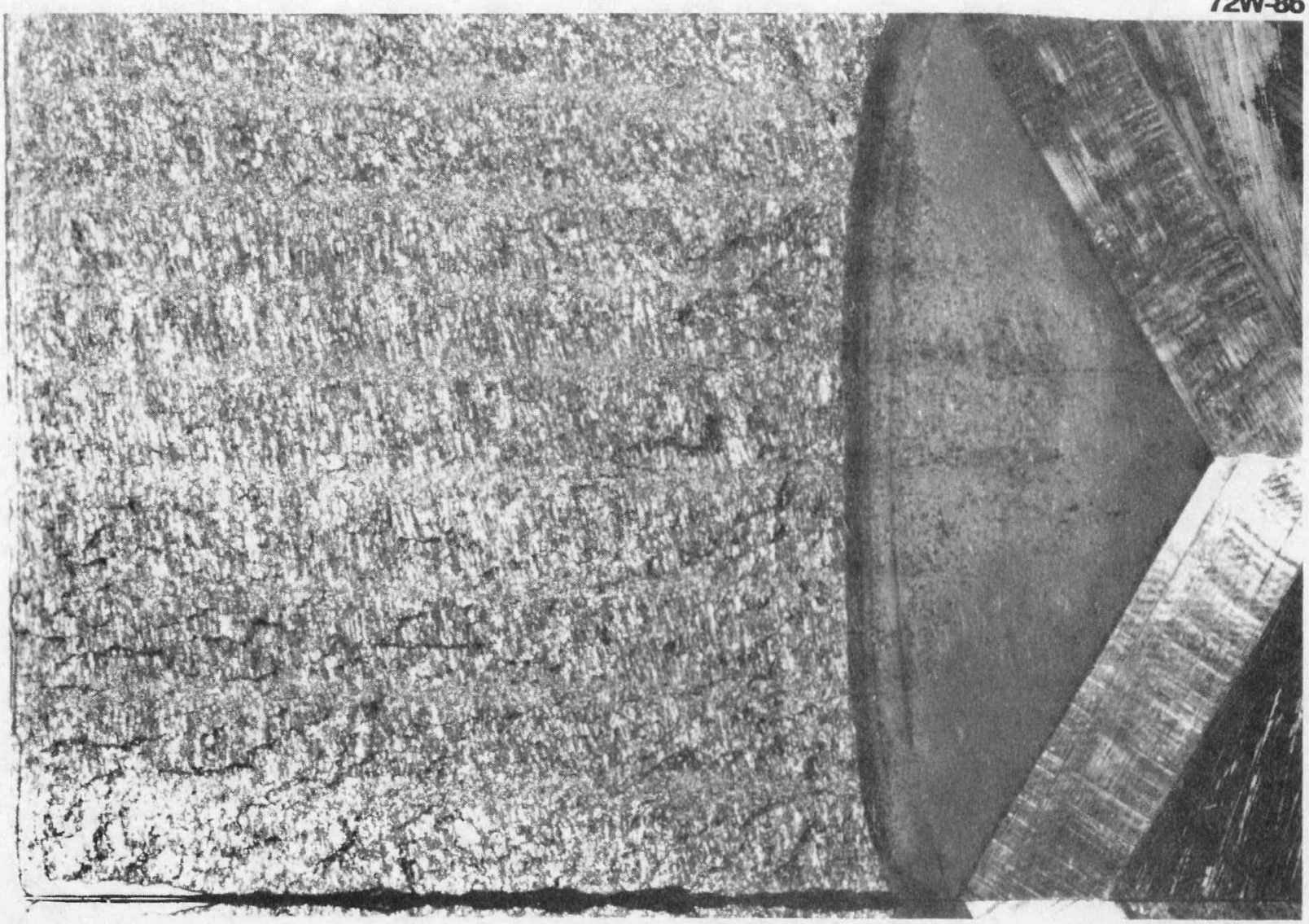

NUREG/CR-5913 


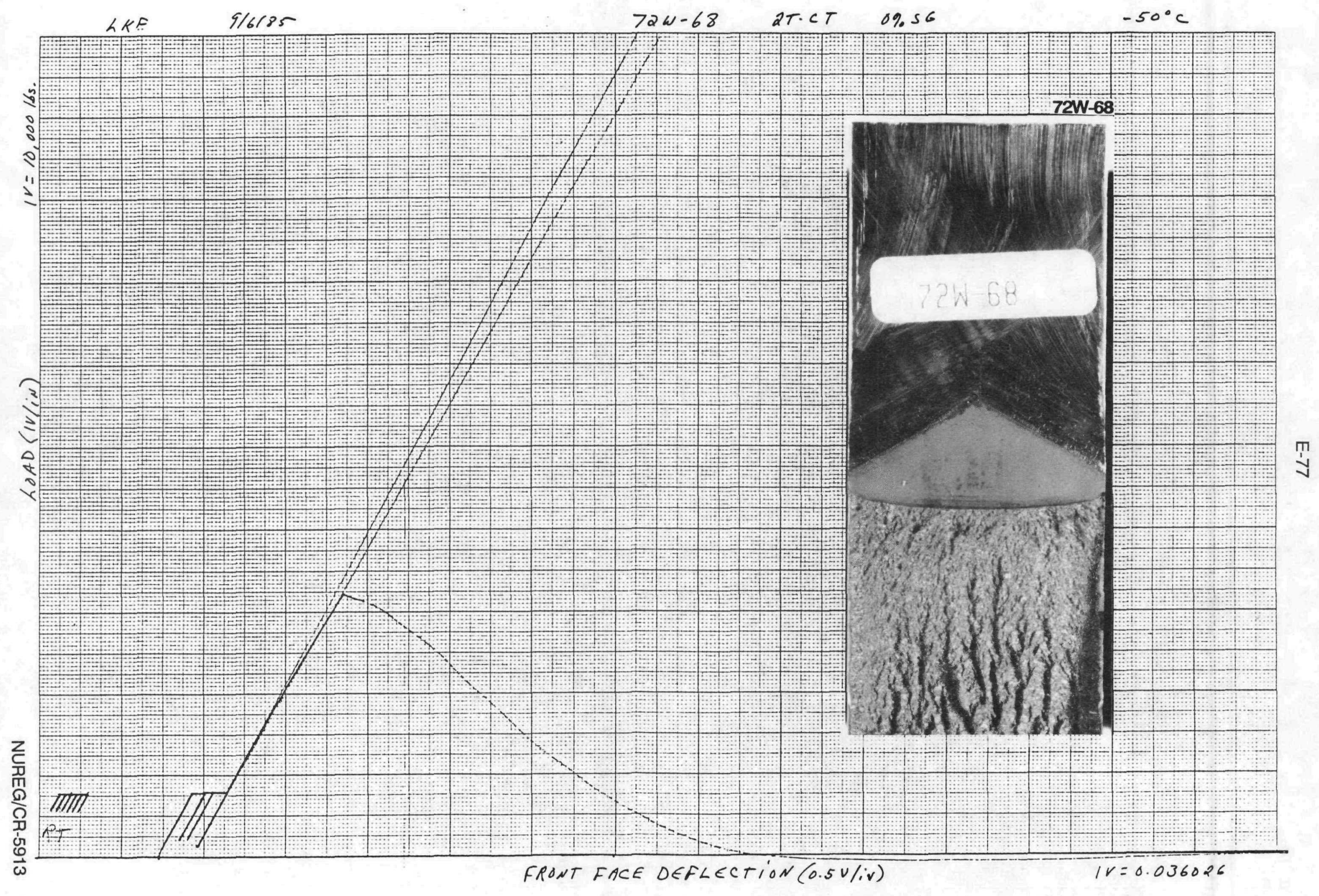




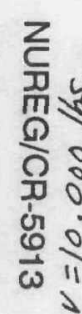

$9 / 10 / 85$

$72 w-69$

2T-CT

$\Delta \% .56$

$-50^{\circ} \mathrm{C}$

APF

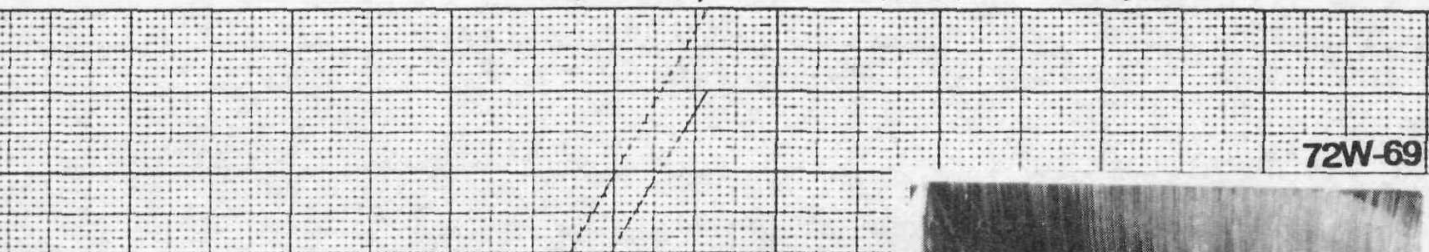

गे
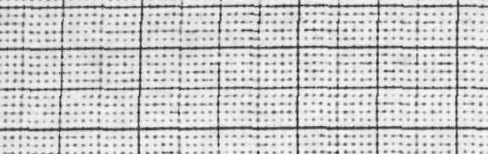

\section{ปี}
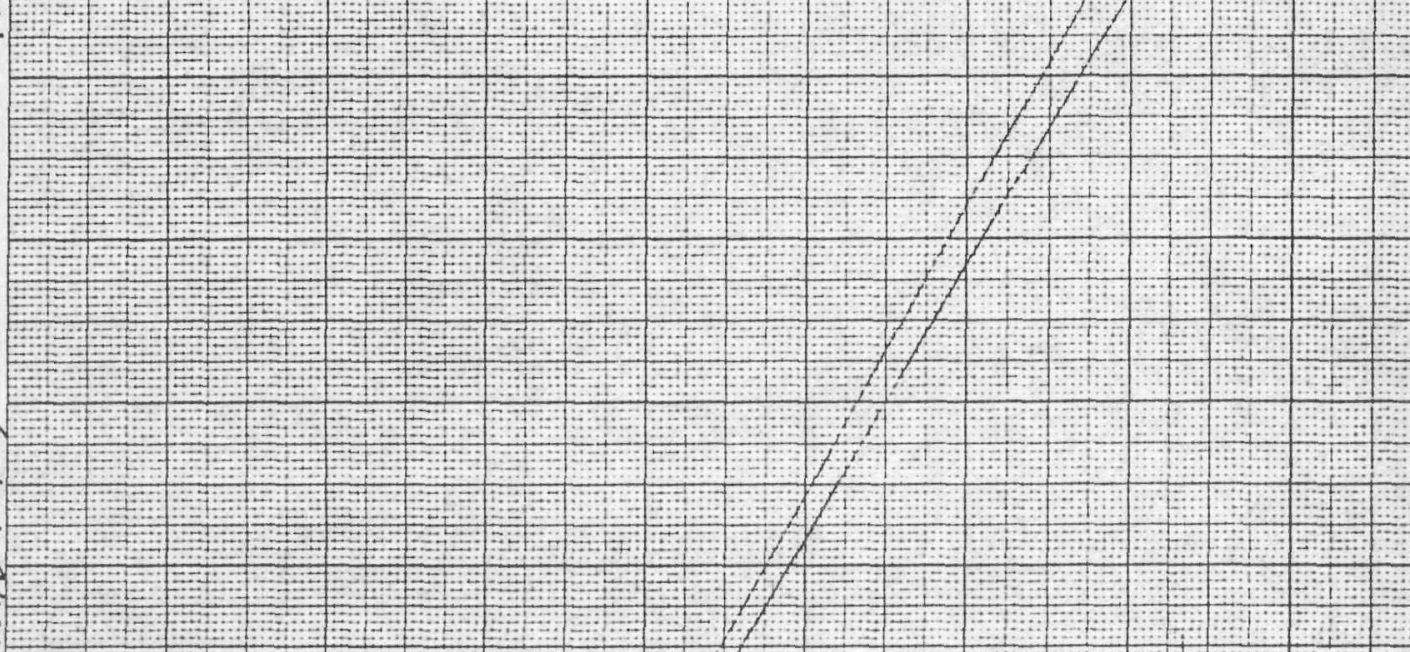

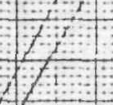

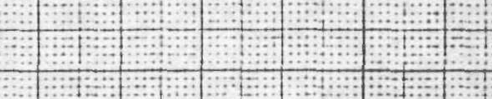

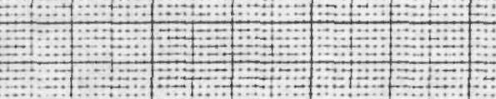

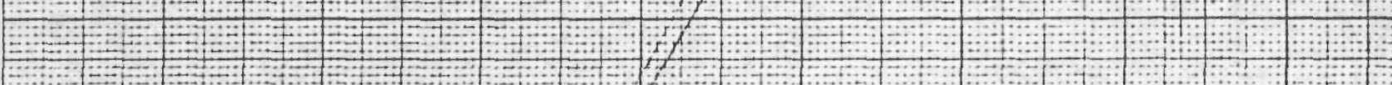
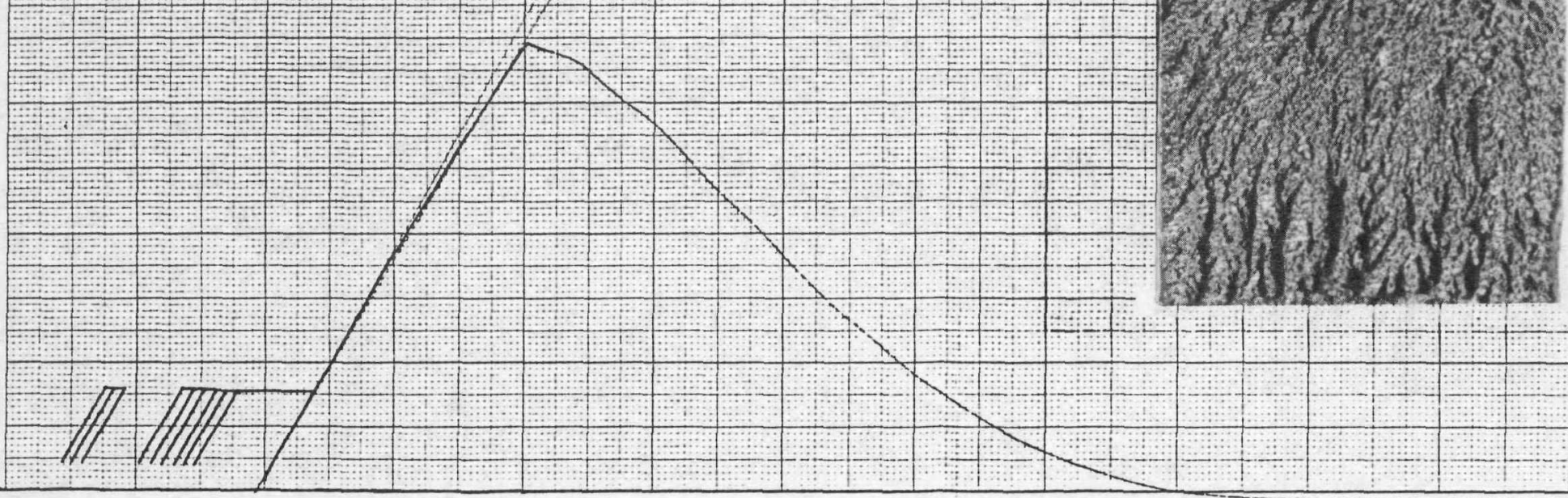

- 4505

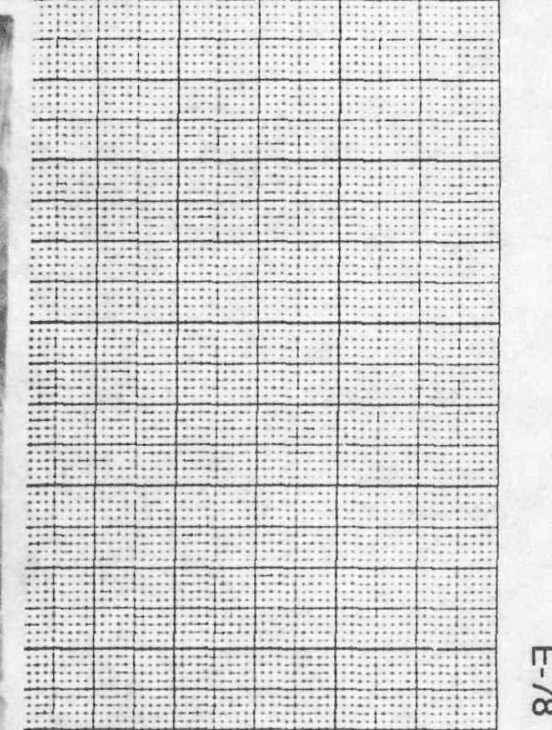




\section{E-79}

SPECIMEN ID $=72$ W8 1

MATERIAL TYPE = MCUWELD

TEST TEMPERATURE $=-50$ DEG C

THICKNESS $=50.9 \mathrm{~mm}$

SIDE GROOUES $=0 \%$

INITIAL CRACK LENGTH $=52.9 \mathrm{~mm}$

$a / W) i=.521$

MEASURED DUETILE DELTA-A $=.1 \mathrm{~mm}$

$Y$ IELD STRENGTH $=547 \mathrm{MPa}$

FLOW STRENGTH $=614 \mathrm{MPa}$

ESTIMATED YOUNG'S MODULUS $=210 \mathrm{GPa}$

SPECIMEN CLEAUED AT KQ= $124.7 \mathrm{MPa}$ SQR $[\mathrm{m}]$ (NDT UALID $K I c$ )

UALUES AT MAXIMUM LOAD

$\mathrm{J}-\mathrm{INTEGRAL}=77.3 \mathrm{~kJ} / \mathrm{m}^{\wedge} 2$

$\mathrm{K} J \mathrm{c}=127.4 \mathrm{MPa} \operatorname{SQR}[\mathrm{m}]$

$K$-beta-c $=100.4 \mathrm{MPa} \operatorname{SQR}[\mathrm{m}]$
UaLUeS at CLEaUAGE LOAD

J-INTEGRAL $=77.3 \mathrm{~kJ} / \mathrm{m}^{\wedge} 2$

$\mathrm{K} \cdot \mathrm{C}=127.4 \mathrm{MPa} \operatorname{SQR}[\mathrm{m}]$

$K$-beta-c $=100.4 \mathrm{MPa} S Q R[\mathrm{~m}]$ 
E-80

ORNL-DWG 92-11552
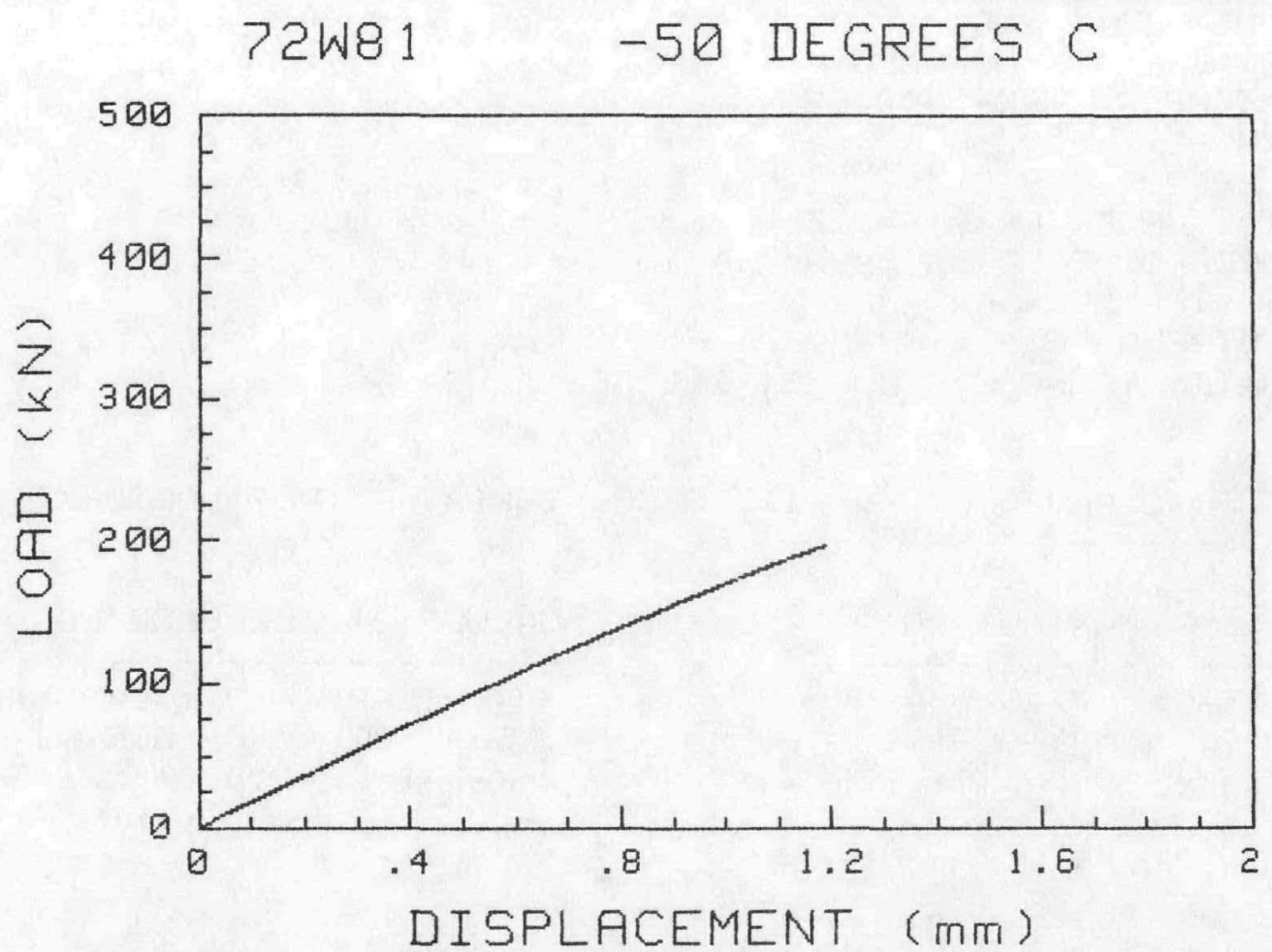

$72 W-81$

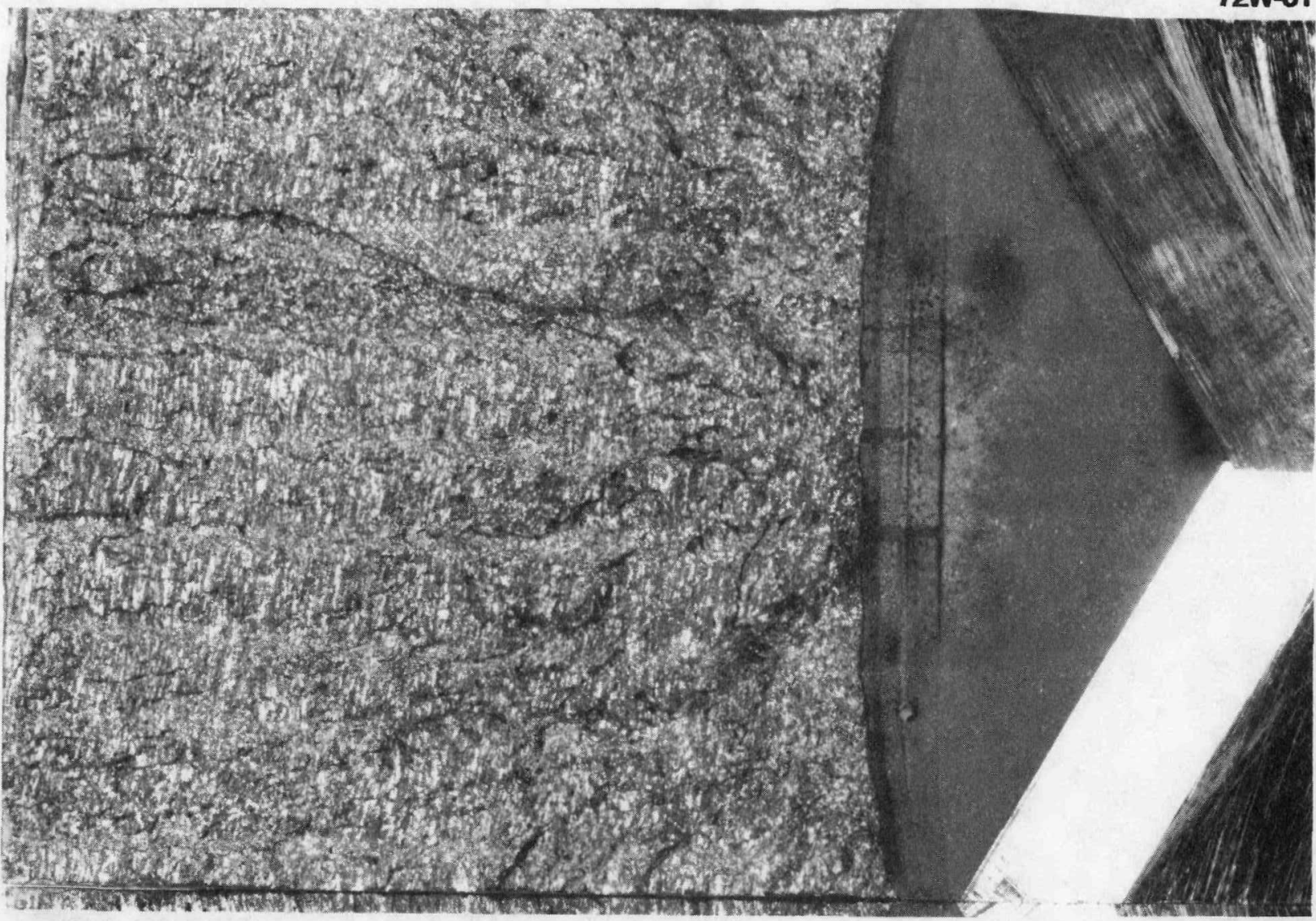

NUREG/CR-5913 
SPECIMEN ID $=72 W>6$

MATERIAL TYPE $=$ MCUWELD

TEST TEMPERATURE $=-30$ DEG C

THICKNESS $=50.8 \mathrm{~mm}$

SIDE GROOUES $=0 \%$

INITIAL CRACK LENGTH $=54.3 \mathrm{~mm}$

$a / w) i=.535$

MEASURED DUCTILE DELTA-A $=.07 \mathrm{~mm}$

$Y$ IELD STRENGTH $=534 \mathrm{MPa}$

FLOW STRENGTH $=598 \mathrm{MPa}$

ESTIMATED YOUNG'S MODULUS $=209 \mathrm{GPa}$

SPECIMEN CLEAUED AT KQ= $126.3 \mathrm{MPa}$ SQR $[\mathrm{m}]$ (NOT UALID $K I \mathrm{c}$ )

UALUES AT MAXIMUM LDAD

J-INTEGRAL $=80.6 \mathrm{~kJ} / \mathrm{m}^{\wedge} 2$

$\mathrm{KJ} c=129.7 \mathrm{MPa} S Q R[\mathrm{~m}]$

K-beta-c $=100.3 \mathrm{MPa} \operatorname{SQR}[\mathrm{m}]$

\author{
VALUES AT CLEAUAGE LOAD \\ $J$-INTEGRAL $=81 \mathrm{~kJ} / \mathrm{m}^{\wedge} 2$ \\ $K J c=130.1 \mathrm{MPa} \operatorname{SQR}[\mathrm{m}]$ \\ $K$-beta-c $=100.5 \mathrm{MPa} S Q R[\mathrm{~m}]$
}


E-82

ORNL-DWG 92-11553

72W76 -30 DEGREES C

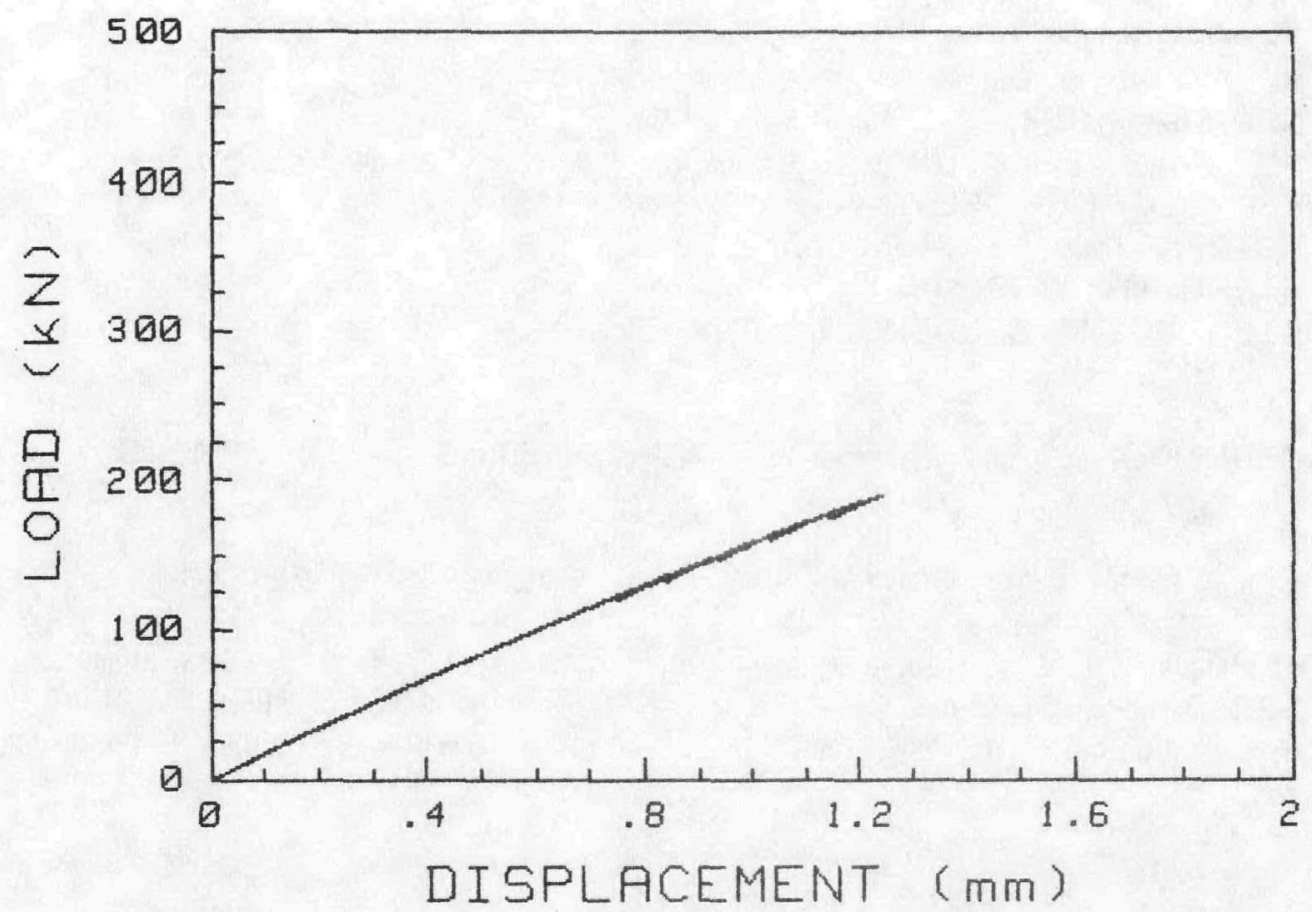

$72 W-76$

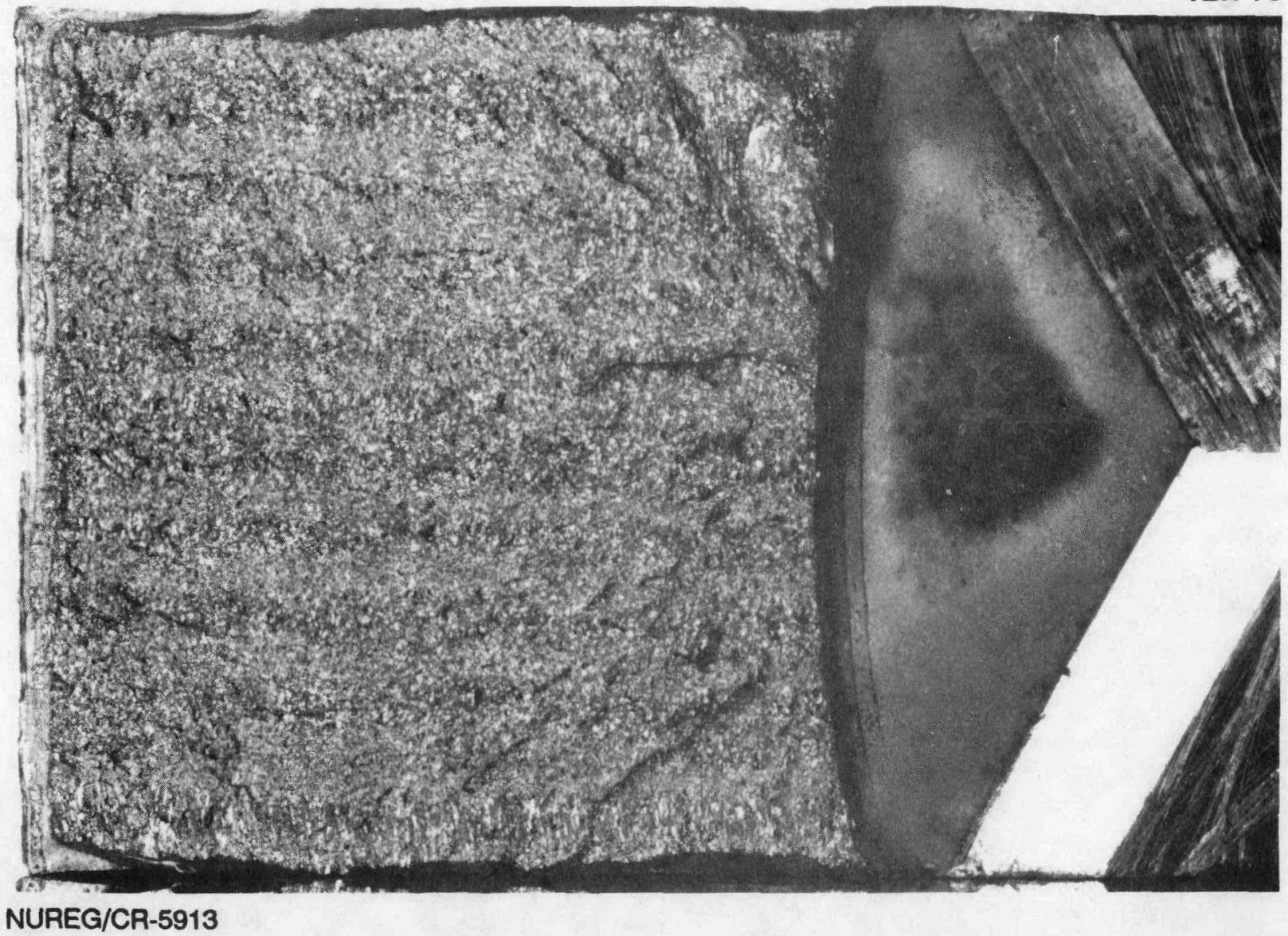

NUREG/CR-5913 
SPECIMEN ID $=72 W>5$

MATERIAL TYPE = MCUWELD

TEST TEMPERATURE $=-30$ DEG $C$

THICKNESS $=50.5 \mathrm{~mm}$

SIDE GRODUES $=0 \%$

INITIAL CRACK LENGTH $=54.4 \mathrm{~mm}$

$a / W) i=.532$

MEASURED DUCTILE DELTA-A $=.05 \mathrm{~mm}$

$Y$ IELD STRENGTH $=534 \mathrm{MPa}$

FLOW STRENGTH $=598 \mathrm{MPa}$

ESTIMATED YOUNG'S MODULUS $=209 \mathrm{GPa}$

SPECIMEN CLEAUED AT KQ= $130.6 \mathrm{MPa} S Q R[\mathrm{~m}]$ (NDT UALID KIc)

UALUES AT MAXIMUM LDAD

J-INTEGRAL $=87.4 \mathrm{~kJ} / \mathrm{m}^{\wedge} 2$

$\mathrm{K} \cdot]_{\mathrm{c}}=135.2 \mathrm{MPa} \operatorname{SQR}[\mathrm{m}]$

$K$-beta-c= $102.5 \mathrm{MPa} \operatorname{SQR}[\mathrm{m}]$
UALUES AT CLEAUAGE LOAD

J-INTEGRAL $=87.4 \mathrm{~kJ} / \mathrm{m}^{\wedge} 2$

$\mathrm{KJ} c=135.2 \mathrm{MPa} S Q R[\mathrm{~m}]$

K-beta-c $=102.5 \mathrm{MPa} S Q R[\mathrm{~m}]$ 
E-84

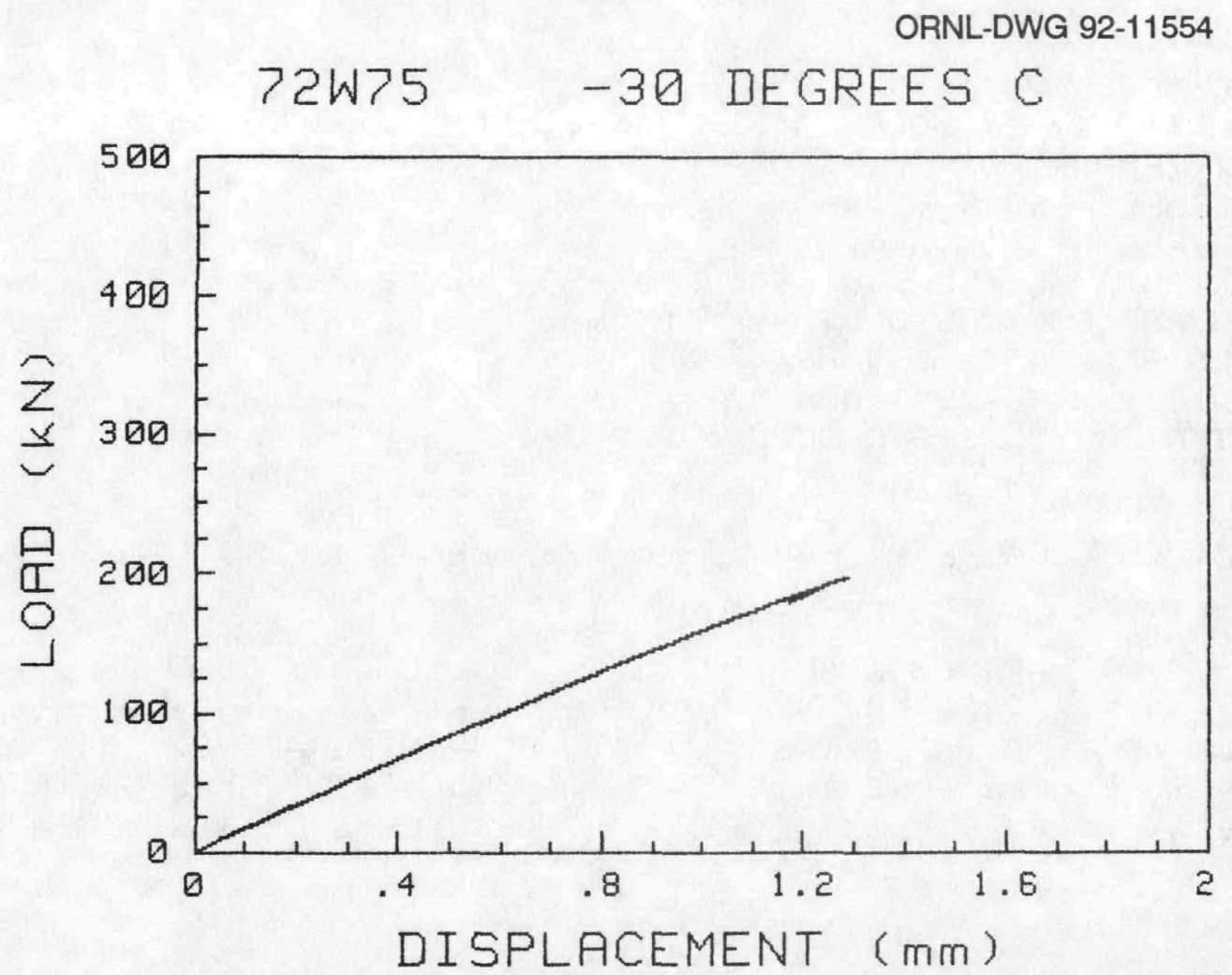

72W-75

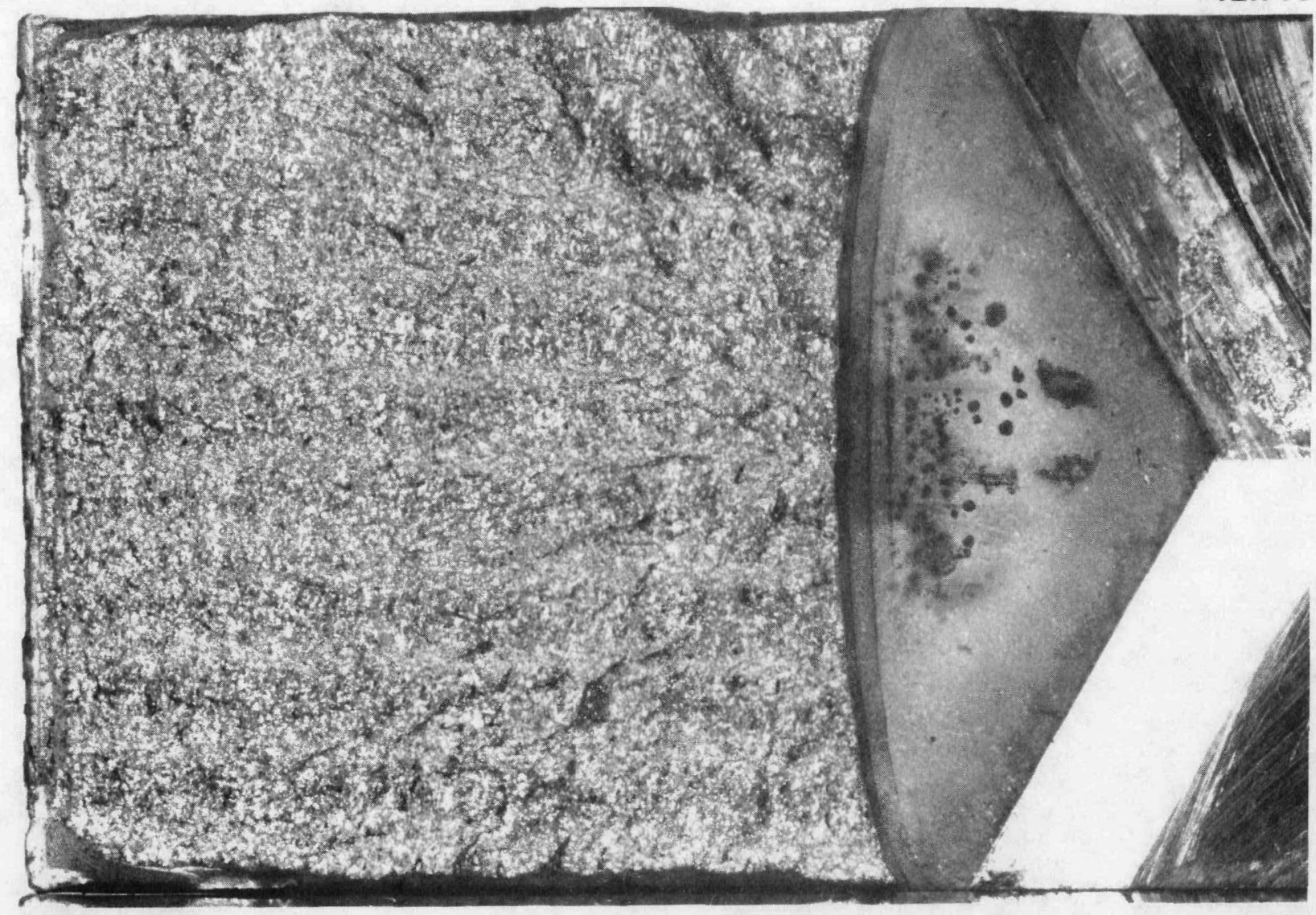

NUREG/CR-5913 


\section{E-85}

SPECIMEN ID $=72 W 85$

MATERIAL TYPE = MCUWELD

TEST TEMPERATURE $=-30$ DEG $C$

THICKNESS $=50.9 \mathrm{~mm}$

SIDE GROOUES = $0 \%$

INITIAL CRACK LENGTH $=52.6 \mathrm{~mm}$

MEASURED DUCTILE DELTA-A $=.16 \mathrm{~mm}$

$a / W) i=.518$

YIELD STRENGTH $=534 \mathrm{MPa}$

FLOW STRENGTH $=598 \mathrm{MPa}$

ESTIMATED YOUNG'S MODULUS $=209 \mathrm{GPa}$

SPECIMEN CLEAUED AT KQ= $152.5 \mathrm{MPa}$ SQR [m] (NOT UALID $K I \mathrm{c}$ )

UALUES AT MAXIMUM LOAD

J-INTEGRAL $=130.4 \mathrm{~kJ} / \mathrm{m}^{\wedge} 2$

$K J c=165.1 \mathrm{MPa} S Q R[\mathrm{~m}]$

$K$-bet $a-c=113.7 \mathrm{MPa} \operatorname{SQR}[\mathrm{m}]$
Ualues at cleavage load

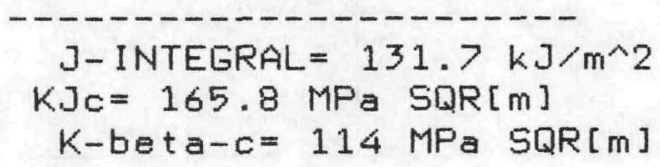


E-86

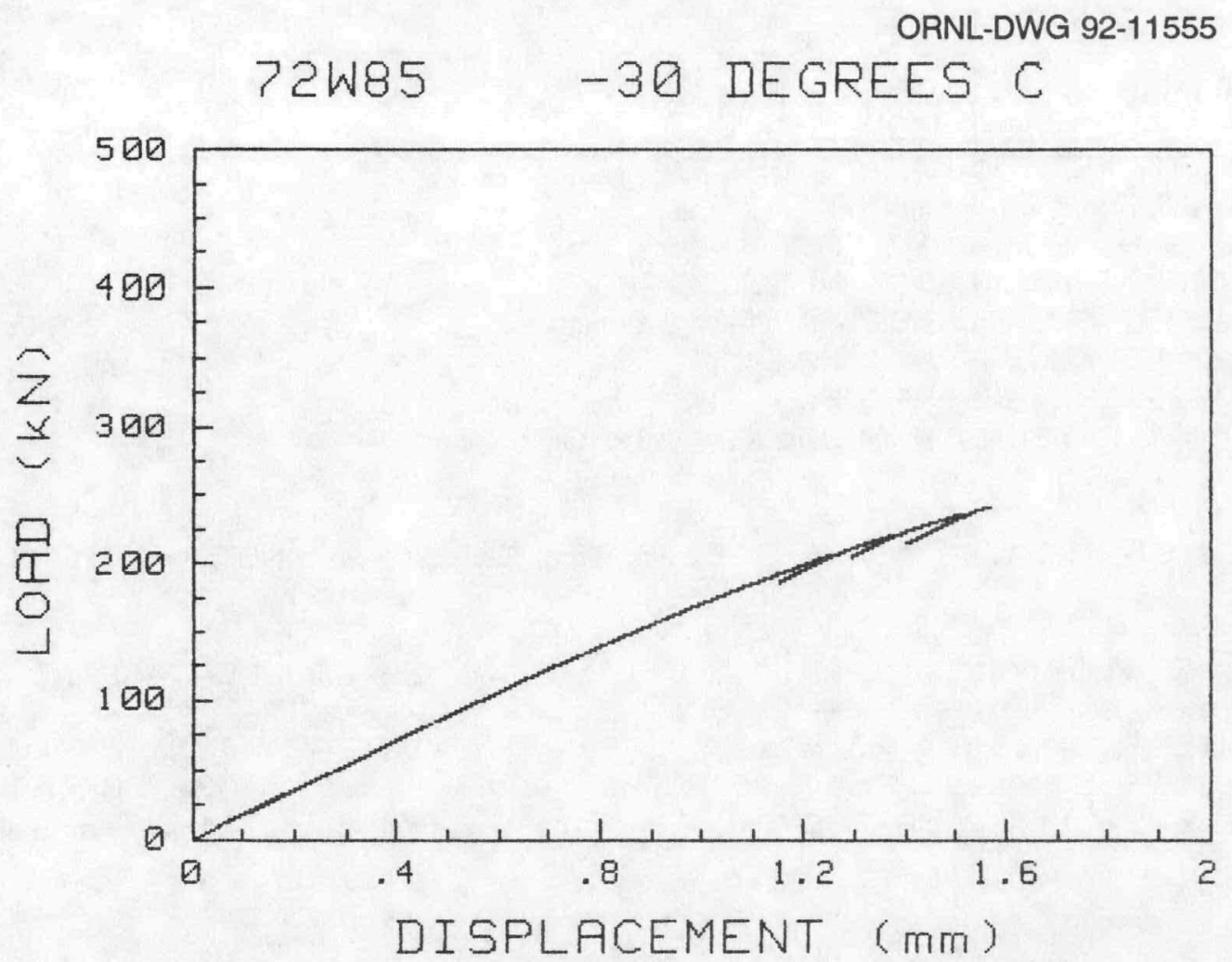

$72 W-85$

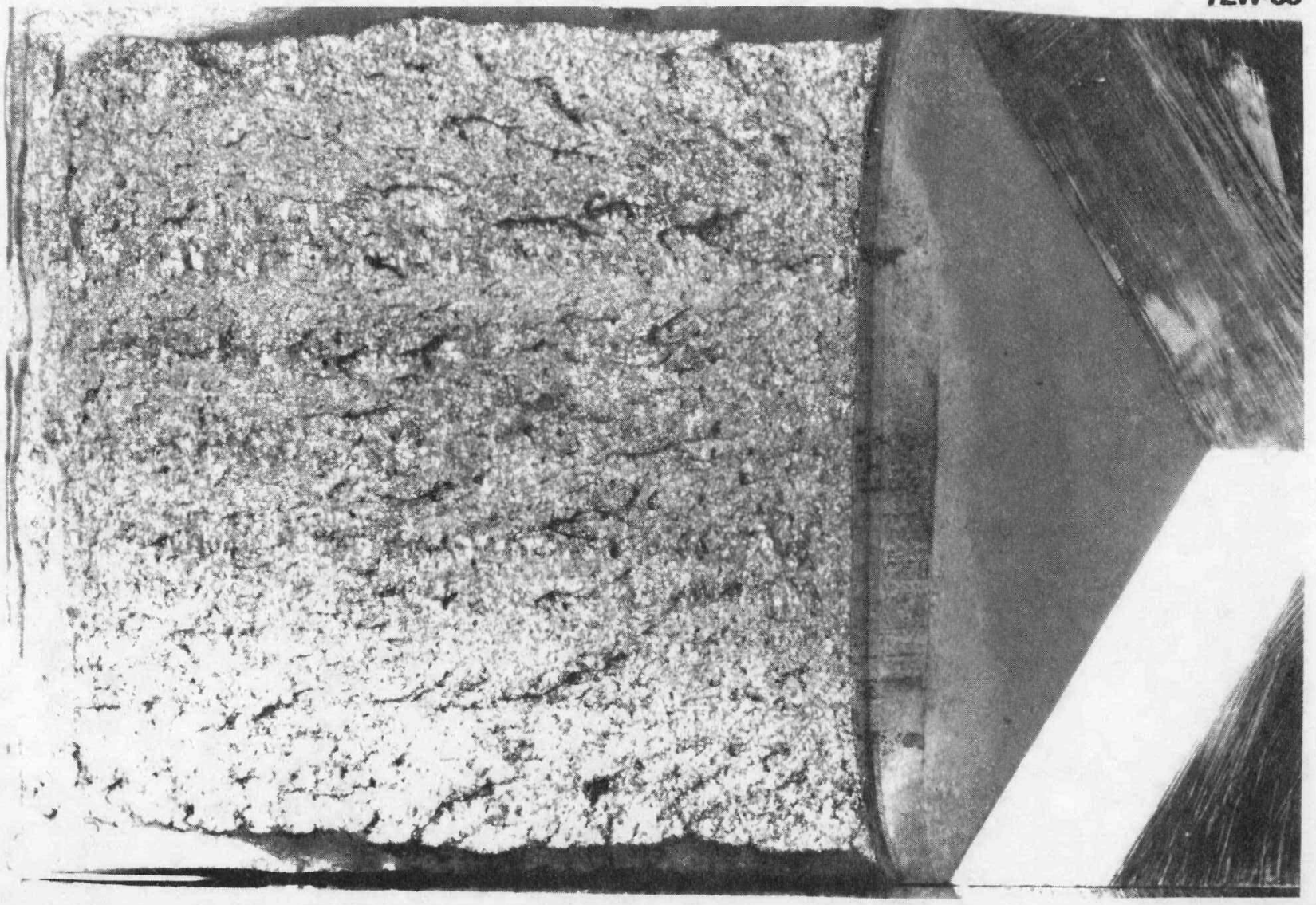


SPECIMEN ID = $72 W 50$

MATERIAL TYPE $=$ MCUWELD

TEST TEMPERATURE $=-15$ DEG $\mathrm{C}$

THICKNESS $=50.9 \mathrm{~mm}$

SIDE GROOUES = $0 \%$

INITIAL CRACK LENGTH $=54.8 \mathrm{~mm}$

$a / W) i=.54$

MEASURED DUCTILE DELTA-A $=.06 \mathrm{~mm}$

$Y$ IELD STRENGTH $=526 \mathrm{MPa}$

FLOW STRENGTH $=586 \mathrm{MPa}$

ESTIMATED YOUNG'S MODULUS $=208 \mathrm{GPa}$

SPECIMEN CLEAUED AT KQ= $98.4 \mathrm{MPa}$ SQR $[\mathrm{m}]$ (NOT UALID KIc)

UALUES AT MAXIMUM LDAD

J-INTEGRAL $=46.6 \mathrm{~kJ} / \mathrm{m}^{\wedge} 2$

$K \cdot{ }_{c}=98.5 \mathrm{MPa} S Q R[\mathrm{~m}]$

$K-b e t a-c=84.5 \mathrm{MPa} \operatorname{SQR}[\mathrm{m}]$
UALUES at CLEAUAGE LDAD

J-INTEGRAL $=46.6 \mathrm{~kJ} / \mathrm{m}^{\wedge} 2$

$K J c=98.5 \mathrm{MPa} S Q R[\mathrm{~m}]$

$K$-bet $a-c=84.5 \mathrm{MPa} \operatorname{SQR}[\mathrm{m}]$ 
E-88

ORNL-DWG 92-11556

72W50 -15 DEGREES C

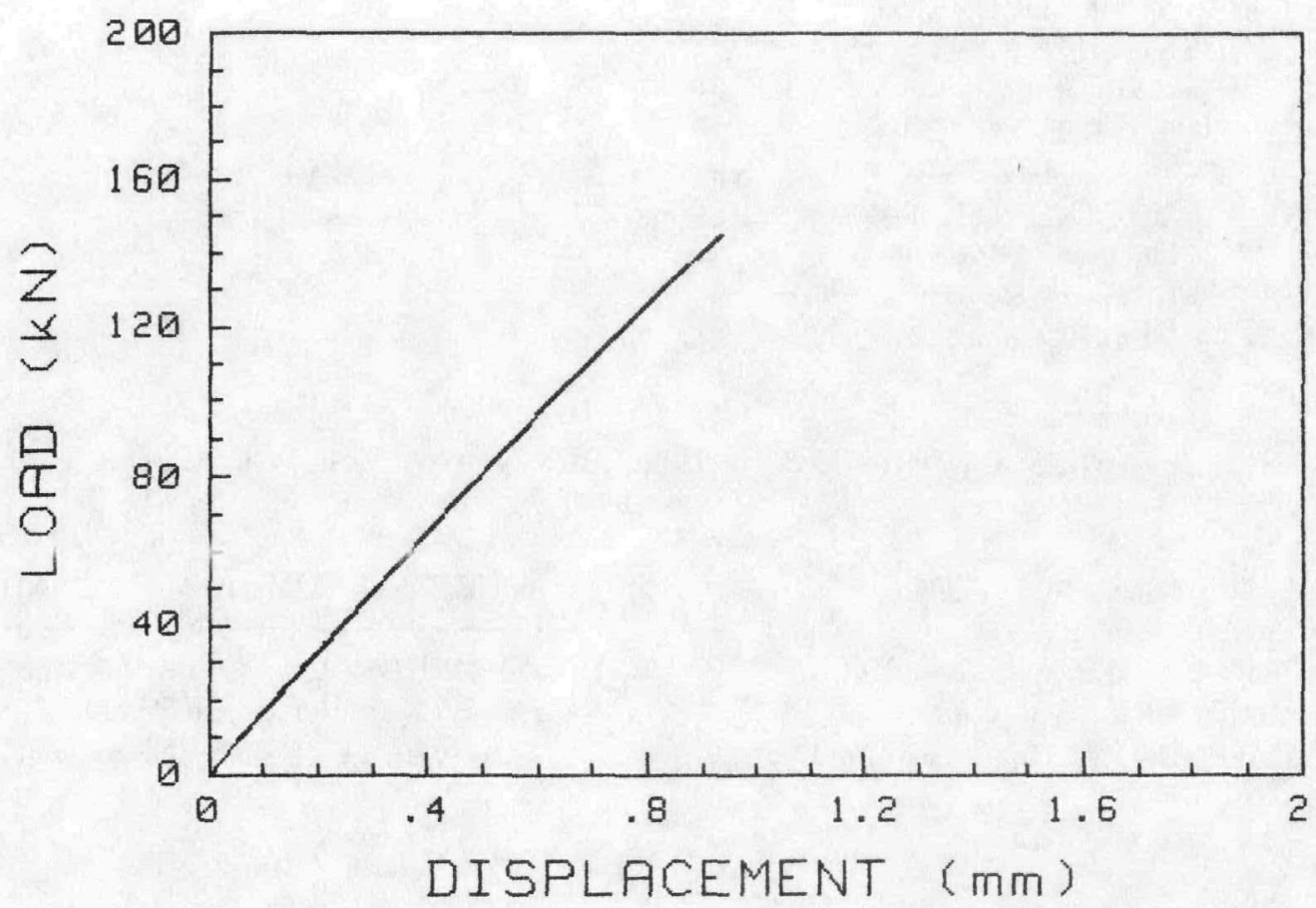

$72 W-50$

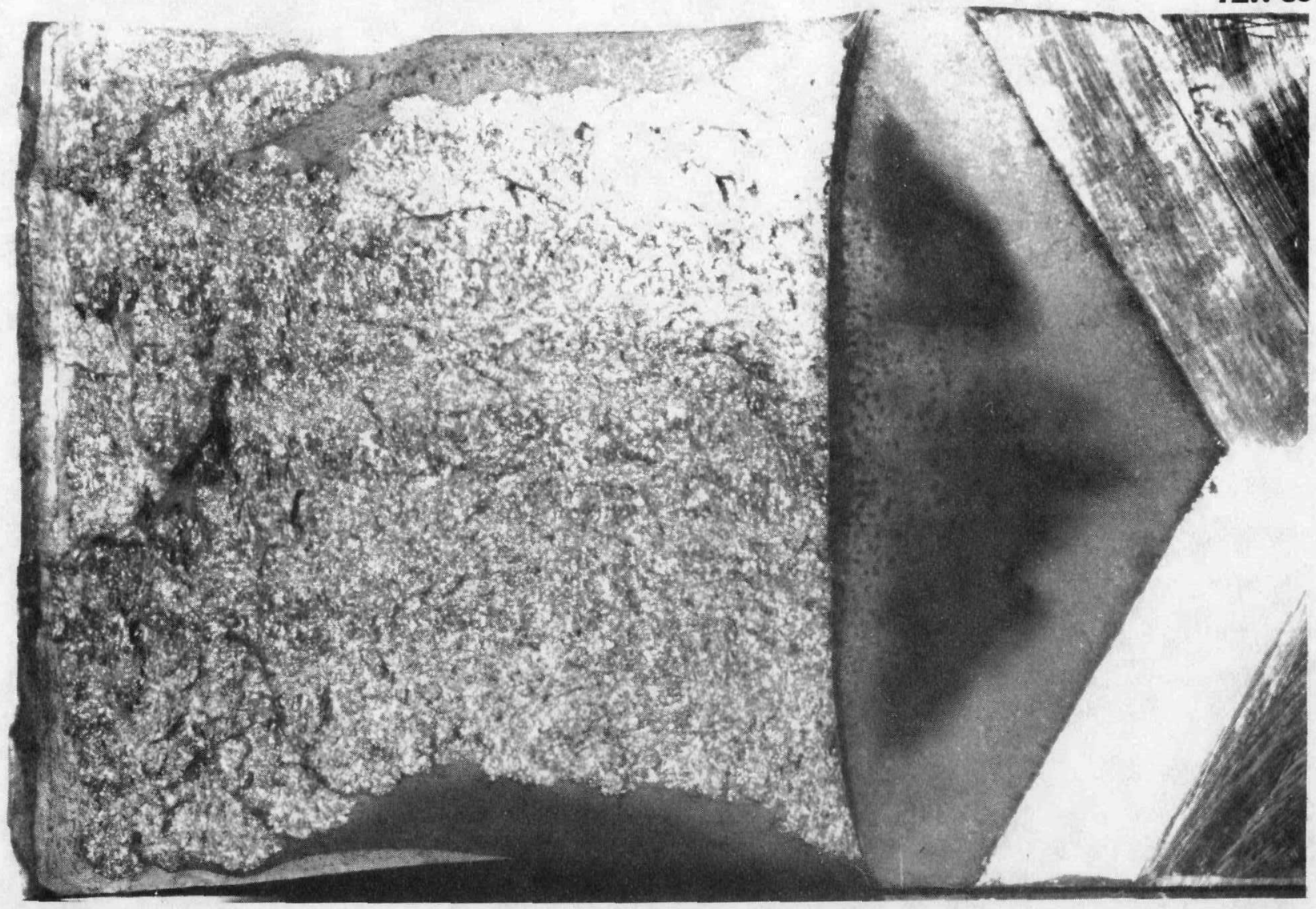


SPECIMEN ID $=72 \mathrm{~W} 87$

MATERIAL TYPE = MCUWELD

TEST TEMPERATURE $=-15$ DEG $C$

THICKNESS $=50.8 \mathrm{~mm}$

SIDE GROOUES $=0 \%$

INITIAL CRACK LENGTH $=53.2 \mathrm{~mm}$

$a / W) i=.524$

MEASURED DUCTILE DELTA-A $=.1 \mathrm{~mm}$

$Y$ IELD STRENGTH $=526 \mathrm{MPa}$

FLOW STRENGTH $=586 \mathrm{MPa}$

ESTIMATED YOUNG'S MODULUS $=208 \mathrm{GPa}$

SPECIMEN CLEAUED AT KQ= $117.6 \mathrm{MPa}$ SQR[m] (NOT UALID KIc)

UALUES AT MAXIMUM LOAD

J-INTEGRAL $=68.5 \mathrm{~kJ} / \mathrm{m}^{\wedge} 2$

$\mathrm{KJ} c=119.4 \mathrm{MPa} \operatorname{SQR}[\mathrm{m}]$

$K$-beta-c $=95 \mathrm{MPa} \operatorname{SQR}[\mathrm{m}]$
UALUES AT CLEAUAGE LDAD

J-INTEGRAL $=68.5 \mathrm{~kJ} / \mathrm{m}^{\wedge} 2$

$\mathrm{KJ}=119.4 \mathrm{MPa} \operatorname{SQR}[\mathrm{m}]$

$K$-beta-c $=95 \mathrm{MPa} \operatorname{SQR}[\mathrm{m}]$ 


\section{E-90}

ORNL-DWG 92-11557

72W87 - 15 DEGREES $C$

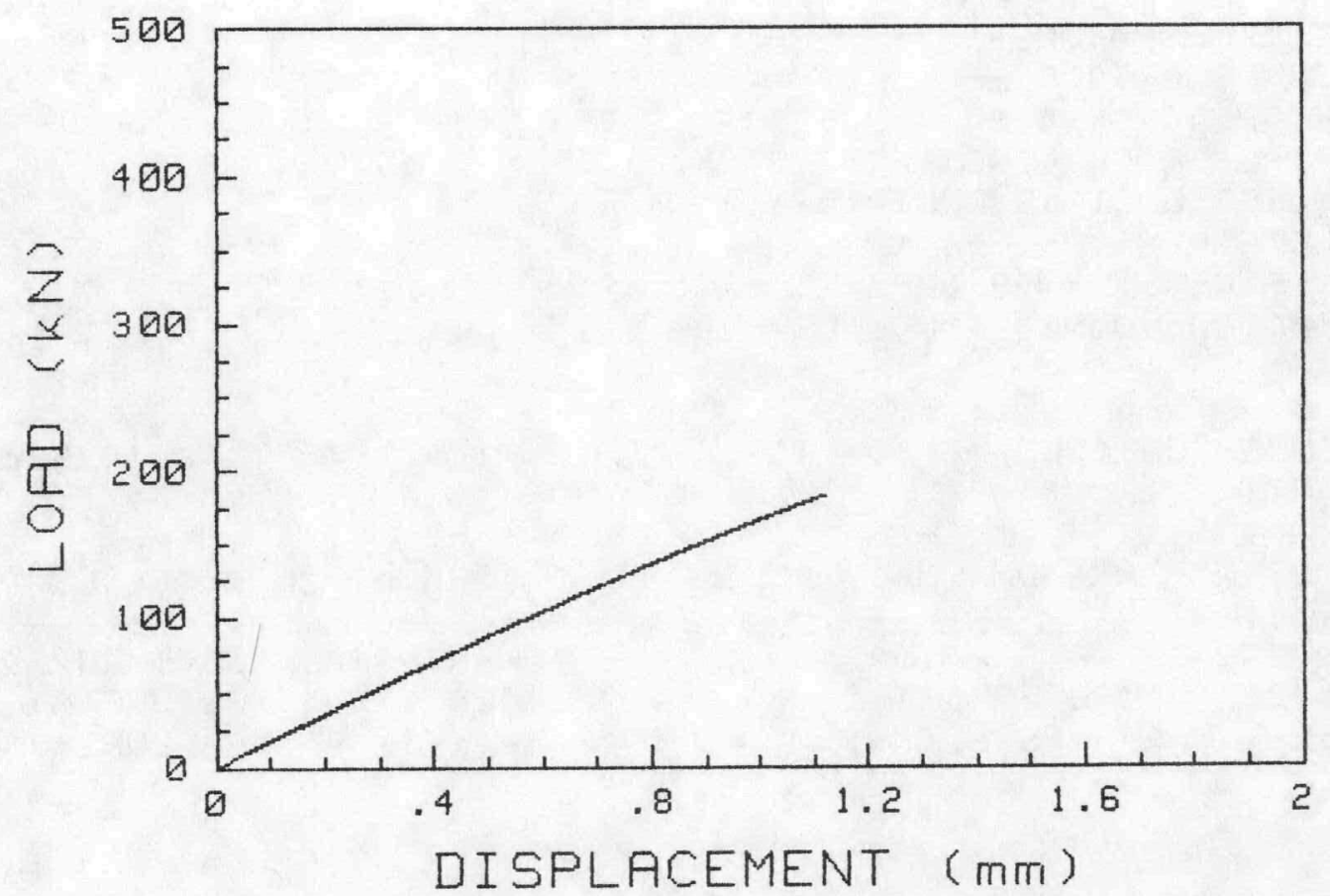

72W-87

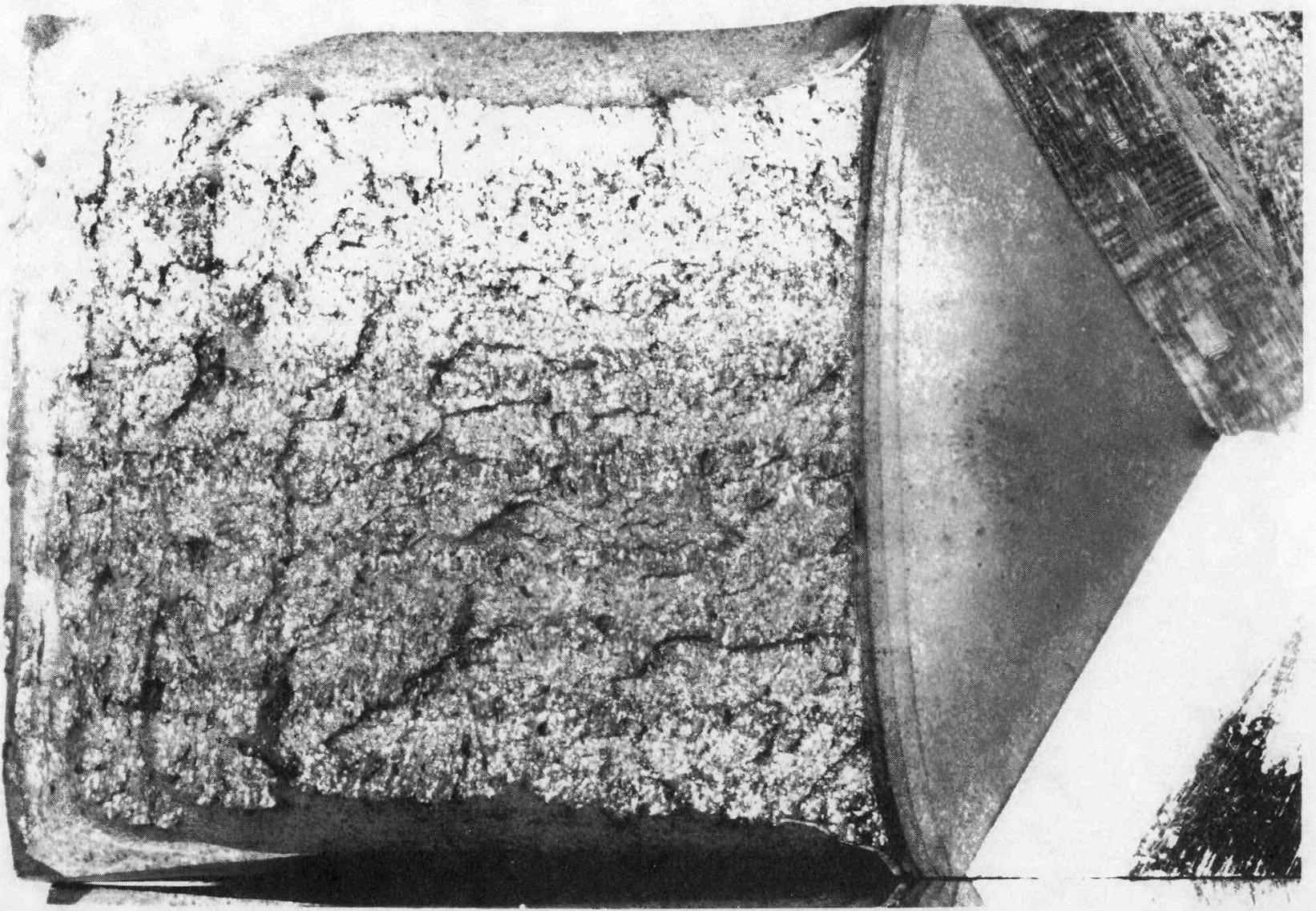


SPECIMEN ID $=72 W 62$

MATERIAL TYPE = MCUWELD

TEST TEMPERATURE $=-15$ DEG $C$

THICKNESS $=50.8 \mathrm{~mm}$

SIDE GROOUES = $0 \%$

INITIAL CRACK LENGTH $=53.7 \mathrm{mrn}$

$a / W) \mathrm{i}=.529$

MEASURED DULTILE DELTA-A $=.15 \mathrm{~mm}$

YIELD STRENGTH $=526 \mathrm{MPa}$

FLDW STRENGTH $=586 \mathrm{MPa}$

EST IMATED YOUNG'S MODULUS $=208$ GPa

SPECIMEN CLEAUED AT KQ= $150.5 \mathrm{MPa}$ SQR [m] (NOT UALID KIc)

UALUES AT MAXIMUM LOAD

J-INTEGRAL $=138.5 \mathrm{~kJ} / \mathrm{m}^{\wedge} 2$

$K J c=169.8 \mathrm{MPa} S Q R[\mathrm{~m}]$

$K$-beta-c $=114.3 \mathrm{MPa} \operatorname{SQR}[\mathrm{m}]$
UALUES AT CLEaUAGE LDAD

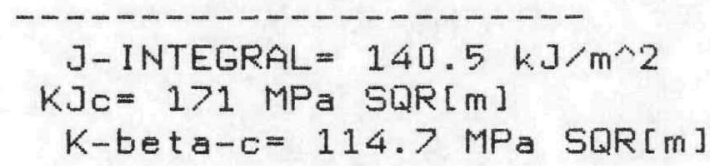




\section{E-92}

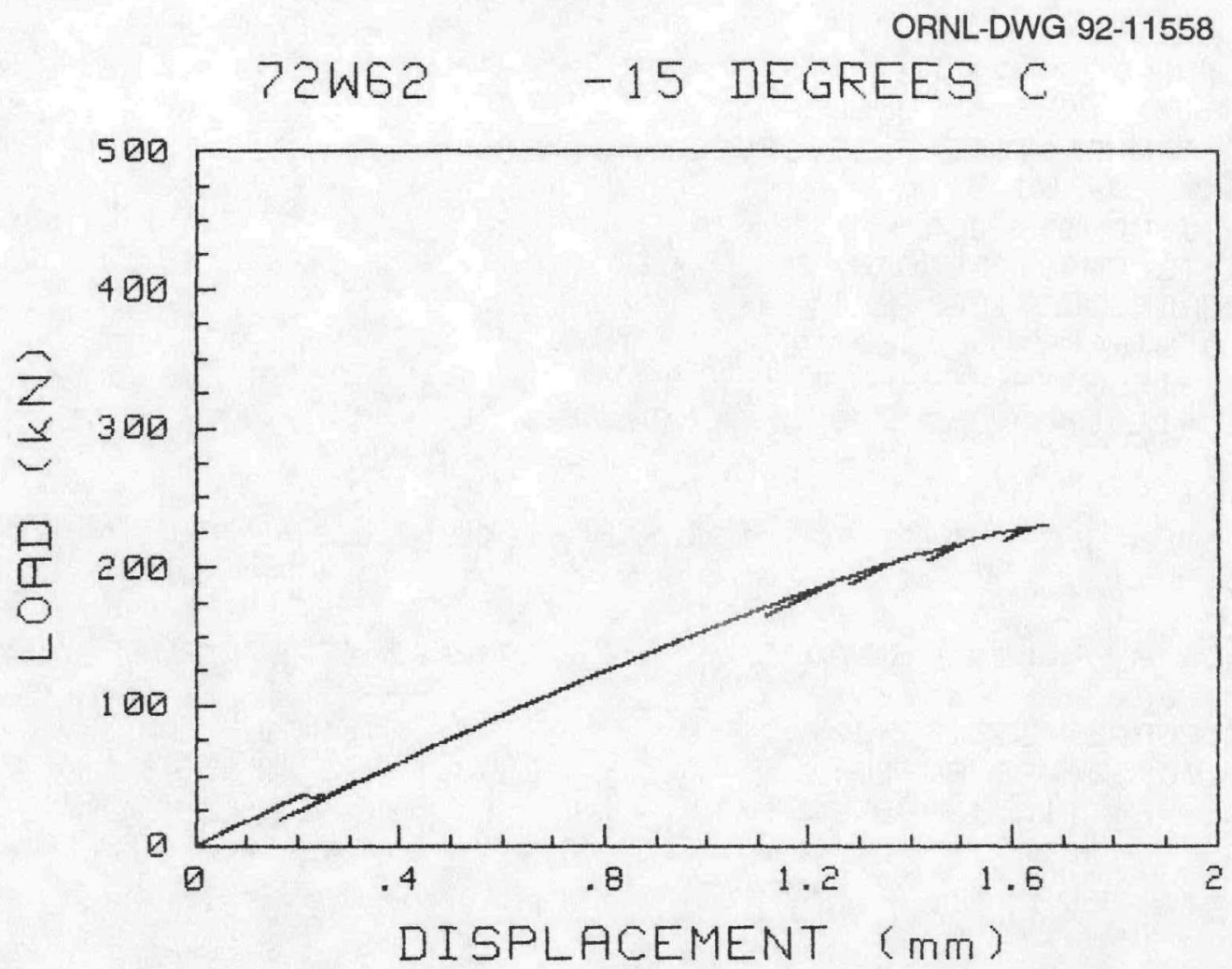

72W-62

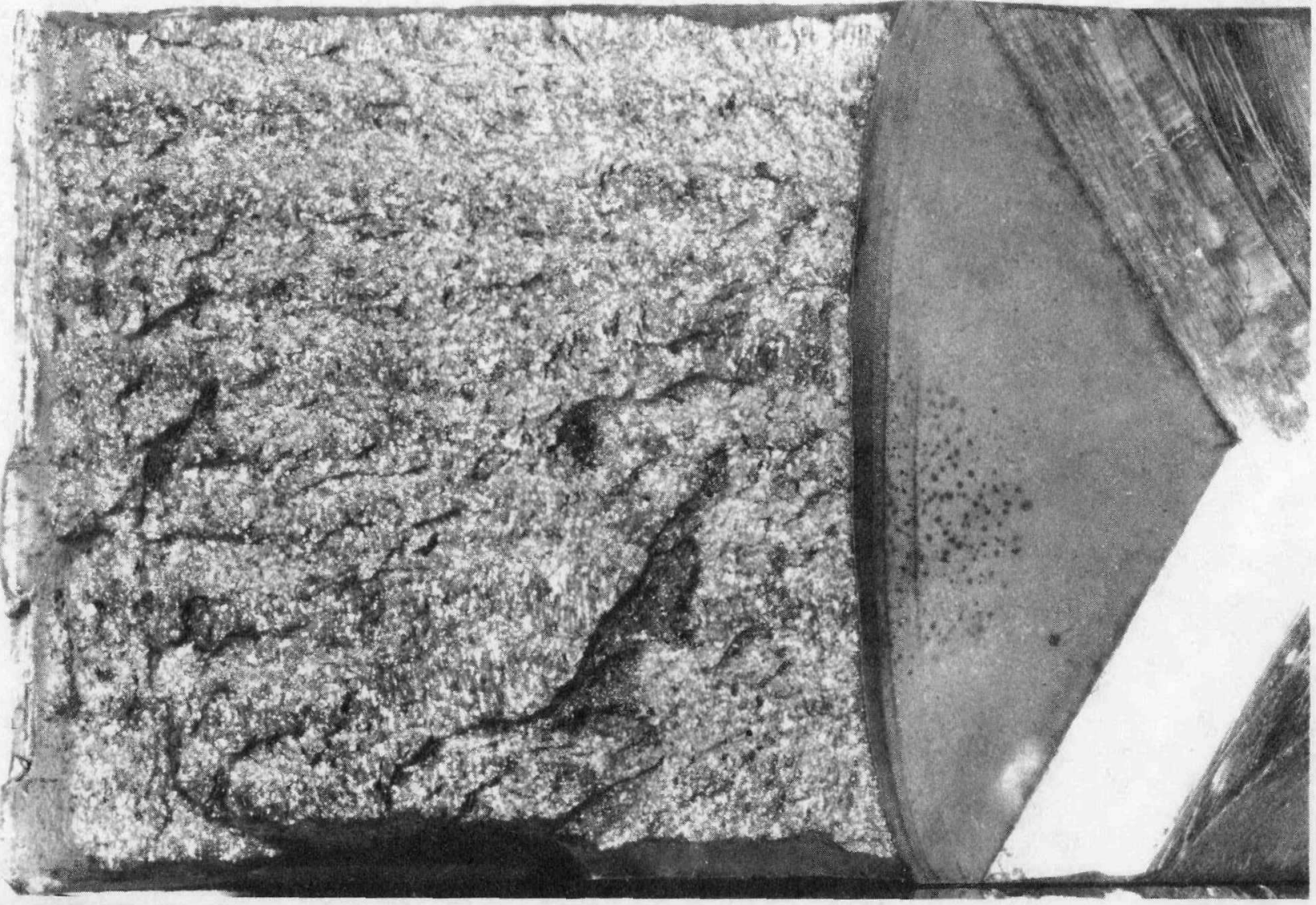




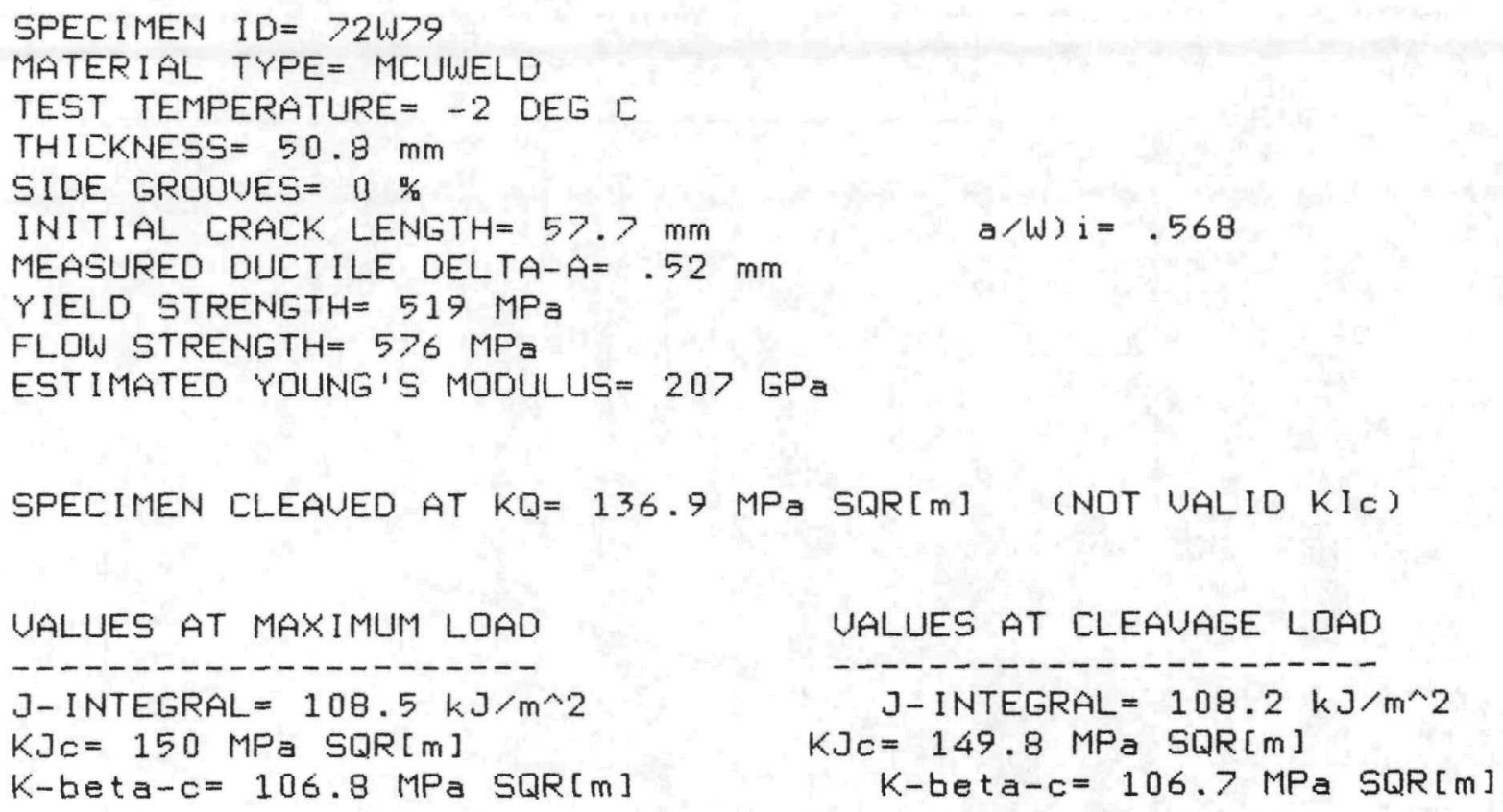


E-94

ORNL-DWG 92-11559

72W79 -2 DEGREES C

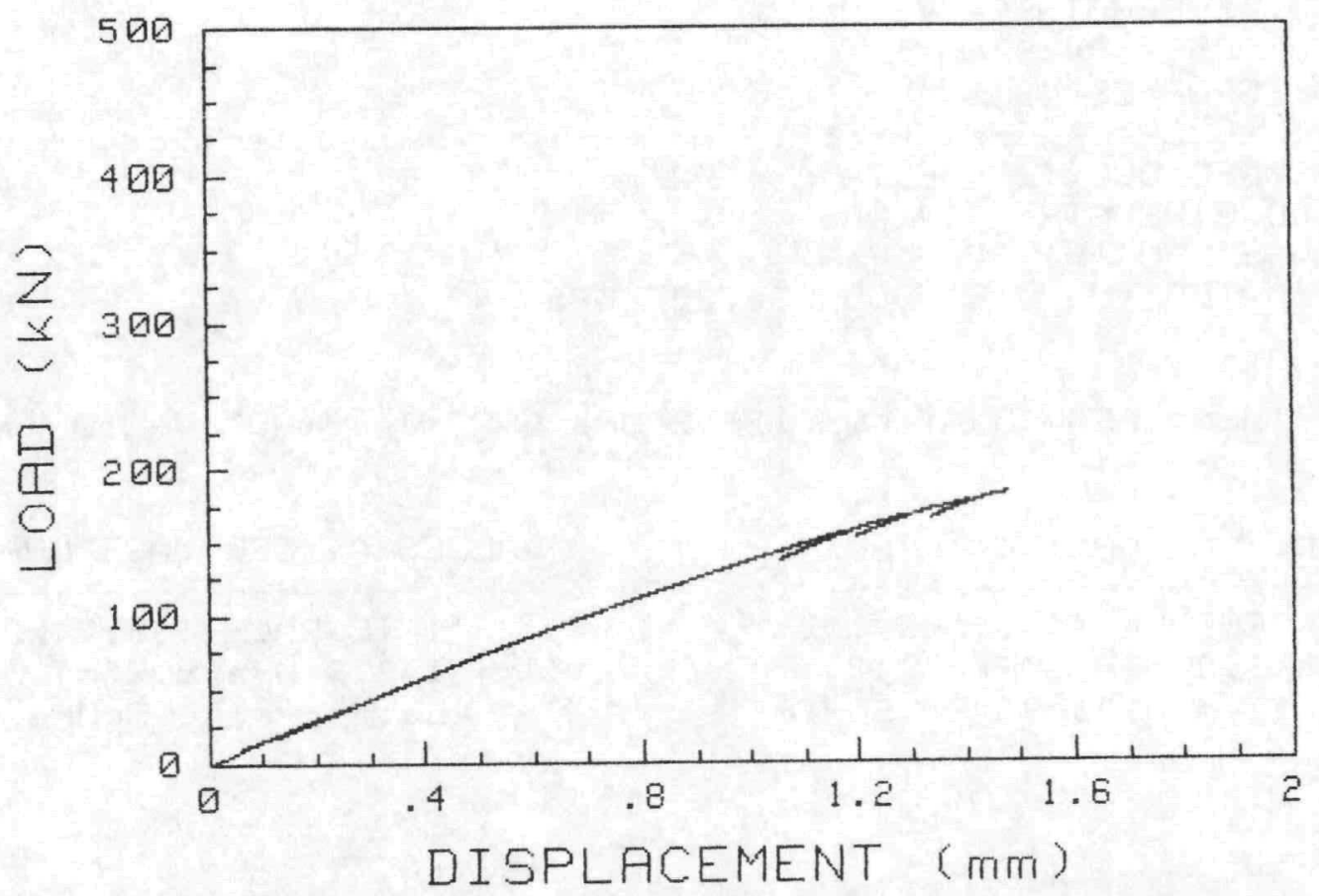

$72 W-79$

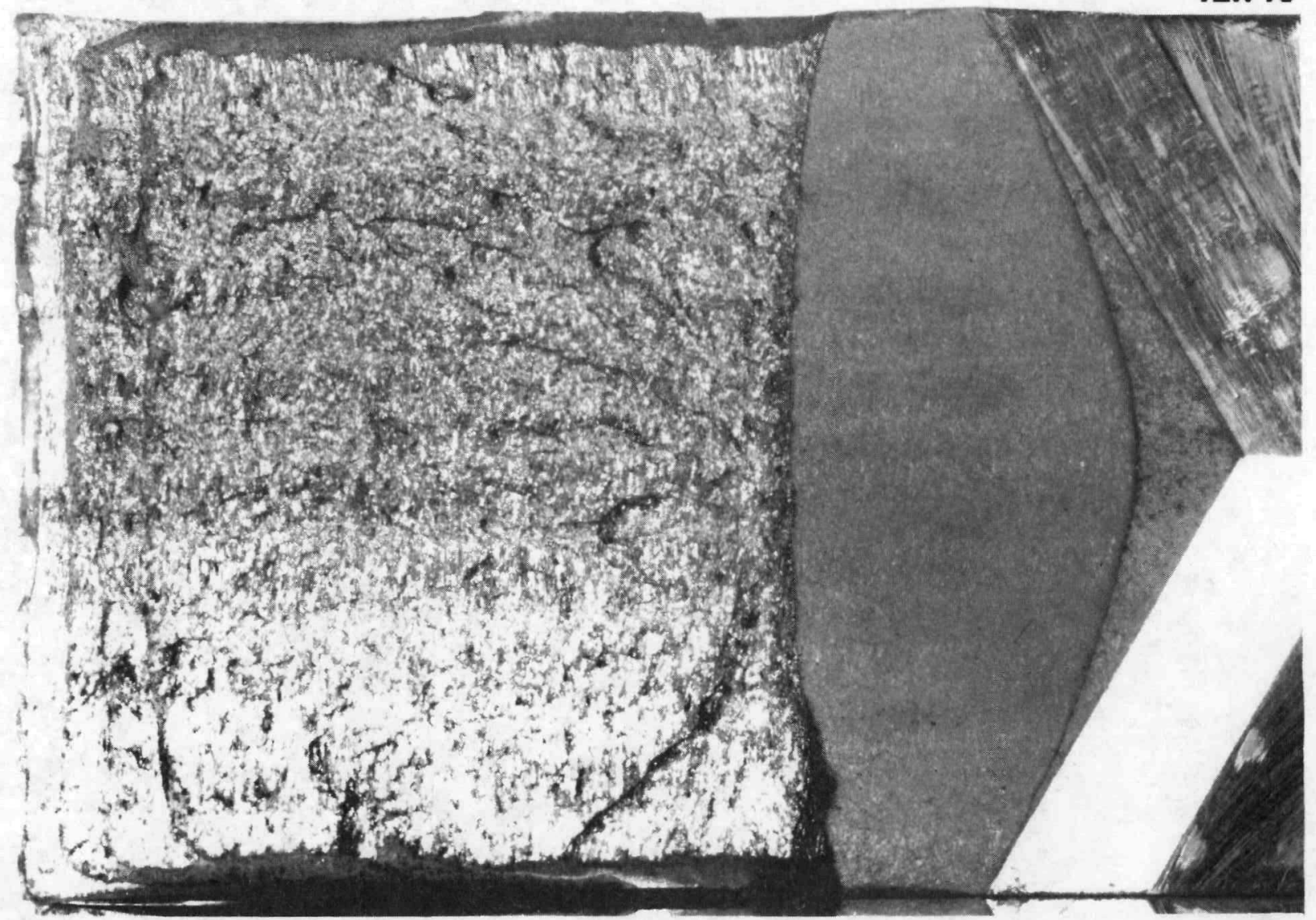


Specimen Crackmouth Def lection (in .) ORNL-DWG 92-11560

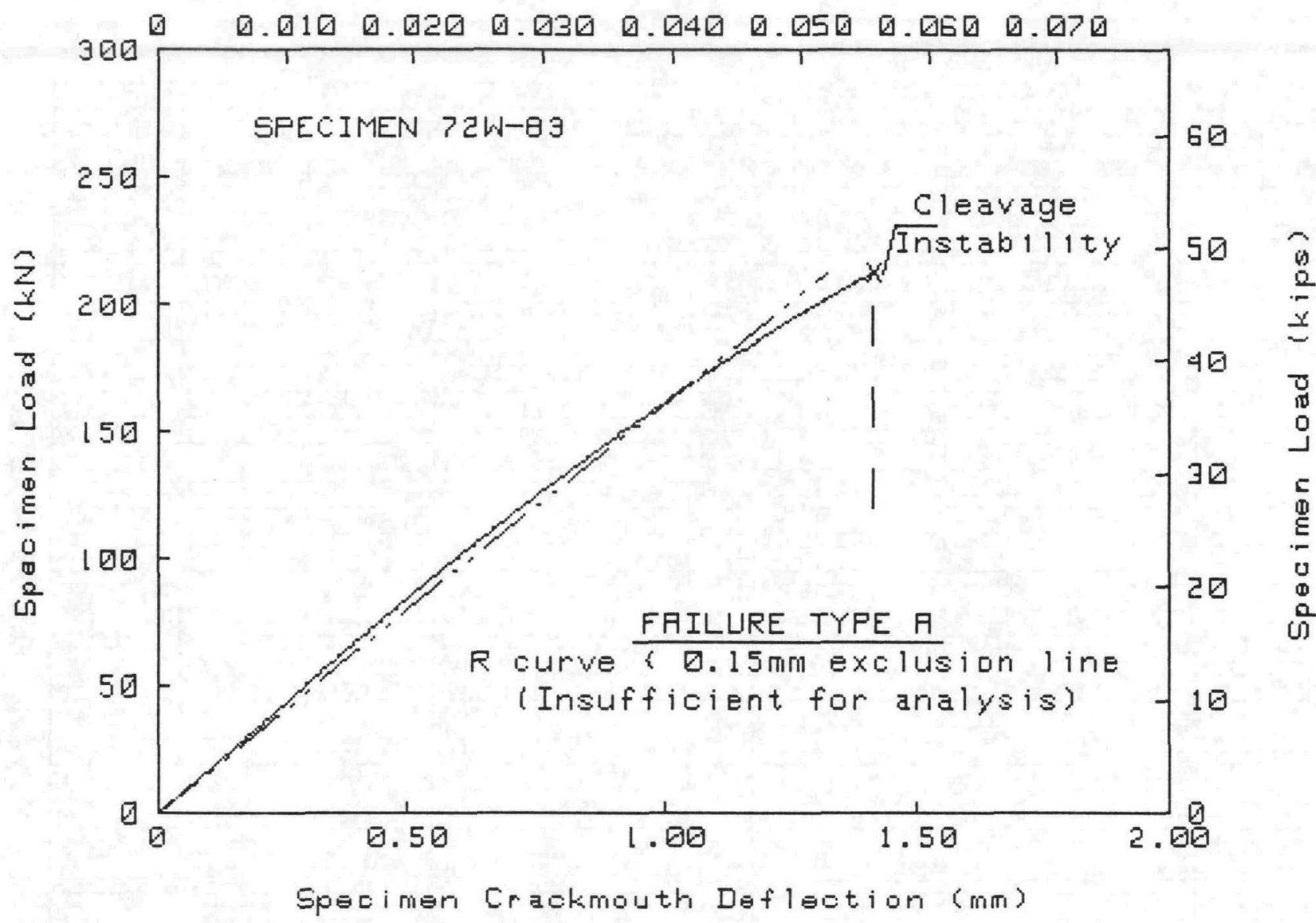

TEST SFECIMEN DATA

Material Type
Test Temperature
Percent Side Groove
Specimen Thickness B
Initial a/W
Init crack length a
Flow strength
Yield strength
Youngs modulus
Poisson's Ratio ( E)
J-INTEGRAL ANALYSIS

= HSST WELD

$=10^{\circ} \mathrm{C}$

$=0 \%$

$=50.8 \mathrm{~mm}$

$=.52$

$=52.8 \mathrm{~mm}$

$=566.7 \mathrm{MPa}$

$=510.9 \mathrm{MPa}$

$=206600 \mathrm{MPa}$

$=0$

J-INTEGRAL ANALYSIS

Je

[ Failure Type $A$ (J at instability = JC〉]

$=\frac{\text { Merkle-Corten }}{108.4 \mathrm{~kJ} / \mathrm{m}^{2}}$

$106.9 \mathrm{~kJ} / \mathrm{m}^{2}$

$=149.7 \mathrm{MPa} \sqrt{\mathrm{m}}$

$148.6 \mathrm{MPa} \sqrt{\mathrm{m}}$

$=105.8 \mathrm{MPa} \sqrt{\mathrm{m}}$

$105.5 \mathrm{MPa} \sqrt{\mathrm{m}}$

Ke (Beta Corrected)

ASTM E399-81 ANALYSIS

Modified Kic Equation; $B=\langle B B n\rangle 1 / 2$

Maximum Load $P=212.71 \mathrm{kN}$

$5 \%$ Secant offset $\mathrm{Pq}=161.89 \mathrm{kN}$

$\mathrm{Kq}$

Validity

$K$ at Maximum Load

$=102.7 \mathrm{MPa} \sqrt{\mathrm{m}}$

$=$ INYALID--1, 2, 3

$=135 \mathrm{MPa} \sqrt{\mathrm{m}}$

DATA CORRECTIOHS USED FOR J-INTEGRAL DATA

Merkle-Corten correction

Correction to J for crack extension (Deformation-J)

OFFSET LOAD $=0 \mathrm{kN}$ OFFSET DEFLECTION $=.016 \mathrm{~mm}$

Fitted elastic load range $=25 \mathrm{kN}$ TO $75 \mathrm{kN}$

Elastic slope $=1.698 \mathrm{E}+02 \mathrm{kN} / \mathrm{mm}$ 


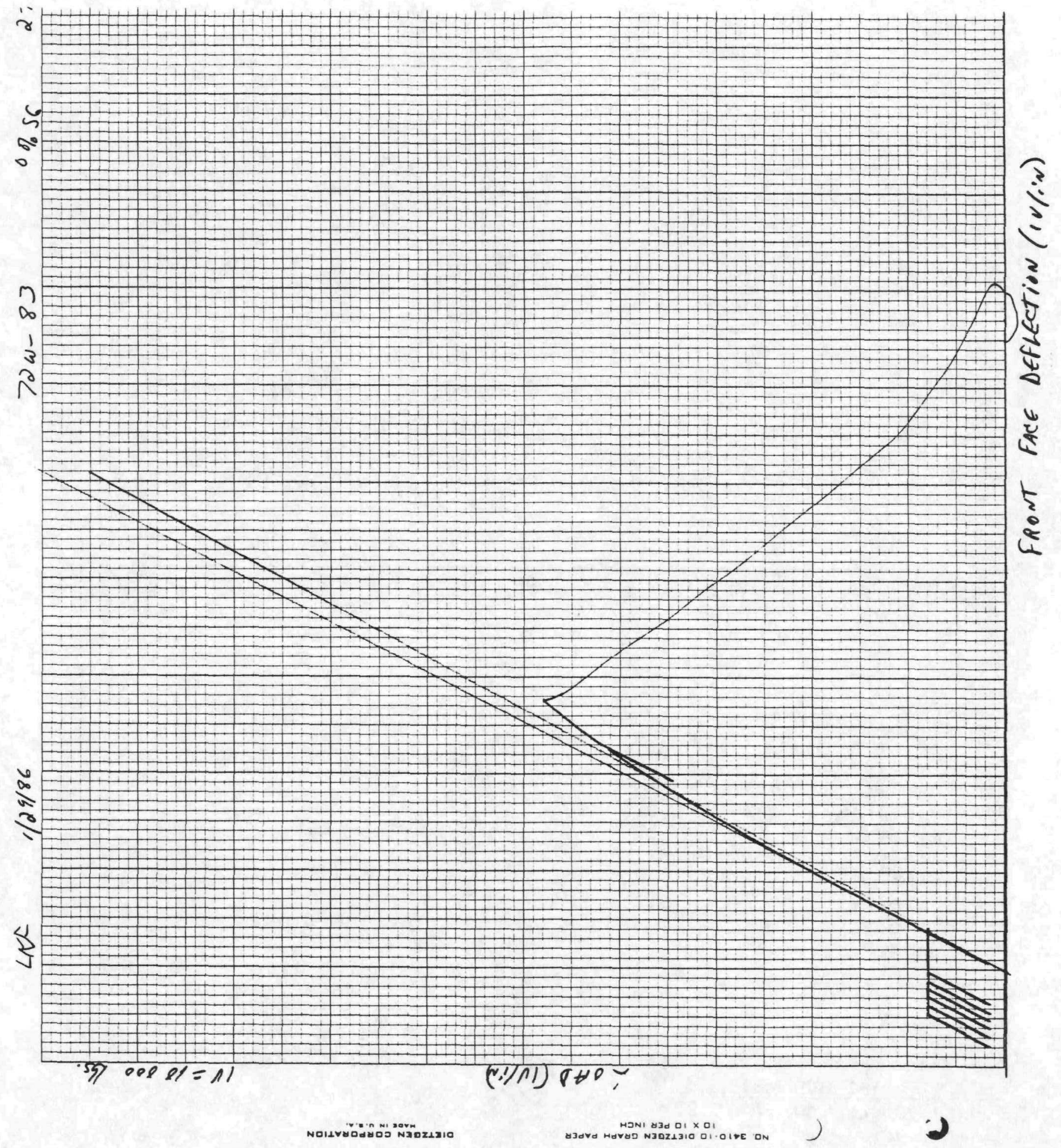


Specimen Crackmouth Deflection (in.)

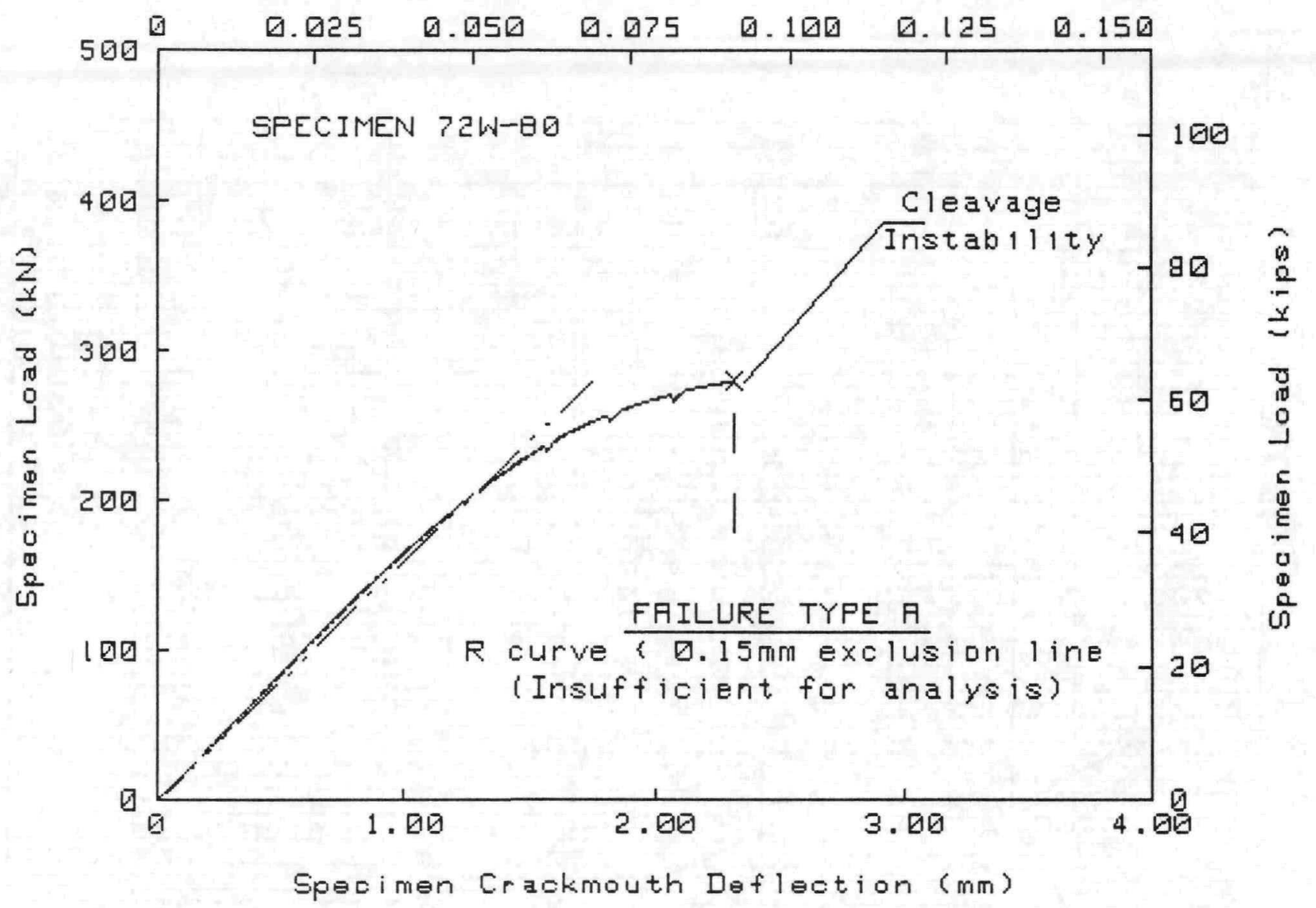

\section{TEST SPECIMEN DATA}

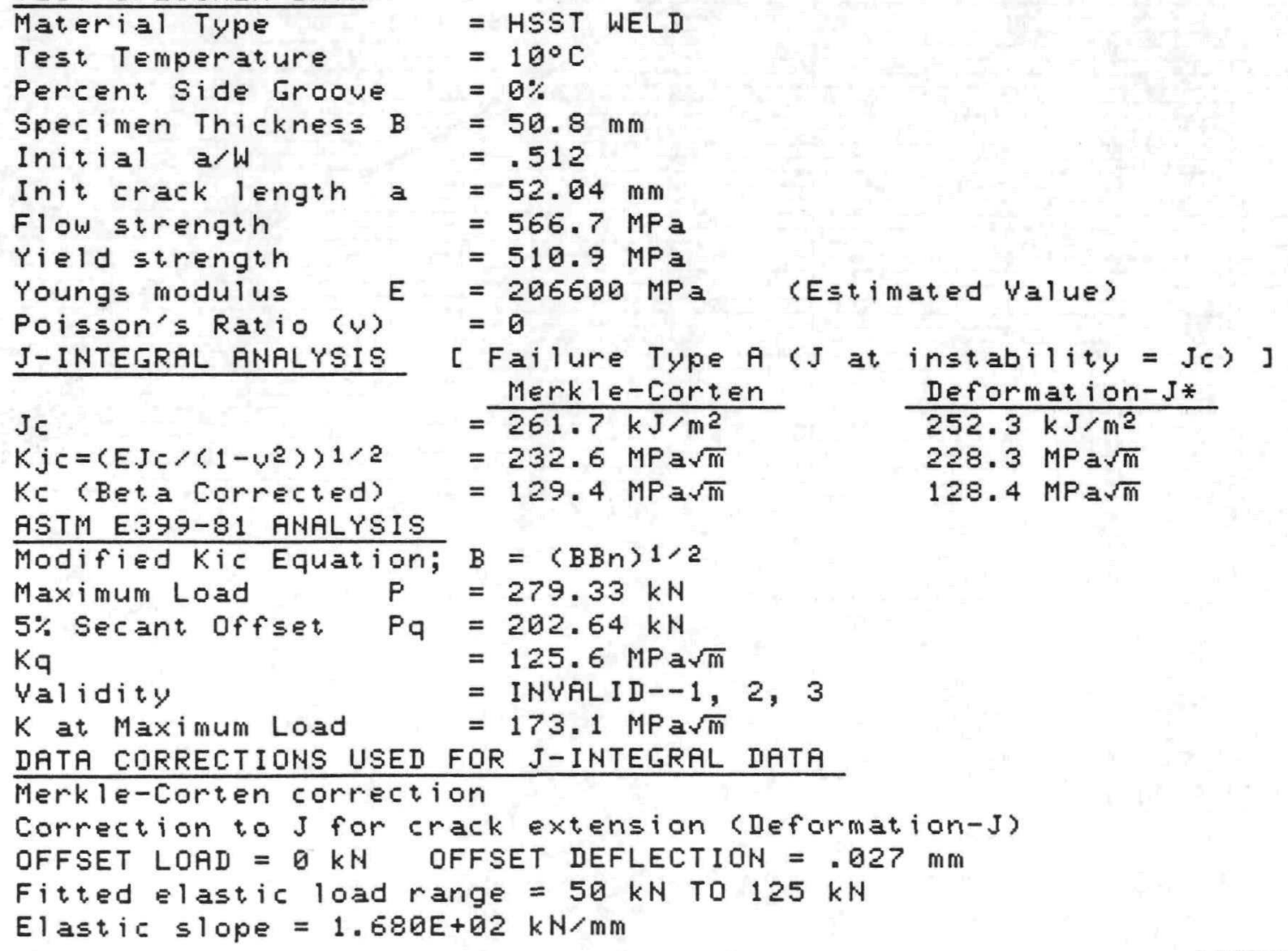




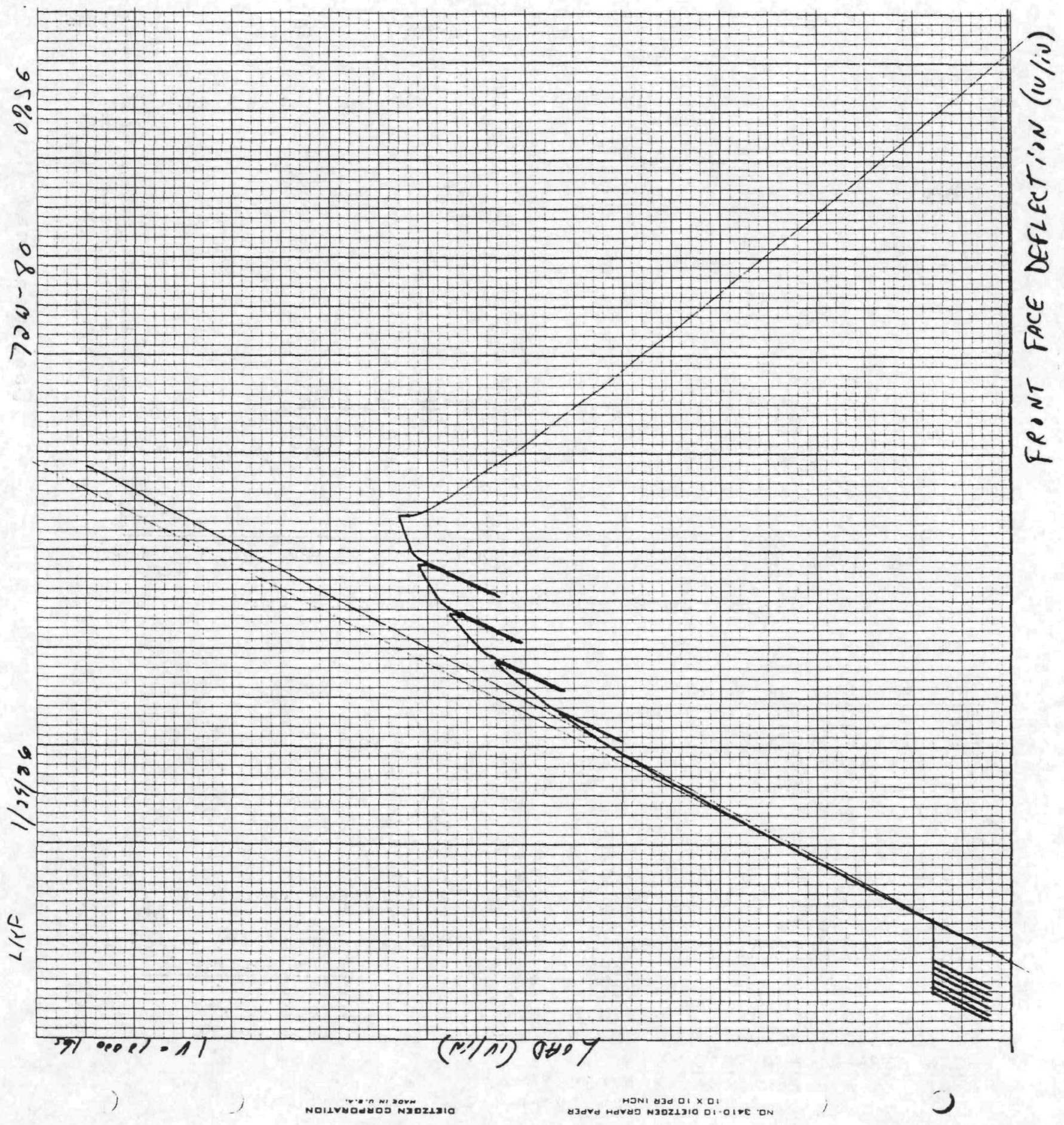


Specimen Crackmouth Def lection (in.)

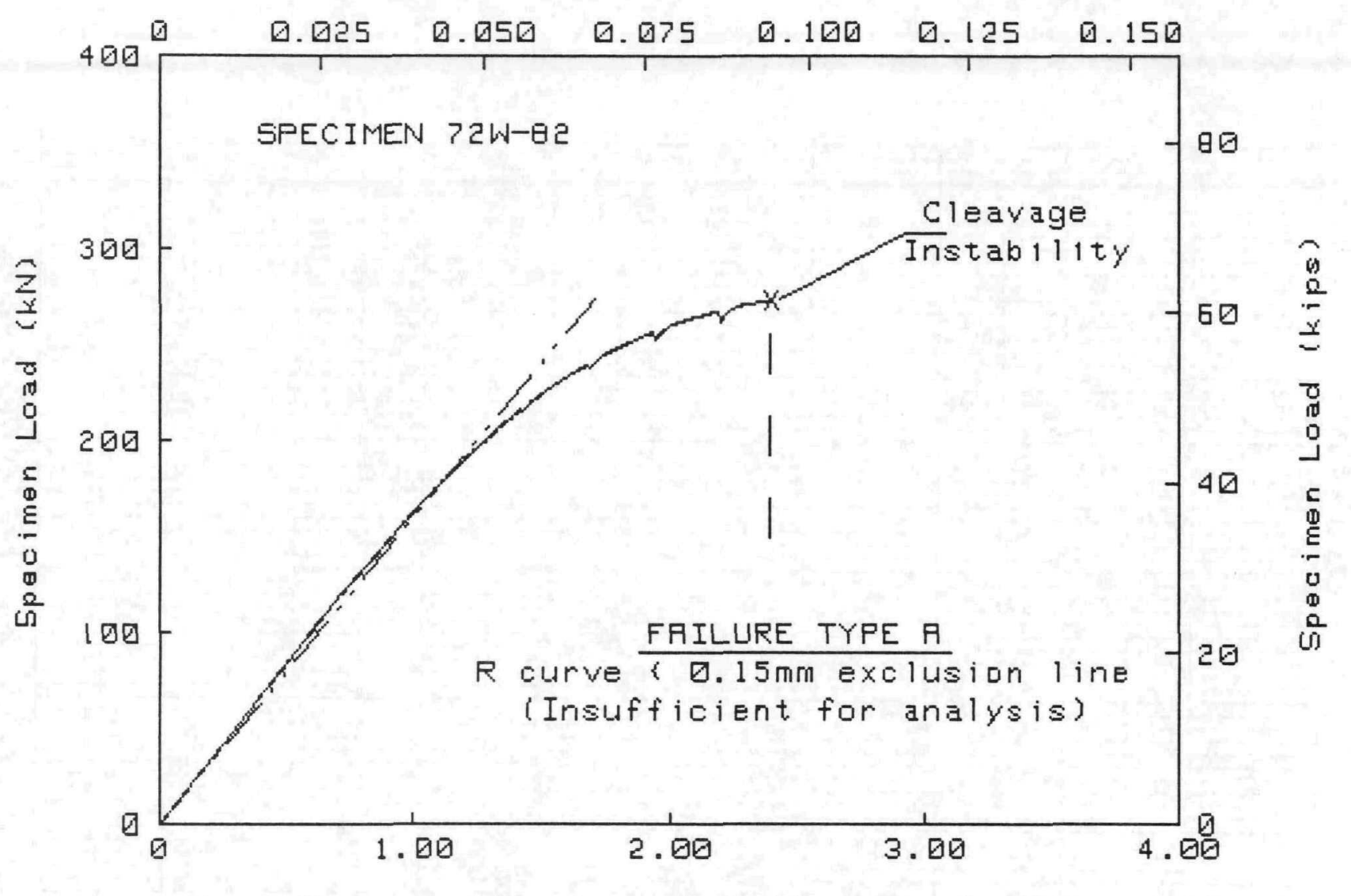

Specimen Crackmouth Defleotion (mm)

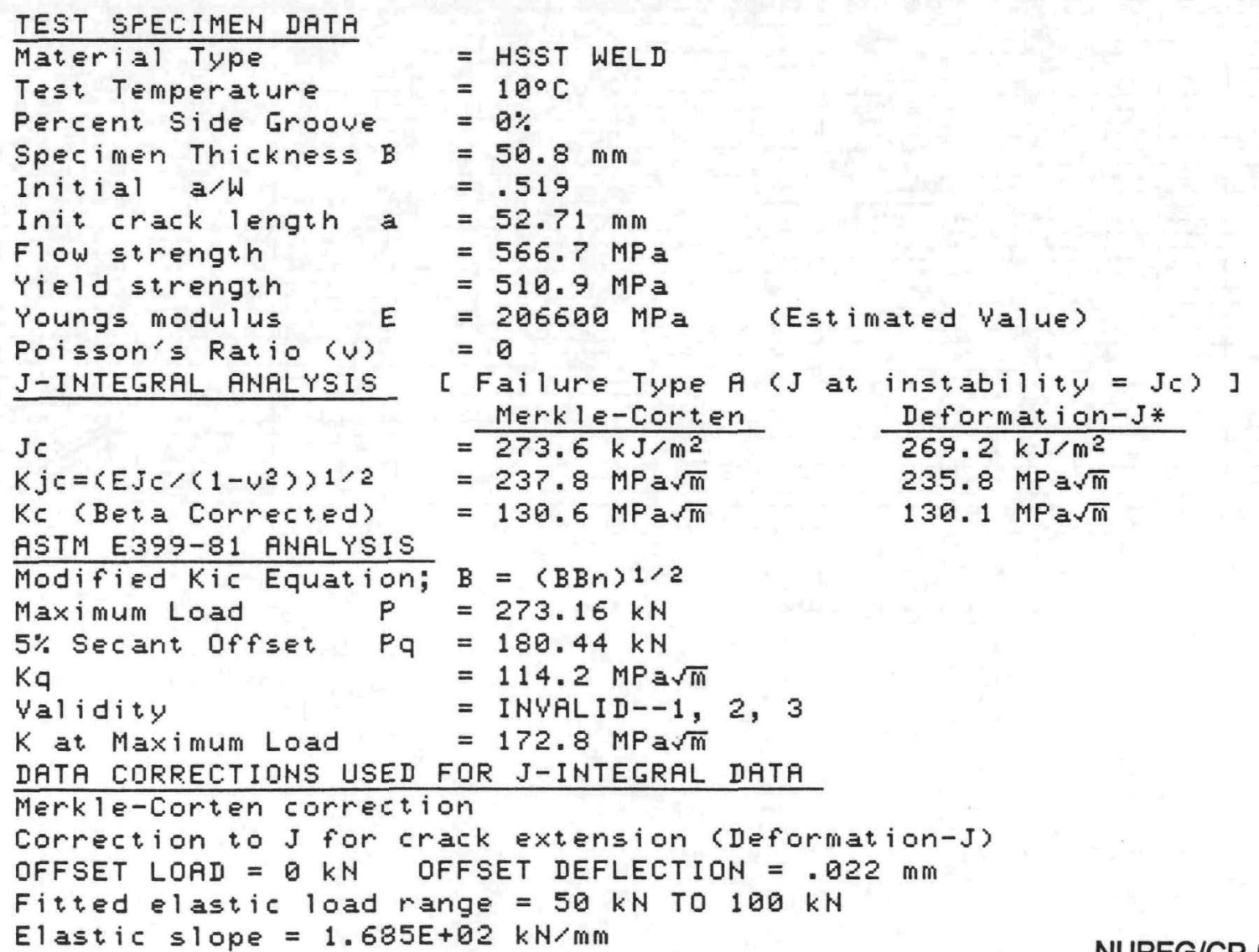




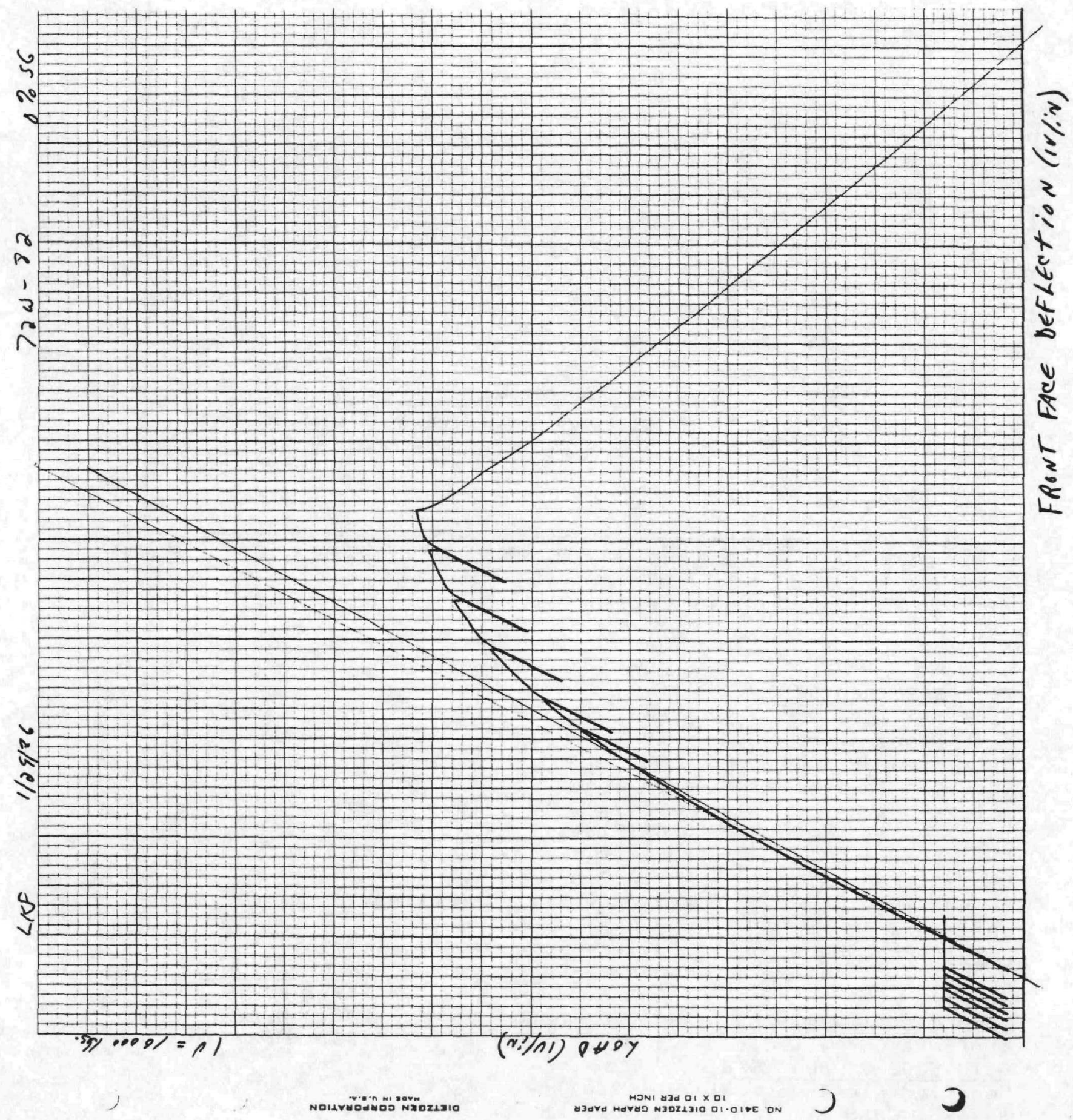


Specimen Crackmauth Def lection (in.) ORNL-DWG 92-11563

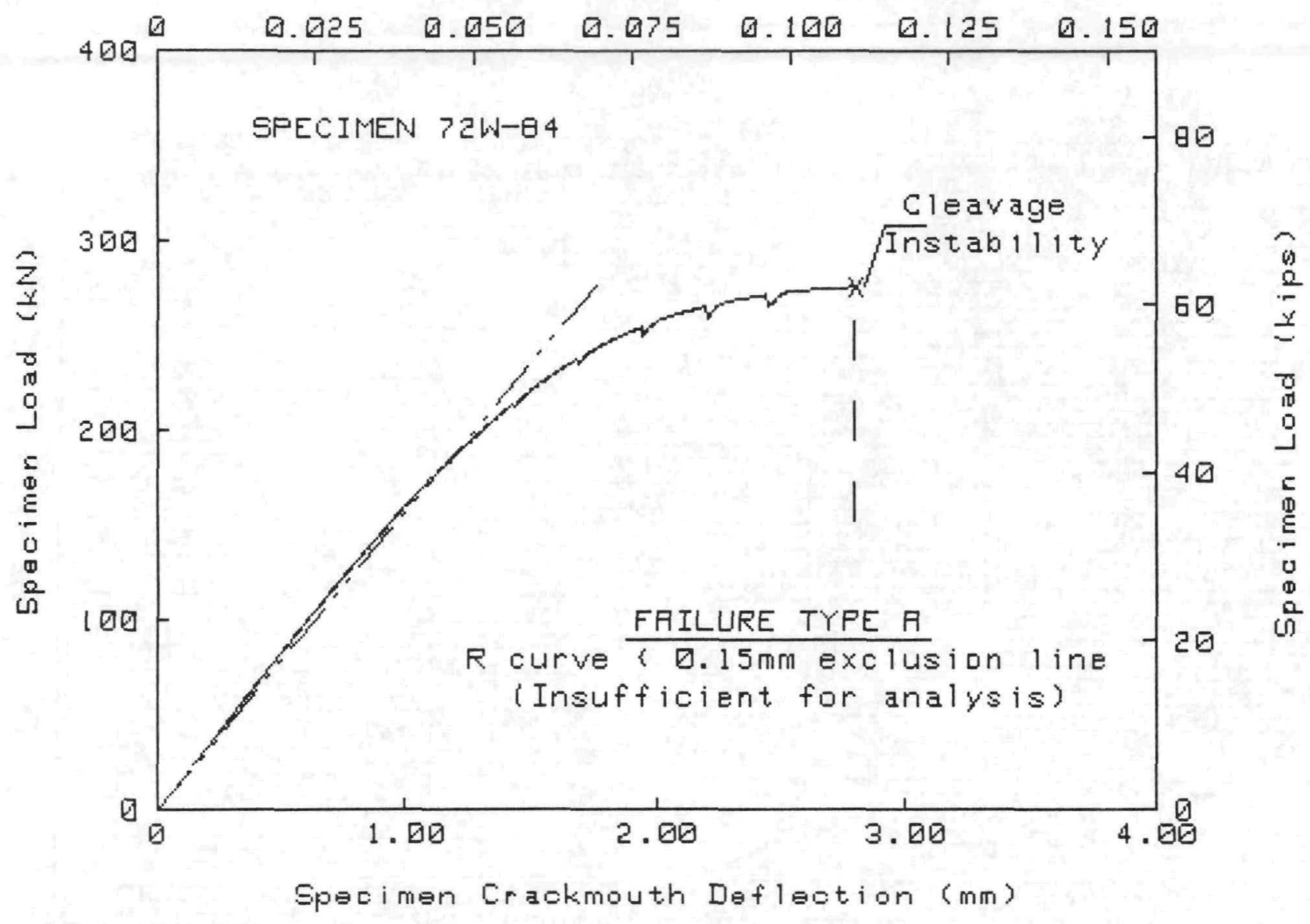

TEST SPECIMEN DATA

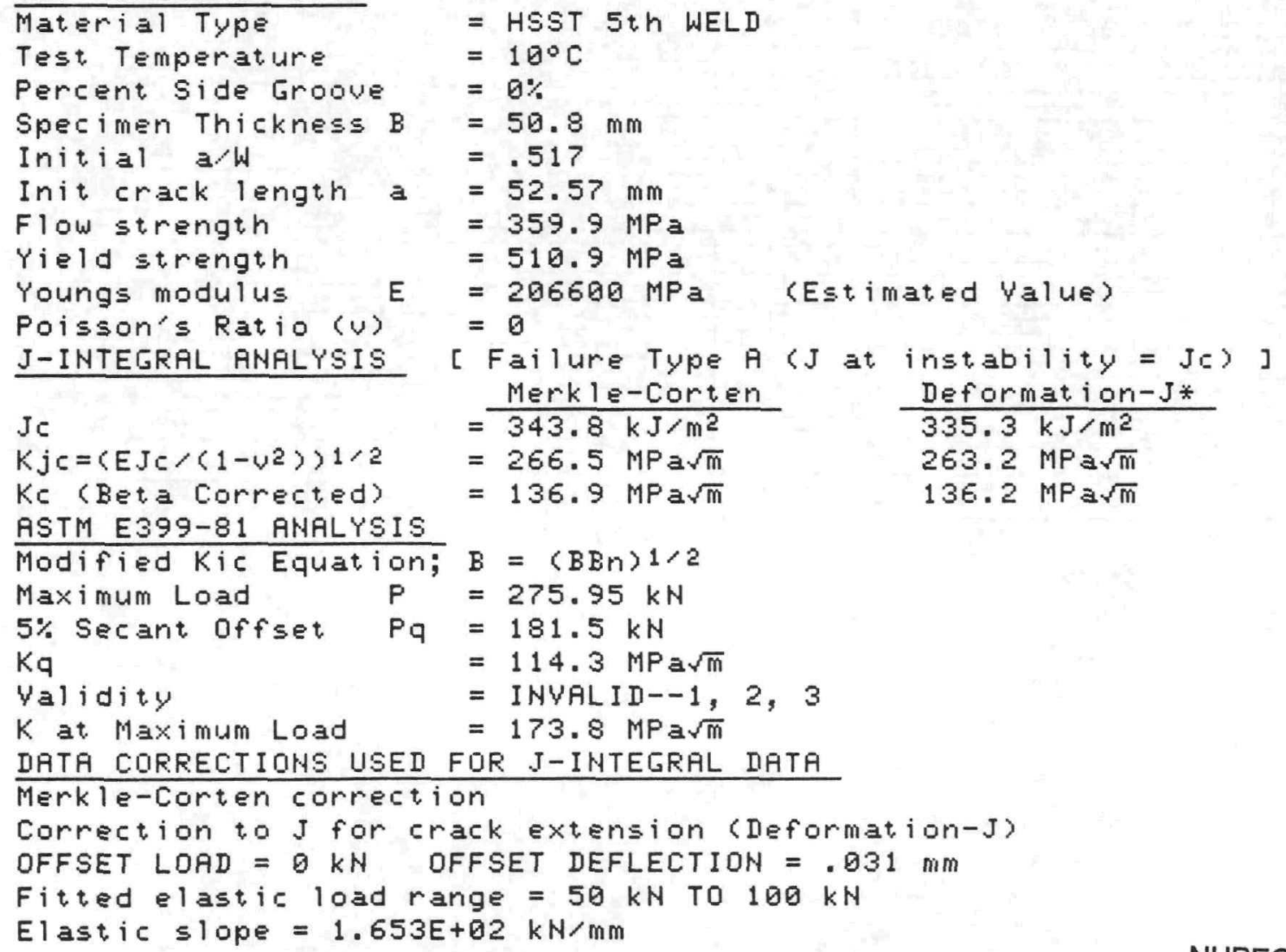


E-102

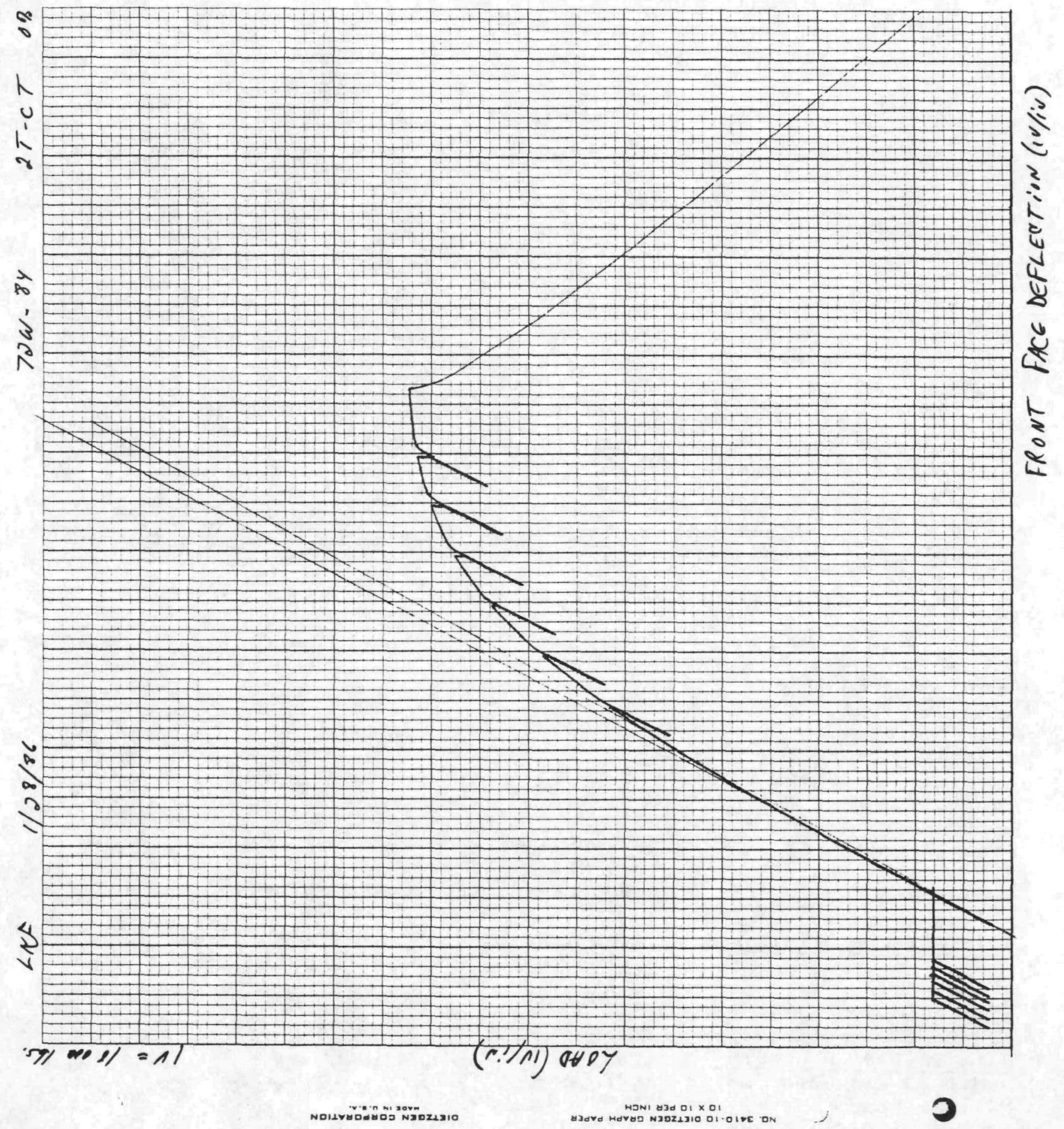


SPECIMEN ID $=72 W 12$

MATERIAL TYPE $=$ MCUWELD

TEST TEMPERATURE $=-50$ DEG $C$

THICKNESS $=101.9 \mathrm{~mm}$

SIDE GROOUES = $0 \%$

INITIAL CRACK LENGTH $=106.6 \mathrm{~mm}$

$a / W)_{i}=.524$

MEASURED DUCTILE DELTA-A $=.05 \mathrm{~mm}$

$Y$ IELD STRENGTH $=547 \mathrm{MPa}$

FLOW STRENGTH $=614 \mathrm{MPa}$

ESTIMATED YOUNG'S MODULUS $=210 \mathrm{GPa}$

SPECIMEN CLEAUED AT KIc= $83.8 \mathrm{MPa}$ SQR $[\mathrm{m}]$ (UALID ASTM E399)

UALUES AT MAXIMUM LOAD

J-INTEGRAL $=31.4 \mathrm{~kJ} / \mathrm{m}^{\wedge} 2$

$\mathrm{KJ} c=81.2 \mathrm{MPa} S Q R[\mathrm{~m}]$

K-beta-c $=78.9 \mathrm{MPa} \operatorname{SQR}[\mathrm{m}]$
WALUES AT CLEAUAGE LOAD

J-INTEGRAL $=31.4 \mathrm{~kJ} / \mathrm{m}^{\wedge} 2$

$\mathrm{KJ} c=81.2 \mathrm{MPa} \operatorname{SQR}[\mathrm{m}]$

$\mathrm{K}$-bet $\mathrm{a}-\mathrm{c}=78.9 \mathrm{MPa} \operatorname{SQR}[\mathrm{m}]$ 


\section{E-104}

ORNL-DWG 92-11564

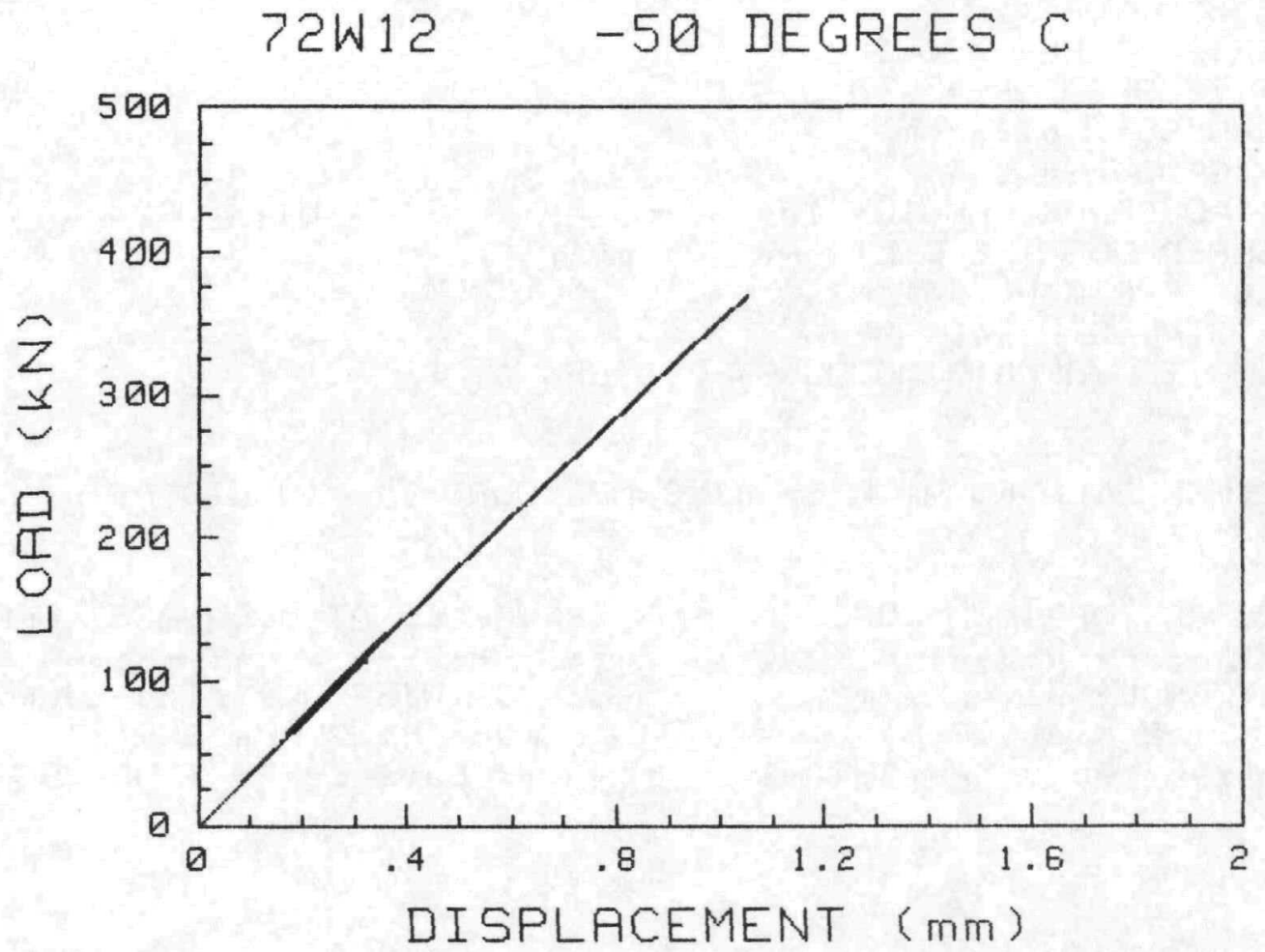

72W-12

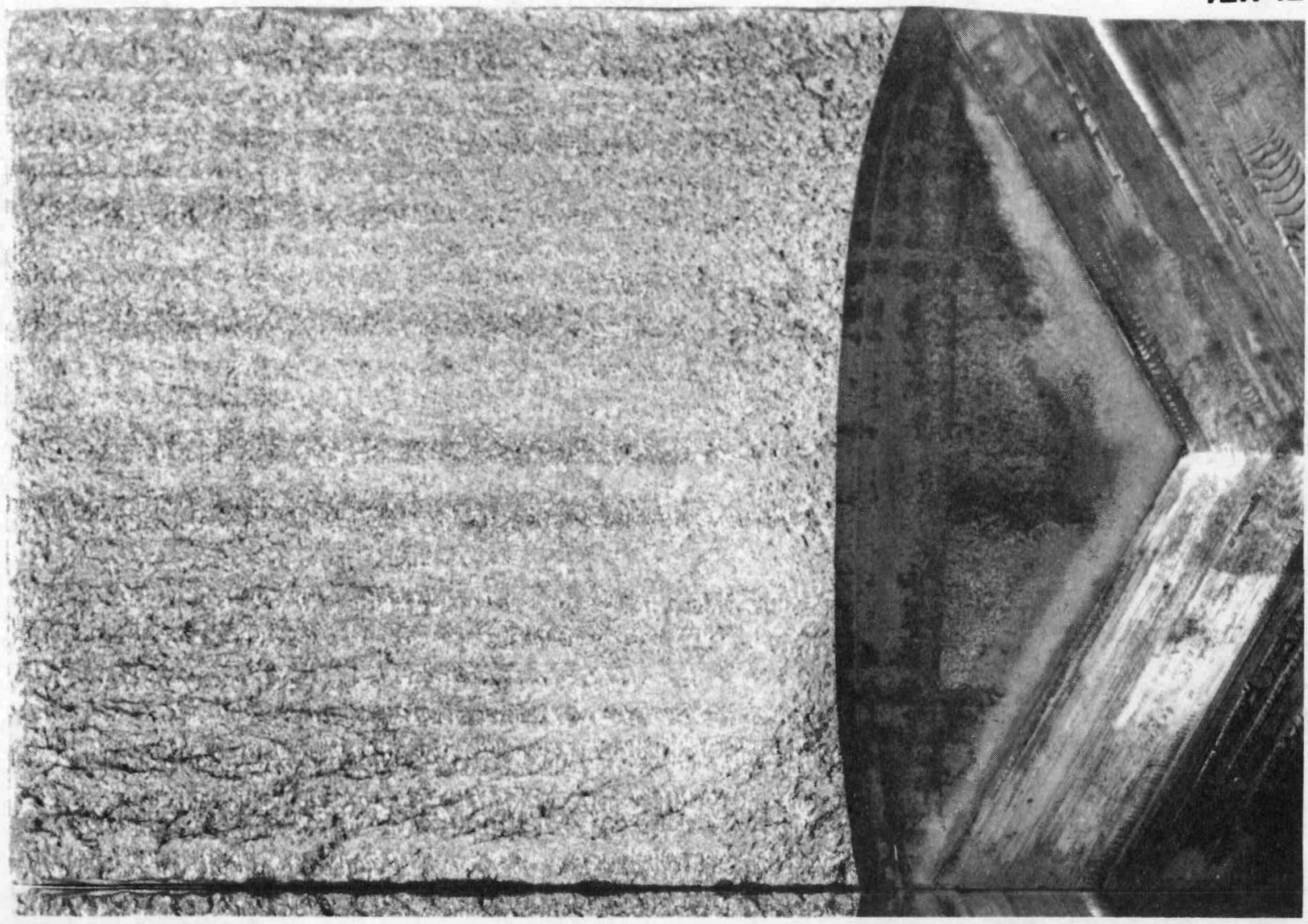


SPECIMEN ID $=72 W 21$

MATERIAL TYPE = MCUWELD

TEST TEMPERATURE $=-50$ DEG $\mathrm{C}$

THICKNESS $=101.6 \mathrm{~mm}$

SIDE GROOUES = $0 \%$

INITIAL CRACK LENGTH $=106.7 \mathrm{~mm}$

$a / w) i=.526$

MEASURED DUCTILE DELTA-A $=.05 \mathrm{~mm}$

$Y I E L D$ STRENGTH $=547 \mathrm{MPa}$

FLDW STRENGTH $=614 \mathrm{MPa}$

ESTIMATED YOUNG'S MODULUS $=210 \mathrm{GPa}$

SPECIMEN CLEAUED AT $K I c=102.4 \mathrm{MPa}$ SQR $[\mathrm{m}]$ (UALID ASTM E399)

UALUES AT MAXIMUM LDAD

J-INTEGRAL $=47.6 \mathrm{~kJ} / \mathrm{m}^{\wedge} 2$

$\mathrm{K} \cdot \mathrm{c}=100 \mathrm{MPa} \operatorname{SQR}[\mathrm{m}]$

K-beta-c $=94.5 \mathrm{MPa} \operatorname{SQR}[\mathrm{m}]$
UALUES AT CLEAUAGE LDAD

$$
\begin{aligned}
& J \text { - INTEGRAL }=47.6 \mathrm{~kJ} / \mathrm{m}^{\wedge} 2 \\
& \mathrm{KJc}=100 \mathrm{MPa} \operatorname{SQR}[\mathrm{m}] \\
& \mathrm{K} \text {-beta-c=94.5 MPa SQR }[\mathrm{m}]
\end{aligned}
$$


E-106

ORNL-DWG 92-11565

72W21 -50 DEGREES C

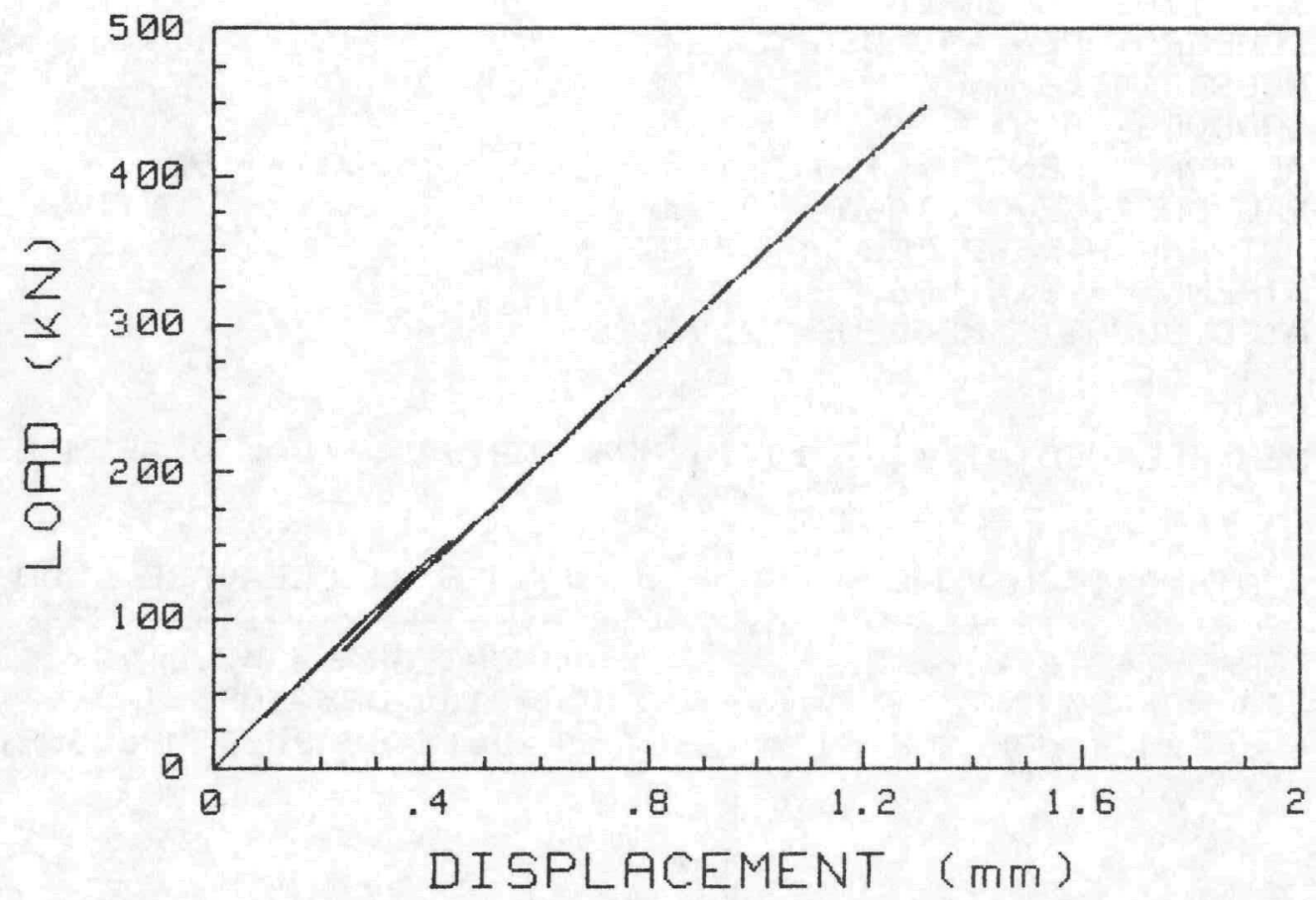

72W-21

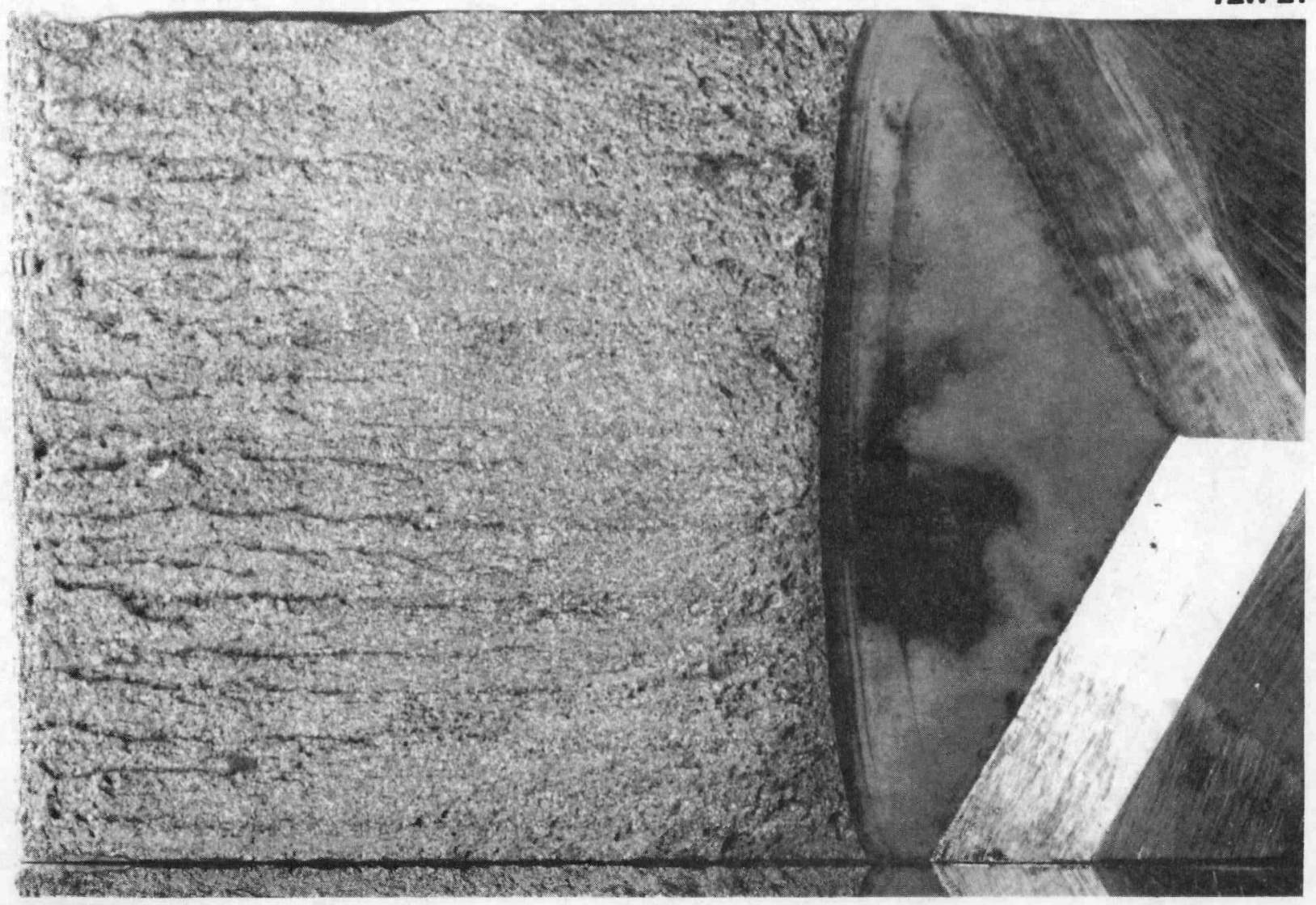

NUREG/CR-5913 
SPECIMEN ID $=72 W 23$

MATERIAL TYPE = MCUWELD

TEST TEMPERATURE $=-30$ DEG $C$

THICKNESS $=101.6 \mathrm{~mm}$

SIDE GROOUES $=0 \%$

INITIAL CRACK LENGTH $=106.2 \mathrm{~mm}$

$a / W) i=.522$

MEASURED DUCTILE DELTA-A $=.05 \mathrm{~mm}$

$Y$ IELD STRENGTH $=534 \mathrm{MPa}$

FLOW STRENGTH $=598 \mathrm{MPa}$

ESTIMATED YOUNG'S MODULUS= $209 \mathrm{GPa}$

SPECIMEN CLEAUED AT $K I c=105.2 \mathrm{MPa}$ SQR $[\mathrm{m}]$ (WALID ASTM E399)

UALUES AT MAXIMUM LOAD

J-INTEGRAL $=50.6 \mathrm{~kJ} / \mathrm{m}^{\wedge} 2$

$K J c=102.8 \mathrm{MPa} S Q R[\mathrm{~m}]$

K-beta-c $=96.2 \mathrm{MPa} \operatorname{SDR}[\mathrm{m}]$
Ualues at CLEaUage LDaD

J-INTEGRAL $=50.6 \mathrm{~kJ} / \mathrm{m}^{\wedge} 2$

$K J c=102.8 \mathrm{MPa} \operatorname{SQR}[\mathrm{m}]$

K-bet $a-c=96.2 \mathrm{MPa} \operatorname{SQR}[\mathrm{m}]$ 
E-108

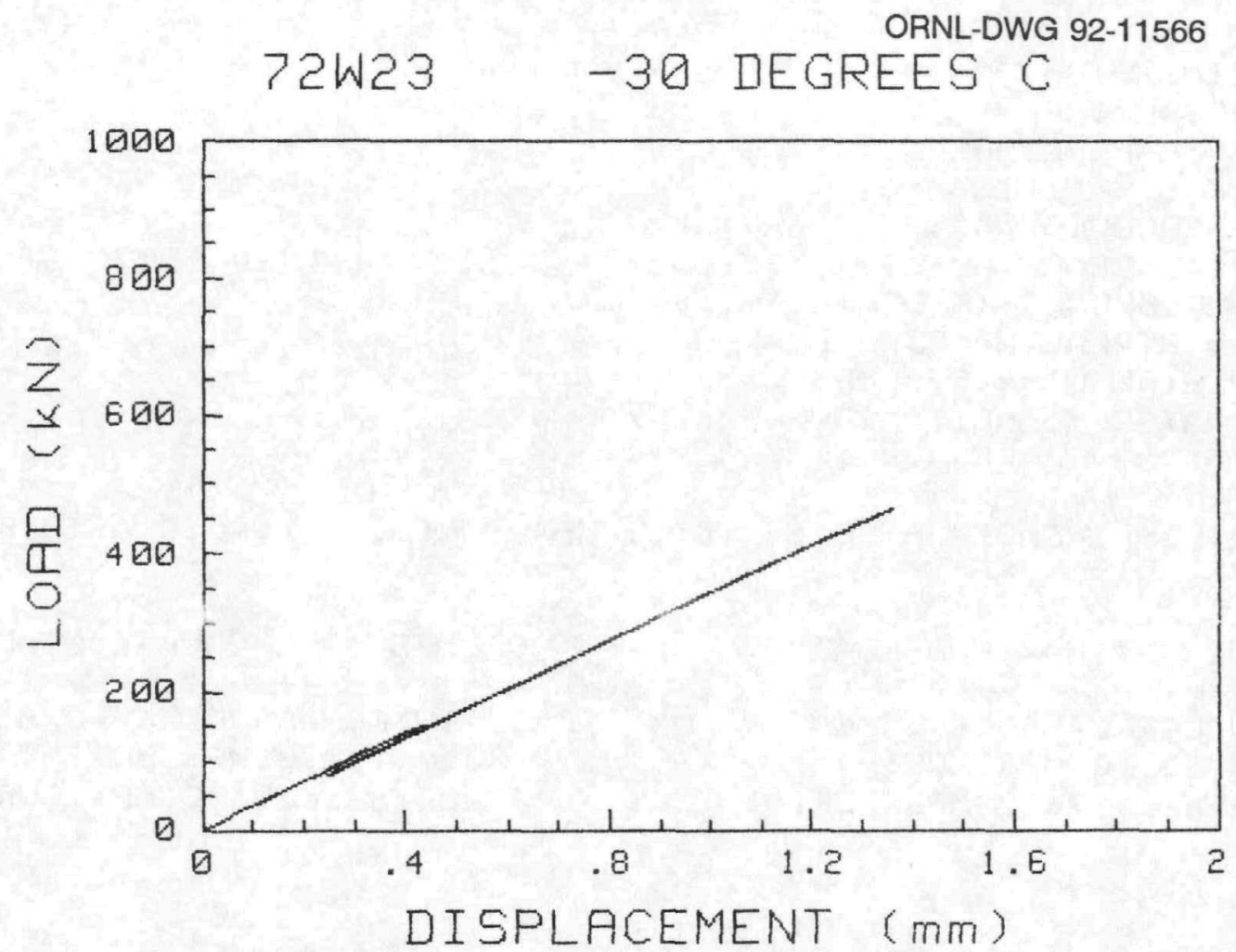

72W-23

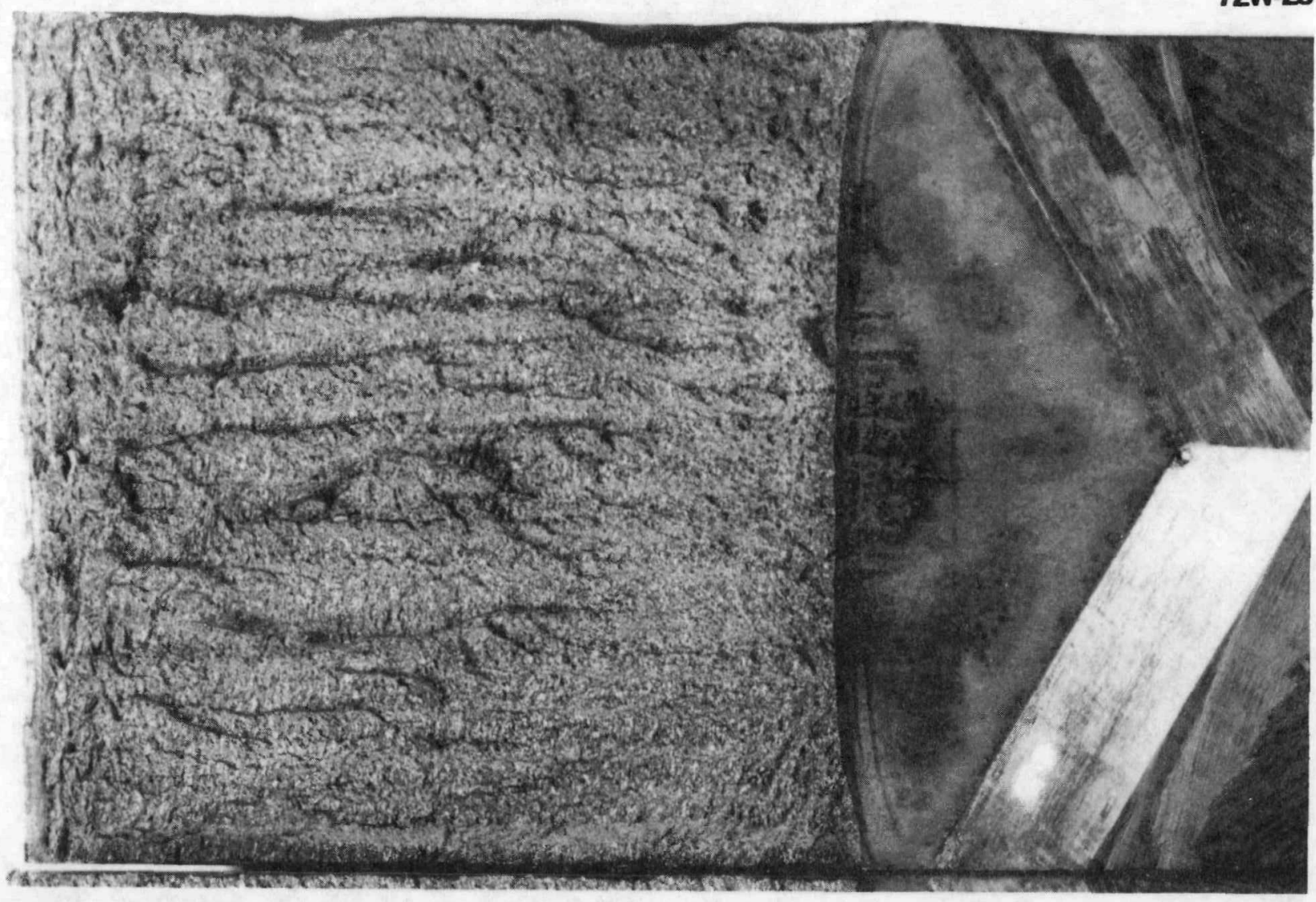

NUREG/CR-5913 
SPECIMEN ID $=72 W 10$

MATERIAL TYPE $=$ A533BW

TEST TEMPERATURE $=-30$ DEG C

THICKNESS $=101.6 \mathrm{~mm}$

SIDE GROOUES $=0 \%$

INITIAL CRACK LENGTH $=109 \mathrm{~mm}$

MEASURED DUCTILE DELTA-A $=.05 \mathrm{~mm}$

$a / W) i=.536$

YIELD STRENGTH $=534 \mathrm{MPa}$

FLOW STRENGTH $=598 \mathrm{MPa}$

ESTIMATED YOUNG'S MODULUS $=209 \mathrm{GPa}$

SPECIMEN CLEAUED AT KQ= $144.9 \mathrm{MPa}$ SQR $[\mathrm{m}]$ (NDT UALID KIC)

UALUES AT MAXIMUM LOAD

J-INTEGRAL $=101.2 \mathrm{~kJ} / \mathrm{m}^{\wedge} 2$

$\mathrm{KJc}=145.4 \mathrm{MPa} \operatorname{SDR}[\mathrm{m}]$

K-beta-c $=123.5 \mathrm{MPa} \operatorname{SQR}[\mathrm{m}]$
Ualues at CLEaUage LoAd

J-INTEGRAL $=101.2 \mathrm{~kJ} / \mathrm{m}^{\wedge} 2$

$K J c=145.4 \mathrm{MPa} \operatorname{SQR}[\mathrm{m}]$

$K$-beta-c $=123.5 \mathrm{MPa} S Q R[\mathrm{~m}]$ 
E-110

ORNL-DWG 92-11567

72W10 -30 DEGREES C

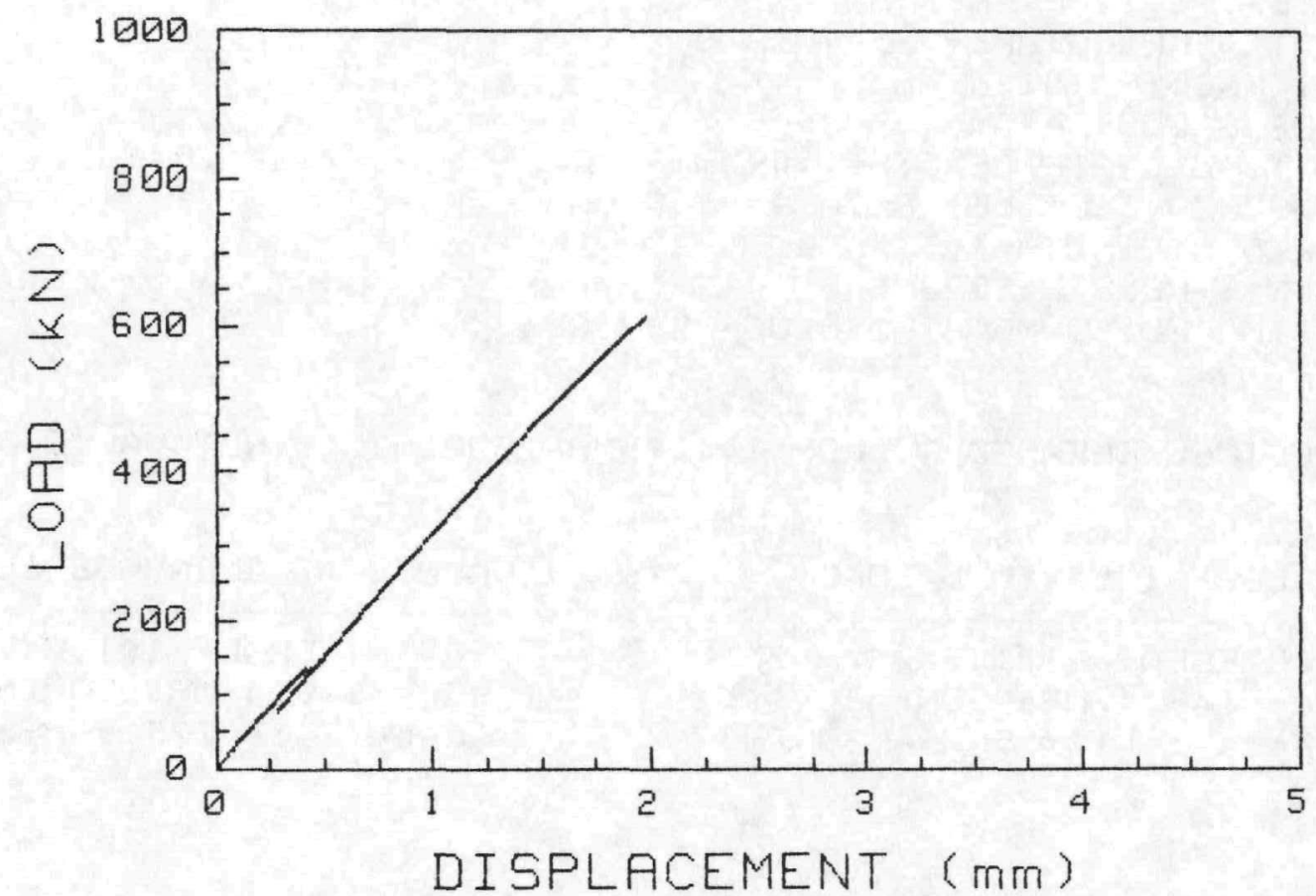

$72 W-10$

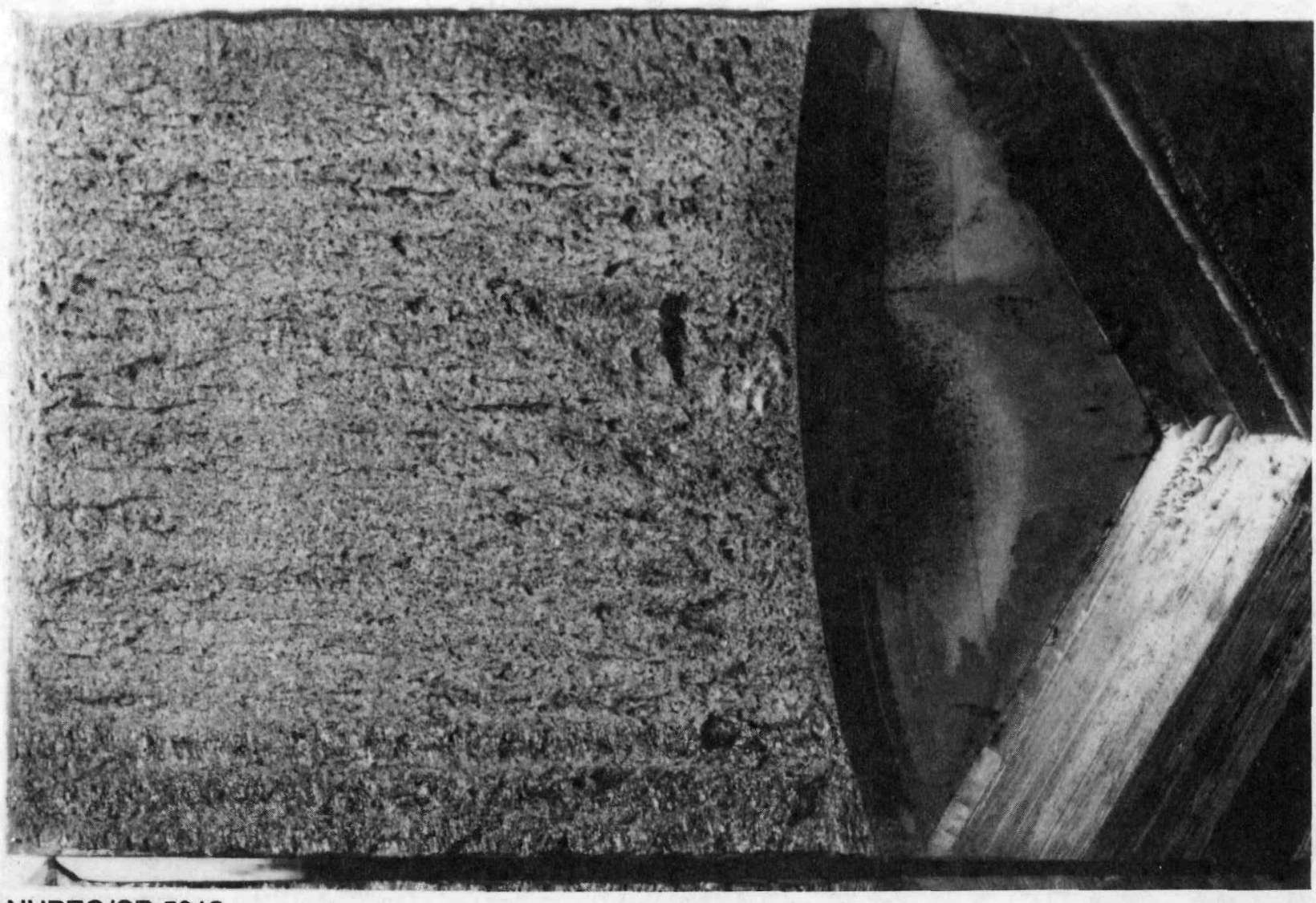


SPECIMEN ID $=72 W 24$

MATERIAL TYPE = MCUWELD

TEST TEMPERATURE $=-15$ DEG $C$

THICKNESS $=101.6 \mathrm{~mm}$

SIDE GRODUES $=0 \%$

INITIAL CRACK LENGTH $=110.5 \mathrm{~mm}$

$\mathrm{a} / \mathrm{W}\rangle \mathrm{i}=.544$

MEASURED DUCTILE DELTA-A $=.05 \mathrm{~mm}$

YIELD STRENGTH $=526 \mathrm{MPa}$

FLOLA STRENGTH $=586 \mathrm{MPa}$

ESTIMATED YOUNG'S MDDULUS= 208 GPa

SPECIMEN CLEAUED AT $K I c=35.8$ MPa SQR $[\mathrm{m}]$ (UALID ASTM E399)

VALUES AT MAXIMUM LOAD

J-INTEGRAL $=32.4 \mathrm{~kJ} / \mathrm{m}^{\wedge} 2$

$\mathrm{KJ} \mathrm{c}=82.1 \mathrm{MPa} \operatorname{SQR}[\mathrm{m}]$

$K$-beta-c $=79.4 \mathrm{MPa} S Q R[\mathrm{~m}]$
UALUES AT CLEAUAGE LDAD

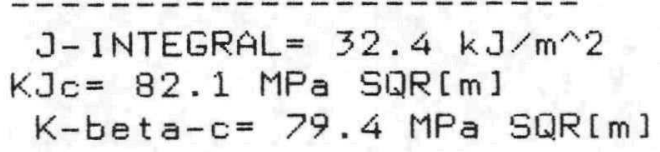




\section{E-112}

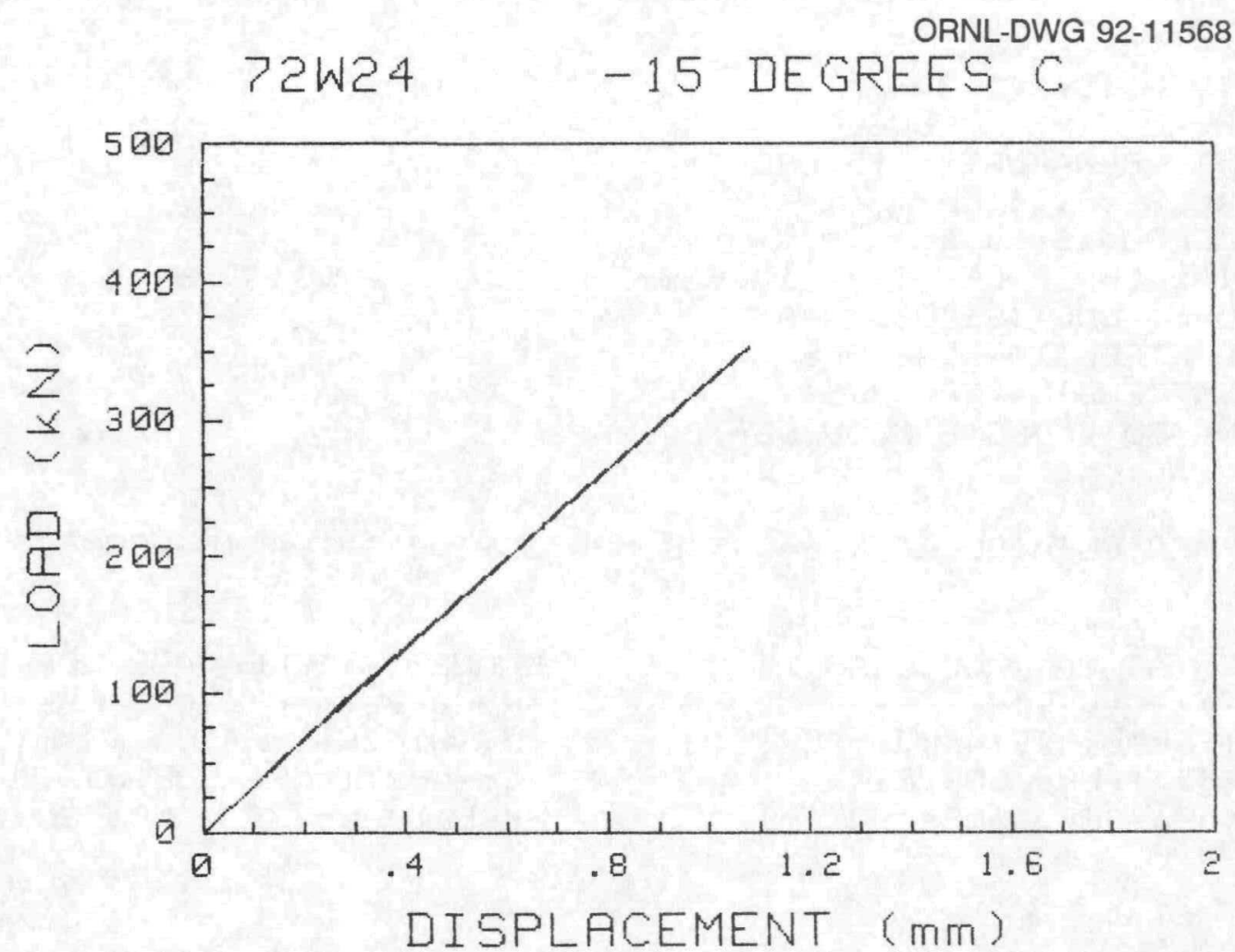

72W-24

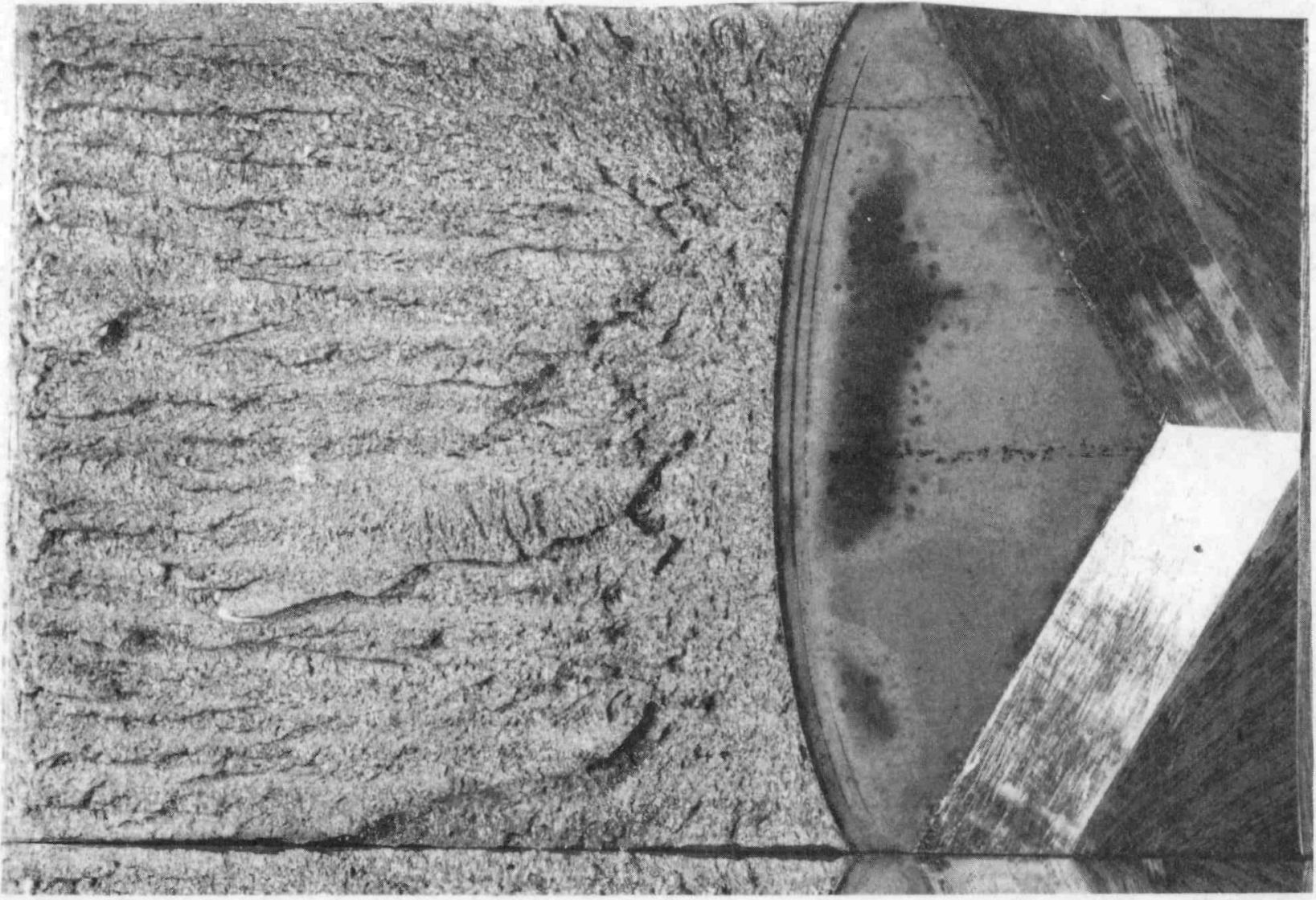


SPECIMEN ID $=72 W 15$

MATERIAL TYPE = MCUWELD

TEST TEMPERATURE $=-15$ DEG C

THICKNESS $=101.8 \mathrm{~mm}$

SIDE GROOUES $=0 \%$

INITIAL CRACK LENGTH $=108.4 \mathrm{~mm}$

$a / W) i=.533$

MEASURED DULTILE DELTA-A $=.05 \mathrm{~mm}$

$Y$ IELD STRENGTH $=526 \mathrm{MPa}$

FLOW STRENGTH $=586 \mathrm{MPa}$

ESTIMATED YOUNG'S MODULUS $=208$ GPa

SPECIMEN CLEAUED AT KD= $165.6 \mathrm{MPa}$ SDR[m] (NOT UALID KIc)

UALUES AT MAXIMUM LOAD

J-INTEGRAL $=134.2 \mathrm{~kJ} / \mathrm{m}^{\wedge} 2$

$K J c=167.1 \mathrm{MPa} S Q R[\mathrm{~m}]$

$K$-beta-c $=133.6 \mathrm{MPa}$ SDR $[\mathrm{m}]$
UALUES AT CLEAUAGE LDAD

J-INTEGRAL $=134.2 \mathrm{~kJ} / \mathrm{m}^{\wedge} 2$

$K] \mathrm{c}=167.1 \mathrm{MPa} S \mathrm{SQR}[\mathrm{m}]$

$K$-beta-c $=133.6 \mathrm{MPa} S Q R[\mathrm{~m}]$ 
$E-114$

ORNL-DWG 92-11569

\section{W15 - 15 DEGREES C}

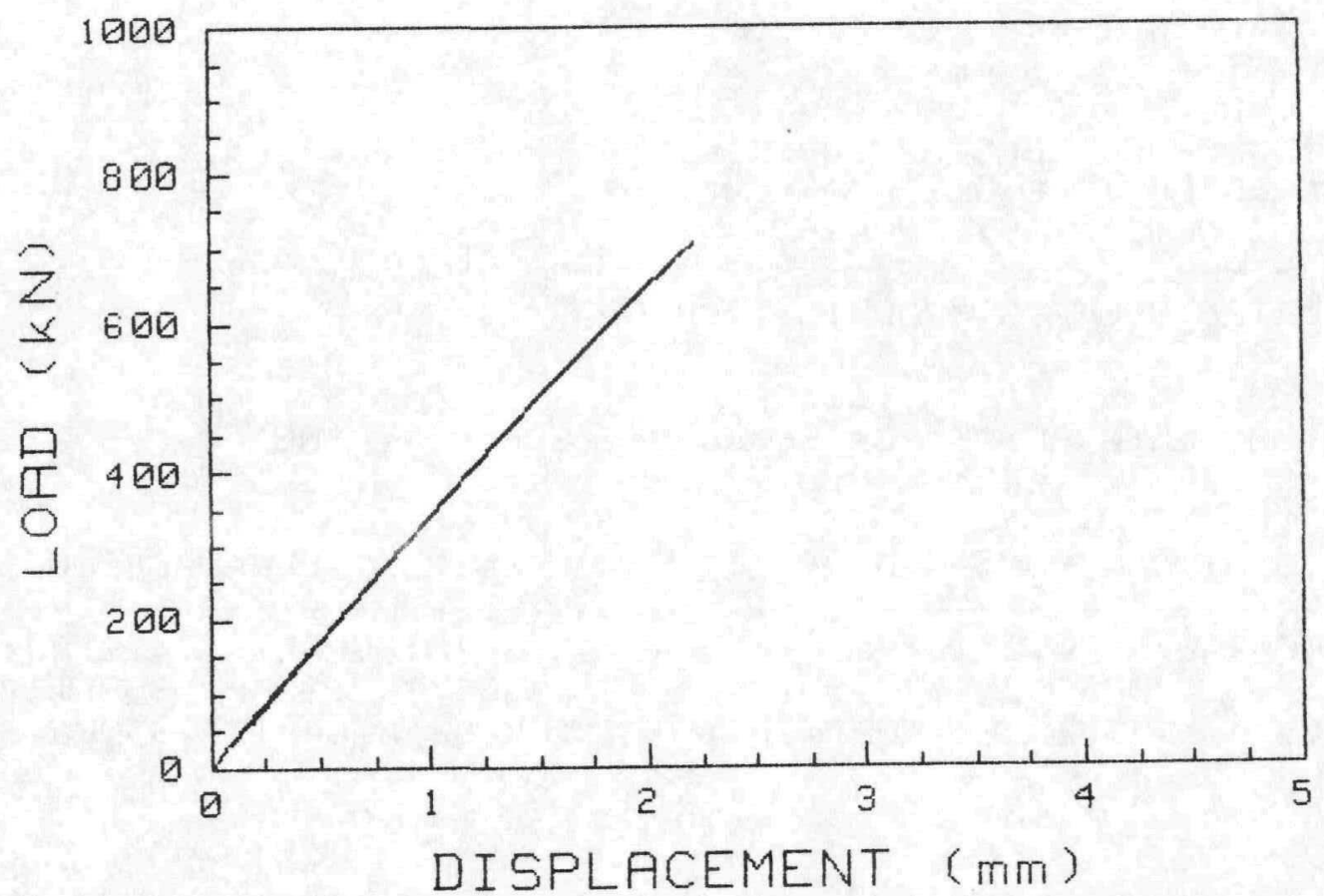

72W-15

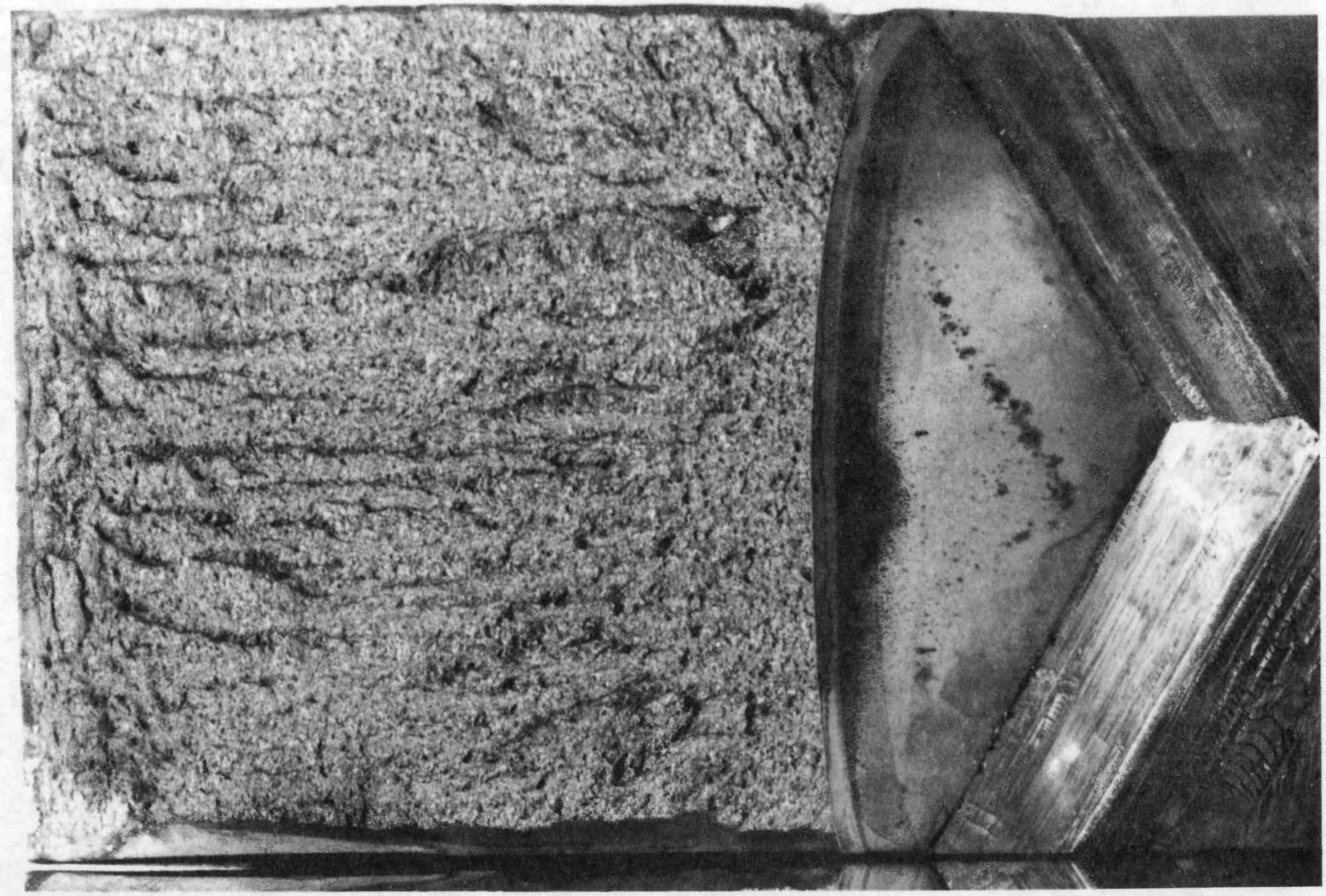




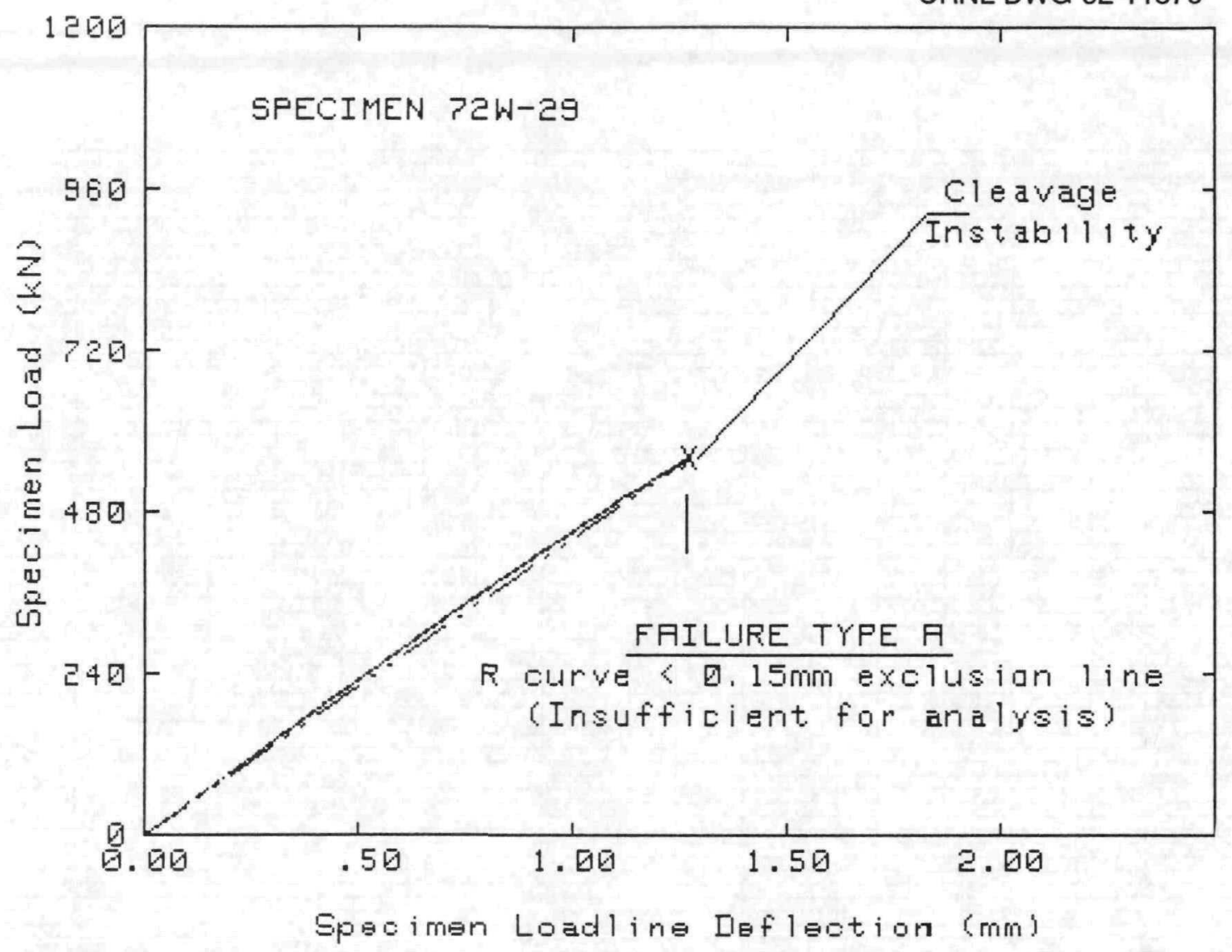

TEST SFECIMEN DATA

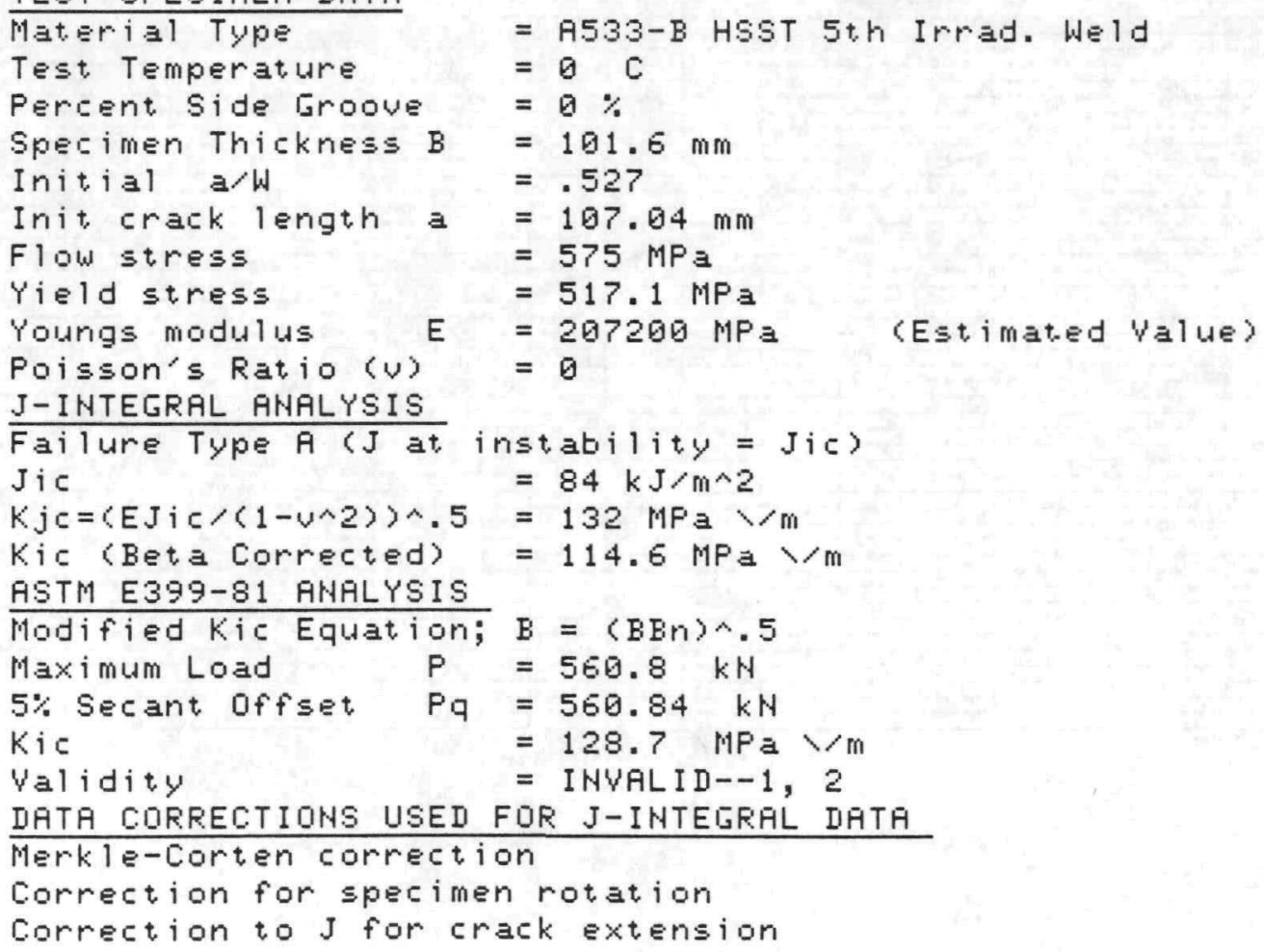




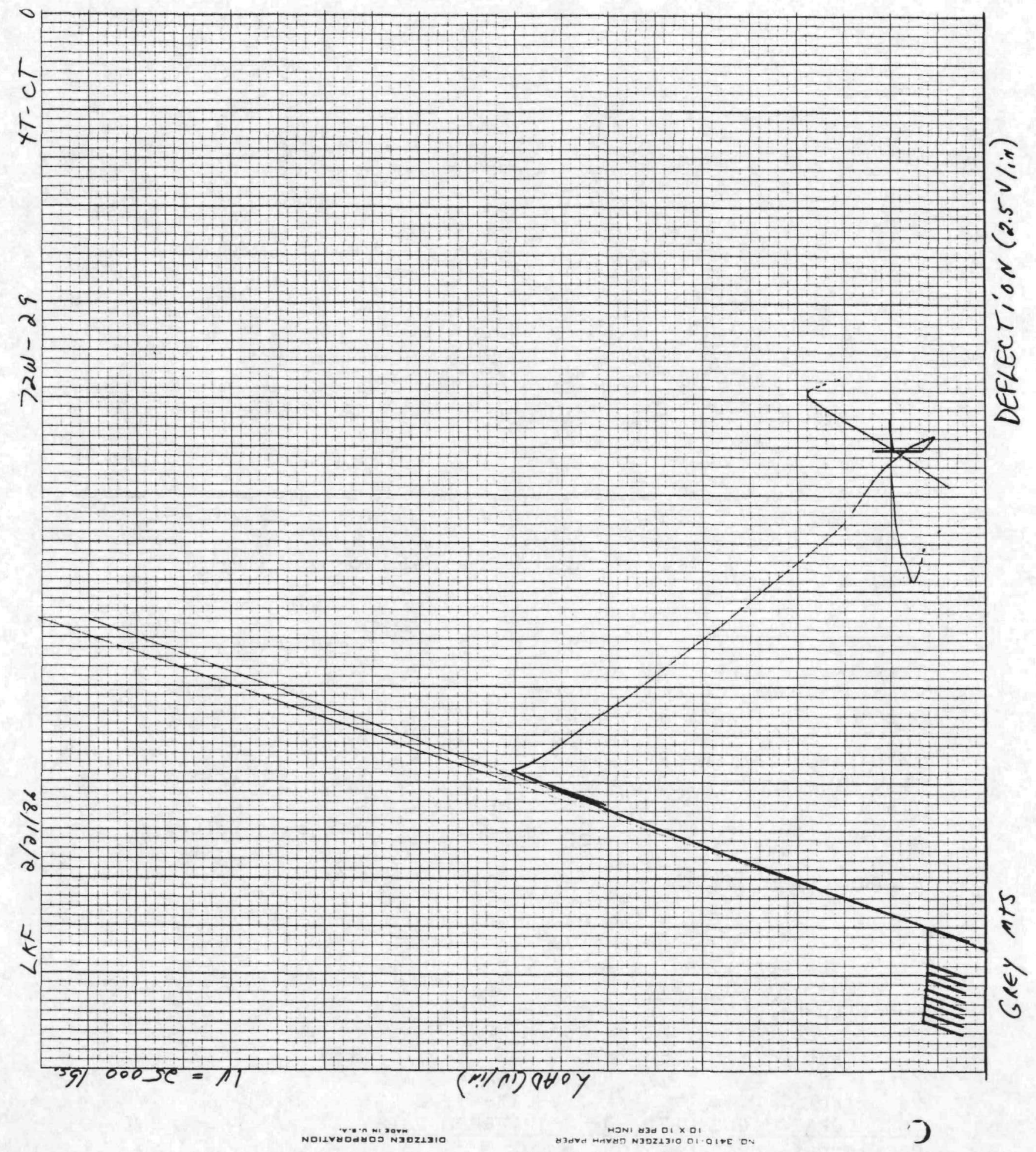



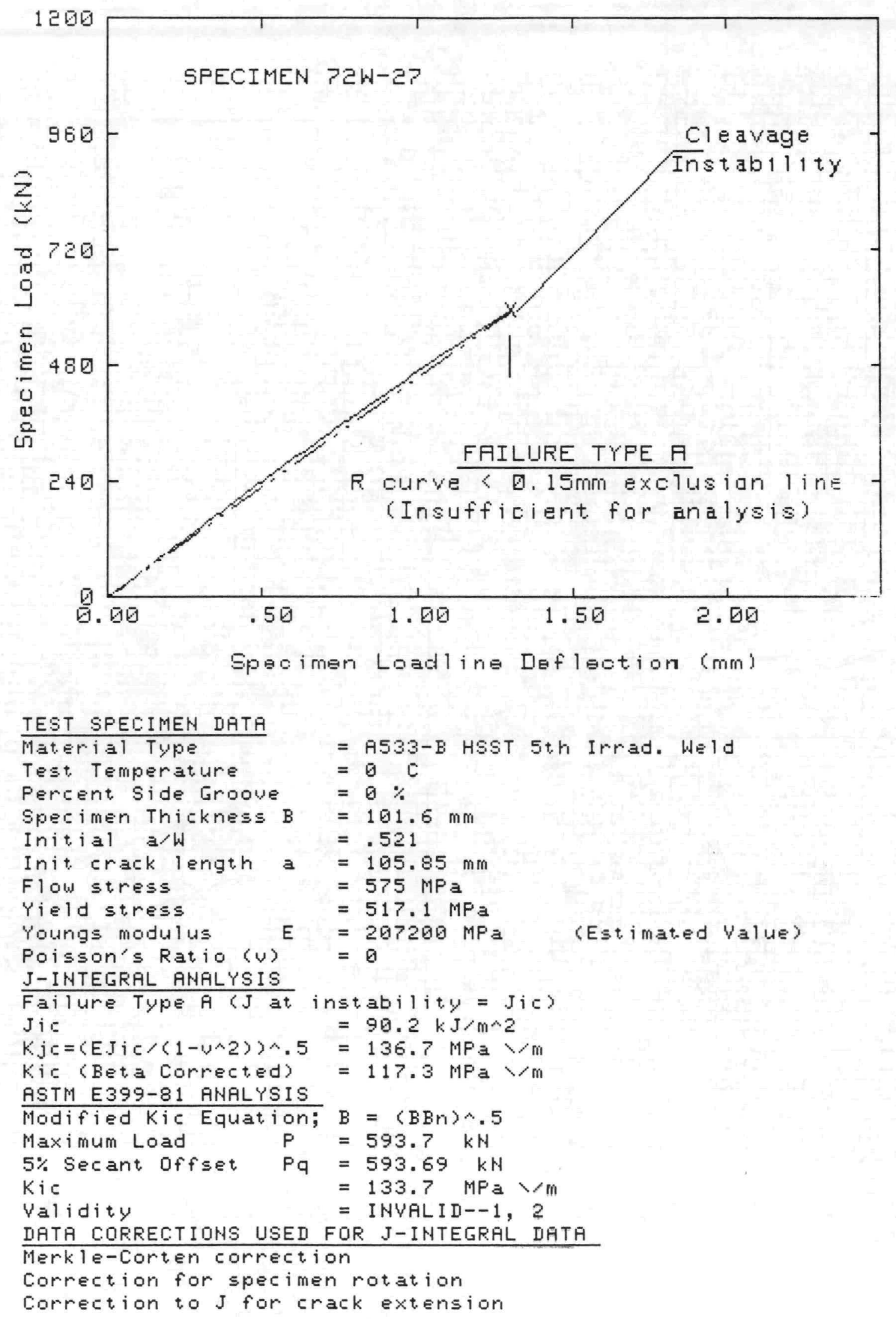


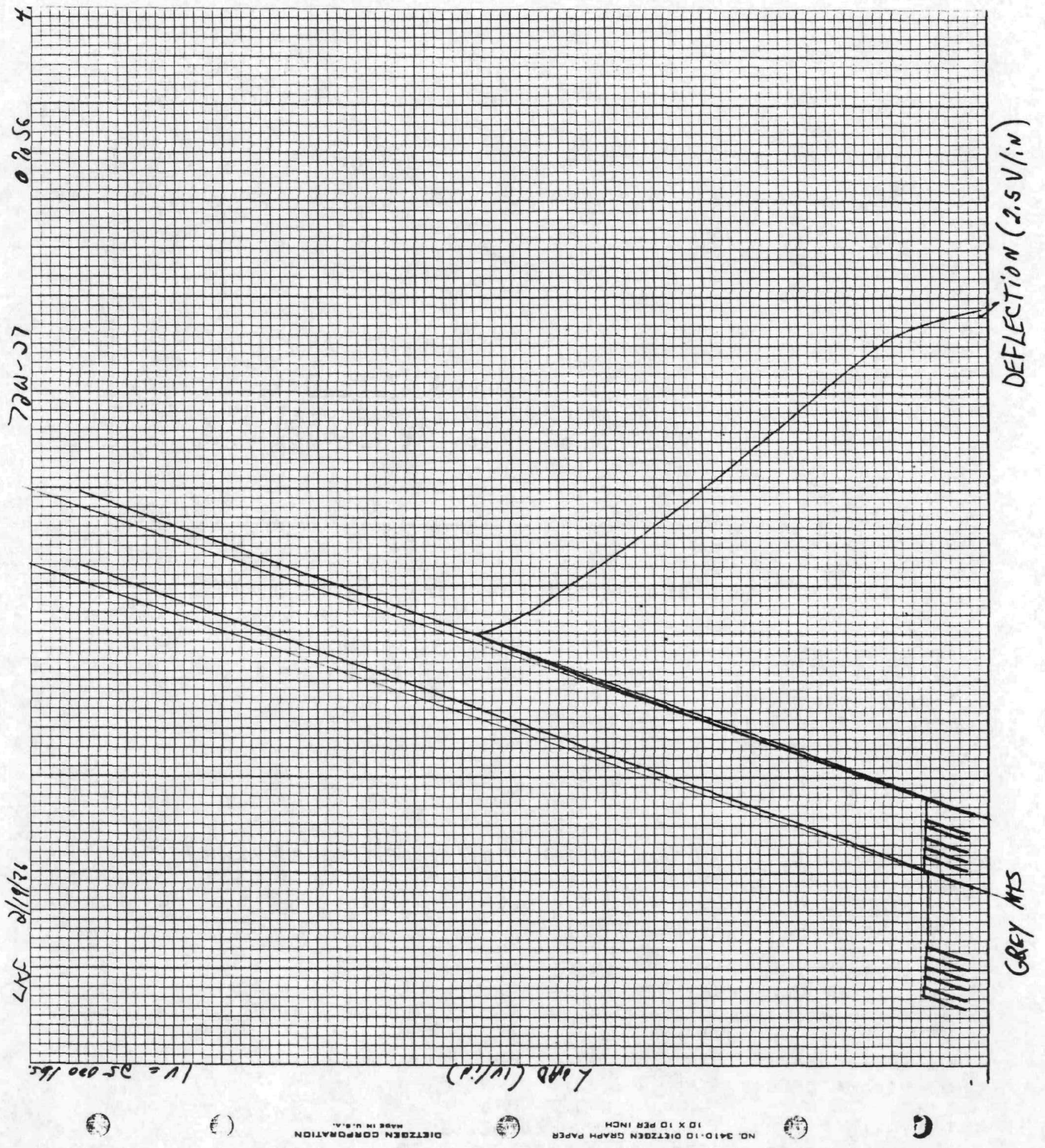




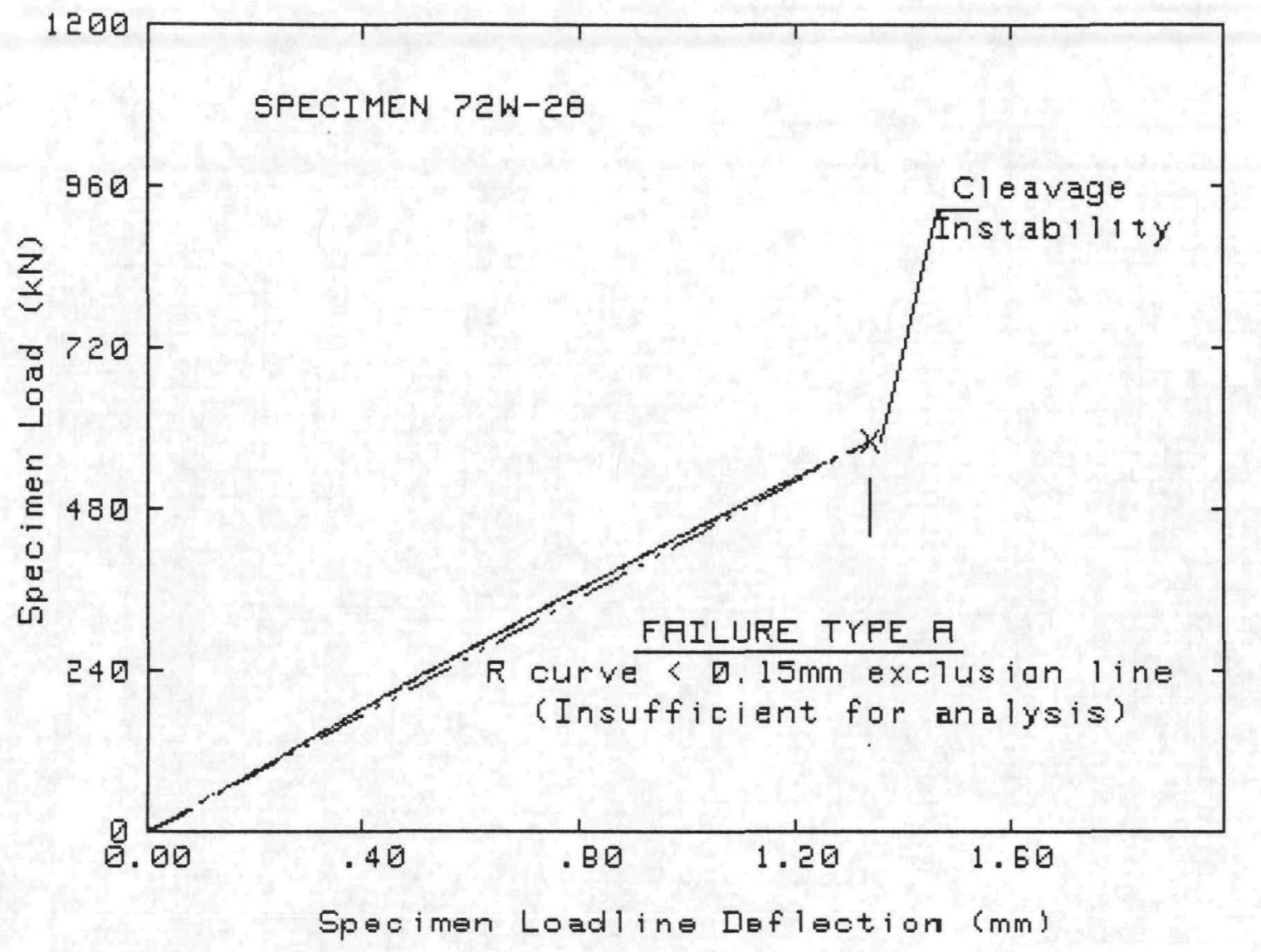

\section{TEST SPECIMEN DATA}

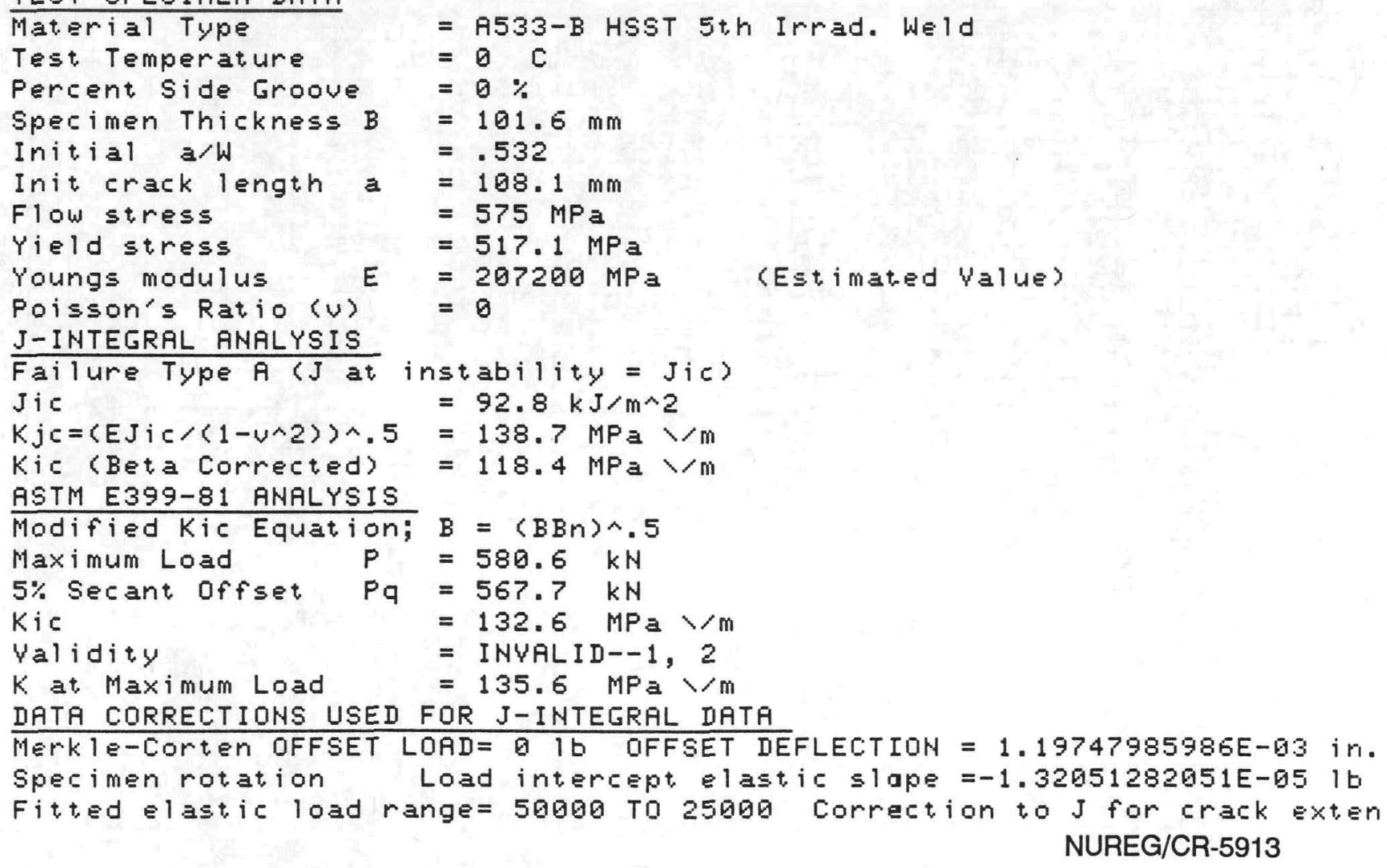




\section{E-120}

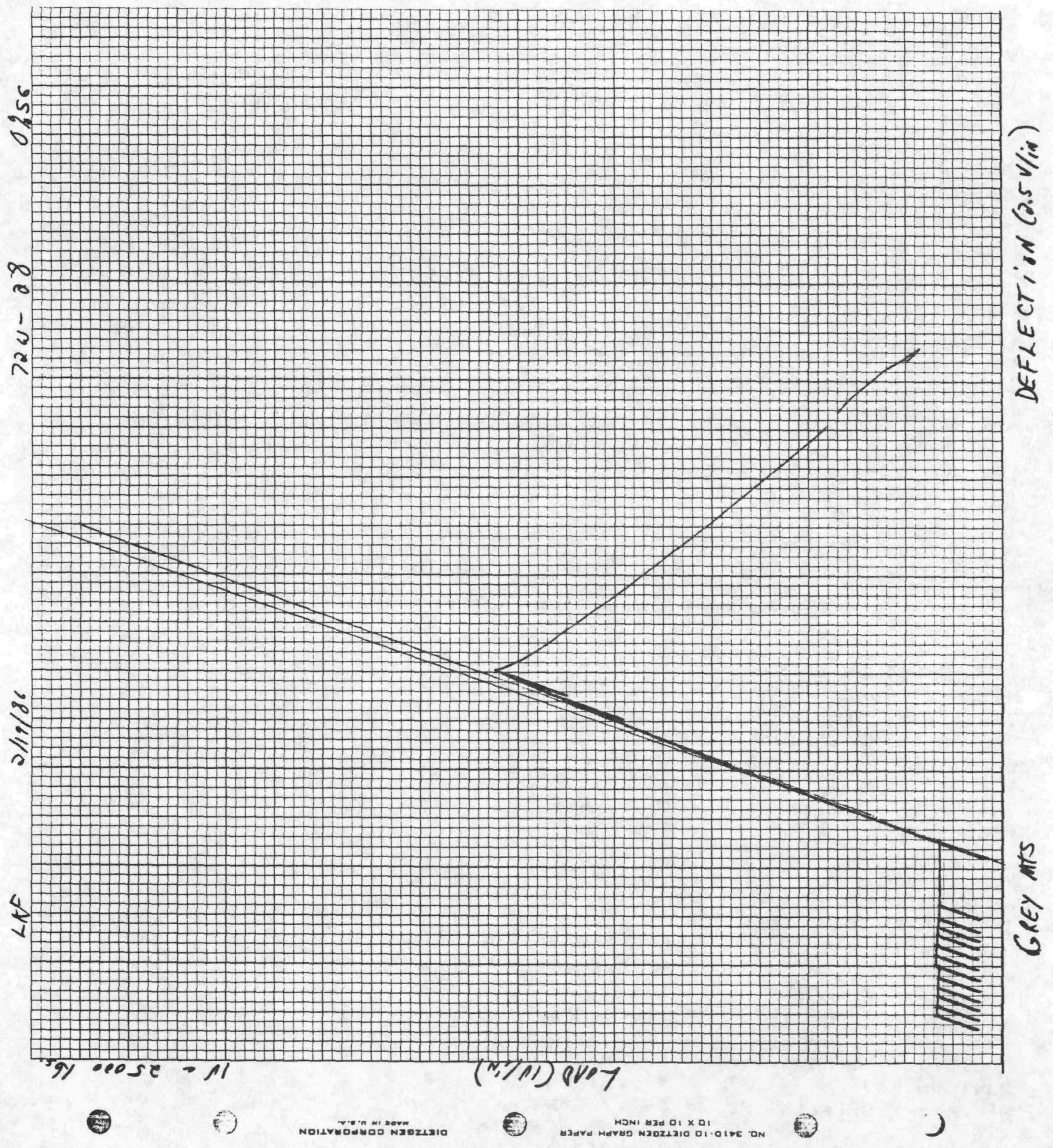




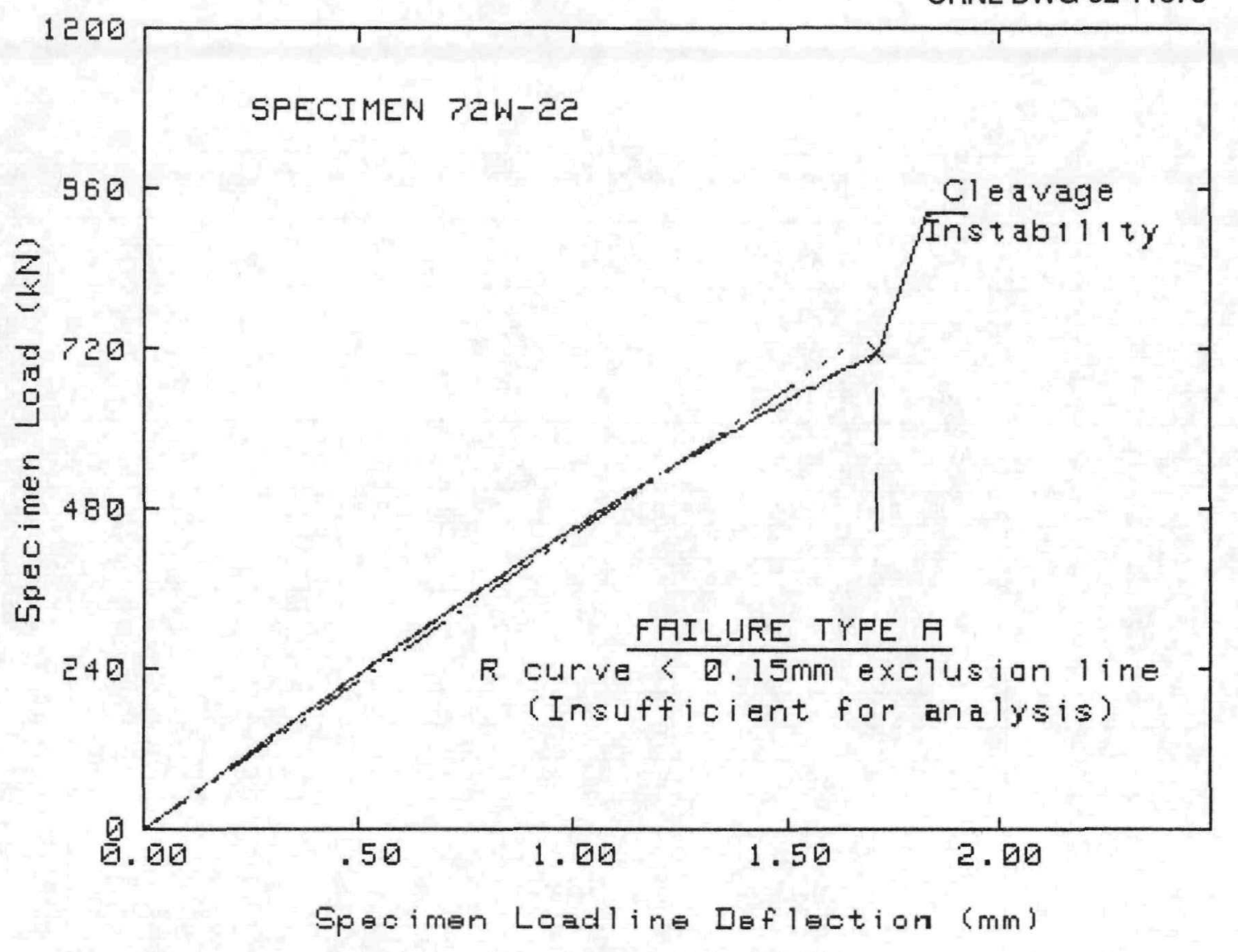

TEST SFECIMEN DATA

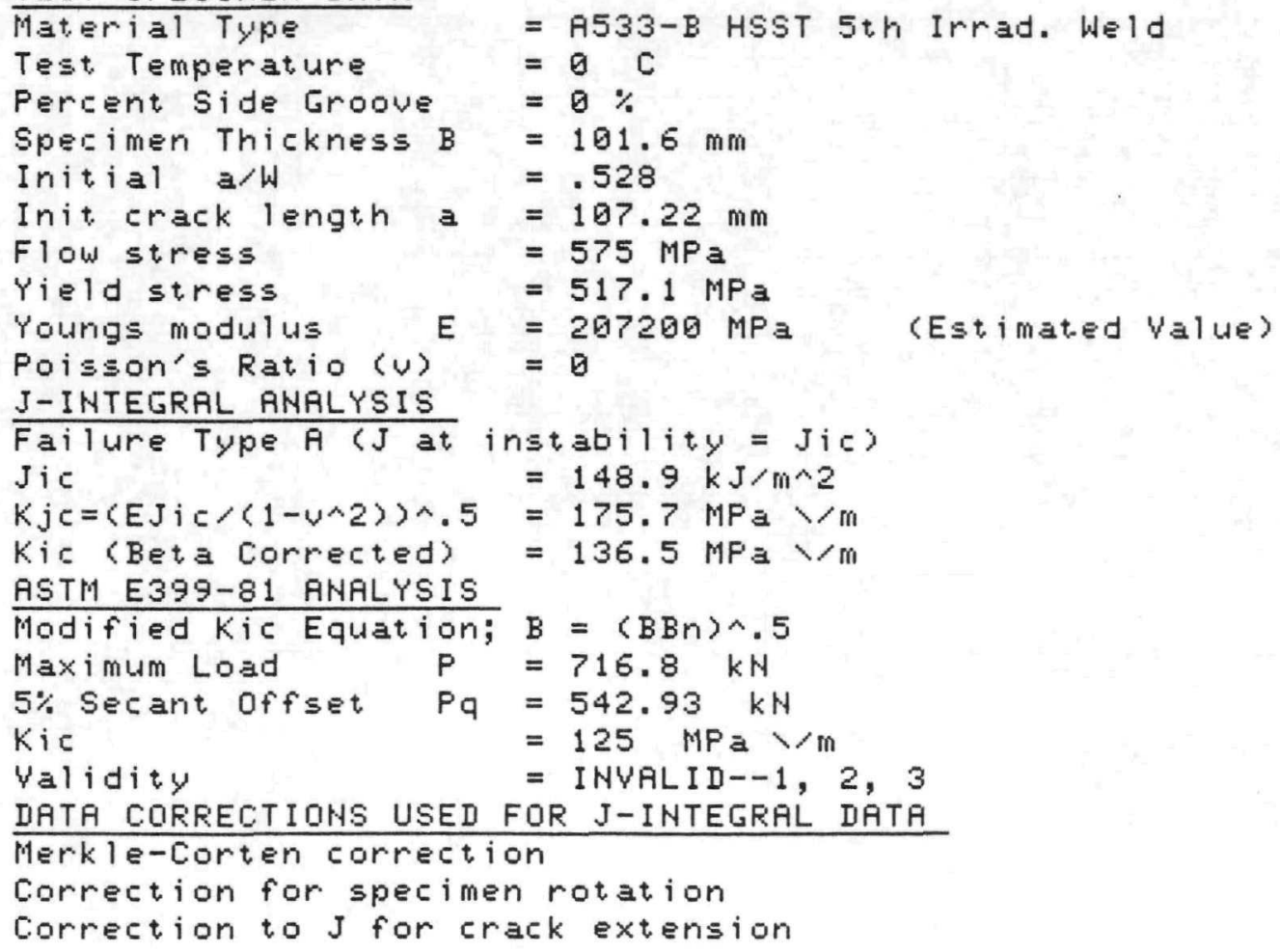

Validity

Merkle-Corten correction

Correction for specimen rotation

Correction to $\mathrm{J}$ for crack extension 


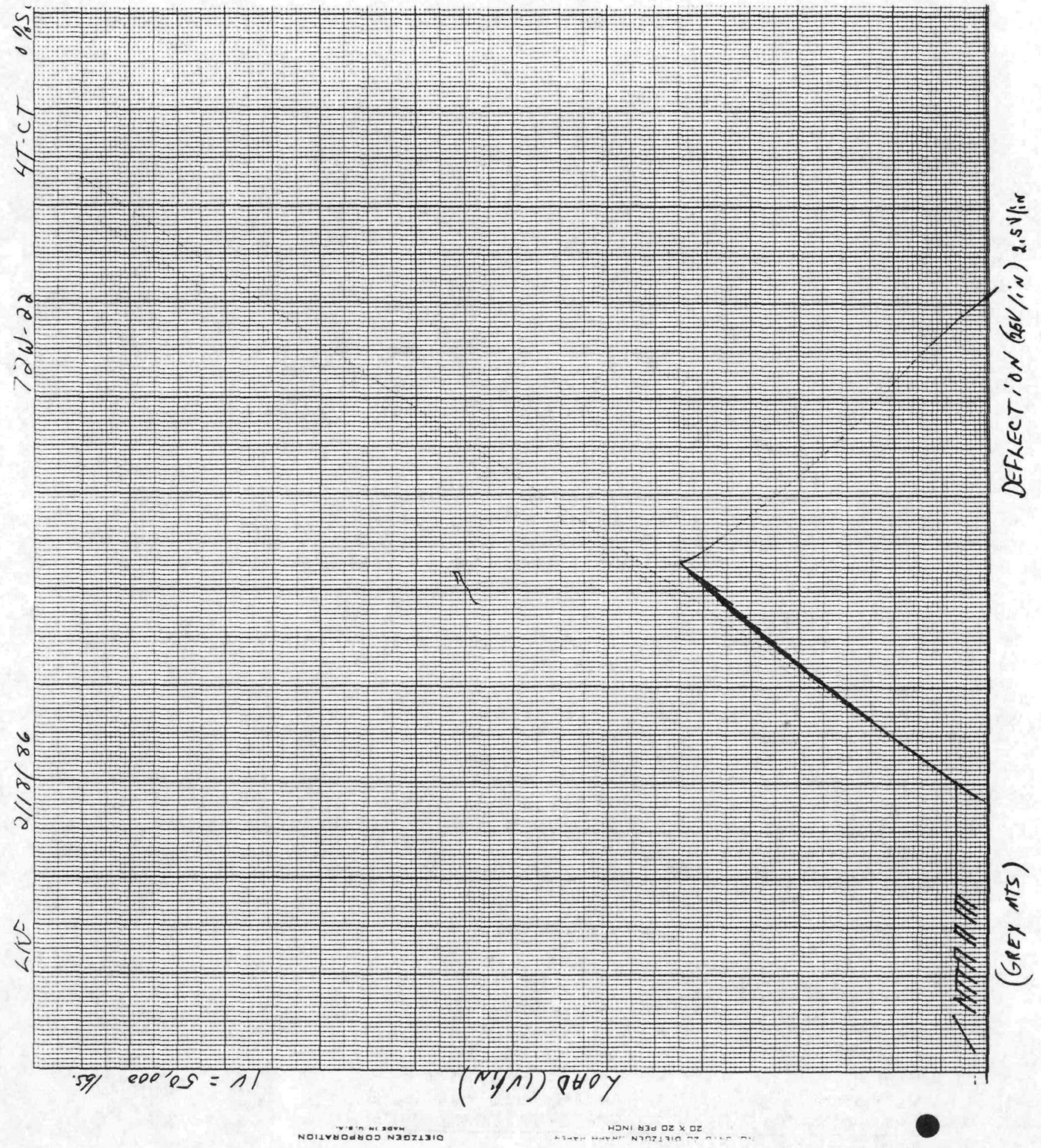


ORNL-DWG 92-11574

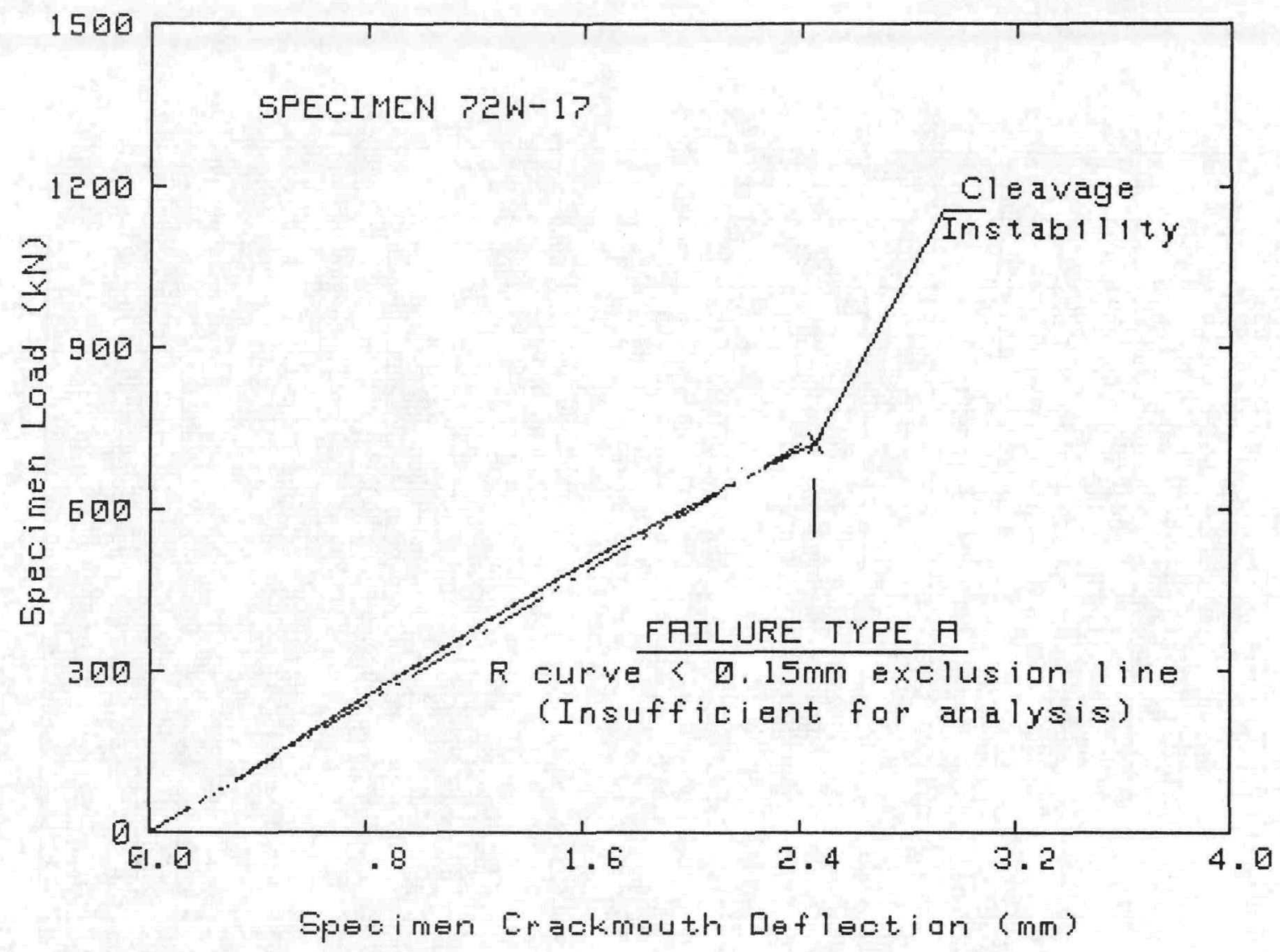

TEST SPECIMEN DATA

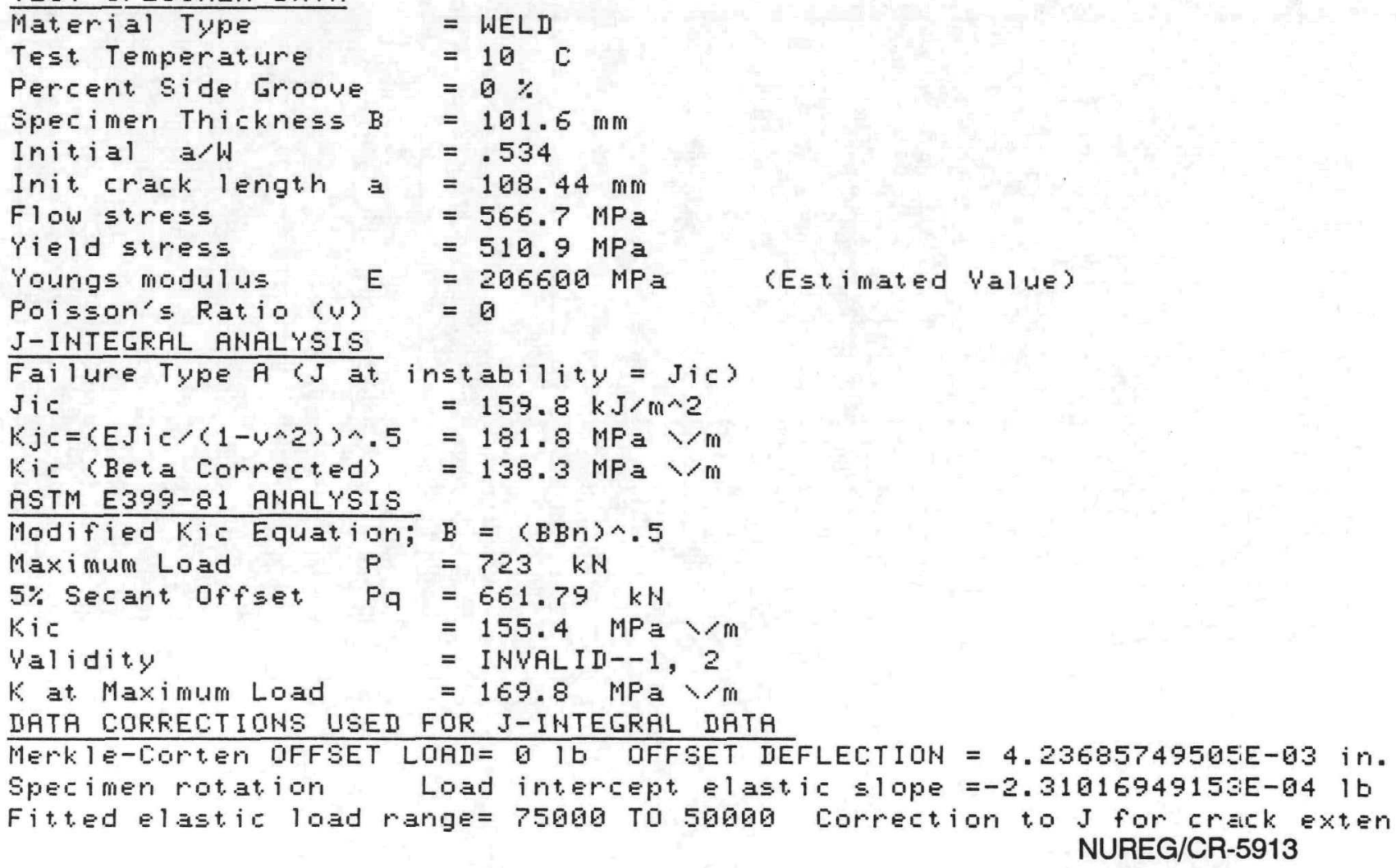




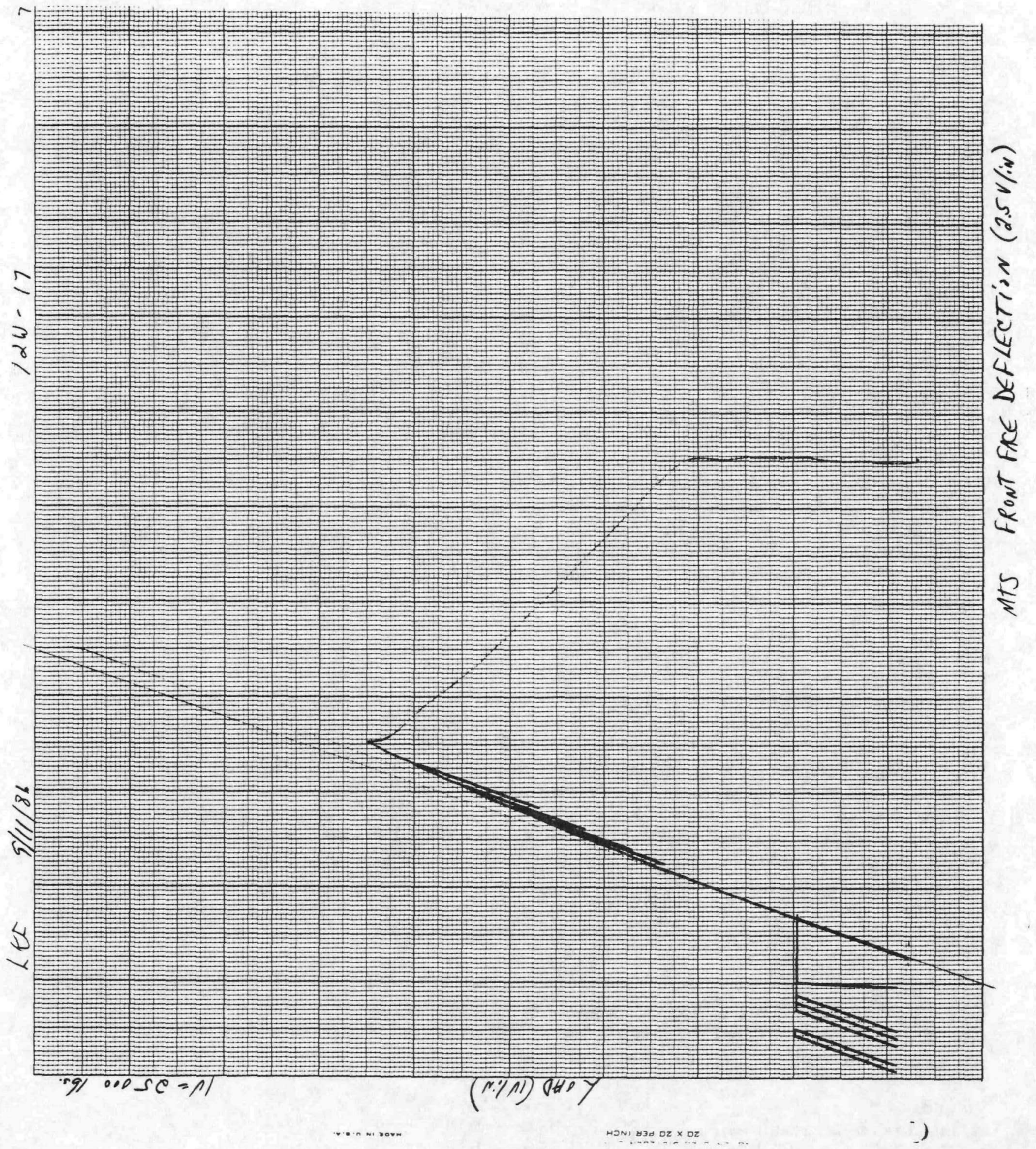




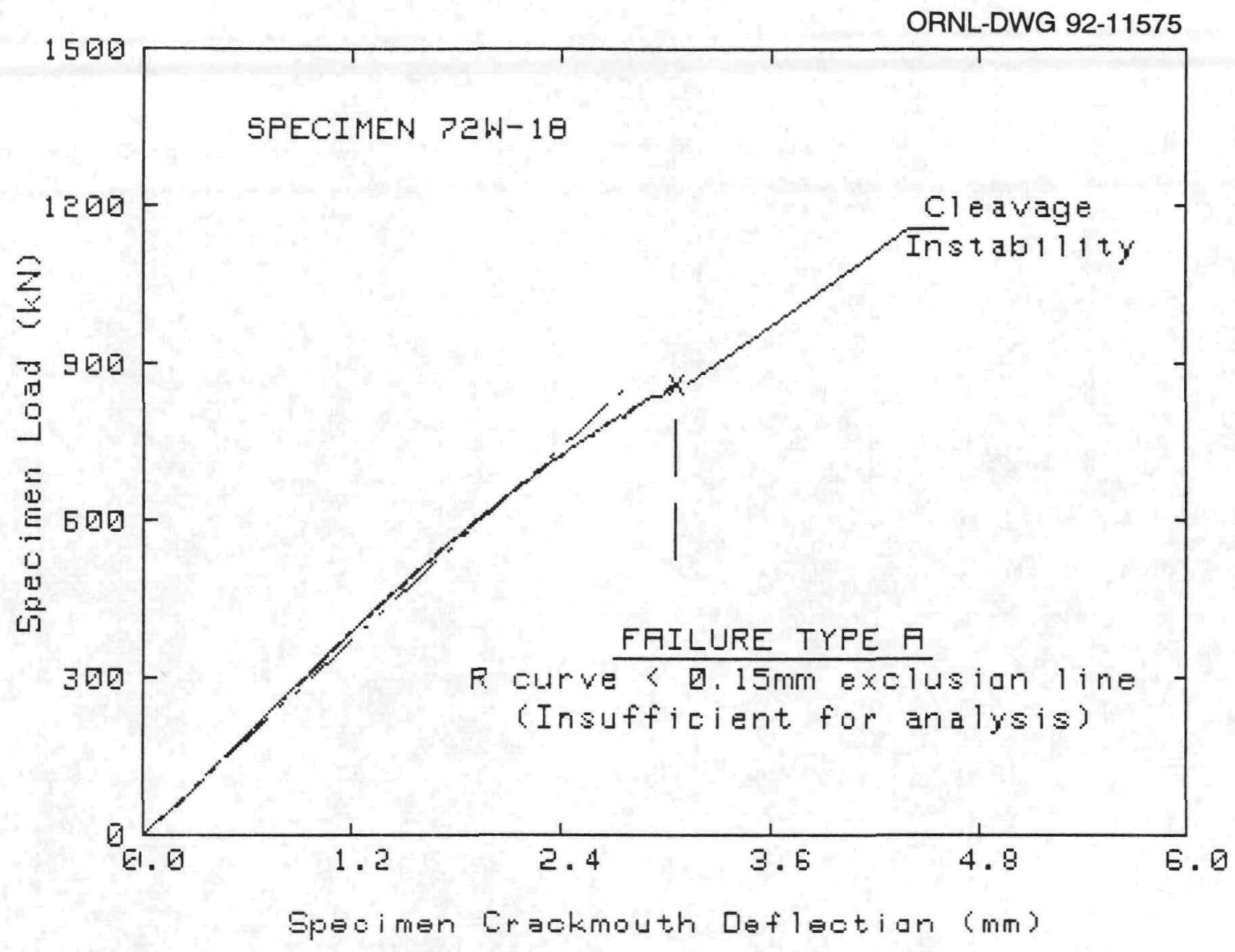

TEST SPECIMEN DATA

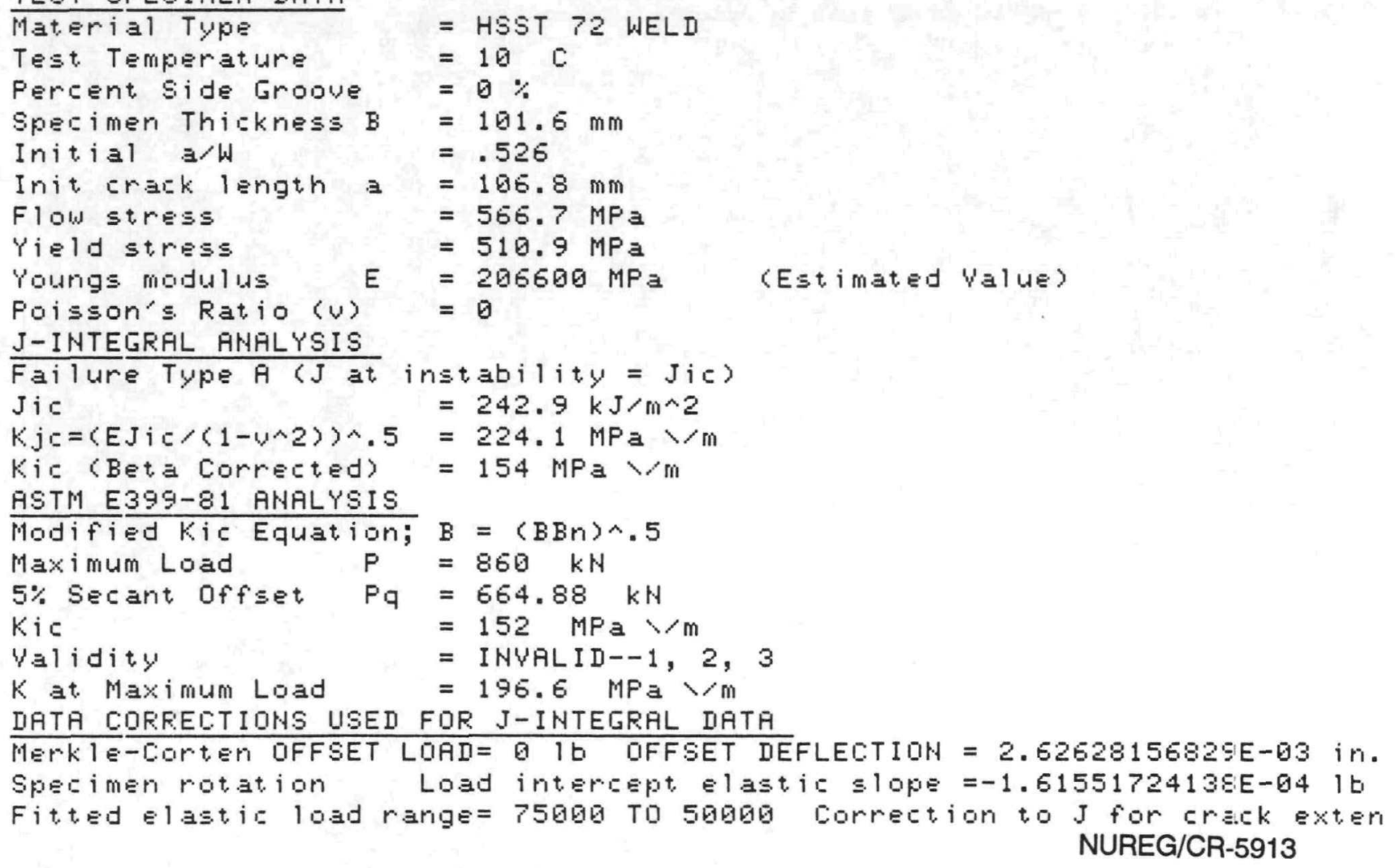




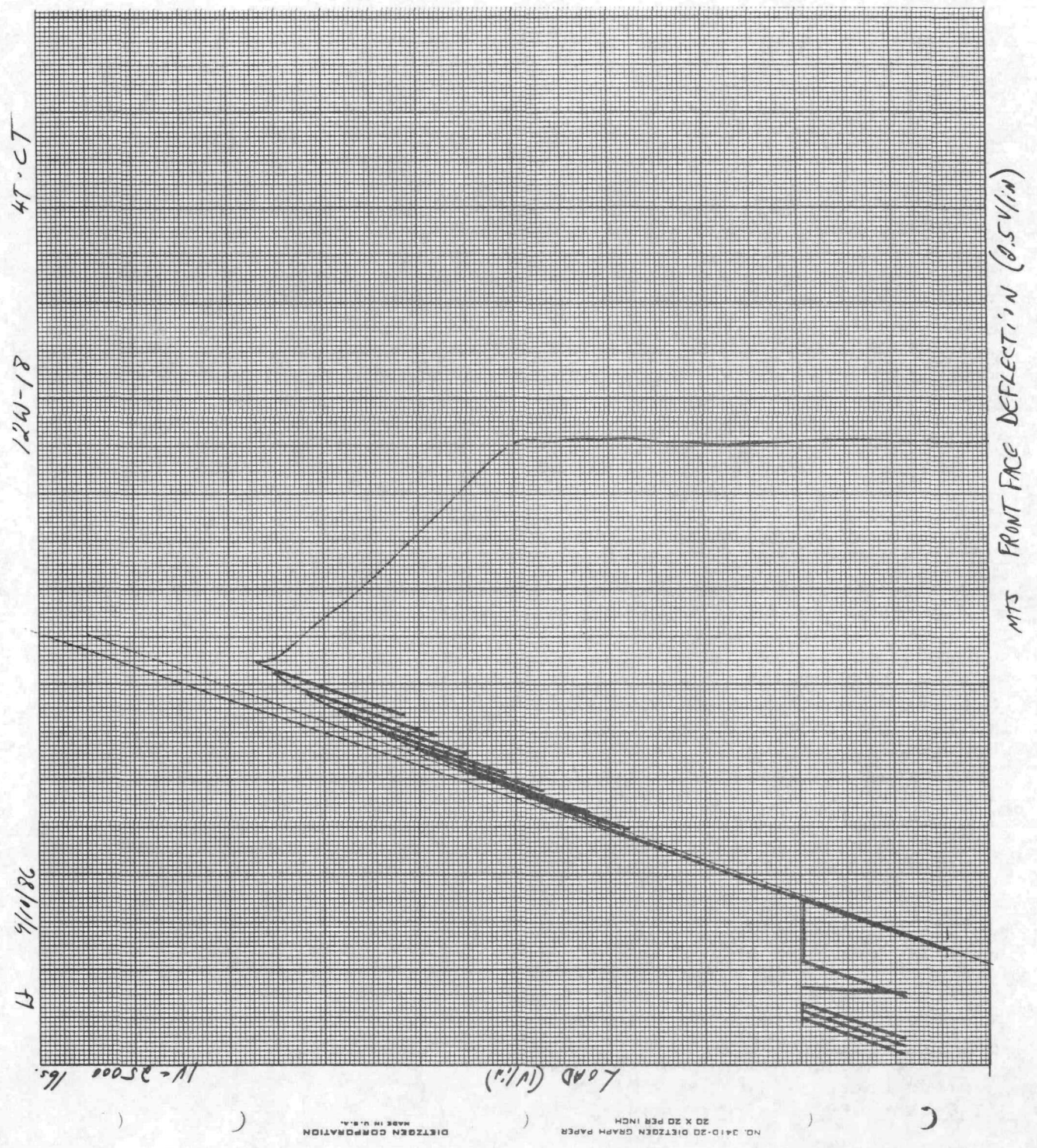


132910

591.18

148030

658.46

0.005

0.127

29885

206.06

124.82

137.17

139.03

152.78

0

0

$-100$

$P Q$ (LB)

$P Q \quad(K N)$

MAX LOAD (LB)

MAX LOAD (KN)

MEASURED FINAL CRACK EXTENSION (IN)

MEASURED FINAL CRACK EXTENSION (MM)

REFERENCE MODULUS (KSI)

REFERENCE MODULUS (GPA)

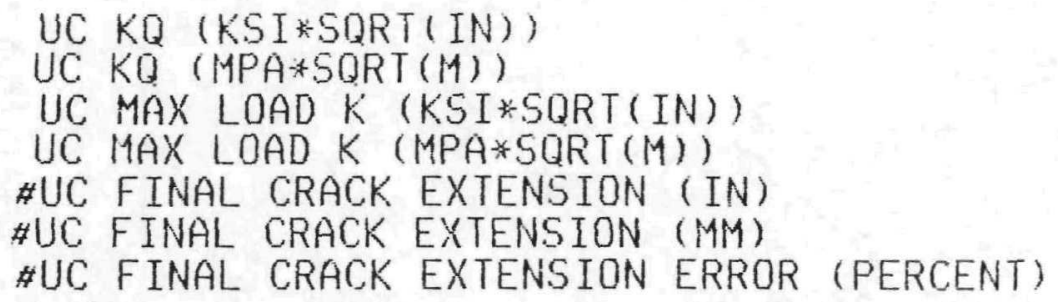

INVALID UC KIC TEST

696.4

121.97

144.27

158.54

115.56

126.99

144.27

158.54

115.56

126.99

671.5

117.59

141.66

155.67

114.33

125.64

141.66

155.67

114.33

125.64

671.5

117.59

141.66

155.67

114.33

125.64

141.66

155.67

114.33

125.64
UC MERKLE-CORTEN MAX LOAD J-INTEGRAL (IN*LB/IN`2)

UC MERKLE-CORTEN MAX LOAD J-INTEGRAL (KJ/M`2)

UC MERKLE-CORTEN MAX LOAD KJ (KSI*SQRT(IN))

UC MERKLE-CORTEN MAX LOAD KJ (MPA*SQRT(M))

UC MERKLE-CORTEN BETA-ADJ MAX LOAD KJ (KSI*SQRT (IN))

UC MERKLE-CORTEN BETA-ADJ MAX LOAD KJ (MPA*SQRT(M))

UC MERKLE-CORTEN FINAL KJ (KSI*SQRT(IN))

UC MERKLE-CORTEN FINAL K.J (MPA*SQRT(M))

UC MERKLE-CORTEN BETA-ADJ FINAL KJ (KSI*SQRT(IN))

UC MERKLE-CORTEN BETA-ADJ FINAL KJ (MPA*SQRT(M))

\#UC ERNST MAX LOAD J-INTEGRAL (IN*LB/IN*2)

\#UC ERNST MAX LOAD J-INTEGRAL (KJ/M^2)

\#UC ERNST MAX LOAD KJ (KSI*SQRT(IN))

\#UC ERNST MAX LOAD K.J (MPA*SQRT(M))

\#UC ERNST BETA-ADJ MAX LOAD KJ (KSI*SQRT (IN))

\#UC ERNST BETA-ADJ MAX LOAD KJ (MPA*SQRT(M))

\#UC ERNST FINAL KJ (KSI*SQRT(IN))

\#UC ERNST FINAL KJ (MPA*SQRT (M))

\#UC ERNST BETA-ADJ FINAL KJ (KSI*SQRT (IN))

\#UC ERNST BETA-ADJ FINAL KJ (MPA*SQRT (M))

\#UC MODIFIED ERNST MAX LOAD J-INTEGRAL (IN*LB/IN*2)

\#UC MODIFIED ERNST MAX LOAD J-INTEGRAL (KJ/M^2)

\#UC MODIFIED ERNST MAX LOAD KJ (KSI*SQRT(IN))

\#UC MODIFIED ERNST MAX LOAD KJ (MPA*SQRT(M))

\#UC MODIFIED ERNST BETA-ADJ MAX LOOAD KJ (KSI*SQRT(IN))

\#UC MODIFIED ERNST BETA-ADJ MAX LOAD KJ (MPA*SQRT(M))

\#UC MODIFIED ERNST FINAL KJ (KSI*SQRT(IN))

\#UC MODIFIED ERNST FINAL KJJ (MPA*SQRT (M))

\#UC MODIFIED ERNST BETA-ADJ FINAL KJ (KSI*SQRT(IN))

\#UC MODIFIED ERNST BETA-ADJ FINAL KJ (MPA*SQRT(M))

ESTIMATED VALUE FOR FINAL UC CRACK EXTENSION

UC MERKLE-CORTEN JIC,CEIC,KJIC AND TAVG NOT DEFINED--NOT ENOUGHT DATA POINTS FOR POWER LAW FIT 
$E-128$

ORNL-DWG 92-11576

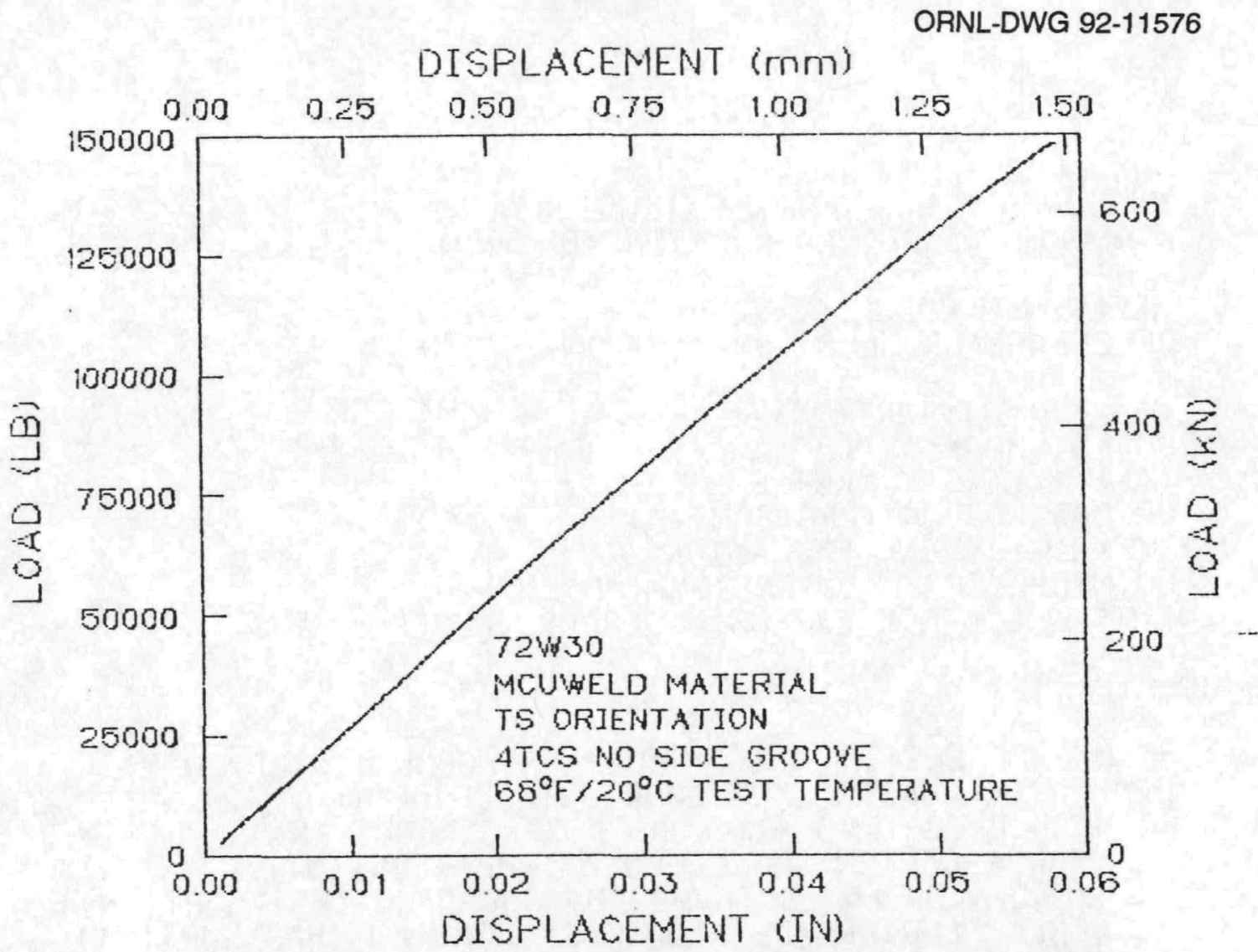

72W-30

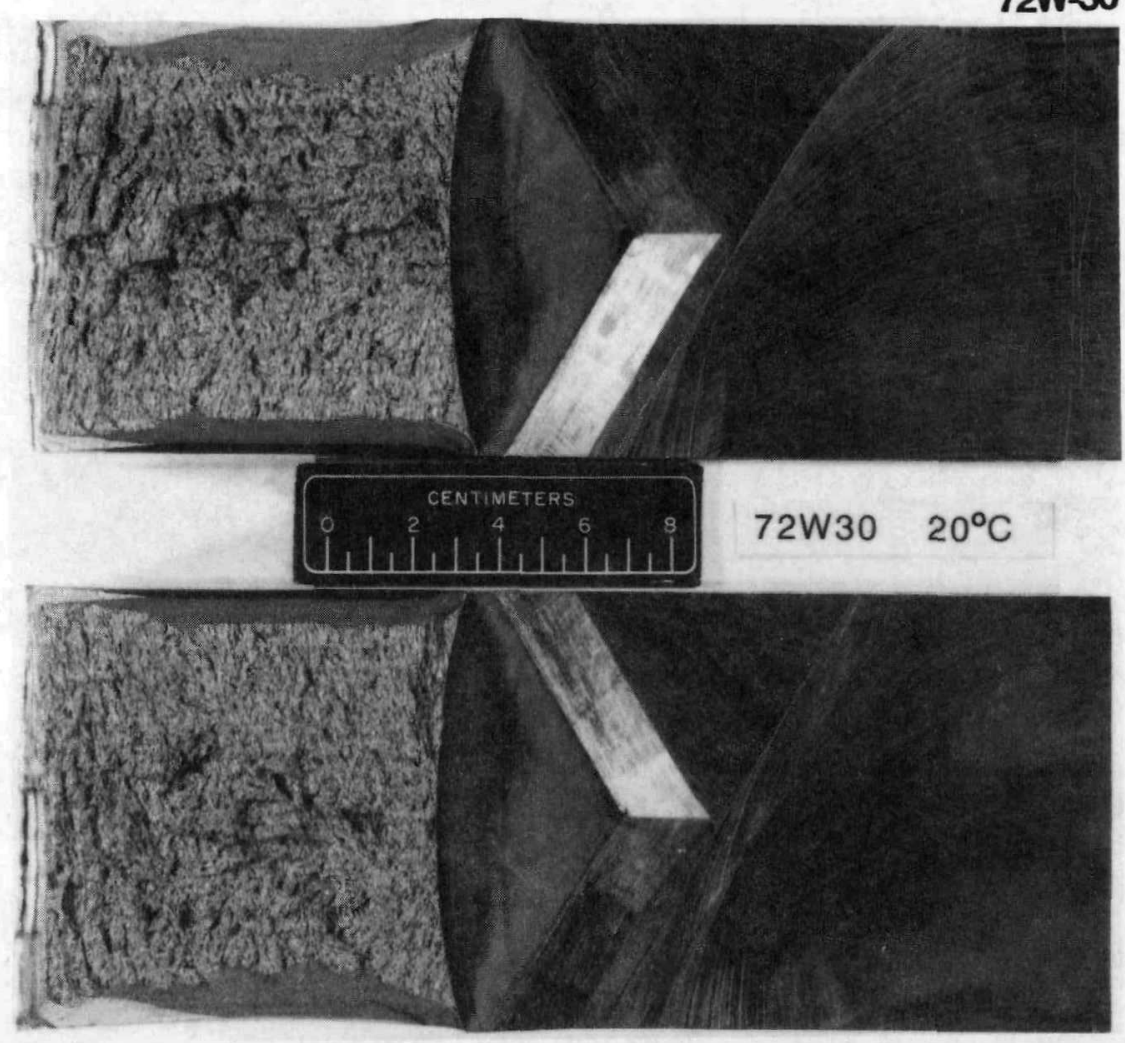


SPECIMEN CLEAVED

$\begin{array}{ll}134110 & P Q(L B) \\ 596.5 & P Q(K N) \\ 162750 & \text { MAX LOAD (LB) } \\ 723.93 & \text { MAX LOAD (KN) } \\ 0.005 & \text { MEASURED FINAL CRACK EXTENSION (IN) } \\ 0.127 & \text { MEASURED FINAL CRACK EXTENSION (MM) } \\ 29885 & \text { REFERENCE MODULUS (KSI) } \\ 206.06 & \text { REFERENCE MODULUS (GPA) } \\ 121.43 & \text { UC KQ (KSI*SQRT(IN)) } \\ 133.44 & \text { UC KQ (MPA*SQRT(M)) } \\ 147.37 & \text { UC MAX LOAD K (KSI*SQRT(IN)) } \\ 161.95 & \text { UC MAX LOADK (MPA*SQRT(M)) } \\ -0.047 & \text { \#UC FINAL CRACK EXTENSION (IN) } \\ -1.194 & \text { \#UC FINAL CRACK EXTENSION (MM) } \\ -1040.5 & \text { \#UC FINAL CRACK EXTENSION ERROR (PERCENT) } \\ \text { INUALID UC KIC TEST }\end{array}$

799.4

140

154.57

UC MERKLE-CORTEN

IAAX LOAD J-INTEGRAL (IN*LB/IN*2)

UC MERKLE-CORTEN

MAX LOAD J-INTEGRAL (KJ/M*2)

UC MERKLE-CORTEN

MAX LOAD KJ (KSI*SORT (IN))

169.86

UC MERKLE-CORTEN

MAX LOAD KJ (MPA*SQRT (M))

120.19

UC MERKLE-CORTEN

BETA-ADJ MAX LOAD KJ (KSI*SQRT (IN))

132.08

UC MERKLE-CORTEN

154.57

UC MERKLE-CORTEN

BETA-ADJ MAX LOAD KJ (MPA*SQRT (M))

169.86

UC MERKLE-CORTEN

FINAL KJ (KSI*SQRT (IN))

120.19

UC HERKLE-CORTEN

FINAL KJ (MPA*SQRT $(M))$

132.08

UC MERKLE-CORTEN

BETA-ADJ FINAL KJ (KSI*SQRT (IN))

BETA-ADJ FINAL KJ (MPA $* S Q R T(M))$

779.2

136.46

152.6

167.69

119.33

131.14

152.6

167.69

119.33

\#UC ERNST

\#UC ERNST

HUC ERNST

\#UC ERNST

\#UC ERNST

\#UC ERNST

\#UC ERNST

\#UC ERNST

MAX LOAD

J-INTEGRAL (IN*LB/IN*2)

MAX LOAD J-INTEGRAL (KJ/M^2)

MAX LOAD KJ (KSI*SORT (IN))

MAX LOAD KJ (MPAKSORT (M))

BETA-ADJ MAX LOAD KJ (KSI*SORT(IN))

BETA-ADJ MAX LOAD KJ (MPA $K Q R T(M))$

\#UC ERNST

FINAL KJ (KSI*SQRT (IN))

FINAL KJ (MPA*SQRT(M))

131.14

BETA-ADJ FINAL KJ (KSI*SQRT (IN))

\#UC ERNST

BETA-ADJ FINAL KJ (MPA*SQRT (M))

787

137.82

153.36

168.53

119.67

131.5

153.36

168.53

119.67

131.5
\#UC MODIFIED ERNST

\#UC MODIFIED ERNST

\#UC MODIFIED ERNST

\#UC MODIFIED ERNST

\#UC MODIFIED ERNST

\#UC MODIFIED ERNST

\#UC MODIF IED ERNST

\#UC MODIFIED ERNST

AUC MODIFIED ERNST

\#UC MODIFIED ERNST
MAX LOAD J-INTEGRAL (IN*LB/IN`2)

MAX LOAD J-INTEGRAL (KJ/M`2)

MAX LOAD KJ (KSI*SQRT (IN))

MAX LOAD KJ (MPA*SQRT (M))

BETA-ADJ MAX LOAD KJ (KSI*SQRT(IN))

BETA-ADJ MAX LOAD KJ (MPA*SQRT(M))

FINAL KJ (KSI*SQRT (IN))

FINAL KJ (MPA*SQRT $(M))$

BETA-ADJ FINAL KJ (KSI*SQRT (IN))

BETA-ADJ FINAL KJ (MPA*SQRT $(M)$ )

\# ESTIMATED VALUE FOR FINAL UC CRACK EXTENSION

GET POWER LAW FIT TO UC MERKLE-CORTEN TEST RESULIS W NO 


\section{E-130}

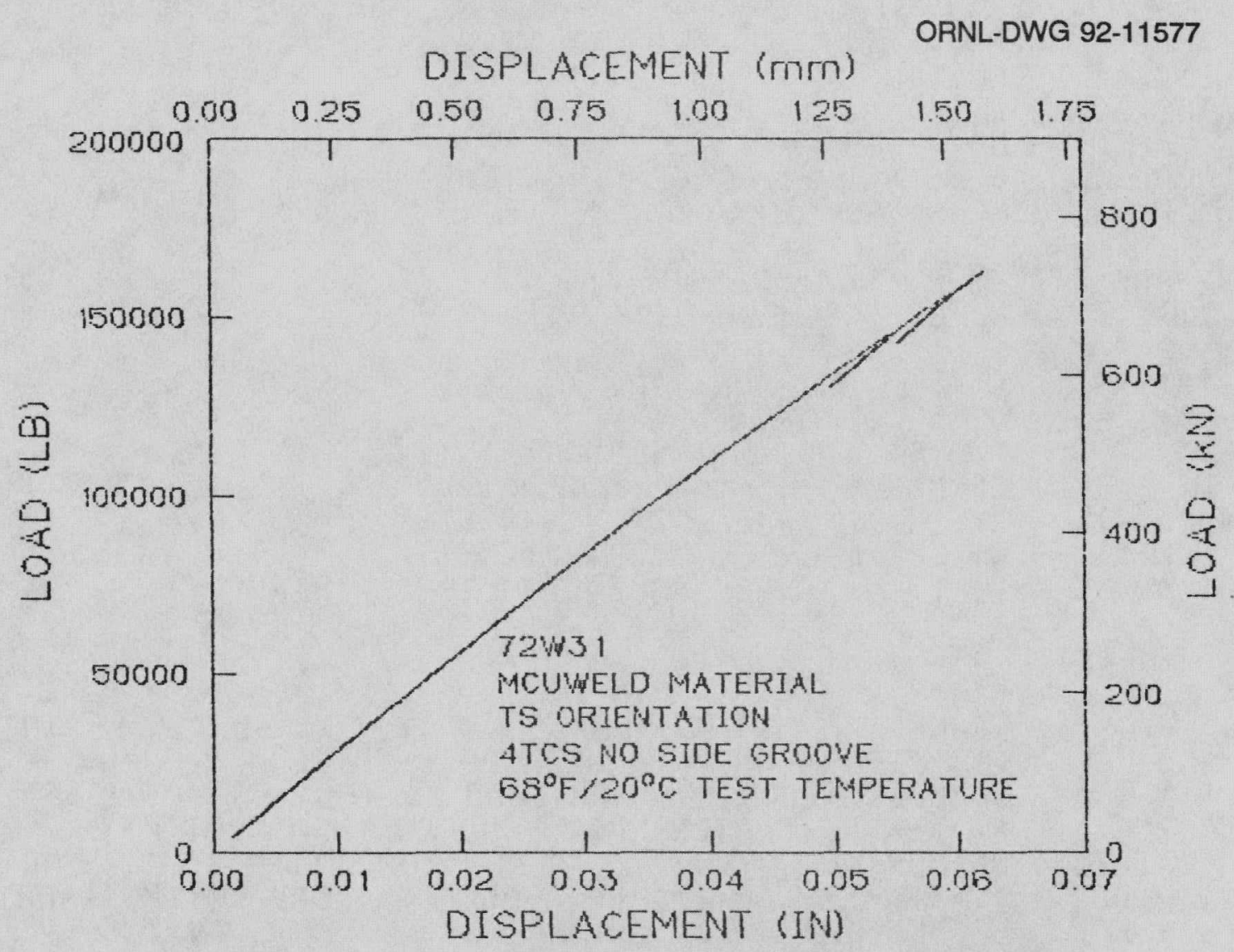

72W-31

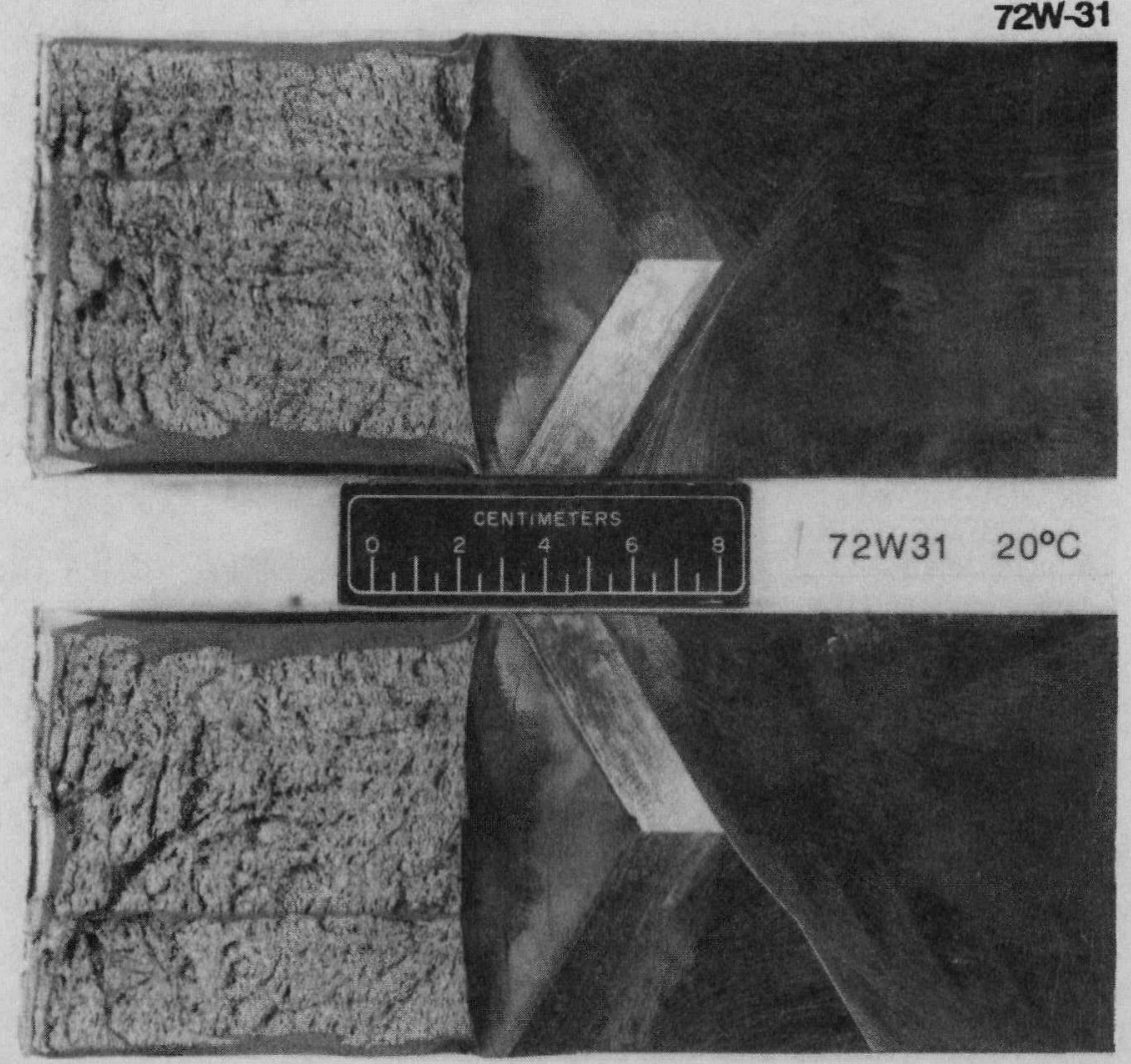


SPECIMEN CLEAVED

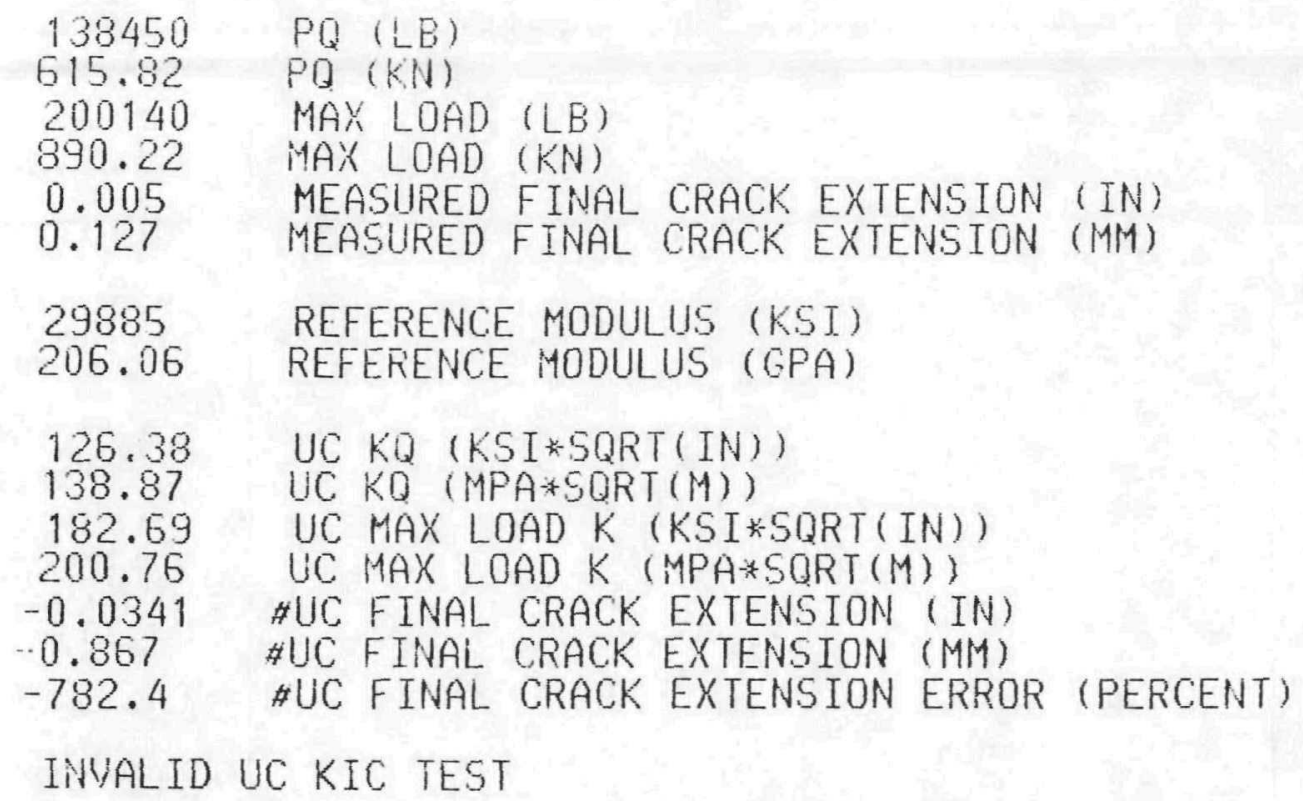

INUALID UC KIC TEST

1388.5 UC MERKLE-CORTEN MAX LOAD J-INTEGRAL (IN*LB/IN`2)

243.17 UC MERKLE-CORTEN MAX LOAD J-INTEGRAL. (KJ/M“2)

203.7

223.85

138.67

152.38

216.42

237.82

142.72

156.83

1351.6 236.69

200.98

220.85

137.76

151.39

213.66

234.79

141.86

155.89

1361

238.35

201.68

221.62

138

151.64

214.32

235.51

142.06

156.11
UC MERKLE-CORTEN

UC MERKLE-CORTEN

UC MERKLE-CORTEN

UC MERKLE-CORTEN

UC MERKLE-CORTEN

UC MERKLE-CORTEN

UC MERKLE-CORTEN

UC MERKLE-CORTEN
MAX LOAD KJ (KSI*SQRT (IN))

MAX LOAD KJ (MPA*SQRT (M))

BETA-ADJ MAX LOAD KJ (KSI*SQRT (IN))

BETA-ADJ MAX LOAD KJ (MPA*SQRT(M))

FINAL K.J $(K S I * S O R T(I N))$

FINAL KJ (MPA*SQRT (M))

BETA-ADJ FINAL KJ (KSI*SQRT (IN))

BETA-ADJ FINAL KJ (MPA*SQRT(M))

\section{\#UC ERNST MAX LOAD J-INTEGRAL (IN*LB/IN*2)}

\#UC ERNST MAX LOAD J-INTEGRAL (KJ/M^2)

\#UC ERNST MAX LOAD K.J (KSI*SORT(IN))

\#UC ERNST MAX LOAD K.J (MPA*SQRT(M))

\#UC ERNST BETA-ADJ MAX LOAD KJ (KSI*SQRT (IN))

AUC ERNST BETA-ADJ MAX LOAD KJ (MPA*SQRT(M))

\#UC ERNST FINAL KJ (KSI*SQRT(IN))

\#UC ERNST FINAL KJ (MPA*SQRT(M))

\#UC ERNST BETA-ADJ FINAL KJJ (KSI*SQRT(IN))

\#UC ERNST BETA-ADJ FINAL KJ (MPA*SQRT(M))

\# ESTIMATED VALUE FOR FINAL UC CRACK EXTENSION

GET POWER LAW FIT TO UC MERKLE-CORTEN TEST RESULTS N NO 
E-132
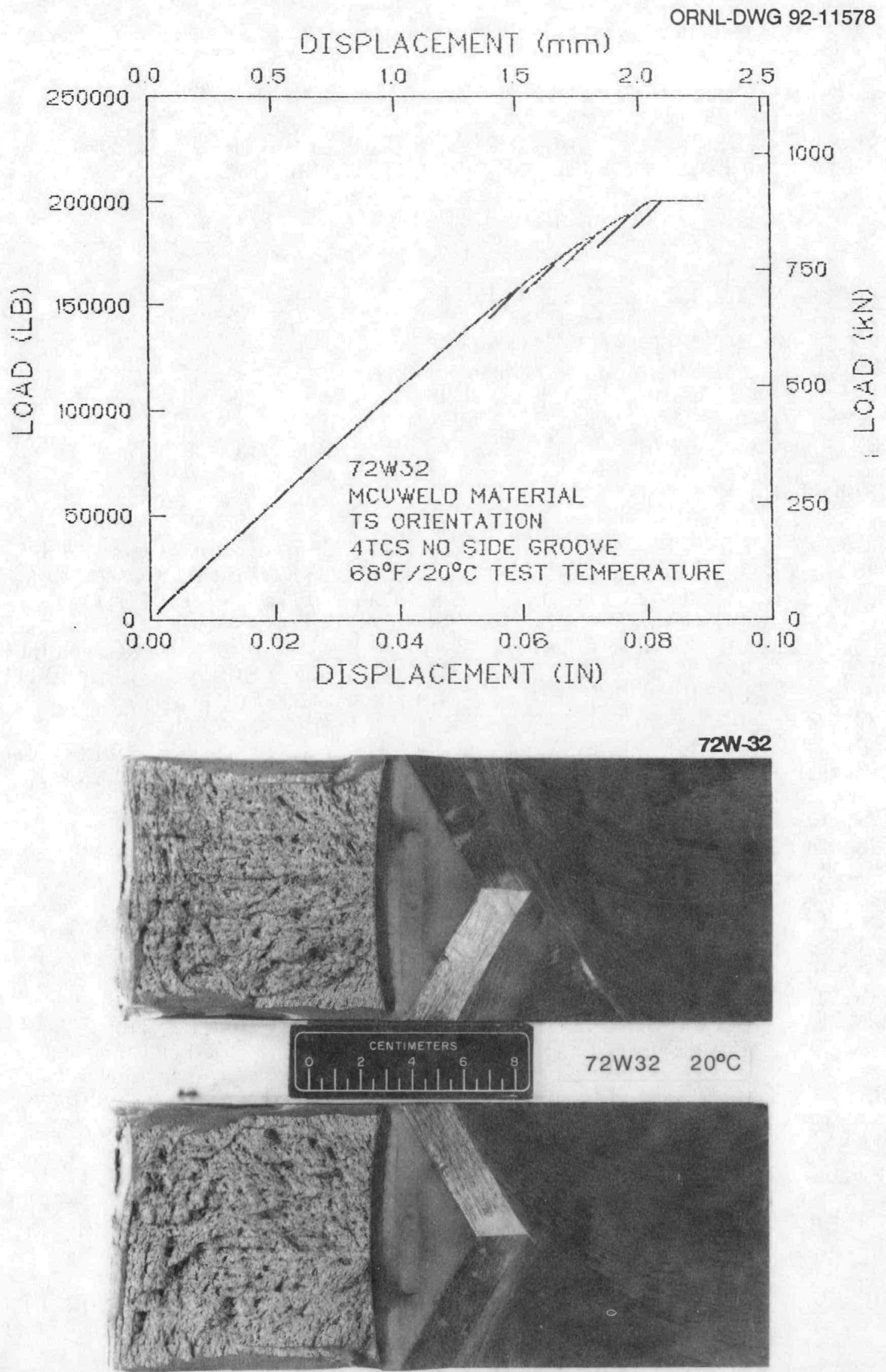

144310
541.89
149800
666.33
0.005
0.127
29885
206.06
128.61
141.33
133.5
146.71
0
100

\author{
P0) (LB) \\ $P$ (KN) \\ MAX LOAD (LB) \\ MAX LOAD (KN) \\ MEASURED FINAL CRACK EXTENSION (IN) \\ MEASURED FINAL CRACK EXTENSION (MM)
}

INVALID UC KIC TEST

631.6

110.61

137.39

150.97

112.27

123.37

137.39

150.97

112.27

123.37

608.3

106.53

134.83

148.16

111

121.98

134.83

148.16

111

121.98

608.3

106.53

134.83

148.16

111

121.98

134.83

148.16

111

121.98
UC MERKLE-CORTEN

UC MERKLE-CORTEN

UC MERKLE-CORTEN

UC MERKLE-CORTEN

UC MERKLE-CORTEN

UC MERKLE-CORTEN

UC MERKLE-CORTEN

UC MERKLE-CORTEN

UC MERKLE-CORTEN

UC MERKLE-CORTEN
MAX LOAD J-INTEGRAL (IN*LB/IN`2)

MAX LOAD J-INTEGRAL (KJ/M`2)

MAX LOAD KJ (KSI*SORT (IN))

MAX LOAD KJ (MPA*SQRT (M))

BETA-ADJ MAX LOAD KJ (KSI*SQRT(IN))

BETA-ADJ MAX LOAD KJ (MPA*SQRT (M))

FINAL KJ (KSI*SQRT (IN))

FINAL K.J (MPA*SQRT (M))

BETA-ADJ FINAL KJ (KSI*SQRT (IN))

BETA-AD.J FINAL KJ (MPA*SQRT (M))

\#UC ERNST MAX LOAD J-INTEGRAL (IN*LB/IN*2)

\#UC ERNST MAX LOAD J-INTEGRAL (K.J/M^2)

\#UC ERNST MAX LOAD KJ (KSI*SQRT(IN))

\#UC ERNST MAX LOAD KJ (MPA*SQRT(M))

\#UC ERNST BETA-ADJ MAX LOAD KJ (KSI*SORT(IN))

\#UC ERNST BETA-ADJ MAX LOAD KJ (MPA*SQRT(M))

\#UC ERNST FINAL KJ (KSI*SQRT(IN))

\#UC ERNST FINAL KJ (MPAKSQRT(M))

\#UC ERNST BETA-ADJ FINAL KJ (KSI*SQRT(IN))

\#UC ERNST BETA-ADJ FINAL KJ (MPA*SQRT $(M)$ )

\#UC MODIFIED ERNST MAX LOAD J-INTEGRAL (IN*LB/IN`2)

\#UC MODIFIED ERNST MAX LOAD J-INTEGRAL (KJ/M“2)

\#UC MODIFIED ERNST MAX LOAD KJ (KSI*SORT(IN))

\#UC MODIFIED ERNST MAX LOAD KJ (MPA*SQRT(M))

\#UC MODIFIED ERNST BETA-ADJ MAX LOAD KJ (KSI*SQRT(IN))

\#UC MODIFIED ERNST BETA-ADJ MAX LOAD KJ (MPA*SQRT(M))

\#UC MODIFIED ERNST FINAL KJ (KSI*SQRT(IN))

\#UC MODIFIED ERNST FINAL KJ (MPA*SQRT(M))

\#UC MODIFIED ERNST BETA-ADJ FINAL KJ (KSI*SQRT(IN))

\#UC MODIFIED ERNST BETA-ADJ FINAL KJ (MPA*SQRT(M))

\# ESTIMATED VALUE FOR FINAL UC CRACK EXTENSION

UC MERKLE-CORTEN JIC,CEIC,KJIC AND TAVG NOT DEFINED--NOT ENOUGHT DATA POINTS FOR POWER LAW FIT 
E-134

ORNL-DWG 92-11579

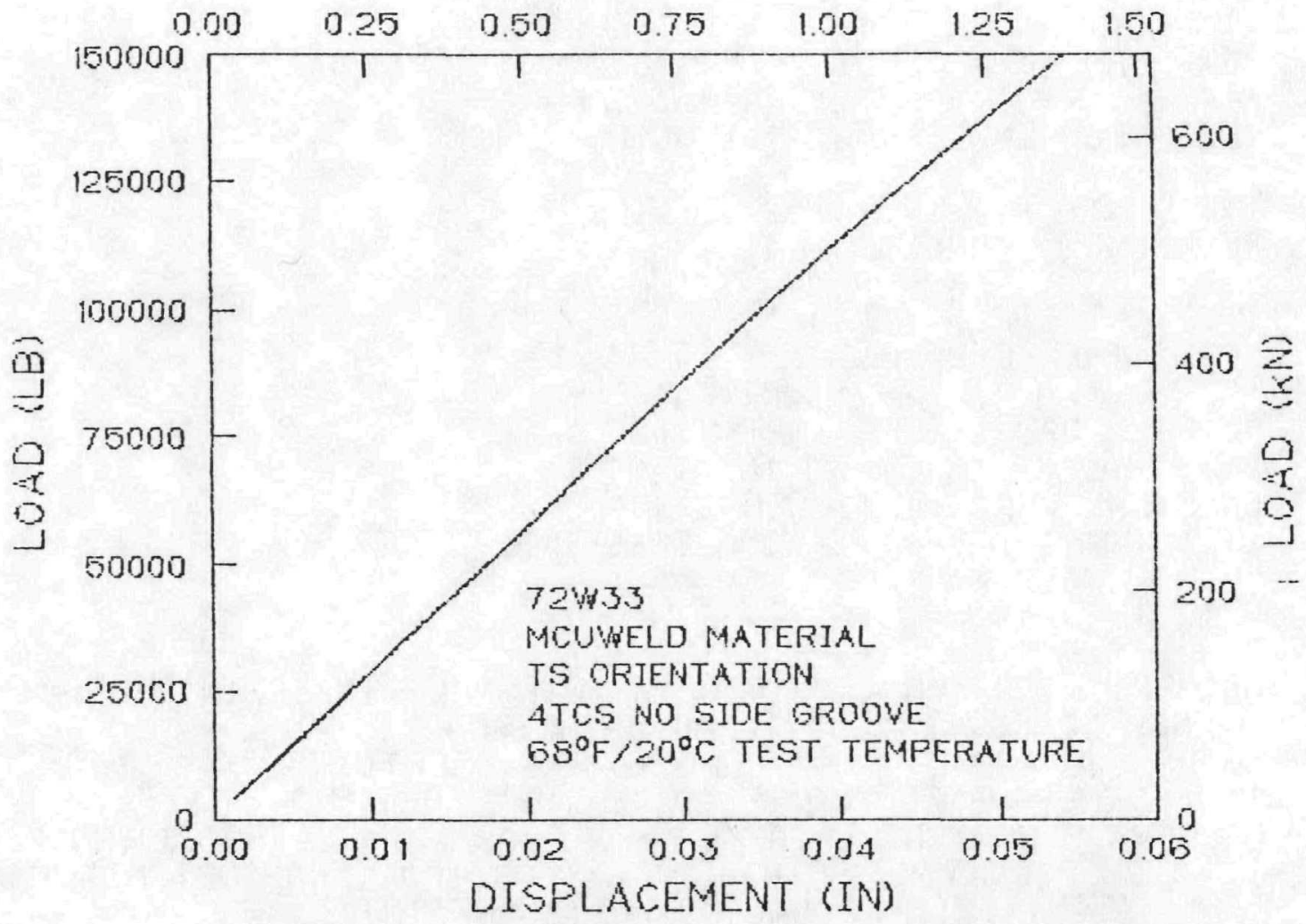

$72 W-33$
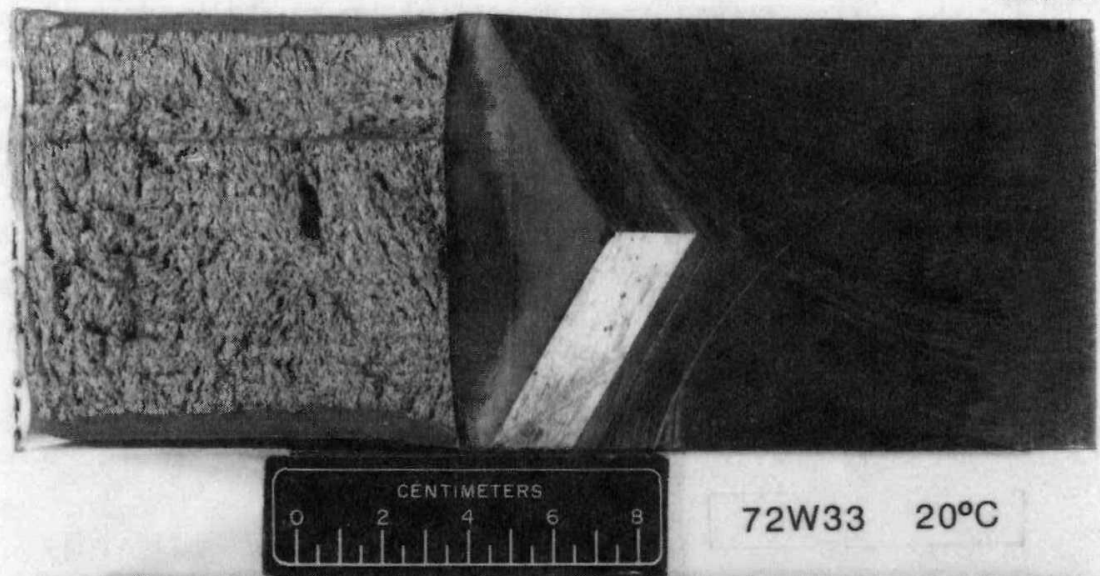
Specimen Loadline Deflection (in.)
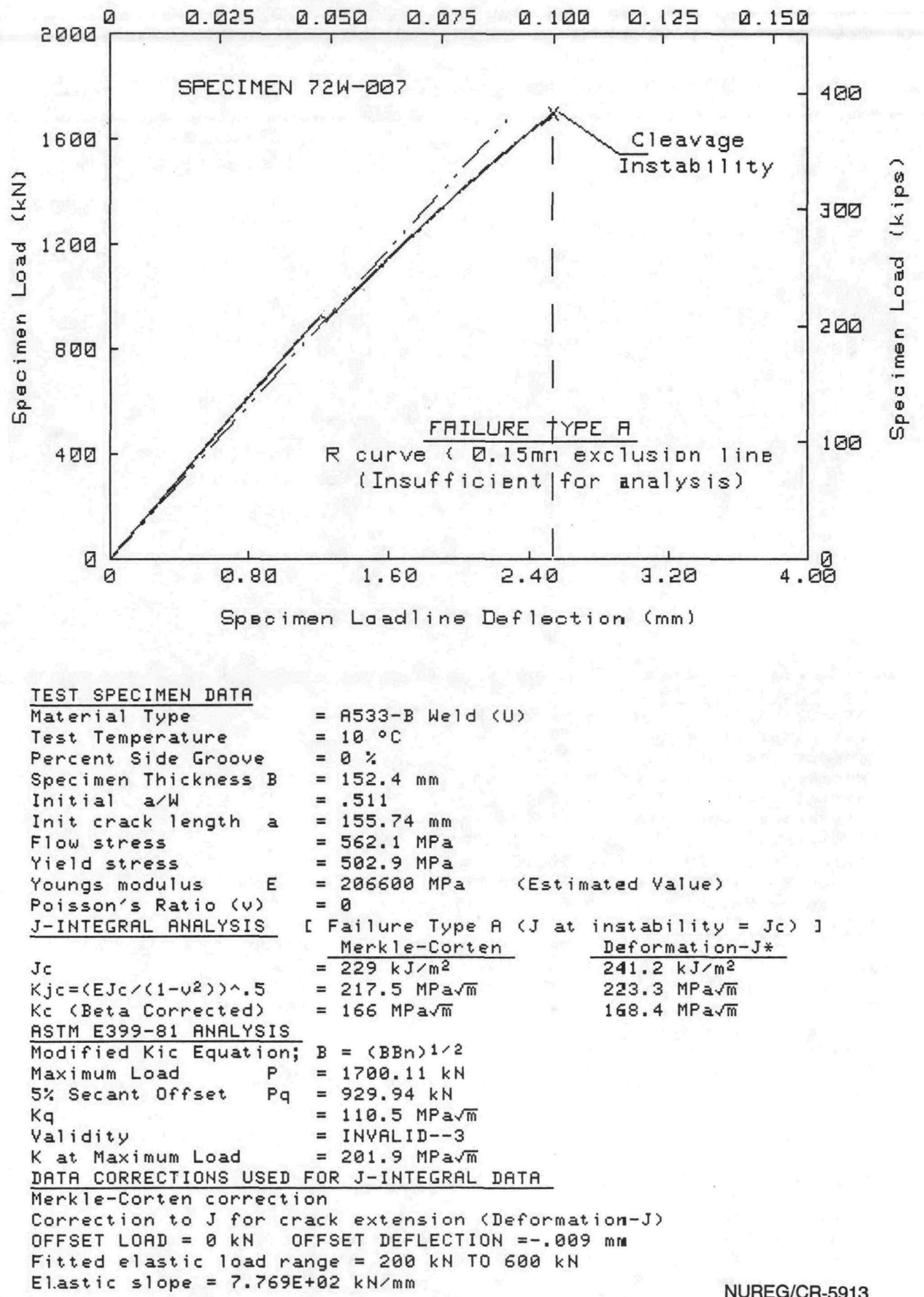
Specimen Loadline Deflection (in.) ORNL-DWG 92-11581

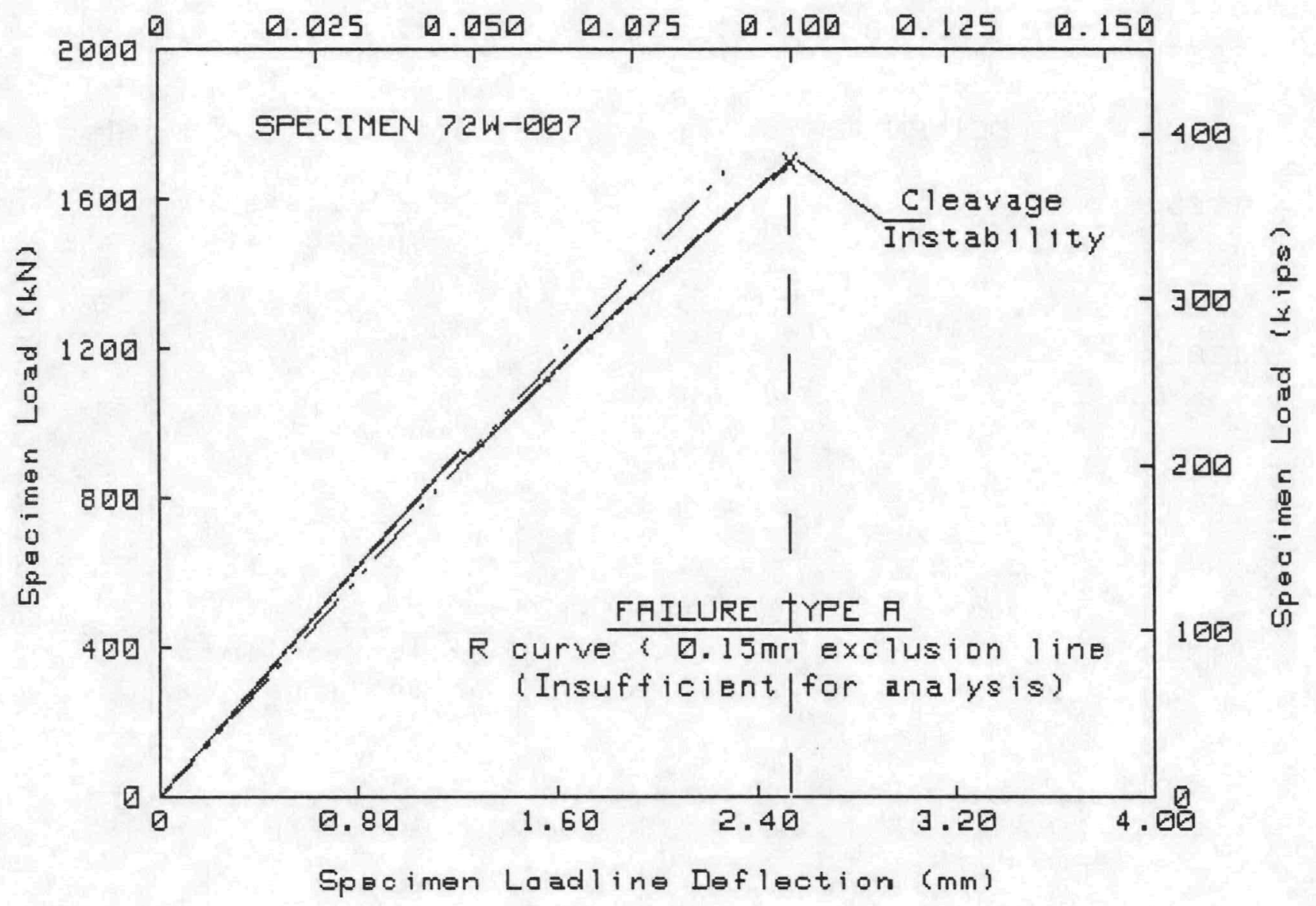

TEST SPECIMEN DATA

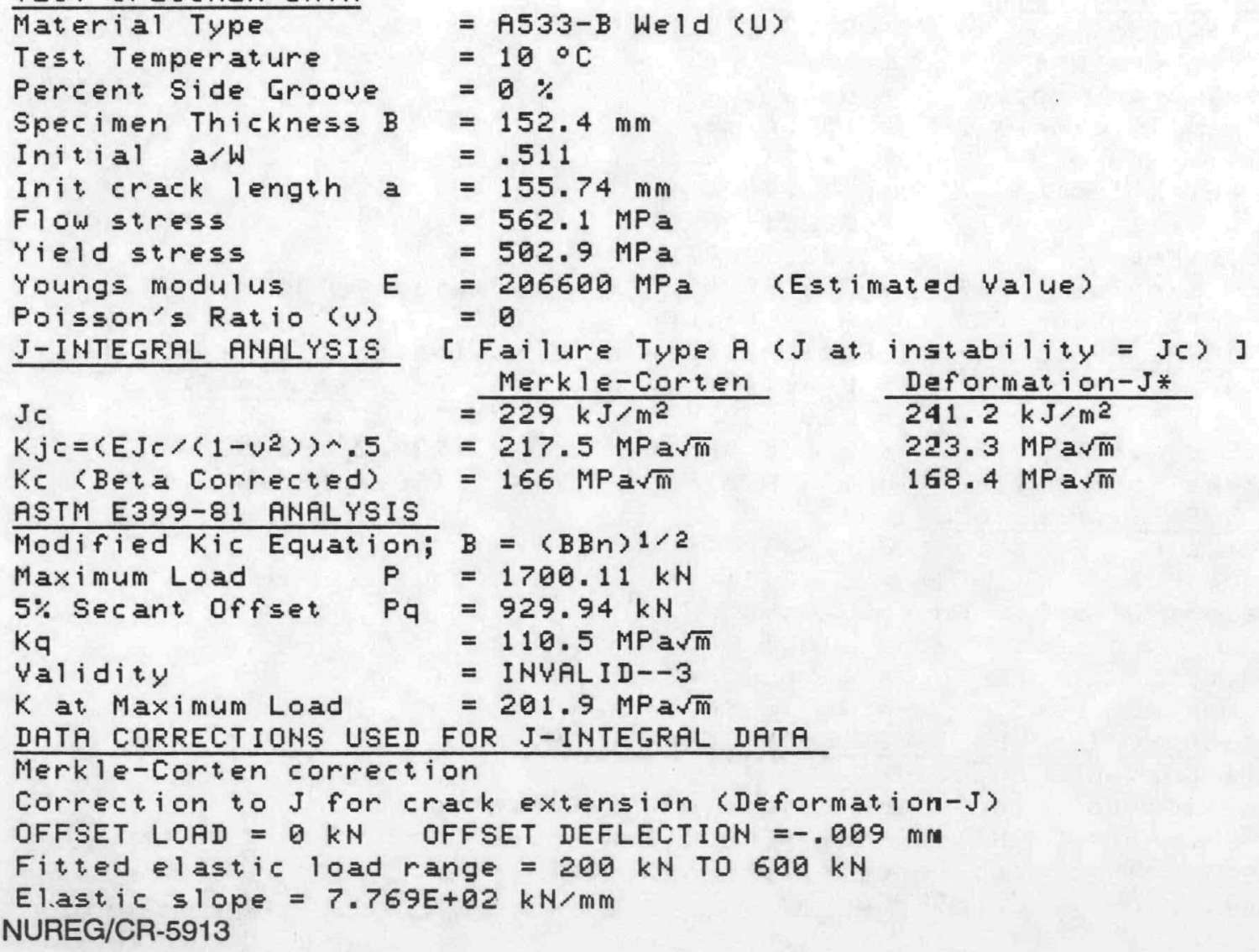


Specimen Crackmauth Def lection (in.) ORNL-DWG 92-11582

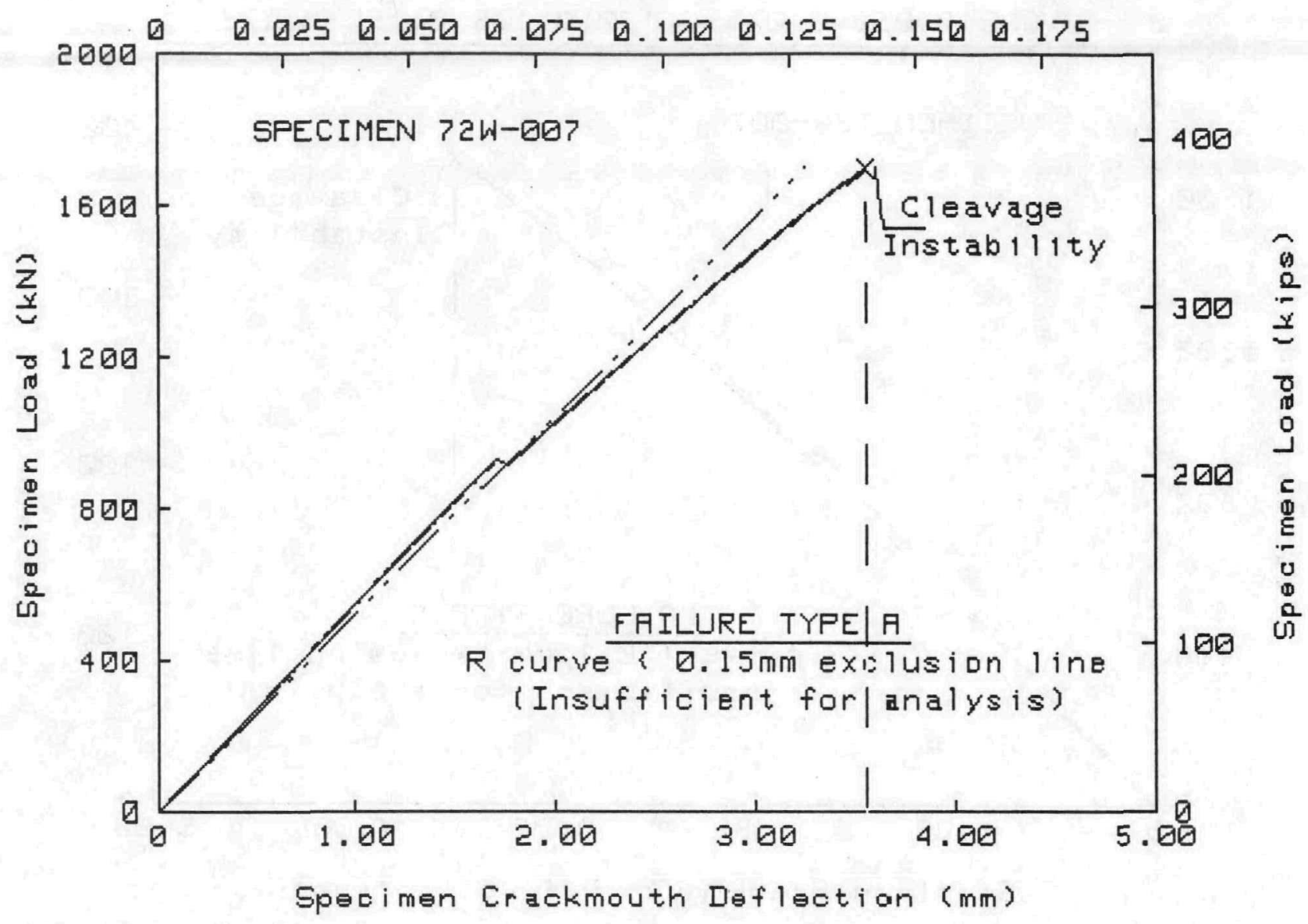

TEST SPECIMEN DATA

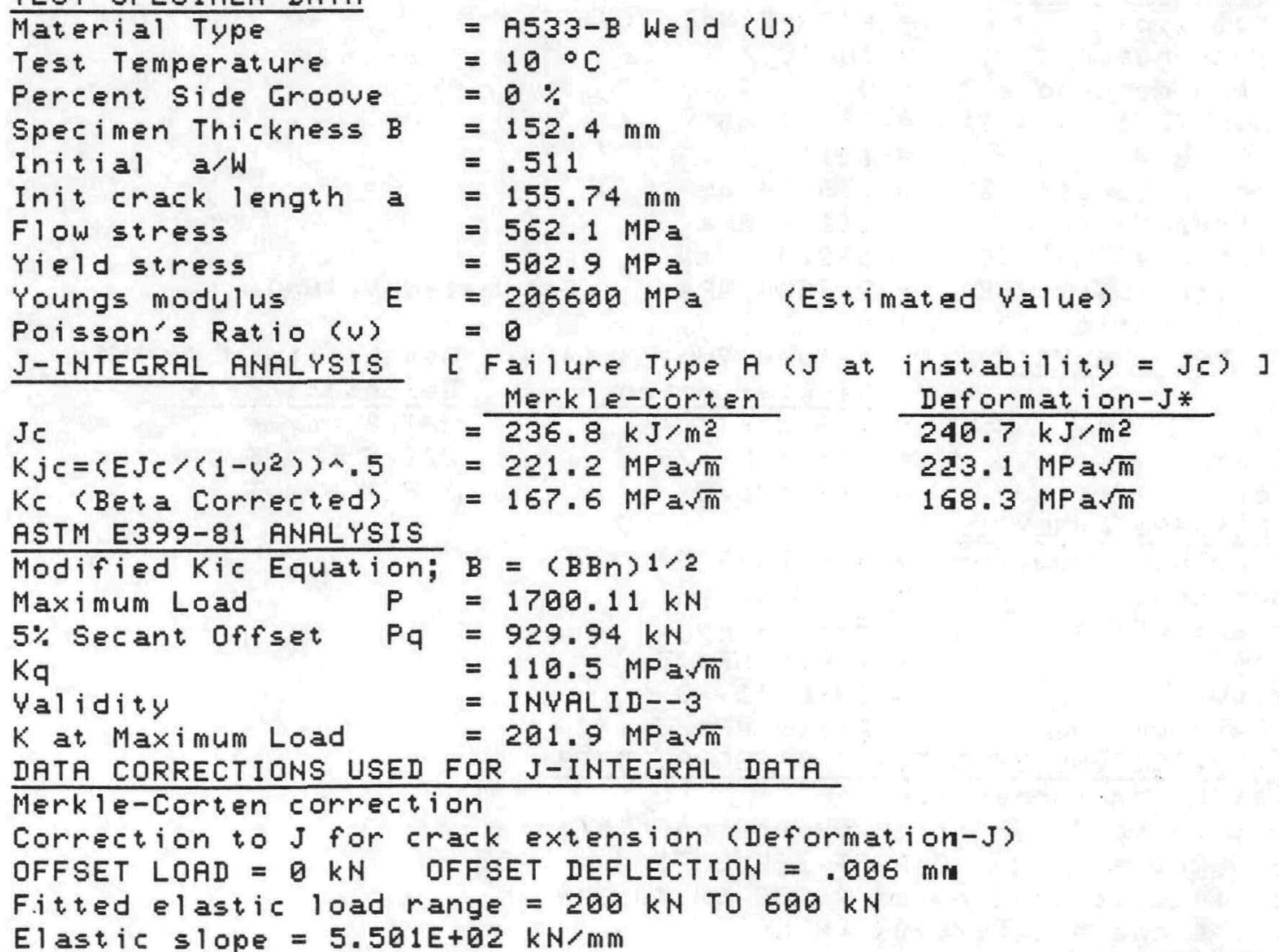


Specimen Crackmouth Deflection (in.) ORNL-DWG 92-11583

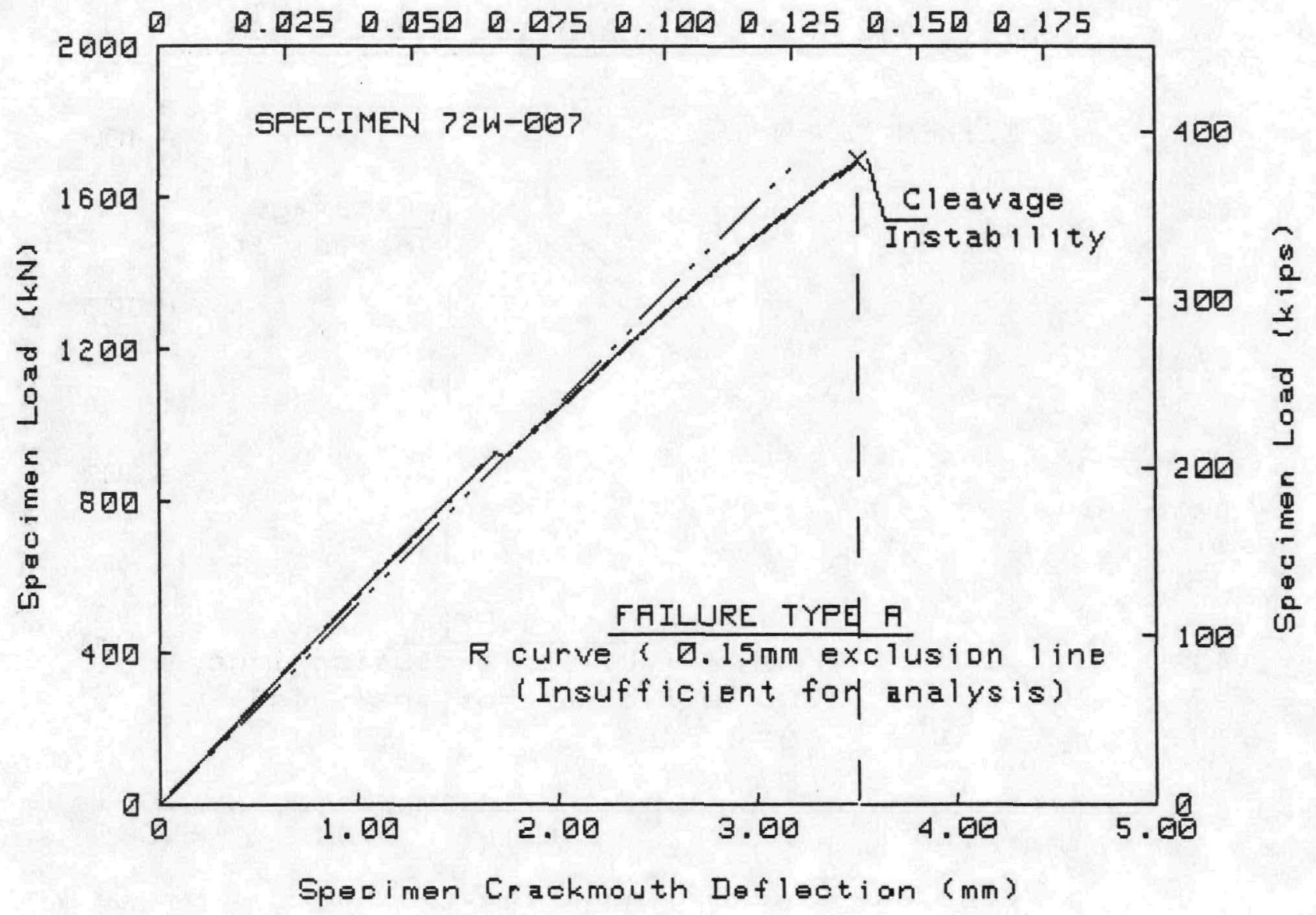

TEST SPECIMEN DATA

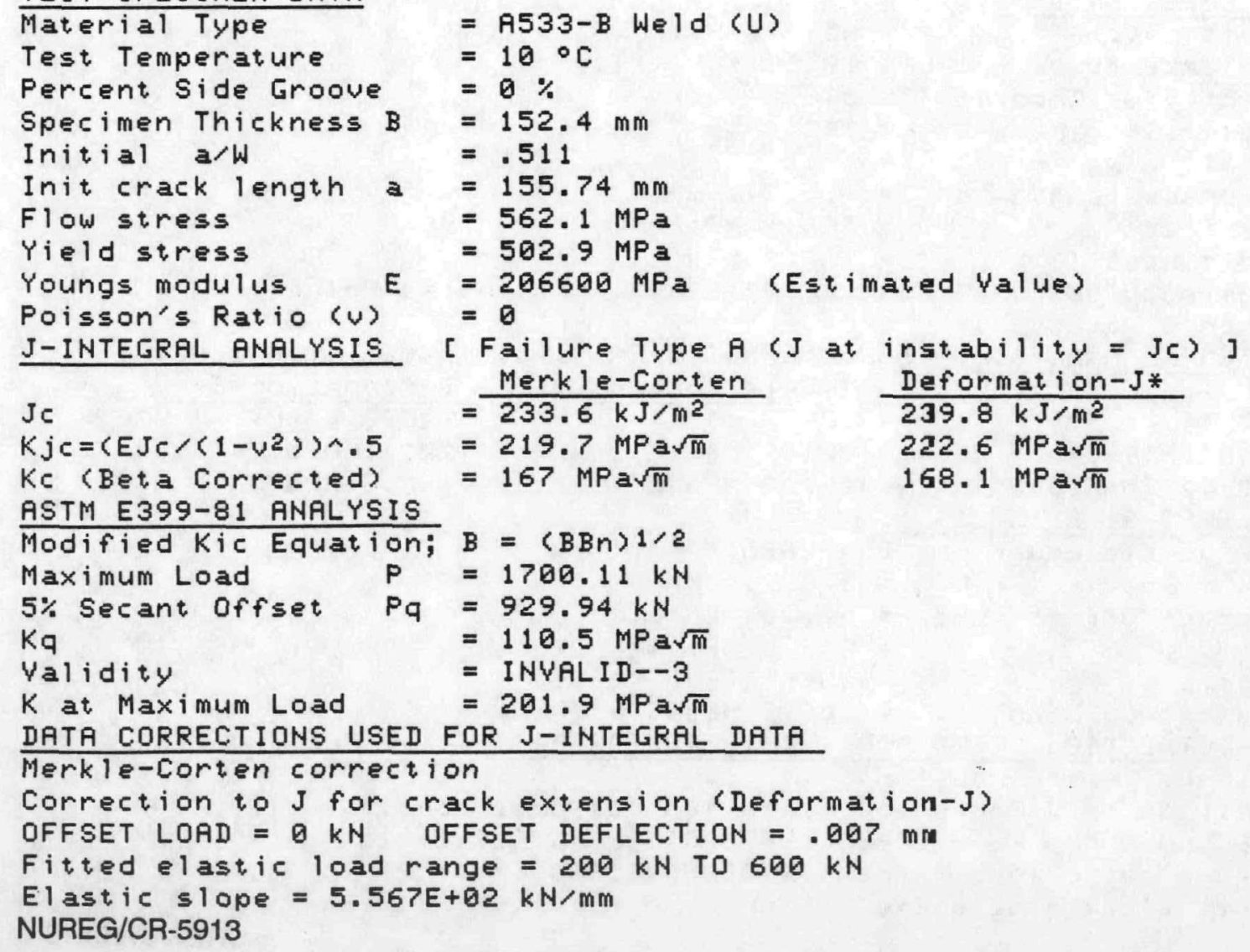




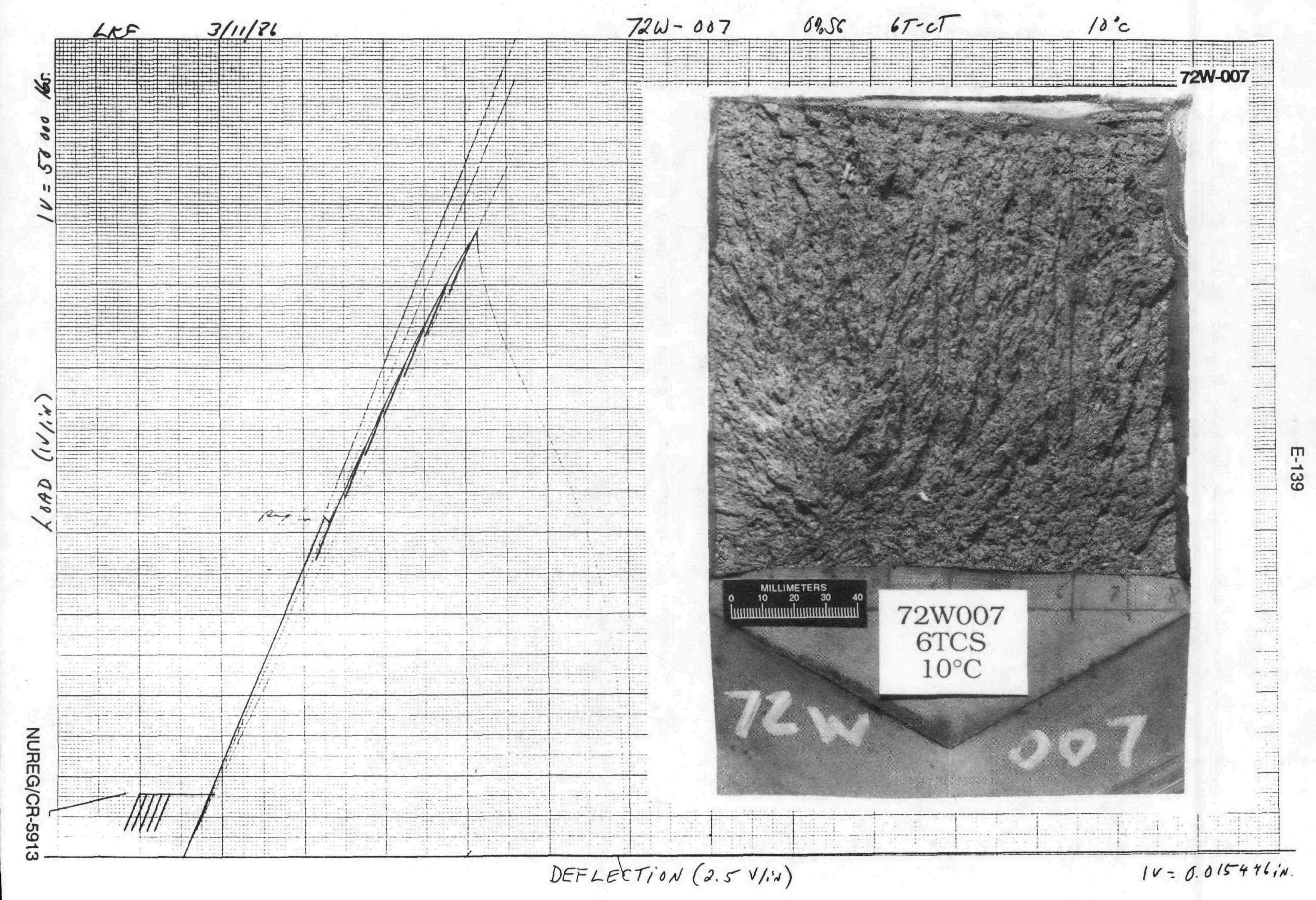


Specimen Crackmouth Deflection (in.) ORNL-DWG 92-11584

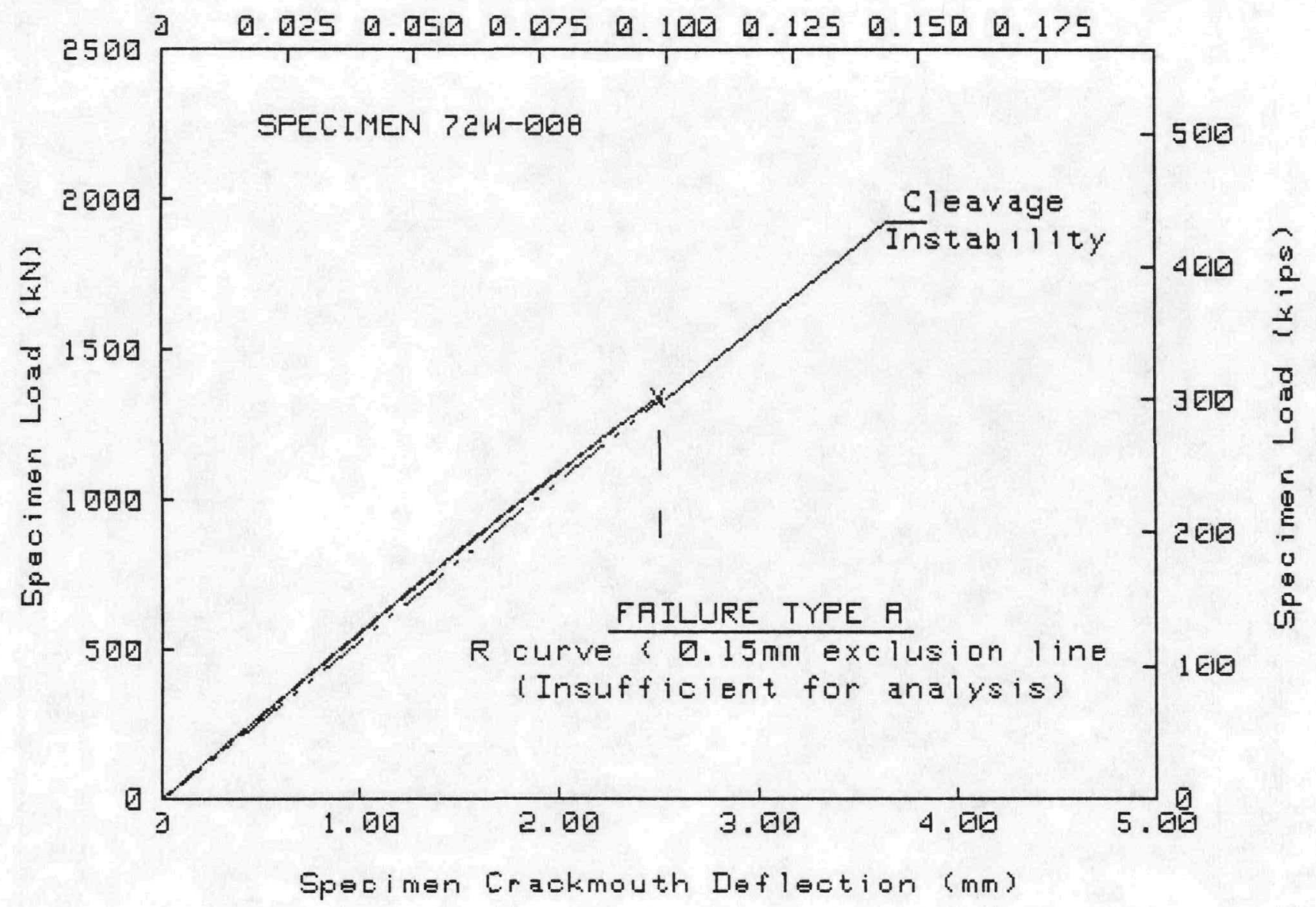

TEST SPECIMEN IATA

Material Type
Test Temperature
Percent Side Groove
Specimen Thiekness B
Initial a/W
Init erack length a
Flow stress
Yield stress
Youngs modulus E
Poisson's Ratio (U)
J-INTEGRAL ANALYSIS

= A533-B HSST 5th Irrad. Weld

$=10 \circ \mathrm{C}$

$=0 \%$

$=152.4 \mathrm{~mm}$

$=.512$

$=156.08 \mathrm{~mm}$

$=566.7 \mathrm{MPa}$

$=510.9 \mathrm{MPa}$

$=206600 \mathrm{MPa}$ (Estimated Value)

I-INTEGRAL ANALYSIS

$\mathrm{Jc}$

$=0$

$K j c=\left\langle E . J c /\left(1-w^{2}\right)\right\rangle \wedge .5$

[ Failure Type A ( $J$ at instatility = Je) ]

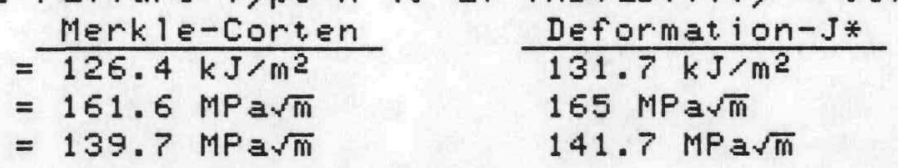

Ke (Bet.a Corrected)

ASTM E399-81 ANALYSIS

Modified Kic Equation; $B=\langle B B n\rangle 1 / 2$

Maximum Load $P=1339.68 \mathrm{kN}$

$5 \%$ Secant offset $\mathrm{Pq}=1339.68 \mathrm{kH}$

$\mathrm{Kq}$

$=159.7 \mathrm{MPa} \sqrt{\mathrm{m}}$

= INVALID- $-1,2$

Validity

$=159.7 \mathrm{MPa} \sqrt{\mathrm{m}}$

DATA CORRECTIONS USED FOR J-INTEGRAL DATA

Merkle-Carten correction

Correction to J for crack extension (Deformation-J)

OFFSET LOAD $=0 \mathrm{kN}$ DFFSET DEFLECTION $=.004 \mathrm{~mm}$

Fitted elastic load range $=222 \mathrm{kN}$ To $444 \mathrm{kN}$

Elastic slope $=5.606 E+02 \mathrm{kN} / \mathrm{mm}$

NUREG/CR-5913 


\section{LNA $3 / 13 / 86$}

$72 w-008$

$0 \% 56$

$6 T-C$

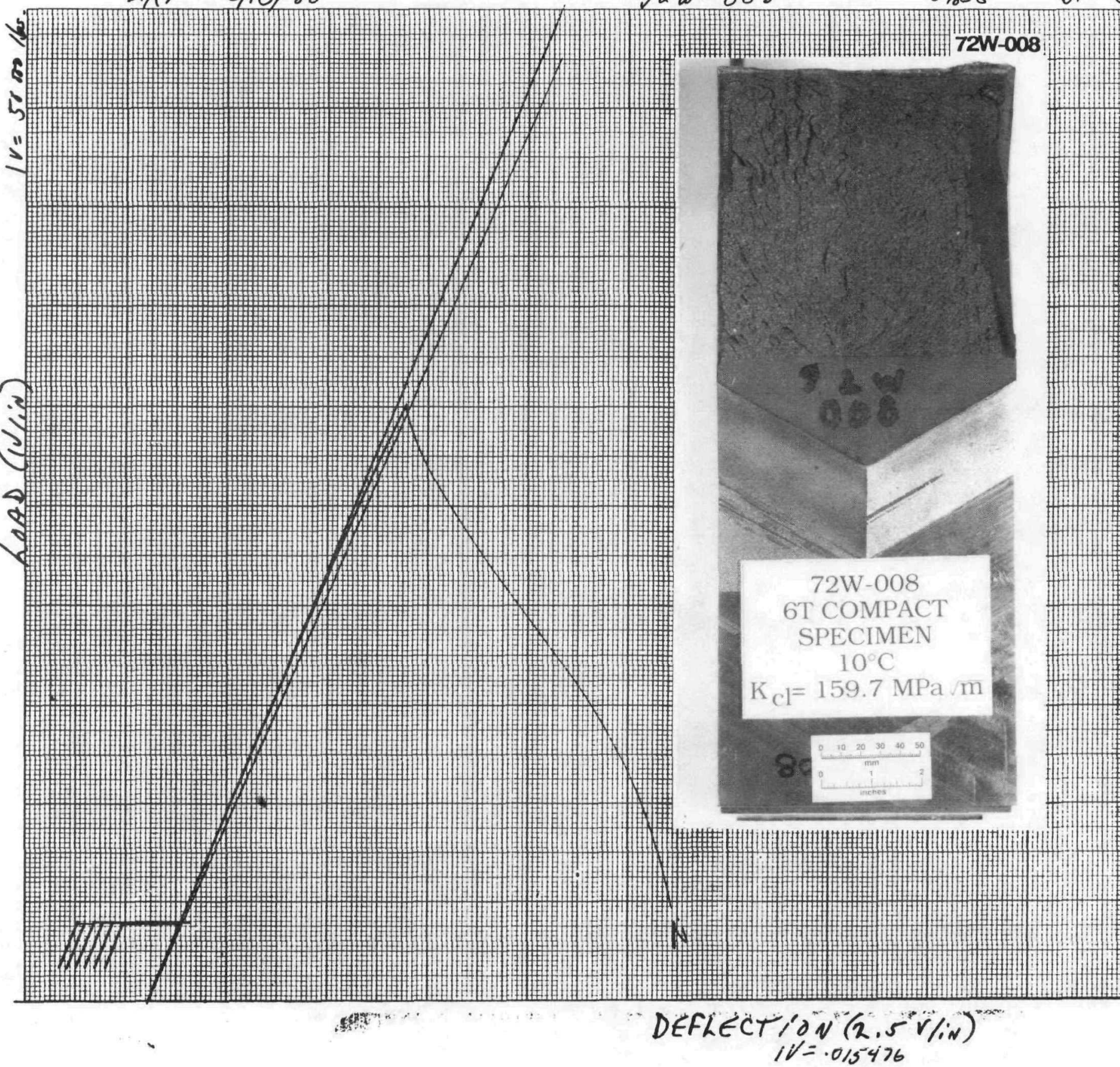


Specimen Crackmouth Deflection (in.) ORNL-DWG 92-11585
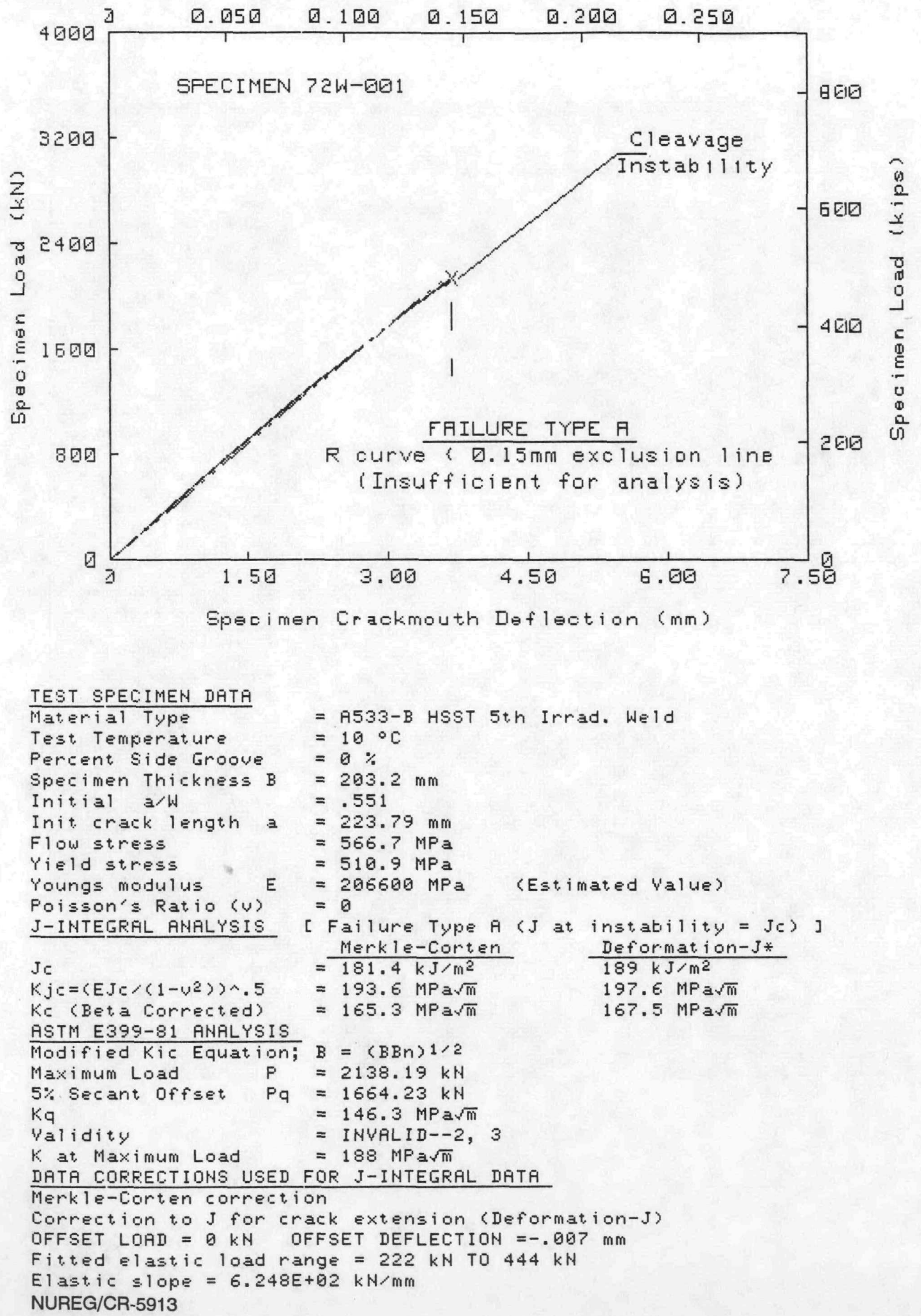
LES

$4 / 2 / 86$

$72 \omega-001$

$8 T-C 7$

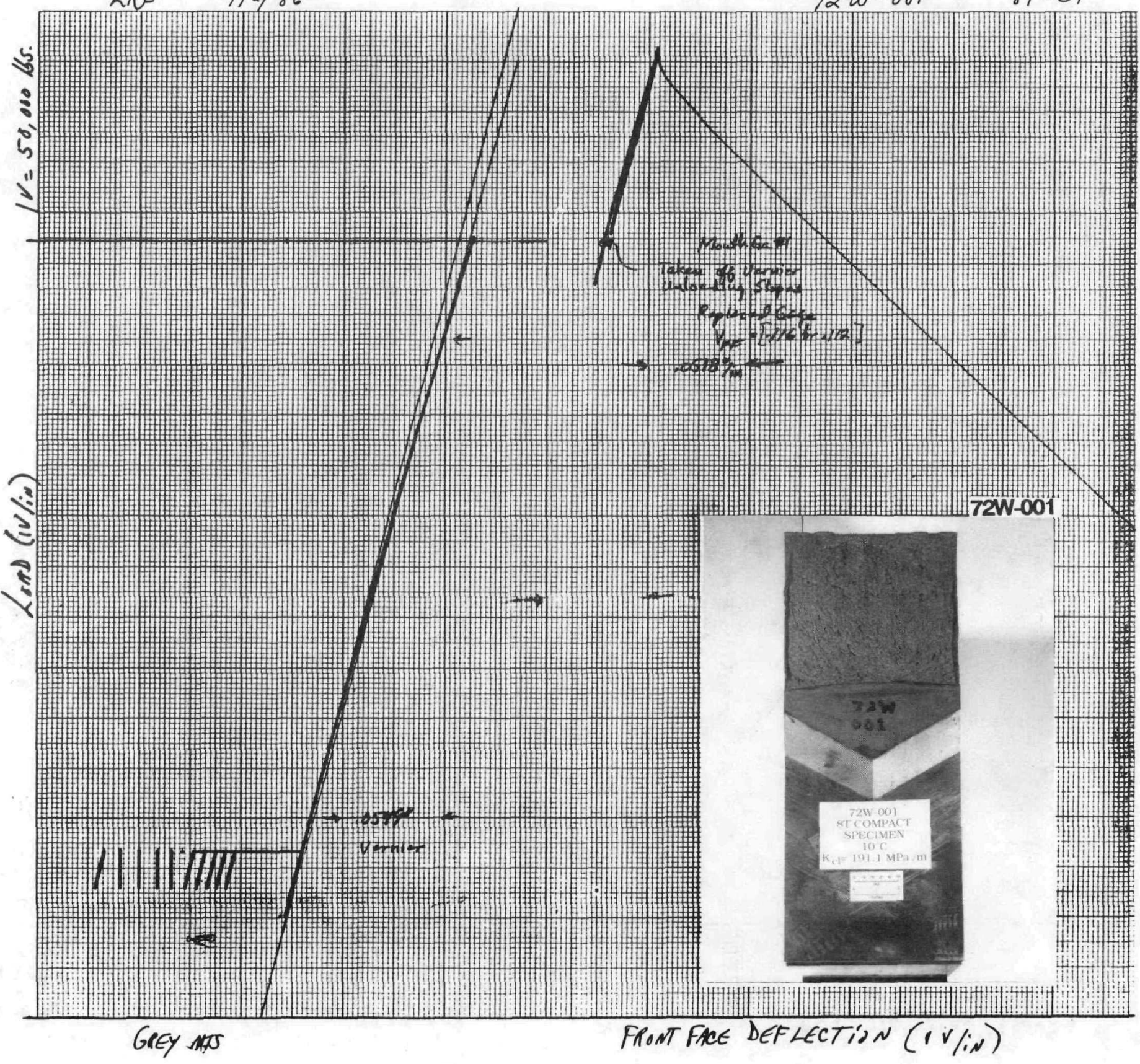

$\frac{\prod_{i}^{+}}{\vec{\omega}}$

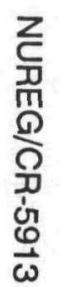

FRONT FACE DEFLECTION (I V/iN) 
Specimen Crackmouth Def lection (in.)

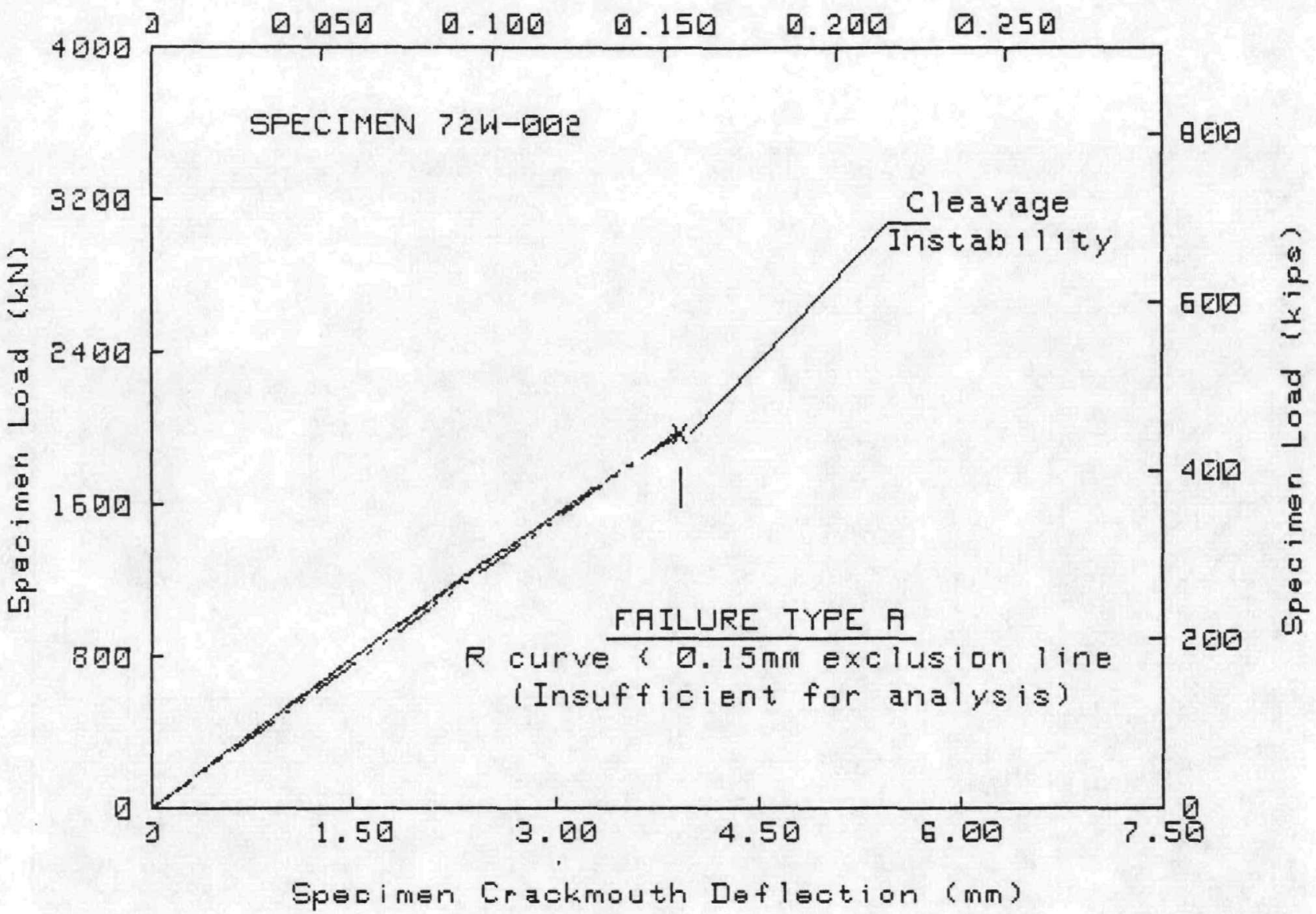

TEST SPECIMEN DATA

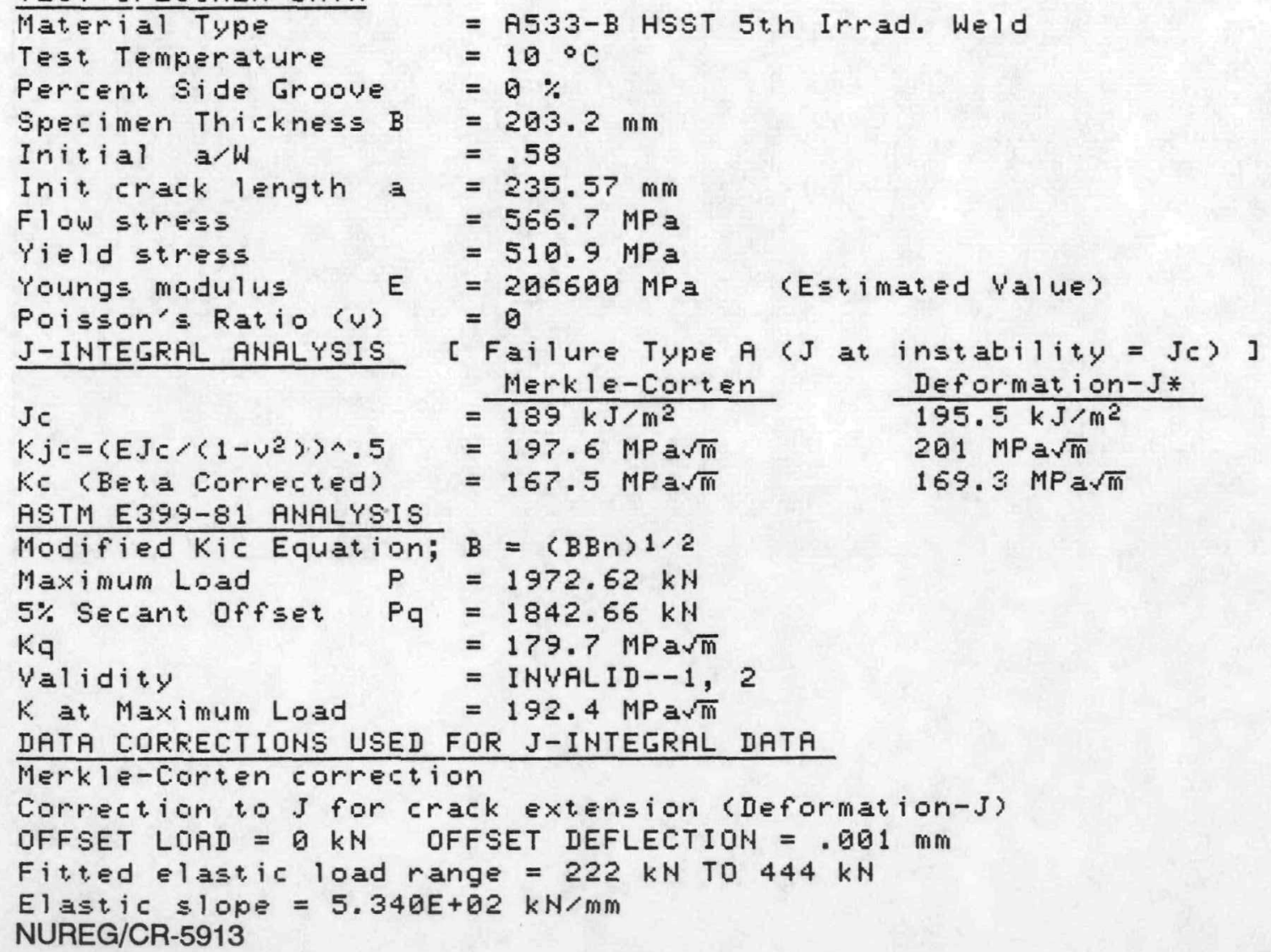


$\angle N F$

$4 / 25786$

$1 \alpha w-002$

$\gamma 7-c /$

$0 \% 56$

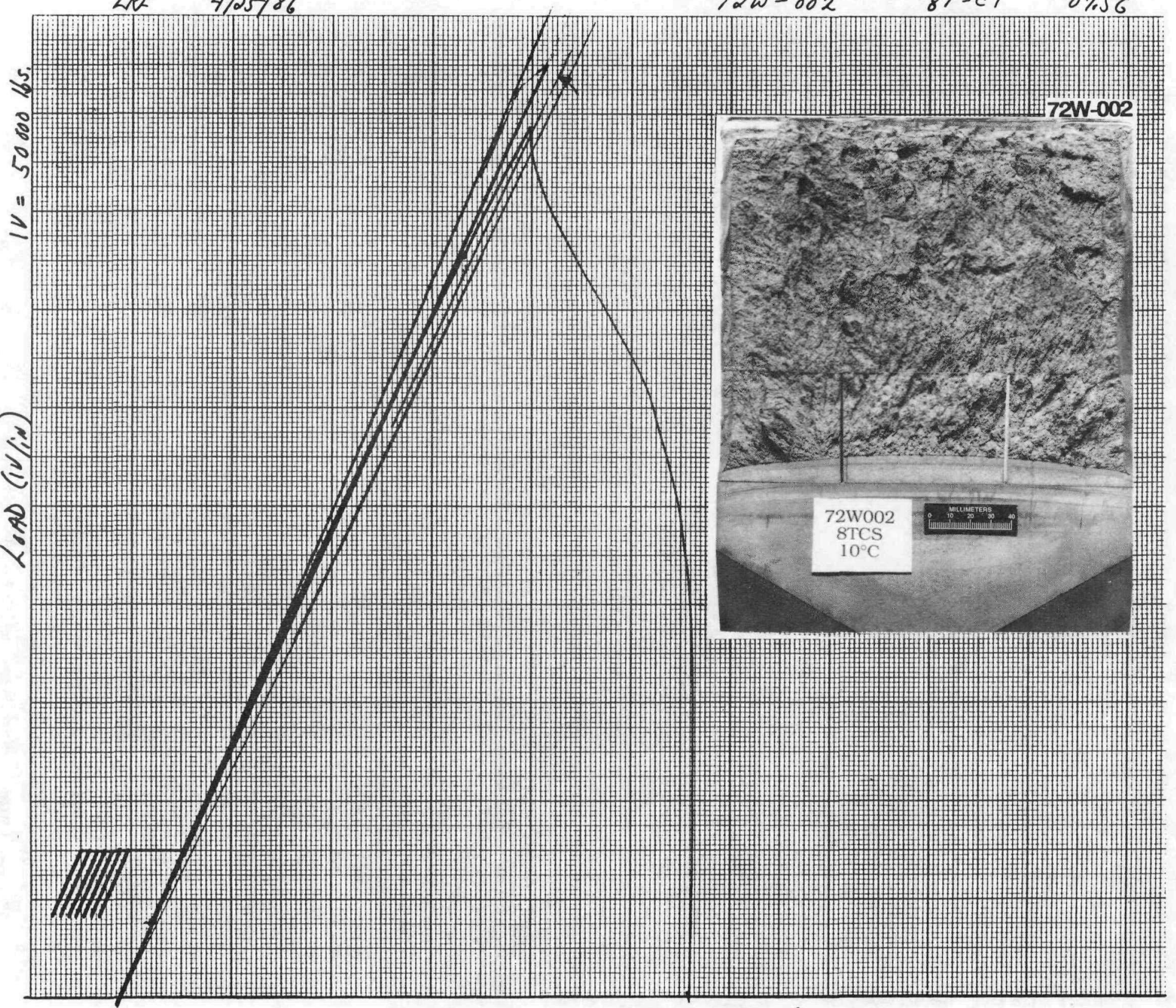

GREY $\$ / 01$

DEFLECTION (2.5V/iv) 
ORNL-DWG 92-11587

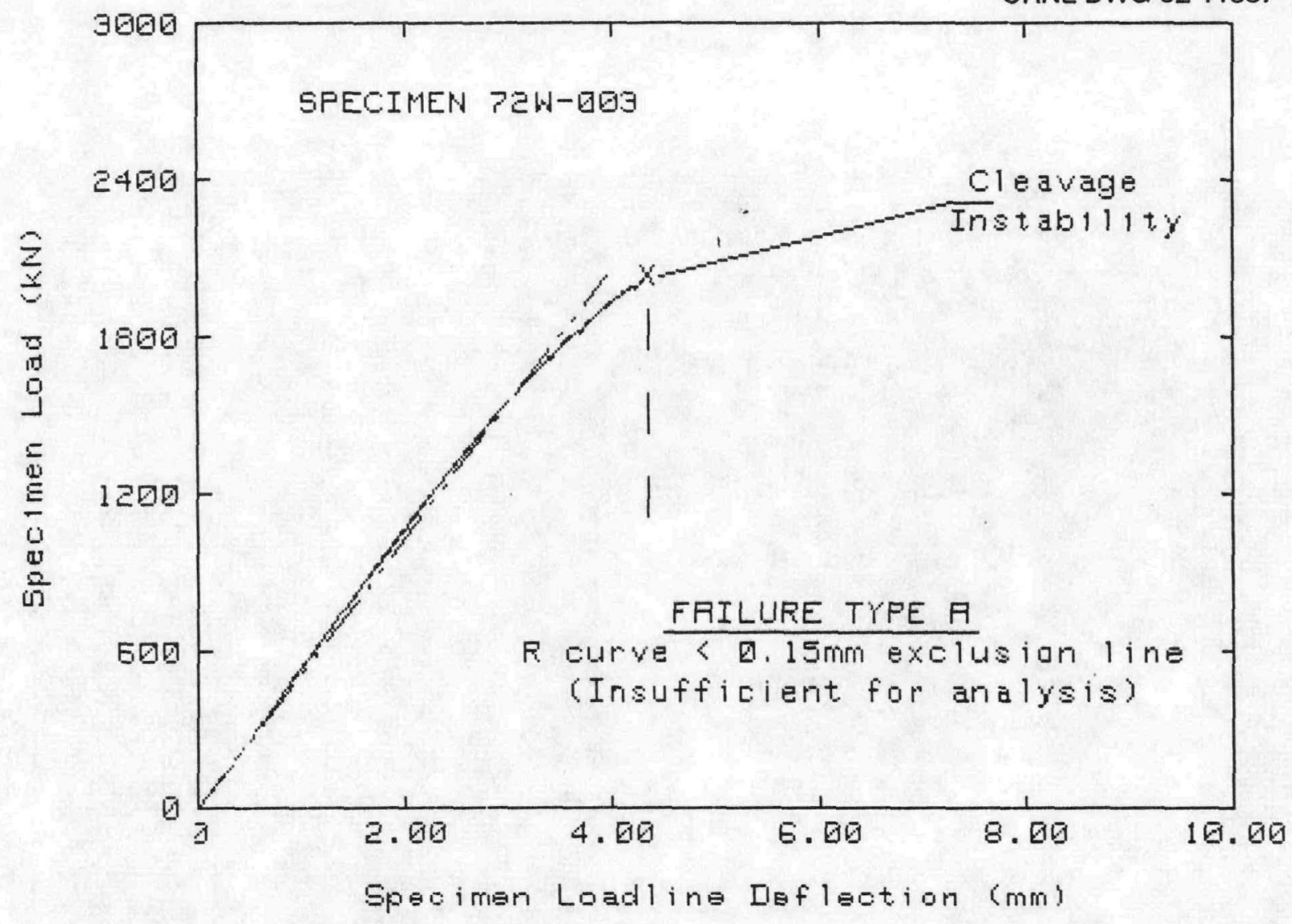

TEST SFECIMEN IATA

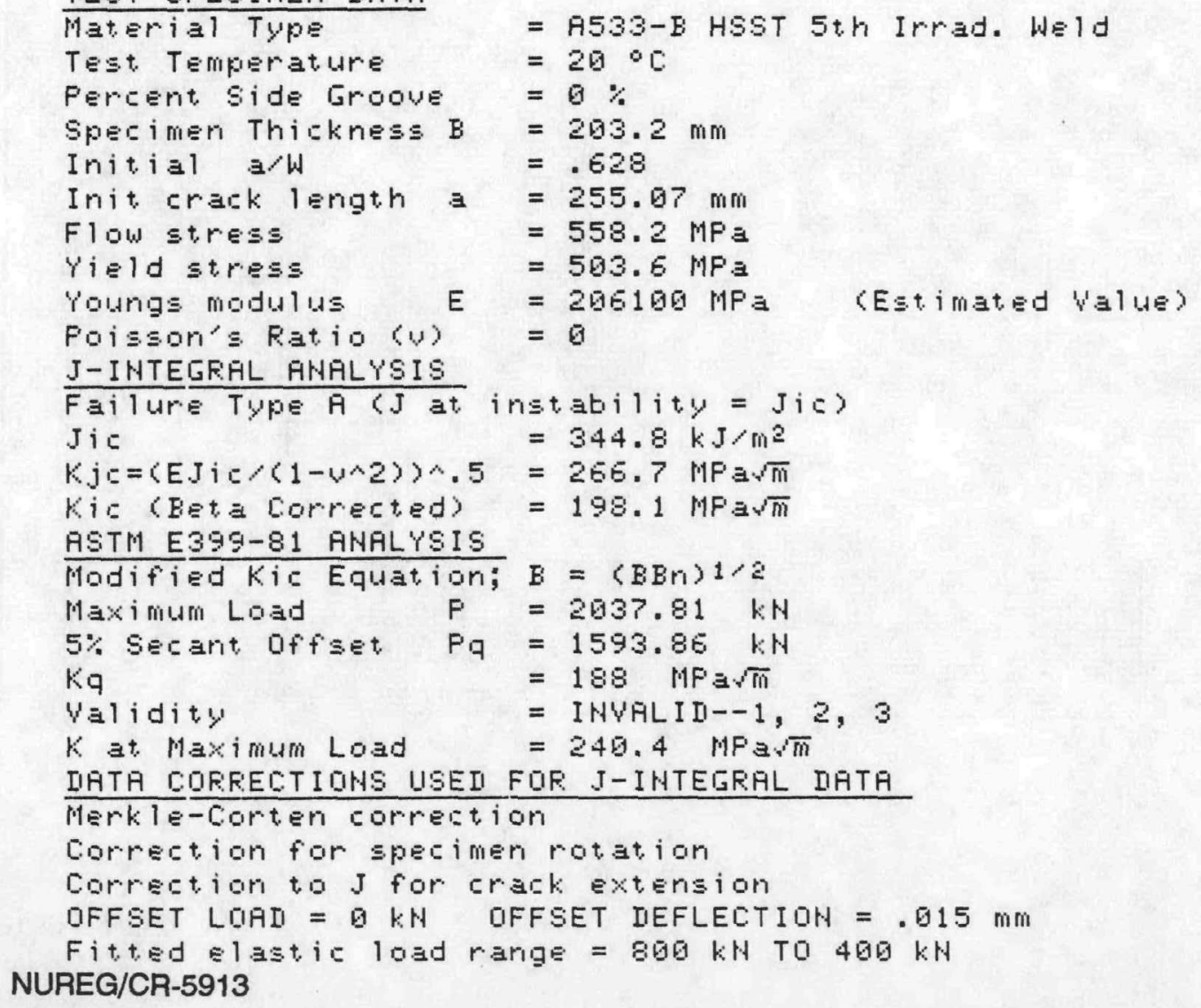




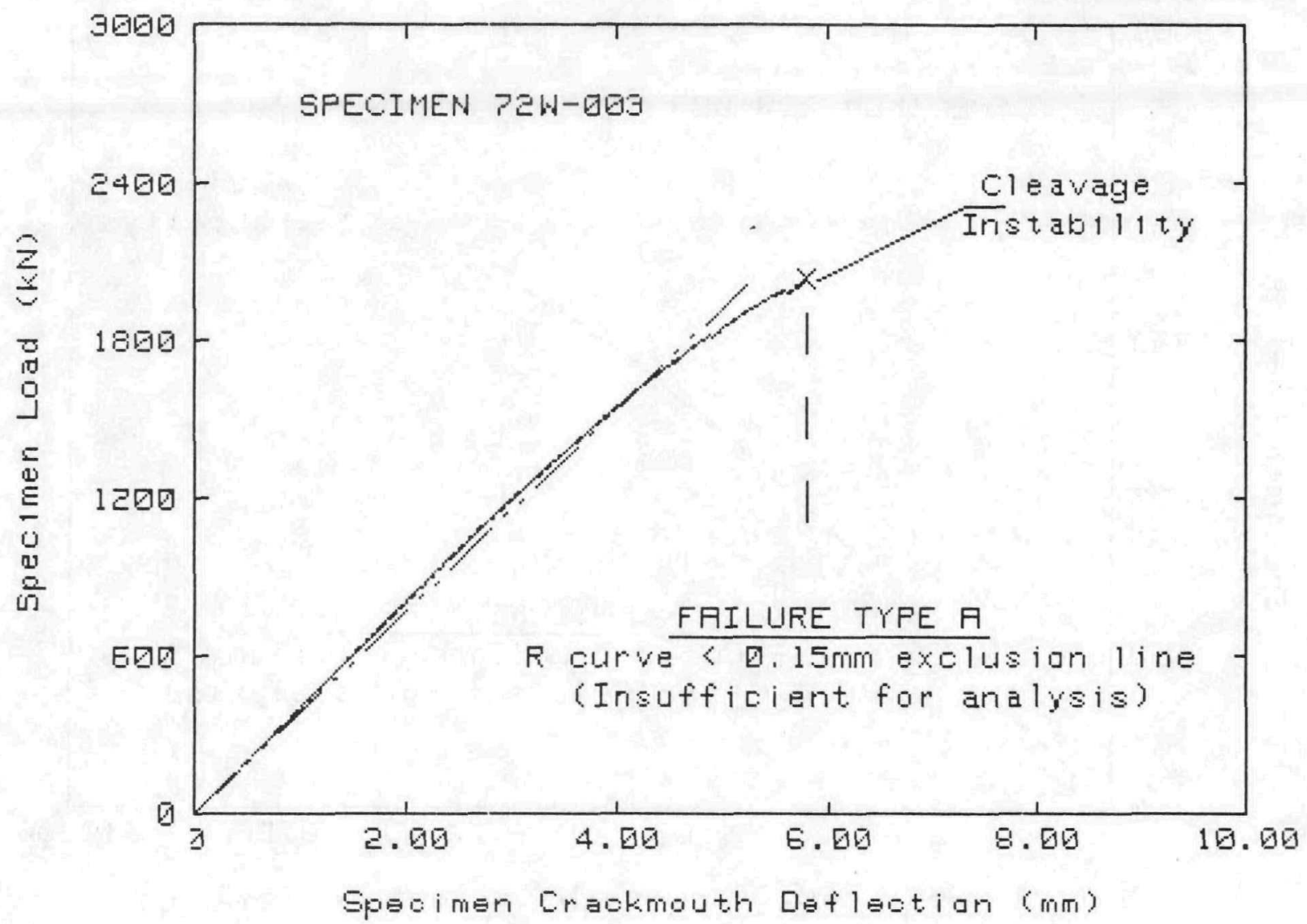

TEST SFELIMEN IATH

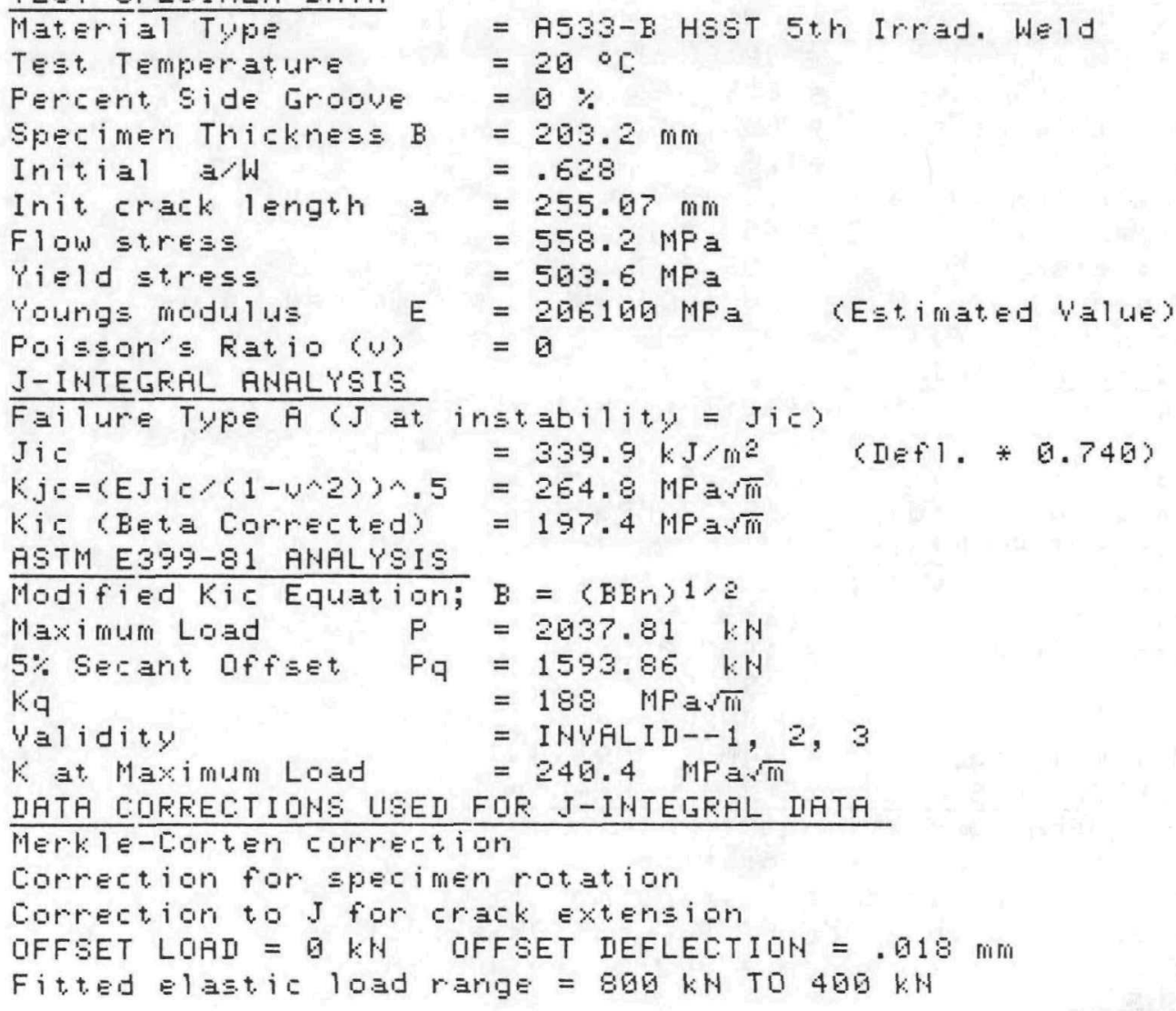




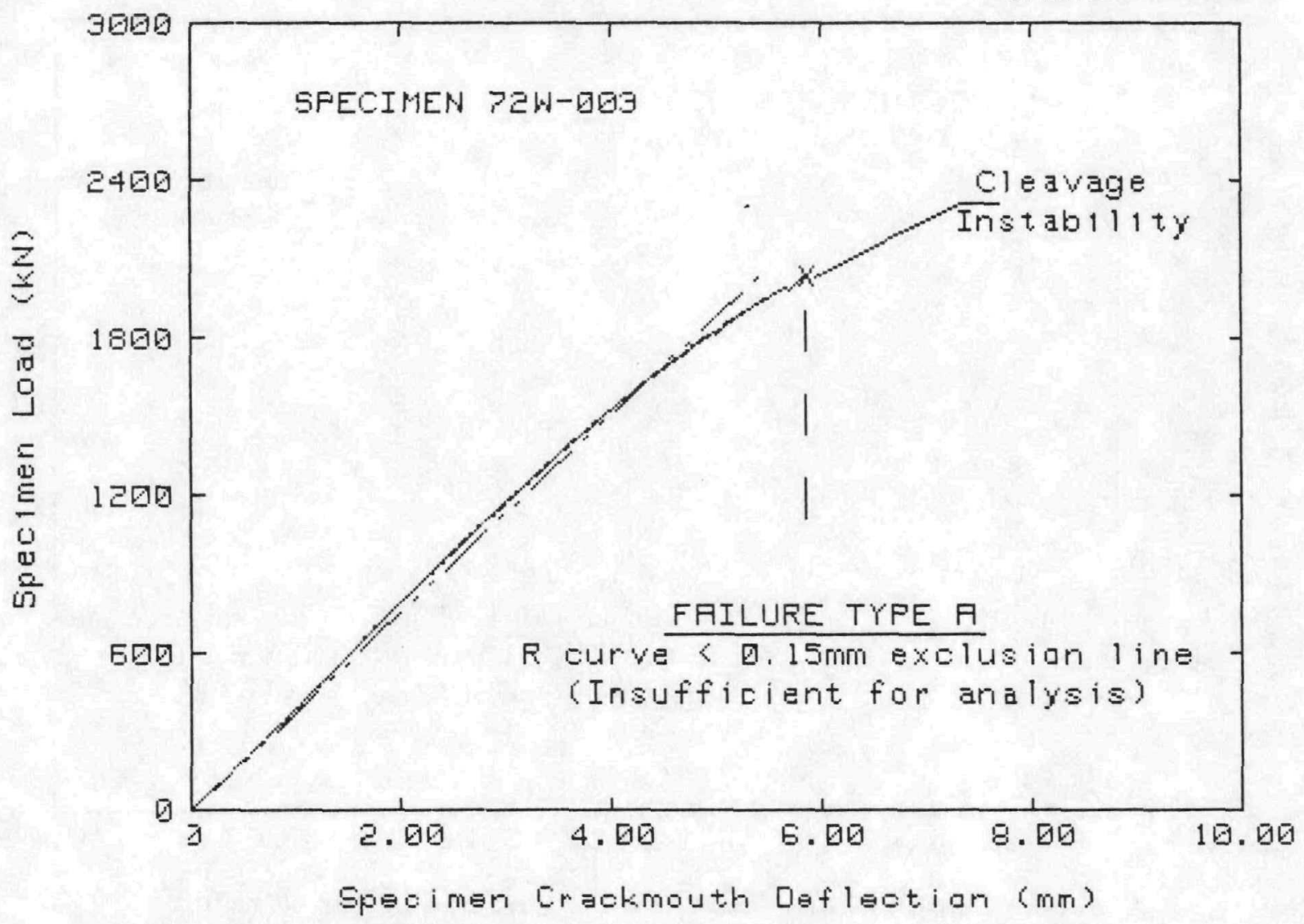

TEST SFECIMEN DATA

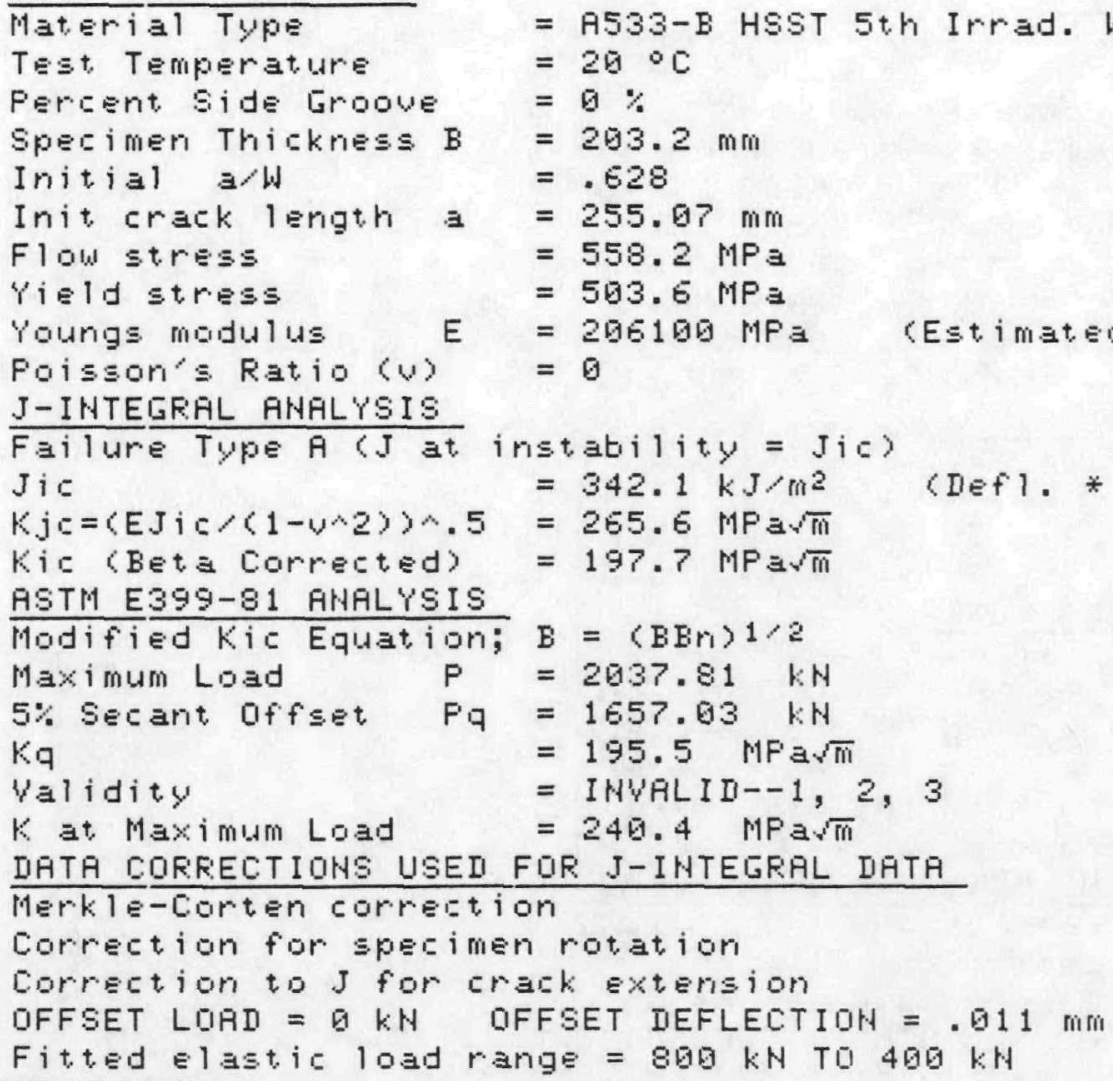




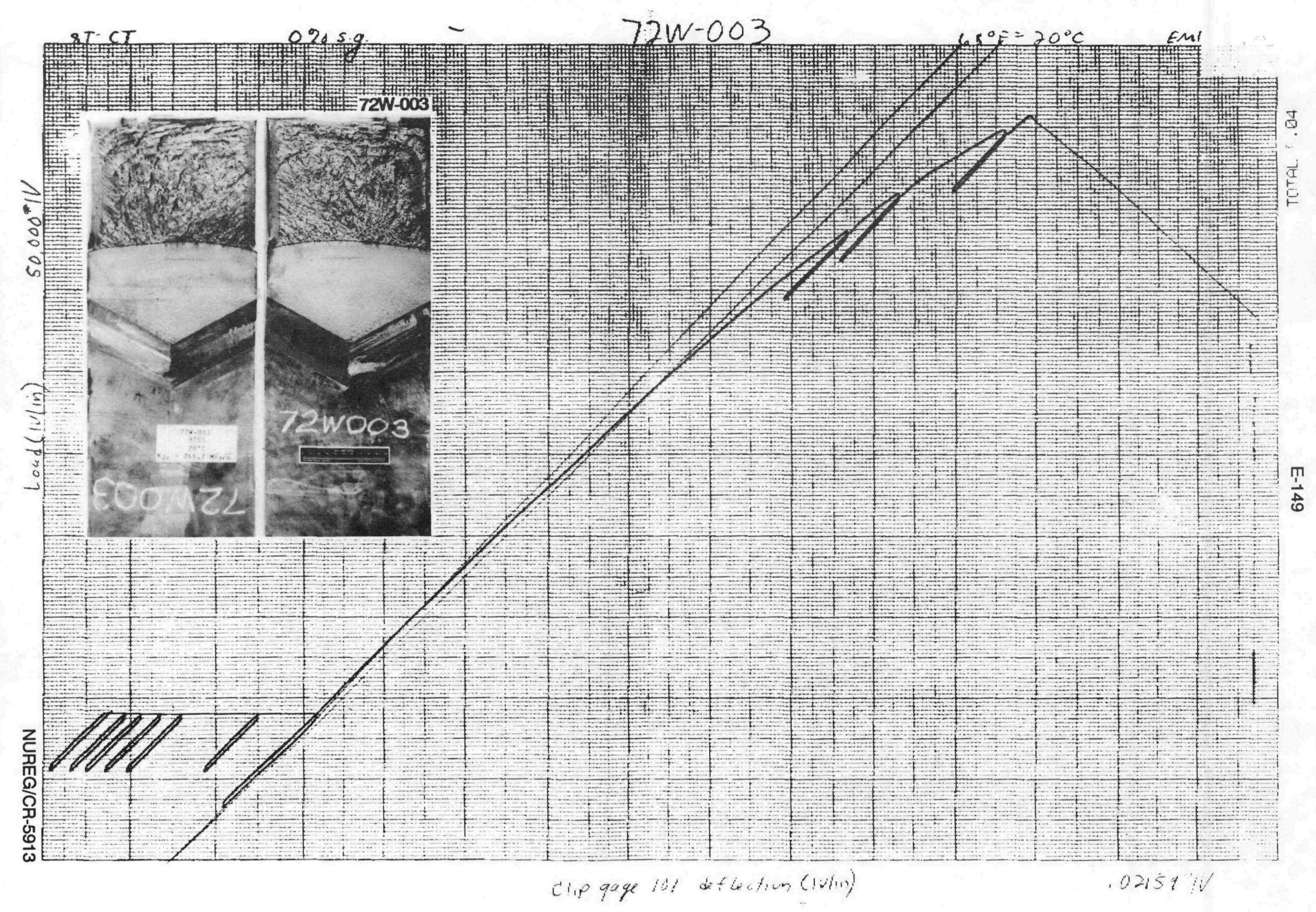




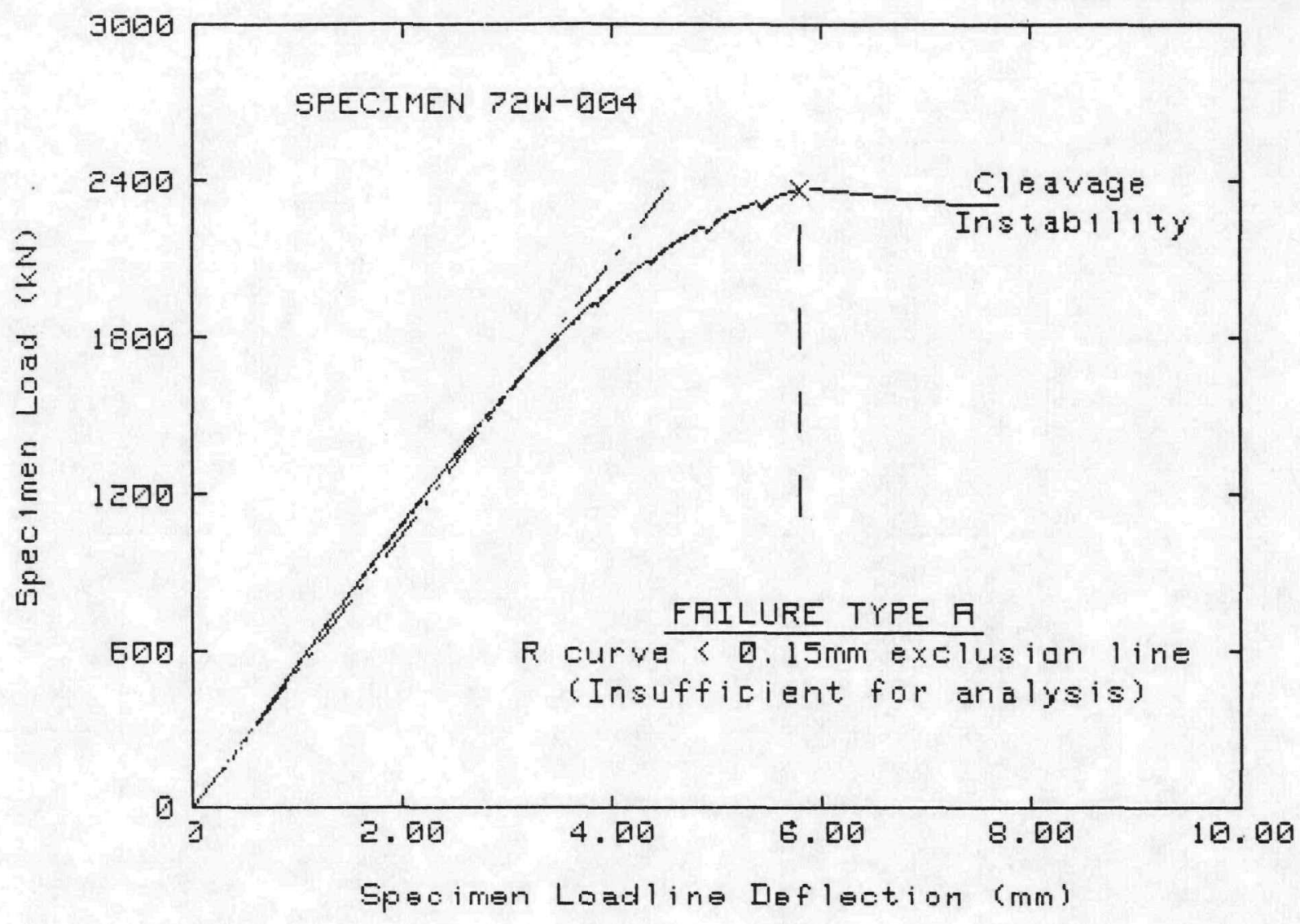

TEST SFELIMEN IIATA

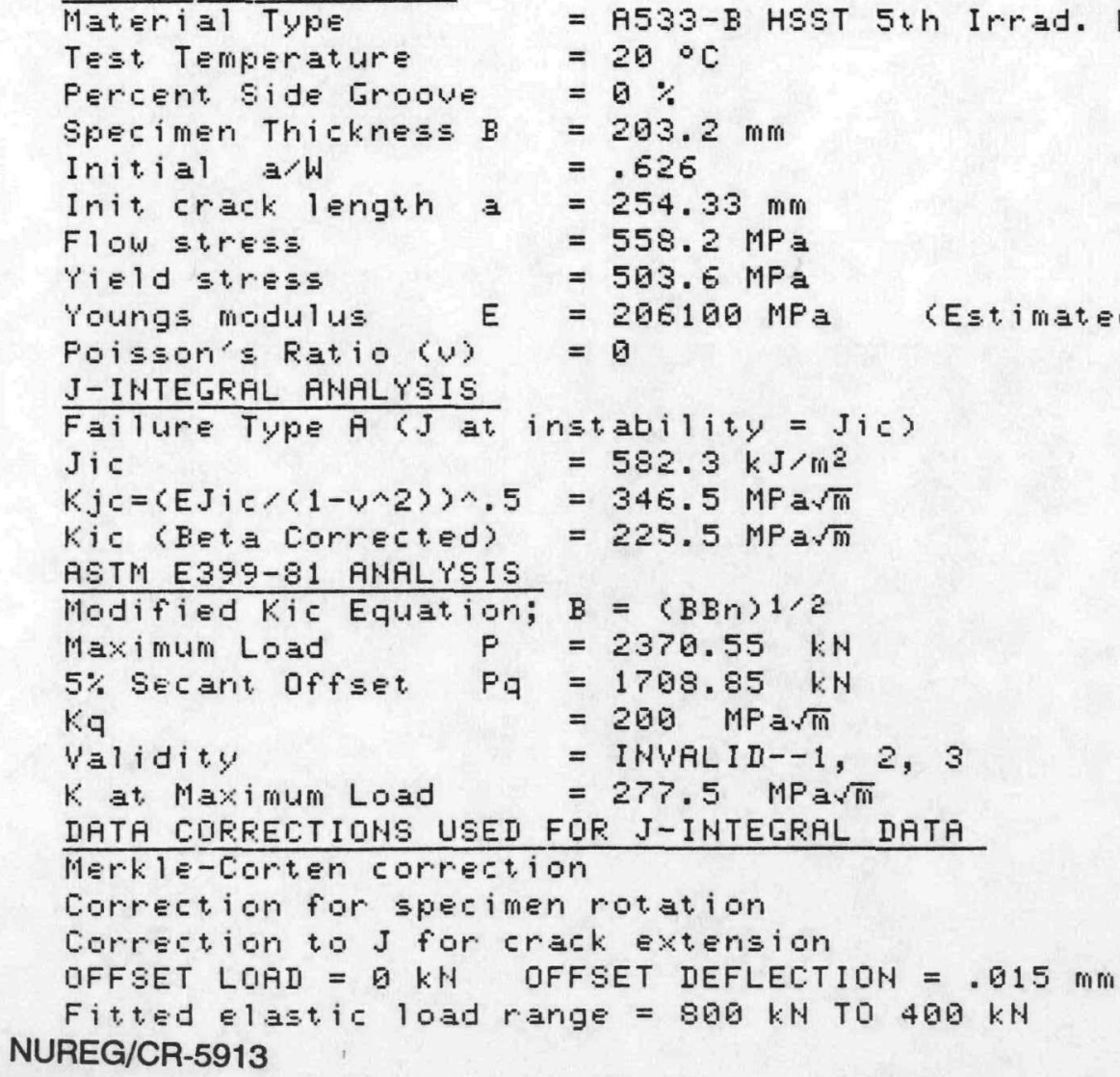




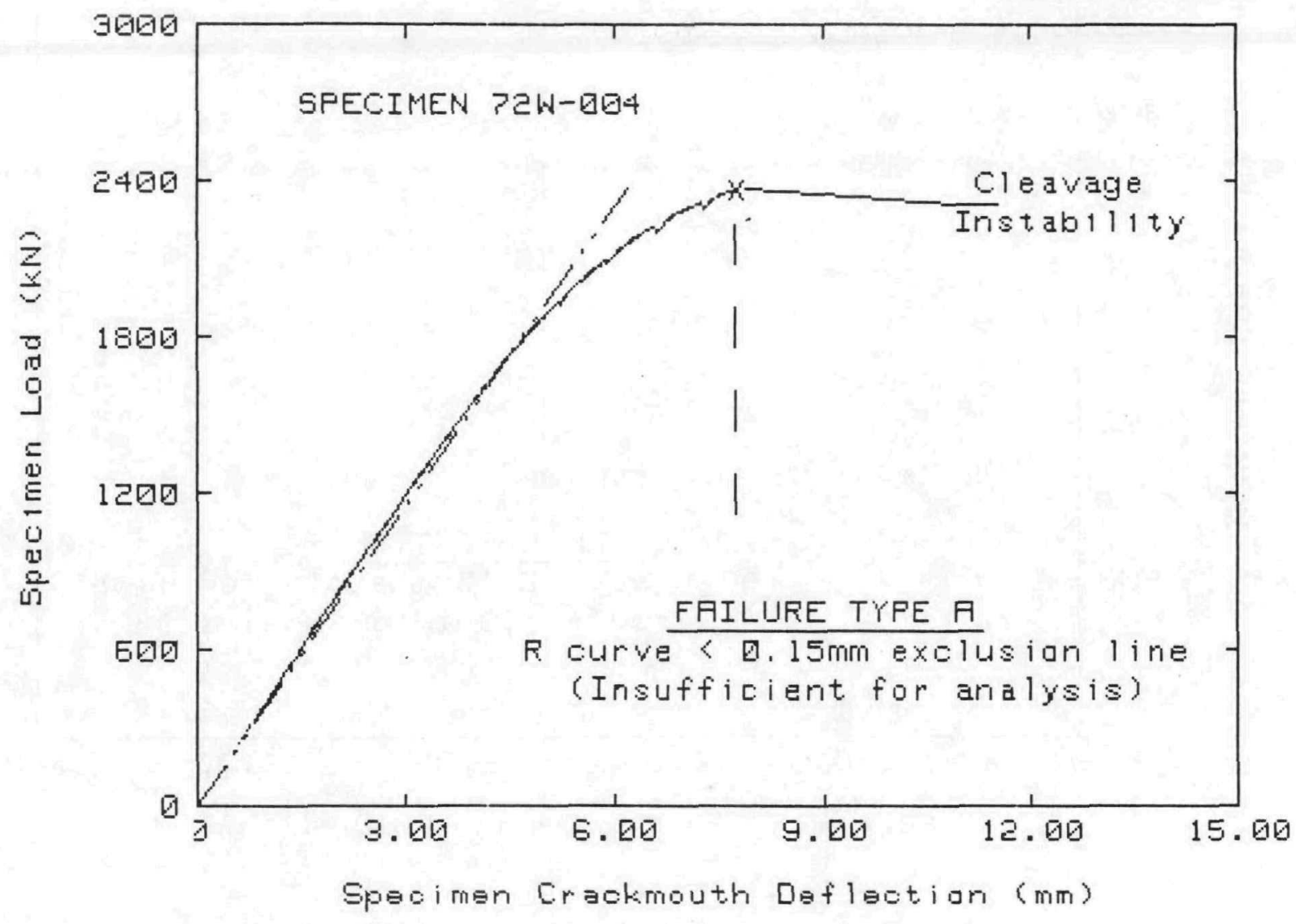

TEST SFEL: IMEN DATH

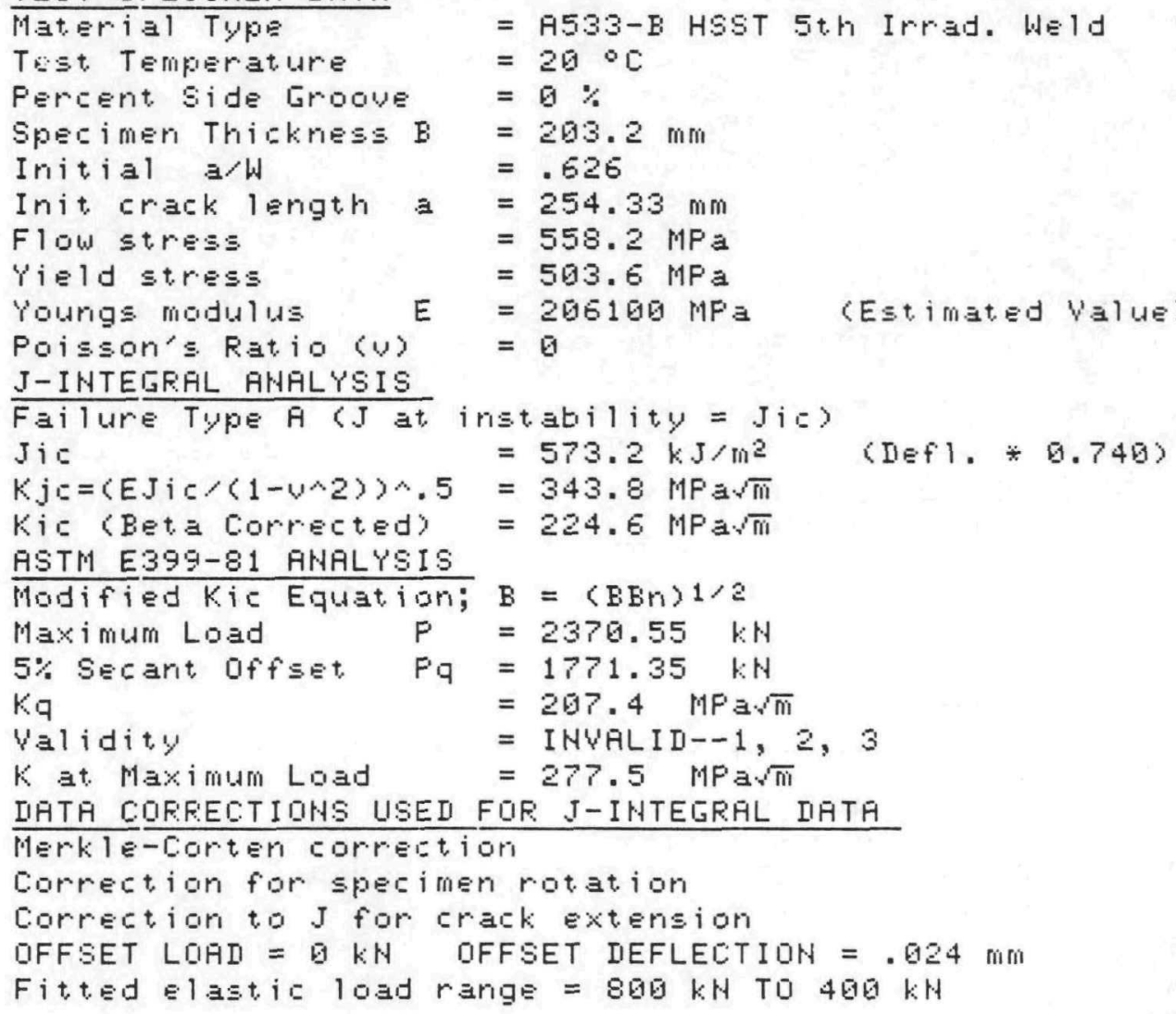




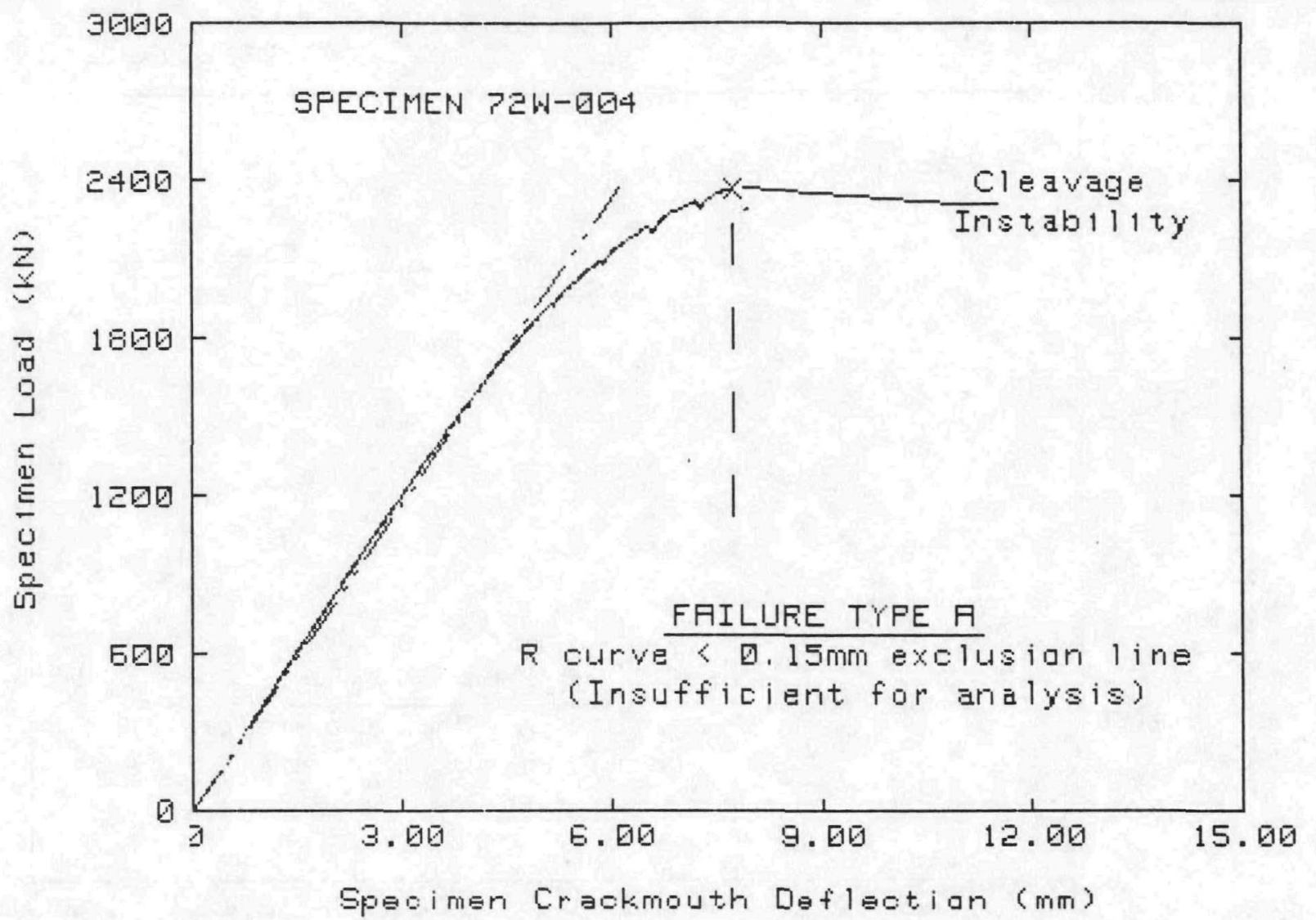

TEST SFELIMEN IATA

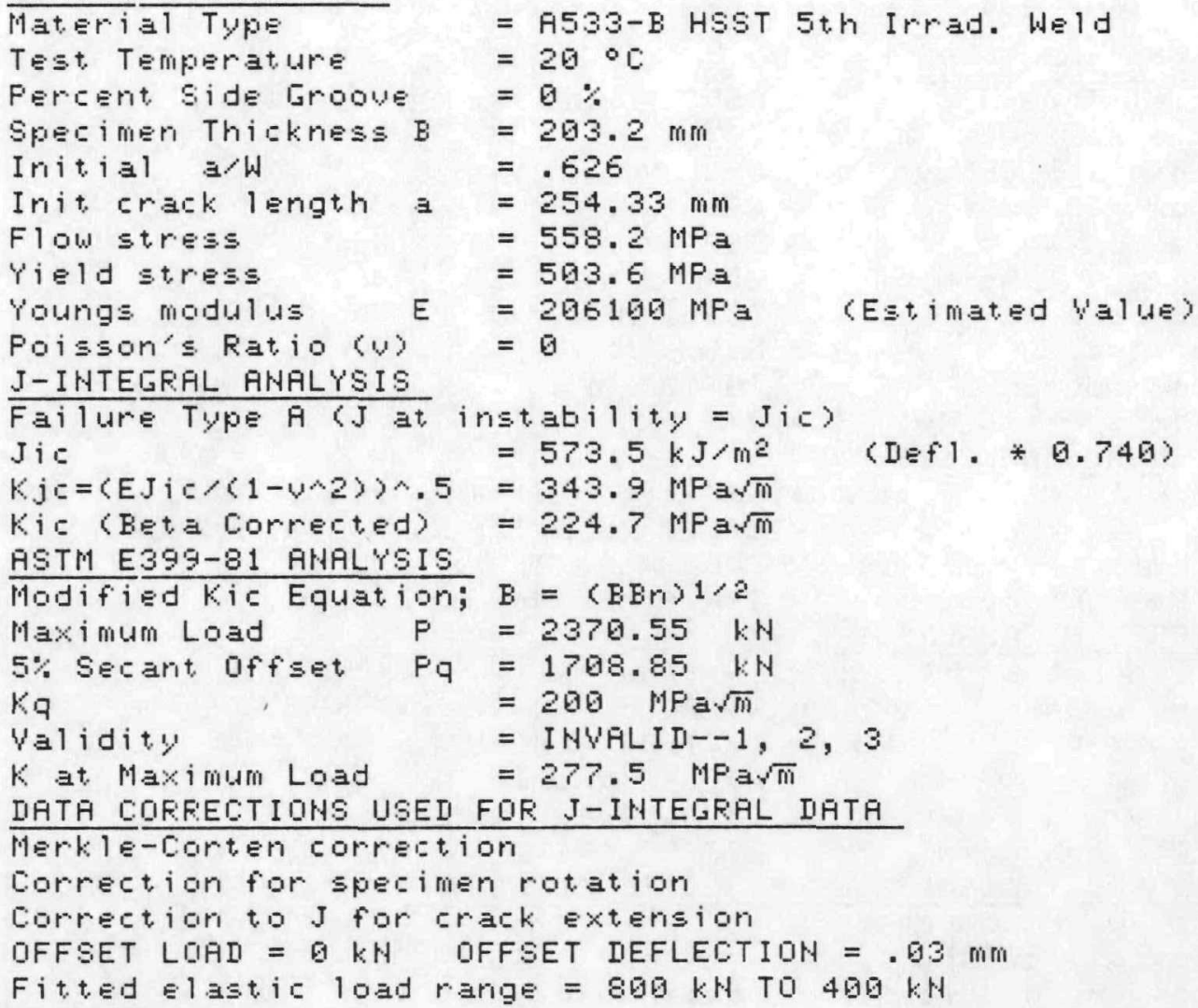




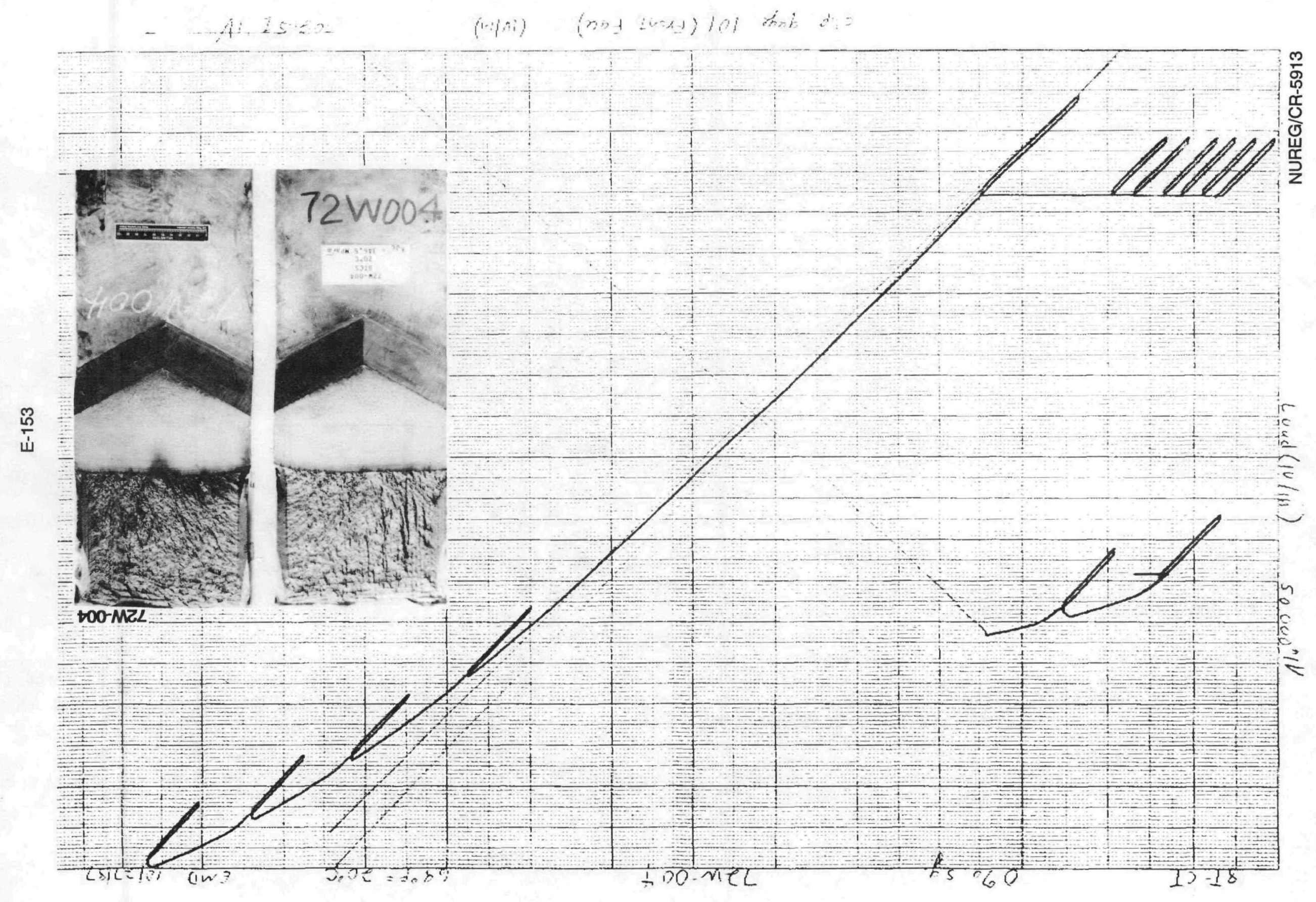


Load-Displacement Curves and Photographs of the Fracture Toughness Specimens from the Irradiated $72 \mathrm{~W}$ Weld (0.23 wt\% Cu) 
SPECIMEN ID $=72 W 137$

MATERIAL TYPE $=$ MCUWELD

TEST TEMPERATURE $=-75$ DEG $C$

THICKNESS $=25.4 \mathrm{~mm}$

SIDE GROOUES $=0 \%$

INITIAL CRACK LENGTH $=26.5 \mathrm{~mm}$

$a / W) i=.522$

MEASURED DUCTILE DELTA-A $=.05 \mathrm{~mm}$

$Y$ IELD STRENGTH $=621 \mathrm{MPa}$

FLOW STRENGTH $=672 \mathrm{MPa}$

ESTIMATED YOUNG'S MODULUS $=211 \mathrm{GPa}$

SPECIMEN CLEAUED AT $K I c=40.8 \mathrm{MPa}$ SQR[m] (UALID ASTM E399)

UALUES AT MAXIMUM LOAD

J-INTEGRAL $=9 \mathrm{~kJ} / \mathrm{m}^{\wedge} 2$

$\mathrm{KJ} c=43.6 \mathrm{MPa} \operatorname{SQR}[\mathrm{m}]$

$\mathrm{K}$-bet $\mathrm{a}-\mathrm{c}=42.6 \mathrm{MPa} \operatorname{SQR}[\mathrm{m}]$

\author{
Walues at cleauage load \\ $J-I N T E G R A L=9 \mathrm{~kJ} / \mathrm{m}^{\wedge} 2$ \\ $\mathrm{KJc}=43.6 \mathrm{MPa} \operatorname{SQR}[\mathrm{m}]$ \\ K-beta-c $=42.6 \mathrm{MPa} \operatorname{SQR}[\mathrm{m}]$
}


E-156

ORNL-DWG 92-11593

72W137 -75 DEGREES C

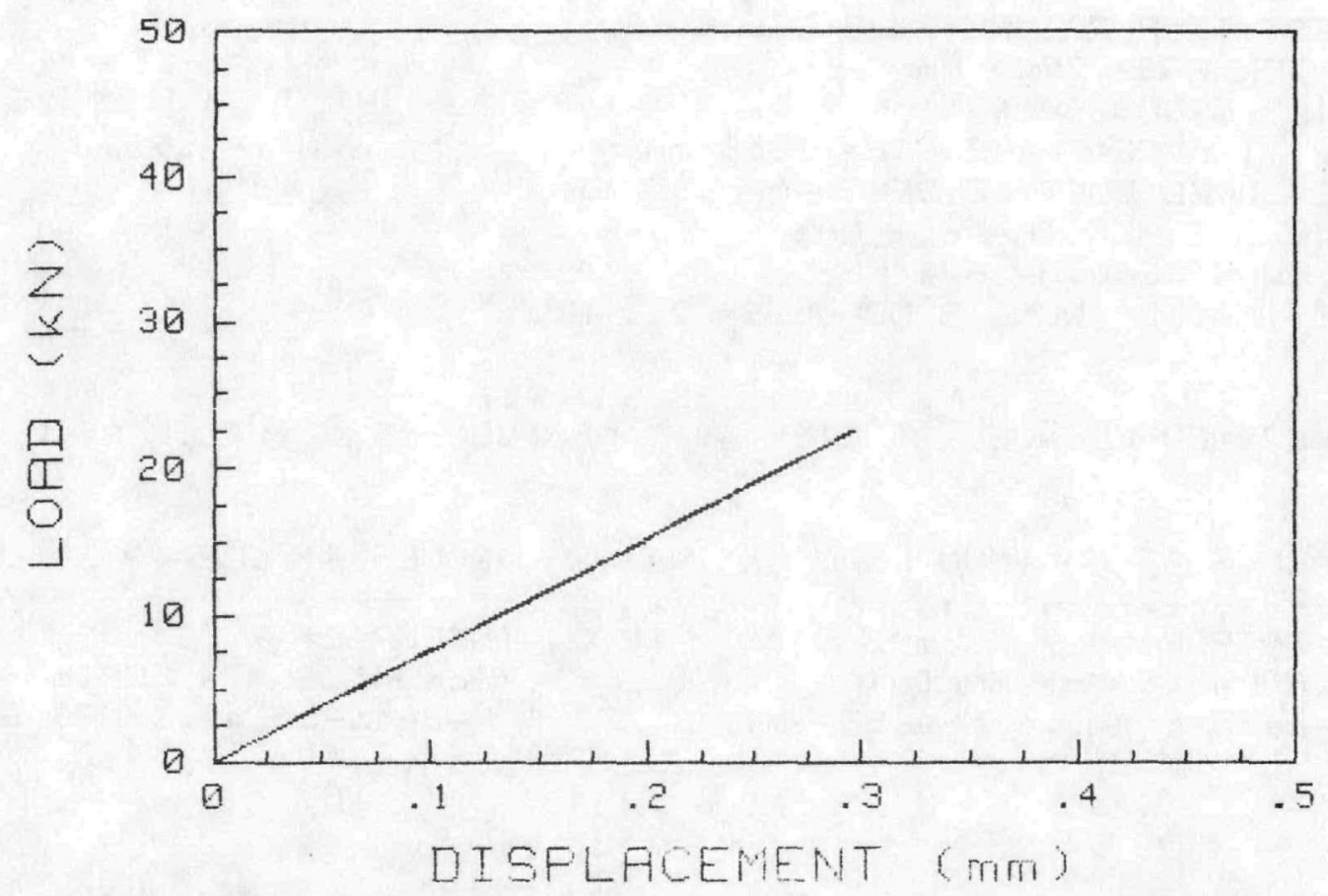

$72 W 137$

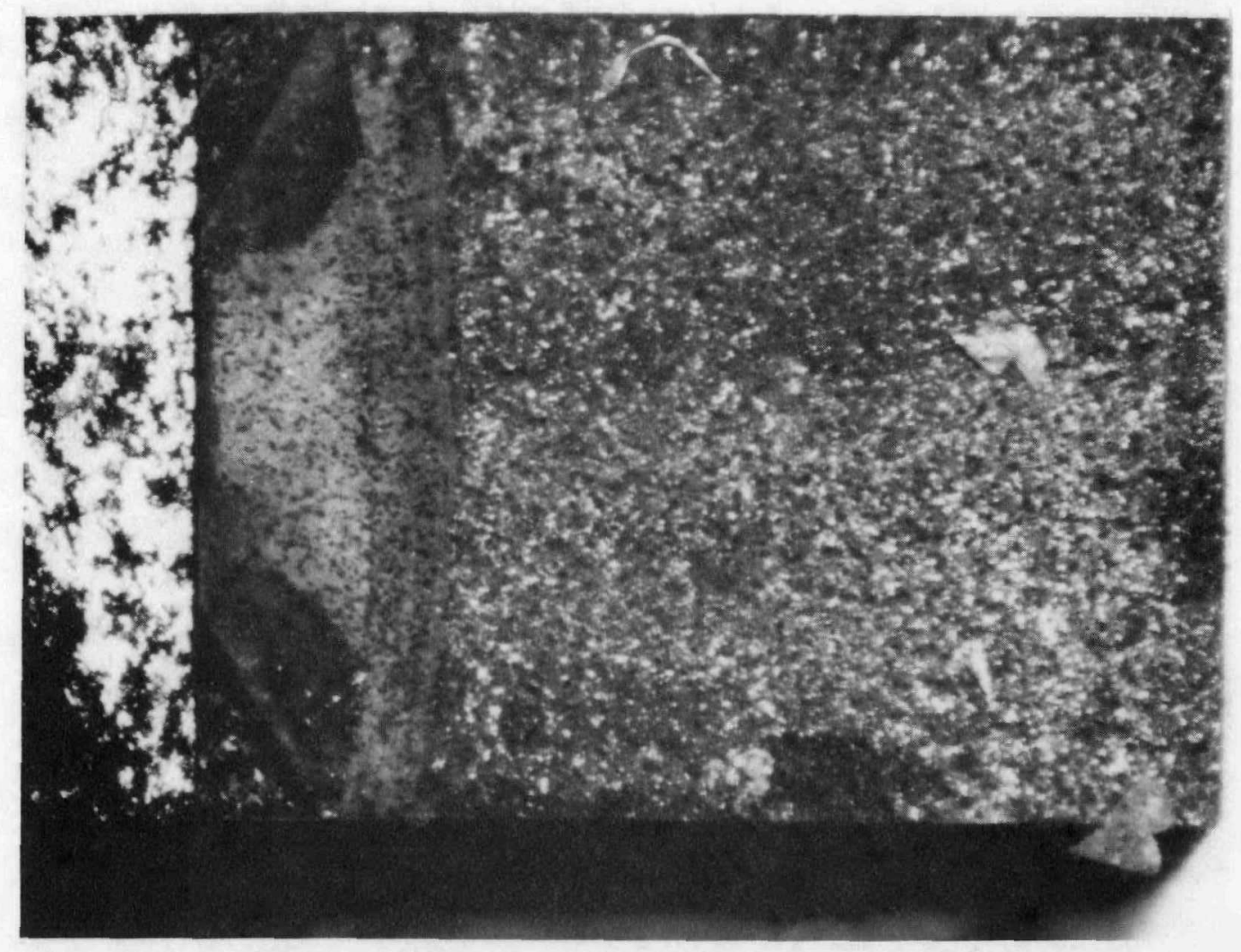

NUREG/CR-5913 
SPECIMEN ID $=72 W 141$

MATERIAL TYPE = MCUWELD

TEST TEMPERATURE $=-25$ DEG C

THICKNESS $=25.4 \mathrm{~mm}$

SIDE GRDDUES $=0 \%$

INITIAL CRACK LENGTH $=27.1 \mathrm{~mm}$

$a / W) i=.534$

MEASURED DUCTILE DELTA-A $=.05 \mathrm{~mm}$

YIELD STRENGTH $=621 \mathrm{MPa}$

FLOW STRENGTH $=672 \mathrm{MPa}$

ESTIMATED YOUNG'S MODULUS $=209 \mathrm{GPa}$

SPECIMEN CLEAUED AT KQ= $68.9 \mathrm{MPa}$ SQR $[\mathrm{m}]$ (NDT VALID KIC)

UALUES AT MAXIMUM LOAD

J-INTEGRAL $=26.9 \mathrm{~kJ} / \mathrm{m}^{\wedge} 2$

$\mathrm{KJc}=74.9 \mathrm{MPa} \operatorname{SQR}[\mathrm{m}]$

K-beta-c $=66.2 \mathrm{MPa} \operatorname{SQR}[\mathrm{m}]$
UALUES at CLEavage LDAD

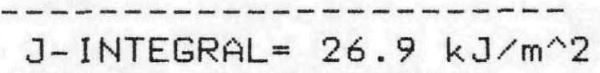

$\mathrm{KJc}=74.9 \mathrm{MPa} \operatorname{SQR}[\mathrm{m}]$

$\mathrm{K}$-beta-c $=66.2 \mathrm{MPa} \operatorname{SQR}[\mathrm{m}]$ 
ORNL-DWG 92-11594
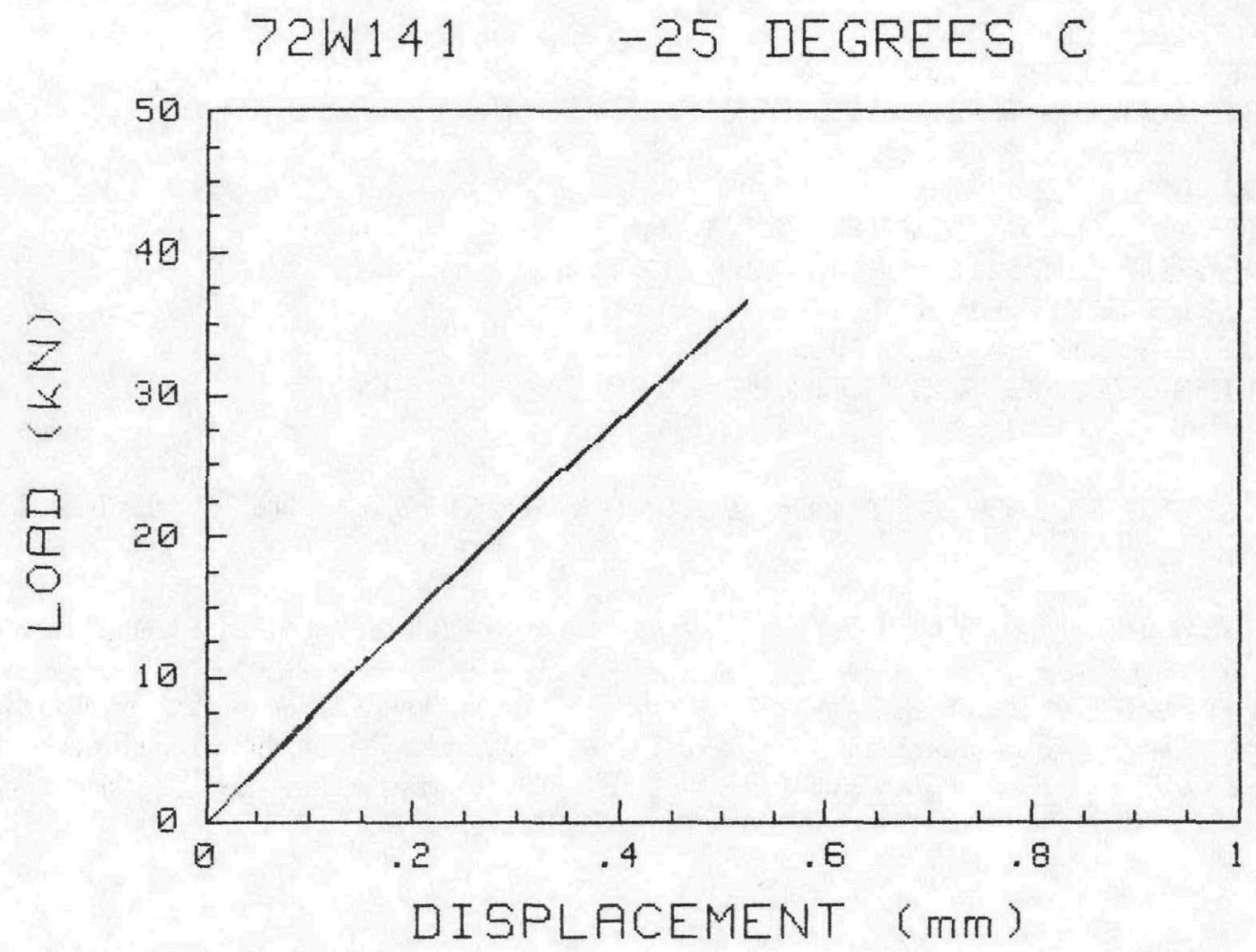

NUREG/CR-5913 
Specimen Loadline Deflection (in.)

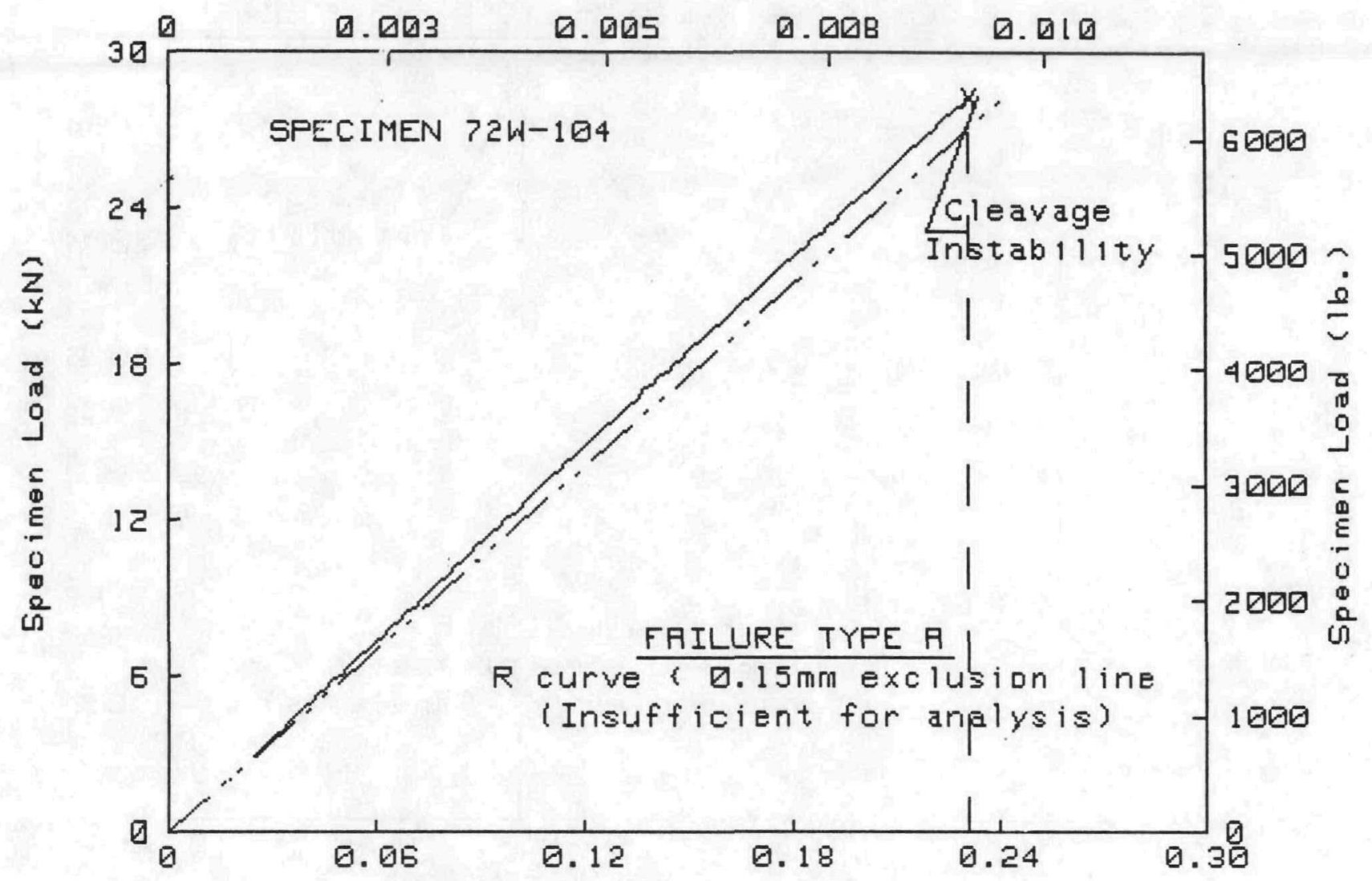

Spocimen Laadline Defleotion (mm)

\section{TEST SPECIMEN DATA}

Material Type
Test Temperature
Percent Side Groove
Specimen Thickness B
Initial a/W
Init crack length a
Flow stress
Yield stress
Youngs modulus
Poisson's Ratio (
J-INTEGRAL ANALYSIS

$=$ A533-B WELD (I)

$=-25^{\circ} \mathrm{C}$

$=0 \%$

$=25.4 \mathrm{~mm}$

$=.506$

$=25.69 \mathrm{~mm}$

$=704.2 \mathrm{MPa}$

$=655 \mathrm{MPa}$

$=208600 \mathrm{MPa}$ (Estimated Value)

J-INTEGRAL ANALYSIS

$$
=0
$$

[ Failure Type $A$ ( $J$ at instability = Jc) ]

\section{$\mathrm{Jc}$}

$K j c=\left\langle E J c /\left(1-v^{2}\right\rangle\right) \wedge .5$

Ke (Beta Corrected)

$=\frac{\text { Merkle-Corten }}{11.7 \mathrm{~kJ} / \mathrm{m}^{2}}$

$=49.3 \mathrm{MPa} \sqrt{\mathrm{m}}$

$=47.9 \mathrm{MPa} \sqrt{\mathrm{m}}$ $\frac{\text { Deformation }-\mathrm{J} *}{11.5 \mathrm{~kJ} / \mathrm{m}^{2}}$

$48.9 \mathrm{MPa} \sqrt{\mathrm{m}}$

$47.5 \mathrm{MPa} \sqrt{\mathrm{m}}$

ASTM E399-81 ANALYSIS

Modified Kic Equation; $B=(B B n) 1 / 2$

Maximum Load $P=28.31 \mathrm{kN}$

$5 \%$ Secant Offset $\mathrm{Pq}=28.31 \mathrm{kN}$

$\mathrm{Kq}$

$=48.6 \mathrm{MPa} \sqrt{\mathrm{m}}$

Validity

$K$ at Maximum Load

= VALID Kic value

$=48.6 \mathrm{MPa} \sqrt{\mathrm{m}}$

DATA CORRECTIONS USED FOR J-INTEGRAL DATA

\section{Merkle-Corten correction}

Correction to $\mathrm{J}$ for crack extension (Deformation-J)

OFFSET LOAD $=0 \mathrm{kN}$ OFFSET DEFLECTION $=0 \mathrm{~mm}$

Fitted elastic load range $=5 \mathrm{kN}$ To $25 \mathrm{kN}$

Elastic slope $=1.233 \mathrm{E}+02 \mathrm{kN} / \mathrm{mm}$ 
Specimen Crackmouth Deflection (in.)

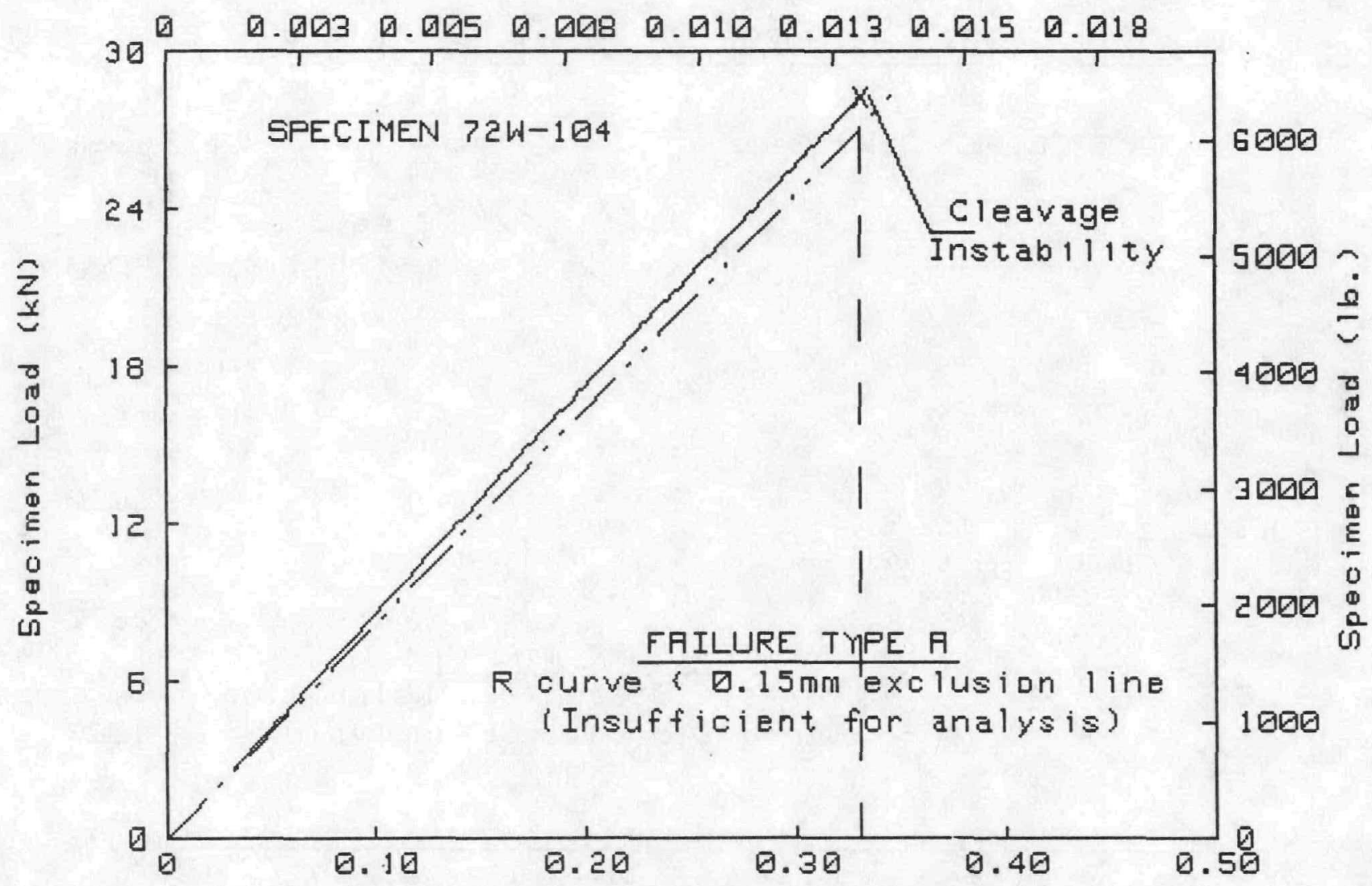

Specimen Derstanch Defleotion (mm)

\section{TEST SPECIMEN DATA}

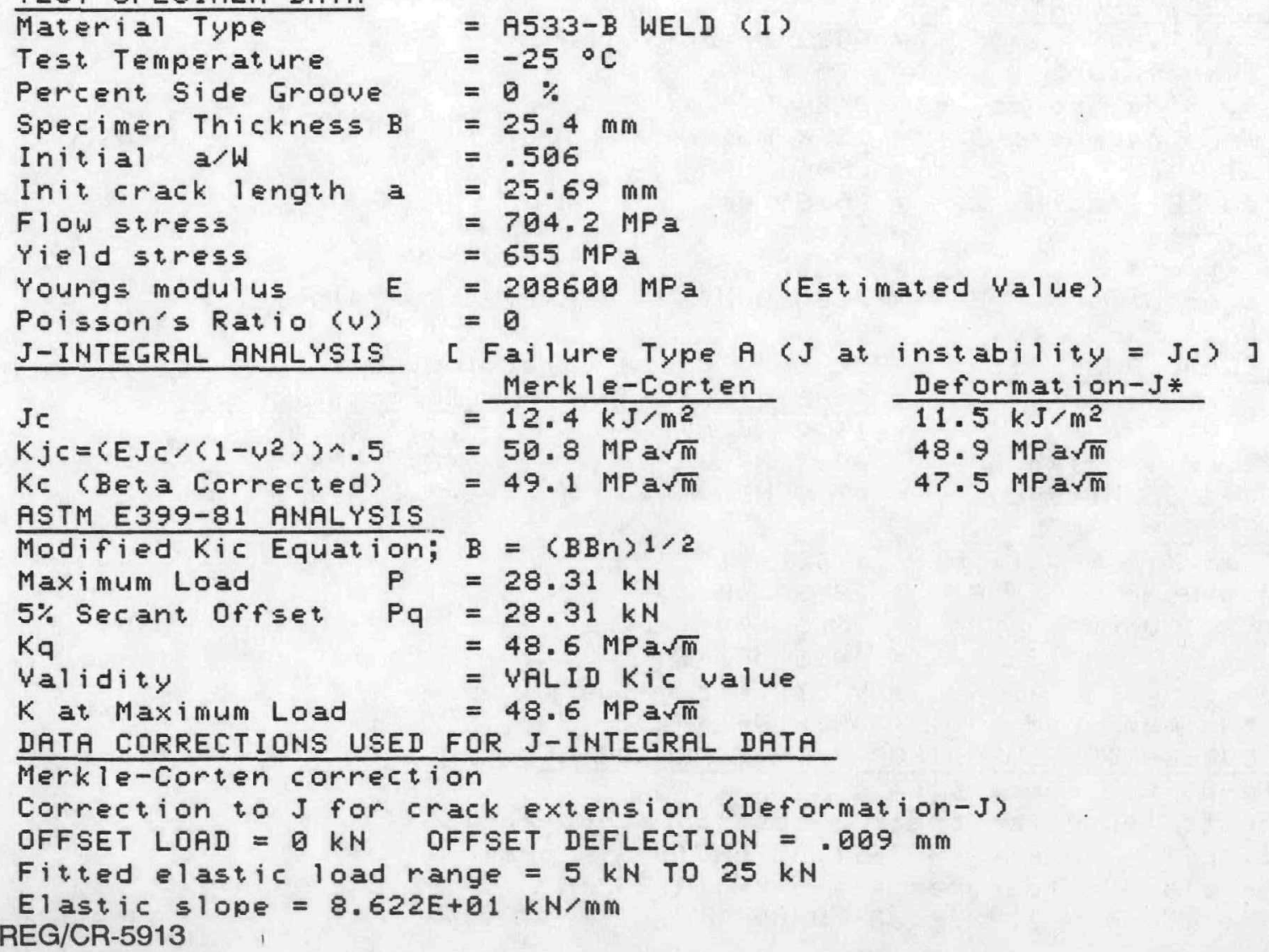




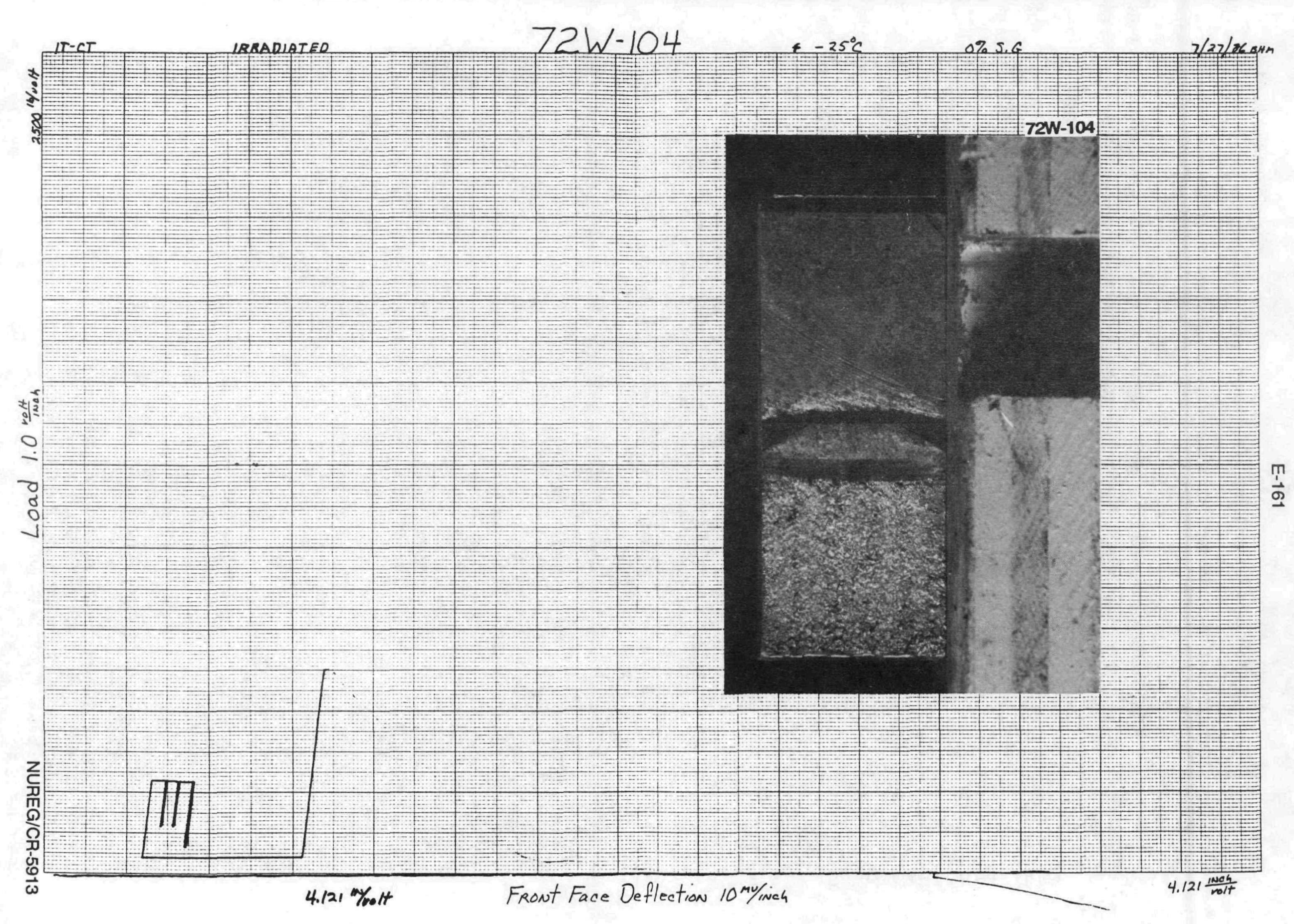


Specimen Loadline Deflection (in.)

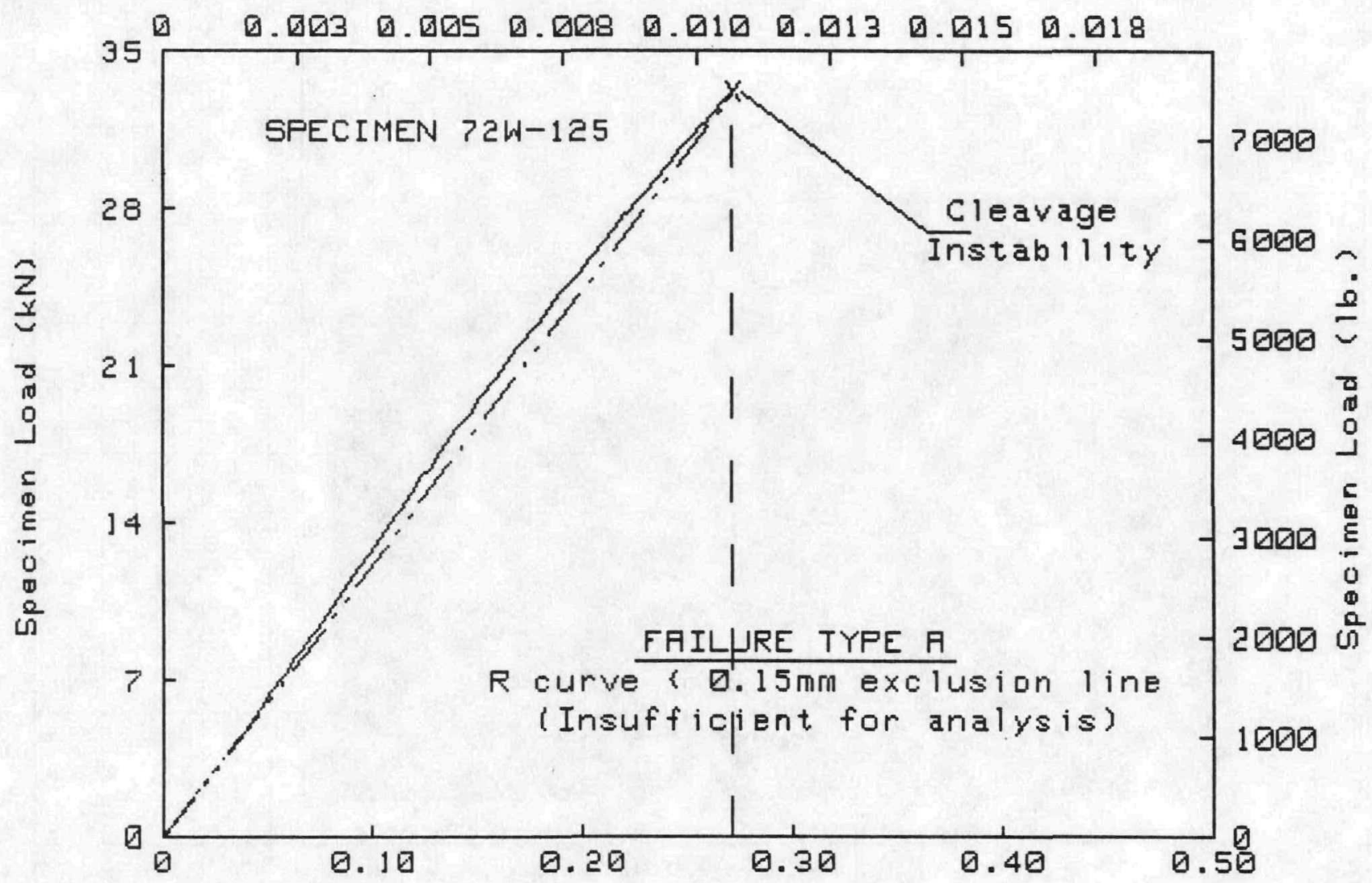

Speoimen Laadline Deflection (mm)

\section{TEST SPECIMEN DATA}

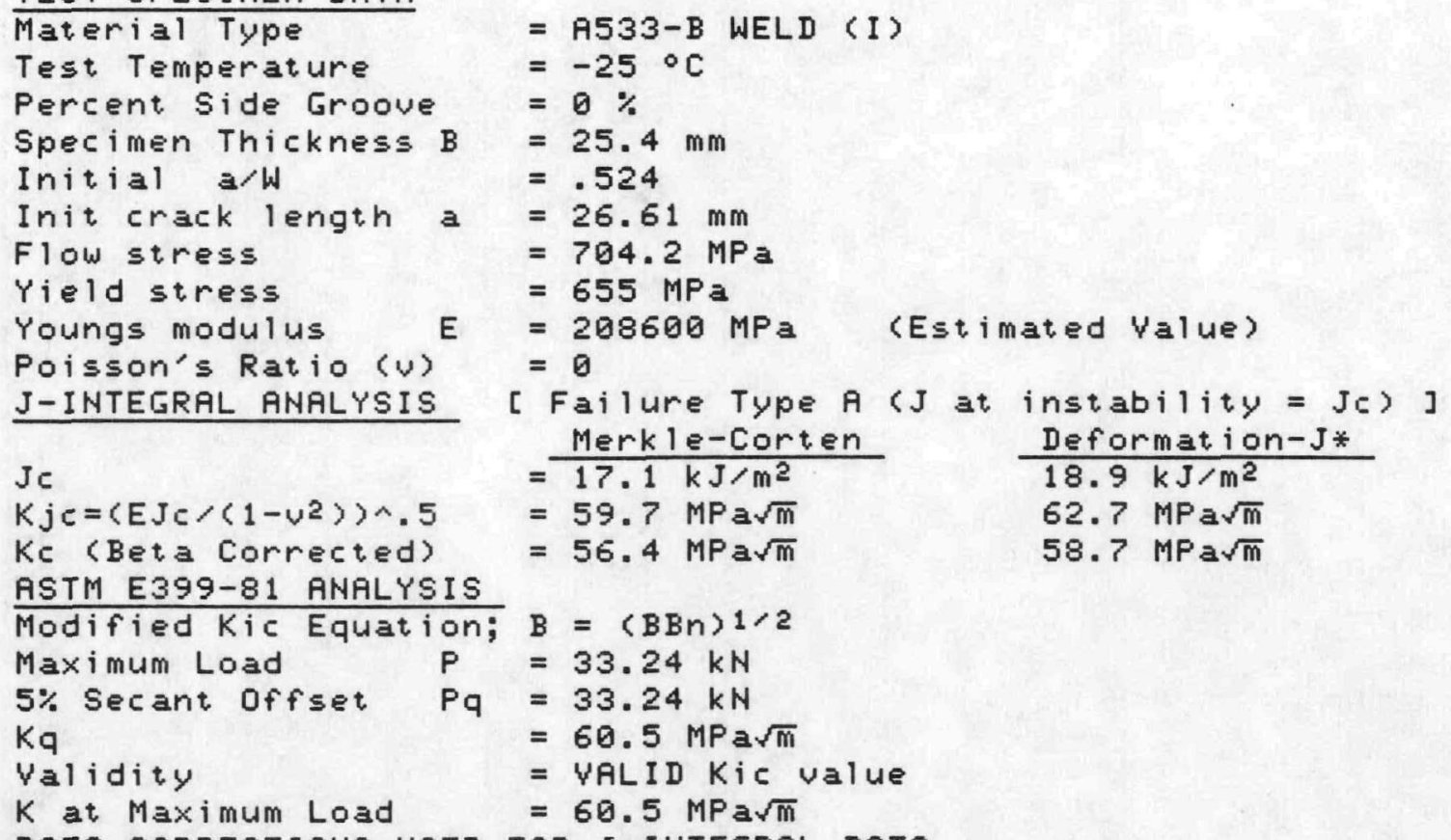

DATA CORRECTIONS USED FOR J-INTEGRAL DATA

Merkle-Corten correction

Correction to J for crack extension (Deformation-J)

OFFSET LOAD $=0 \mathrm{kN}$ OFFSET DEFLECTION $=.005 \mathrm{~mm}$

Fitted elastic load range $=5 \mathrm{kN}$ TO $25 \mathrm{kN}$

Elastic slope $=1.287 \mathrm{E}+02 \mathrm{kN} / \mathrm{mm}$ 
Specimen Crackmouth Deflection (in.)

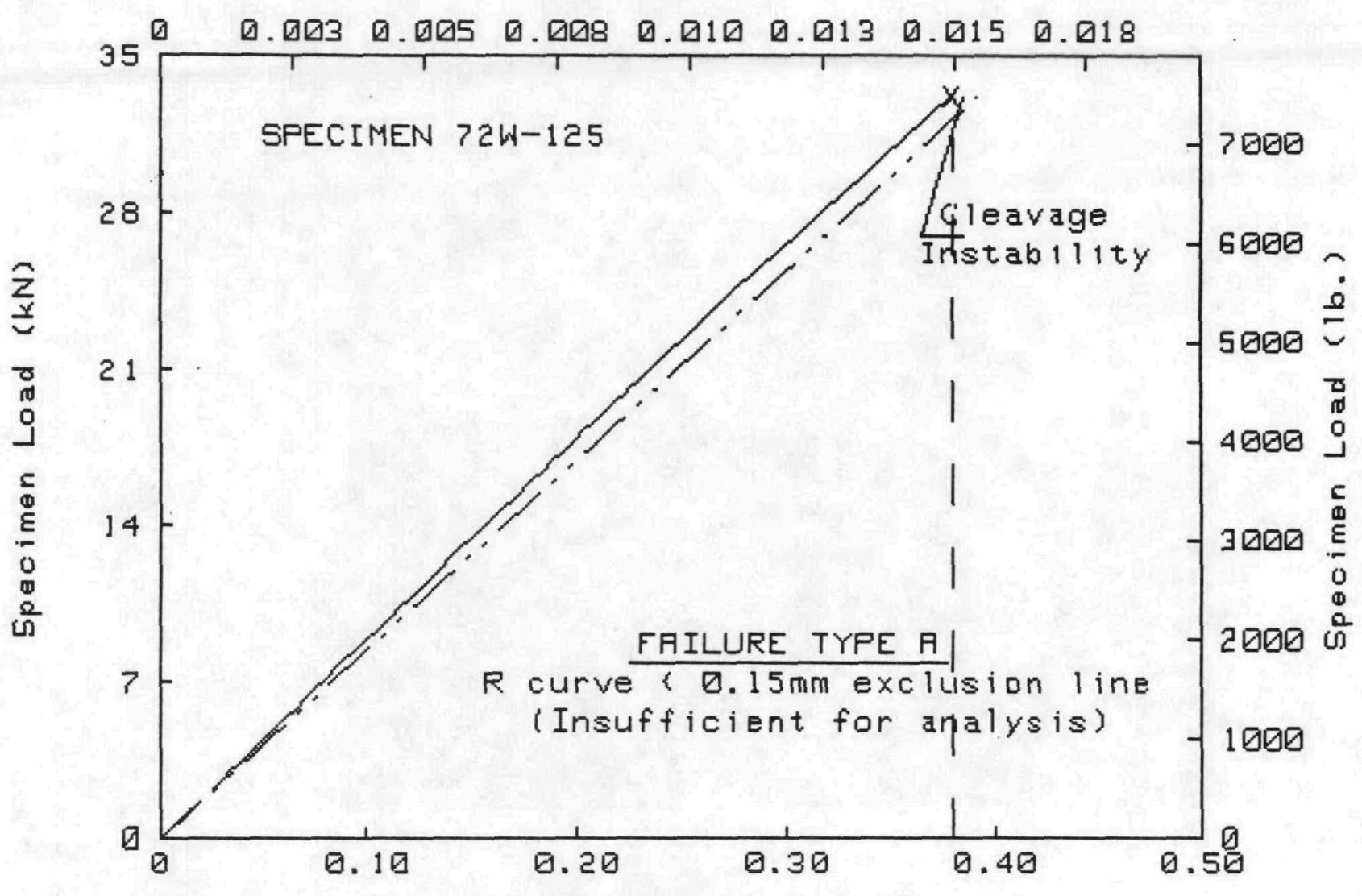

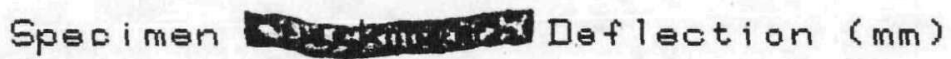

TEST SPECIMEN DATA

Material Type
Test Temperature
Percent Side Groove
Specimen Thickness B
Initial a/W
Init crack length a
Flow stress
Yield stress
Youngs modulus
Poisson's Ratio (U)
J-INTEGRAL ANALYSIS

$=$ A533-B WELD (I)

$=-25^{\circ} \mathrm{C}$

$=0 \%$

$=25.4 \mathrm{~mm}$

$=.524$

$=26.61 \mathrm{~mm}$

$=704.2 \mathrm{MPa}$

$=655 \mathrm{MPa}$

$=208600 \mathrm{MPa}$ (Estimated Value)

$=0$

[ Failure Type A ( $J$ at, instability = JC) ]

$\mathrm{Jc}$ $=\frac{\text { Merkle-Corten }}{17.4 \mathrm{~kJ} / \mathrm{m}^{2}}$ Deformation $-J *$

$=60.2 \mathrm{MPa} \sqrt{\mathrm{m}}$ $18.1 \mathrm{~kJ} / \mathrm{m}^{2}$

$K j c=\left\langle E J c /\left\langle 1-v^{2}\right\rangle\right\rangle \wedge .5$

$=56.8 \mathrm{MPa} \sqrt{\mathrm{m}}$

$61.5 \mathrm{MPa} \sqrt{\mathrm{m}}$

$57.8 \mathrm{MPa} \sqrt{\mathrm{m}}$

ASTM E399-81 ANALYSIS

Modified Kic Equation; $B=(B B n) 1 / 2$

Maximum Load $P=33.24 \mathrm{kN}$

$5 \%$ Secant offset $\mathrm{Pq}=33.24 \mathrm{kN}$

$\mathrm{Kq}$

$=60.5 \mathrm{MPa} \sqrt{\mathrm{m}}$

= VALID Kic value

Validity

$=60.5 \mathrm{MPa} \sqrt{\mathrm{m}}$

DATA CORRECTIONS USED FOR J-INTEGRAL DATA

Merkle-Corten correction

Correction to J for crack extension (Deformation-J)

OFFSET LOAD $=0 \mathrm{kN}$ OFFSET DEFLECTION $=.013 \mathrm{~mm}$

Fitied elastic load range $=5 \mathrm{kN}$ T0 $25 \mathrm{kN}$

Elastic slope $=8.949 E+01 \mathrm{kN} / \mathrm{mm}$ 


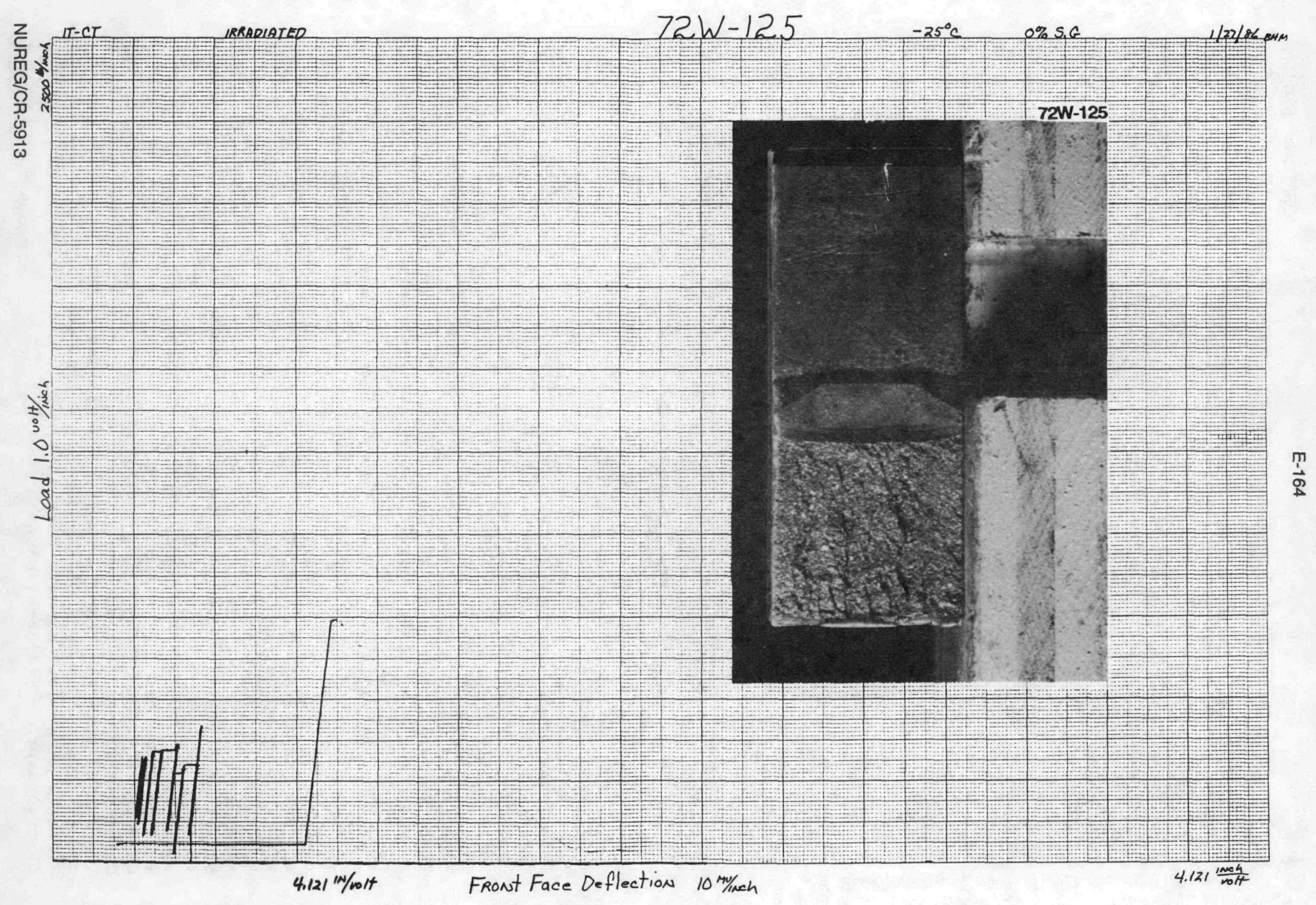


SPECIMEN ID $=72 W 124$

MATER IAL TYPE = MCUWELD

TEST TEMPERATURE $=25$ DEG C

THICKNESS $=25.4 \mathrm{~mm}$

SIDE GROOUES $=0 \%$

INITIAL CRACK LENGTH $=26.7 \mathrm{~mm}$

$a / W)_{i}=.526$

MEASURED DUCTILE DELTA-A $=.05 \mathrm{~mm}$

YIELD STRENGTH $=621 \mathrm{MPa}$

FLOW STRENGTH $=672 \mathrm{MPa}$

ESTIMATED YOUNG'S MODULUS $=206 \mathrm{GPa}$

SPECIMEN CLEAUED AT KQ= $96.8 \mathrm{MPa} S Q R[\mathrm{~m}]$ (NOT WALID KIc)

UALUES AT MAXIMUM LDAD

J-INTEGRAL $=55.5 \mathrm{~kJ} / \mathrm{m}^{\wedge} 2$

$\mathrm{KJc}=106.9 \mathrm{MPa}$ SQR $[\mathrm{m}]$

$K$-beta-c $=82.5 \mathrm{MPa} \operatorname{SQR}[\mathrm{m}]$
UALUES AT CLEAUAGE LDAD

J-INTEGRAL $=55.5 \mathrm{~kJ} / \mathrm{m}^{\wedge} 2$

$\mathrm{KJc}=106.9 \mathrm{MPa} \operatorname{SQR}[\mathrm{m}]$

K-beta-c $=82.5 \mathrm{MPa} \operatorname{SQR}[\mathrm{m}]$ 


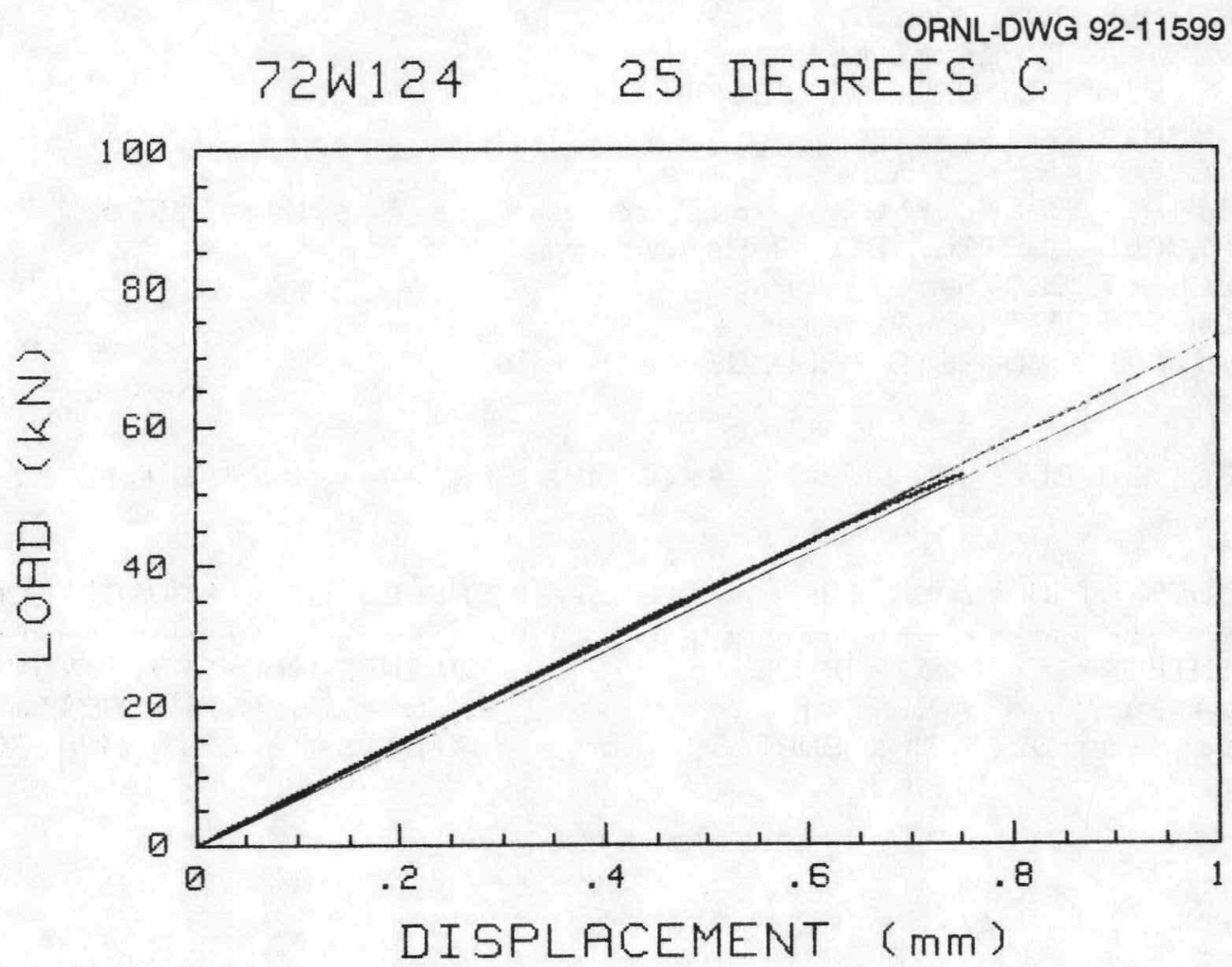


Specimen Loadline Deflection (in.)

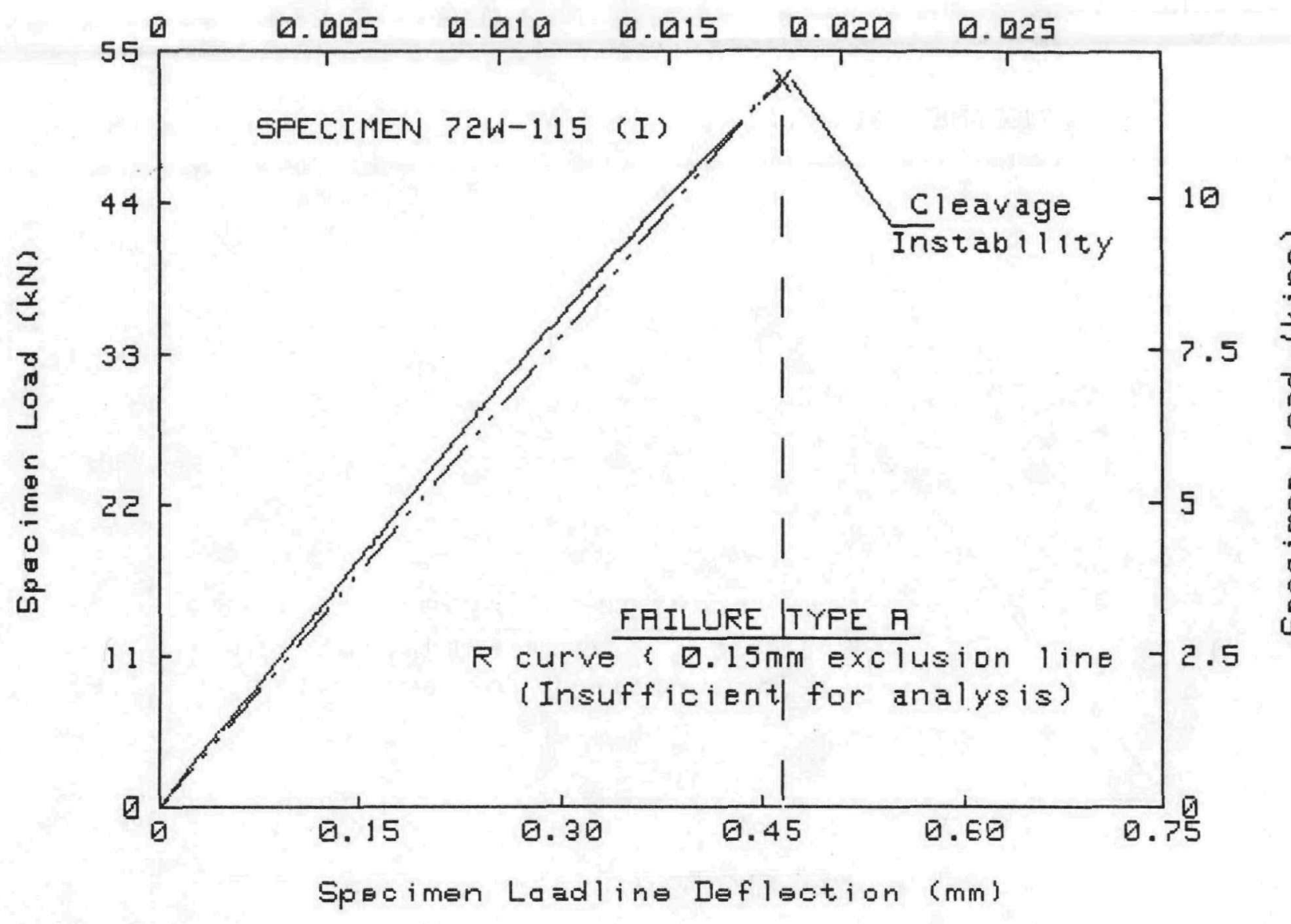

\section{TEST SPECIMEN DATA}

Material Type
Test Temperature
Percent Side Groove
Specimen Thickness B
Initial a/W
Init crack length a
Flow stress
Yield stress
Youngs modulus E
Poisson's Ratio (W)
J-INTEGRAL RNALYSIS

$\mathrm{Jc}$

$K j c=\left\langle E J c /\left(1-v^{2}\right)\right\rangle \wedge .5$

$\mathrm{Ke}$ (Beta Corrected)

ASTM E399-81 ANALYSIS

$=$ A533-B WELD $\langle I\rangle$

$=29^{\circ} \mathrm{C}$

$=0 \%$

$=25.4 \mathrm{~mm}$

$=.522$

$=26.5 \mathrm{~mm}$

$=666 \mathrm{MPa}$

$=617.8 \mathrm{MPa}$

$=205600 \mathrm{MPa}$

$$
=0
$$

[ Failure Type $A$ ( $J$ at instability = JC) ]

$=\frac{\text { Merkle-Corten }}{46.6 \mathrm{~kJ} / \mathrm{m}^{2}}$

$=97.9 \mathrm{MPa} \sqrt{\mathrm{m}}$

$=78.4 \mathrm{MPa} \sqrt{\mathrm{m}}$ $\frac{\text { Deformation-J* }}{48.7 \mathrm{~kJ} / \mathrm{m}^{2}}$

$100 \mathrm{MPa} \sqrt{\mathrm{m}}$

$79.3 \mathrm{MPa} \sqrt{\mathrm{m}}$

Modified Kic Equation; $B=\langle B B n\rangle 1 / 2$

Maximum Load $P=52.87 \mathrm{kN}$

$5 \%$ Secant offset $\mathrm{Pq}=51.2 \mathrm{kN}$

$\mathrm{Kq} \quad=92.5 \mathrm{MPa} \sqrt{\mathrm{m}}$

Validity

$K$ at Maximum Load

= INVALID--1, 2

$=95.5 \mathrm{MPa} \sqrt{\mathrm{m}}$

DATA CORRECTIONS USED FOR J-INTEGRAL IATA

Merkle-Corten correction

Correction to J for erack extension (Deformation-J)

OFFSET LOAD $=0 \mathrm{kN}$ OFFSET DEFLECTION $=-.002 \mathrm{~mm}$

Fitted elastic load range $=5 \cdot \mathrm{kN}$ TO $25 \mathrm{kN}$

Elastic slope $=1.206 \mathrm{E}+02 \mathrm{kN} / \mathrm{mm}$ 
Specimen Crackmouth Deflection (in.)

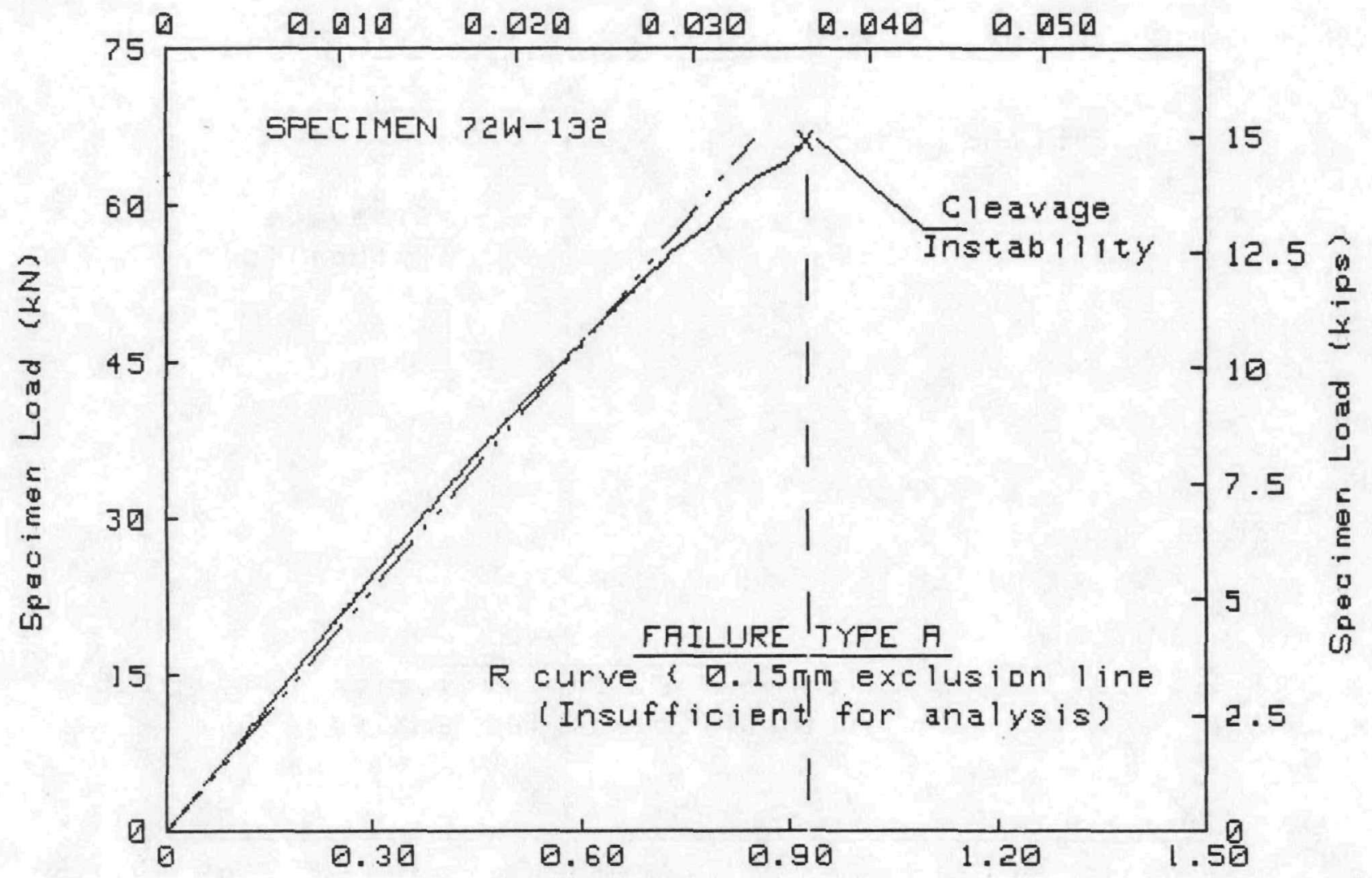

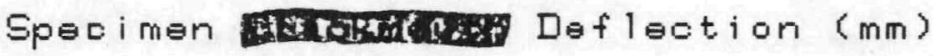

TEST SPECIMEN DATA

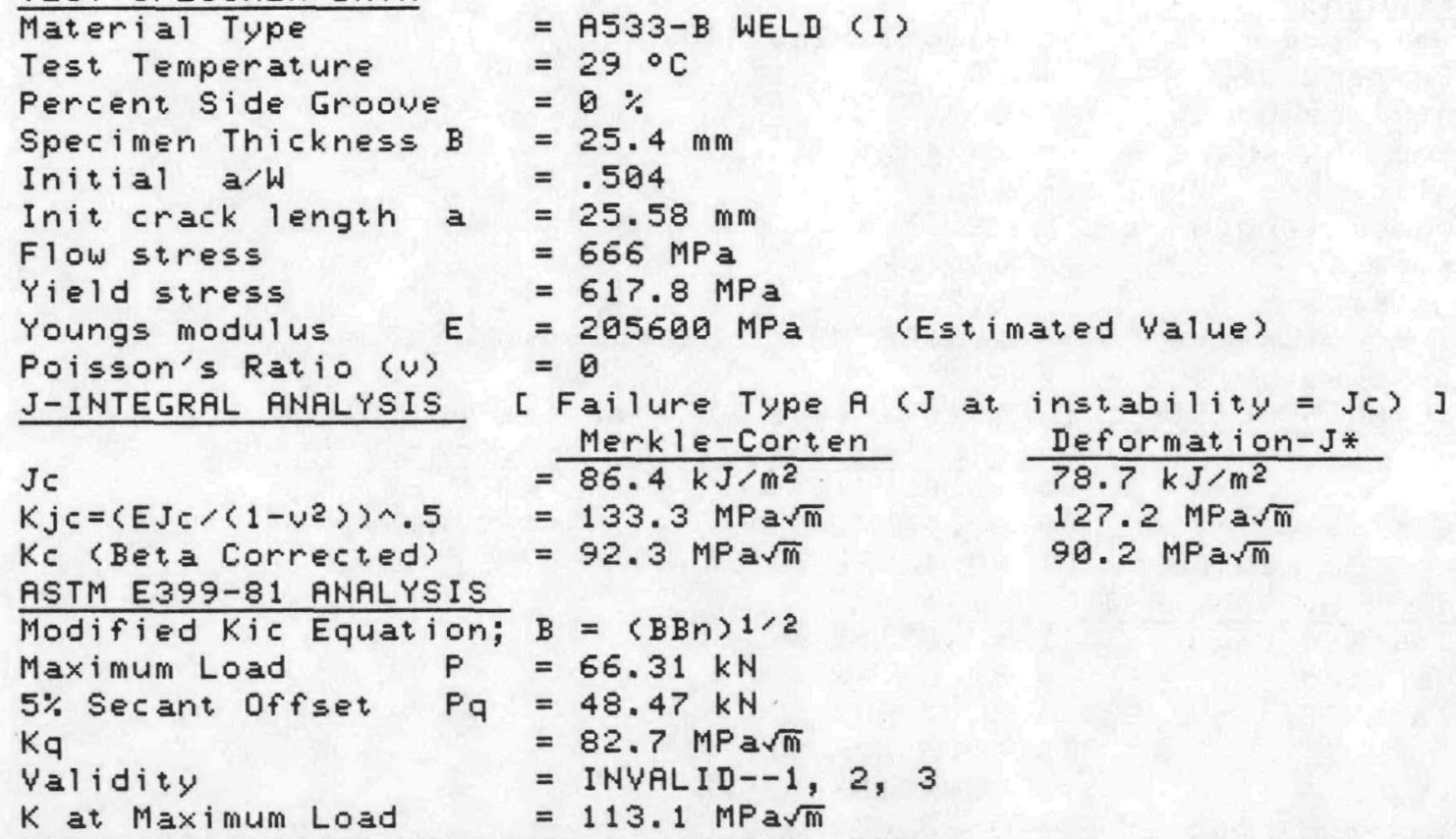

DATA CORRECTIONS USED FOR J-INTEGRAL DATA

Merkle-Corten correction

Correction to $\mathrm{J}$ for crack extension (Deformation-J)

OFFSET LOAD $=0 \mathrm{kN}$ OFFSET DEFLECTION $=.018 \mathrm{~mm}$

Fitted elastic load range $=5 \mathrm{kN}$ TO $25 \mathrm{kN}$

Elastic slope $=8.216 \mathrm{E}+\emptyset 1 \mathrm{kN} / \mathrm{mm}$

NUREG/CR-5913 


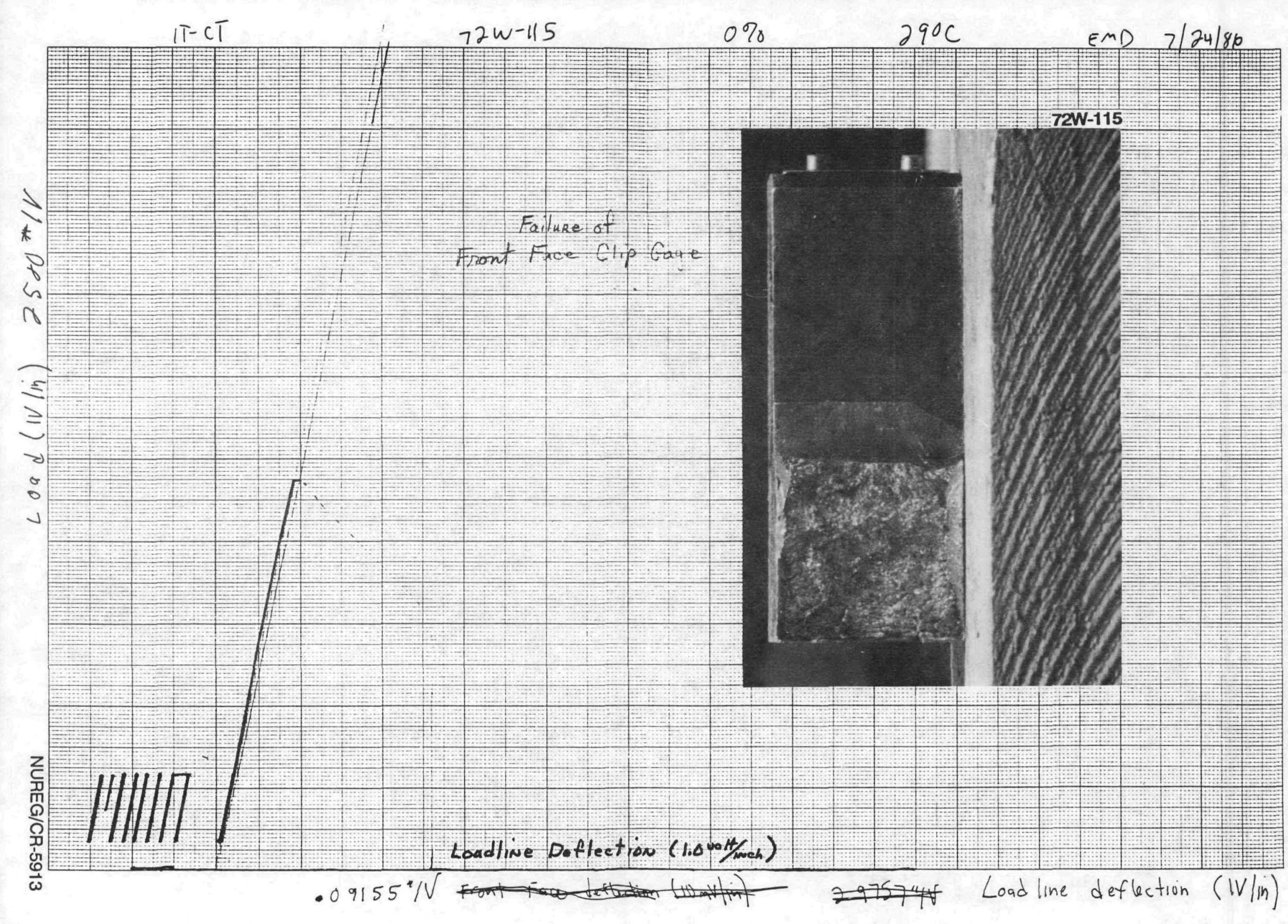


Specimen Loadline Deflection (in.)

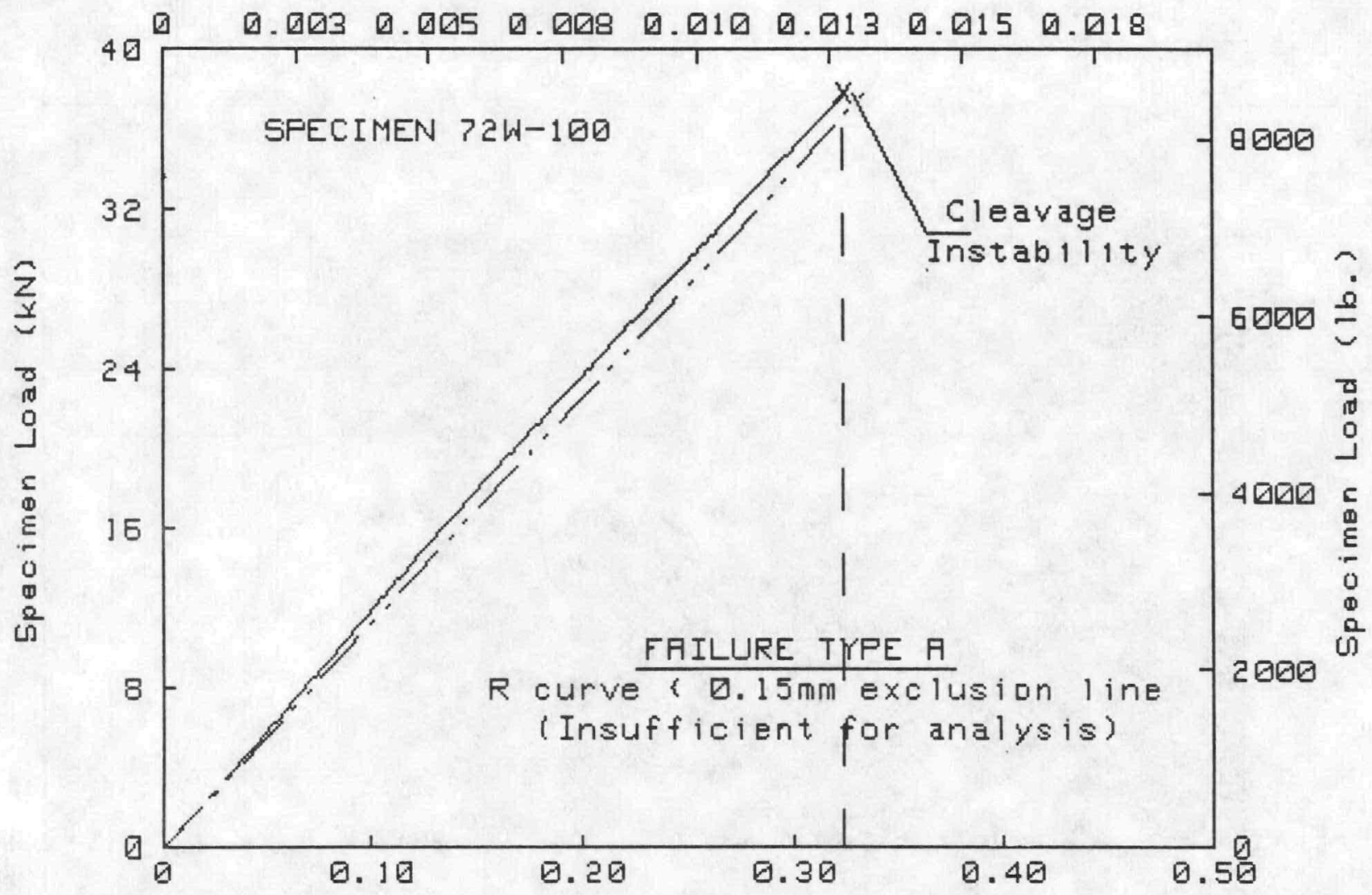

Specimen Laadline Deflection (mm)

\section{TEST SFECIMEN DATA}

Material Type
Test Temperature
Percent Side Groove
Specimen Thickness B
Initial a/W
Init crack length a
Flow stress
Yield stress
Youngs modulus E
Poisson's Ratio (U)
J-INTEGRAL ANALYSIS

$\mathrm{Je}$

$K j c=\left\langle E \cdot J c\left\langle\left(1-v^{2}\right)\right\rangle \wedge .5\right.$

Ke (Beta Corrected)

$$
\begin{aligned}
& =\text { F533-B WELD (I) } \\
& =29{ }^{\circ} \mathrm{C} \\
& =9 \% \\
& =25.4 \mathrm{~mm} \\
& =.52 \\
& =26.42 \mathrm{~mm} \\
& =666 \mathrm{MPa} \\
& =617.8 \mathrm{MPa} \\
& =205600 \mathrm{MPa} \quad \text { (Est imated Value) } \\
& =0 \\
& {[\text { Failure Type A (J at instability = JC) ] }}
\end{aligned}
$$
$=\frac{\text { Merkle-Corten }}{22.7 \mathrm{~kJ} / \mathrm{m}^{2}}$

$=68.2 \mathrm{MPa} \sqrt{\mathrm{m}}$

$=61.8 \mathrm{MPa} \sqrt{\mathrm{m}}$

\section{Deformation-J*$$
69.2 \mathrm{MPa} \sqrt{\mathrm{m}}
$$

$62.4 \mathrm{MPa} \sqrt{\mathrm{m}}$

ASTM E399-81 ANALYSIS

$$
\begin{array}{lll}
\text { Modified Kic Equation; } & B=(B B n) 1 / 2 \\
\text { Maximum Load } & P & =37.83 \mathrm{kN} \\
5 \% \text { Secant Offset } \mathrm{Pq} & =37.83 \mathrm{kN} \\
\mathrm{Kq} & & =68 \mathrm{MPa} \sqrt{\mathrm{m}} \\
\text { Validity } & =I N V A L I D--1,2 \\
\text { Kat Maximum Load } & =68 \mathrm{MPa} \sqrt{\mathrm{m}}
\end{array}
$$

DATA CORRECTIONS USED FOR J-INTEGRAL DATA

Merkle-Corten correction

Correction to $J$ for erack extension (Deformation-J)

OFFSET LOAD $=0 \mathrm{kN}$ OFFSET DEFLECTION $=0 \mathrm{~mm}$

Fitted elastic load range $=5 \mathrm{kN}$ TO $25 \mathrm{kN}$

Elastic slope $=1.191 \mathrm{E}+02 \mathrm{kN} / \mathrm{mm}$ 
Specimen Crackmouth Deflection (in.)

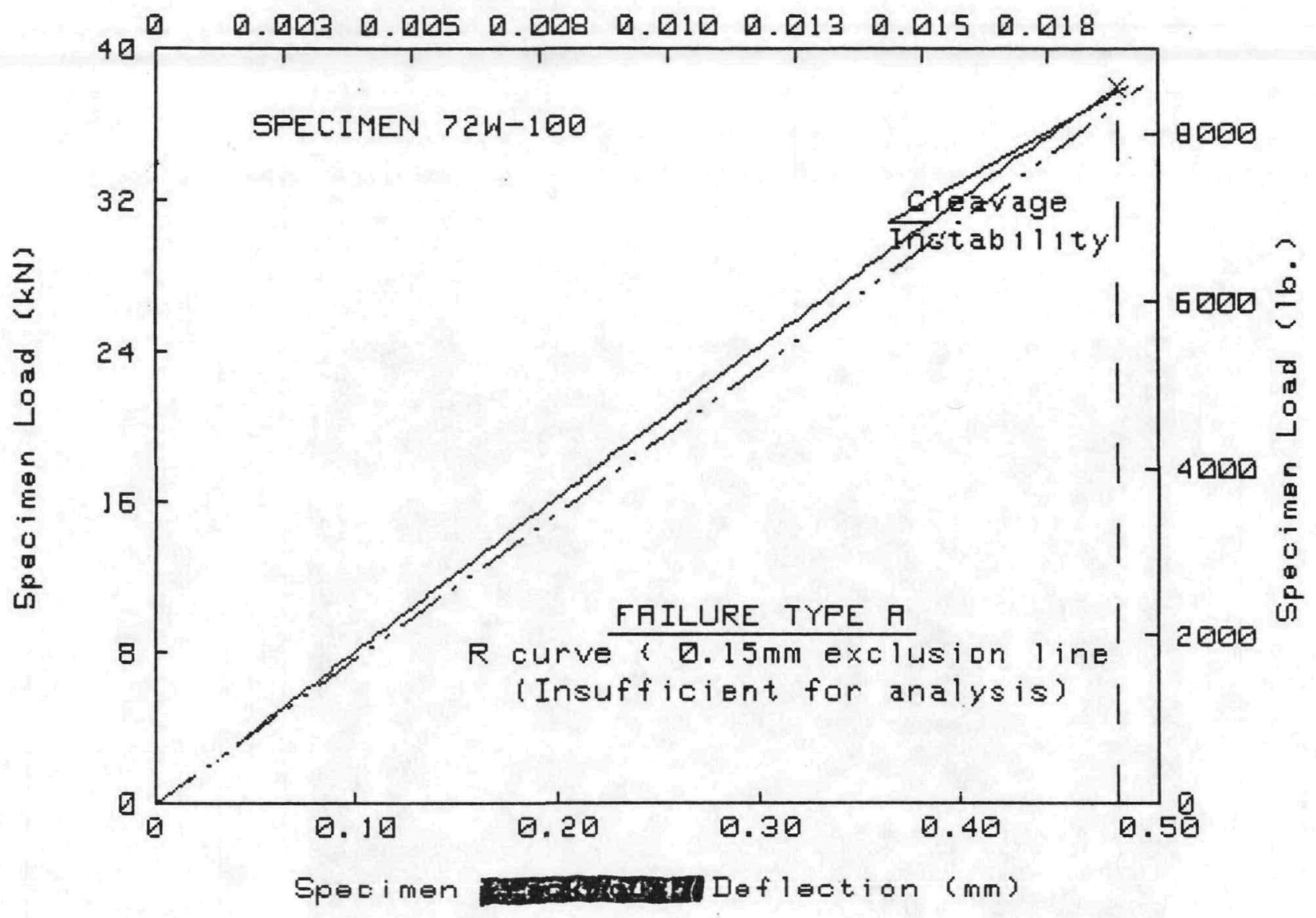

TEST SPECIMEN DATA

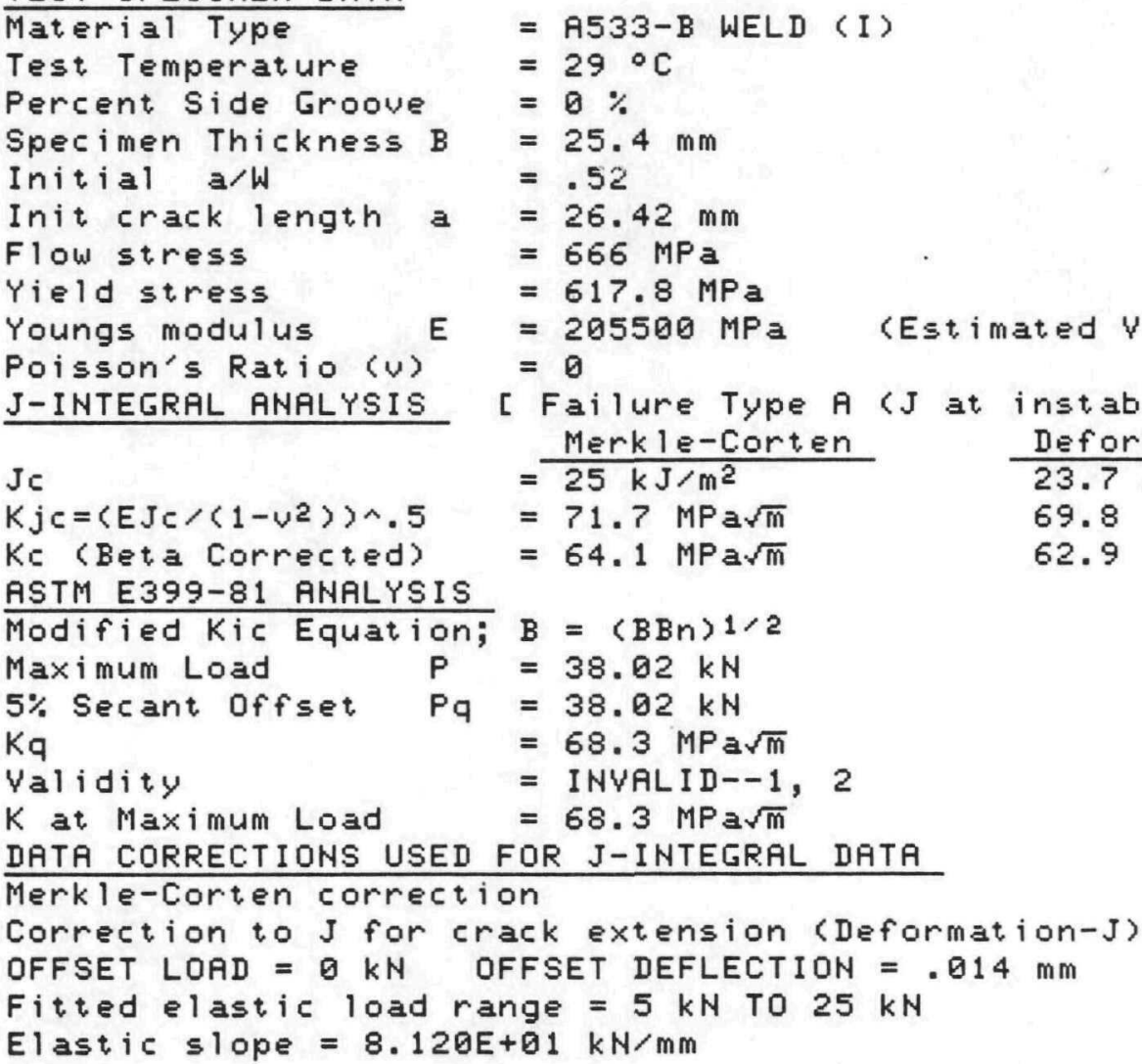




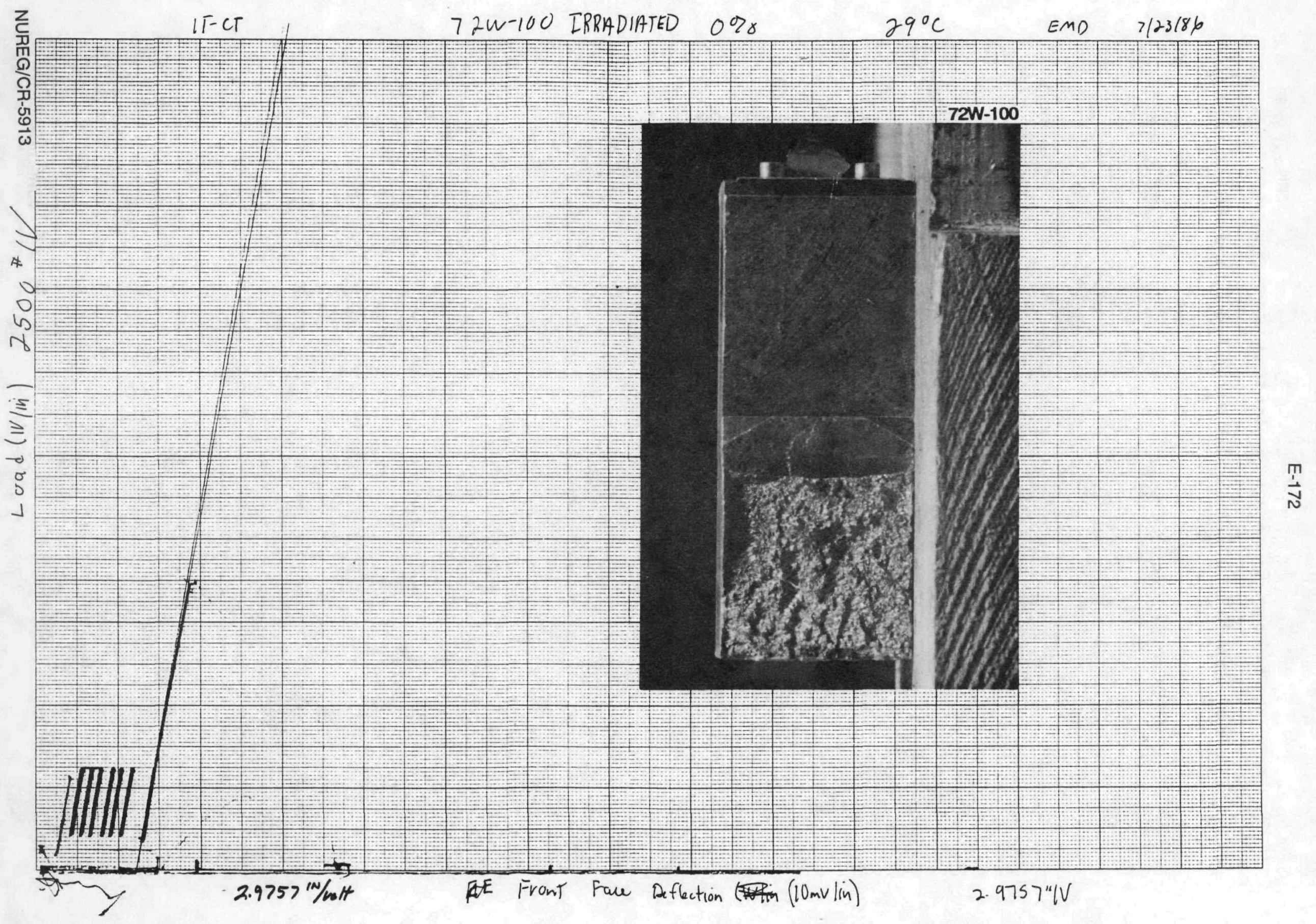


Specimen Loadline Deflection (in.)

ORNL-DWG 92-11604

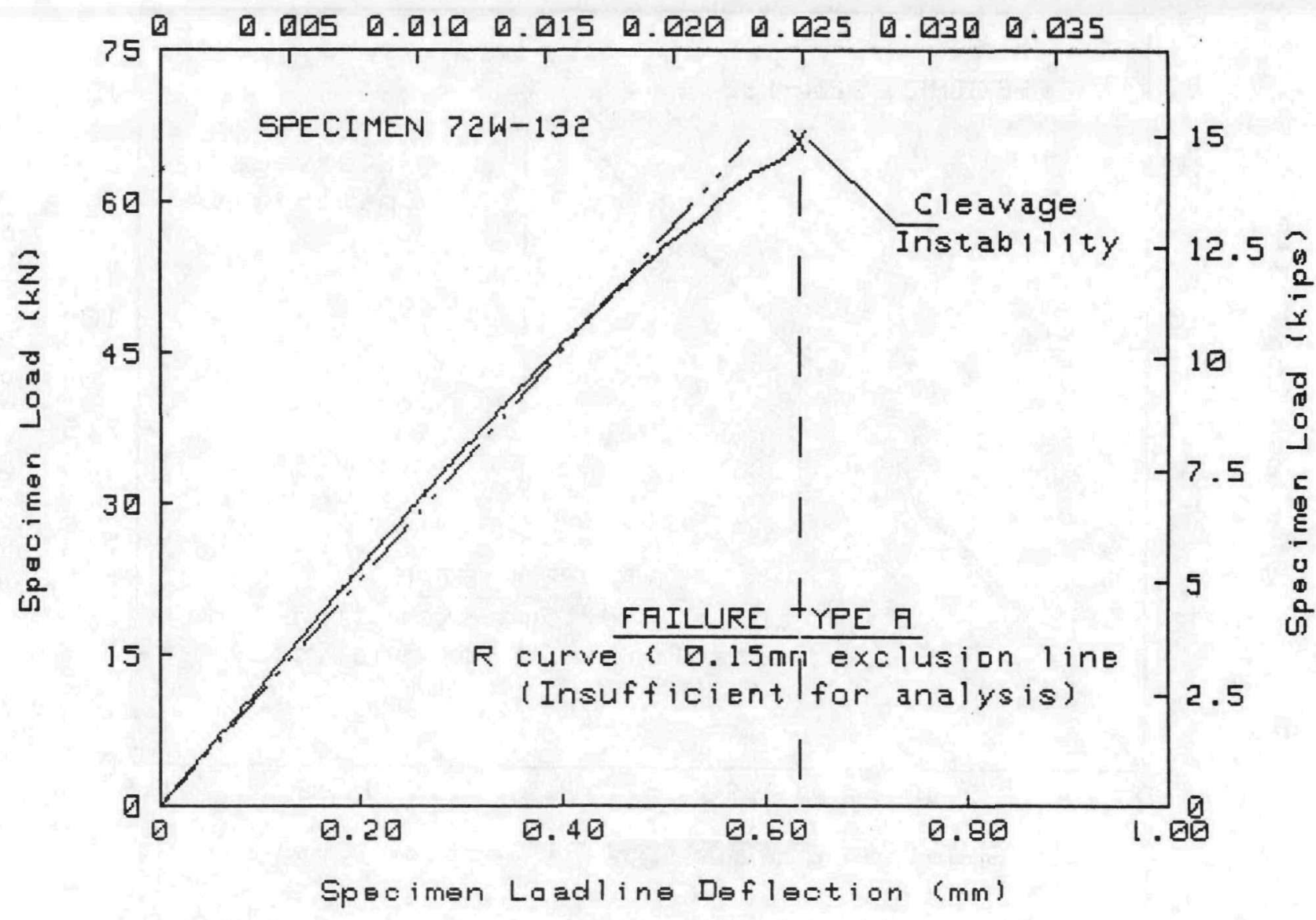

TEST SPECIMEN DATA

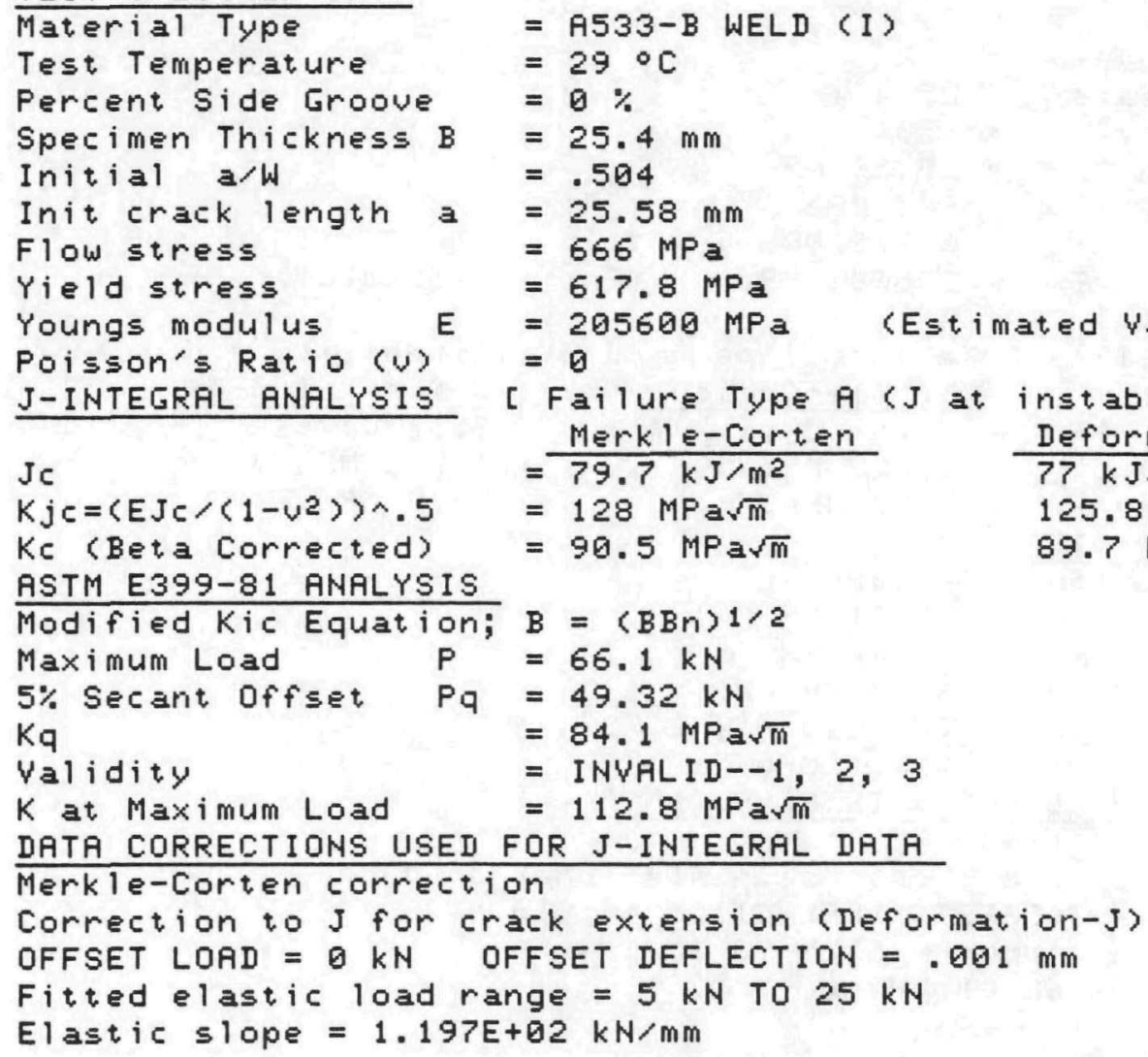


Specimen Crackmouth Deflection (in.)

ORNL-DWG 92-11605

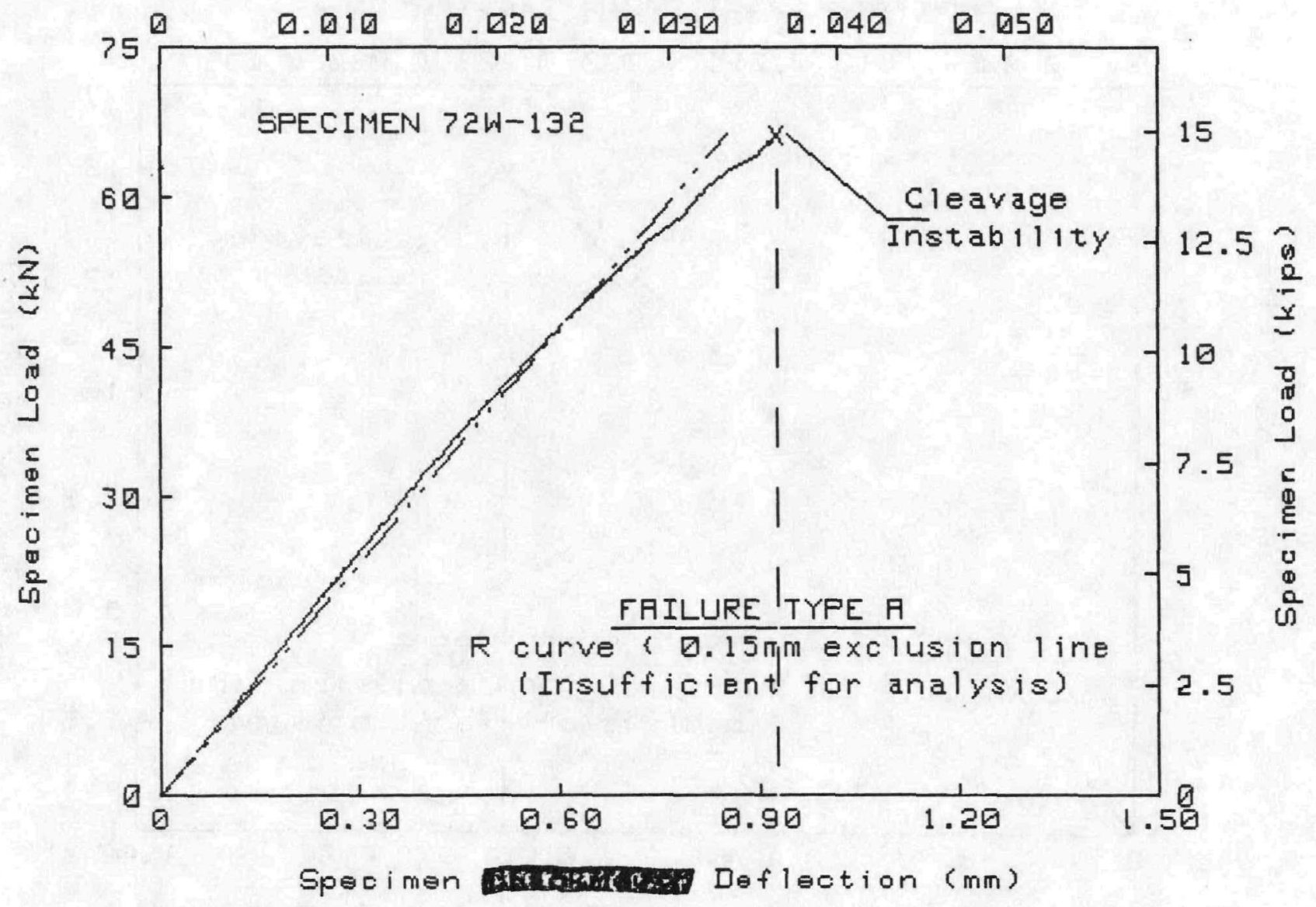

TEST SPECIMEN DATA

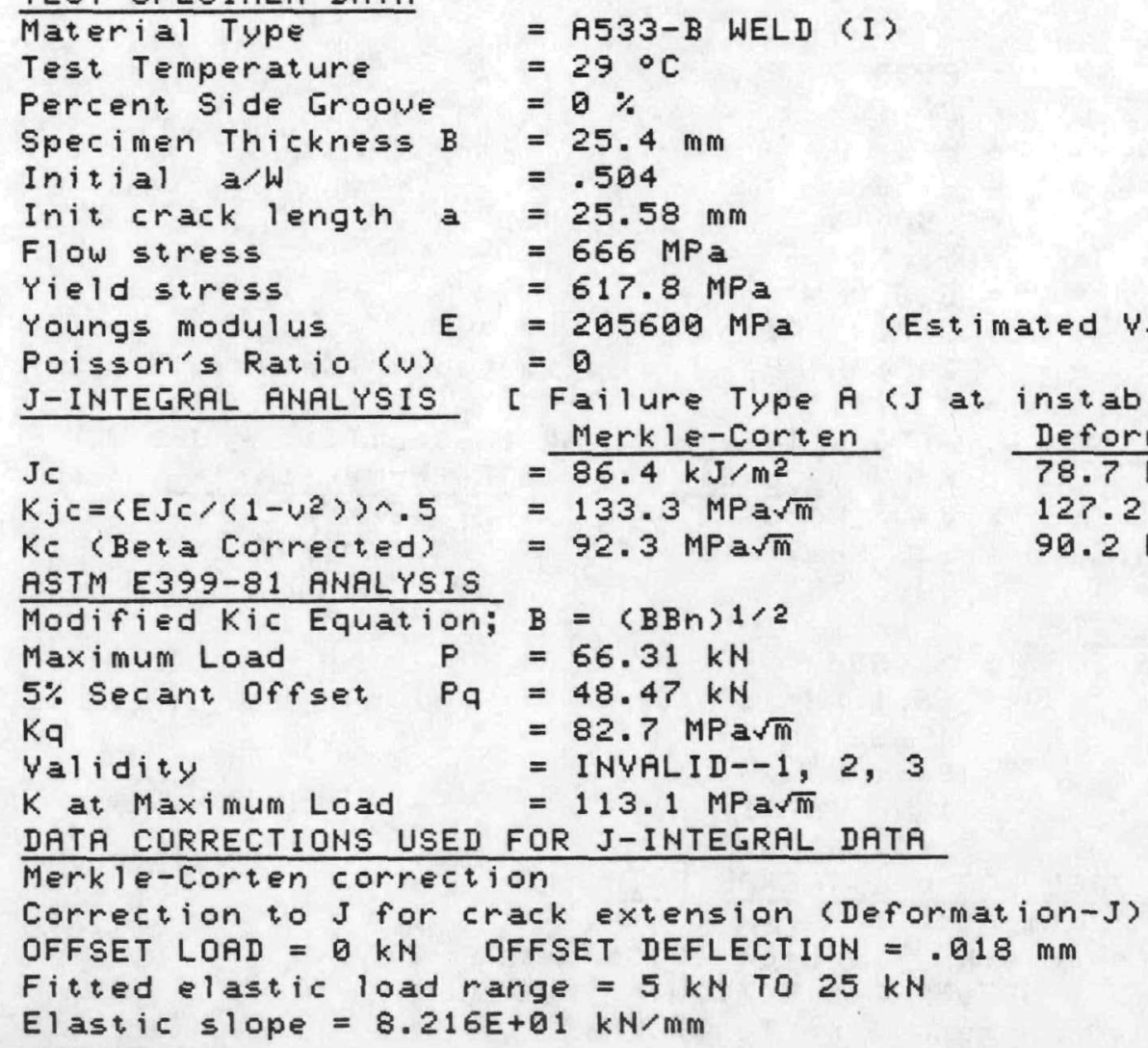

NUREG/CR-5913 


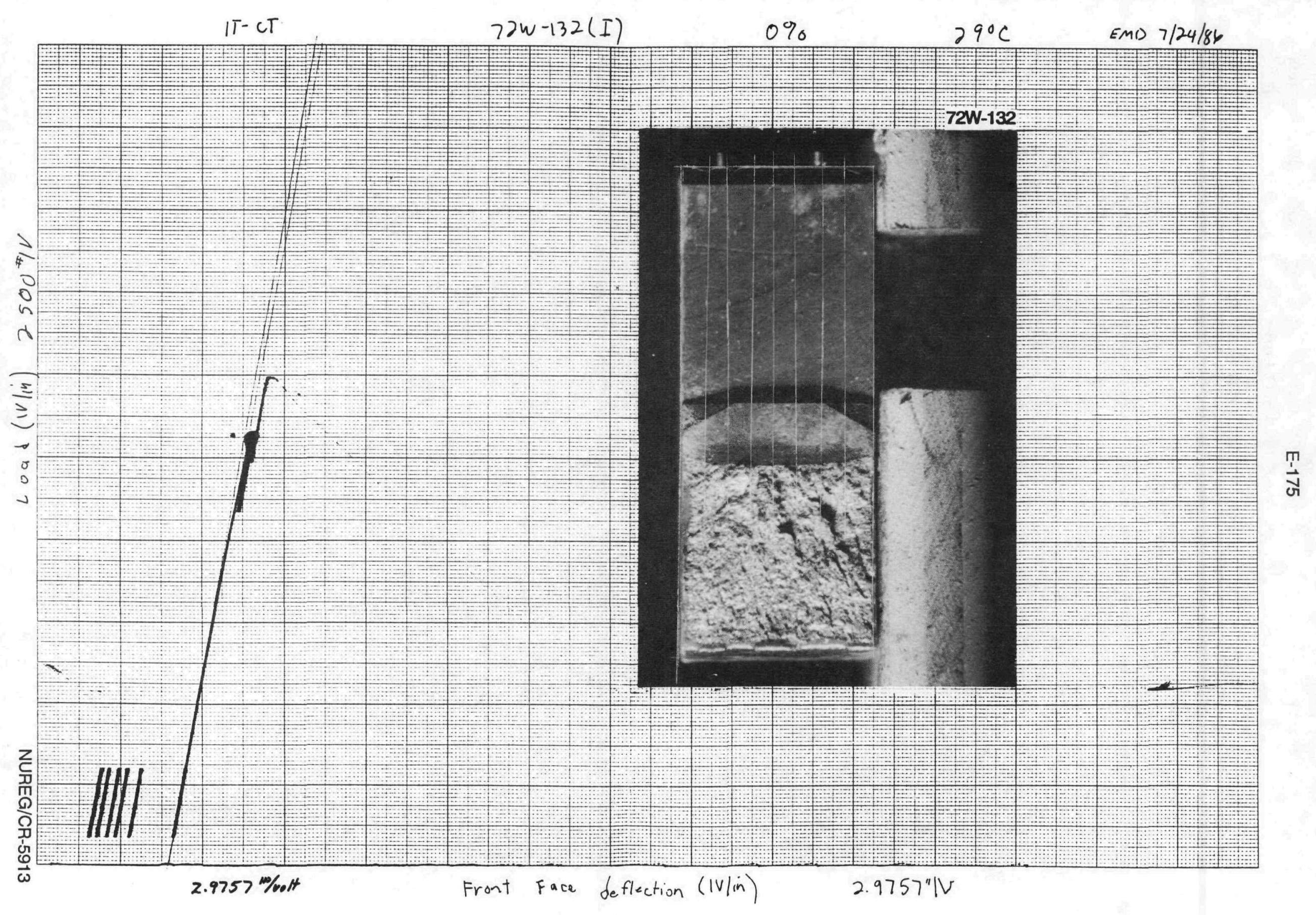


Specimen Loadline Deflection (in.)

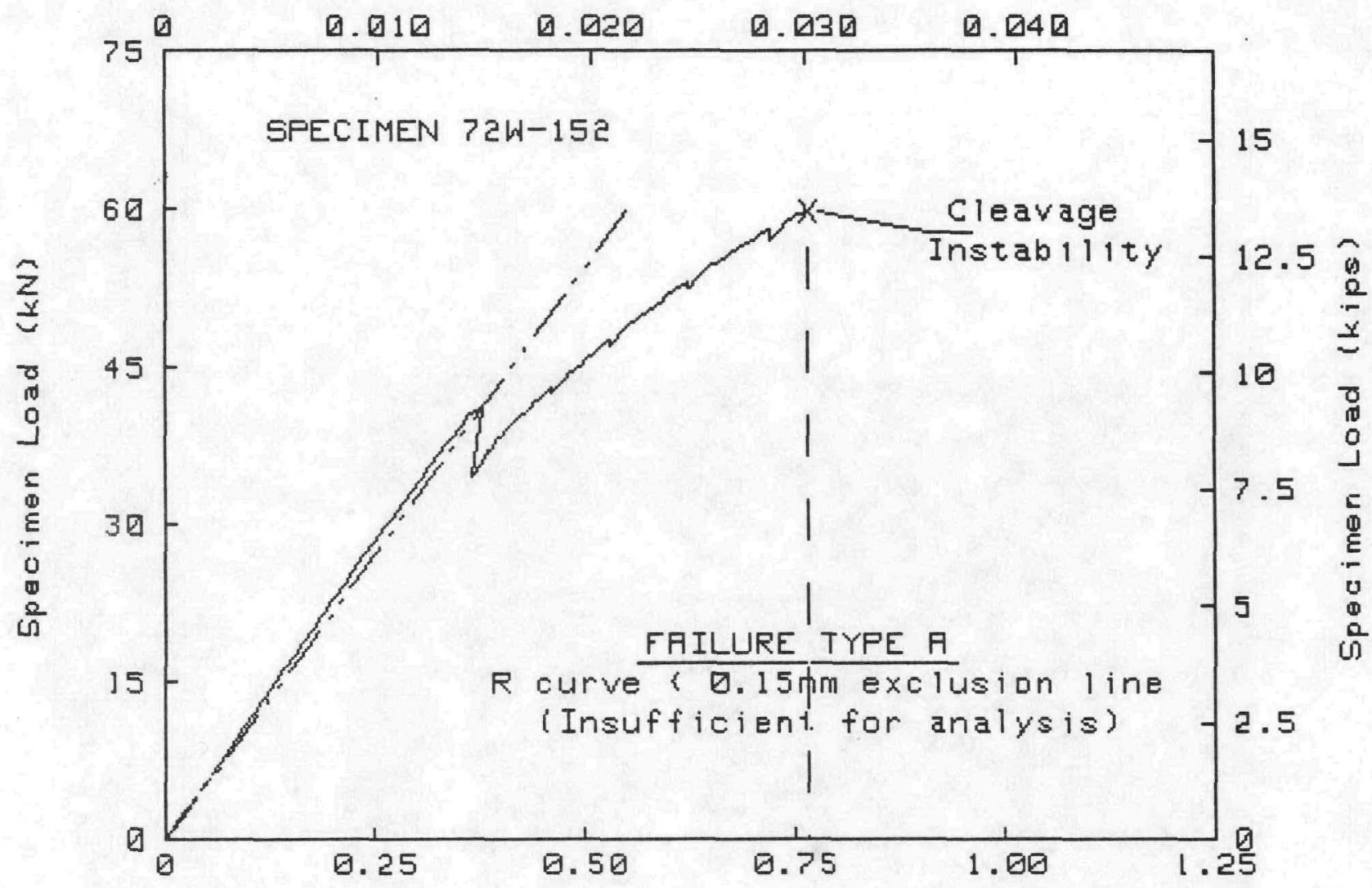

Spooimen Laadline Defleotion (mm)

\section{TEST SPECIMEN DATA}

Material Type
Test Temperature
Percent Side Groove
Specimen Thickness B
Initial a/W
Init crack length a
Flow stress
Yield stress
Youngs modulus
Poisson's Ratio (W)
J-INTEGRAL ANALYSIS

$=A 533-B$ WELD (I)

$=50^{\circ} \mathrm{C}$

$=0 \%$

$=25.4 \mathrm{~mm}$

$=.55$

$=27.94 \mathrm{~mm}$

$=654.5 \mathrm{MPa}$

$=607.1 \mathrm{MPa}$

$=204400 \mathrm{MPa}$

$$
=0
$$

[ Failure Type $A$ (J at instability = JC) ]

\section{$\mathrm{Jc}$}

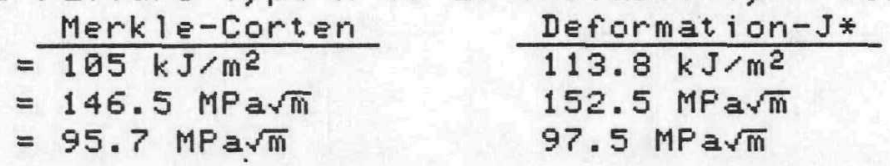

Ke (Beta Corrected)

ASTM E399-81 ANALYSIS

Modified Kic Equation; $B=(B B \cap) 1 / 2$

Maximum Load $P=59.86 \mathrm{kN}$

$5 \%$ Secant offset $\mathrm{Pq}=41.12 \mathrm{kN}$

$\mathrm{Kq}$

Validity

$K$ at Maximum Load

$=81.6 \mathrm{MPa} \sqrt{\mathrm{m}}$

$=$ INYAL ID--1, 2, 3

DATA CORRECTIONS USED FOR J-INTEGRAL DATA

Merkle-Corten correction

Correction to J for crack extension (Deformation-J)

OFFSET LOAD $=0 \mathrm{kN}$ OFFSET DEFLECTION $=.001 \mathrm{~mm}$

Fitted elastic load range $=5 \mathrm{kN}$ TO $25 \mathrm{kN}$

Elastic slope $=1.148 E+02 \mathrm{kN} / \mathrm{mm}$ 
Specimen Loadline Deflection (in.)

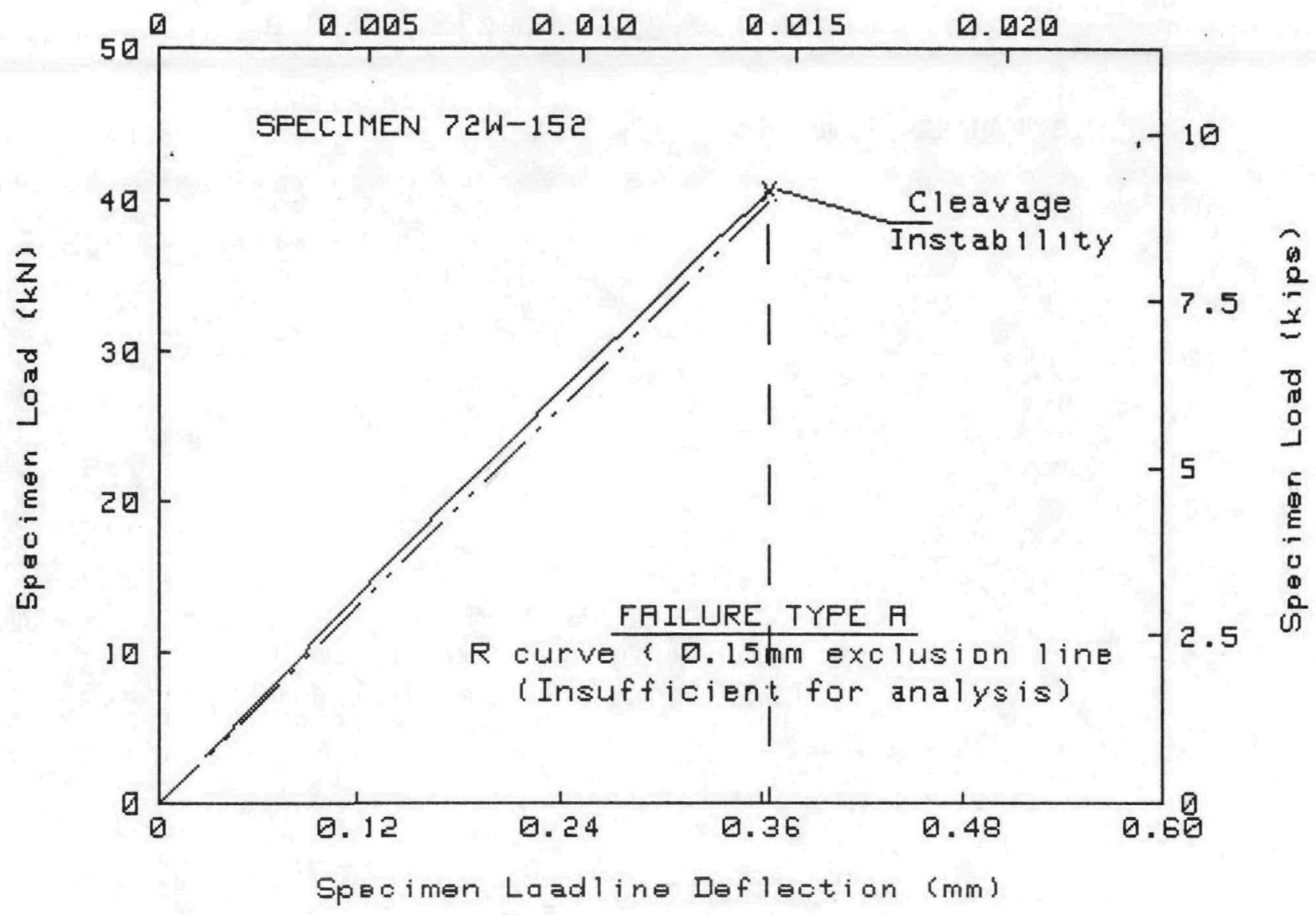

\section{TEST SPECIMEN DATA}

Material Type
Test Temperature
Percent Side Groove
Specimen Thickness B
Initial a/W
Init crack length a
Flow stress
Yield stress
Youngs modulus E
Poisson's Ratio (U)
J-INTEGRAL ANALYSIS

$\mathrm{Je}$

$K j c=\left\langle E J c /\left(1-v^{2}\right)\right\rangle \wedge .5$

Kc (Beta Corrected)

ASTM E399-81 ANALYSIS

Modified Kic Equation;

Maximum Load

$5 \%$ secant off set

$\mathrm{Kq}$

Validity

$K$ at Maximum Load

$P=40.66 \mathrm{kN}$

$\mathrm{Pq}=40.66 \mathrm{kN}$

$=80.7 \mathrm{MPa} \sqrt{\mathrm{m}}$

= INVALID--1, 2

$=80.7 \mathrm{MPa} \sqrt{\mathrm{m}}$
$=$ A533-B WELD (I)

$=50^{\circ} \mathrm{C}$

$=25.4 \mathrm{~mm}$

$=.55$

$=27.94 \mathrm{~mm}$

$=654.5 \mathrm{MPa}$

$=607.1 \mathrm{MPa}$

$=204400 \mathrm{MPa} \quad$ (Estimated Value)

$$
=0
$$

Failure Type $A$ ( $J$ at instability = JC) J

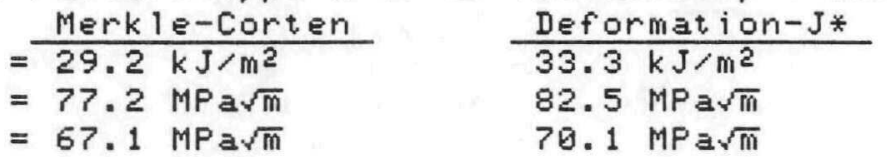

DATA CORRECTIONS USED FOR J-INTEGRAL DATA

Merkle-Corten correction

Correction to J for crack extension (Deformation-J)

OFFSET LOAD $=0 \mathrm{kN}$ OFFSET DEFLECTION $=.001 \mathrm{~mm}$

Fitted elastic load range $=5 \mathrm{kN}$ To $25 \mathrm{kN}$

Elastic slope $=1.148 E+02 \mathrm{kN} / \mathrm{mm}$ 
Specimen Crackmauth Deflection (in.)

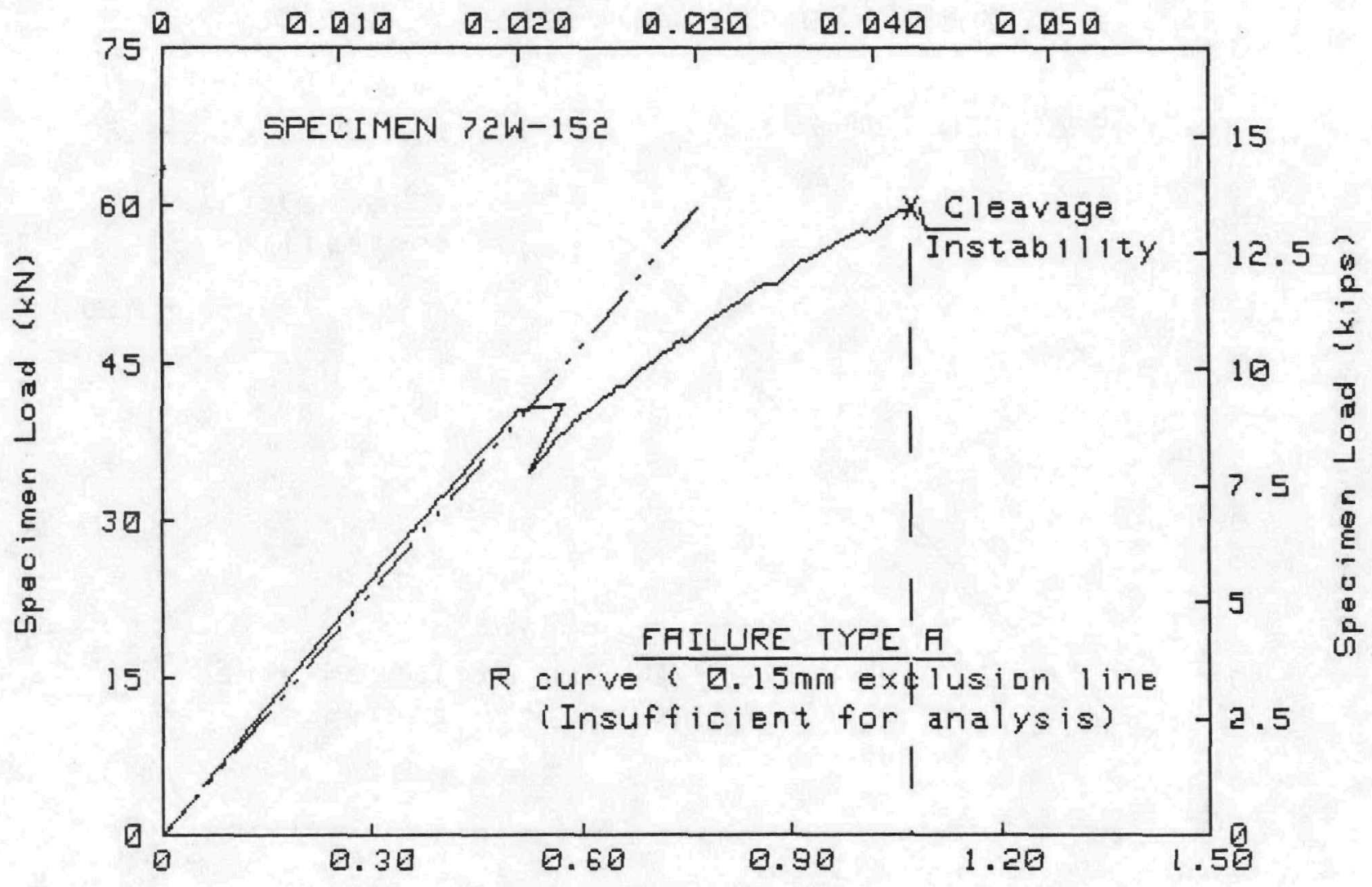

Specimen

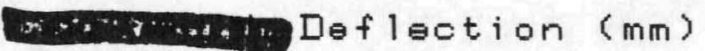

TEST SPECIMEN DATA

Material Type
Test Temperature
Percent Side Groove
Specimen Thickness B
Initial a/W
Init crack length a
Flow stress
Yield stress
Youngs modulus
Poisson's Ratio (U)
J-INTEGRAL RNALYSIS

$=$ A533-B WELD (I)

$=50^{\circ} \mathrm{C}$

$=0 \%$

$=25.4 \mathrm{~mm}$

$=.55$

$=27.94 \mathrm{~mm}$

$=654.5 \mathrm{MPa}$

$=607.1 \mathrm{MPa}$

$=204400 \mathrm{MPa}$

$=0$

[ Failure Type $A$ ( $J$ at instability = Jc) ]

Jc $=\frac{\text { Merkle-Corten }}{108.8 \mathrm{~kJ} / \mathrm{m}^{2}}$ Deformation-J*

$=149.1 \mathrm{MPa} \sqrt{\mathrm{m}}$

$=96.5 \mathrm{MPa} \sqrt{\mathrm{m}}$ $115.4 \mathrm{~kJ} / \mathrm{m}^{2}$ $153.6 \mathrm{MPa} \sqrt{\mathrm{m}}$ $97.8 \mathrm{MPa} \sqrt{\mathrm{m}}$

Ke (Beta Corrected) ASTM E399-81 ANALYSIS

Modified Kic Equation; $B=(B B n) 1 / 2$

Maximum Load $P=59.86 \mathrm{kN}$

$5 \%$ Secant offset $\mathrm{Pq}=41.12 \mathrm{kN}$

$\mathrm{Kq}$

$=81.6 \mathrm{MPa} \sqrt{\mathrm{m}}$

Validity

$K$ at Maximum Load

= INVALID--1, 2, 3

DATA CORRECTIONS USED FOR J-INTEGRAL DATA

Merkle-Corten correction

Correction to J for crack extension (Deformation-J)

OFFSET LOAD $=0 \mathrm{kN}$ OFFSET DEFLECTION $=.011 \mathrm{~mm}$

Fitted elastic load range $=5 \mathrm{kN}$ TO $25 \mathrm{kN}$

Elastic slope $=8.203 E+01 \mathrm{kN} / \mathrm{mm}$

NUREG/CR-5913 


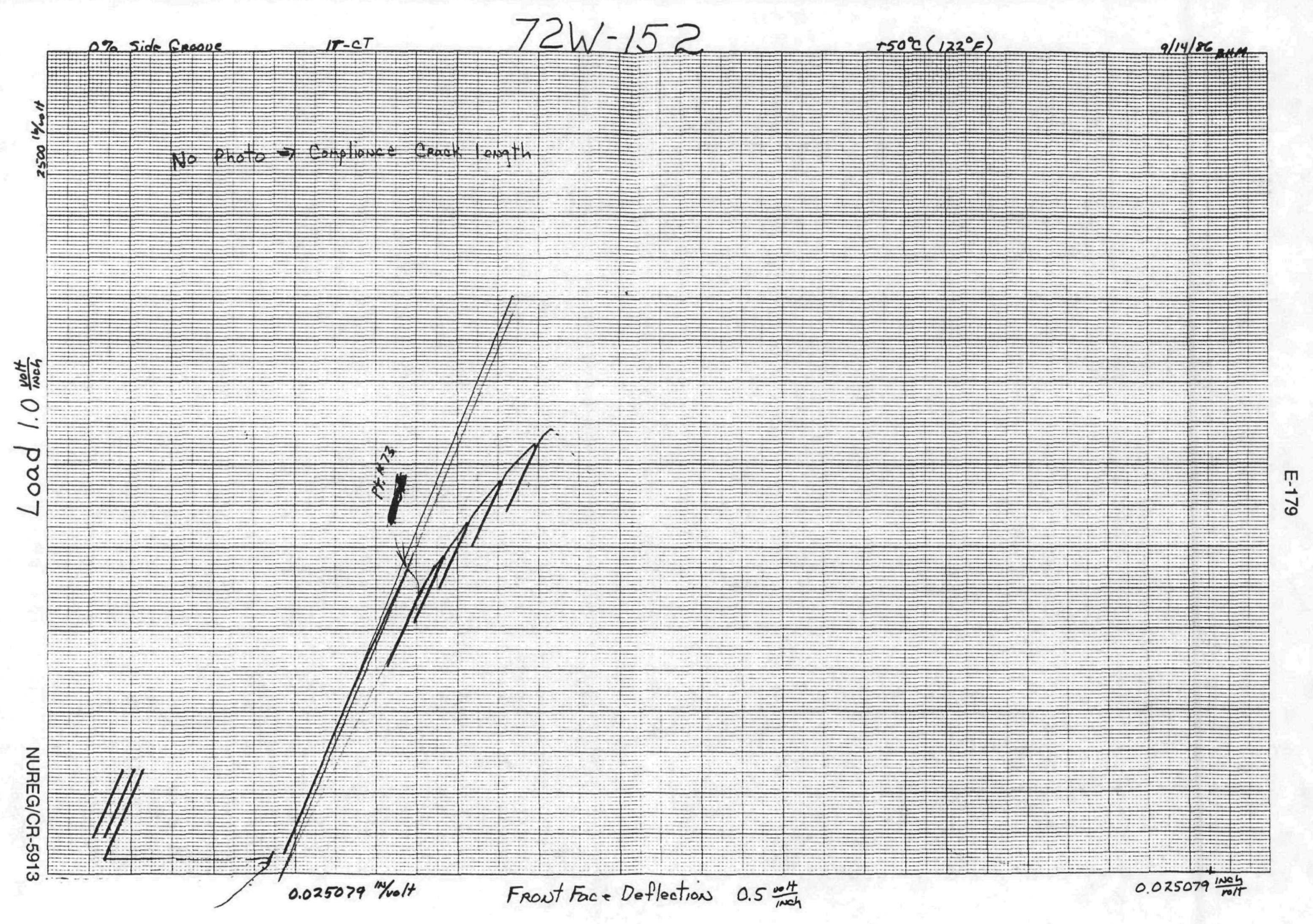


Specimen Laadline Deflection (in.)

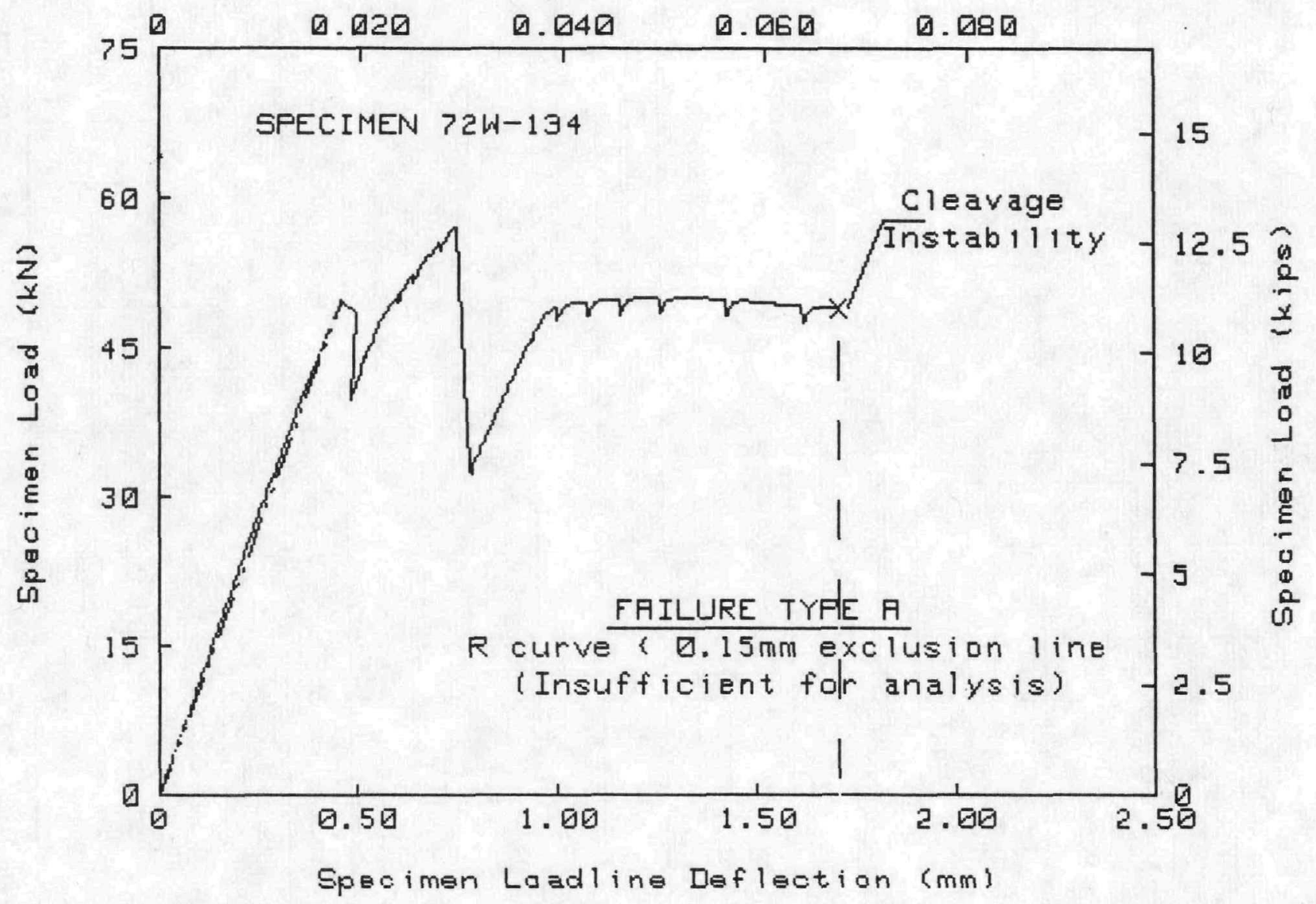

TEST SPECIMEN DATA

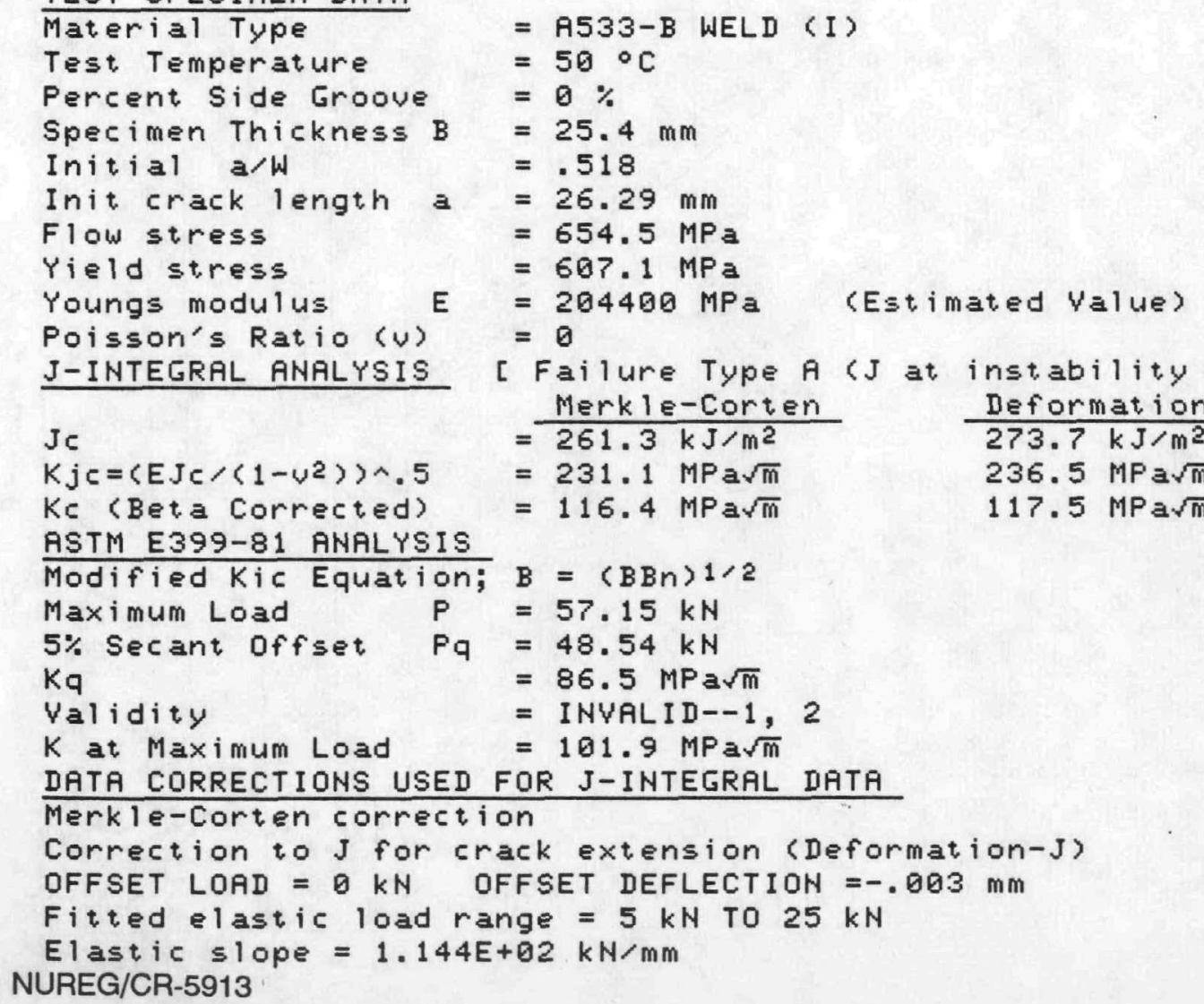


Specimen Loadline Deflection (in.)

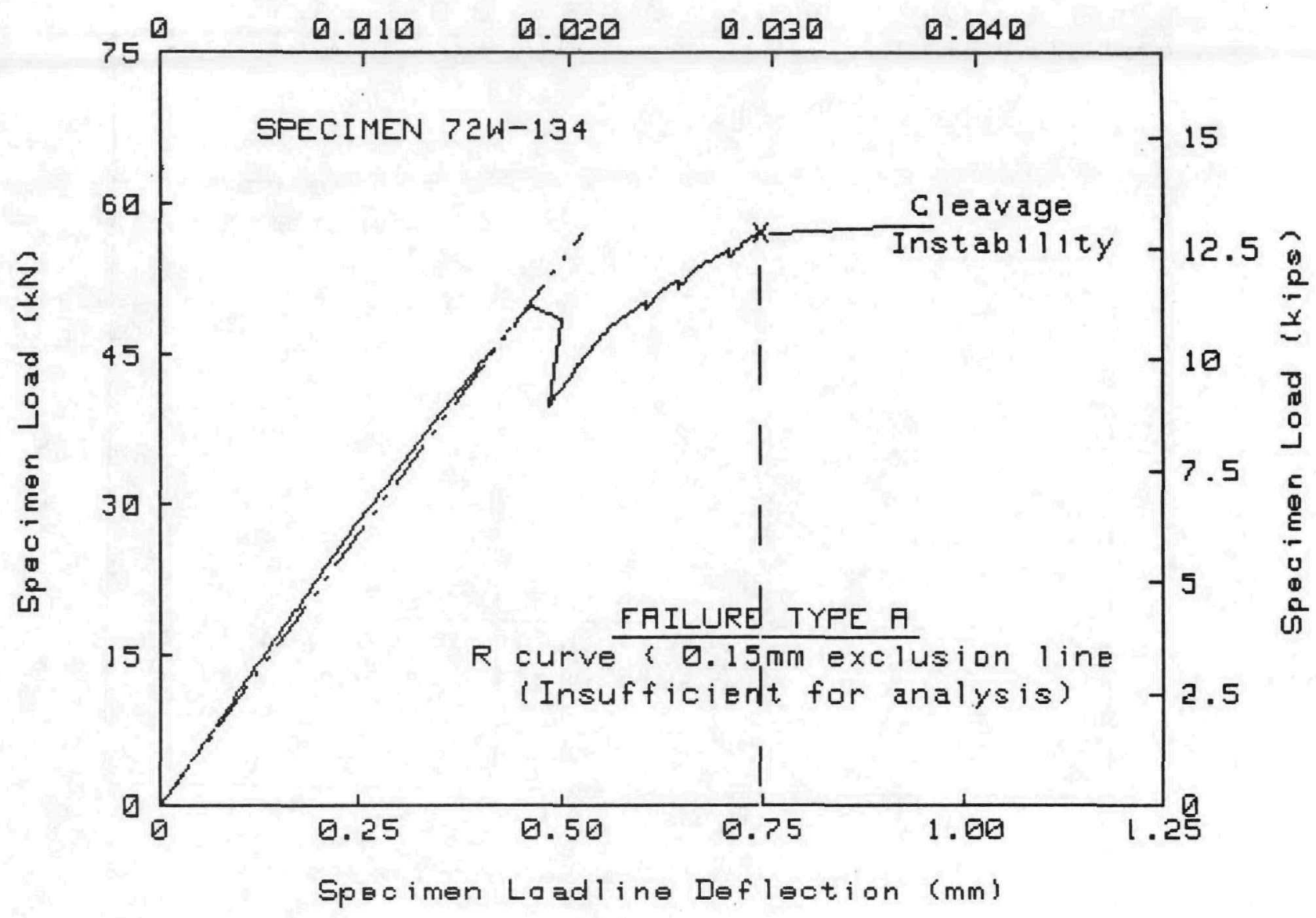

TEST SPECIMEN DATA

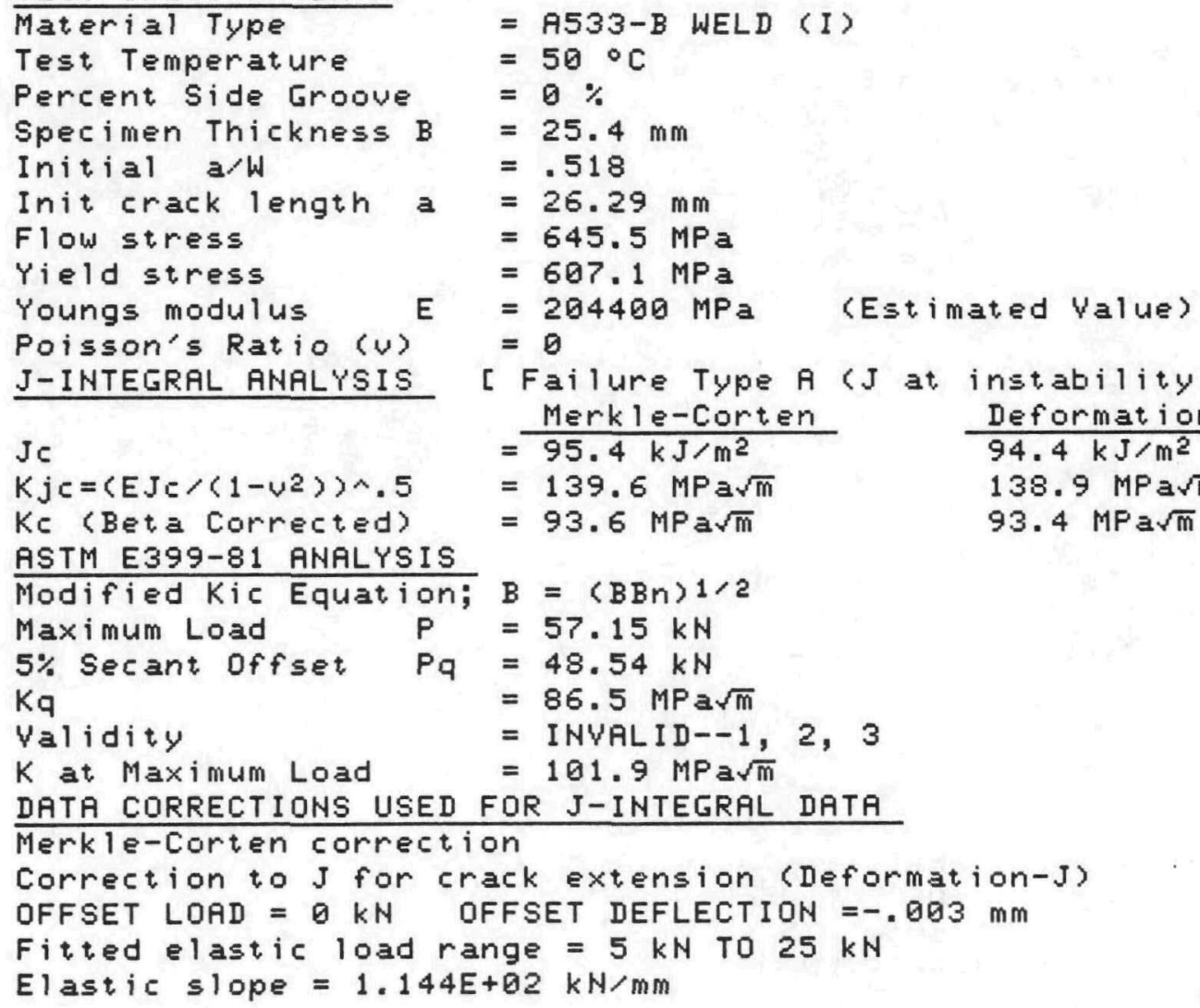


Specimen Loadline Deflection (in.)

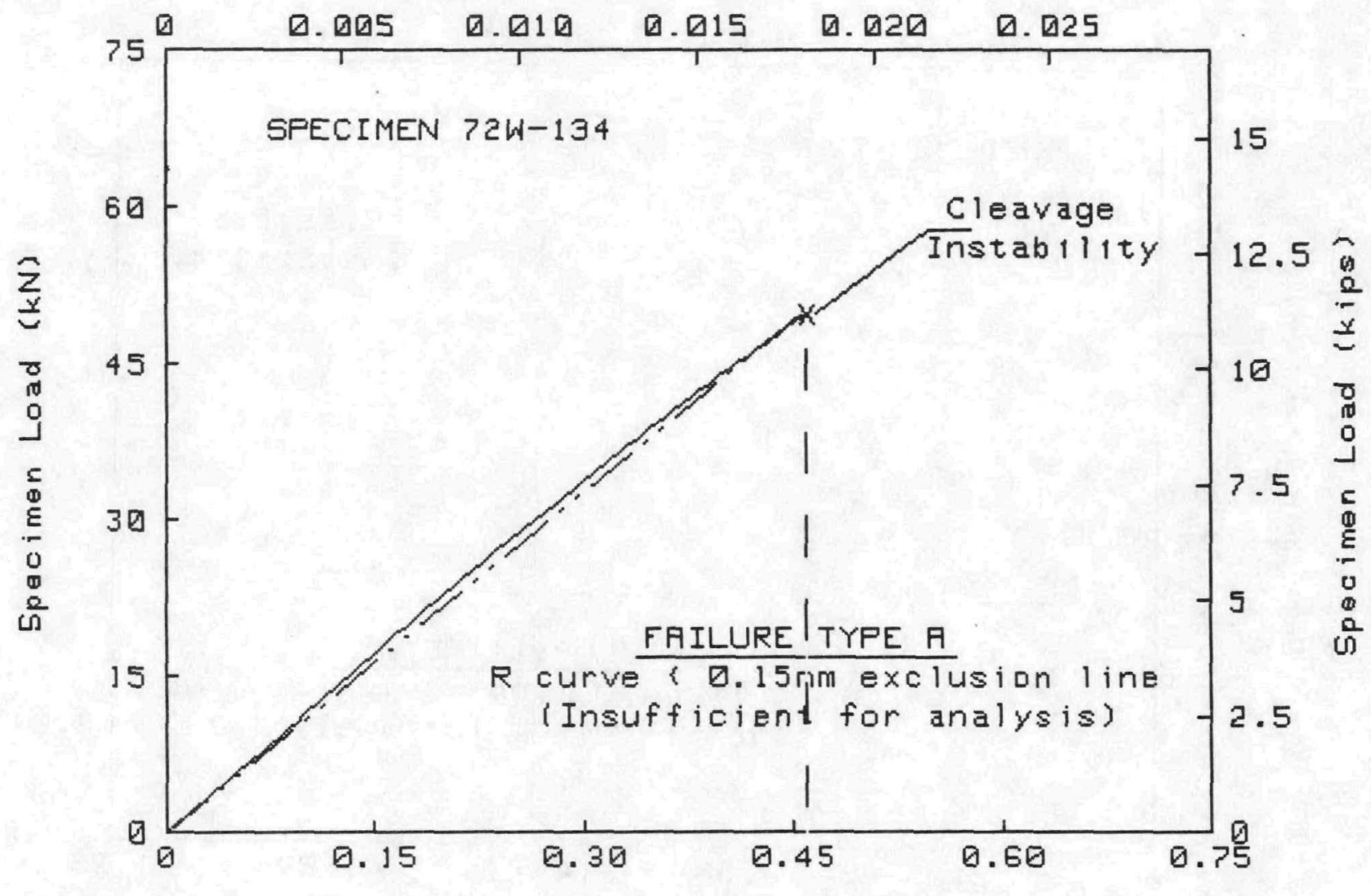

Specimen Laadline Deflection (mm)

\section{TEST SPECIMEN DATA}

Material Type
Test Temperature
Percent Side Groove
Specimen Thickness B
Initial a/W
Init crack length a
Flow stress
Yield stress
Youngs modulus
Poisson's Ratio (U)
J-INTEGRAL ANALYSIS

$\mathrm{Jc}$

$K j c=\left\langle E J c /\left(1-v^{2}\right\rangle\right) \wedge .5$

Ke (Beta Corrected)

ASTM E399-81 ANALYSIS

Modified Kic Equation; $B=\langle$ (BBn) $1 / 2$
Maximum Load $P$

$5 \%$ Secant offset $P q=48.54 \mathrm{kN}$

$\mathrm{Kq}$

Validity

$K$ at Maximum Load

$=A 533-B$ WELD

(I)

$=50^{\circ} \mathrm{C}$

$=0 \%$

$=25.4 \mathrm{~mm}$

$=.518$

$=26.29 \mathrm{~mm}$

$=654.5 \mathrm{MPa}$

$=607.1 \mathrm{MPa}$

$=204400 \mathrm{MPa}$

$=0$

[ Failure Type $A$ ( $J$ at instability = JC) ]

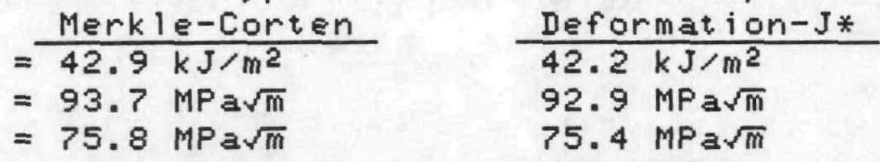

DATA CORRECTIONS USED FOR J-INTEGRAL DATA

Merkle-Corten correction

Correction to J for crack extension (Deformation-J)

OFFSET LOAD $=0 \mathrm{kN}$ OFFSET DEFLECTION $=-.003 \mathrm{~mm}$

Fitted elastic load range $=5 \mathrm{kN}$ TO $25 \mathrm{kN}$

Elastic slope $=1.144 E+02 \mathrm{kN} / \mathrm{mm}$

NUREG/CR-5913 
Specimen Crackmouth Deflection (in.)

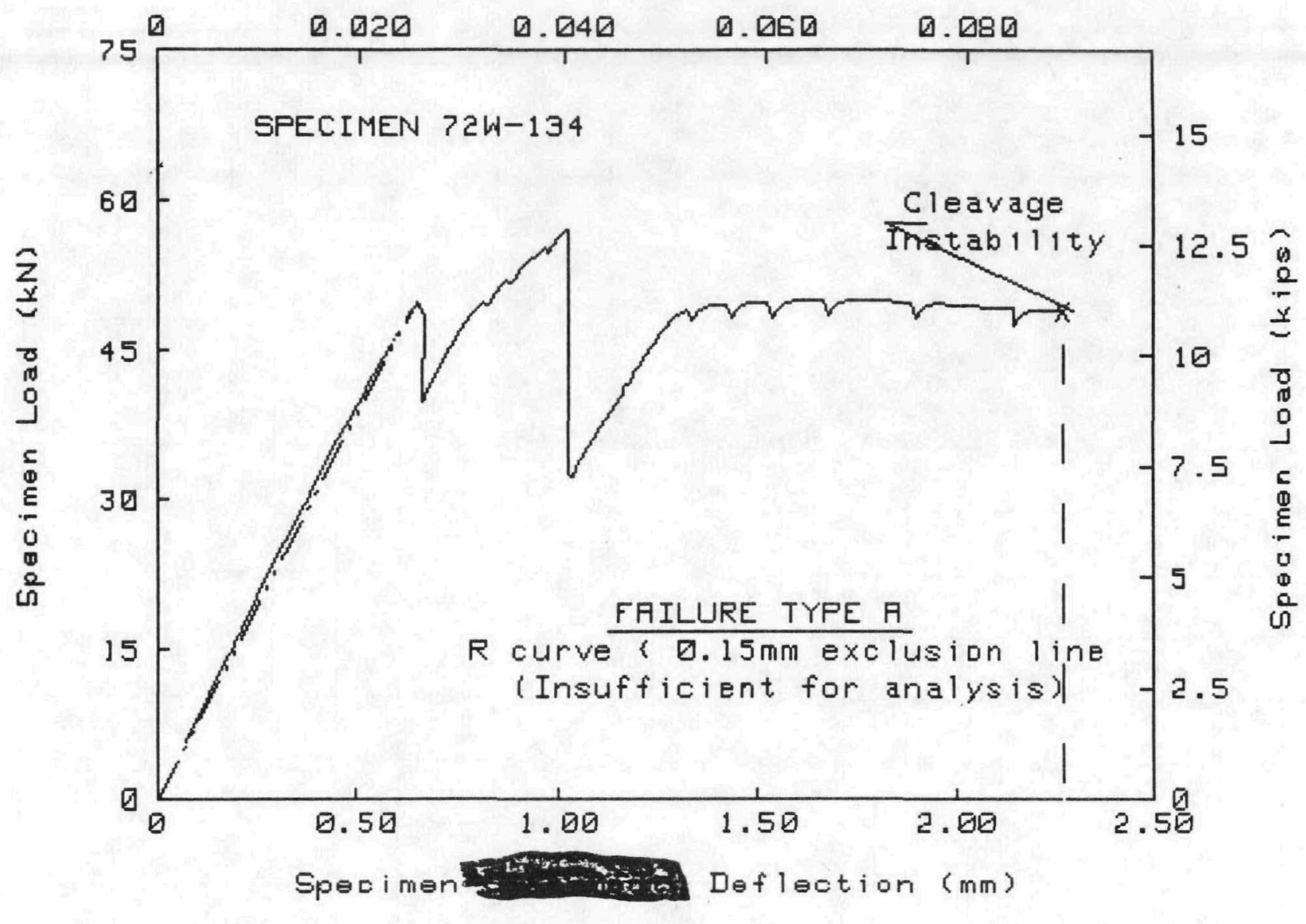

TEST SPECIMEN DATA

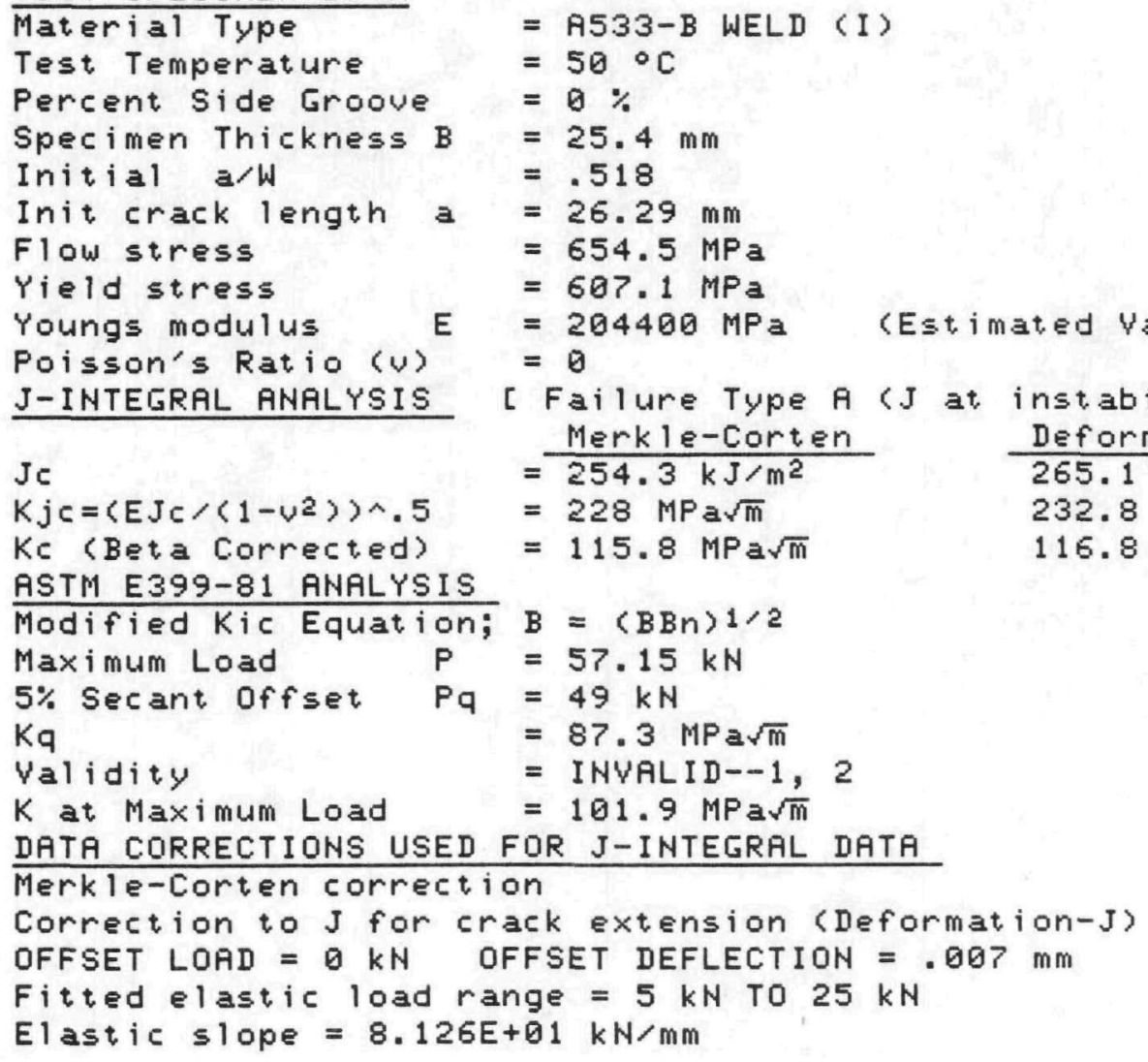




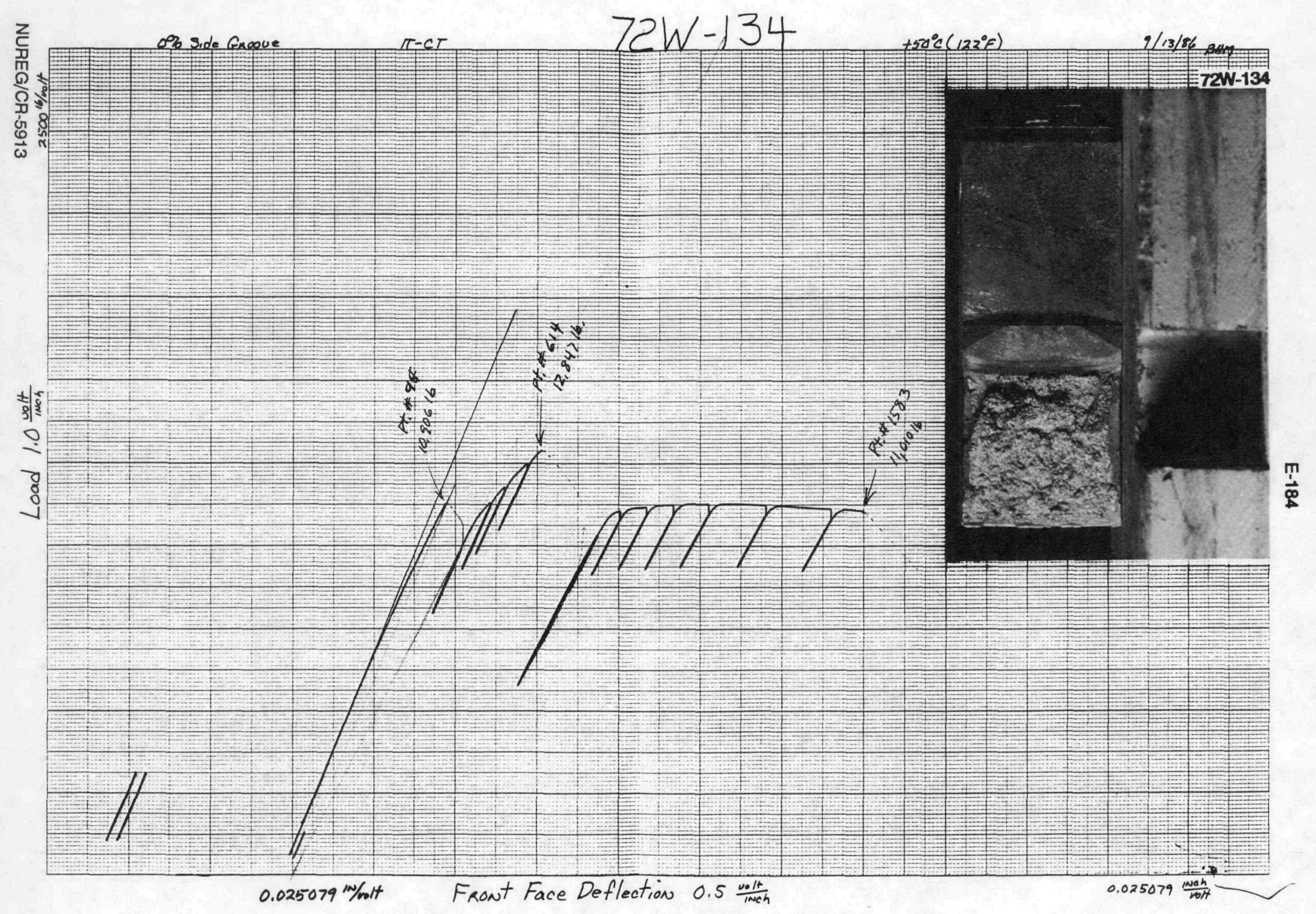




\section{E-185}

SPEC IMEN ID $=72 W 116$

MATERIAL TYPE $=$ MCUWELD

TEST TEMPERATURE $=75$ DEG $\mathrm{C}$

THICKNESS $=25.4 \mathrm{~mm}$

SIDE GRDDUES = $0 \%$

INITIAL CRACK LENGTH $=26.8 \mathrm{~mm}$

$a / W) i=.527$

MEASURED DUCTILE DELTA-A $=.05 \mathrm{~mm}$

YIELD STRENGTH $=621 \mathrm{MPa}$

FLOW STRENGTH $=672 \mathrm{MPa}$

ESTIMATED YOUNG'S MODULUS $=203$ GPa

SPECIMEN CLEAUED AT KQ= $119 \mathrm{MPa}$ SQR $[\mathrm{m}]$ (NOT UALID KIc)

UALUES AT MAXIMUM LDAD

J-INTEGRAL $=94.8 \mathrm{~kJ} / \mathrm{m}^{\wedge} 2$

$\mathrm{KJc}=138.7 \mathrm{MPa} \operatorname{SQR}[\mathrm{m}]$

$K$-beta-c $=94.3 \mathrm{MPa} \operatorname{SQR}[\mathrm{m}]$
UALUES at CLEaUAGE LDAD

J-INTEGRAL $=94.8 \mathrm{~kJ} / \mathrm{m}^{\wedge} 2$

$K J c=138.7 \mathrm{MPa} S Q R[\mathrm{~m}]$

K-beta-c $=94.3 \mathrm{MPa} S Q R[\mathrm{~m}]$ 



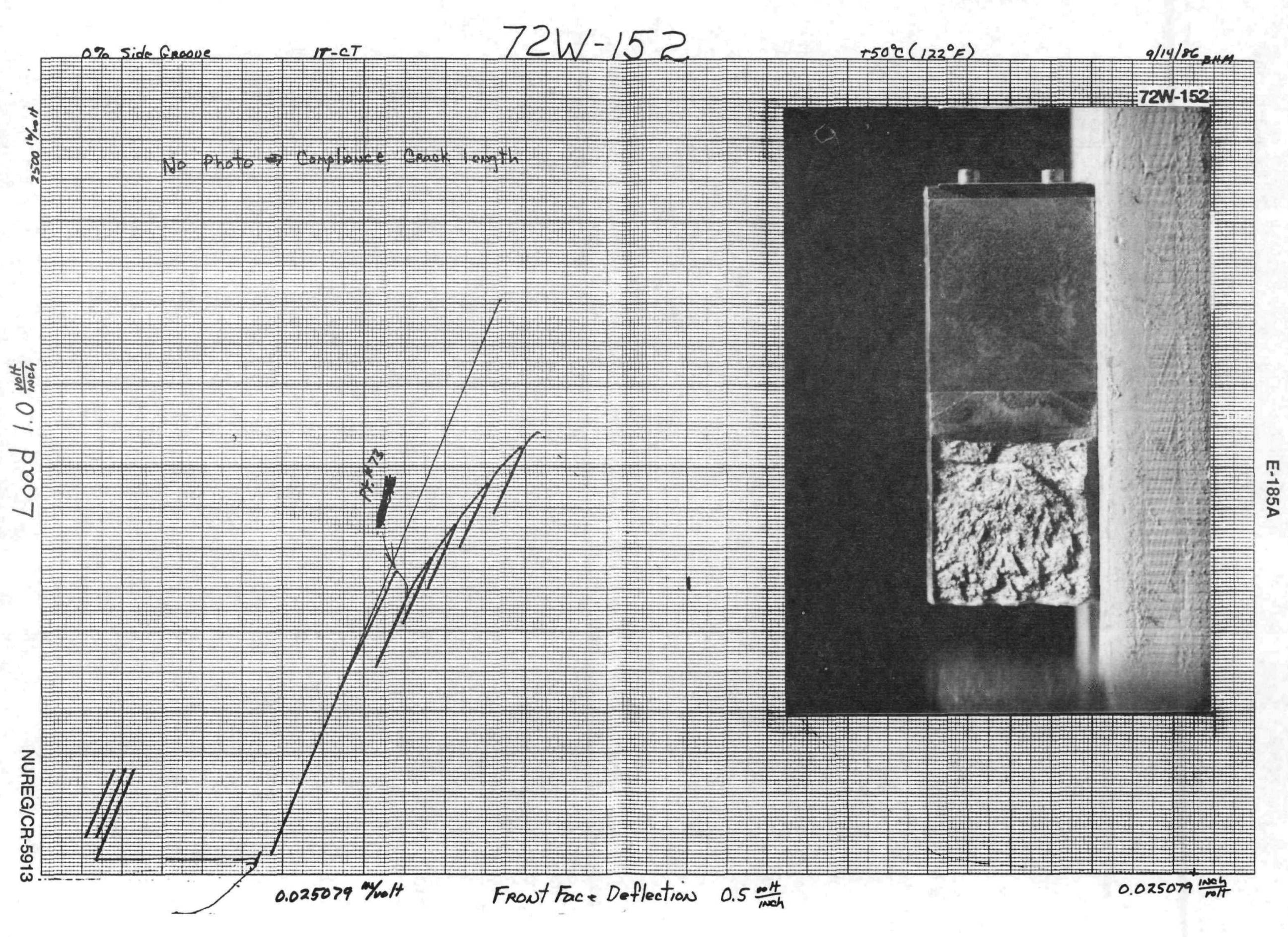




\section{$E-185 B$}

. ecimen Crackmauth Def lection (in.)

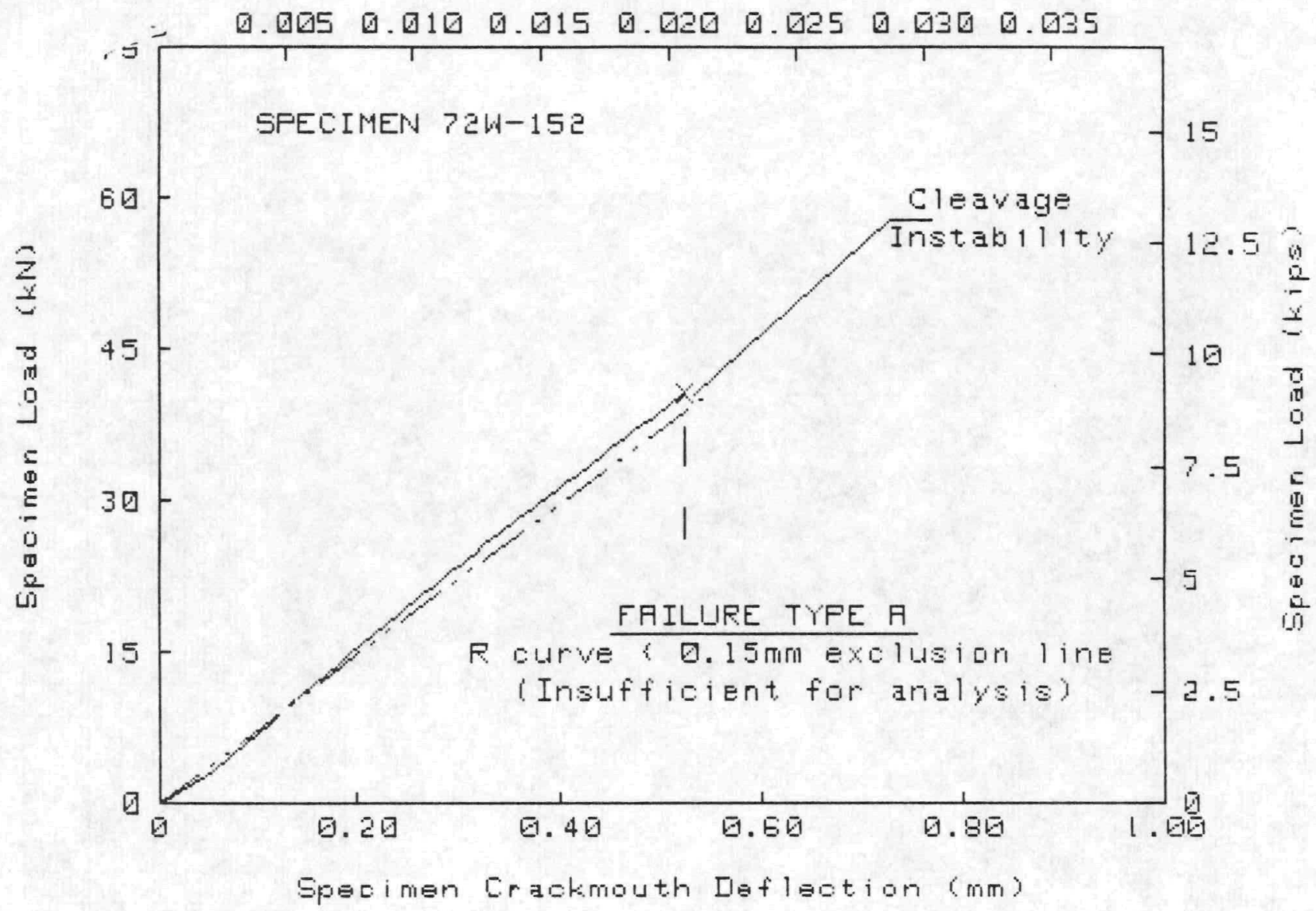

TEST SFECIMEN DATA

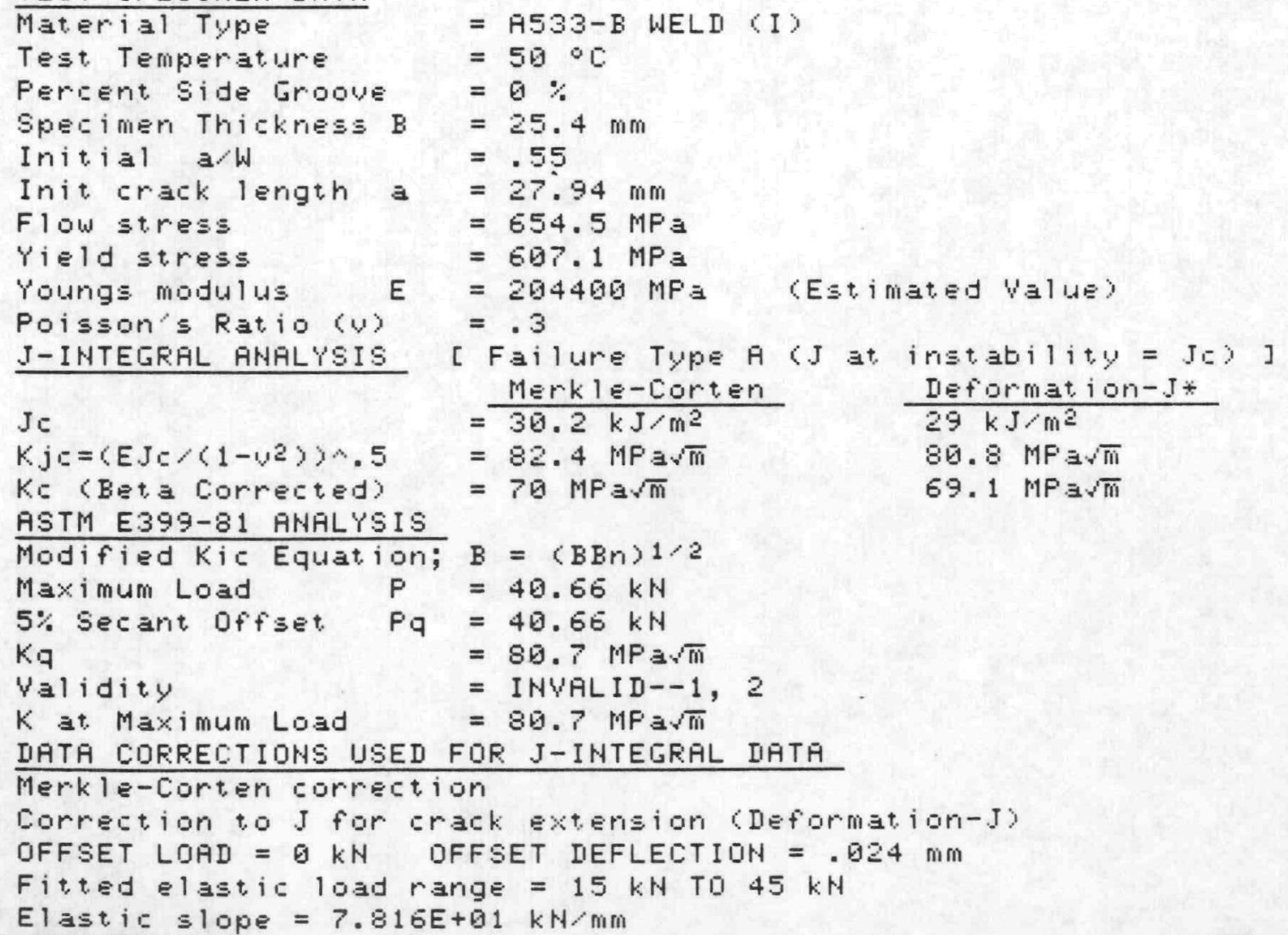




\section{E-185C}

Specimen Erackmouth Deflection (in.)

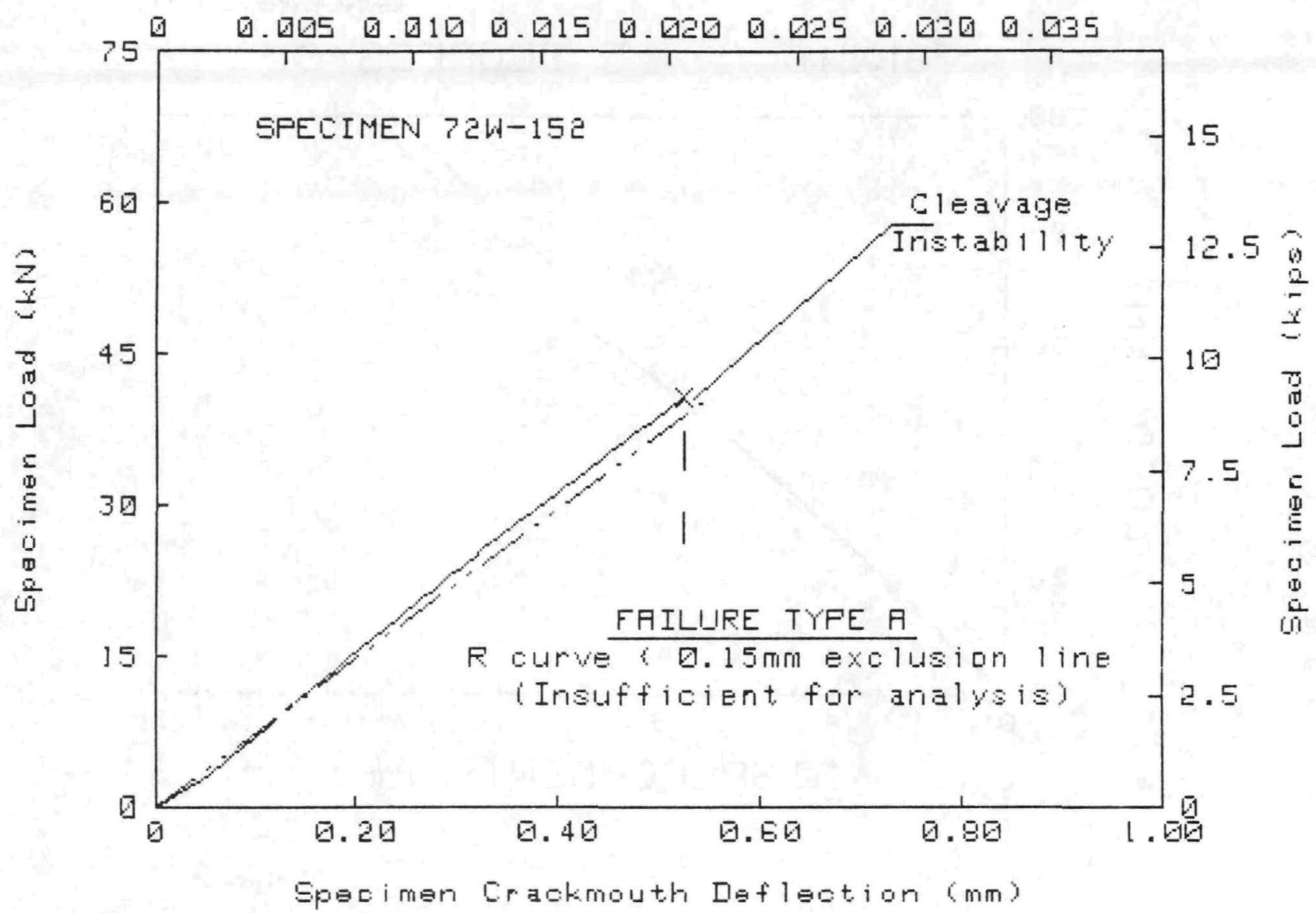

TEST SPECIMEN IATA
Material TYPe
Test Temperature
Fercent Side Groque
Spetimen Thickness B
Initial a/W
Init crack length a
Floi stress
Yield stress
Youngs modulus
Poisson's Ratio (U)
J-INTEGRAL HWALYSIS

$\mathrm{Jc}$

$K j c=\left\langle E J c /\left(1-v^{2}\right\rangle\right) \cdots .5$

Kc (Beta Corrected)

ASTM E399-81 ANALYSIS

Modified Kic Equation;

5\% Secant offset.

$\mathrm{Kq}$

Validity

$K$ at Maximum Load

$$
\begin{aligned}
& \mathrm{P}=40.66 \mathrm{kN} \\
& \mathrm{Pq}=40.66 \mathrm{kN} \\
& =80.7 \mathrm{MPa} \sqrt{\mathrm{m}} \\
& \text { = INVALID--1, } 2 \\
& =80.7 \mathrm{MPa} \text {. } \\
& =\frac{\text { Merkle-Corten }}{30.2 \mathrm{~kJ} / \mathrm{m}^{2}} \\
& =78.6 \mathrm{MPa} \cdot \mathrm{m} \\
& =67.9 \mathrm{MPa} \sqrt{\mathrm{m}}
\end{aligned}
$$$$
=A 533-\mathrm{E} \text { WELD (I) }
$$$$
=500 \mathrm{C}
$$$$
=0 \%
$$$$
=25.4 \mathrm{~mm}
$$$$
=.55
$$$$
=27.94 \mathrm{~mm}
$$$$
=654.5 \mathrm{MPa}
$$$$
=607.1 \mathrm{MPa}
$$$$
=204400 \mathrm{MPa}
$$$$
=0
$$

(Estimated Value)

DATA CORRECTIONS USED FOR J-INTEGRAL IIATA

Merkle-Corten Eorrection

Corregtion to J for crack extension (IEformation-J)

DFFSET LOAD $=0 \mathrm{kN}$ OFFSET DEFLECTIDH $=.024 \mathrm{~mm}$

Fitted Elastic load range $=15 \mathrm{kH} \mathrm{TD} 45 \mathrm{kH}$

Elastic slope $=7.816 E+01 \mathrm{kH} / \mathrm{mm}$ 


\section{E-186}

ORNL-DWG 92-11615

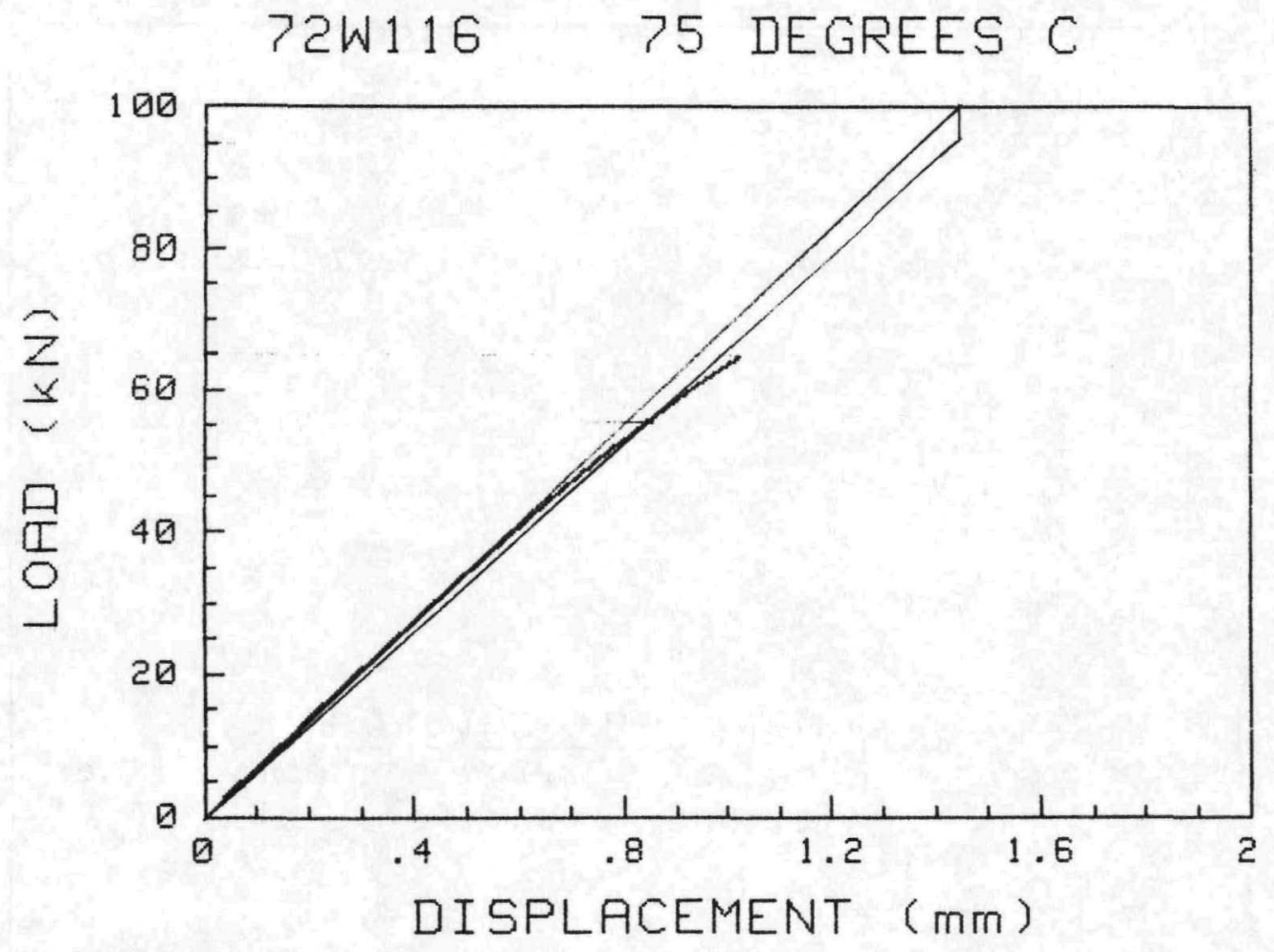


Specimen Loadline Deflection (in.)

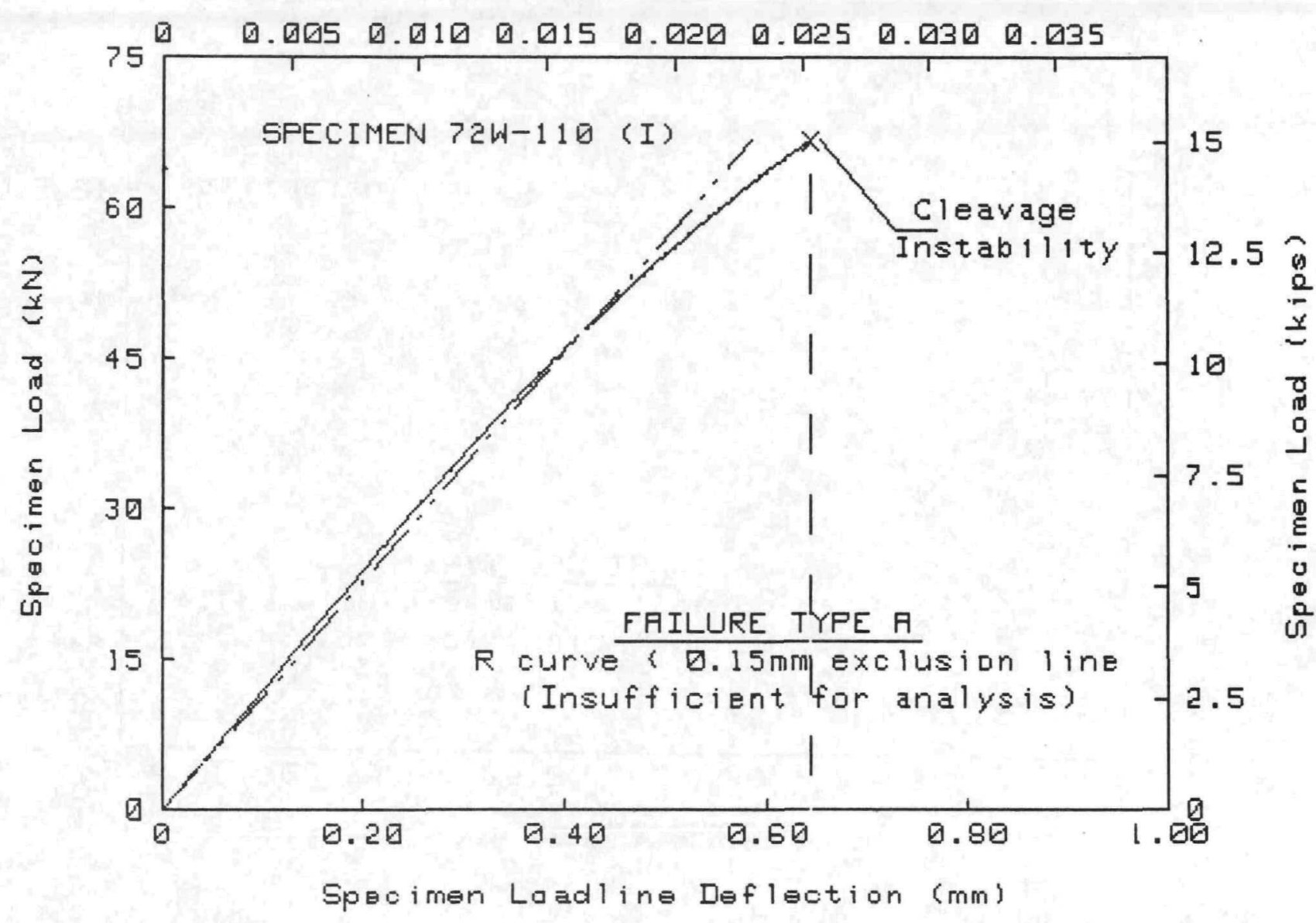

TEST SPECIMEN DATA

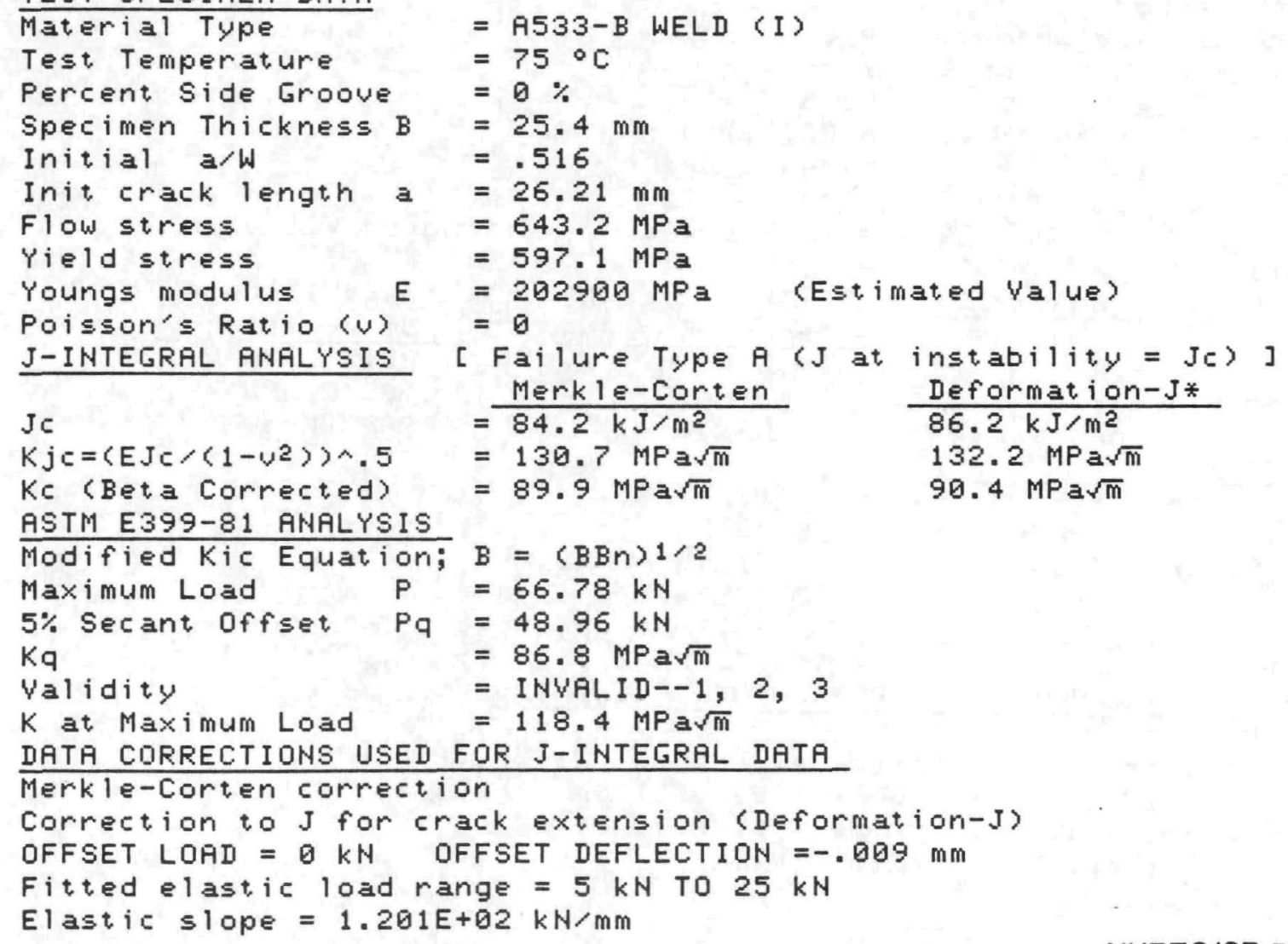


Specimen Crackmouth Deflection (in.)

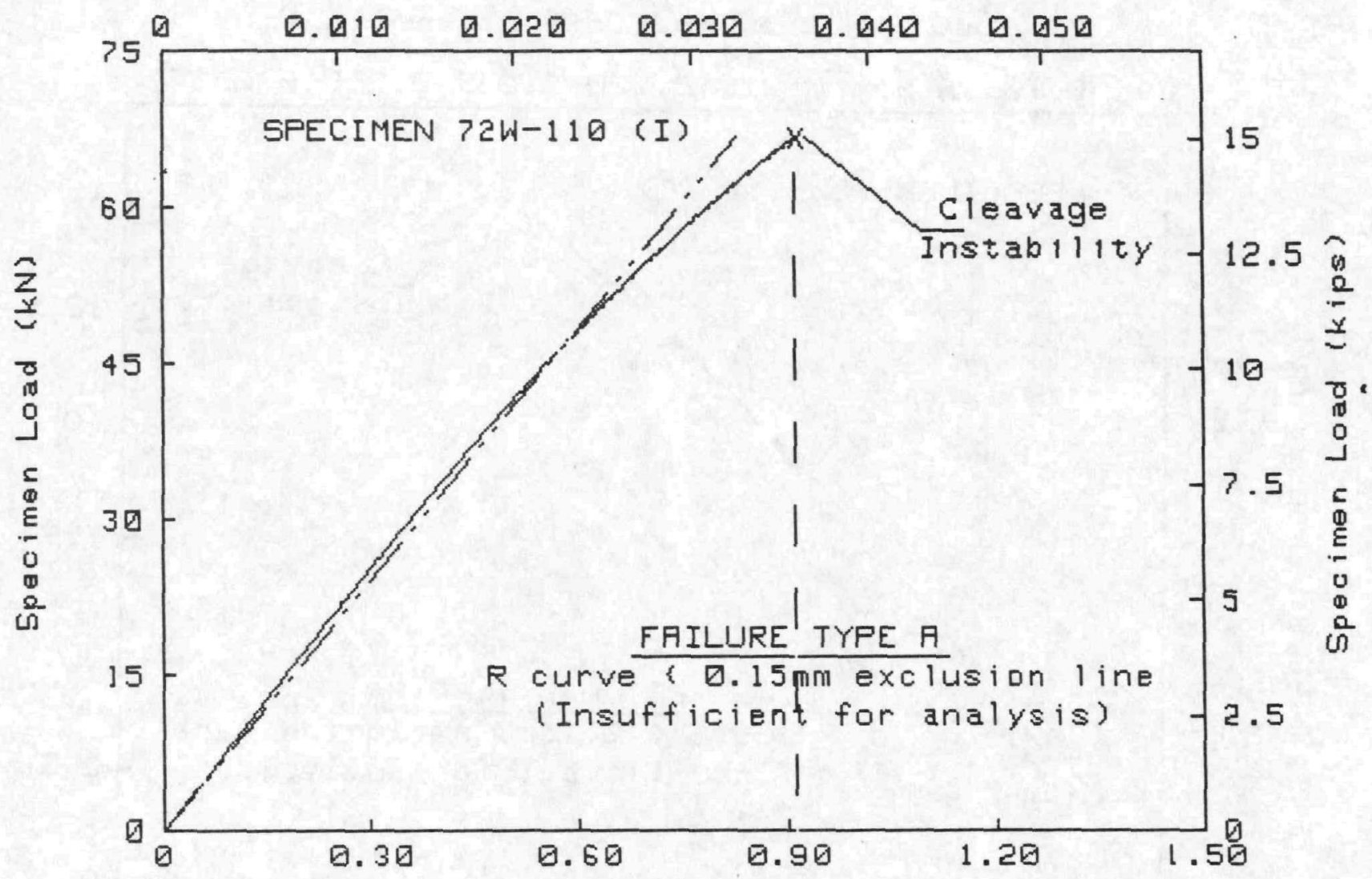

Specimen Fistis: Def leotion (mm)

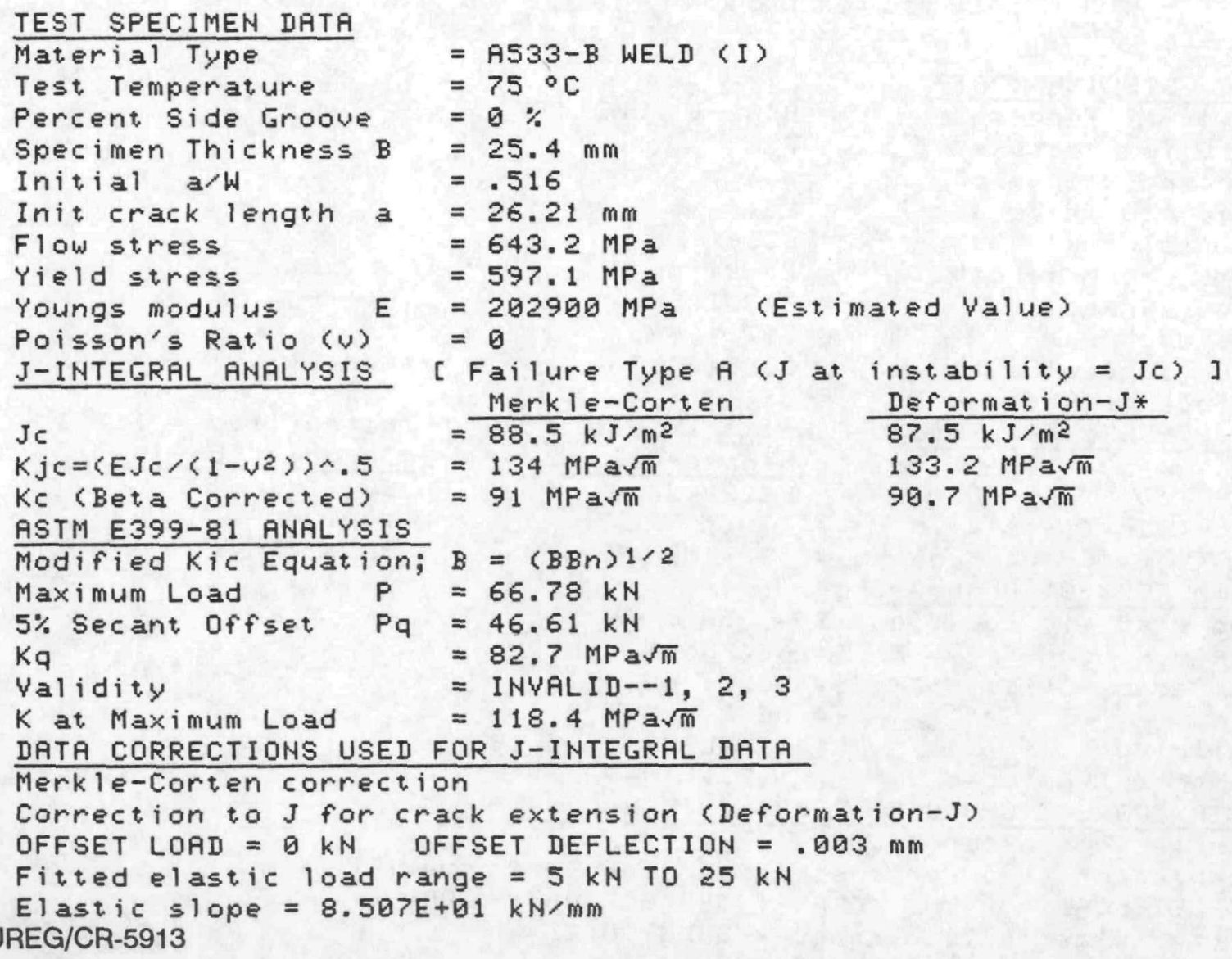




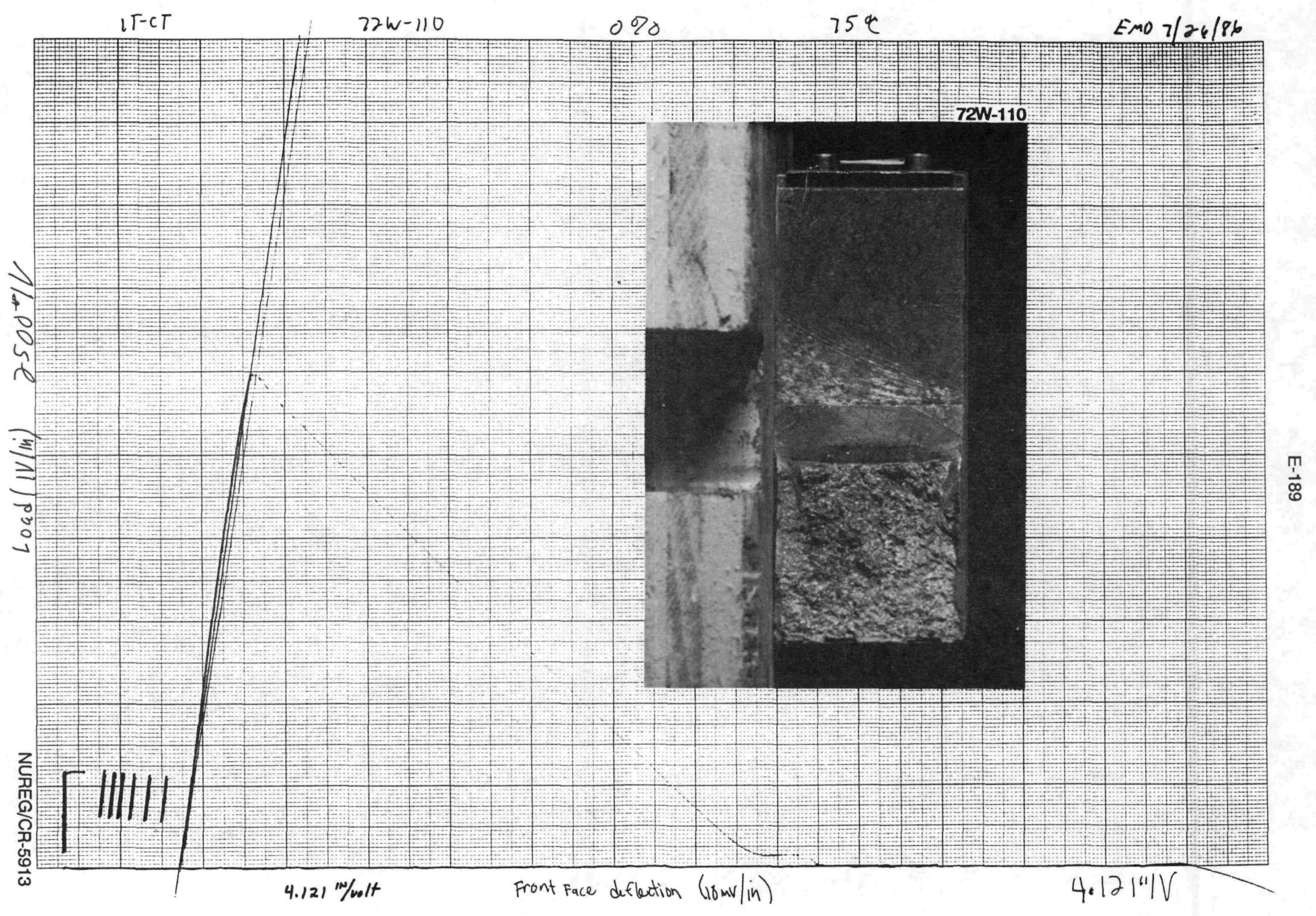


Specimen Loadline Deflection (in.)

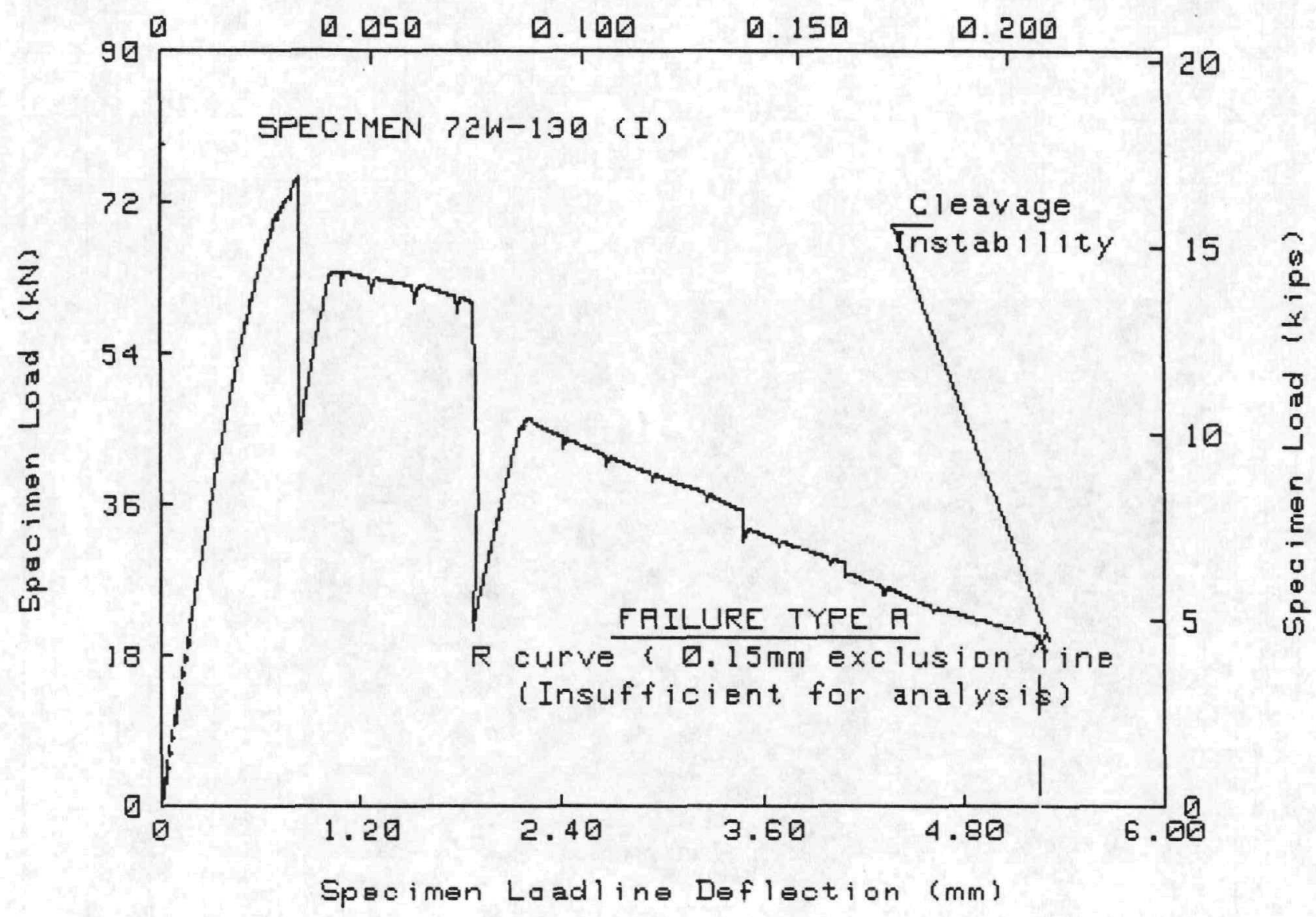

TEST SFECIMEN DATA

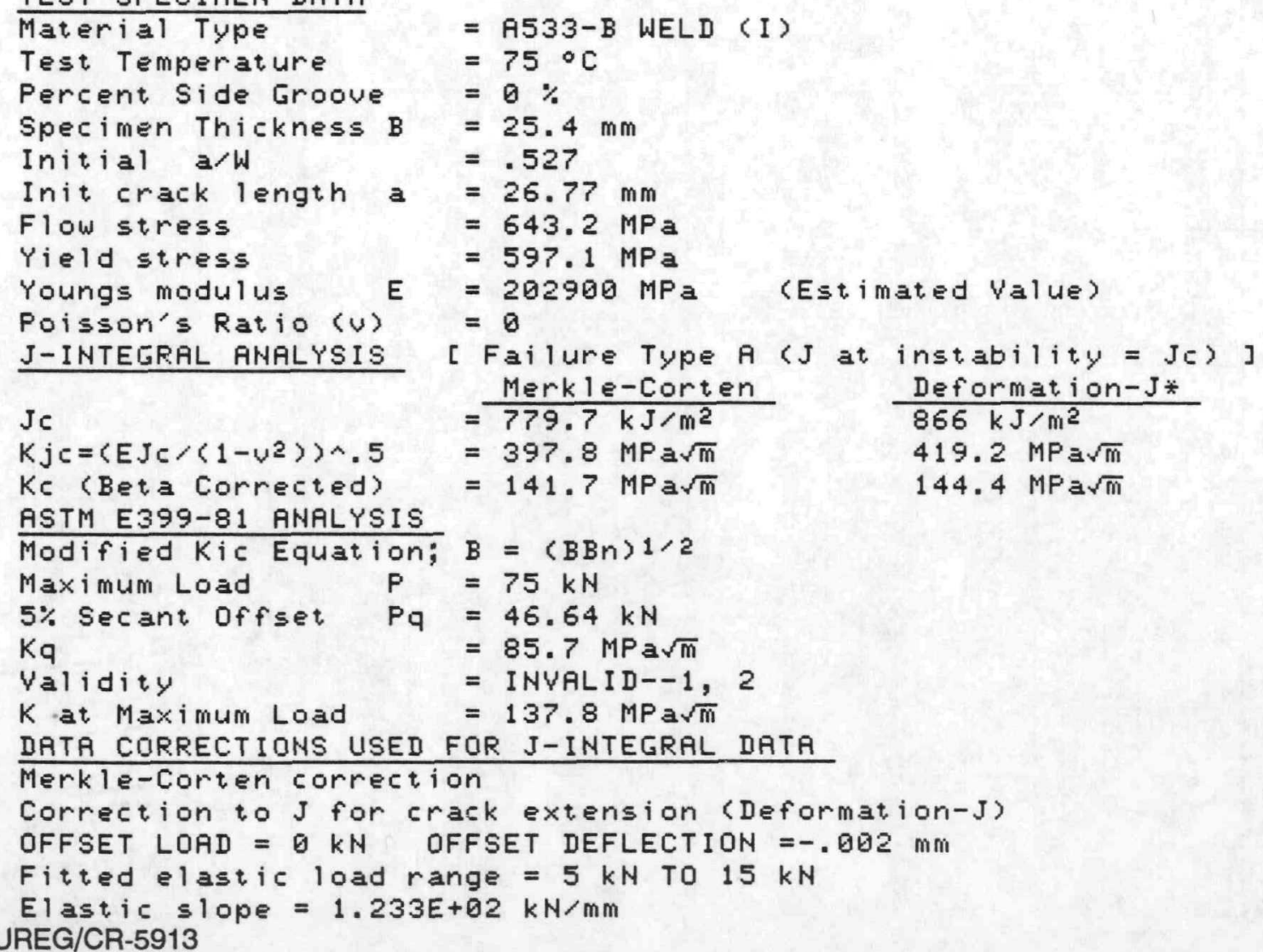


Specimen Loadline Deflection (in.)

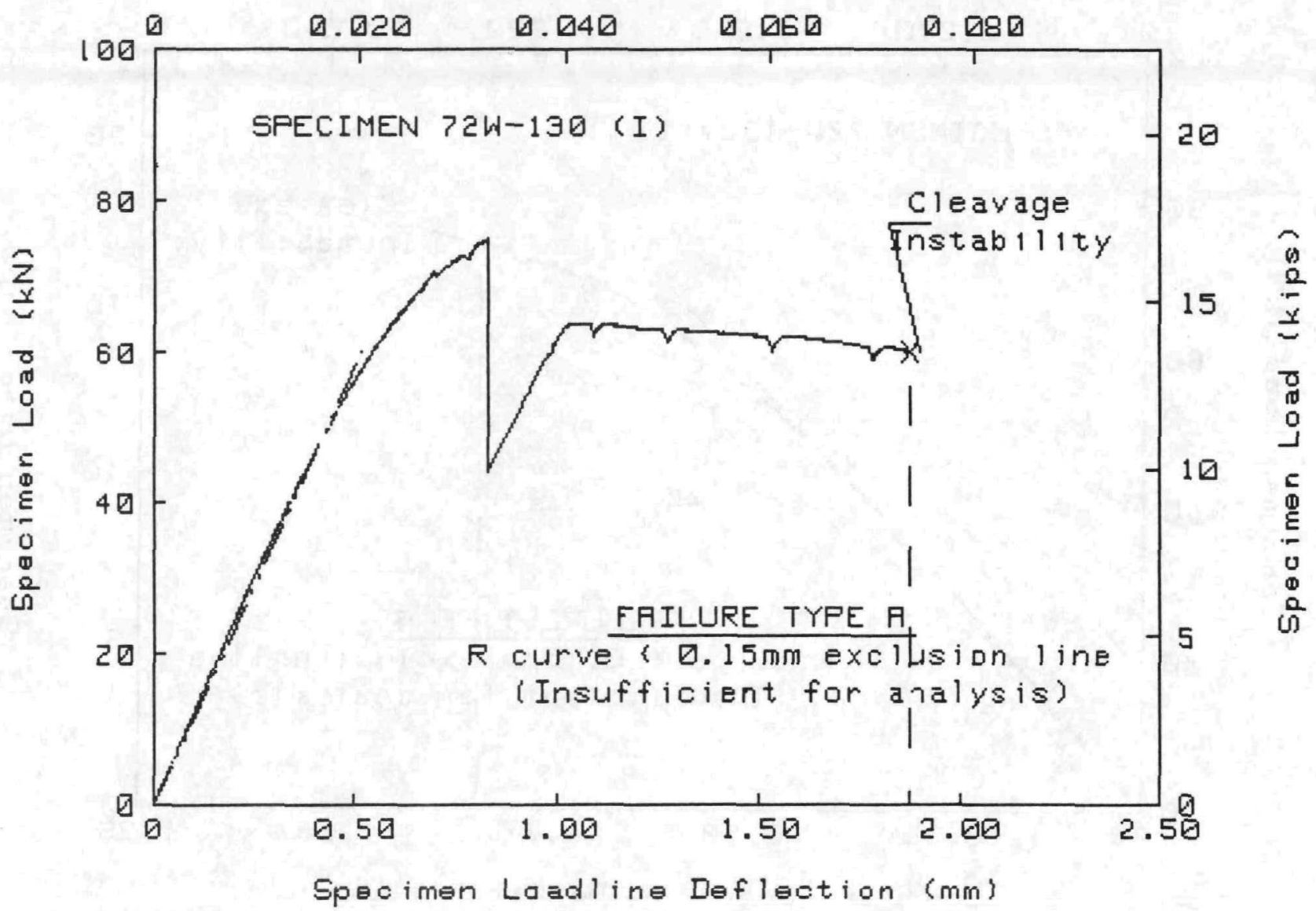

TEST SPECIMEN DATA

Material Type
Test Temperature
Percent Side Groove
Specimen Thickness B
Initial a/W
Init crack length a
Flow stress
Yield stress
Youngs modulus
Poisson's Ratio ( $)$
J-INTEGRA ANALYSIS

$=$ A533-B WELD (I)

$=75^{\circ} \mathrm{C}$

$=0 \%$

$=25.4 \mathrm{~mm}$

$=.527$

$=26.77 \mathrm{~mm}$

$=643.2 \mathrm{MPa}$

$=567.1 \mathrm{MPa}$

$=202900 \mathrm{MPa}$ (Estimated Value)

J-INTEGRAL ANALYSIS

$\mathrm{Jc}$

$=0$

[ Failure Type $A$ ( $J$ at instability = Jc) ]

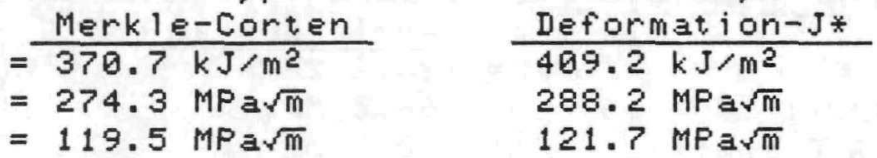

$K \mathrm{j} c=\left\langle\mathrm{E} \cdot \mathrm{J} c /\left(1-v^{2}\right\rangle\right\rangle^{\wedge} .5$

Kc (Beta Corrected)

ASTM E399-81 ANALYSIS

Modified Kic Equation; $B=\langle(B B n) 1 / 2$

Maximum Load $P=75 \mathrm{kN}$

$5 \%$ Secant offset. $\mathrm{Pq}=48.84 \mathrm{kN}$

$\mathrm{Kq}$

$=89.7 \mathrm{MPa} \sqrt{\mathrm{m}}$

Validity

= INVALID--1, 2, 3

$K$ at Maximum Load

$=137.8 \mathrm{MPa} \sqrt{\mathrm{m}}$

DATA CORRECTIONS USED FOR J-INTEGRAL DATA

Merkle-Corten correction

Correction to J for crack extension (Deformation-J)

DFFSET LOAD $=0 \mathrm{kN}$ OFFSET DEFLECTION $=-.001 \mathrm{~mm}$

Fitted elastic load range $=5 \mathrm{kN}$ TO $25 \mathrm{kN}$

Elastic slope $=1.222 E+02 \mathrm{kN} / \mathrm{mm}$ 
Specimen Loadline Deflection (in.)

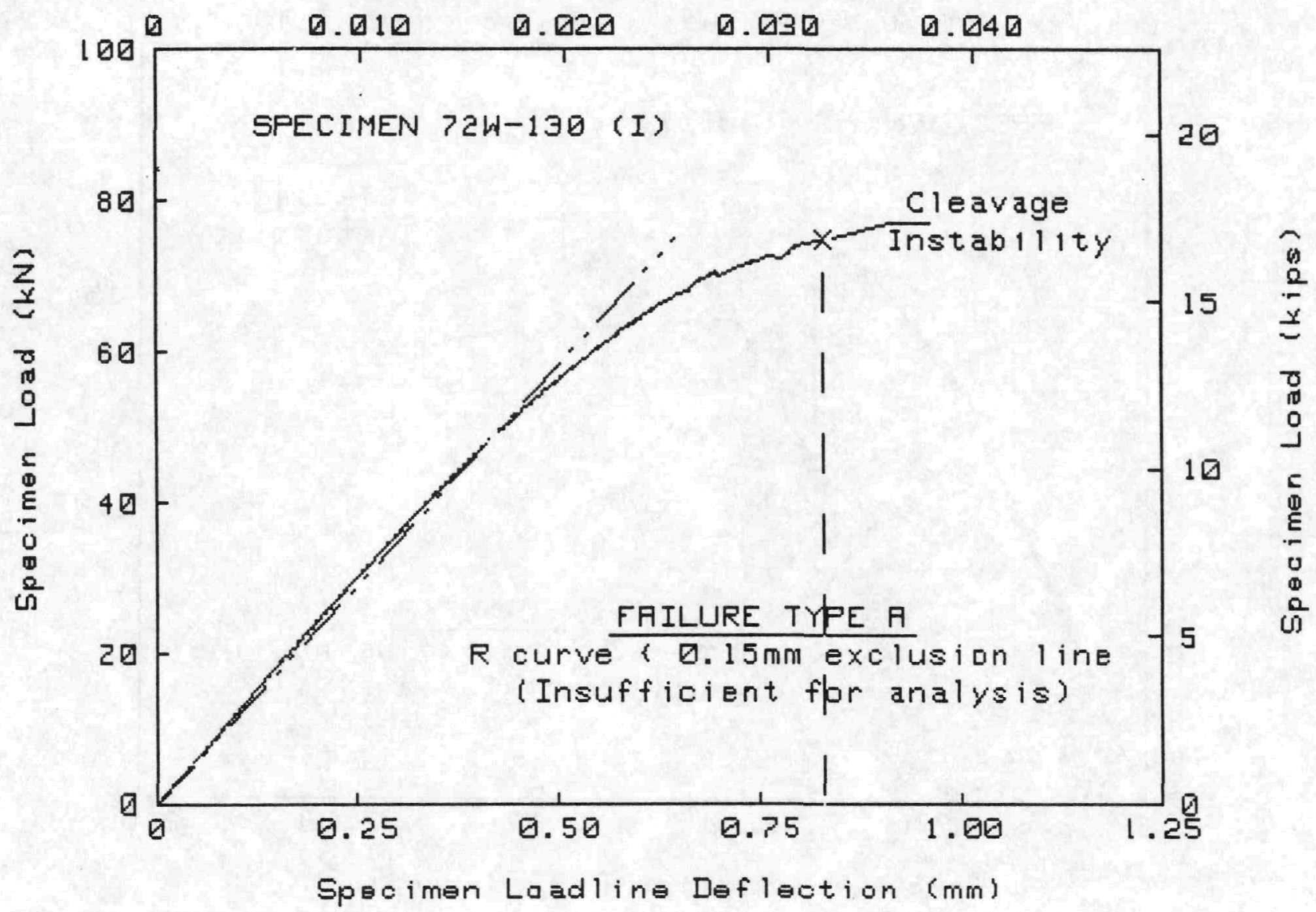

TEST SPECIMEN DATA

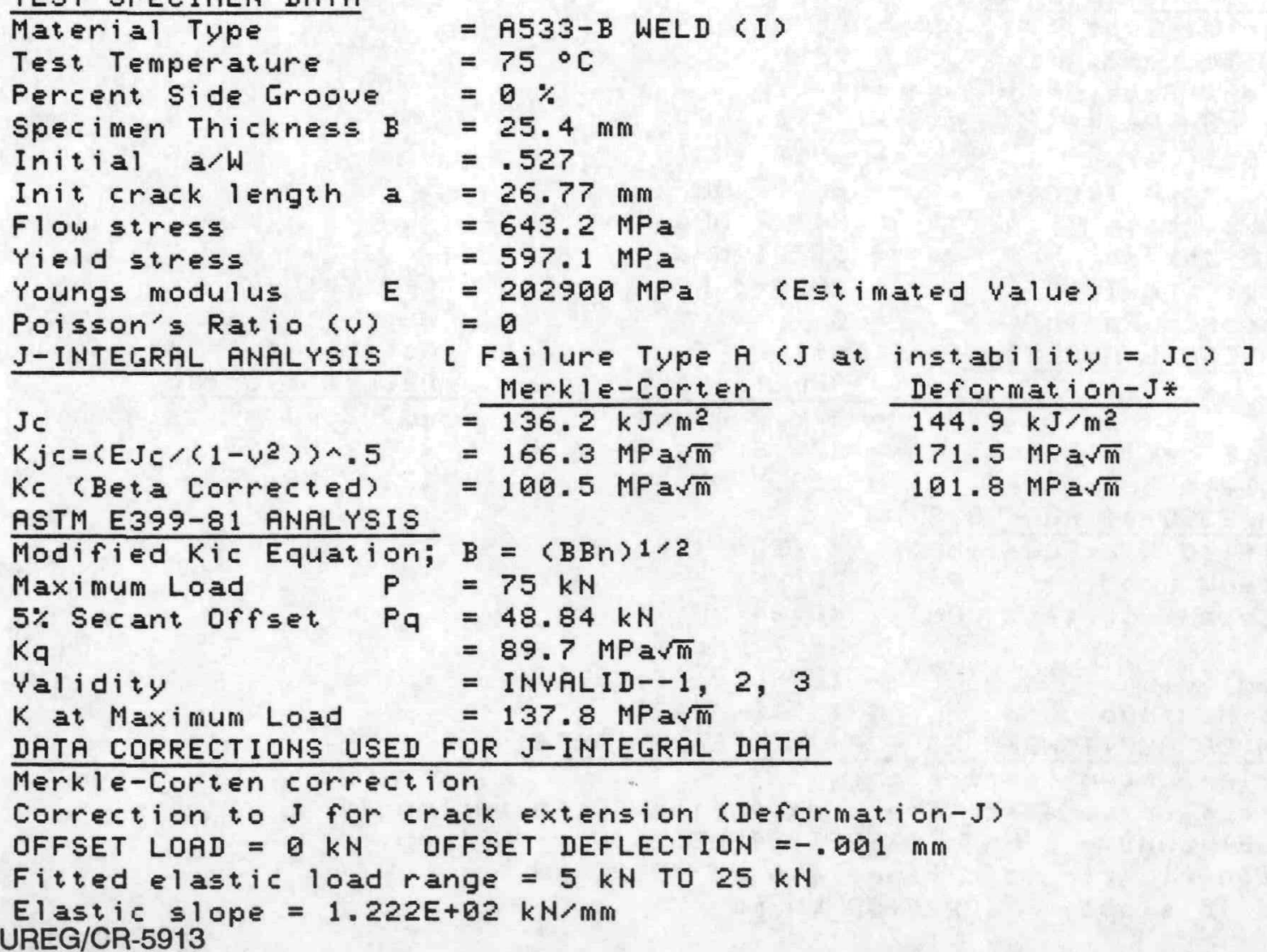


Specimen Crackmouth Deflection (in.)

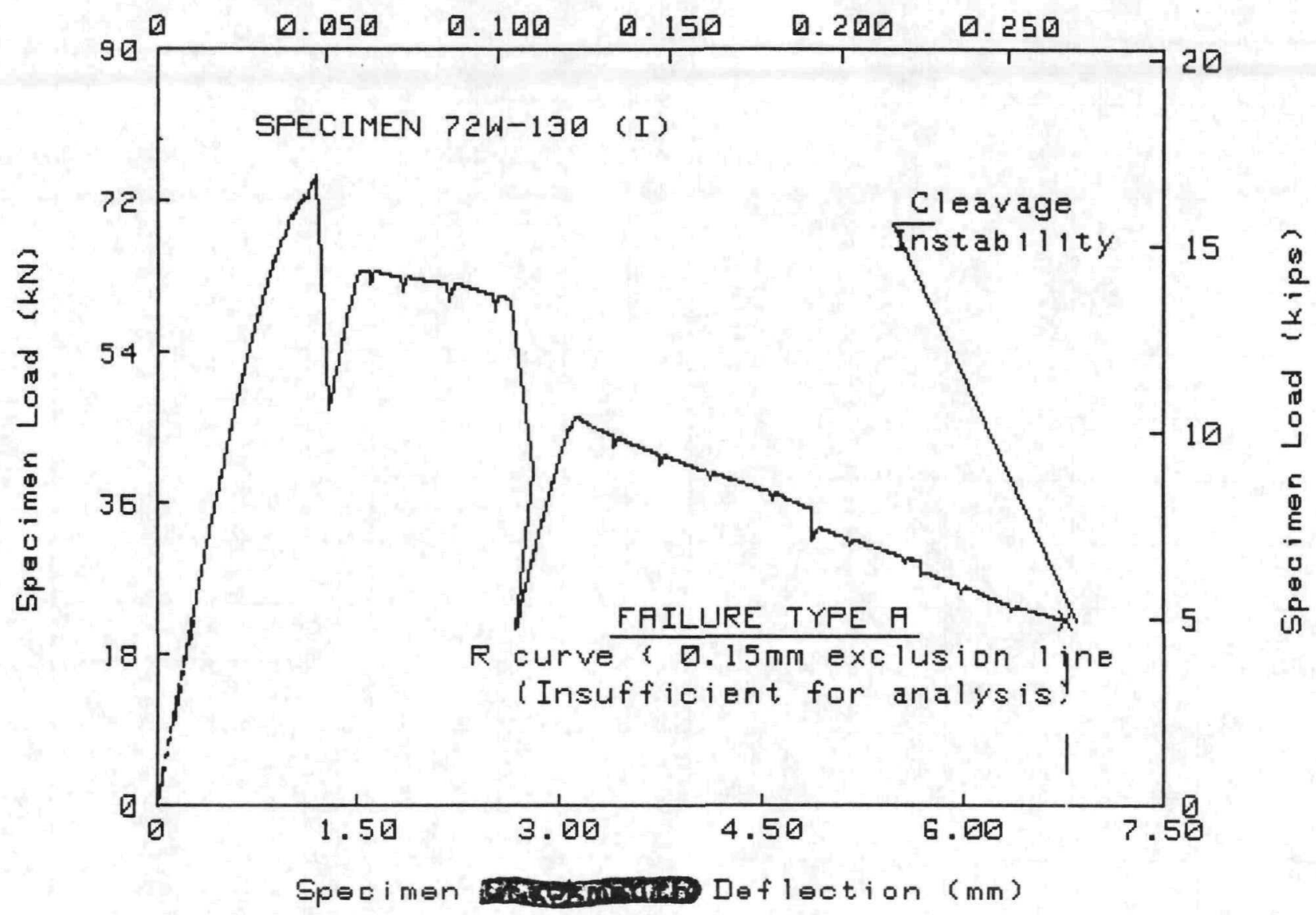

TEST SPECIMEN DATA

Material Type
Test Temperature
Percent Side Groove
Specimen Thickness B
Initial a/W
Init crack length a
Flow stress
Yield stress
Youngs modulus
Poisson's Ratio (U)
J-INTEGRAL ANALYSIS

$=$ A533-B WELD $(I)$

$=75 \circ \mathrm{C}$

$=0 \%$

$=25.4 \mathrm{~mm}$

$=.527$

$=26.77 \mathrm{~mm}$

$=643.2 \mathrm{MPa}$

$=597.1 \mathrm{MPa}$

Yield stress

$=202900 \mathrm{MPa}$

(Estimated Value)

J-INTEGRAL ANALYSIS

$\mathrm{Jc}$

$$
=0
$$

[ Failure Type $A$ ( $J$ at instability $=J C$ ) ]

$K j c=\left\langle E J c /\left(1-v^{2}\right)\right\rangle \wedge .5$

$=\frac{\text { Merkle-Corten }}{776.6 \mathrm{~kJ} / \mathrm{m}^{2}}$

$=397 \mathrm{MPa} \sqrt{\mathrm{m}}$

$=141.6 \mathrm{MPa} \sqrt{\mathrm{m}}$ $\frac{\text { Deformat i on-J* }}{861.1 \mathrm{~kJ} / \mathrm{m}^{2}}$

$418.1 \mathrm{MPa} \sqrt{\mathrm{m}}$

$144.3 \mathrm{MPa} \sqrt{\mathrm{m}}$

ASTM E399-81 ANALYSIS

Modified Kic Equation; $B=\langle B B n\rangle 1 / 2$

Maximum Load $P=75 \mathrm{kN}$

$5 \%$ Secant offset $P q=43.29 \mathrm{kN}$

$\mathrm{Kq}$

$=79.6 \mathrm{MPa} \sqrt{\mathrm{m}}$

Validity

$K$ at Maximum Load

= INVALID--1, 2

$=137.8 \mathrm{MPa} \sqrt{\mathrm{m}}$

DATA CORRECTIONS USED FOR J-INTEGRAL DATA

Merkle-Corten correction

Correction to J for crack extension (Deformation-J)

OFFSET LOAD $=0 \mathrm{kN}$ OFFSET DEFLECTION $=.01 \mathrm{~mm}$

Fitted elastic load range $=5 \mathrm{kN}$ TO $15 \mathrm{kN}$

Elastic slope $=8.630 E+01 \mathrm{kN} / \mathrm{mm}$

NUREG/CR-5913 


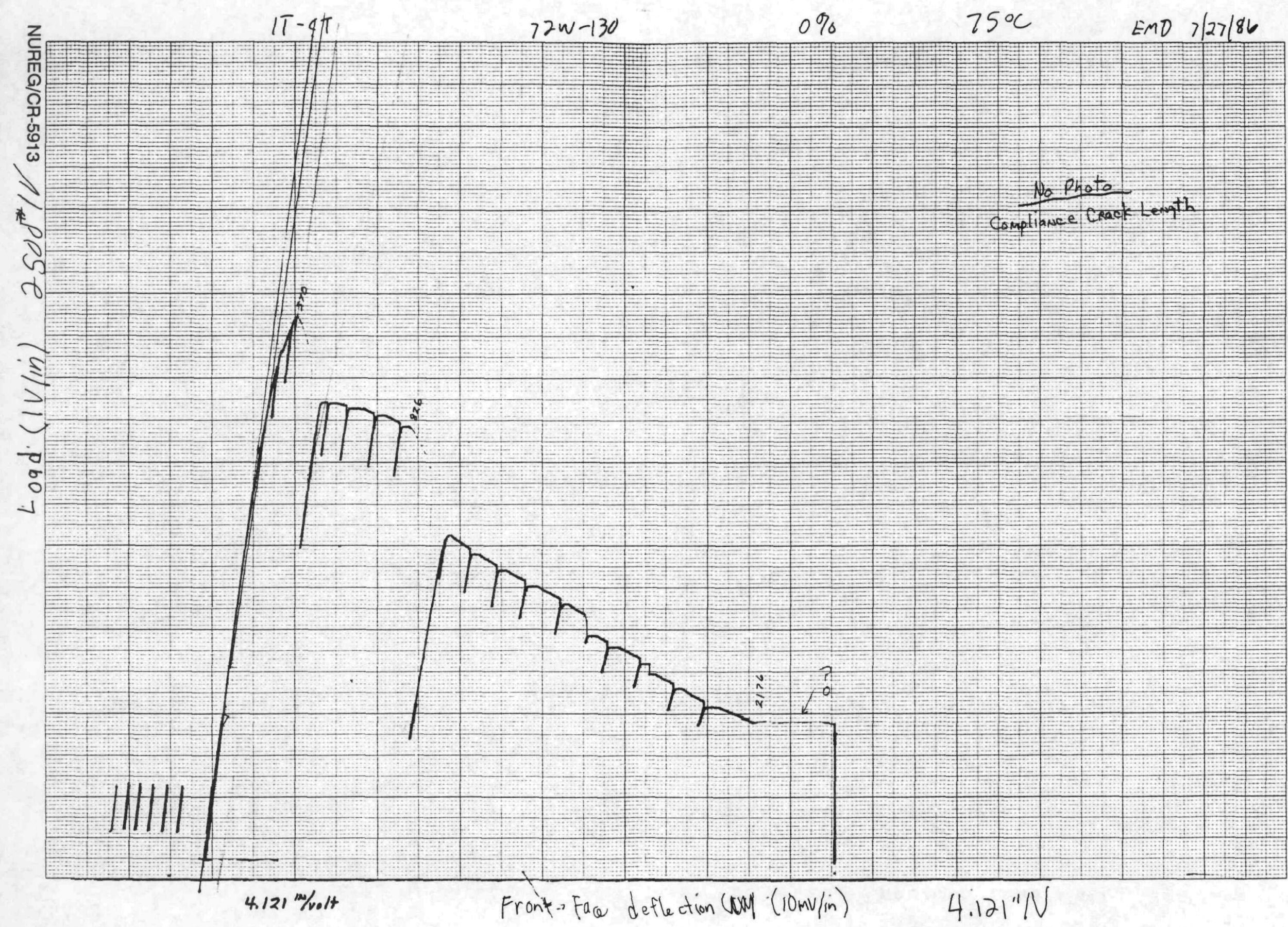


E-195

Specimen Laadline Deflection (in.)

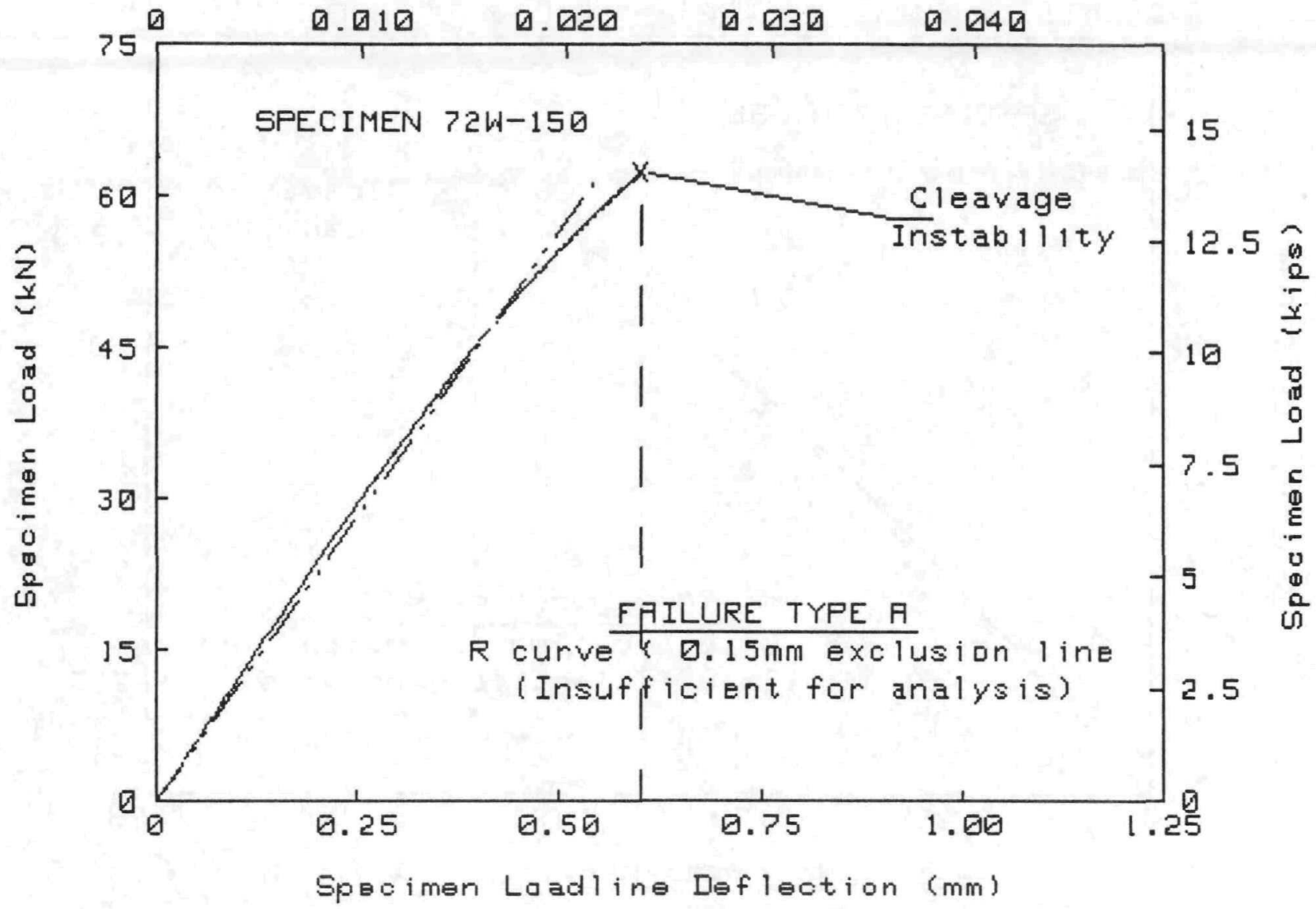

TEST SPECIMEN DATA

Material Type
Test Temperature
Percent Side Groove
Specimen Thickness B
Initial a/w
Init crack length a
Flow stress
Yield stress
Youngs modulus
Poisson's Ratio ( $)$
J-INTEGRA ANALYSIS

$=$ A533-B WELD (I)

$=75 \circ \mathrm{C}$

$=0 \%$

$=25.4 \mathrm{~mm}$

$=.524$

$=26.63 \mathrm{~mm}$

$=643.2 \mathrm{MPa}$

$=597.1 \mathrm{MPa}$

$=202900 \mathrm{MPa}$

$=0$

J-INTEGRAL ANALYSIS

[ Failure Type $A$ ( $J$ at instability = JC) ]

$\mathrm{Jc}$

$=\frac{\text { Merkle-Corten }}{74.2 \mathrm{~kJ} / \mathrm{m}^{2}}$

Deformat ion-J*

$=122.7 \mathrm{MPa} \sqrt{\mathrm{m}}$

$78.1 \mathrm{~kJ} / \mathrm{m}^{2}$

$125.9 \mathrm{MPa} \sqrt{\mathrm{m}}$

$K j c=\left\langle E J c /\left(1-v^{2}\right\rangle\right) \wedge .5$

$=87.1 \mathrm{MPa} \sqrt{\mathrm{m}}$

$88.3 \mathrm{MPa} \sqrt{\mathrm{m}}$

ASTM E399-81 ANALYSIS

Modified Kic Equation;

Maximum Load $P=62.4 \mathrm{kN}$

$5 \%$ Secant offset $\mathrm{Pq}=46.48 \mathrm{kN}$

$\mathrm{Kq}$

$=84.7 \mathrm{MPa} \sqrt{\mathrm{m}}$

Validity

$=$ INYALID--1, 2, 3

$K$ at Maximum Load

$=113.6 \mathrm{MPa} \sqrt{\mathrm{m}}$

DATA CORRECTIONS USED FOR J-INTEGRAL DATA

Merkle-Corten correction

Correction to J for crack extension (Deformation-J)

OFFSET LOAD $=0 \mathrm{kN}$ OFFSET DEFLECTION $=.002 \mathrm{~mm}$

Fitted elastic load range $=5 \mathrm{kN}$ TO $25 \mathrm{kN}$

Elastic slope $=1.197 \mathrm{E}+02 \mathrm{kN} / \mathrm{mm}$ 
Specimen Crackmouth Deflection (in.)

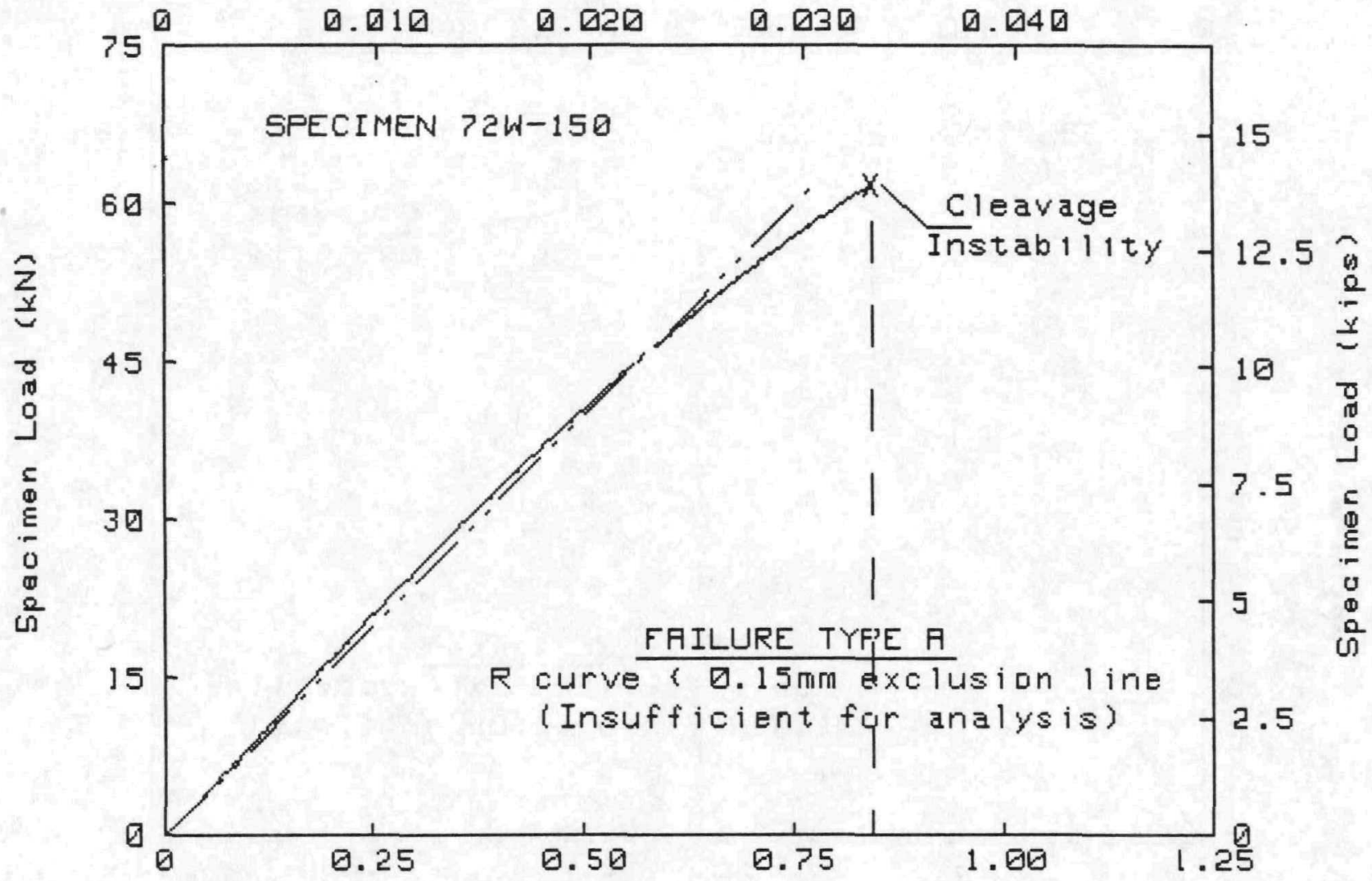

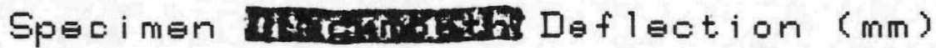

TEST SFECIMEN DATA

Material Type
Test Temperature
Percent Side Groove
Specimen Thickness B
Initial a/W
Init crack length a
Flow stress
Yield stress
Youngs modulus B
Poisson's Ratio (U)
J-INTEGRAL ANALYSIS

$=A 533-B$ WELD (I)

$=75^{\circ} \mathrm{C}$

$=0 \%$

$=25.4 \mathrm{~mm}$

$=.524$

$=26.63 \mathrm{~mm}$

$=643.2 \mathrm{MPa}$

$=597.1 \mathrm{MPa}$

(Est imated Value)

$=202900 \mathrm{MPa}$

(Est imated Value)

$=0$

(Estimated Value)

$\mathrm{Jc}$

$K j c=\left\langle E J c /\left(1-v^{2}\right\rangle\right) \wedge .5$

$\mathrm{Kc}$ (Beta Corrected)

ASTM E399-81 ANALYSIS

Modified Kic Equation;

5\% Secant offset

$\mathrm{Kq}$

Validity

$K$ at Maximum Load

$\mathrm{PQ}$

$$
=0
$$

[ Failure Type

$$
\begin{aligned}
= & \text { Merkle-Corten } \\
= & 76.5 \mathrm{~kJ} / \mathrm{m}^{2} \\
= & 124.6 \mathrm{MPa} \sqrt{\mathrm{m}} \\
= & 87.8 \mathrm{MPa} \sqrt{\mathrm{m}}
\end{aligned}
$$

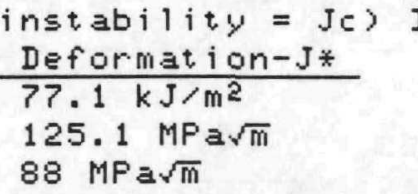

DATA CORRECTIONS USED FOR J-INTEGRAL DATA

Merkle-Corten correction

Correction to $J$ for crack extension (Deformation-J)

OFFSET LOAD $=0 \mathrm{kN}$ OFFSET DEFLECTION $=.008 \mathrm{~mm}$

Fitted elastic load range $=5 \mathrm{kN}$ TO $25 \mathrm{kN}$

Elast ic slope $=8.392 \mathrm{E}+01 \mathrm{kN} / \mathrm{mm}$ 


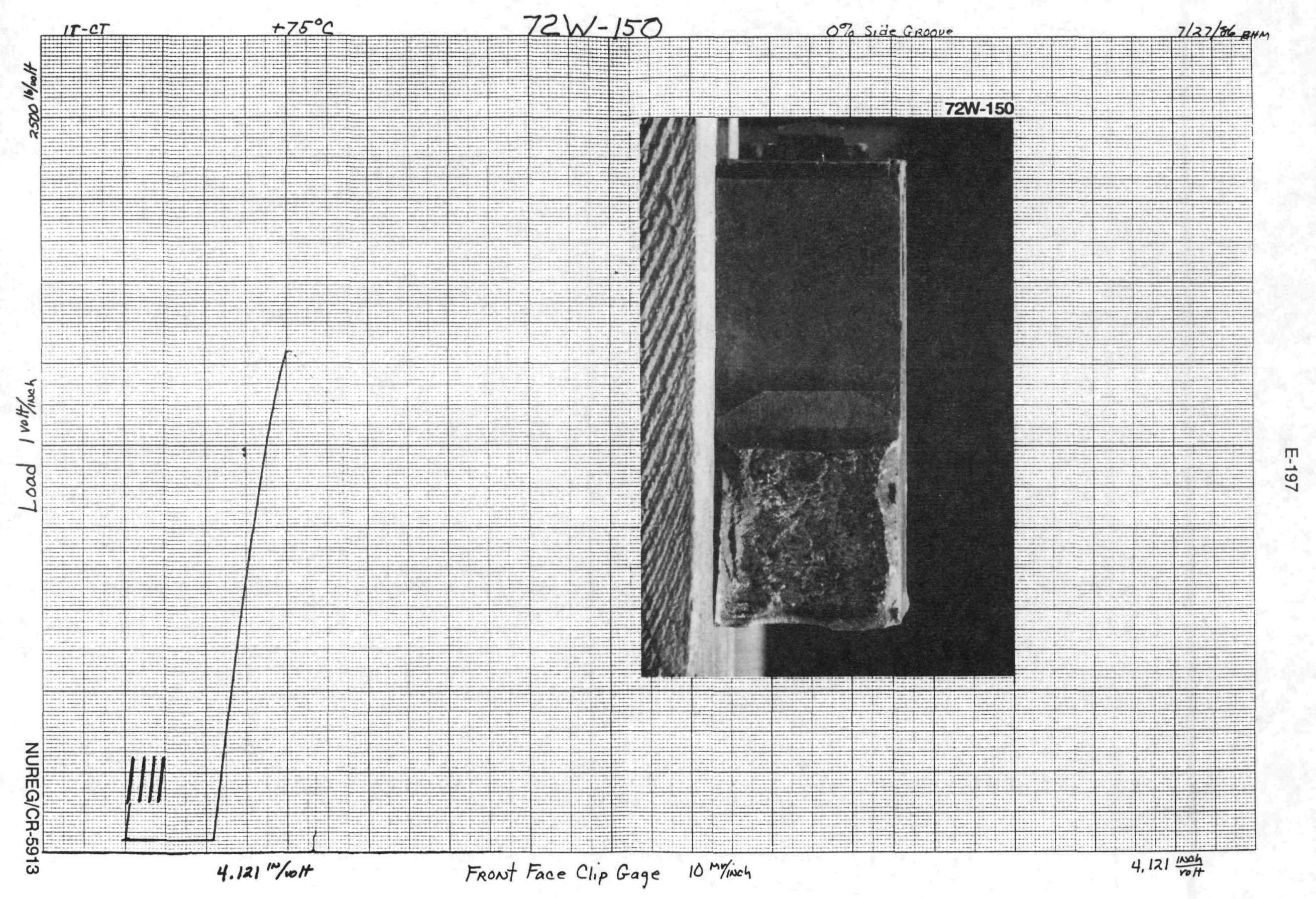




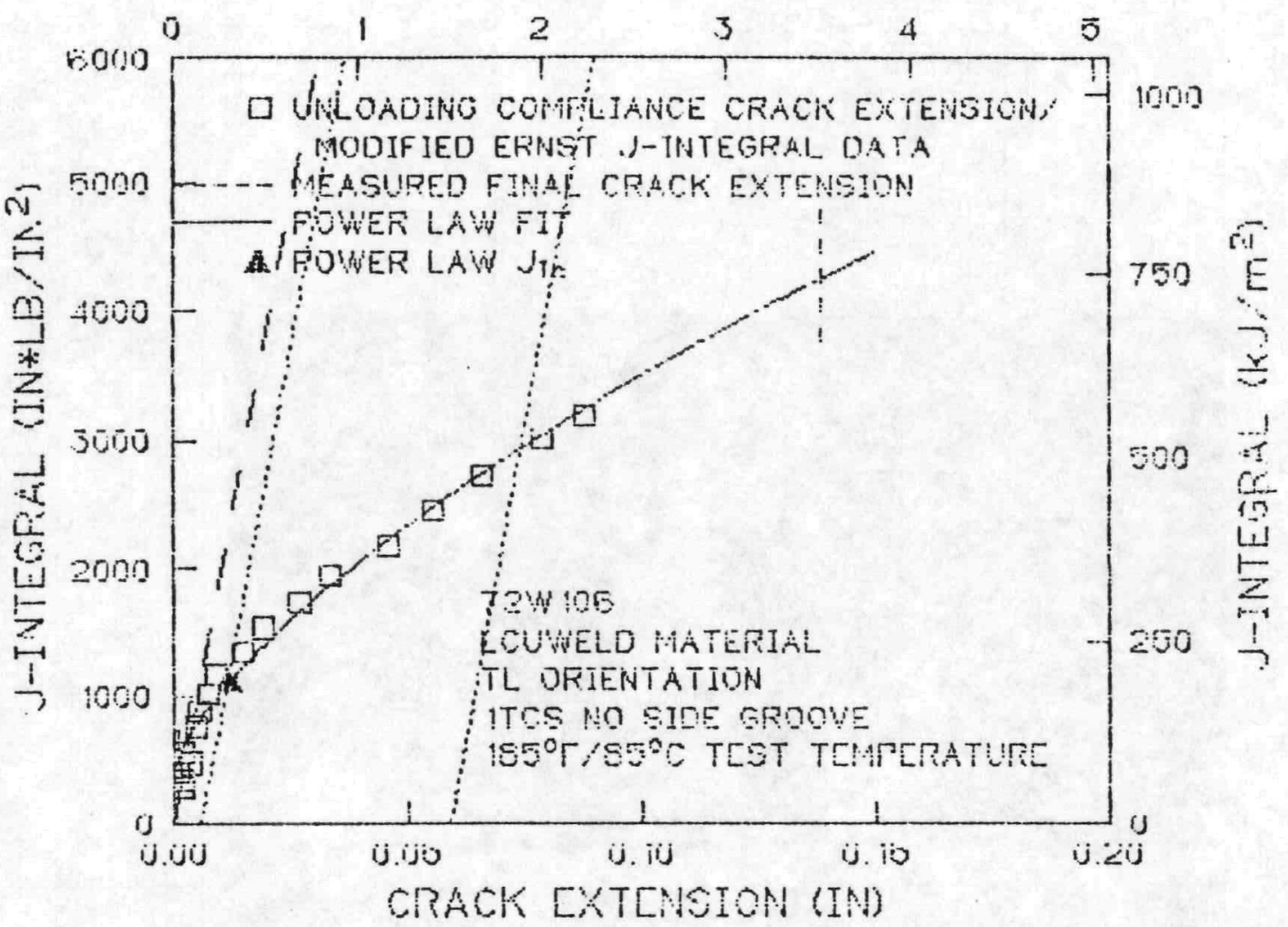

DISPLACEMENT (mM)

ORNL-DWG 92-11625

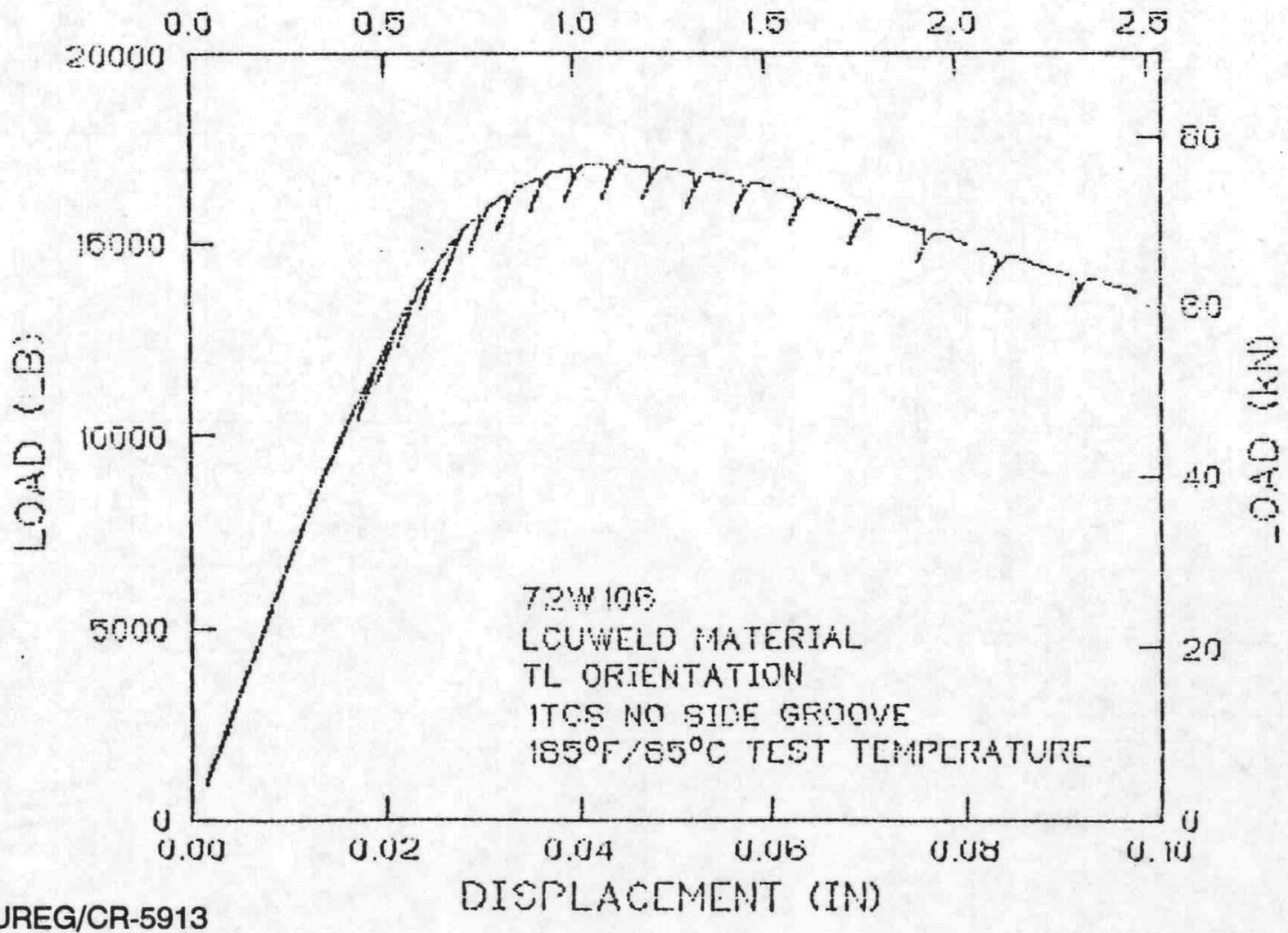




\section{E-199}

72W-106

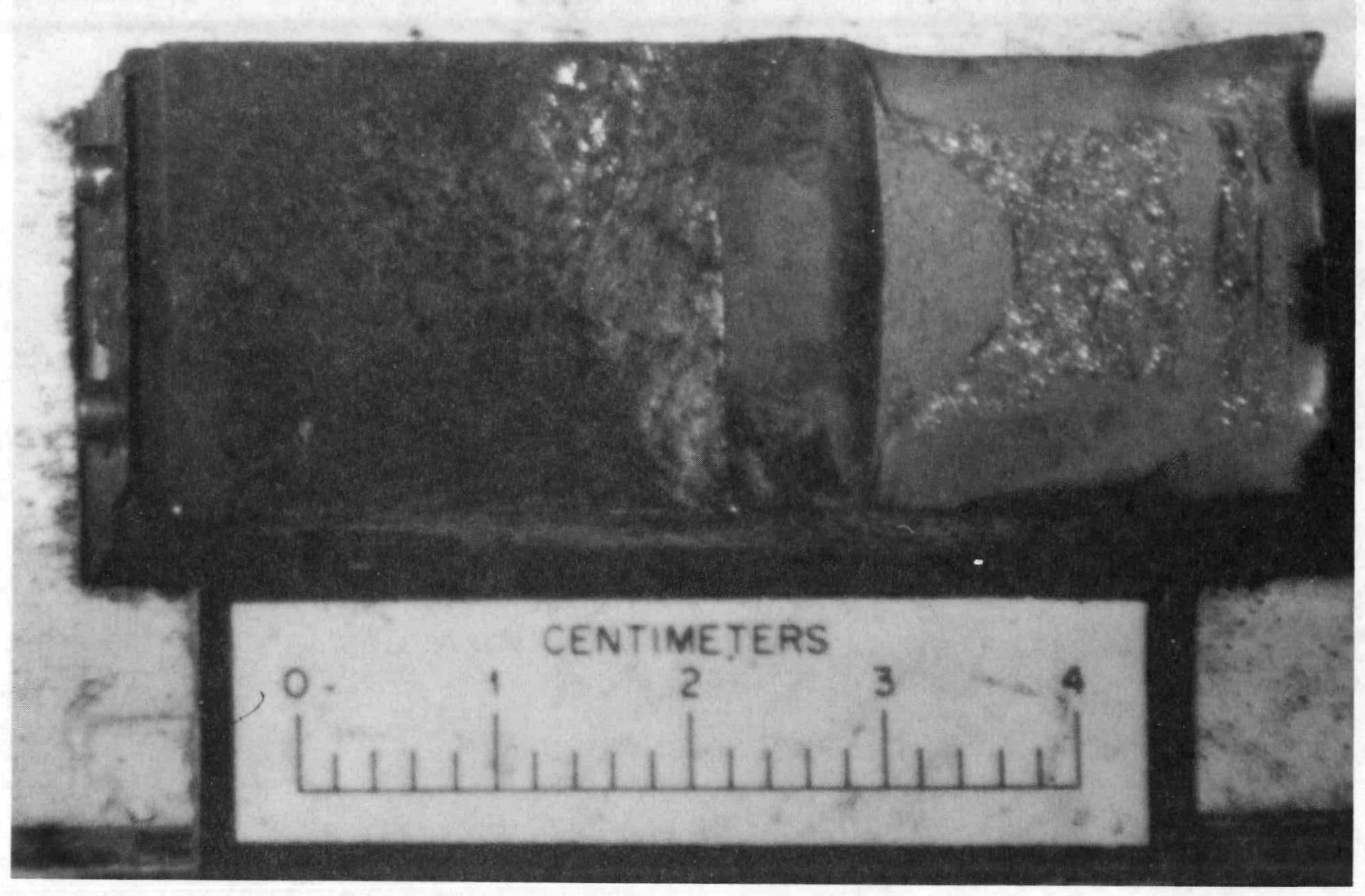

NUREG/CR-5913 


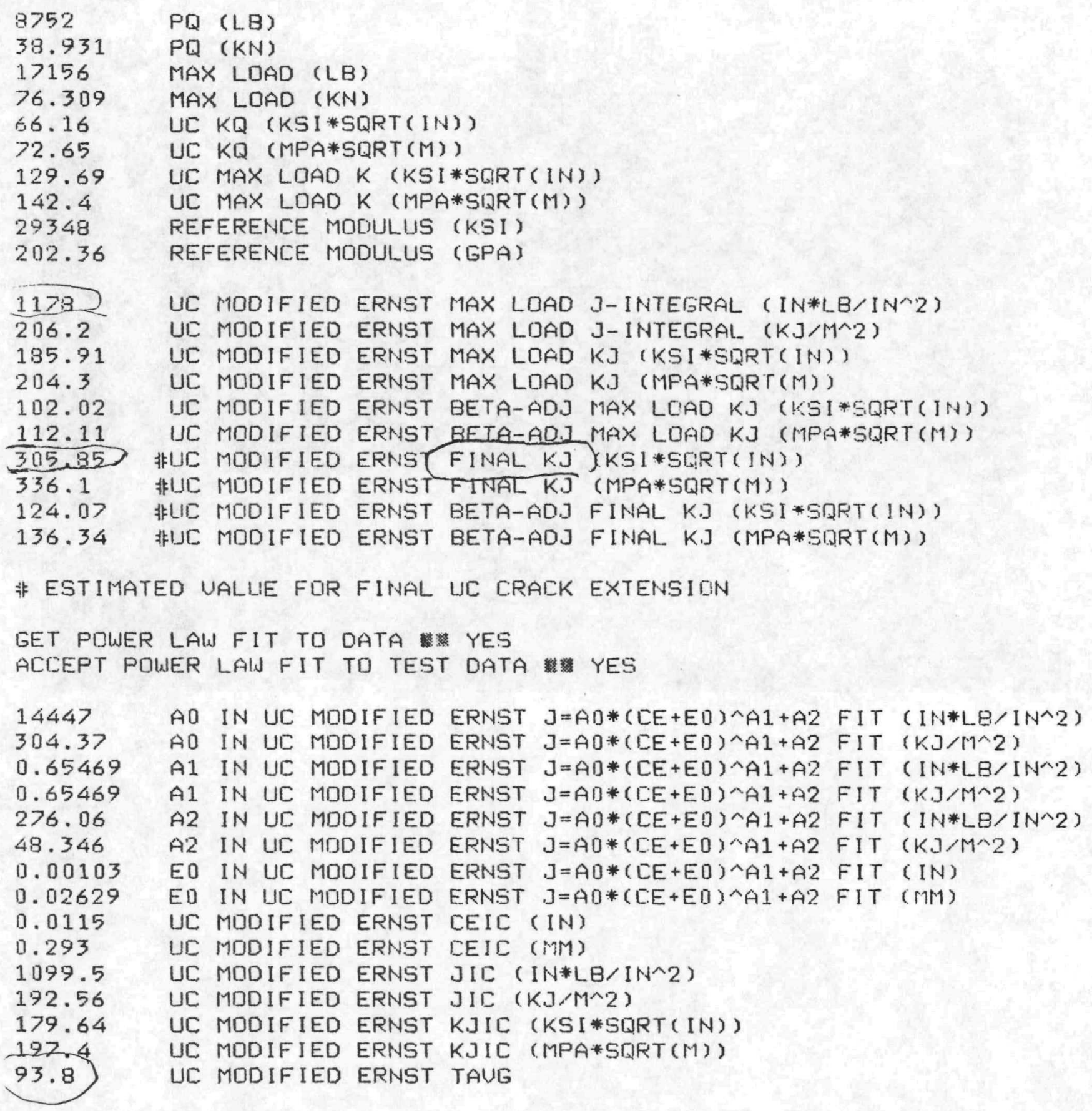


E-201

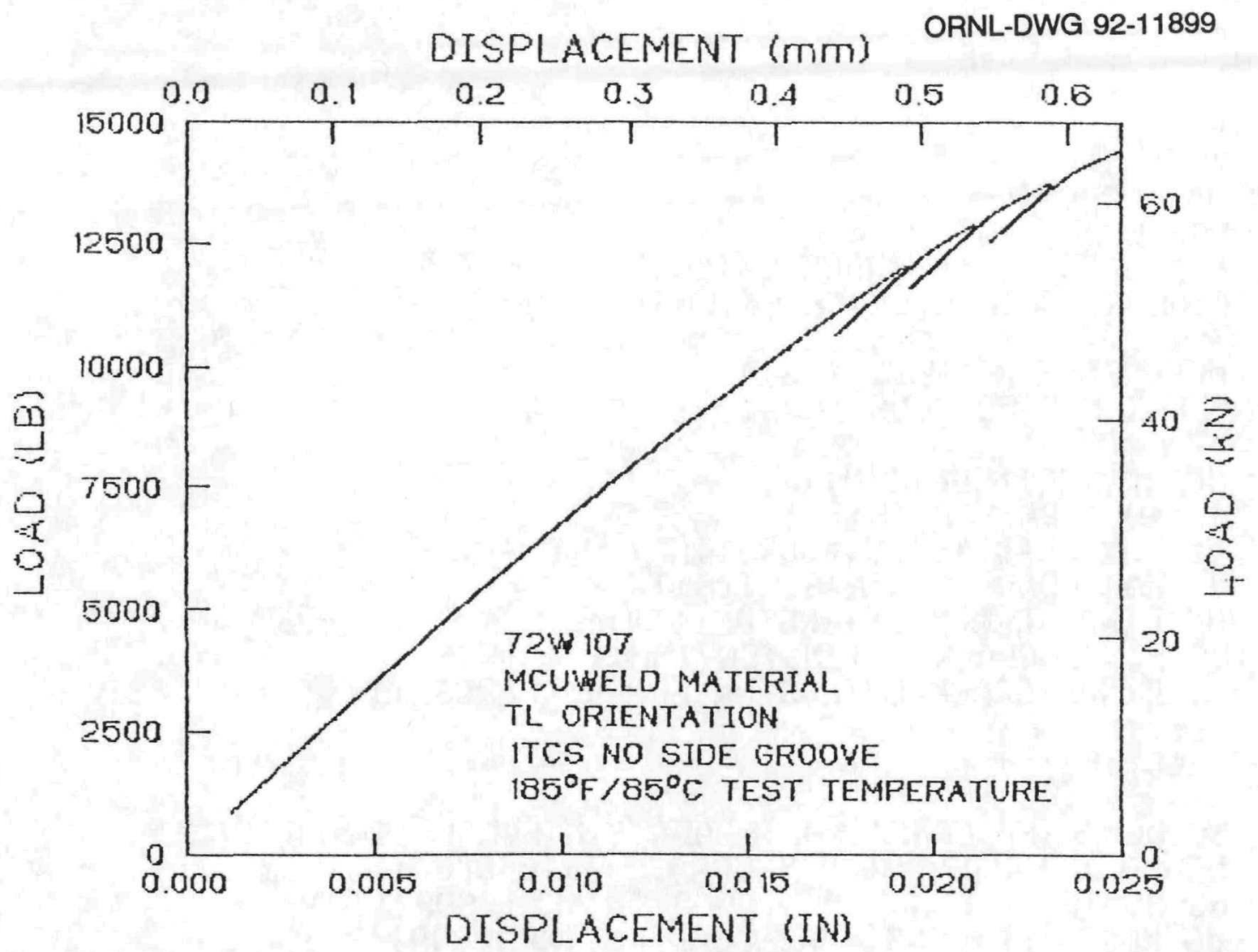

72W-107

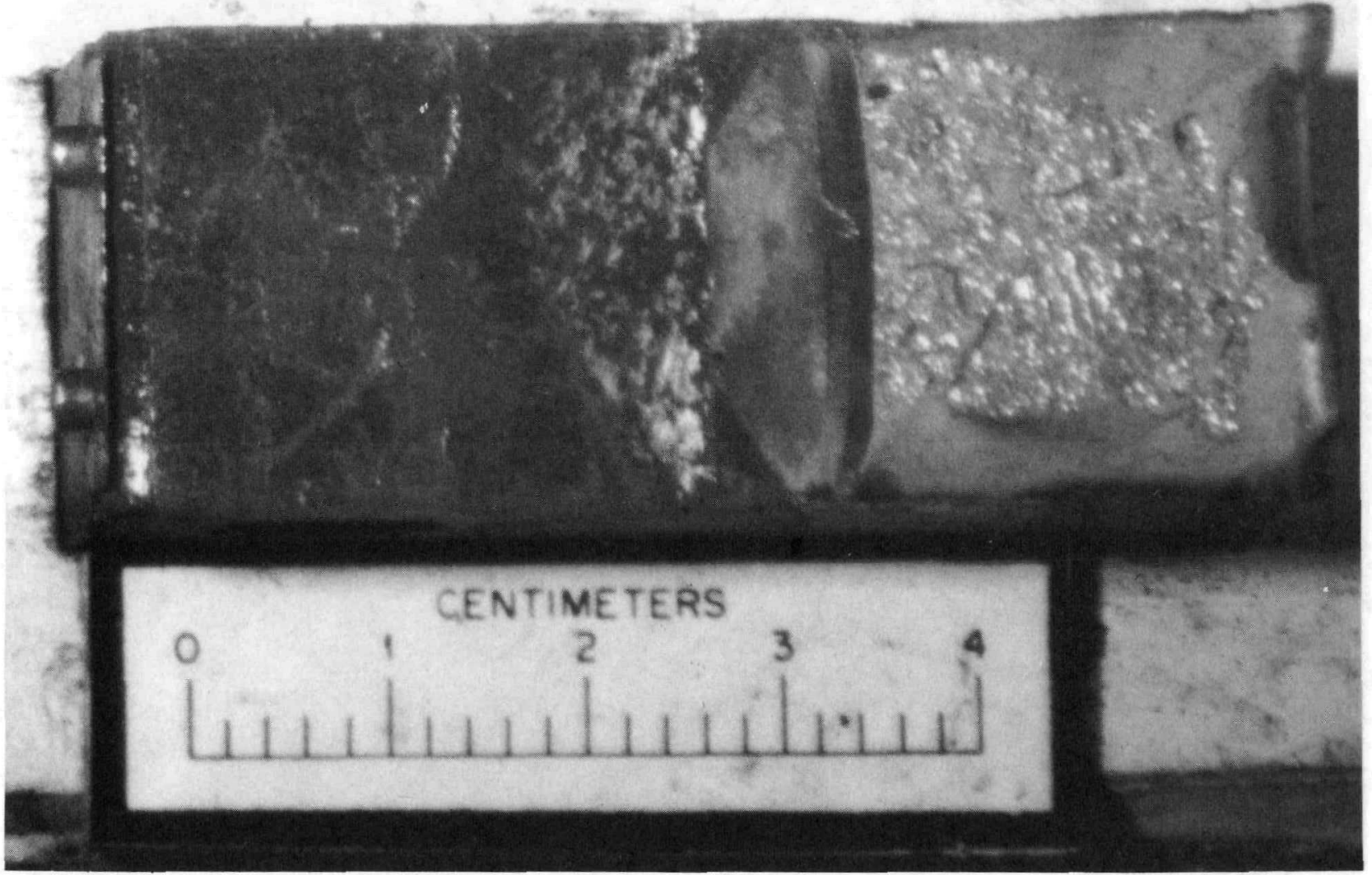

NUREG/CR-5913 
SPECIMEN DID NOT CLEAVE

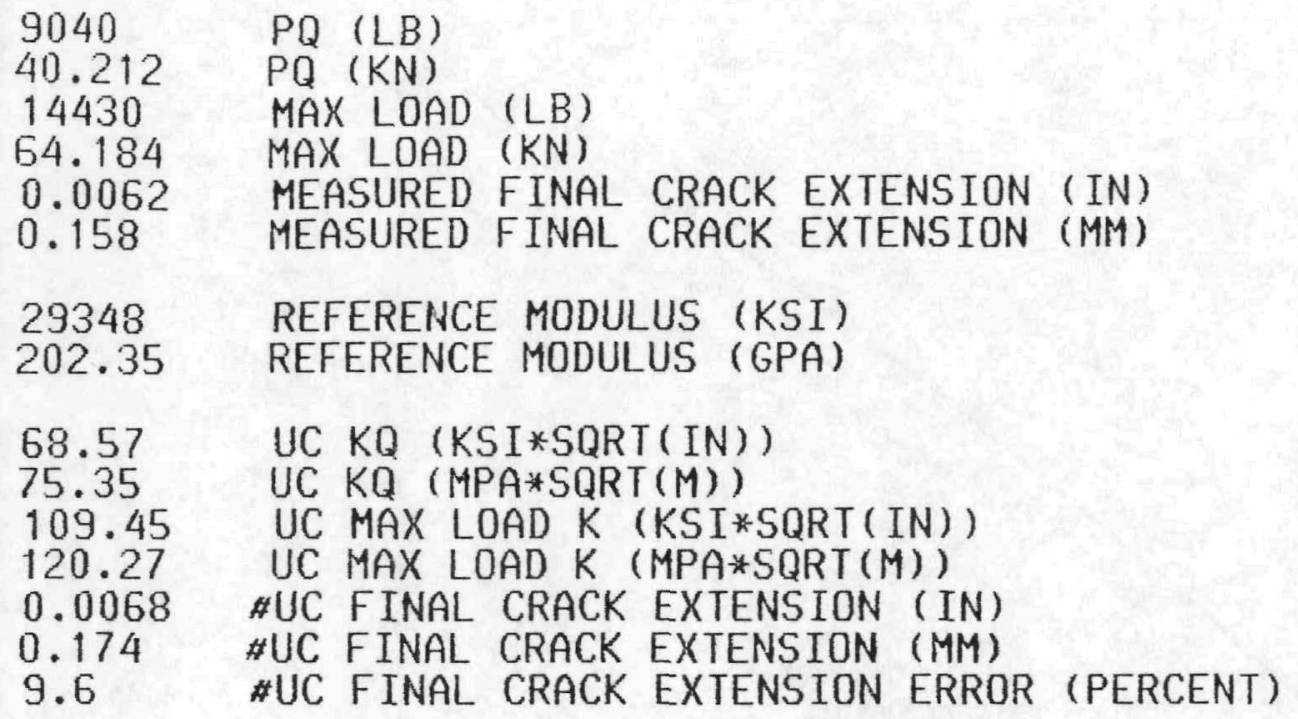




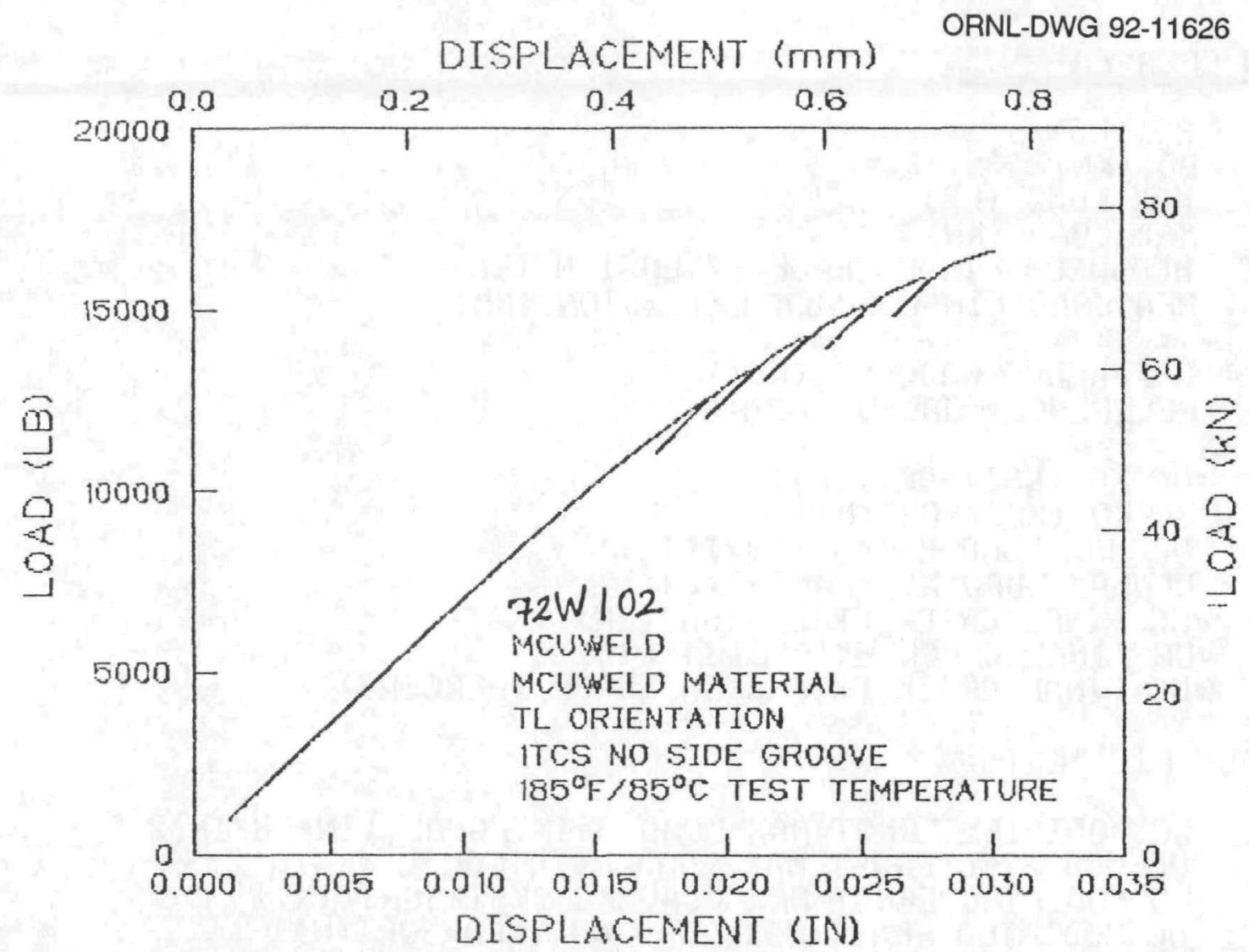

72W-102
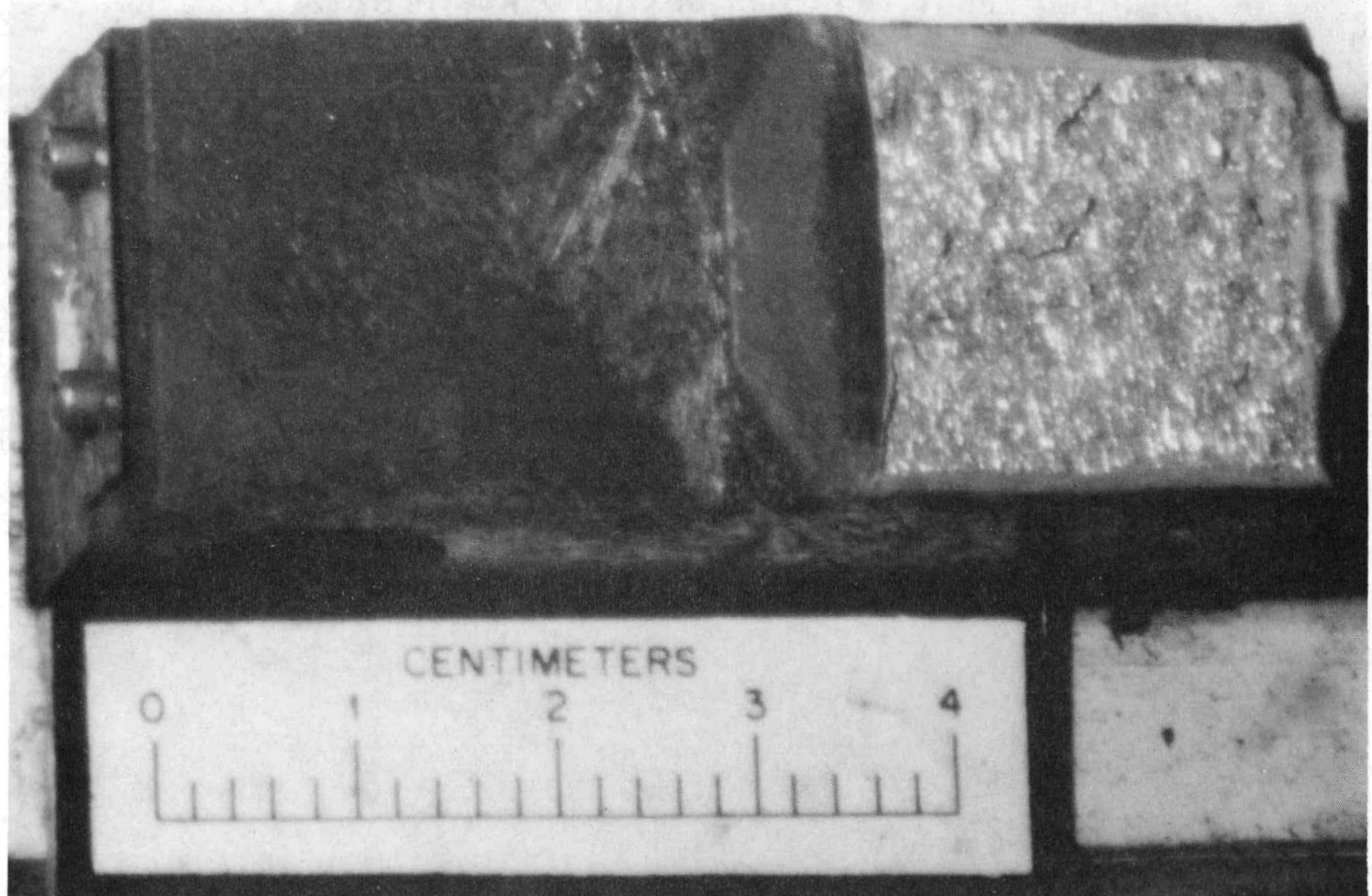
SPECIMEN CLEAVED

$\begin{array}{ll}9002 & P Q(L B) \\ 40.043 & P Q \text { (KN) } \\ 16605 & \text { MAX LOAD (LB) } \\ 73.859 & \text { MAX LOAD (KN) } \\ 0.0099 & \text { MEASURED FINAL CRACK EXTENSION (IN) } \\ 0.252 & \text { MEASURED FINAL CRACK EXTENSION (MM) } \\ 29348 & \text { REFERENCE MODULUS (KSI) } \\ 202.35 & \text { REFERENCE MODULUS (GPA) } \\ 66.69 & \text { UC KQ (KSI*SQRT(IN)) } \\ 73.29 & \text { UC KQ (MPA*SQRT(M)) } \\ 123.02 & \text { UC MAX LOAD K (KSI*SQRT (IN)) } \\ 135.18 & \text { UC MAX LOAD K (MPA*SQRT(M)) } \\ 0.0001 & \text { \#UC FINAL CRACK EXTENSION (IN) } \\ 0.002 & \text { \#UC FINAL CRACK EXTENSION (MM) } \\ -99.1 & \text { \#UC FINAL CRACK EXTENSION ERROR (PERCENT) }\end{array}$

INUALID UC KIC TEST

686.9 UC MODIFIED ERNST MAX LOAD J-INTEGRAL (IN*LB/IN`2)

120.3 UC MODIFIED ERNST MAX LOAD J-INTEGRAL $\left(K J / M^{\wedge} 2\right)$

141.99 UC MODIFIED ERNST MAX LOAD KJ (KSI*SQRT(IN))

156.03 UC MODIFIED ERNST MAX LOAD KJ (MPA*SQRT(M))

89.09 UC MODIFIED ERNST BETA-ADJ MAX LOAD KJ (KSI*SQRT(IN))

97.91 UC MODIFIED ERNST BETA-ADJ MAX LOAD KJ (MPA*SQRT(M))

141.99 UC MODIFIED ERNST FINAL KJ (KSI*SQRT(IN))

156.03 UC MODIFIED ERNST FINAL KJ (MPA*SQRT(M))

89.09 UC MODIFIED ERNST BETA-ADJ FINAL KJ (KSI*SQRT(IN))

97.91 UC MODIFIED ERNST BETA-ADJ FINAL KJ (MPA*SQRT(M))

\# ESTIMATED VALUE FOR FINAL UC CRACK EXTENSION

GET POWER LAW FIT TO UC MODIFIED ERNST TEST RESULTS YA YES 51 ITERATIONS--STOP AND RETURN CURRENT COEFFICIENTS 疋 YES ACCEPT POWER LAW FIT TO UC MODIFIED ERNST TEST RESULTS NO NO SUPPRESS SELECTED DATA AND REFIT DATA NO NO

UC MODIFIED ERNST JIC,CEIC,KJIC AND TAVG NOT DEFINED--POWER LAW FIT REJECTED BY TECHNICIAN 


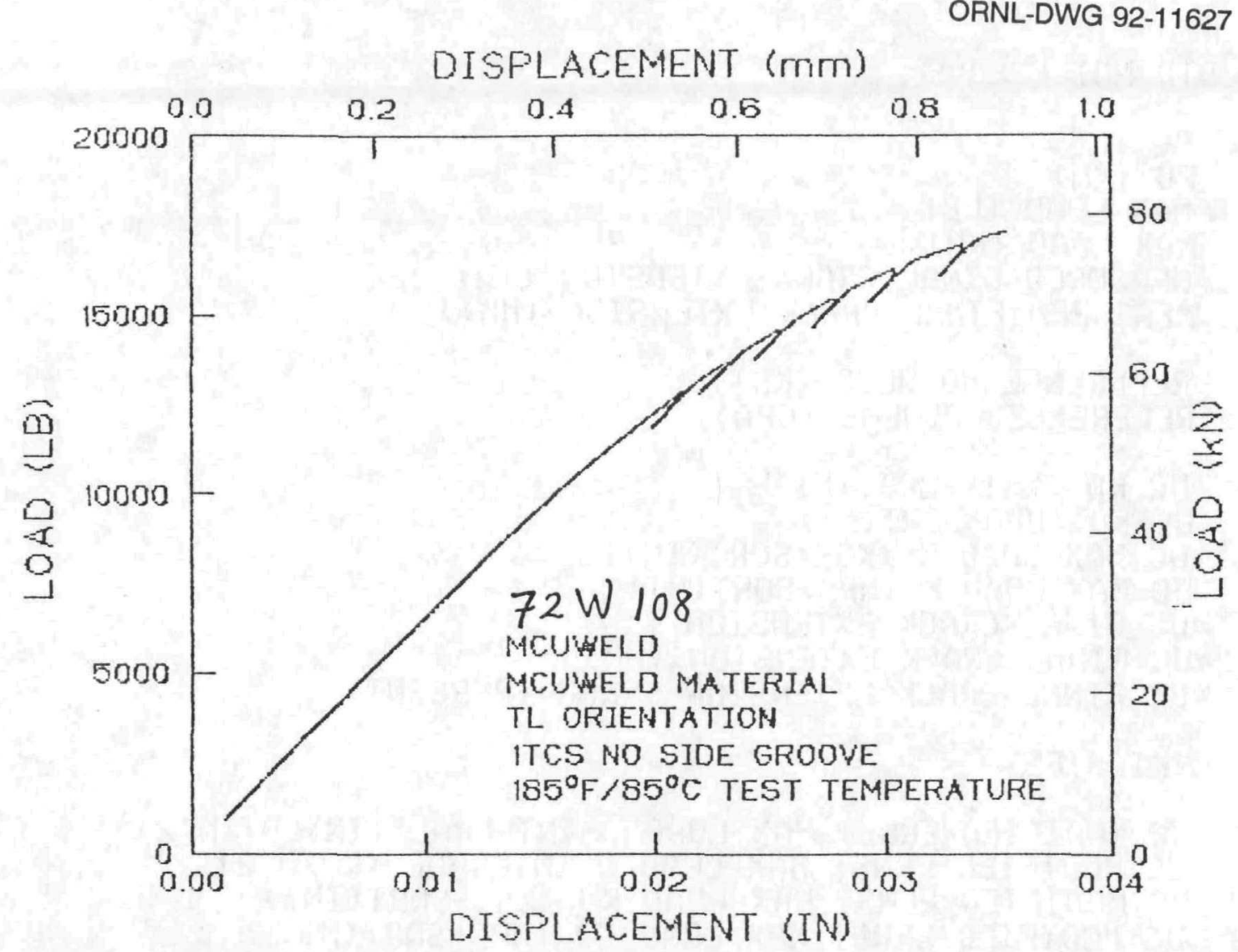

72W-108

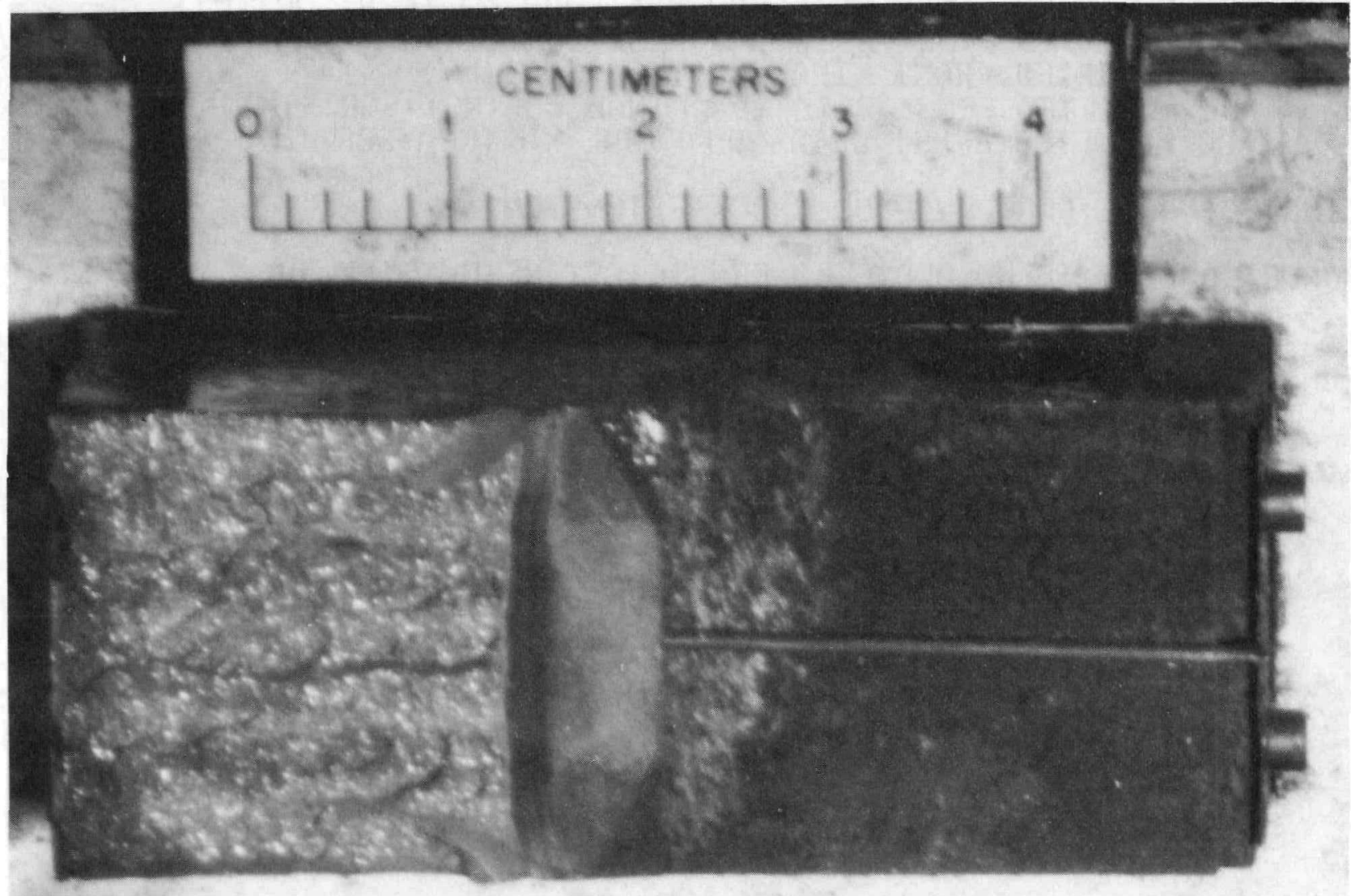


SPECIMEN DID NOT CLEAVE

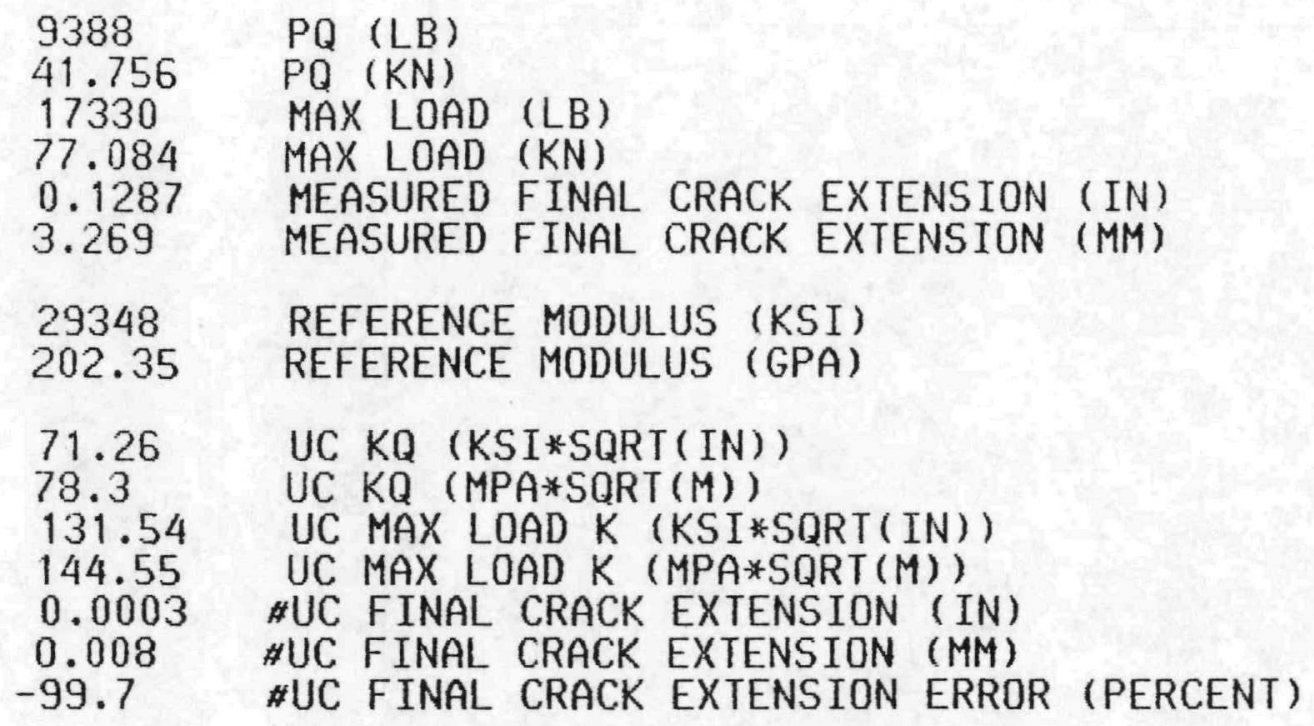




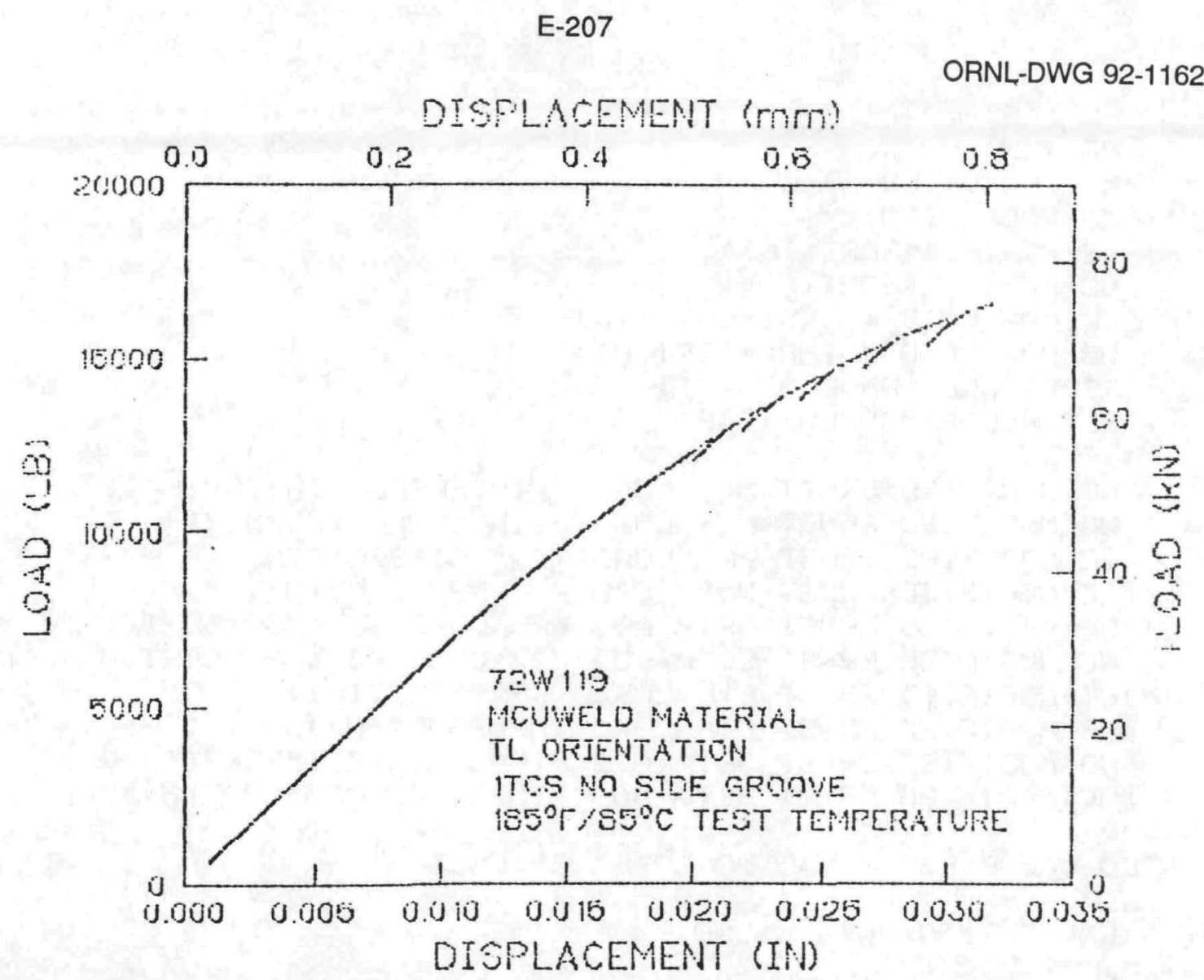

72W-119

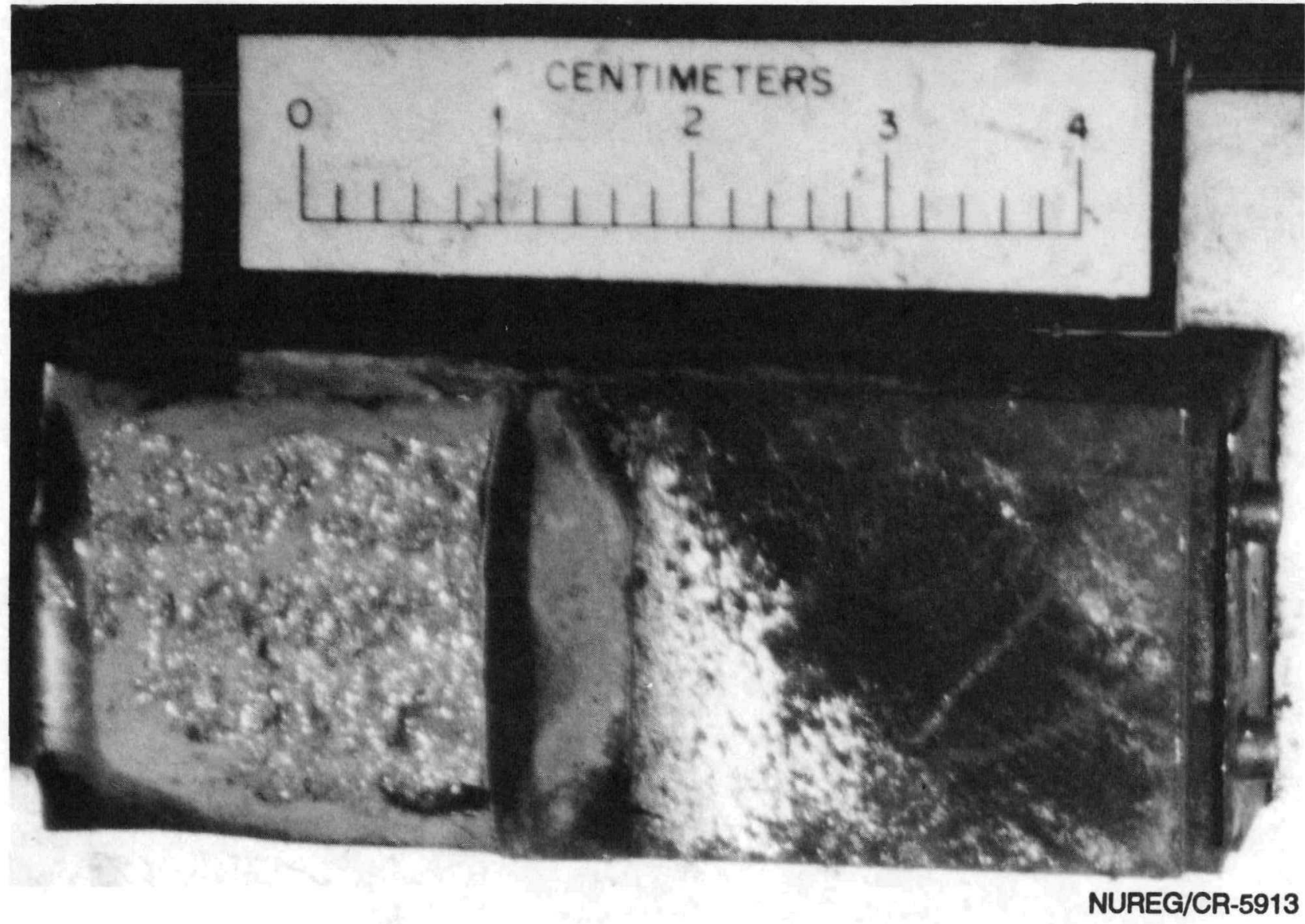




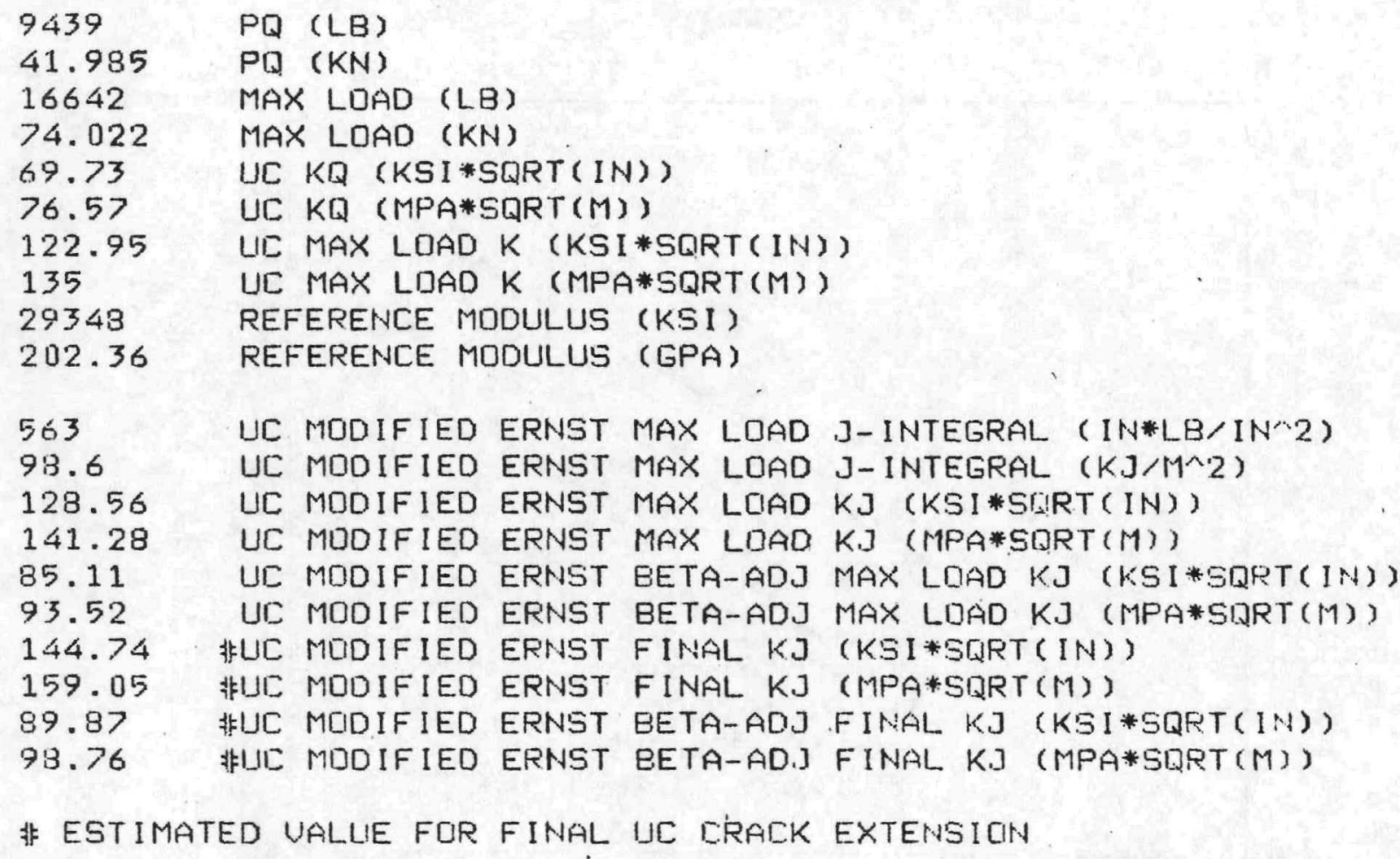



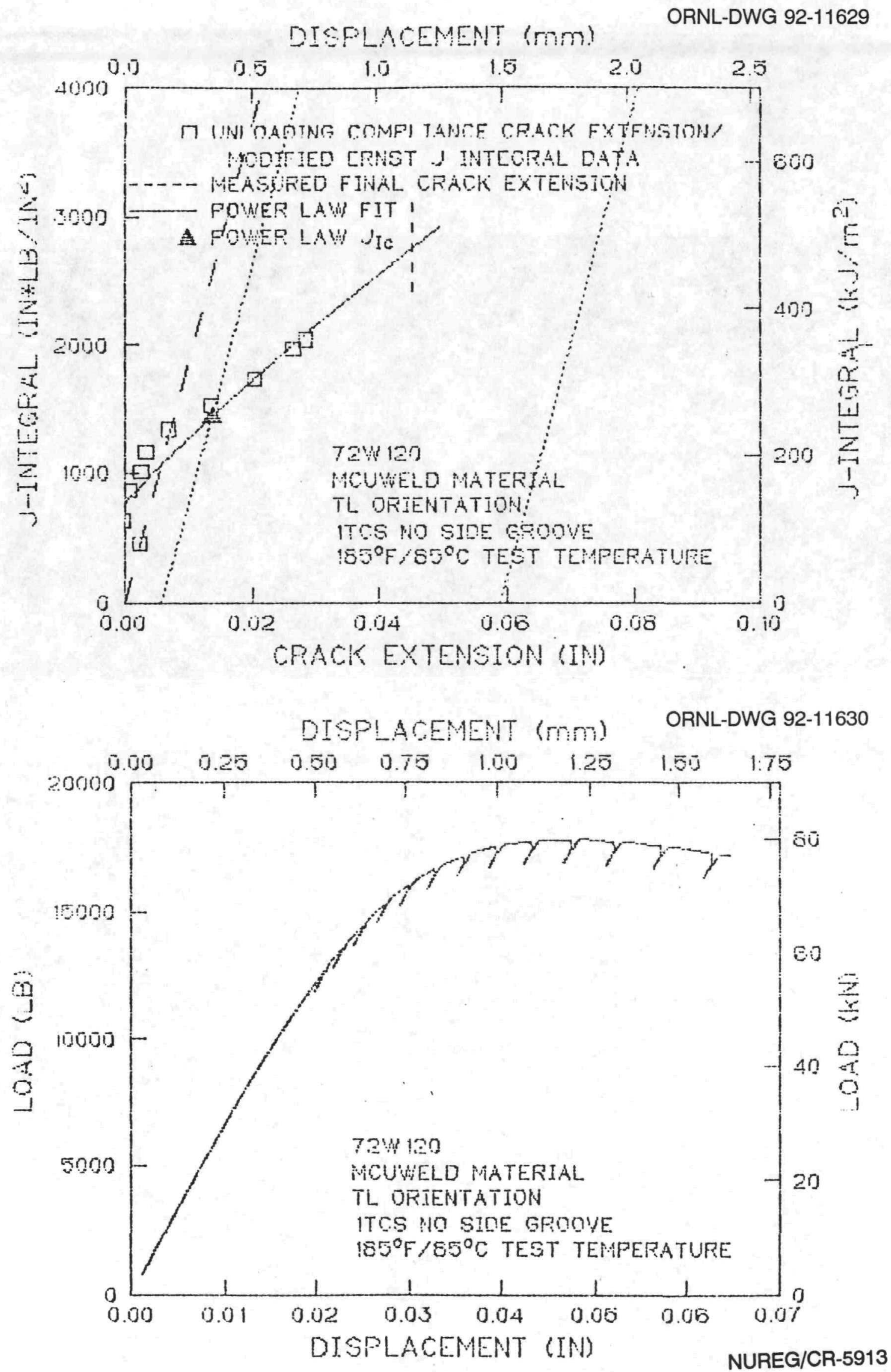


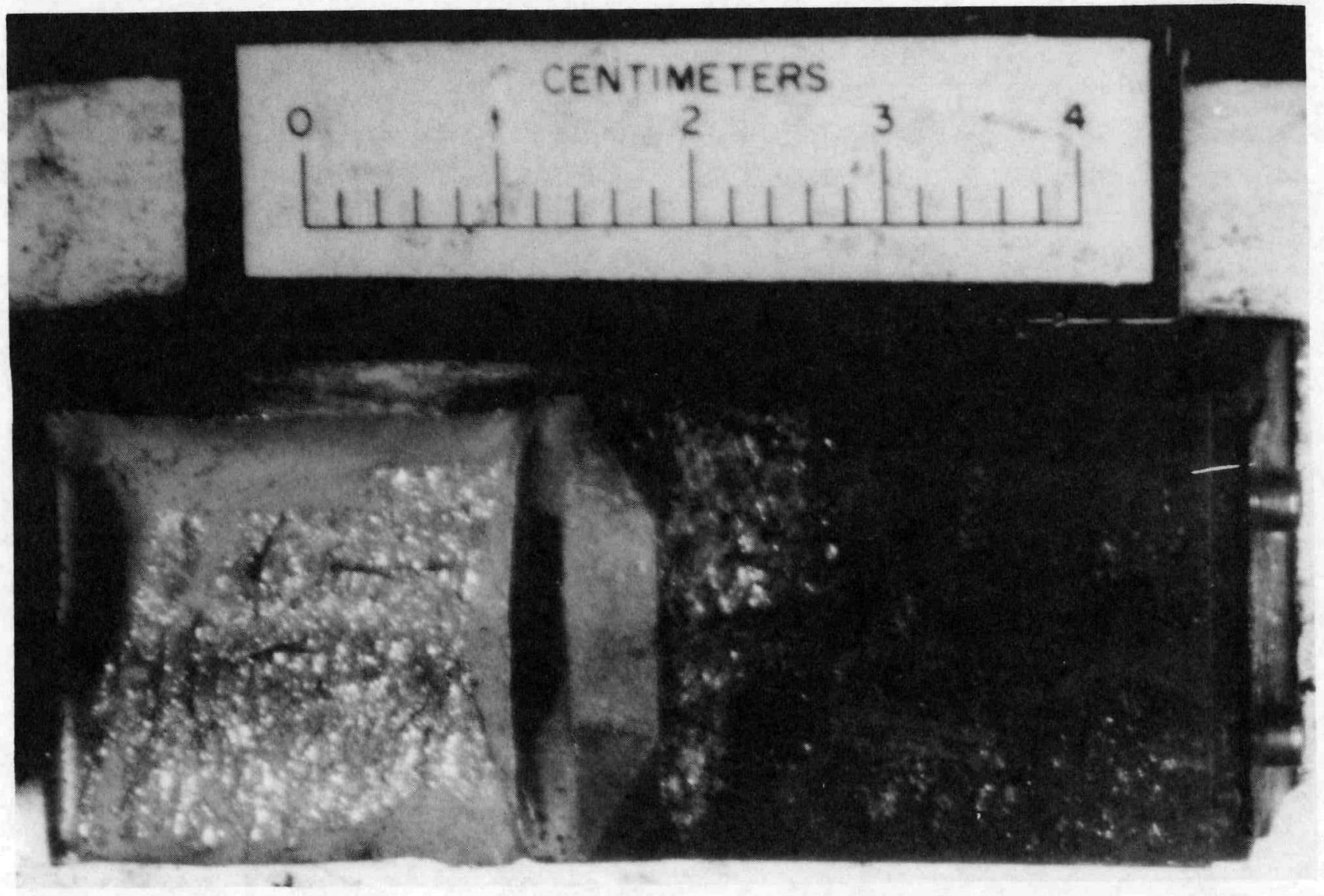




$\begin{array}{ll}9559 & P Q \text { (LB) } \\ 42.52 & \text { PQ (KN) } \\ 17818 & \text { MAX LOAD (LB) } \\ 79.254 & \text { MAX LDAD (KN) } \\ 70.04 & \text { UC KQ (KSI*SQRT (IN)) } \\ 76.9 & \text { UC KQ (MPA*SQRT(M)) } \\ 130.54 & \text { UC MAX LOADK (KSI*SART(IN)) } \\ 143.33 & \text { UC MAX LDADK (MPA*SQRT(M)) } \\ 29348 & \text { REFERENCE MODULUS (KSI) } \\ 202.36 & \text { REFERENICE MODULUS (GPA) }\end{array}$

1343

235.3.

193.56

218.2

102.81

112.98

244.67

268.89

111.68

122.72
UC MDDIFIED ERNST MAX LDAD J-INTEGRAL (IN*LG/IN^2)

UC MODIFIED ERNST MAX LOAD ]-INTEGRAL ( $\left.\mathrm{KJ} / \mathrm{M}^{\wedge} 2\right)$

UC MODIFIED ERNST MAX. LOAD K.J (KSI*SGRT (IN))

UC MIDOIFIED ERNST MAX LOAD KJ (MPA*SQRT (M))

UC MDDIFIED ERNST BETA-ADJ MAX LOAD KJ (KSI*SDRT(INI))

UL MDOIFIED ERNST BETA-ADJ MAX LDAD KJ (MPA*SQRT (M)

非UC MODIFIED ERNST FINAL KJ (KSI*SGRT (IN))

非UC MDDIFIED ERNST FINAL KJ (MPA*SQRT(M))

非C MODIFIED ERNST BETA-ADJ FINAL K.J, (KSI*SQRT (IN))

非C MDDIFIED ERNST BETA-ADJ FINAL KJ (MPA*SQRT(M))

非 ESTIMATED VALLE FOR FINAL UC CRACK EXTENSION

GET PDWER LAW FIT TO DATA 彎 YES

ALCEPT POWER LALN FIT TD TEST DATA

\begin{tabular}{|c|c|c|c|c|c|c|}
\hline 28288 & AO & IN UC MODIFIED & ERNST & $J=A D *(C E+E D) \wedge A 1+A 2$ & FIT & $(I N * L B / I N \wedge 2)$ \\
\hline 335.42 & $A O$ & UE MODIFIED & ERNST & $J=A D *(C E+E D) \wedge A 1+A 2$ & FIT & $\left(K J / M^{\wedge} 2\right)$ \\
\hline .83239 & A1 & IN UE MODIFIED & ERNST & $J=A D *(C E+E D) \wedge A 1+A^{\prime} 2$ & FIT & $\left(I N * L B / I N^{\wedge} 2\right)$ \\
\hline .83239 & Al & MODIF IED & ERNST & $J=A D *(C E+E 0) \wedge A 1+A 2$ & FIT & $\left(K J / M^{\wedge} 2\right)$ \\
\hline 77.13 & $A 2$ & UC MOOIFIED & ERNST & $J=A 0 *(C E+E O) \wedge A 1+A 2$ & FIT & $\left(\mathrm{IN} * \mathrm{LB} / \mathrm{IN} \mathrm{N}^{\wedge} \mathrm{C}\right.$ \\
\hline 6.046 & $A 2$ & UI MODIFIED & ERNST & $J=A D *(C E+E 0) \wedge A 1+A 2$ & FIT & $\left(K \cdot J / M^{\wedge} 2\right)$ \\
\hline .0058 & E0 & UC MOOIFIED & ERNST & $J=A 0 *(C E+E D) \wedge A 1+A 2$ & FIT & $(I N)$ \\
\hline 4742 & EO & UI MODIFIED & ERNST & $J=A D *(C E+E D) \times A 1+A 2$ & FIT & $(\mathrm{mM})$ \\
\hline 136 & UC & MODIFIED ERNST & CEIC & $(\mathrm{IN})$ & & \\
\hline .346 & UC & MODIFIED ERNST & CEIC & (MM) & & \\
\hline 1440.3 & UC & MODIFIED ERNST & JIC 6 & IN*LB $/ \mathrm{IN}^{\prime} 2$ ) & & \\
\hline 52.23 & $\mathrm{UC}$ & MODIFIED ERNST & JIC & $K J / M \cdots 2)$ & & \\
\hline & UC & MODIFIED ERNST & $\mathrm{KJIC}$ & (KSI*SORT (IN) & & \\
\hline & $\triangle C$ & MDDIFIED ERNST. & $\mathrm{KJIC}$ & (MPA*SQRT (M) & & \\
\hline & $1 \mathrm{DL}$ & MODIFIED ERNST & TAUG & & & \\
\hline
\end{tabular}



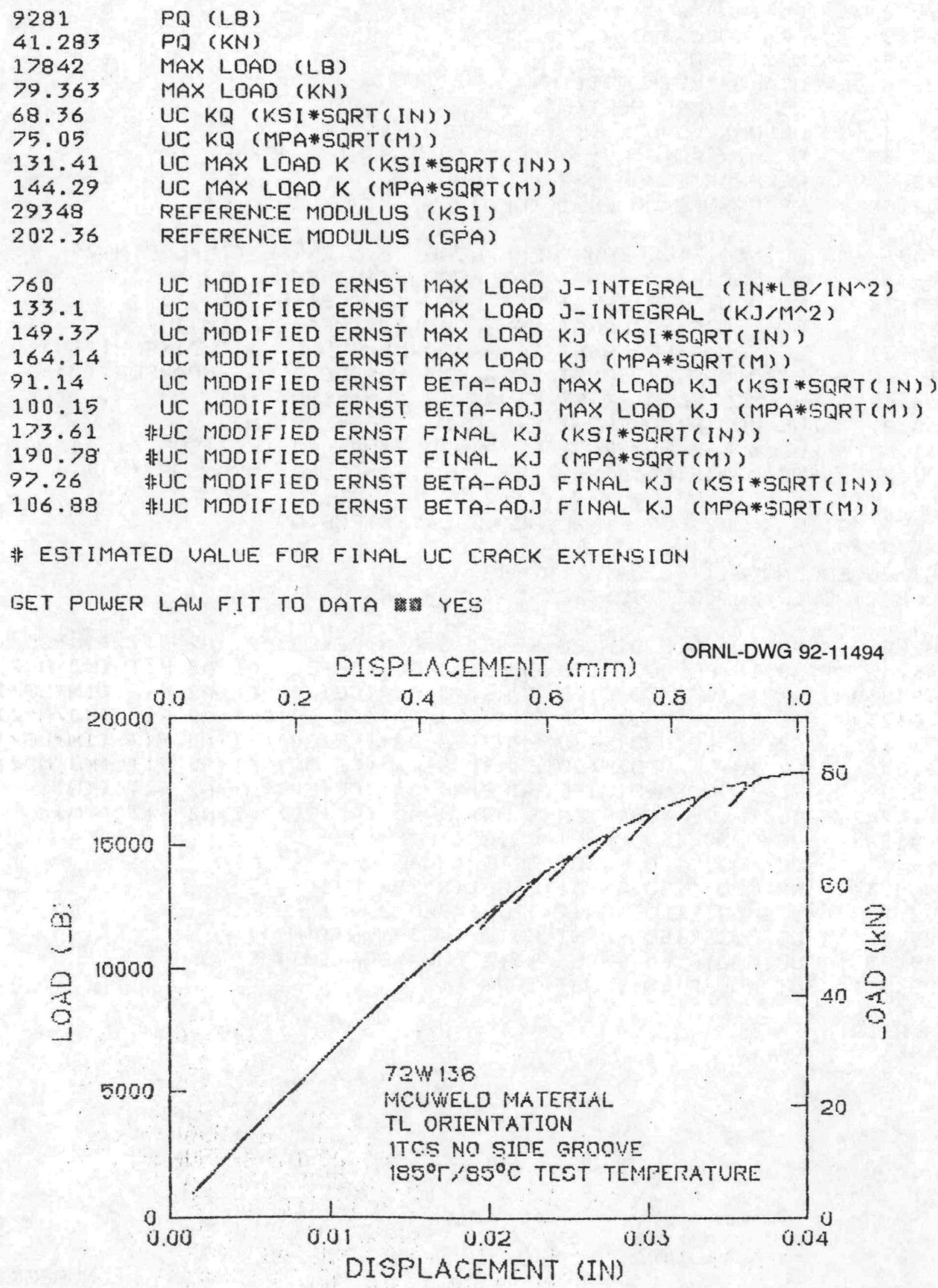

NUREG/CR-5913 


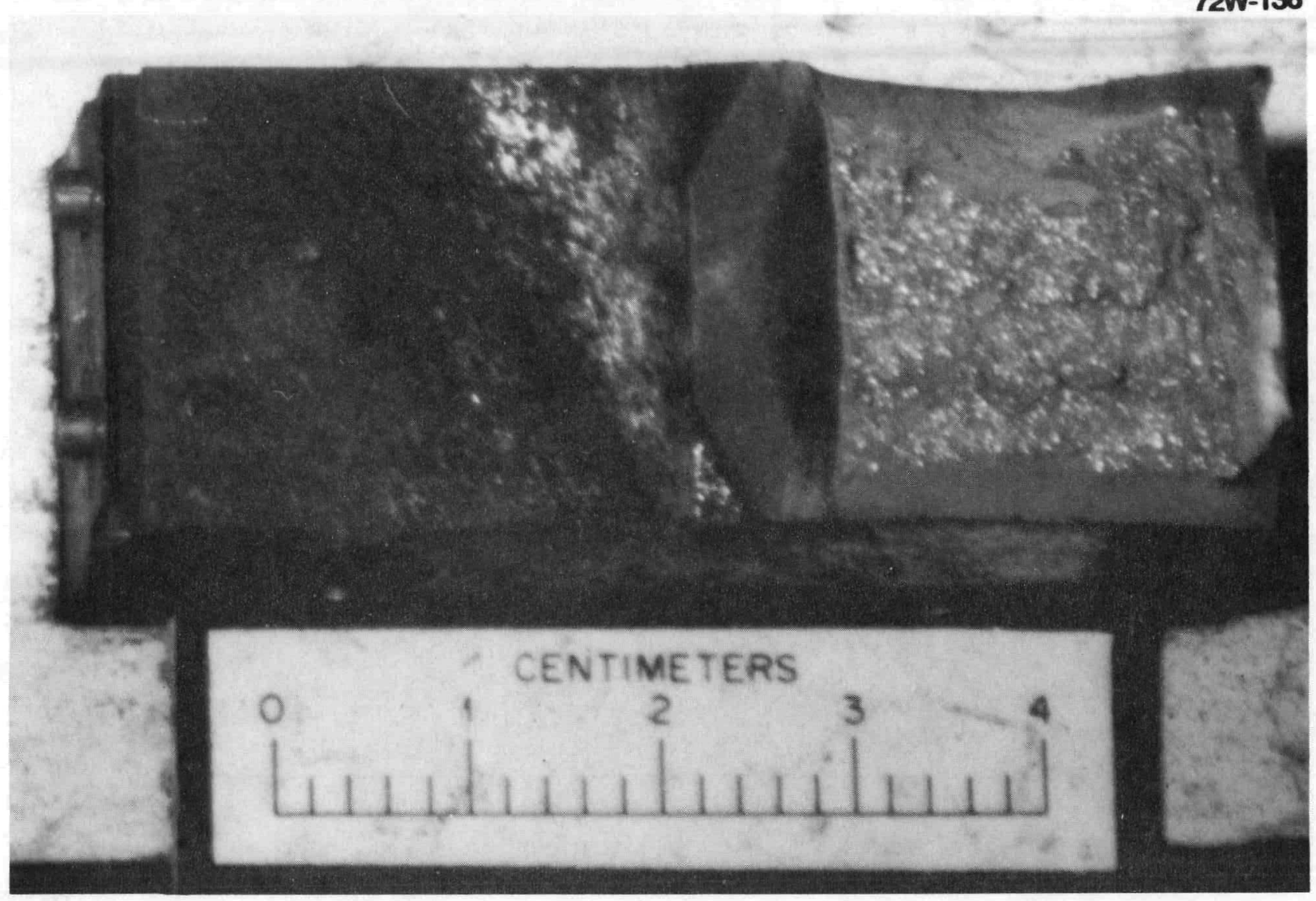




\section{E-214}

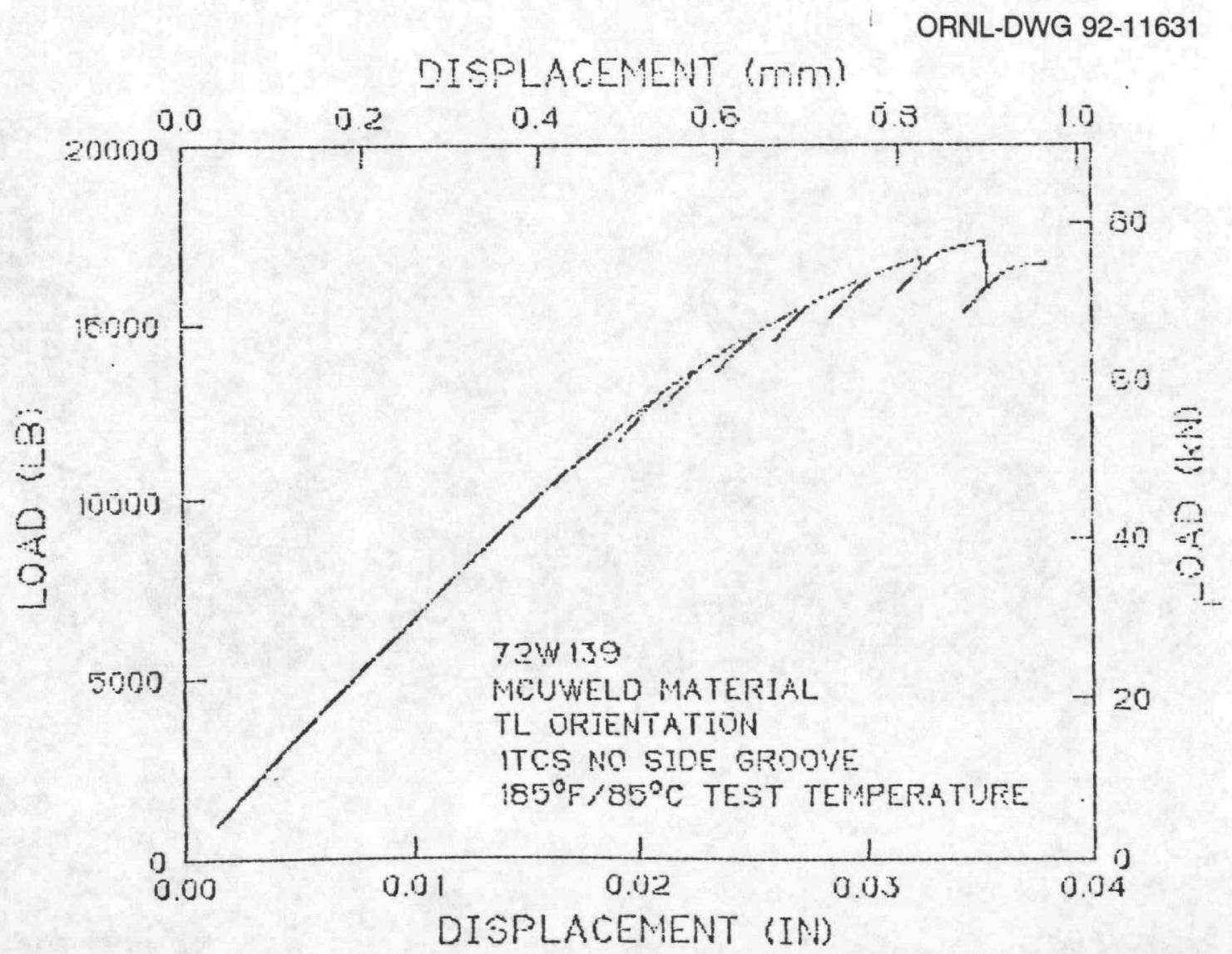

$72 W-139$

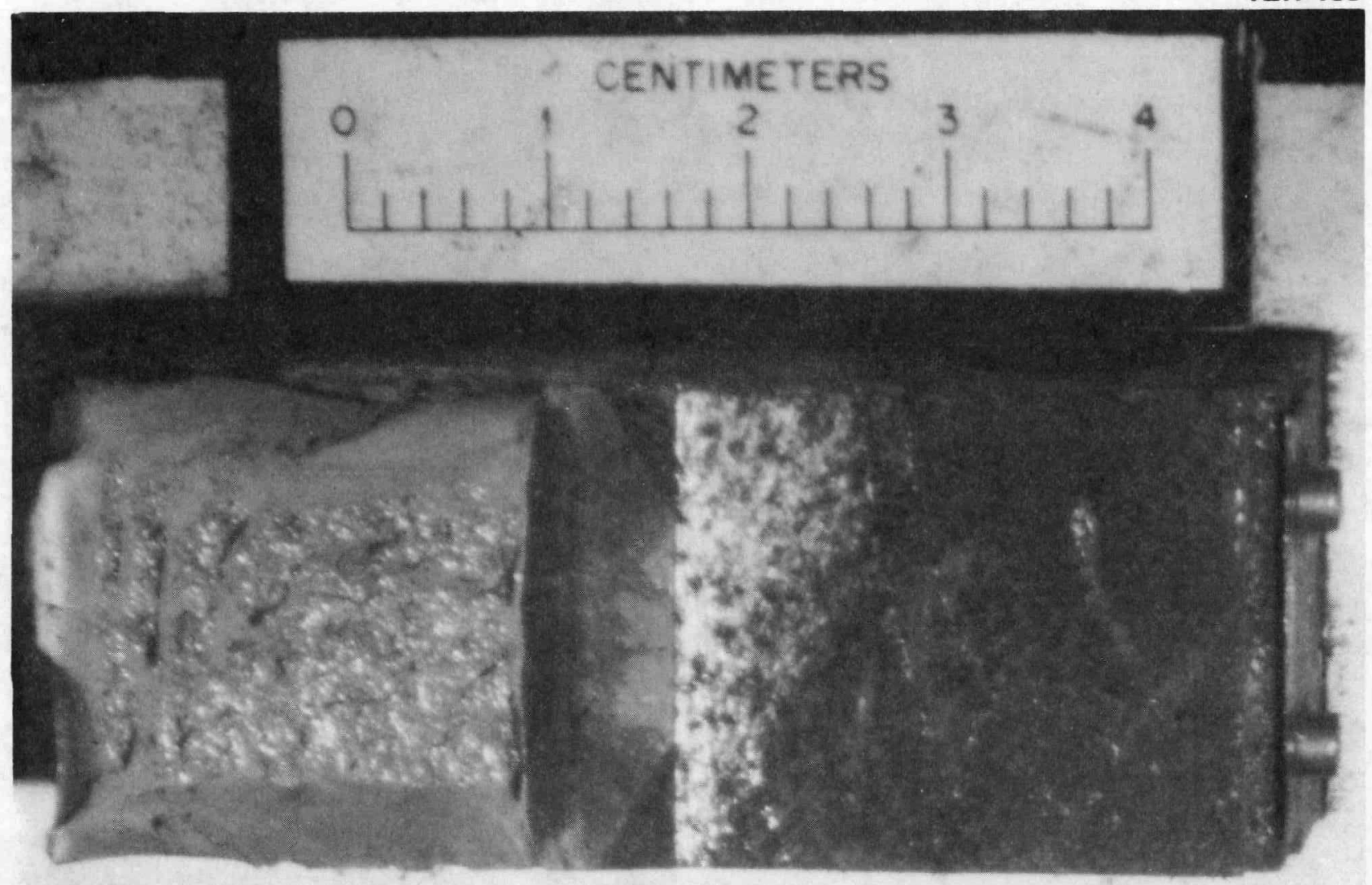




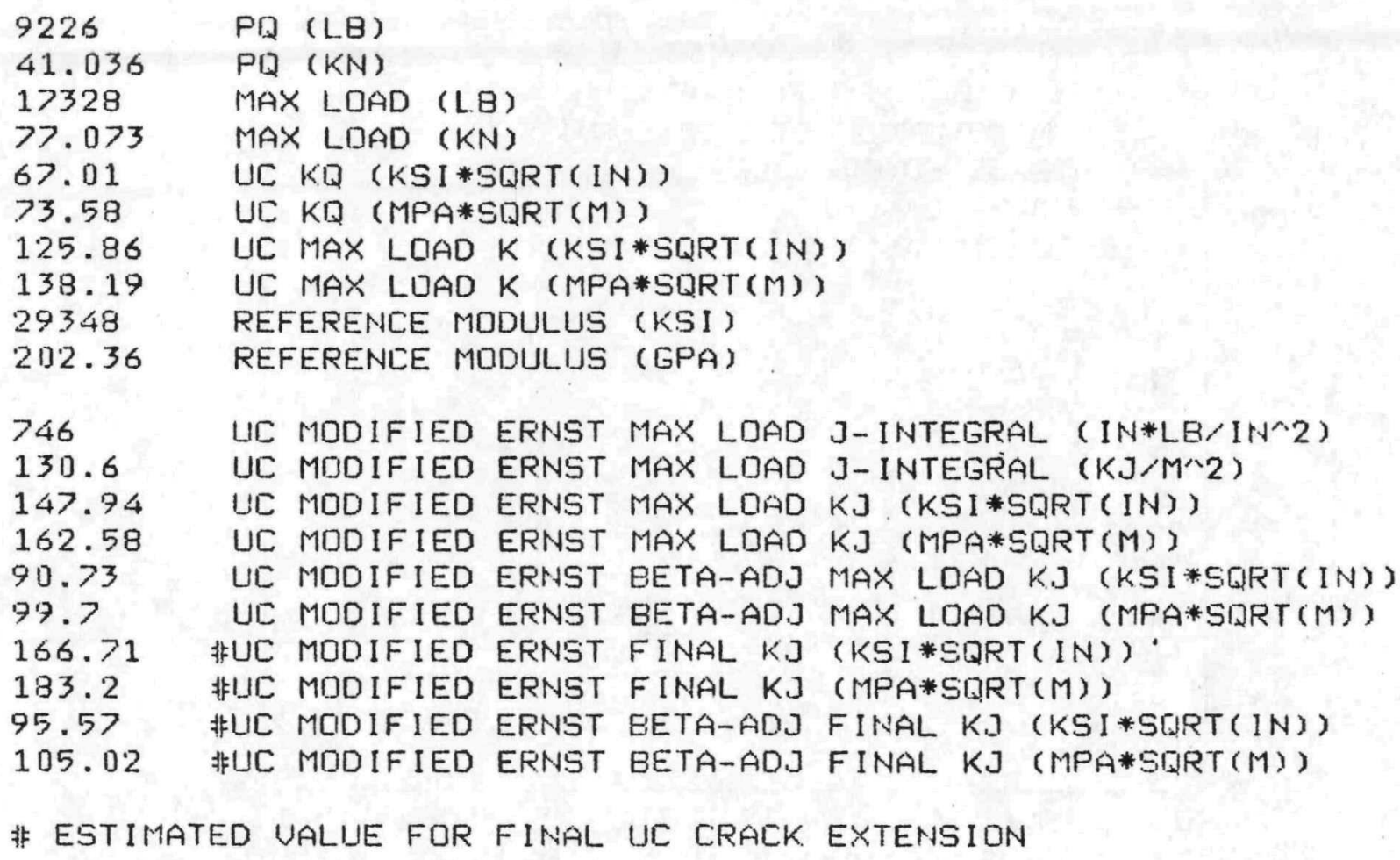


E-216
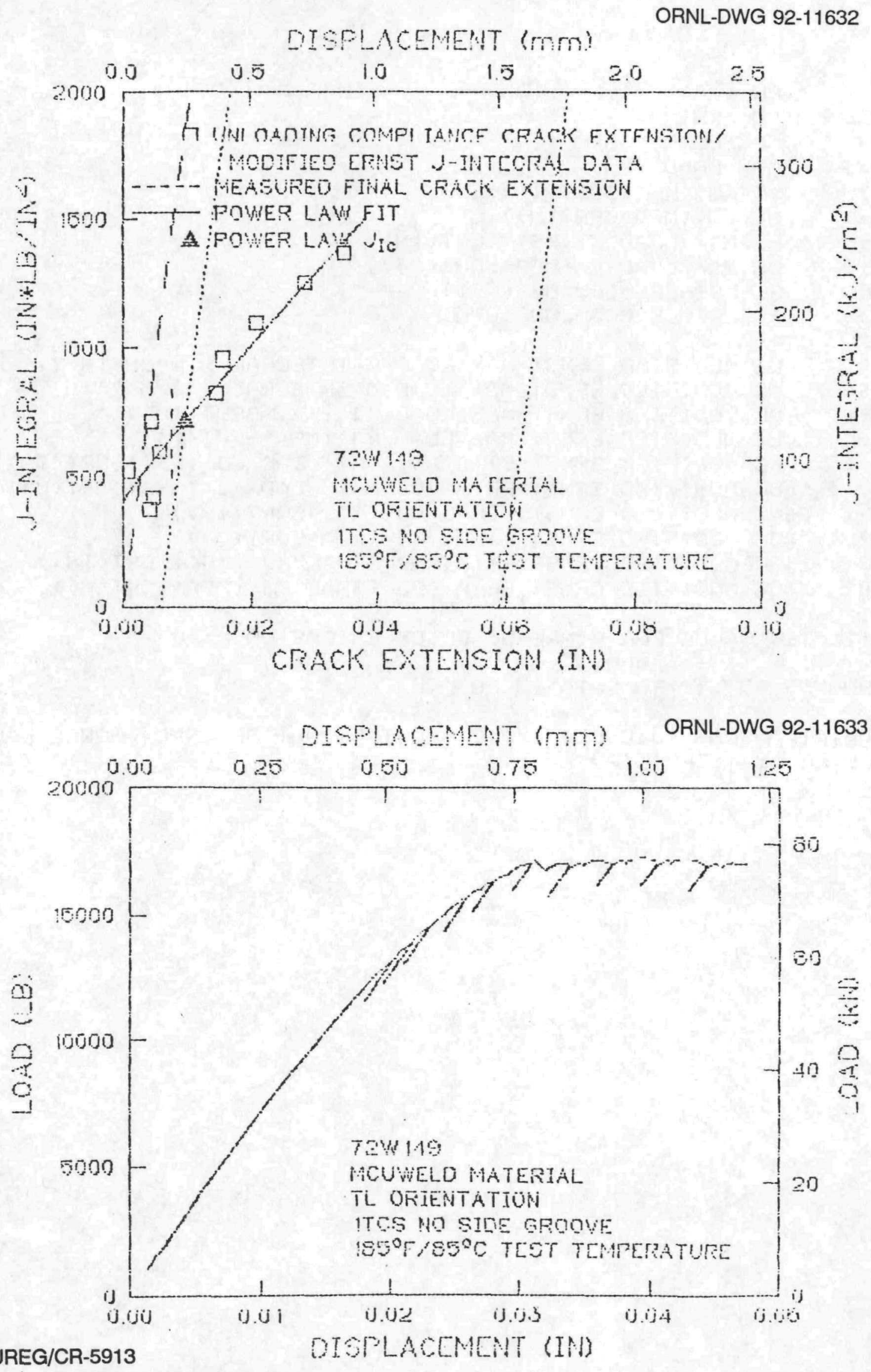


\section{E-217}

$72 W-149$

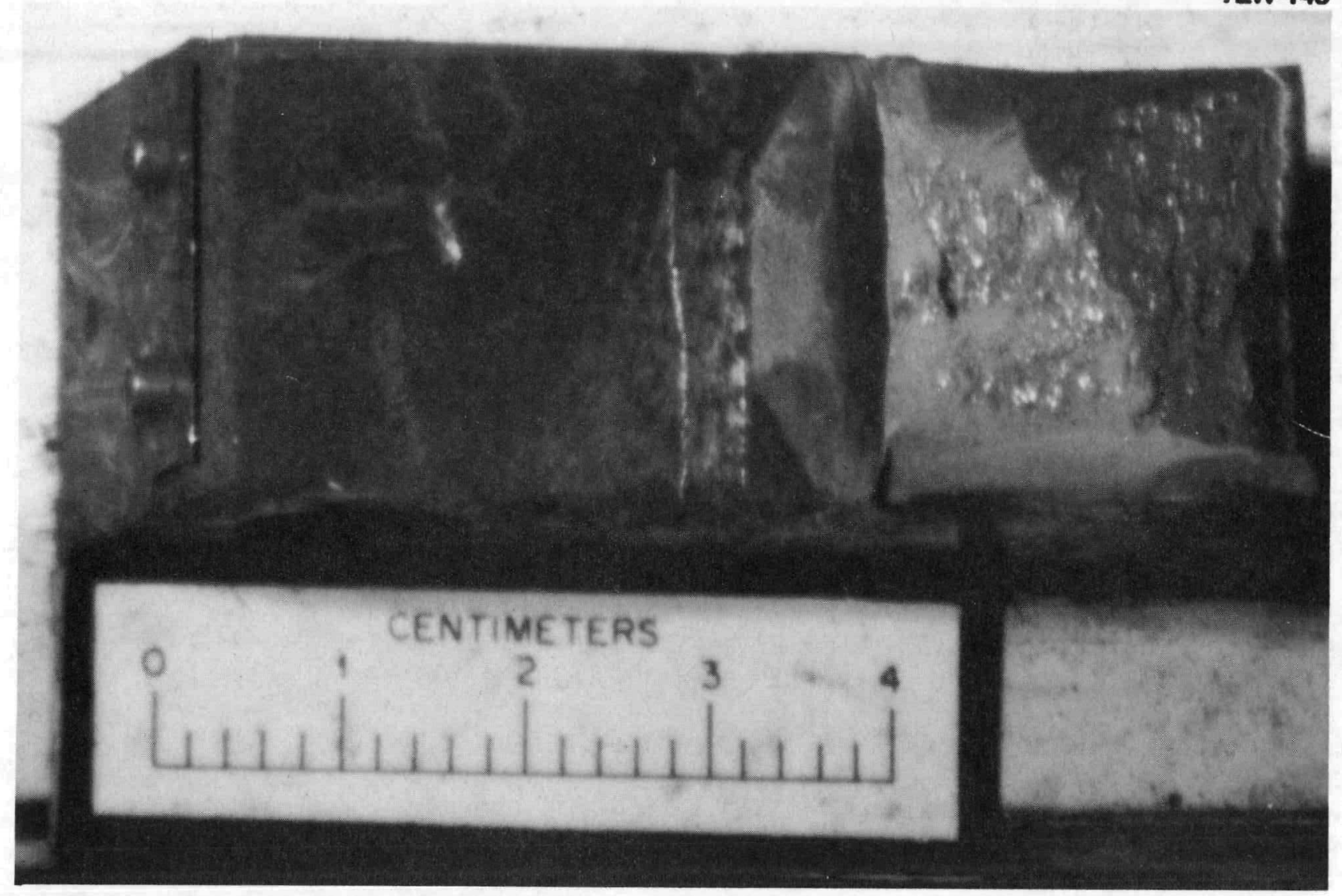

NUREG/CR-5913 


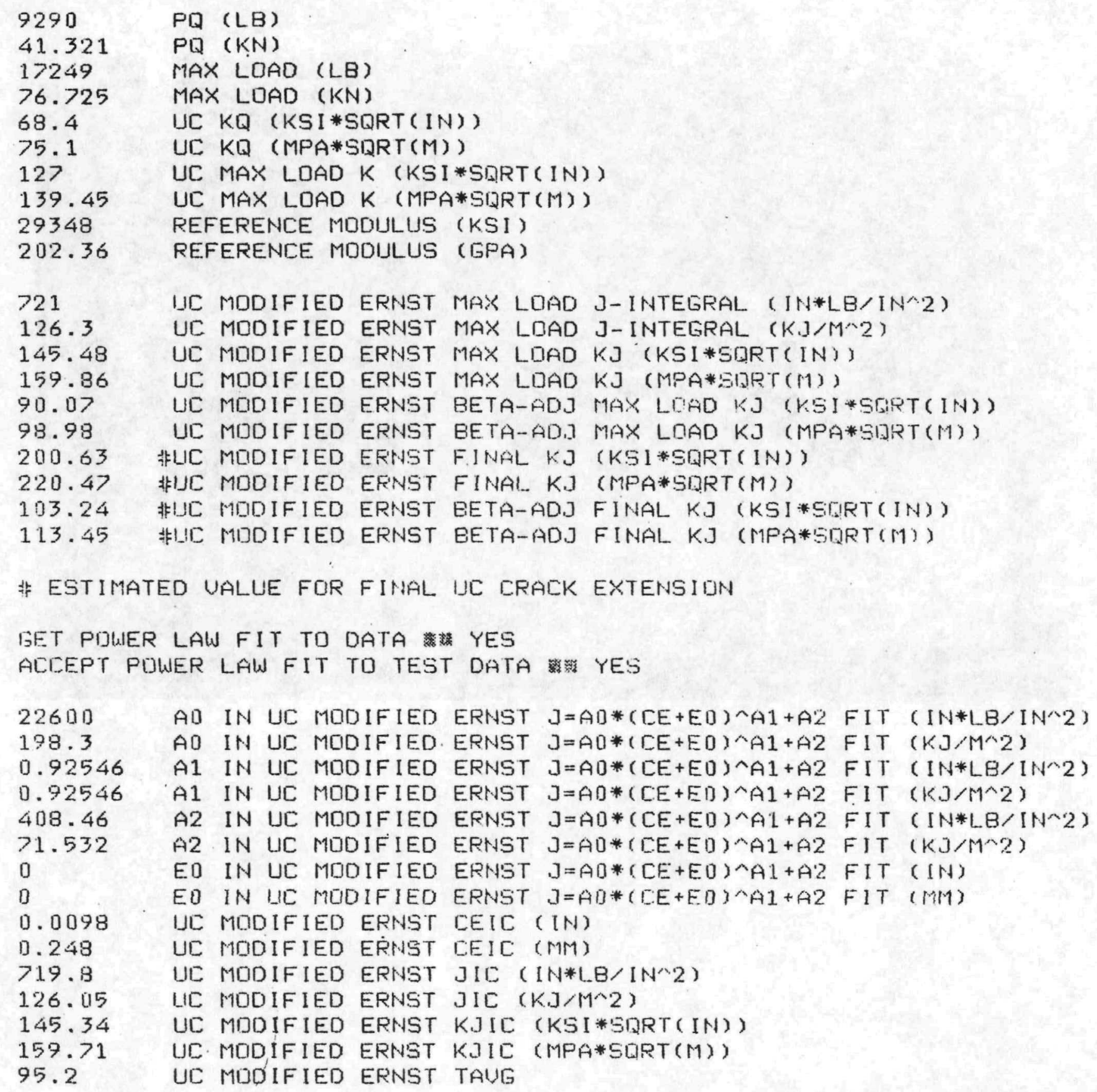



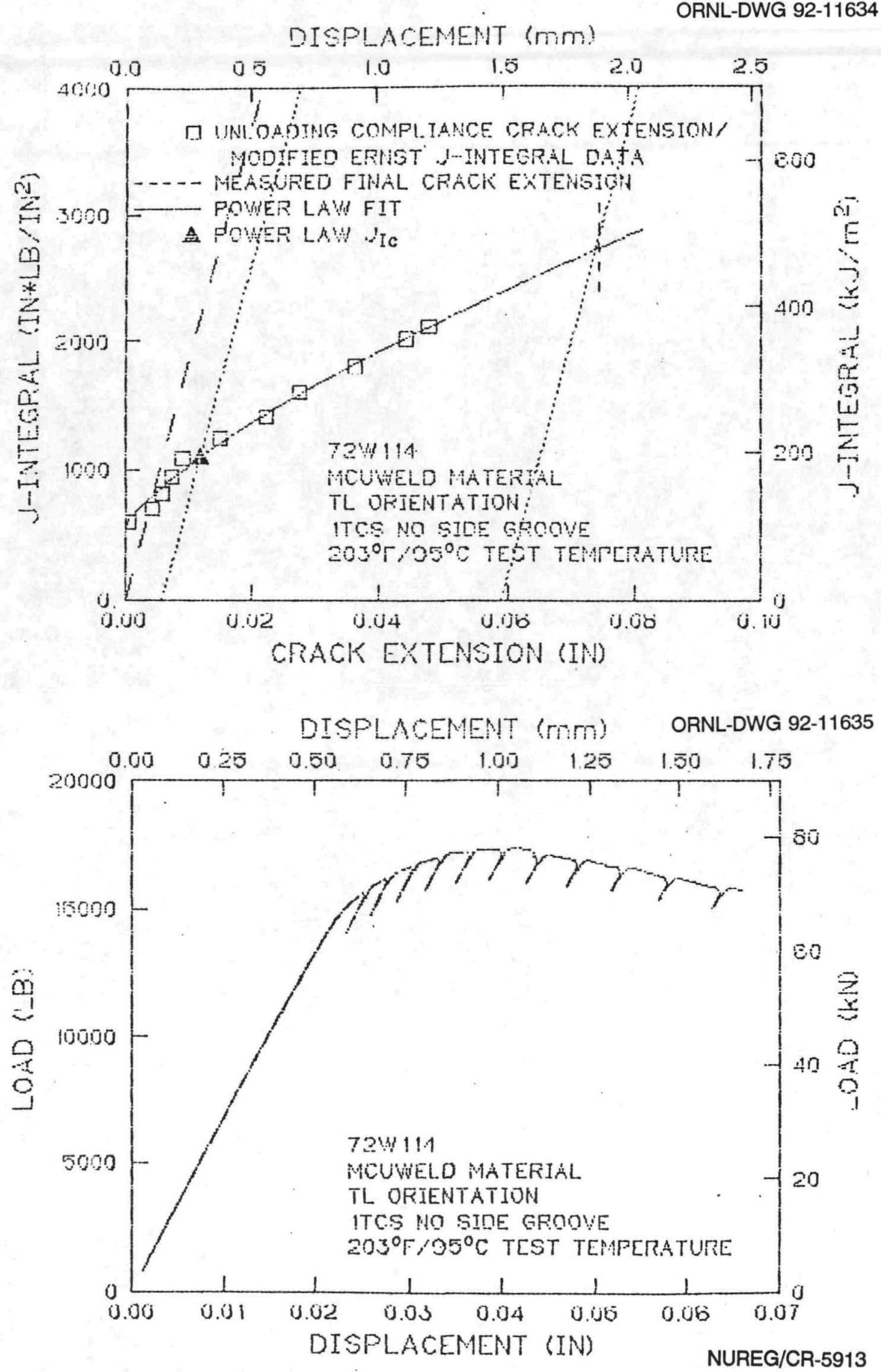


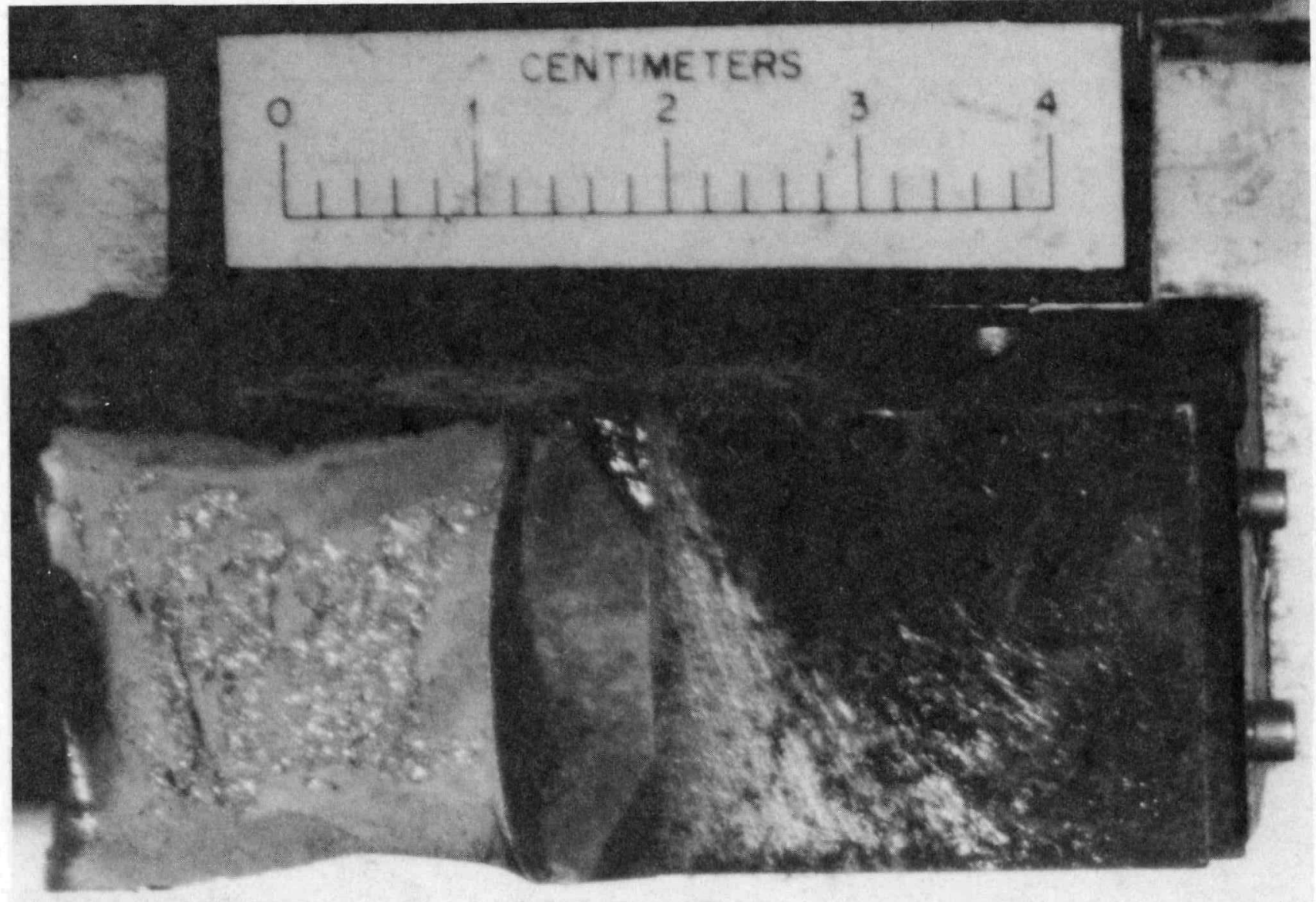




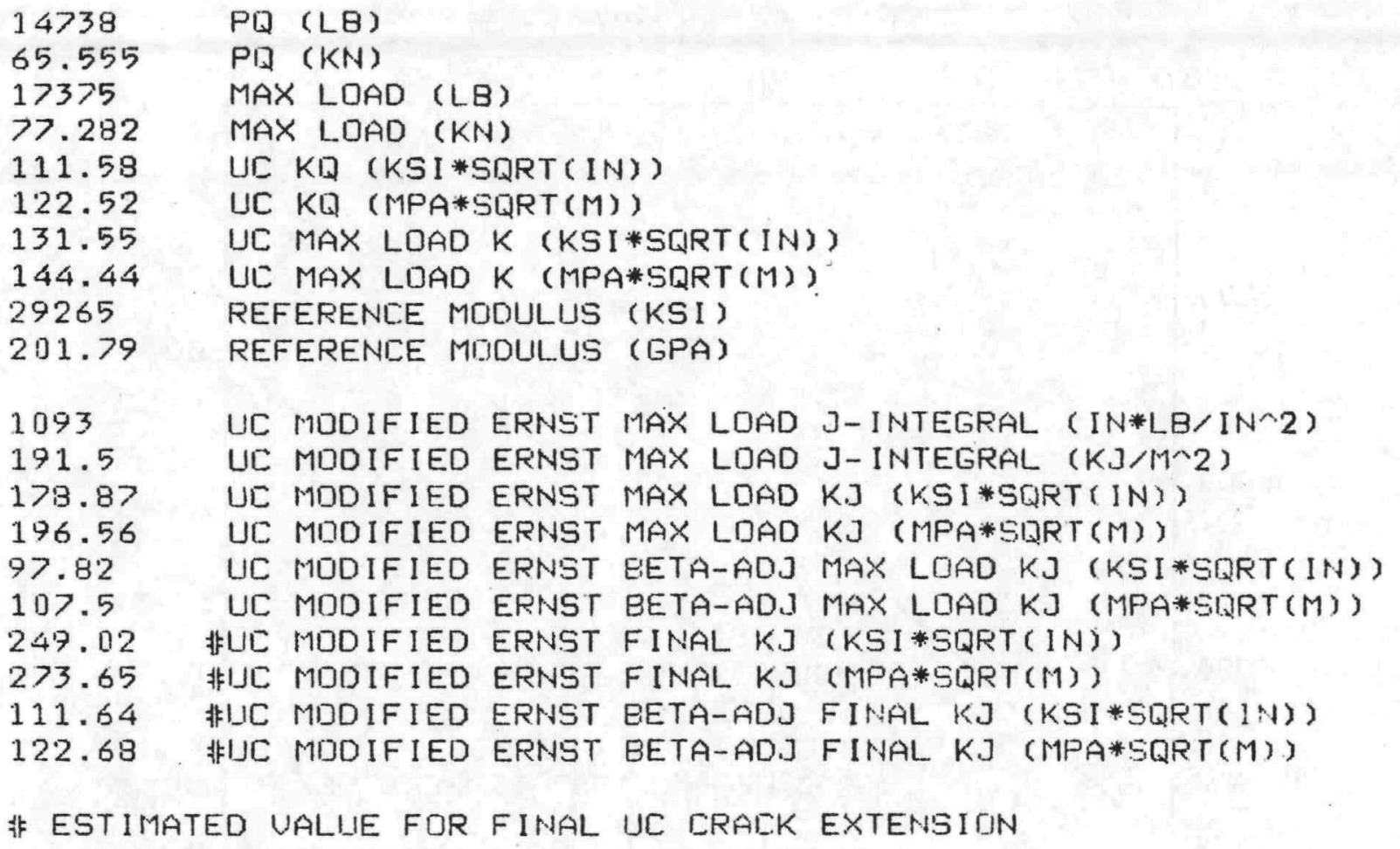

GET POWER LAW FIT TO DATA Y YES

ACCEPT POWER LAW FIT TO TEST DATA 紋 YES.

\begin{tabular}{|c|c|c|c|c|c|}
\hline 15104 & AD & UE MOOIFIED & ERNST $J=A D *(E E+E D) \wedge A 1+A 2$ & FIT & $(I N * L B / I N \wedge 2)$ \\
\hline 246.12 & AO & IN LIC MODIFIED & $J=A D *(C E+E 0) \wedge A 1+A 2$ & FIT & $(K J / M \wedge 2)$ \\
\hline .73411 & A1 & MODIFIED & $J=A O *(C E+E 0) \wedge A 1+A^{\prime} 2$ & FIT & $(I N * L B / I N \wedge 2$ \\
\hline$? 3411$ & A1 & MODIF IED & $J=A 0 *(C E+E D) \wedge A 1+A 2$ & FIT & $(K J / M \wedge 2)$ \\
\hline 74.5 & $A 2$ & MODIF IED & $J=A O *(C E+E D) \wedge A 1+A 2$ & FIT & (IN*LB/IN^ \\
\hline 097 & $A 2$ & MODIFIED & $J=A 0 *(C E+E 0) \wedge A 1+A 2$ & FIT & $(K J / M \wedge 2)$ \\
\hline 00111 & ED & MOOIF IED & $J=A 0 *(C E+E 0) \wedge A 1+A 2$ & FIT & $(I N)$ \\
\hline 02813 & EO & IN UC MODIFIED & ERNST $J=A D *(C E+E 0)^{\wedge} A 1+A 2$ & FIT & $(M M)$ \\
\hline 0118 & UC & MODIFIED ERNST & CEIC & & \\
\hline & $\Delta C$ & MDDIFIED ERNST & CEIC (MM) & & \\
\hline 995.2 & UE & MDDIF IED ERNST & JIC (IN*LB/IN`2) & & \\
\hline 1.8 & UIE & MODIFIED ERNST & $J I C\left(K J / M^{\wedge} 2\right)$ & & \\
\hline 79.03 & UE & MODIFIED ERNST & KJIC (KSI*SQRT (IN)) & & \\
\hline .73 & $U C$ & MODIFIED ERNST & (MPA*SCLRT (M) & & \\
\hline & UE & MODIFIED ERNST & TAUG & & \\
\hline
\end{tabular}




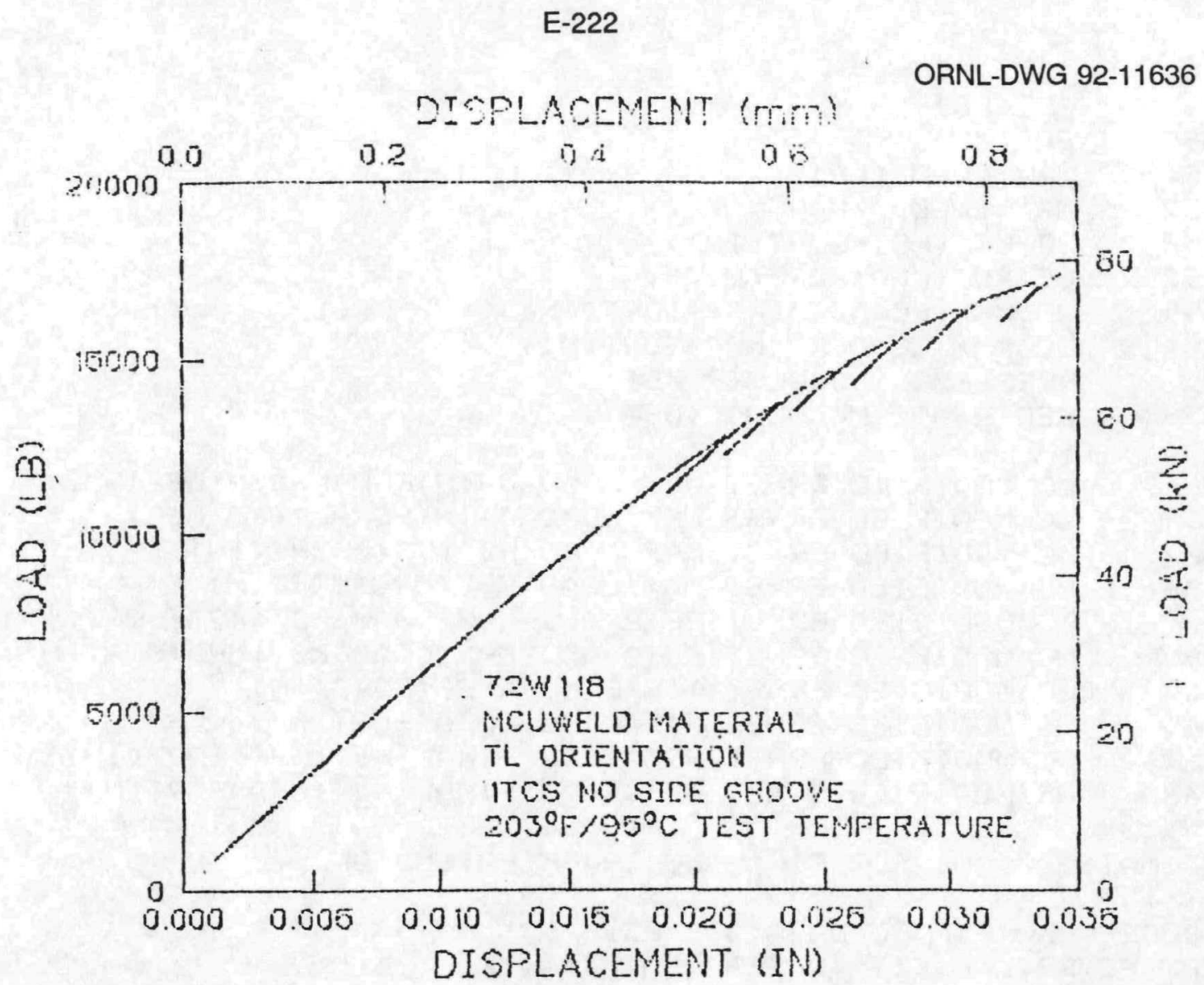

$72 W-118$

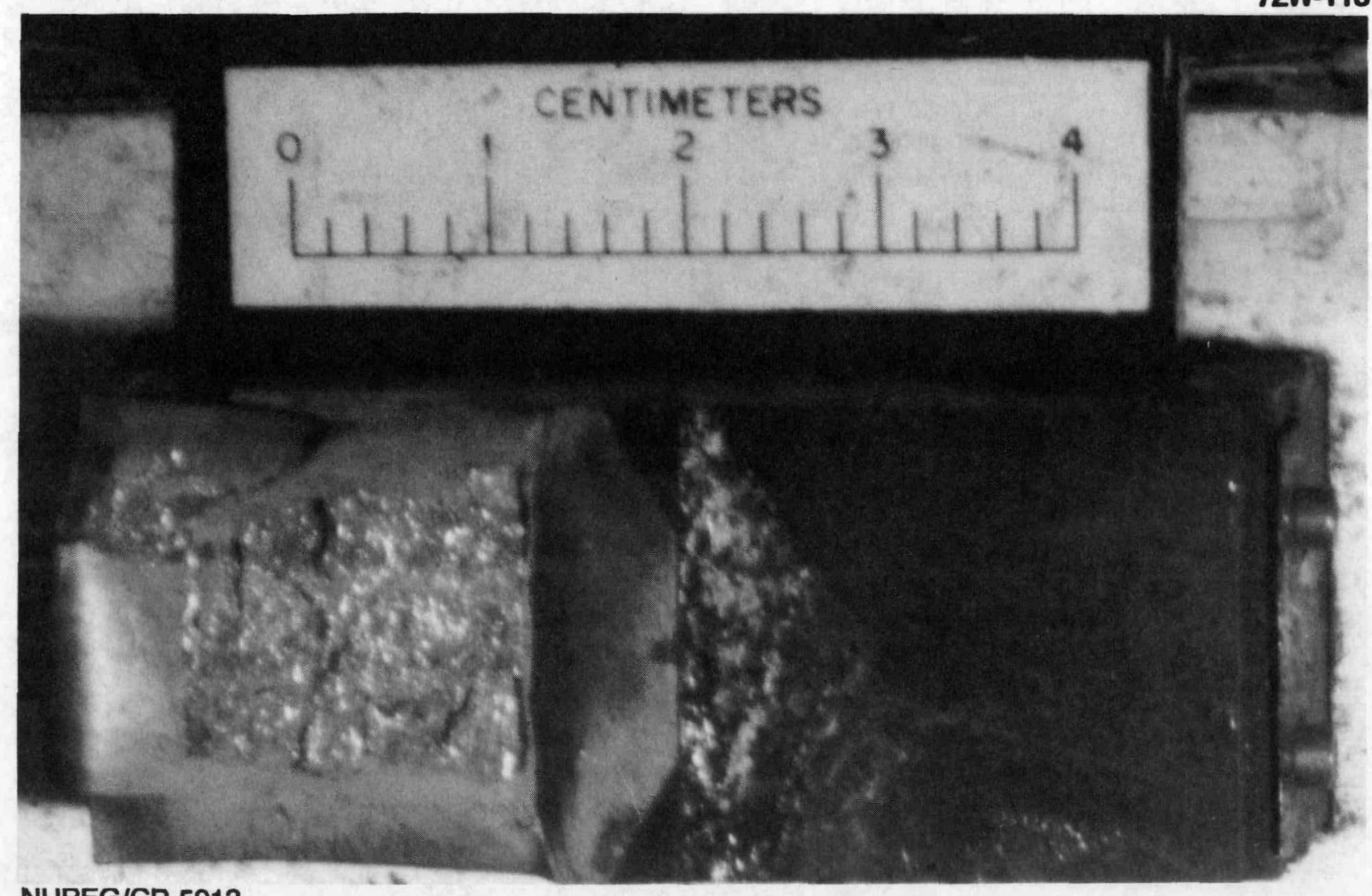




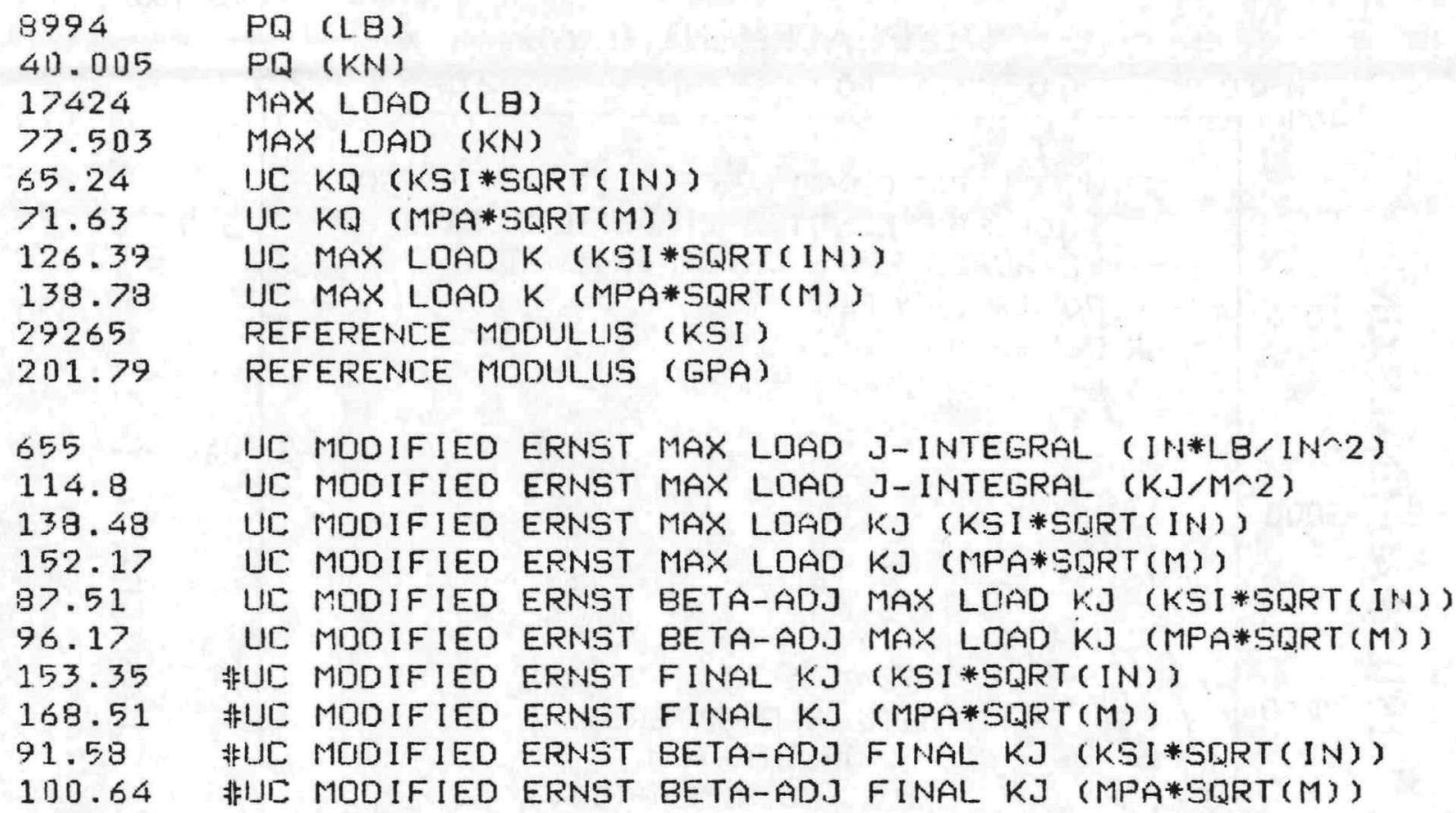


DISPLACEMENT (mm)

ORNL-DWG 92-11637
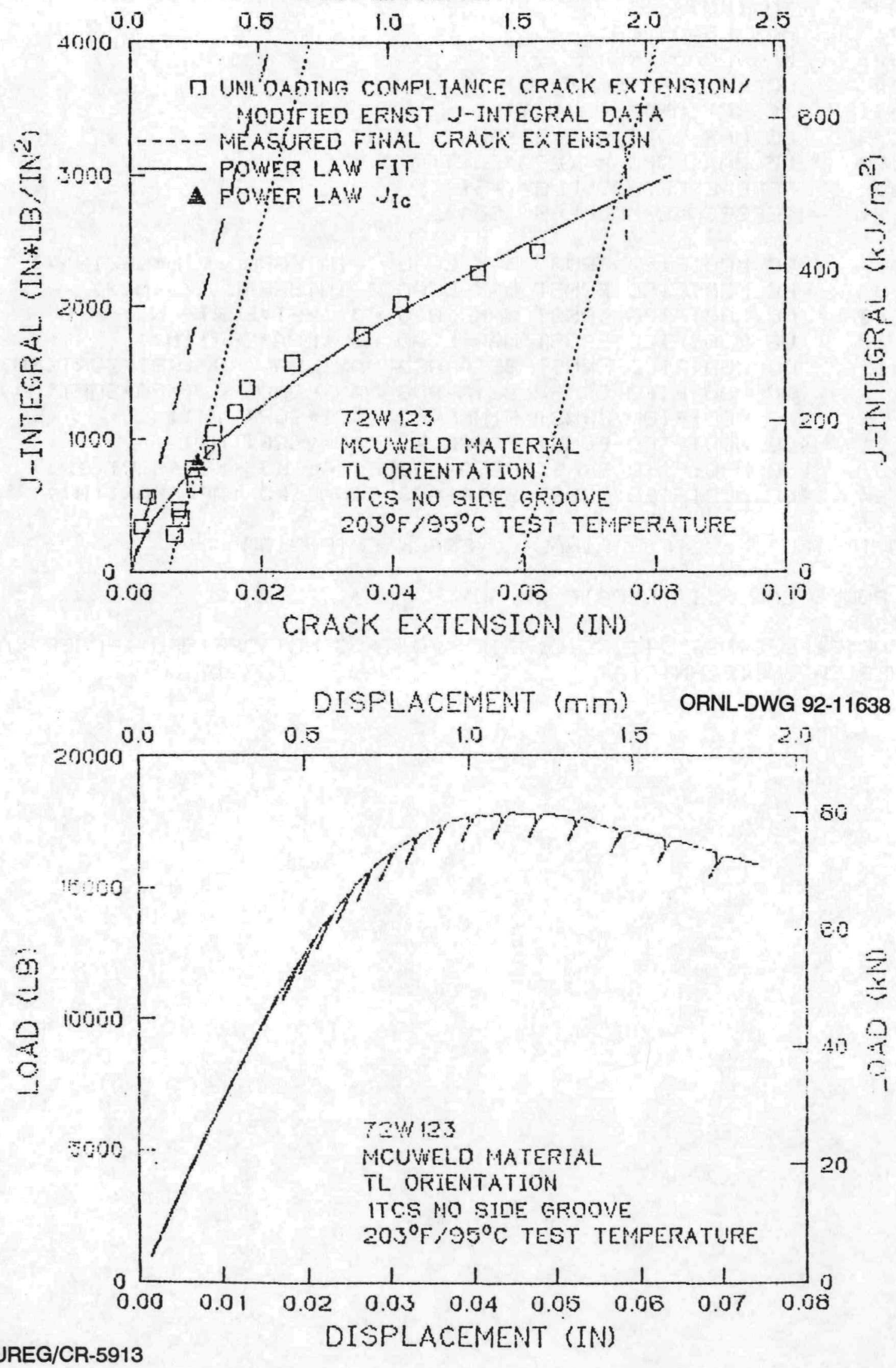


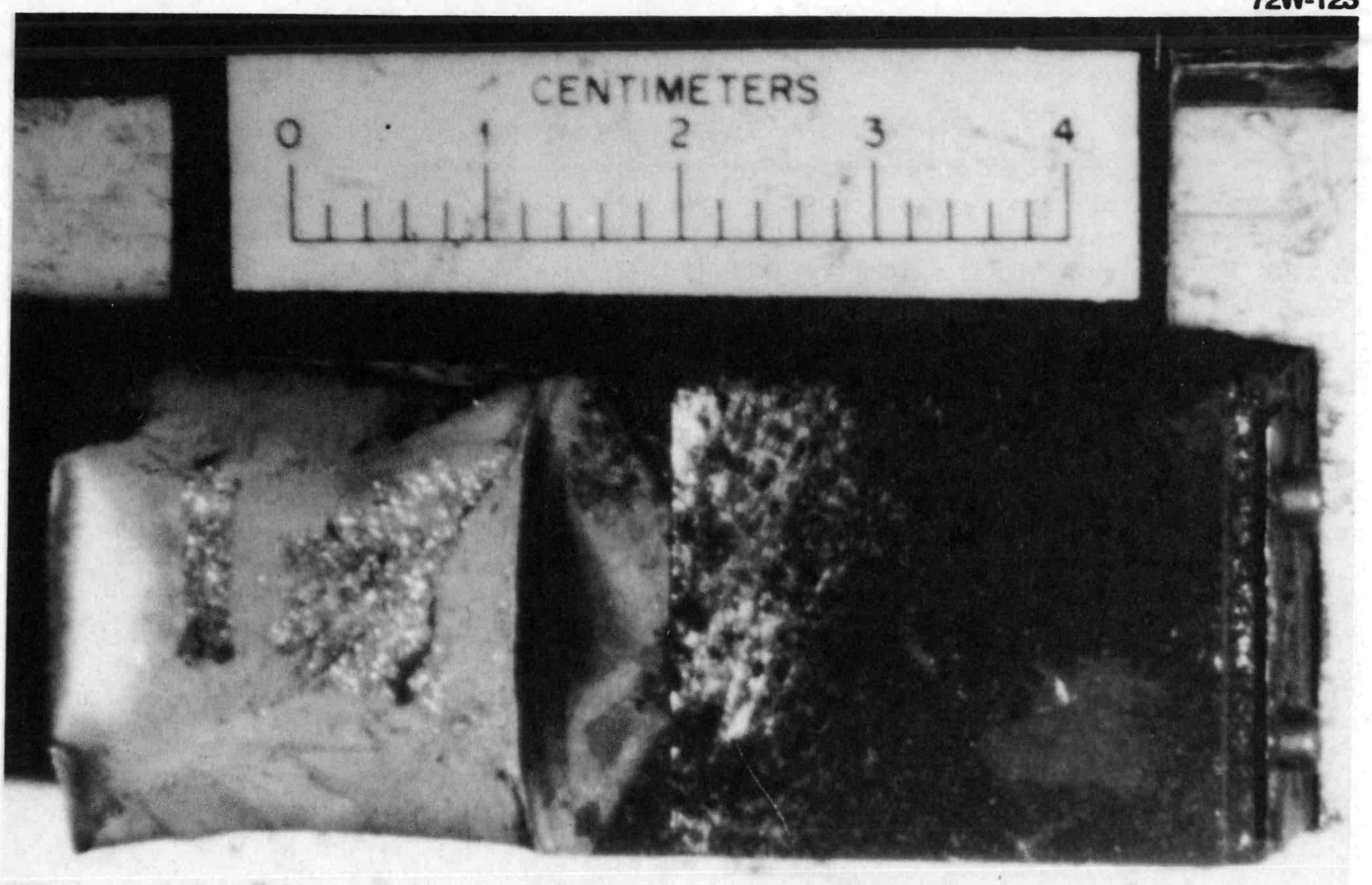




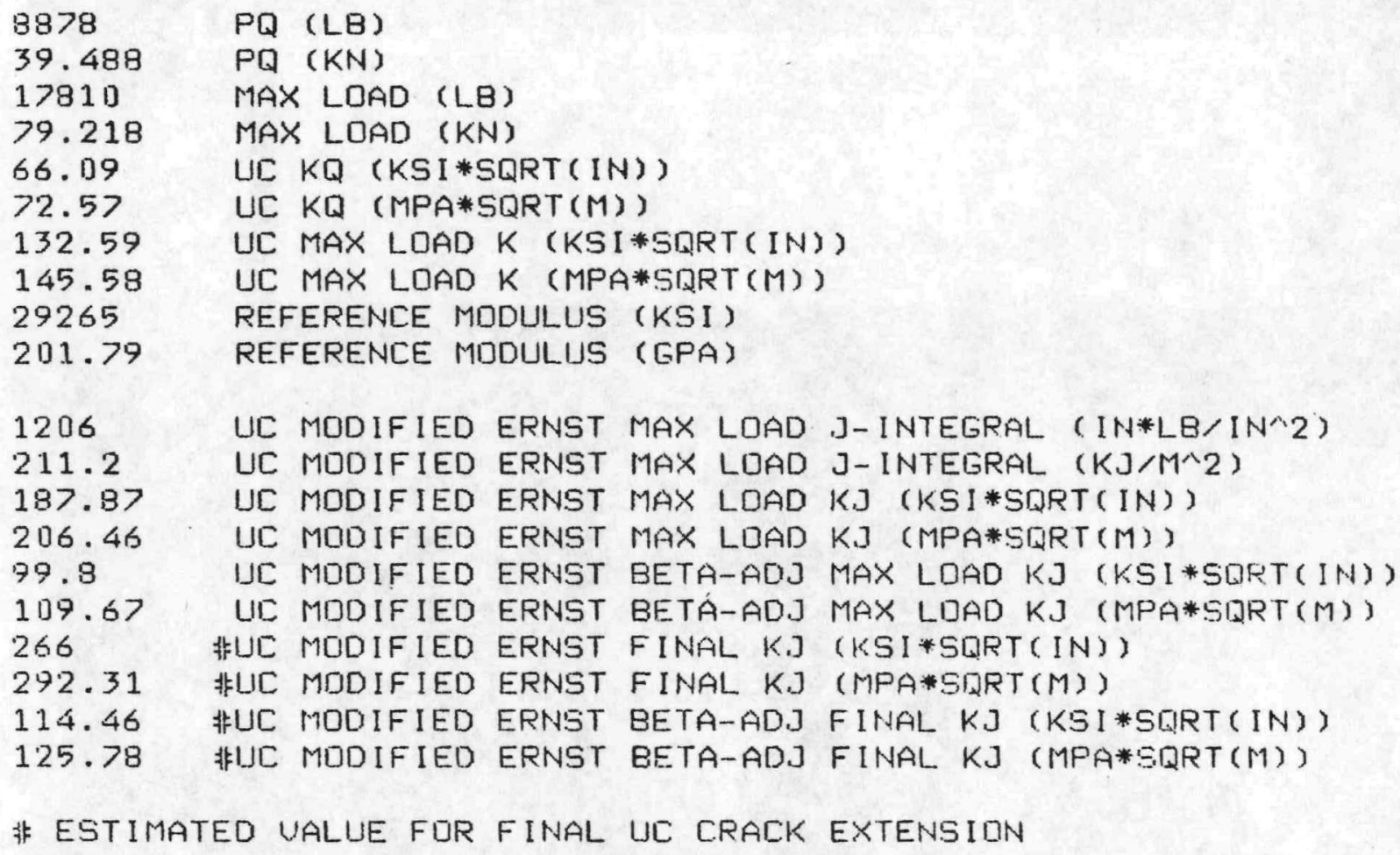



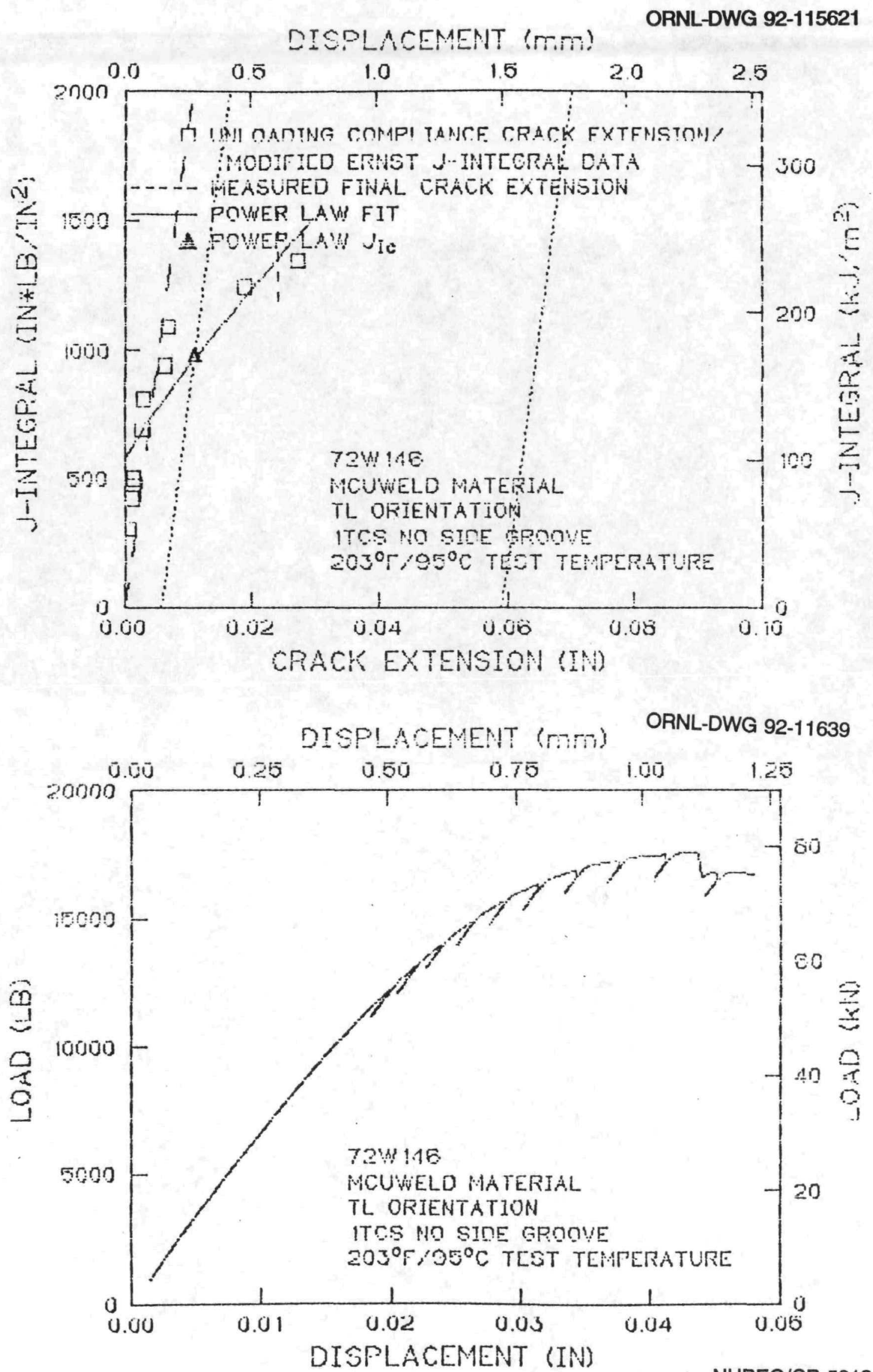
E-228

$72 W-146$

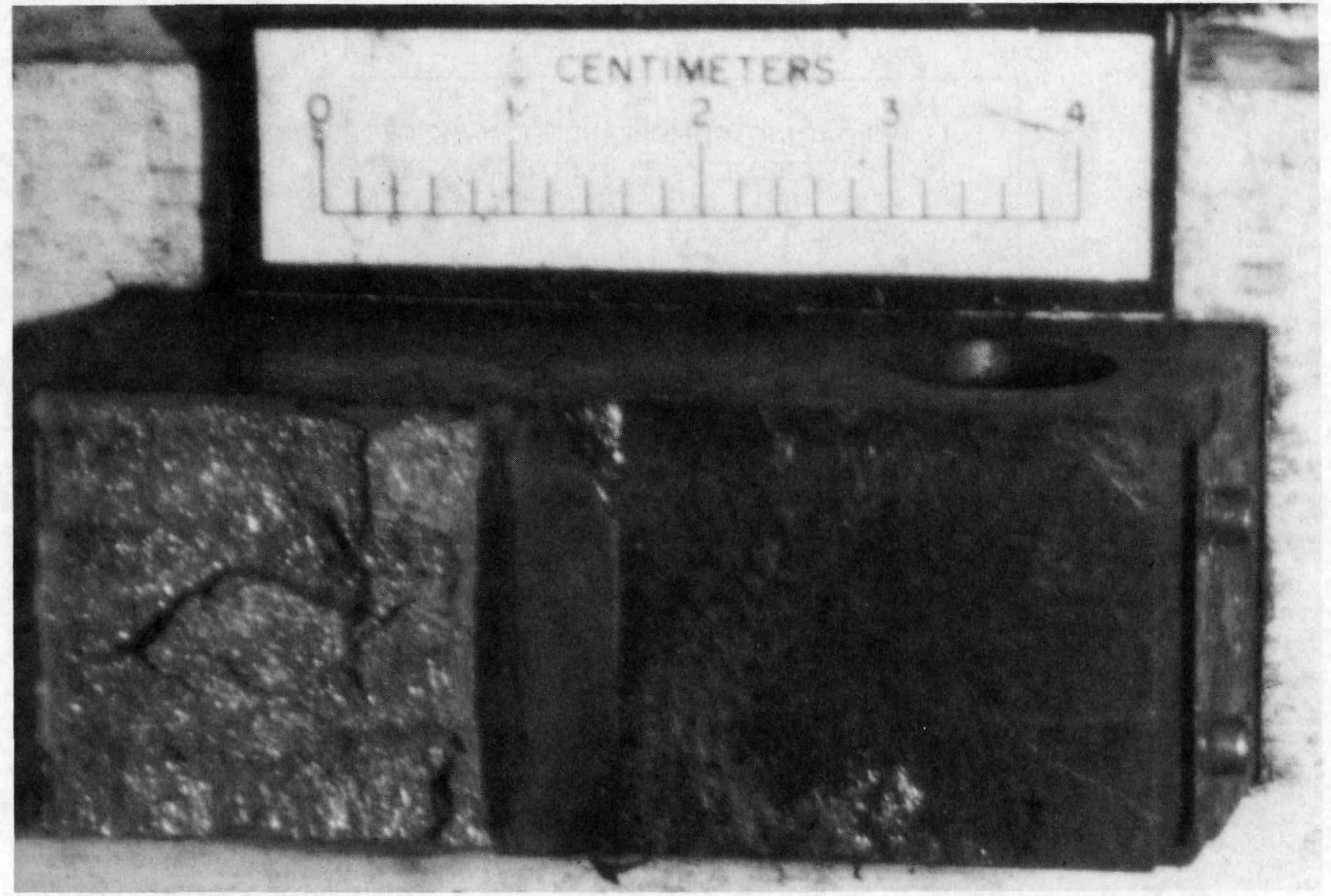

NUREG/CR-5913 


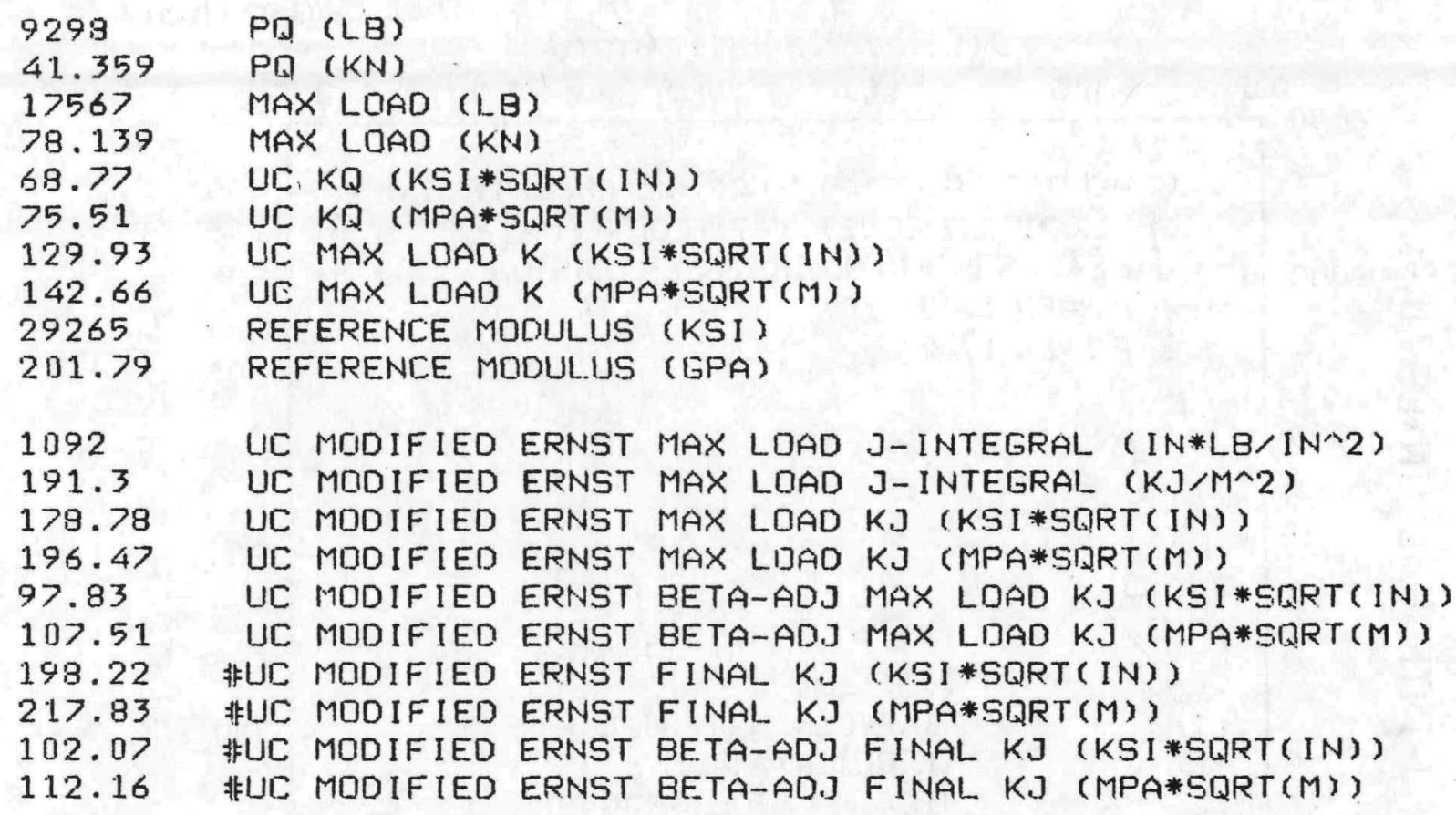

非 ESTIMATED UALUE FDR FINAL UC CRACK EXTENSION

GET POWER LAW FIT TO DATA 霖 YES ALCEPT POWER LAW FIT TO TEST DATA YES

\begin{tabular}{|c|c|c|c|c|c|}
\hline 17345 & $A D$ & IN UC MODIFIED & $J=A 0 *(C E+E 0)^{\wedge} A 1+A 2$ & FIT & $(I N * L B / I N \wedge 2)$ \\
\hline 223.69 & AO & MOD IF IED & $J=A 0 *(C E+E 0) \wedge A 1+A 2$ & FIT & $\left(K . J / M^{\wedge} 2\right)$ \\
\hline 0.81641 & A1 & MODIF IED & $J=A D *(C E+E D)^{\wedge} A 1+A 2$ & FIT & $\left(I N * L B / I N^{\wedge} 2\right)$ \\
\hline 0.80641 & A1 & MDDIF IED & $J=A O *(C E+E D) \wedge A 1+A 2$ & FIT & $\left(K J / M^{\wedge} 2\right)$ \\
\hline 365.66 & A2 & MODIF IED & $J=A Q *(C E+E O)^{\wedge} A 1+A 2^{\prime}$ & FIT & ( IN*LB/IN^? \\
\hline 64.037 & $A 2$ & MOD IF IED & $J=A 0 *(C E+E 0) \wedge A 1+A 2$ & F IT & $\left(K J / M^{\wedge} 2\right)$ \\
\hline 0.00439 & EO & UC MODIFIED & $J=A D *(C E+E D) \wedge A 1+A 2$ & FIT & $($ IN) \\
\hline 0.1114 & EO & IN UC MODIFIED & $J=A 0 *(C E+E 0) \wedge A 1+A 2$ & F I T & $(M M)$ \\
\hline 0.0111 & UC & MODIFIED ERNST & CEIC (IN) & & \\
\hline 0.283 & UC & MDD IF IED & CEIC (MM) & & \\
\hline 968.9 & UC & MDOIF IED & JIL (IN*LB $/ I N \wedge 2)$ & & \\
\hline 169.68 & UC & MODIF IED & $J I C\left(K J / M^{\wedge} 2\right)$ & & \\
\hline 168.39 & UC & MODIFIED ERNST & KJII (KSI*SQRT (IN)) & & \\
\hline 185.04 & UC & MOD IF IED & (MPA*SQRT (M)) & & \\
\hline 89.5. & UC & MDDIFIED ERNST & TAUG & & \\
\hline
\end{tabular}


DISPLACEMENT (mM)

ORNL-DWG 92-11640
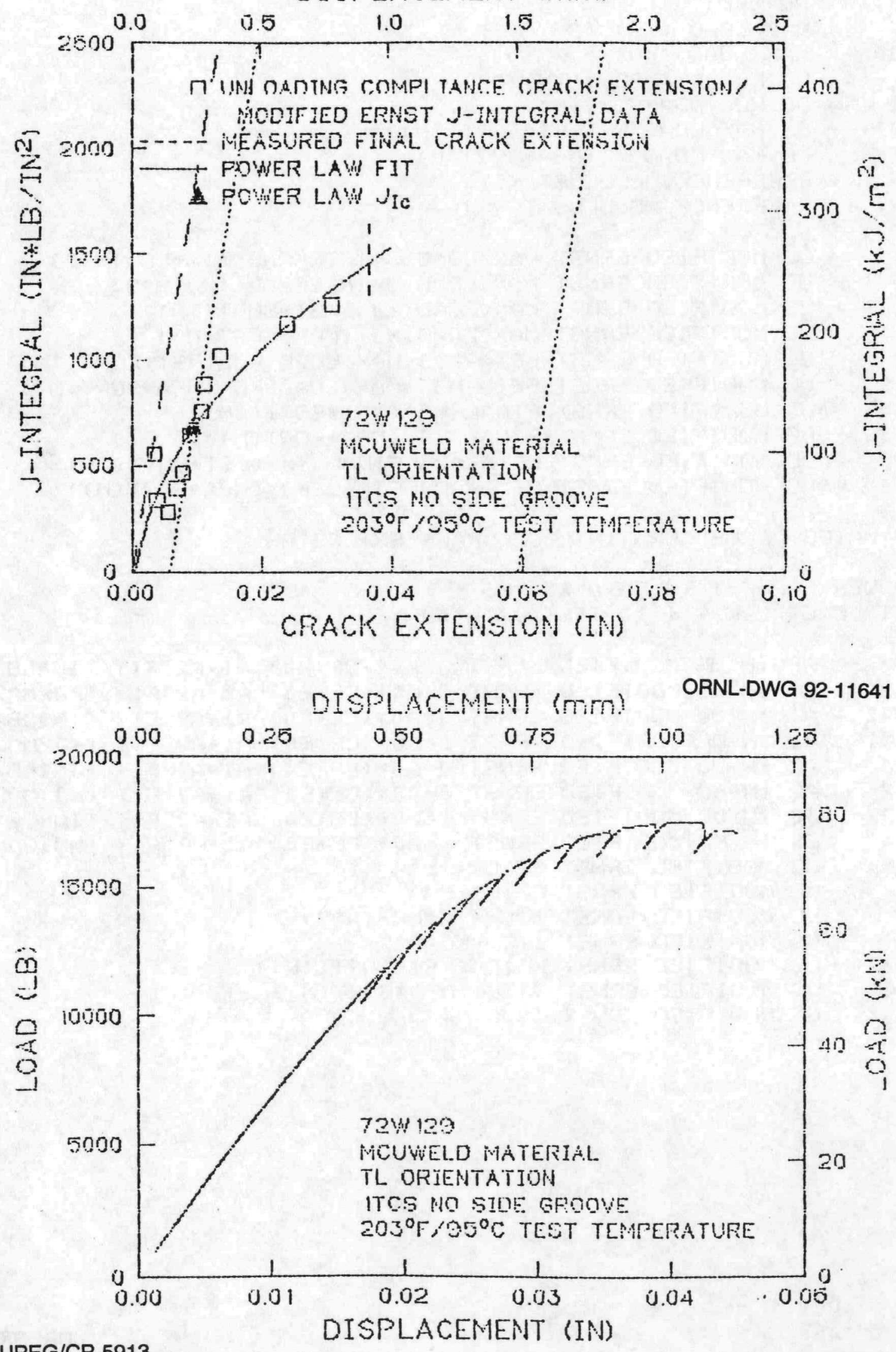


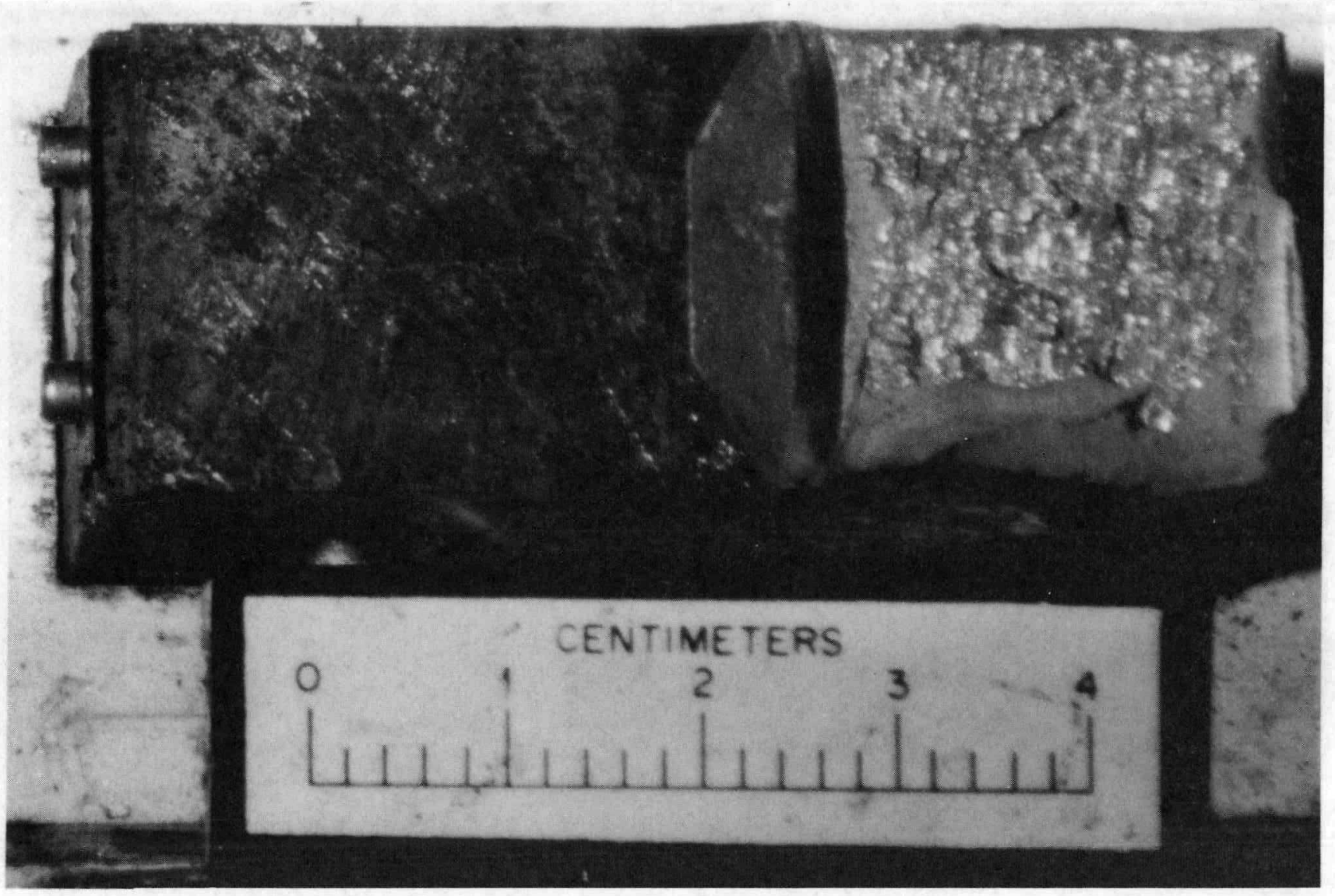




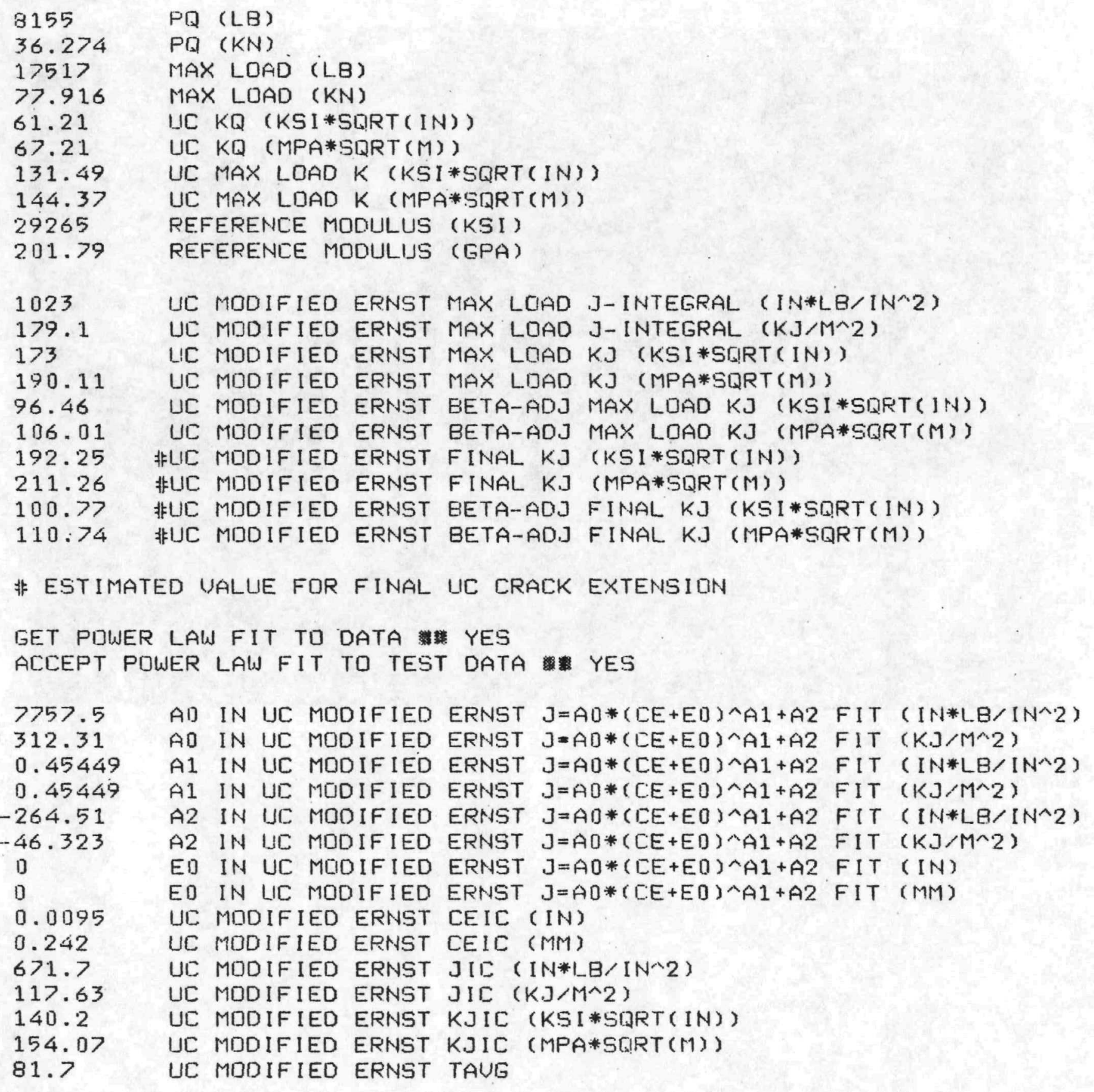




\section{E-233}

SPECIMEN ID $=72 W 112$

TEST TEMPERATURE $=125$ DEG C

THICKNESS $=25.4 \mathrm{~mm}$

SIDE GROOUES $=0 \%$

INITIAL CRACK LENGTH $=26.8 \mathrm{~mm}$

MEASURED DUCTILE DELTA-A $=3.81 \mathrm{~mm}$

$a / W) i=.528$

YIELD STRENGTH $=621 \mathrm{MPa}$

FLOW STRENGTH $=672 \mathrm{MPa}$

ESTIMATED YOUNG'S MODULUS $=200 \mathrm{GPa}$

SPECIMEN DID NDT CLEAUE, J-MAX LDAD $=260.4 \mathrm{~kJ} / \mathrm{m}^{\wedge} 2$

$K J-M A X L D A D=228.3 \quad M P a \operatorname{SQR}[\mathrm{m}]$

POWER LAW PROCEDURE

JI $=379.9 \mathrm{~kJ} / \mathrm{m}^{\wedge}$

$\mathrm{K}-J \mathrm{Ic}=275.7 \mathrm{MPa} \operatorname{SQR}[\mathrm{m}]$

$\mathrm{POWER}=.368$

COEFFICIENT $=517.1 \mathrm{~kJ} / \mathrm{m}^{\wedge} 2$

$T$ (AUERAGE) $=78$

JI-R CURUE CALCULATIONS

$\mathrm{J}($ 回 $\mathrm{J} / \mathrm{T}=8.8)=537.8 \mathrm{~kJ} / \mathrm{m}^{\wedge} 2$

POWER $=\quad .2641$
$J-M A X$ ALLOWED $=805.4 \mathrm{~kJ} / \mathrm{m}^{\wedge} 2$

RCURUE INUALID, 23
ASTM LINEAR PROCEDURE

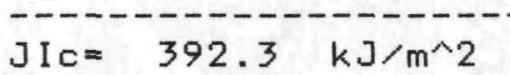

$K-J I c=280.2 \quad M P a \operatorname{SQR}[\mathrm{m}]$

$S L O P E=159700 \mathrm{~kJ} / \mathrm{m}^{\wedge} 3$

INTERCEPT $=345.7 \mathrm{~kJ} / \mathrm{m}^{\wedge} 2$

$T(A S T M)=71$

INUALID, 6

COEFFICIENT $=533.7 \mathrm{~kJ} / \mathrm{m}^{\wedge} 2$

DELTA-A MAX ALLOWED $=2.4 \mathrm{~mm}$ 


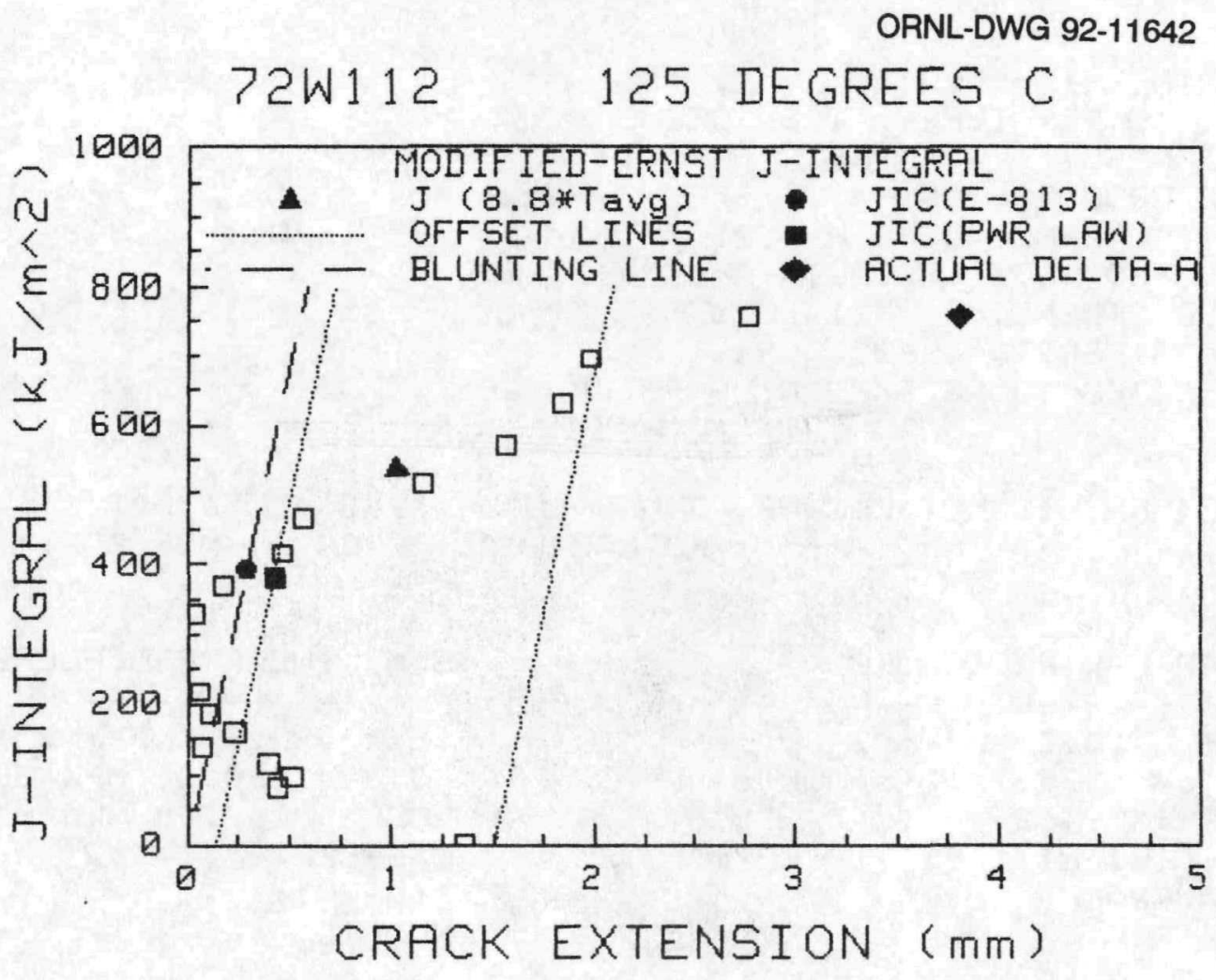




\section{W112 125 DEGREES C}

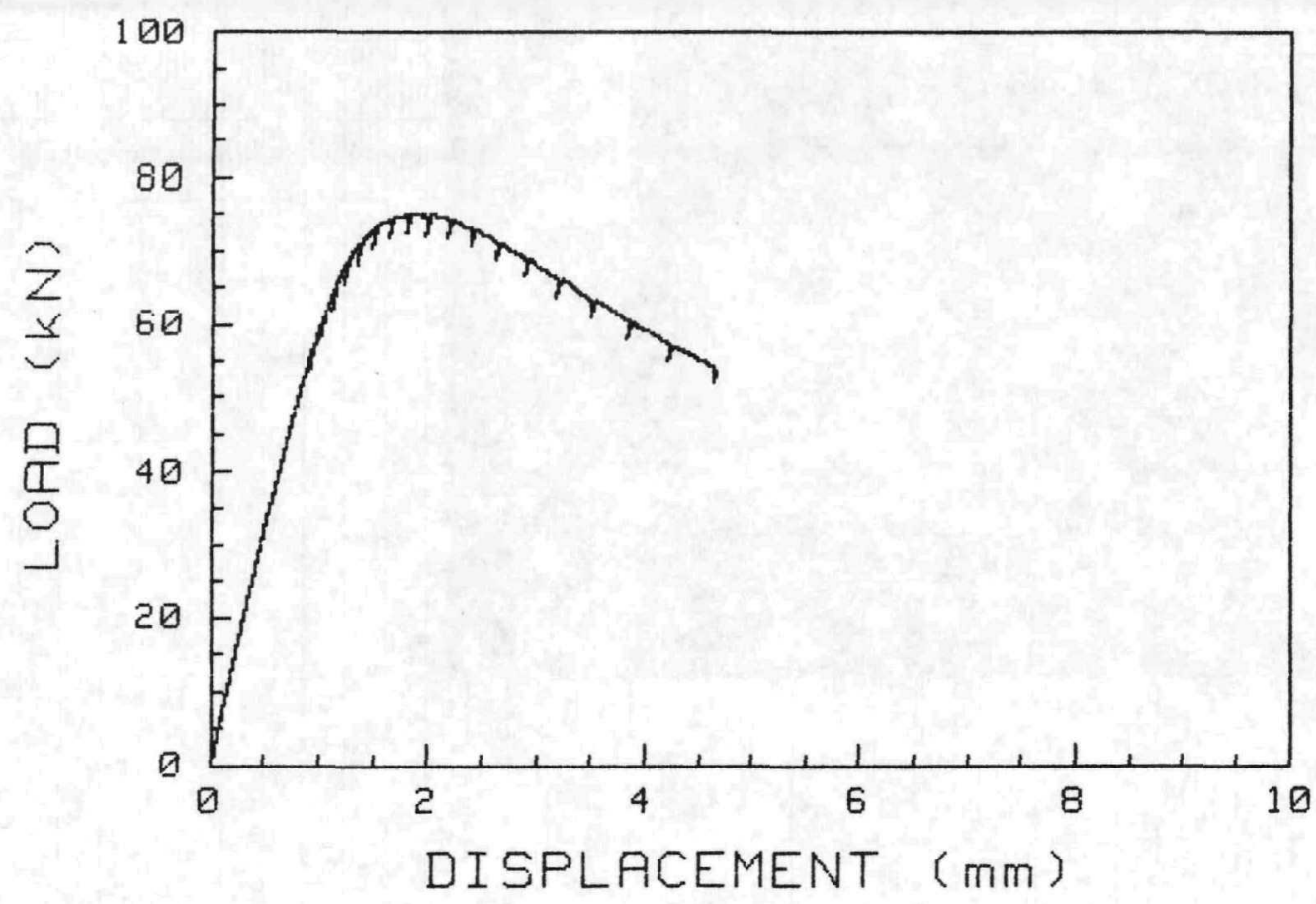




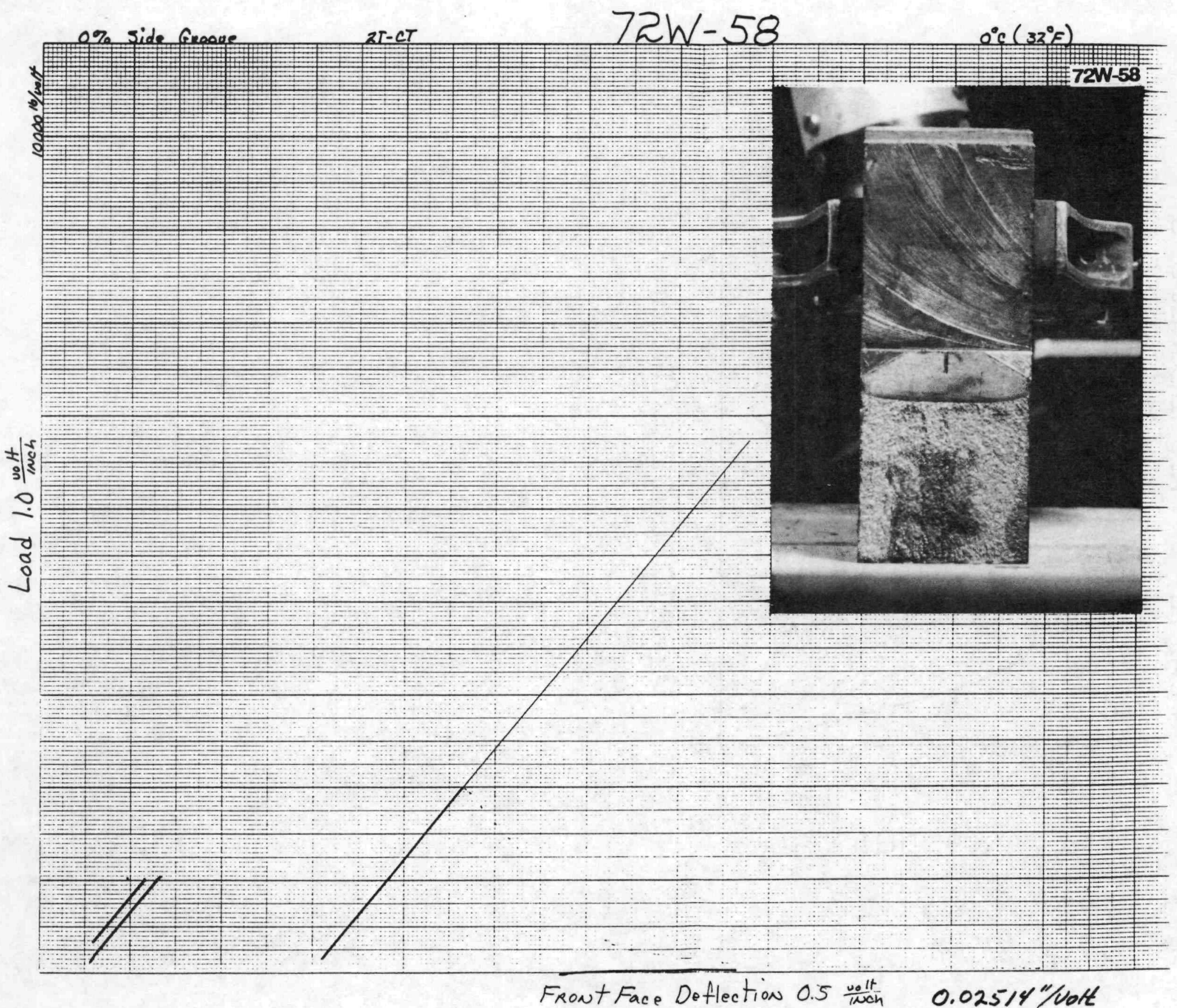

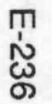




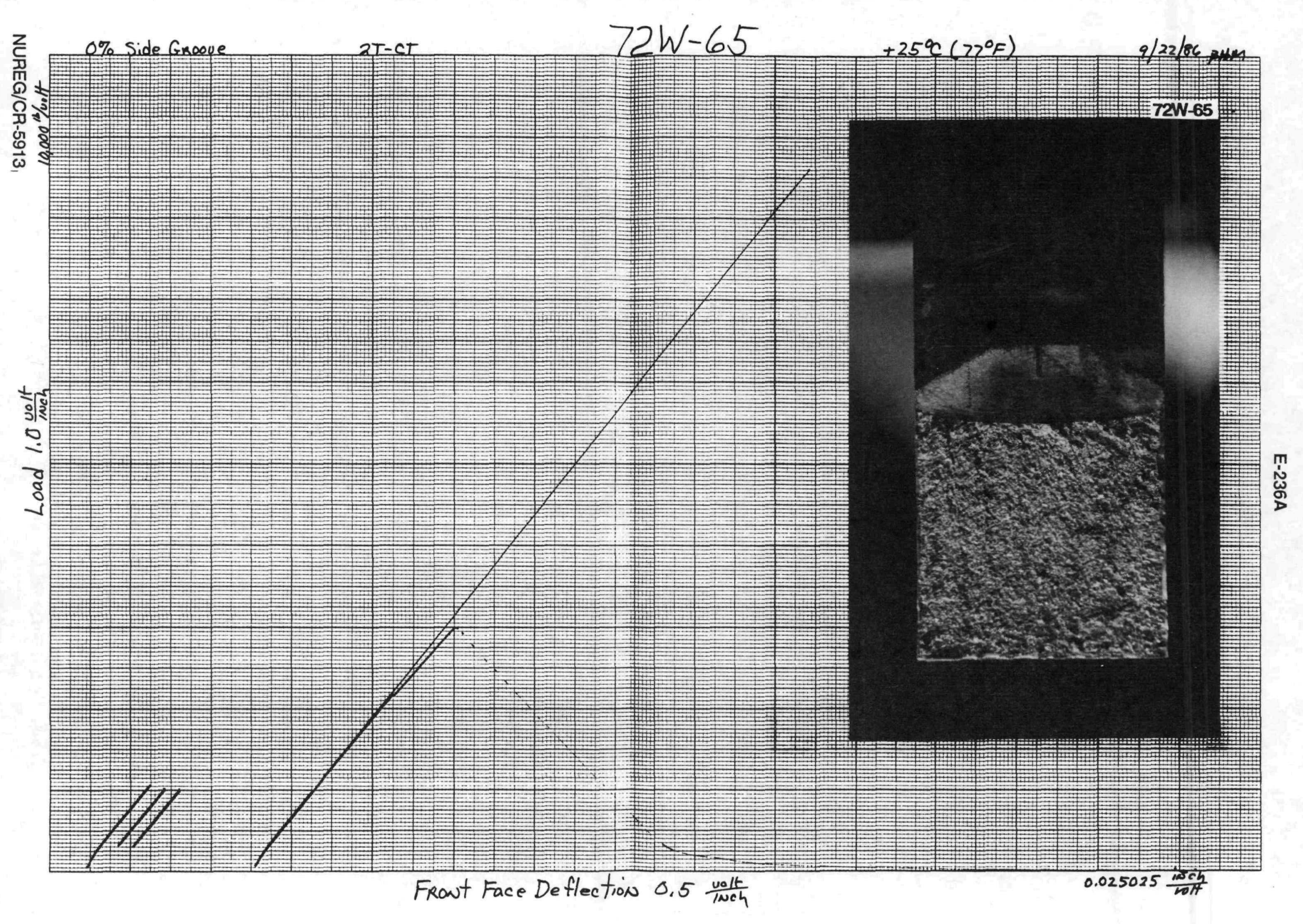


E-236B

Specimen Crackmauth Def lectian (in.) ORNL-DWG 92-11644

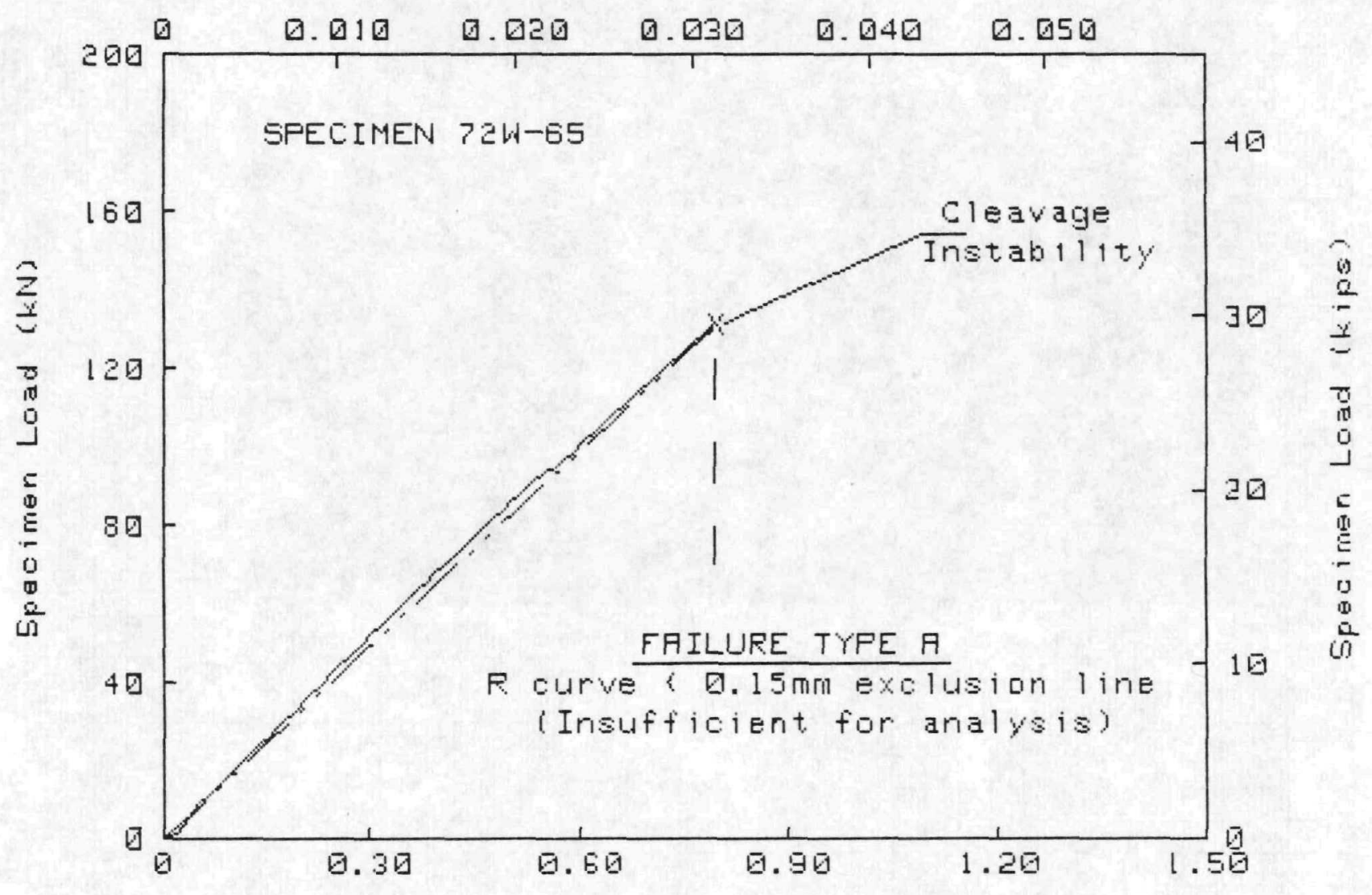

Spesimen Craokmouth Defleotion (mm)

\section{TEST SFELIMEN IATA

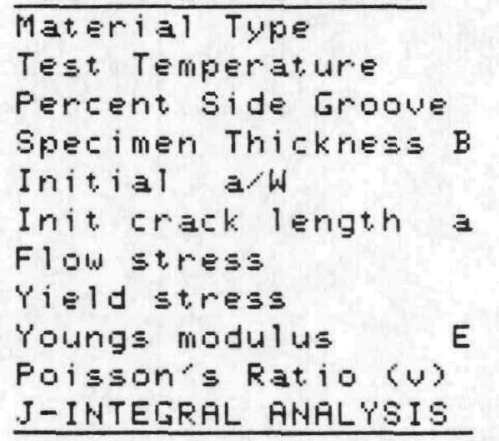

$\mathrm{Jc}$

$K j c=\left\langle E J c\left\langle\left(1-v^{2}\right\rangle\right\rangle\right) .5$

$\mathrm{Ke}$ (Beta Corrected)

ASTM E399-81 ANALYSIS

Modified Kic Equation;

Maximum Load $P$

$5 \%$ Secant offset $P q$

$\mathrm{Kq}$

Validity

$K$ at. Maximum Load

DATA CDRRECTIDNS USED FOR J-INTEGRAL IATA

Merkle-Corten correction

Correction to J for crack Extension (Deformation-J)

OFFSET LDAD $=0 \mathrm{kN}$ OFFSET DEFLECTIDH $=-.026 \mathrm{~mm}$

Fitted elastic load range $=15 \mathrm{kN}$ To $45 \mathrm{kN}$

Elastic slope $=1.734 \mathrm{E}+02 \mathrm{kN} / \mathrm{mm}$
$=A 533-B$ WEI

$=50.8 \mathrm{~mm}$

$=.523$

$=53.14 \mathrm{~mm}$

$=620.5 \mathrm{MPa}$

$=551.6 \mathrm{MPa}$

= $205800 \mathrm{MPa}$ (Estimated Value)

[ Failupe Type $A$ (J at instability = Ja) ]

\begin{tabular}{|c|c|}
\hline Merkle-cort & DEt ormat $10 n-1$ t \\
\hline $36.2 \mathrm{~kJ} / \mathrm{m}^{2}$ & $37 \mathrm{~kJ} / \mathrm{m}^{2}$ \\
\hline $86.3 \mathrm{MP} a \sqrt{\mathrm{m}}$ & $M P \equiv \sqrt{m}$ \\
\hline$M P a \sqrt{m}$ & $M P a \sqrt{T}$ \\
\hline
\end{tabular}

$B=\langle B B \cap\rangle 1\rangle 2$

= INVALID--1, 2

= $34.3 \mathrm{MPa}$

TE T 


\section{E-236C}

Gpecimen Erackmauth Def lectian (in.) ORNL-DWG 92-11645

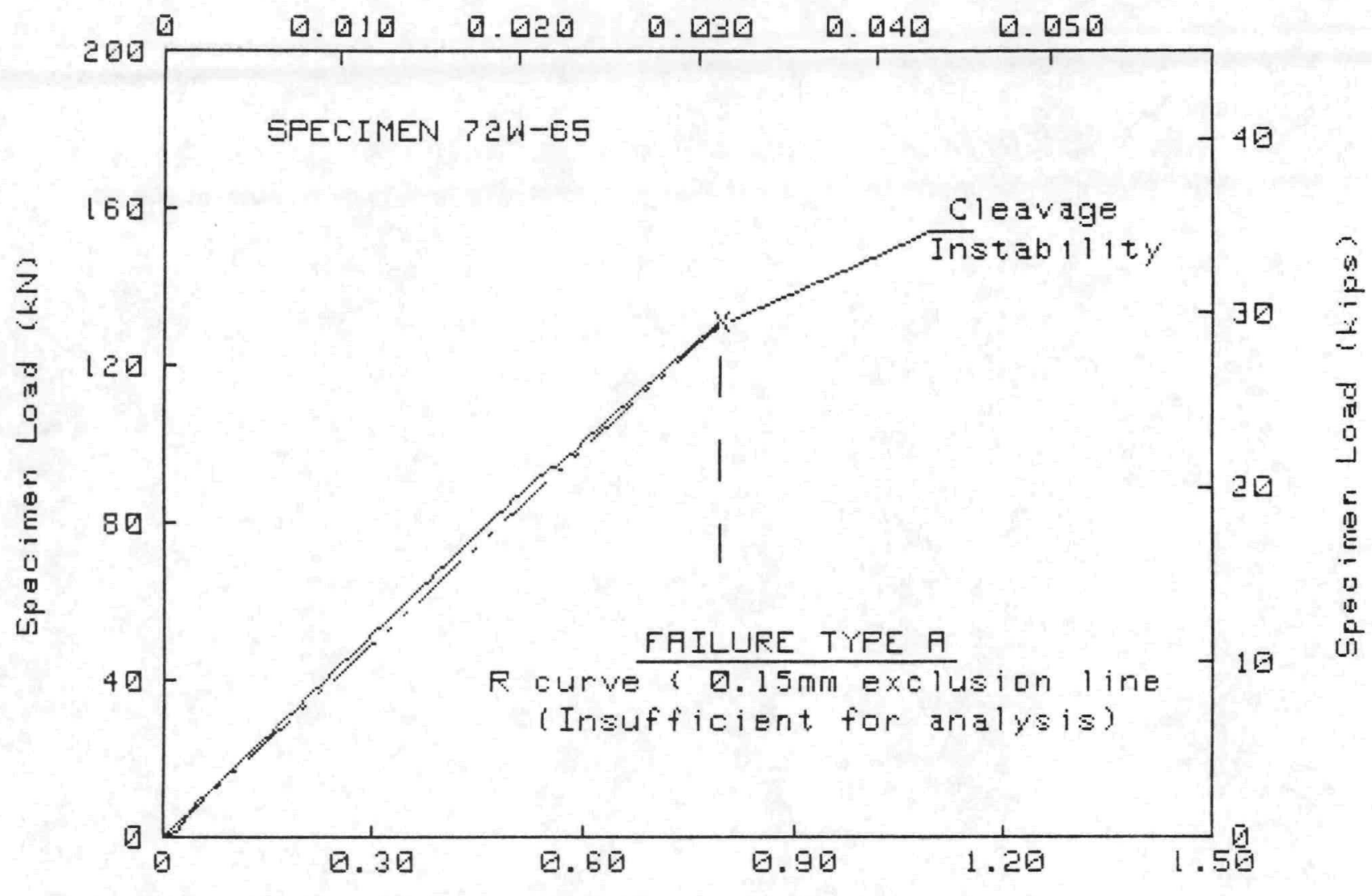

Specimen Crackmouth Defleotion (mm)

TEST SPECIMEN DATA

Material Type
Test Temperature
Percent Side Groove
Specimen Thickness B
Initial a/w
Init crack length a
Flow stress
Yield stress
Youngs modulus
Poisson's Ratio (U)
J-IHTEGRAL ANALYSIS

Je

$K j c=\langle E J c /(1-v 2)\rangle \wedge .5$

Kc (Beta Corrected)

$=A 533-\mathrm{B}$ Weld

$=25 \circ \mathrm{C}$

$=\overline{0} \%$

$=50.8 \mathrm{~mm}$

$=.523$

$=53.14 \mathrm{~mm}$

$=620.5 \mathrm{MFa}$

$=551.6 \mathrm{MPa}$

$=205800 \mathrm{MPa}$ (Estimated Value)

$=.3$

[ Failure Type $A$ (J at instability = J J ) ]

$=\frac{\text { Merkle-Corten }}{36.2 \mathrm{~kJ} / \mathrm{m}^{2}} \quad \frac{\text { Deformation-J* }}{33.9 \mathrm{~kJ} / \mathrm{m}^{2}}$

$=90.4 \mathrm{MPa} \sqrt{\mathrm{m}} \quad 87.5 \mathrm{MPa} \sqrt{\mathrm{m}}$

$=80.9 \mathrm{MFa} / \mathrm{m}$

ASTM E399-81 ANALYSIS

Modified Kic Equation; $B=(B B n) 1 / 2$

Maximum Load $P=131.35 \mathrm{kN}$

$5 \%$ Secant offset $\mathrm{Pq}=131.35 \mathrm{kN}$

$\mathrm{Kq}$

$=84.3 \mathrm{MPa} \sqrt{\mathrm{m}}$

Validity

$K$ at Maximum Load

= INVALID--1, 2

$=84.3 \mathrm{MPa} \sqrt{\mathrm{m}}$

DATA CORRECTIONS USED FOR J-INTEGRAL DATA

Merkle-Corten correction

Correction to J for crack extension (Deformat ion-J)

DFFSET LOAD $=\square \mathrm{kN}$ OFFSET DEFLECTION $=-.026 \mathrm{~mm}$

Fitted elastic load range $=15 \mathrm{kH} \mathrm{TO} 45 \mathrm{kN}$

Elastic slope $=1.734 E+02 \mathrm{kN} / \mathrm{mm}$ 



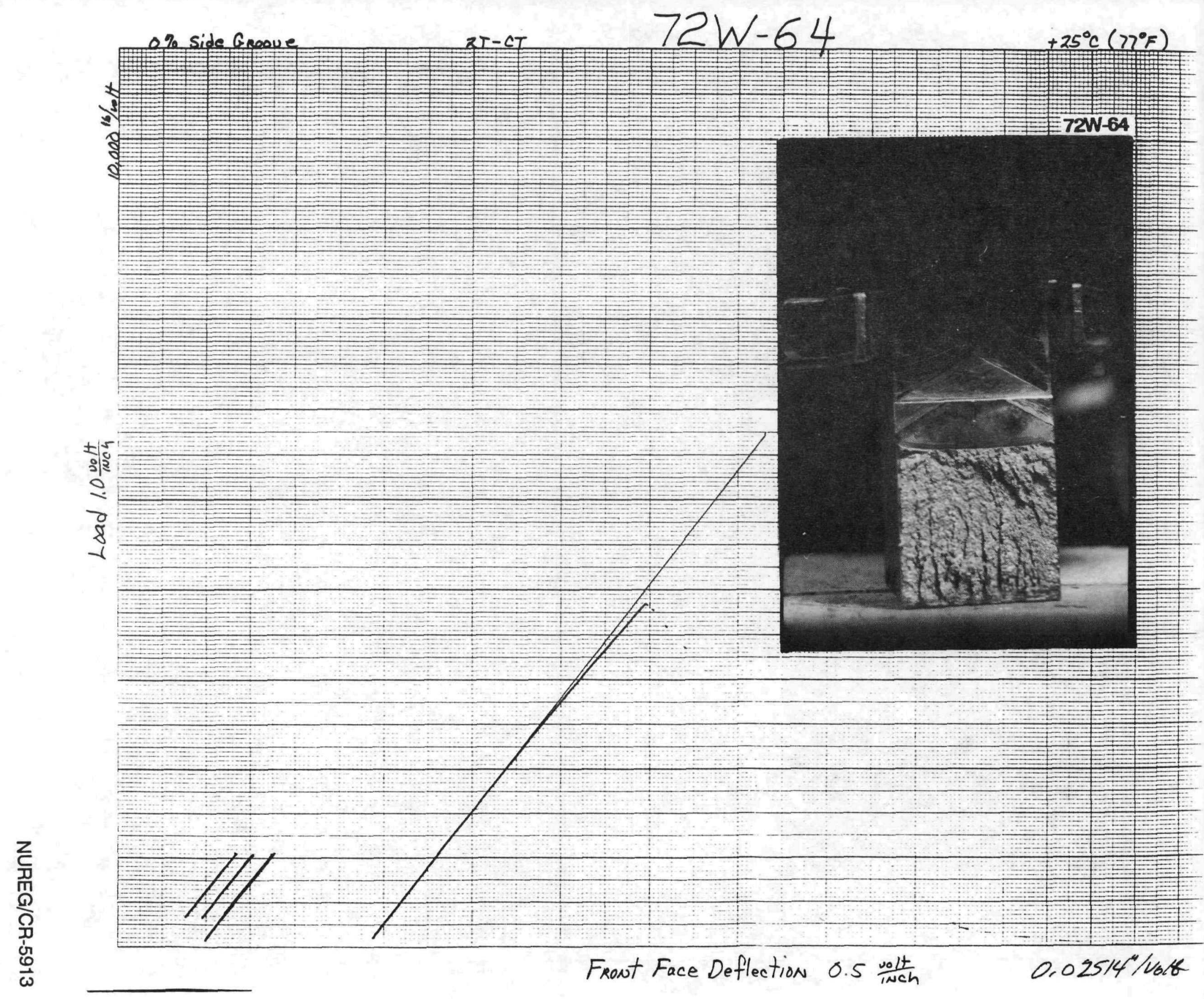




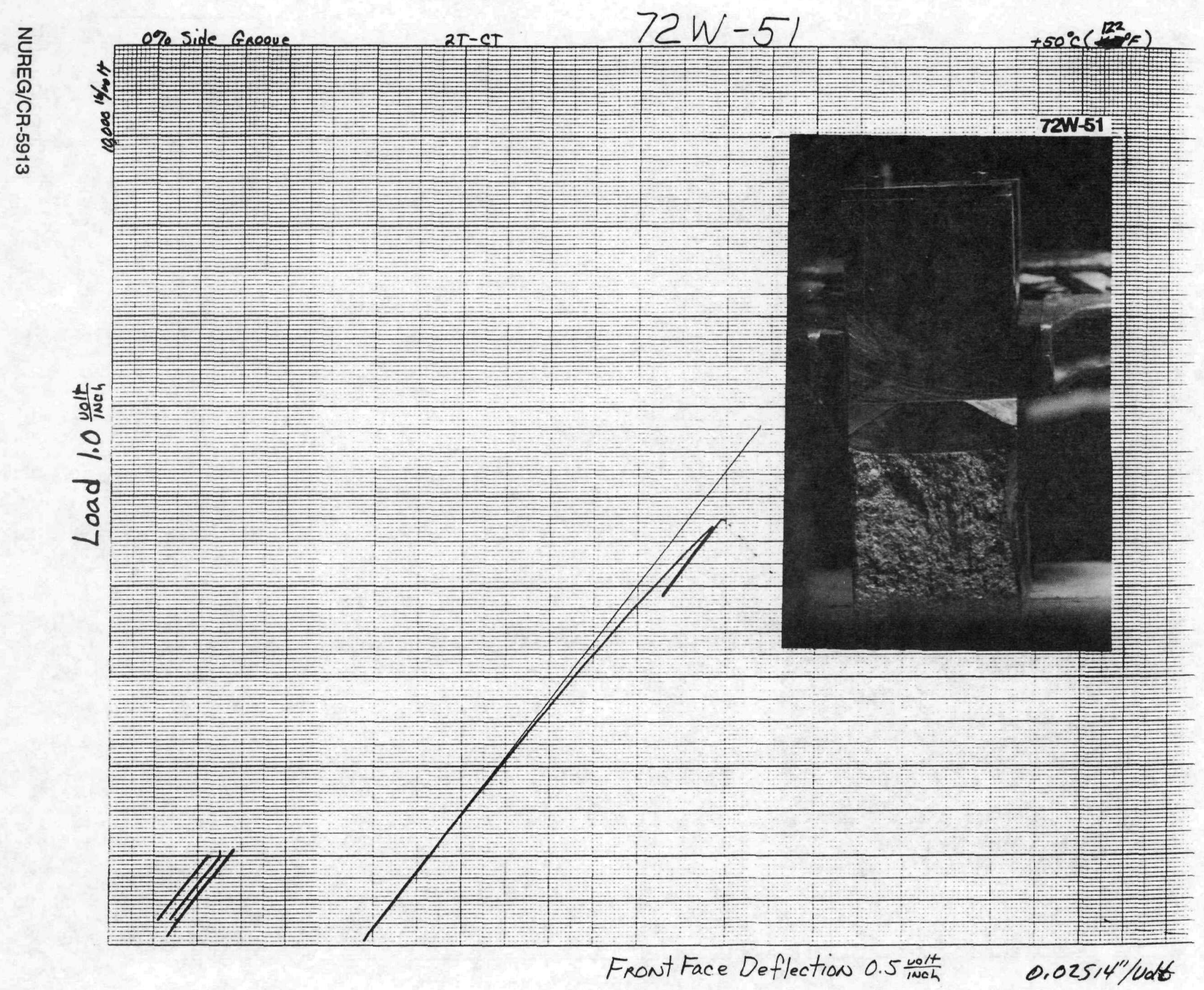




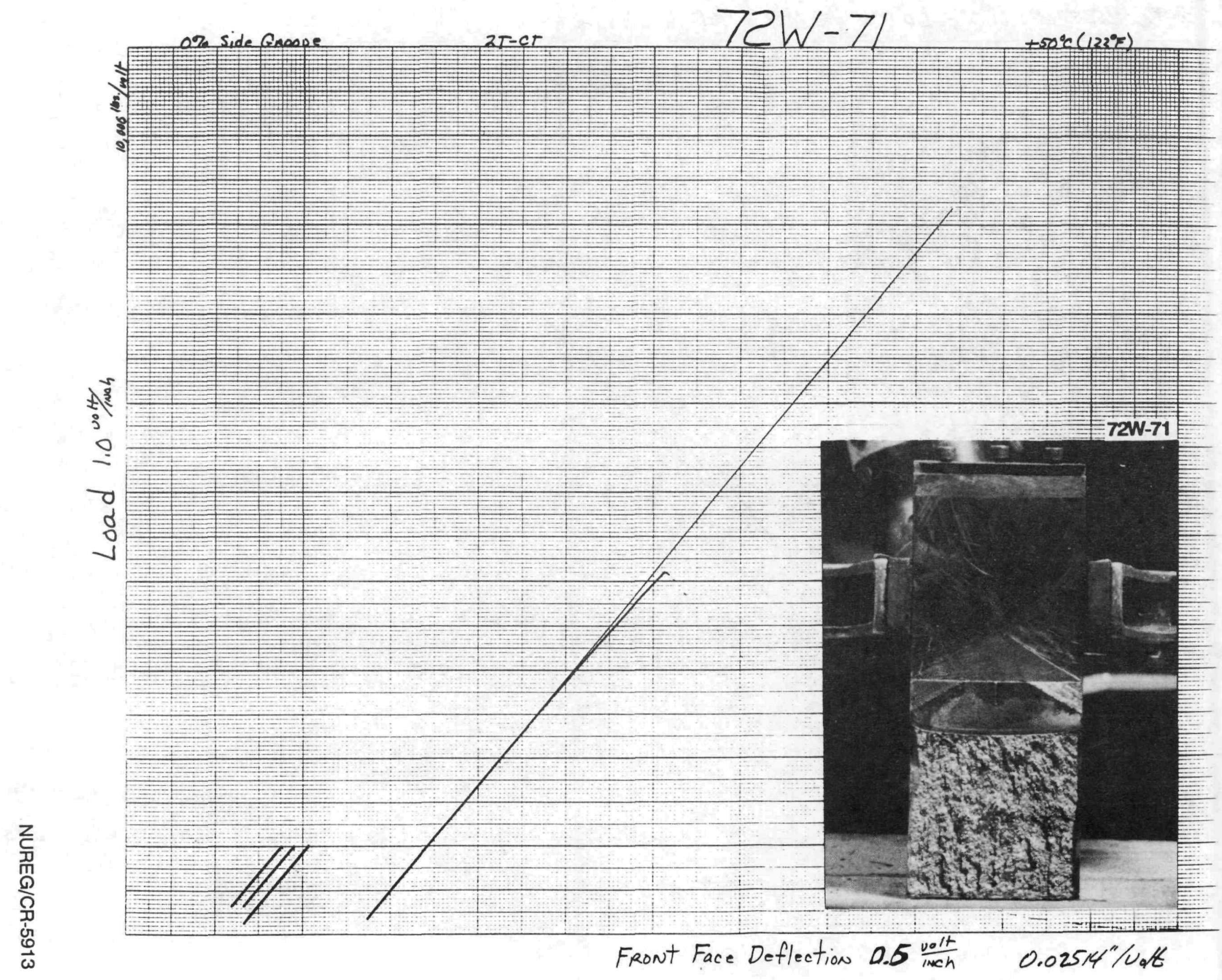




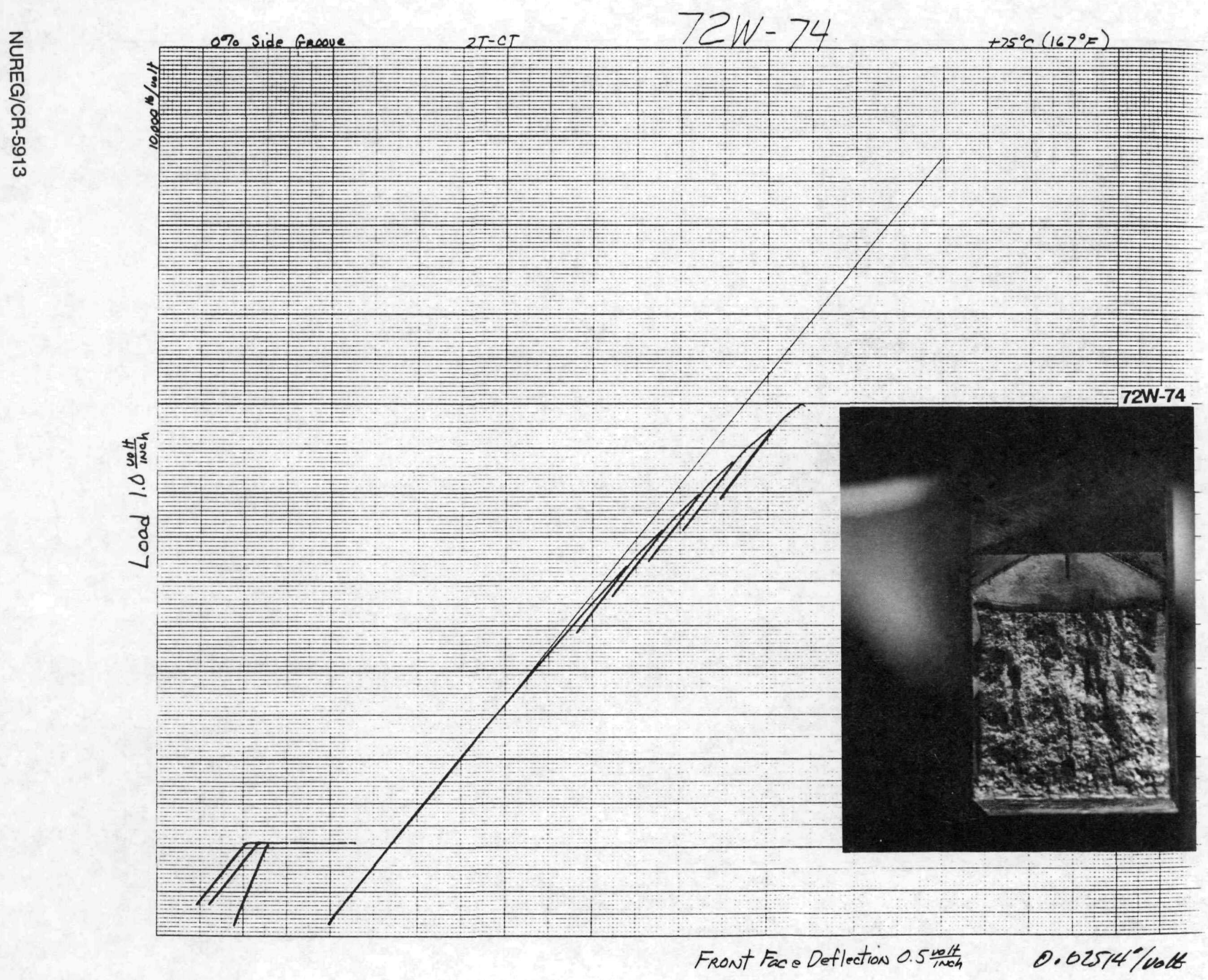




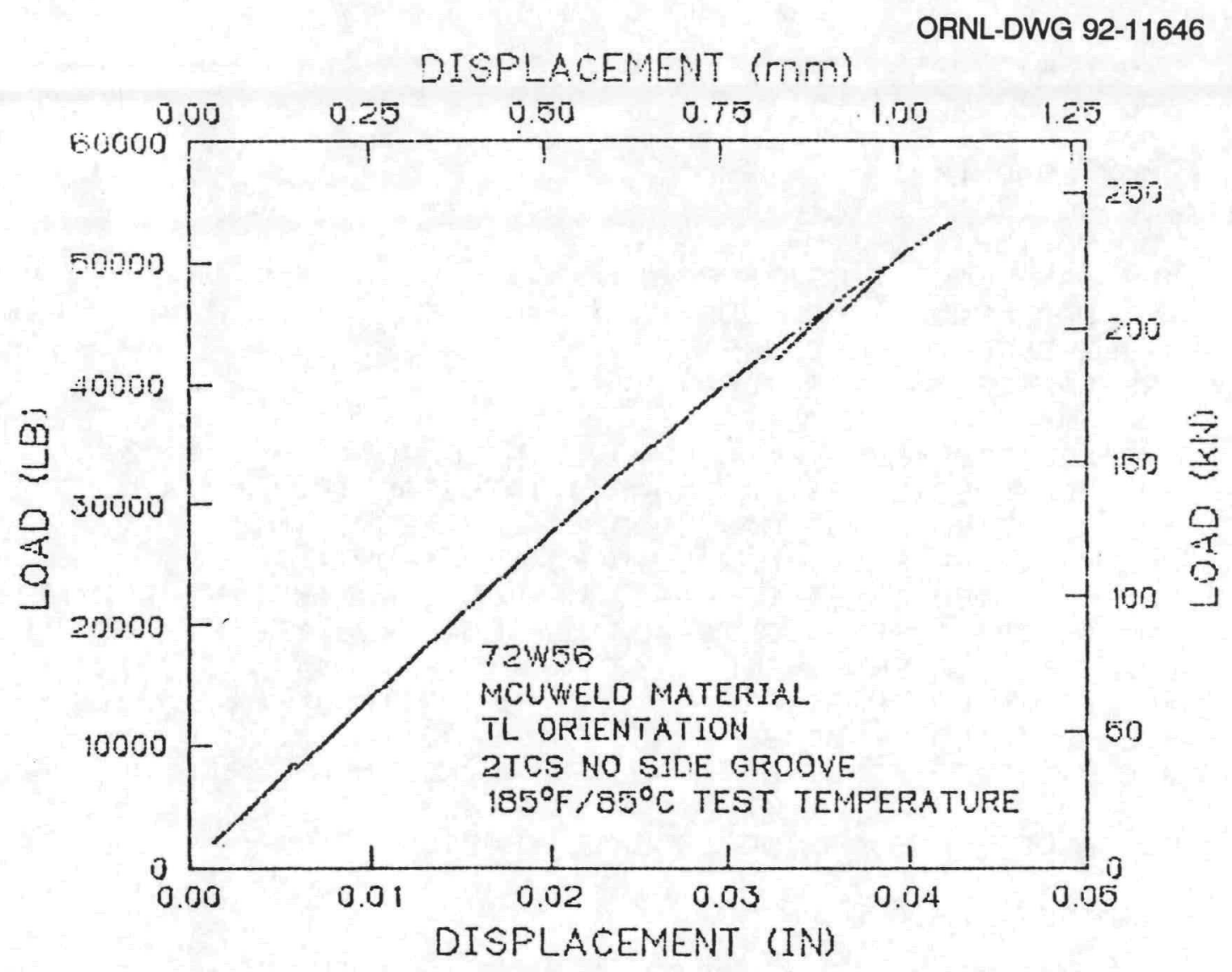

$72 W-56$

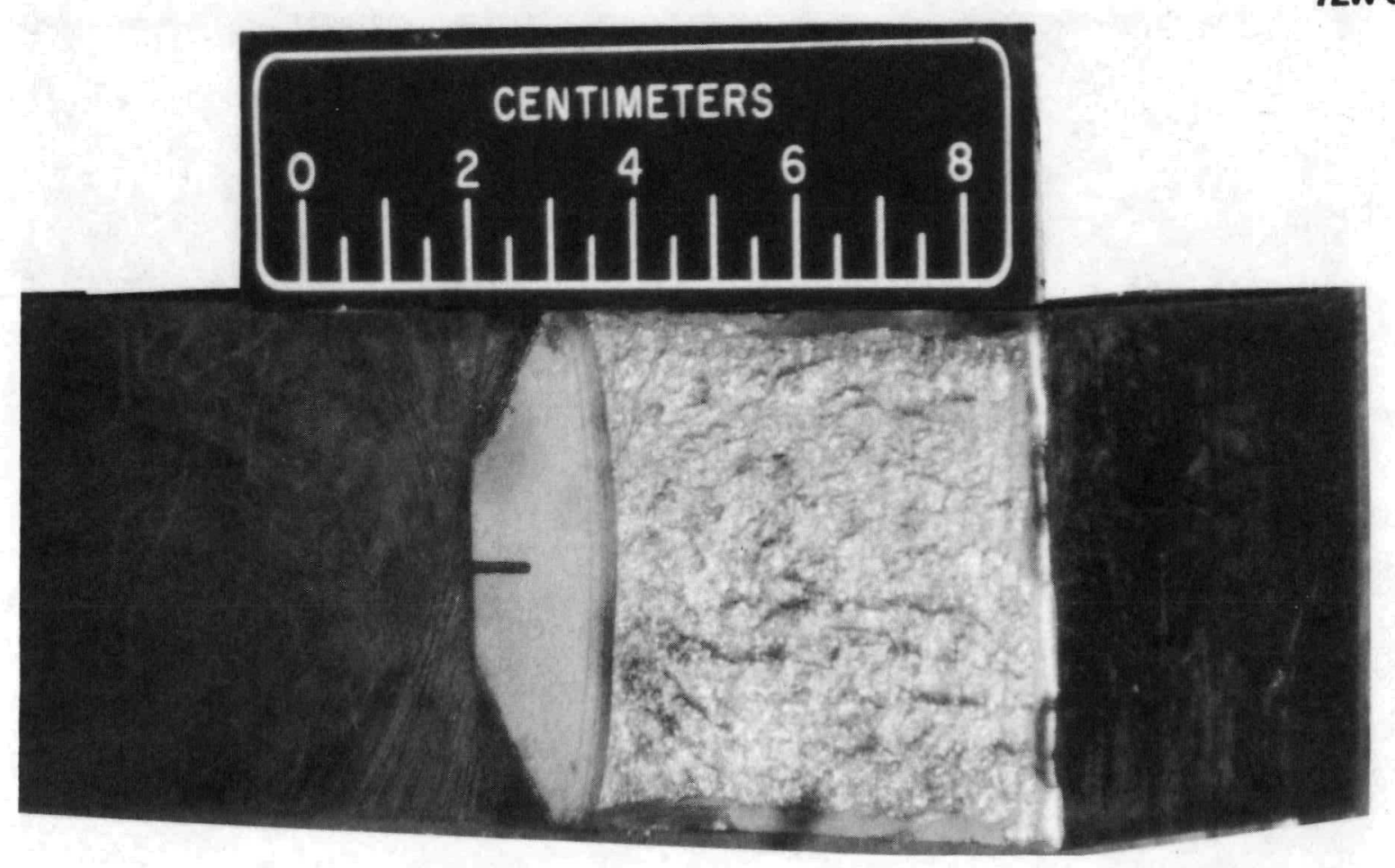




\section{$\mathrm{E}-242$}

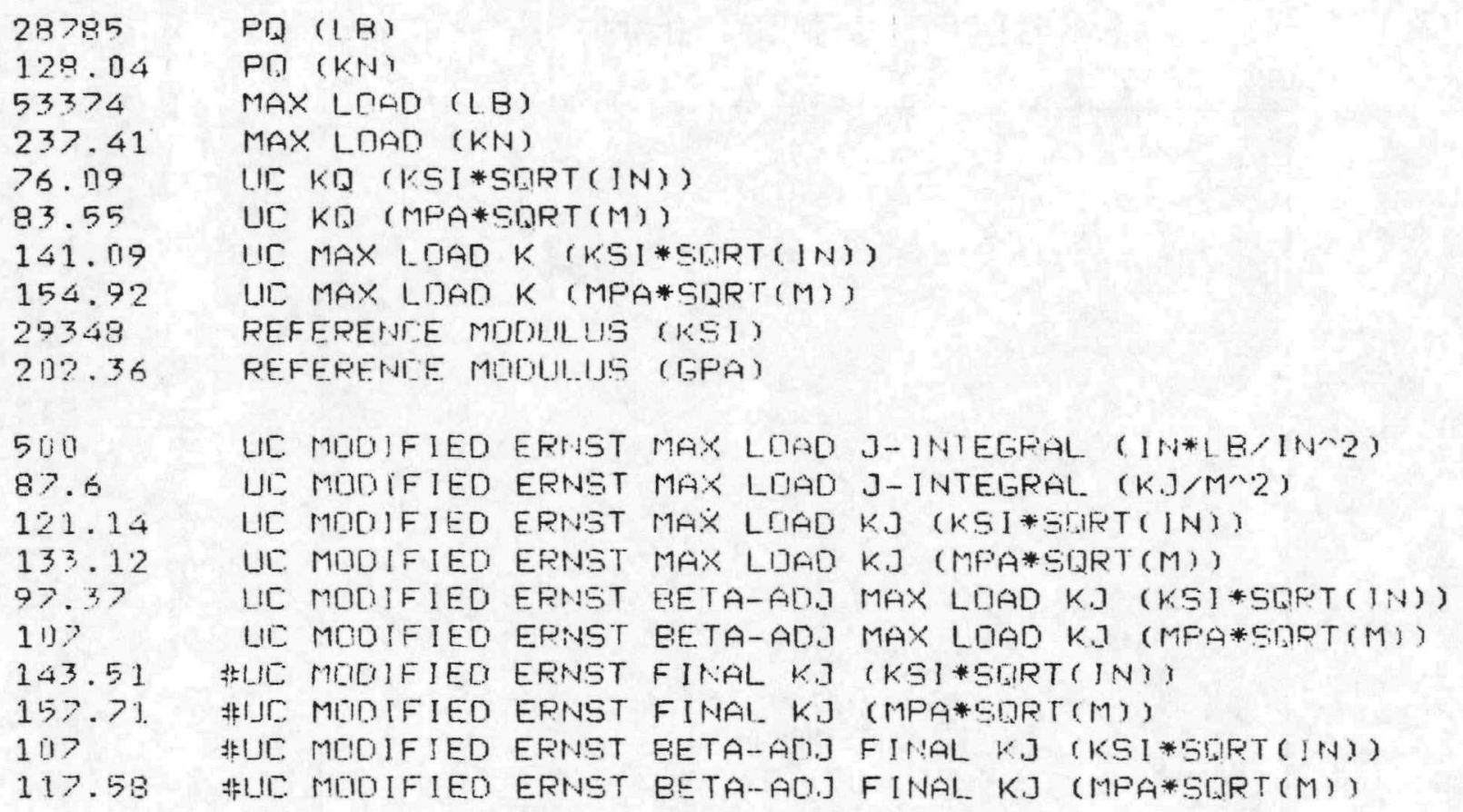

\# ESTJMATED UALIJE FDR FINAL IU CRACK EXTENSIIM

GET FDLWER LALI FIT TO DATA NO

WI MOIFIED ERNGT ,IIC, CEIC, K.]IC AND TAUG NOT DEFINED--POUER LAW FIT NOT REQLIESTED BY TE[HN]CIAN 

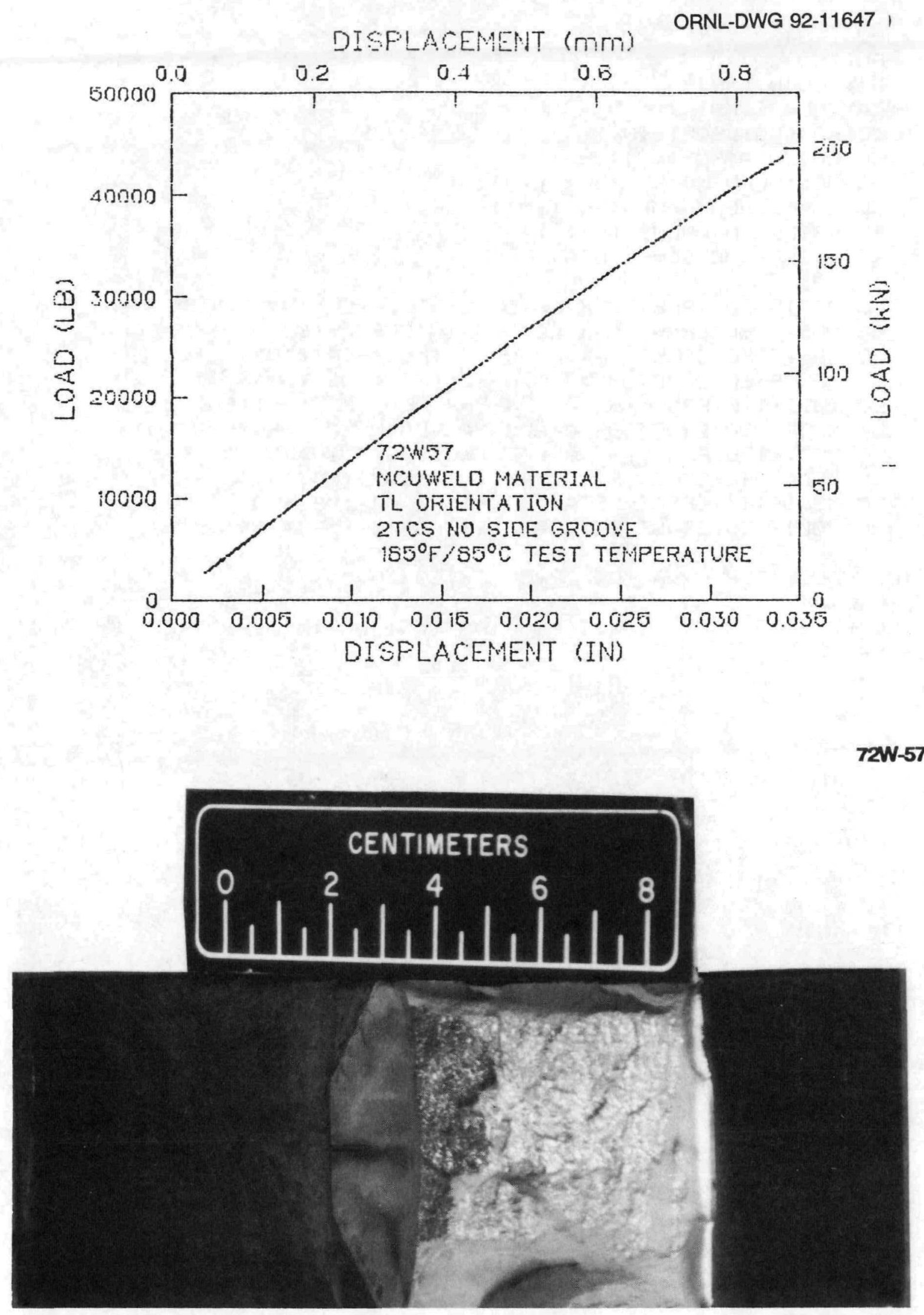


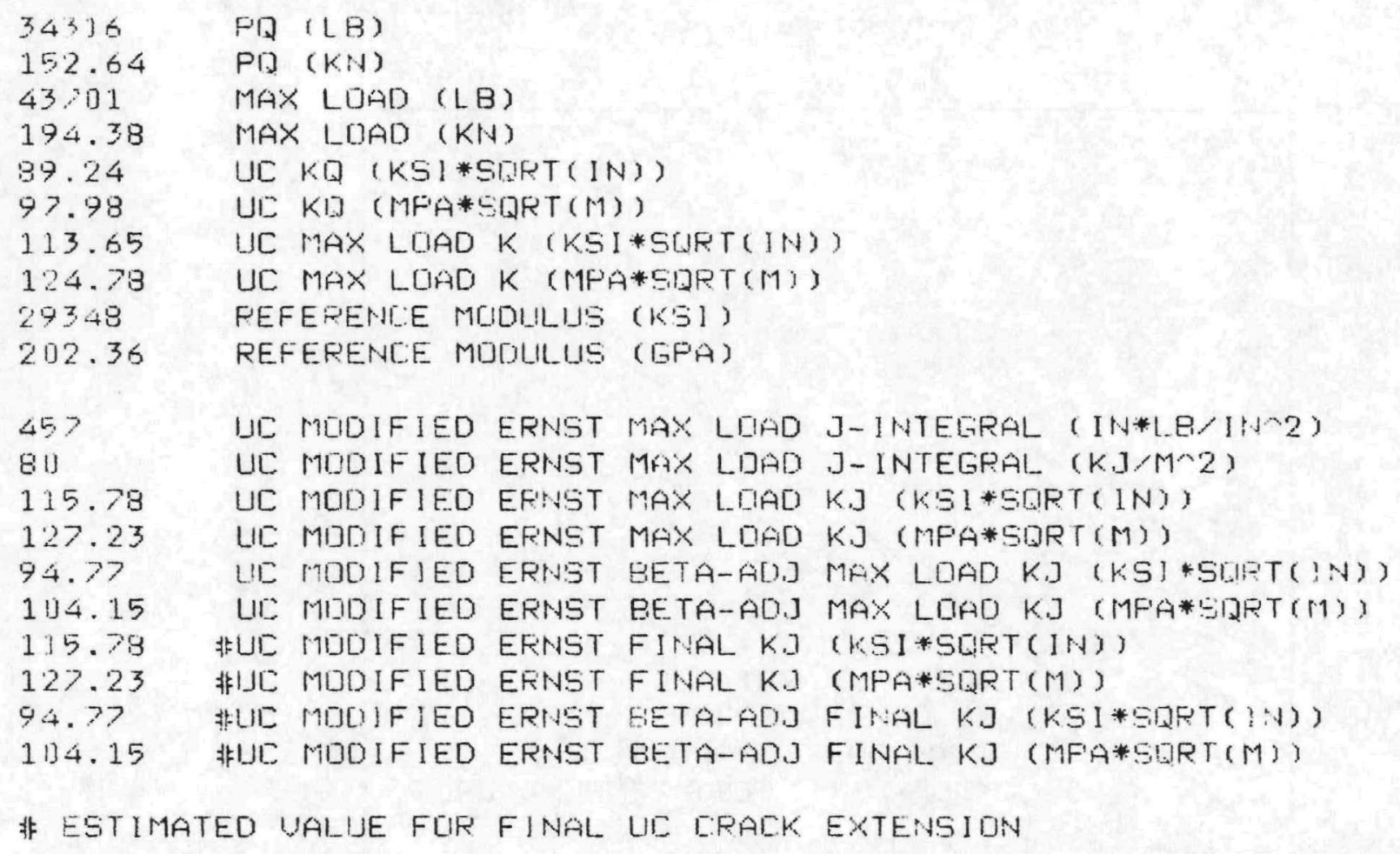




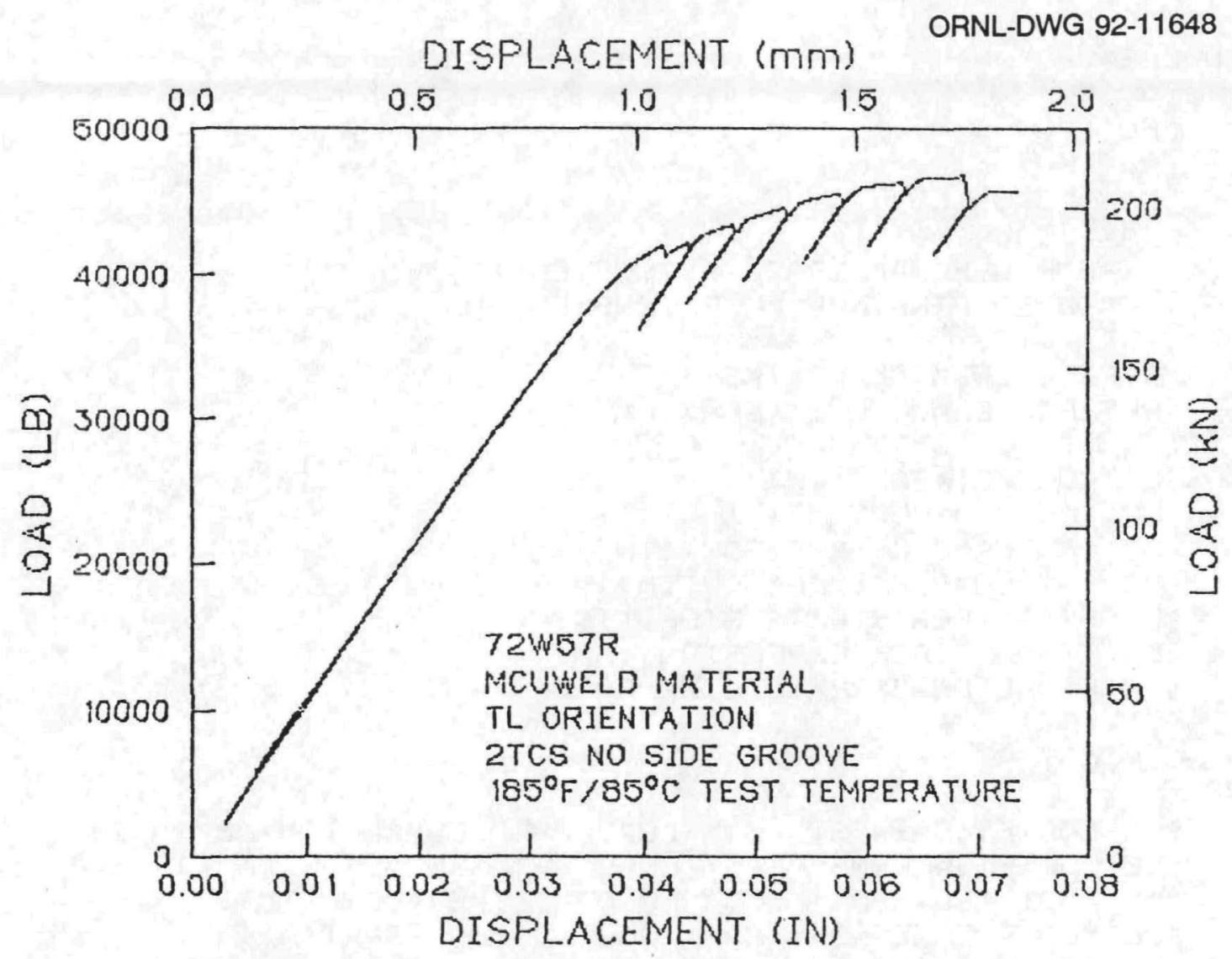

YP5282
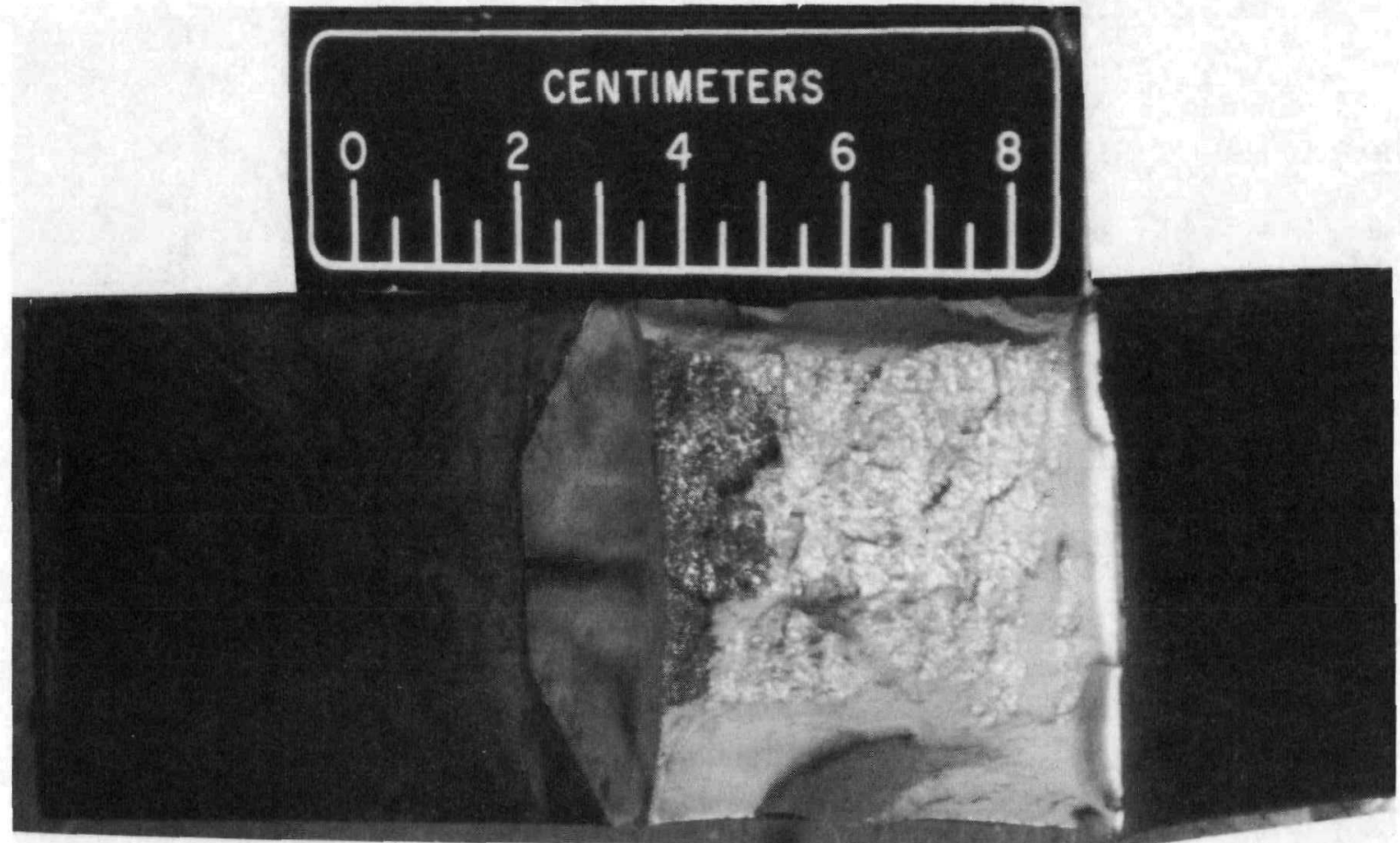

NUREG/CR-5913 


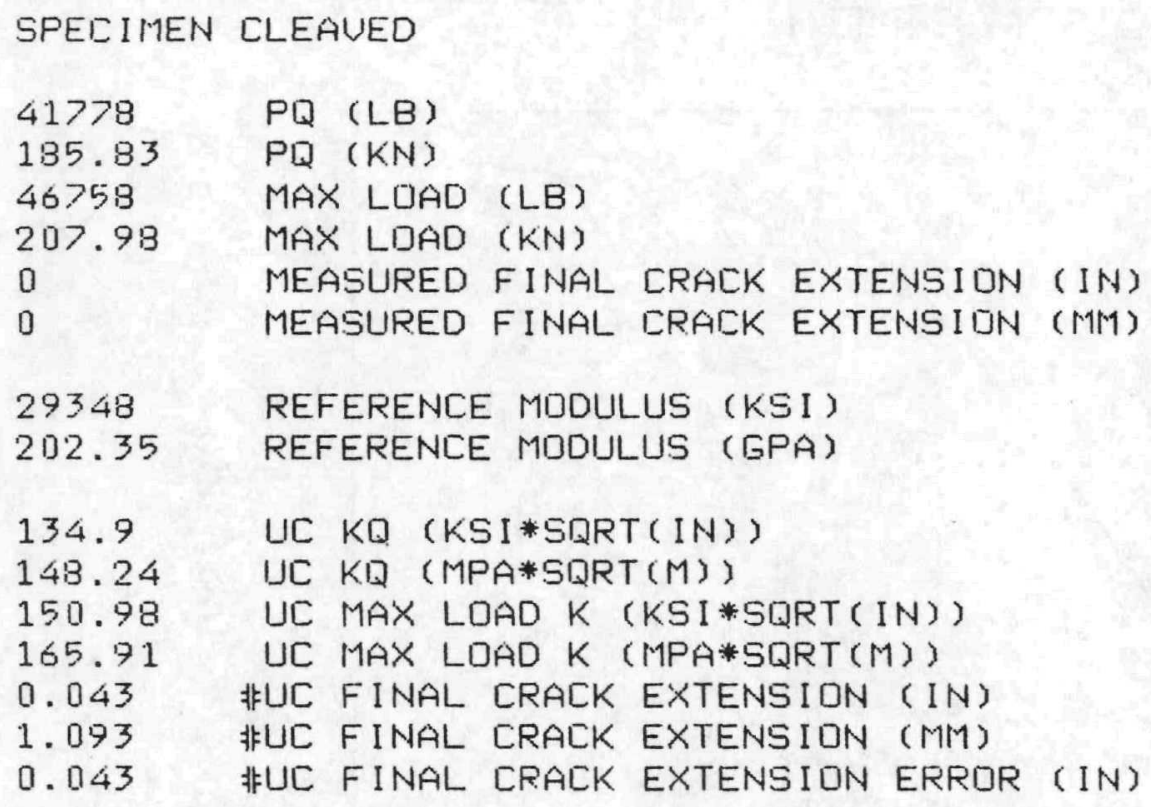




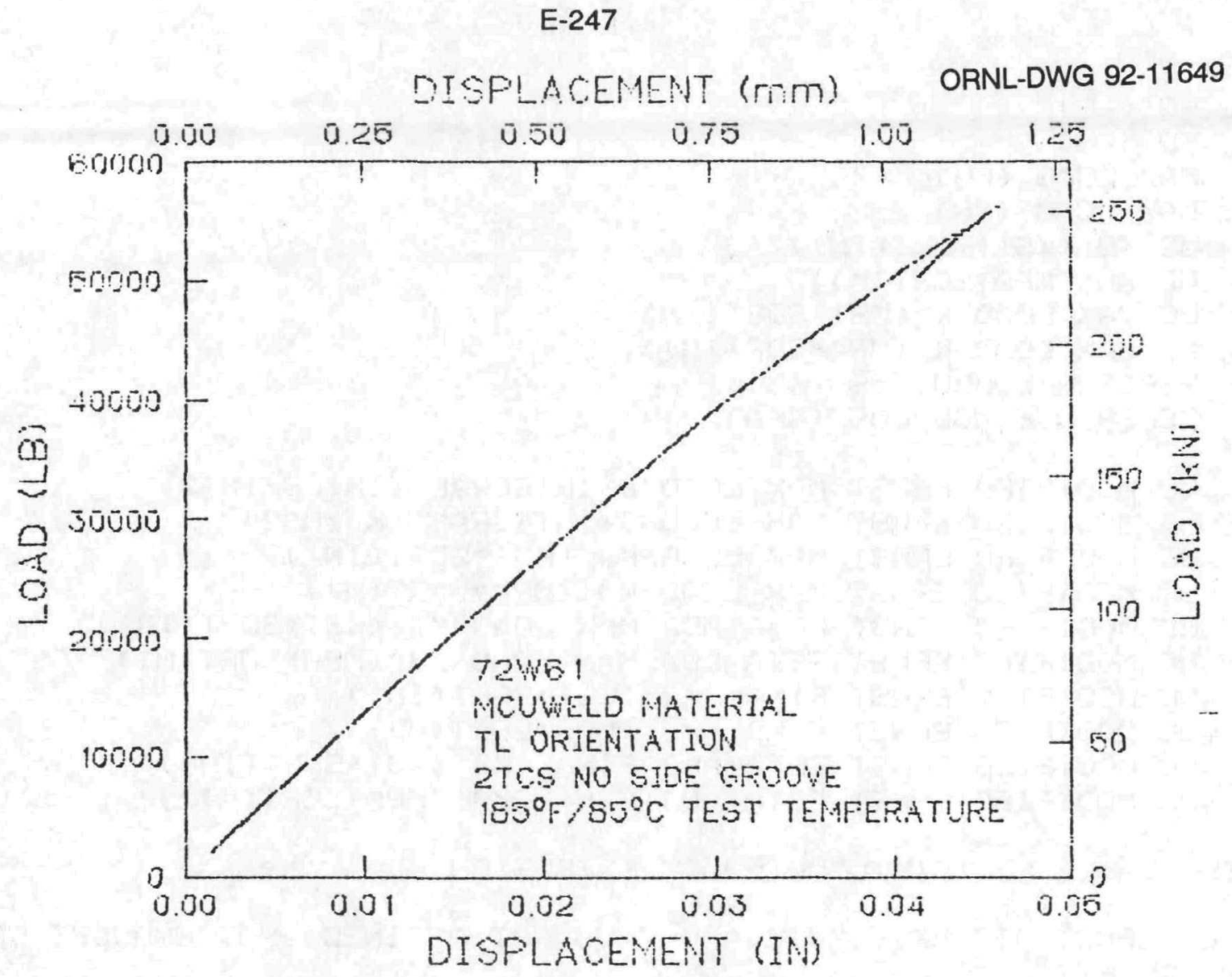

COMMAND
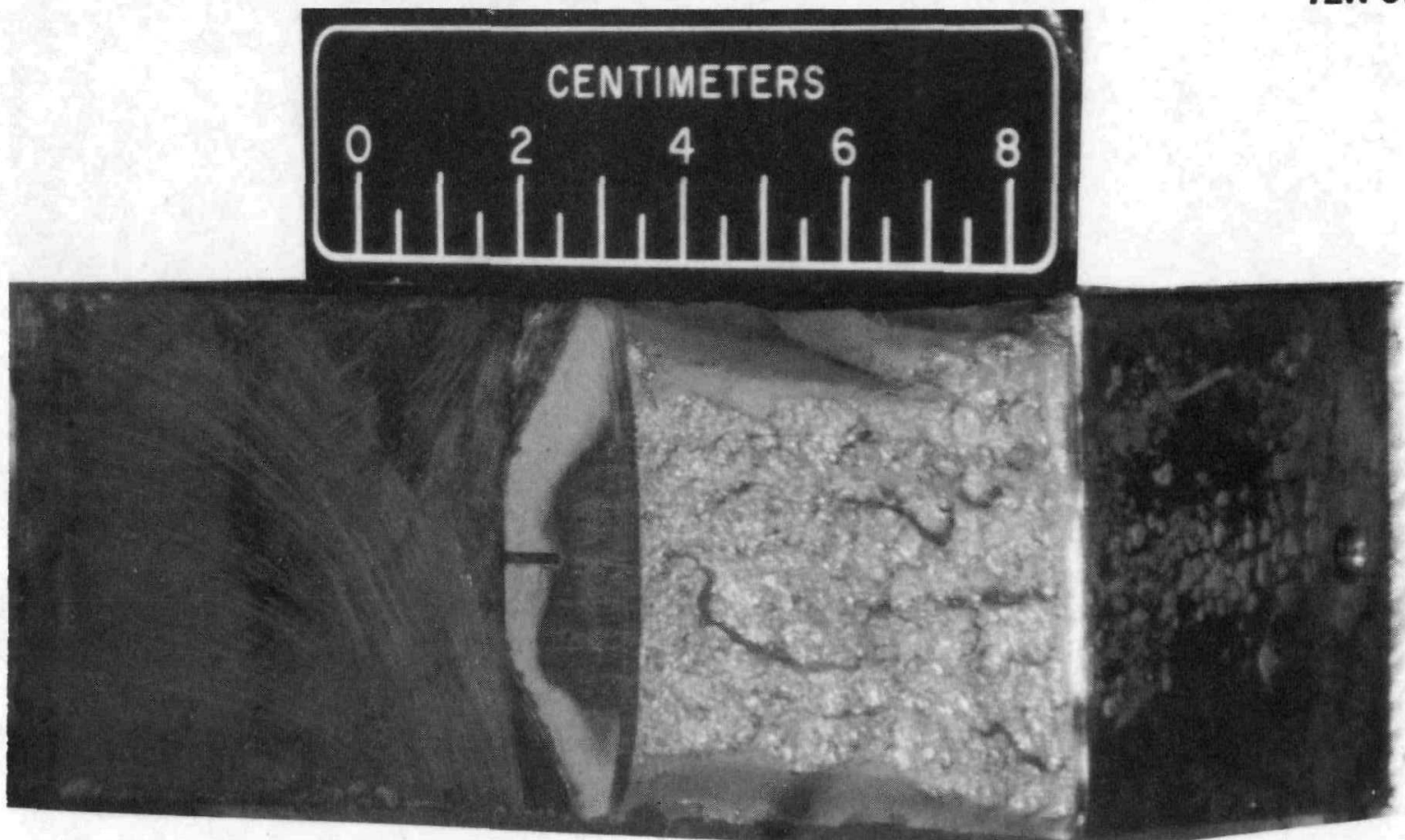


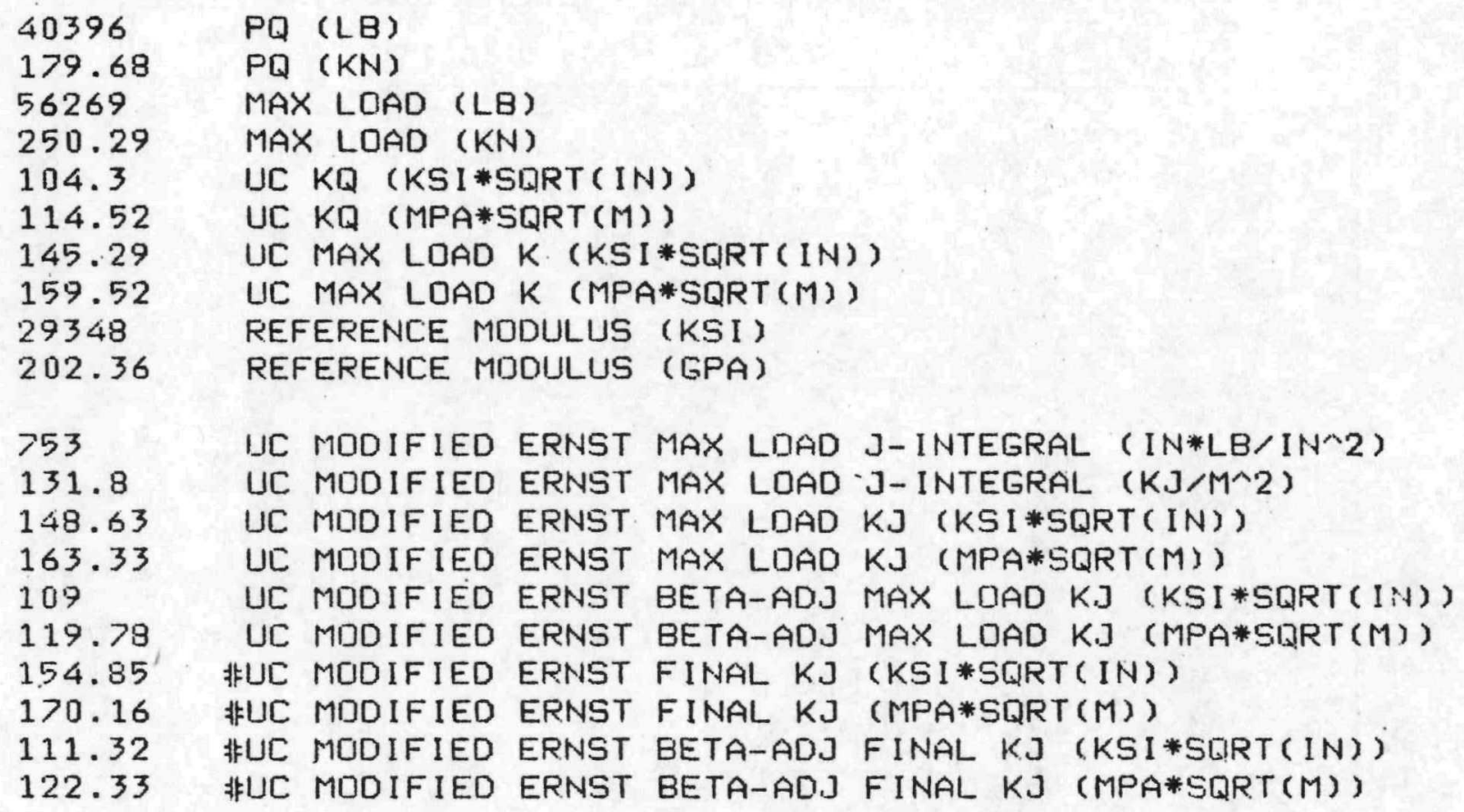




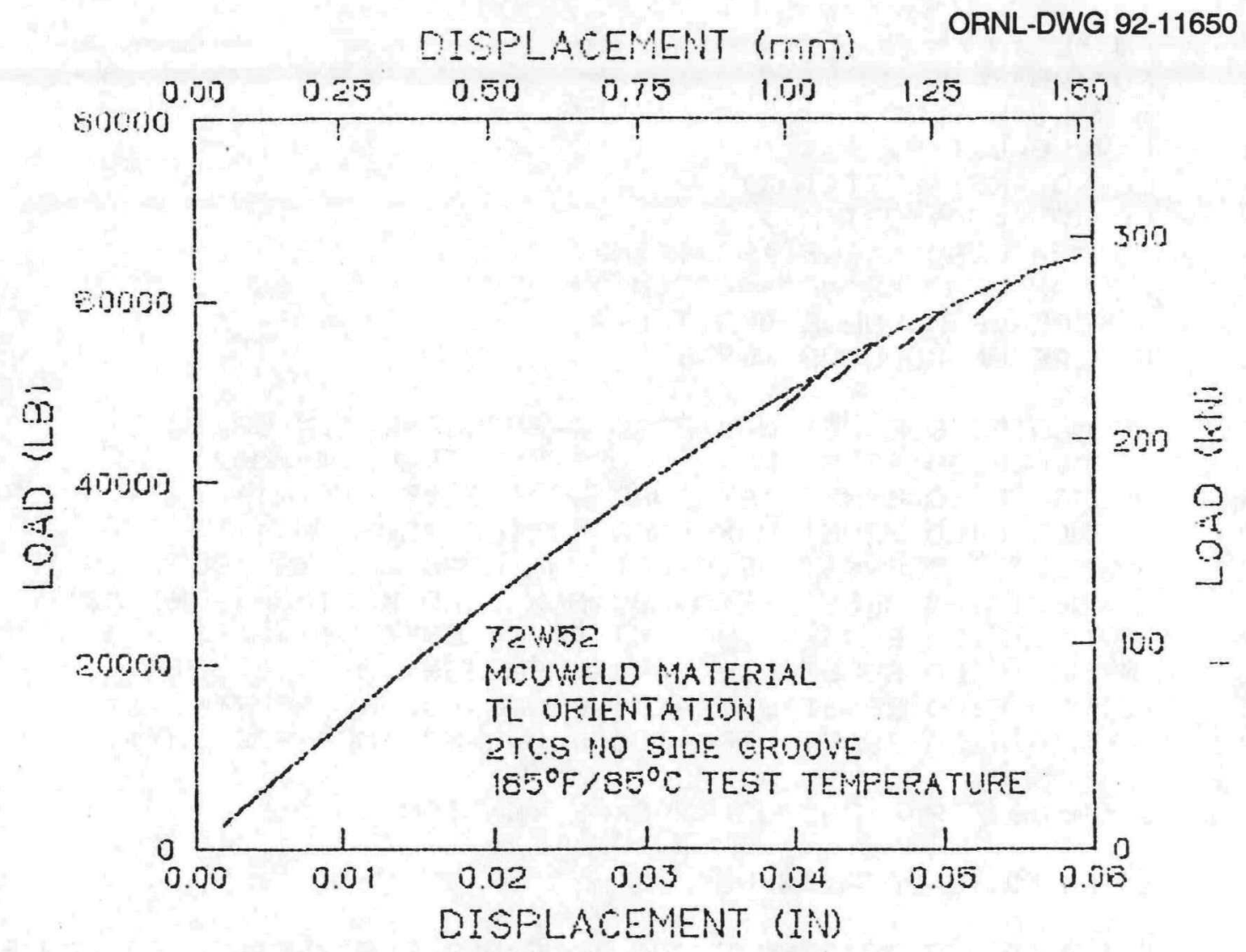

72W-52

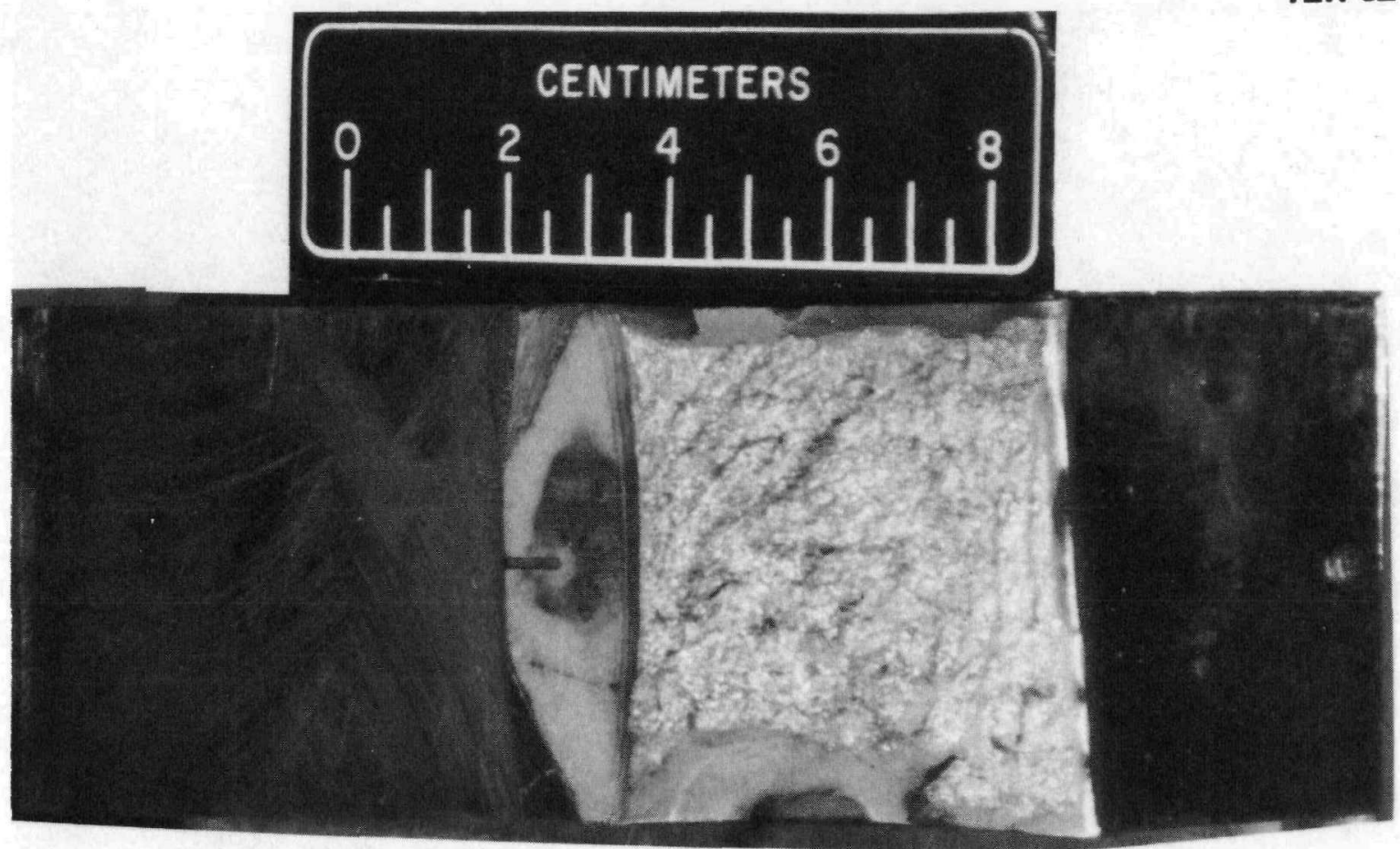

NUREG/CR-5913 


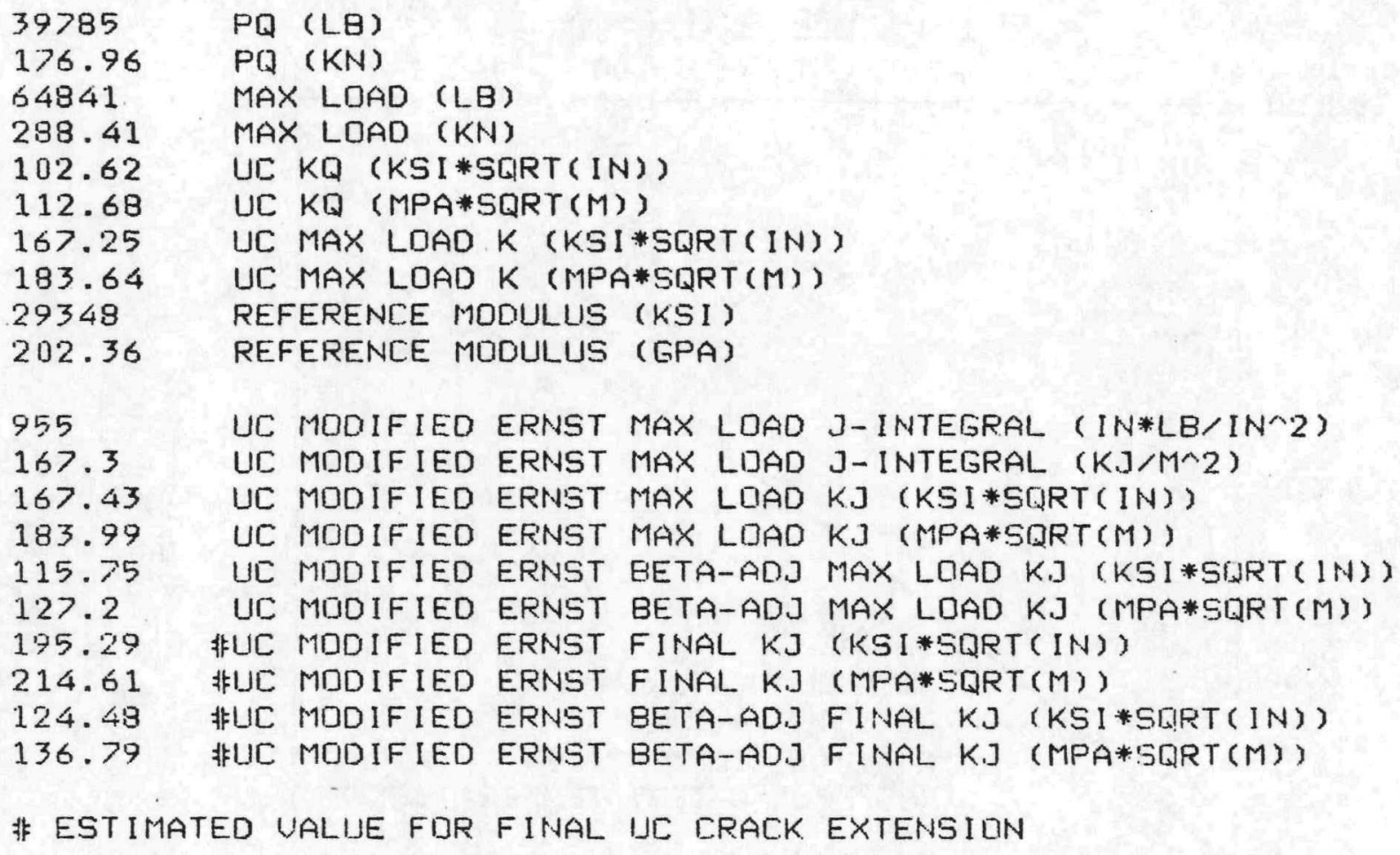


Specimen Laadline Deflection (in.) ORNL-DWG 92-11651

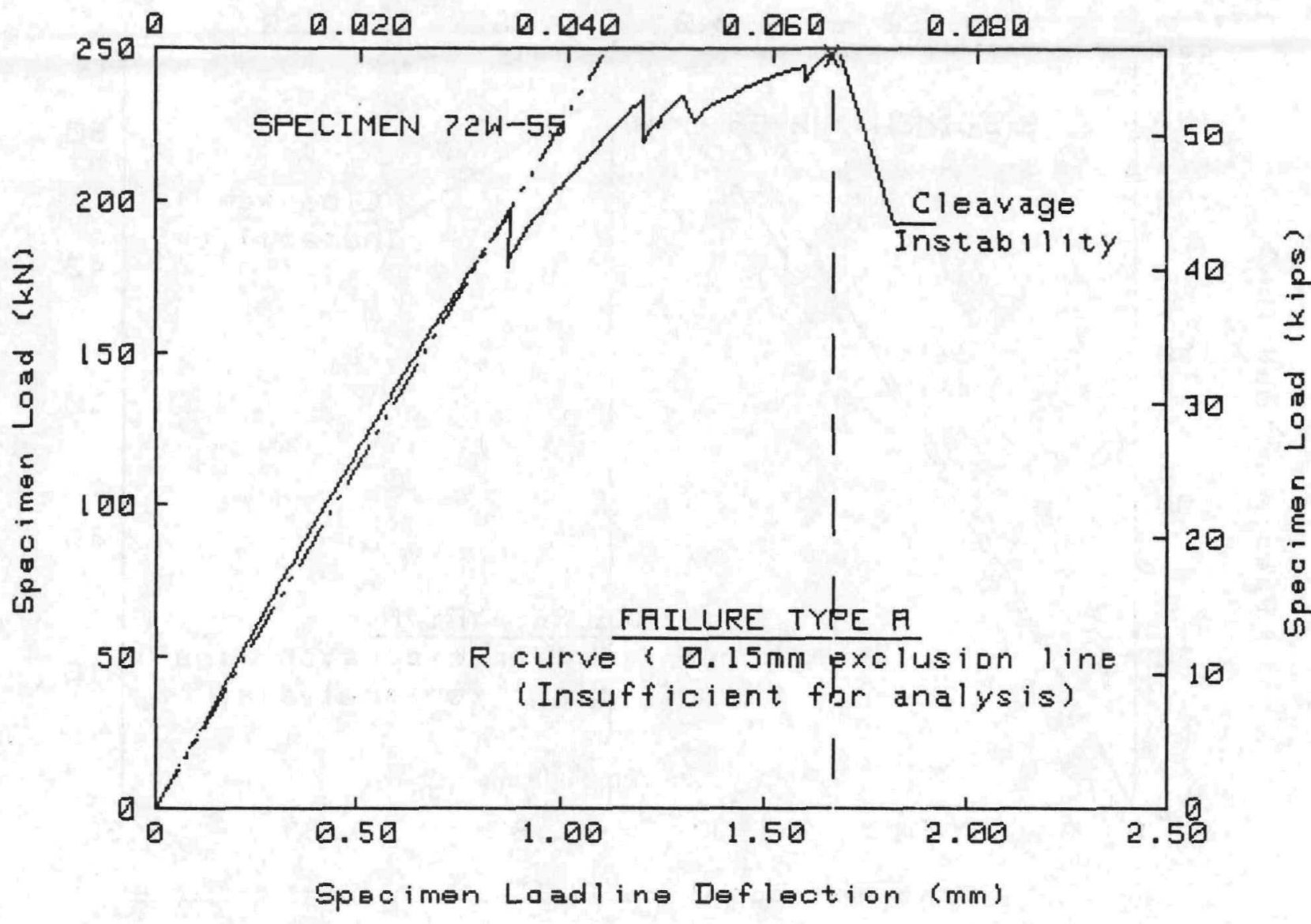

TEST SPECIMEN DATA

Material Type
Test Temperature
Percent Side Groove
Specimen Thickness B
Initial a/W
Init crack length a
Flow stress
Yield stress
Youngs modulus E
Poisson's Ratio (U)
J-INTEGRAL ANALYSIS

$=A 533-B$ Weld (I)

$=95^{\circ} \mathrm{C}$

$=0 \%$

$=50.8 \mathrm{~mm}$

$=.526$

$=53.4 \mathrm{~mm}$

$=636 \mathrm{MPa}$

$=591.3 \mathrm{MPa}$

$=201800 \mathrm{MPa}$ (Estimated Value)

J-INTEGRAL ANALYSIS

Jc

$$
=0
$$

[ Failure Type $A$ ( $J$ at instability = JC) ]

$K j c=\left\langle E J c /\left(1-v^{2}\right)\right\rangle \wedge .5$

Ke (Beta Corrected)

$=\frac{\text { Merkle-Corten }}{246 \mathrm{~kJ} / \mathrm{m}^{2}}$

$=222.8 \mathrm{MPa} \sqrt{\mathrm{m}}$

$=138 \mathrm{MPa} \sqrt{\mathrm{m}}$
Deformation-J*

$226.5 \mathrm{MPa} \sqrt{\mathrm{m}}$

$139 \mathrm{MPa} \sqrt{\mathrm{m}}$

ASTM E399-81 ANALYSIS

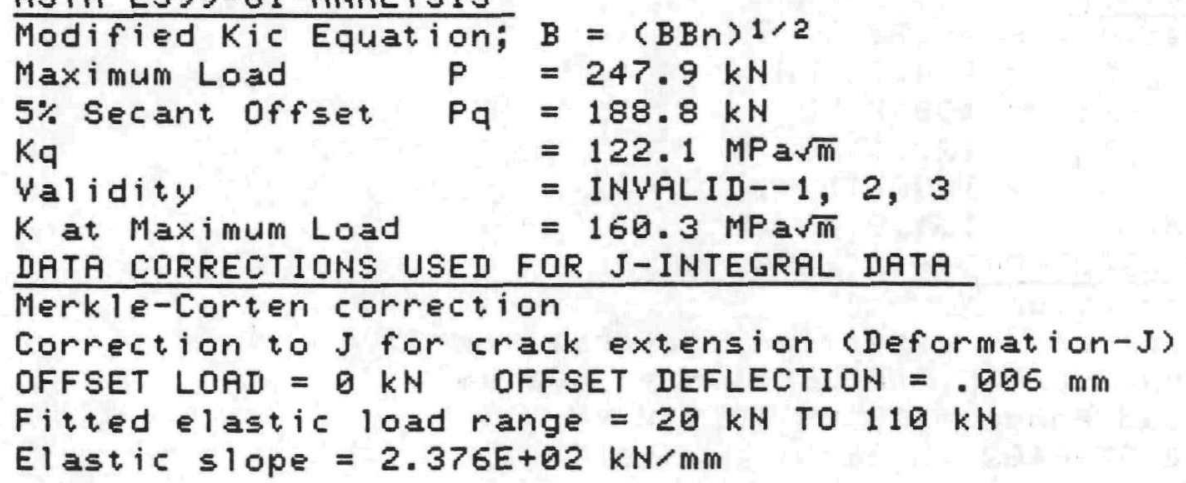

Elastic slope $=2.376 E+02 \mathrm{kN} / \mathrm{mm}$ 
Specimen Laadline Deflection (in.) ORNL-DWG 92-11652

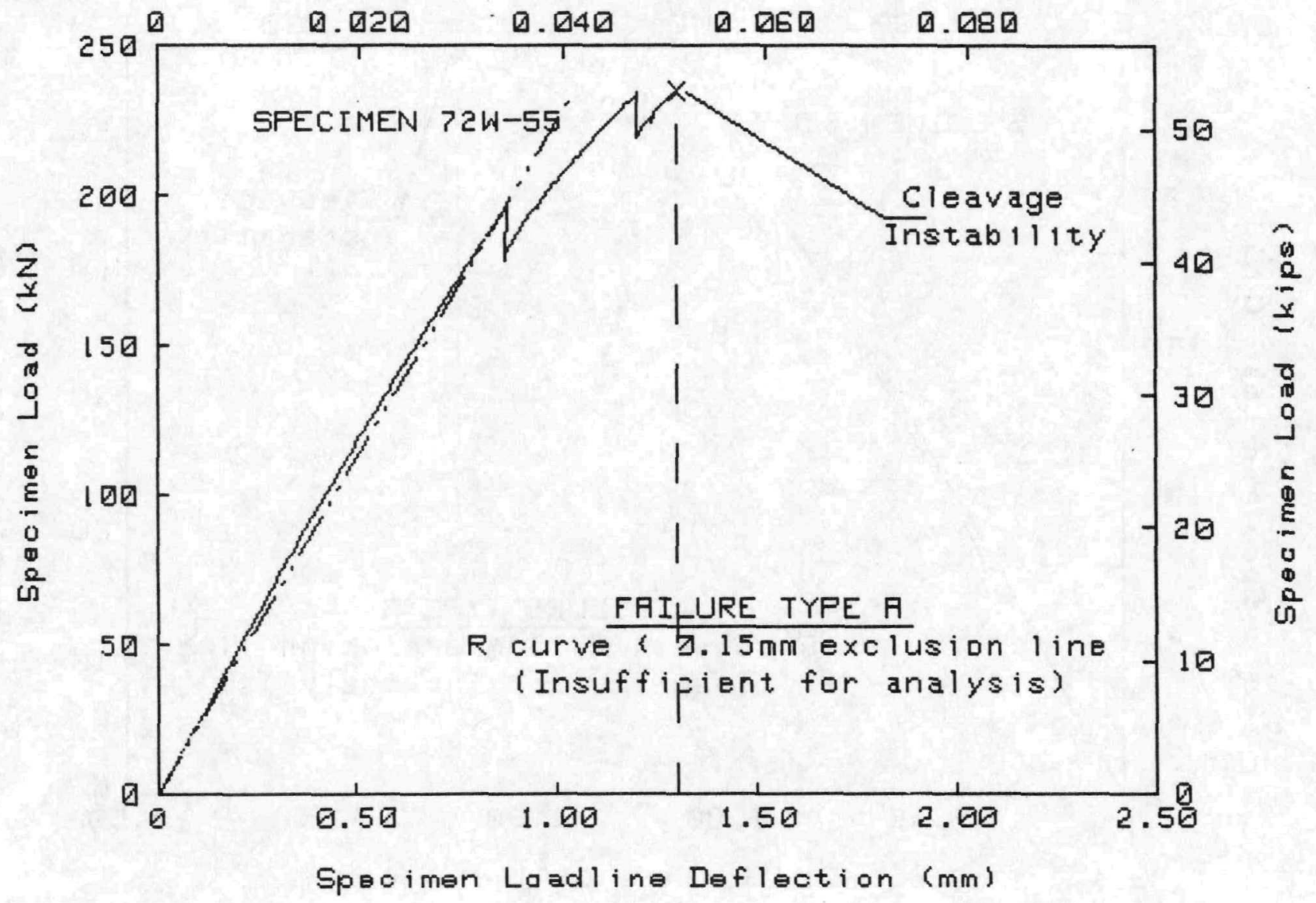

TEST SPECIMEN DATA

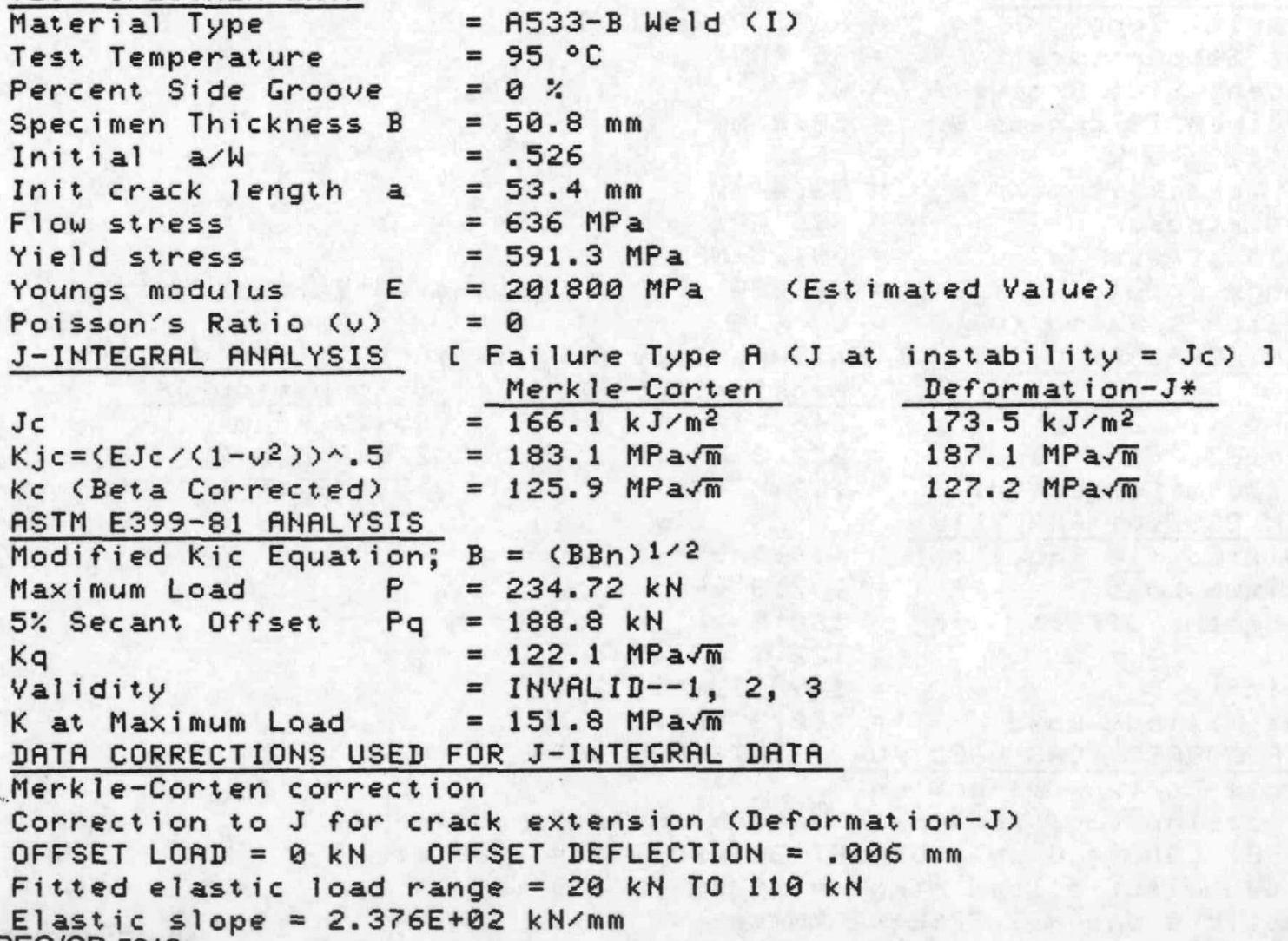


Specimen Loadline Deflection (in.) ORNL-DWG 92-11653

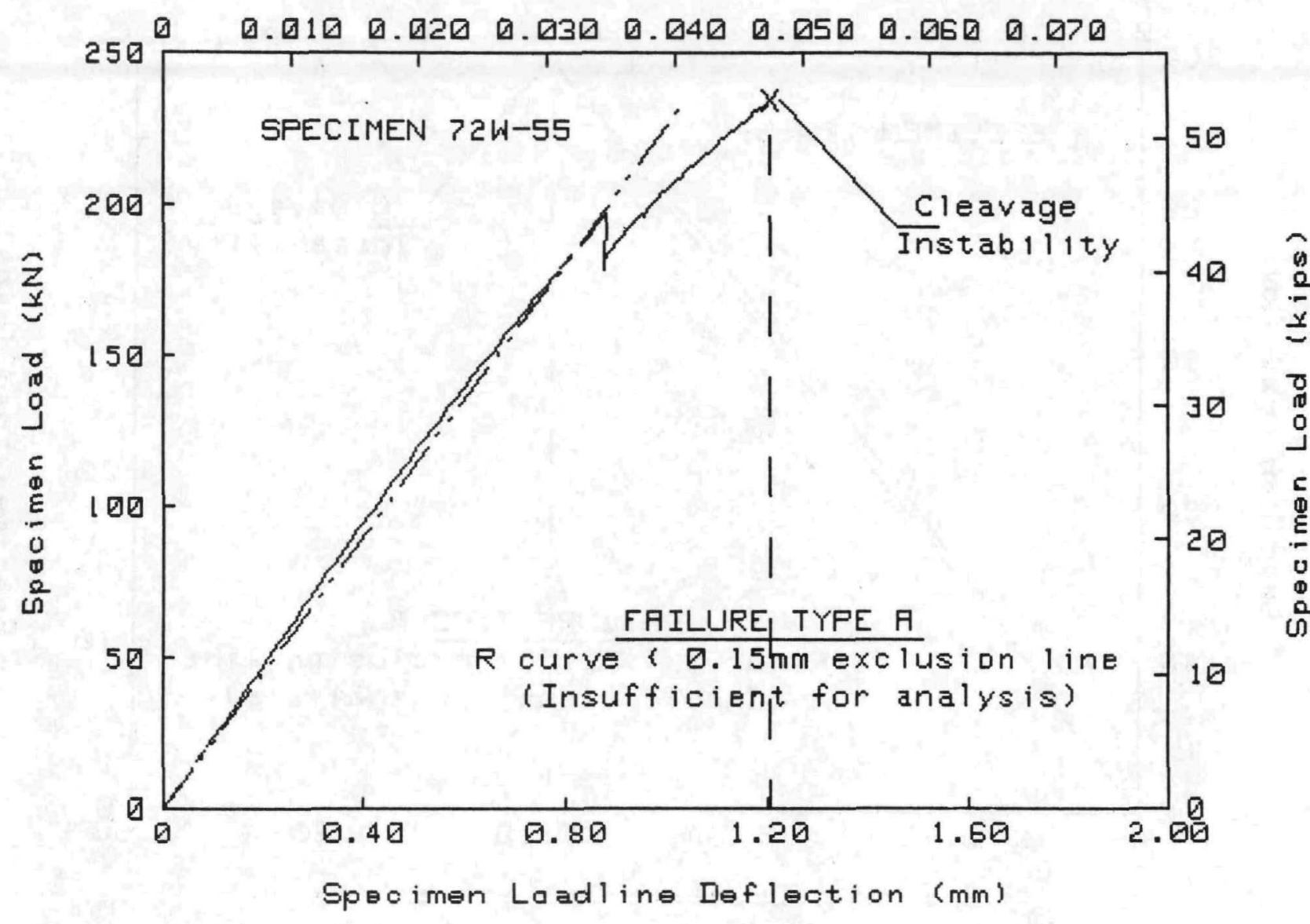

TEST SPECIMEN DATA

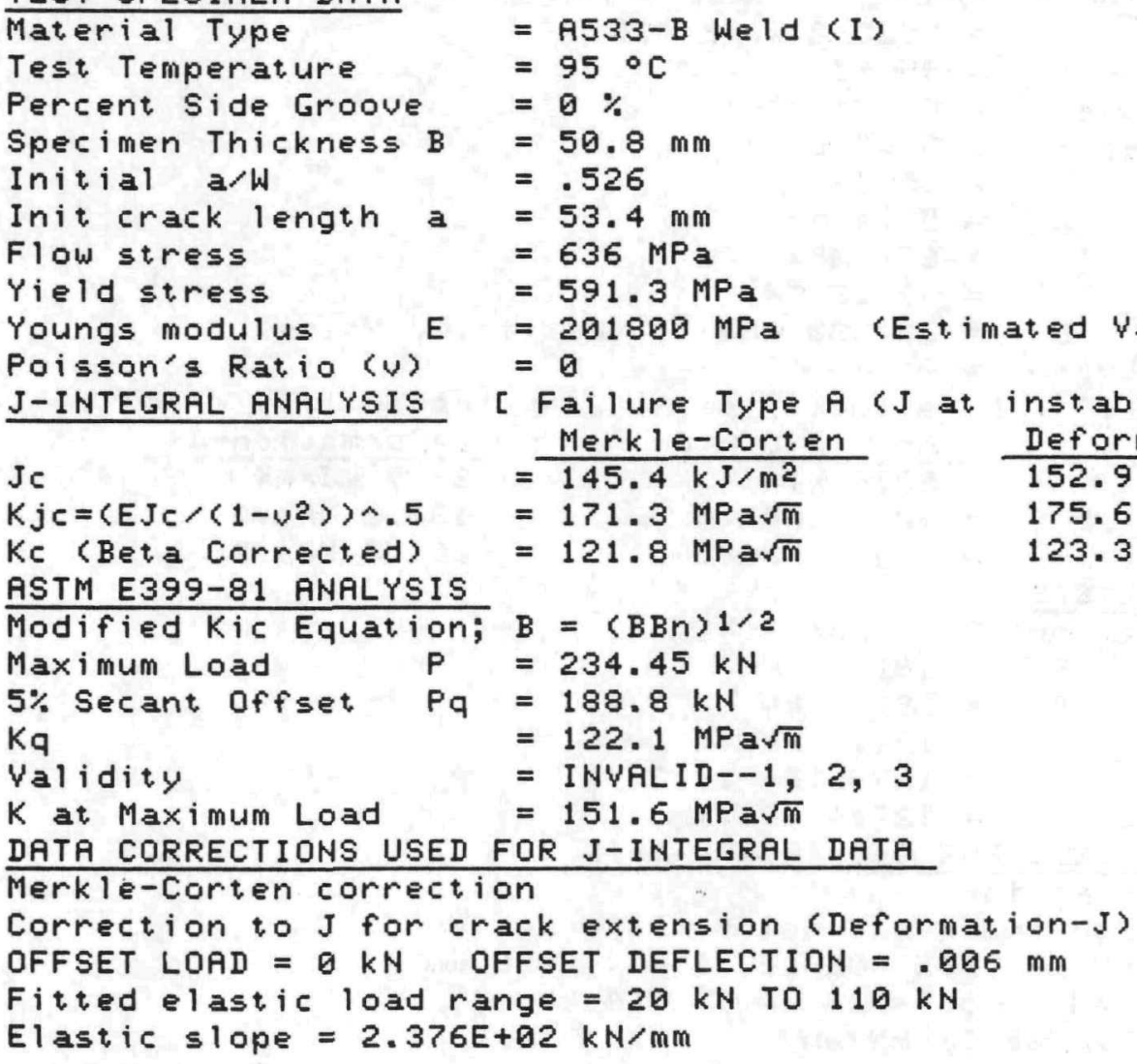


Specimen Loadline Deflection (in.) ORNL-DWG 92-11654

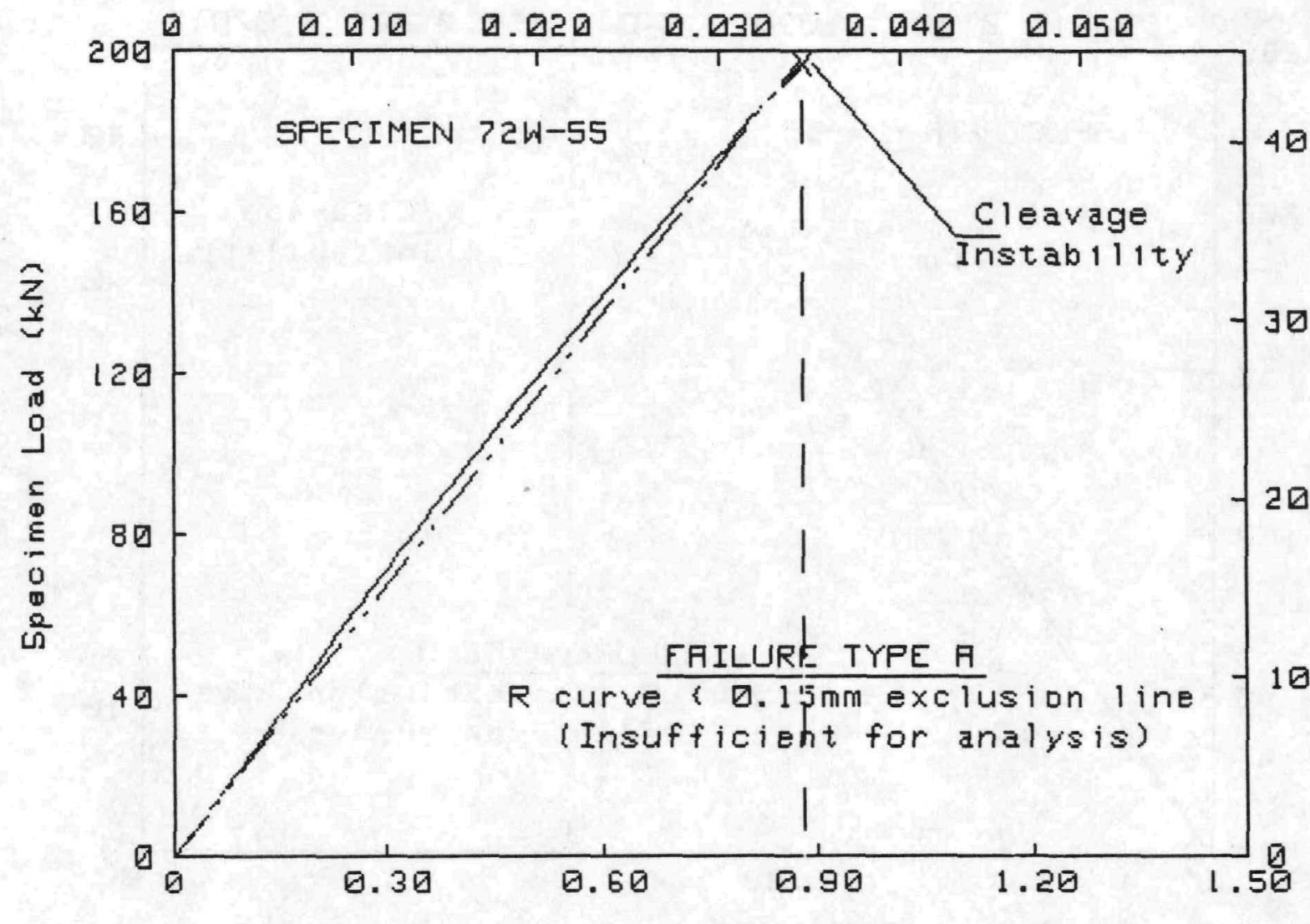

Specimen Laadline Defleotion (mm)

TEST SPECIMEN DATA

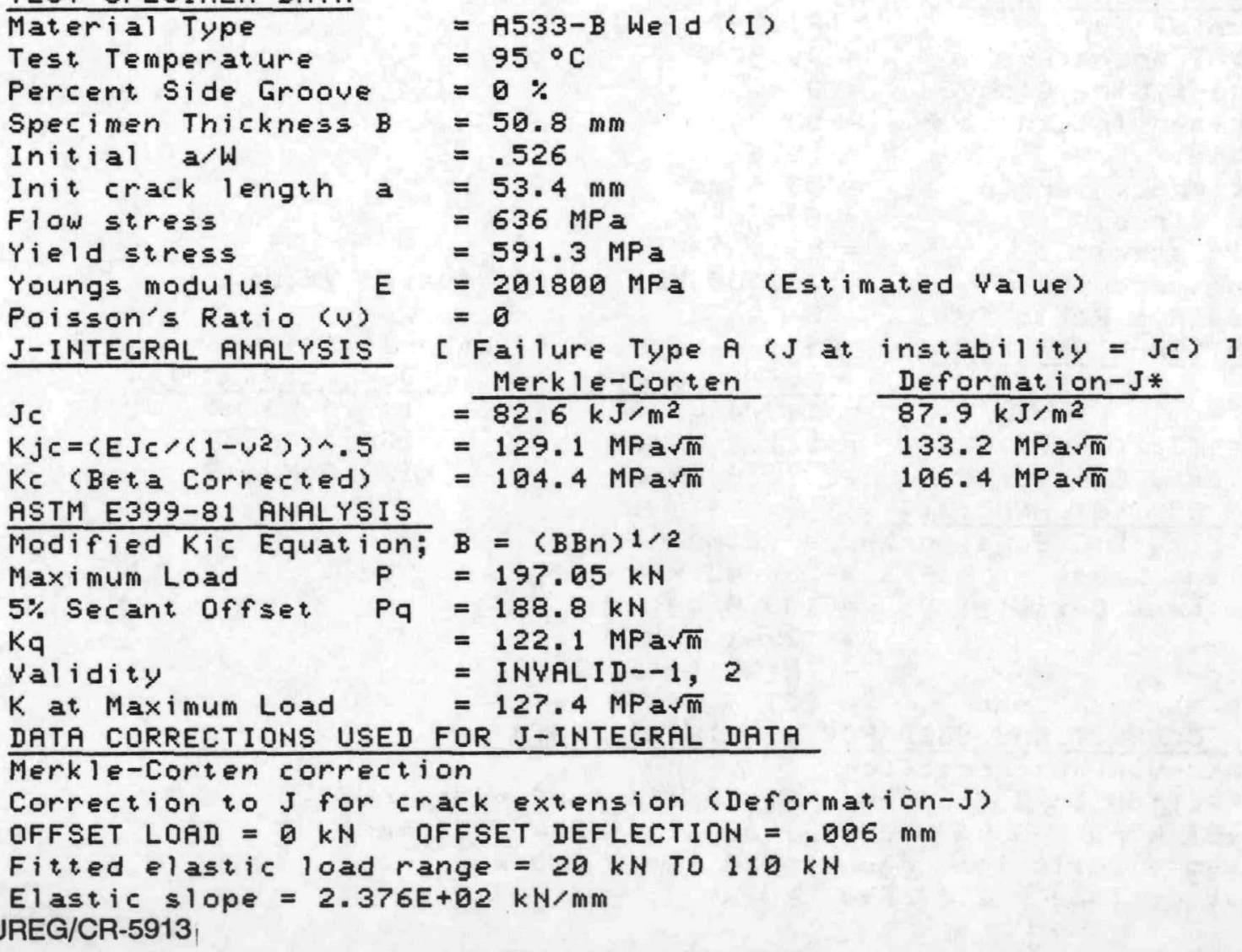


Specimen Crackmouth Def lection (in.) ORNL-DWG 92-11655

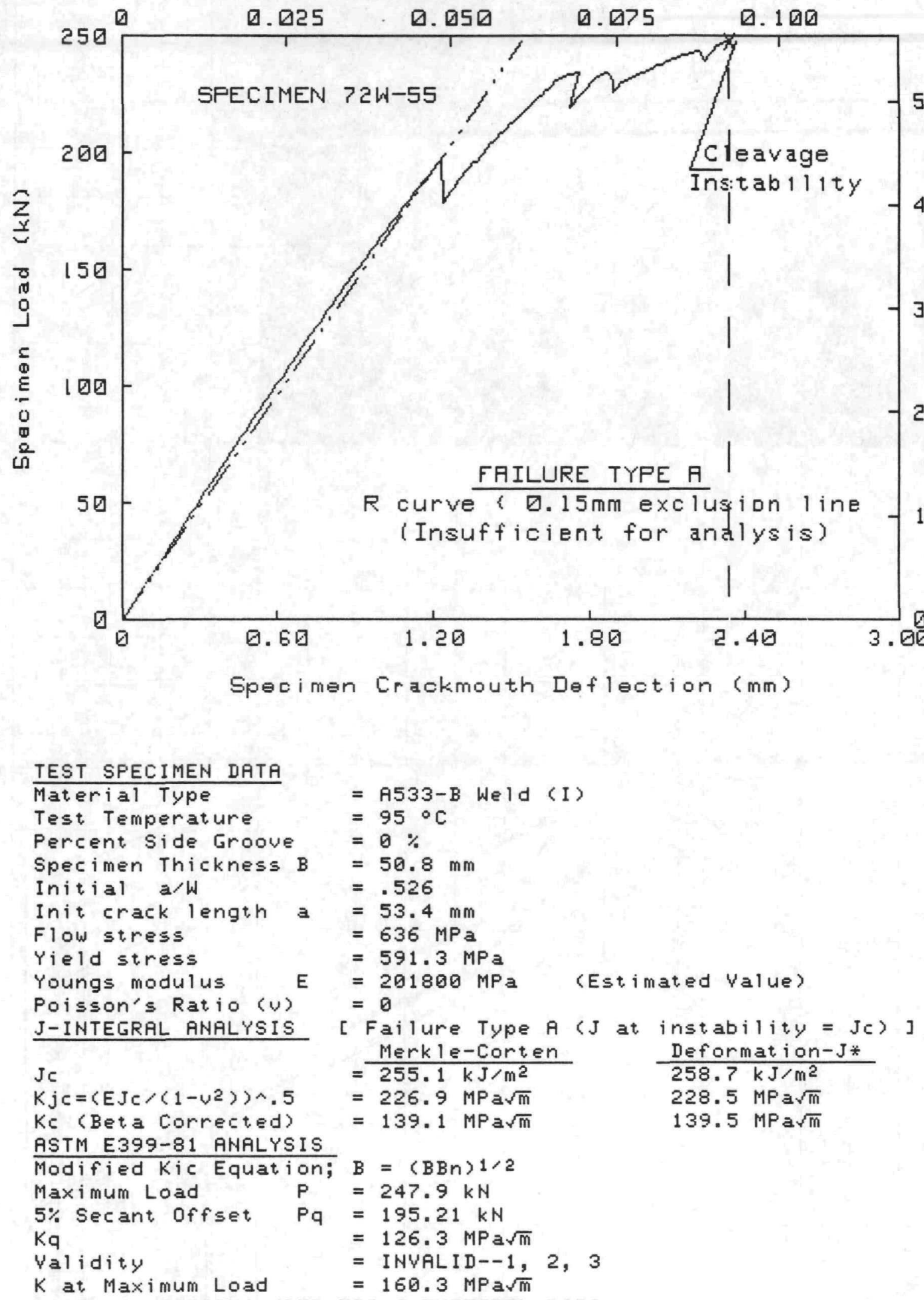

DATA CORRECTIONS USED FOR J-INTEGRAL DATA

Merkle-Corten correction

Correction to J for crack extension (Deformation-J)

OFFSET LOAD $=0 \mathrm{kN}$ OFFSET DEFLECTION $=.017 \mathrm{~mm}$

Fitted elastic load range $=20 \mathrm{kN}$ TO $110 \mathrm{kN}$

Elastic slope $=1.692 \mathrm{E}+02 \mathrm{kN} / \mathrm{mm}$ 


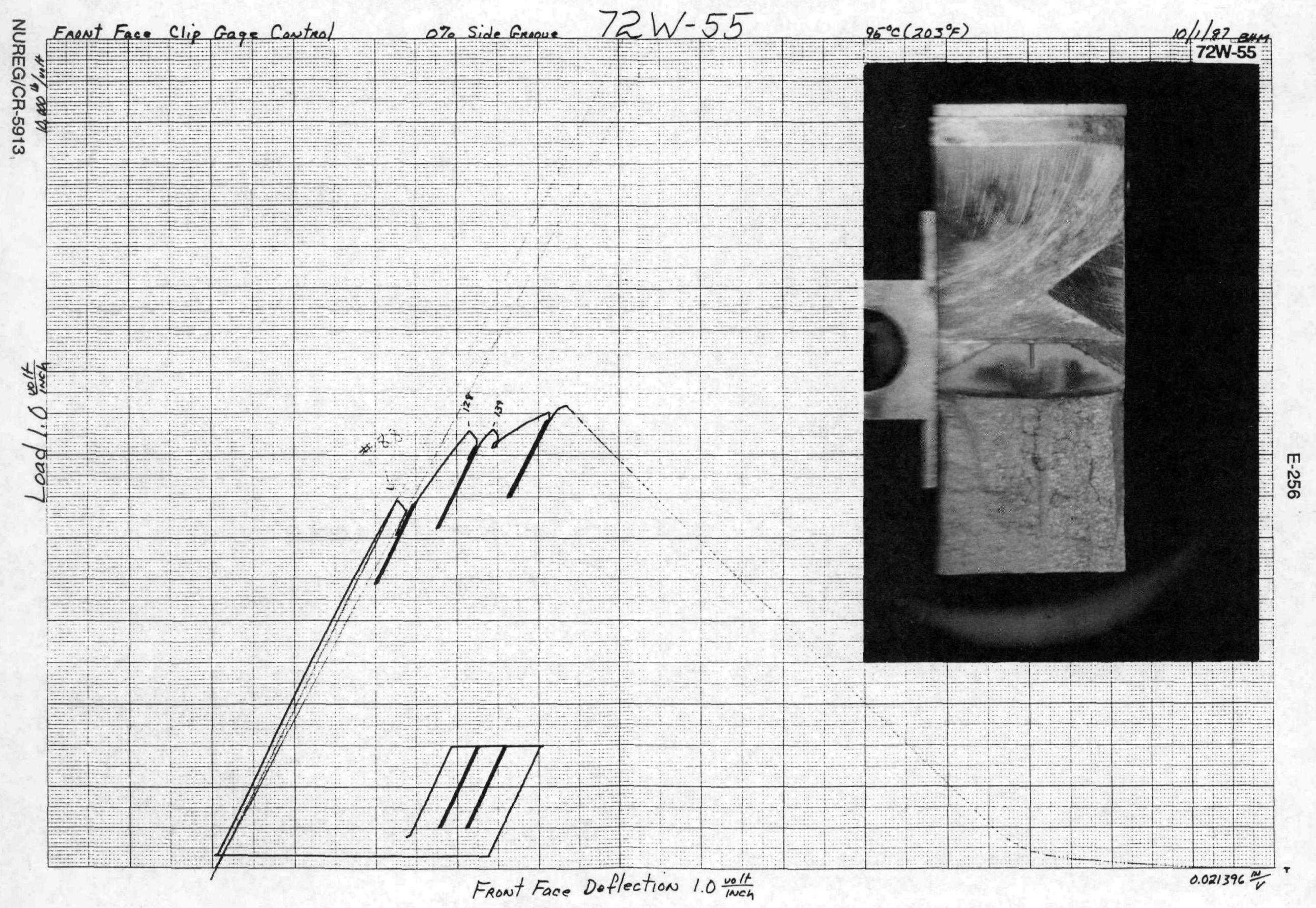




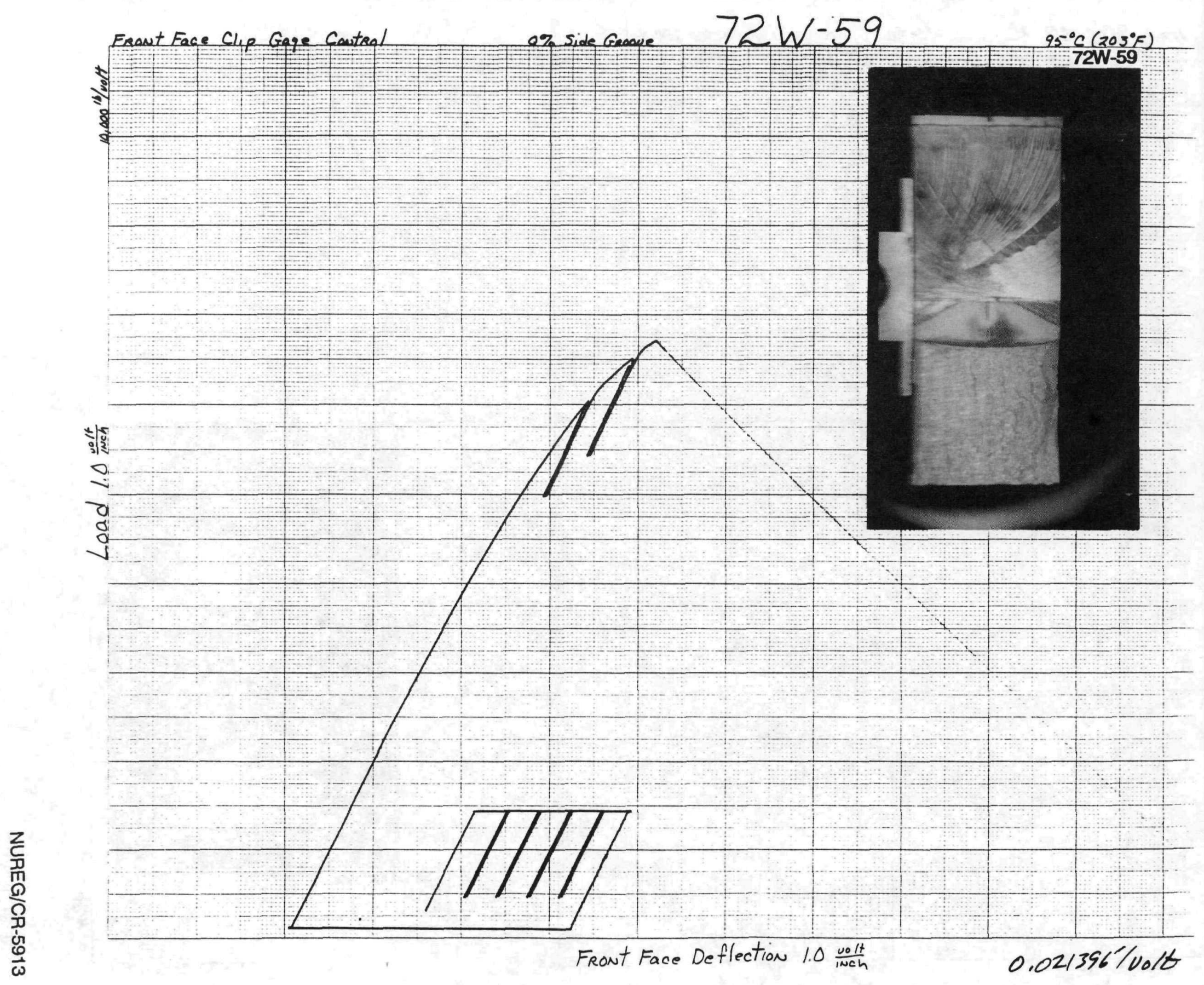




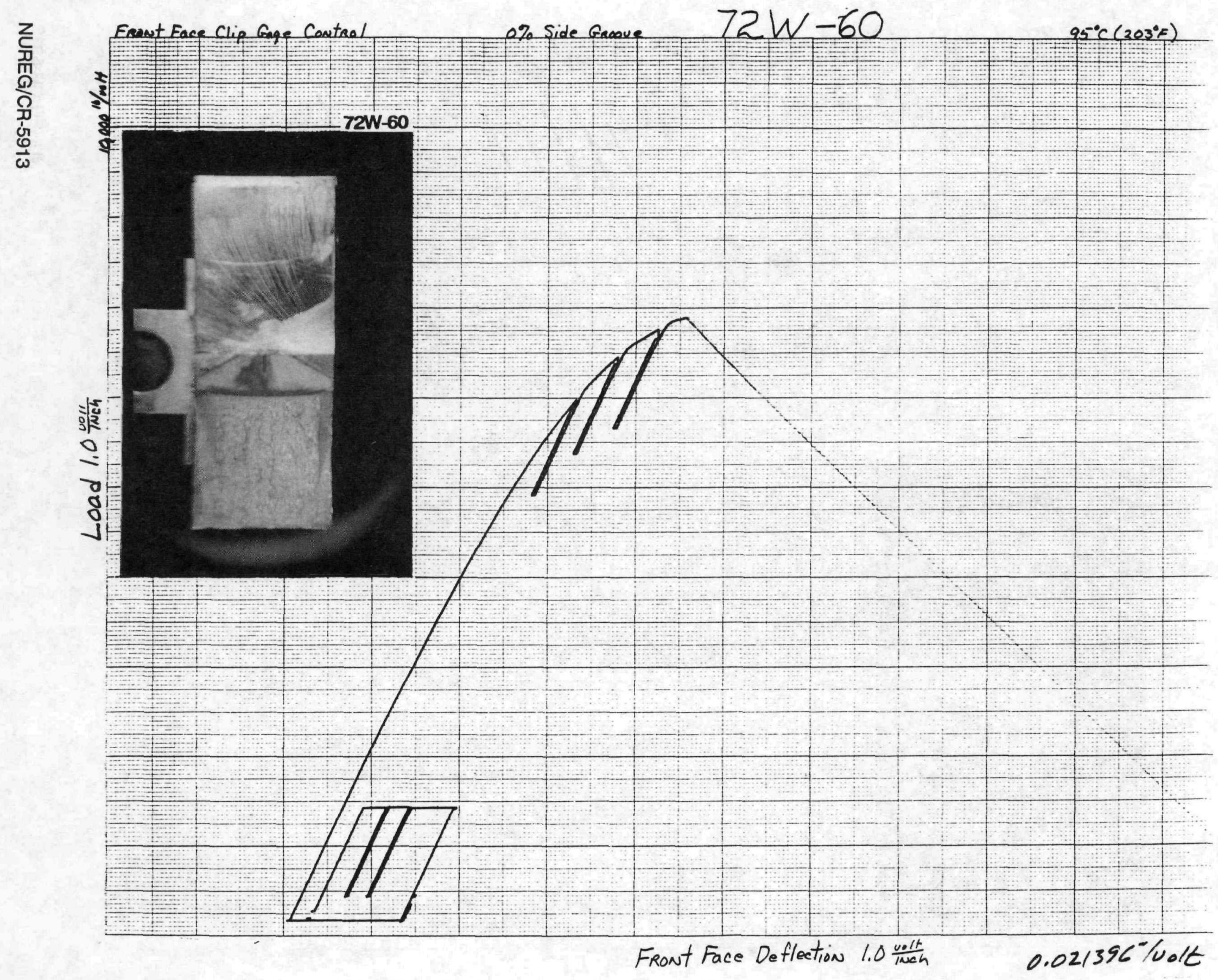




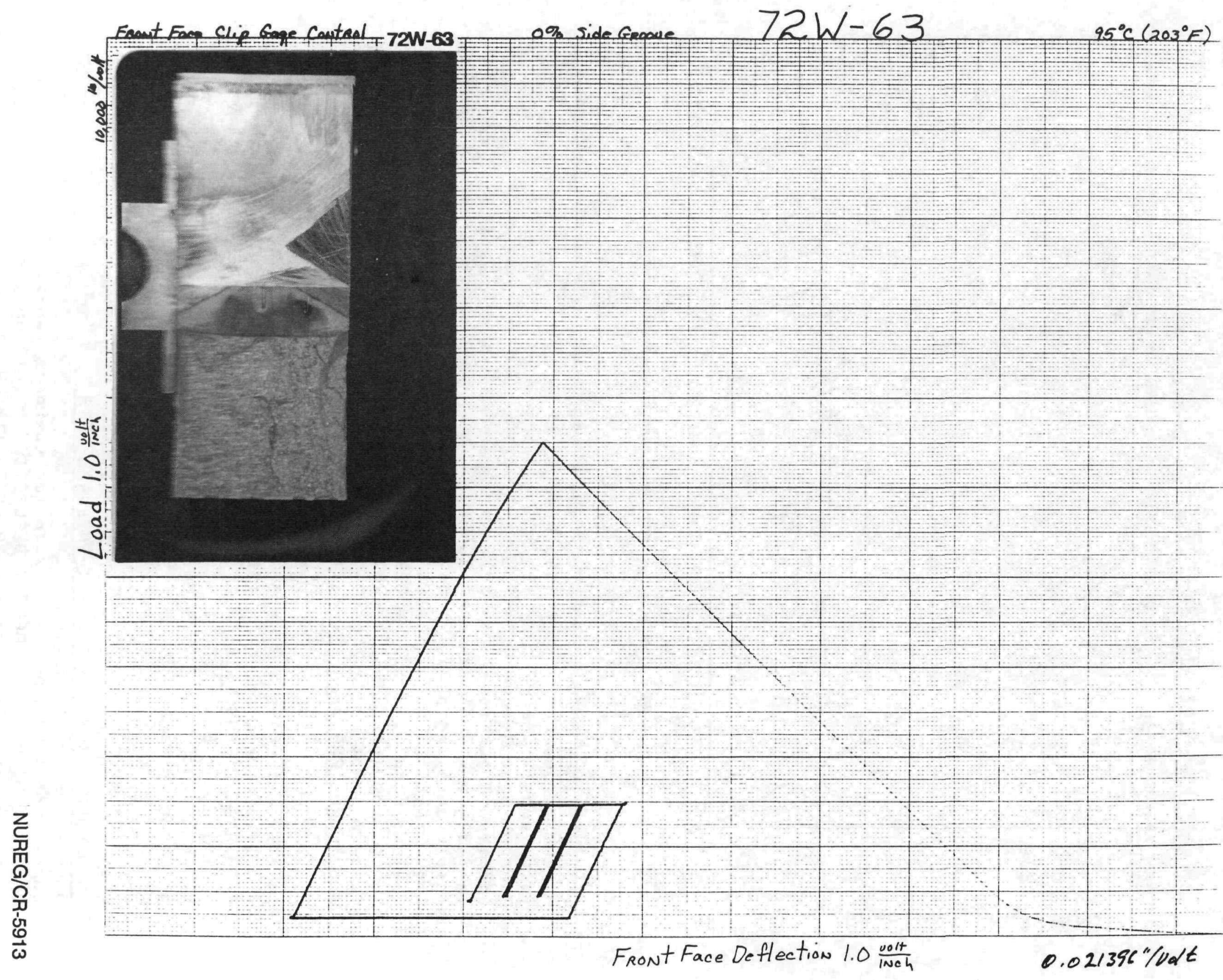


Specimen Crackmouth Deflection (in.) ORNL-DWG 92-11656

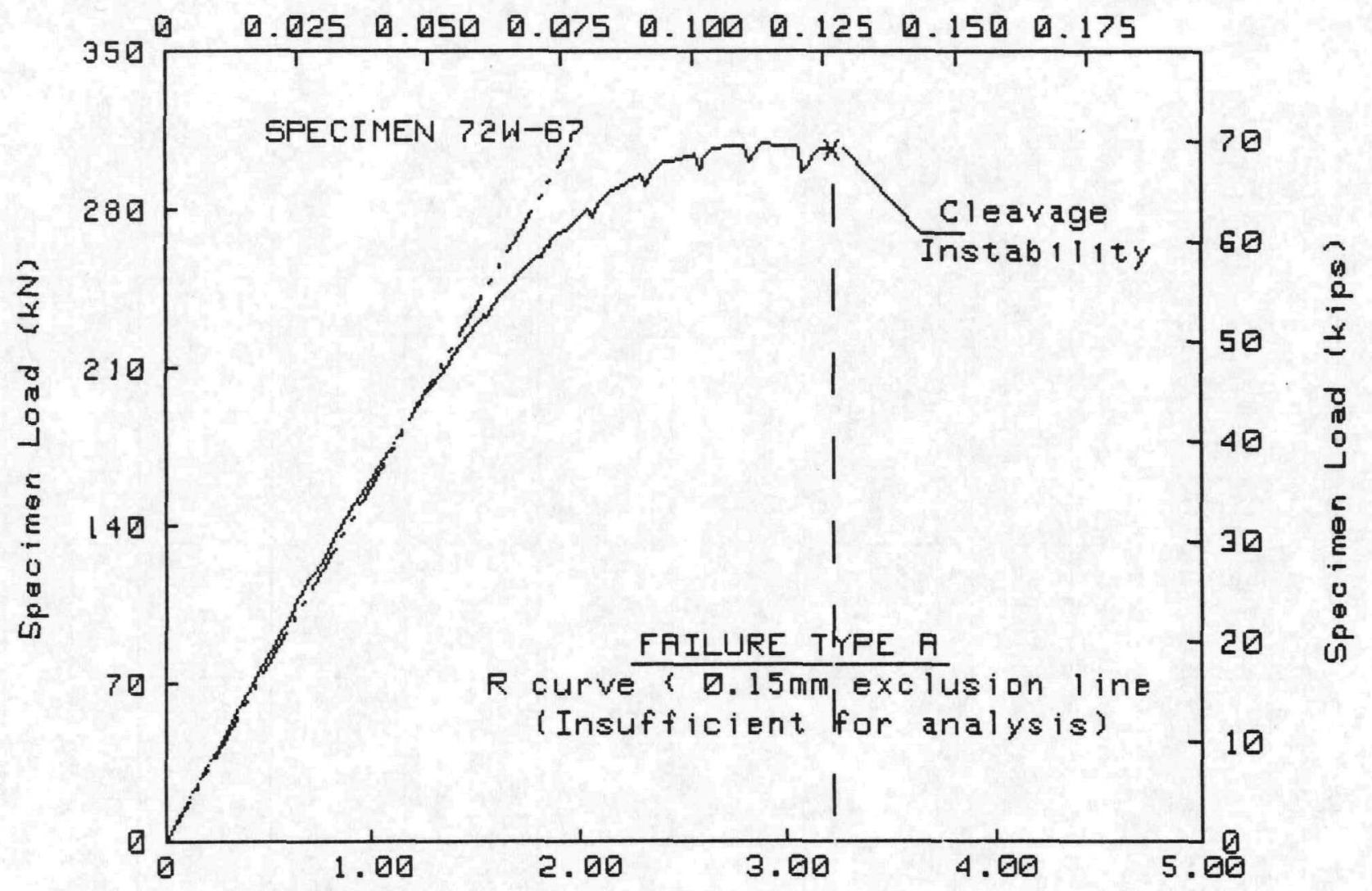

Specimen Crackmouth Defleotion (mm)

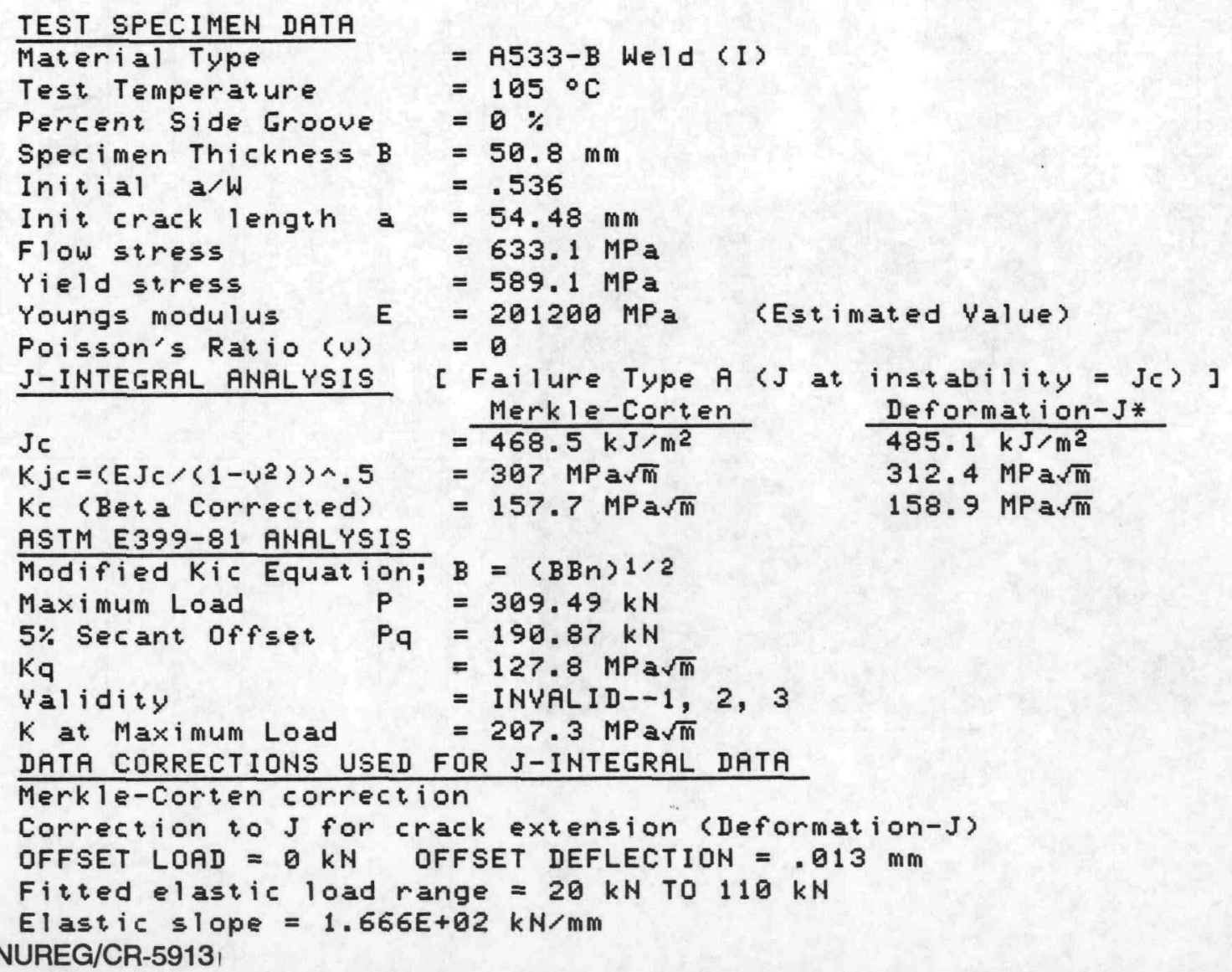


Specimen Laadline Deflection (in.) ORNL-DWG 92-11657

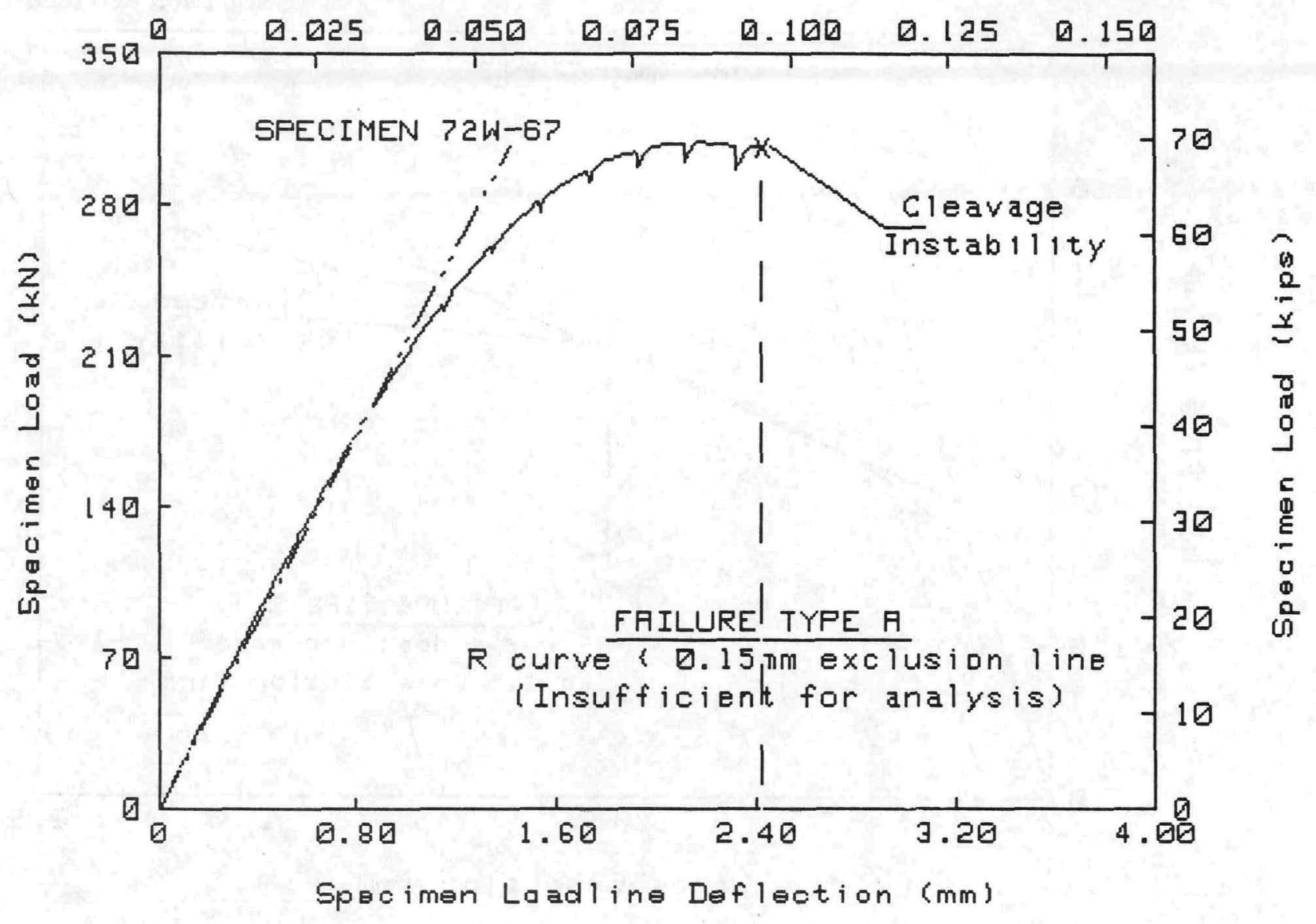

TEST SFECIMEN DATA

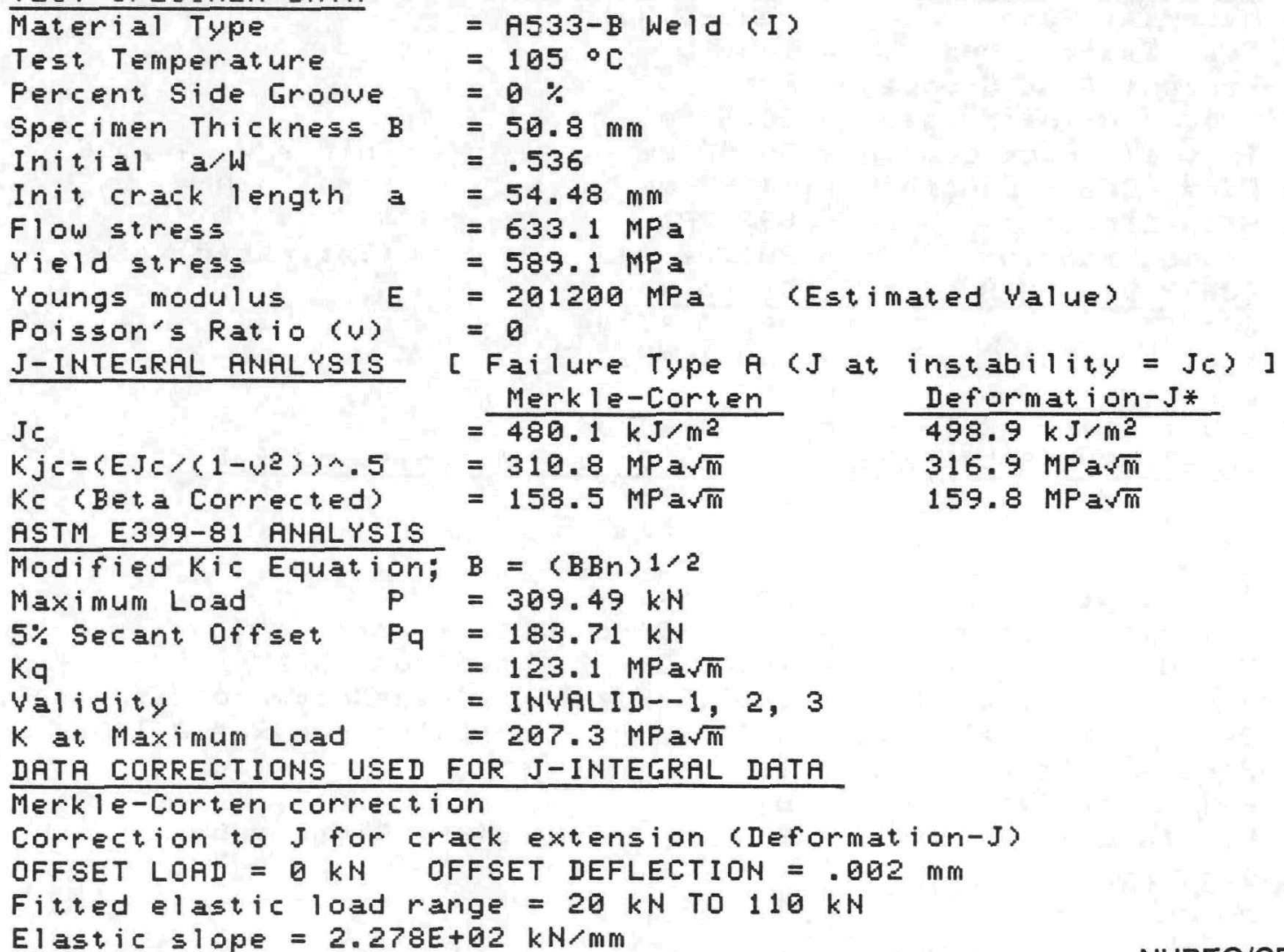


ORNL-DWG 92-11658

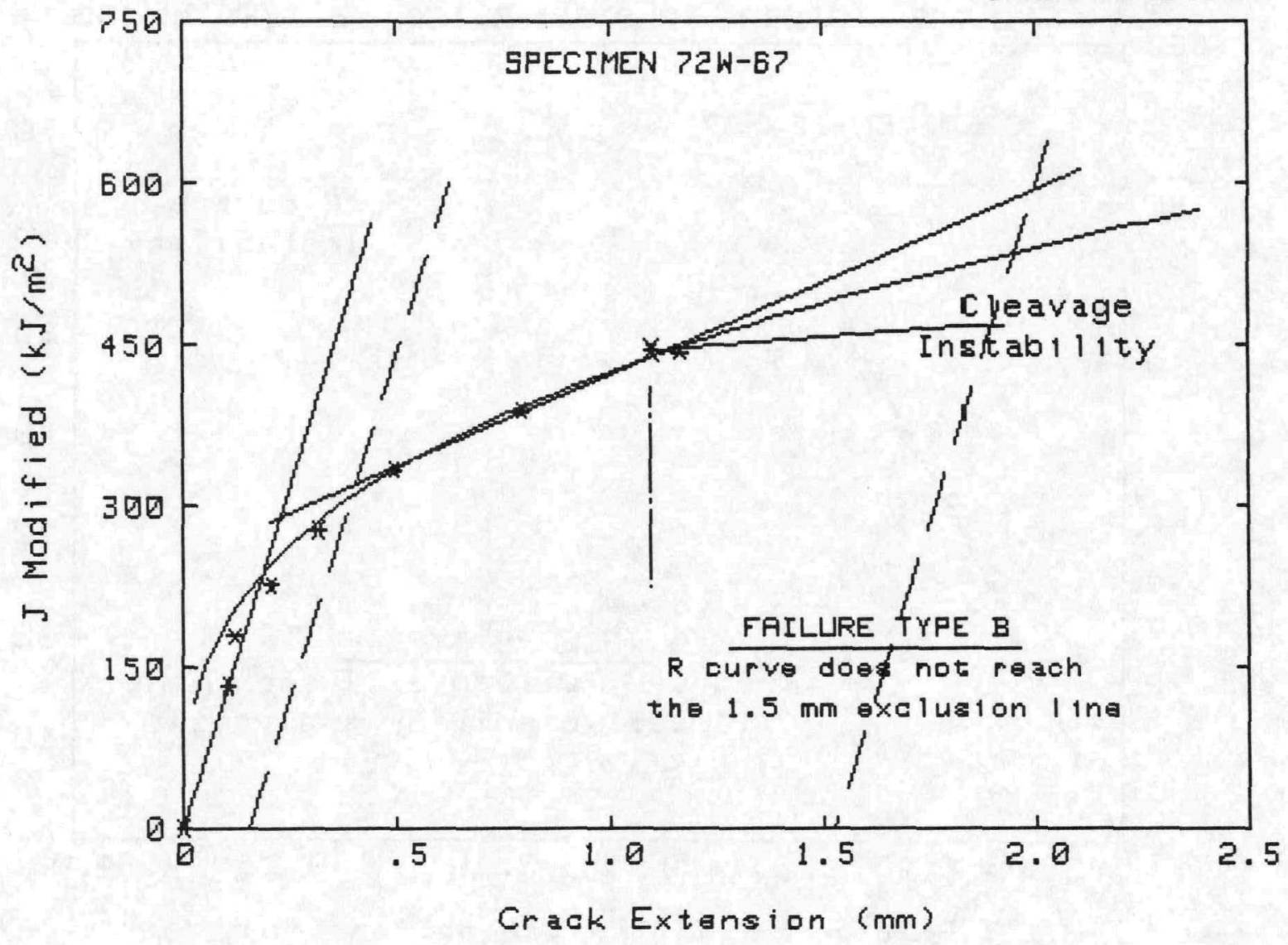

TEST SPECIMEN DATA

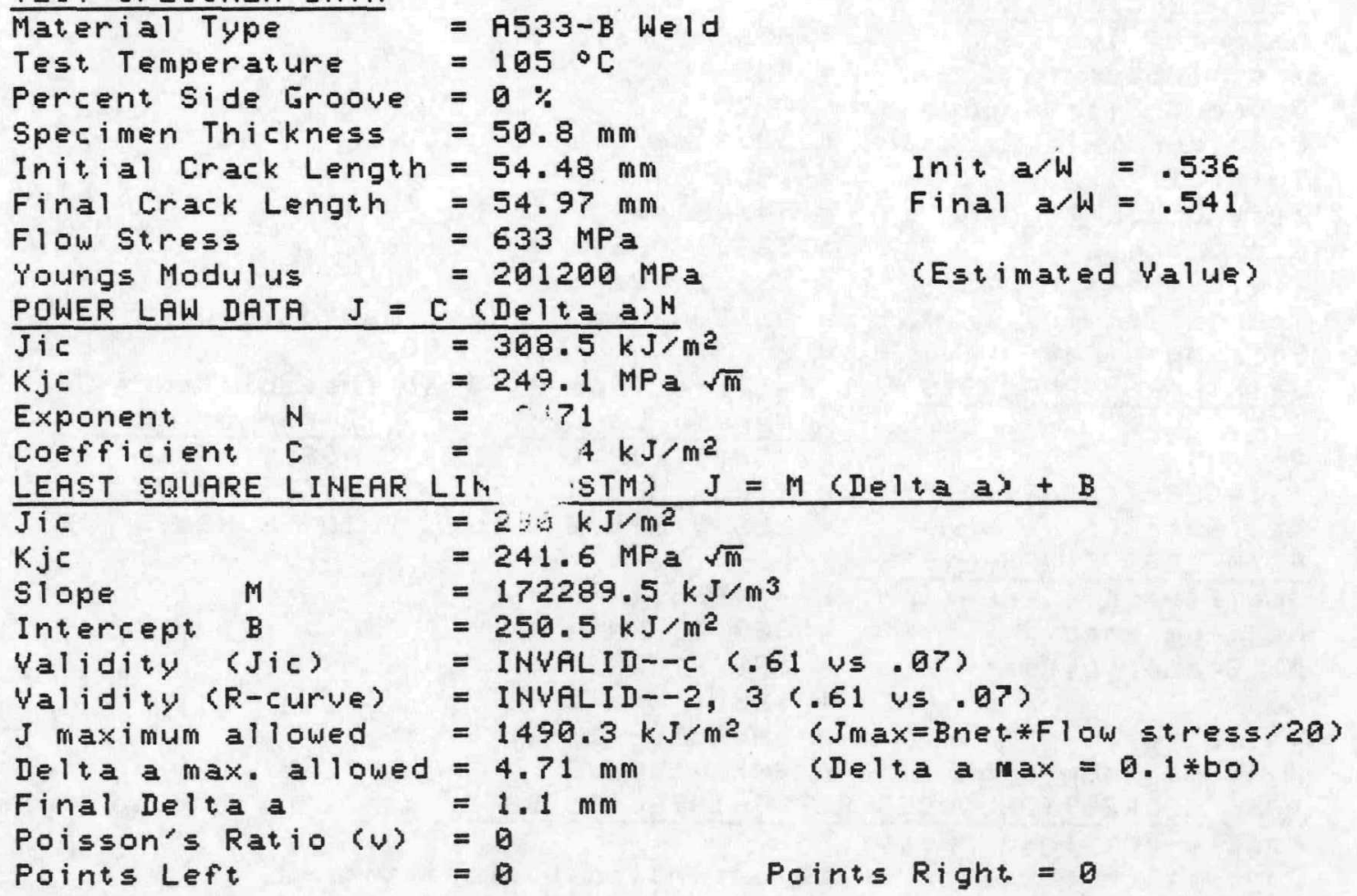

NUREG/CR-5913 


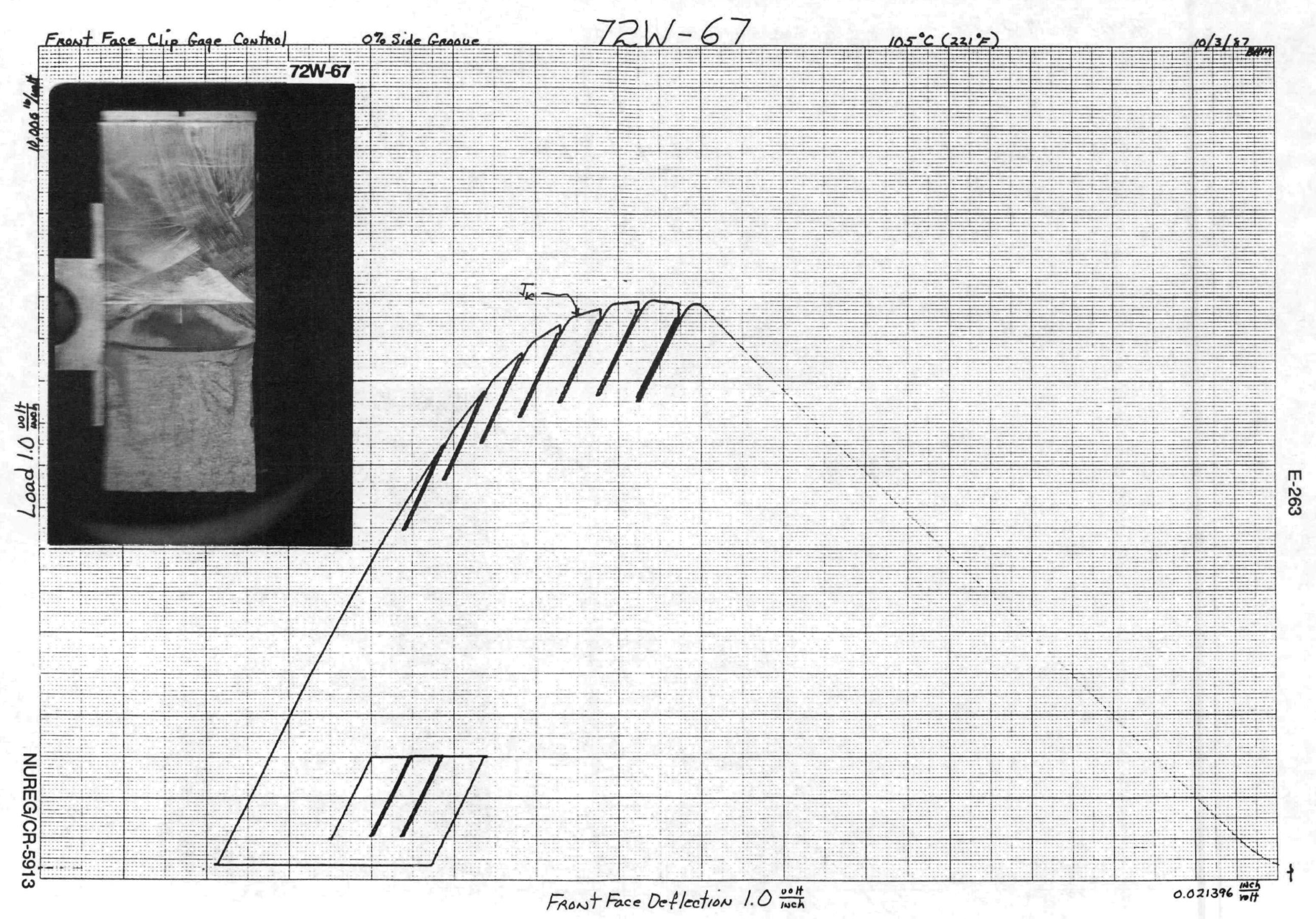




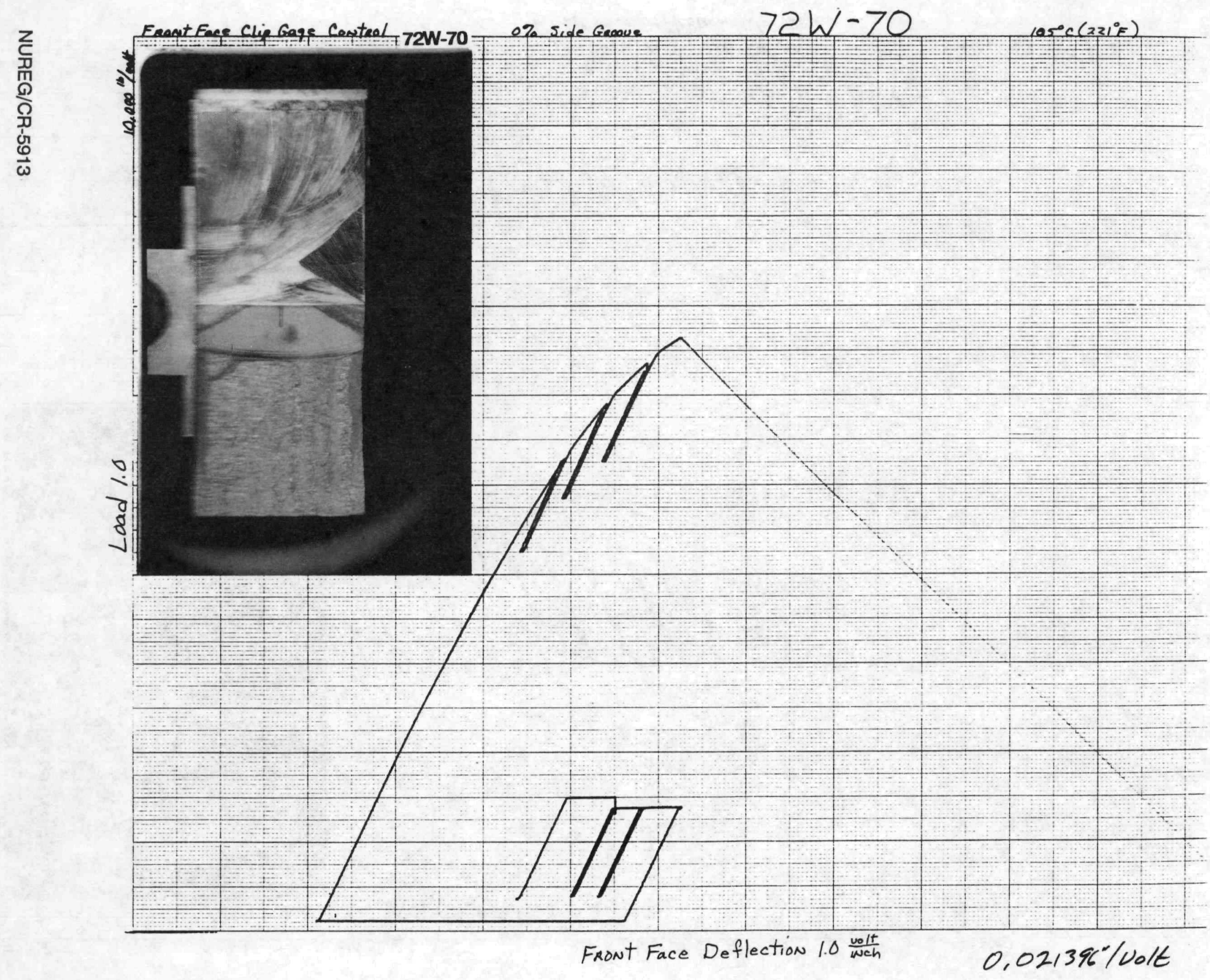




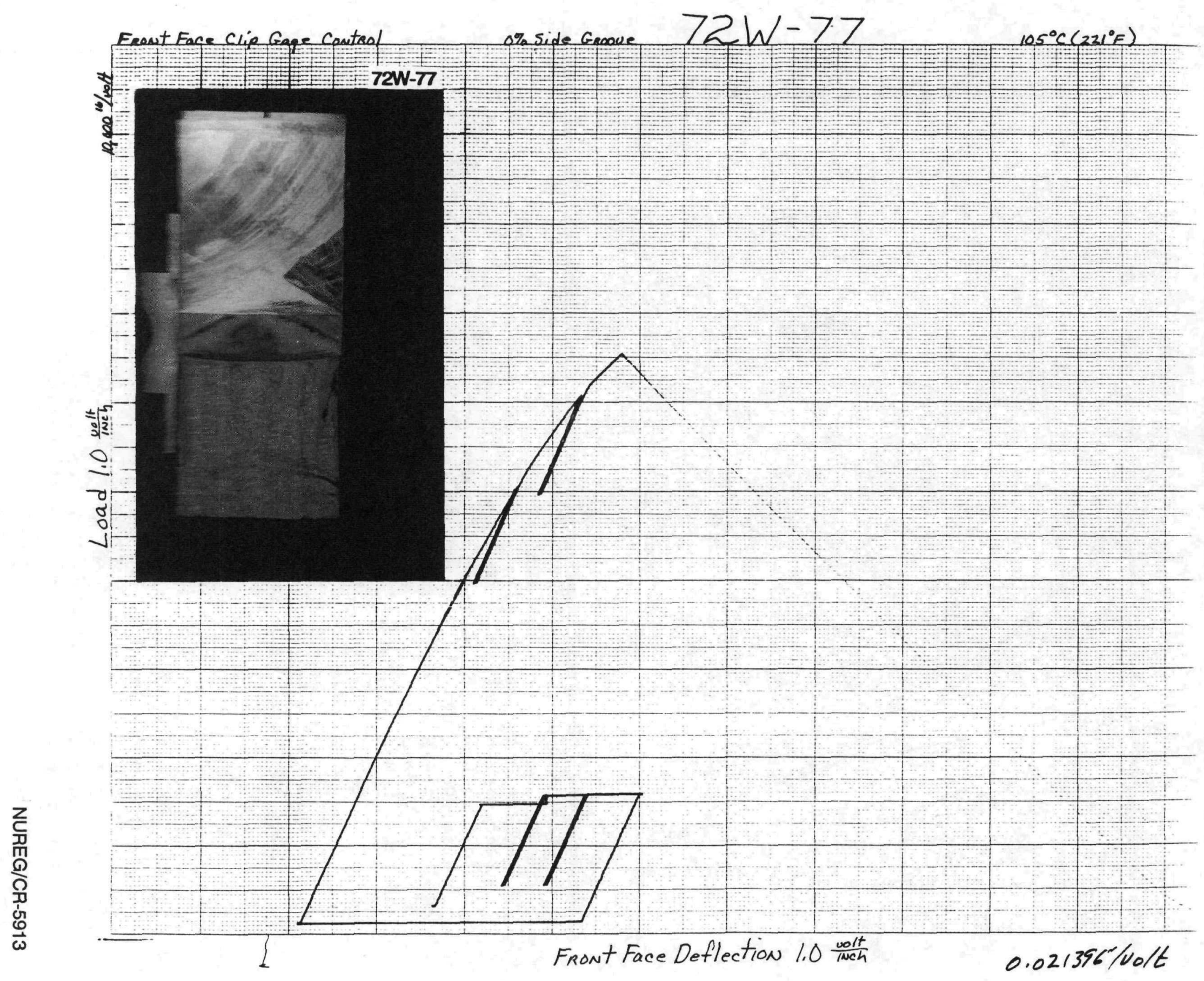




\section{E-266}

ORNL-DWG 92-11659
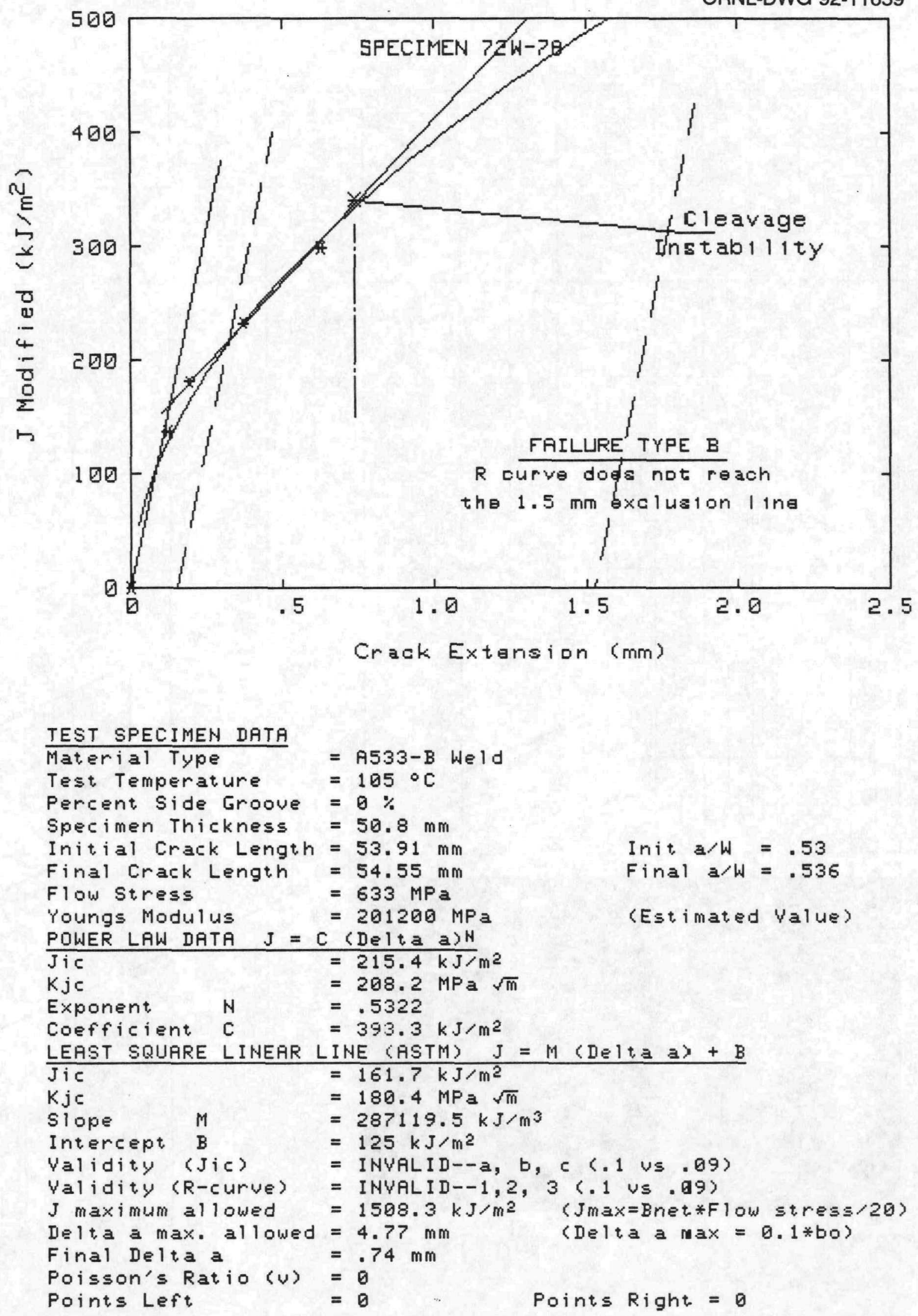
Spec imen Laadline Deflection (in.) ORNL-DWG 92-11660

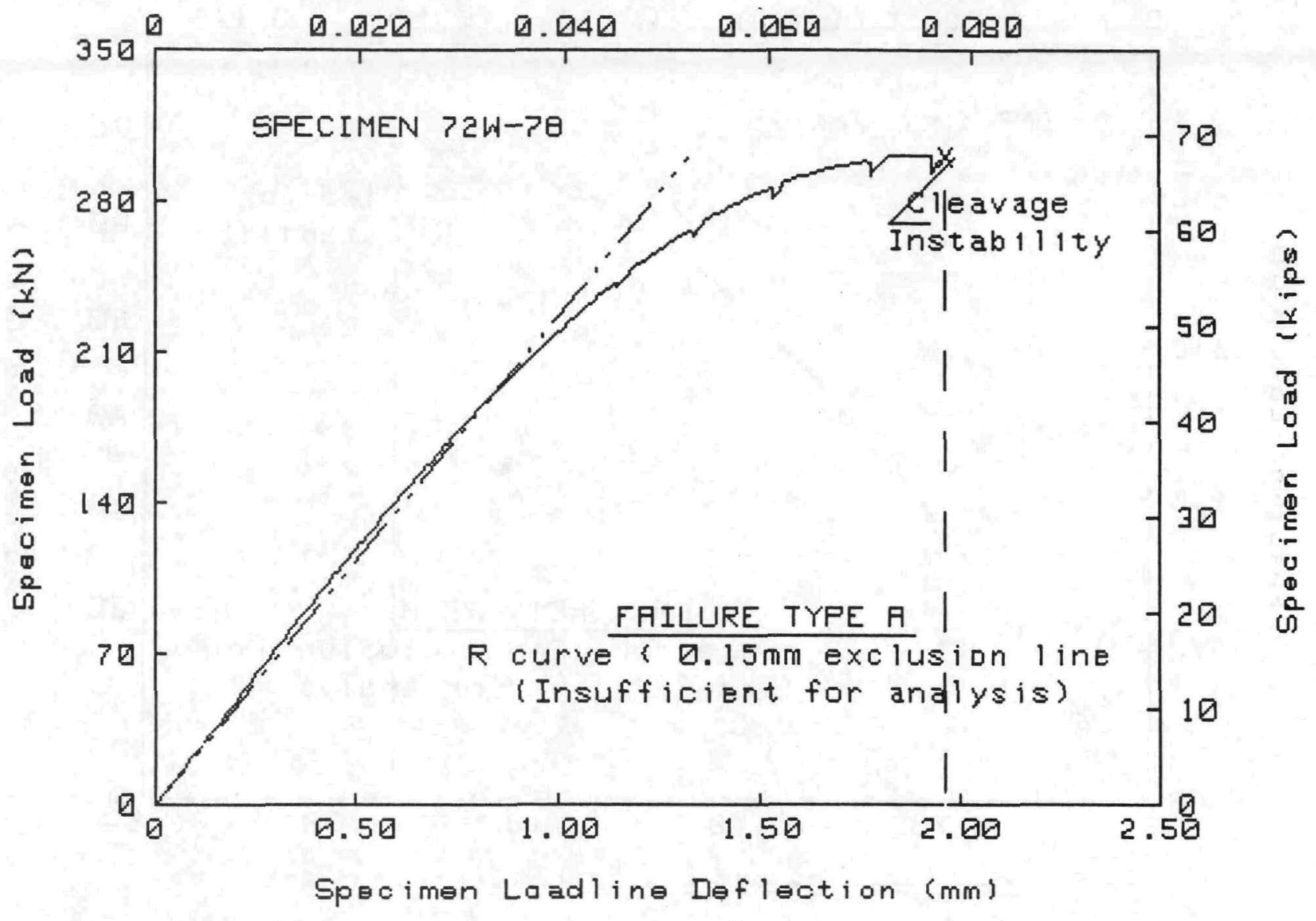

TEST SPECIMEN DATA

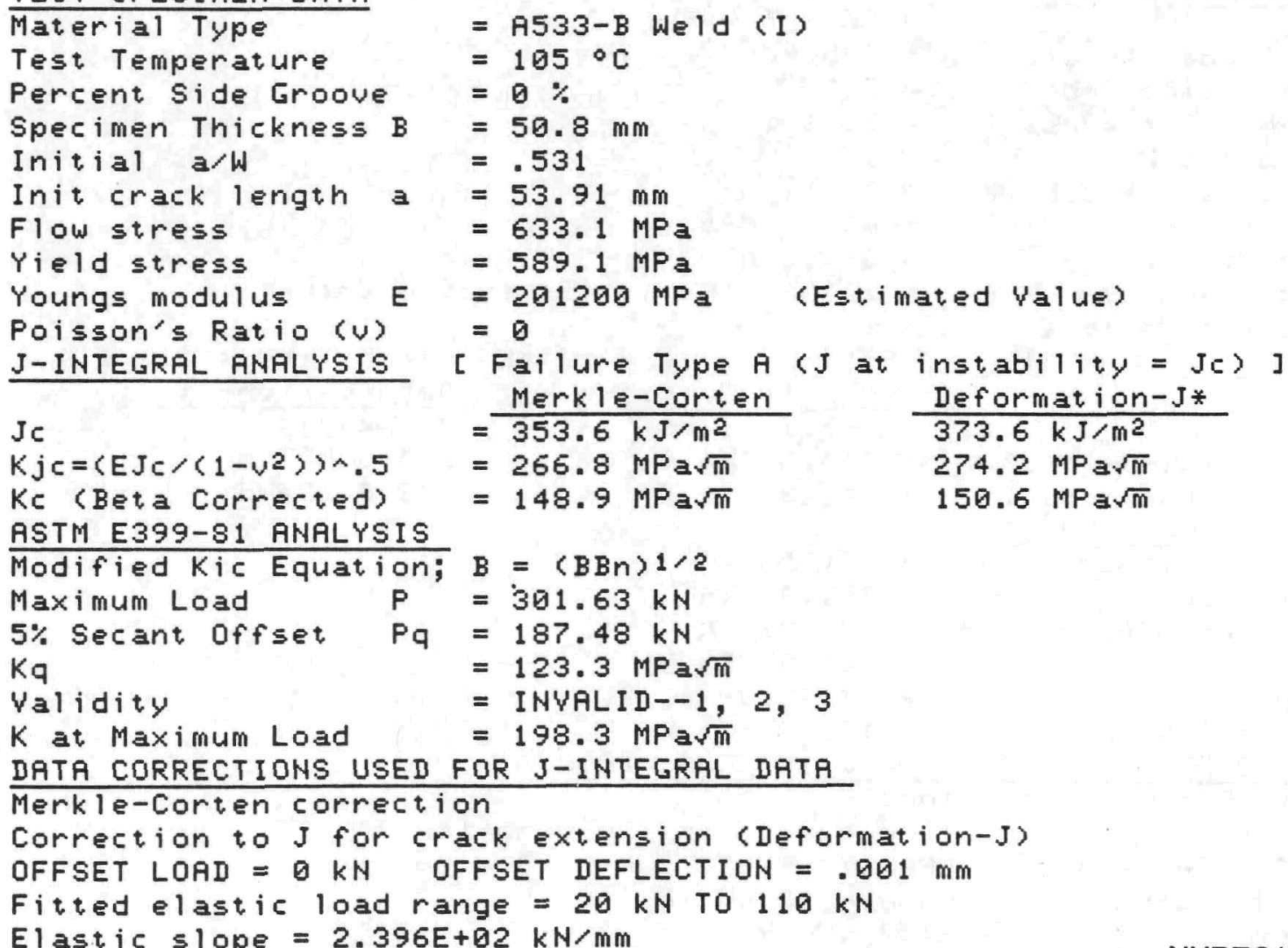


Specimen Crackmauth Def lection (in.) ORNL-DWG 92-11661

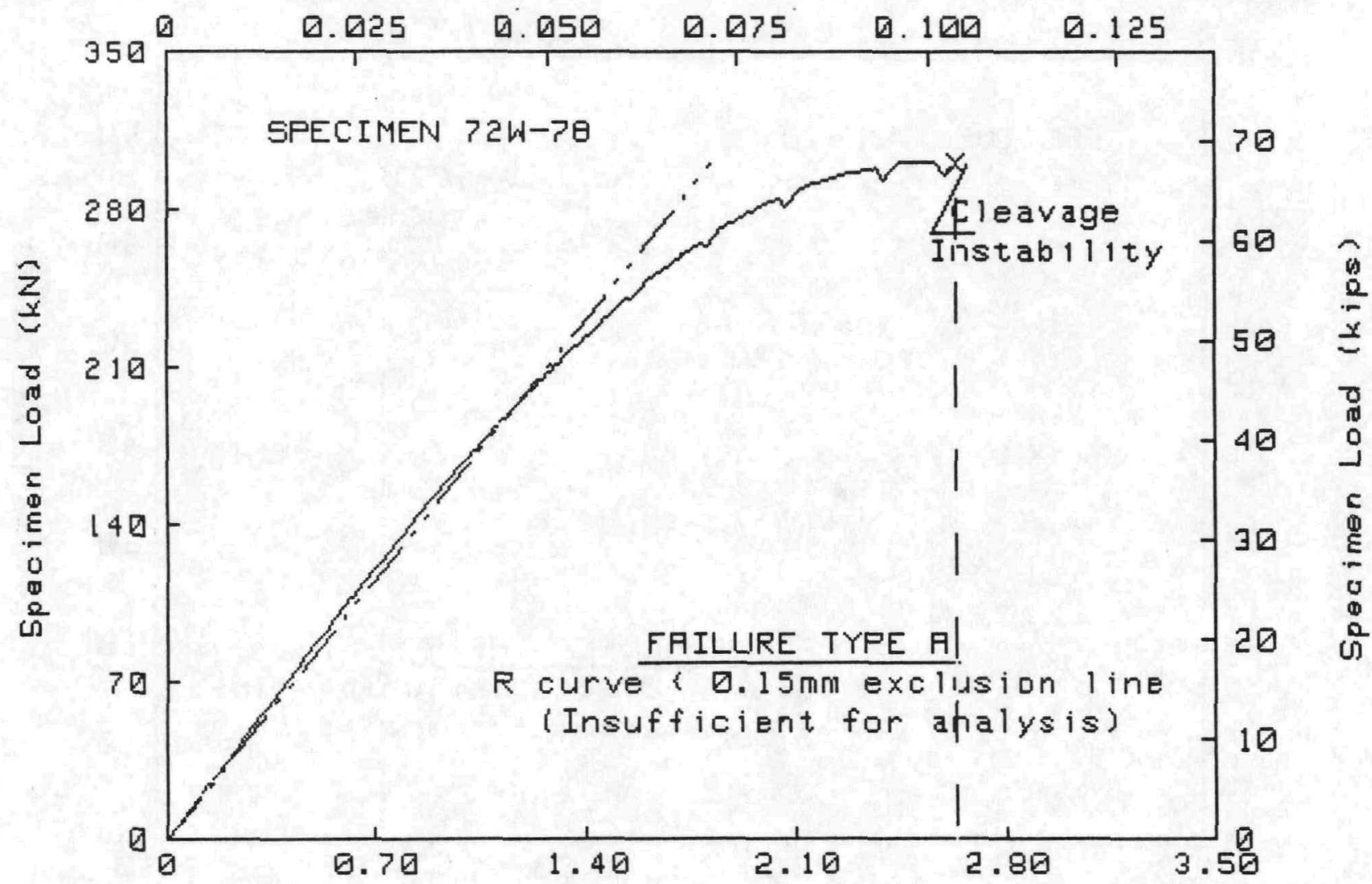

Specimen Crackmouth Defleotion ( $m m$ )

TEST SPECIMEN DATA

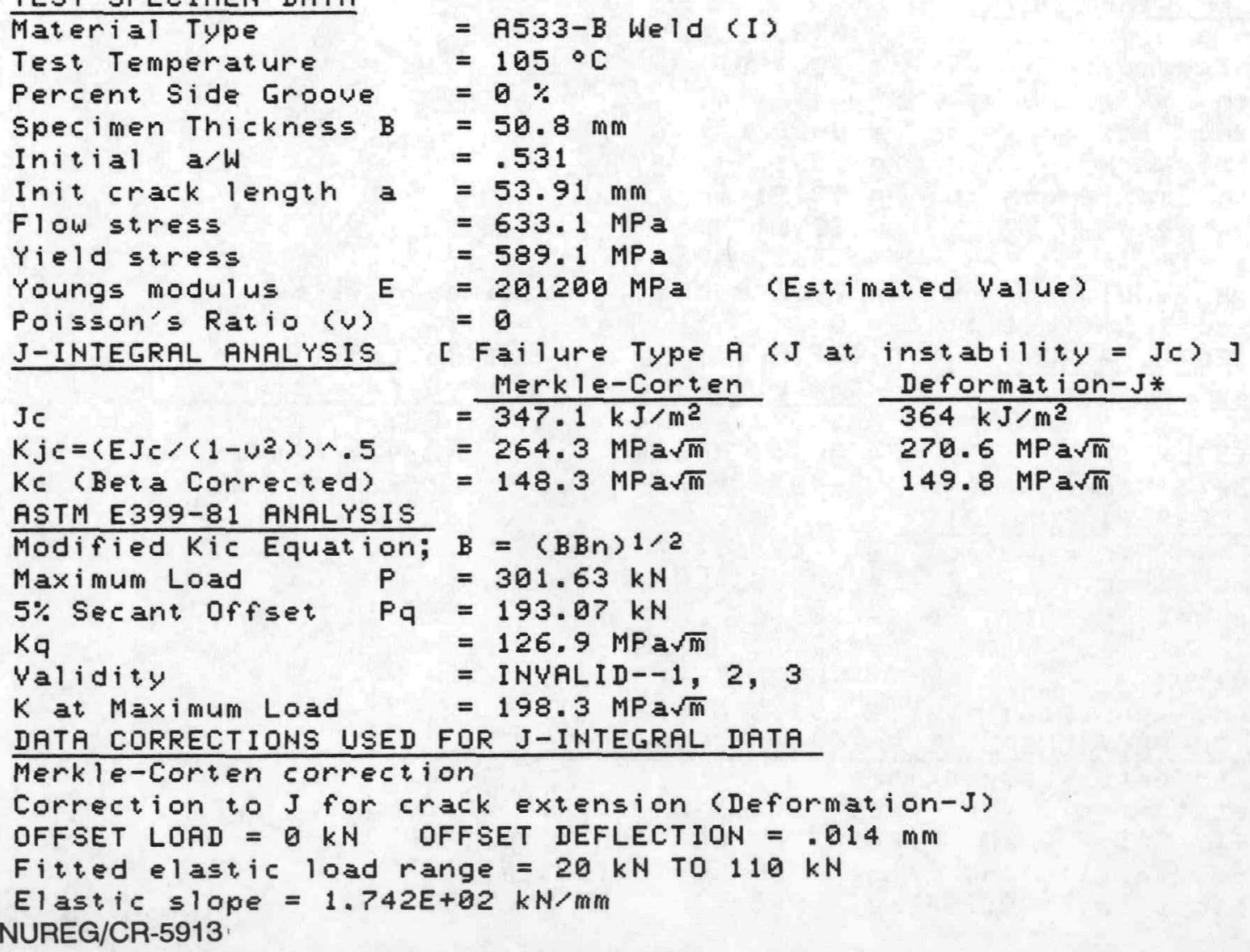


Spec imen Loadline Def lection (in.) ORNL-DWG 92-11662

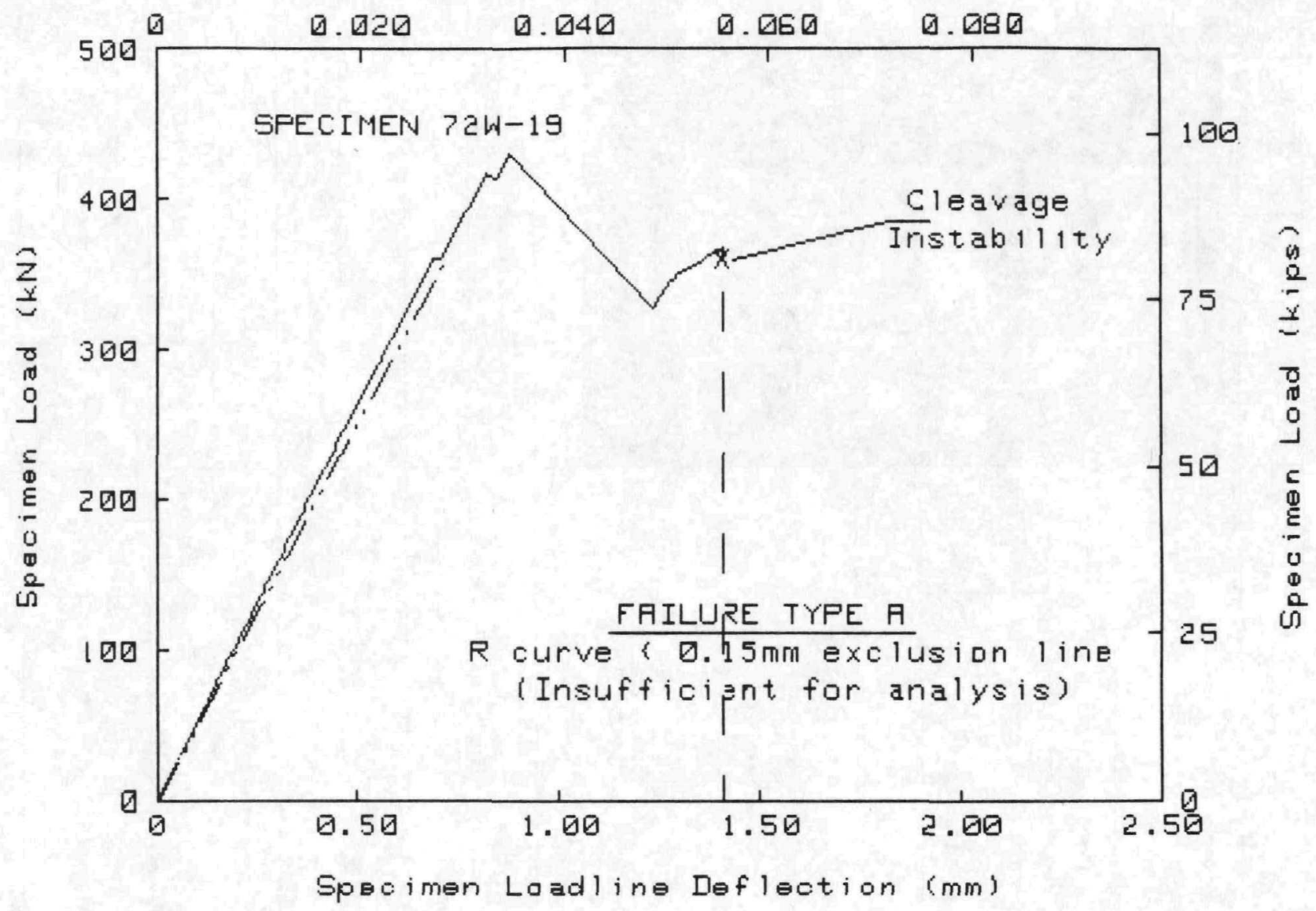

TEST SPECIMEN DATA

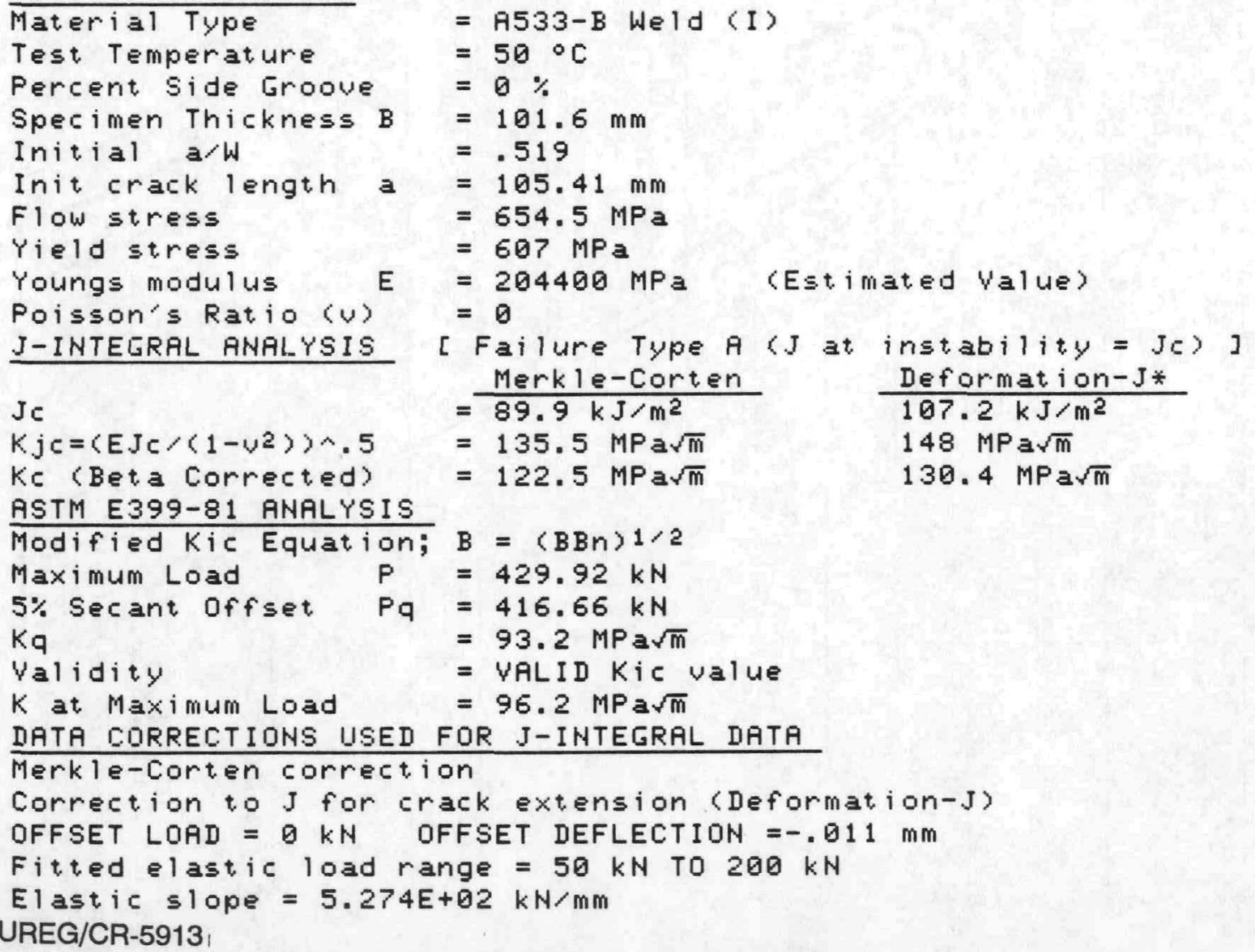


Specimen Laadline Deflection (in.) ORNL-DWG 92-11663

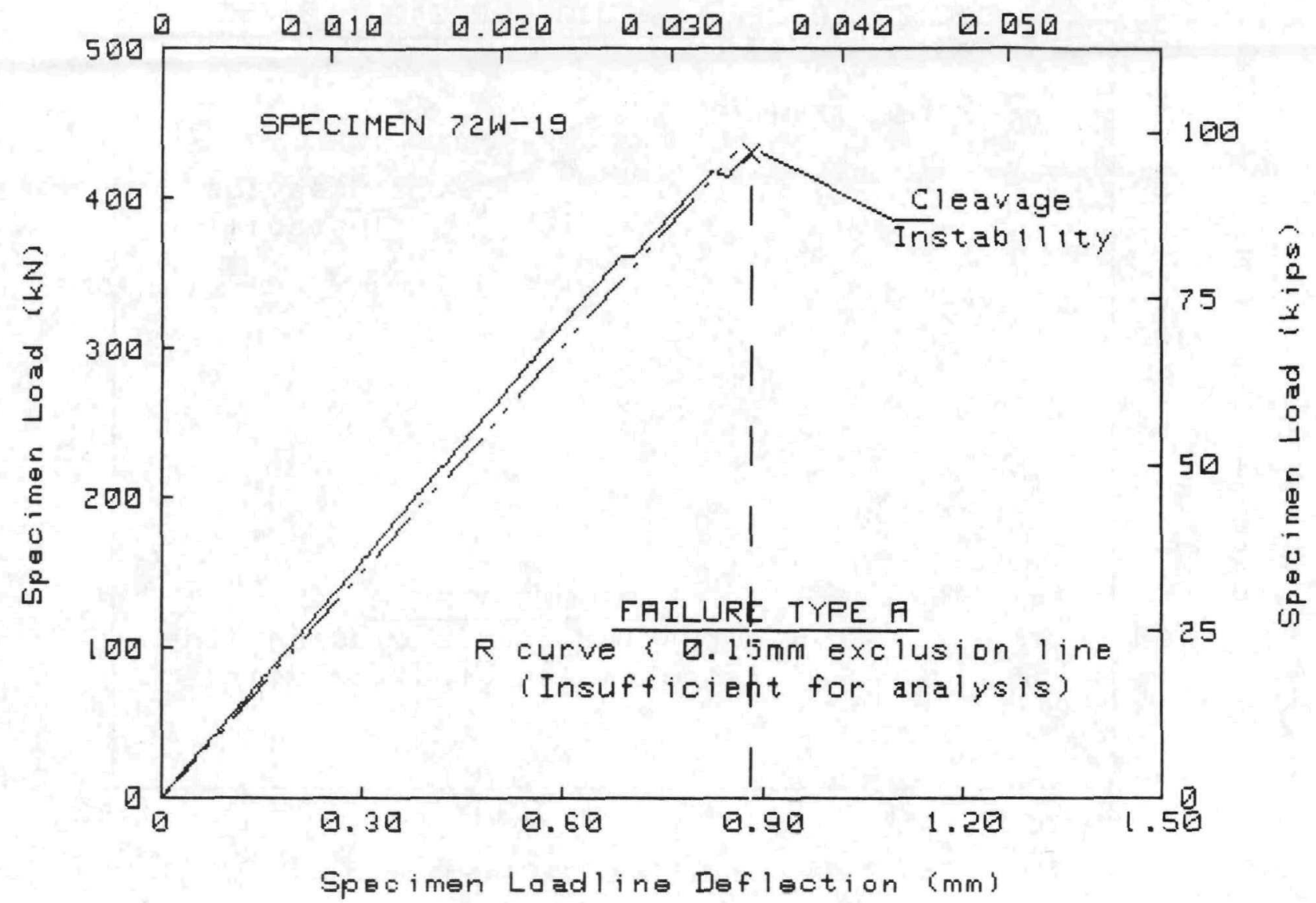

TEST SPECIMEN DATA

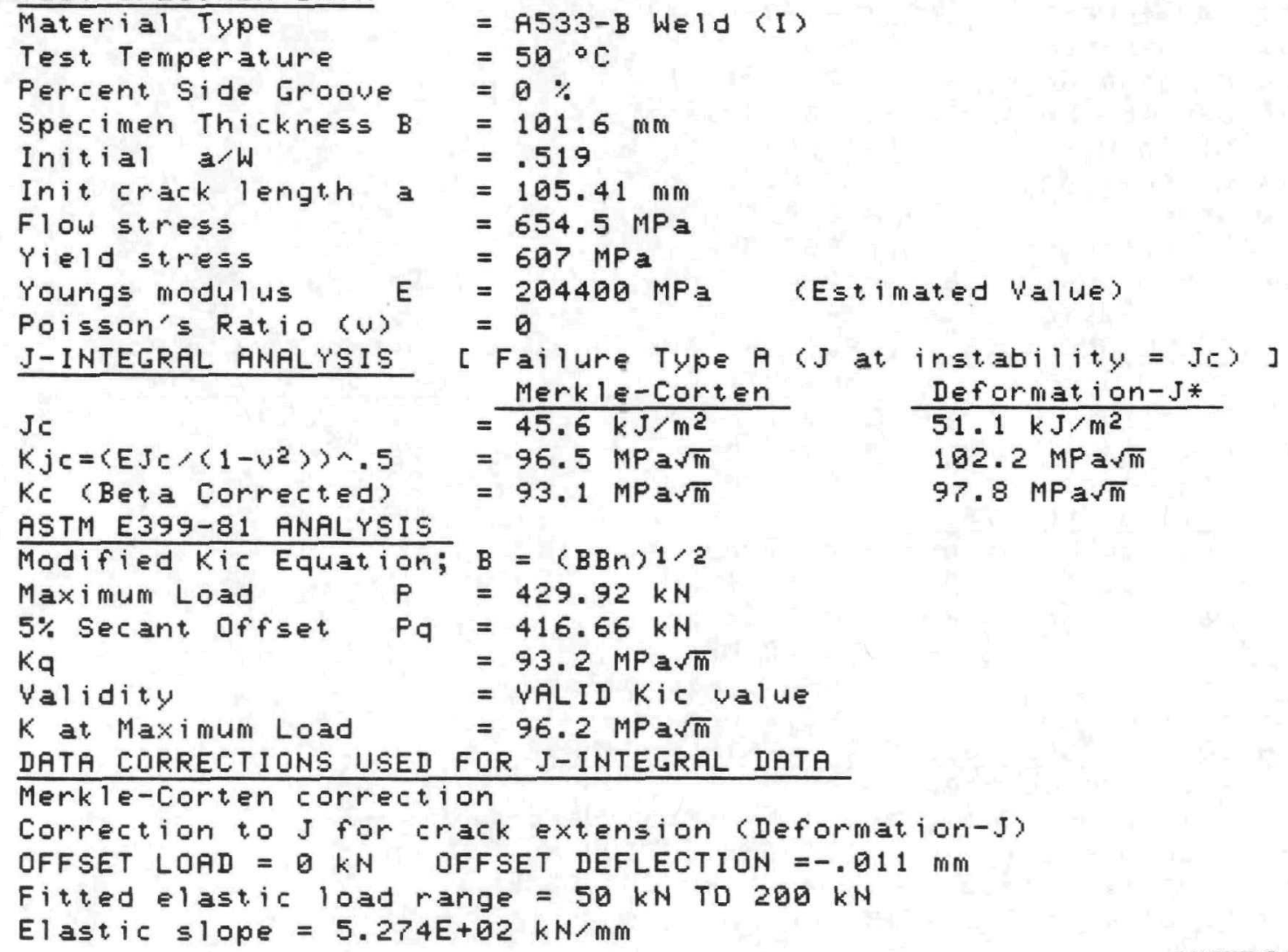


Spec imen Loadline Def lection (in. ) ORNL-DWG 92-11664

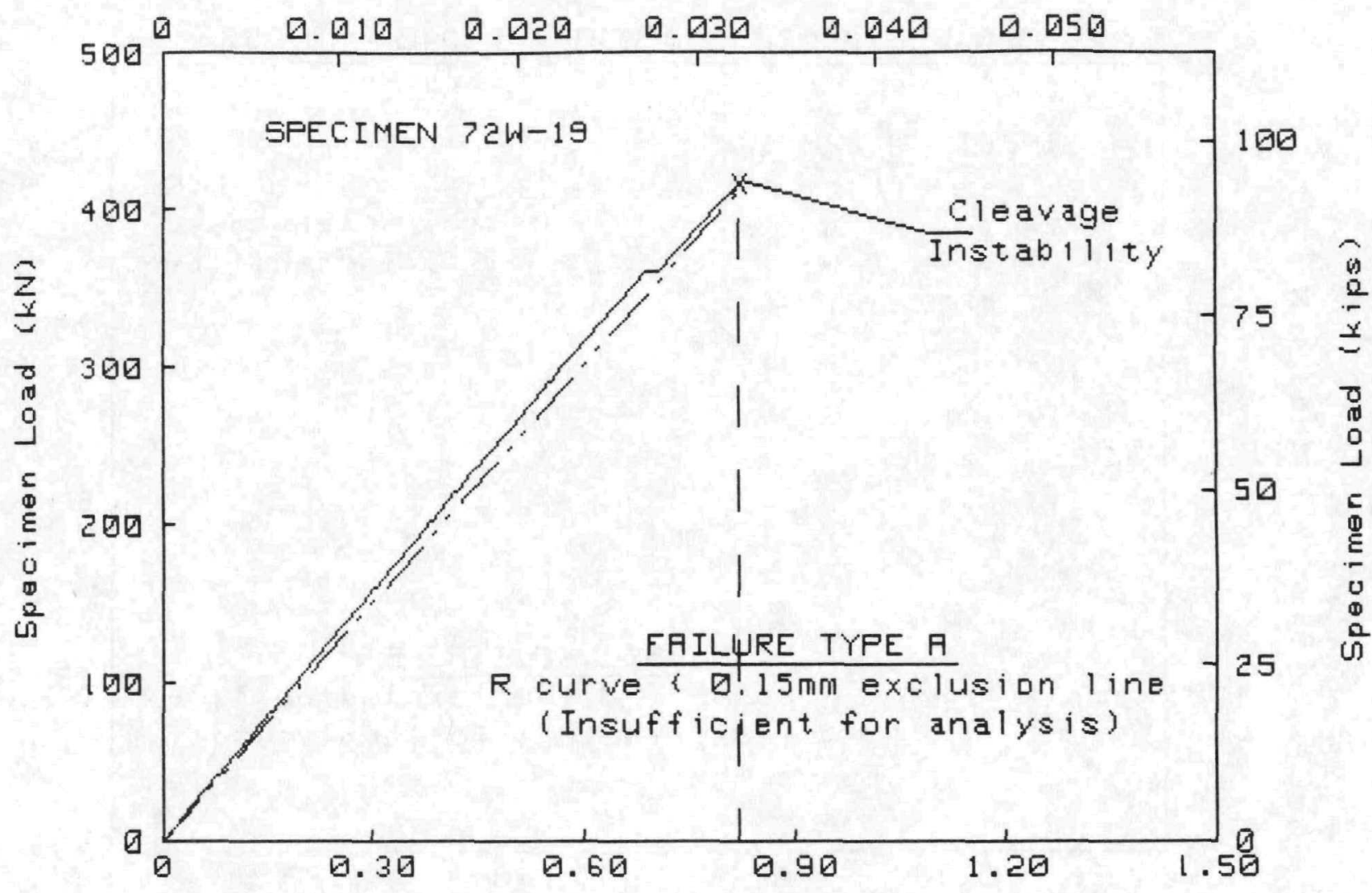

Speoimen Laadine Defleotion (mm)

TEST SFECIMEN DATA

Material Type
Test Temperature
Percent Side Groove
Specimen Thickness B
Initial a/W
Init crack length a
Flow stress
Yield stress
Youngs modulus
Poisson's Ratio (U)
J-INTEGRAL ANALYSIS

$=A 533-B$ Weld (I)

$=50^{\circ} \mathrm{C}$

$=0 \%$

$=101.6 \mathrm{~mm}$

$=.519$

$=105.41 \mathrm{~mm}$

$=654.5 \mathrm{MPa}$

$=607 \mathrm{MPa}$

$=204400 \mathrm{MPa}$ (Estimated Value)

Poisson's Ratio (v)

$=0$

Je

[ Failure Type $A$ ( $J$ at instability = Je)] $=\frac{\text { Merkle-Corten }}{39.9 \mathrm{~kJ} / \mathrm{m}^{2}}$ Deformation-J*

$=90.3 \mathrm{MPa} \sqrt{\mathrm{m}}$ $45.1 \mathrm{~kJ} / \mathrm{m}^{2}$

$K j c=\left\langle E J c /\left(1-v^{2}\right)\right\rangle \wedge .5$

$=87.8 \mathrm{MPa} \sqrt{\mathrm{m}}$ $96 \mathrm{MPa} \sqrt{\mathrm{m}}$

$92.6 \mathrm{MPa} \sqrt{\mathrm{m}}$

ASTM E399-81 ANALYSIS

Modified Kic Equation; $B=\langle B B n\rangle 1 / 2$

Maximum Load $P=416.66 \mathrm{kN}$

$5 \%$ Secant offset $P q=416.66 \mathrm{kN}$

$\mathrm{Kq}$

$=93.2 \mathrm{MPa} \sqrt{\mathrm{m}}$

= VALID Kic value

Validity

$=93.2 \mathrm{MPa} \sqrt{\mathrm{m}}$

$K$ at Maximum Load

DATA CORRECTIONS USED FOR J-INTEGRAL DATA

Merkle-Corten correction

Correction to J for crack extension (Deformation-J)

OFFSET LOAD $=0 \mathrm{kN}$ OFFSET DEFLECTION $=-.011 \mathrm{~mm}$

Fitted elastic load range $=50 \mathrm{kN}$ T0 $200 \mathrm{kN}$

Elastic slope $=5.274 E+02 \mathrm{kN} / \mathrm{mm}$

NUREG/CR-5913 
Specimen Loadline Deflection (in.) ORNL-DWG 92-11665

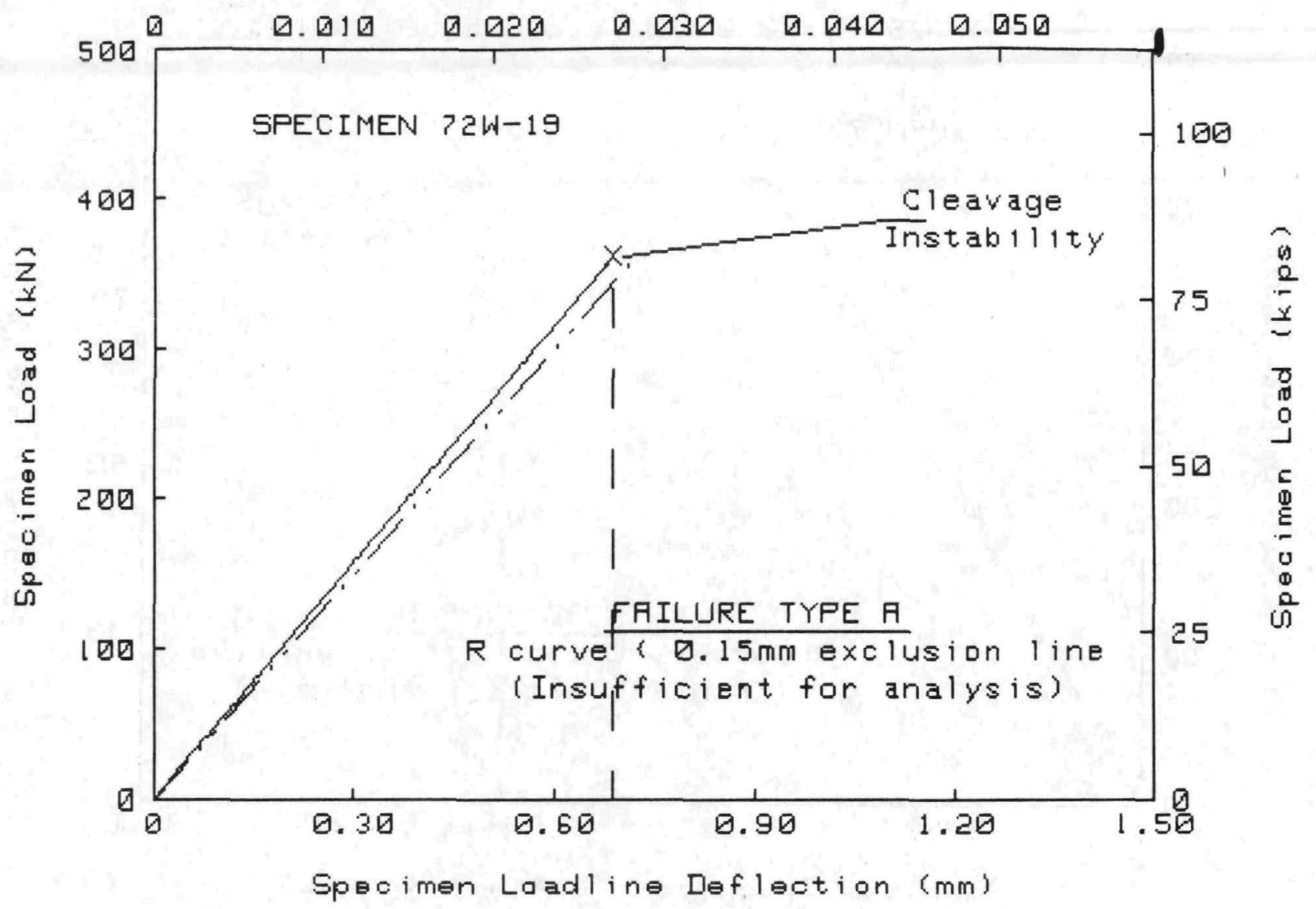

TEST SFECIMEN DATA

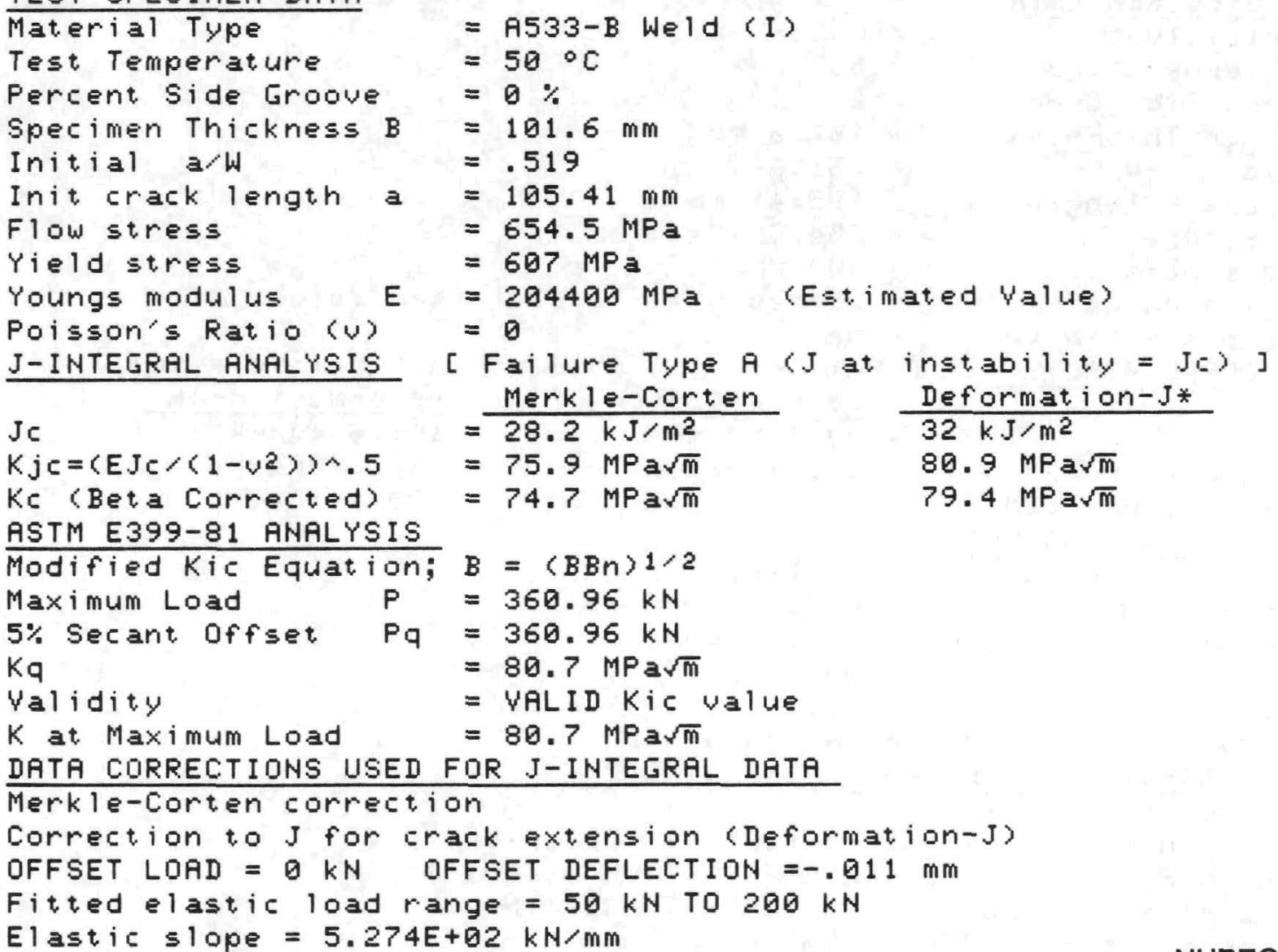


Spec imen Crackmauth Def lect i on ( i n.) ORNL-DWG 92-11666
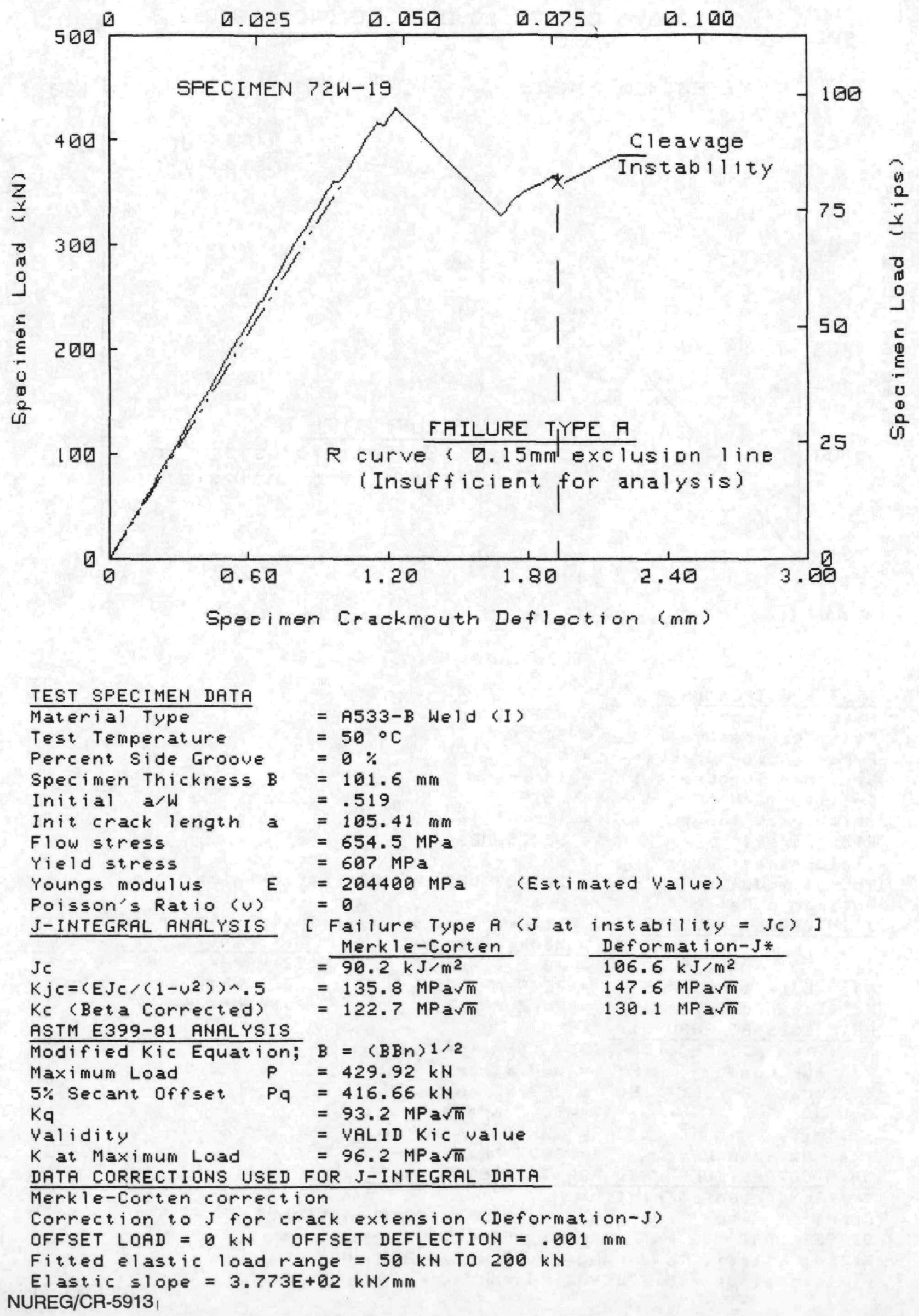
Specimen Crackmauth Def lection (in.) ORNL-DWG 92-11667

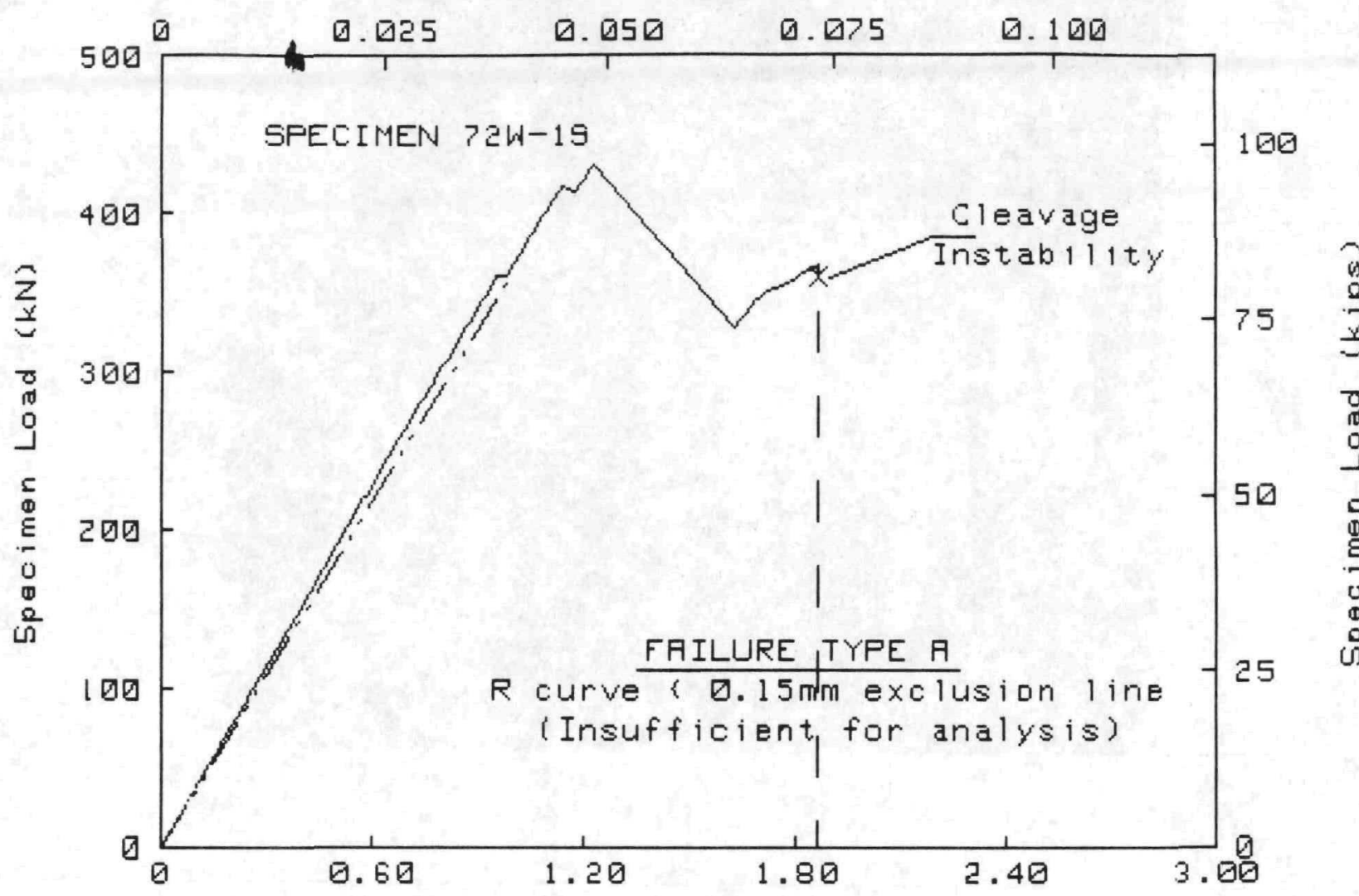

Specimen Crackmouth Defleotion ( $m m$ )

TEST SPECIMEN DATA

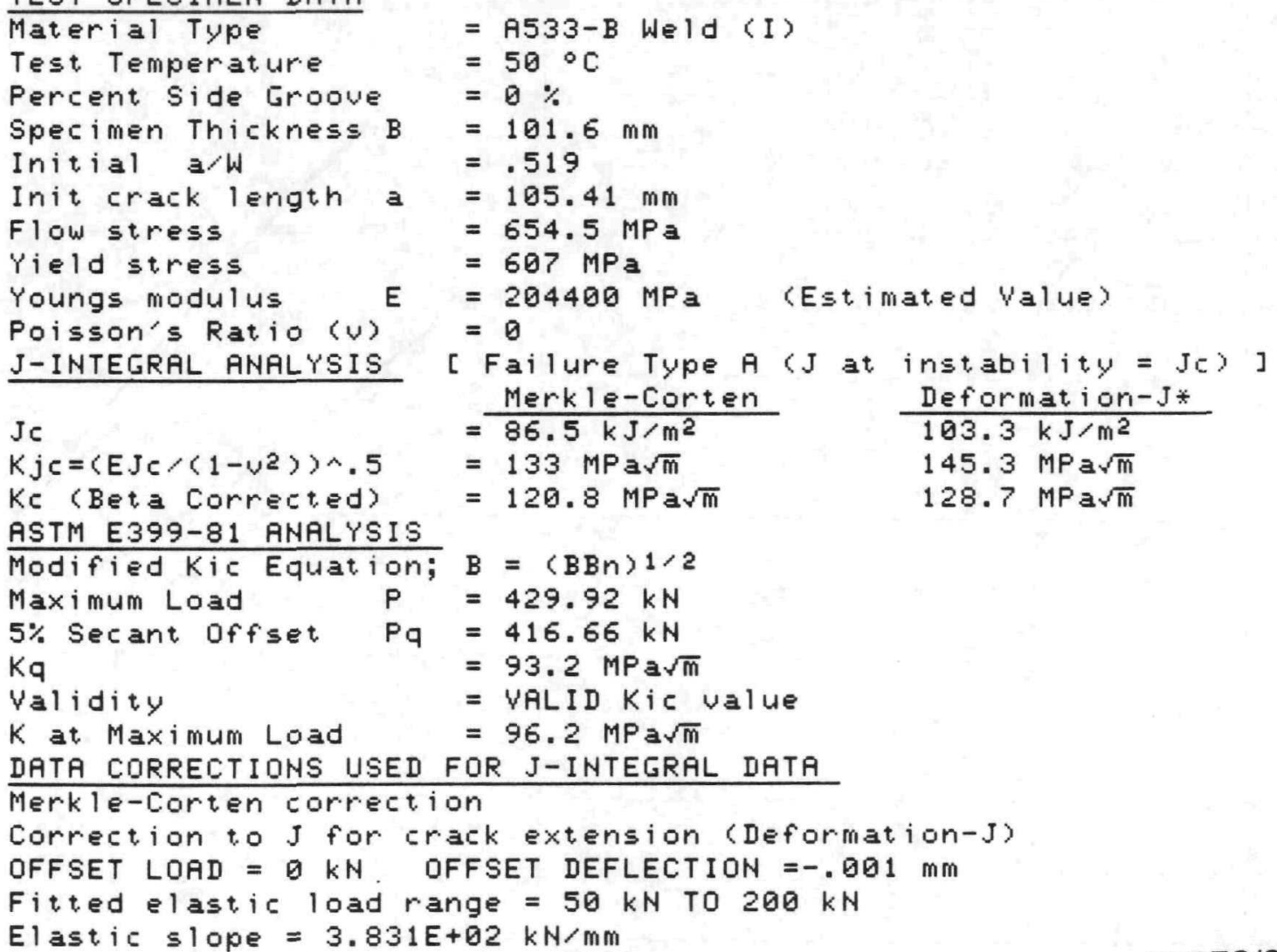




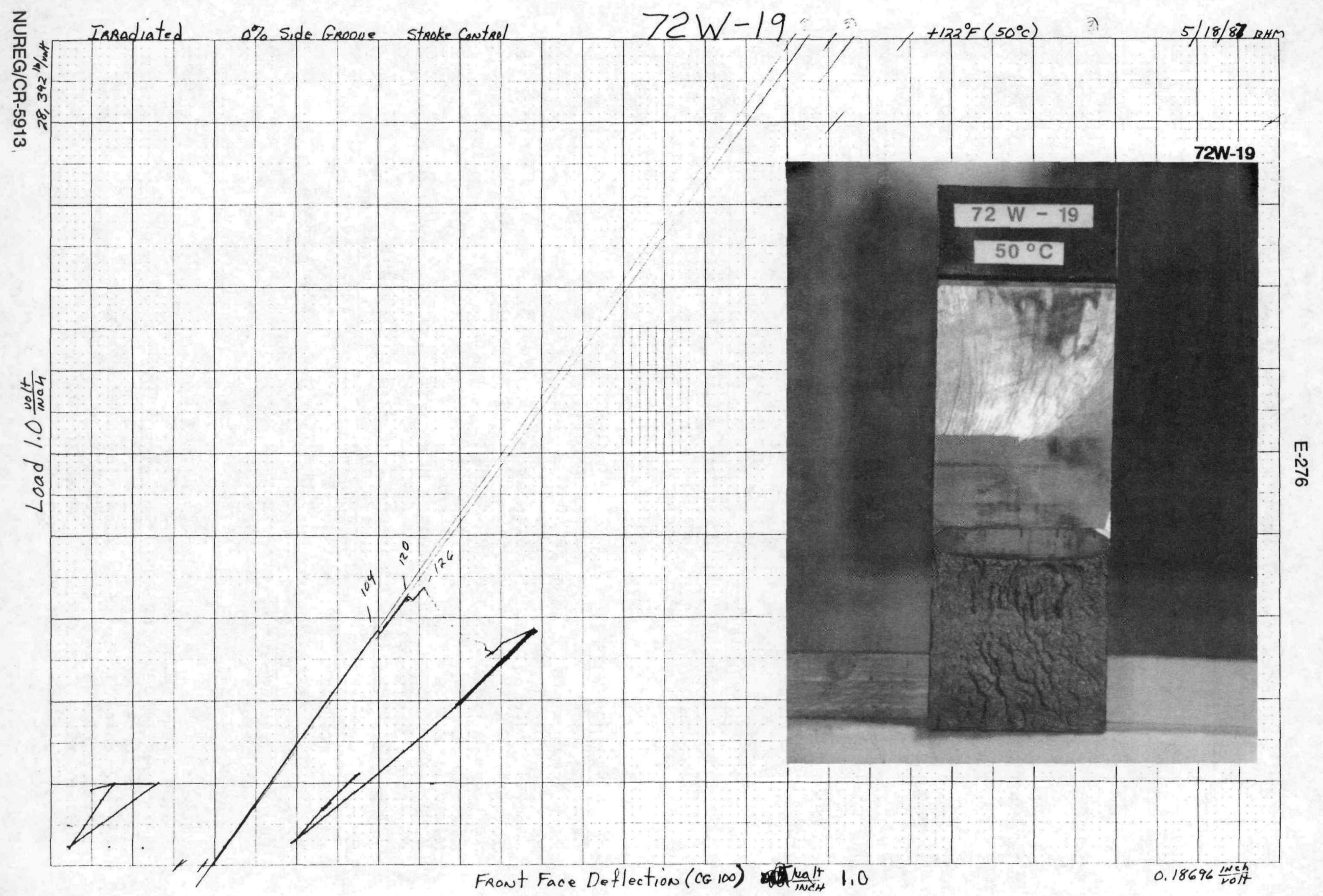




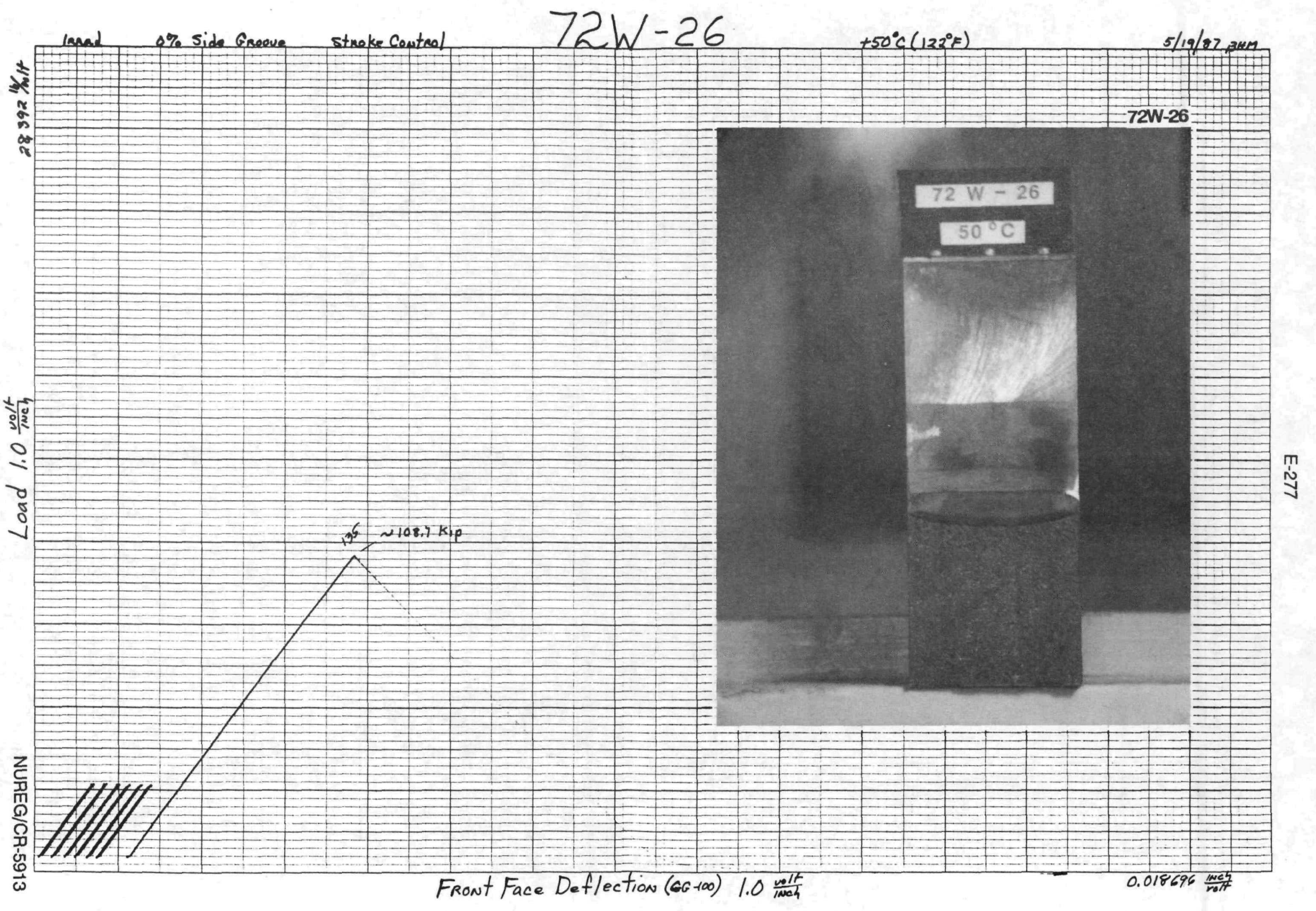


Specimen Laadline Def lection ( in. ) ORNL-DWG 92-11668

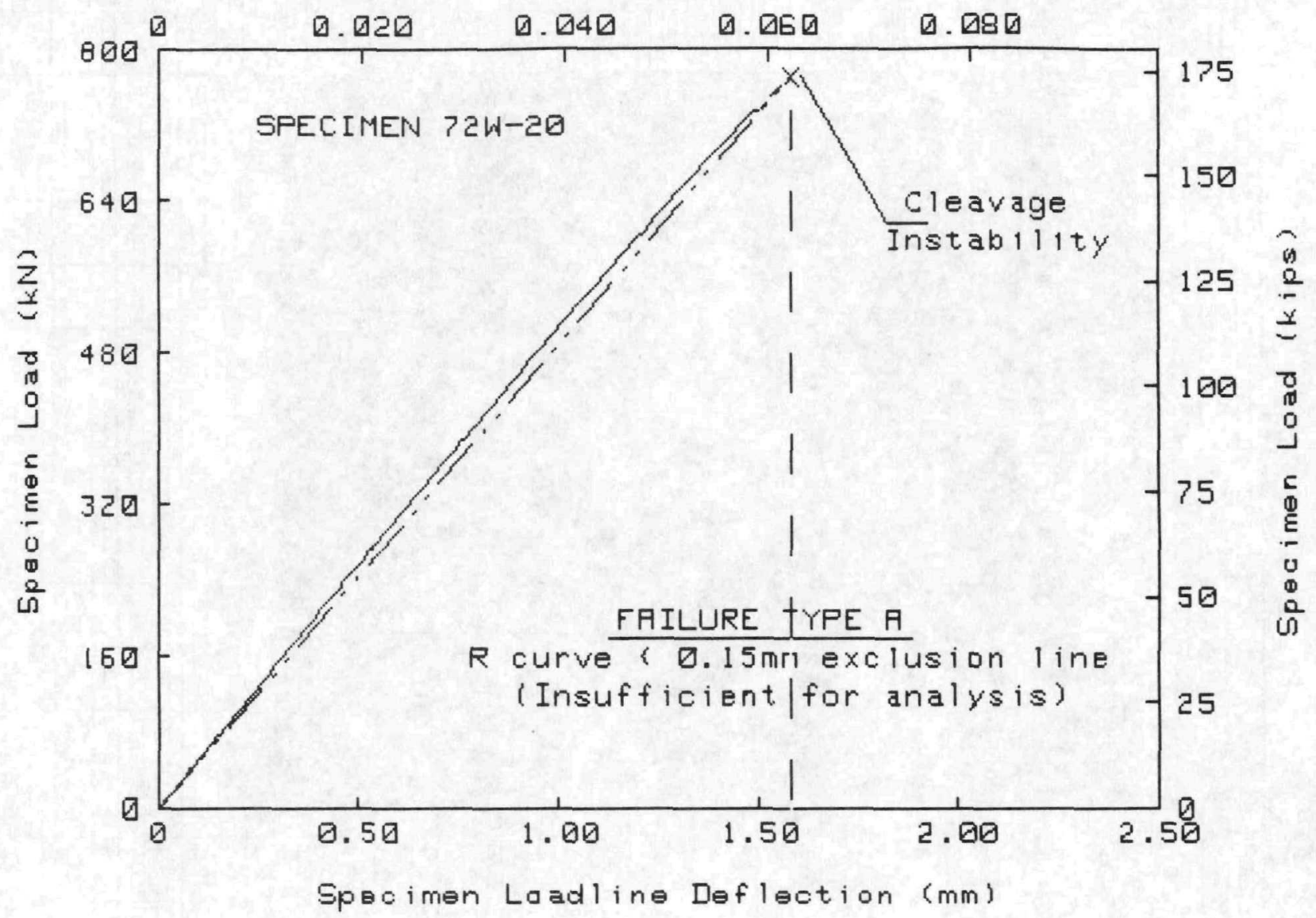

TEST SPECIMEN DATA

Material Type
Test Temperature
Percent Side Groove
Specimen Thickness B
Initial a/W
Init crack length a
Flow stress
Yield stress
Youngs modulus
PoisSon's Ratio (U)
J-INTEGRAL ANALYSIS

$=A 533-B$ Weld (I)

$=75 \circ \mathrm{C}$

$=0 \%$

$=101.6 \mathrm{~mm}$

$=.526$

$=106.78 \mathrm{~mm}$

$=643.2 \mathrm{MPa}$

$=597.1 \mathrm{MPa}$

$=202900 \mathrm{MPa}$

$=0$

$\mathrm{Jc}$

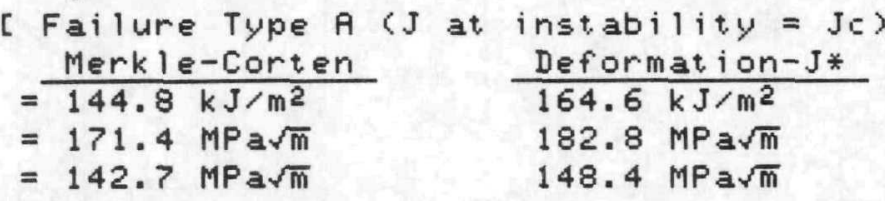

$K, j c=\left\langle E J c /\left(1-w^{2}\right)\right\rangle \wedge .5$

Ke (Beta Corrected)

$=142.7 \mathrm{MPa} \sqrt{\mathrm{m}}$

(Est imated Value)

ASTM E399-81 ANALYSIS

Modified Kic Equation; $B=\langle B B n\rangle 1 / 2$

Maximum Load $P=771.05 \mathrm{kN}$

$5 \%$ Secant offset $\mathrm{Pq}=771.05 \mathrm{kN}$

$\mathrm{Kq}$

$=176.3 \mathrm{MPa} \sqrt{\mathrm{m}}$

Validity

$K$ at Maximum Load

= INVALID- $-1,2$

DATA CORRECTIONS USED FOR J-INTEGRAL DATA

Merkle-Corten correction

Correction to J for erack extension (Deformation-J)

OFFSET LORD $=0 \mathrm{kN}$ OFFSET DEFLECTION $=0 \mathrm{~mm}$

Fitted elastic load range $=50 \mathrm{kN}$ TO $200 \mathrm{kN}$

Elastic slope $=5.127 E+Q 2 \mathrm{kN} / \mathrm{mm}$

NUREG/CR-5913. 
Specimen Loadline Deflection (in. ) ORNL-DWG 92-11669

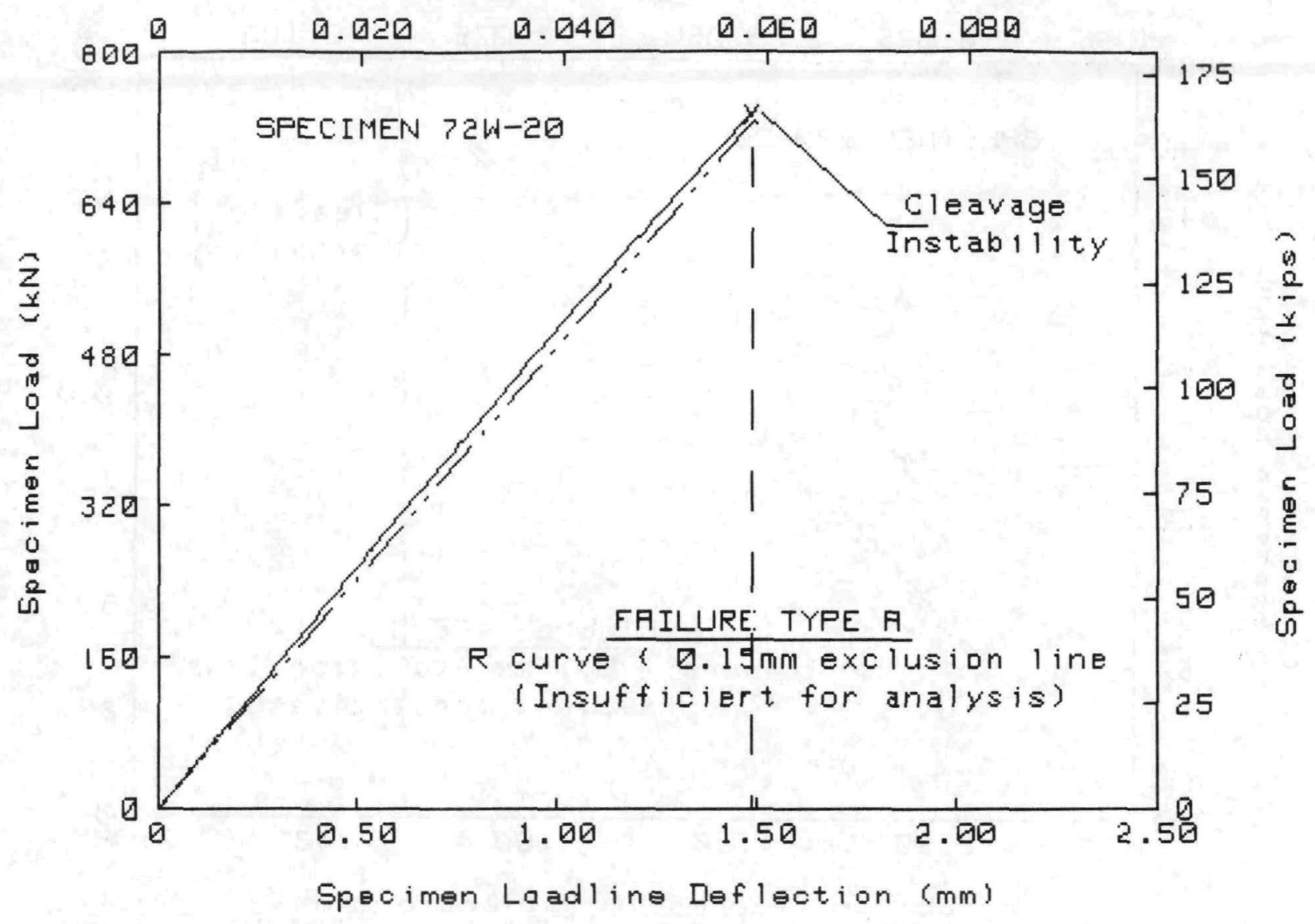

TEST SPECIMEN DATA

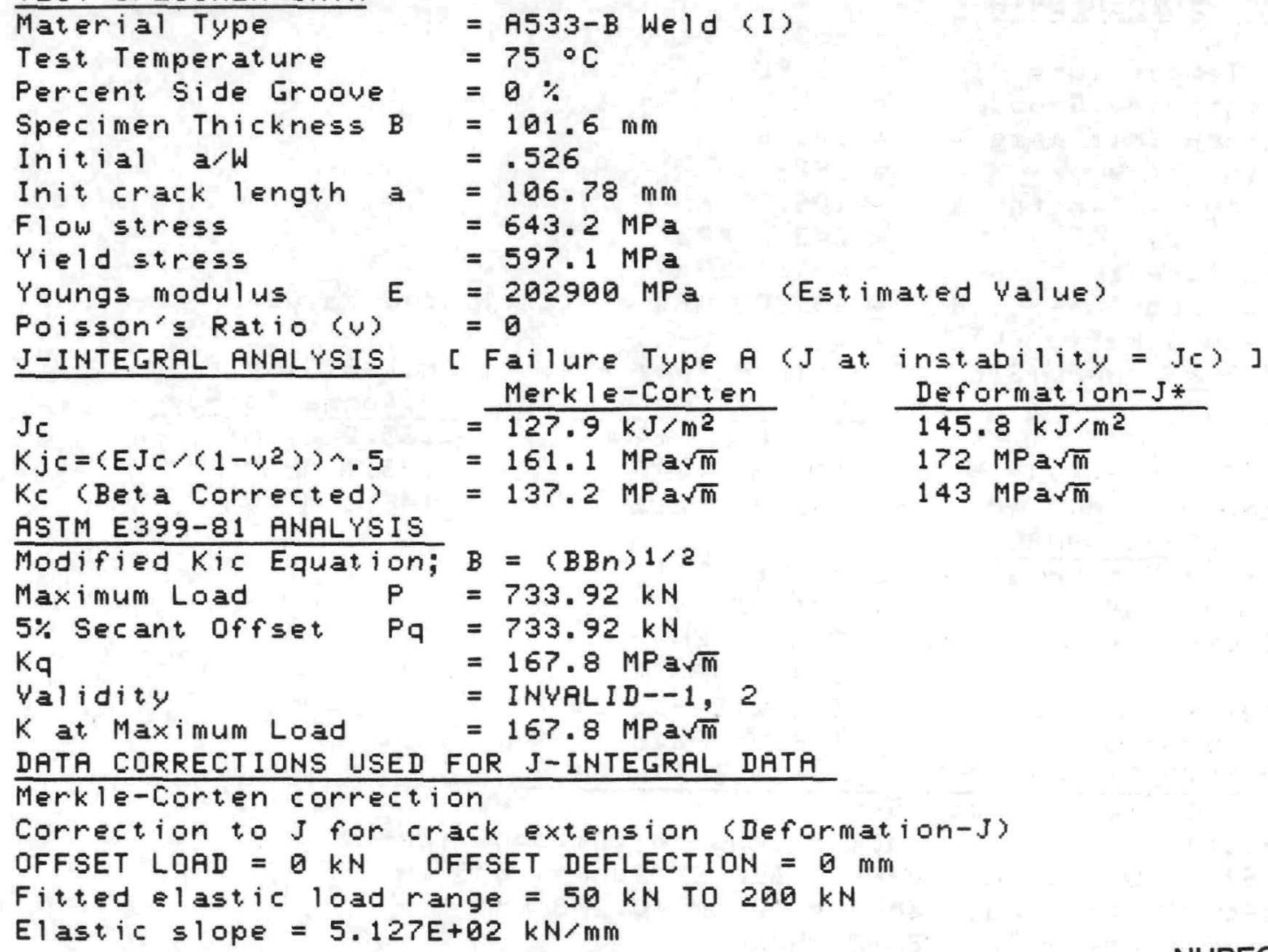


Spec imen Crackmouth Def lect ion ( i n.) ORNL-DWG 92-11670

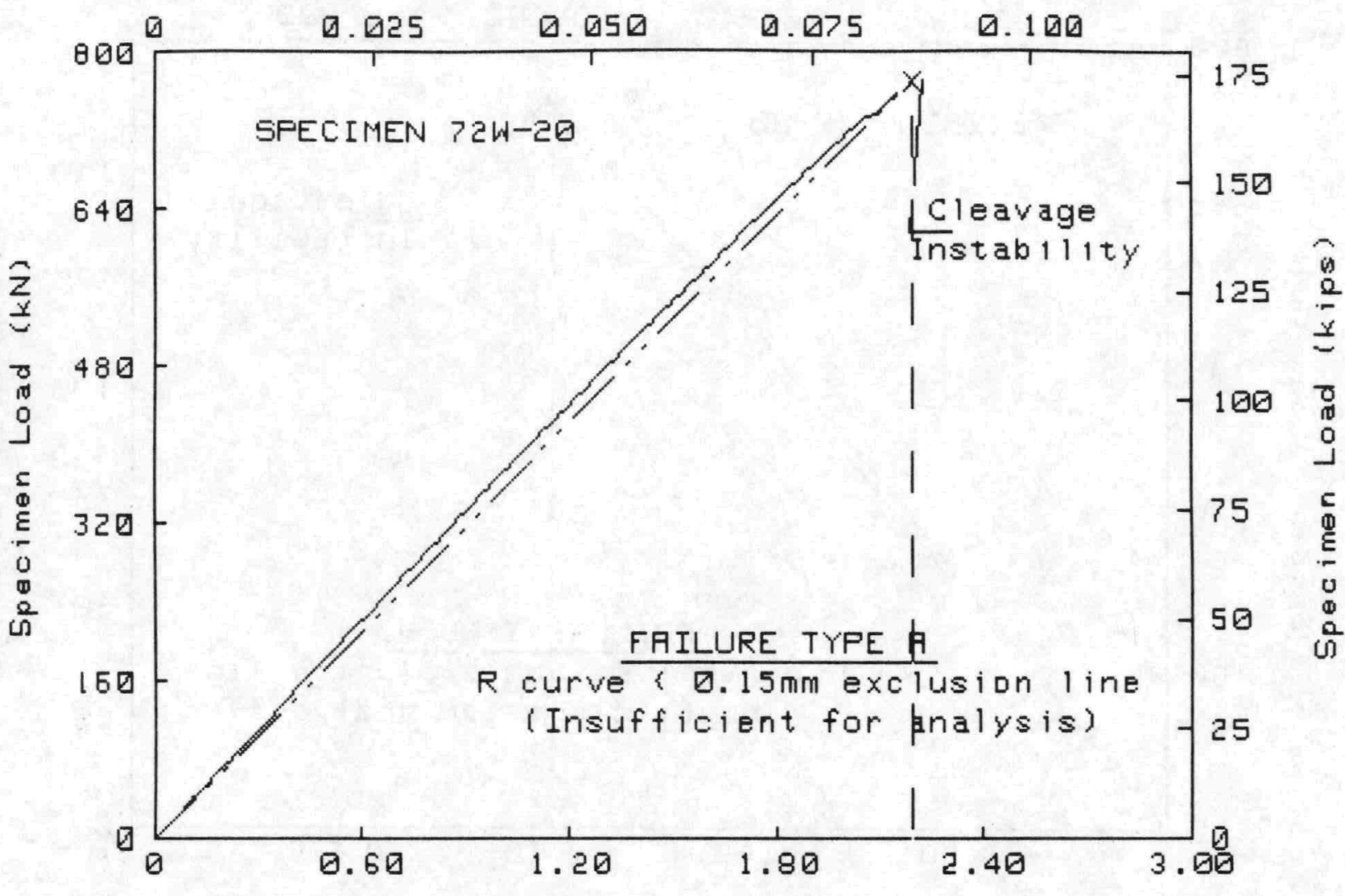

Specimen Crackmouth Deflection ( $m$ )

TEST SPECIMEN DATA

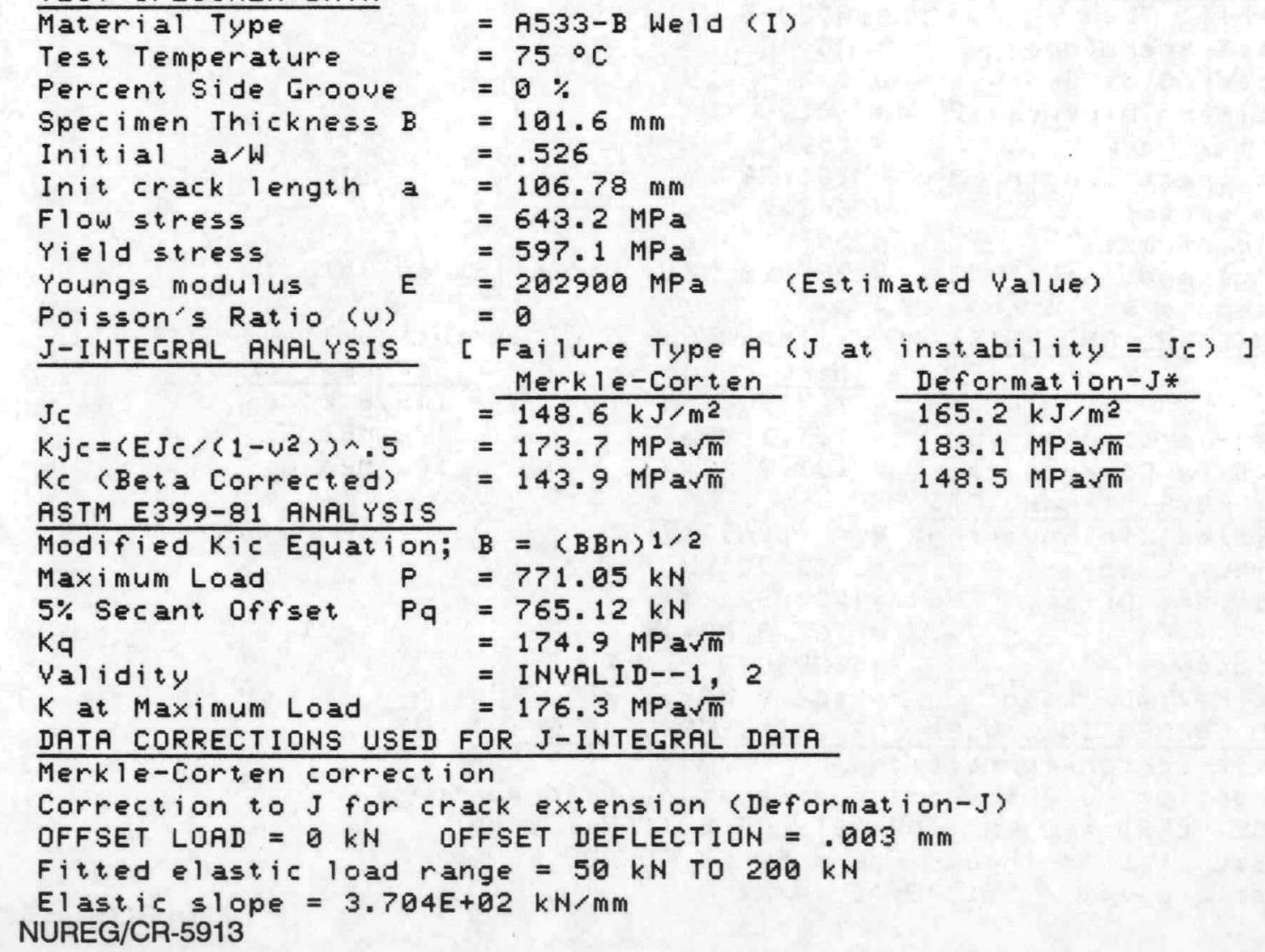


Gpecimen Crackmouth Def lection (in.) ORNL-DWG 92-11671

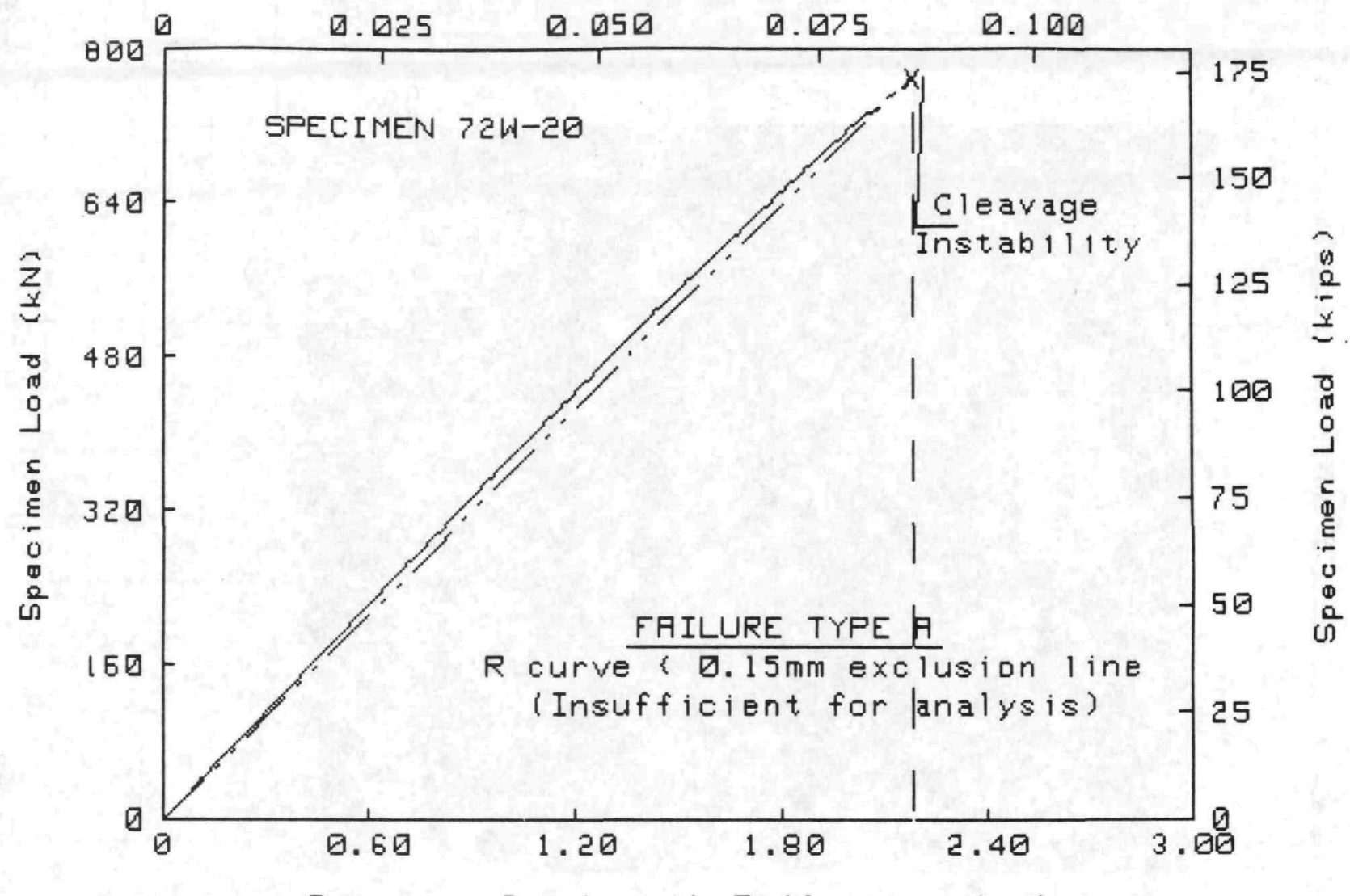

Specimen Crackmouth Defleotion ( $\mathrm{mm}$ )

\section{TEST SPECIMEN DATA}

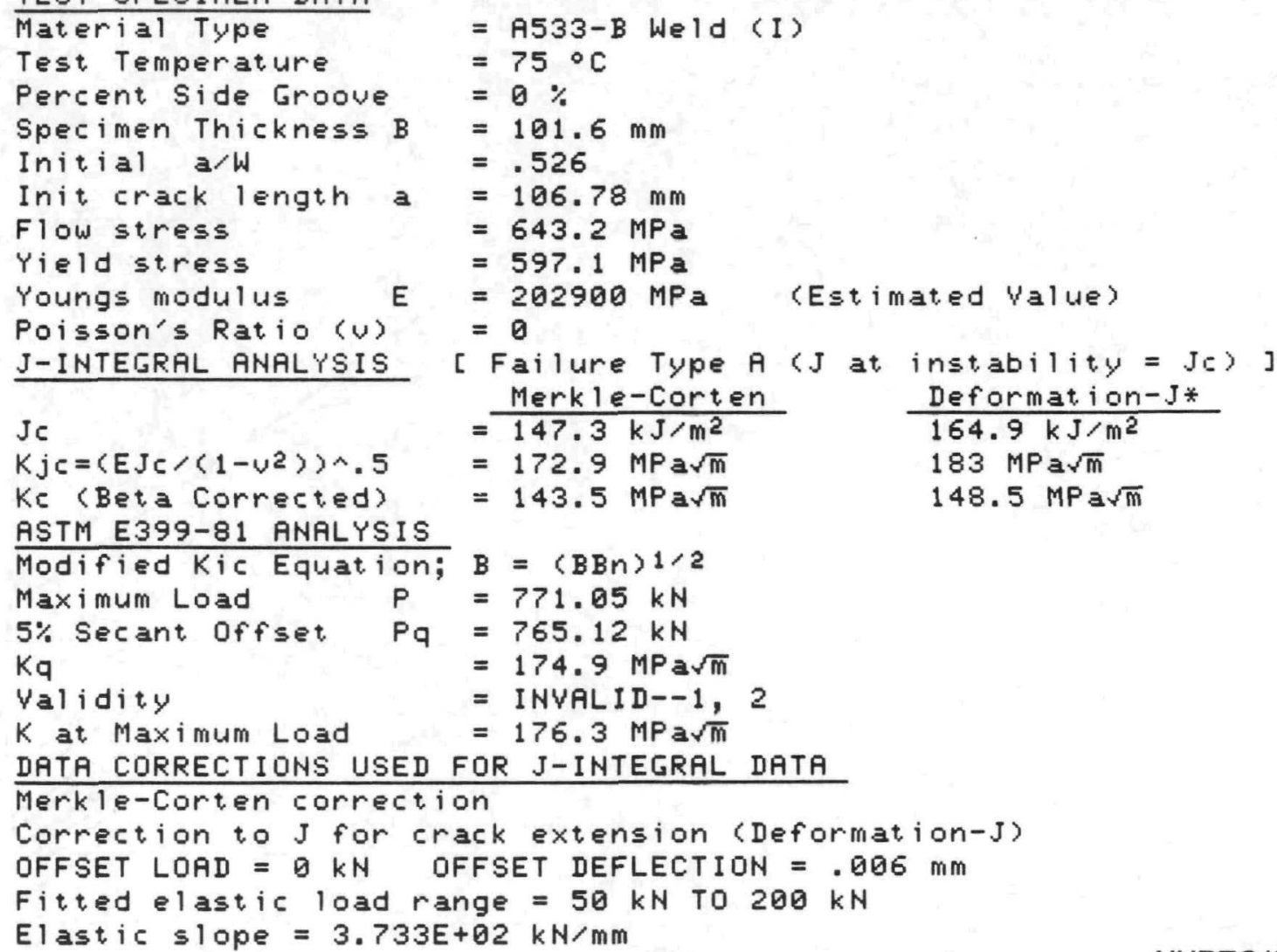




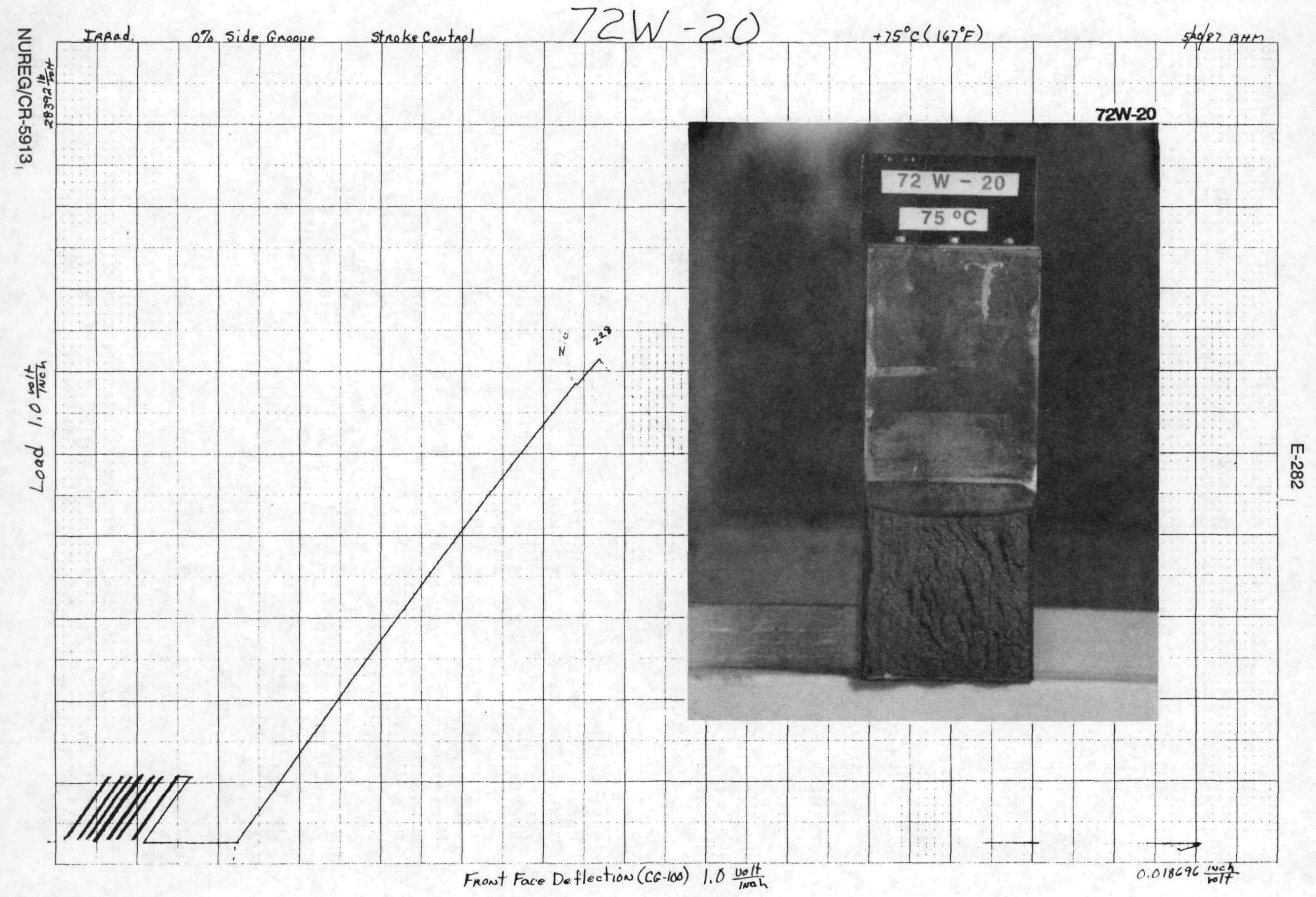


Inadiated $45-C T$

o7. Side Qsean

stroke contal $72 W-25$

$75^{\circ} \mathrm{C}\left(147^{\circ} \mathrm{F}\right)$

$5 / 21870$ men

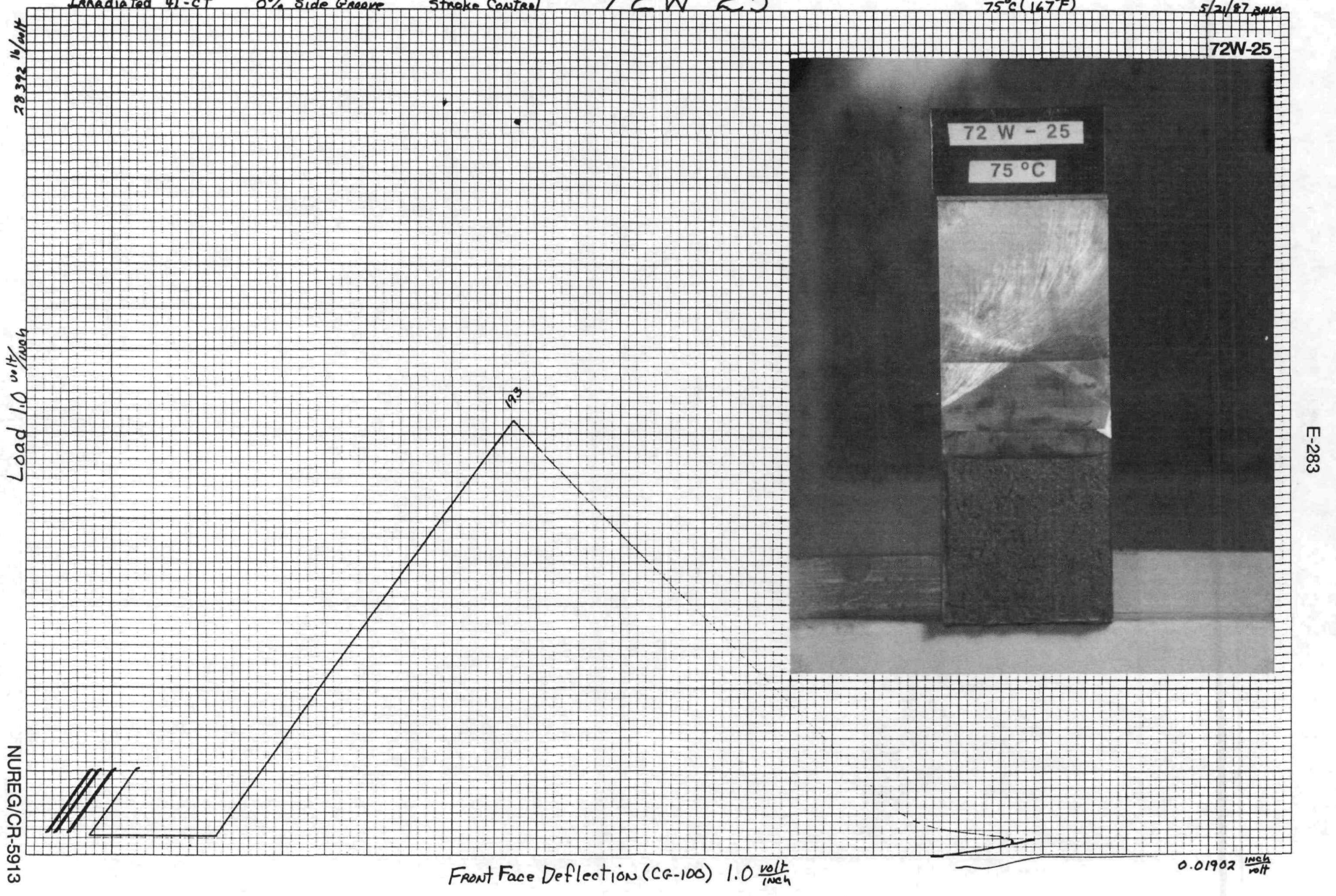




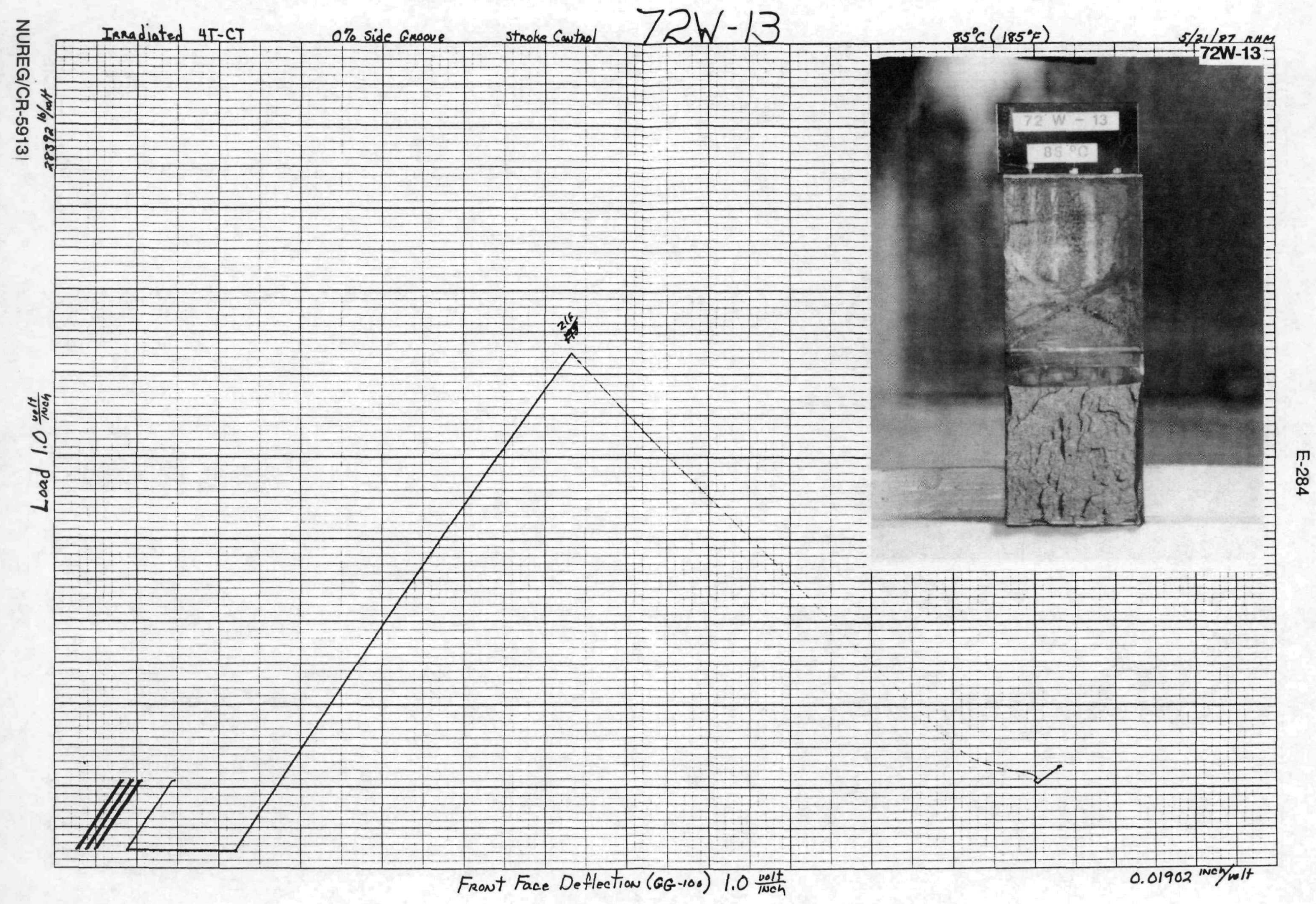


Specimen Loadline Deflection (in., ORNL-DWG 92-11672
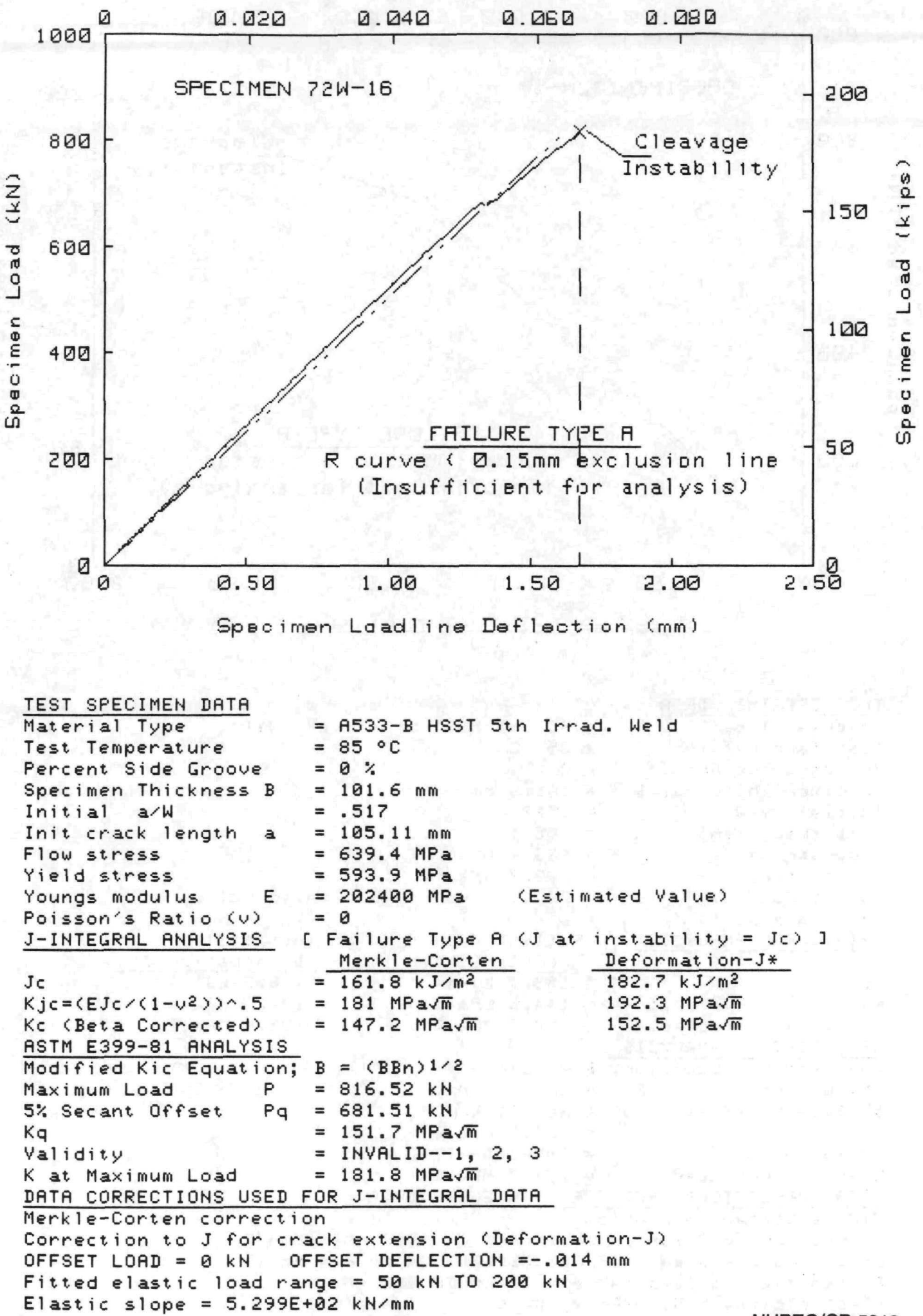
Spec imen Loadline Deflection (in.) ORNL-DWG 92-11673

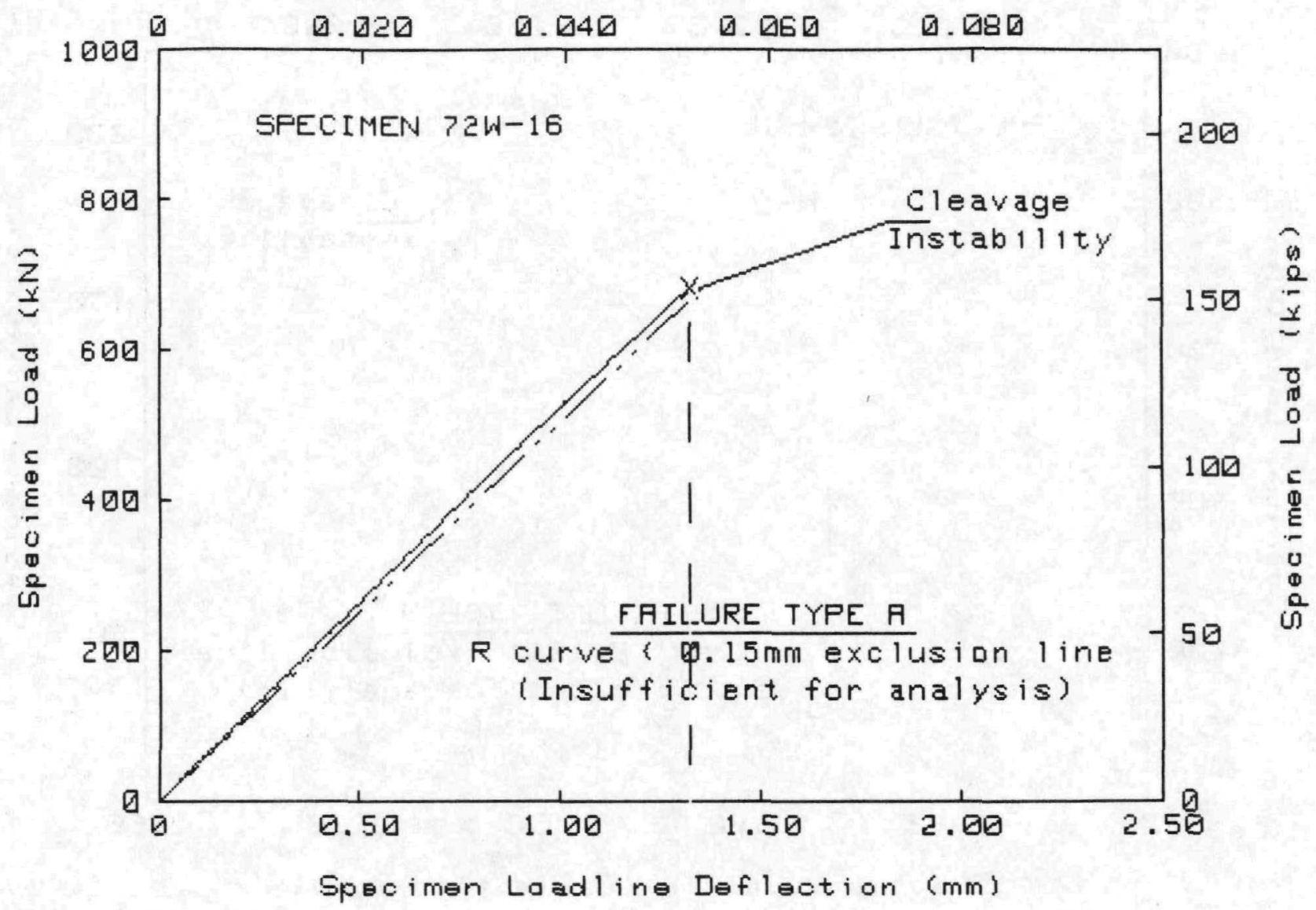

TEST SFECIMEN DATA

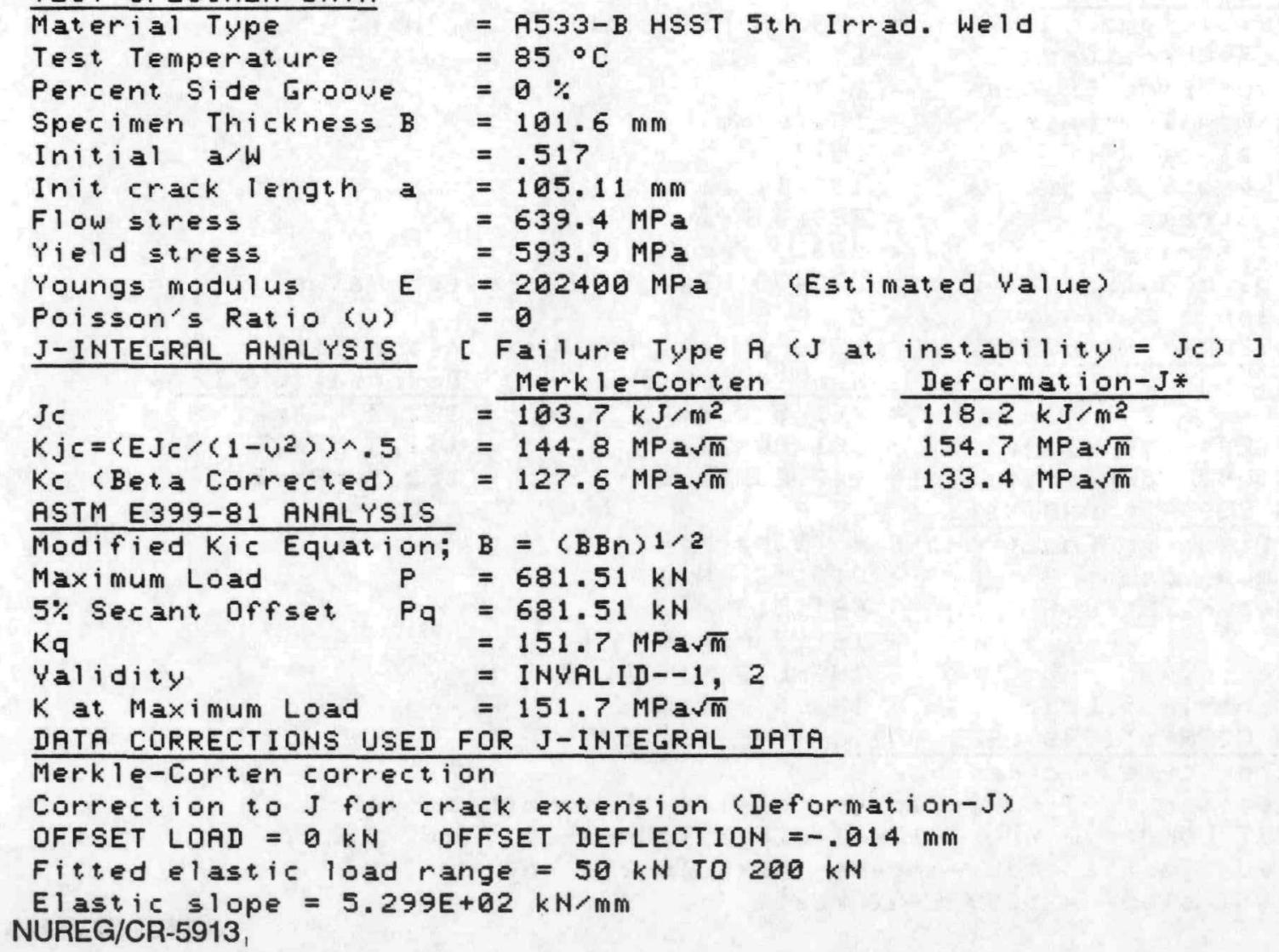


Specimen Crackmouth Def lection (in.) ORNL-DWG 92-11674

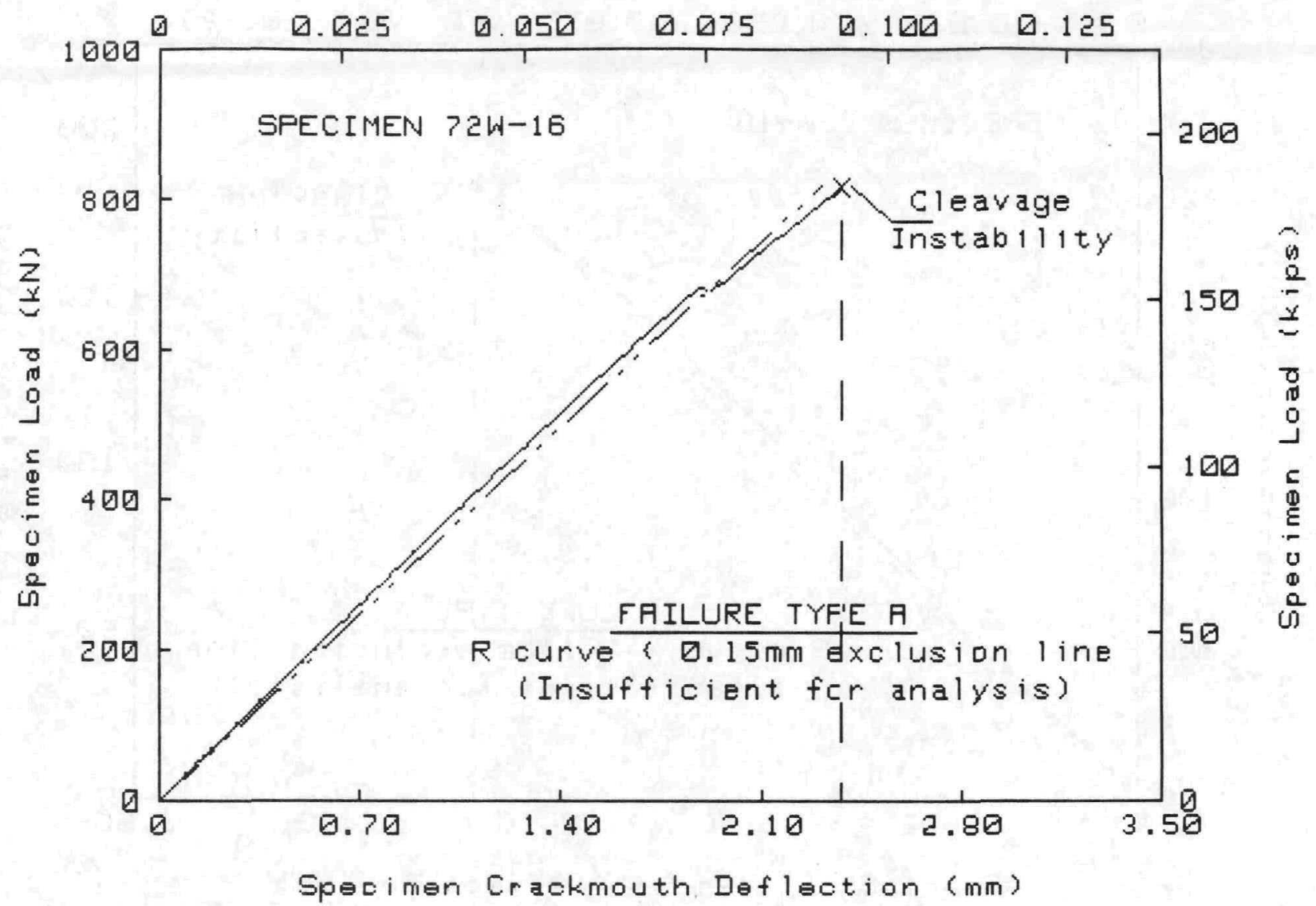

\section{TEST SPECIMEN DATA}

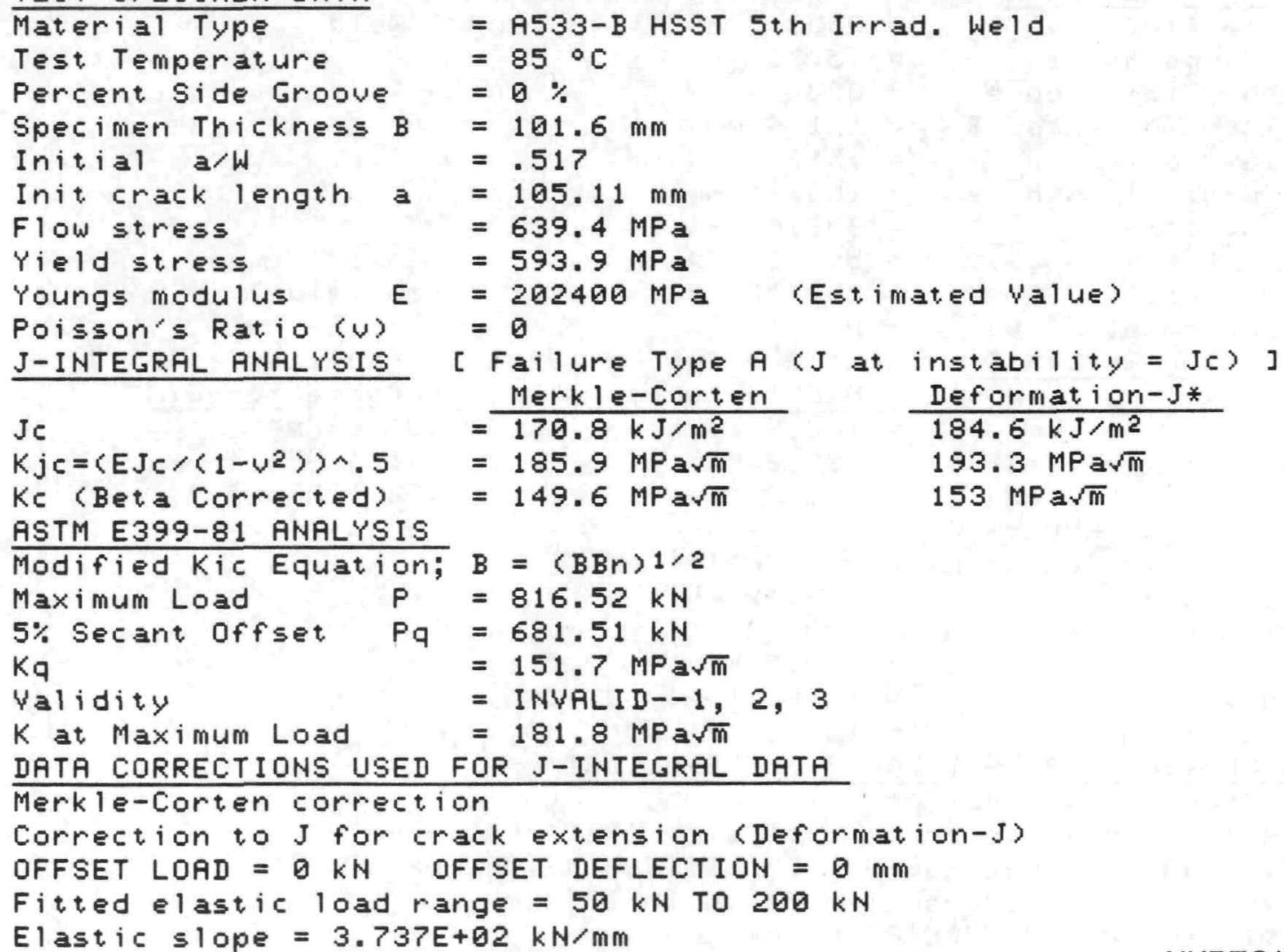


Specimen Crackmouth Def lection (in.) ORNL-DWG 92-11675

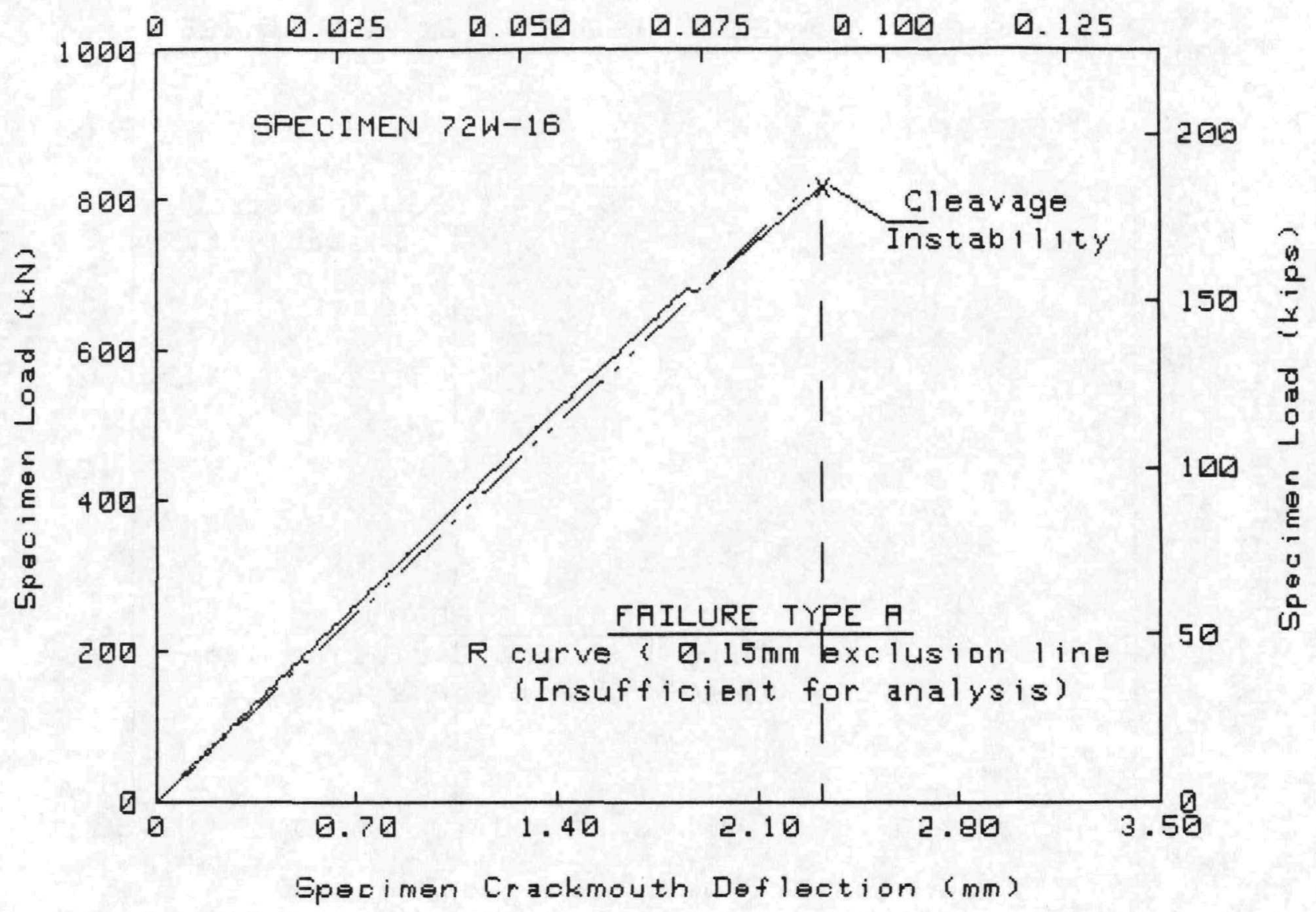

TEST SPECIMEN DATA

Material Type
Test Temperature
Percent Side Groove
Specimen Thickness B
Initial a/W
Init crack length a
Flow stress
Yield stress
Youngs modulus E
Poisson's Ratio (U)
J-INTEGRAL ANALYSIS

$\mathrm{Jc}$

$K j c=\left\langle E J c /\left(1-v^{2}\right\rangle\right) \wedge .5$

Ke (Beta Corrected)

ASTM E399-81 ANALYSIS
= A533-B HSST 5th Irrad. Weld

$=85^{\circ} \mathrm{C}$

$=0 \%$

$=101.6 \mathrm{~mm}$

$=.517$

$=105.11 \mathrm{~mm}$

$=639.4 \mathrm{MPa}$

$=593.9 \mathrm{MPa}$

$=202400 \mathrm{MPa}$ (Estimated Value)

$=0$

[ Failure Type A ( $J$ at instability = JC) ]

$$
\begin{aligned}
& =\frac{\text { Merkle-Corten }}{165.8 \mathrm{~kJ} / \mathrm{m}^{2}} \\
& =183.2 \mathrm{MPa} \sqrt{\mathrm{m}} \\
& =148.3 \mathrm{MPa} \sqrt{\mathrm{m}}
\end{aligned}
$$

Deformat ion $-\mathrm{J} *$

$191.4 \mathrm{MPa} \sqrt{\mathrm{m}}$

152. $1 \mathrm{MPa} \sqrt{\mathrm{m}}$ 


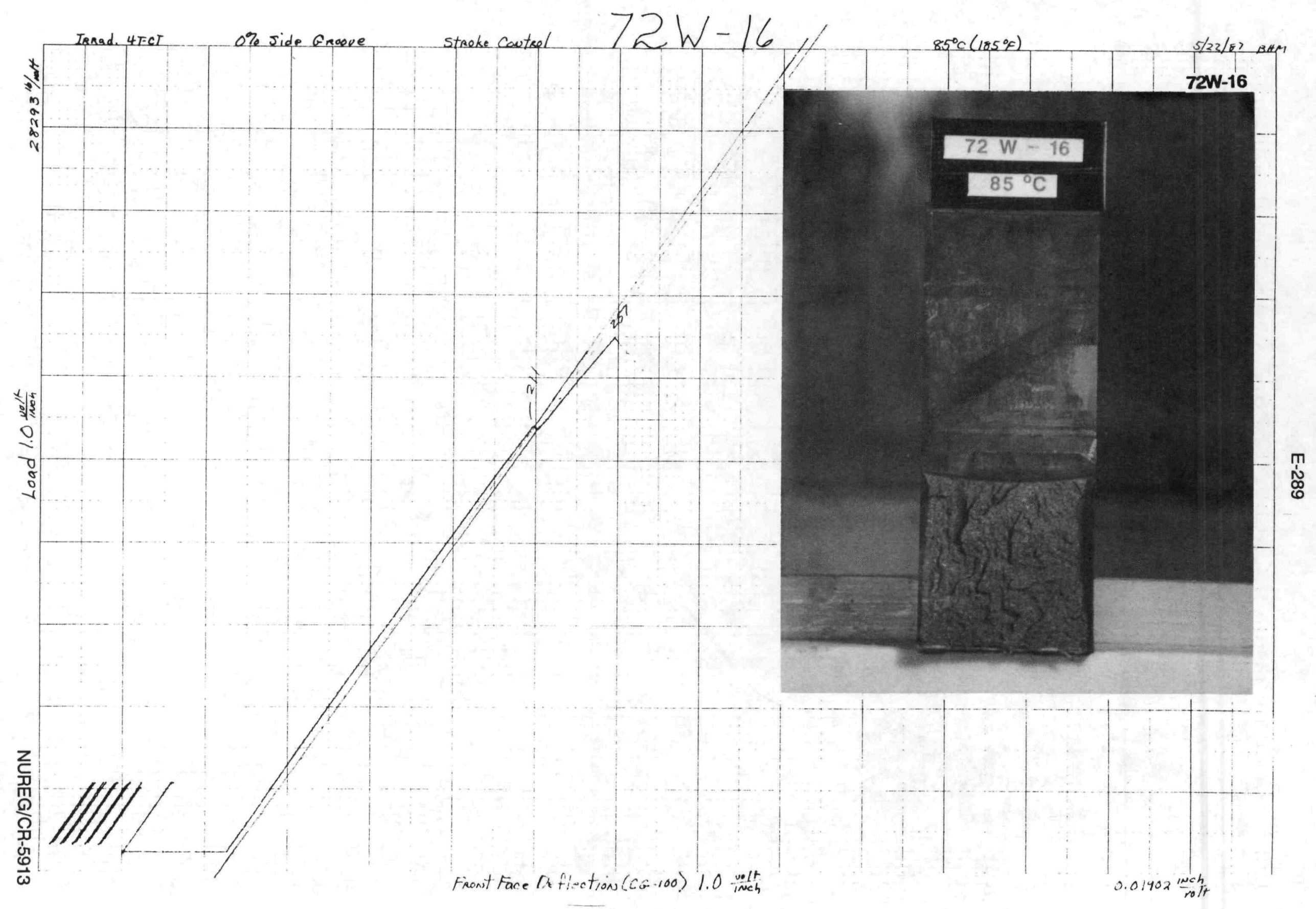




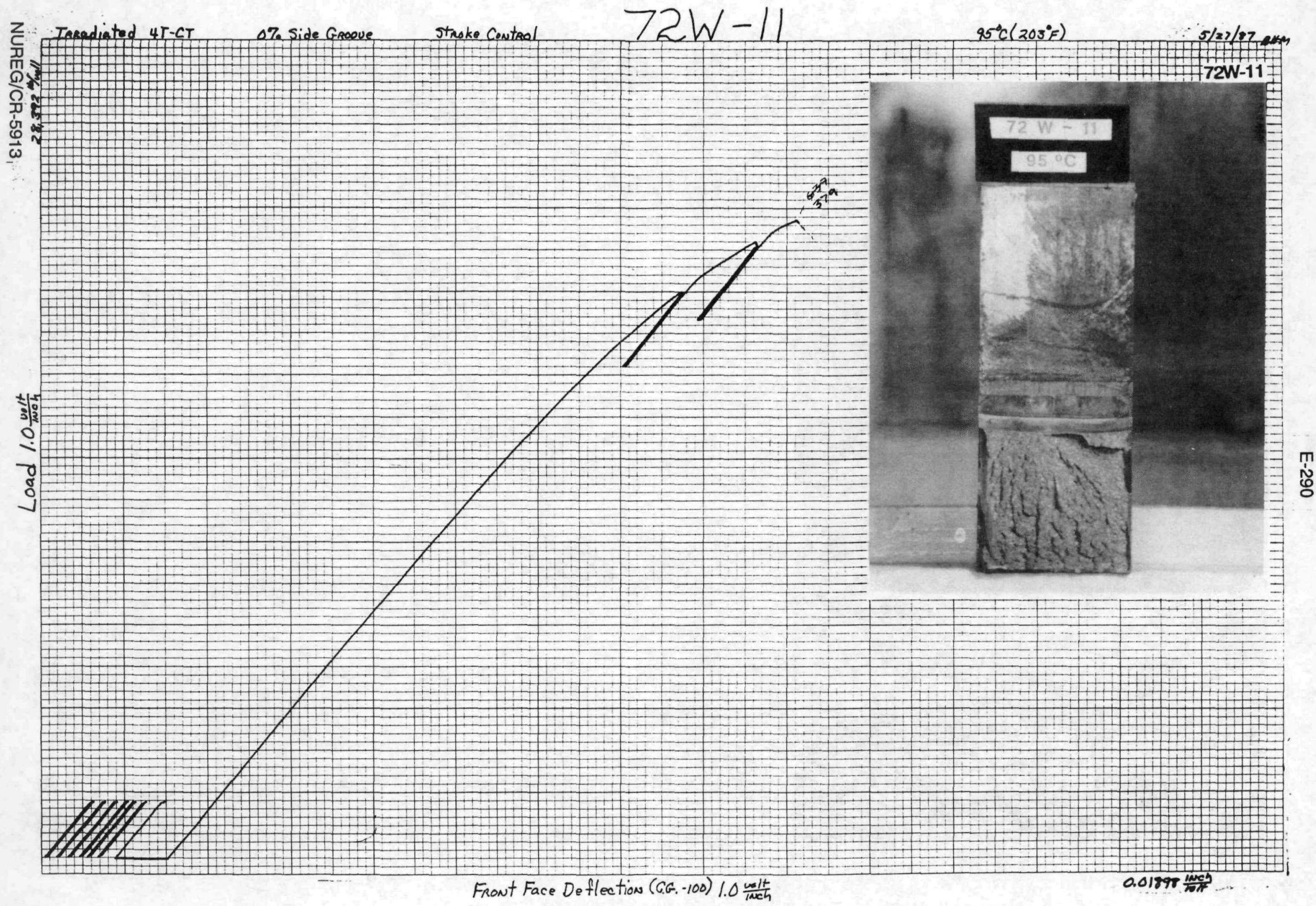


Specimen Loadline Deflection (in., ORNL-DWG 92-11676

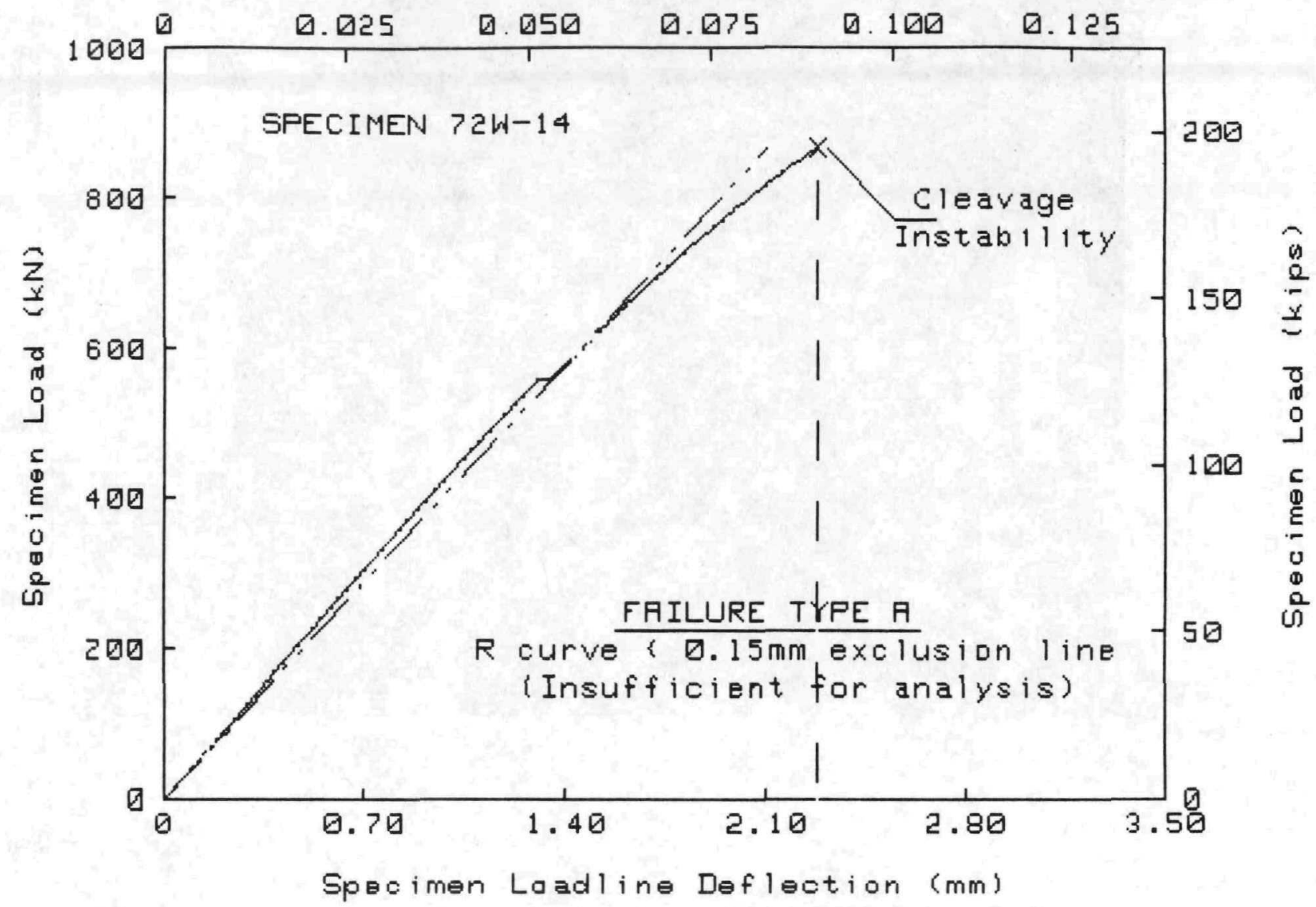

TEST SPECIMEN DATA

Material Type
Test Temperature
Percent Side Groove
Specimen Thickness B
Initial a/W
Init crack length a
Flow stress
Yield stress
Youngs modulus
Poisson's Ratio (U)
J-INTEGRAL ANALYSIS

$=$ A533-B HSST 5 th I rrad. Weld

$=95{ }^{\circ} \mathrm{C}$

$=0 \%$

$=101.6 \mathrm{~mm}$

$=.556$

$=112.95 \mathrm{~mm}$

$=636 \mathrm{MPa}$

$=591.3 \mathrm{MPa}$

$=201800 \mathrm{MPa}$ (Estimated Value)

$=0$

[ Failure Type $A$ ( $J$ at instability = JC) ]

Je

$=\frac{\text { Merkle-Corten }}{259 \mathrm{~kJ} / \mathrm{m}^{2}}$

$\frac{\text { Deformation }-\mathrm{J} *}{286.7 \mathrm{~kJ} / \mathrm{m}^{2}}$

$=228.6 \mathrm{MPa} \cdot \sqrt{\mathrm{m}}$

$=167.3 \mathrm{MPa} \sqrt{\mathrm{m}}$

$240.5 \mathrm{MPa} \sqrt{\mathrm{m}}$

$171.7 \mathrm{MPa} \sqrt{\mathrm{m}}$

Ke (Beta Corrected)

ASTM E399-81 ANALYSIS

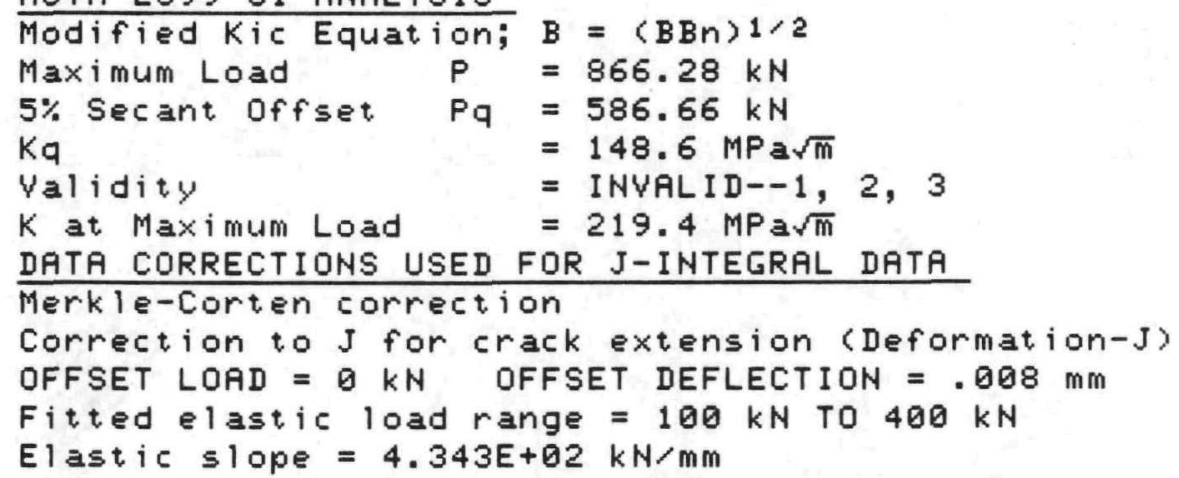

Elastic slope $=4.343 E+02 \mathrm{kN} / \mathrm{mm}$ 


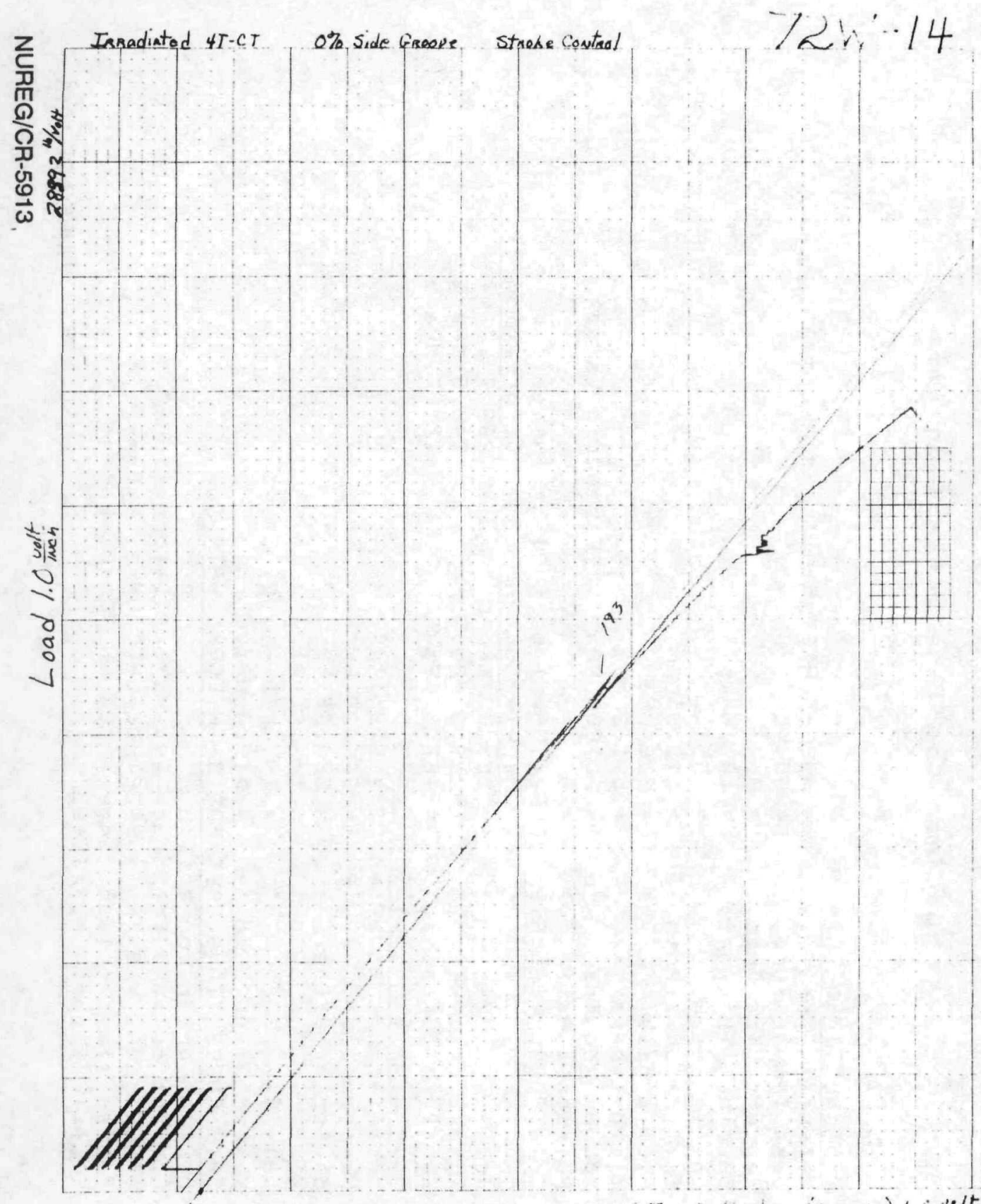


Spec imen Loadl ine Deflect i an ( in. I ORNL-DWG 92-11677

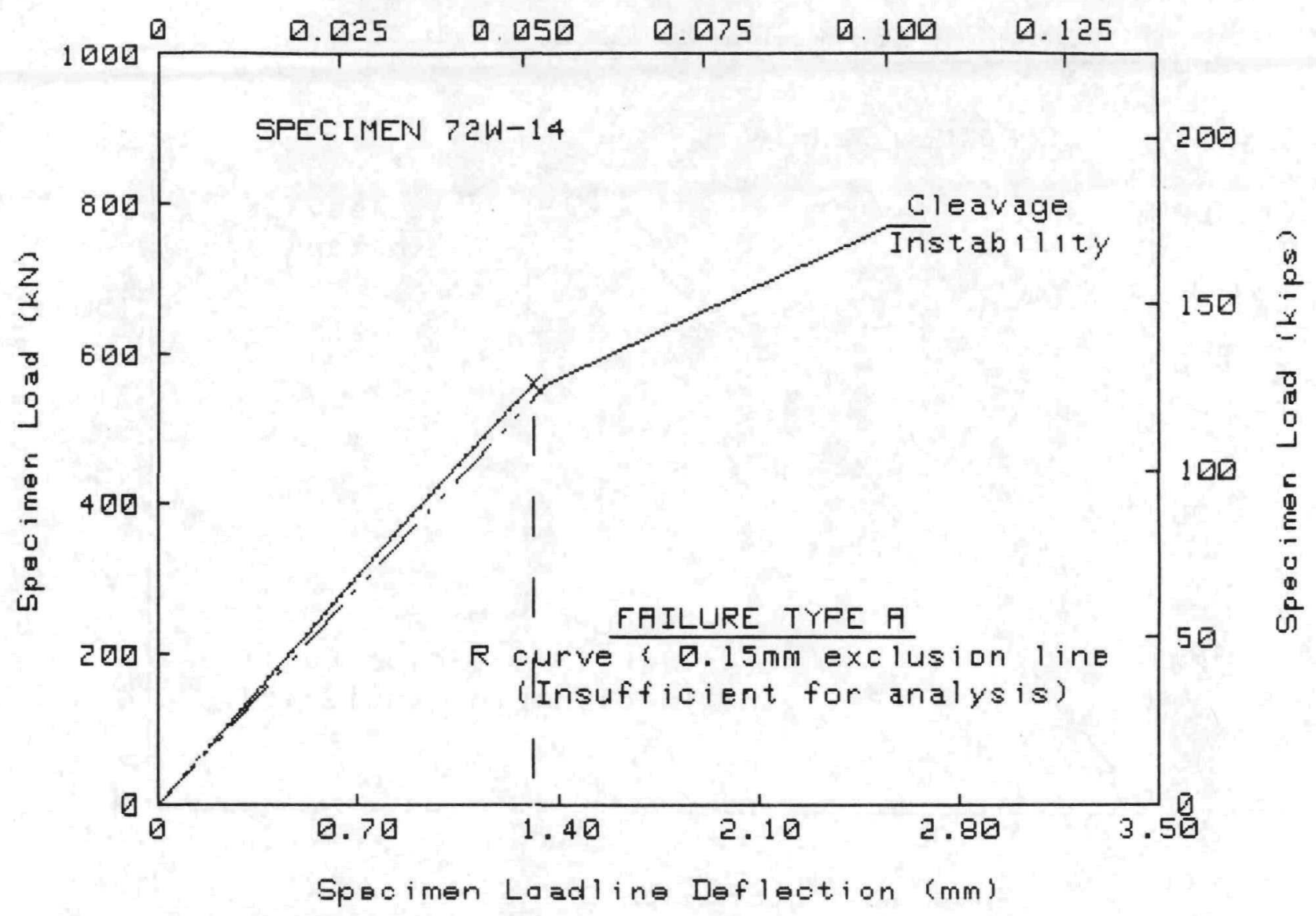

TEST SPECIMEN DATA

Material Type
Test Temperature
Percent Side Groove
Specimen Thickness B
Initial a/W
Init crack length a
Flow stress
Yield stress
Youngs modulus
Poisson's Ratio (U)
J-INTEGRAL ANALYSIS

$=$ A533-B HSST 5th Irrad. Weld

$=95 \circ \mathrm{C}$

$=0 \%$

$=101.6 \mathrm{~mm}$

$=.556$

$=112.95 \mathrm{~mm}$

$=636 \mathrm{MPa}$

$=591.3 \mathrm{MPa}$

$=201800 \mathrm{MPa}$ (Estimated Value)

$=0$

[ Failure Type $A$ ( $J$ at instability = Jc) ]

$\mathrm{Jc}$

$=\frac{\text { Merkle-Corten }}{90.3 \mathrm{~kJ} / \mathrm{m}^{2}}$

Deformation-J*

$K, j c=\left\langle E J c /\left(1-w^{2}\right)\right\rangle .5=135 \mathrm{MPa} \sqrt{\mathrm{m}}$

$\mathrm{Ke}$ (Beta Corrected) $=121.3 \mathrm{MPa} \sqrt{\mathrm{m}}$

$101.9 \mathrm{~kJ} / \mathrm{m}^{2}$

$143.4 \mathrm{MPa} \sqrt{\mathrm{m}}$

$126.5 \mathrm{MPa} \sqrt{\mathrm{m}}$

ASTM E399-81 ANALYSIS

Modified Kic Equation;

Maximum Load $P=559.25 \mathrm{kN}$

$5 \%$ Secant offset $\mathrm{Pq}=559.25 \mathrm{kN}$

$\mathrm{Kq}$

Validity

$K$ at Maximum Load

$=141.6 \mathrm{MPa} \sqrt{\mathrm{m}}$

= INVALID--1, 2

$=141.6 \mathrm{MPa} \sqrt{\mathrm{m}}$

DATA CORRECTIONS USED FOR J-INTEGRAL DATA

Merkle-Corten correction

Correction to J for crack extension (Deformation-J)

OFFSET LOAD $=0 \mathrm{kN}$ OFFSET DEFLECTION $=.008 \mathrm{~mm}$

Fitied elastic load range $=100 \mathrm{kN}$ TO $400 \mathrm{kN}$

Elastic slope $=4.343 E+02 \mathrm{kN} / \mathrm{mm}$ 
Specimen Crackmauth Def lection (in.) ORNL-DWG 92-11678

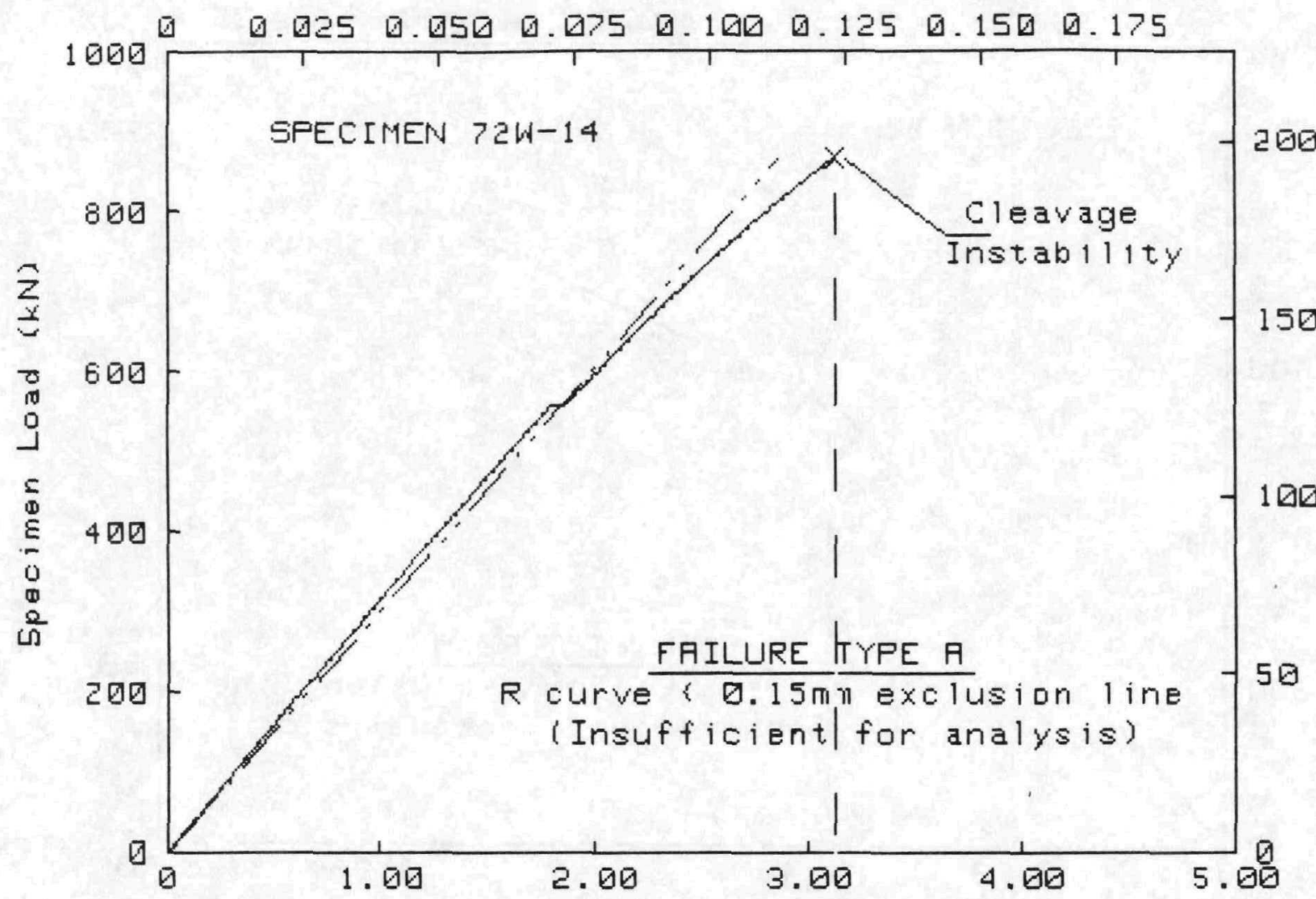

Specimen Crackmouth Defleotion (mm)

\section{TEST SFECIMEN DATA}

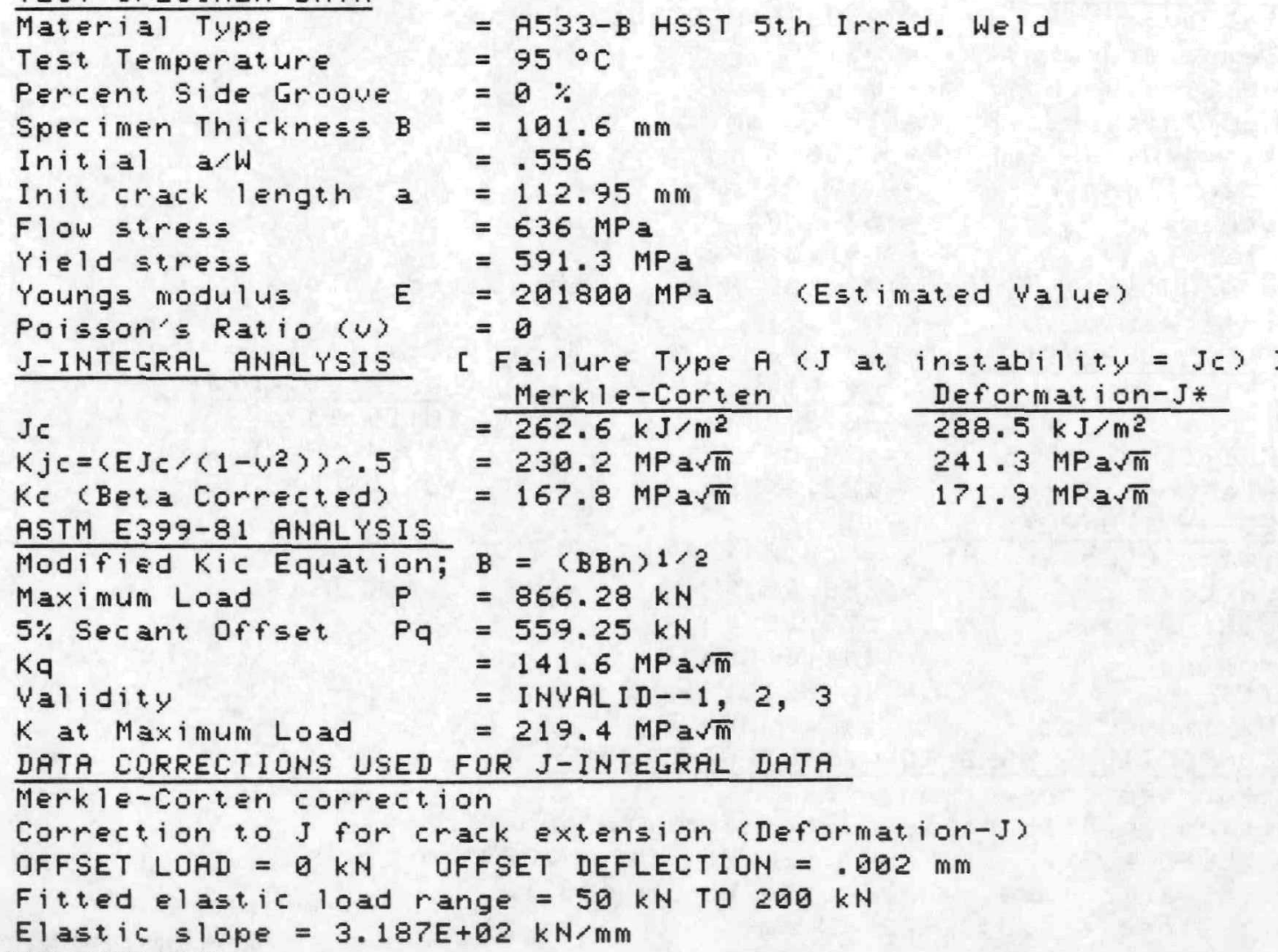


Specimen Crackmauth Def lection (in.) ORNL-DWG 92-11679

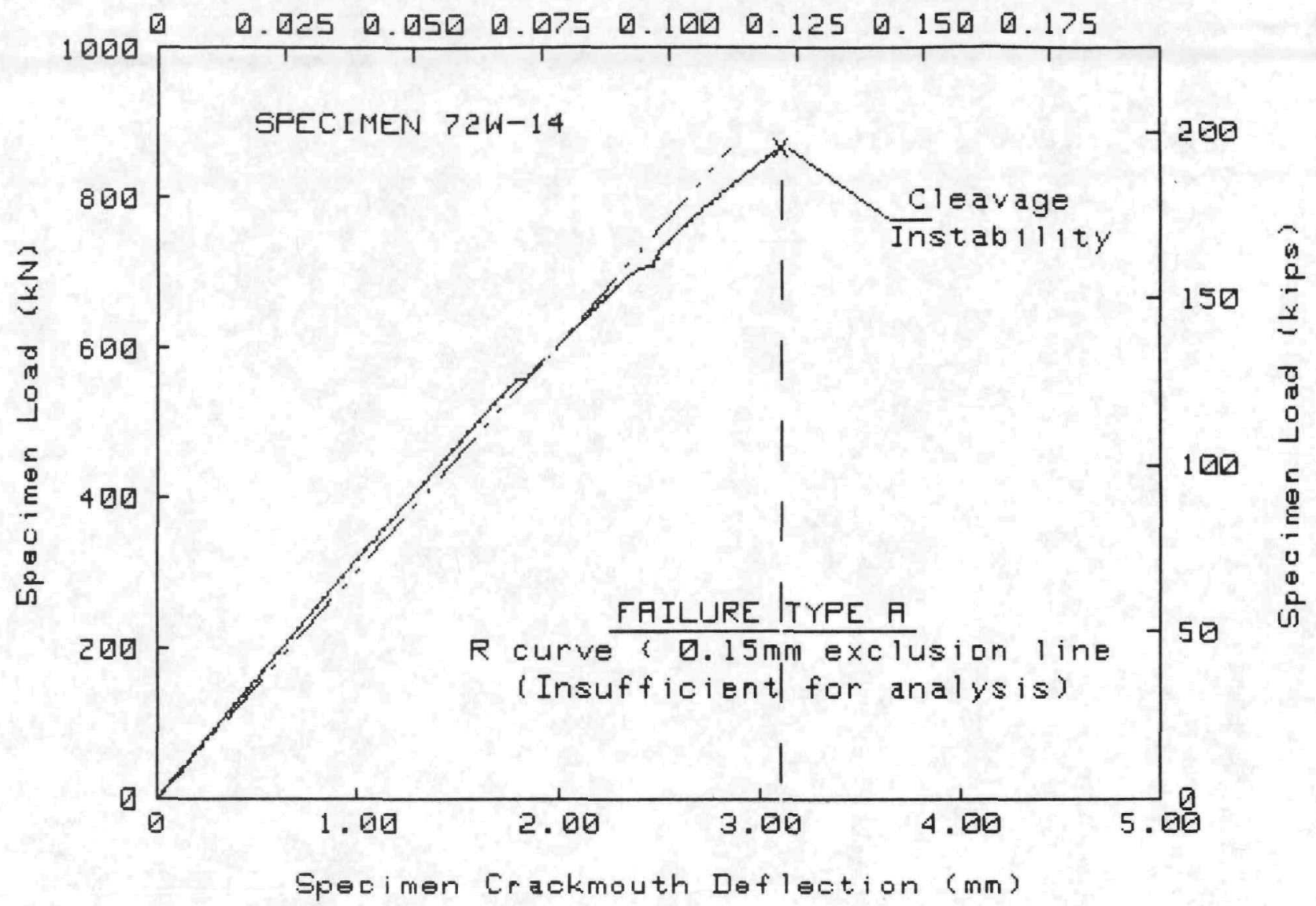

TEST SPECIMEN DATA

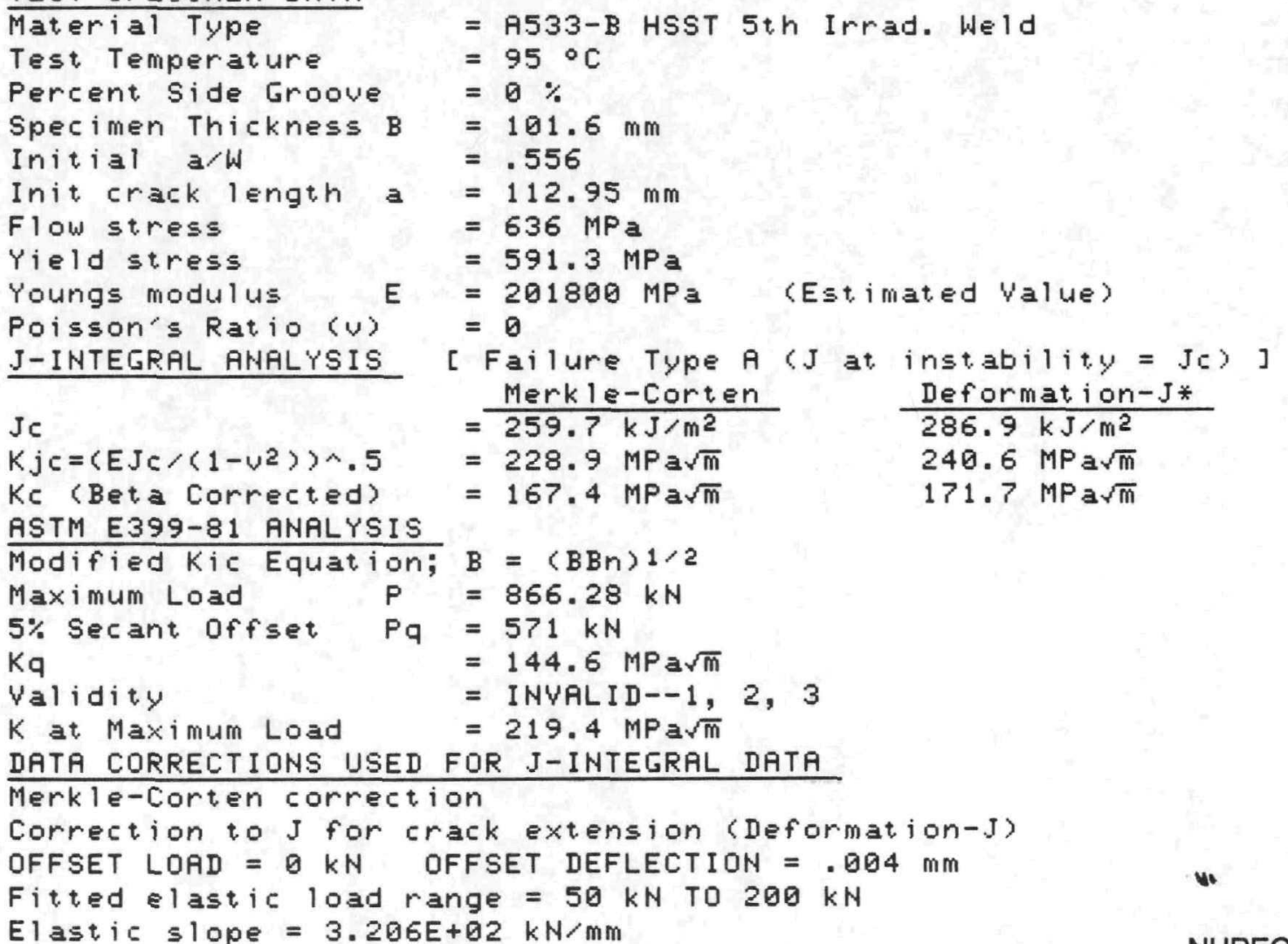



Appendix F

Load-Displacement Curves and Photographs of the Fracture Toughness Specimens From the $73 \mathrm{~W}$ Weld $(0.31 \mathrm{wt} \% \mathrm{Cu})$ 
SPEC IMEN ID $=73 W 137$

MATERIAL TYPE = HCUWELD

TEST TEMPERATURE $=-150$ DEG C

THICKNESS $=25.5 \mathrm{~mm}$

SIDE GROOUES $=0 \%$

INITIAL CRACK LENGTH $=27.5 \mathrm{~mm}$

MEASURED DUCTILE DELTA-A $=.05 \mathrm{~mm}$

$a / W\rangle i=.541$

YIELD STRENGTH $=712 \mathrm{MPa}$

FLOW STRENGTH $=760 \mathrm{MPa}$

ESTIMATED YOUNG'S MODULUS $=216 \mathrm{GPa}$

SPECIMEN CLEAUED AT $K I c=39.3 \mathrm{MPa}$ SQR [m] (UALID ASTM E399)

UALUES AT MAXIMUM LOAD

J-INTEGRAL $=6.7 \mathrm{~kJ} / \mathrm{m}^{\wedge} 2$

$\mathrm{KJ} c=38.1 \mathrm{MPa} \operatorname{SQR}[\mathrm{m}]$

K-bet $a-c=37.8 \mathrm{MPa} S Q R[\mathrm{~m}]$
UALUES AT CLEAUAGE LDAD

J-INTEGRAL $=6.7 \mathrm{~kJ} / \mathrm{m}^{\wedge} 2$

$\mathrm{KJ} c=38.1 \mathrm{MPa} \operatorname{SQR}[\mathrm{m}]$

$K$-beta-c $=37.8 \mathrm{MPa} \operatorname{SQR}[\mathrm{m}]$ 
F-3

ORNL-DWG 92-11315

73W137 -150 DEGREES C

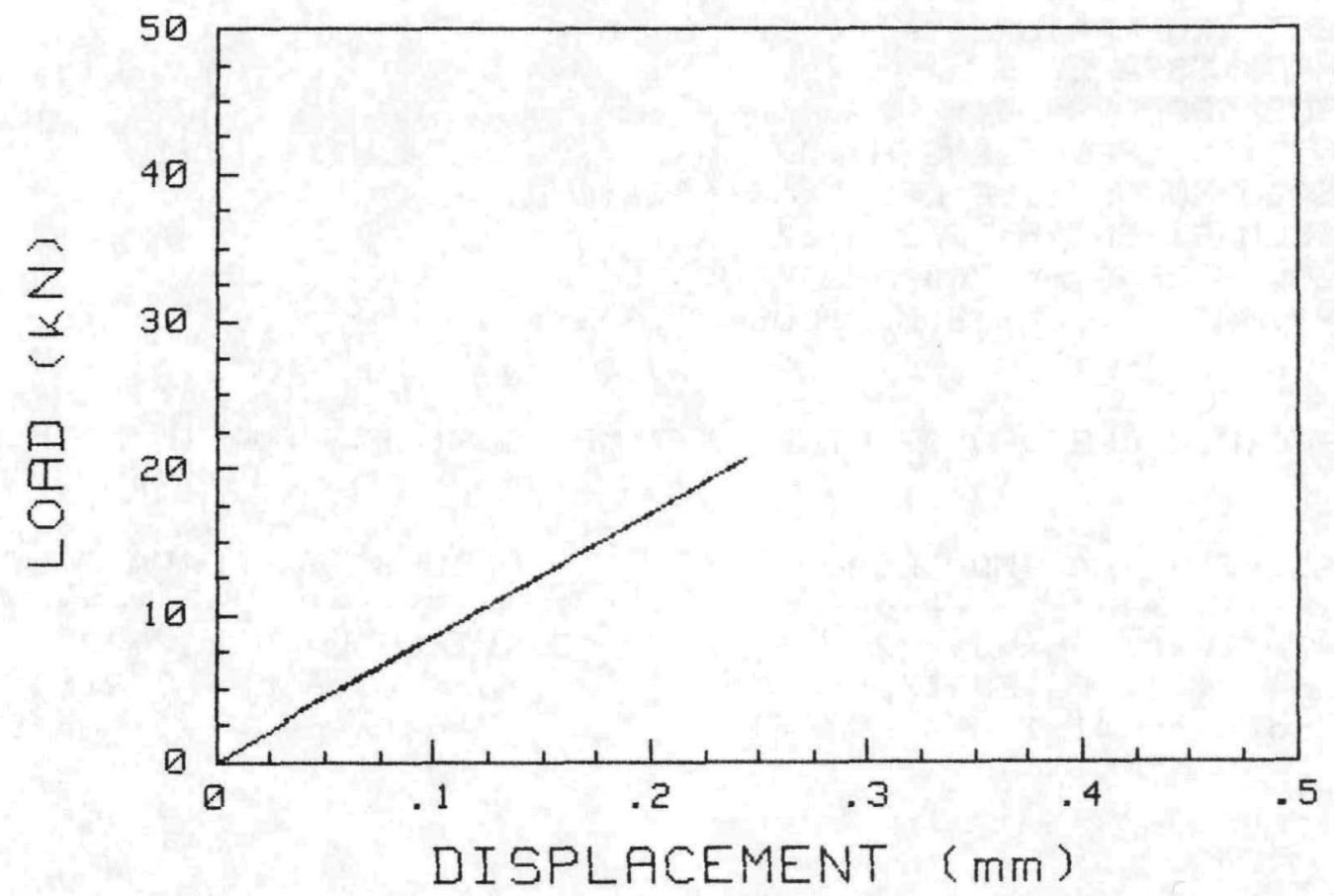

$73 W-137$

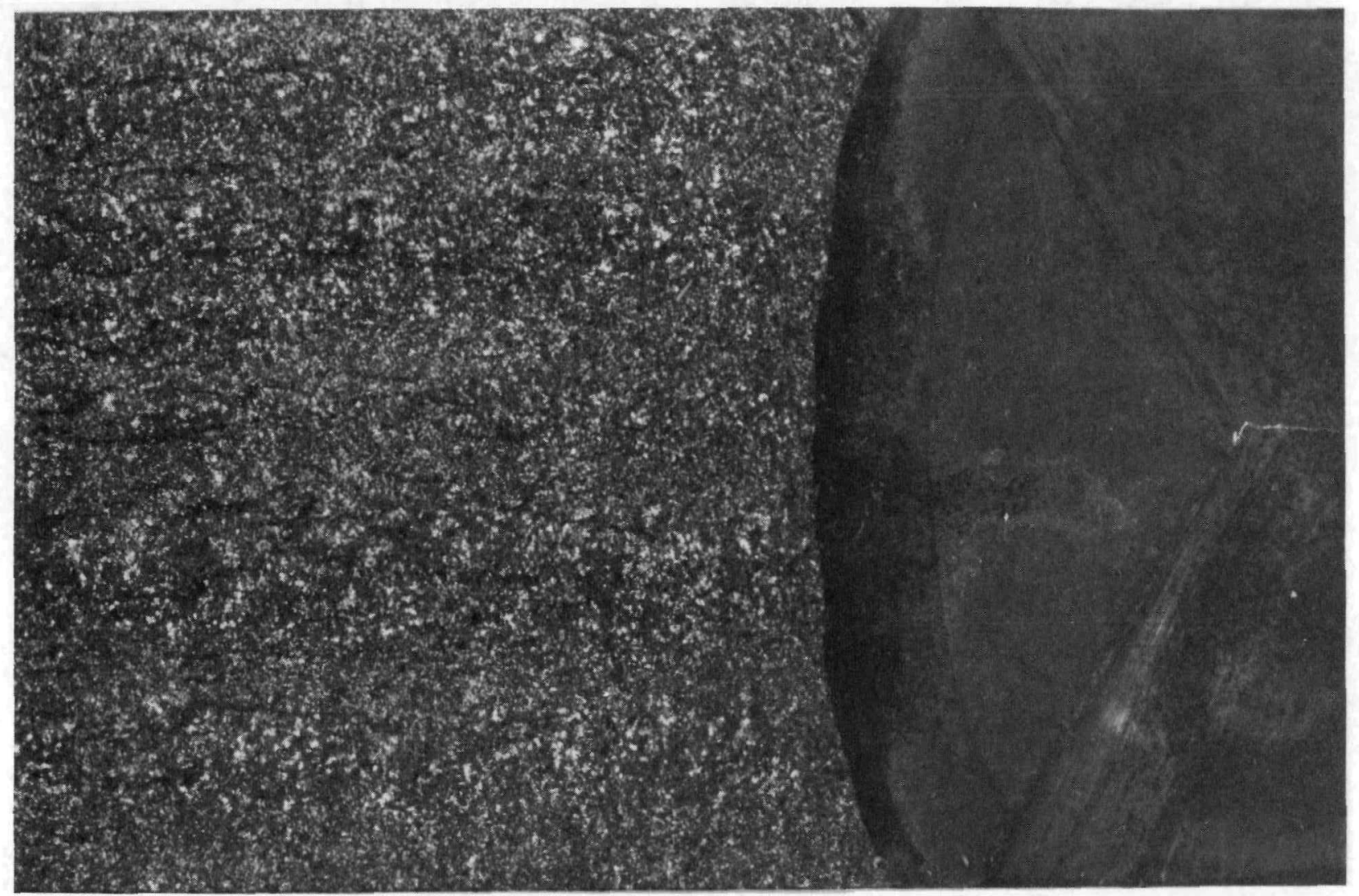


SPECIMEN ID $=73$ W 141

MATERIAL TYPE = HCUWELD

TEST TEMPERATURE $=-150$ DEG C

THICKNESS $=25.3 \mathrm{~mm}$

SIDE GROOUES = $0 \%$

INITIAL CRACK LENGTH $=27 \mathrm{~mm}$

MEASURED DUCTILE DELTA-A $=.05 \mathrm{~mm}$

$a / W) i=.531$

YIELD STRENGTH $=712 \mathrm{MPa}$

FLOW STRENGTH $=760 \mathrm{MPa}$

ESTIMATED YOUNG'S MODULUS $=216 \mathrm{GPa}$

SPECIMEN CLEAUED AT $K I c=41.2 \mathrm{MPa} S Q R[\mathrm{~m}]$ (UALID ASTM E399)

UALUES AT MAXIMUM LOAD

J-INTEGRAL $=8 \mathrm{~kJ} / \mathrm{m}^{\wedge} 2$

$\mathrm{KJ} c=41.6 \mathrm{MPa} \operatorname{SQR}[\mathrm{m}]$

$K$-beta-c $=41.1 \mathrm{MPa} \operatorname{SQR}[\mathrm{m}]$
NaLUES at CLEaUAGE LOAD

J-INTEGRAL $=8 \mathrm{~kJ} / \mathrm{m}^{\wedge} 2$

$K J c=41.6 \mathrm{MPa} \operatorname{SQR}[\mathrm{m}]$

$\mathrm{K}$-bet $a-c=41.1 \mathrm{MPa} S Q R[\mathrm{~m}]$ 


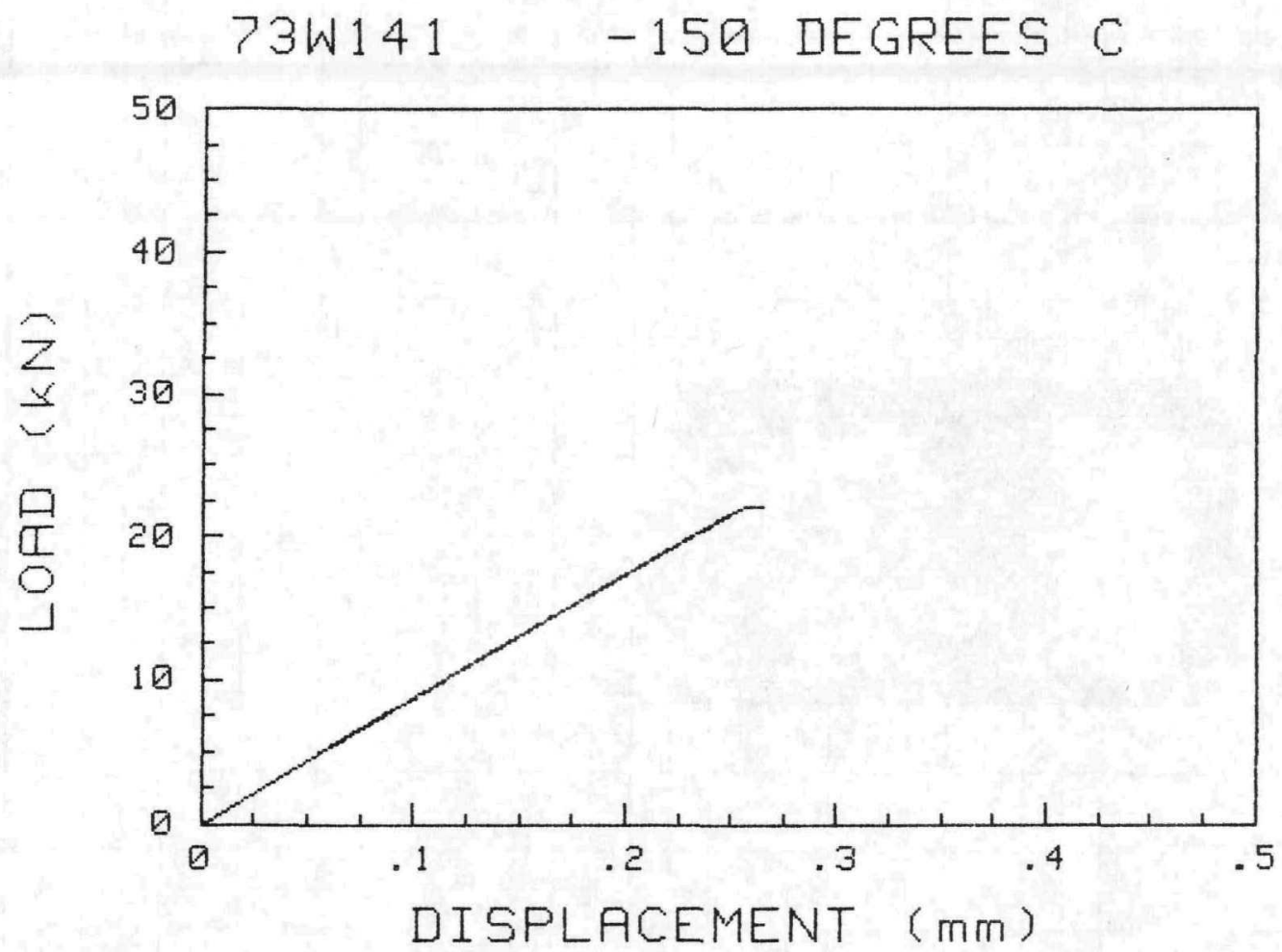

$73 W-141$

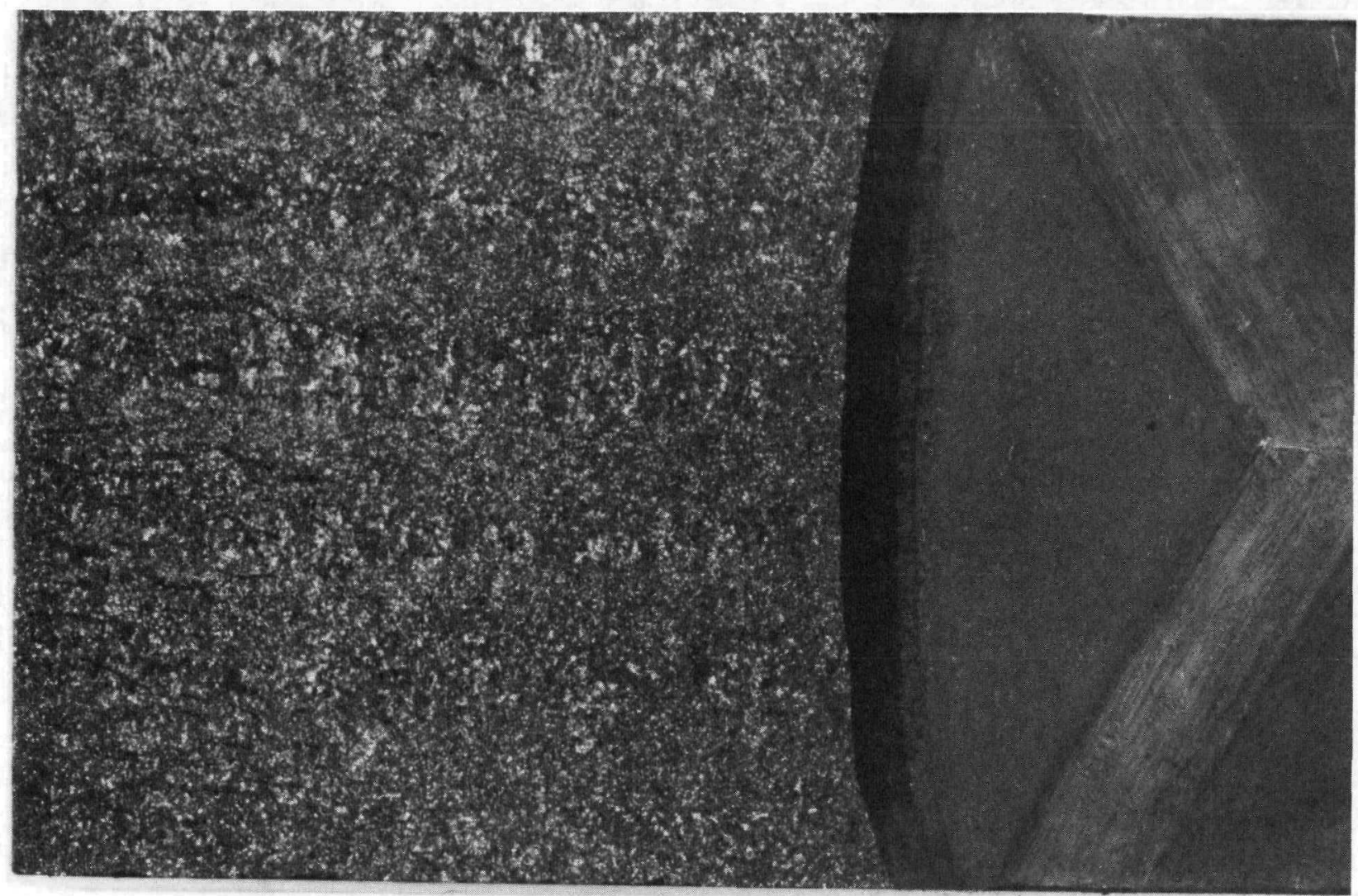




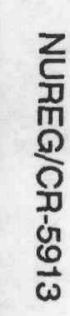

$-150^{\circ} \mathrm{C} \quad 8 / 2 \phi / 85 \mathrm{BHM} / \mathrm{MJL}$

$73 w-157$

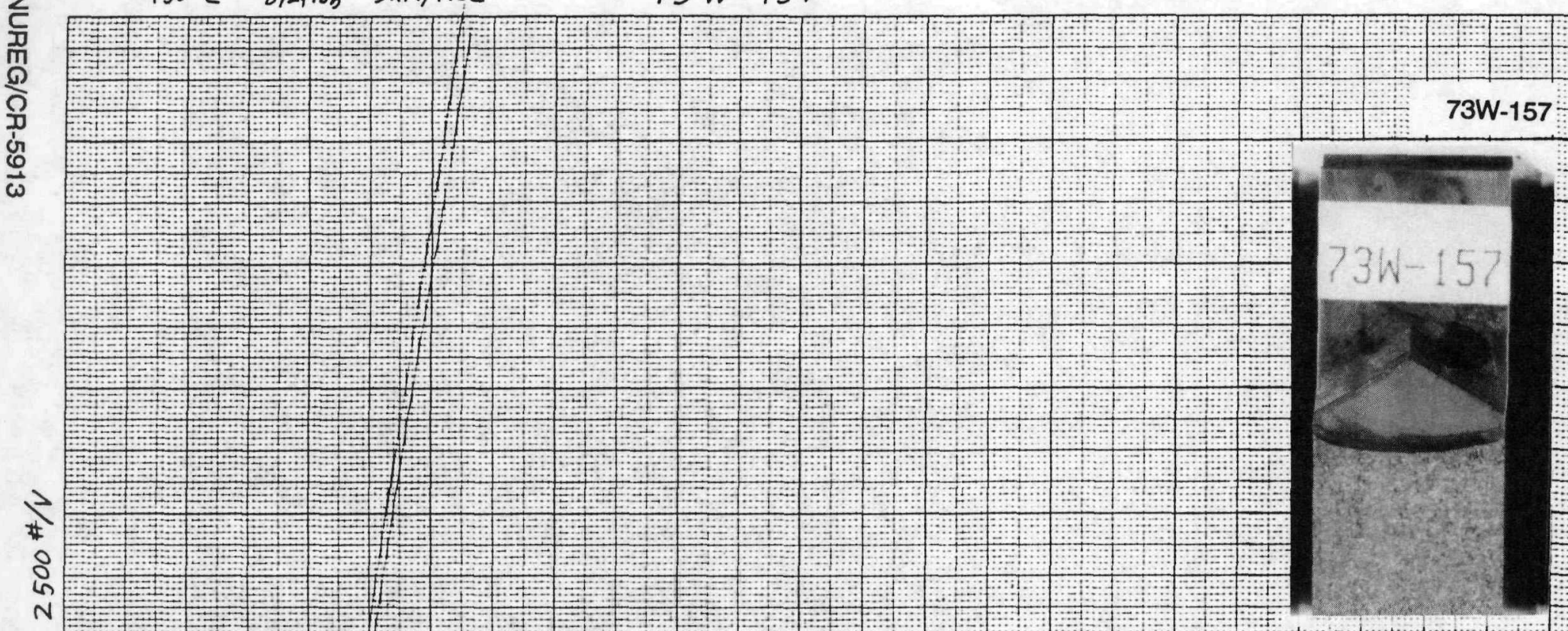

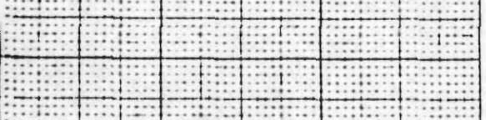

$\div \quad \div \quad \square$

$+10$

$\sqrt[0]{0.0}$

40

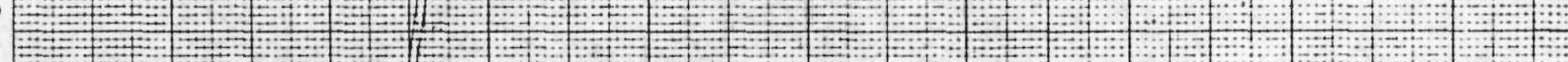

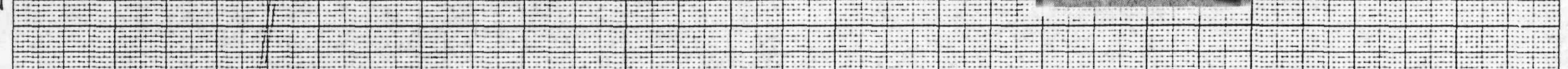

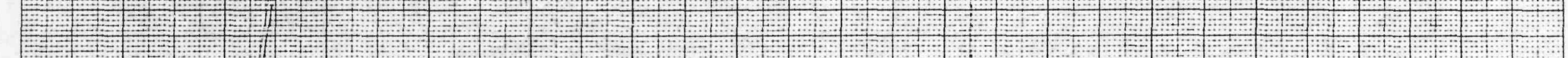

I

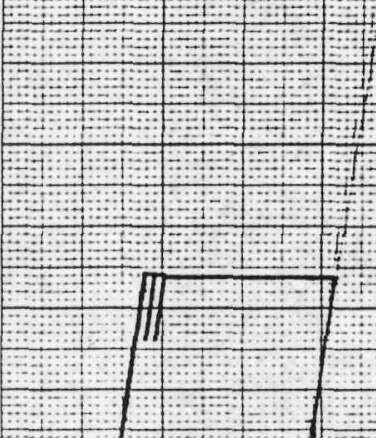

$+1+1:+1$

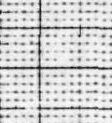

$\because: 1 .+1:$

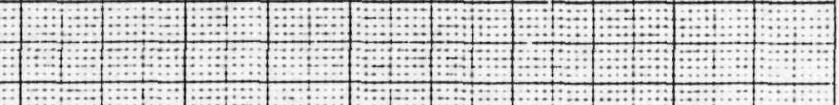

$+1$
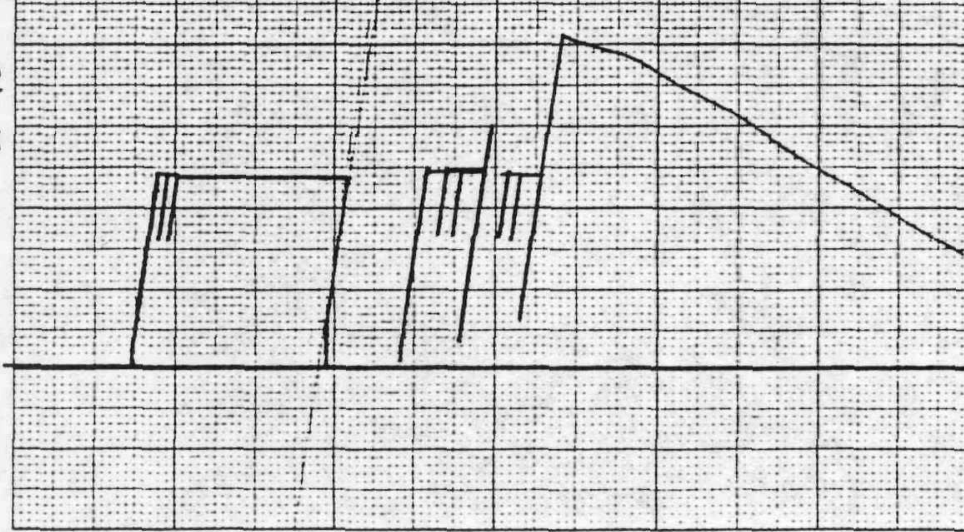
$+11$ $-1$

$$
\text { FRONT FACE DEFLECTION IV/inp } \quad .0358105 \mathrm{in} / \mathrm{V}
$$


$73 \mathrm{~W} 153 \quad-150^{\circ} \mathrm{C} \quad 0 \% 56 \quad 1 \mathrm{~T}-\mathrm{CT} \quad 8 / 22 / 85 \mathrm{MCP} / \mathrm{MJL}$

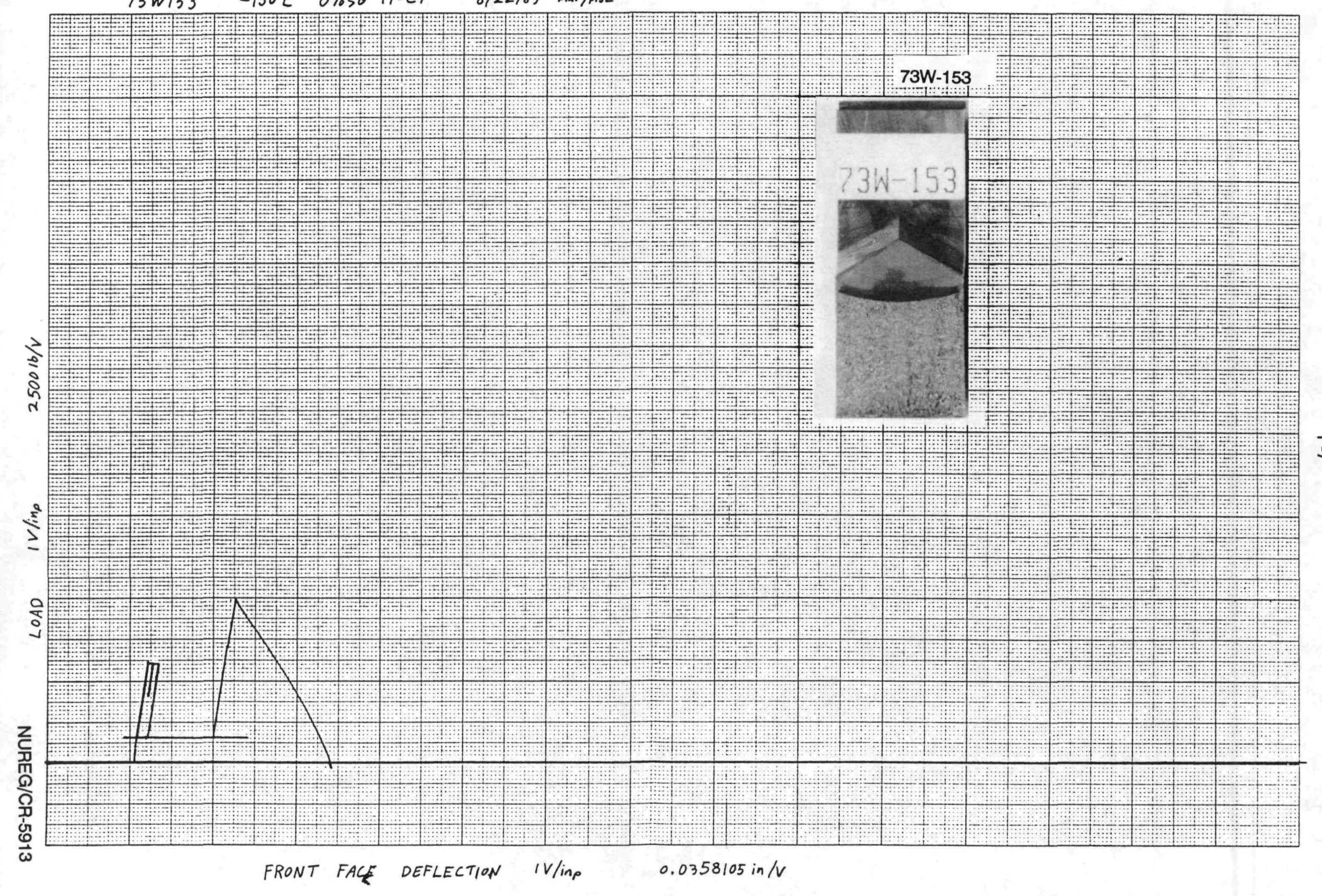


SPECIMEN ID $=73 W 127$

MATERIAL TYPE $=$ HCUWELD

TEST TEMPERATURE $=-101$ DEG $C$

THICKNESS $=25.4 \mathrm{~mm}$

SIDE GROOUES $=0 \%$

INITIAL CRACK LENGTH $=28 \mathrm{~mm}$

MEASURED DUCTILE DELTA-A $=.05 \mathrm{~mm}$

$a / W) i=.552$

YIELD STRENGTH $=600 \mathrm{MPa}$

FLDW STRENGTH $=666 \mathrm{MPa}$

ESTIMATED YOUNG'S MODULUS $=213$ GPa

SPECIMEN CLEAUED AT KQ $=65.8 \mathrm{MPa}$ SQR $[\mathrm{m}]$ (NDT WALID KIc)

UALUES AT MAXIMUM LOAD

J-INTEGRAL $=19.8 \mathrm{~kJ} / \mathrm{m}^{\wedge} 2$

$K J \mathrm{C}=64.9 \mathrm{MPa} S Q R[\mathrm{~m}]$

$K$-bet $a-c=59.2 \mathrm{MPa} \operatorname{SQR}[\mathrm{m}]$ values at cleanage load

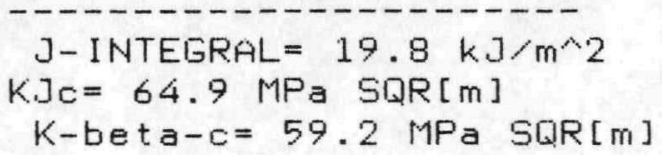



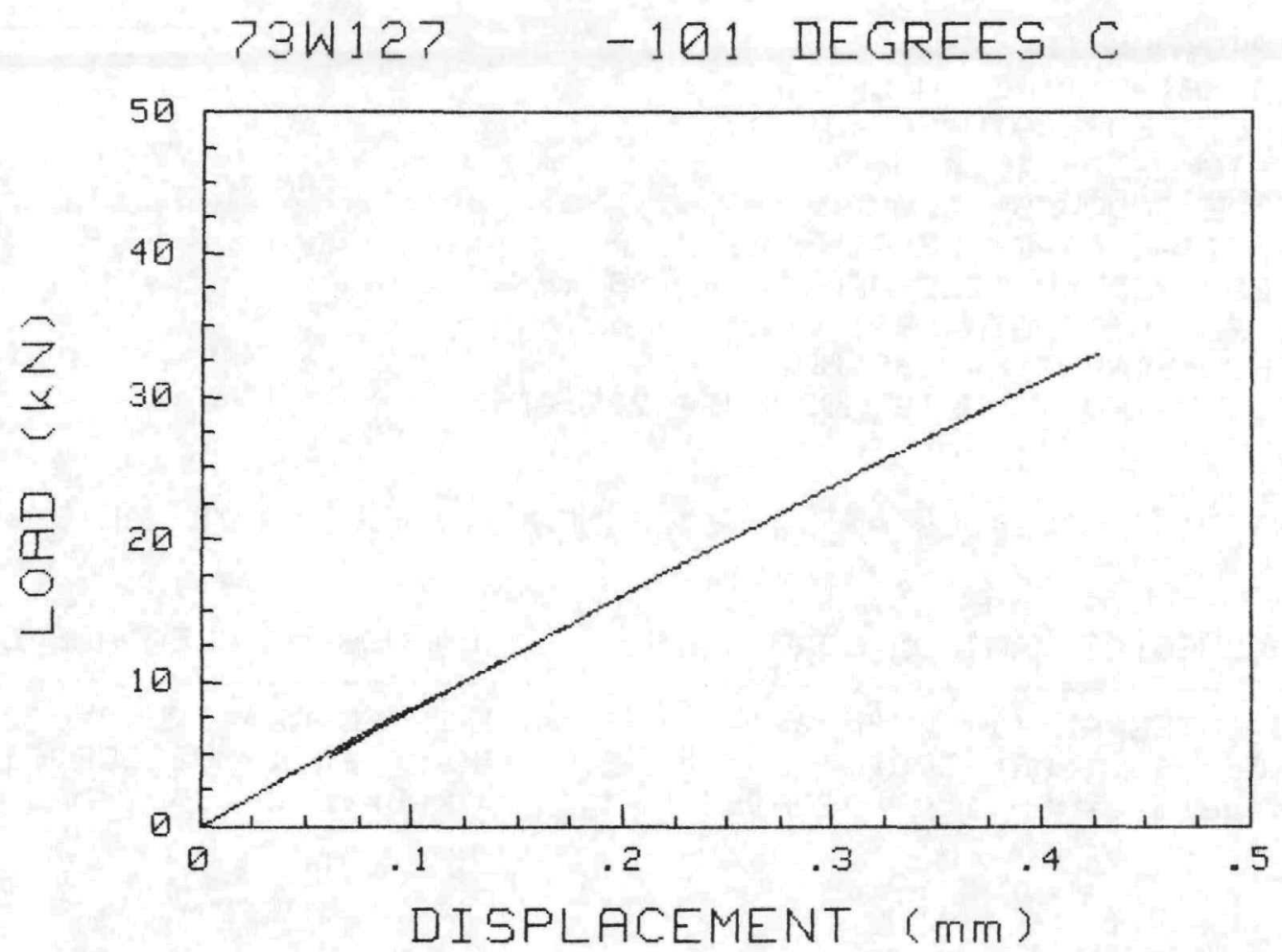

$73 W-127$

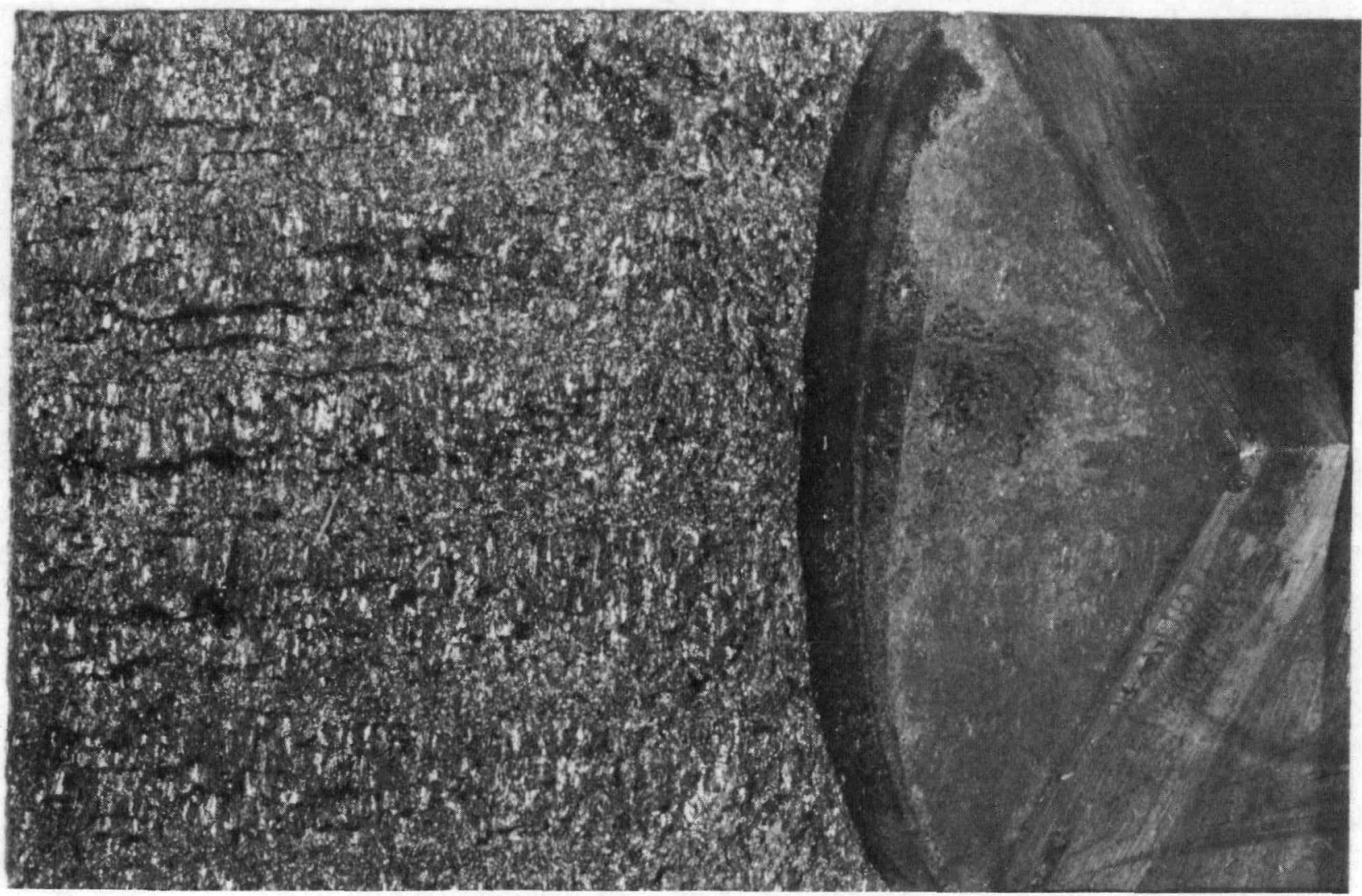


SPECIMEN ID $=73 W 123$

MATERIAL TYPE = HCUWELD

TEST TEMPERATURE $=-80$ DEG $C$

THICKNESS $=25.4 \mathrm{~mm}$

SIDE GROOUES $=0 \%$

INITIAL CRACK LENGTH $=26.7 \mathrm{~mm}$

MEASURED DUCTILE DELTA-A $=.05 \mathrm{~mm}$

$a / W)_{i}=.525$

YIELD STRENGTH $=571 \mathrm{MPa}$

FLOW STRENGTH $=639 \mathrm{MPa}$

ESTIMATED YOUNG'S MODULUS= 212 GPa

SPECIMEN CLEAUED AT KQ=60.6 MPa SQR $[\mathrm{m}]$ (NOT UALID KIC)

UALUES AT MAXIMUM LDAD

J-INTEGRAL $=17 \mathrm{~kJ} / \mathrm{m}^{\wedge} 2$

$\mathrm{KJc}=60.1 \mathrm{MPa} \operatorname{SQR}[\mathrm{m}]$

K-beta-c $=55.1 \mathrm{MPa} \operatorname{SQR}[\mathrm{m}]$

\author{
UALUES AT CLEAUAGE LDAD \\ - - - - - - - - - - - - - - \\ $J-I N T E G R A L=17 \mathrm{~kJ} / \mathrm{m}^{\wedge} 2$ \\ $\mathrm{KJc}=60.1 \mathrm{MPa} \operatorname{SQR}[\mathrm{m}]$ \\ $K-$ bet $a-c=55.1 \mathrm{MPa} \operatorname{SQR}[\mathrm{m}]$
}




\section{F-11}

ORNL-DWG 92-11318

\section{W123 -80 DEGREES C}

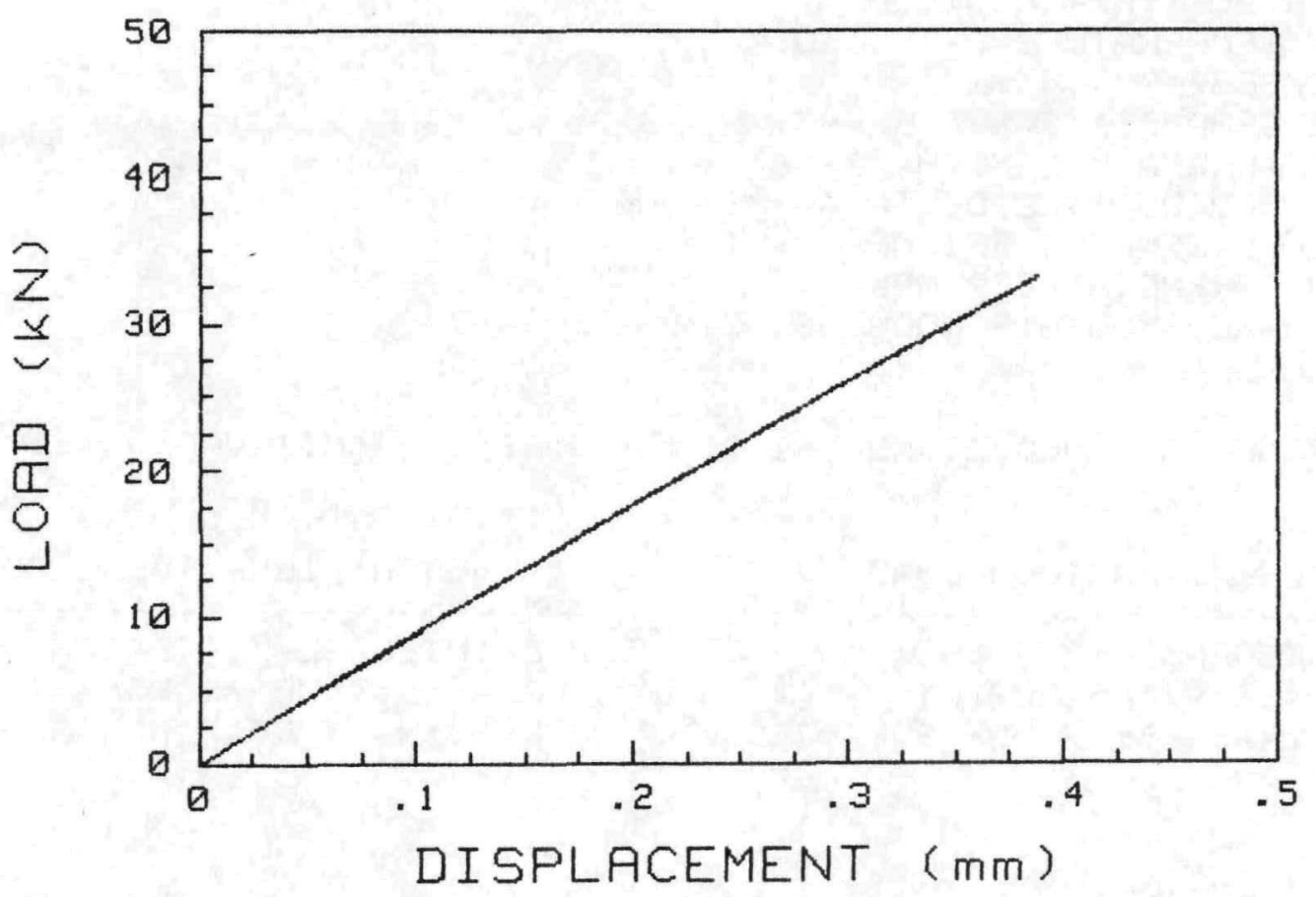

$73 W-123$

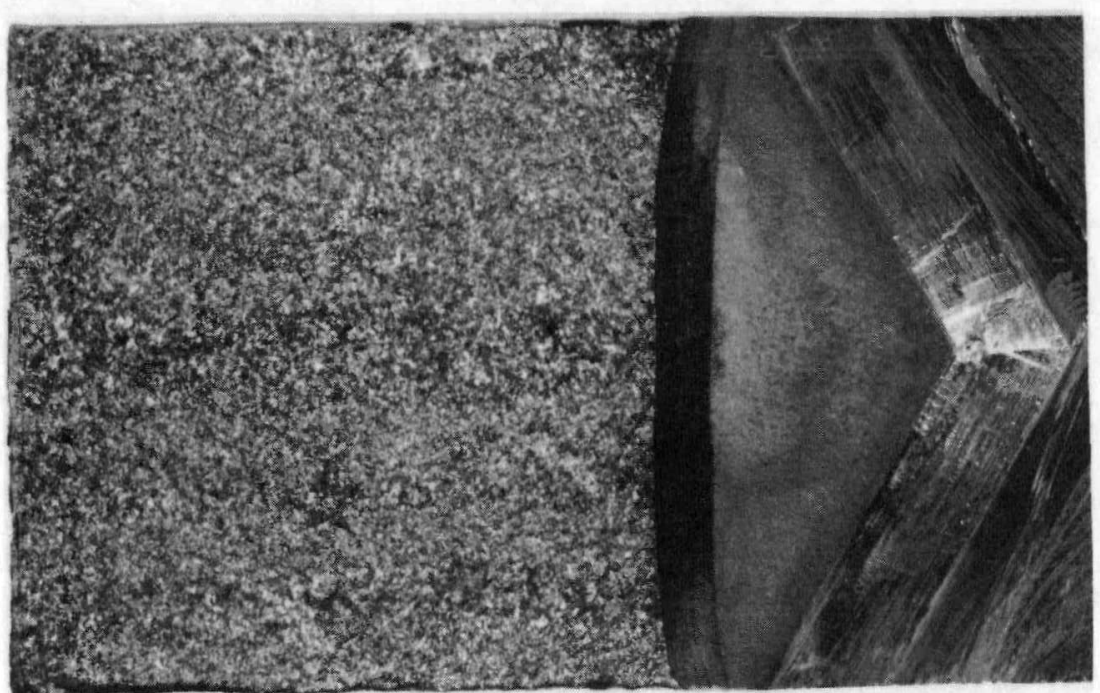


SPECIMEN ID $=73 W 121$

MATERIAL TYPE = HCUWELD

TEST TEMPERATURE $=-80$ DEG C

THICKNESS $=25.4 \mathrm{~mm}$

SIDE GROOUES $=0 \%$

INITIAL CRACK LENGTH $=27.6 \mathrm{~mm}$

MEASURED DUCTILE DELTA-A $=.05 \mathrm{~mm}$

$a / W) i=.543$

YIELD STRENGTH $=571 \mathrm{MPa}$

FLOW STRENGTH $=639 \mathrm{MPa}$

ESTIMATED YOUNG'S MODULUS $=212 \mathrm{GPa}$

SPECIMEN CLEAUED AT KQ $=61.8 \mathrm{MPa} S Q R[\mathrm{~m}]$ (NOT VALID KIc)

UALUES AT MAXIMUM LDAD

J-INTEGRAL $=17.5 \mathrm{~kJ} / \mathrm{m}^{\wedge} 2$

$K J c=60.9 \mathrm{MPa} S \mathrm{SQR}[\mathrm{m}]$

K-beta-c $=55.7 \mathrm{MPa} S Q R[\mathrm{~m}]$
UALUES at CLEAUAGE LOAD

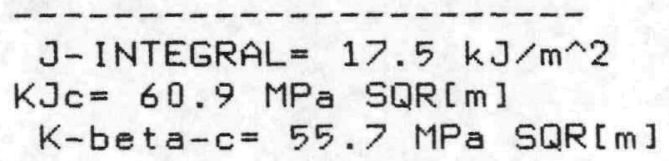




\section{$\mathrm{F}-13$}

ORNL-DWG 92-11319

73W121 -80 DEGREES C

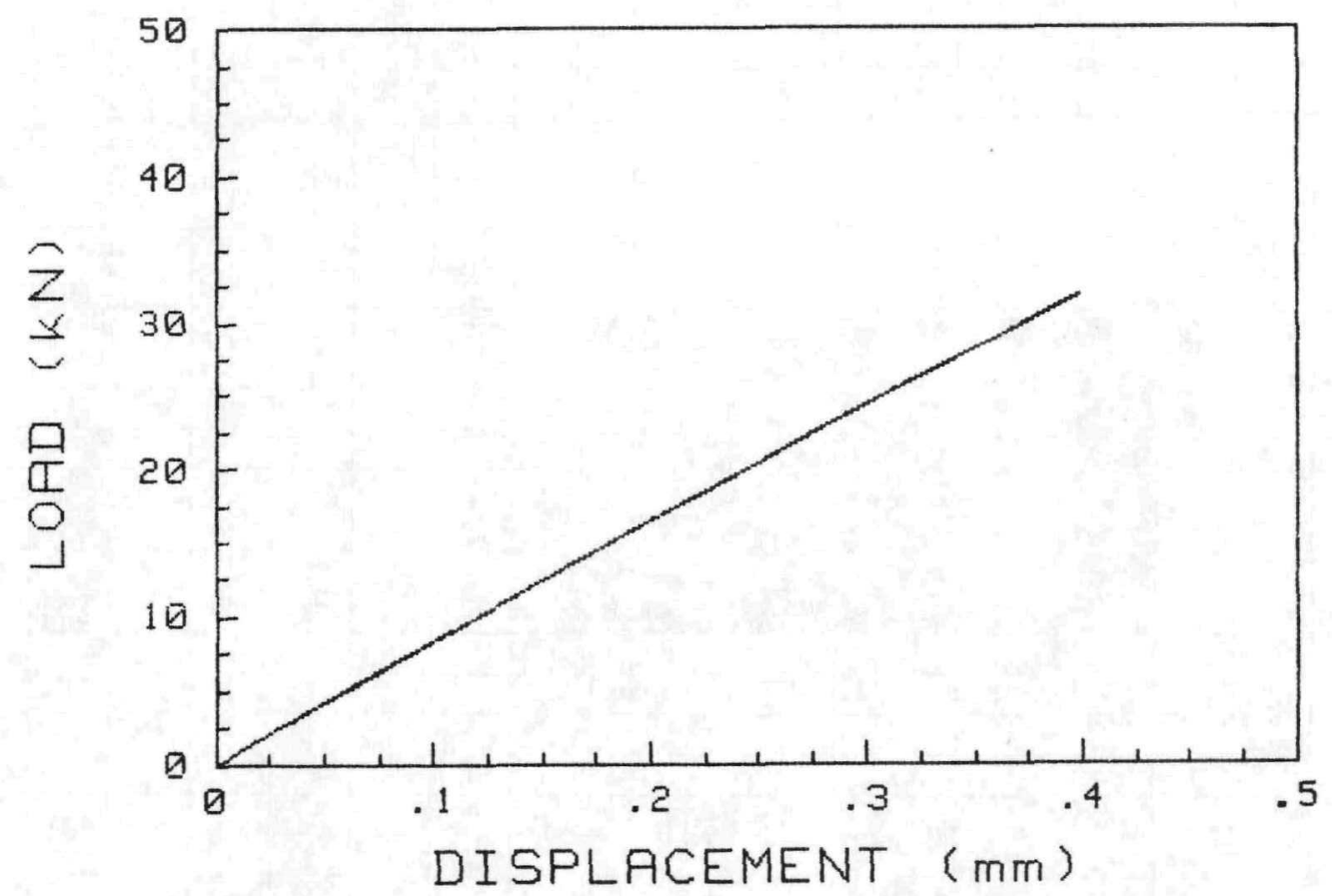

$73 W-121$

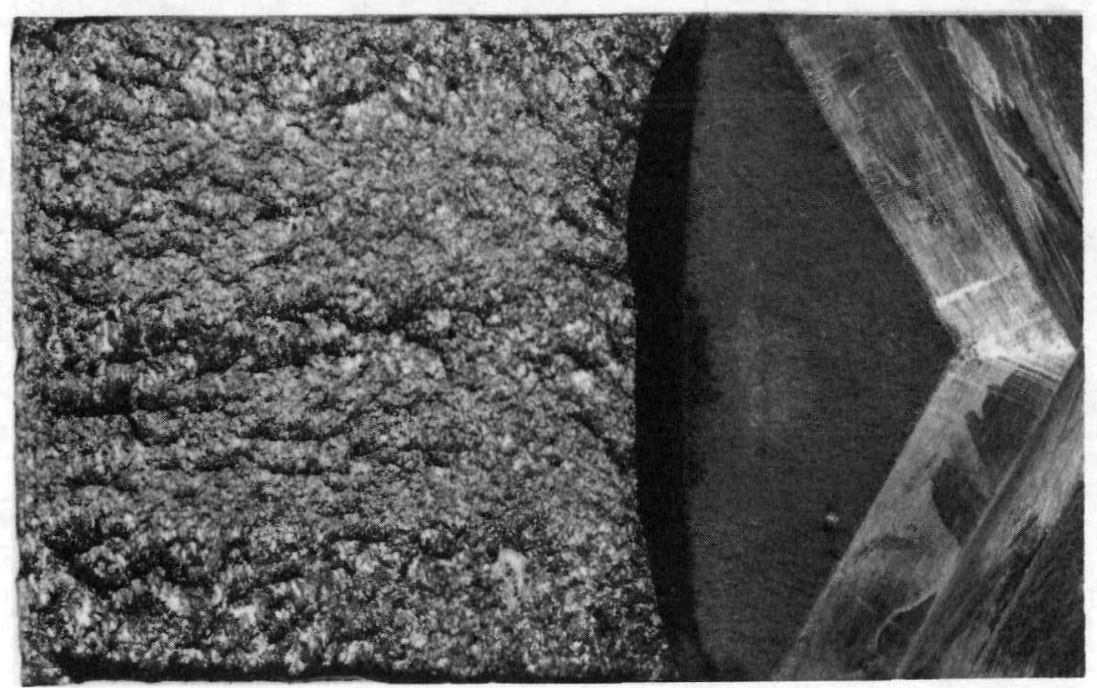




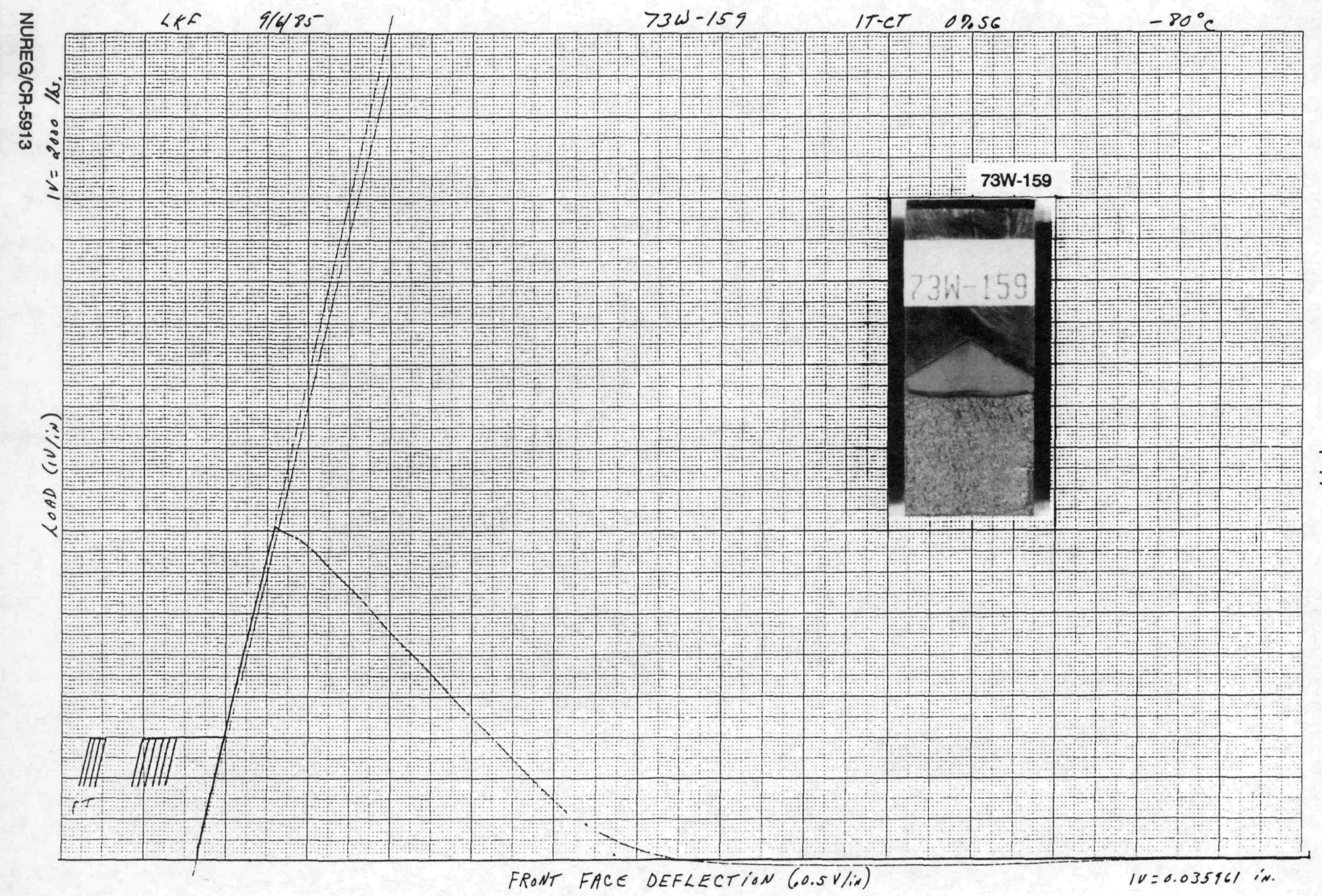




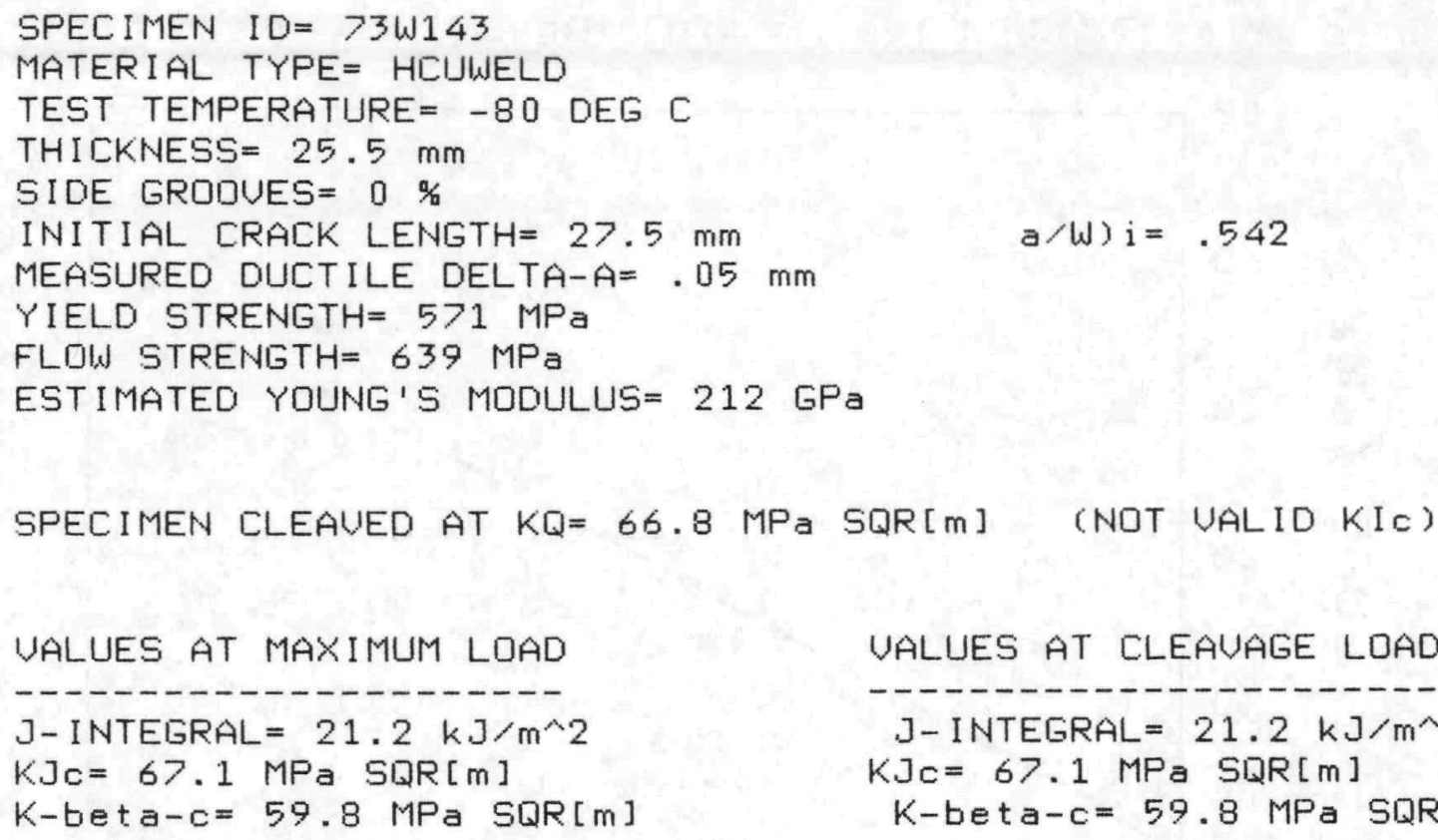

UALUES AT MAXIMUM LDAD

J-INTEGRAL $=21.2 \mathrm{~kJ} / \mathrm{m}^{\wedge} 2$

$K J c=67.1 \mathrm{MPa} S \mathrm{SQR}[\mathrm{m}]$

$K$-beta-c $=59.8 \mathrm{MPa} \operatorname{SQR}[\mathrm{m}]$ 
F-16

ORNL-DWG 92-11320

73W143 -80 DEGREES C

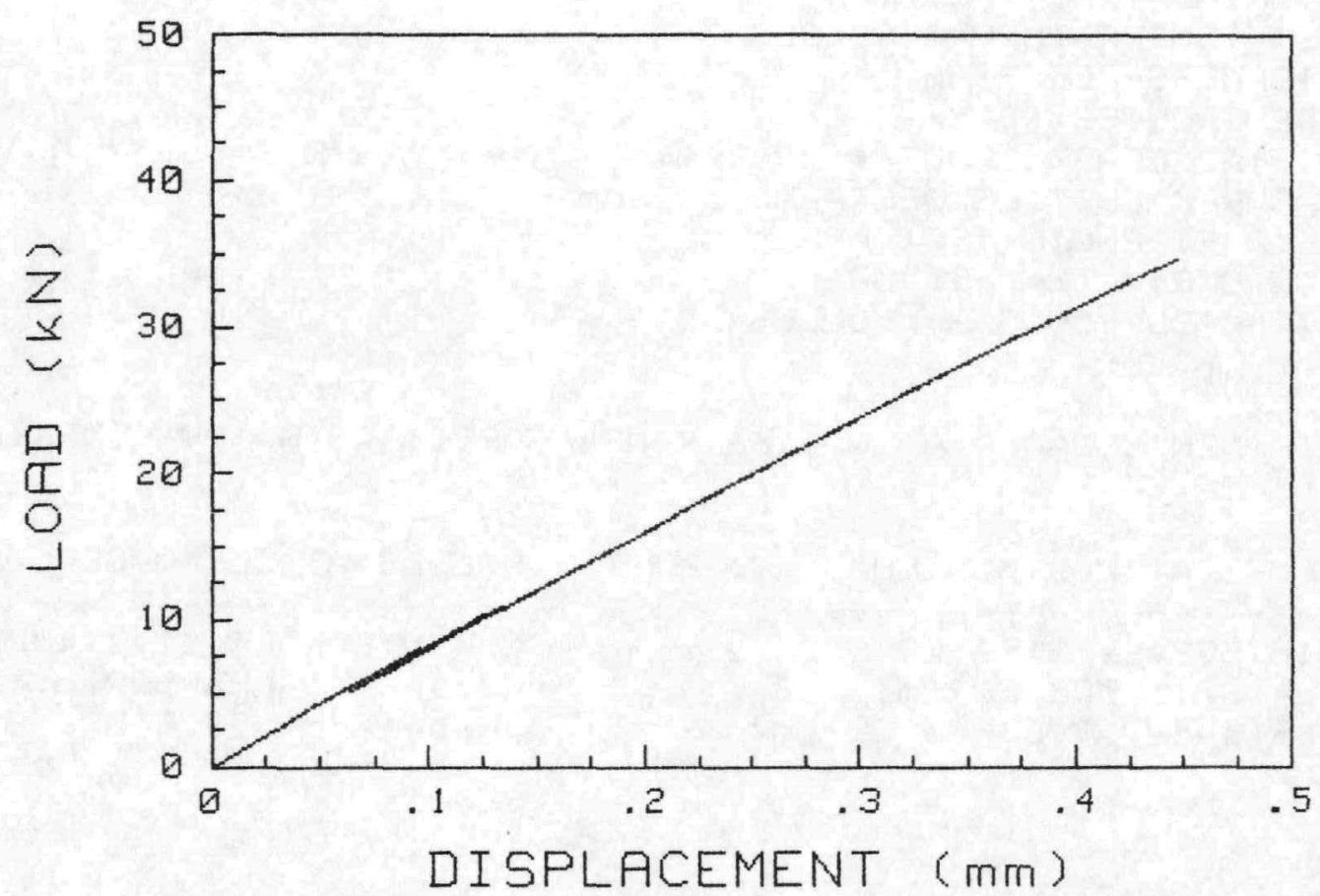

$73 W-143$

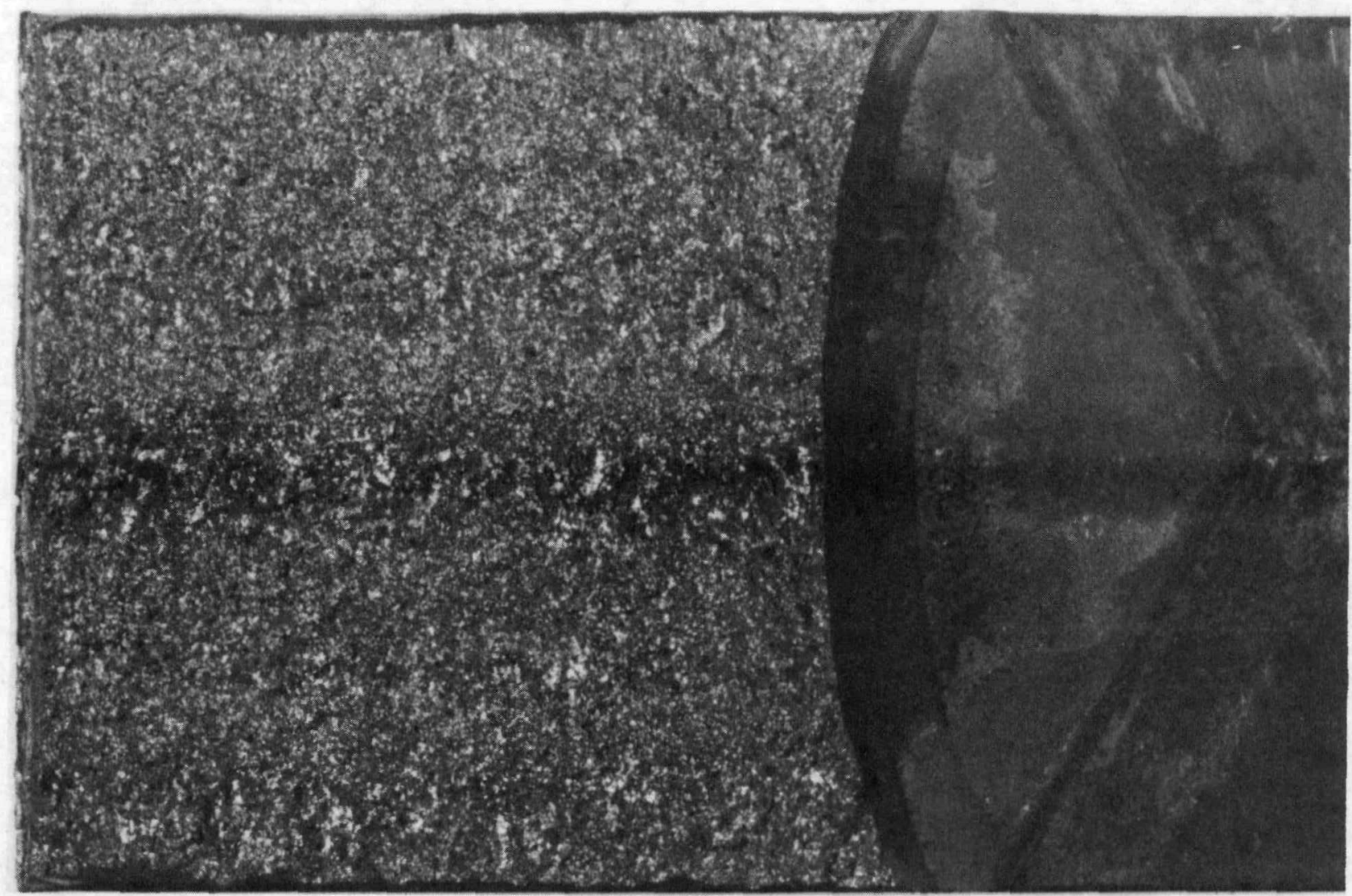

NUREG/CR-5913 
$73 \omega 161$

$-112^{\circ} \mathrm{F}$

$-80^{\circ} \mathrm{C}$

$8 / 19 / 85$

3
$n$
0
0
0
$n$
$n$

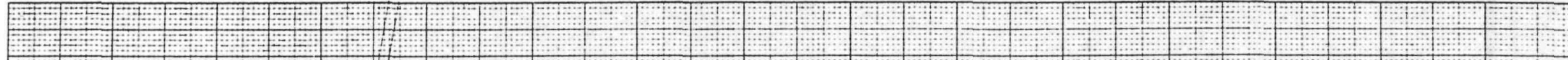

产

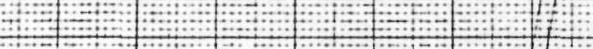
$+\cdots \cdots \cdots$

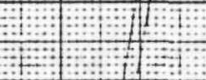

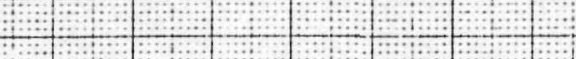

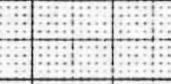

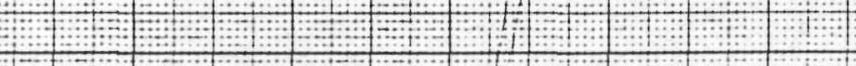

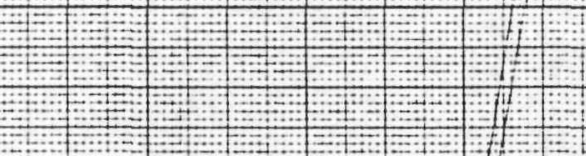

$+7+2+2$

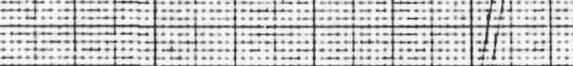

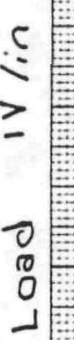

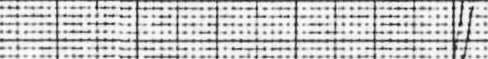
$-7+\square$

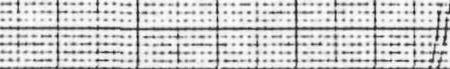

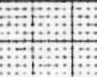

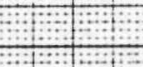

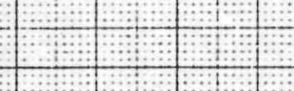

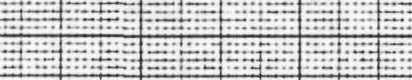

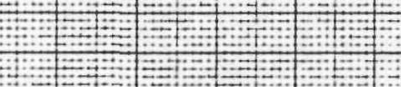
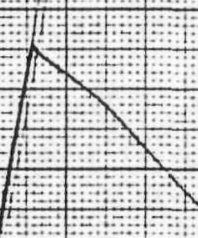

z

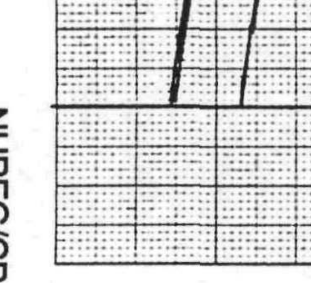
$\int \sqrt{1 ! n !}$
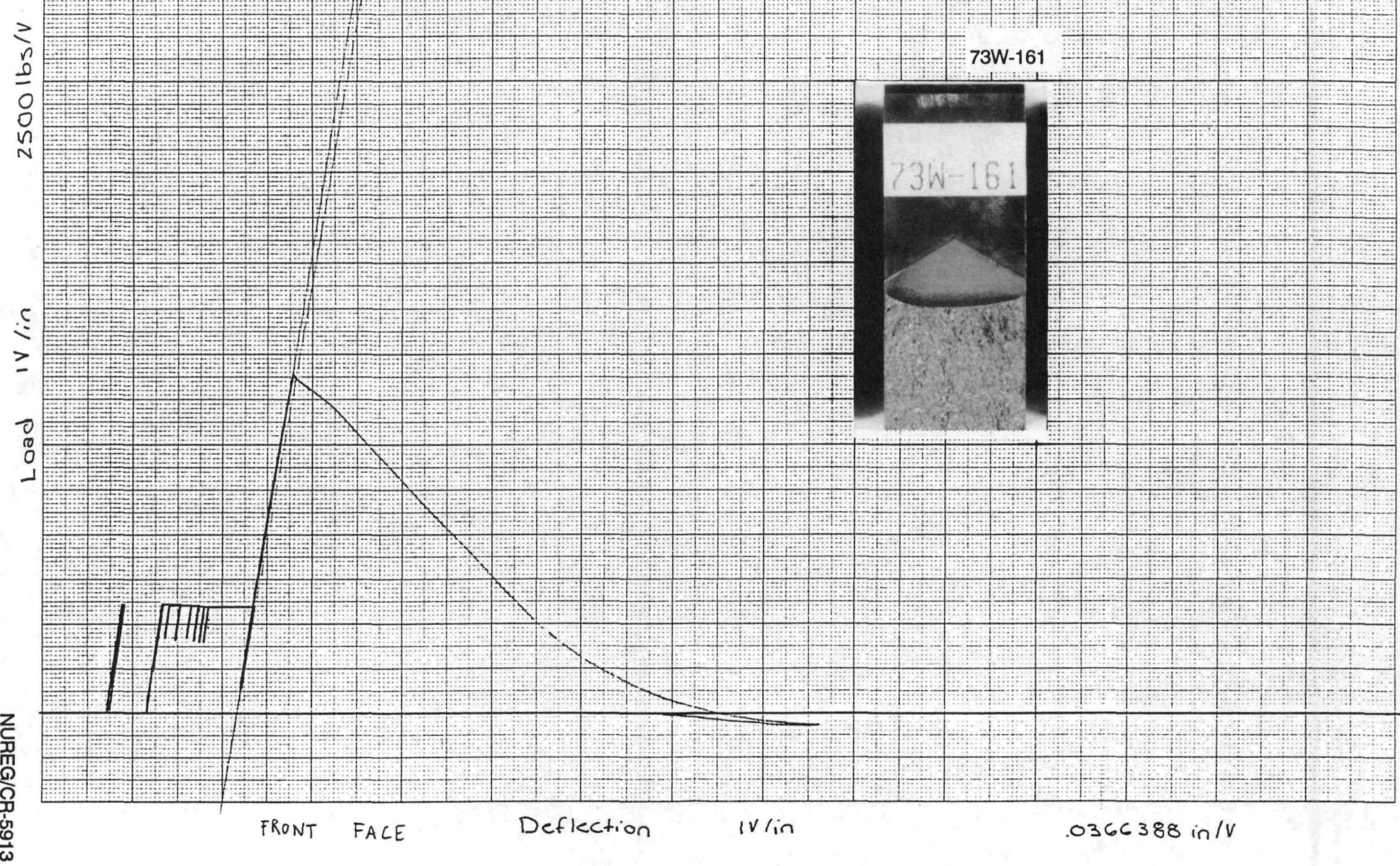

Deflection

IV/in

$.0366388 \mathrm{in} / \mathrm{V}$ 
SPECIMEN ID $=73 W 122$

MATERIAL TYPE = HCUWELD

TEST TEMPERATURE $=-80$ DEG $C$

THICKNESS $=25.4 \mathrm{~mm}$

SIDE GROOUES = $0 \%$

INITIAL CRACK LENGTH $=26.1 \mathrm{~mm}$

$a / W\rangle \mathrm{i}=.515$

MEASURED DUCTILE DELTA-A $=.05 \mathrm{~mm}$

YIELD STRENGTH $=571 \mathrm{MPa}$

FLOW STRENGTH $=639 \mathrm{MPa}$

ESTIMATED YOUNG'S MODULUS $=212 \mathrm{GPa}$

SPECIMEN CLEAUED AT KQ= $78.2 \mathrm{MPa} S Q R[\mathrm{~m}]$ (NOT VALID KIc)

VALUES AT MAXIMUM LOAD

J-INTEGRAL $=30.1 \mathrm{~kJ} / \mathrm{m}^{\wedge} 2$

$\mathrm{KJ} c=79.8 \mathrm{MPa} \operatorname{SQR}[\mathrm{m}]$

$K-$ bet $a-c=67.1 \mathrm{MPa} \operatorname{SQR}[\mathrm{m}]$
UALUES AT CLEAUAGE LOAD

J-INTEGRAL $=30.1 \mathrm{~kJ} / \mathrm{m}^{\wedge} 2$

$\mathrm{KJ} c=79.8 \mathrm{MPa} \operatorname{SQR}[\mathrm{m}]$

K-beta-c $=67.1 \mathrm{MPa} \operatorname{SQR}[\mathrm{m}]$ 


\section{W122 -80 DEGREES C}

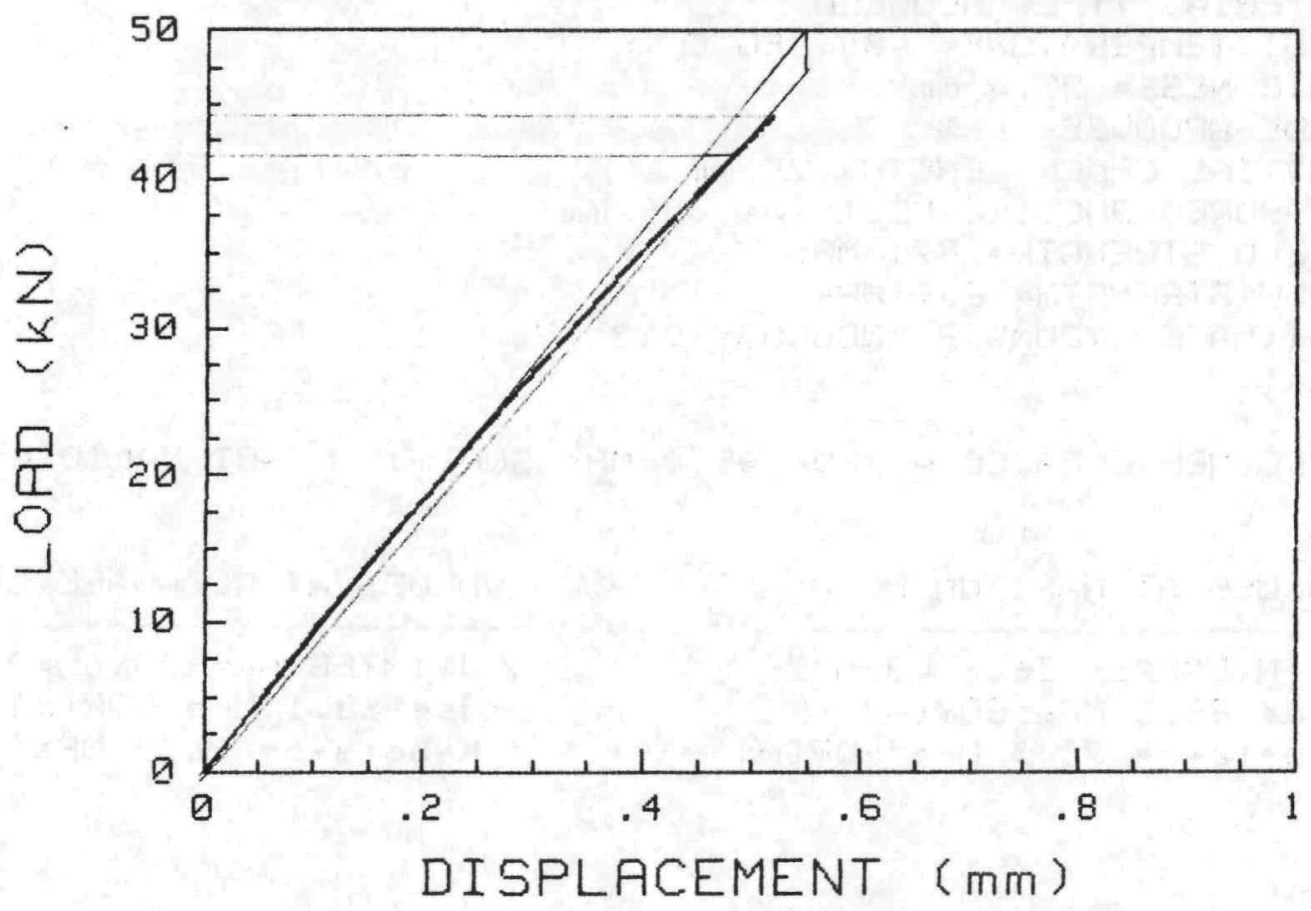

$73 W-122$

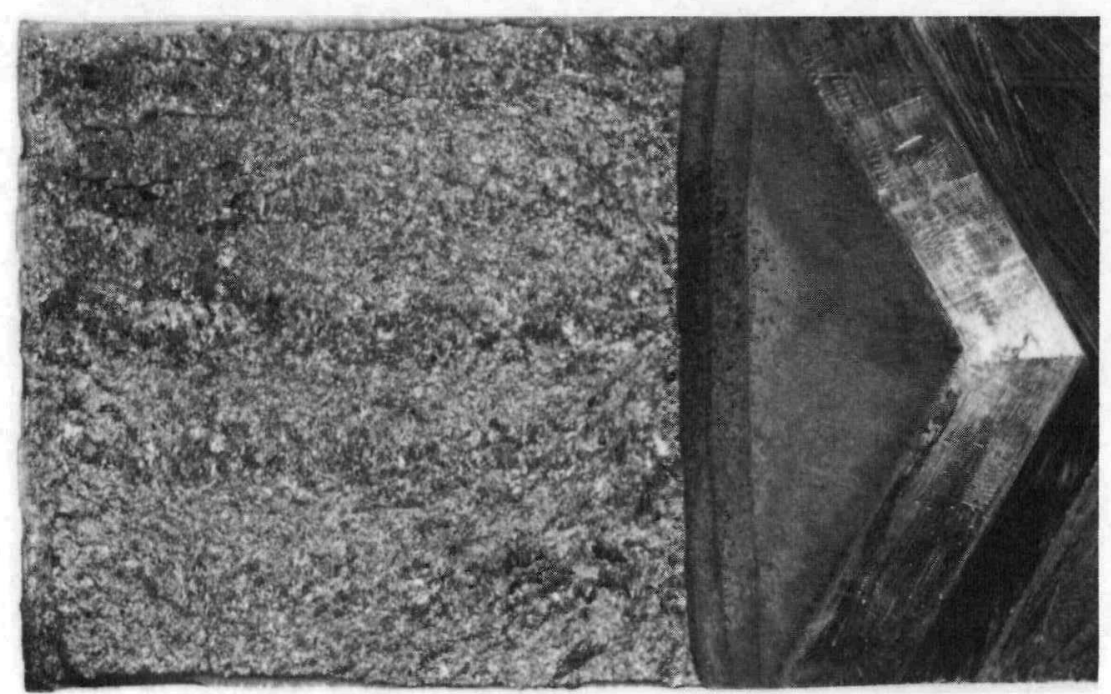




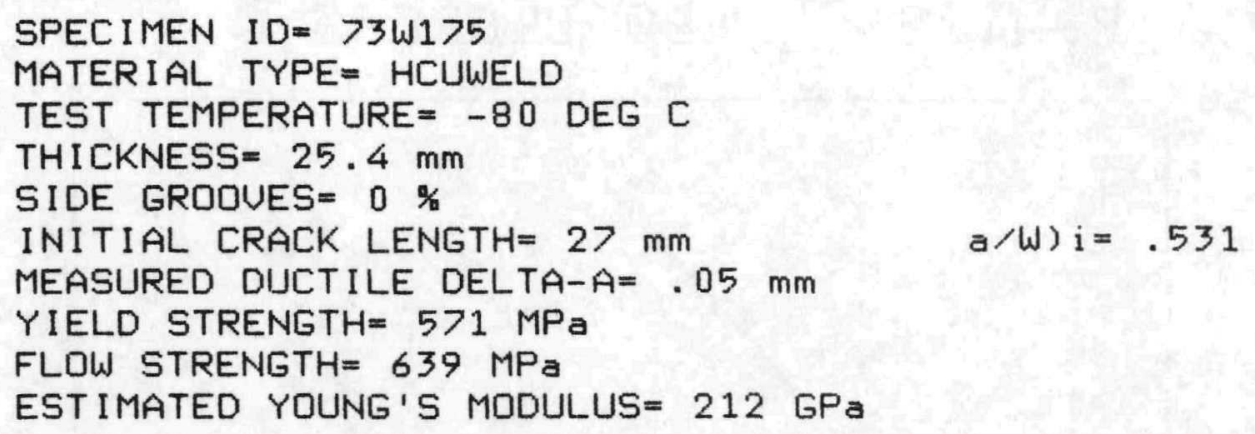

UALUES AT MAXIMUM LOAD

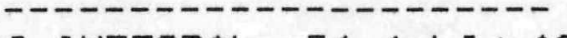

J-INTEGRAL $=36.6 \mathrm{~kJ} / \mathrm{m}^{\wedge} 2$

$\mathrm{KJc}=88.1 \mathrm{MPa} S Q R[\mathrm{~m}]$

K-bet $a-c=71.3 \mathrm{MPa} \operatorname{SQR}[\mathrm{m}]$
VALUES at CLEaUAGE LDAD

J-INTEGRAL $=36.6 \mathrm{~kJ} / \mathrm{m}^{\wedge} 2$

$K J c=88.1 \mathrm{MPa} \operatorname{SQR}[\mathrm{m}]$

$K$-beta-c $=71.3 \mathrm{MPa} \operatorname{SQR}[\mathrm{m}]$ 


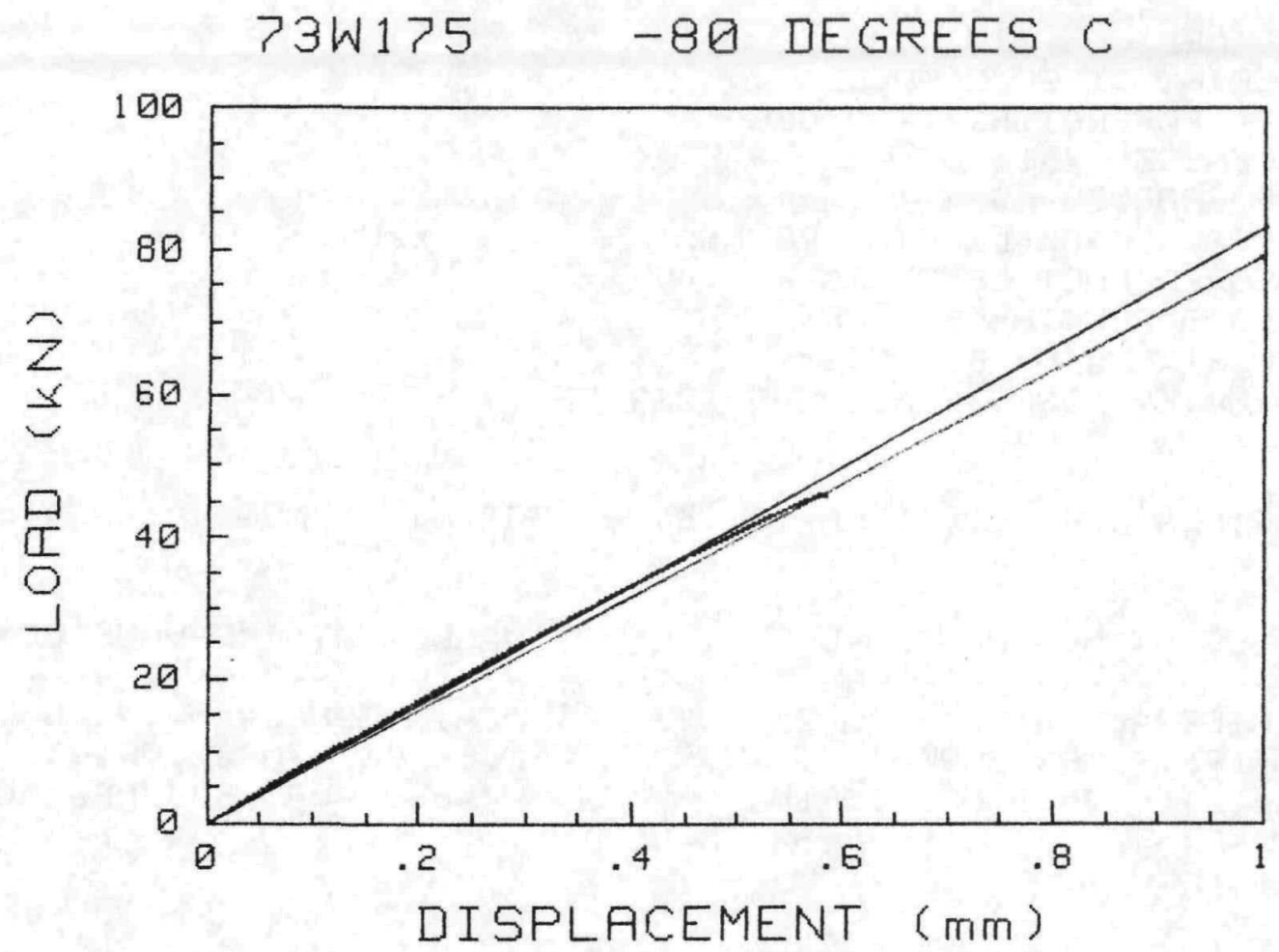

$73 W-175$

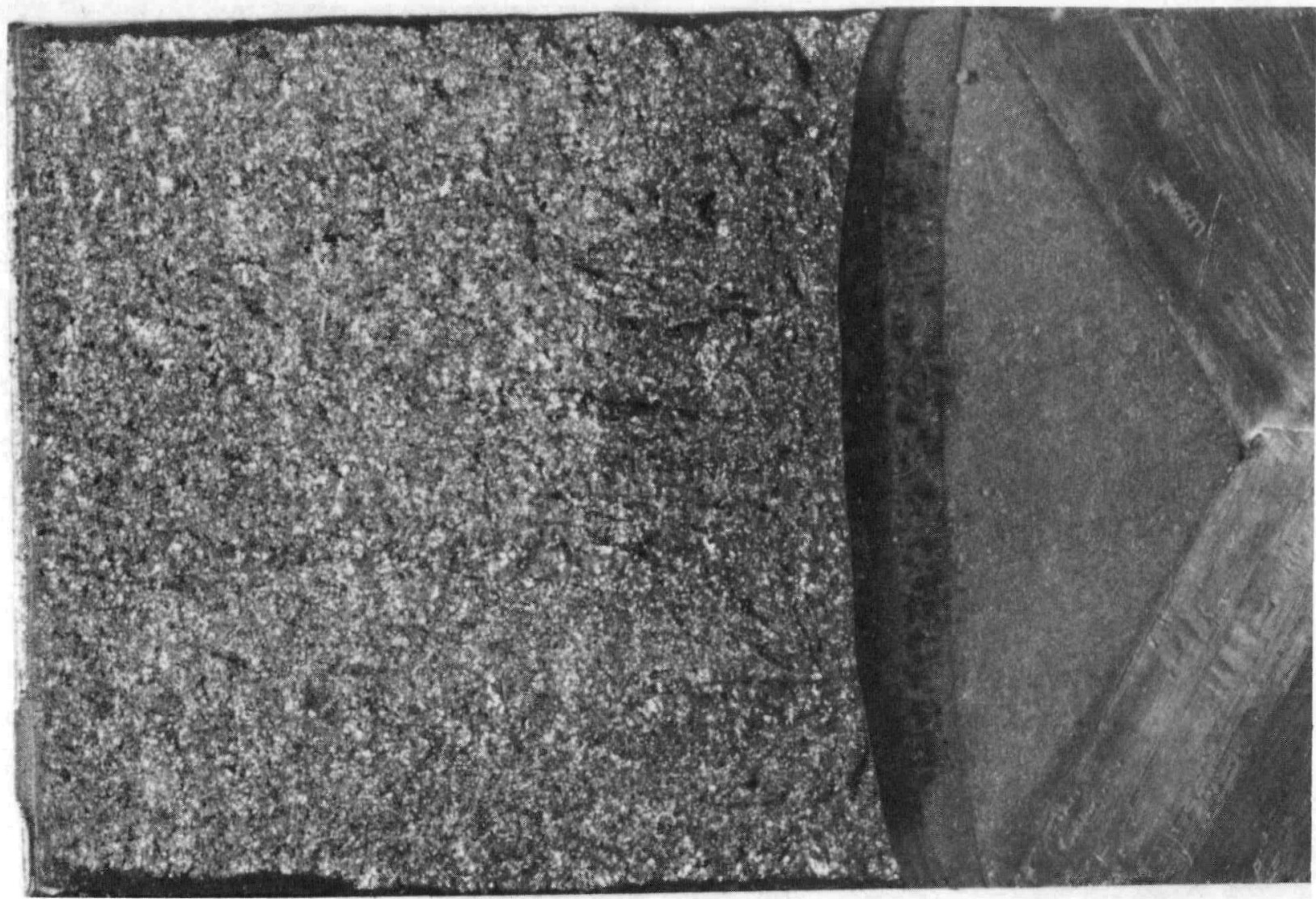


SPECIMEN ID $=73 W 145$

MATERIAL TYPE = HCIWELD

TEST TEMPERATURE $=-80$ DEG $C$

THICKNESS $=25.4 \mathrm{~mm}$

SIDE GROOUES $=0 \%$

INITIAL CRACK LENGTH $=28 \mathrm{~mm}$

$a / W) i=.551$

MEASURED DUCTILE DELTA-A $=.05 \mathrm{~mm}$

$Y I E L D$ STRENGTH $=571 \mathrm{MPa}$

FLOW STRENGTH $=639 \mathrm{MPa}$

ESTIMATED YOUNG'S MODULUS= $212 \mathrm{GPa}$

SPECIMEN CLEAUED AT KQ $=92.6 \mathrm{MPa} S Q R[\mathrm{~m}]$ (NOT UALID KIc)

UALUES AT MAXIMUM LOAD

J-INTEGRAL $=44.4 \mathrm{~kJ} / \mathrm{m}^{\wedge} 2$

$K J c=96.9 \mathrm{MPa} \operatorname{SQR}[\mathrm{m}]$

K-beta-c $=75.3 \mathrm{MPa} \operatorname{SQR}[\mathrm{m}]$
UALUES at CLEaUAGE LDAD

J-INTEGRAL $=44.4 \mathrm{~kJ} / \mathrm{m}^{\wedge} 2$

$K \cdot J c=96.9 \mathrm{MPa} \operatorname{SQR}[\mathrm{m}]$

K-bet $a-c=75.3 \mathrm{MPa} S Q R[\mathrm{~m}]$ 
F-23

ORNL-DWG 92-11323

73W145 -80 DEGREES C

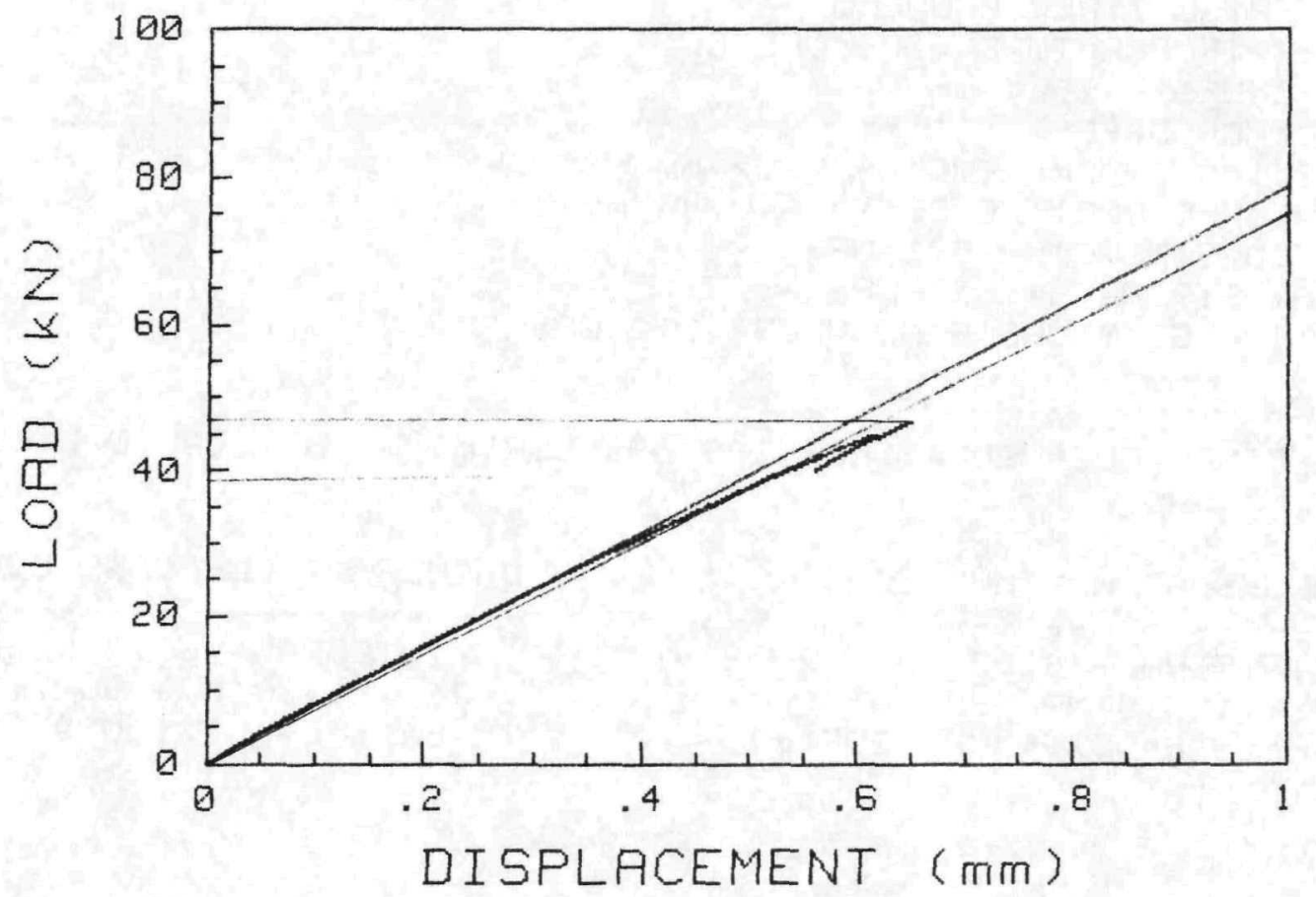

$73 W-145$

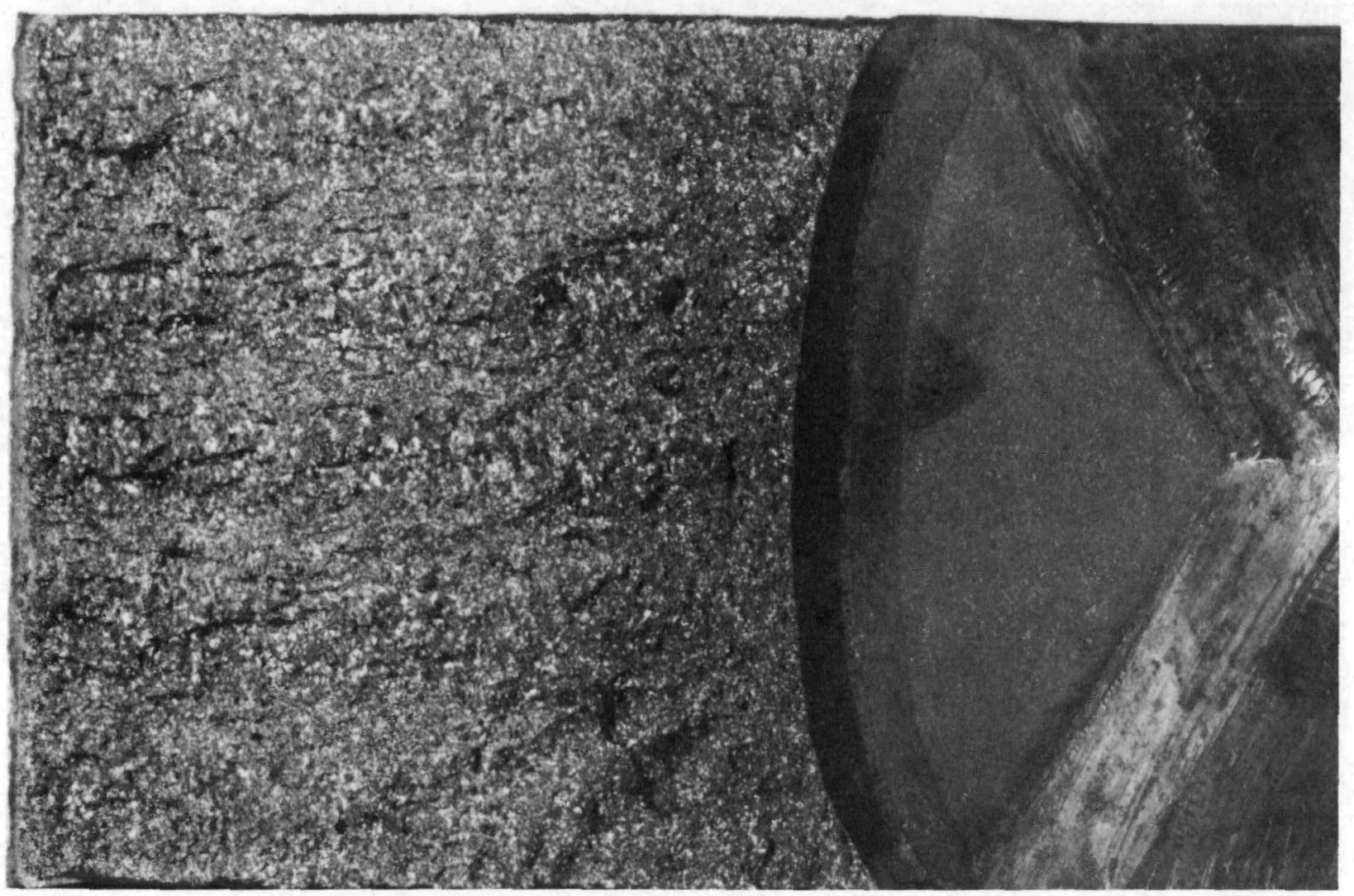

NUREG/CR-5913 
SPECIMEN ID $=73 W 174$

MATERIAL TYPE $=$ HCUWELD

TEST TEMPERATURE $=-80$ DEG $C$

THICKNESS $=25.4 \mathrm{~mm}$

SIDE GRDOUES = $0 \%$

INITIAL CRACK LENGTH $=26.7 \mathrm{~mm}$

MEASURED DUCTILE DELTA-A $=.05 \mathrm{~mm}$

$a / W)_{i}=.525$

$Y$ IELD STRENGTH $=571 \mathrm{MPa}$

FLOW STRENGTH $=639 \mathrm{MPa}$

ESTIMATED YOUNG'S MODULUS $=212 \mathrm{GPa}$

SPECIMEN CLEAUED AT $K Q=105 \mathrm{MPa} S Q R[\mathrm{~m}]$ (NDT UALID $\mathrm{KIc}$ )

UALUES AT MAXIMUM LDAD

J-INTEGRAL $=61.2 \mathrm{~kJ} / \mathrm{m}^{\wedge} 2$

$K J c=113.8 \mathrm{MPa} S Q R[\mathrm{~m}]$

K-beta-c $=82.1 \mathrm{MPa} \operatorname{SQR}[\mathrm{m}]$
UALUES AT CLEaUage LDAD

J-INTEGRAL $=61.2 \mathrm{~kJ} / \mathrm{m}^{\wedge} 2$

$\mathrm{KJ} \mathrm{c}=113.8 \mathrm{MPa} \operatorname{SQR}[\mathrm{m}]$

K-beta-c $=82.1$ MPa $\operatorname{SQR}[\mathrm{m}]$ 
F-25

ORNL-DWG 92-11324

73W174 -80 DEGREES C

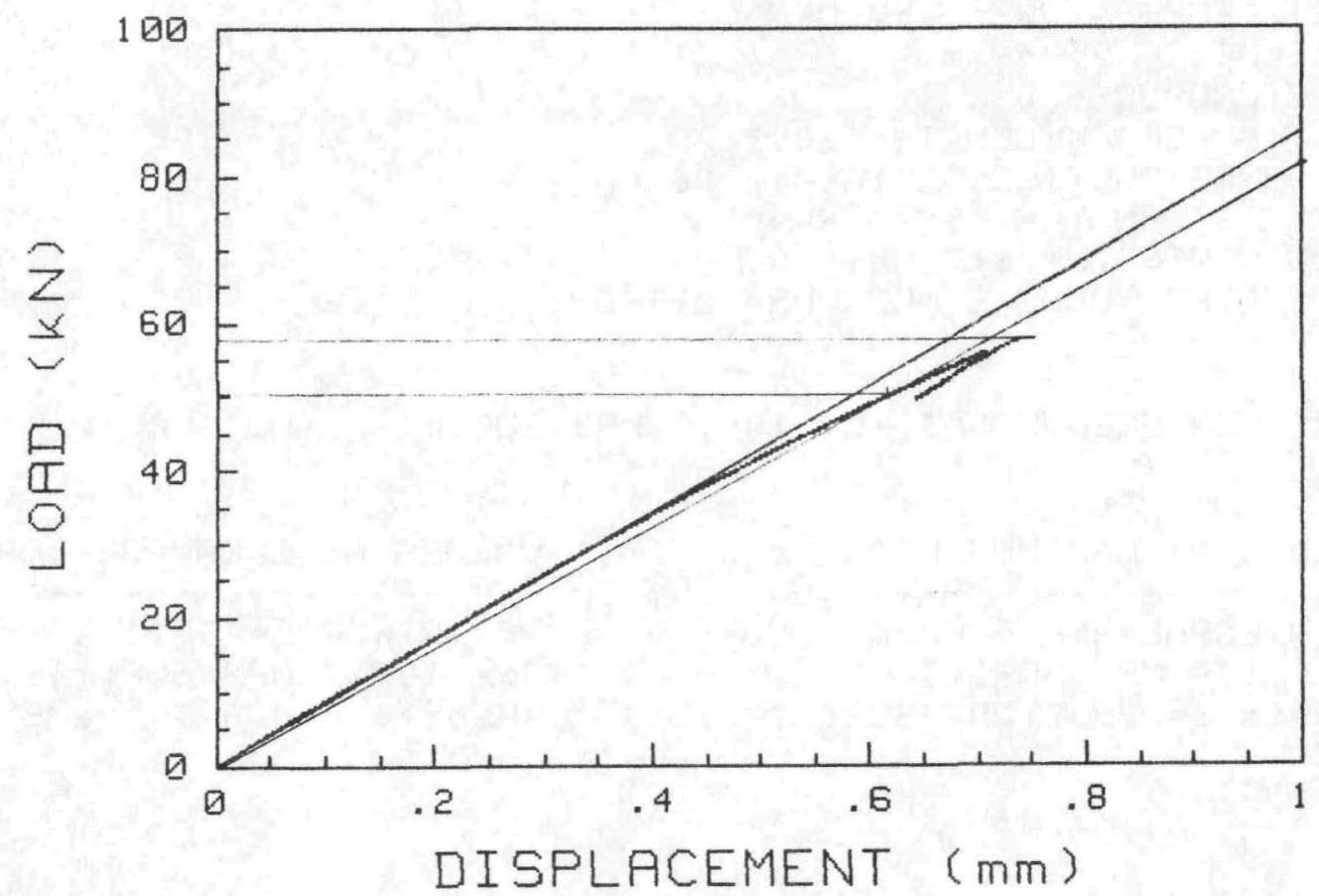

$73 W-174$

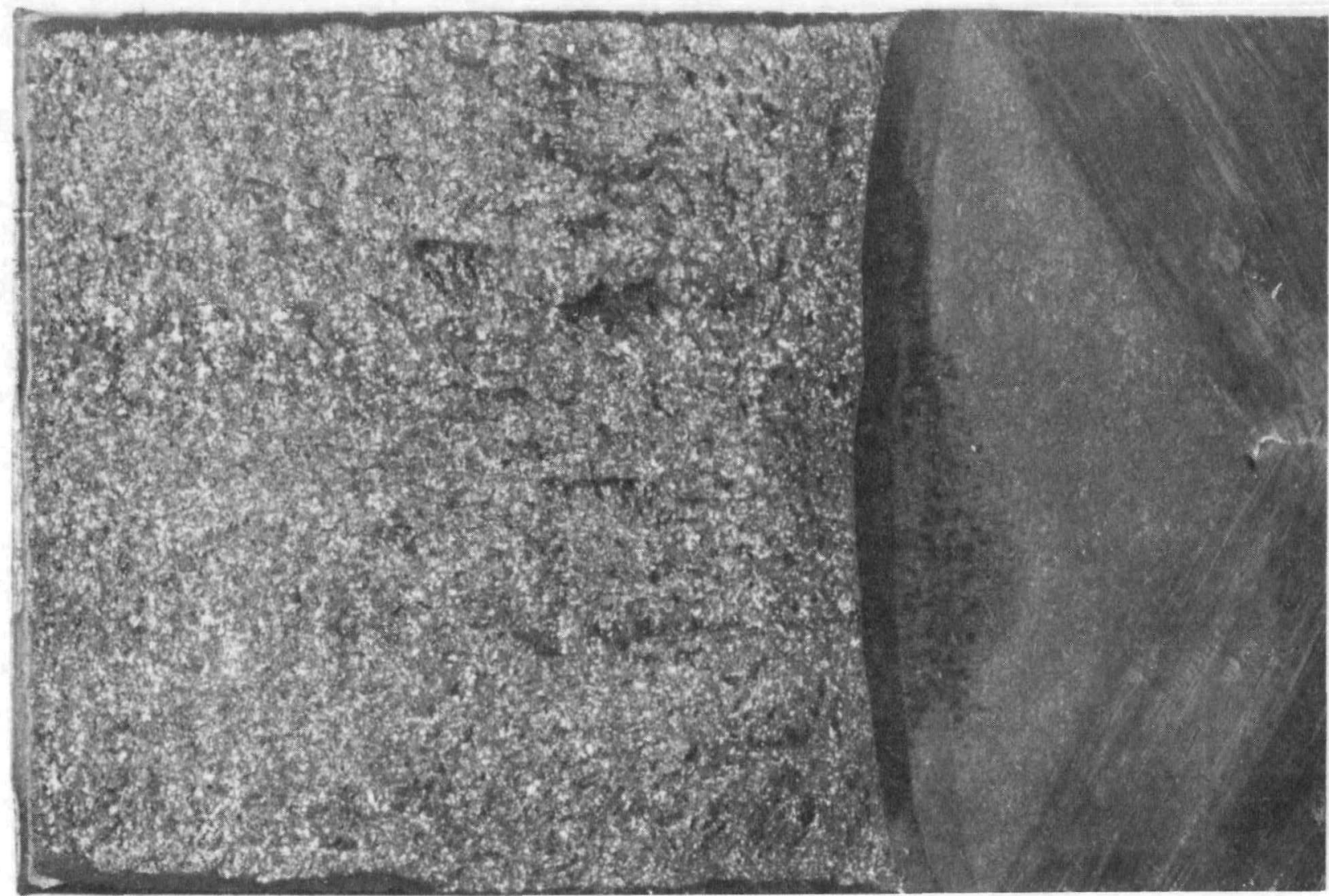


SPECIMEN ID $=73 W 129$

MATERIAL TYPE = HCUWELD

TEST TEMPERATURE $=-59$ DEG C

THICKNESS $=25.4 \mathrm{~mm}$

SIDE GROOUES $=0 \%$

INITIAL CRACK LENGTH $=28.3 \mathrm{~mm}$

$a / W\rangle i=.557$

MEASURED DUCTILE DELTA-A $=.06 \mathrm{~mm}$

$Y$ IELD STRENGTH $=550 \mathrm{MPa}$

FLOW STRENGTH $=617 \mathrm{MPa}$

ESTIMATED YOUNG'S MODULUS $=211 \mathrm{GPa}$

SPECIMEN CLEAUED AT KQ $=100.8 \mathrm{MPa} S Q R[\mathrm{~m}]$ (NOT UALID KIc)

WALUES AT MAXIMUM LOAD

J-INTEGRAL $=56.5 \mathrm{~kJ} / \mathrm{m}^{\wedge} 2$

$\mathrm{KJc}=109 \mathrm{MPa} \operatorname{SQR}[\mathrm{m}]$

$K$-bet $a-c=78.8 \mathrm{MPa} \operatorname{SQR}[\mathrm{m}]$
UALUES AT CLEAUAGE LDAD

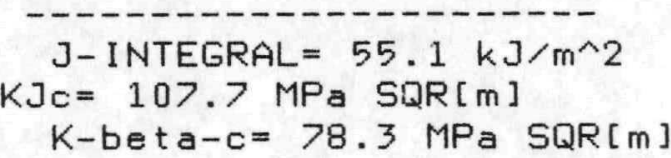


F-27

ORNL-DWG 92-11325

73W129 -59 DEGREES C

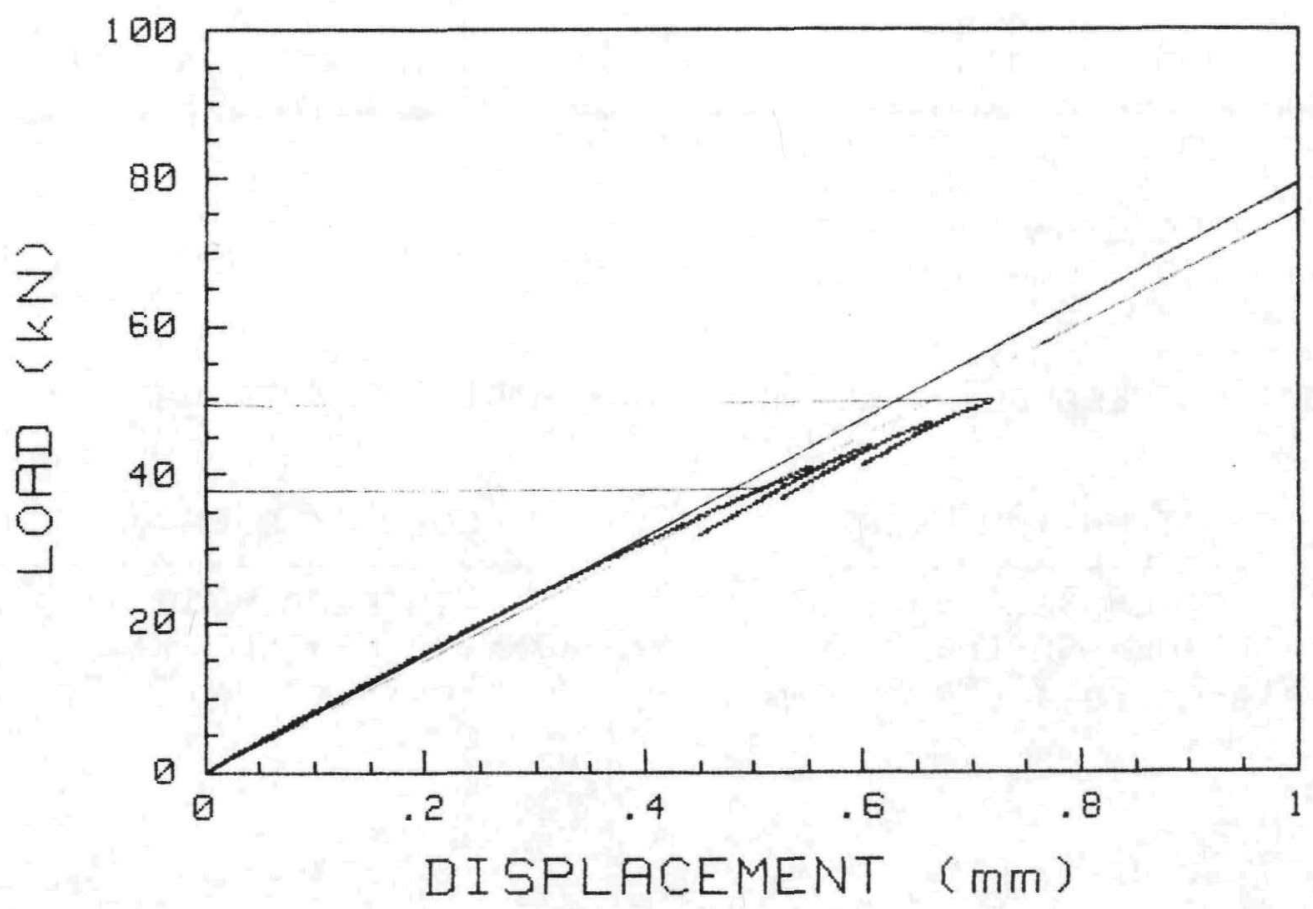

$73 W-129$

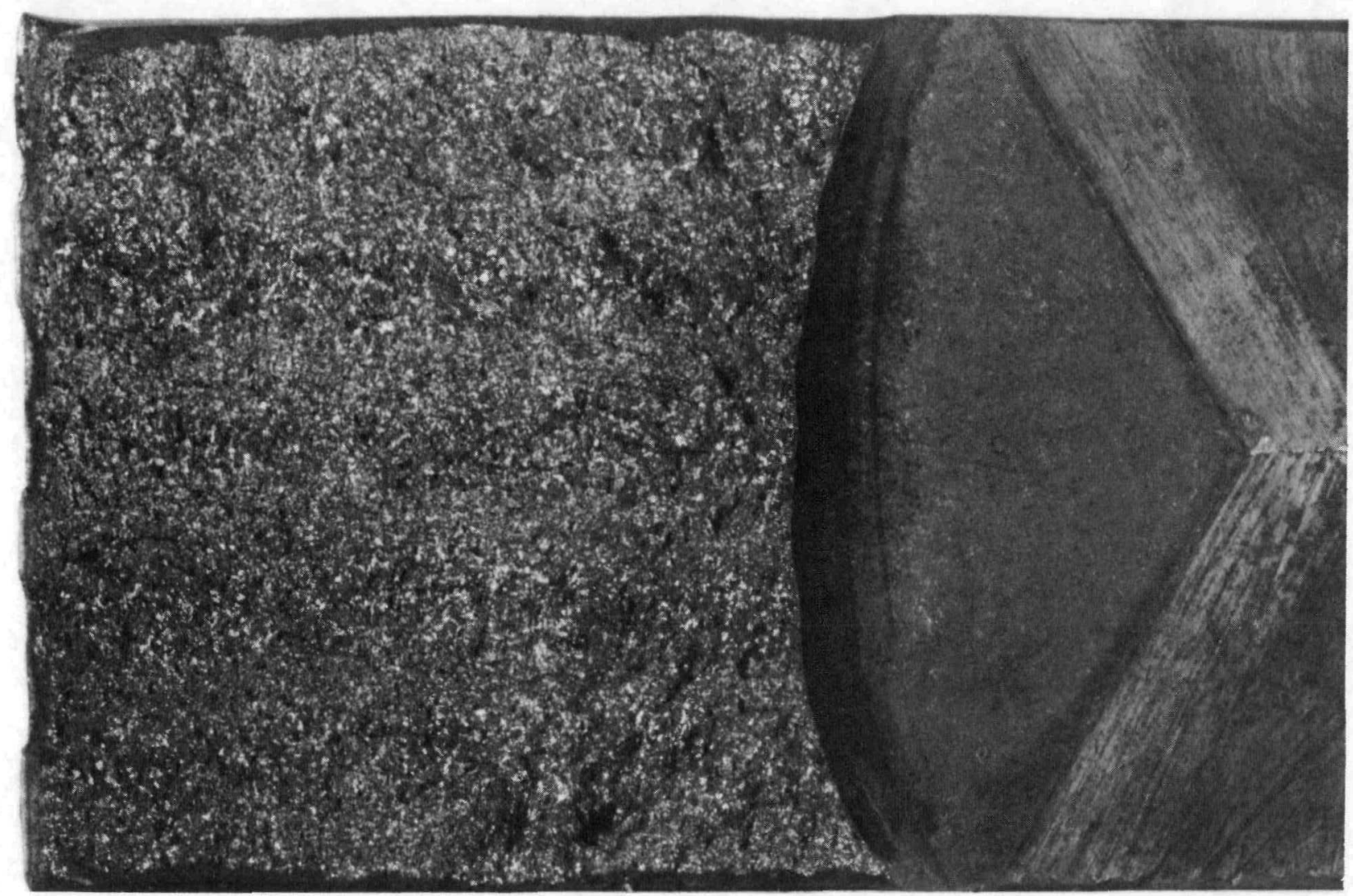




\section{F-29}

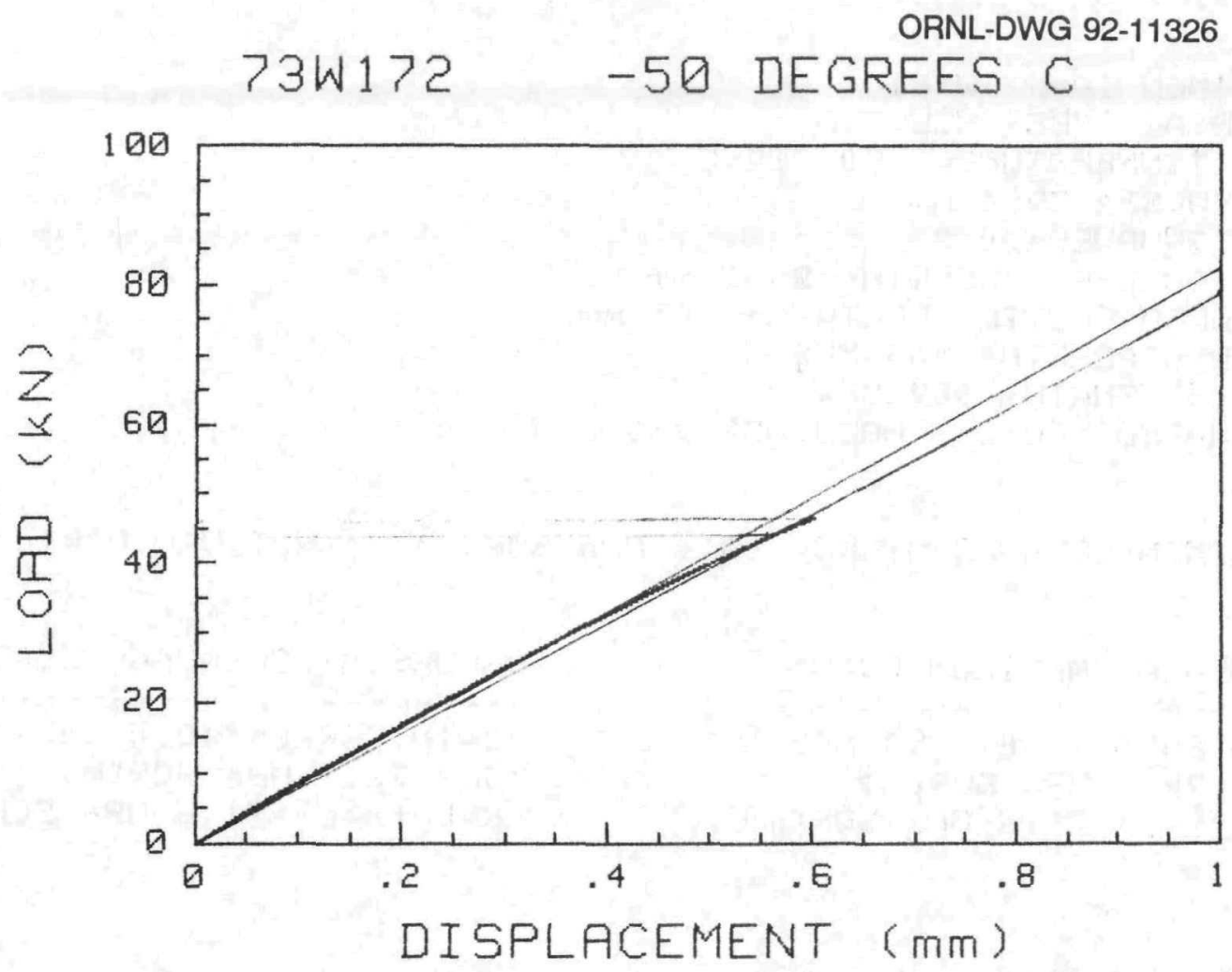

$73 W-172$

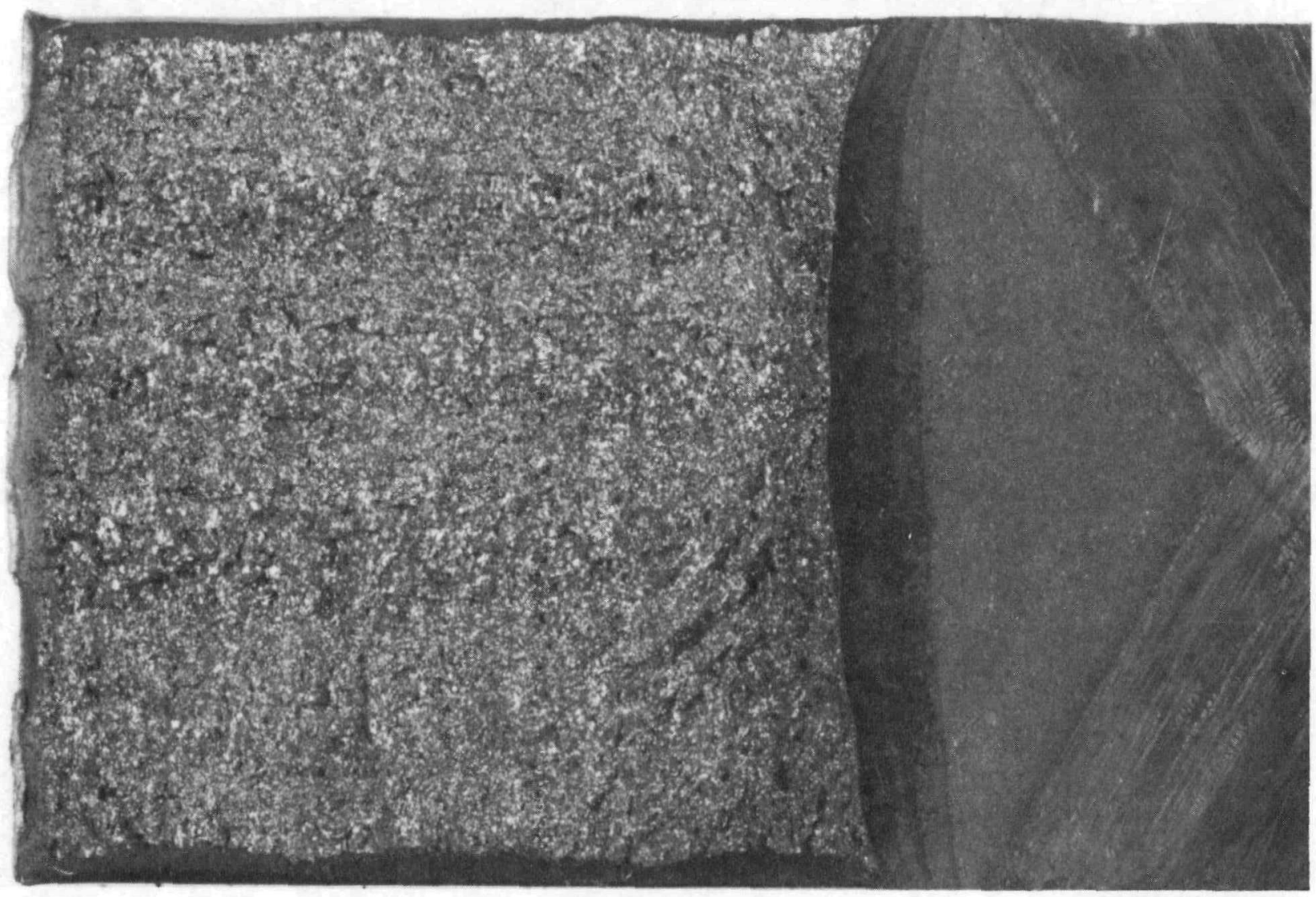


SPECIMEN ID $=73 W 181$

MATERIAL TYPE $=$ HCUWELD

TEST TEMPERATURE $=-50$ DEG C

THICKNESS $=25.4 \mathrm{~mm}$

SIDE GROOUES = $0 \%$

INITIAL CRACK LENGTH $=26.7 \mathrm{~mm}$

$a / W) i=.526$

MEASURED DUCTILE DELTA-A $=.05 \mathrm{~mm}$

YIELD STRENGTH $=543 \mathrm{MPa}$

FLOW STRENGTH $=609 \mathrm{MPa}$

EST IMATED YOUNG'S MODULUS $=210 \mathrm{GPa}$

SPECIMEN CLEAUED AT KQ $=88.6 \mathrm{MPa}$ SQR $[\mathrm{m}]$ (NOT UALID KIc)

UALUES AT MAXIMUM LOAD

J-INTEGRAL $=40.4 \mathrm{~kJ} / \mathrm{m}^{\wedge} 2$

$\mathrm{KJ} c=92.1 \mathrm{MPa} \operatorname{SQR}[\mathrm{m}]$

$\mathrm{K}$-bet $a-c=71.6 \mathrm{MPa} \operatorname{SQR}[\mathrm{m}]$
UALUES AT CLEAUAGE LOAD

J-INTEGRAL $=40.4 \mathrm{~kJ} / \mathrm{m}^{\wedge} 2$

$\mathrm{KJc}=92.1 \mathrm{MPa} \operatorname{SQR}[\mathrm{m}]$

K-bet $a-c=71.6 \mathrm{MPa} S Q R[\mathrm{~m}]$ 
F-31

ORNL-DWG 92-11327

73W181 -50 DEGREES C

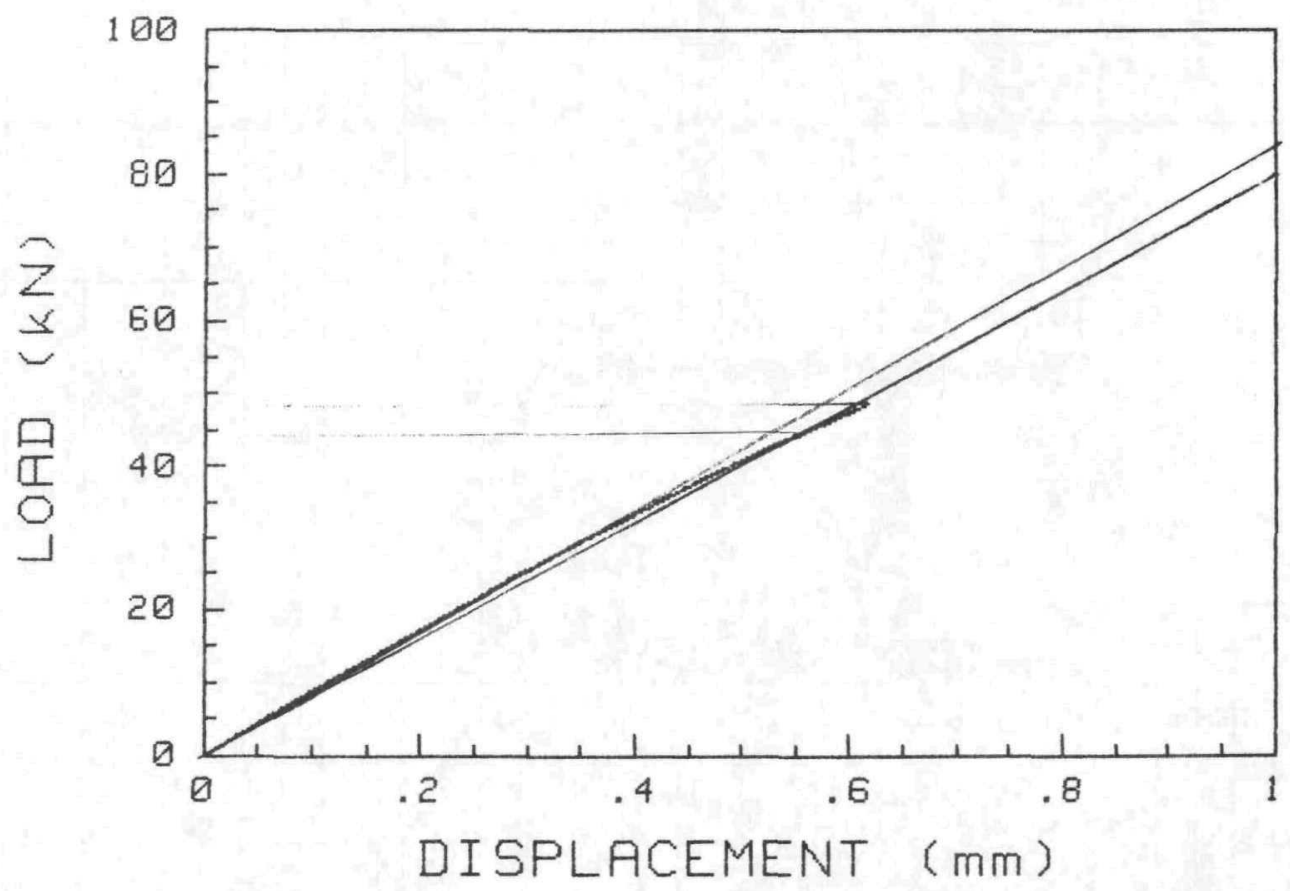

$73 W-181$

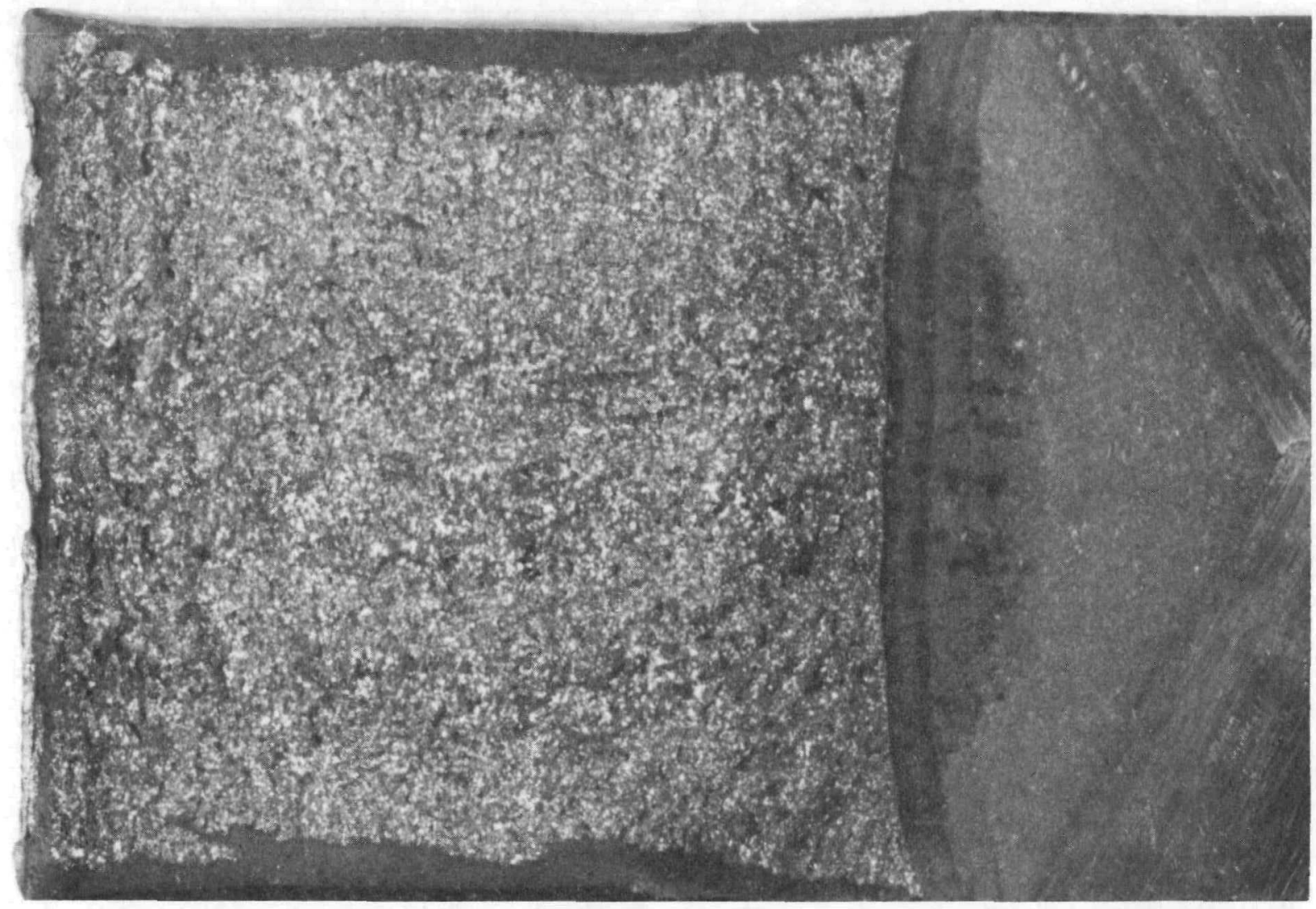




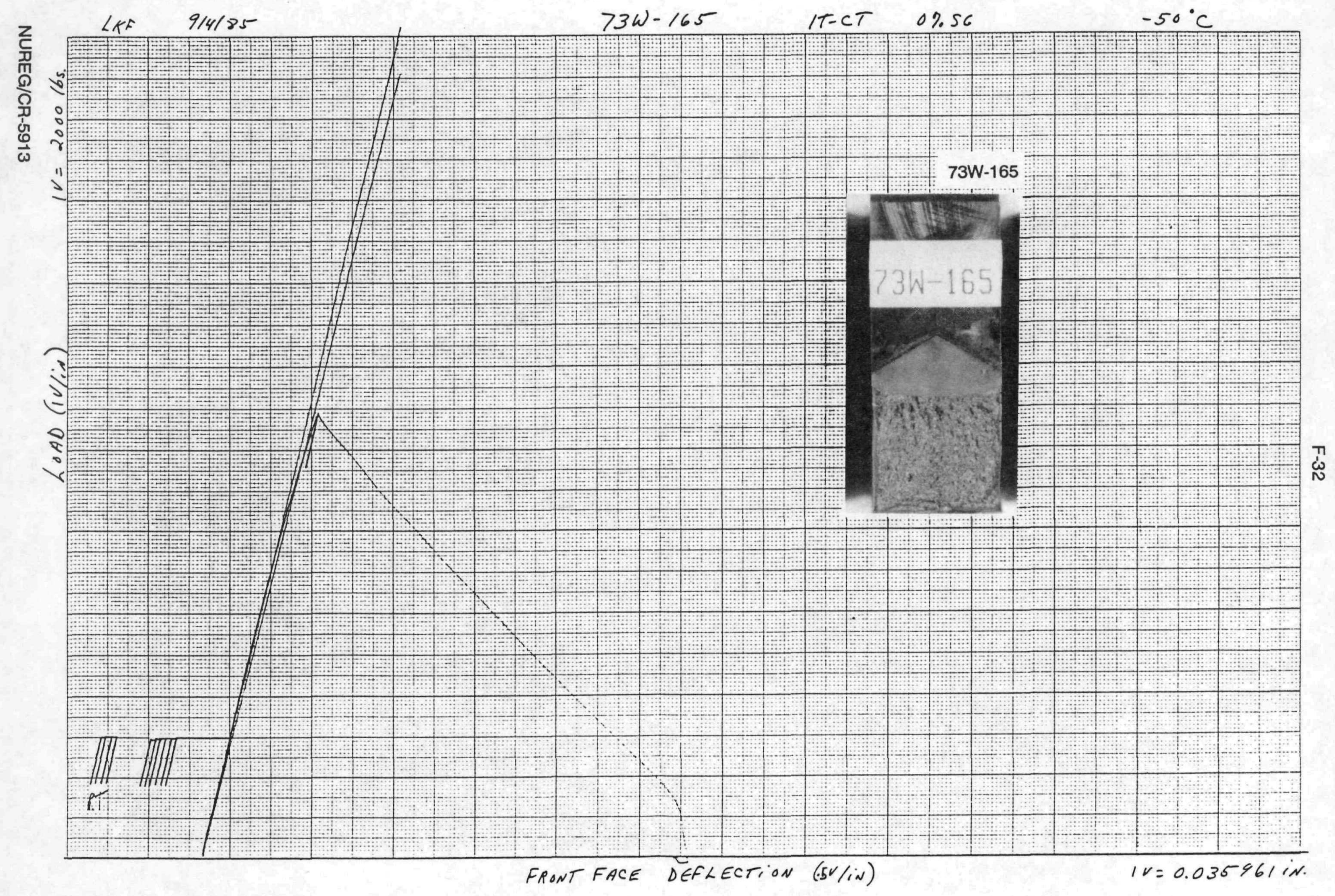


$73 \omega-167$

$-58^{\circ} \%$

$8 / 31 / 85$

MRP/BHM

IT-CT 07.56

2/3/8 $5 * F$

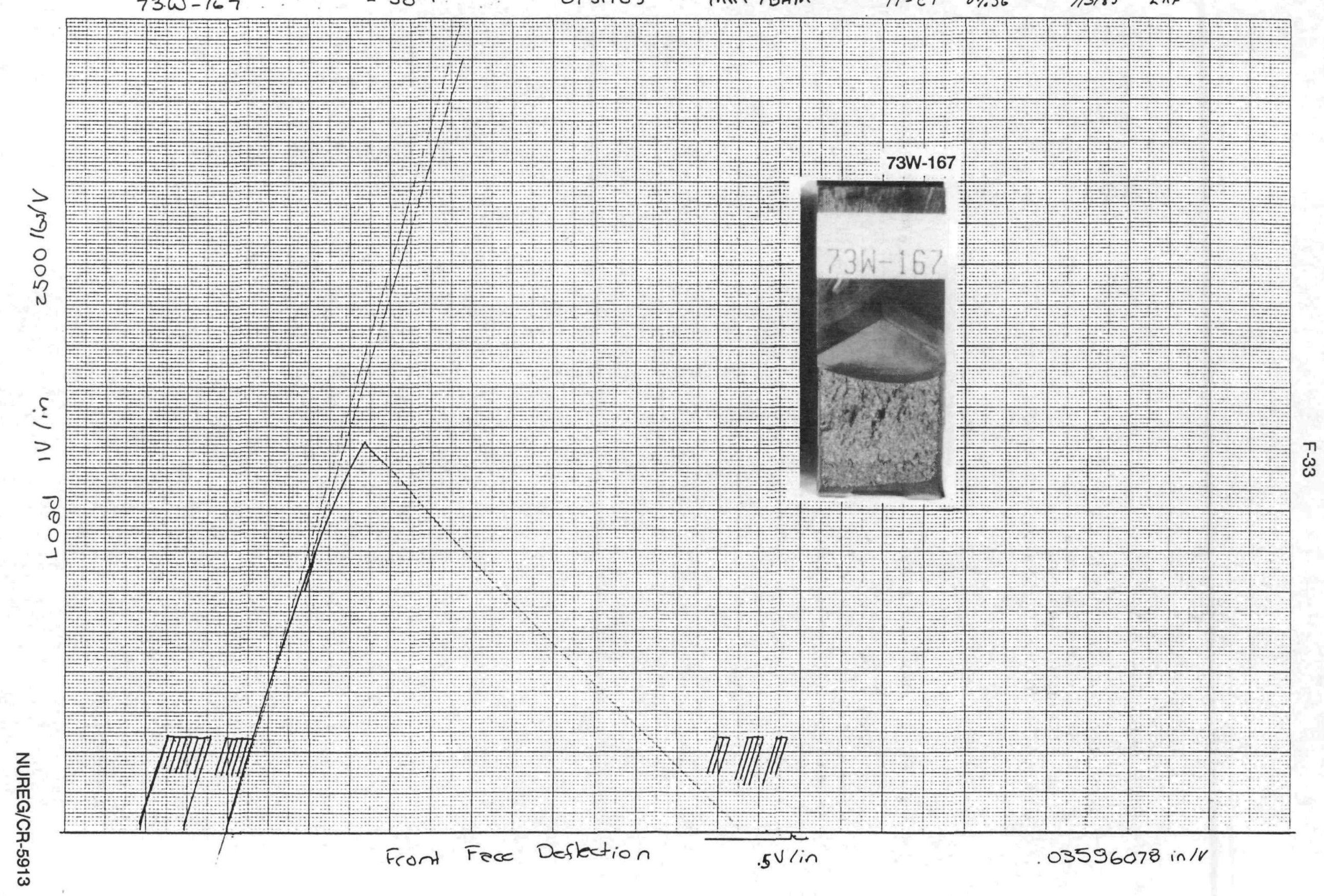


SPECIMEN ID $=73 W 180$

MATERIAL TYPE = HCUWELD

TEST TEMPERATURE $=-50$ DEG $C$

THICKNESS $=25.4 \mathrm{~mm}$

SIDE GROOUES $=0 \%$

INITIAL CRACK LENGTH $=26.7 \mathrm{~mm}$

MEASURED DUCTILE DELTA-A $=.06 \mathrm{~mm}$

$a / W) i=.526$

YIELD STRENGTH $=543 \mathrm{MPa}$

FLOW STRENGTH $=609 \mathrm{MPa}$

ESTIMATED YOUNG'S MODULUS $=210 \mathrm{GPa}$

SPECIMEN CLEAUED AT $K Q=109.3 \mathrm{MPa} S Q R[\mathrm{~m}]$ (NOT UALID $\mathrm{KIc}$ )

UALUES AT MAXIMUM LOAD

J-INTEGRAL $=72.7 \mathrm{~kJ} / \mathrm{m}^{\wedge} 2$

$\mathrm{KJ} c=123.5 \mathrm{MPa} \operatorname{SQR}[\mathrm{m}]$

K-beta-c $=83.3 \mathrm{MPa} \operatorname{SQR}[\mathrm{m}]$
Ualues at CLEaUage load

J-INTEGRAL $=72.5 \mathrm{~kJ} / \mathrm{m}^{\wedge} 2$

$\mathrm{KJ} c=123.4 \mathrm{MPa} \operatorname{SQR}[\mathrm{m}]$

K-beta-c $=83.3 \mathrm{MPa} \operatorname{SQR}[\mathrm{m}]$ 
F-35

\section{ORNL-DWG 92-11328}

73W180 -50 DEGREES C

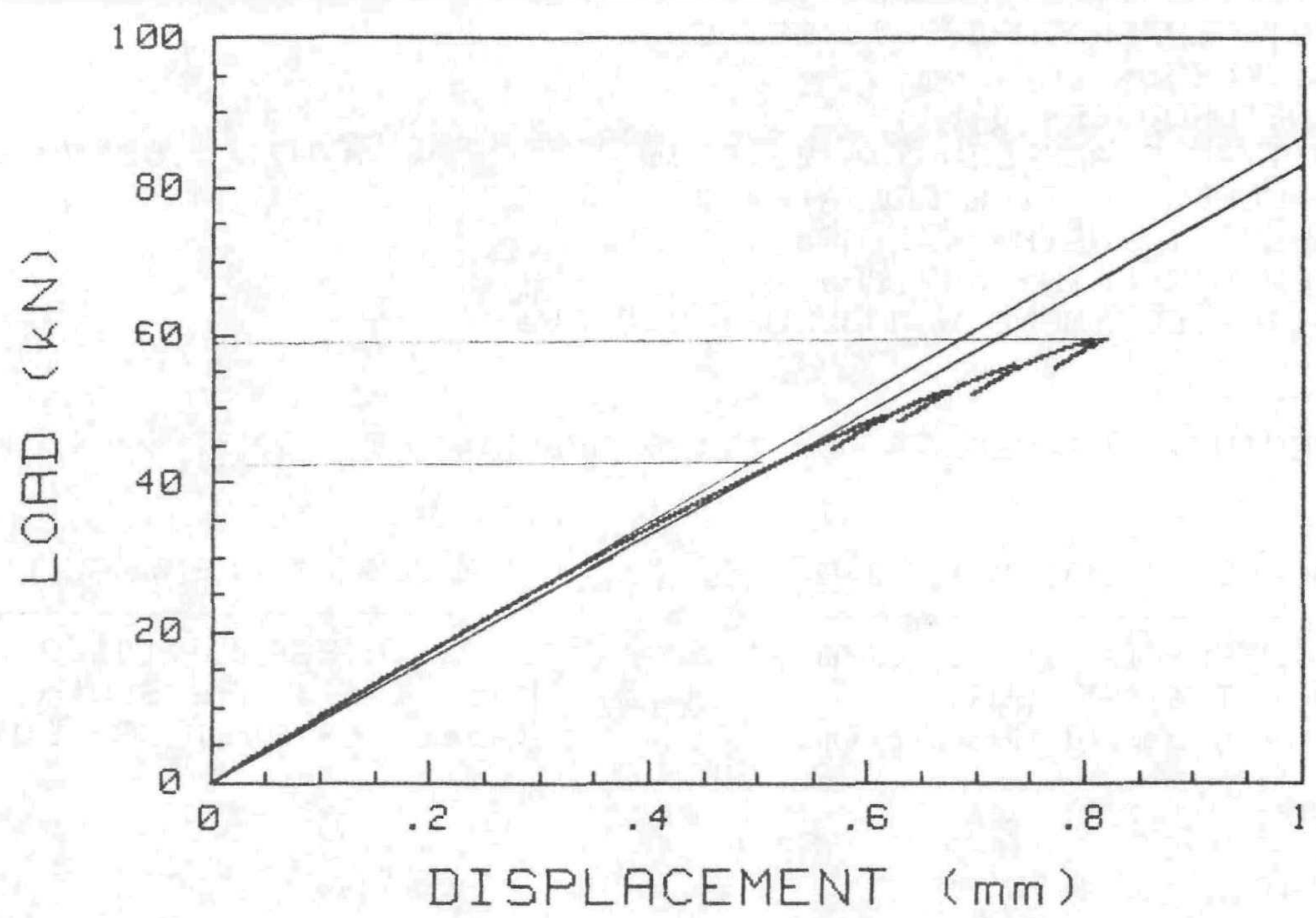

$73 W-180$

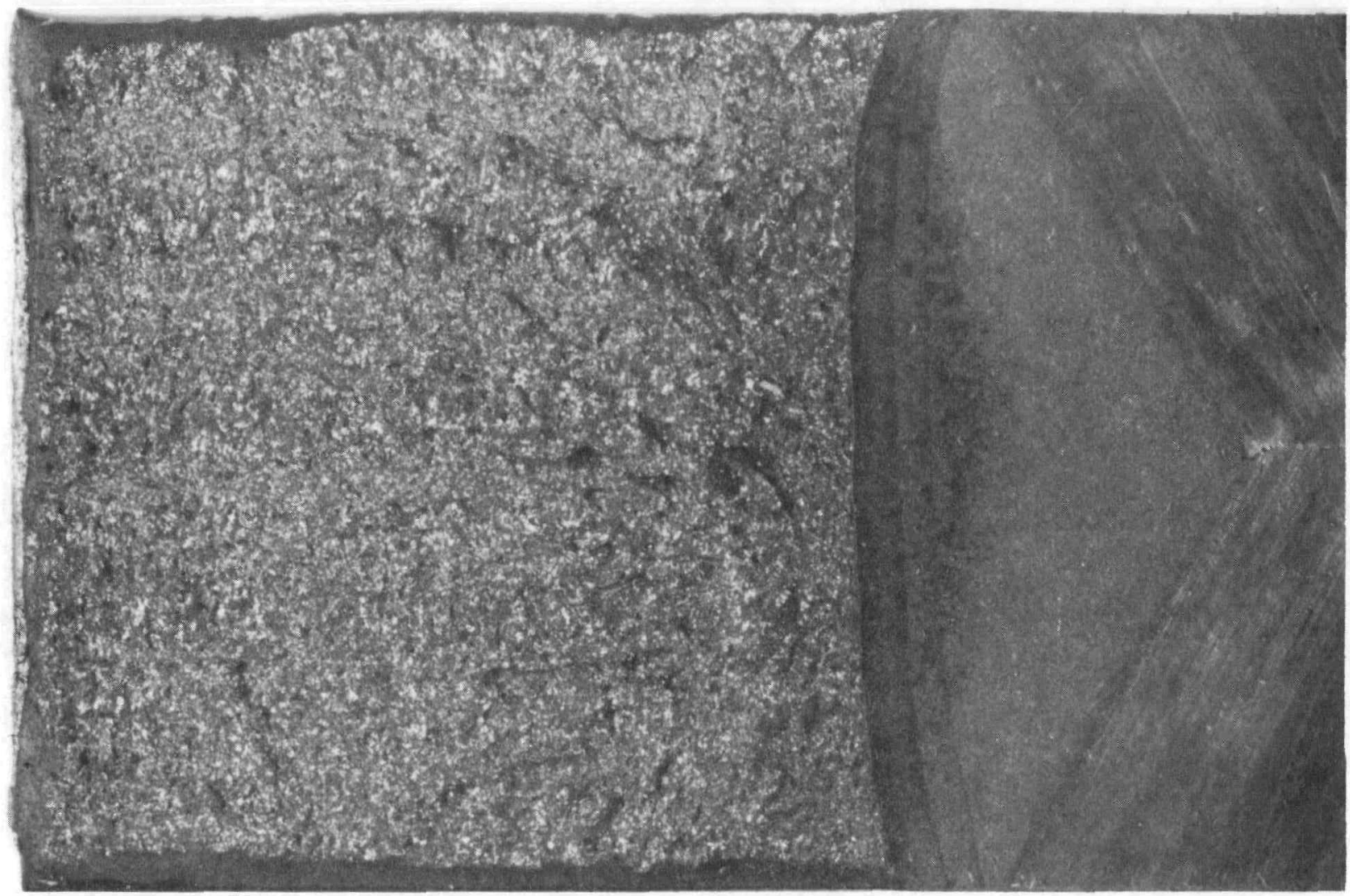


SPECIMEN ID $=73 \omega 176$

MATERIAL TYPE = HCUWELD

TEST TEMPERATURE $=-50$ DEG $C$

THICKNESS $=25.4 \mathrm{~mm}$

SIDE GROOUES $=0 \%$

INITIAL CRACK LENGTH $=26.5 \mathrm{~mm}$

MEASURED DUCTILE DELTA-A $=.1 \mathrm{~mm}$

$a / W) i=.522$

YIELD STRENGTH $=543 \mathrm{MPa}$

FLOW STRENGTH $=609 \mathrm{MPa}$

EST IMATED YOUNG'S MODLLUS $=210 \mathrm{GPa}$

SPECIMEN CLEAUED AT KQ $=121.5 \mathrm{MPa} S Q R[\mathrm{~m}]$ (NOT UALID KIC)

UALUES AT MAXIMUM LOAD

-

J-INTEGRAL $=101.5 \mathrm{~kJ} / \mathrm{m}^{\wedge} 2$

$K \cdot]_{c}=146 \mathrm{MPa} \operatorname{SQR}[\mathrm{m}]$

$K$-beta-c $=90 \mathrm{MPa} S Q R[\mathrm{~m}]$
UALUES AT CLEAUAGE LOAD

J-INTEGRAL $=101.9 \mathrm{~kJ} / \mathrm{m}^{\wedge} 2$

$K \cdot] c=146 \cdot 3 \mathrm{MPa} \operatorname{SQR}[\mathrm{m}]$

$K$-beta-c $=90.1 \mathrm{MPa} S Q R[\mathrm{~m}]$ 
F-37

ORNL-DWG 92-11329

73W176 -50 DEGREES C

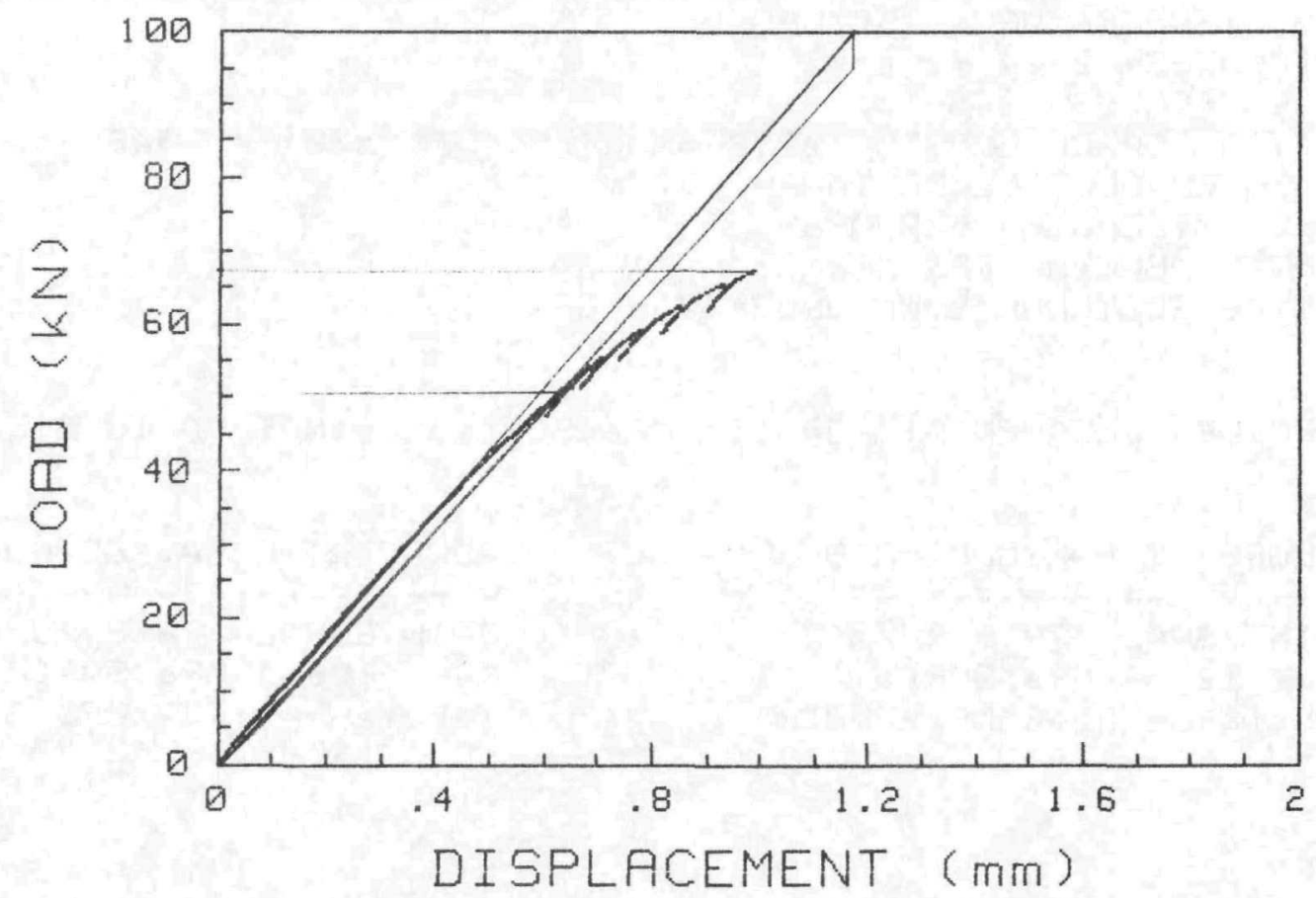

73W-176

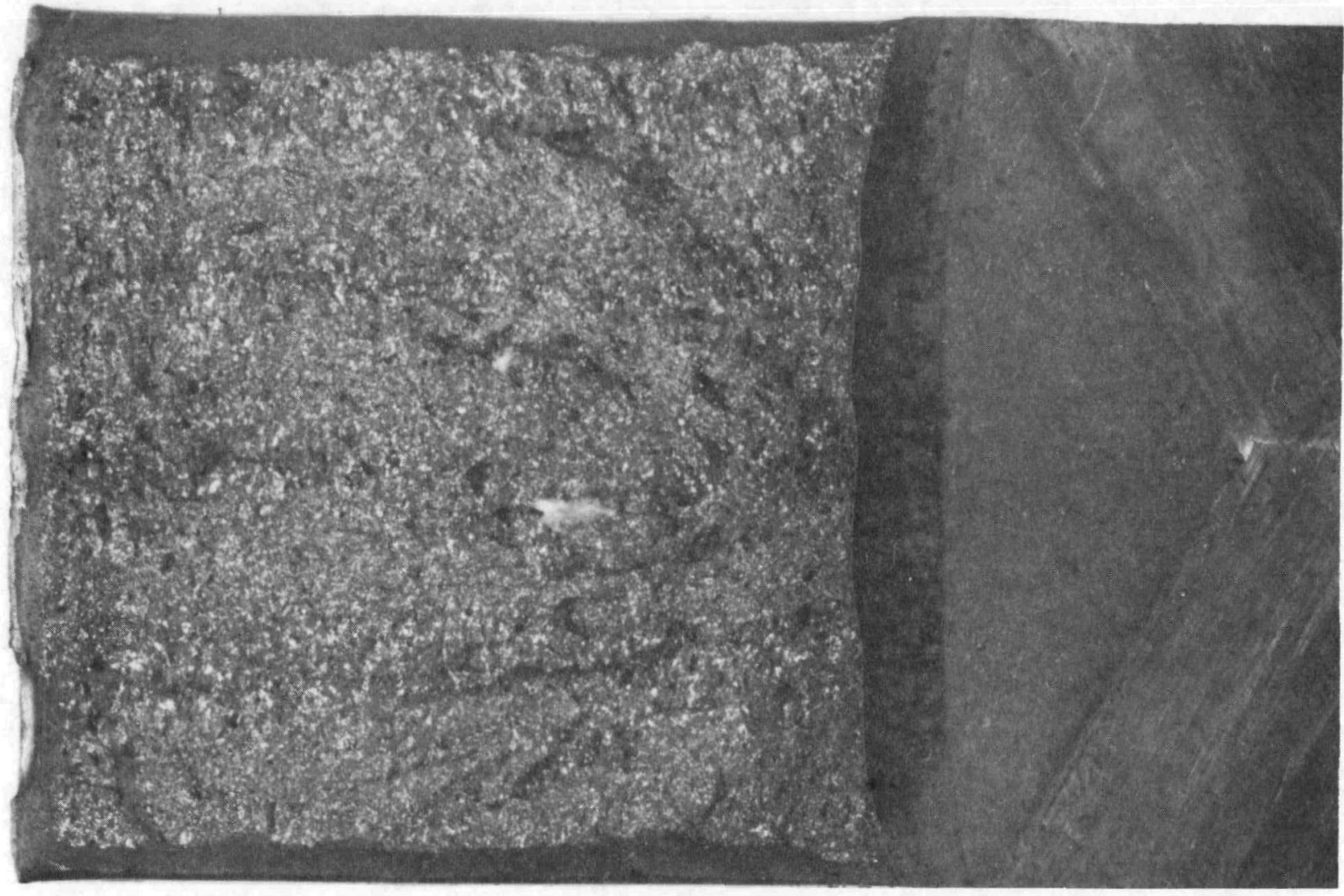


SPEC IMEN ID $=73 W 151$

MATERIAL TYPE $=$ HCUWELD

TEST TEMPERATURE $=-30$ DEG $C$

THICKNESS $=25.4 \mathrm{~mm}$

SIDE GRODUES $=0 \%$

INITIAL CRACK LENGTH $=27.8 \mathrm{~mm}$

MEASURED DUCTILE DELTA-A $=.09 \mathrm{~mm}$

$a / W\rangle \mathrm{i}=.548$

YIELD STRENGTH $=529 \mathrm{MPa}$

FLOW STRENGTH $=593 \mathrm{MPa}$

ESTIMATED YOUNG'S MODULUS $=209 \mathrm{GPa}$

SPECIMEN CLEAUED AT KQ= $110 \mathrm{MPa}$ SQR $[\mathrm{m}]$ (NOT VALID $K I c$ )

UALUES AT MAXIMUM LDAD

-

J-INTEGRAL $=79.5 \mathrm{~kJ} / \mathrm{m}^{\wedge} 2$

$\mathrm{KJ} c=128.8 \mathrm{MPa} \operatorname{SQR}[\mathrm{m}]$

$K$-bet $a-c=83.8 \mathrm{MPa} \operatorname{SQR}[\mathrm{m}]$

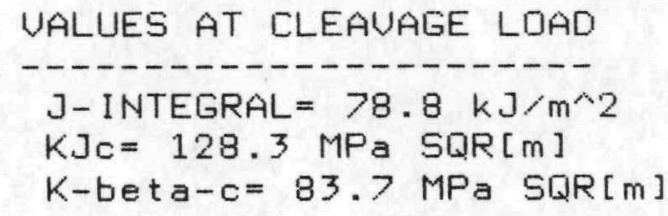




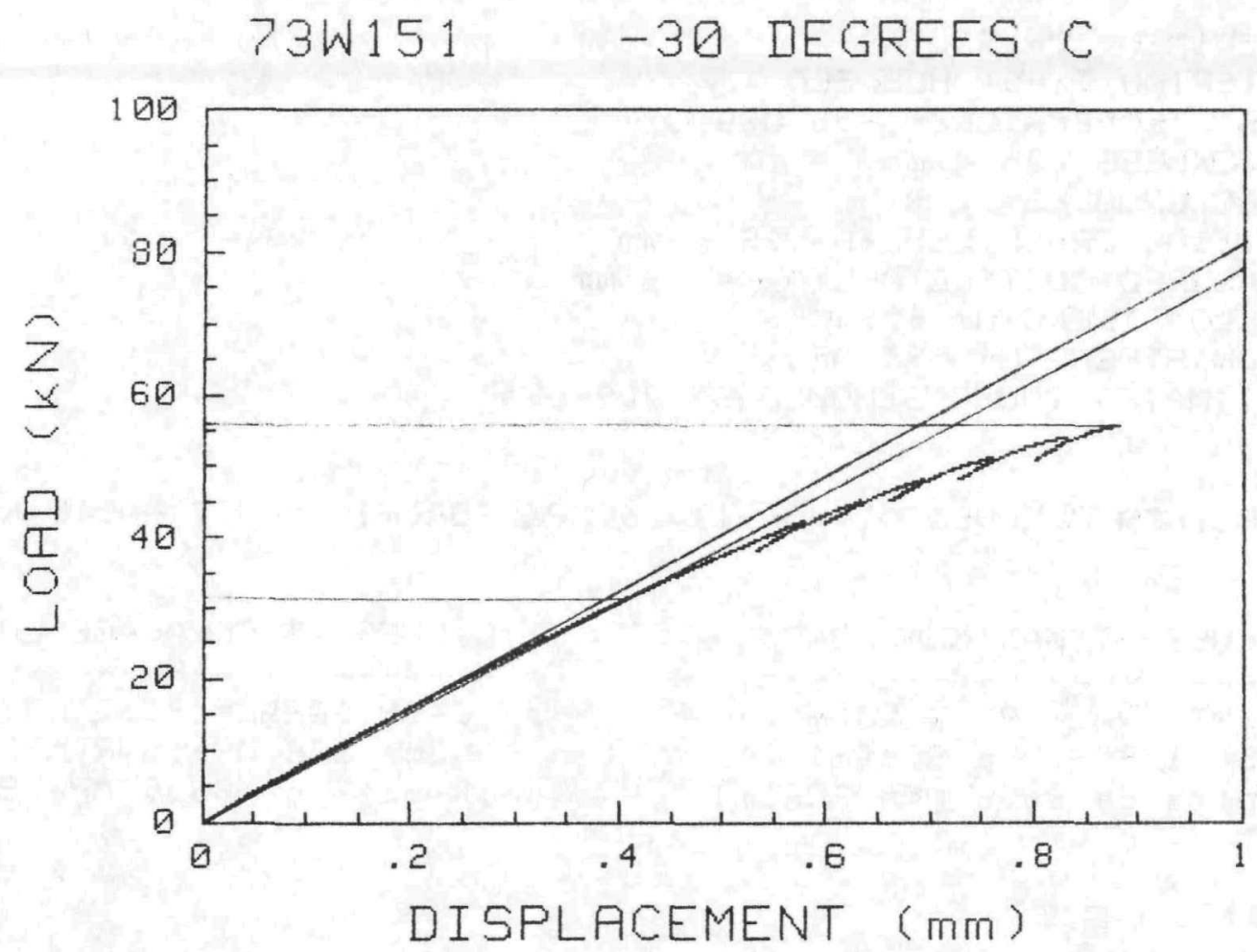

$73 W-151$

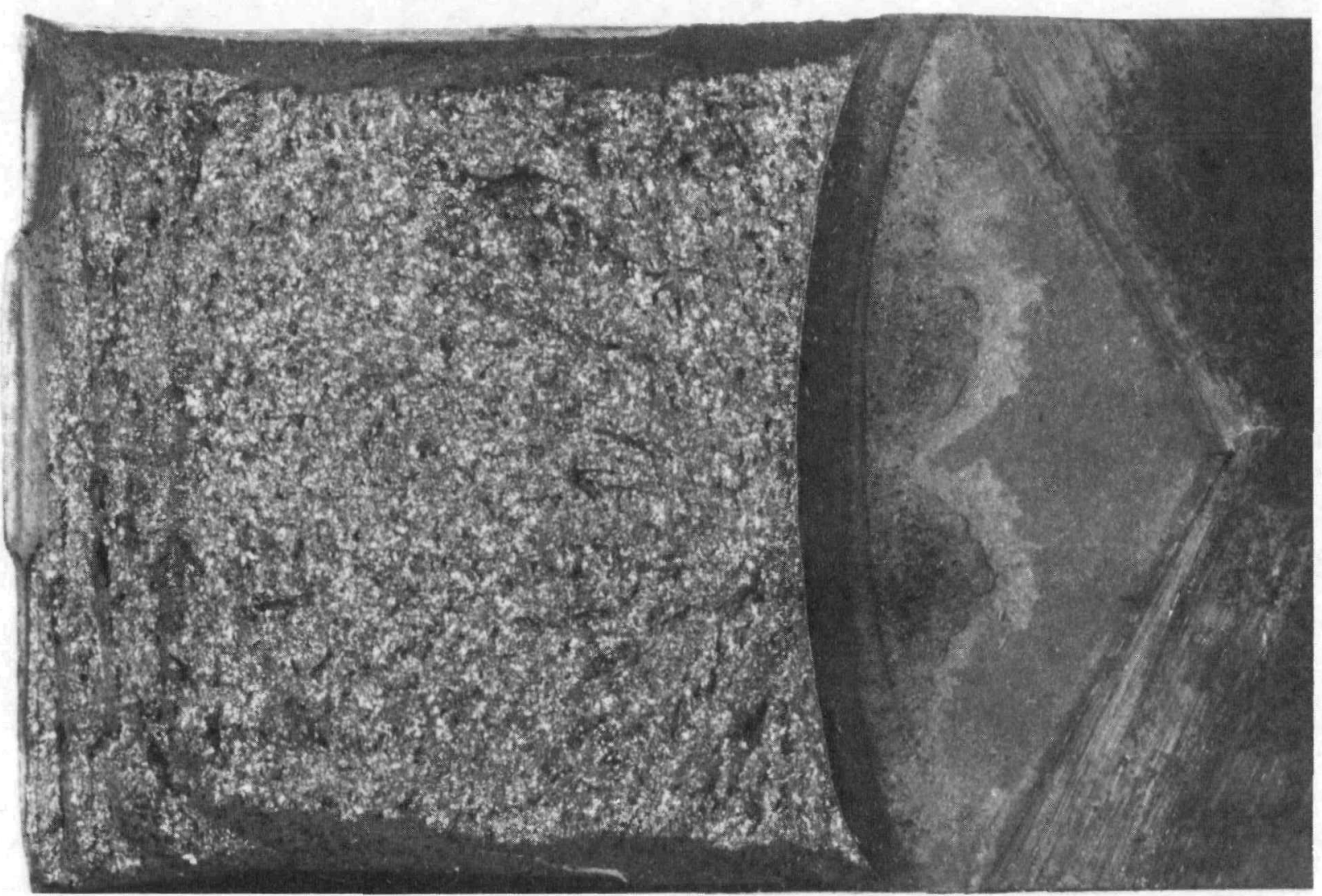


SPECIMEN ID $=73 W 149$

MATERIAL TYPE $=$ HCUWELD

TEST TEMPERATURE $=-30$ DEG C

THICKNESS $=25.4 \mathrm{~mm}$

SIDE GROOUES $=0 \%$

INITIAL CRACK LENGTH $=28.5 \mathrm{~mm}$

$a / W) i=.561$

MEASURED DUCTILE DELTA-A $=.1 \mathrm{~mm}$

YIELD STRENGTH $=529 \mathrm{MPa}$

FLDW STRENGTH $=593 \mathrm{MPa}$

ESTIMATED YOUNG'S MODULUS $=209 \mathrm{GPa}$

SPECIMEN CLEAUED AT KQ= $114.6 \mathrm{MPa}$ SQR $[\mathrm{m}]$ (NOT VALID KIC)

UALUES AT MAXIMUM LOAD

J-INTEGRAL $=91.4 \mathrm{~kJ} / \mathrm{m}^{\wedge} 2$

$\mathrm{KJc}=138.2 \mathrm{MPa} \operatorname{SQR}[\mathrm{m}]$

$K$-beta-c $=86.6 \mathrm{MPa}$ SQR $[\mathrm{m}]$
UALUES at CLEAUAGE LDAD

$J$-INTEGRAL $=91.2 \mathrm{~kJ} / \mathrm{m}^{\wedge} 2$

$\mathrm{KJ} c=138 \mathrm{MPa} \operatorname{SQR}[\mathrm{m}]$

K-beta-c $=86.5 \mathrm{MPa} S Q R[\mathrm{~m}]$ 
F-41

ORNL-DWG 92-11331

?3W149 -30 DEGREES C

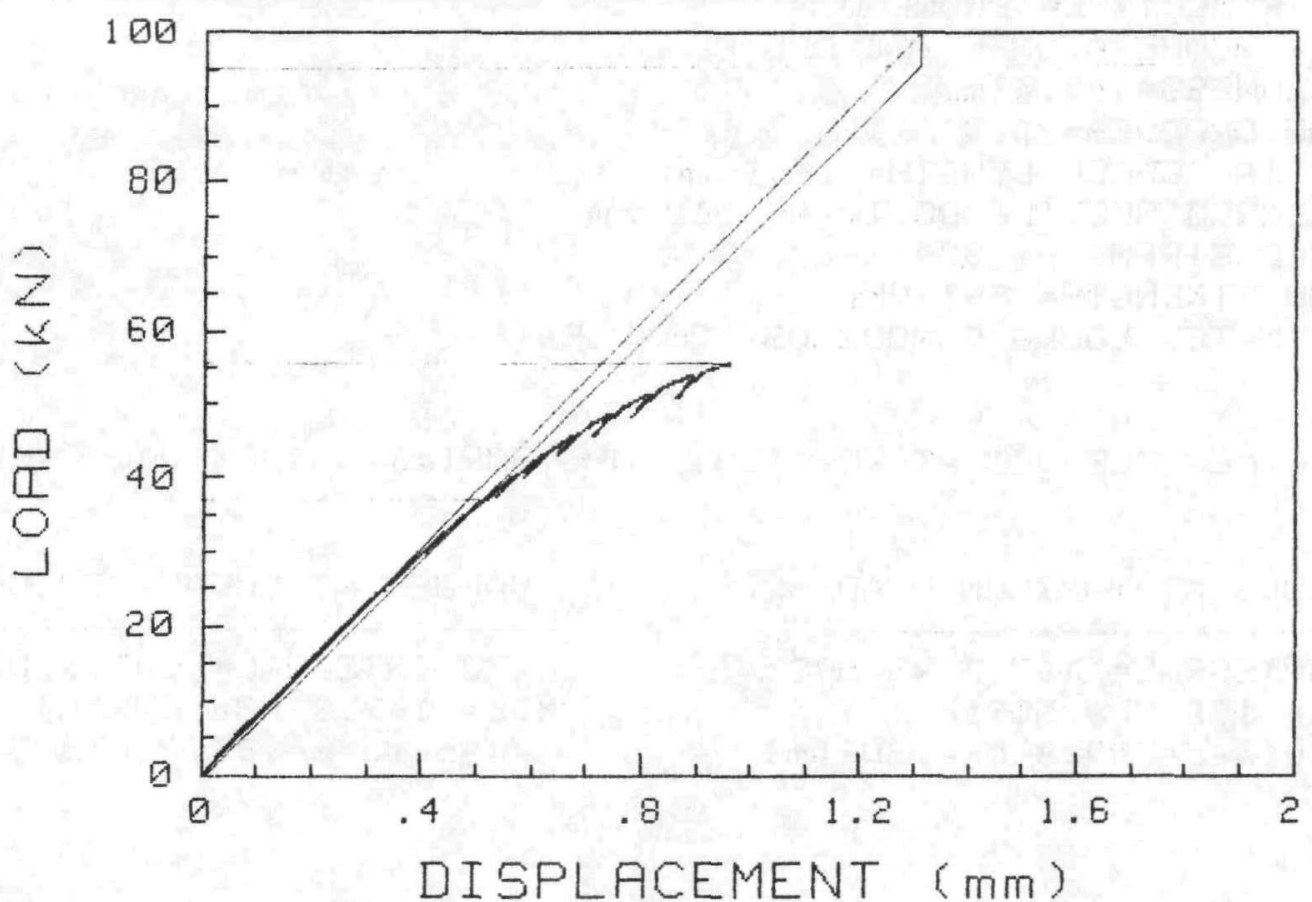

$73 W-149$

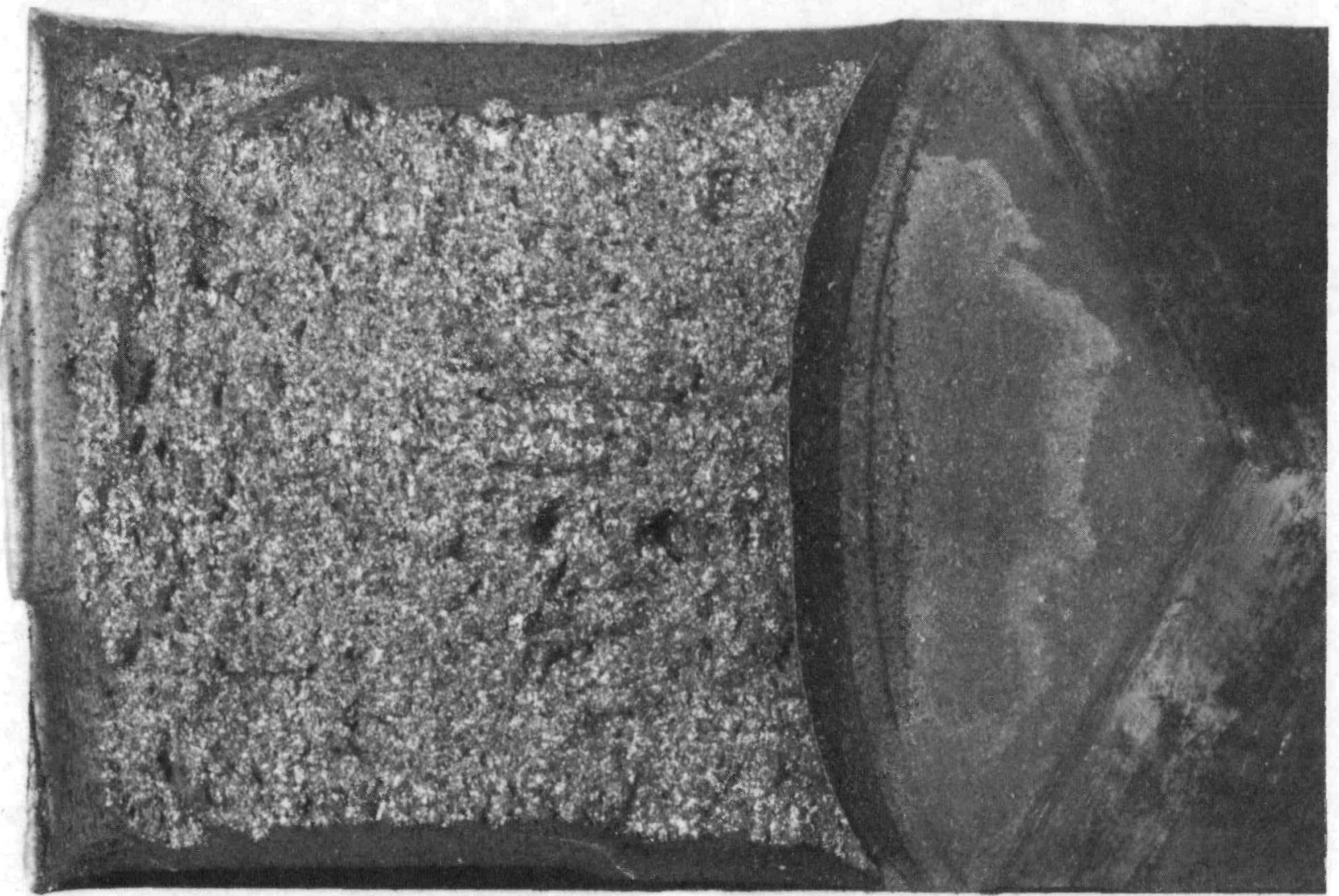

NUREG/CR-5913 
SPECIMEN ID $=73 W 178$

MATERIAL TYPE $=$ HCUWELD

TEST TEMPERATURE $=-30$ DEG C

THICKNESS $=25.4 \mathrm{~mm}$

SIDE GROOUES $=0 \%$

INITIAL CRACK LENGTH $=26.9 \mathrm{~mm}$

$a / w) i=.53$

MEASURED DUCTILE DELTA-A $=.12 \mathrm{~mm}$

YIELD STRENGTH $=529 \mathrm{MPa}$

FLOW STRENGTH $=593 \mathrm{MPa}$

ESTIMATED YOUNG'S MODULUS $=209 \mathrm{GPa}$

SPECIMEN CLEAUED AT KQ $=120.3 \mathrm{MPa} S Q R[\mathrm{~m}]$ (NOT UALID KIC)

UALUES AT MAXIMUM LOAD

J-INTEGRAL $=107.7 \mathrm{~kJ} / \mathrm{m}^{\wedge} 2$

$\mathrm{KJ} c=150 \mathrm{MPa} \operatorname{SQR}[\mathrm{m}]$

$K$-beta-c $=89.8 \mathrm{MPa} \operatorname{SQR}[\mathrm{m}]$
UALUES AT CLEAUAGE LOAD

J-INTEGRAL $=107.5 \mathrm{~kJ} / \mathrm{m}^{\wedge} 2$

$\mathrm{KJc}=149.8 \mathrm{MPa} \operatorname{SQR}[\mathrm{m}]$

K-beta-c $=89.7 \mathrm{MPa} \operatorname{SQR}[\mathrm{m}]$ 
$\mathrm{F}-43$

ORNL-DWG 92-11332

73W178 -30 DEGREES C

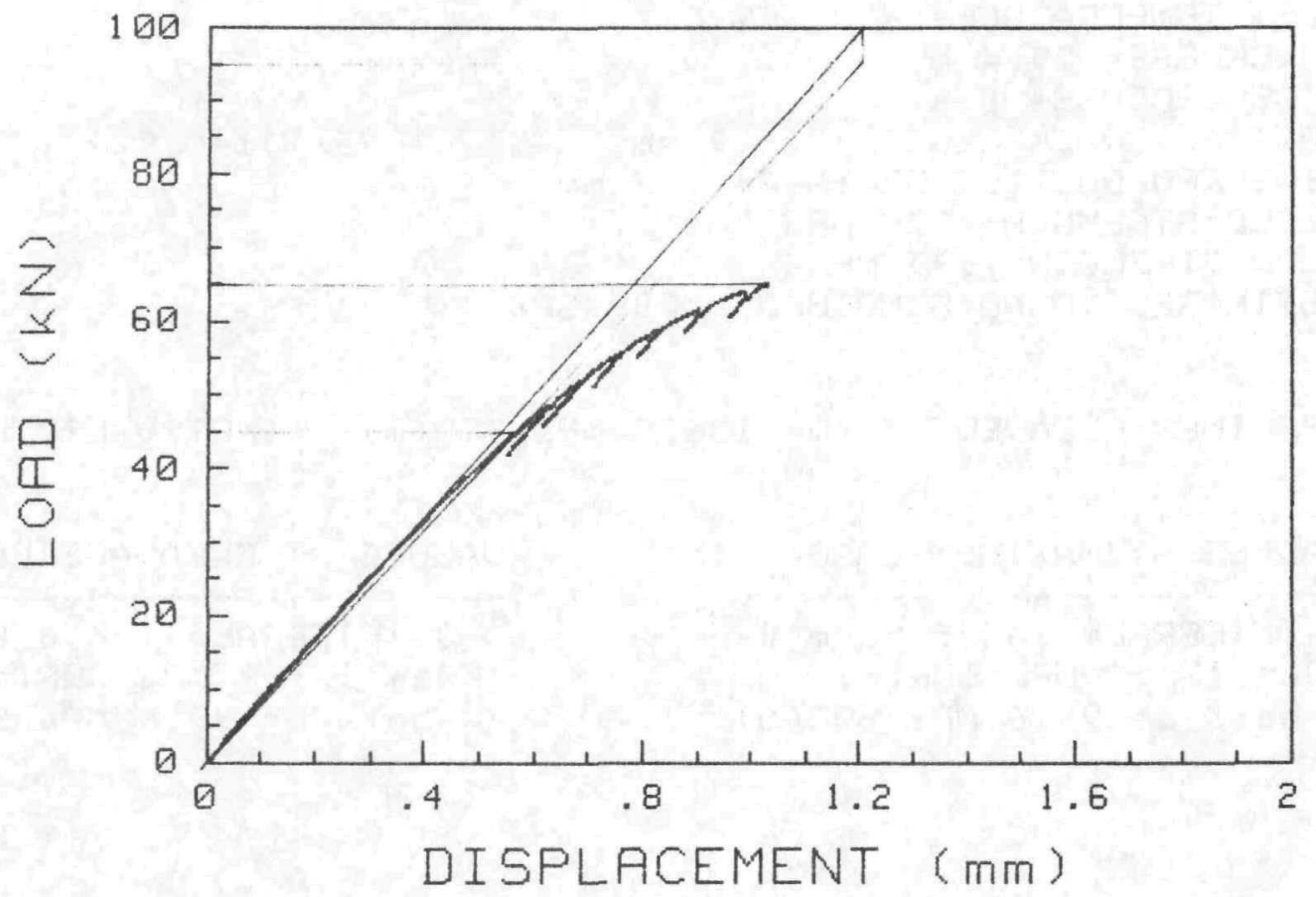

$73 W-178$

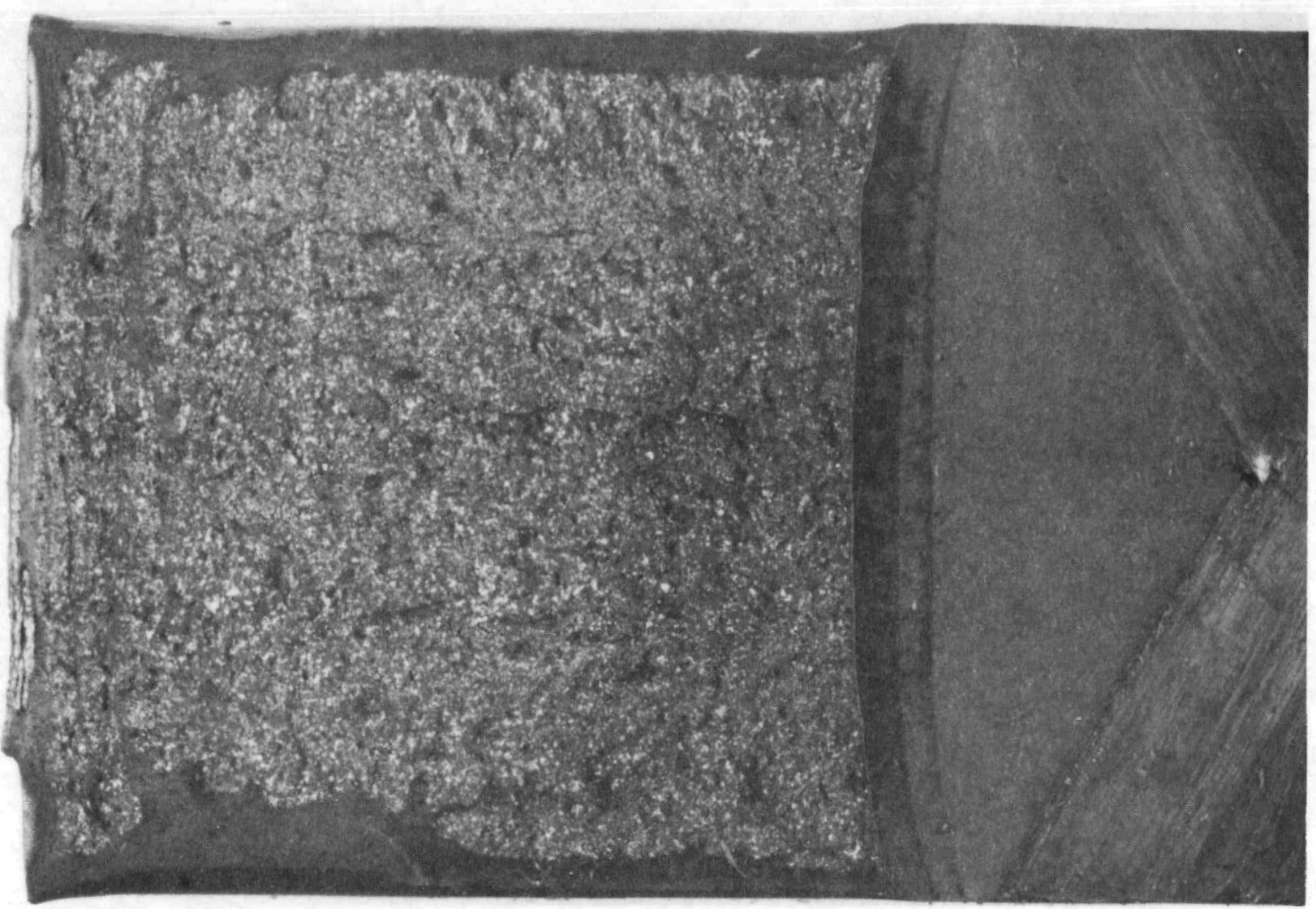


SPEC IMEN ID $=73 W 182$

MATERIAL TYPE = HCUWELD

TEST TEMPERATURE $=-30$ DEG $C$

THICKNESS $=25.4 \mathrm{~mm}$

SIDE GROOUES = $0 \%$

INITIAL CRACK LENGTH $=26.9 \mathrm{~mm}$

$a / W) i=.529$

MEASURED DUCTILE DELTA-A $=.17 \mathrm{~mm}$

YIELD STRENGTH $=529 \mathrm{MPa}$

FLOW STRENGTH $=593 \mathrm{MPa}$

ESTIMATED YOUNG'S MODULUS $=209 \mathrm{GPa}$

SPECIMEN CLEAUED AT KQ $=126.6 \mathrm{MPa}$ SQR $[\mathrm{m}]$ (NOT UALID KIc)

UALUES AT MAXIMUM LOAD

- - - - - - - - - - - - - - - -

J-INTEGRAL $=144.5 \mathrm{~kJ} / \mathrm{m}^{\wedge} 2$

$\mathrm{KJ} c=173.7 \mathrm{MPa} \operatorname{SQR}[\mathrm{m}]$

K-beta-c $=95.6 \mathrm{MPa} \operatorname{SQR}[\mathrm{m}]$
UALUES AT CLEAUAGE LOAD

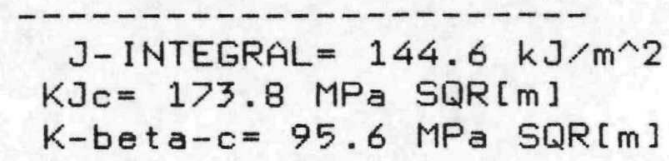




\section{W182 -30 DEGREES C}

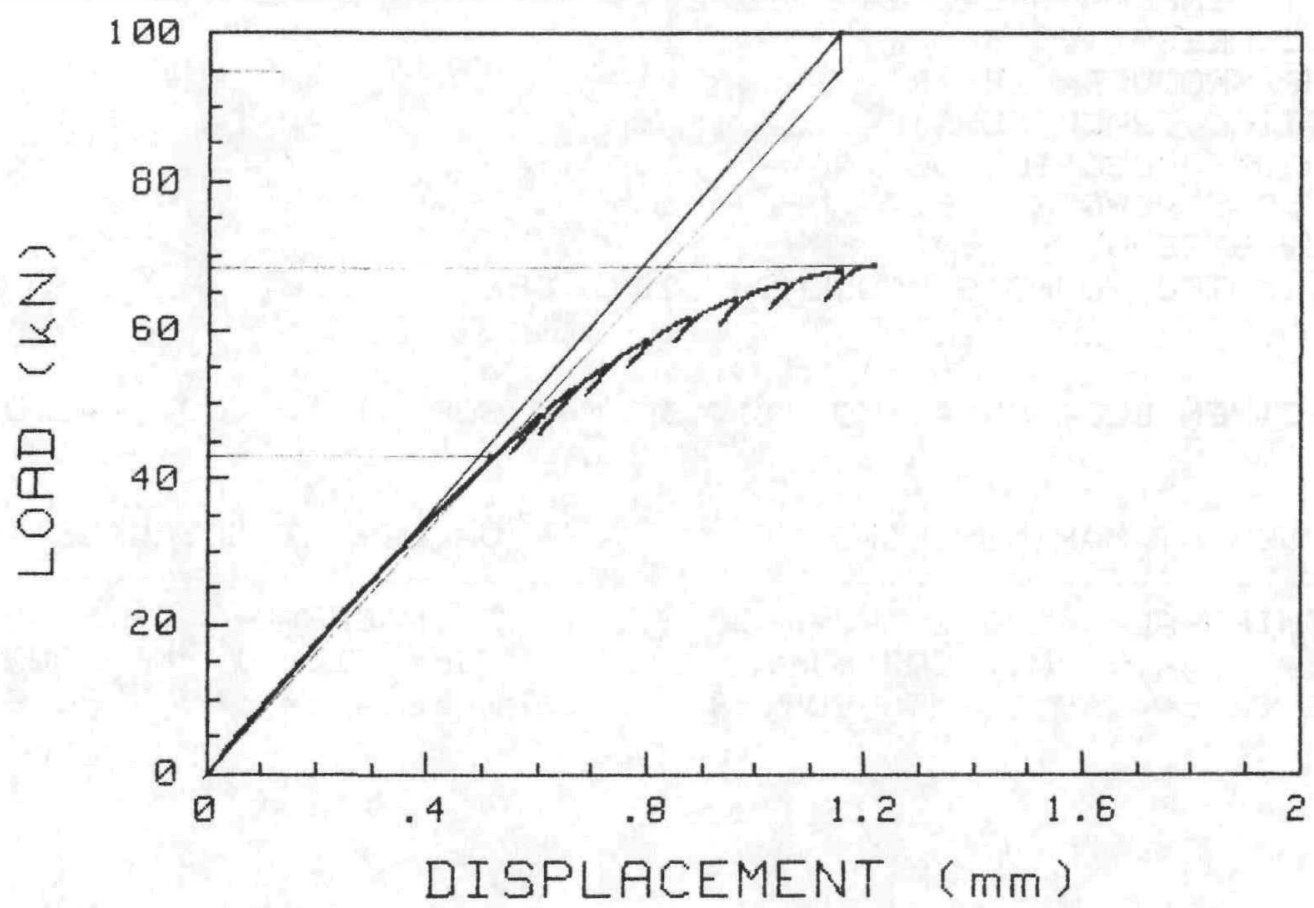

$73 W-182$

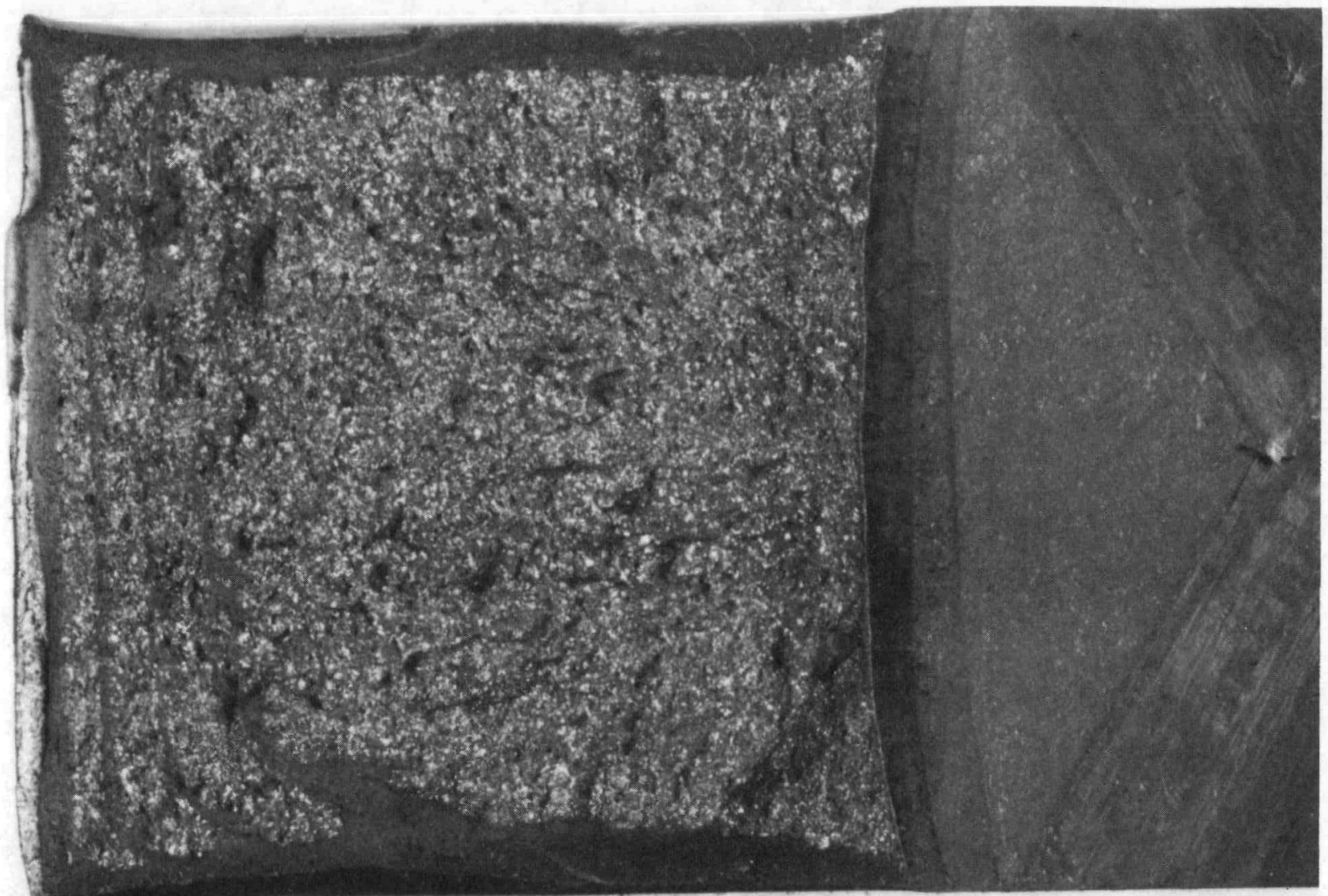




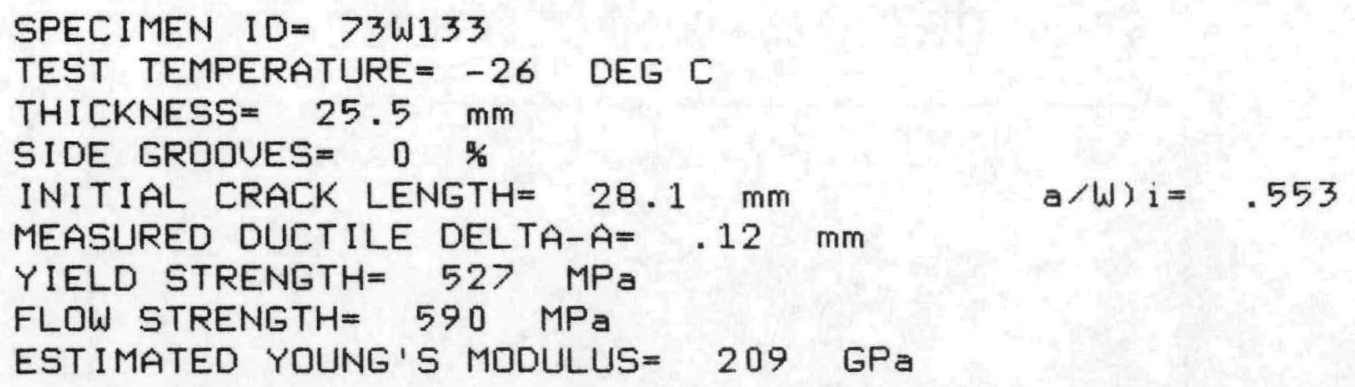

UALUES AT MAXIMUM LOAD

J-INTEGRAL $=100.1 \mathrm{~kJ} / \mathrm{m}^{\wedge} 2$

$K J c=144.5 \quad \mathrm{MPa} S D R[\mathrm{~m}]$

K-beta-c $=88.1$ MPa SQR $[\mathrm{m}]$
UALUES AT CLEAUAGE LDAD

J-INTEGRAL $=99.5 \mathrm{~kJ} / \mathrm{m}^{\wedge} 2$

$K J c=144.1$ MPa SQR $[\mathrm{m}]$

$K-b e t a-c=88$ MPa $S Q R[m]$

JI-R CURUE CALCULATIONS

$J($ 可丁 $/ T=8.8)=0 \mathrm{~kJ} / \mathrm{m}^{\wedge} 2$
POWER $=0$
COEFFICIENT $=0 \mathrm{~kJ} / \mathrm{m}^{\wedge} 2$

$J-M A X$ ALLOWED $=667.8 \mathrm{~kJ} / \mathrm{m}^{\wedge} 2$

RCURUE INUALID, 2

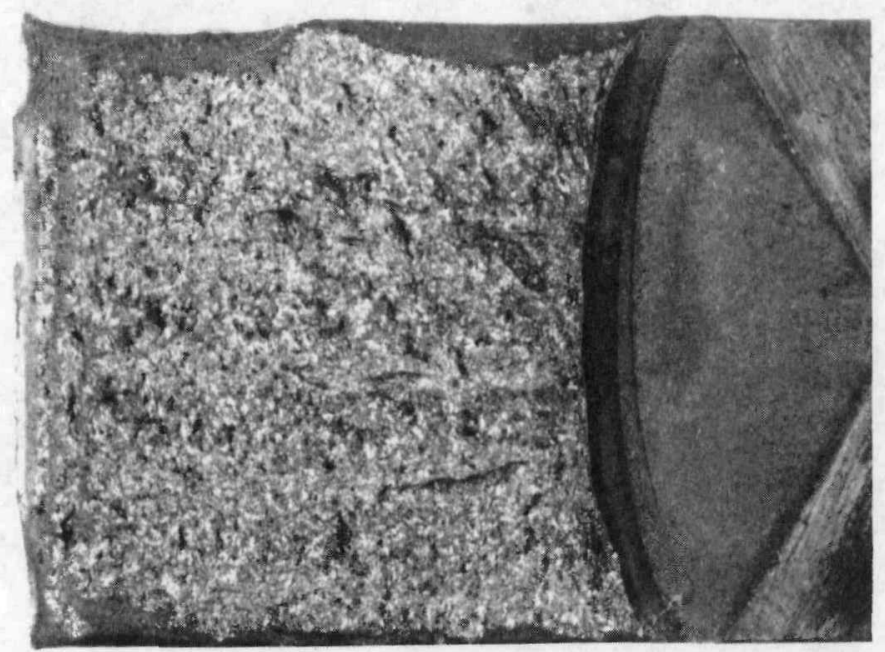


73W133 -26 DEGREES C

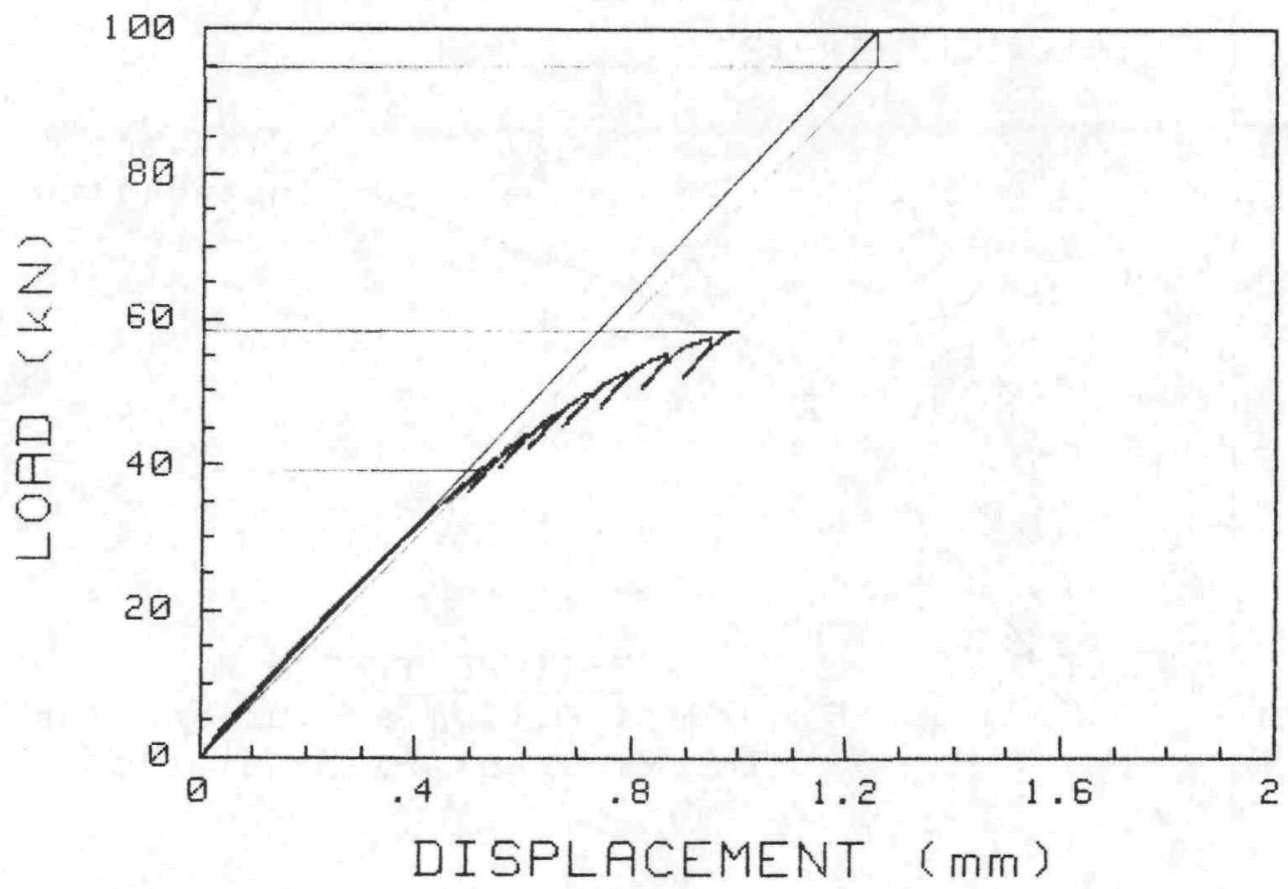

ORNL-DWG 92-11335

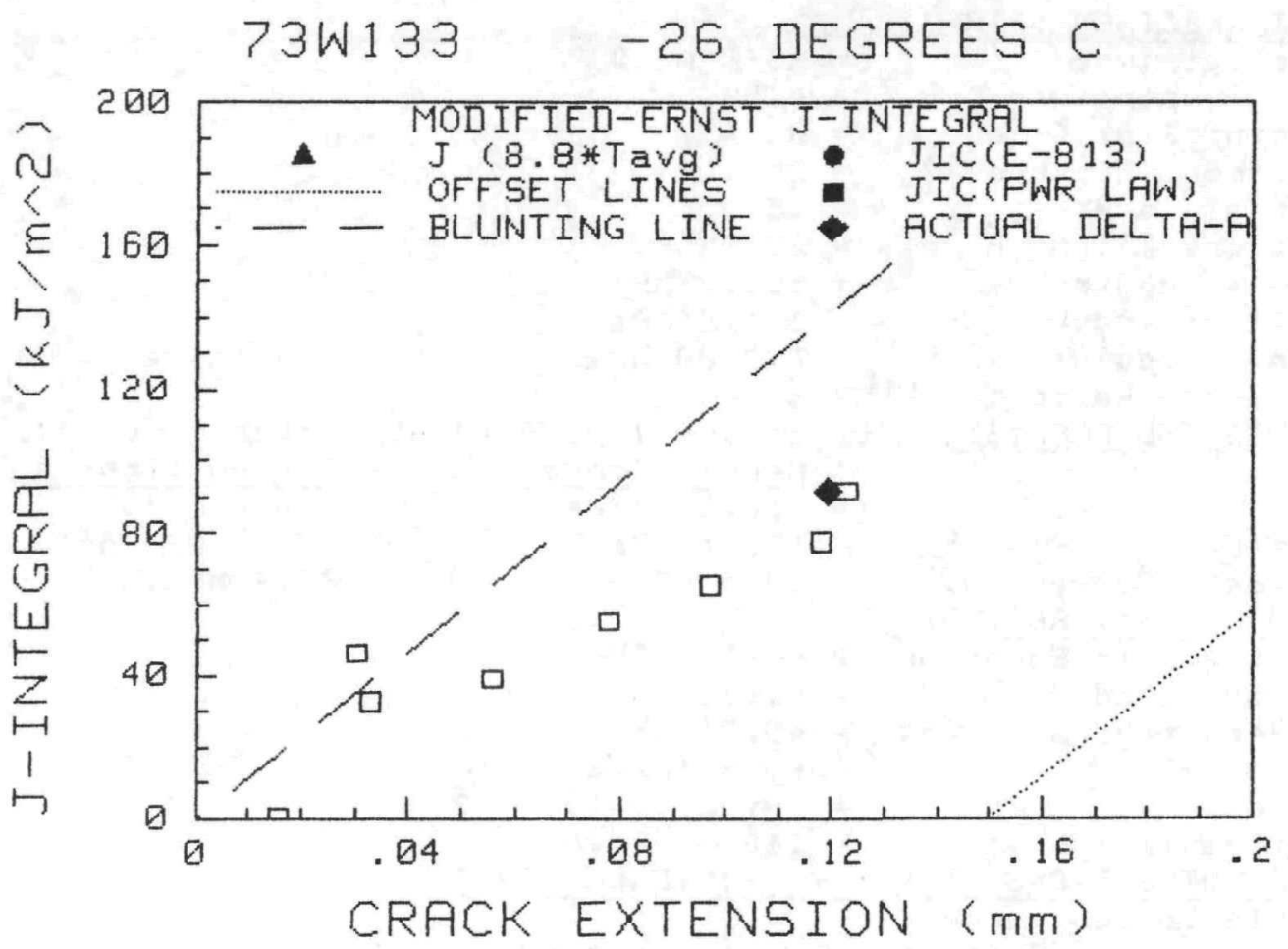

NUREG/CR-5913 
Specimen Crackmouth Deflection (in.)

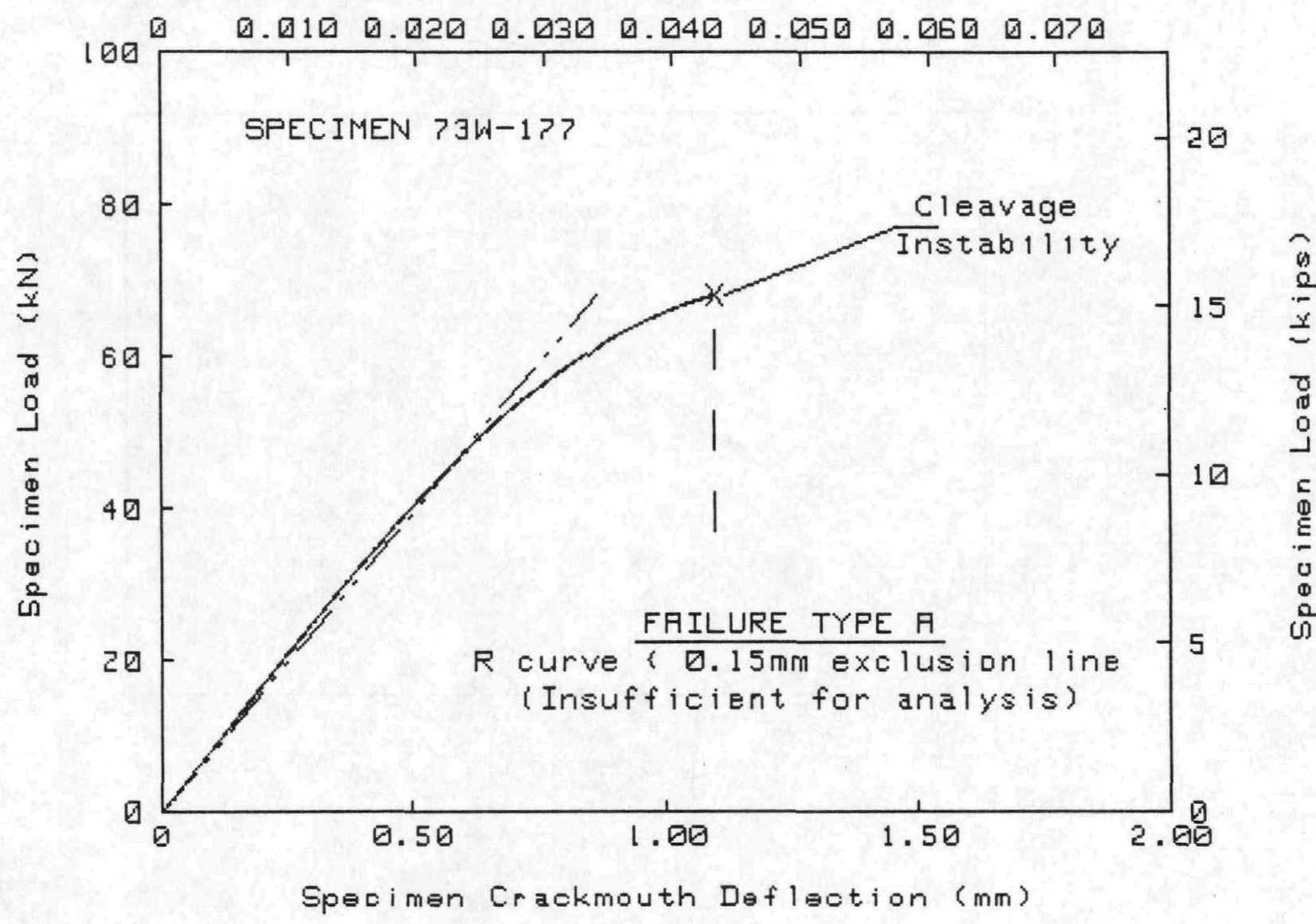

\section{TEST SFECIMEN DATA}

Material Type
Test Temperature
Percent Side Groove
Specimen Thickness B
Initial a/W
Init crack length a
Flow strength
Yield strength
Youngs modulus E
PoisSon's Ratio (U)
J-INTEGRAL ANALYSIS

= HSST WELD

$=-15^{\circ} \mathrm{C}$

$=0 \%$

$=25.4 \mathrm{~mm}$

$=.51$

$=25.92 \mathrm{~mm}$

$=581.2 \mathrm{MPa}$

$=521.2 \mathrm{MPa}$

$=208100 \mathrm{MPa}$

$=0$

J-INTEGRAL ANALYSIS

$\mathrm{Jc}$

[ Failure Type $A$ ( $J$ at in

$$
\begin{aligned}
& =\frac{\text { Merk le-Corten }}{117.1 \mathrm{~kJ} / \mathrm{m}^{2}} \\
& =156.1 \mathrm{MPa} \sqrt{\mathrm{m}} \\
& =90.5 \mathrm{MPa} \sqrt{\mathrm{m}}
\end{aligned}
$$

(Estimated Value)

$K j c=\left\langle E J c /\left(1-v^{2}\right)\right\rangle 1 / 2$

KC (Beta Corrected)

ASTM E399-81 ANALYSIS

Modified Kic Equation;

Maximum Load $P=68.11 \mathrm{kN}$

$5 \%$ Secant offset $\mathrm{Pq}=45.71 \mathrm{kN}$

$\mathrm{Kq}$

$=79.6 \mathrm{MPa} \sqrt{\mathrm{m}}$

Validity

$K$ at Maximum Load

$=$ INVALID--1, 2, 3

$=118.6 \mathrm{MPa} \sqrt{\mathrm{m}}$

DATA CORRECTIONS USED FOR J-INTEGRAL DATA

Merkle-Carten correction

Correction to $J$ for crack extension (Deformation-J)

OFFSET LOAD $=0 \mathrm{kN}$ OFFSET DEFLECTION $=.006 \mathrm{~mm}$

Fitted elastic load range $=10 \mathrm{kN}$ To $25 \mathrm{kN}$

Elast ic slope $=8.294 \mathrm{E}+01 \mathrm{kN} / \mathrm{mm}$ 


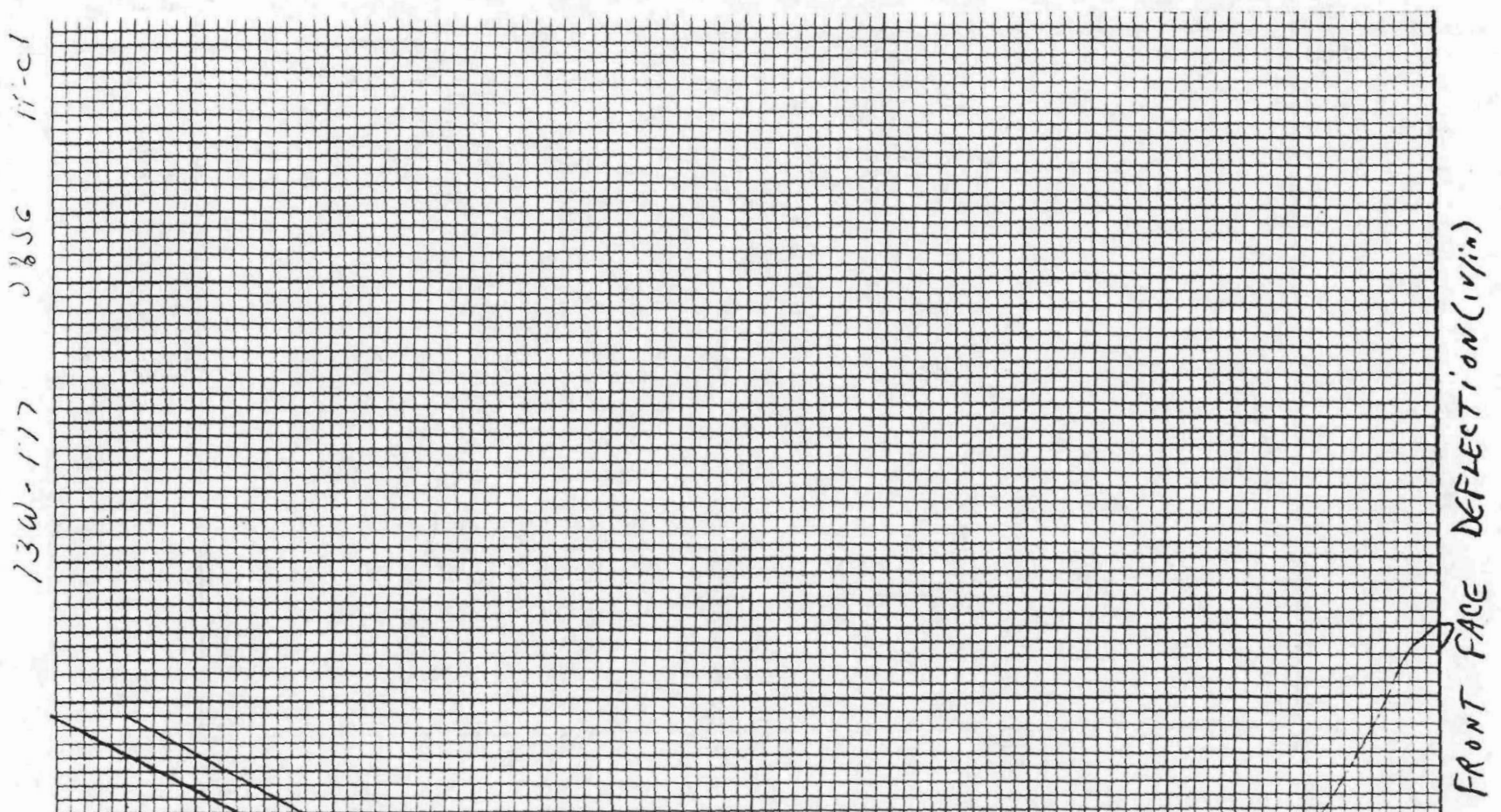
\begin{tabular}{lllll}
\hline & \\
\hline
\end{tabular} $(* 1 / n 1)$ ovey 
Specimen Crackmouth Deflection (in.)

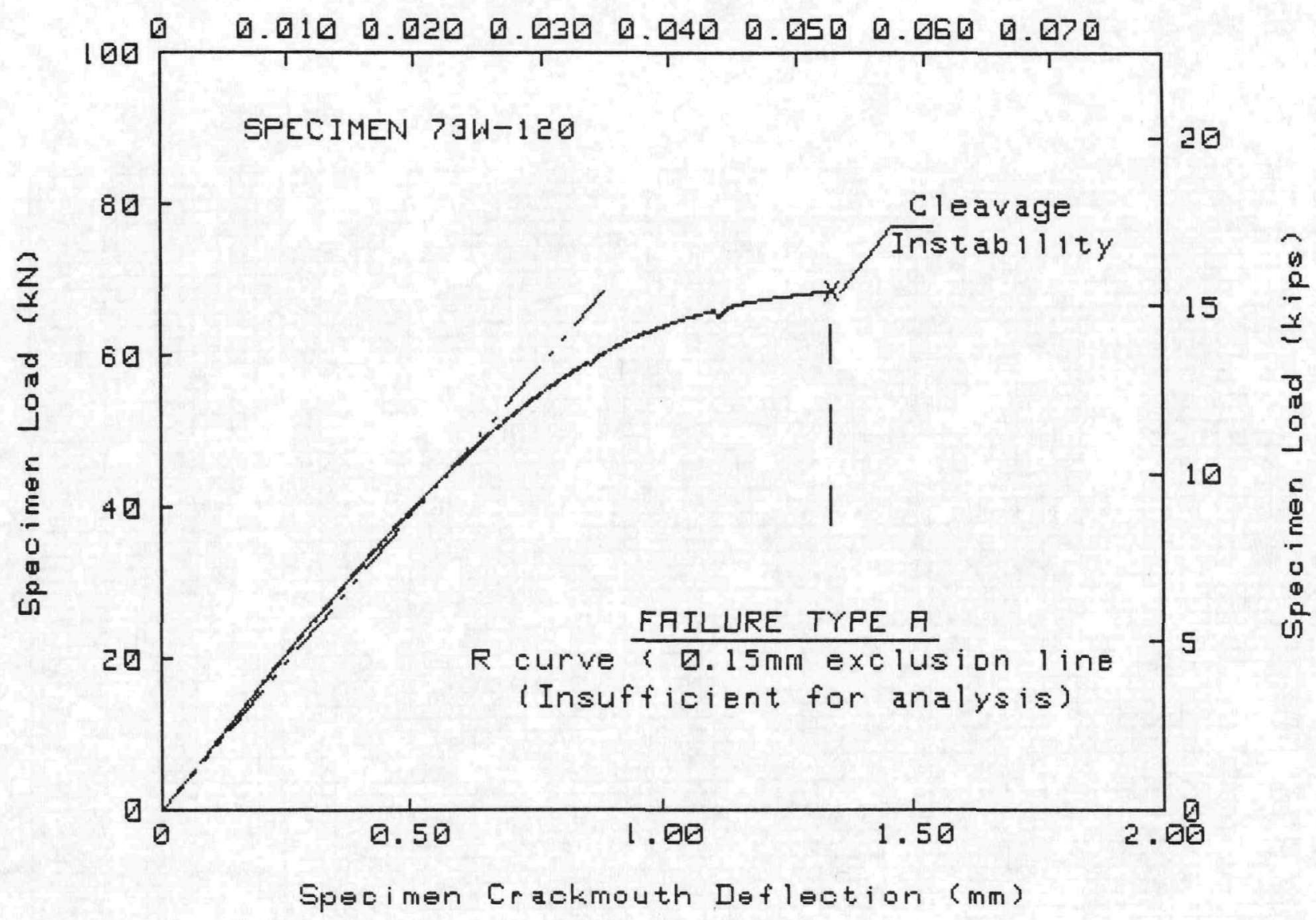

TEST SPECIMEN DATA

Material Type
Test Temperature
Percent Side Groove
Specimen Thickness B
Initial a/W
Init crack length a
Flow strength
Yield strength
Youngs modulus E
PoisSon's Ratio (U)
J-INTEGRAL ANALYSIS

= HSST WELD

$=-15^{\circ} \mathrm{C}$

$=0 \%$

$=25.4 \mathrm{~mm}$

$=.513$

$=26.06 \mathrm{~mm}$

$=581.2 \mathrm{MPa}$

$=521.2 \mathrm{MPa}$

$=208100 \mathrm{MPa}$

(Estimated Yalue)

$$
=0
$$

J-INTEGRAL ANALYSIS

$\mathrm{Je}$

Failure Type $A$ ( $J$ at instability = Je) ]
Merkle-Corten
Deformation-J*

$K j c=\left\langle E J c /\left(1-v^{2}\right)\right\rangle 1 / 2$

$=\frac{\text { Merkle-Corten }}{157.8 \mathrm{~kJ} / \mathrm{m}^{2}}$

$\frac{\text { Deformat ion }-\mathrm{J} *}{150.8 \mathrm{~kJ} / \mathrm{m}^{2}}$

$=181.2 \mathrm{MPa} \sqrt{\mathrm{m}}$

$177.2 \mathrm{MPa}$ m

Ke (Beta Corrected)

$=96.4 \mathrm{MPa} \sqrt{\mathrm{m}}$

$95.5 \mathrm{MPa} \sqrt{\mathrm{m}}$

ASTM E399-81 ANALYSIS

Modified Kic Equation; $B=\langle B B n\rangle 1 / 2$

Maximum Load $P=68.53 \mathrm{kN}$

$5 \%$ Secant offset $\mathrm{Pq}=43.51 \mathrm{kN}$

$\mathrm{Kq}$

$=76.4 \mathrm{MPa} \sqrt{\mathrm{m}}$

Validity

$K$ at Maximum Load

= INVALID--1, 2,3

$=120.4 \mathrm{MPa} \sqrt{\mathrm{m}}$

DATA CORRECTIONS USED FOR J-INTEGRAL DATA

Merkle-Corten correction

Correction to $\mathrm{J}$ for crack Extension (Deformation-J)

OFFSET LOAD $=0 \mathrm{kN}$ OFFSET DEFLECTION $=.018 \mathrm{~mm}$

Fitted elastic load range $=10 \mathrm{kN}$ TO $25 \mathrm{kN}$

Elastic slope $=8.155 E+01 \mathrm{kN} / \mathrm{mm}$

NUREG/CR-5913 


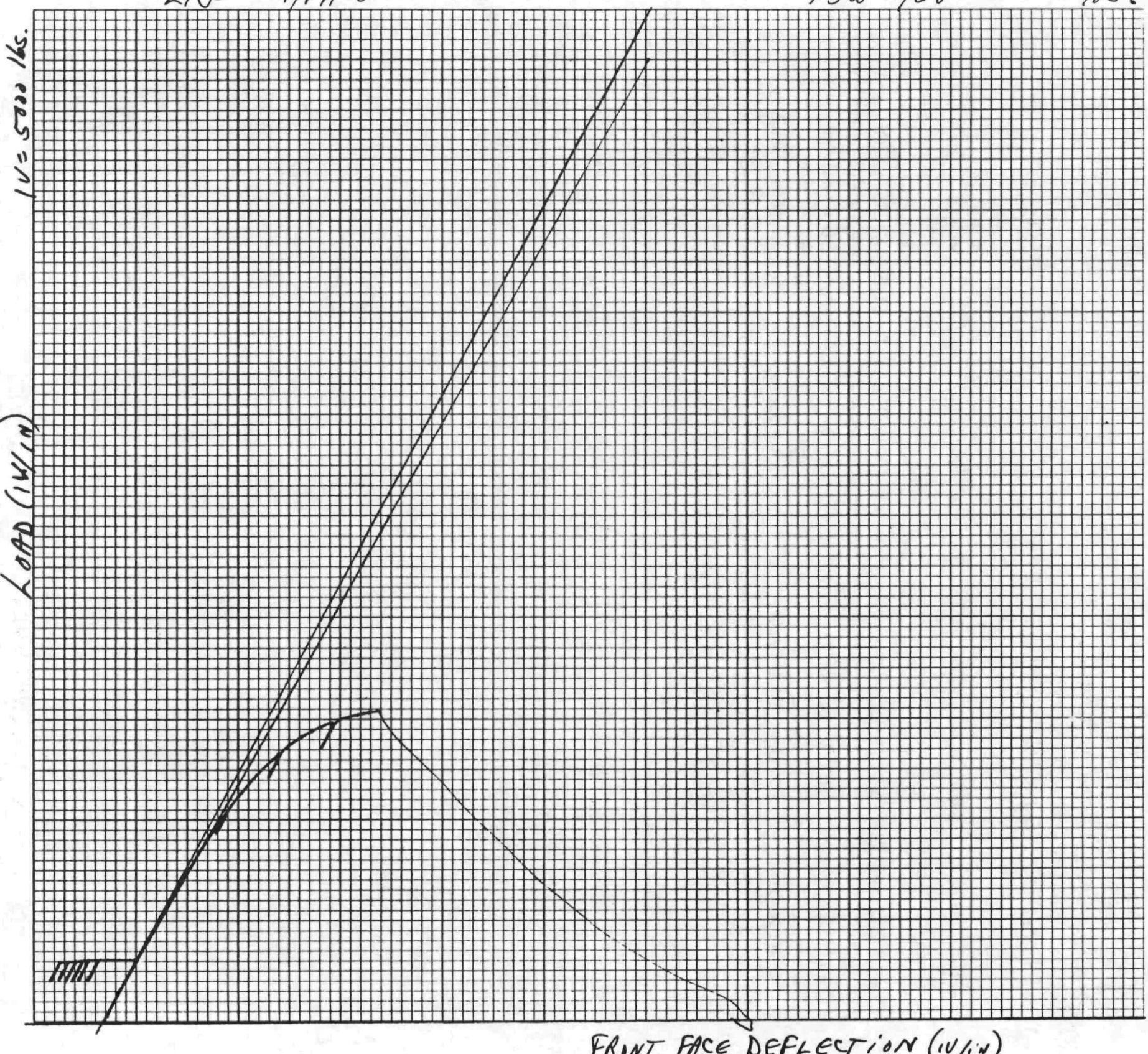


SPECIMEN ID $=73 W 117$

MATERIAL TYPE = HCUWELD

TEST TEMPERATURE $=-15$ DEG C

THICKNESS $=25.4 \mathrm{~mm}$

SIDE GROOUES $=0 \%$

INITIAL CRACK LENGTH $=26.7 \mathrm{~mm}$

$a / W) i=.526$

MEASURED DUCTILE DELTA-A $=.23 \mathrm{~mm}$

YIELD STRENGTH $=520 \mathrm{MPa}$

FLDW STRENGTH $=581 \mathrm{MPa}$

ESTIMATED YOUNG'S MODULUS $=208 \mathrm{GPa}$

SPECIMEN CLEAUED AT KQ $=126.8 \mathrm{MPa} S Q R[\mathrm{~m}]$ (NOT UALID $K I \mathrm{c}$ )

WALUES AT MAXIMUM LOAD.

-

J-INTEGRAL $=188.3 \mathrm{~kJ} / \mathrm{m}^{\wedge} 2$

$\mathrm{KJ} c=198 \mathrm{MPa} S Q R[\mathrm{~m}]$

K-beta-c $=99.8 \mathrm{MPa} \operatorname{SQR}[\mathrm{m}]$
UALUES AT CLEAUAGE LDAD

$$
\begin{aligned}
& \text { J-INTEGRAL }=185 \mathrm{~kJ} / \mathrm{m}^{\wedge} 2 \\
& \mathrm{KJ}=196.2 \mathrm{MPa} \operatorname{SQR}[\mathrm{m}] \\
& \mathrm{K} \text {-beta-c=99.4 MPa SQR }[\mathrm{m}]
\end{aligned}
$$


F-53

ORNL-DWG 92-11338

73W117 -15 DEGREES C

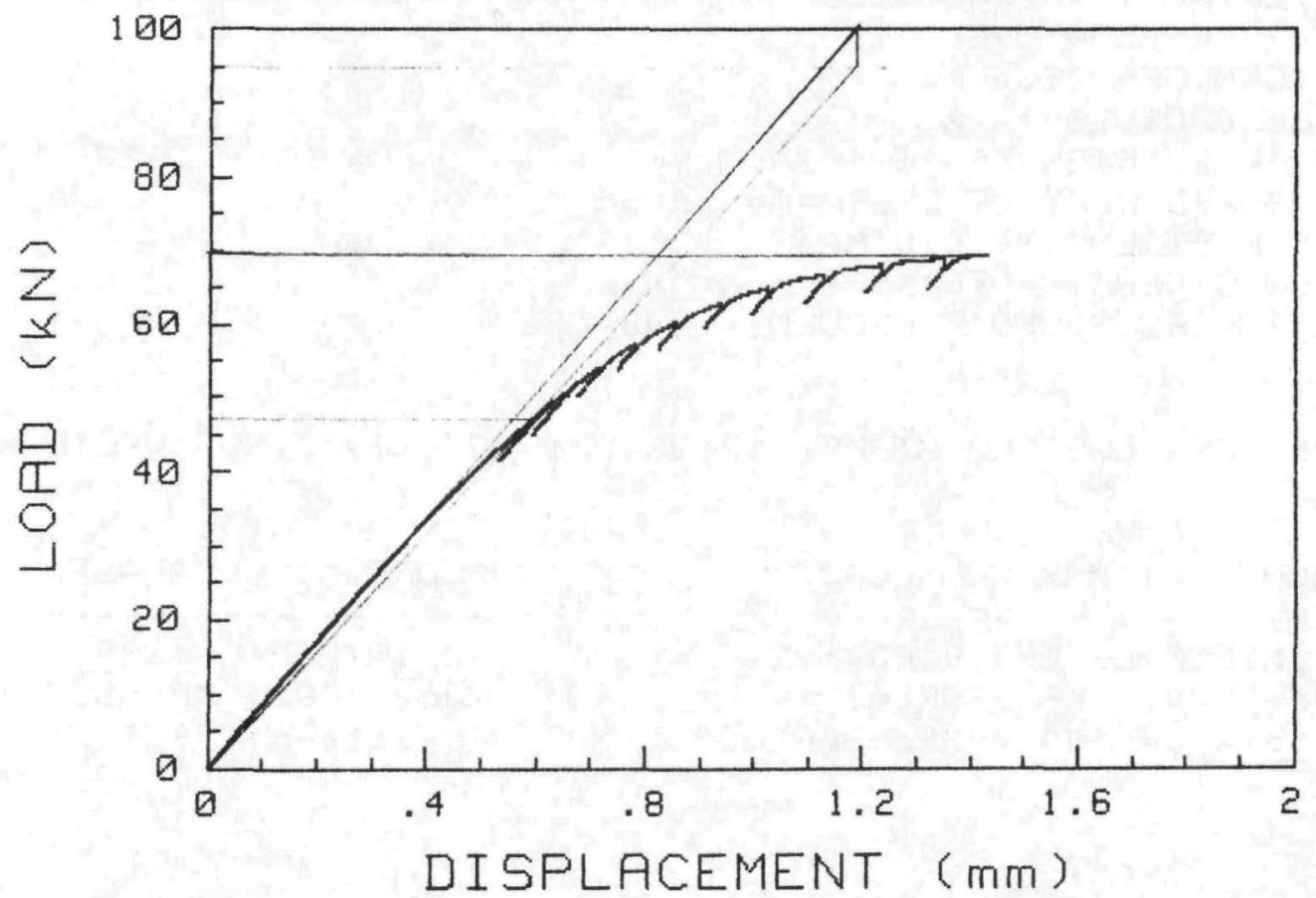

$73 W-117$

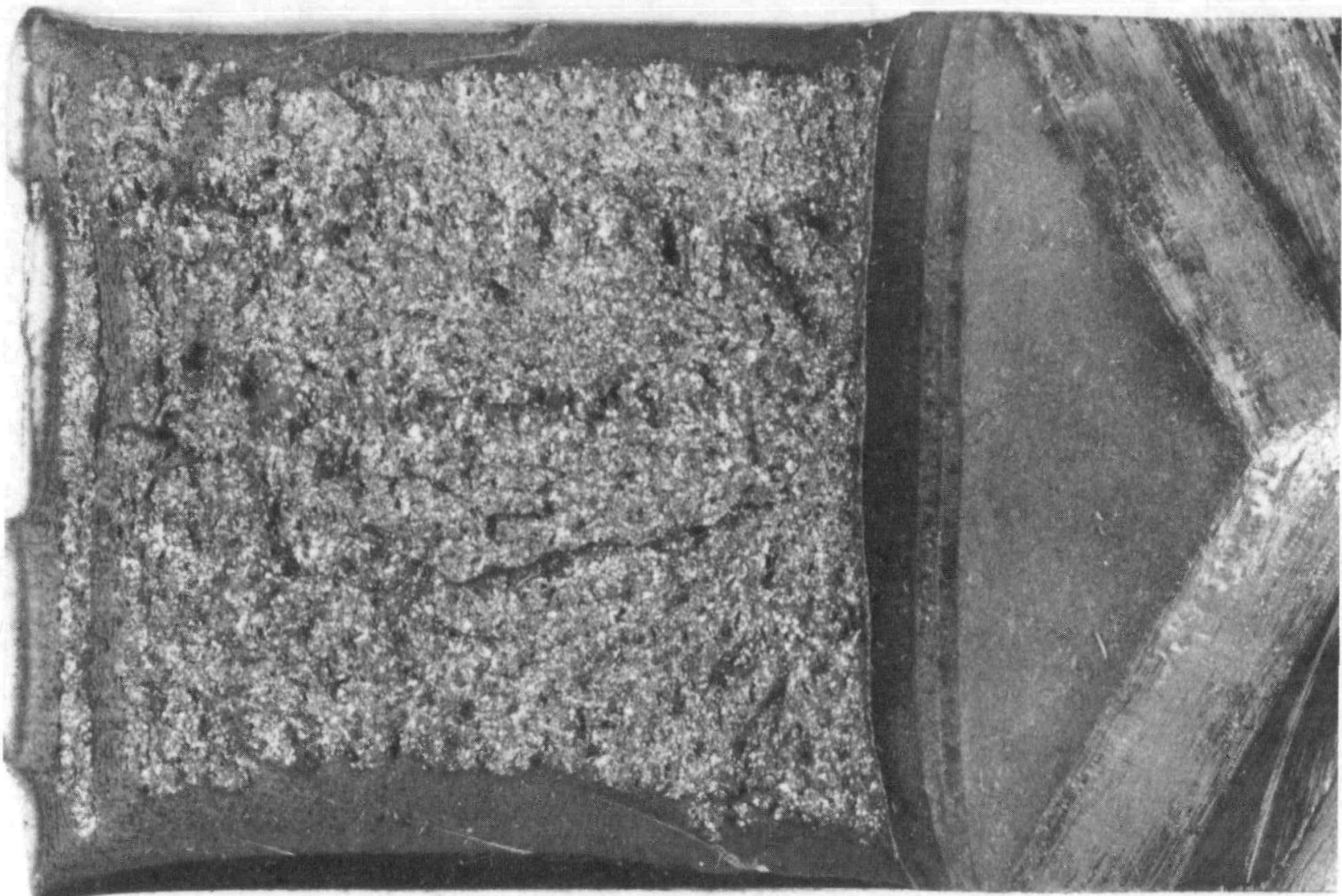


SPECIMEN ID $=73 W 179$

MATERIAL TYPE = HCUWELD

TEST TEMPERATURE $=-15$ DEG $\mathrm{C}$

THICKNESS $=25.4 \mathrm{~mm}$

SIDE GROOUES $=0 \%$

INITIAL CRACK LENGTH $=26.7 \mathrm{~mm}$

$a / W) i=.527$

MEASURED DUCTILE DELTA-A $=.3 \mathrm{~mm}$

$Y$ IELD STRENGTH $=520 \mathrm{MPa}$

FLOW STRENGTH $=581 \mathrm{MPa}$

ESTIMATED YOUNG'S MODULUS $=208 \mathrm{GPa}$

SPECIMEN CLEAUED AT KQ= $126.8 \mathrm{MPa} S Q R[\mathrm{~m}]$ (NOT UALID KIc)

UALUES AT MAXIMUM LOAD

J-INTEGRAL $=193.5 \mathrm{~kJ} / \mathrm{m}^{\wedge} 2$

$K J c=200.6 \mathrm{MPa} \operatorname{SQR}[\mathrm{m}]$

K-beta-c $=100.4 \mathrm{MPa}$ SQR $[\mathrm{m}]$
UALUES AT CLEAUAGE LOAD
J-INTEGRAL $=189.1 \mathrm{~kJ} / \mathrm{m}^{\wedge} 2$
KJc= $198.3 \mathrm{MPa}$ SQR [m]
K-beta-c= $99.9 \mathrm{MPa}$ SQR [m] 


\section{F--55}

ORNL-DWG 92-11339

$$
\text { 73W179 -15 DEGREES C }
$$
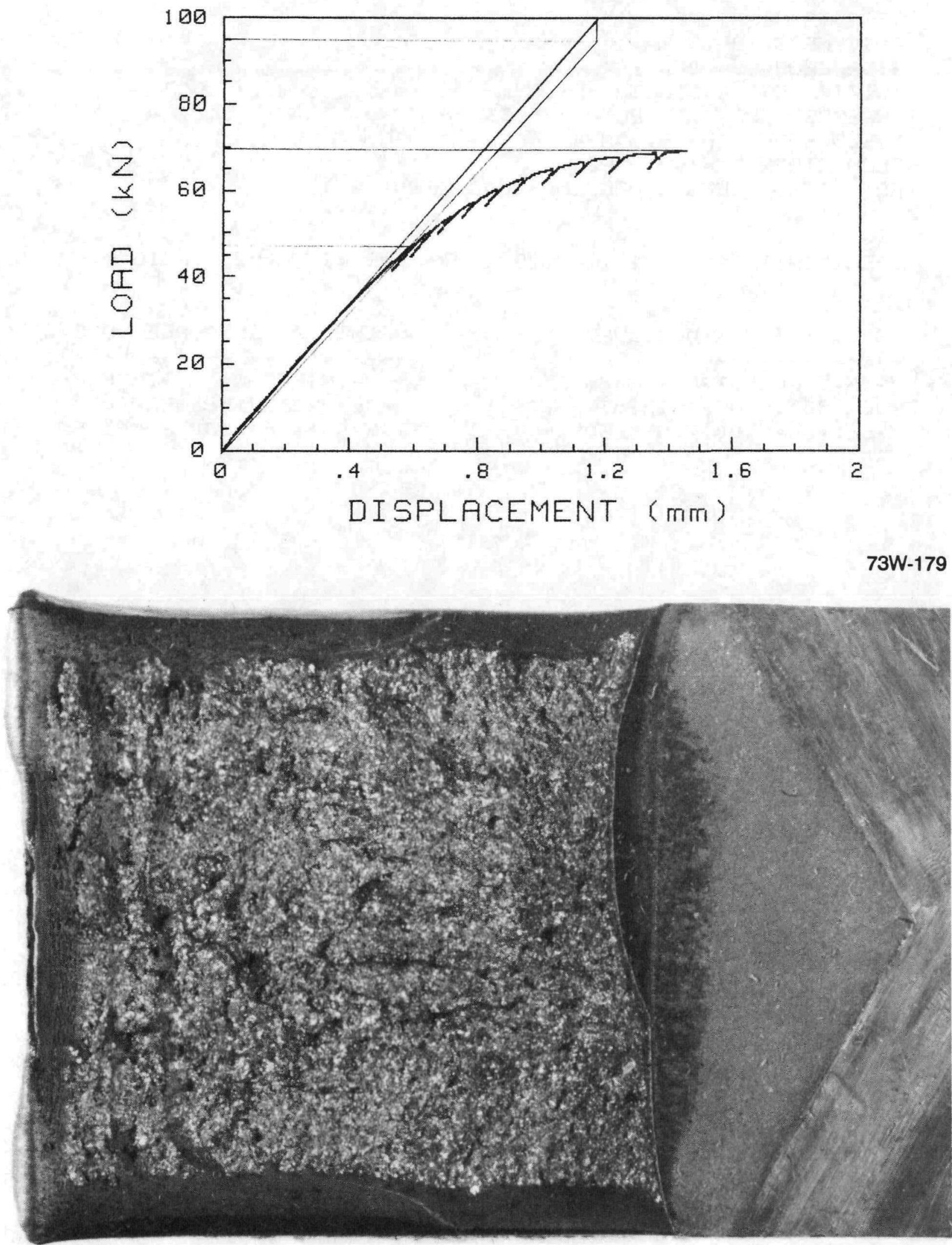


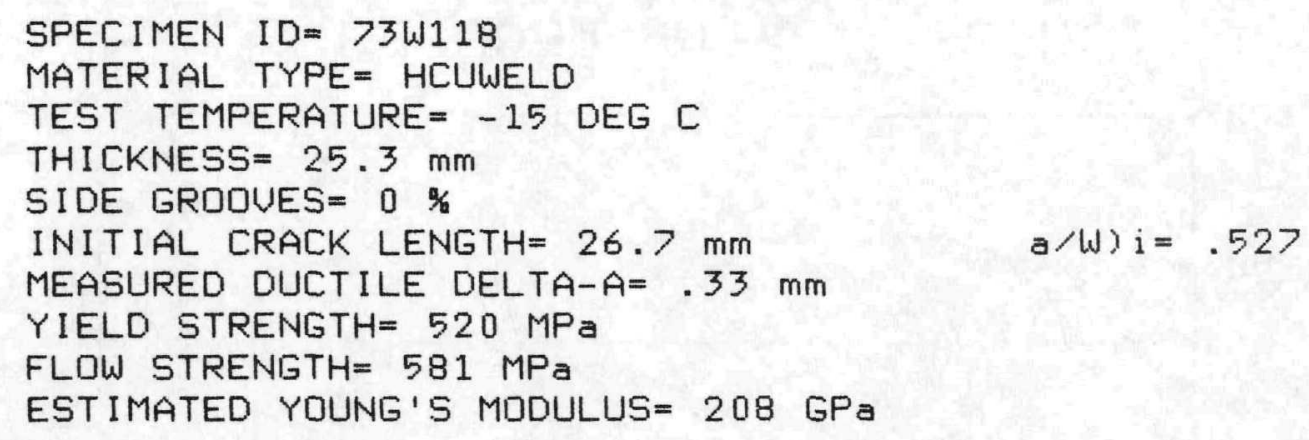

SPECIMEN CLEAUED AT KQ $=125.2 \mathrm{MPa}$ SQR $[\mathrm{m}]$ (NOT WALID KIc)

UALUES AT MAXIMUM LDAD

J-INTEGRAL $=198.9 \mathrm{~kJ} / \mathrm{m}^{\wedge} 2$

$\mathrm{KJ} c=203.5 \mathrm{MPa} S Q R[\mathrm{~m}]$

K-beta-c $=100.7 \mathrm{MPa} \operatorname{SQR}[\mathrm{m}]$
UALUES AT CLEAUAGE LDAD

$J-I N T E G R A L=199.5 \mathrm{~kJ} / \mathrm{m}^{\wedge} 2$
$\mathrm{KJc}=203.7 \mathrm{MPa} \mathrm{SQR}[\mathrm{m}]$
$\mathrm{K}-\mathrm{beta}-\mathrm{c}=100.8 \mathrm{MPa} \operatorname{SQR}[\mathrm{m}]$ 


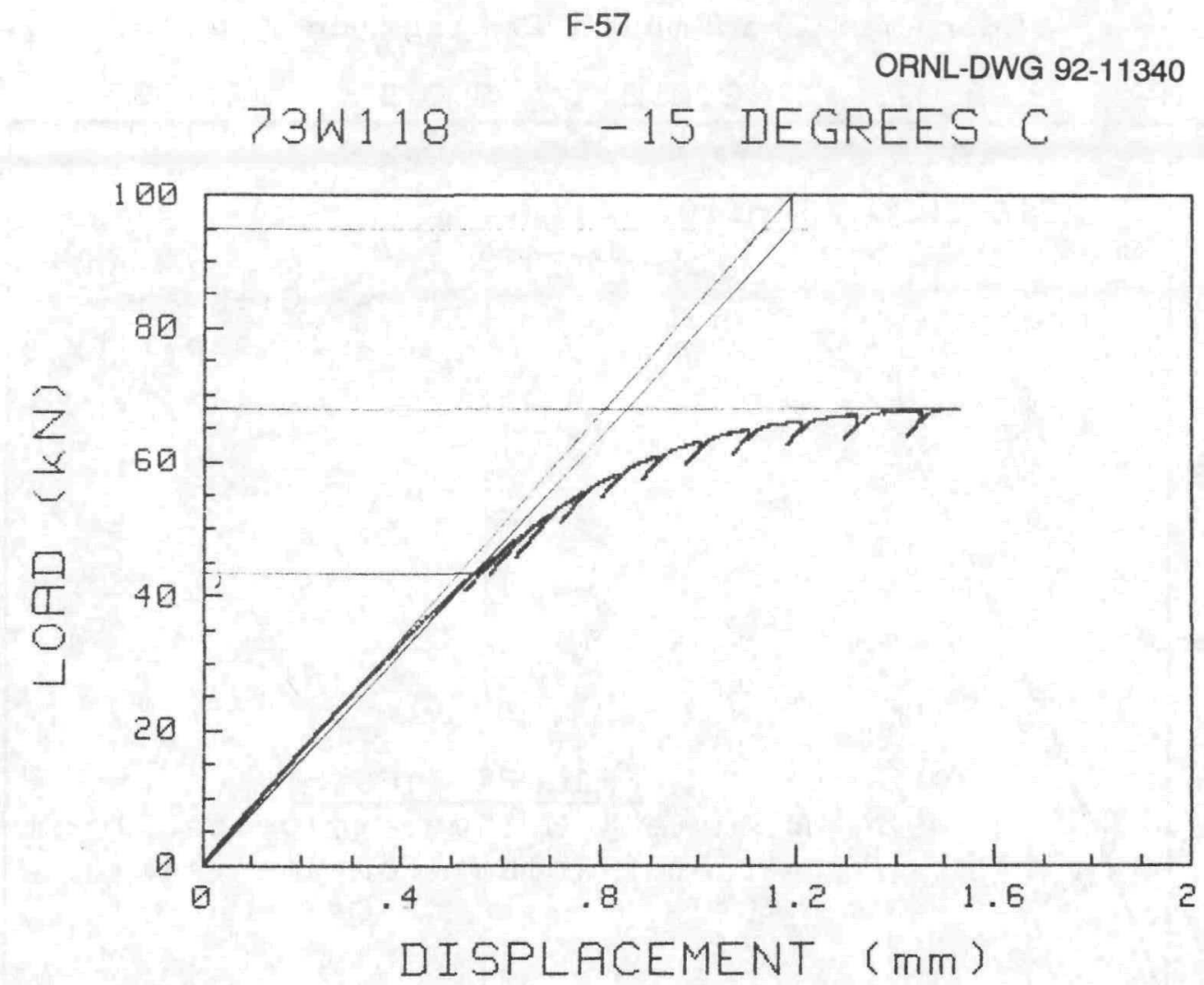

$73 W-118$

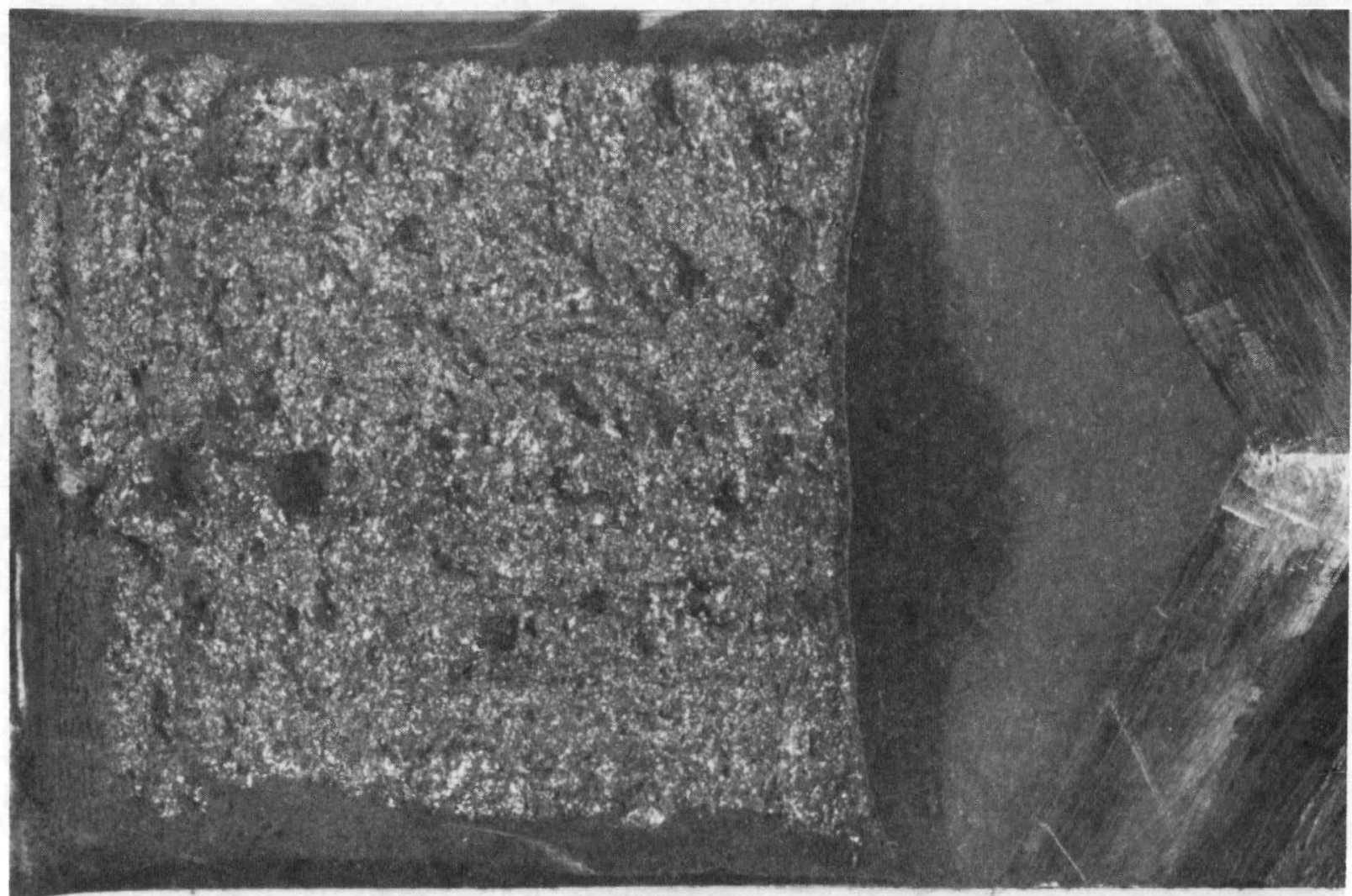

NUREG/CR-5913 
Specimen Crackmouth Deflection (in.)

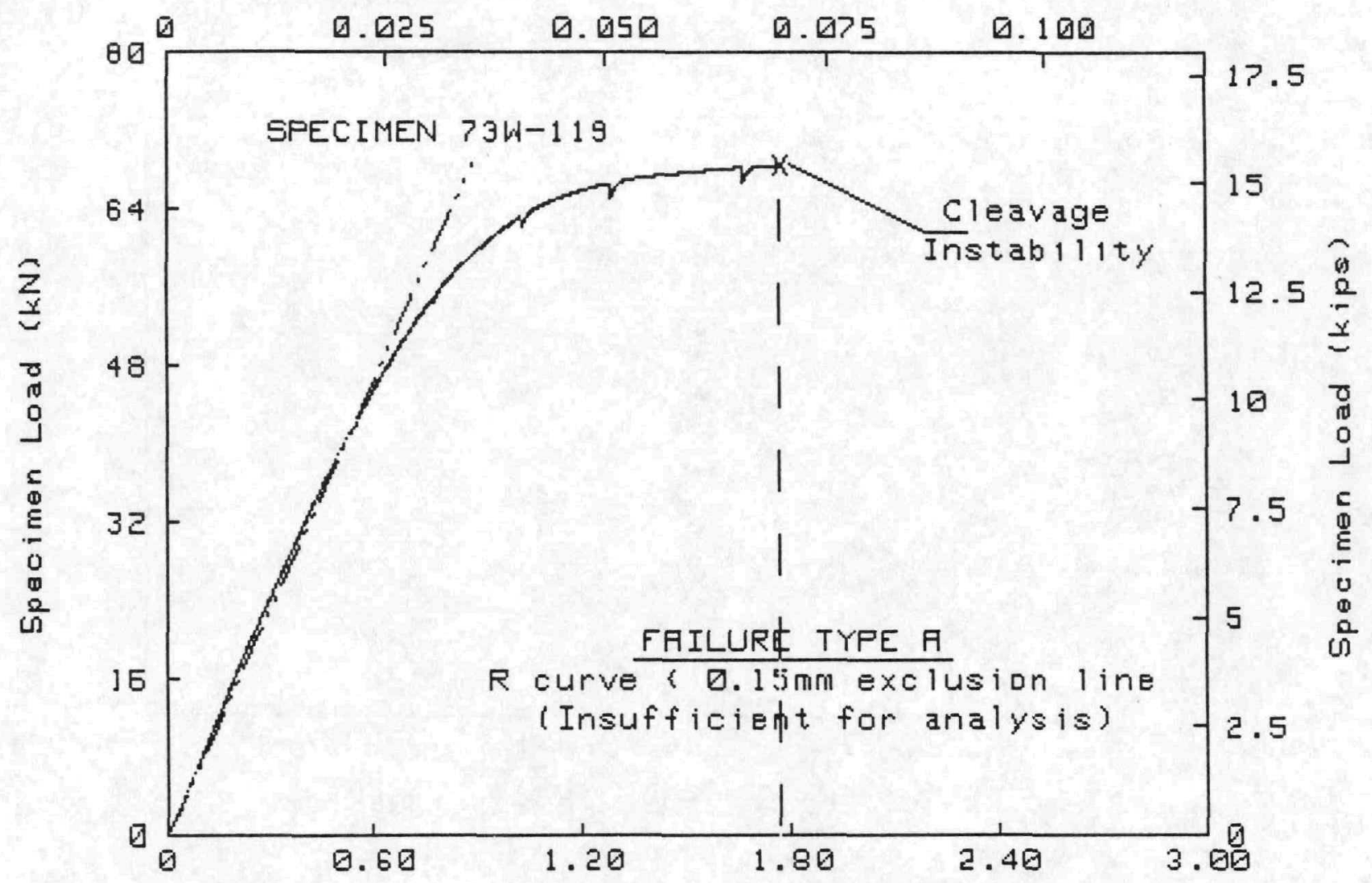

Speoimen Craokmouth Defleotion (mm)

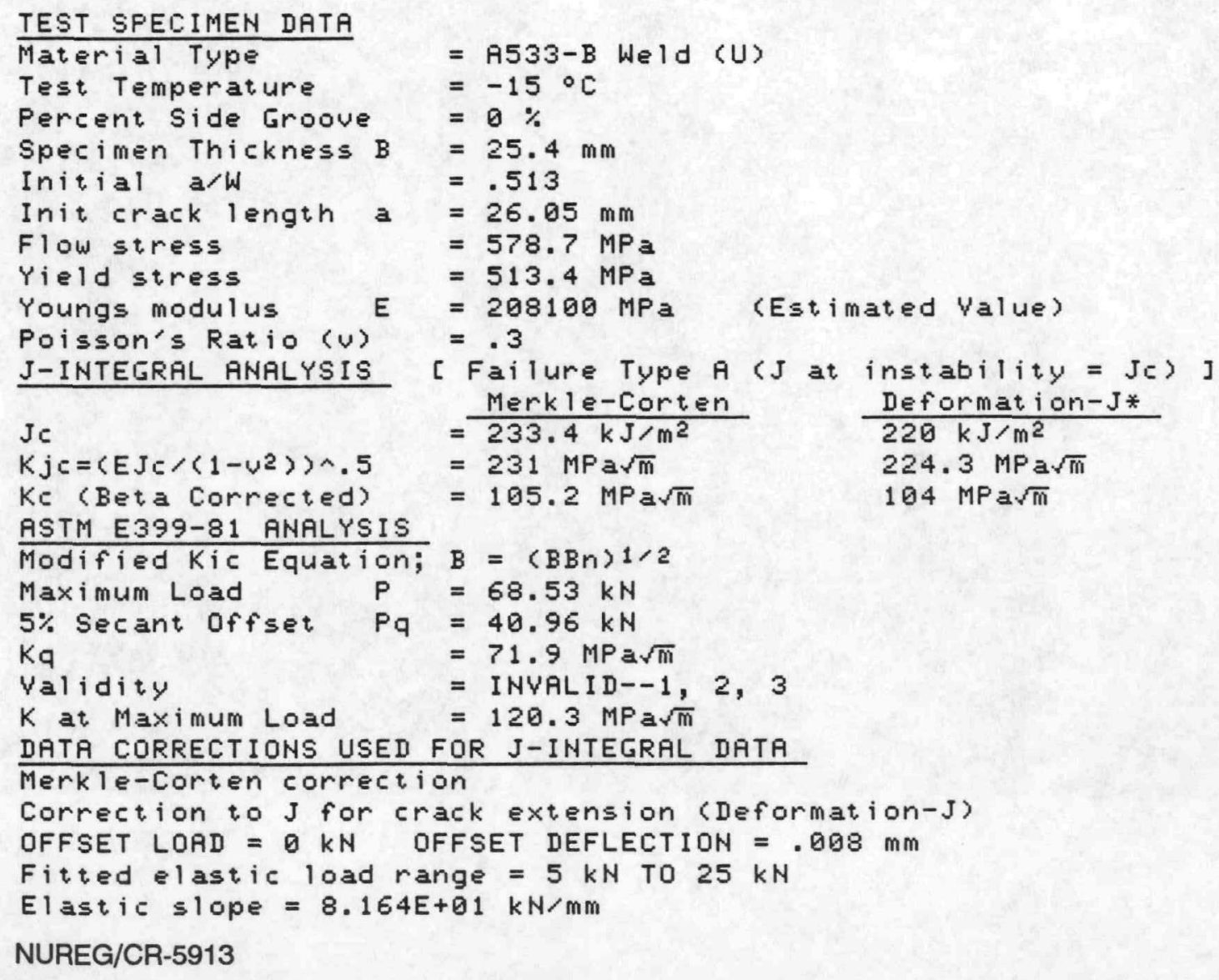


$\angle \pi F$

$1 / 14 / 86$

$13 \omega-119$

$1 T-C T$

$0 \% 5 c$

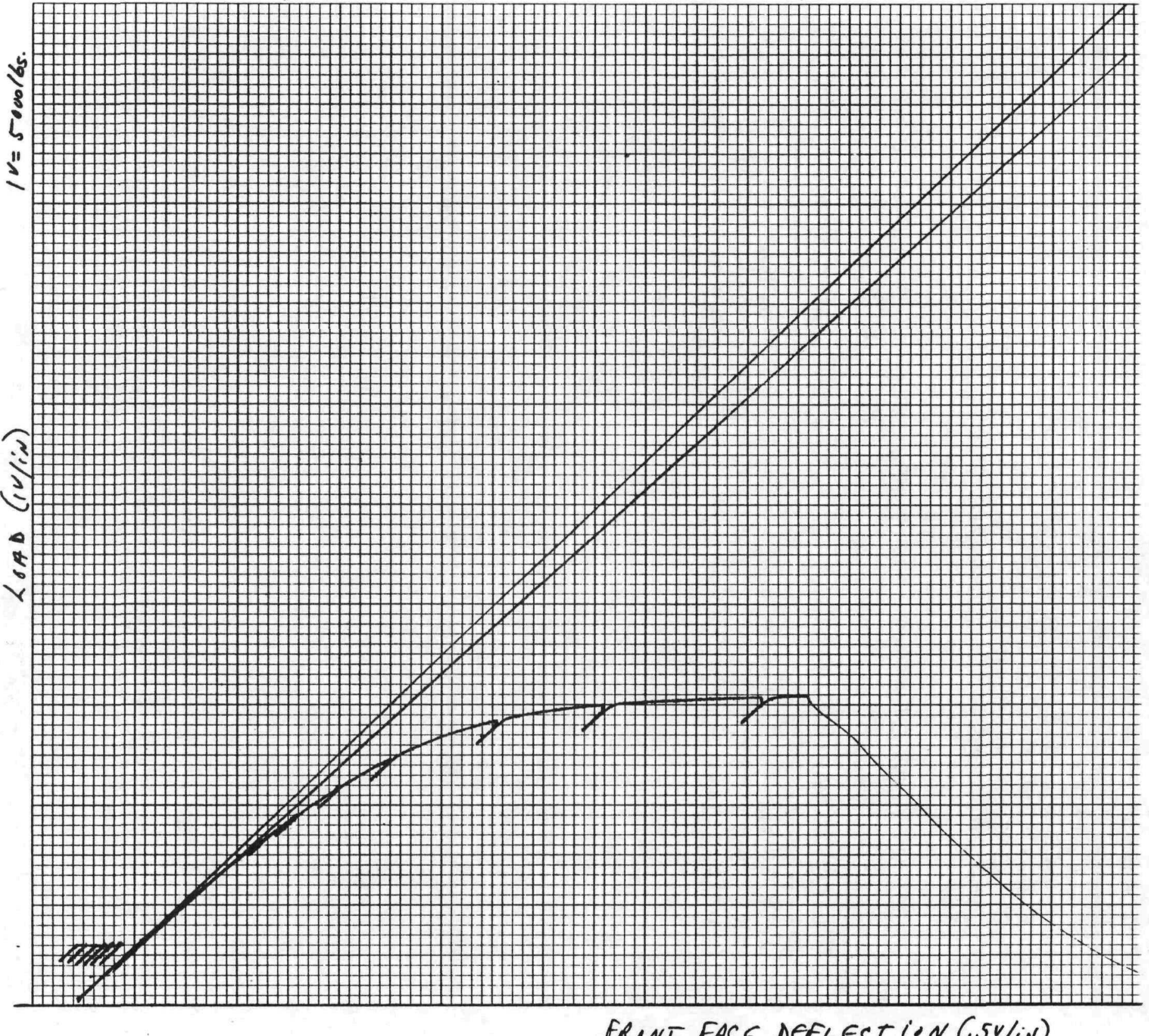




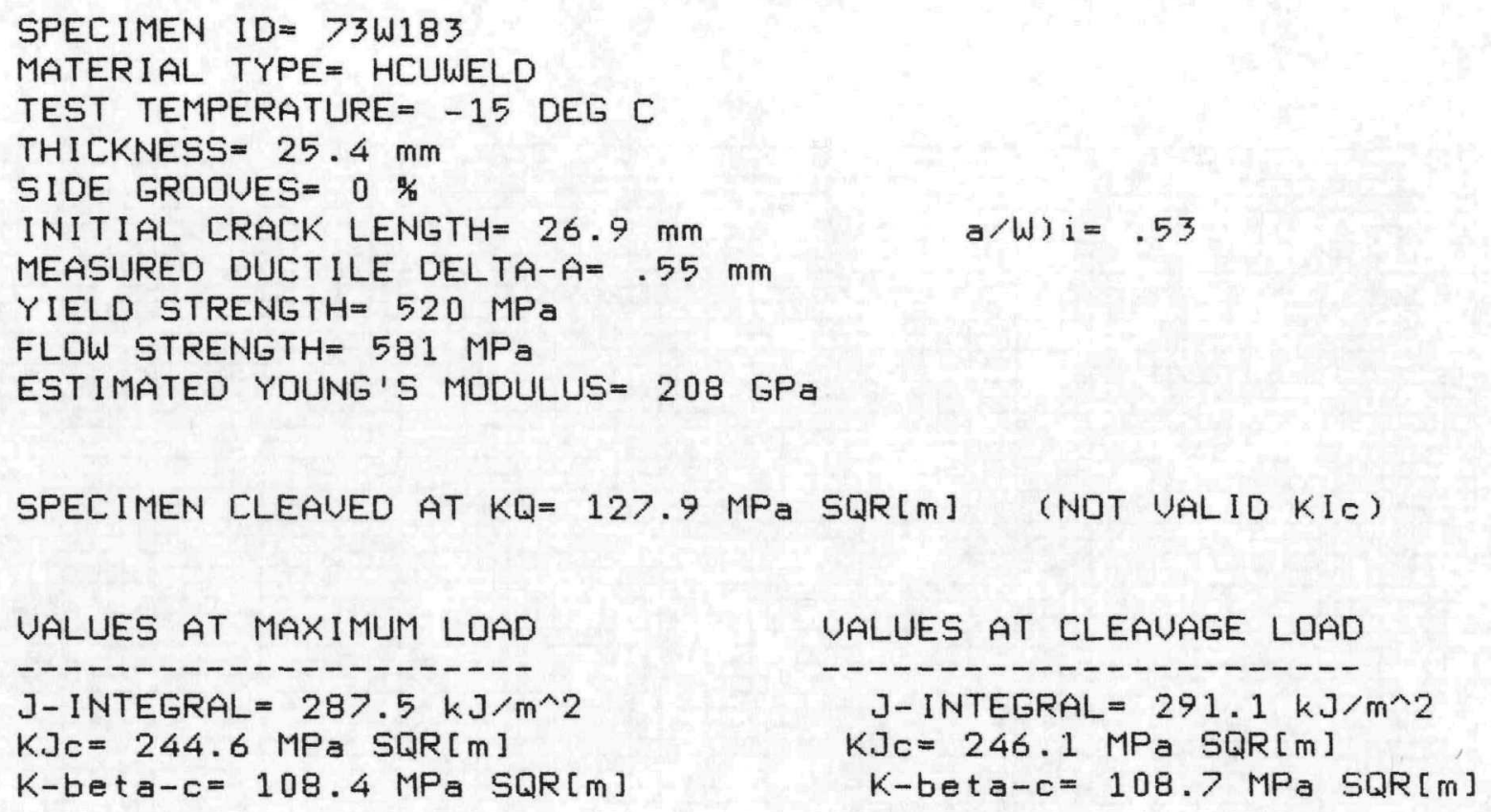




\section{F-61}

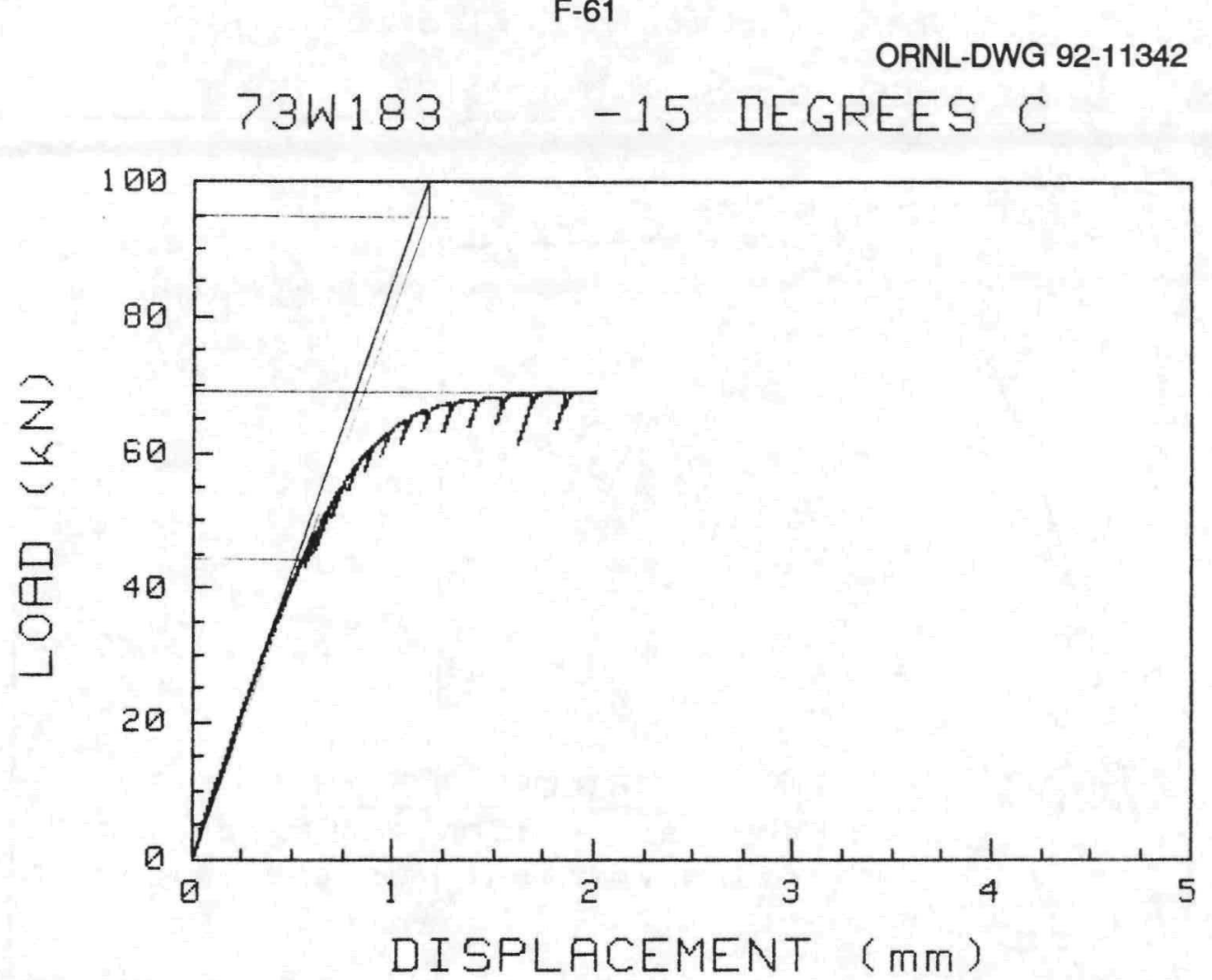

$73 W-183$

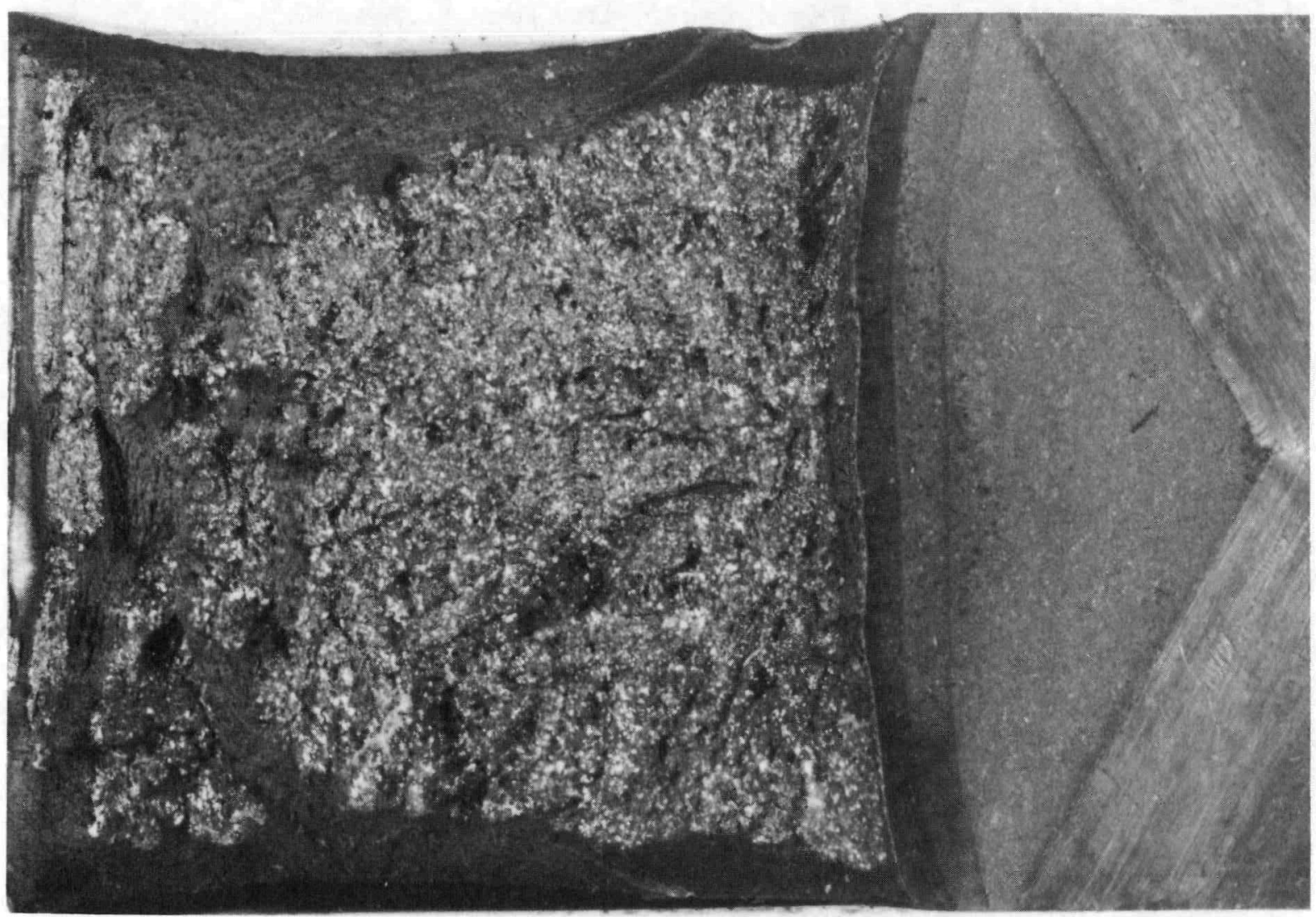


Specimen Crackmauth Deflectian (in.)

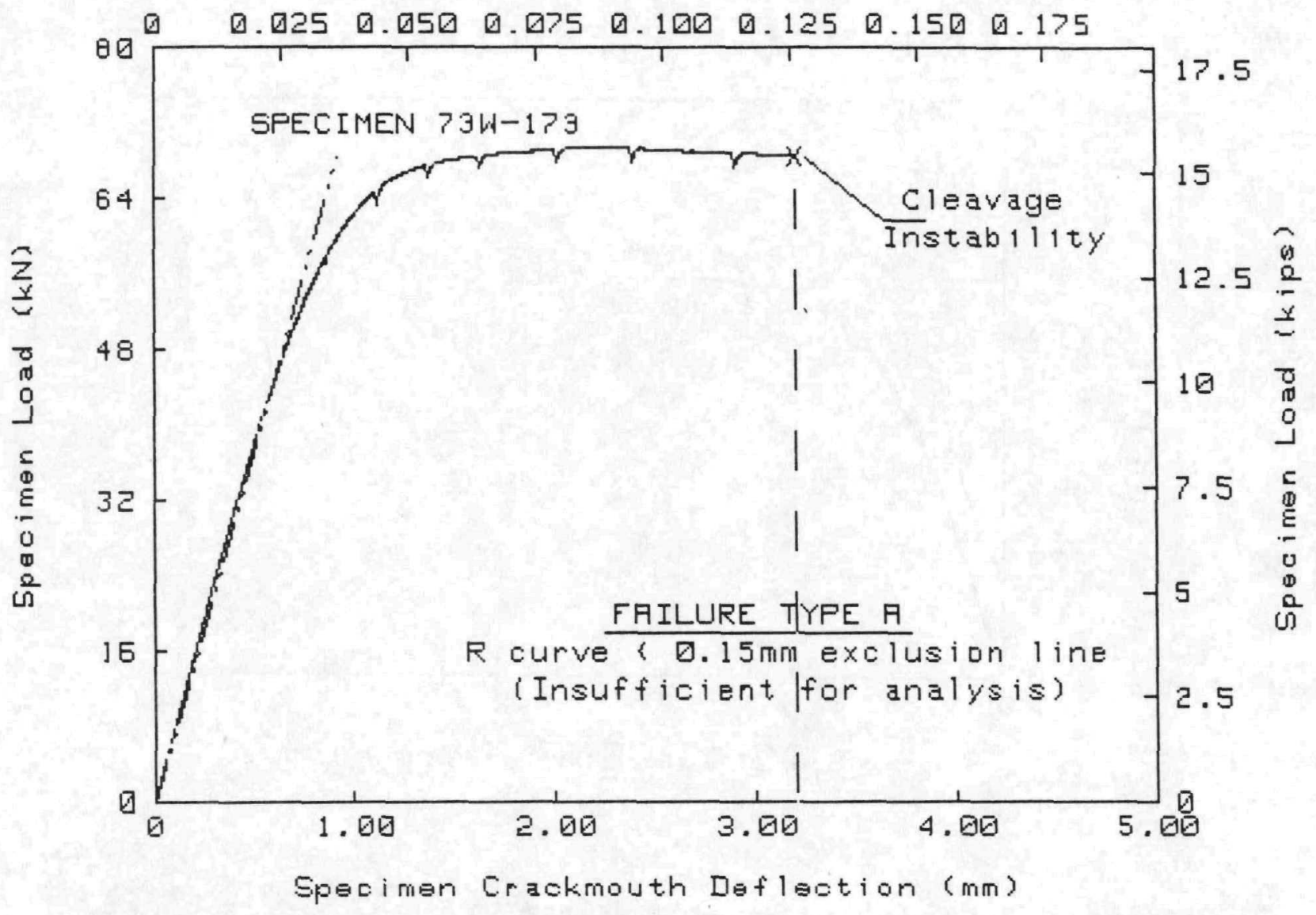

TEST SPECIMEN DATA

Material Type
Test Temperature
Percent Side Groove
Specimen Thickness B
Initial a/W
Init crack length a
Flow stress
Yield stress
Youngs modulus
Poisson's Ratio (U)
J-INTEGRAL RNALYSIS

= A533-B Weld (U)

$=-15 \circ \mathrm{C}$

$=0 \%$

$=25.4 \mathrm{~mm}$

$=.523$

$=26.59 \mathrm{~mm}$

$=578.7 \mathrm{MPa}$

$=513.4 \mathrm{MPa}$

$=208100 \mathrm{MPa}$ (Estimated Value)

$=.3$

$\mathrm{Jc}$

[ Failure Type $A$ ( $J$ at instability = JC) ] $=\frac{\text { Merk 1e-Corten }}{503.6 \mathrm{~kJ} / \mathrm{m}^{2}}$ Deformation-J*
$491.7 \mathrm{~kJ} / \mathrm{m}^{2}$
$335.3 \mathrm{MPa} \sqrt{\mathrm{m}}$

$=339.4 \mathrm{MPa} \sqrt{\mathrm{m}}$

$K J C=\left\langle E J C\left\langle\left\langle 1-v^{2}\right\rangle\right\rangle \wedge\right.$.

$=121.5 \mathrm{MPa} \sqrt{\mathrm{m}}$ $120.9 \mathrm{MPa} \sqrt{\mathrm{m}}$

ASTM E399-81 ANALYSIS

Modified Kic Equation; $B=(B B n) 1 / 2$

Maximum Load $P=69.45 \mathrm{kN}$

$5 \%$ Secant offset $P q=42.26 \mathrm{kN}$

$\mathrm{Kq}$

$=76.8 \mathrm{MPa} \sqrt{\mathrm{m}}$

= INVALID- $-1,2,3$

Wal idity

$K$ at Maximum Load

$=126.1 \mathrm{MPa} \sqrt{\mathrm{m}}$

IATA CORRECTIONS USED FOR J-INTEGRAL DATA

Merkle-Corten correction

Correction to J for crack extension (Deformation-J)

OFFSET LORD $=0 \mathrm{kN}$ OFFSET DEFLECTION $=.02 \mathrm{~mm}$

Fitted elastic load range $=5 \mathrm{kN}$ TO $25 \mathrm{kN}$

Elastic slope $=7.873 E+01 \mathrm{kN} / \mathrm{mm}$

NUREG/CR-5913 


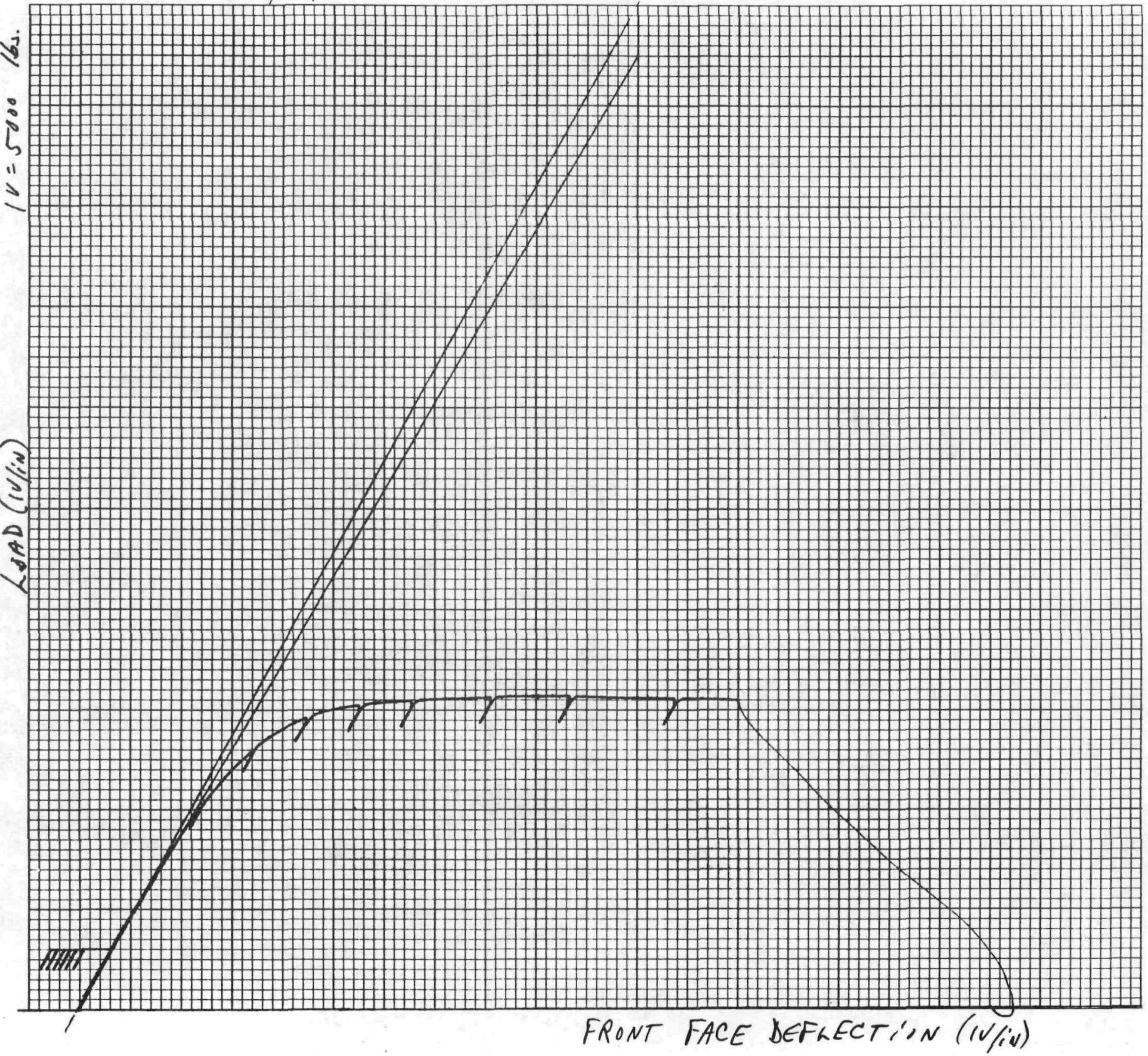


SPECIMEN ID $=73 W 169$

MATERIAL TYPE = HCU WELD

TEST TEMPERATURE $=0$ DEG C

THICKNESS $=25.5 \mathrm{~mm}$

SIDE GRODUES $=0 \%$

INITIAL CRACK LENGTH $=26.5 \mathrm{~mm}$

MEASURED DUCTILE DELTA-A $=.58 \mathrm{~mm}$

$a / W) i=.522$

YIELD STRENGTH $=511 \mathrm{MPa}$

FLOW STRENGTH $=569 \mathrm{MPa}$

ESTIMATED YOUNG'S MODULUS $=207 \mathrm{GPa}$

SPECIMEN CLEAUED AT KQ= $121.3 \mathrm{MPa} S Q R[\mathrm{~m}]$ (NOT VALID KIC)

UALUES AT MAXIMUM LDAD

J-INTEGRAL $=227.8 \mathrm{~kJ} / \mathrm{m}^{\wedge} 2$

$\mathrm{KJc}=217.2 \mathrm{MPa} \operatorname{SQR}[\mathrm{m}]$

K-beta-c $=102.5 \mathrm{MPa}$ SQR $[\mathrm{m}]$
WaLues at cleavage load

J-INTEGRAL $=250.1 \mathrm{~kJ} / \mathrm{m}^{\wedge} 2$

$\mathrm{KJ} c=227.7 \mathrm{MPa} \operatorname{SQR}[\mathrm{m}]$

K-bet $a-c=104.4 \mathrm{MPa} \operatorname{SQR}[\mathrm{m}]$

$73 W-169$

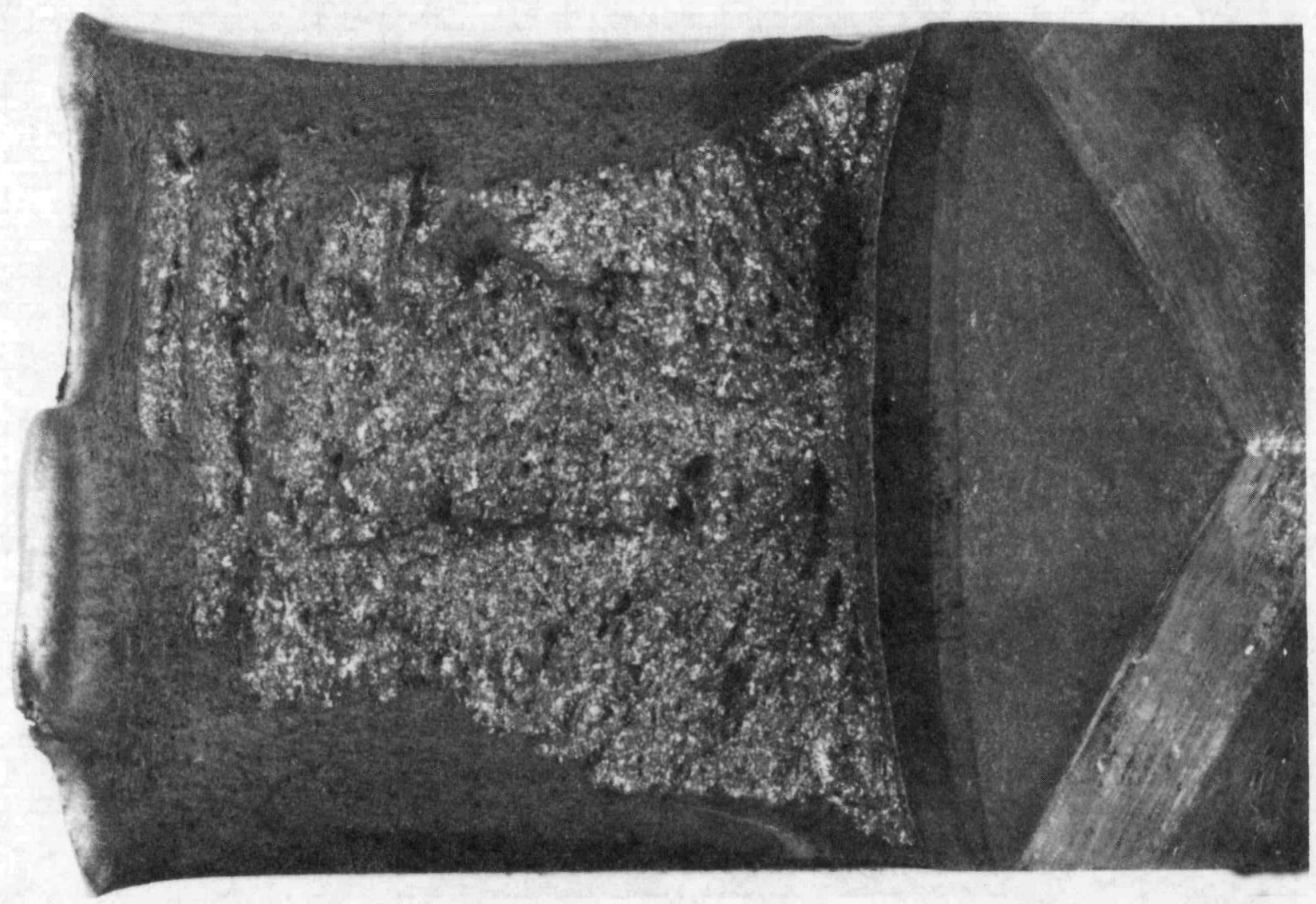




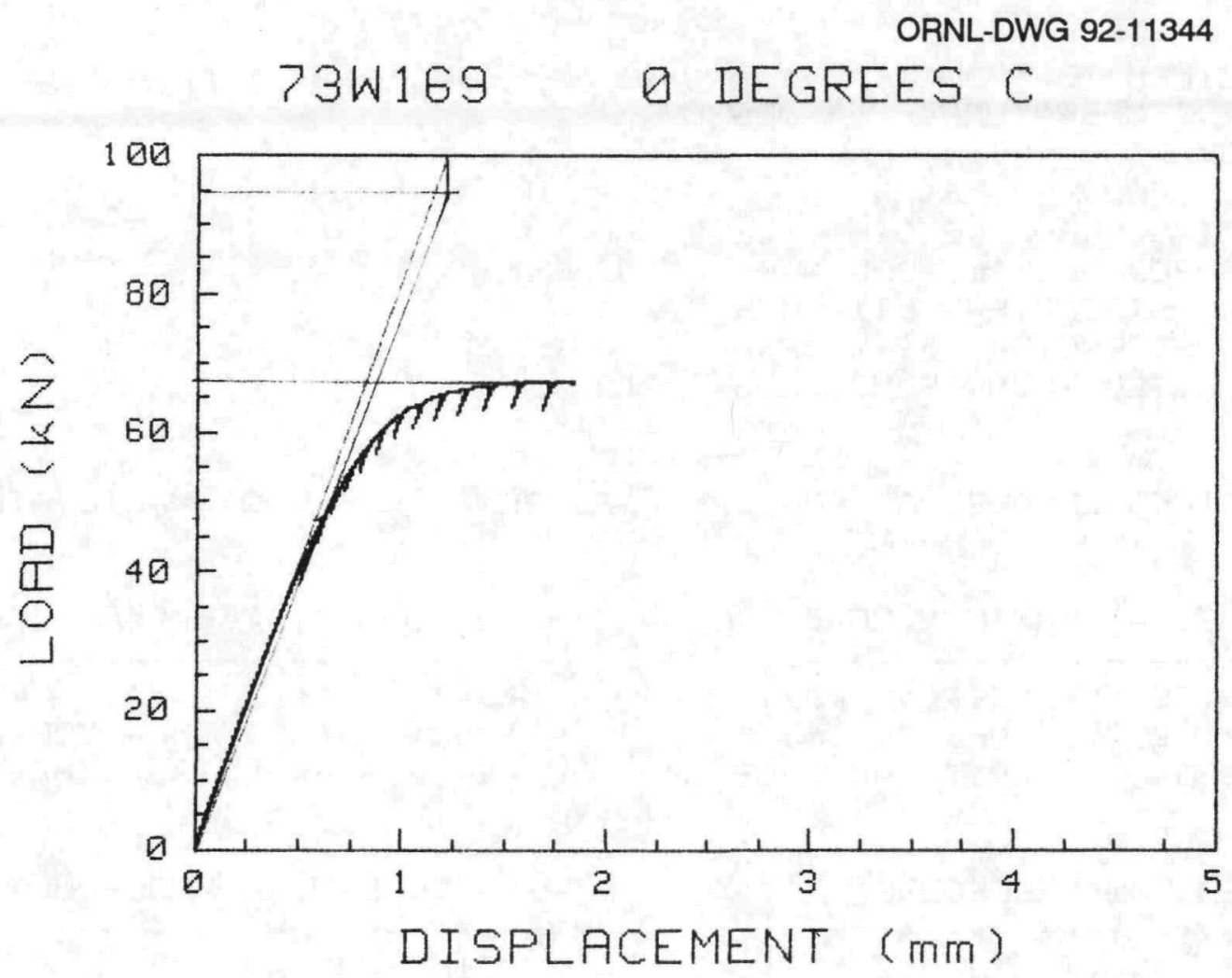

ORNL-DWG 92-11345

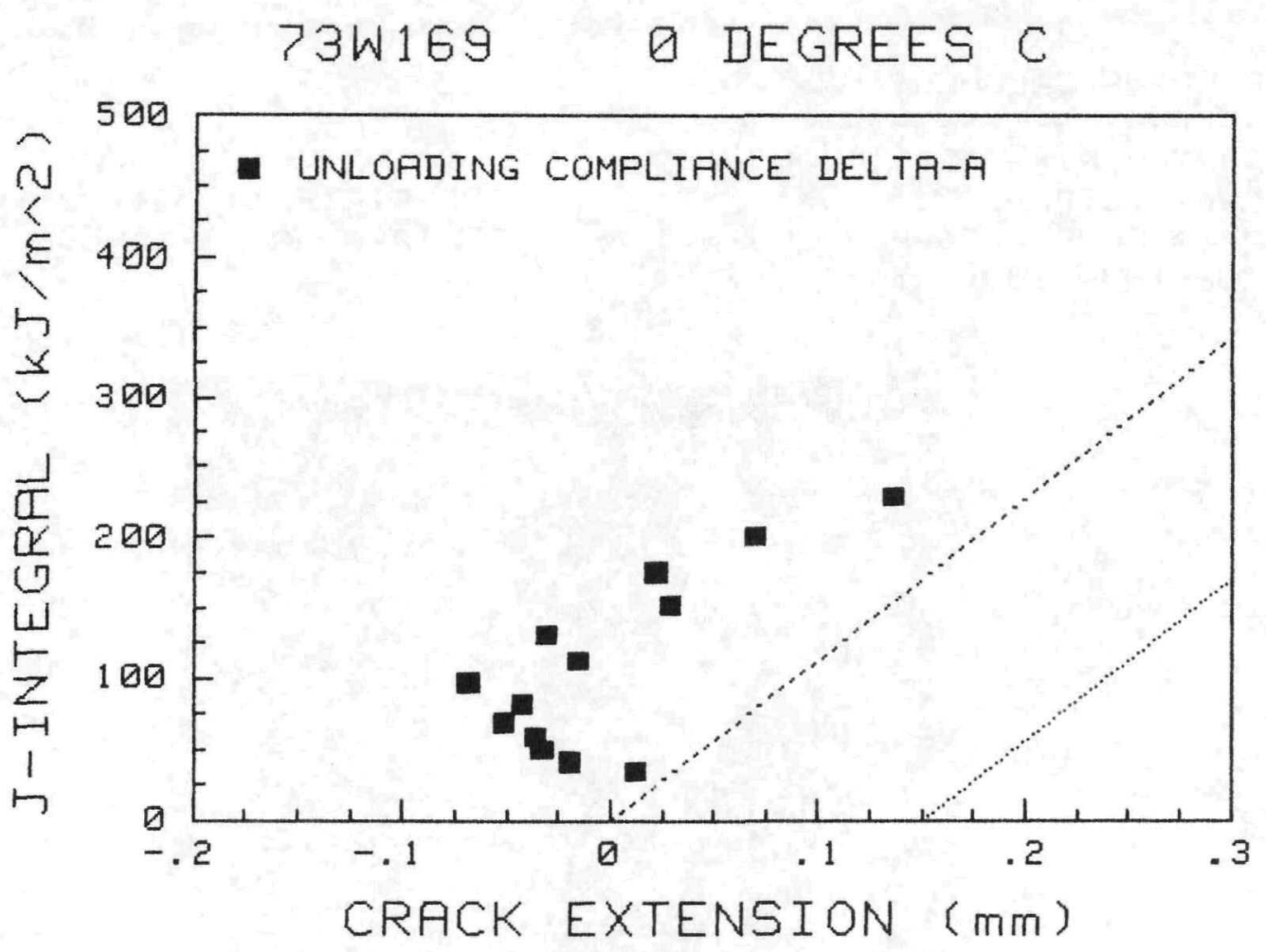


SPECIMEN ID = $73 W 116$

TEST TEMPERATURE $=0$ DEG $C$

THICKNESS $=25.4 \mathrm{~mm}$

SIDE GRODUES $=0 \%$

INITIAL CRACK LENGTH $=26.9 \mathrm{~mm} \quad \mathrm{a} / \mathrm{W}) \mathrm{i}=.529$

MEASURED DUCTILE DELTA-A $=1.02 \mathrm{~mm}$

YIELD STRENGTH $=511 \mathrm{MPa}$

FLOW STRENGTH $=569 \mathrm{MPa}$

ESTIMATED YOUNG'S MODULUS $=207$ GPa

SPECIMEN CLEAUED AT KQ= 125 MPa SQR [m] (NDT UALID KIc)

UALUES AT MAXIMUM LDAD

J-INTEGRAL $=319.4 \mathrm{~kJ} / \mathrm{m}^{\wedge} 2$

$K J c=257.3 \quad M P a S Q R[m]$

K-beta-c $=109.3$ MPa SQR $[\mathrm{m}]$

POWER LAW PRDCEDURE

JIc $=350.7 \mathrm{~kJ} / \mathrm{m}^{\wedge} 2$

$\mathrm{K}-\mathrm{JIC}=269.6 \mathrm{MPa} \operatorname{SQR}[\mathrm{m}]$

POWER $=.5598$

CDEFFICIENT $=542.9 \mathrm{~kJ} / \mathrm{m}^{\wedge} 2$

$T$ (AUERAGE) $=176$
WaLues at cleavage load

J-INTEGRAL $=448.2 \mathrm{~kJ} / \mathrm{m} \wedge 2$

$K J c=304.7$ MPa SQR $[\mathrm{m}]$

$K$-beta-c $=116.4$ MPa SQR [m]

ASTM LINEAR PROCEDURE

$J I c=0 \quad \mathrm{~kJ} / \mathrm{m}^{\wedge} \mathrm{2}$

$K-J I c=0 \quad M P a S Q R[m]$

$S L O P E=0 \mathrm{~kJ} / \mathrm{m}^{\wedge} 3$

INTERCEPT $=0 \mathrm{~kJ} / \mathrm{m}^{\wedge} 2$

$T(A S T M)=\quad D$

INUALID, 16

JI-R CURUE CALCULATIONS

$J($ J $/ T=8.8)=1245.1 \mathrm{~kJ} / \mathrm{m}^{\wedge} 2$
$P$ PWER $=.6306$
$J$-MAX ALLOWED $=680.9 \mathrm{~kJ} / \mathrm{m}^{\wedge} 2$
RCURUE INUALID, 23

COEFFICIENT $=560.2 \mathrm{~kJ} / \mathrm{m}^{\wedge} 2$

DELTA-A MAX ALLOWED $=2.39 \mathrm{~mm}$

$73 W-116$ 

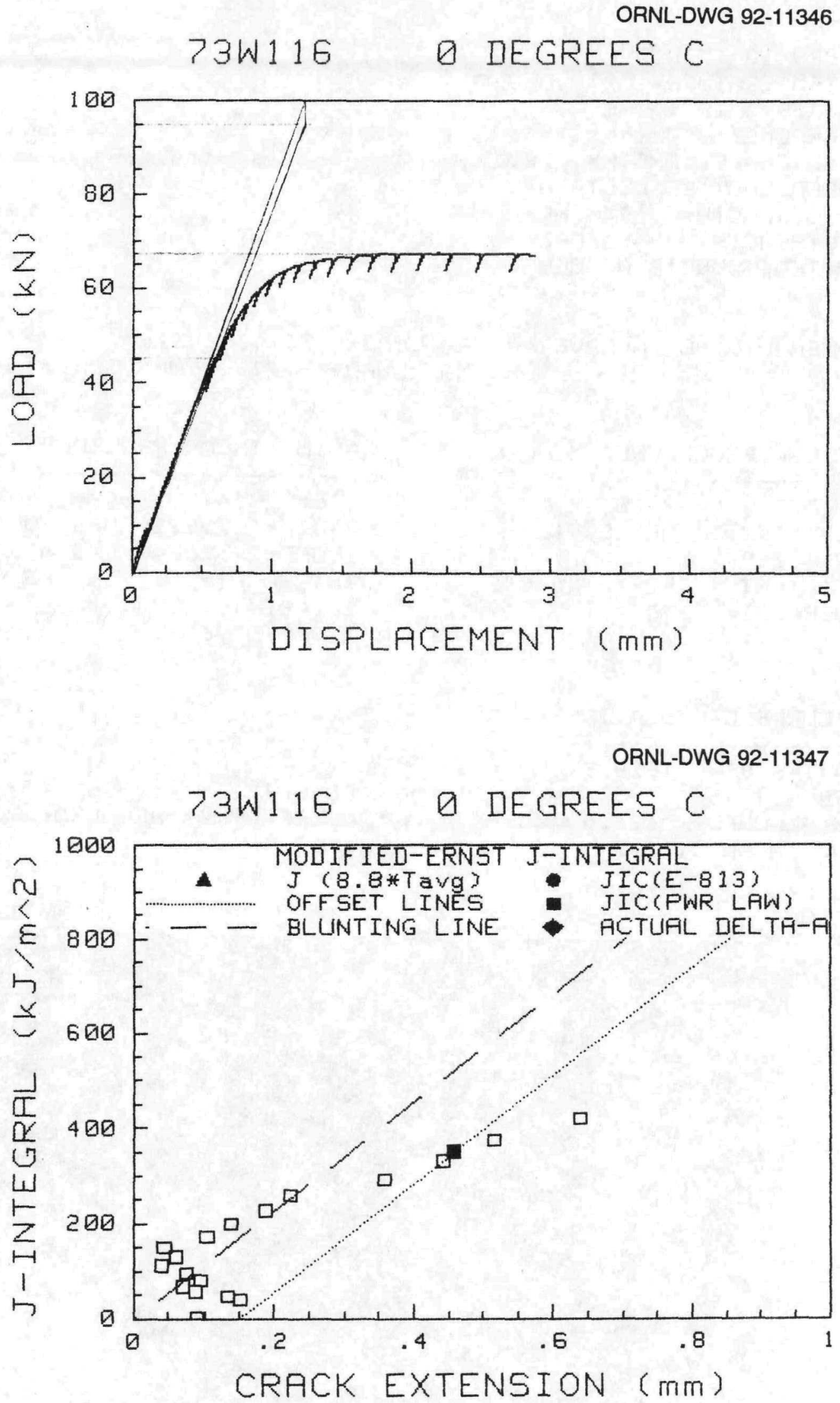


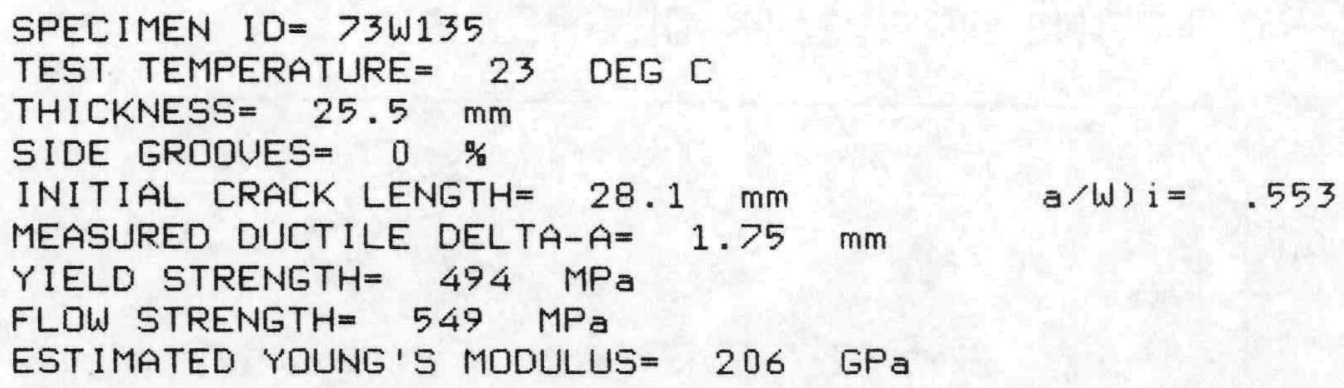

POWER LAW PROCEDURE

$\mathrm{J} \mathrm{IC}=309.2 \mathrm{~kJ} / \mathrm{m}^{\wedge} 2$

$K-J I c=252.3 \quad M P a S Q R[m]$

POWER $=.69$

COEFFICIENT $=552.3 \mathrm{~kJ} / \mathrm{m}^{\wedge} 2$

$T(A U E R A G E)=238$

J I-R CURUE CALCULATIONS

$J($ OJ $/ T=8.8)=1528.5 \mathrm{~kJ} / \mathrm{m}^{\wedge} 2$
POWER $=.7026$
$J$-MAX ALLOWED $=623.5 \mathrm{~kJ} / \mathrm{m}^{\wedge} 2$
RCURUE INUALID, 23

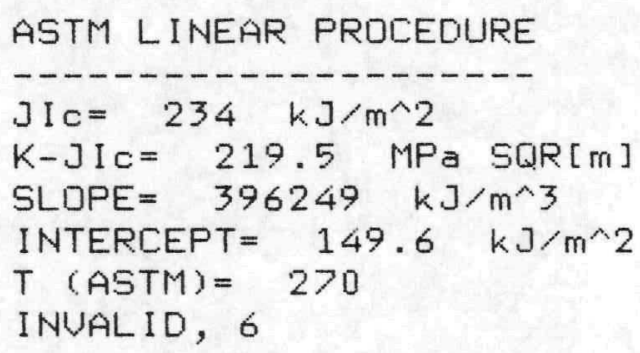

COEFFICIENT $=556 \mathrm{~kJ} / \mathrm{m}^{\wedge} 2$

DELTA-A MAX ALLOWED $=2.27 \mathrm{~mm}$

$73 W-135$

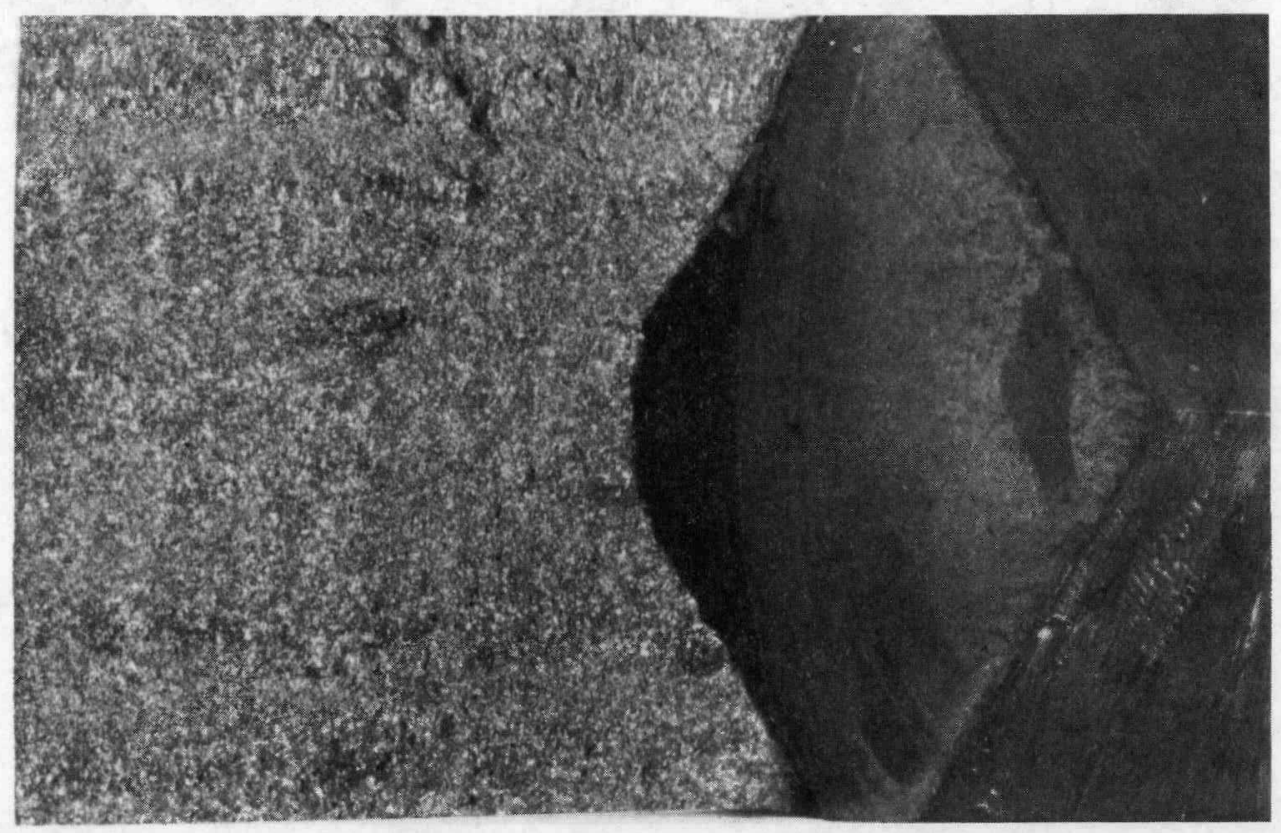




\section{W135 23 DEGREES C}

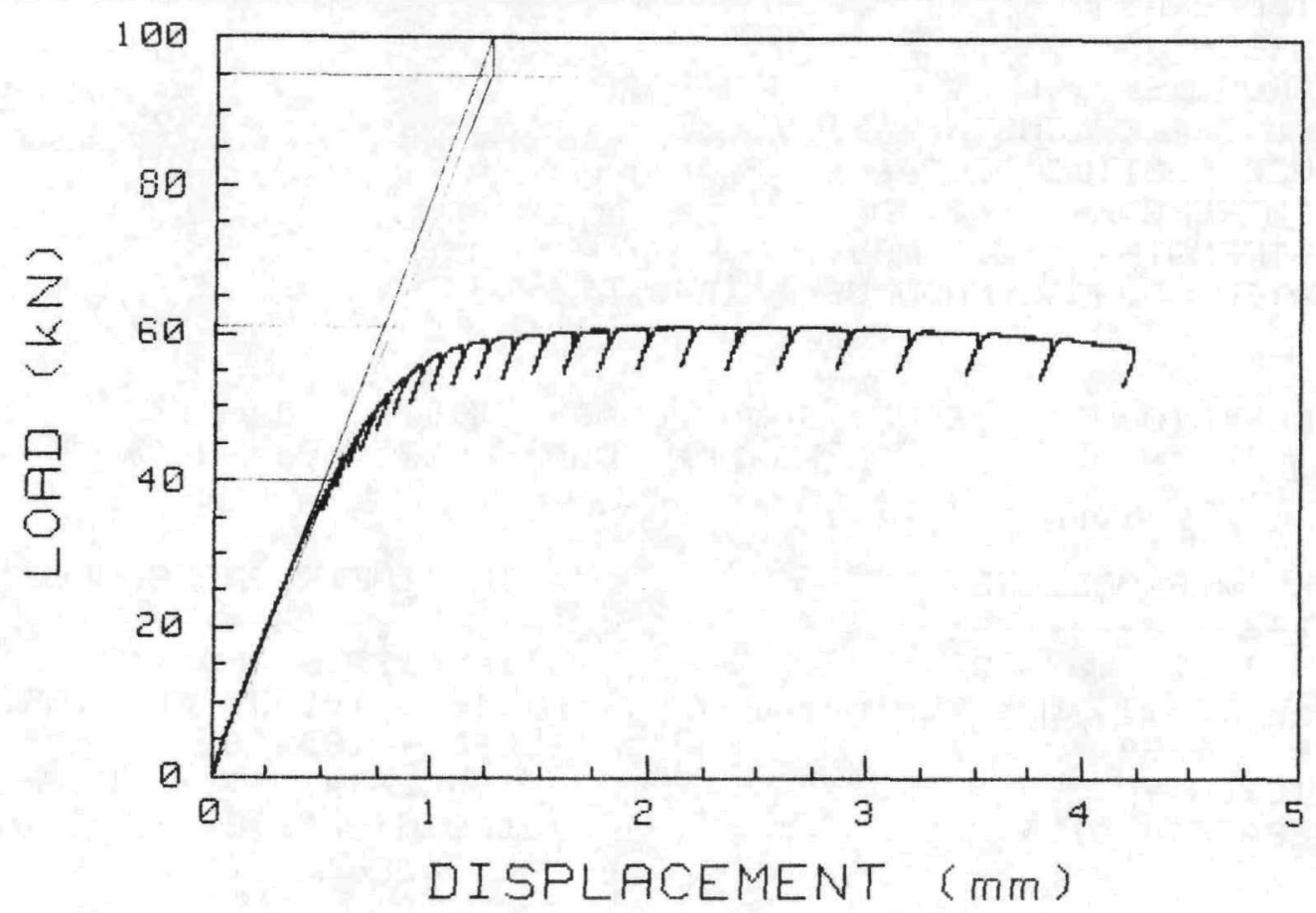

ORNL-DWG 92-11349

73W135 23 DEGREES C

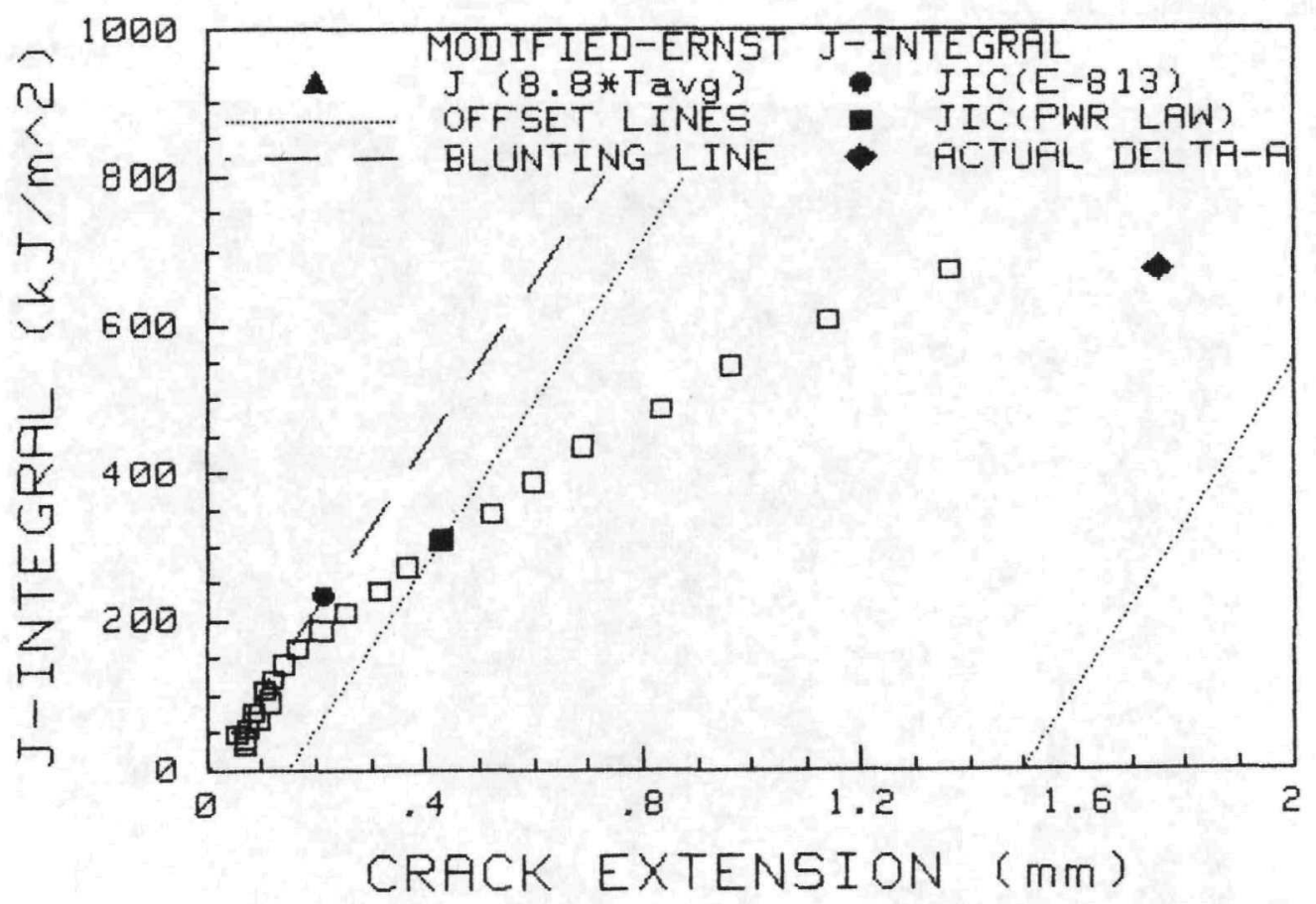




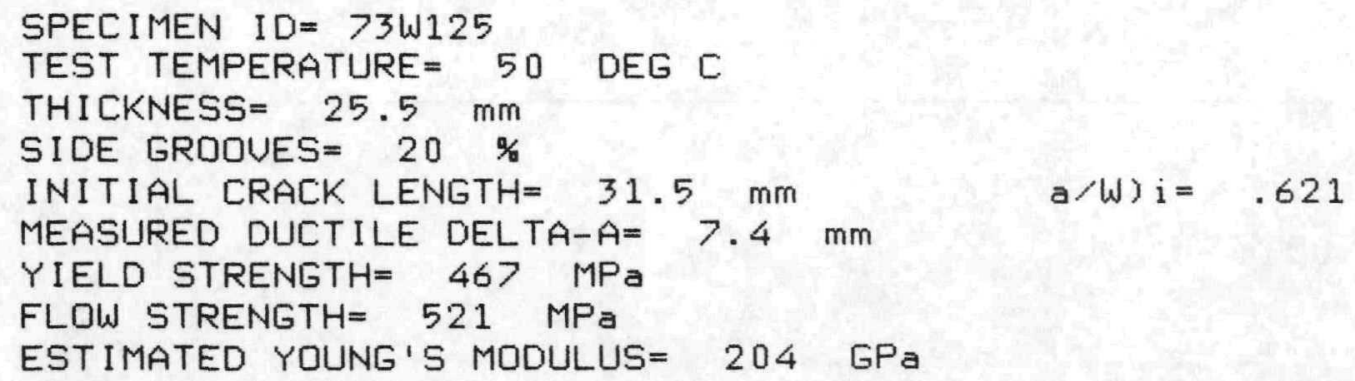

POWER LAW PROCEDURE

J I $=139.2 \mathrm{~kJ} / \mathrm{m}^{\wedge} 2$

$\mathrm{K}-J I_{\mathrm{c}}=168.7 \mathrm{MPa}$ SQR $[\mathrm{m}]$

POWER $=.5759$

COEFFICIENT $=287.7 \mathrm{~kJ} / \mathrm{m}^{\wedge} 2$

$T$ (AUERAGE) $=125$

JI-R CURUE CALCULATIONS

J (国了 $/ T=8.8)=636.9 \mathrm{~kJ} / \mathrm{m}^{\wedge} 2$

POWER $=.5881$
$J-M A X$ ALLOWED $=500.7 \mathrm{~kJ} / \mathrm{m}^{\wedge} 2$ RCURUE INUALID, 2

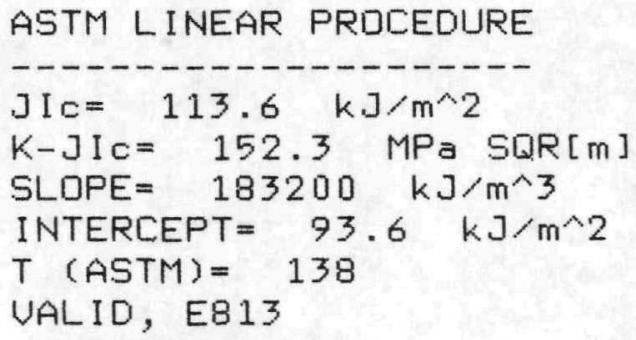

COEFFICIENT $=286.3 \mathrm{~kJ} / \mathrm{m}^{\wedge} 2$

DELTA-A MAX ALLOWED $=1.92 \mathrm{~mm}$

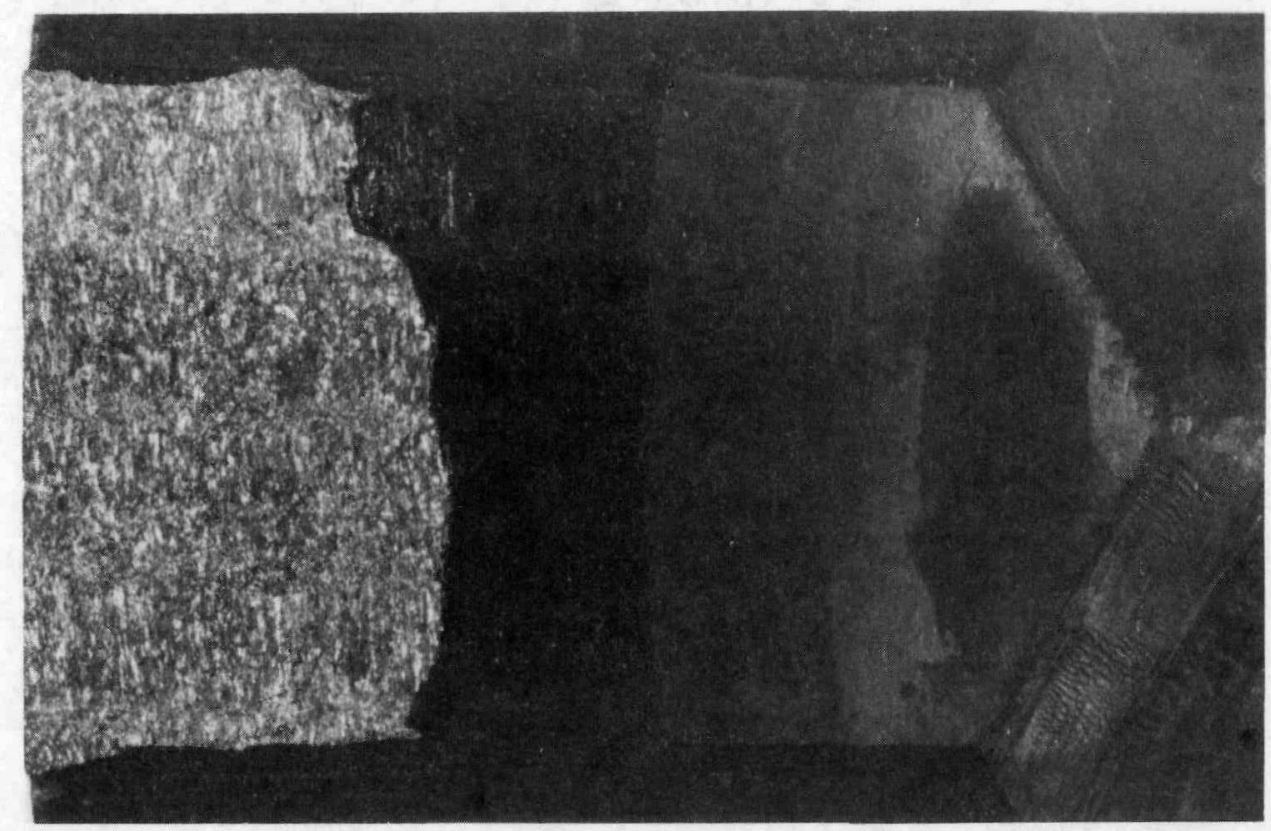




\section{W125 50 DEGREES C}

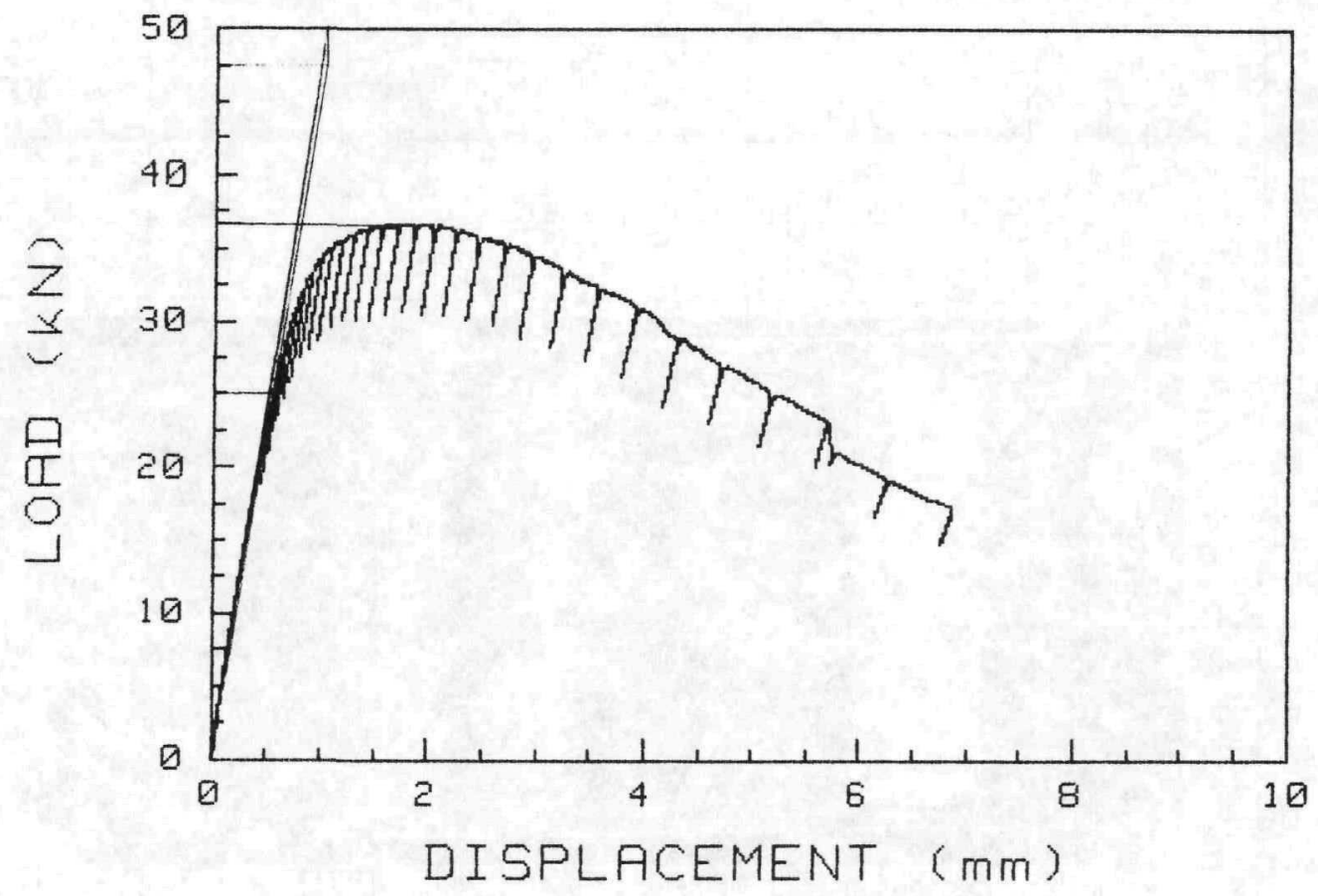

ORNL-DWG 92-11351

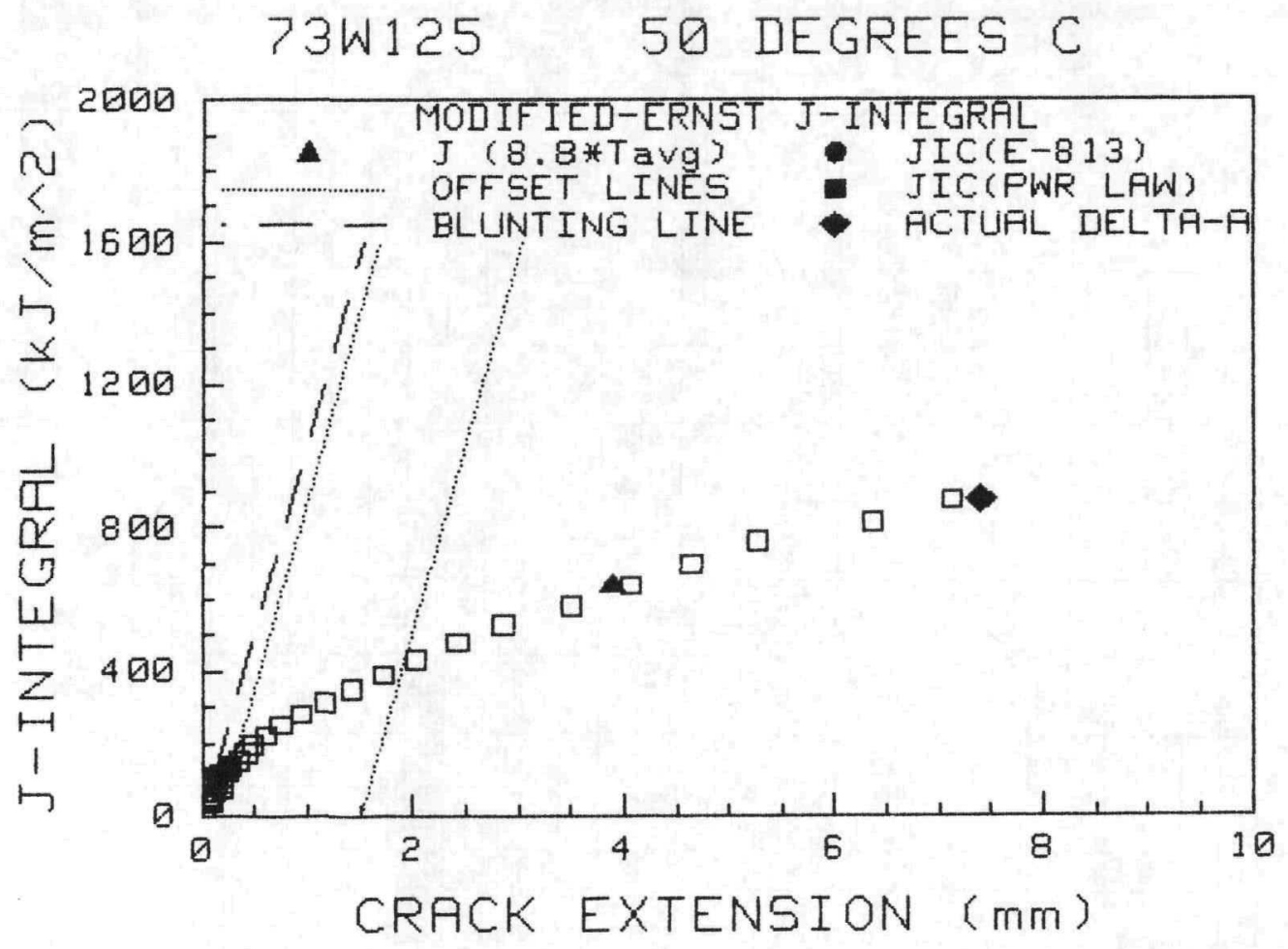




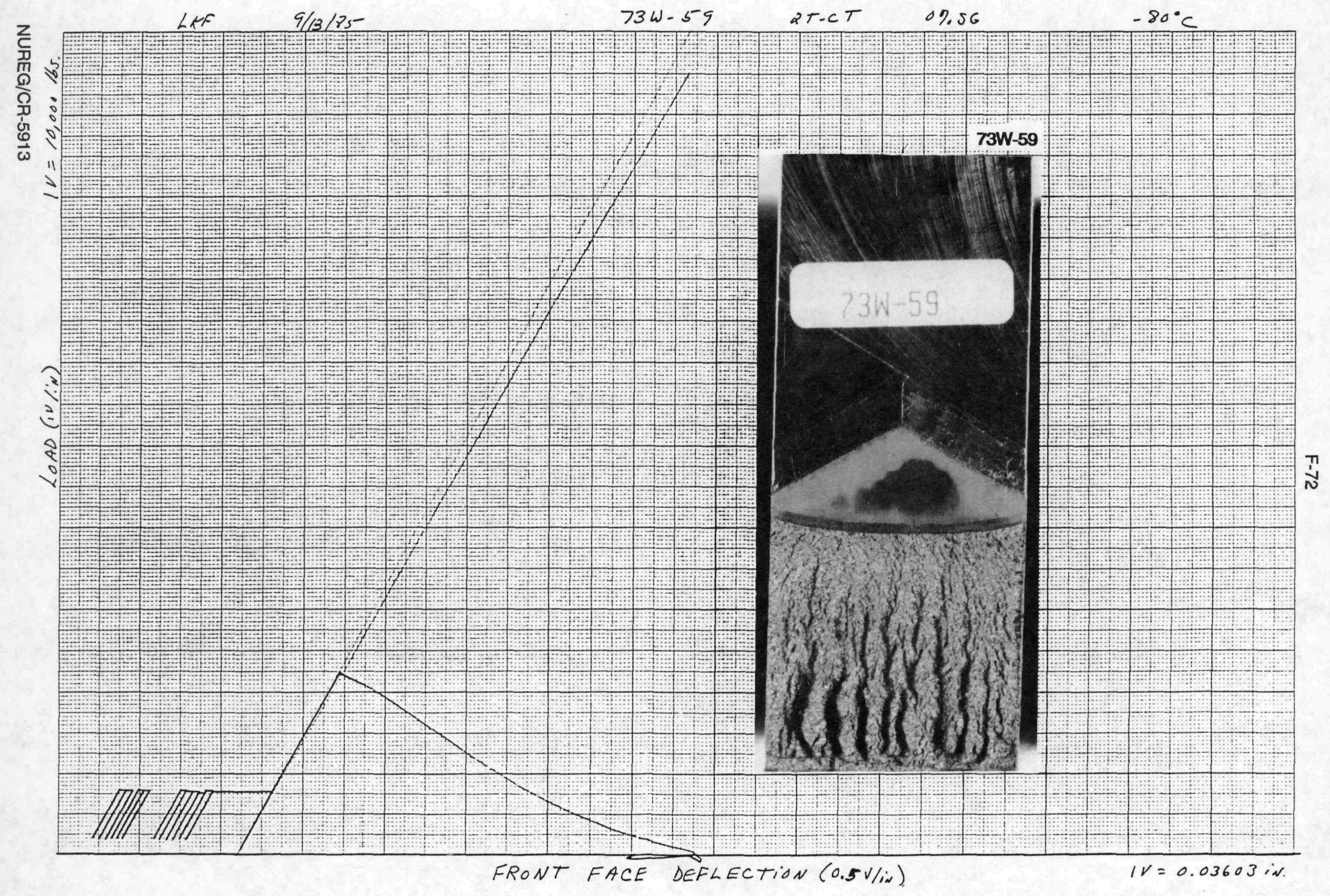




\section{W73 -80 DEGREES C}

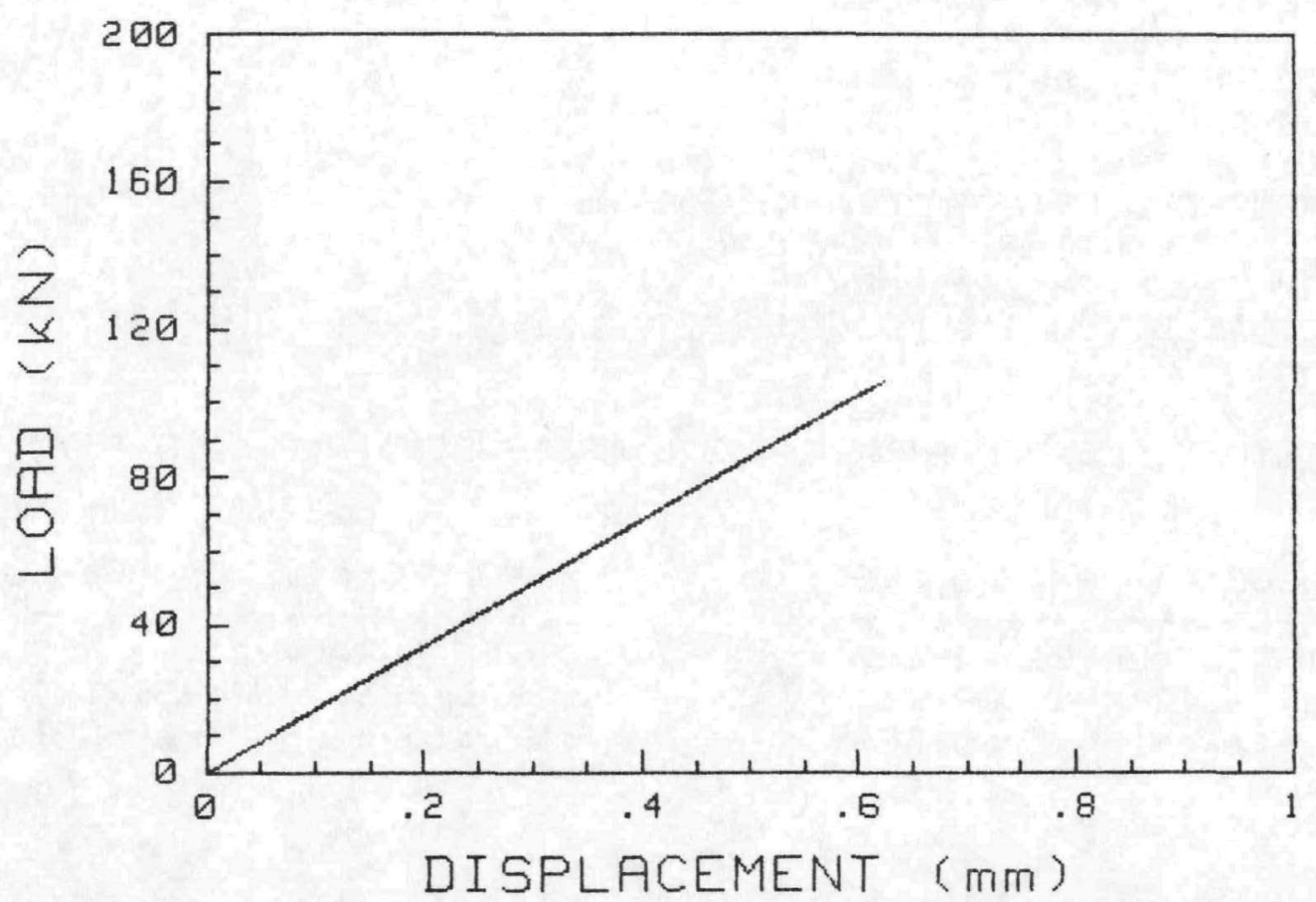

$73 W-73$

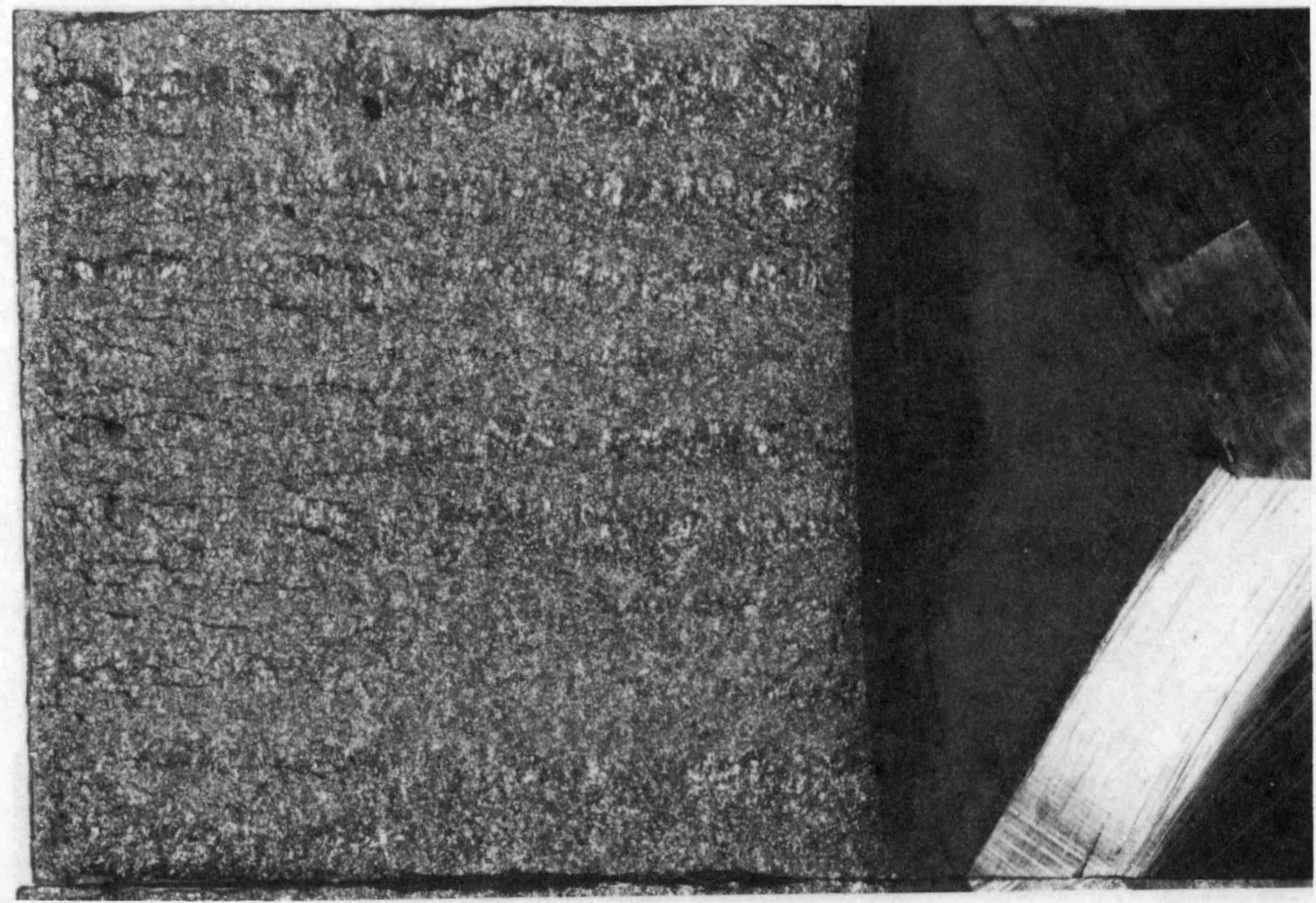

NUREG/CR-5913 


\section{F-75}

SPECIMEN ID $=731666$

MATERIAL TYPE = HCUWELD

TEST TEMPERATURE $=-80$ DEG $C$

THICKNESS $=50.9 \mathrm{~mm}$

SIDE GROOUES = $0 \%$

INITIAL CRACK LENGTH $=53.2 \mathrm{~mm}$

MEASURED DUCTILE DELTA-A $=.05 \mathrm{~mm}$

$a / W) i=.523$

YIELD STRENGTH $=571 \mathrm{MPa}$

FLOW STRENGTH $=639 \mathrm{MPa}$

ESTIMATED YOUNG'S MODULUS $=212 \mathrm{GPa}$

SPECIMEN LLEAUED AT KIC $=72.7$ MPa SQR[m] (UALID ASTM E399)

UALUES AT MAXIMUM LOAD

$J$-INTEGRAL $=24.6 \mathrm{~kJ} / \mathrm{m}^{\wedge} 2$

$\mathrm{KJ} c=72.1 \mathrm{MPa} S Q R[\mathrm{~m}]$

$K$-bet $a-c=68.4 \mathrm{MPa} \operatorname{SQR}[\mathrm{m}]$
UALUES AT CLEAUAGE LOAD

J-INTEGRAL $=24.6 \mathrm{~kJ} / \mathrm{m}^{\wedge} 2$

$\mathrm{K} J \mathrm{c}=72.1 \mathrm{MPa} \operatorname{SQR}[\mathrm{m}]$

$K$-beta-c $=68.4 \mathrm{MPa} \operatorname{SQR}[\mathrm{m}]$ 
F-76

ORNL-DWG 92-11353

73W66 -80 DEGREES C

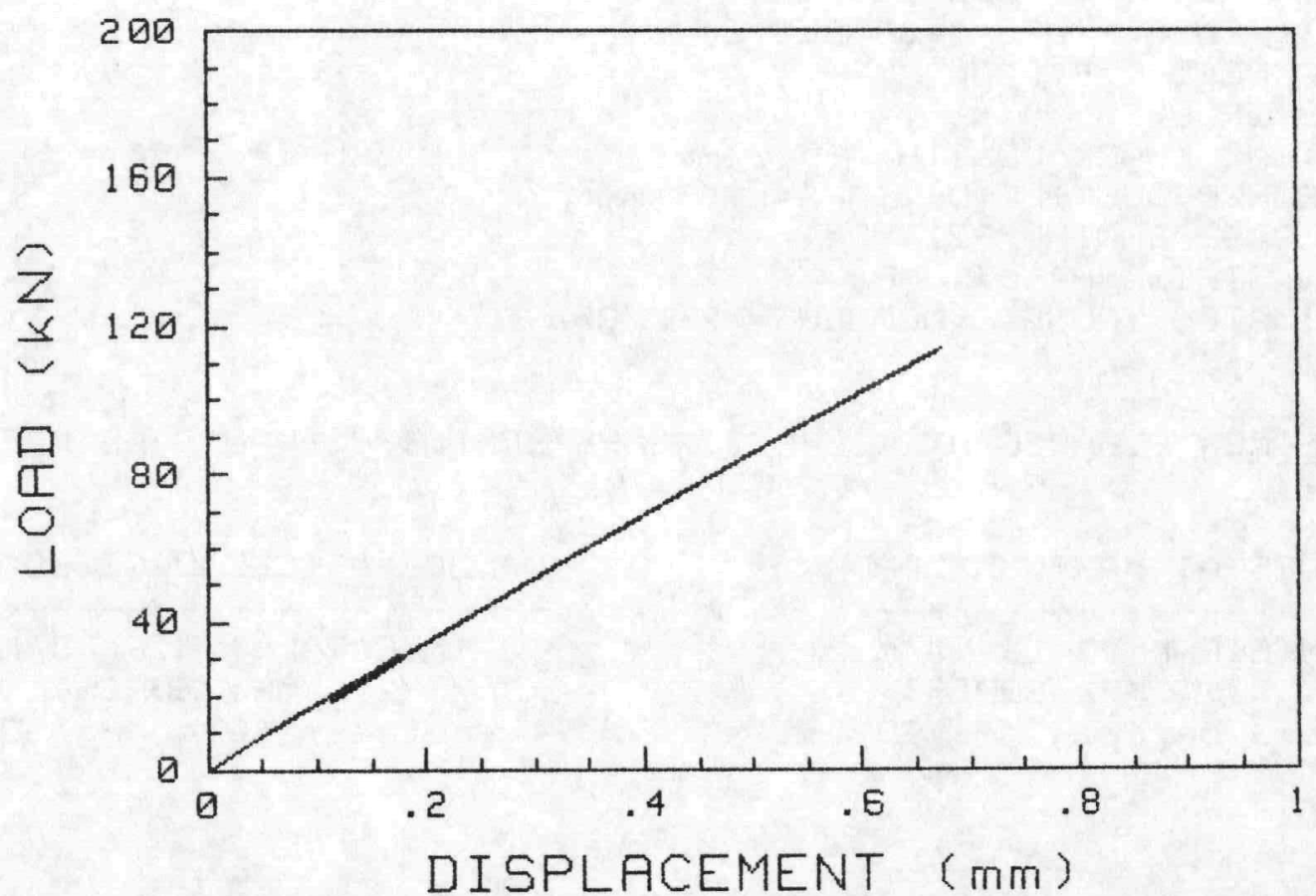

$73 W-66$

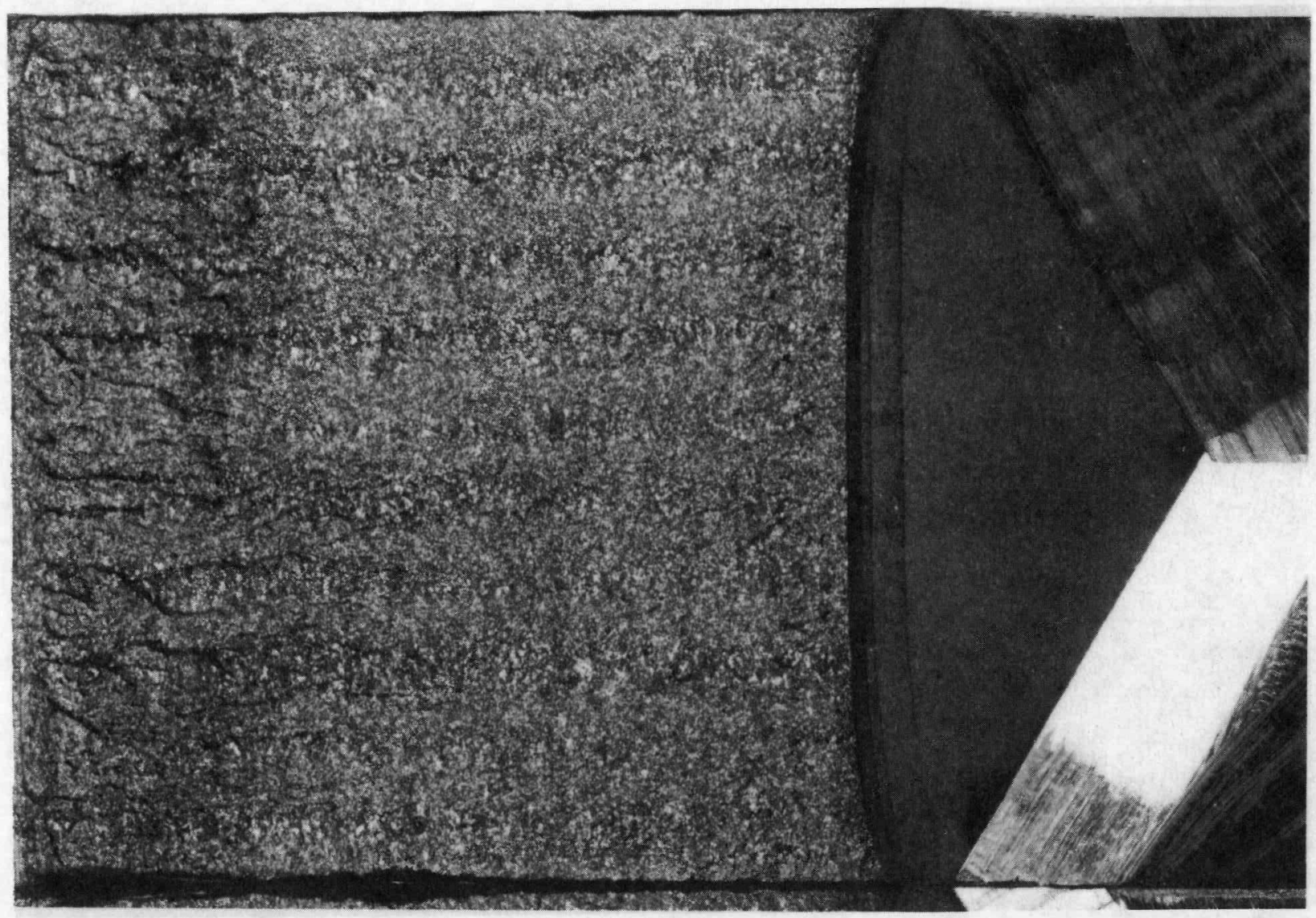




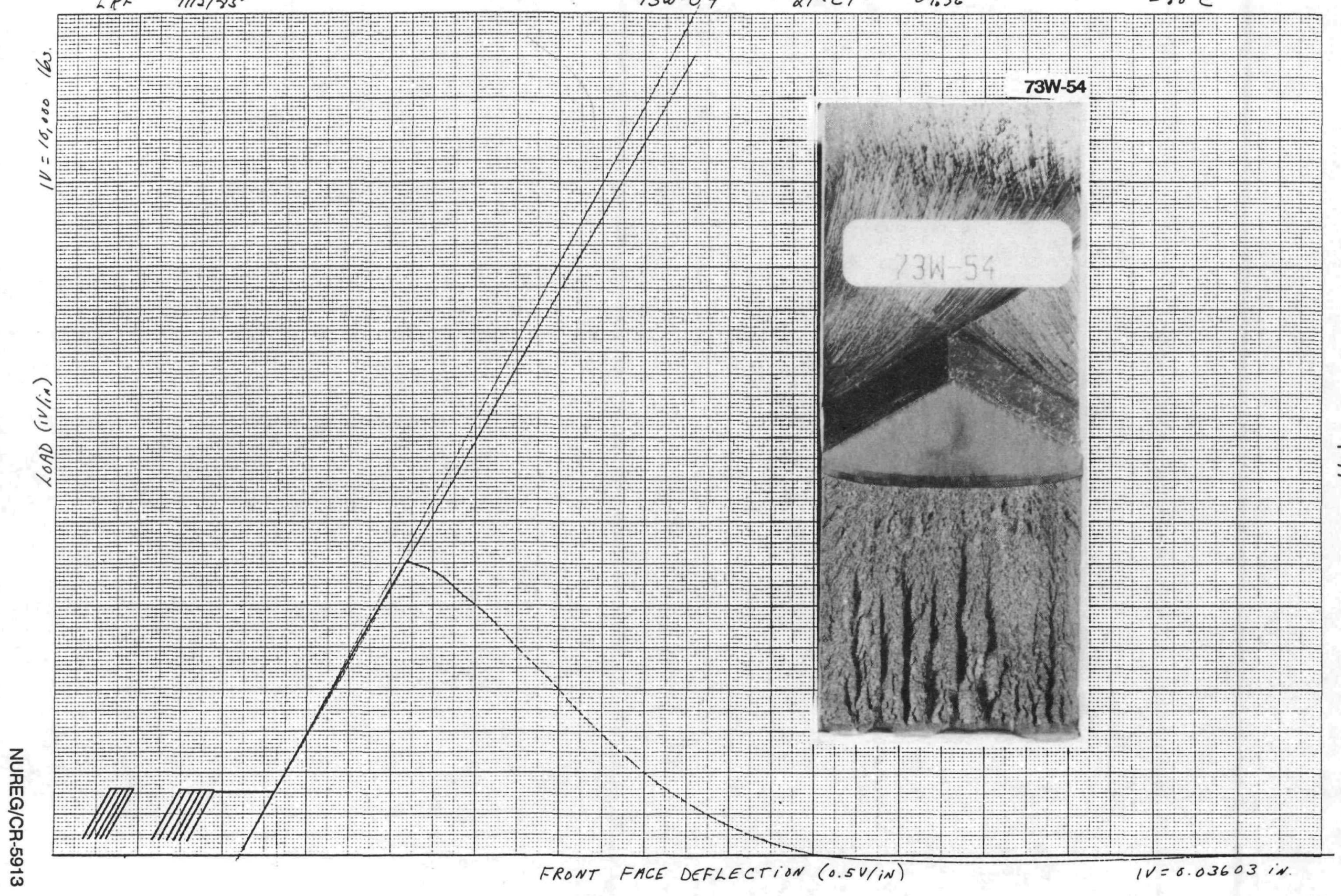


SPECIMEN ID $=73$ W88

MATERIAL TYPE = HCUWELD

TEST TEMPERATURE $=-50$ DEG $C$

THICKNESS $=50.8 \mathrm{~mm}$

SIDE GROOUES = $0 \%$

INITIAL CRACK LENGTH $=53.4 \mathrm{~mm}$

MEASURED DUCTILE DELTA-A $=.05 \mathrm{~mm}$

$a / W) \mathrm{i}=.526$

YIELD STRENGTH $=543 \mathrm{MPa}$

FLOW STRENGTH $=609 \mathrm{MPa}$

EST IMATED YOUNG'S MODULUS $=210 \mathrm{GPa}$

SPECIMEN CLEAUED AT KIC= $74.3 \mathrm{MPa}$ SQR $[\mathrm{m}]$ (UALID ASTM E399)

UALUES AT MAXIMUM LDAD

J-INTEGRAL $=25.2 \mathrm{~kJ} / \mathrm{m}^{\wedge} 2$

$\mathrm{KJ} c=72.7 \mathrm{MPa} \operatorname{SQR}[\mathrm{m}]$

$K$-beta-c $=68.2 \mathrm{MPa} \operatorname{SQR}[\mathrm{m}]$
UALUES AT CLEaUAGE LDAD

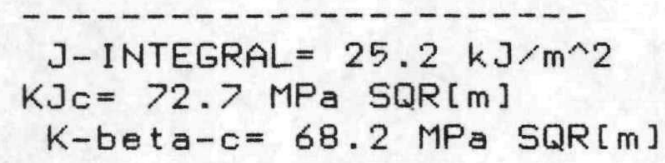


F-79

ORNL-DWG 92-11354

73W88 -50 DEGREES C

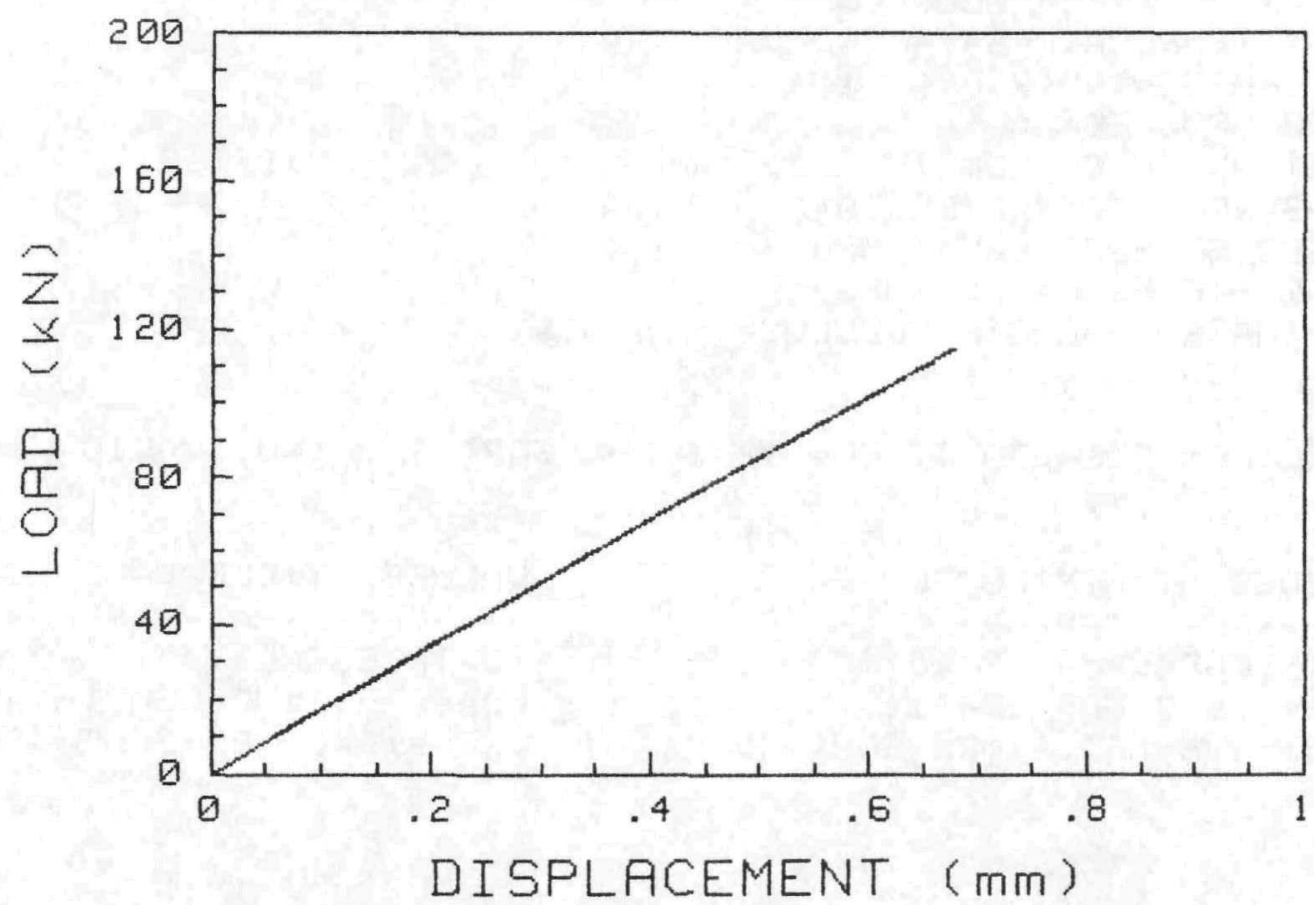

$73 W-88$

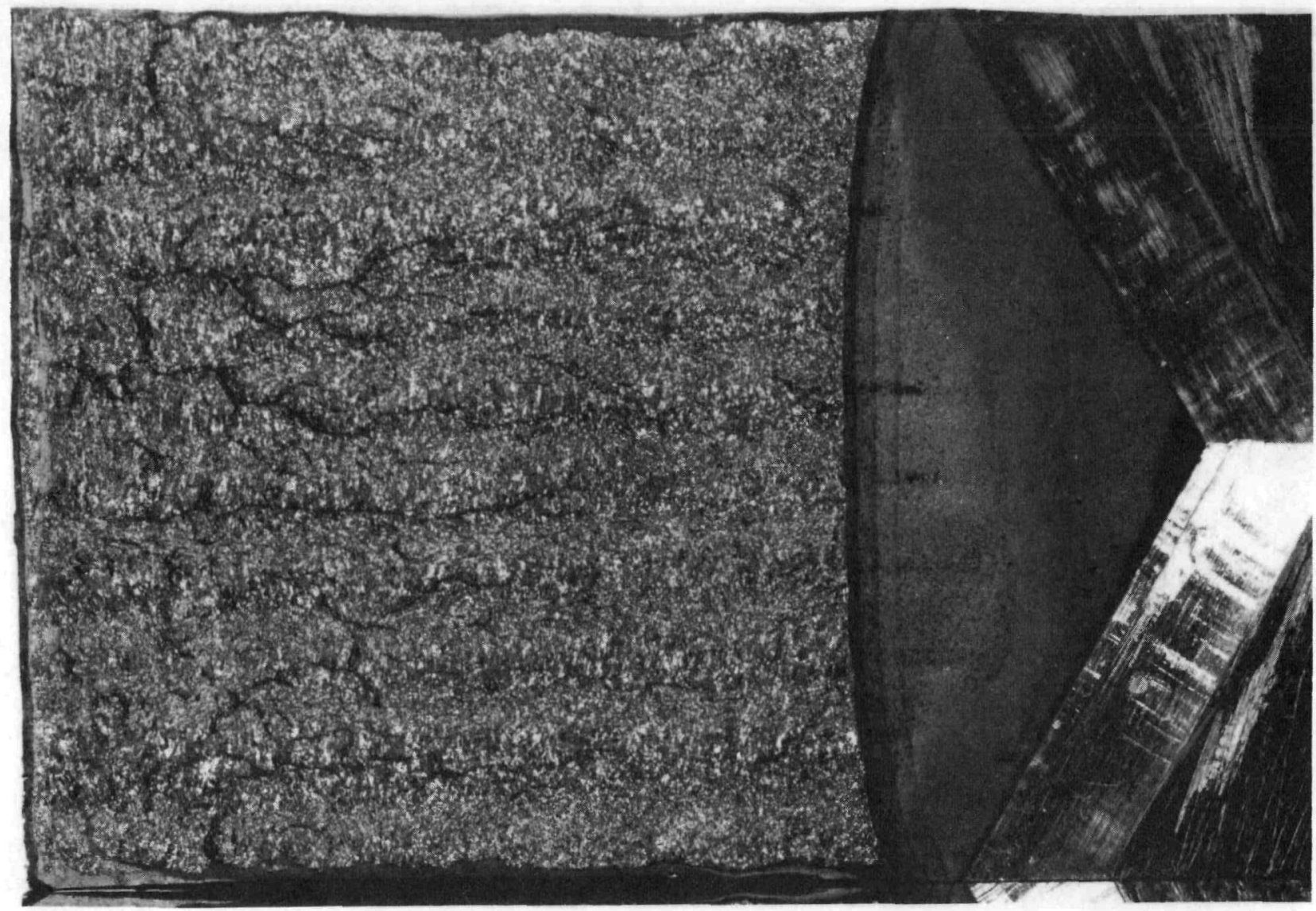

NUREG/CR-5913 


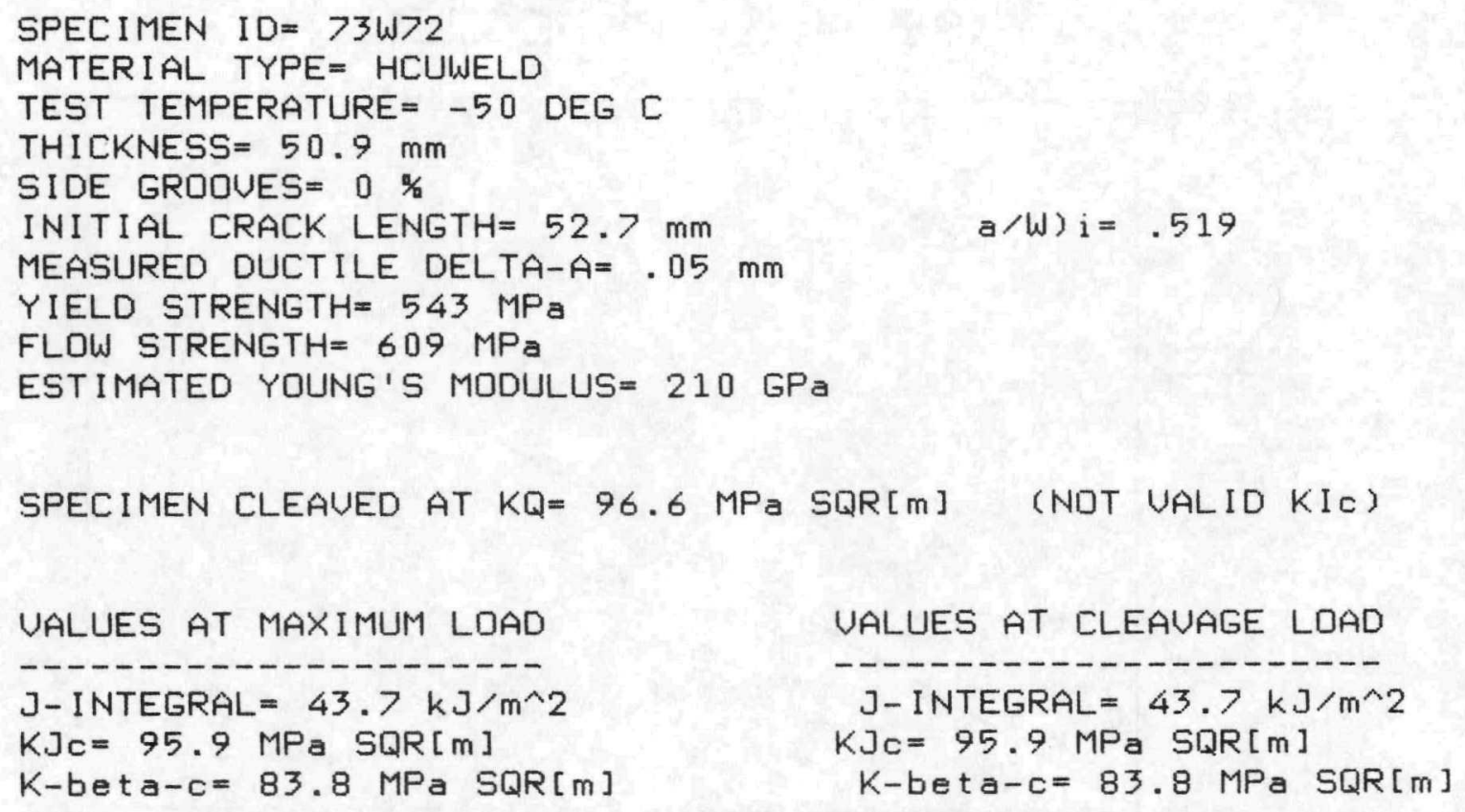


F-81

ORNL-DWG 92-11355

73W72 -50 DEGREES C

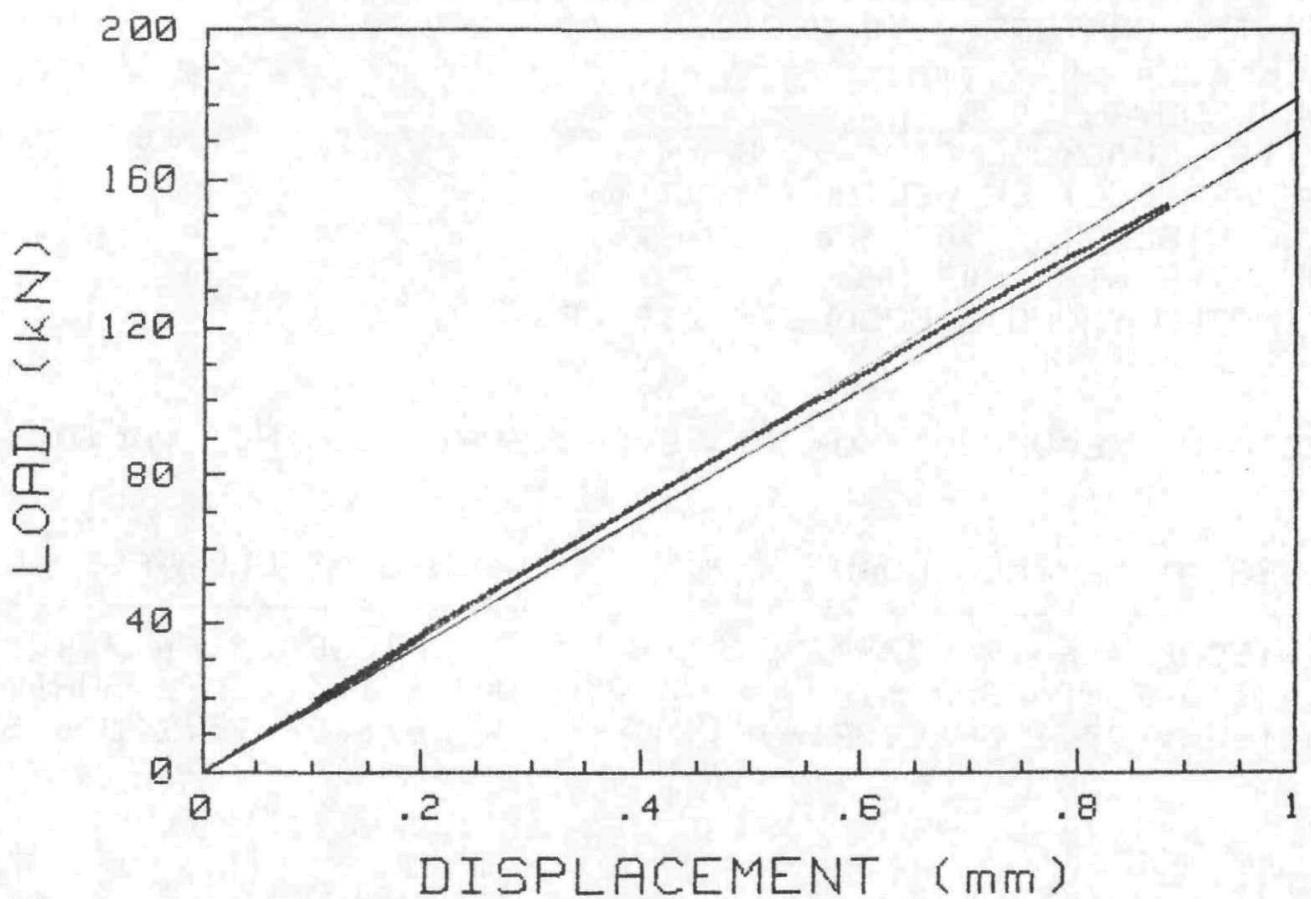

$73 W-72$

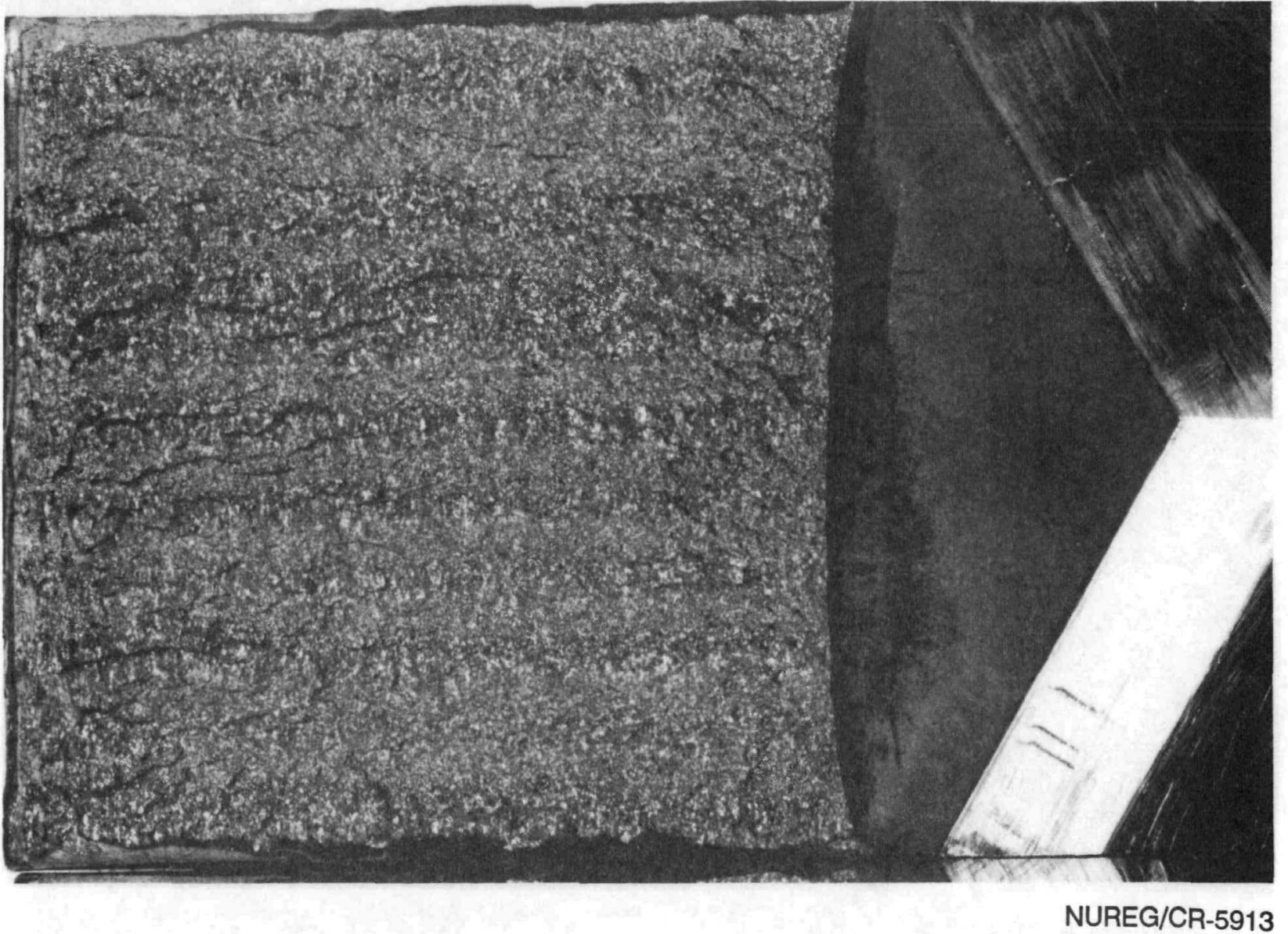


SPECIMEN ID $=73$ W80

MATERIAL TYPE $=$ HCUWELD

TEST TEMPERATURE $=-50$ DEG $\mathrm{C}$

THICKNESS $=50.9 \mathrm{~mm}$

SIDE GROQUES = $0 \%$

INITIAL CRACK LENGTH $=52.8 \mathrm{~mm}$

$a / W) i=.52$

MEASURED DUCTILE DELTA-A $=.07 \mathrm{~mm}$

$Y$ IELD STRENGTH $=543 \mathrm{MPa}$

FLOW STRENGTH $=609 \mathrm{MPa}$

ESTIMATED YOUNG'S MODULUS $=210 \mathrm{GPa}$

SPECIMEN CLEAUED AT KQ= $114.8 \mathrm{MPa}$ SQR $[\mathrm{m}]$ (NOT UALID $K I \mathrm{c}$ )

UALUES AT MAXIMUM LOAD

J-INTEGRAL $=64.9 \mathrm{~kJ} / \mathrm{m}^{\wedge} 2$

$\mathrm{K} \cdot \mathrm{c}=116.7 \mathrm{MPa} \operatorname{SQR}[\mathrm{m}]$

K-beta-c $=95.1 \mathrm{MPa} \operatorname{SQR}[\mathrm{m}]$
UALUES AT CLEAUAGE LDAD

$--------------------$

J-INTEGRAL $=64.9 \mathrm{~kJ} / \mathrm{m}^{\wedge} 2$

$\mathrm{KJc}=116.7 \mathrm{MPa} \operatorname{SQR}[\mathrm{m}]$

K-beta-c $=95.1 \mathrm{MPa} \operatorname{SQR}[\mathrm{m}]$ 
F-83

ORNL-DWG 92-11356

73W80 -50 DEGREES C

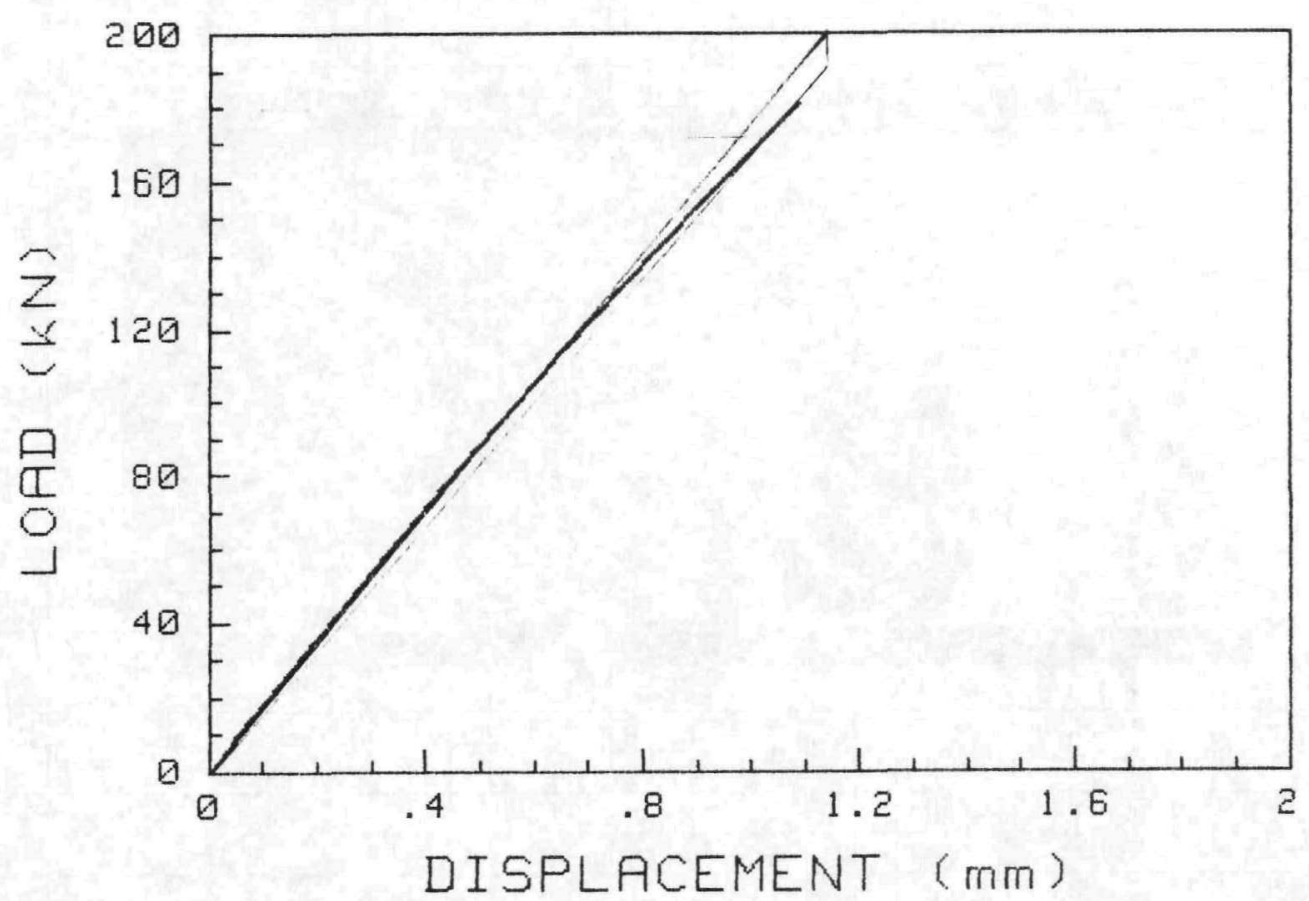

$73 W-80$

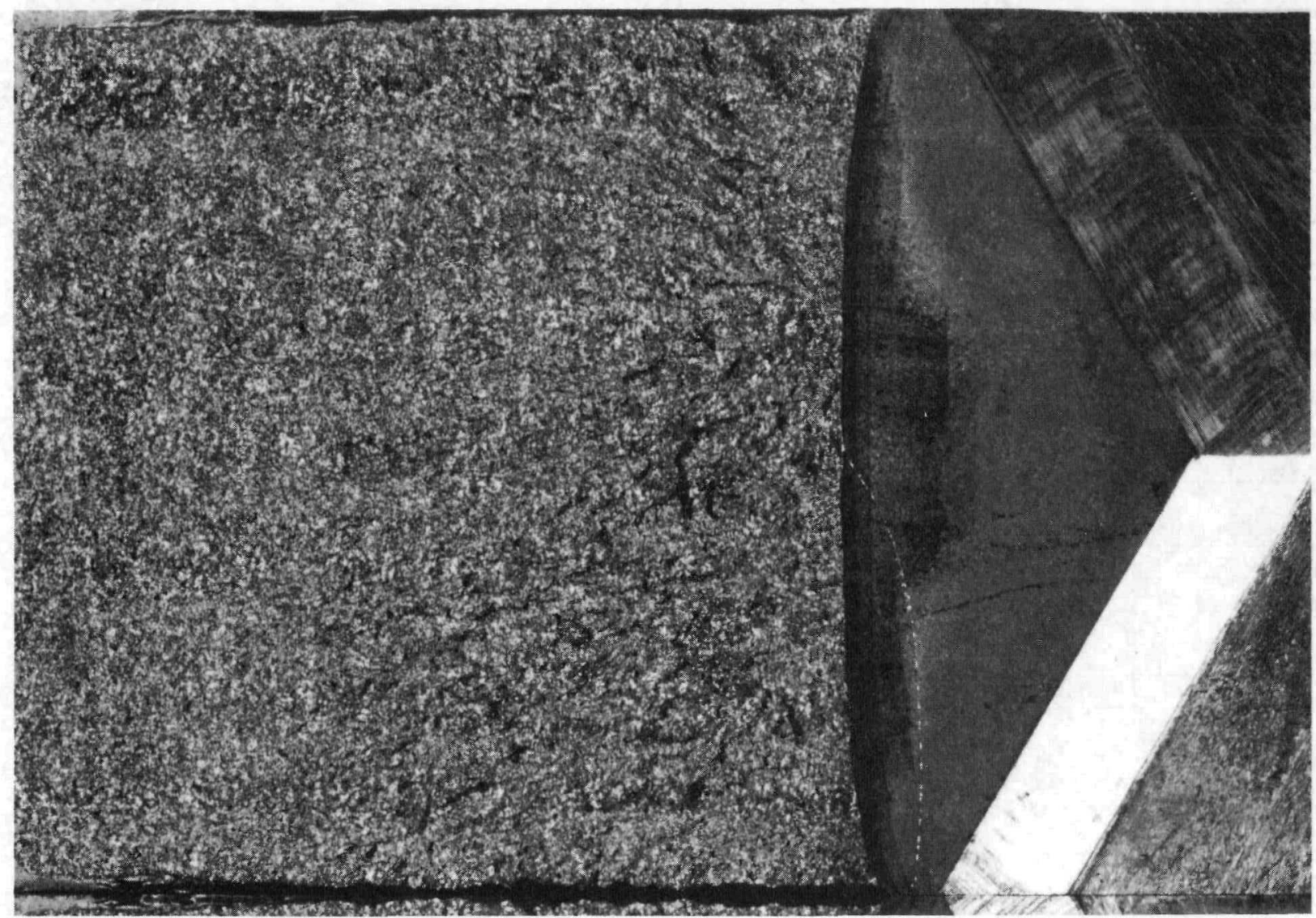




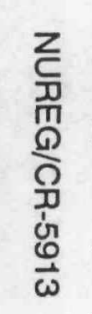

$\angle K A$

$9 / 9 / 85$

$73 w-61$

2T-CT

$0 \% .56$

$-50^{\circ} \mathrm{C}$

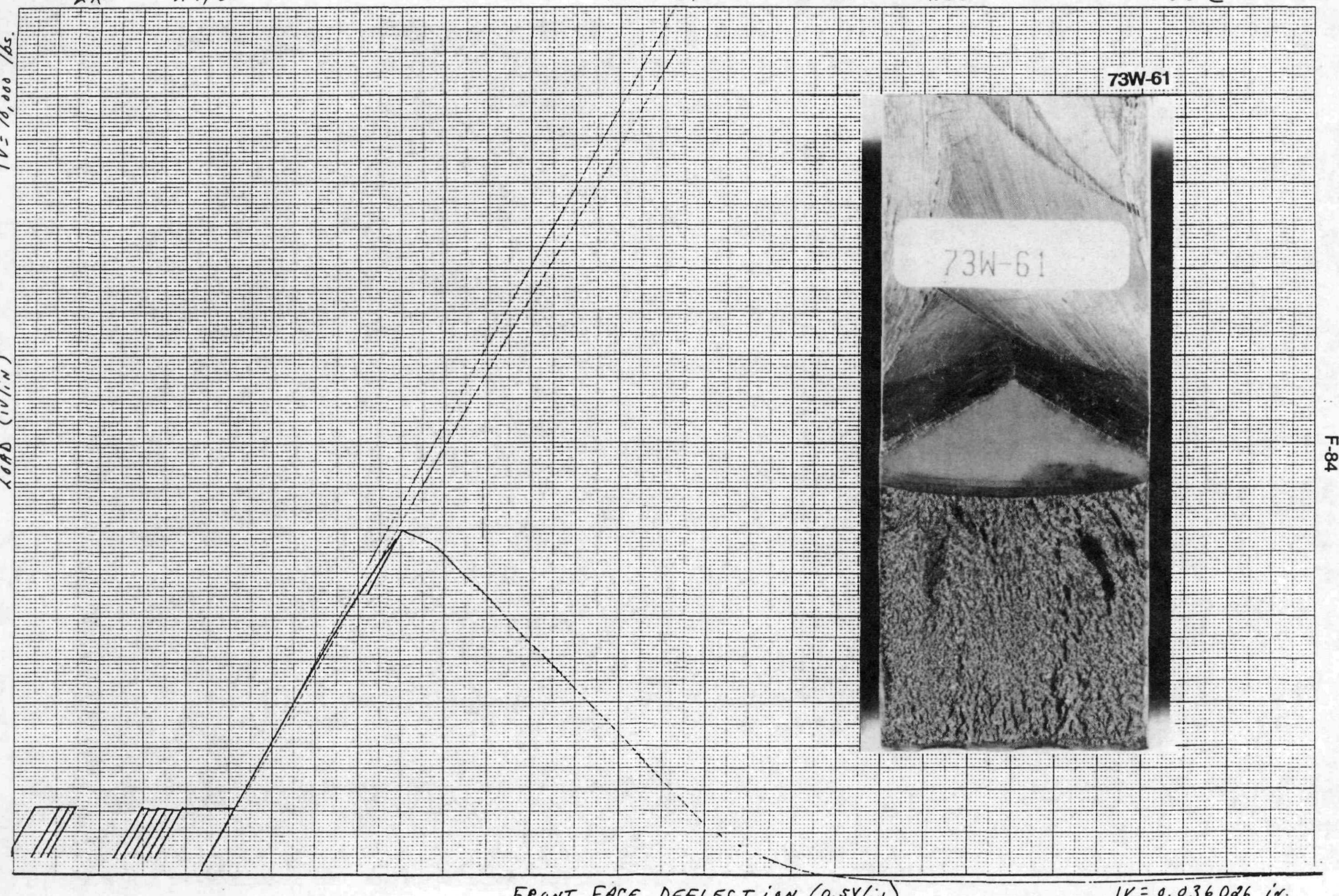

FRONT FACE OEFLECTION (0.5V/iN) 


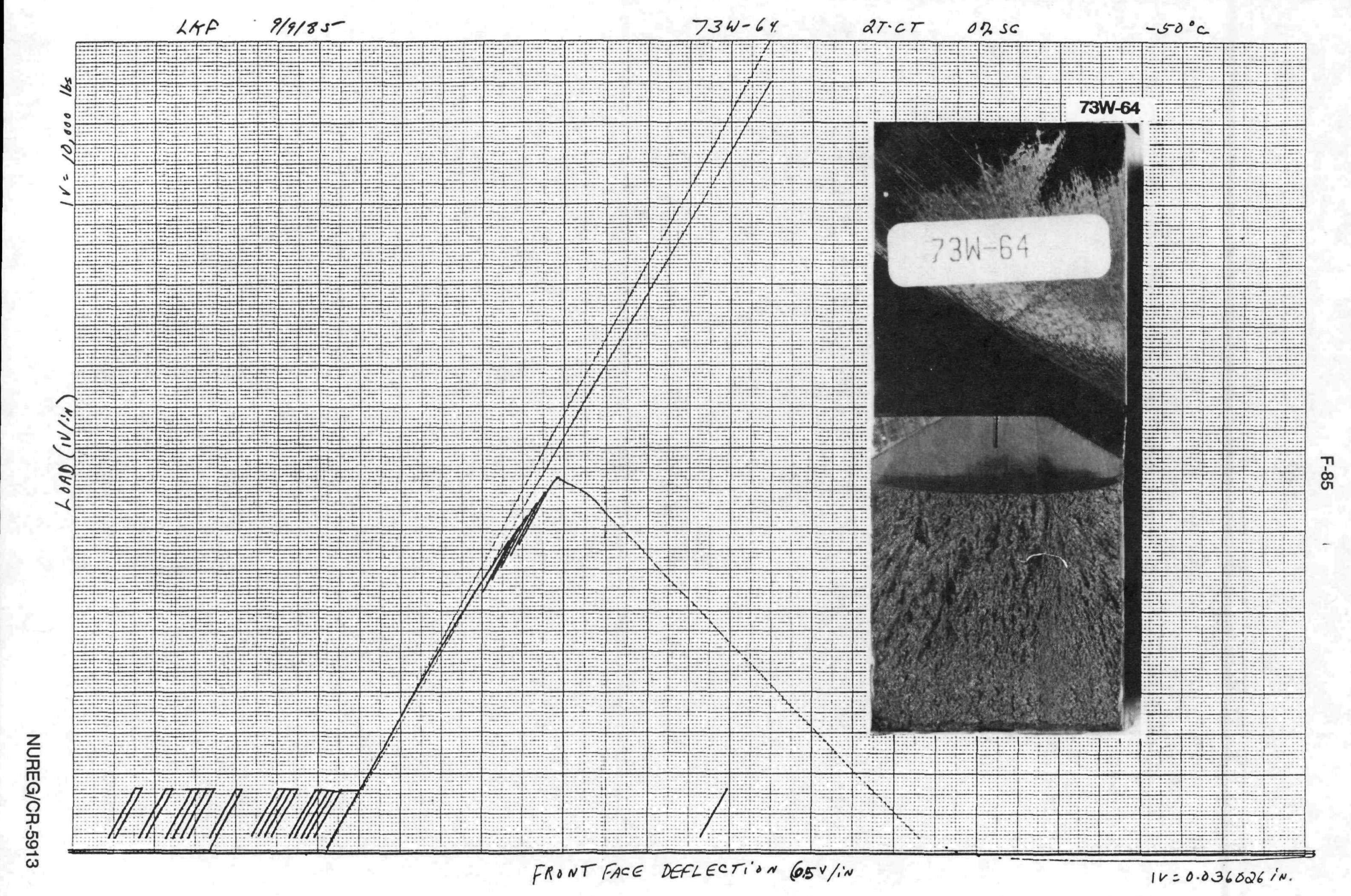




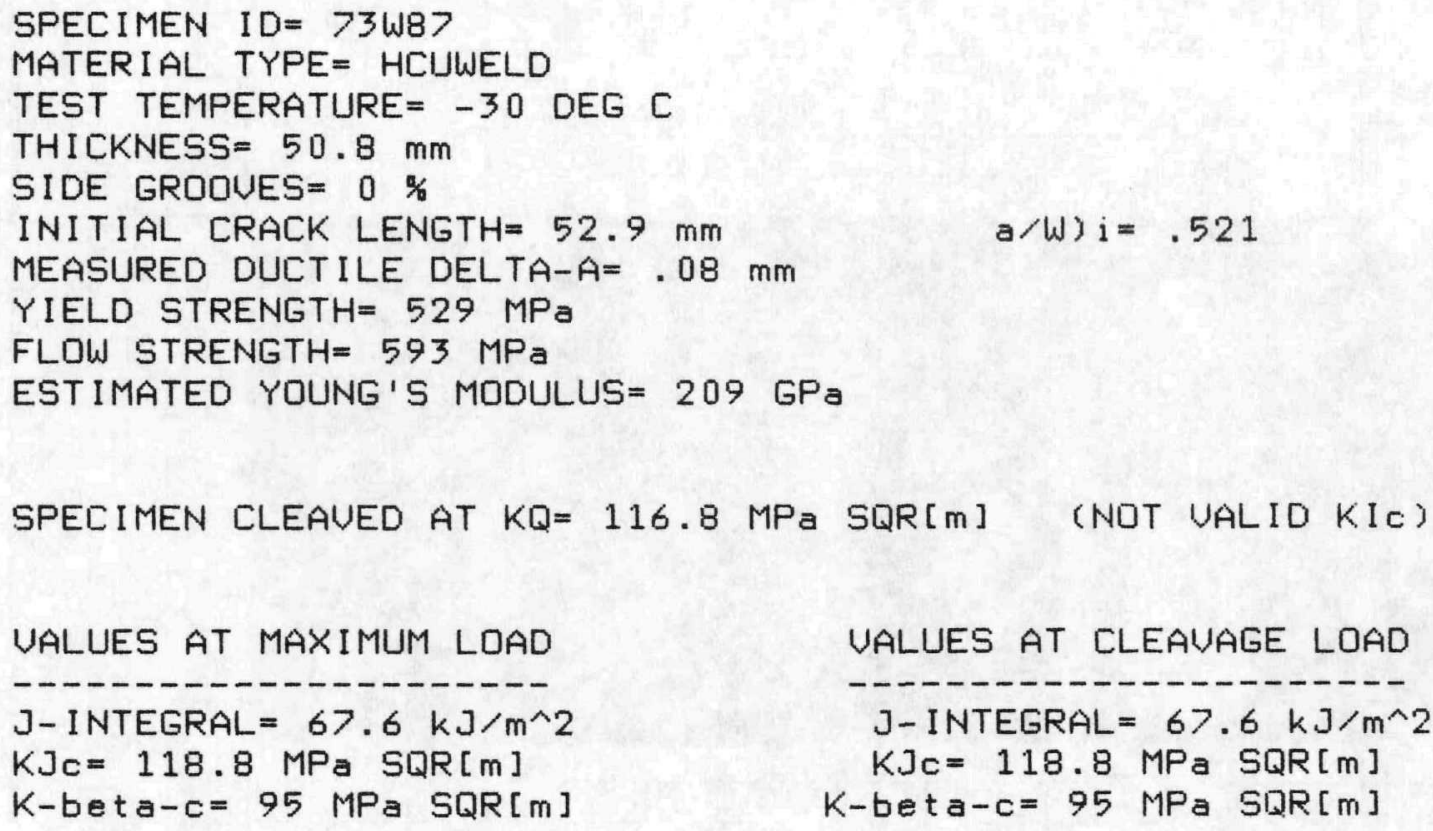

UALUES AT MAXIMUM LOAD

$J$-INTEGRAL $=67.6 \mathrm{~kJ} / \mathrm{m}^{\wedge} 2$

$\mathrm{KJ} \mathrm{c}=118.8 \mathrm{MPa} \operatorname{SQR}[\mathrm{m}]$

K-beta-c $=95 \mathrm{MPa} S Q R[\mathrm{~m}]$

$$
a / W) i=.521
$$

K-beta-c $=95 \mathrm{MPa} \operatorname{SQR}[\mathrm{m}]$ 


\section{F-87}

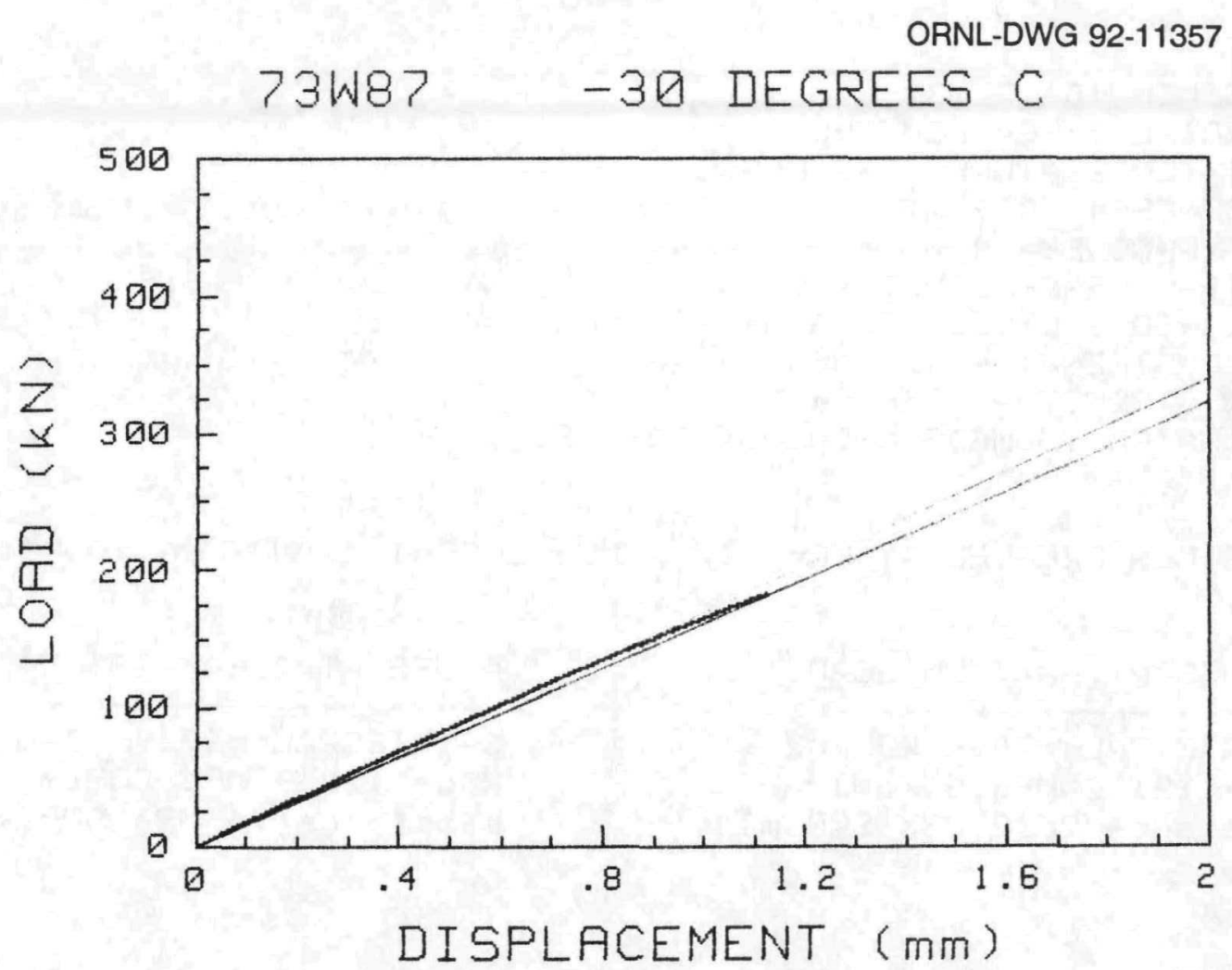

73W-87

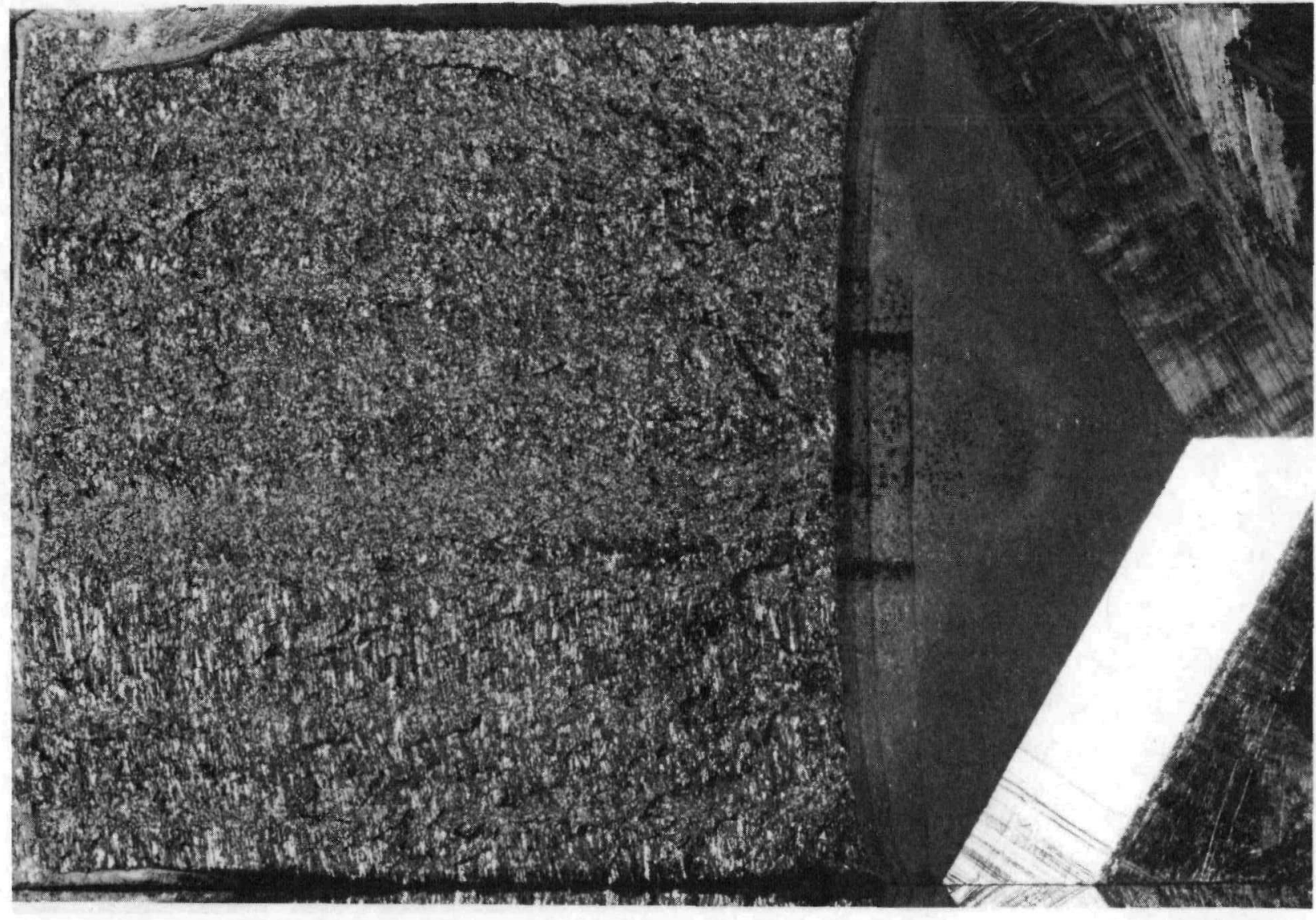


SPECIMEN ID $=73 W 81$

MATERIAL TYPE = HCUWELD

TEST TEMPERATURE $=-30$ DEG C

THICKNESS $=50.9 \mathrm{~mm}$

SIDE GROOUES $=0 \%$

INITIAL CRACK LENGTH $=52.6 \mathrm{~mm}$

MEASURED DUCTILE DELTA-A $=.09 \mathrm{~mm}$

$a / w) i=.519$

YIELD STRENGTH $=529 \mathrm{MPa}$

FLOW STRENGTH $=593 \mathrm{MPa}$

ESTIMATED YOUNG'S MODULUS $=209 \mathrm{GPa}$

SPECIMEN CLEAUED AT KQ $=117.7 \mathrm{MPa} S Q R[\mathrm{~m}]$ (NDT UALID $K I \mathrm{c}$ )

UALUES AT MAXIMUM LOAD

J-INTEGRAL $=70.4 \mathrm{~kJ} / \mathrm{m}^{\wedge} 2$

$\mathrm{KJc}=121.2 \mathrm{MPa} \operatorname{SQR}[\mathrm{m}]$

K-beta-c $=96.2 \mathrm{MPa} \operatorname{SQR}[\mathrm{m}]$
UALUES AT CLEAUAGE LOAD

J-INTEGRAL $=69.9 \mathrm{~kJ} / \mathrm{m}^{\wedge} 2$

$\mathrm{KJc}=120.9 \mathrm{MPa} \operatorname{SQR}[\mathrm{m}]$

$K$-beta-c $=96 \mathrm{MPa} S Q R[\mathrm{~m}]$ 
ORNL-DWG 92-11358
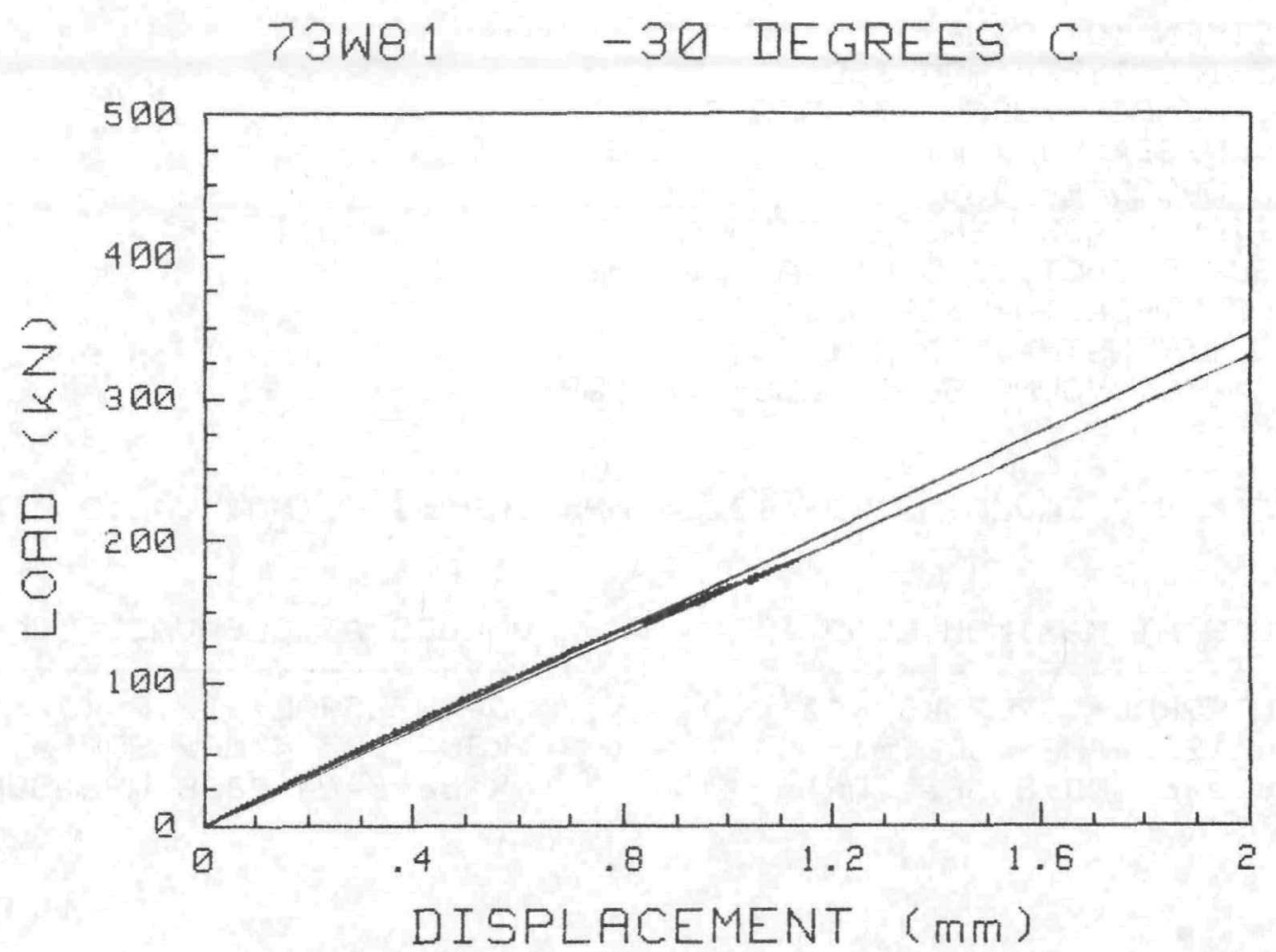

$73 W-81$

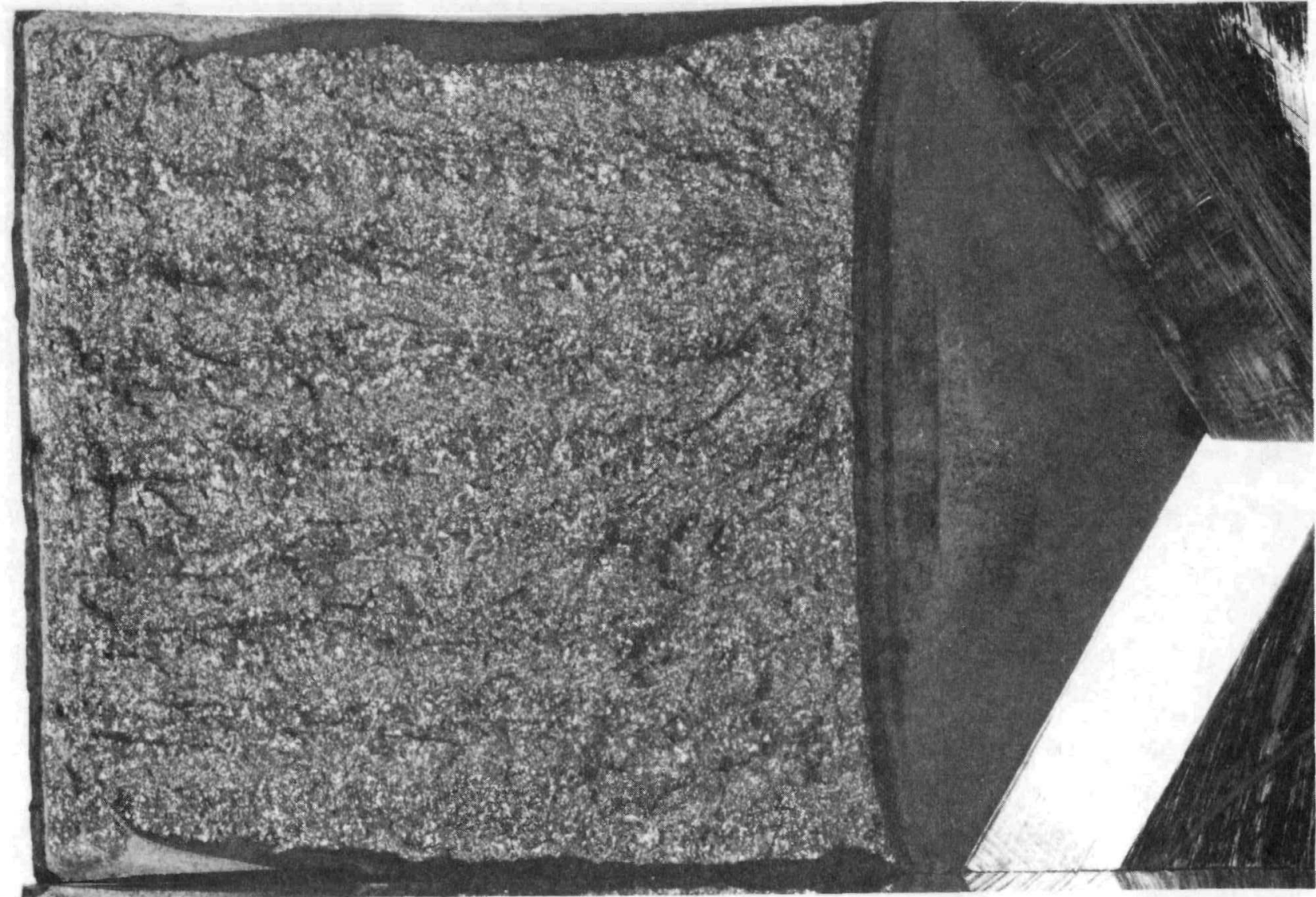


SPECIMEN ID $=73 \mathrm{~W} 67$

MATERIAL TYPE = HCUWELD

TEST TEMPERATURE $=-30$ DEG C

THICKNESS $=50.7 \mathrm{~mm}$

SIDE GROOUES $=0 \%$

INITIAL CRACK LENGTH $=53.4 \mathrm{~mm}$

$a / W) i=.523$

MEASURED DUCTILE DELTA-A $=.05 \mathrm{~mm}$

YIELD STRENGTH $=529 \mathrm{MPa}$

FLOW STRENGTH $=593 \mathrm{MPa}$

ESTIMATED YOUNG'S MODULUS $=209 \mathrm{GPa}$

SPECIMEN CLEAUED AT KQ $=122.4 \mathrm{MPa} S Q R[\mathrm{~m}]$ (NOT UALID KIc)

UALUES AT MAXIMUM LDAD

J-INTEGRAL $=77.7 \mathrm{~kJ} / \mathrm{m}^{\wedge} 2$

$\mathrm{KJ} c=127.4 \mathrm{MPa} \operatorname{SQR}[\mathrm{m}]$

K-beta-c $=98.8 \mathrm{MPa} \operatorname{SQR}[\mathrm{m}]$
UALUES AT CLEAUAGE LDAD

J-INTEGRAL $=77.7 \mathrm{~kJ} / \mathrm{m}^{\wedge} 2$

$\mathrm{KJ} c=127.4 \mathrm{MPa} \operatorname{SQR}[\mathrm{m}]$

K-beta-c $=98.8 \mathrm{MPa} \operatorname{SQR}[\mathrm{m}]$ 
F-91

ORNL-DWG 92-11359

73W6? -3日 DEGREES C

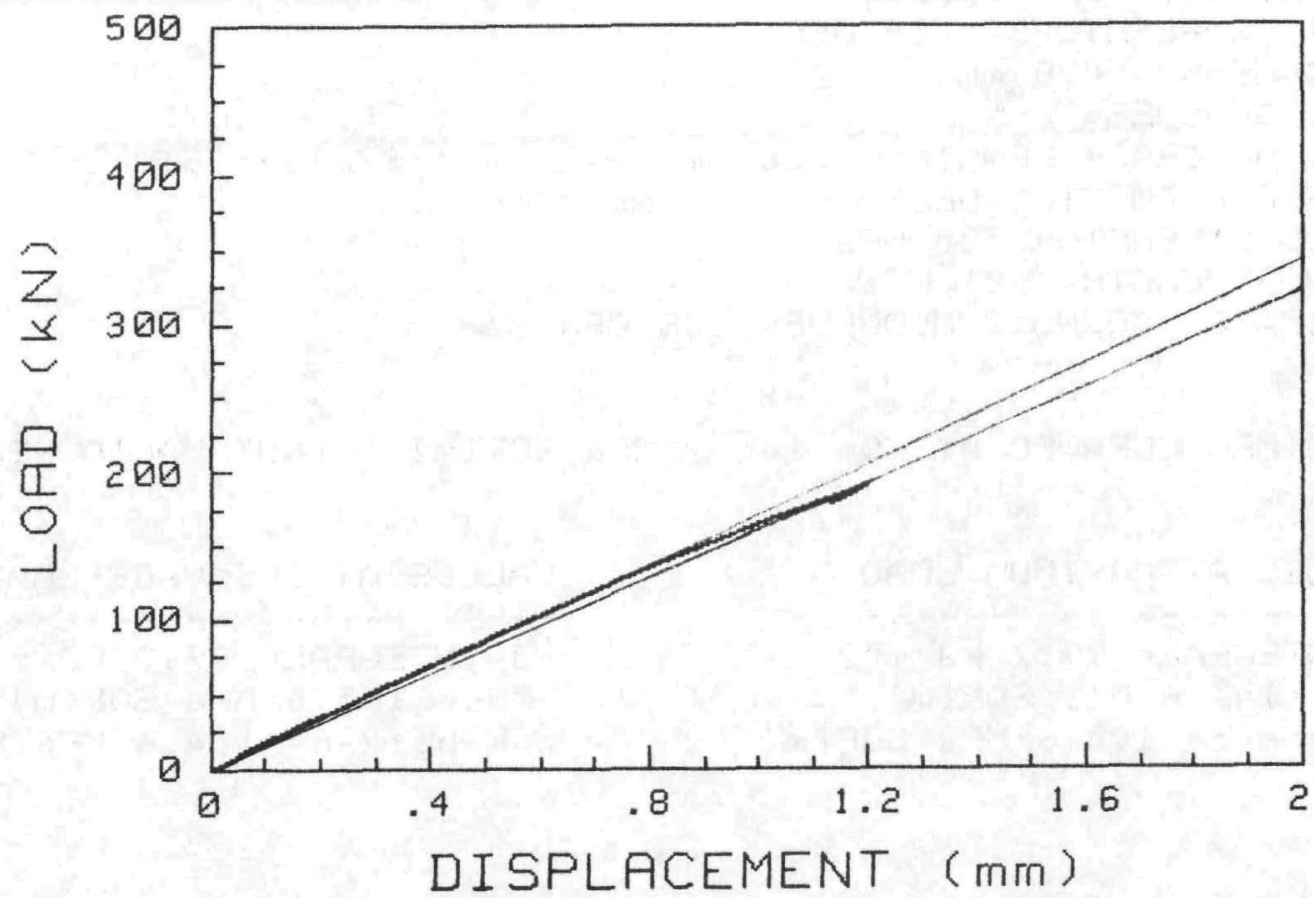

$73 W-67$

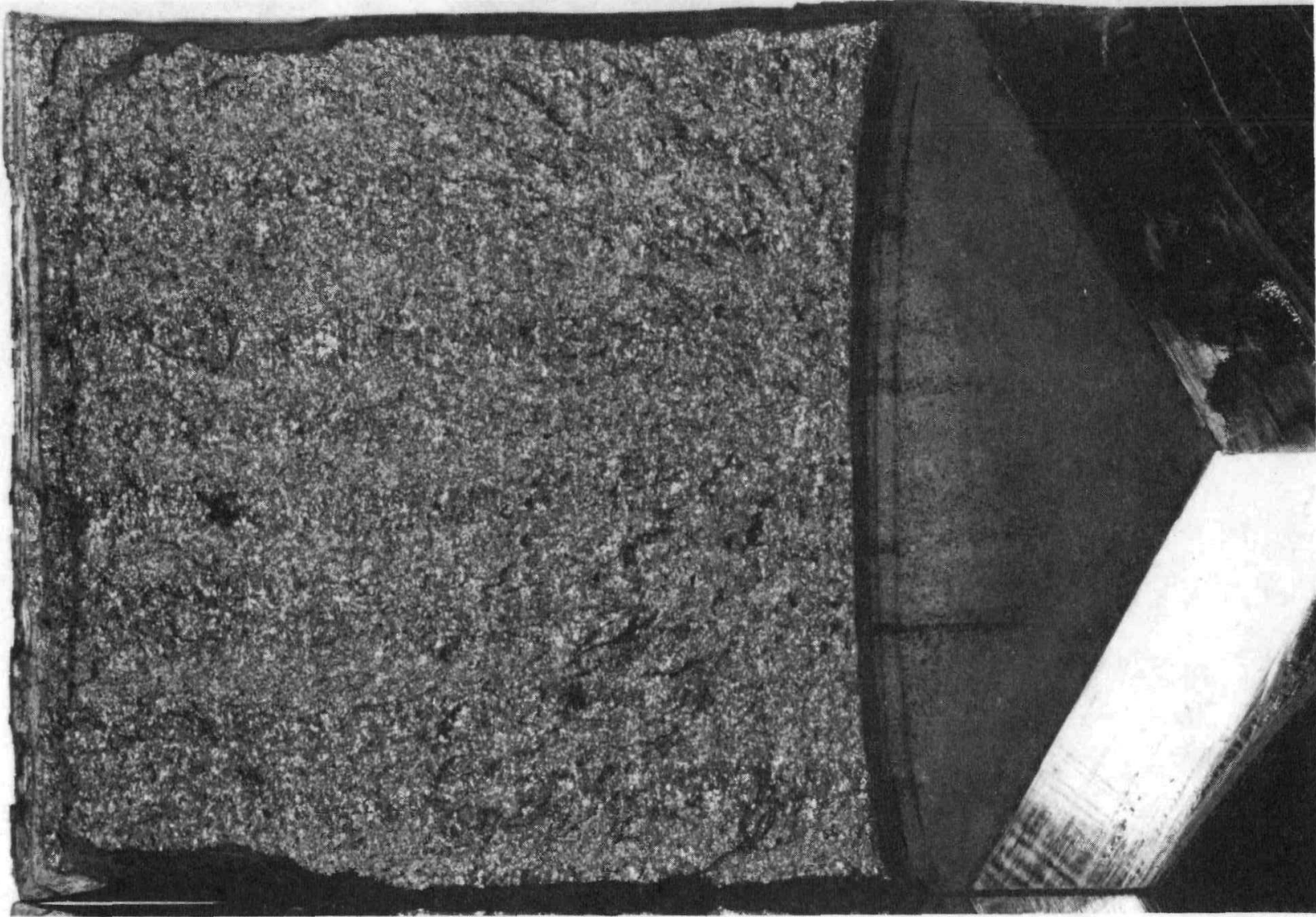


SPECIMEN ID $=73 w 86$

MATERIAL TYPE = HCUWELD

TEST TEMPERATURE $=-15$ DEG $C$

THICKNESS $=50.8 \mathrm{~mm}$

SIDE GRODUES = 0 \%

INITIAL CRACK LENGTH $=52.6 \mathrm{~mm}$

$a / w) i=.519$

MEASURED DUCTILE DELTA-A $=.15 \mathrm{~mm}$

$Y$ IELD STRENGTH $=520 \mathrm{MPa}$

FLOW STRENGTH $=581 \mathrm{MPa}$

ESTIMATED YOUNG'S MODULUS $=208 \mathrm{GPa}$

SPECIMEN CLEAUED AT KQ $=141.7 \mathrm{MPa} S Q R[\mathrm{~m}]$ (NDT UALID KIc)

VALUES AT MAXIMUM LDAD

J-INTEGRAL $=99.2 \mathrm{~kJ} / \mathrm{m}^{\wedge} 2$

$\mathrm{K} J \mathrm{c}=143.6 \mathrm{MPa} \operatorname{SQR}[\mathrm{m}]$

$K$-bet $a-c=104.6 \mathrm{MPa} \operatorname{SQR}[\mathrm{m}]$
UALUES AT CLEAUAGE LDAD

$J-I N T E G R A L=99.2 \mathrm{~kJ} / \mathrm{m}^{\wedge} 2$

$\mathrm{KJ} \mathrm{c}=143.6 \mathrm{MPa} \operatorname{SQR}[\mathrm{m}]$

$K-b e t a-c=104.6 \mathrm{MPa} S Q R[\mathrm{~m}]$ 
F-93

ORNL-DWG 92-11360

73W86 -15 DEGREES C

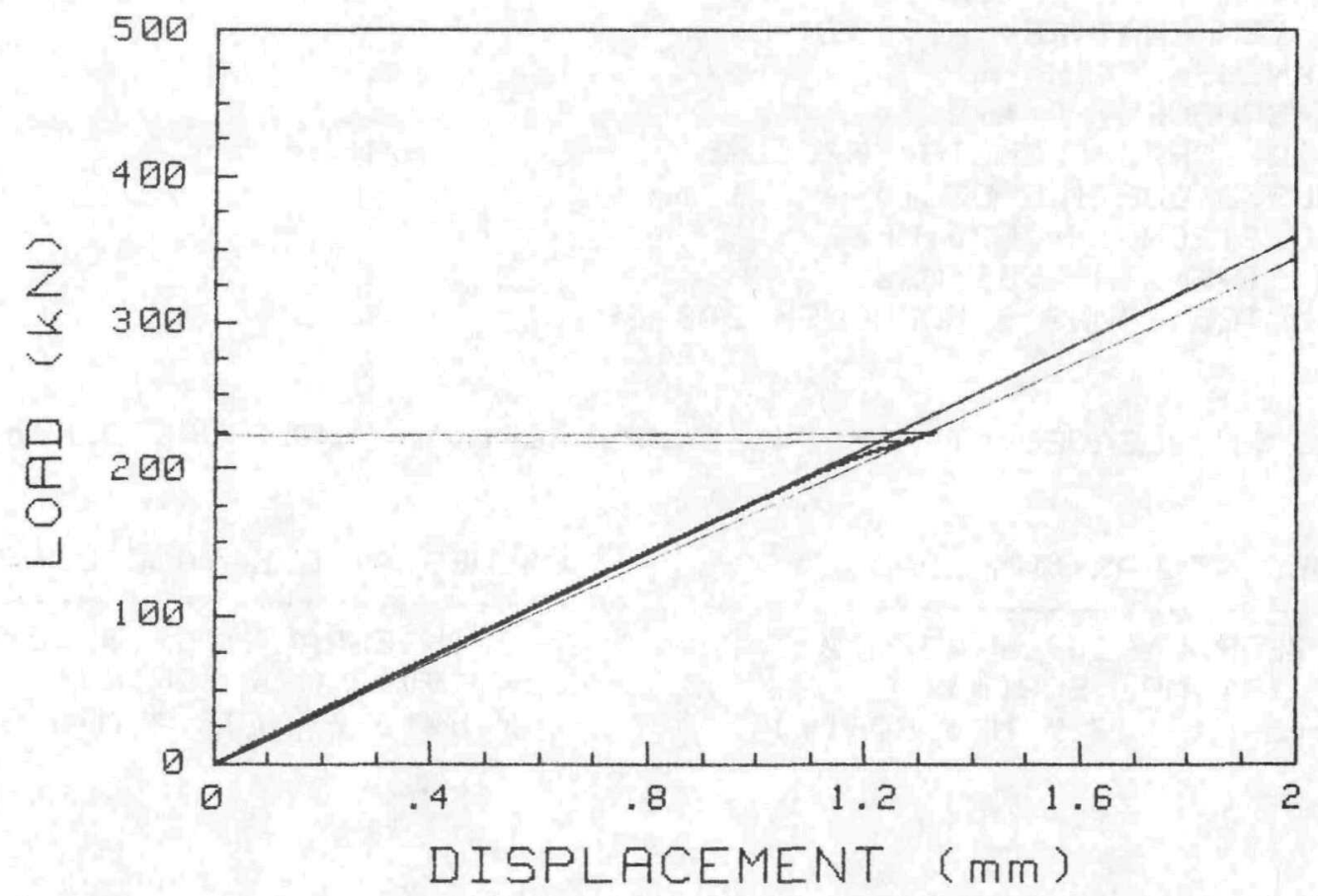

73W-86

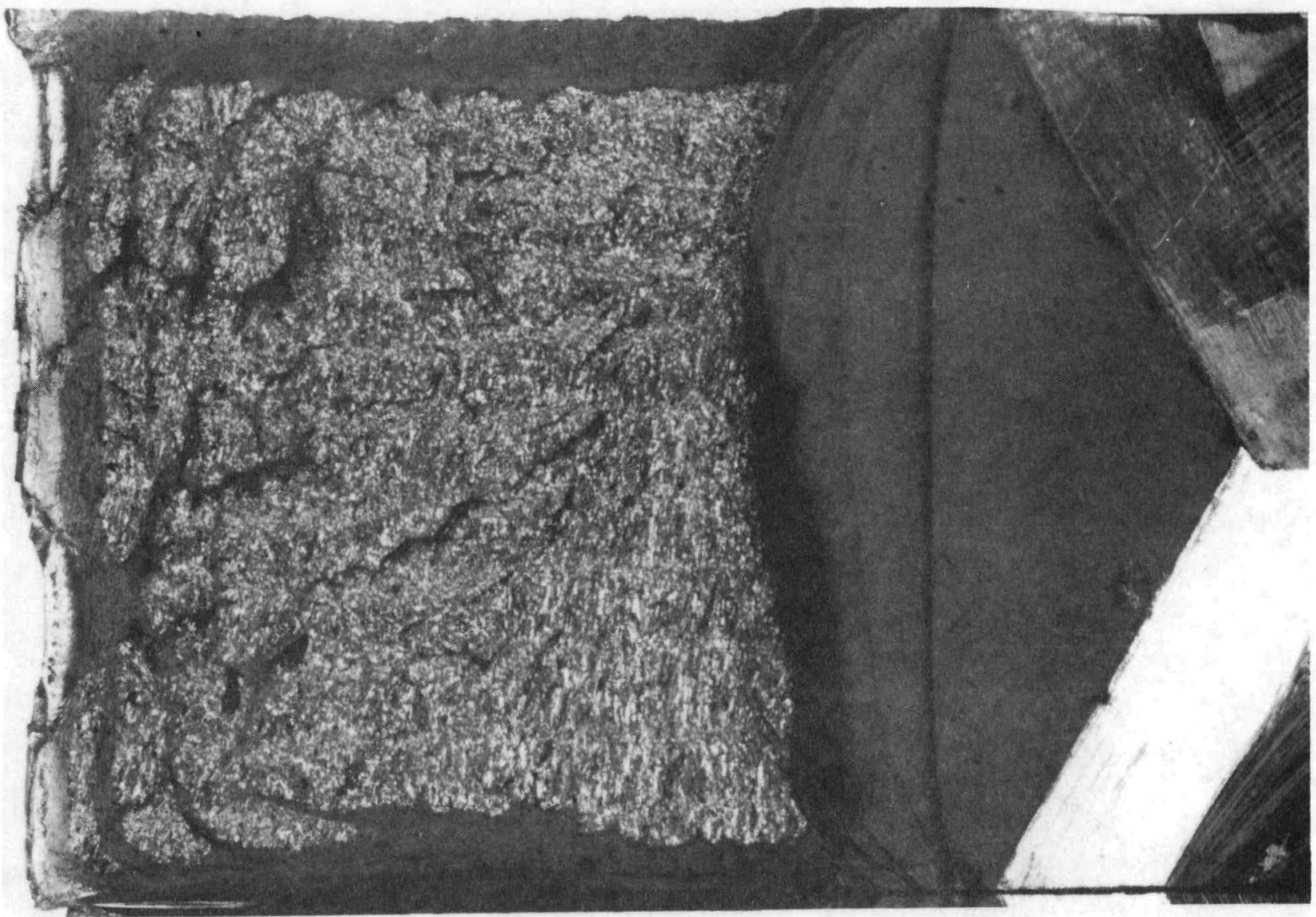


SPECIMEN ID $=73 W>0$

MATERIAL TYPE = HCUWELD

TEST TEMPERATURE $=-15$ DEG $C$

THICKNESS $=50.8 \mathrm{~mm}$

SIDE GROOUES $=0 \%$

INITIAL CRACK LENGTH $=57.6 \mathrm{~mm}$

$a / W\rangle \mathrm{i}=.567$

MEASURED DUCTILE DELTA-A $=.1 \mathrm{~mm}$

$Y$ IELD STRENGTH $=520 \mathrm{MPa}$

FLOW STRENGTH $=581 \mathrm{MPa}$

ESTIMATED YOUNG'S MODULUS $=208 \mathrm{GPa}$

SPECIMEN CLEAUED AT KQ= $141.1 \mathrm{MPa}$ SQR $[\mathrm{m}]$ (NOT UALID KIc)

VALUES AT MAXIMUM LOAD

- - - - - - - -

J-INTEGRAL $=109.7 \mathrm{~kJ} / \mathrm{m}^{\wedge} 2$

$\mathrm{KJ} \mathrm{c}=151 \mathrm{MPa}$ SQR $[\mathrm{m}]$

$K$-beta-c $=107.3 \mathrm{MPa} \operatorname{SQR}[\mathrm{m}]$

\author{
UALUES AT CLEAUAGE LDAD \\ J-INTEGRAL $=109.3 \mathrm{~kJ} / \mathrm{m}^{\wedge} 2$ \\ $\mathrm{KJc}=150.8 \mathrm{MPa} \operatorname{SQR}[\mathrm{m}]$ \\ K-beta-c $=107.2 \mathrm{MPa} \operatorname{SQR}[\mathrm{m}]$
}


F-95

ORNL-DWG 92-11361

73W7ด -15 DEGREES C

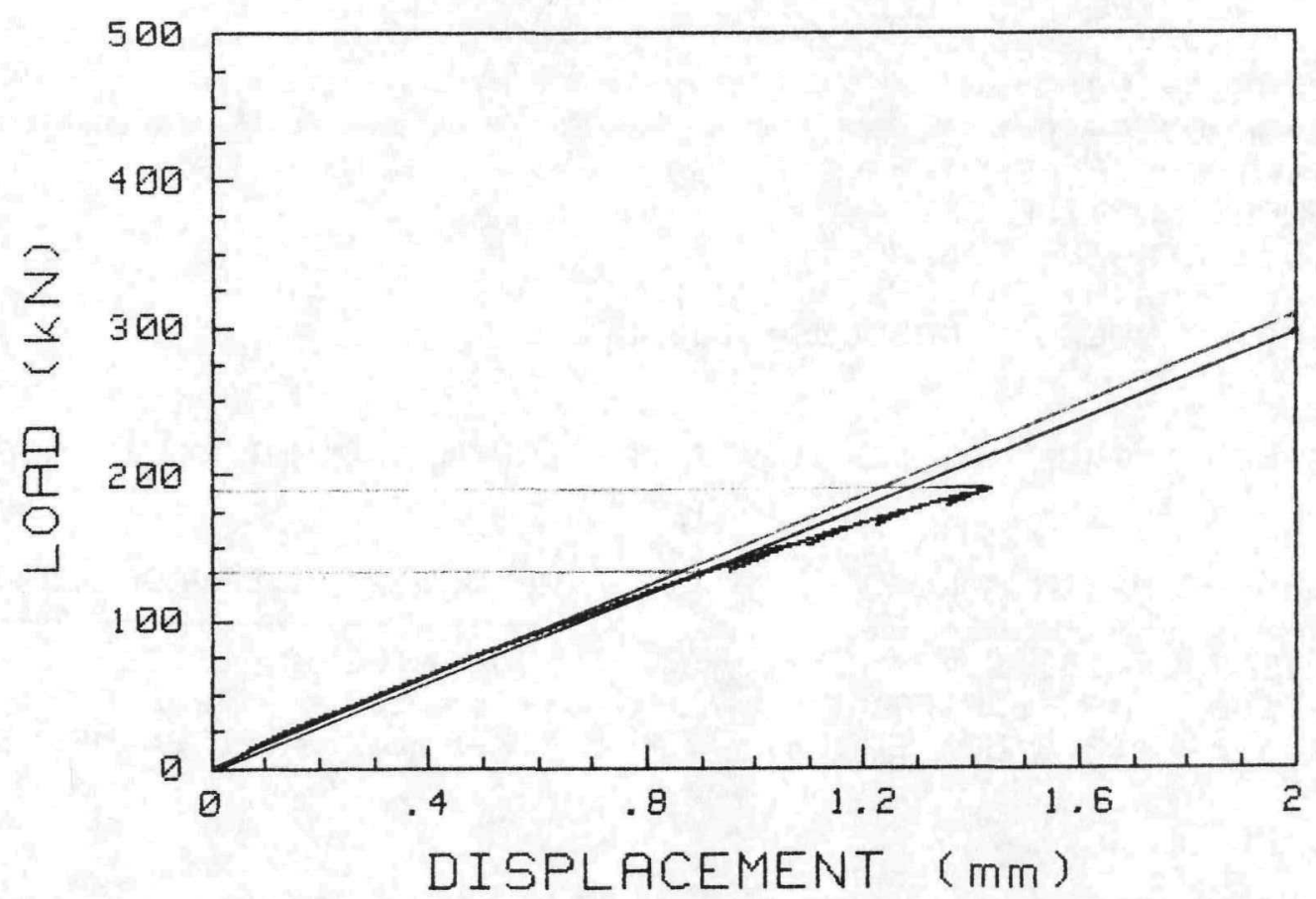

73W-70

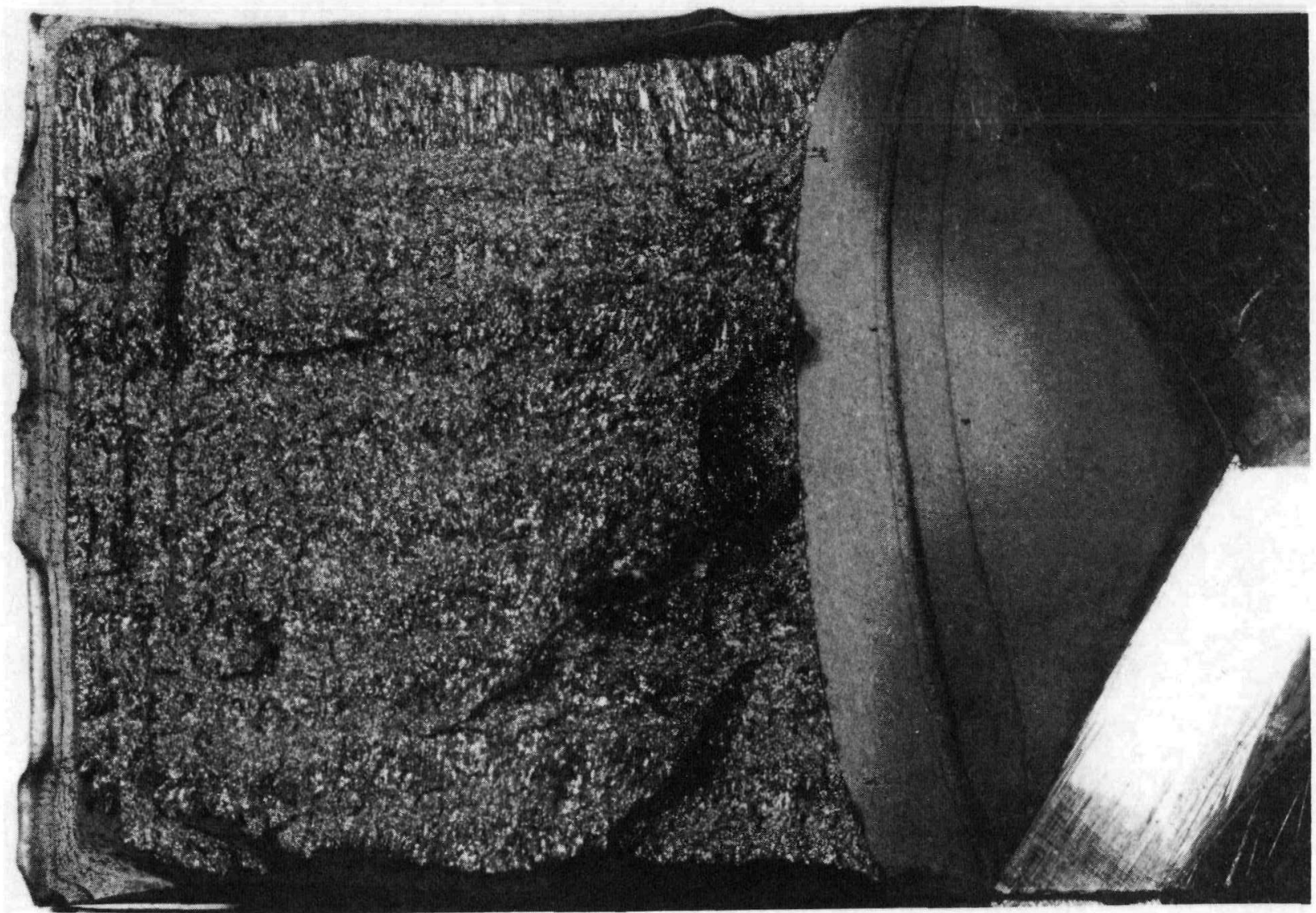


SPECIMEN ID $=73 W>1$

MATERIAL TYPE $=$ HCUWELD

TEST TEMPERATURE $=-15$ DEG C

THICKNESS $=50.8 \mathrm{~mm}$

SIDE GROOUES = $0 \%$

INITIAL CRACK LENGTH $=52.8 \mathrm{~mm}$

MEASURED DUCTILE DELTA-A $=.19 \mathrm{~mm}$

$a(W) i=.52$

YIELD STRENGTH $=520 \mathrm{MPa}$

FLOW STRENGTH $=581 \mathrm{MPa}$

ESTIMATED YOUNG'S MODULUS $=208 \mathrm{GPa}$

SPECIMEN CLEAUED AT KQ $=149.3 \mathrm{MPa} S Q R[\mathrm{~m}]$ (NOT WALID KIc)

UALUES AT MAXIMUM LOAD

J-INTEGRAL $=133.1 \mathrm{~kJ} / \mathrm{m}^{\wedge} 2$

$K J c=166.4 \mathrm{MPa} S Q R[\mathrm{~m}]$

K-beta-c= $112.5 \mathrm{MPa} S Q R[\mathrm{~m}]$
UALUES AT CLEAUAGE LDAD

J-INTEGRAL $=132.4 \mathrm{~kJ} / \mathrm{m}^{\wedge} 2$

$\mathrm{KJC}=165.9 \mathrm{MPa} \operatorname{SQR}[\mathrm{m}]$

K-beta-c $=112.4 \mathrm{MPa} S Q R[\mathrm{~m}]$ 


\section{W71 - 15 DEGREES C}

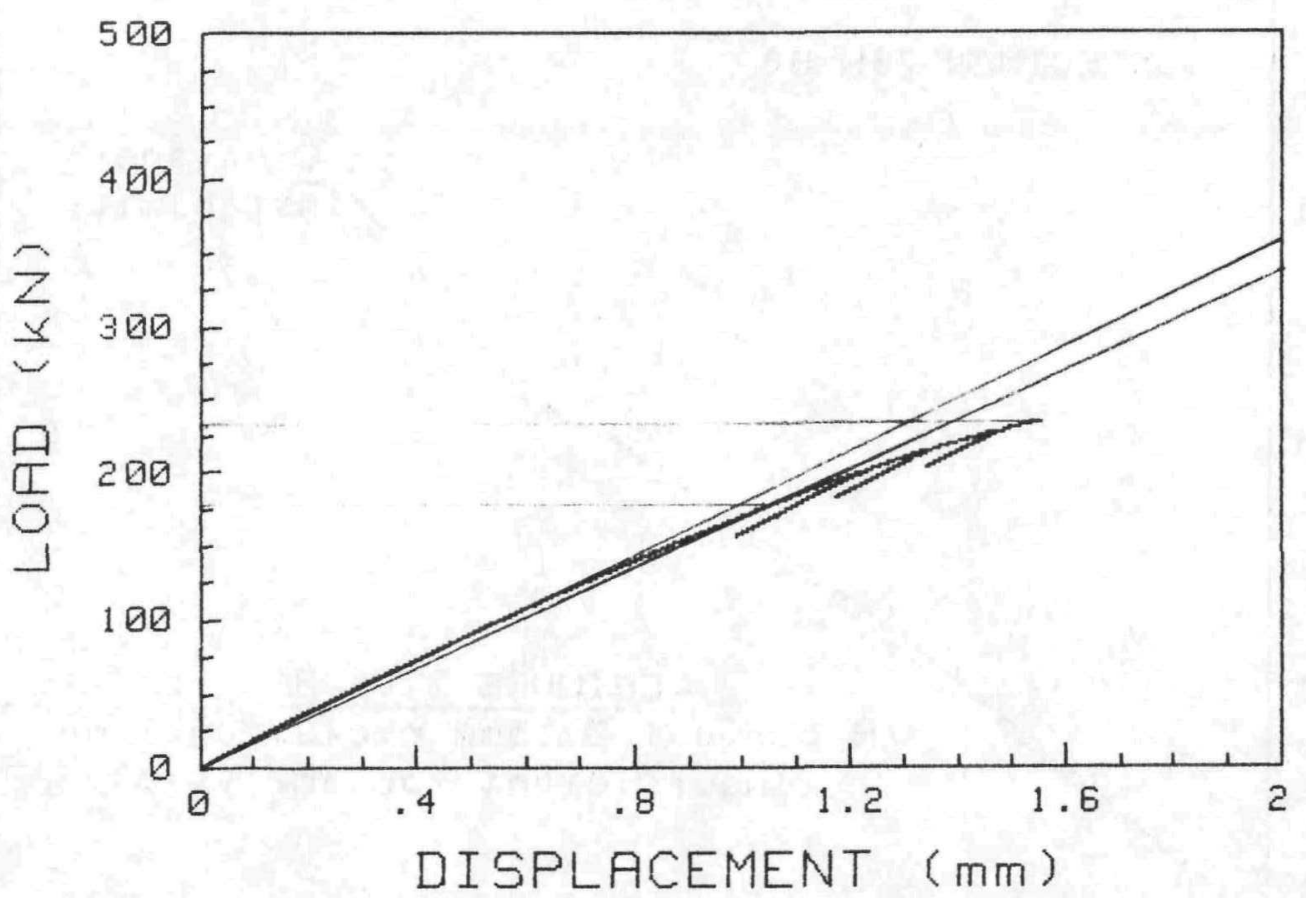

73W-71

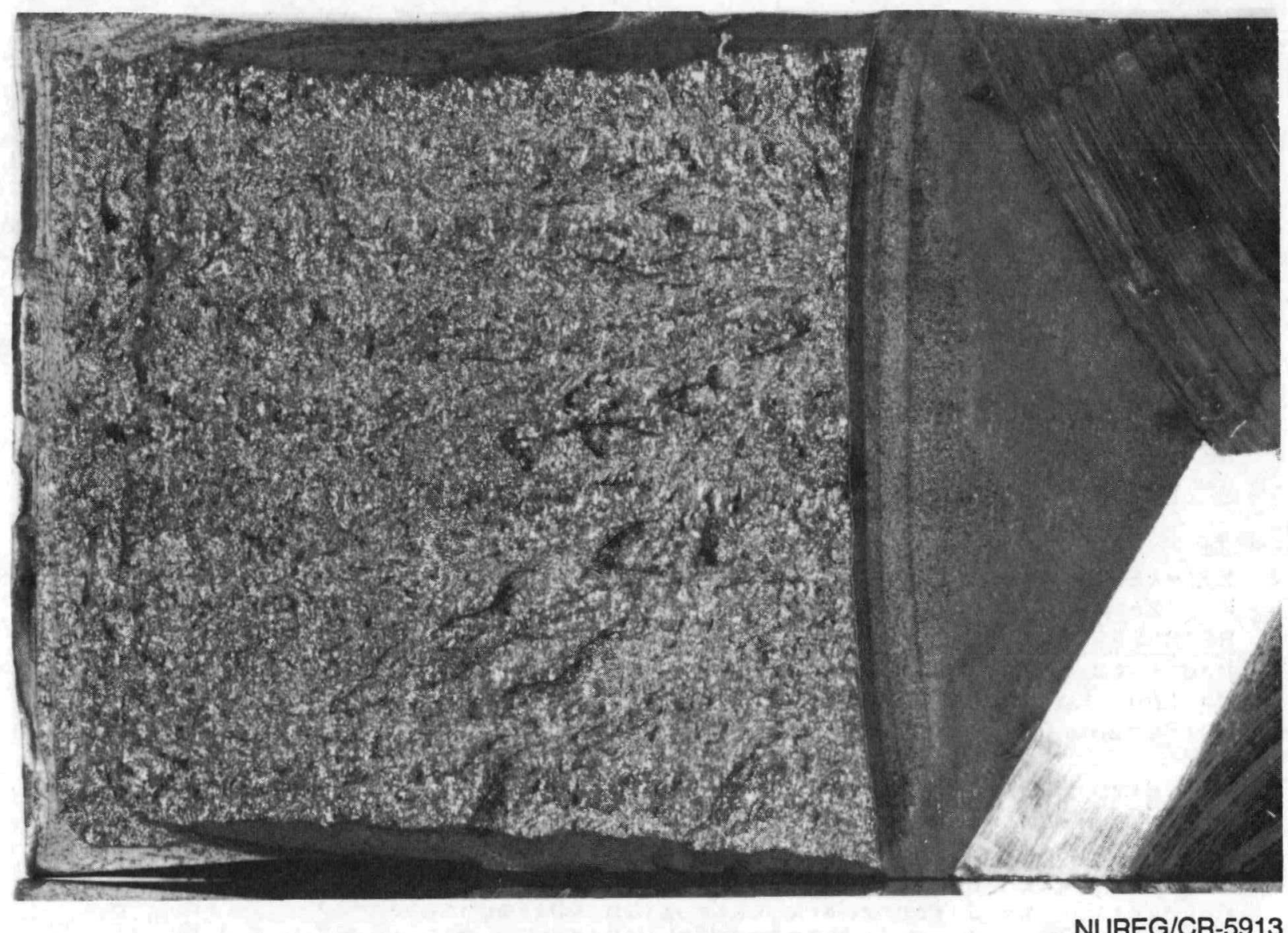


Specimen Crackmouth Deflection (in.)

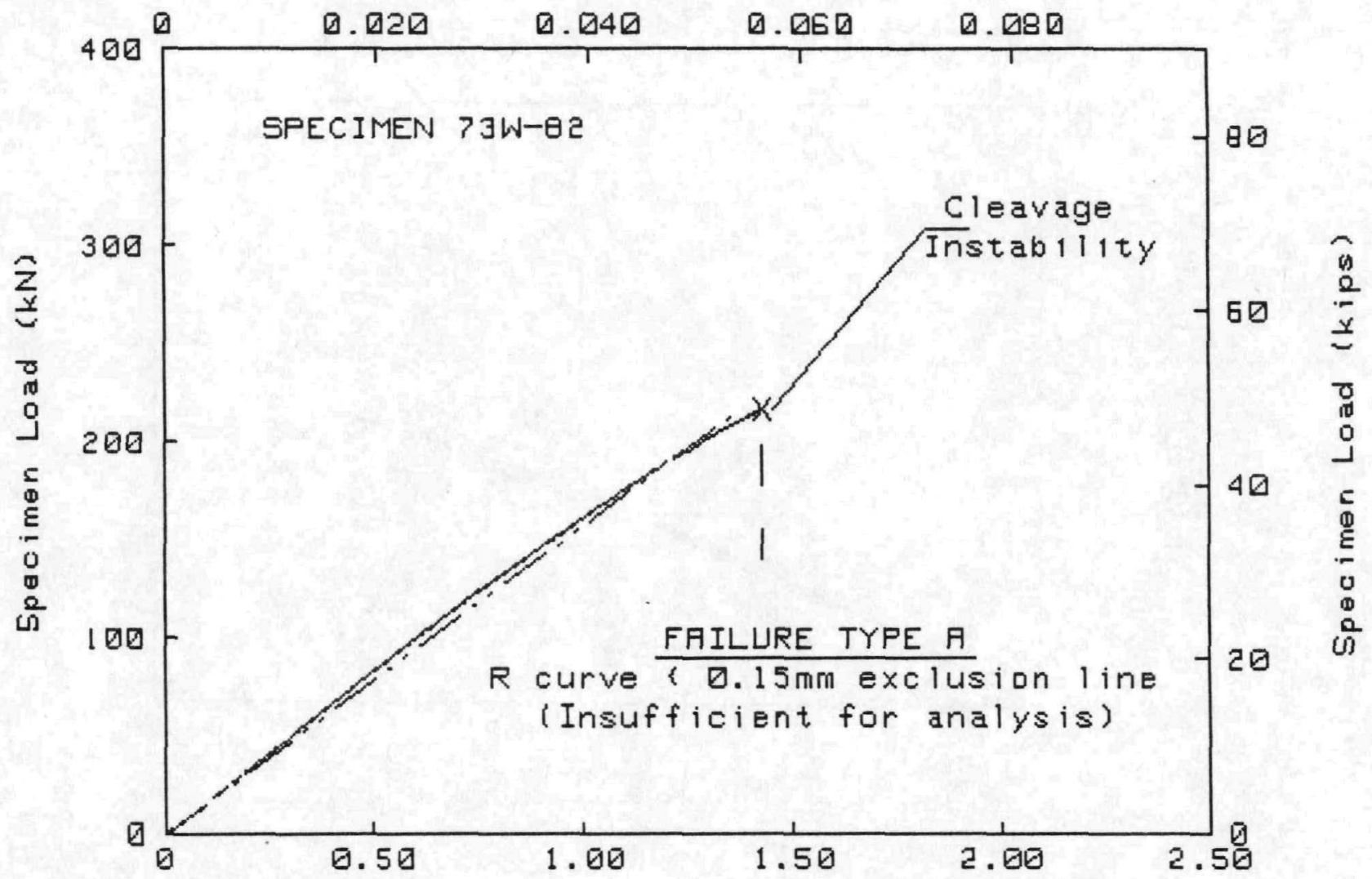

Speoimen Crackmouth Deflection (mm)

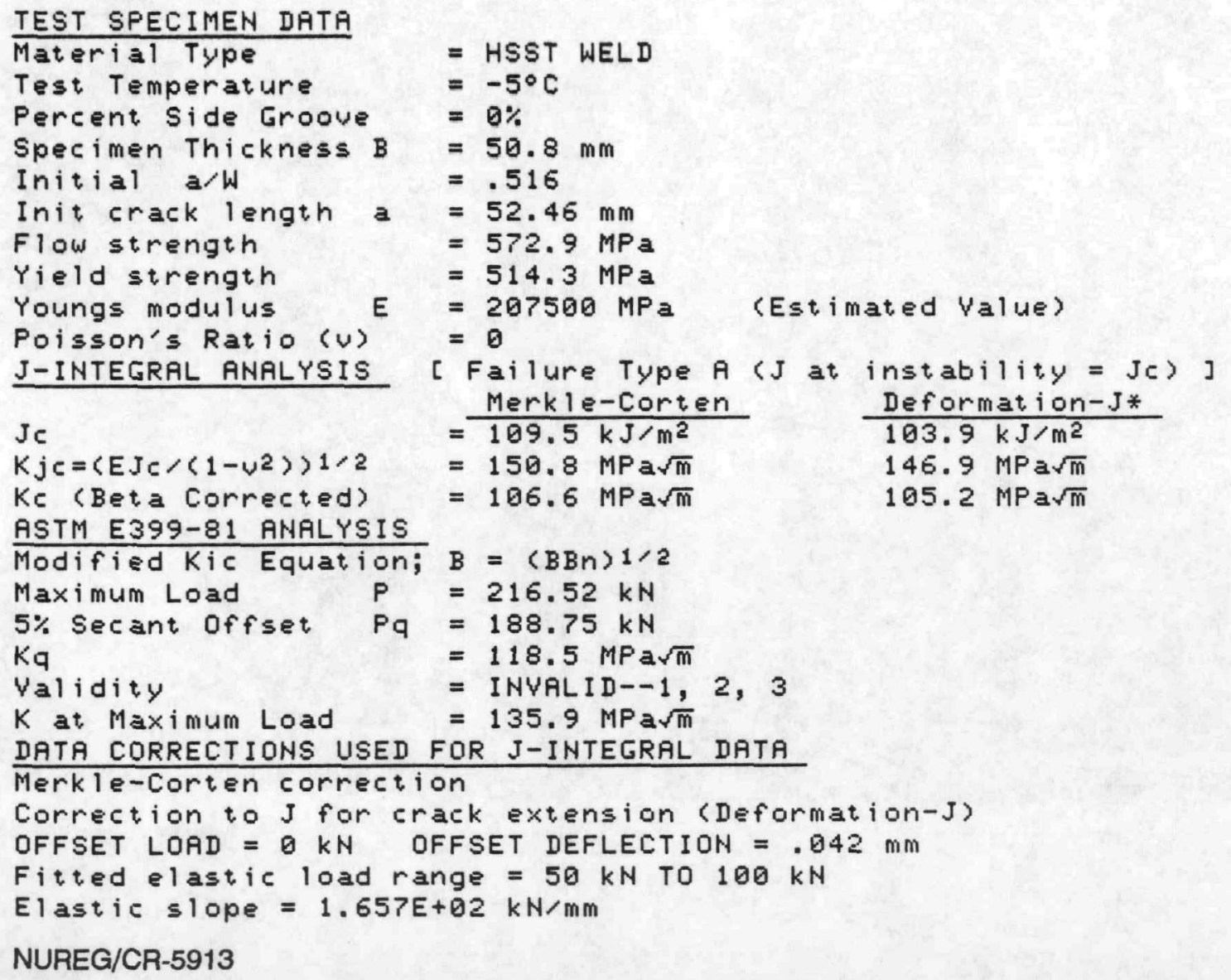




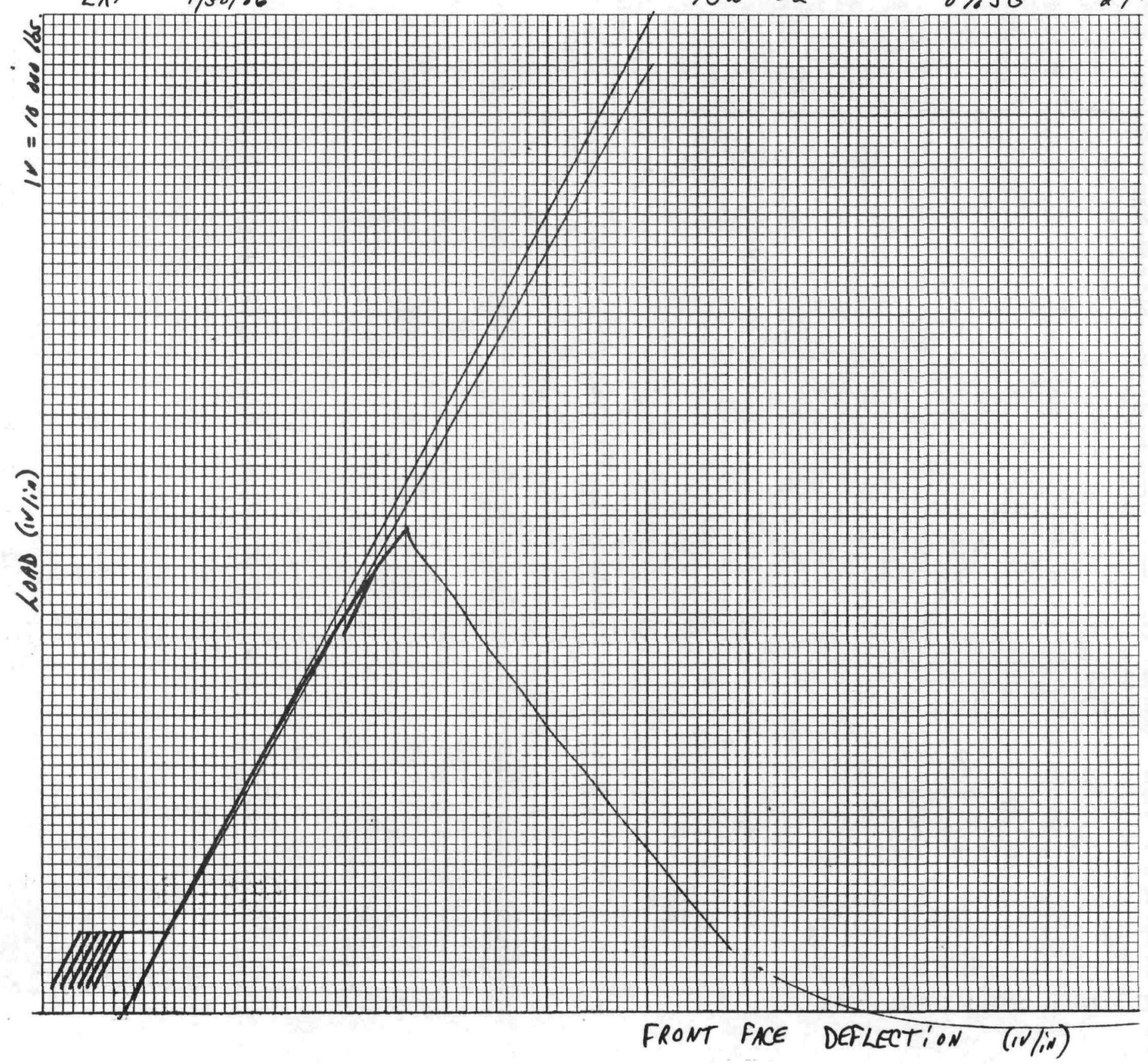


Specimen Crackmouth Deflection (in.)

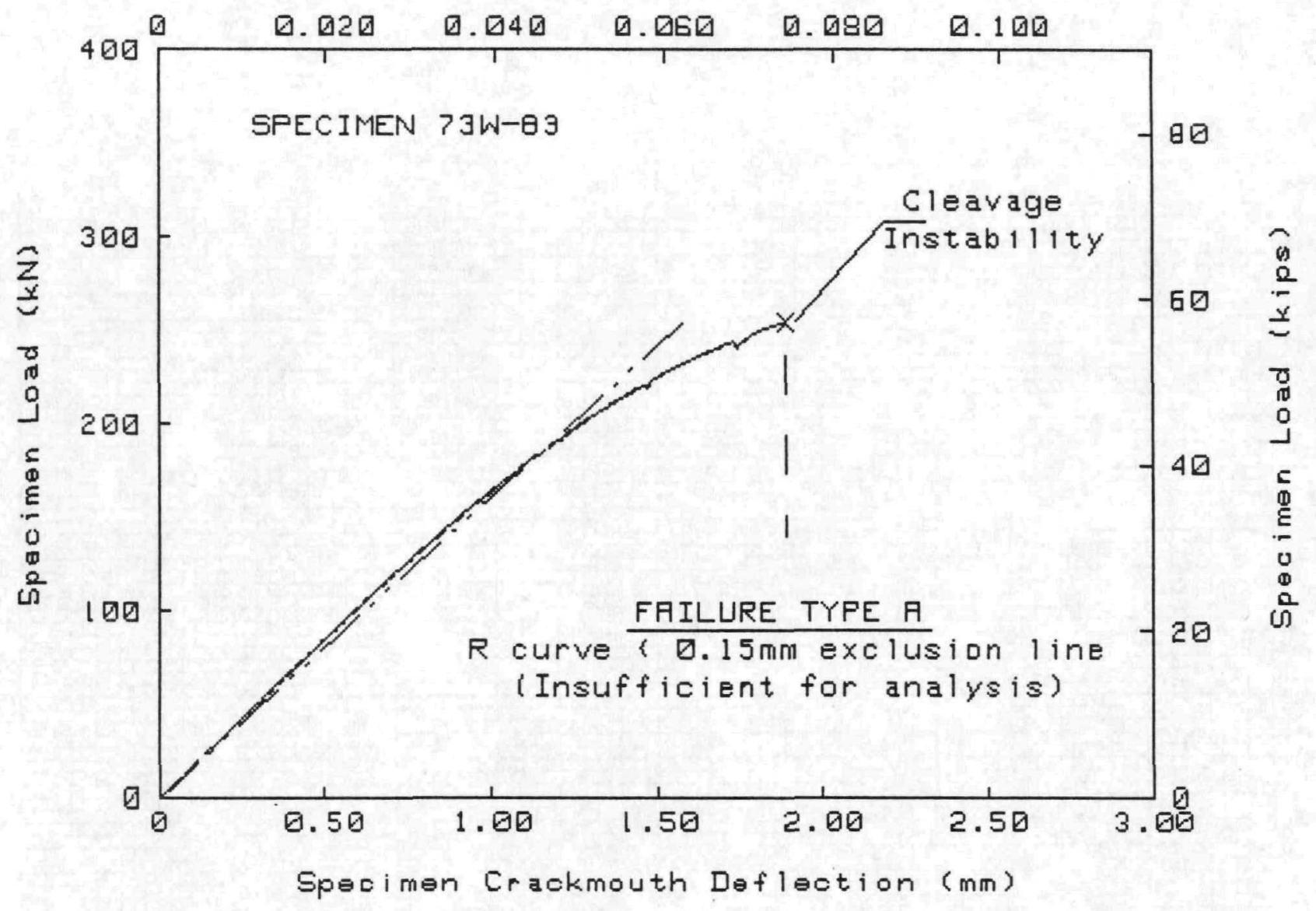

TEST SFECIMEN DATA

Material Type
Test Temperature
Percent Side Groove
Specimen Thickness B
Initial a/W
Init crack length a
Flow strength
Yield strength
Youngs modulus E
Poisson's Ratio ( $)$
J-INTEGRAL ANALYSIS

= HSST WELD

$=-5^{\circ} \mathrm{C}$

$=0 \%$

$=50.8 \mathrm{~mm}$

$=.516$

$=52.39 \mathrm{~mm}$

$=572.9 \mathrm{MPa}$

$=514.3 \mathrm{MPa}$

$=207500 \mathrm{MPa}$

(Est imated Value)

$=0$

[ Failure Type $A$ ( $J$ at instability = JC) ]

$\mathrm{Je}$

$\begin{array}{lll}\text { Merkle-Corten } & & \text { Deformation-J* } \\ =184.4 \mathrm{~kJ} / \mathrm{m}^{2} & & 179 \mathrm{~kJ} / \mathrm{m}^{2} \\ =195.6 \mathrm{MPa} \sqrt{\mathrm{m}} & 192.7 \mathrm{MPa} \sqrt{\mathrm{m}} \\ =120.5 \mathrm{MPa} \sqrt{\mathrm{m}} & 119.7 \mathrm{MPa} \sqrt{\mathrm{m}}\end{array}$

作 $c=\left\langle E J c /\left(1-v^{2}\right\rangle\right) 1 / 2$

$\mathrm{Ke}$ (Beta Corrected)

ASTM E399-81 ANALYSIS

Modified Kic Equation; $B=\langle B B n\rangle 1 / 2$

Maximum Load $P=254.52 \mathrm{kN}$

$5 \%$ Secant offset $\mathrm{Pq}=182.42 \mathrm{kN}$

$\mathrm{Kq}$

$=114.3 \mathrm{MPa} \sqrt{\mathrm{m}}$

Validity

$K$ at Maximum Load

= INVALID--1, 2, 3

$=159.4 \mathrm{MPa} \sqrt{\mathrm{m}}$

DATA CORRECTIONS USEI FOR J-INTEGRAL DATA

Merkle-Corten correction

Correction to J for crack extension (Deformation-J)

OFFSET LOAD $=0 \mathrm{kN}$ OFFSET DEFLECTION $=.01 \mathrm{~mm}$

Fitted elastic load range $=50 \mathrm{kN}$ TO $100 \mathrm{kN}$

Elastic slope $=1.693 \mathrm{E}+02 \mathrm{kN} / \mathrm{mm}$

NUREG/CR-5913 


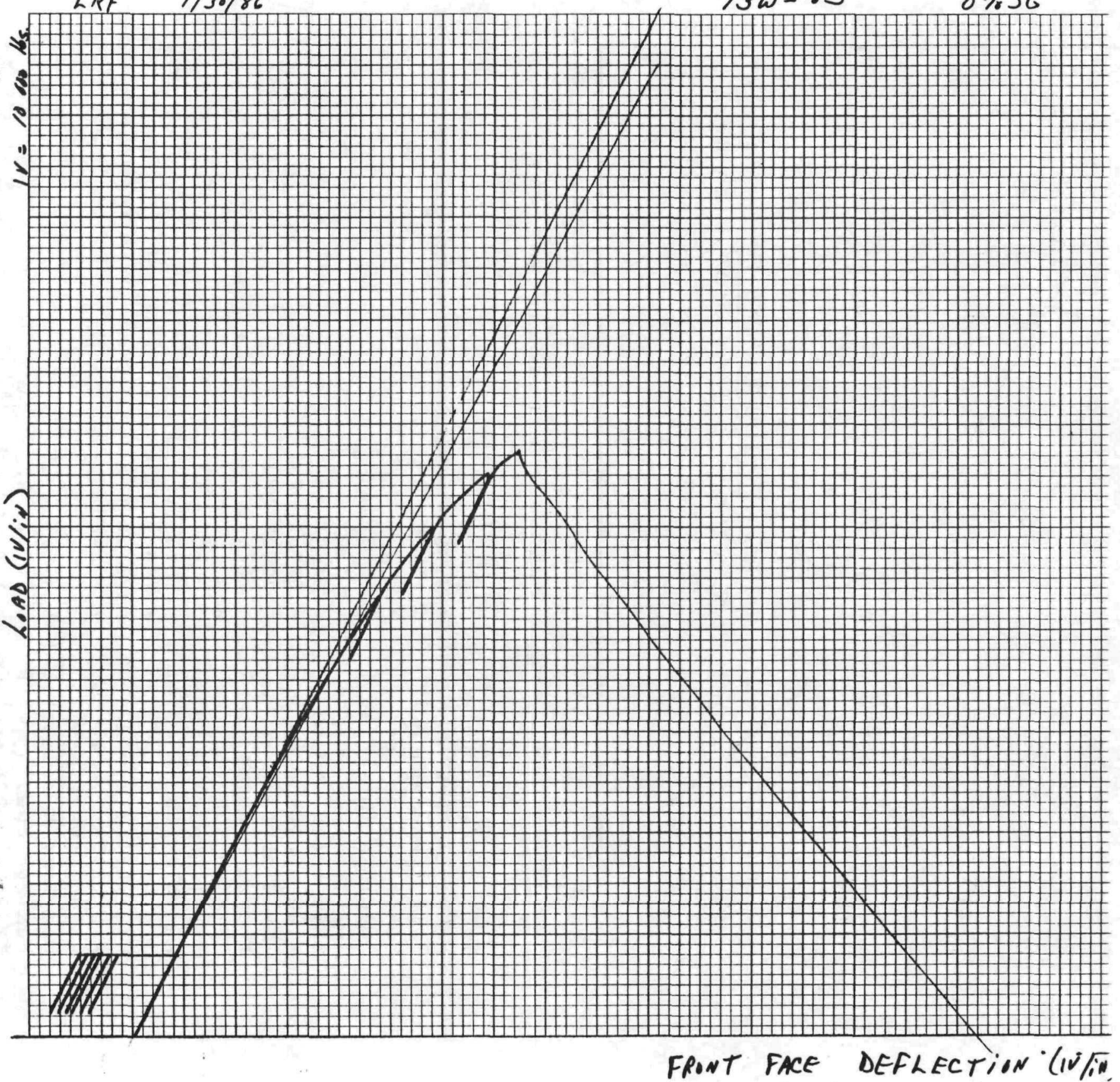




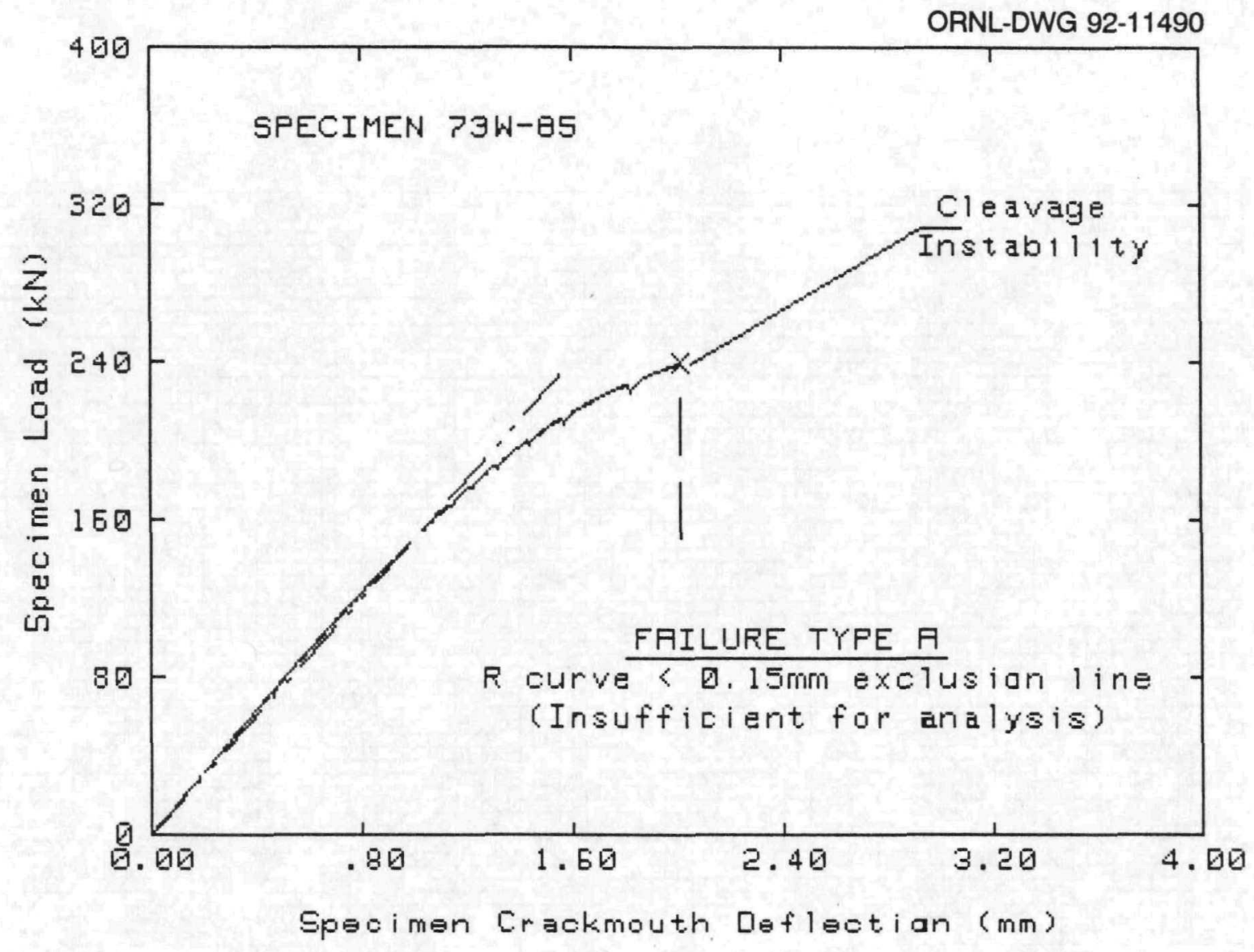

TEST SPECIMEN DATA

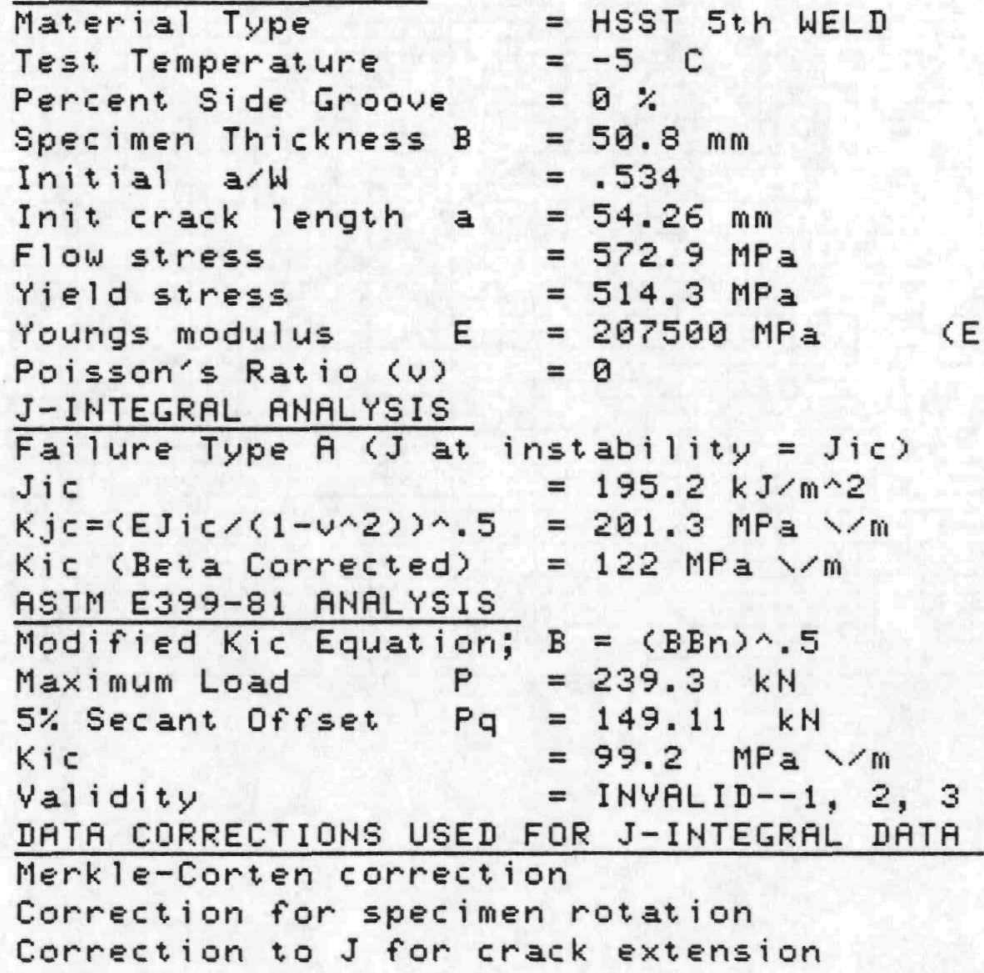




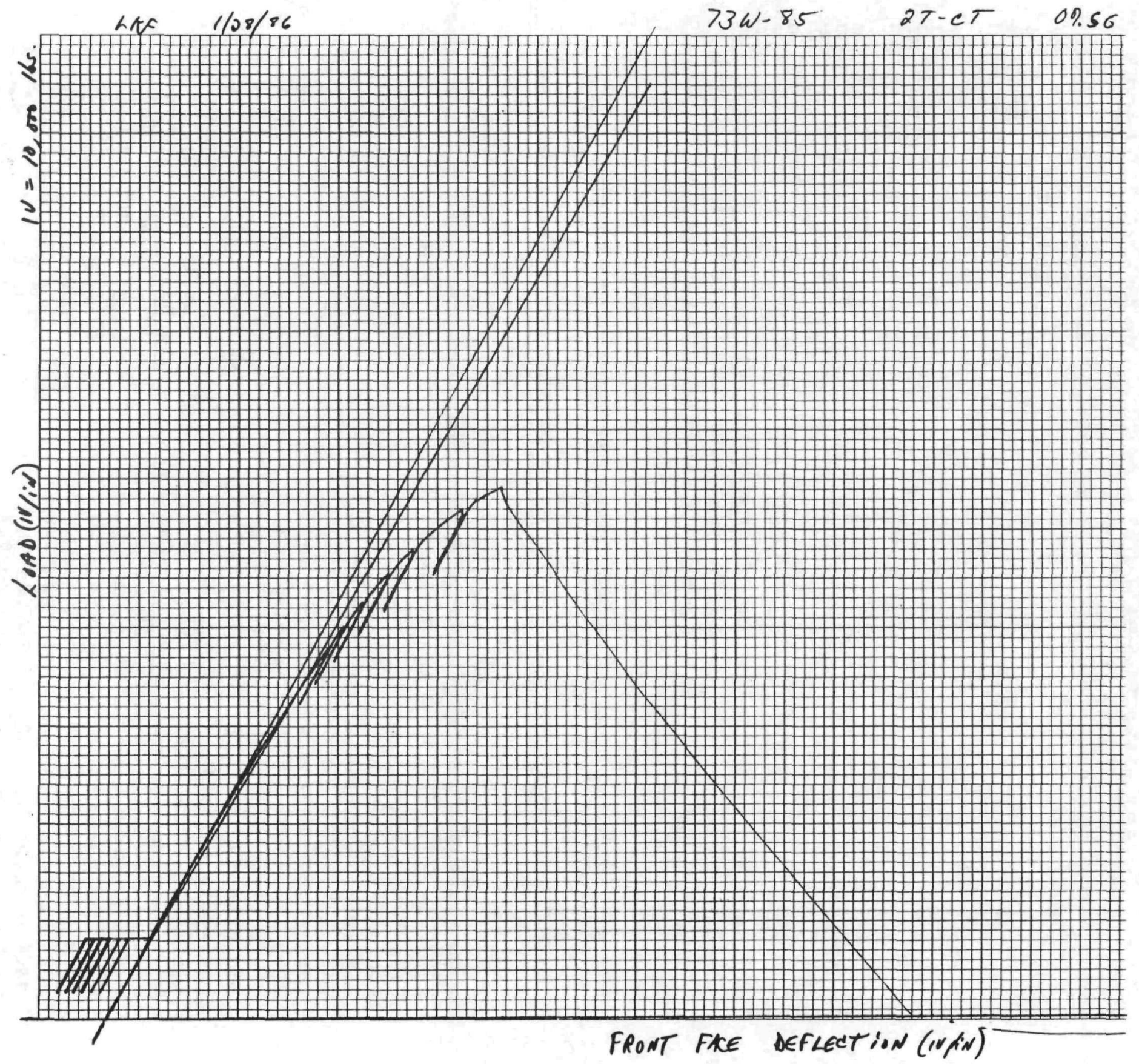


Specimen Crackmouth Deflection (in.)

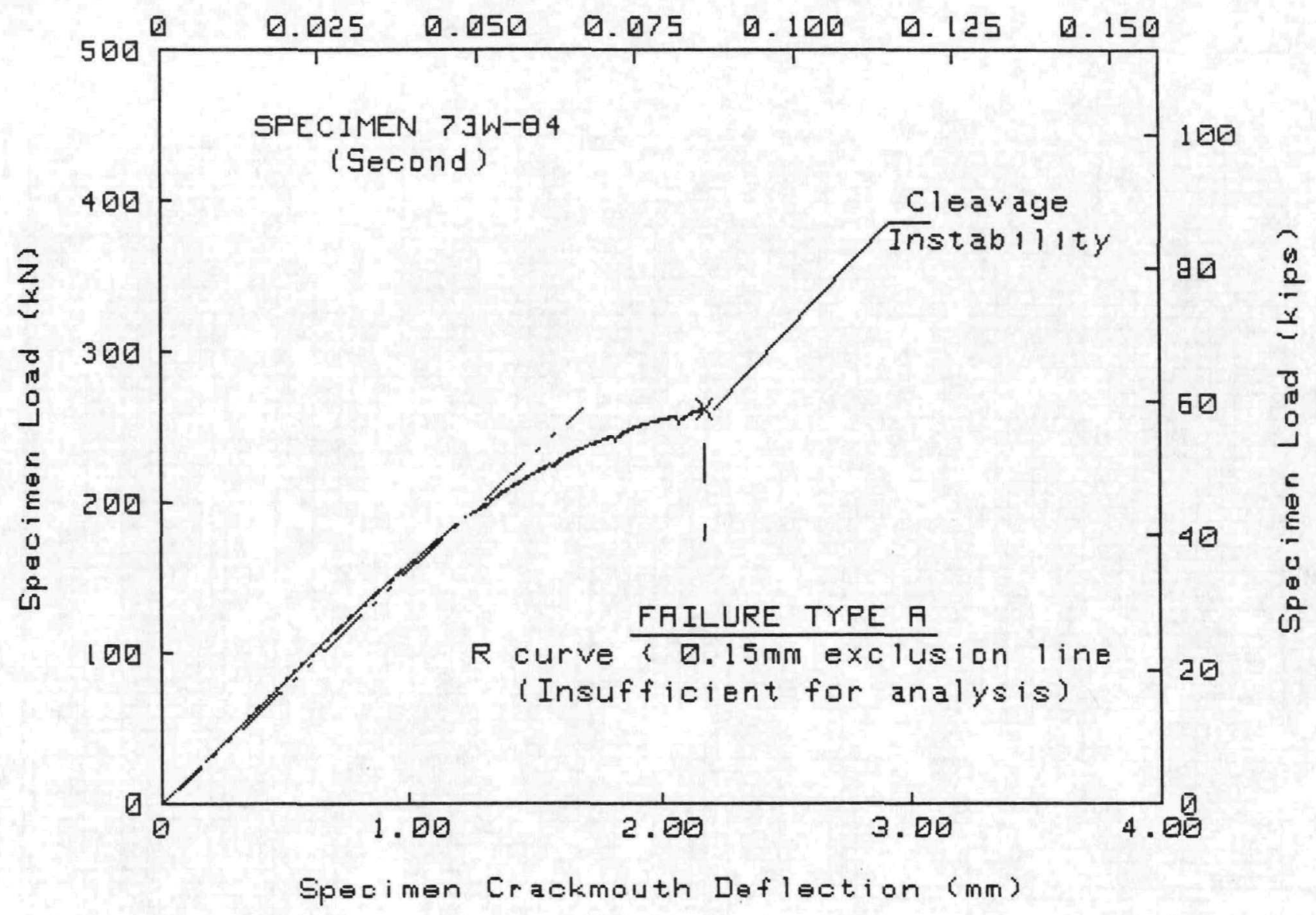

TEST SPECIMEN DATA

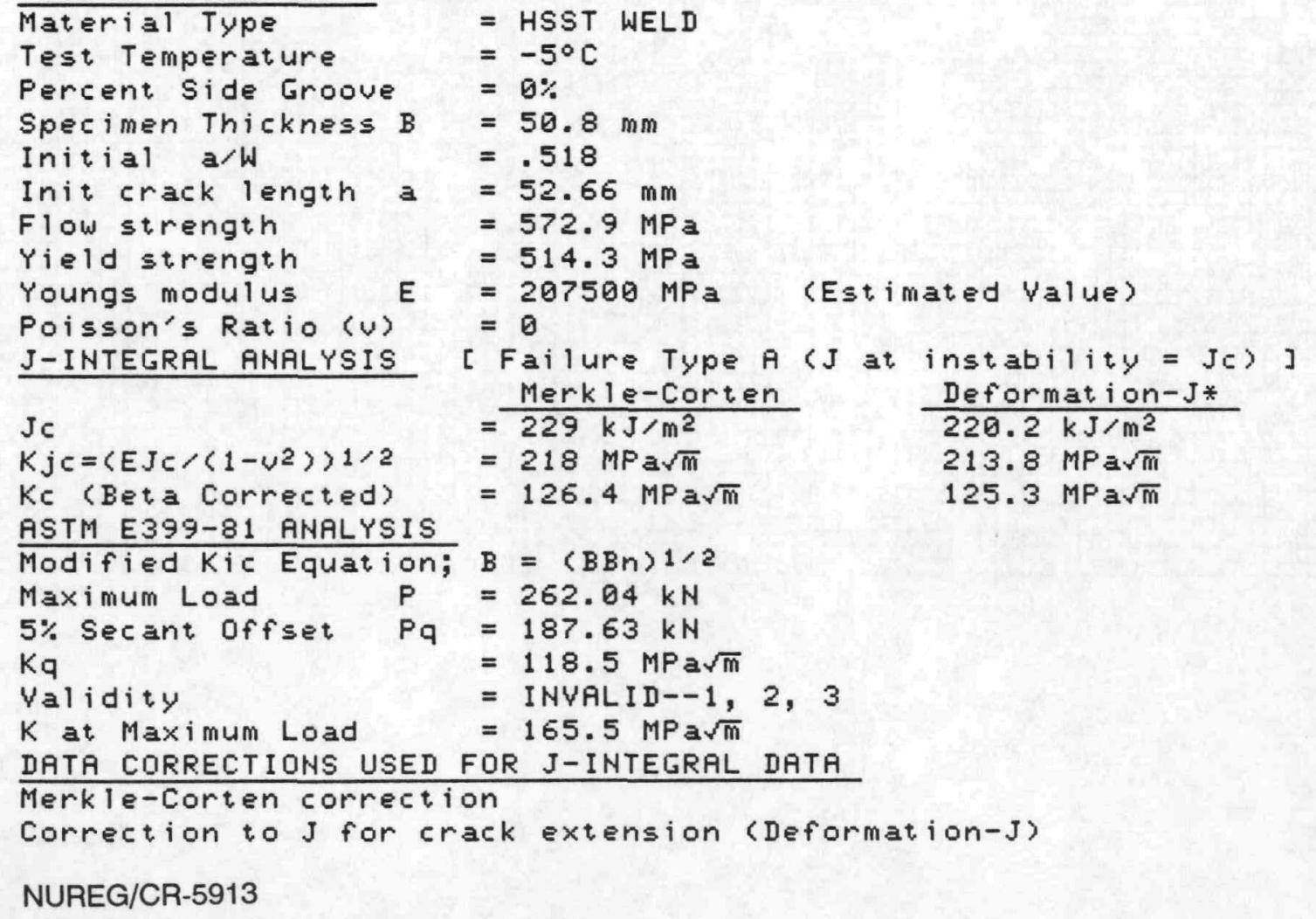


0256

$2 ;$

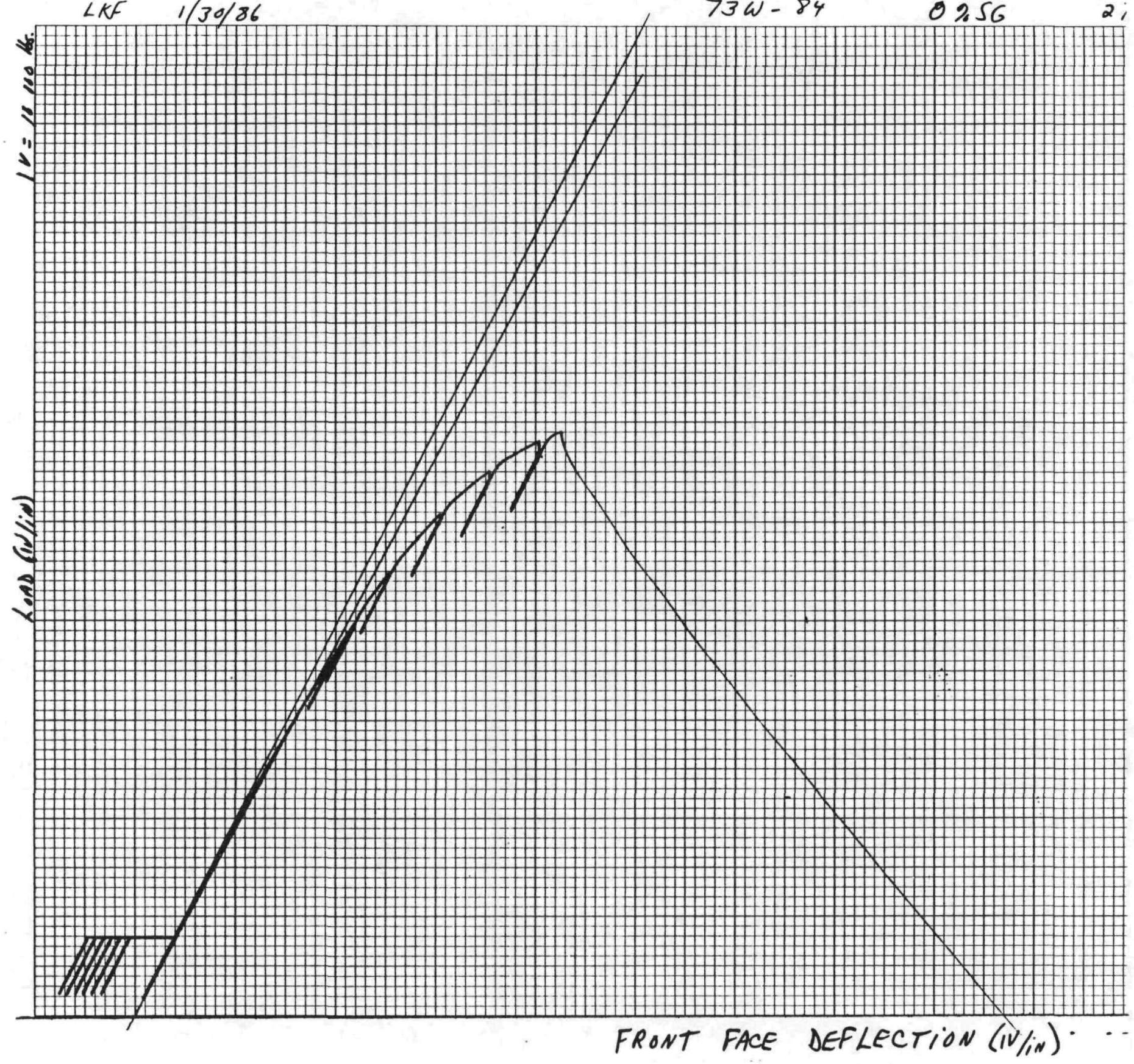


SPECIMEN ID $=73 W 69$

MATERIAL TYPE = HCUWELD

TEST TEMPERATURE $=0$ DEG C

THICKNESS $=50.6 \mathrm{~mm}$

SIDE GROOUES $=0 \%$

INITIAL CRACK LENGTH $=53.1 \mathrm{~mm}$

MEASURED DUCTILE DELTA-A $=.59 \mathrm{~mm}$

$a / W) i=.523$

YIELD STRENGTH $=511 \mathrm{MPa}$

FLOW STRENGTH $=569 \mathrm{MPa}$

ESTIMATED YOUNG'S MODULUS $=207 \mathrm{GPa}$

SPECIMEN CLEAUED AT KQ $=171.7 \mathrm{MPa} S Q R[\mathrm{~m}]$ (NOT VALID KIc)

UALUES AT MAXIMUM LOAD

J-INTEGRAL $=293.2 \mathrm{~kJ} / \mathrm{m}^{\wedge} 2$

$\mathrm{KJc}=246.5 \mathrm{MPa} \operatorname{SQR}[\mathrm{m}]$

$K-b e t a-c=132.4 \mathrm{MPa}$ SQR $[\mathrm{m}]$
Ualues at CLEaUaGe LDAD

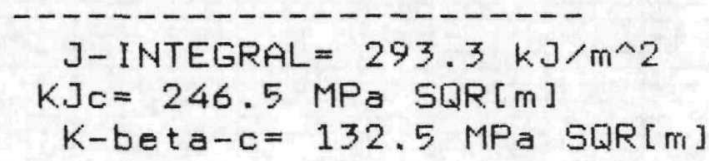


73W69 D DEGREES C

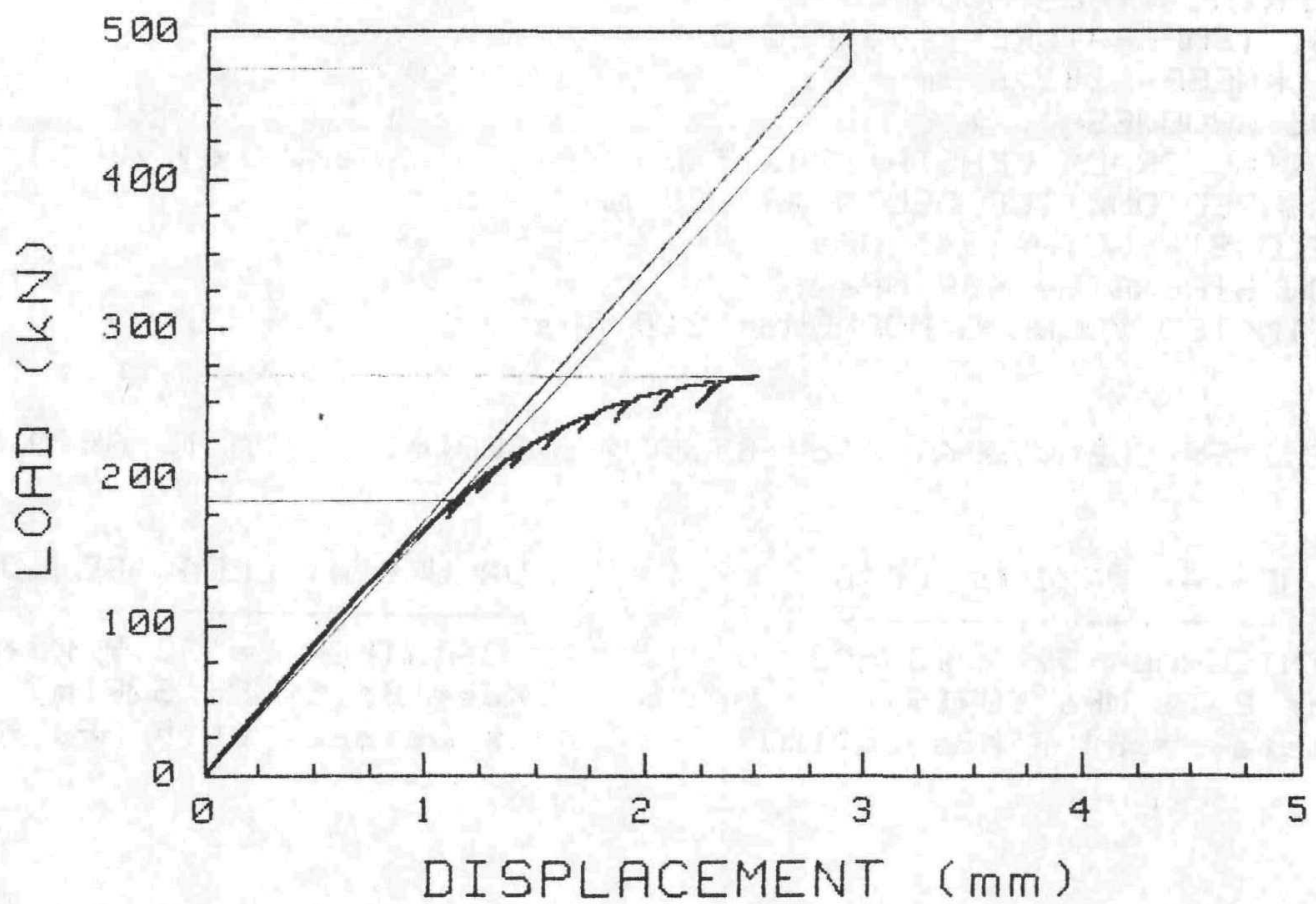

73W-69

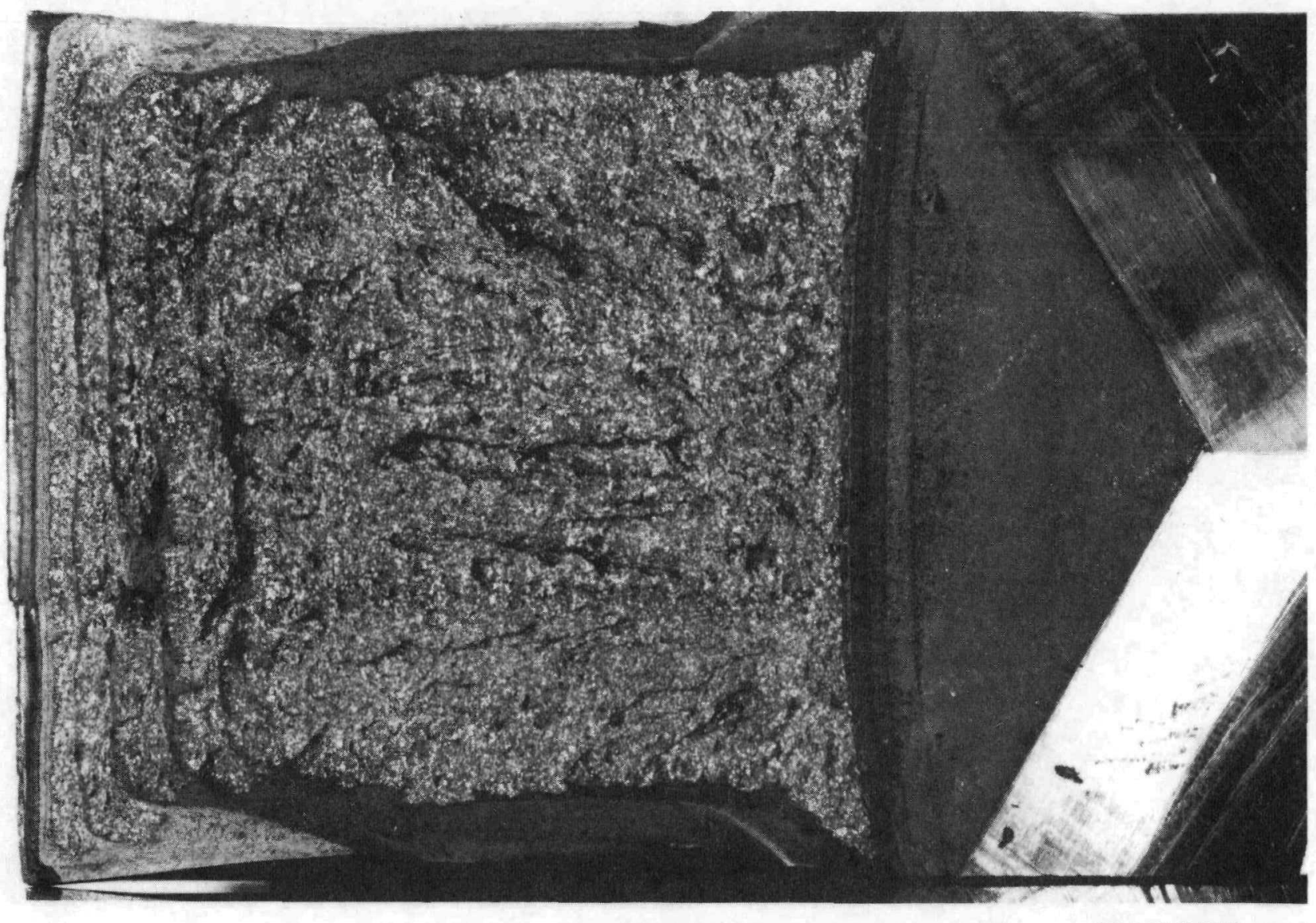


SPECIMEN ID $=73 \mathrm{~W} 20$

MATERIAL TYPE = HLUWELD

TEST TEMPERATURE $=-50$ DEG $C$

THICKNESS $=101.6 \mathrm{~mm}$

SIDE GROOUES $=0 \%$

INITIAL CRACK LENGTH $=103.3 \mathrm{~mm}$

$a / W\rangle i=.508$

MEASURED DUCTILE DELTA-A $=.05 \mathrm{~mm}$

$Y I E L D$ STRENGTH $=543 \mathrm{MPa}$

FLOW STRENGTH $=609 \mathrm{MPa}$

ESTIMATED YOUNG'S MODULUS= $210 \mathrm{GPa}$

SPECIMEN CLEAUED AT KIC $=85.5 \mathrm{MPa}$ SQR $[\mathrm{m}]$ (UALID ASTM E399)

UALUES AT MAXIMUM LDAD

J-INTEGRAL $=32.9 \mathrm{~kJ} / \mathrm{m}^{\wedge} 2$

$\mathrm{KJ} c=83.1 \mathrm{MPa} S Q R[\mathrm{~m}]$

K-beta-c $=80.5 \mathrm{MPa} \operatorname{SQR}[\mathrm{m}]$
UALUES AT CLEAUAGE LOAD

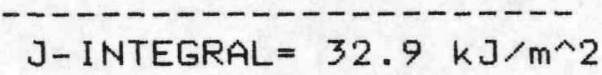

$K J \mathrm{~J}=83.1 \mathrm{MPa} \operatorname{SQR}[\mathrm{m}]$

K-beta-c $=80.5 \mathrm{MPa} \operatorname{SQR}[\mathrm{m}]$ 


\section{F-109}

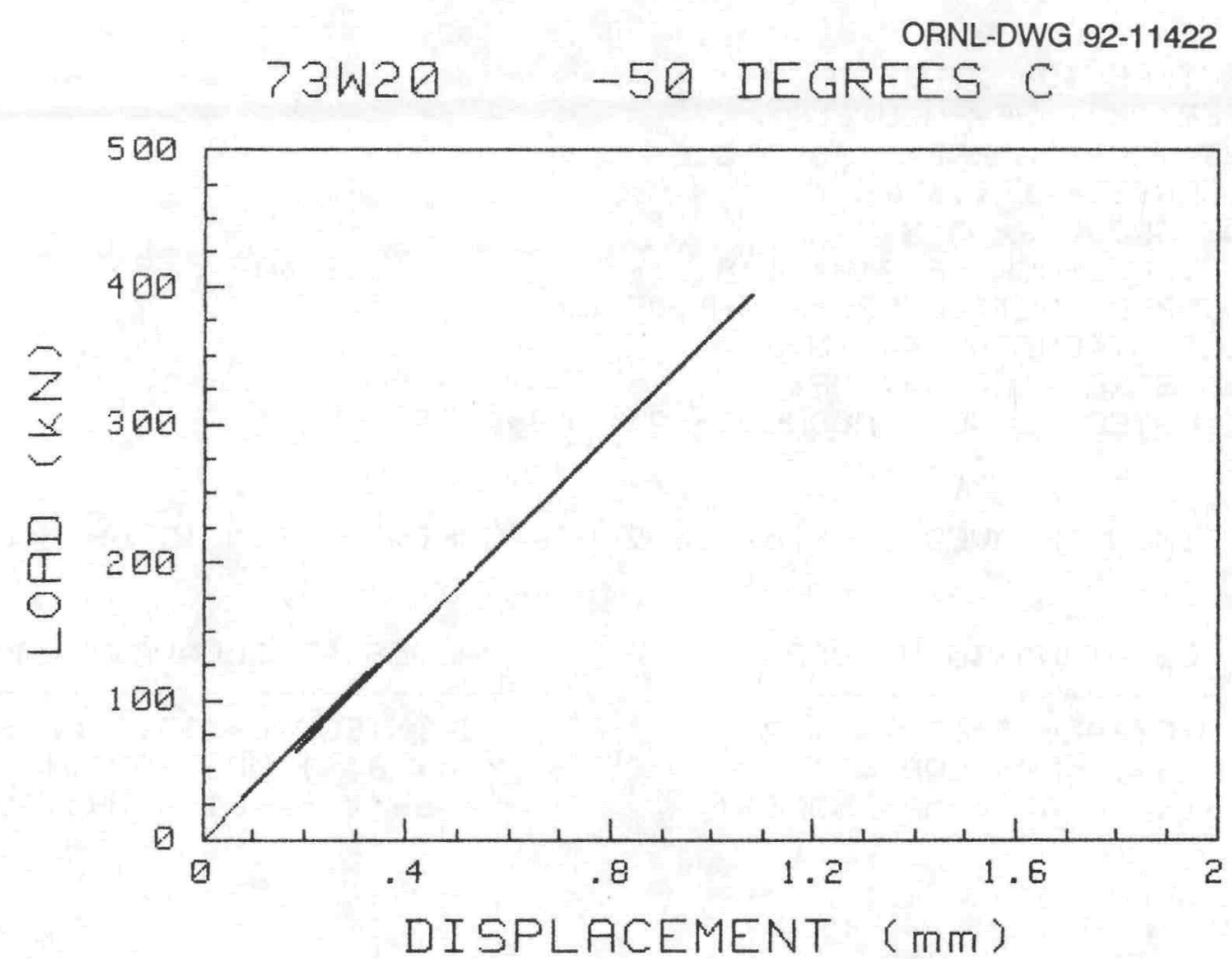

73W-20

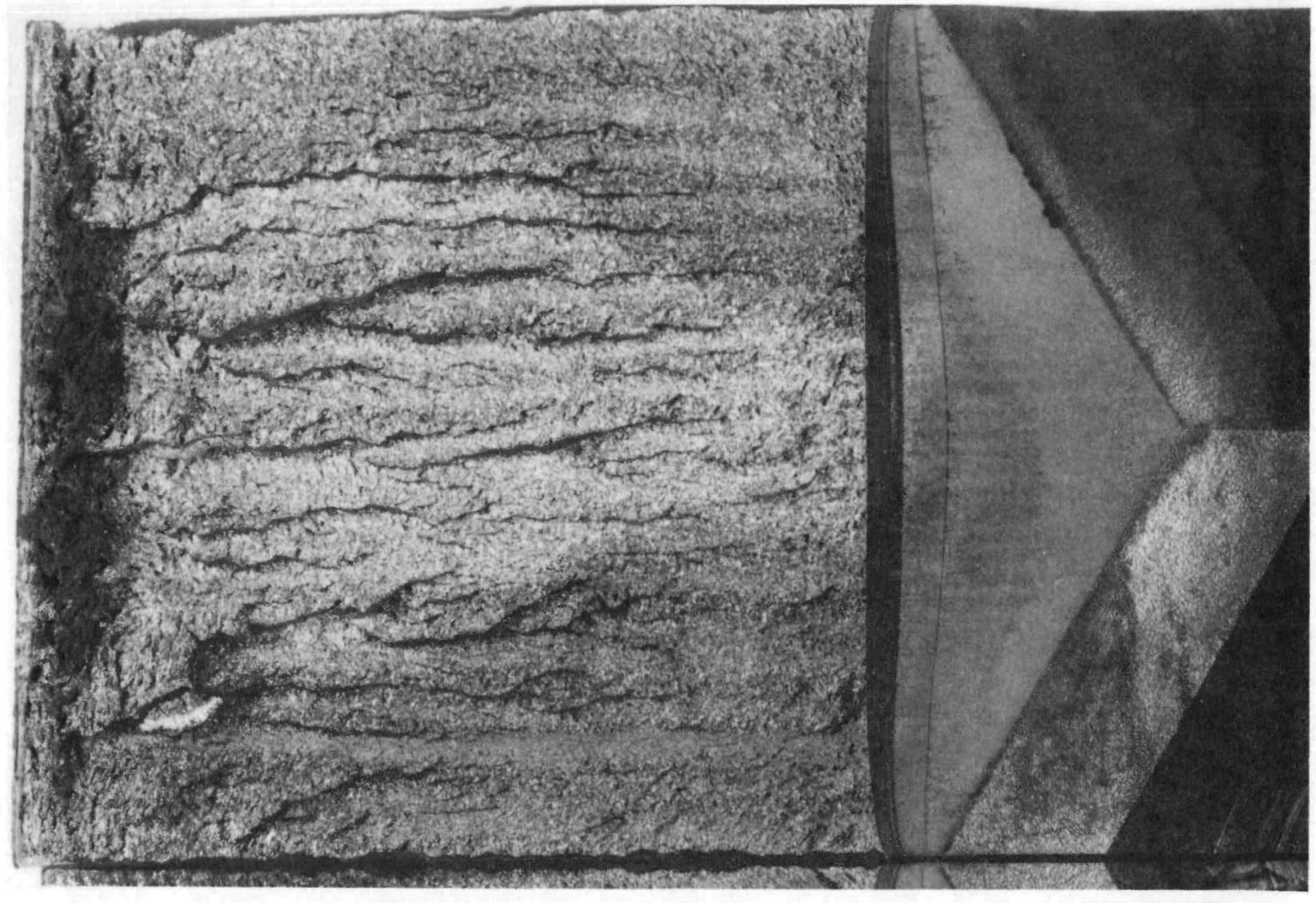


SPEC IMEN ID $=73 \mathrm{~W} 24$

MATERIAL TYPE = HCUWELD

TEST TEMPERATURE $=-50$ DEG C

THICKNESS $=101.6 \mathrm{~mm}$

SIDE GROOUES $=0 \%$

INITIAL CRACK LENGTH $=108.2 \mathrm{~mm}$

$a / W) i=.532$

MEASURED DUCTILE DELTA-A $=.05 \mathrm{~mm}$

YIELD STRENGTH $=543 \mathrm{MPa}$

FLOW STRENGTH $=609 \mathrm{MPa}$

ESTIMATED YOUNG'S MODULUS $=210 \mathrm{GPa}$

SPECIMEN CLEAUED AT KIc= 36.9 MPa SQR [m] (UALID ASTM E399)

UALUES AT MAXIMUM LOAD

J-INTEGRAL $=33.7 \mathrm{~kJ} / \mathrm{m}^{\wedge} 2$

$\mathrm{KJc}=84.1 \mathrm{MPa} \operatorname{SQR}[\mathrm{m}]$

K-beta-c $=81.4 \mathrm{MPa} \operatorname{SQR}[\mathrm{m}]$
Ualues at cleavage LoAd

J-INTEGRAL $=33.7 \mathrm{~kJ} / \mathrm{m}^{\wedge} 2$

$\mathrm{KJc}=84.1 \mathrm{MPa} \operatorname{SQR}[\mathrm{m}]$

K-beta-c $=81.4 \mathrm{MPa} \operatorname{SQR}[\mathrm{m}]$ 
F-111

ORNL-DWG 92-11423

73W24 -50 DEGREES C

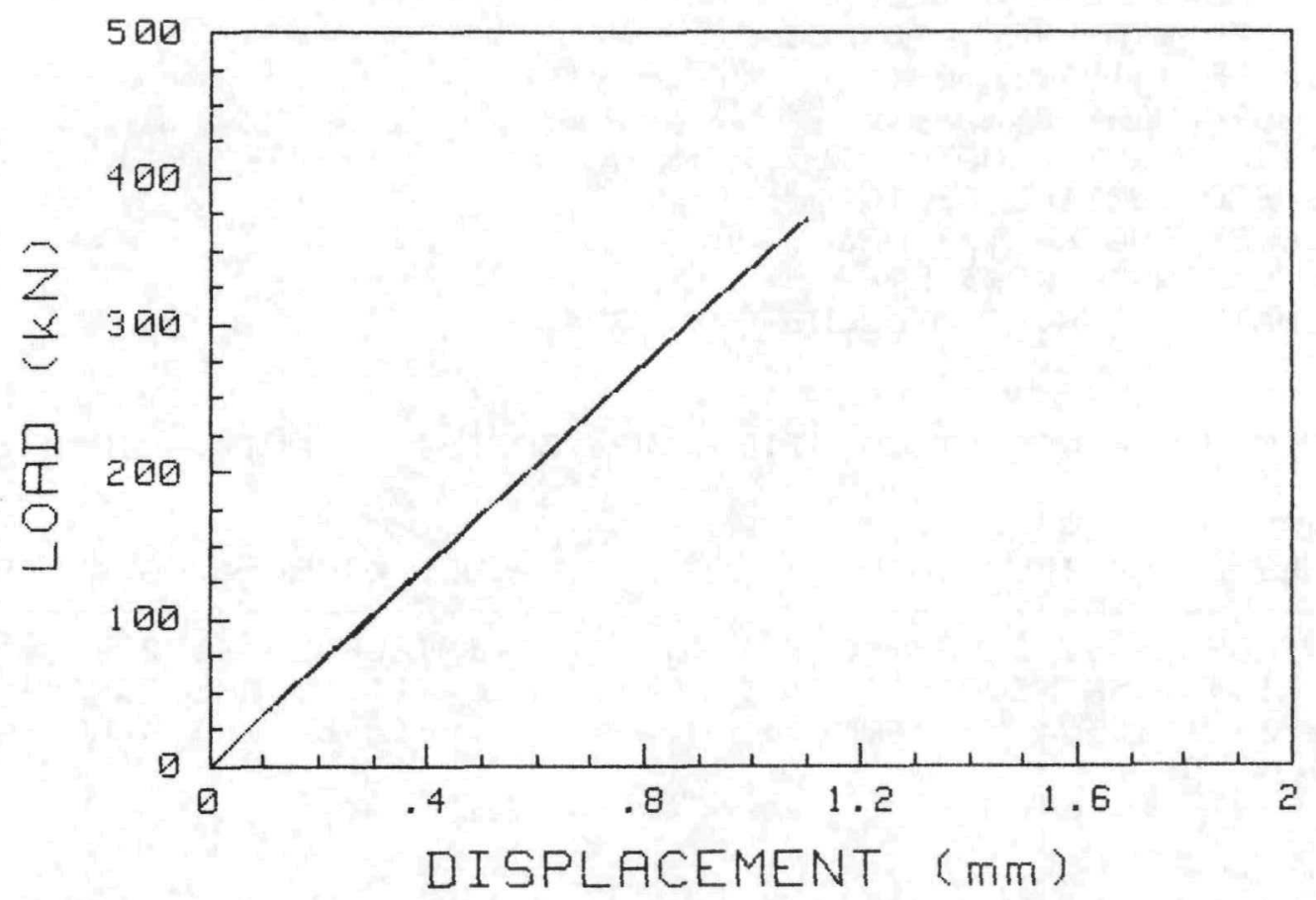

$73 W-24$

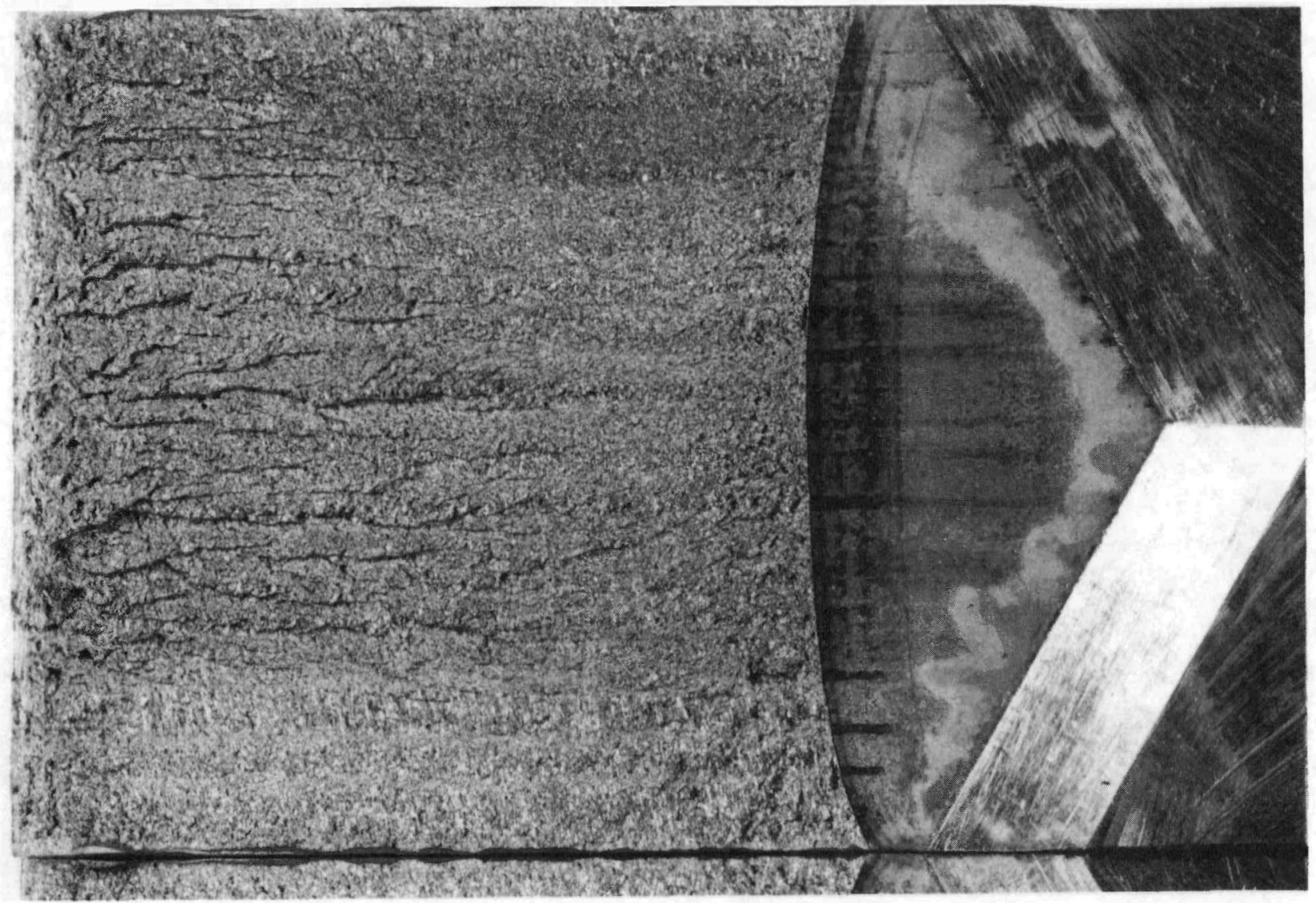




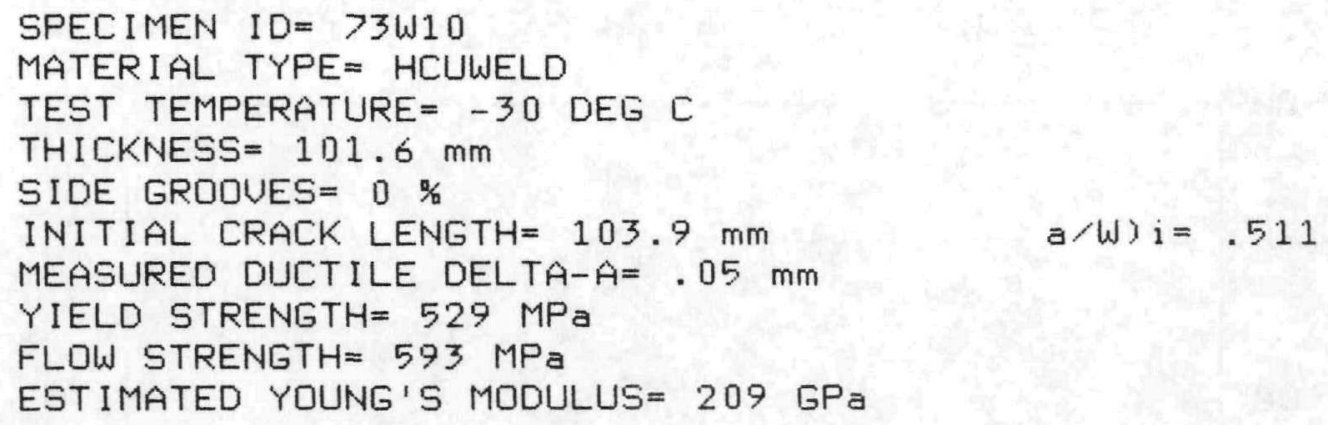


F-113

ORNL-DWG 92-11424

73W10 -3日 DEGREES C

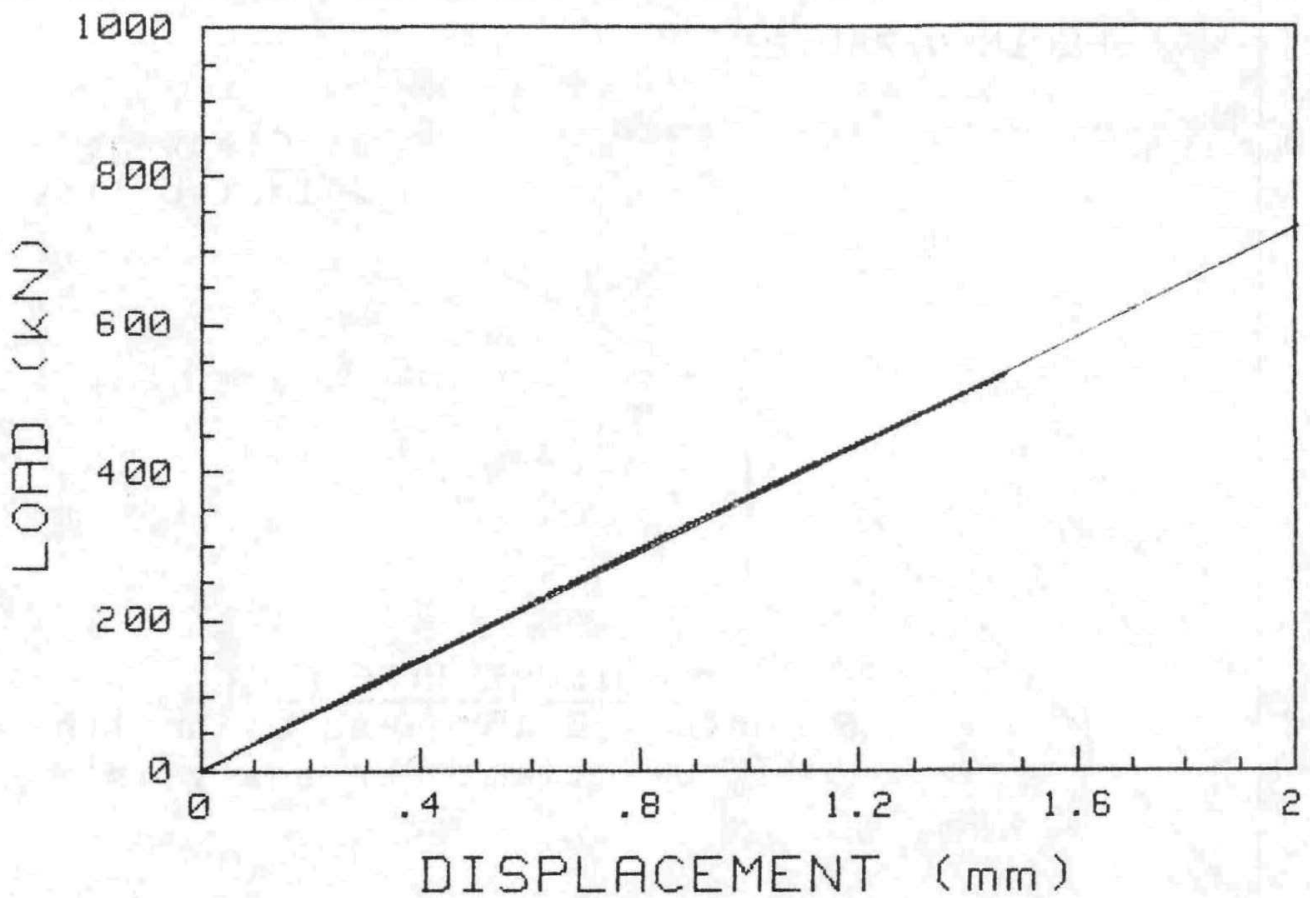

$73 W-10$

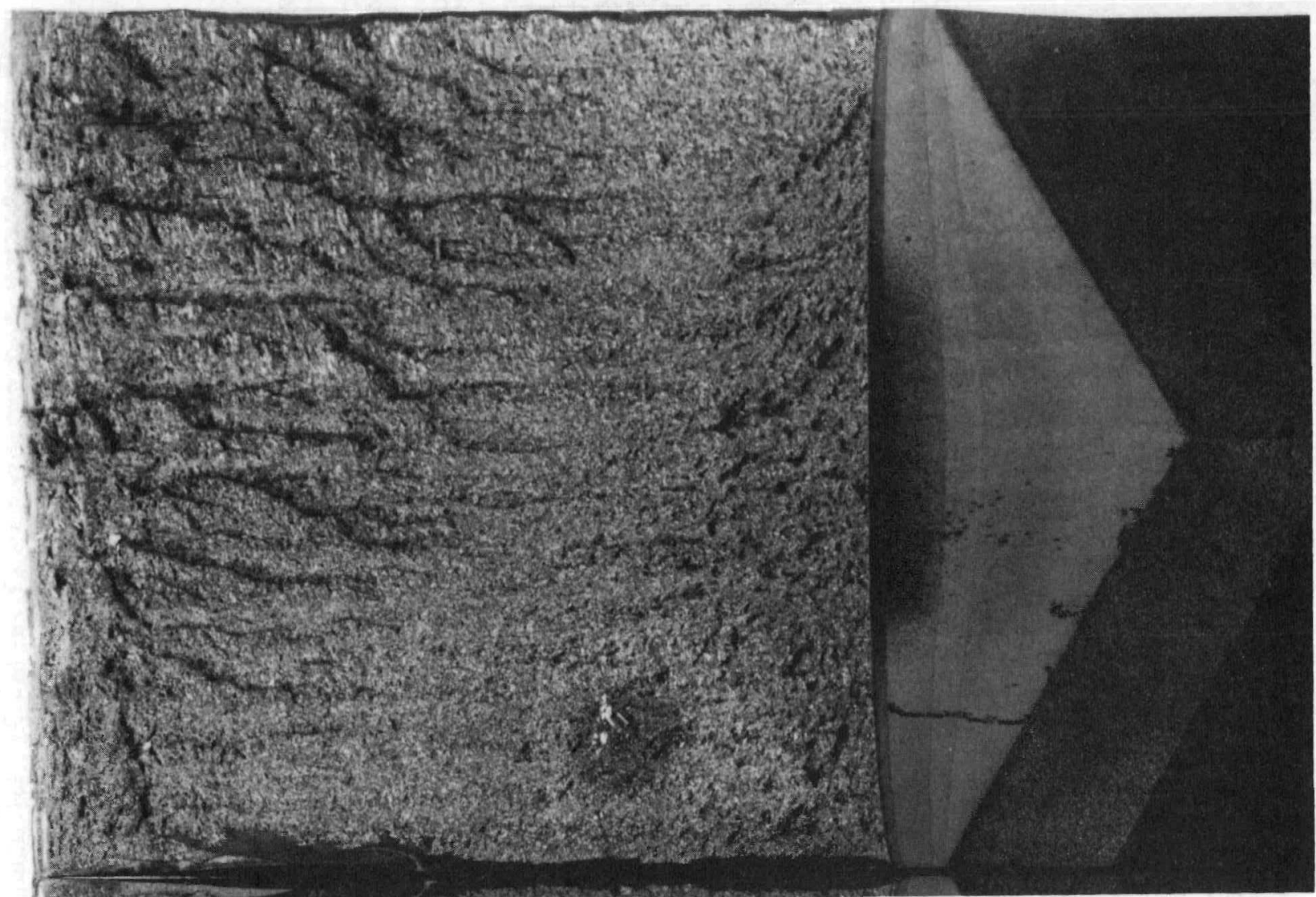

NUREG/CR-5913 


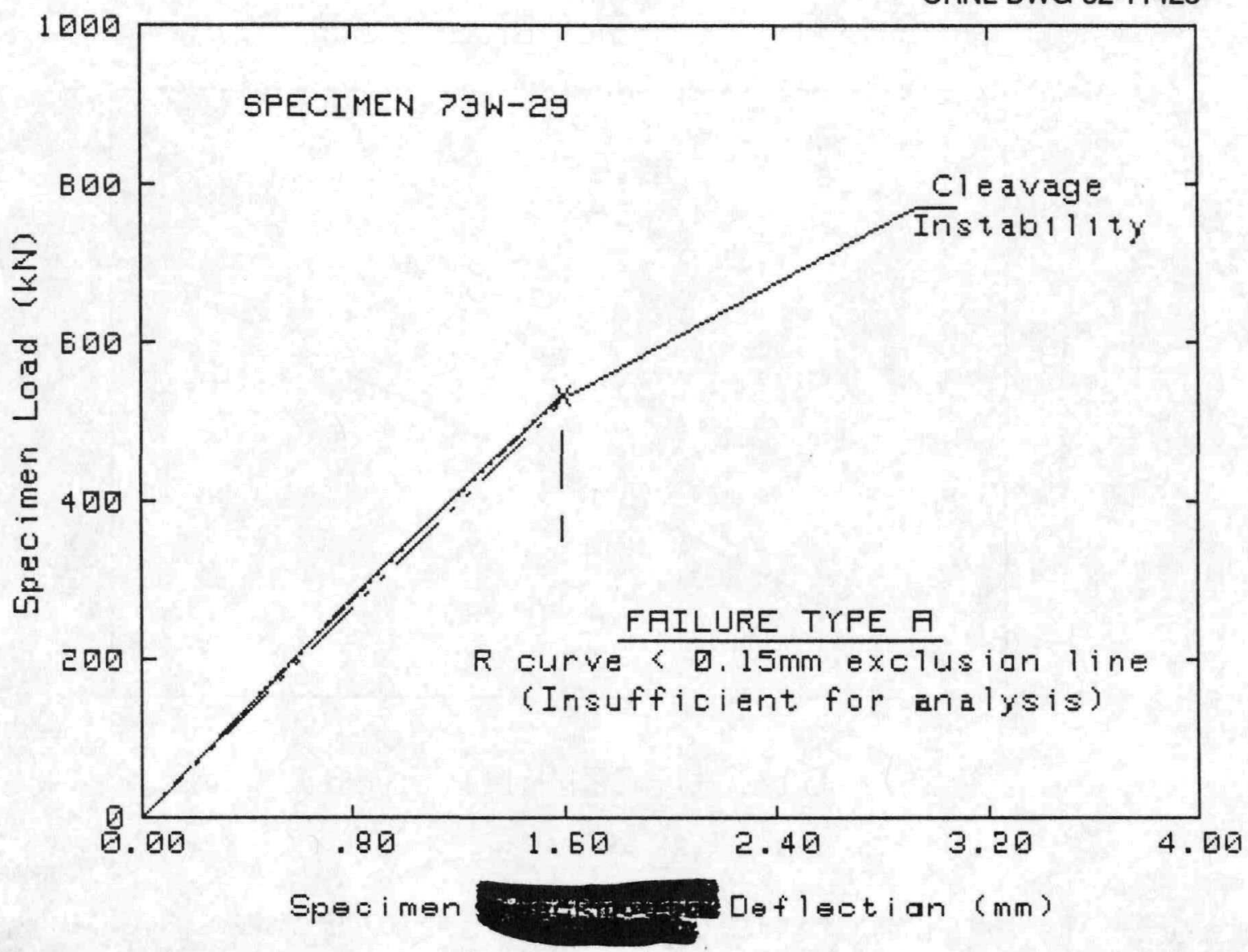

TEST SPECIMEN DATA

Material Type
Test Temperature
Percent Side Groove
Specimen Thickness B
Initial a/W
Init crack length a
Flow stress
Yield stress
Youngs modulus E
Poisson's Ratio (u)

$=$ A533-B HSST $5 \mathrm{th}$ Irrad. Weld

$=-30 \mathrm{C}$

$=0 \%$

$=101.6 \mathrm{~mm}$

$=.517$

$=105.03 \mathrm{~mm}$

$=592.7 \mathrm{MPa}$

$=533.6 \mathrm{MPa}$

Poisson's Ratio(v)

$=298900 \mathrm{MPa}$

J-INTEGRAL ANALYSIS

$=0$

(Est imated Value)

Failure Type $A$ ( $J$ at instability = Jic)

Jic

$=72.4 \mathrm{~kJ} / \mathrm{m} \cap 2$

$K j c=\left\langle E J i c /\left(1-v^{\wedge} 2\right\rangle\right\rangle \wedge .5$

$=123 \mathrm{MPa} \backslash \mathrm{m}$

Kic (Beta Corrected)

$=110.2 \mathrm{MPa} \backslash \mathrm{m}$

ASTM E399-81 ANALYSIS

Modified Kic Equation; $B=\langle$ BBn〉^.5

Maximum Load $P=533.1 \mathrm{kN}$

$5 \%$ Secant offset $\mathrm{Pq}=533.12 \mathrm{kN}$

Kic

$=118.5 \mathrm{MPa} \backslash \mathrm{m}$

Validity

= INVALID $--1,2$

$K$ at Maximum Load

$=118.5 \mathrm{MPa} \backslash \mathrm{m}$

DATA CORRECTIONS USED FOR J-INTEGRAL DATA

Merkle-Corten OFFSET LOAD $=0$ lb OFFSET DEFLECTION $=7.55527742376 E-05 \mathrm{in}$ Specimen rotation Load intercept elastic slape $=-9.44838709677 E-05$ it

Fitted elastic load range $=35000$ To 25000 Correction to J for crack exten 


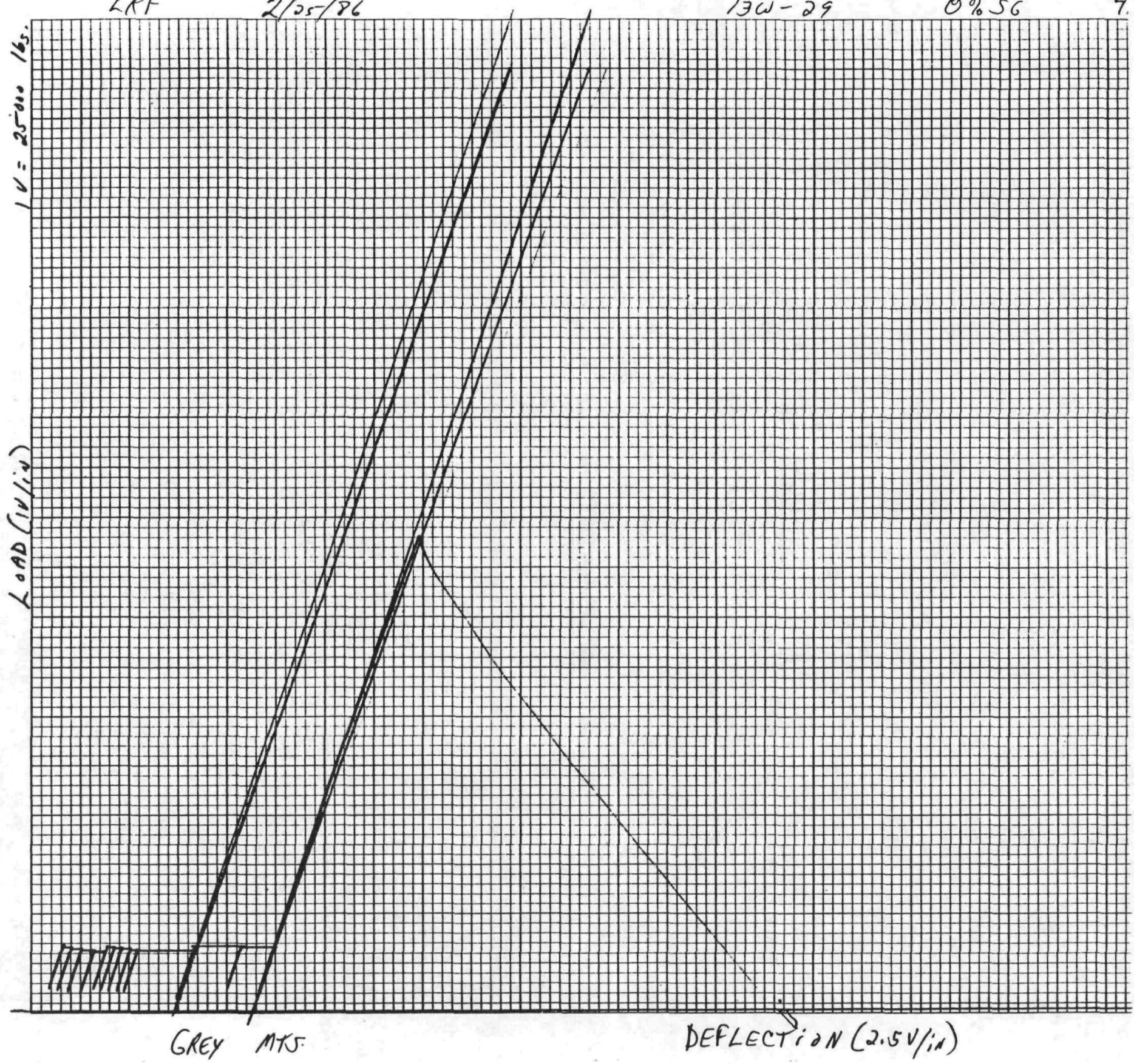




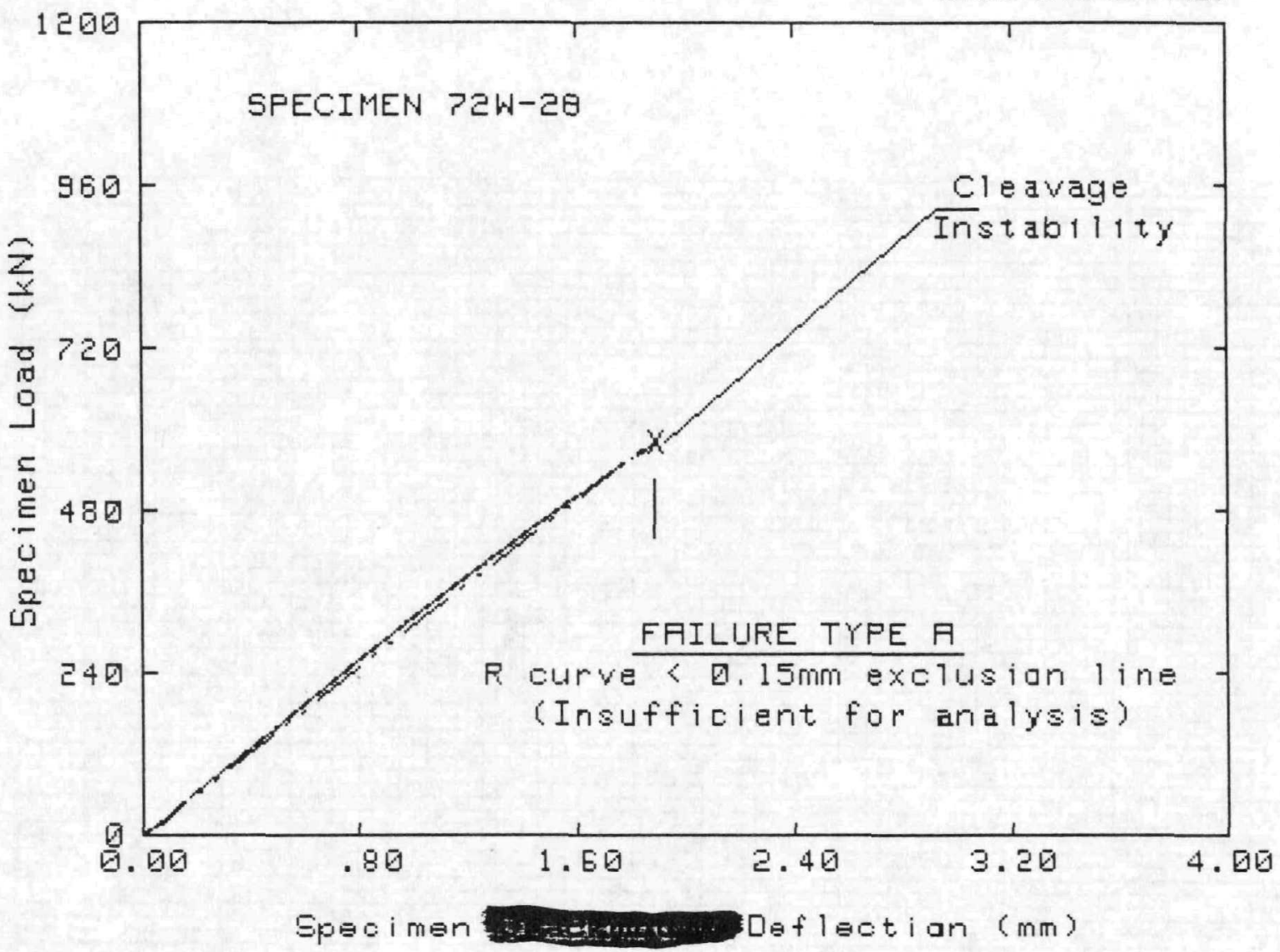

TEST SPECIMEN DATA

Material Type
Test Temperature

Percent Side Groove

Specimen Thickness B

Initial $a / W$

Init erack length a

Flow stress

Yield stress

Youngs modulus

Poisson's Ratio (v)

J-INTEGRAL ANALYSIS

Failure Type $A$ (J at

Jic

$K j c=\left\langle E J i c /\left(1-v^{\wedge} 2\right\rangle\right) \wedge .5$

Kic (Beta Corrected)

ASTM E399-81 ANALYSIS

Modified Kic Equation;

$5 \%$ secant offset

$\mathrm{Kic}$

Validity

$K$ at Maximum Load

$\mathrm{P}=580.6 \mathrm{kN}$

$\mathrm{Pq}=546.95 \mathrm{kN}$

$=127.5 \mathrm{MPa} \backslash / \mathrm{m}$

= INVALID--1, 2

$=135.3 \mathrm{MFa} \backslash \mathrm{m}$

IATA CORRECTIONS USED FOR J-INTEGRAL IATA

MErkle-Corten OFFSET LOAD= g it OFFSET DEFLECTION $=3.11977423278 E-94$ in.

Specimen rotation Load intercept elastic slape = .00005990625 ib

sion

Fitted elastic load range= 35000 To 25000 Correction to J for crack exten 


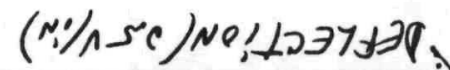

stan tay

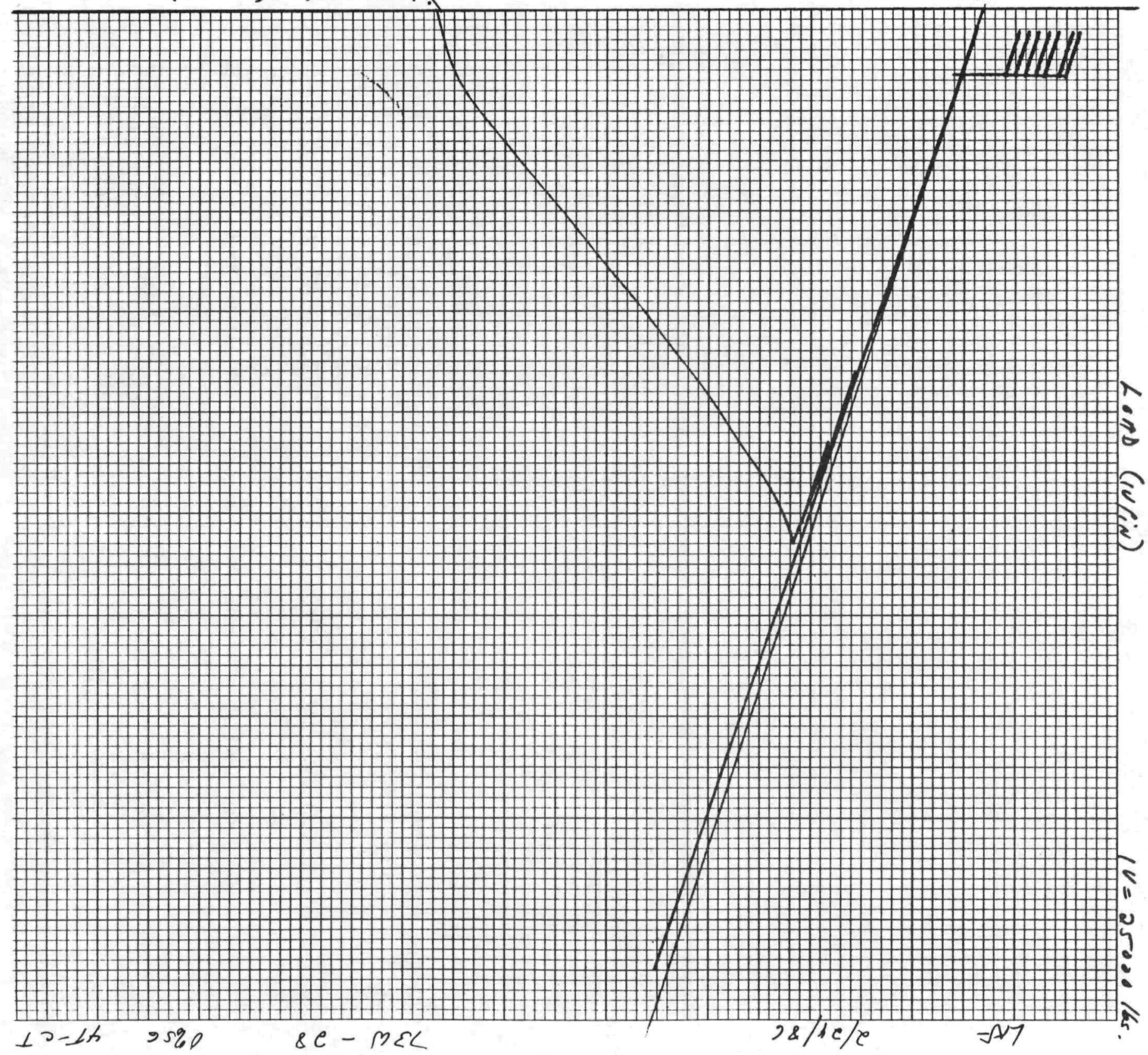

$\frac{N}{1}$

12 -th $95 \% 0 \quad \& C-M E<$

$3 \& / \mathrm{c} / \mathrm{c}$

$-17$ 


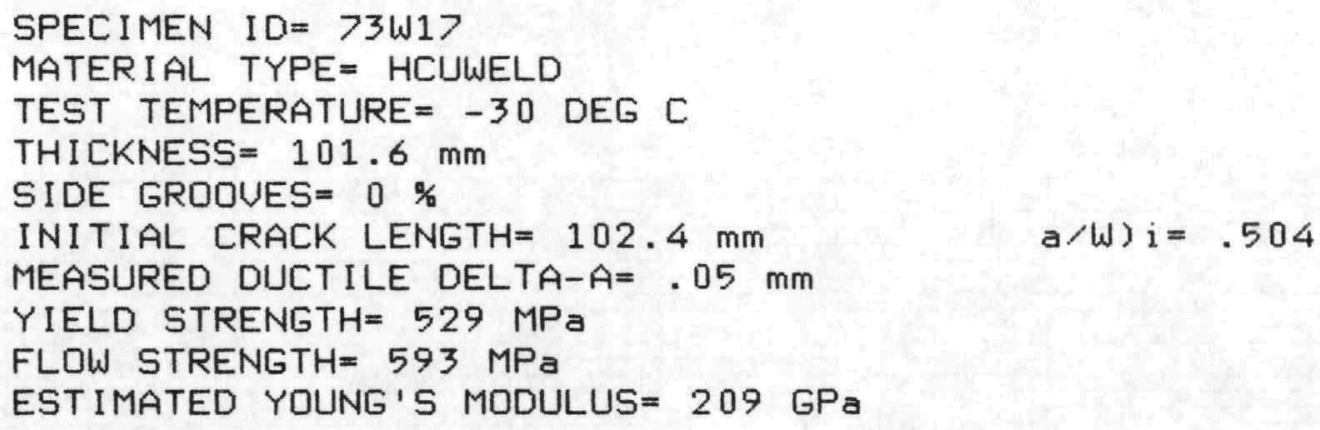

UALUES AT MAXIMUM LDAD

]-INTEGRAL $=149.3 \mathrm{~kJ} / \mathrm{m}^{\wedge} 2$

$\mathrm{KJc}=176.6 \mathrm{MPa} \operatorname{SDR}[\mathrm{m}]$

$K$-bet a-c $=138.3 \mathrm{MPa} \operatorname{SQR}[\mathrm{m}]$
UALUES AT CLEAUAGE LOAD

J-INTEGRAL $=149.3 \mathrm{~kJ} / \mathrm{m}^{\wedge} 2$

$\mathrm{KJc}=176.6 \mathrm{MPa} \operatorname{SQR}[\mathrm{m}]$

K-beta-c $=138.3 \mathrm{MPa} \operatorname{SQR}[\mathrm{m}]$ 
F-119

ORNL-DWG 92-11427

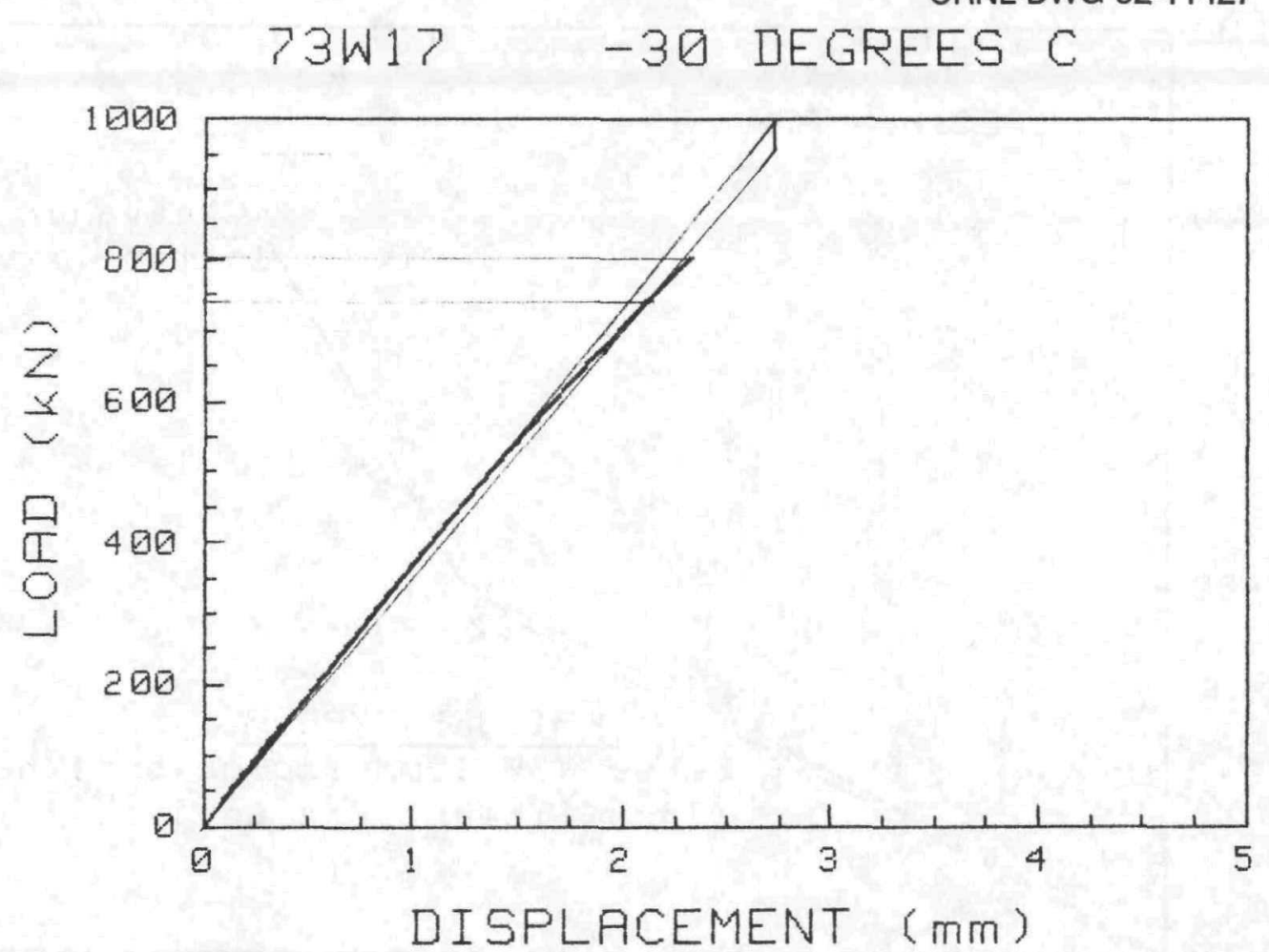

73W-17

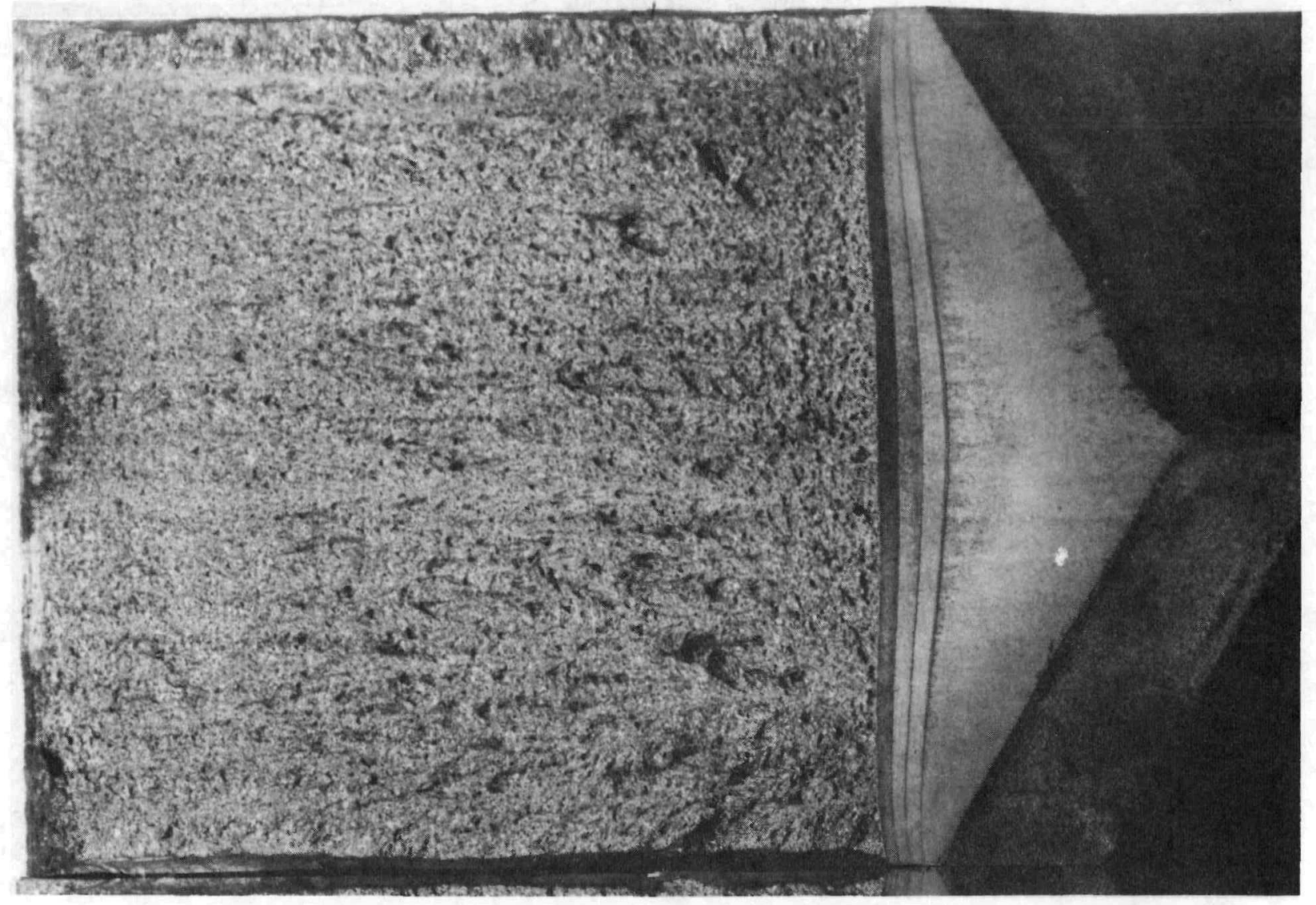




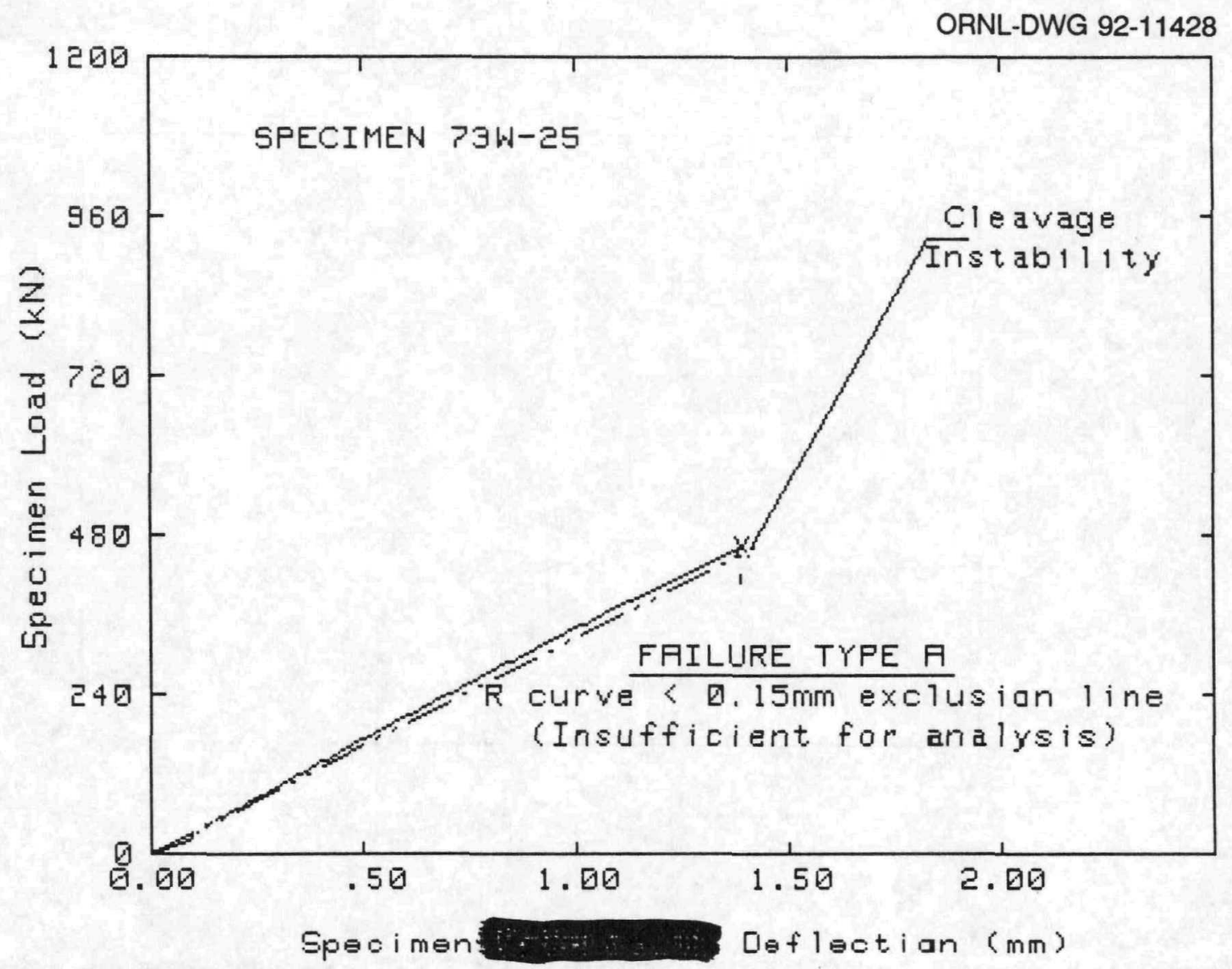

TEST SPECIMEN DATA

Material Type

Test Temperature

Percent Side Groove

Specimen Thickness B

Initial a/w

Init crack length a

Flow stress

Yield stress

Youngs modulus $E$

Poisson's Ratio ( $u$ )

J-INTEGRAL ANALYSIS

Failure Type $A$ < $J$ at

\section{$\mathrm{Jic}$}

$K j c=\langle E J i c /(1-\psi \wedge 2\rangle) \wedge .5$

Kic (Beta Corrected)

= A533-B HSST 5th Irrad. Weld

$=-15 \mathrm{C}$

$=0 \%$

$=101.6 \mathrm{~mm}$

$=.524$

$=106.55 \mathrm{~mm}$

$=581.2 \mathrm{MPa}$

$=521.2 \mathrm{MPa}$

$=208100 \mathrm{MPa} \quad$ (Estimated Value)

$=0$

BSTM E399-81 ANALYSIS

Modified Kic Equation; $B=\langle$ BBn $\rangle \wedge .5$

Maximum Load $P=462.6 \mathrm{kN}$

$5 \%$ Secant offset $\mathrm{Pq}=462.63 \mathrm{kN}$

$\mathrm{Kic}$

Validity

$=105.4 \quad \mathrm{MPa} \backslash \mathrm{m}$

$=$ INVALI ID--2

$K$ at Maximum Load

$=105.4 \mathrm{MPa} \backslash \mathrm{m}$

DATA CORRECTIONS USED FOR J-INTEGRAL IATA

Merkle-Corten OFFSET LOAD $=0$ lb OFFSET DEFLECTION $=1.00682895179 E-03 \mathrm{in}$. Specimen rotation Load intercept Elastic slape $=-3.39769230769 E-05$ it sion

Fitted elastic load range $=35000$ To 25000 Correction to J for crack exten 


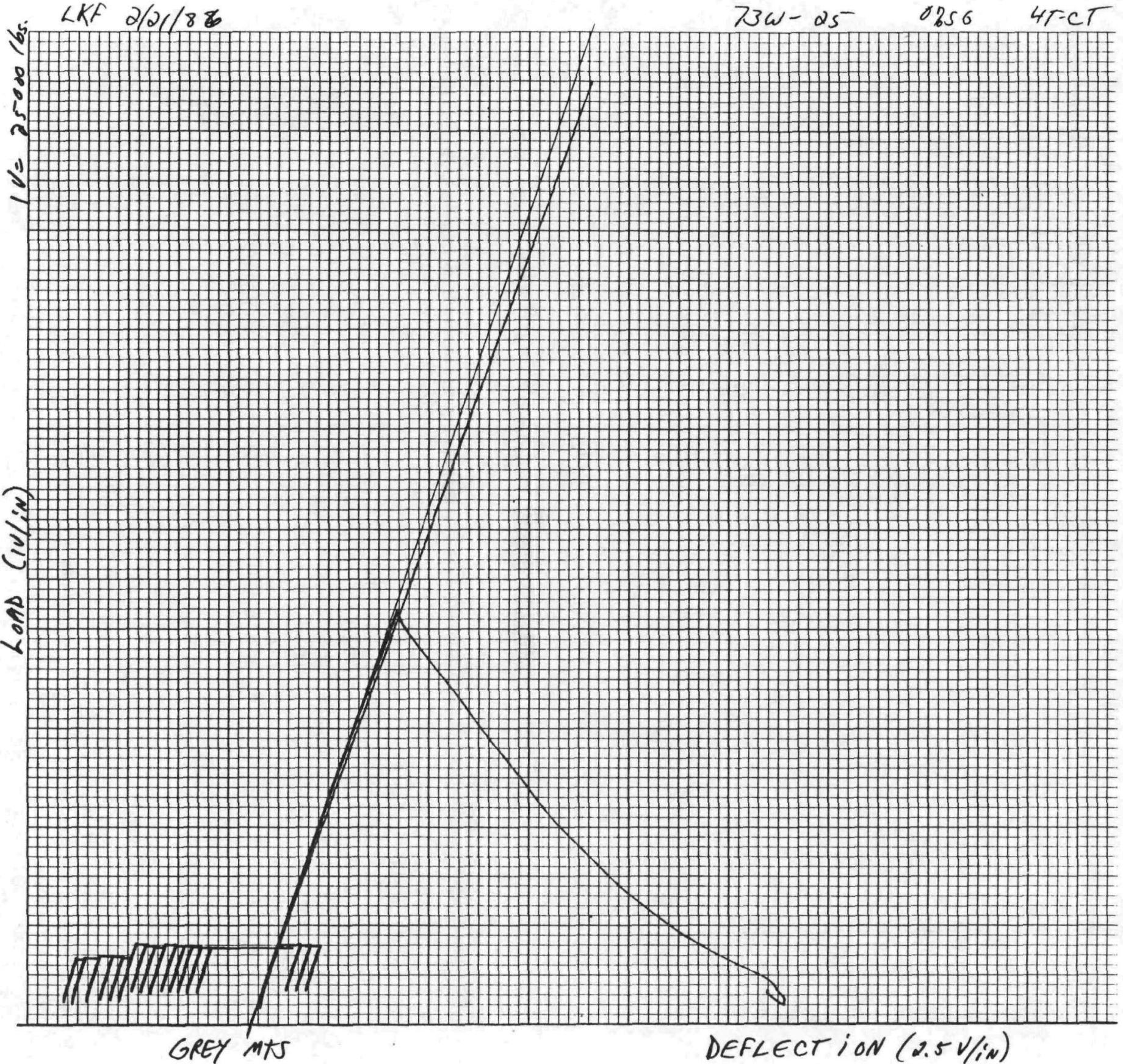




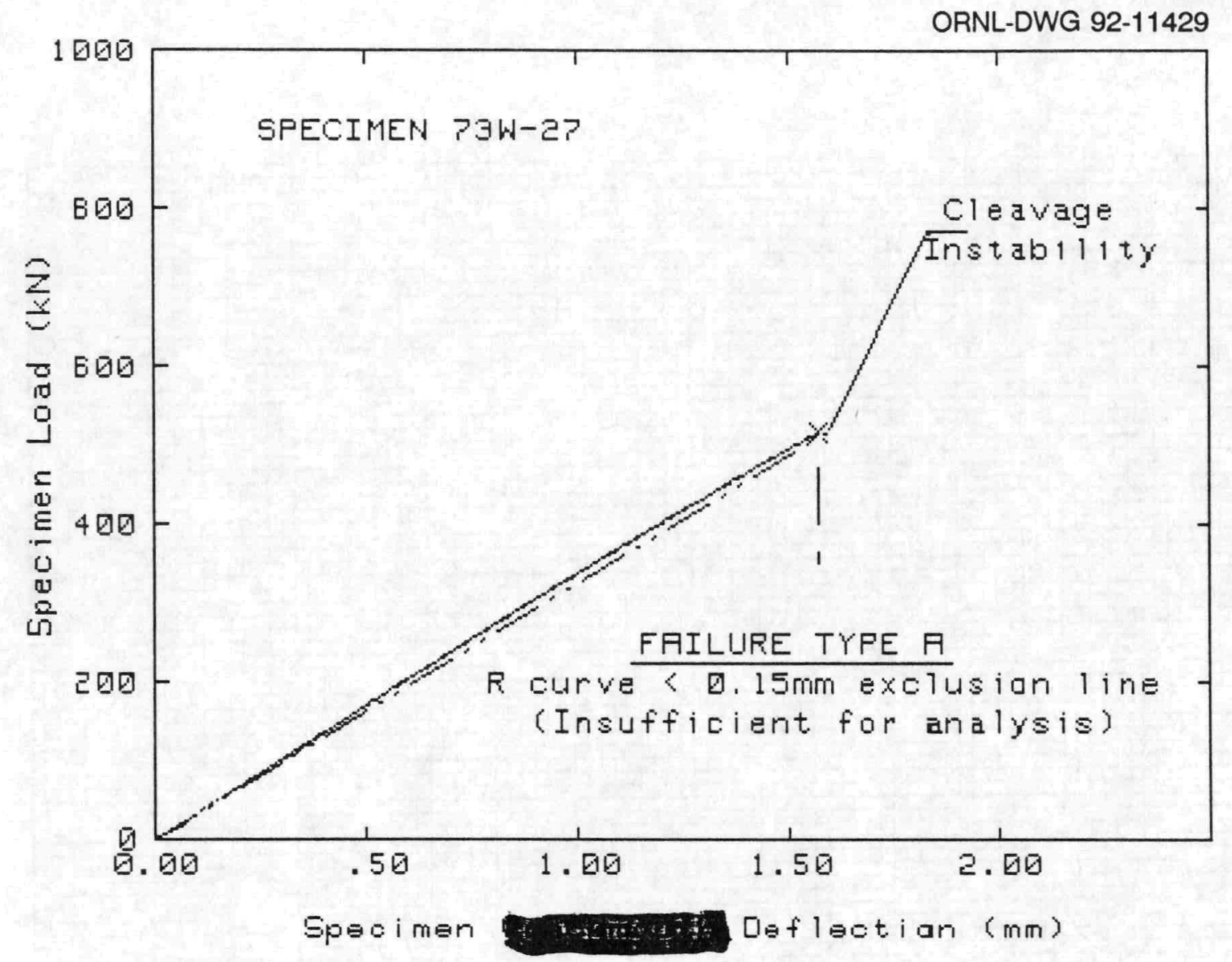

TEST SPECIMEN DATA

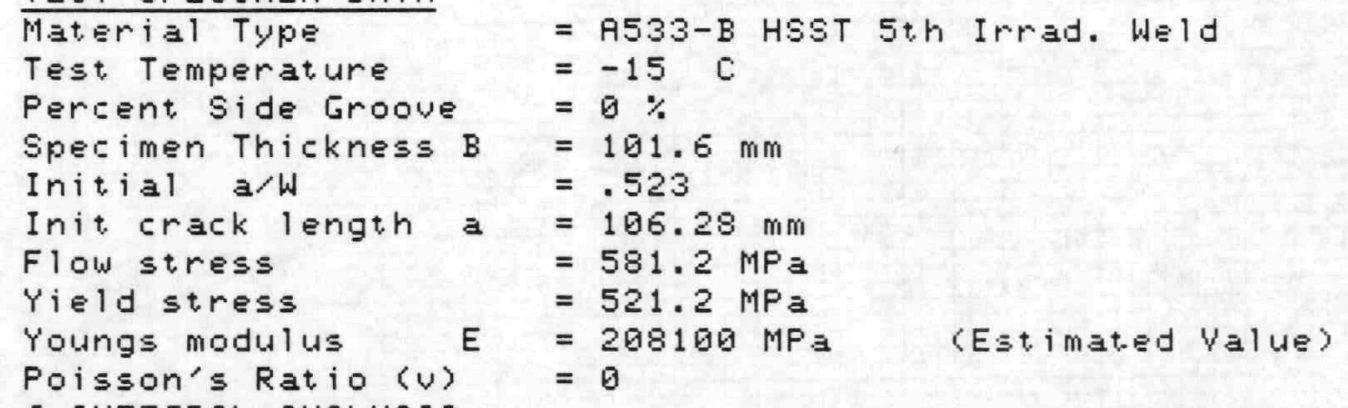

J-INTEGRAL ANALYSIS

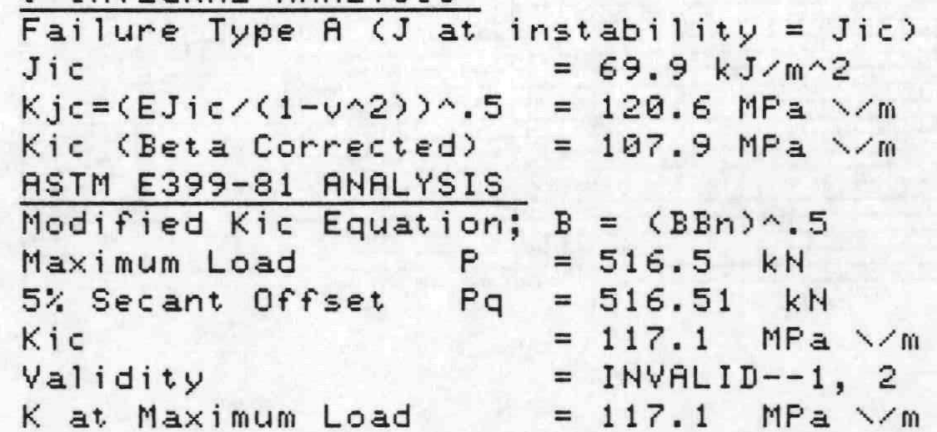

DATA CORRECTIONS USED FOR J-INTEGRAL IATA

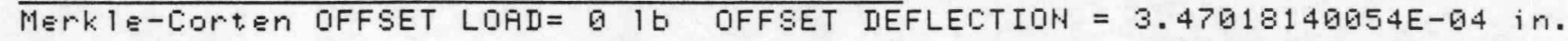
Specimen rotation Load intercept elastic $\$ 1$ ape = 2.76451612903E-G5 ib

Fitted elastic load range= 35000 To 25090 Correction to J for crack exten 


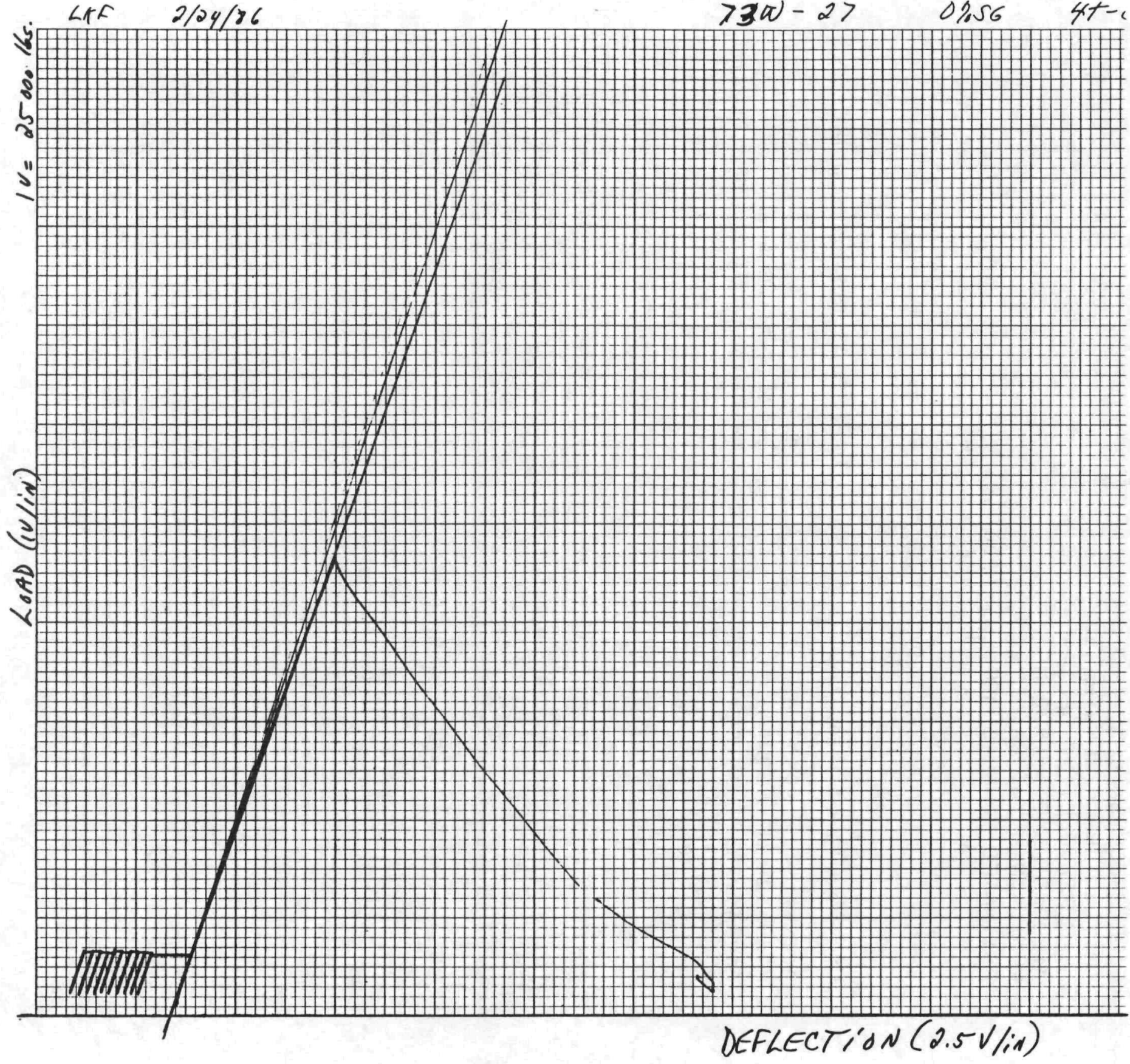


SPECIMEN ID $=73 W 26$

MATERIAL TYPE $=$ HCUWELD

TEST TEMPERATURE $=-15$ DEG $C$

THICKNESS $=101.6 \mathrm{~mm}$

SIDE GROOUES $=0 \%$

INITIAL CRACK LENGTH $=107.7 \mathrm{~mm}$

$a / w) i=.531$

MEASURED DUCTILE DELTA-A $=.05 \mathrm{~mm}$

$Y$ IELD STRENGTH $=520 \mathrm{MPa}$

FLOW STRENGTH $=581 \mathrm{MPa}$

ESTIMATED YOUNG'S MODULUS $=208 \mathrm{GPa}$

SPECIMEN CLEAUED AT KQ= $122.2 \mathrm{MPa} S Q R[\mathrm{~m}]$ (NOT UALID KIC)

UALUES AT MAXIMUM LOAD

J-INTEGRAL $=69.2 \mathrm{~kJ} / \mathrm{m}^{\wedge} 2$

$\mathrm{KJ} c=120 \mathrm{MPa} \operatorname{SQR}[\mathrm{m}]$

$K$-beta-c= $107.5 \mathrm{MPa} S Q R[\mathrm{~m}]$

\section{UALUES AT CLEAUAGE LDAD}

J-INTEGRAL $=69.2 \mathrm{~kJ} / \mathrm{m}^{\wedge} 2$

$\mathrm{KJ} c=120 \mathrm{MPa} S Q R[\mathrm{~m}]$

K-beta-c $=107.5 \mathrm{MPa} \operatorname{SQR}[\mathrm{m}]$ 
F-125

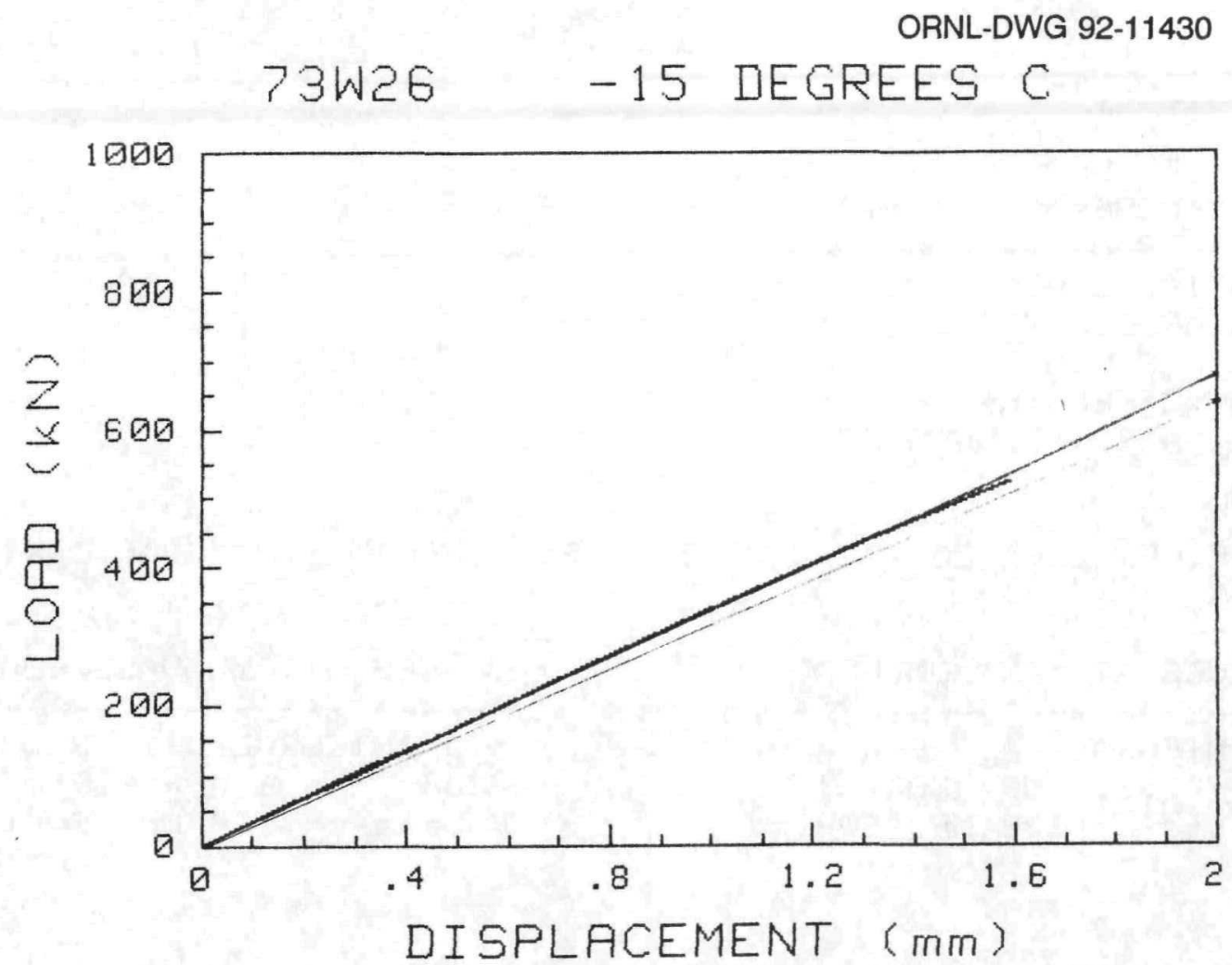

$73 W-26$

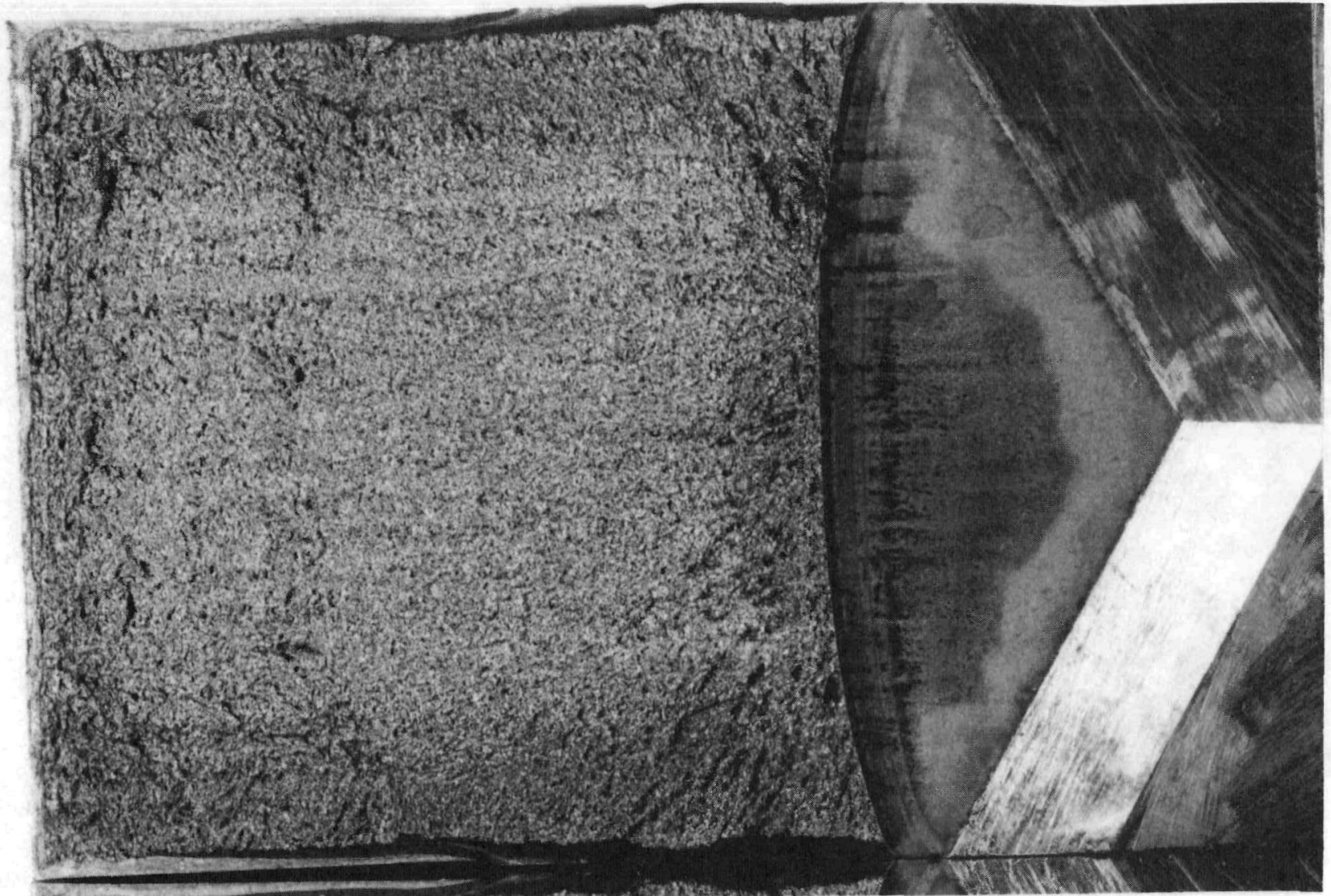


SPECIMEN ID $=73 W 23$

MATERIAL TYPE $=$ HCUWELD

TEST TEMPERATURE $=-15$ DEG C

THICKNESS $=101.7 \mathrm{~mm}$

SIDE GROOUES $=0 \%$

INITIAL CRACK LENGTH $=103.1 \mathrm{~mm}$

MEASURED DUCTILE DELTA- $A=.05 \mathrm{~mm}$

$a /(w) i=.508$

YIELD STRENGTH $=520 \mathrm{MPa}$

FLOW STRENGTH $=581 \mathrm{MPa}$

ESTIMATED YOUNG'S MODULUS $=208$ GPa

SPECIMEN CLEAUED AT KQ $=155.5 \mathrm{MPa}$ SQR $[\mathrm{m}]$ (NOT UALID $K I \mathrm{c}$ )

UALUES AT MAXIMUM LDAD

-

J-INTEGRAL $=117.8 \mathrm{~kJ} / \mathrm{m}^{\wedge} 2$

$\mathrm{KJc}=156.6 \mathrm{MPa} \operatorname{SQR}[\mathrm{m}]$

$K$-bet $a-c=128 \mathrm{MPa} \operatorname{SQR}[\mathrm{m}]$
VALUES at CLEaUage LoAD

-

J-INTEGRAL $=117.8 \mathrm{~kJ} / \mathrm{m}^{\wedge} 2$

$\mathrm{KJc}=156.6 \mathrm{MPa} \operatorname{SQR}[\mathrm{m}]$

$K$-beta-c $=128$ MPa $\operatorname{SQR}[\mathrm{m}]$ 


\section{W23 - 15 DEGREES C}

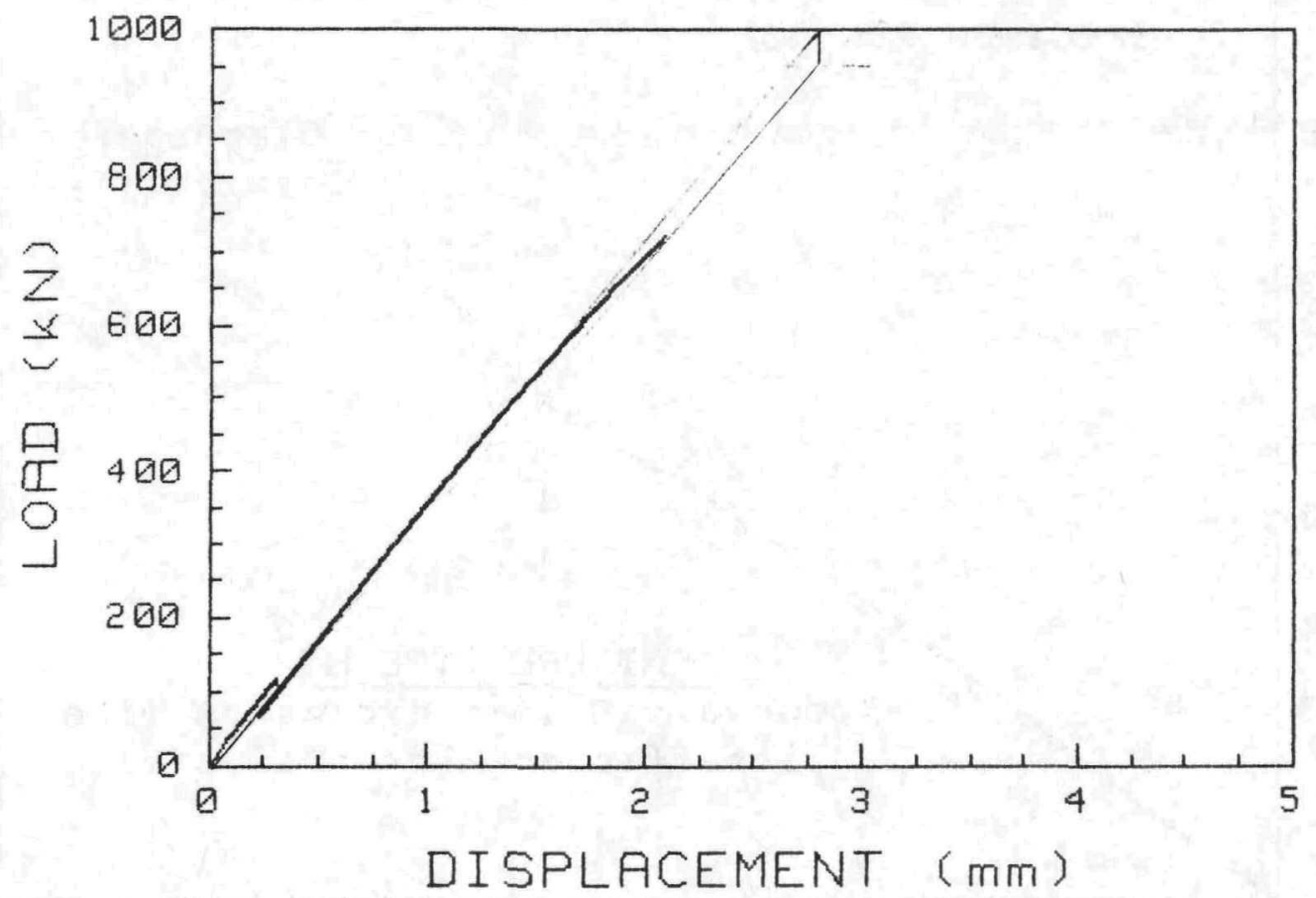

73W-23

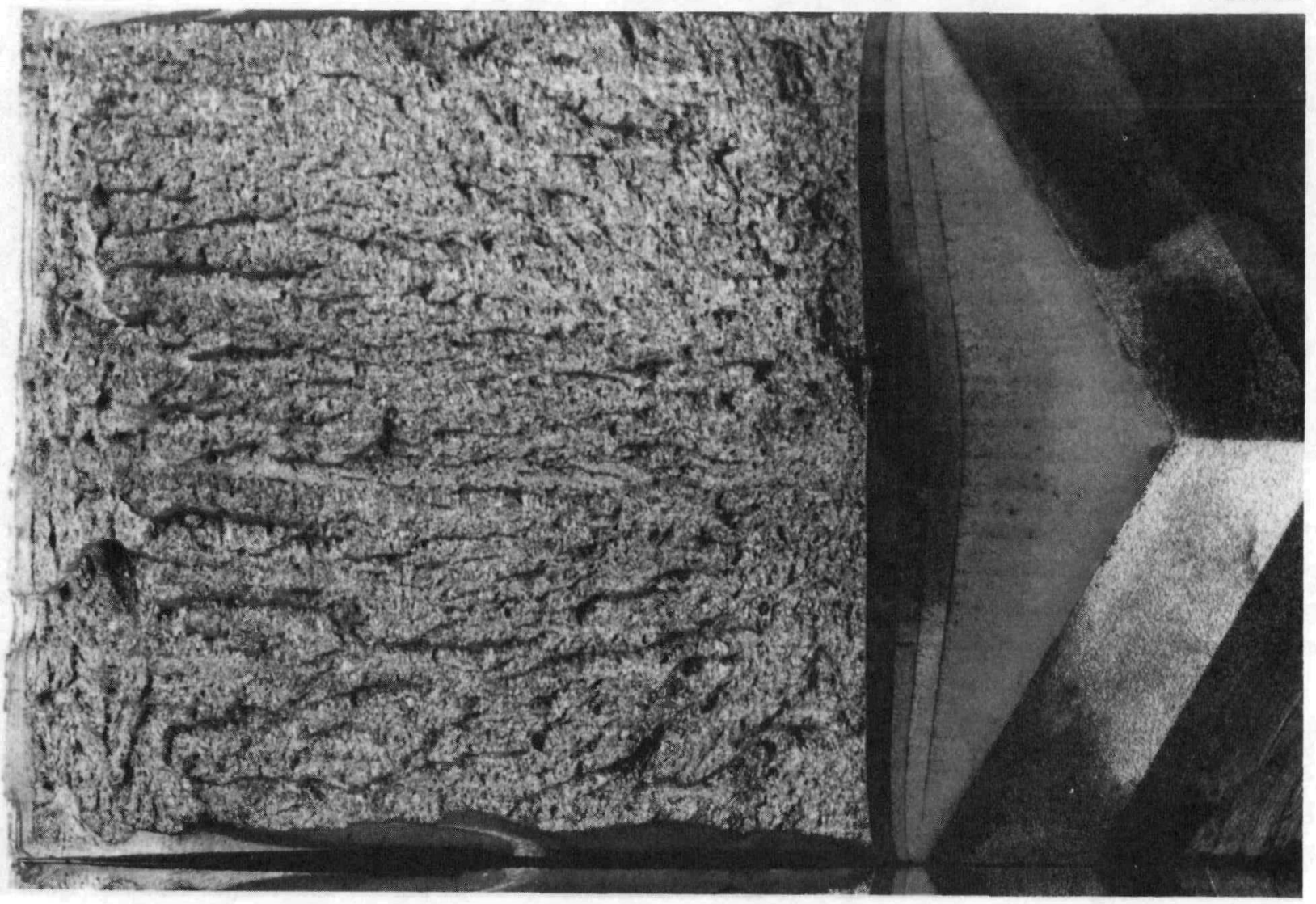




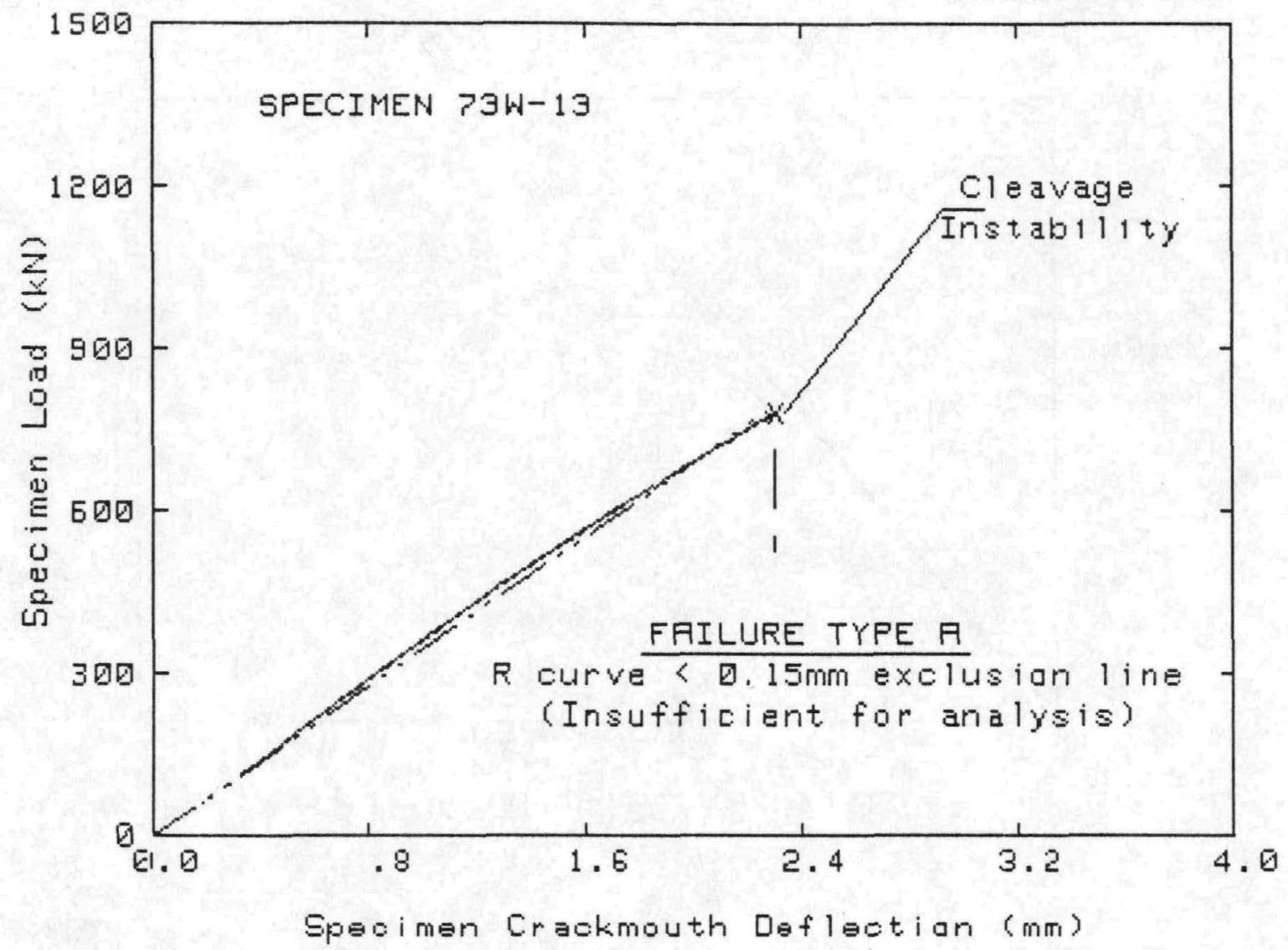

\section{TEST SPECIMEN DATA}

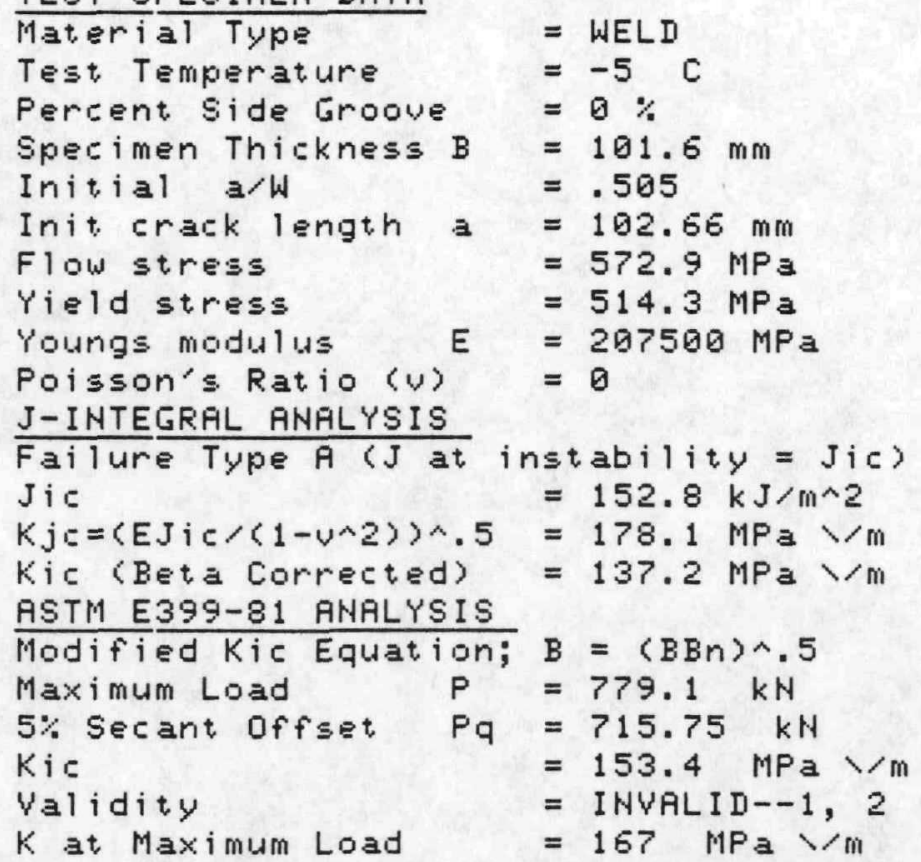

\section{DATA CORRECTIONS USED FOR J-INTEGRAL INTA}

Merk le-COrten DFFSET LOAD $=0$ it OFFSET DEFLECTION $=3.93959963441 \mathrm{E}-03 \mathrm{in}$. Specimen rotation Load intercept elastic slope $=1.62641509434 \mathrm{E}-04$ ib sion

Fitted elastic load range = 75090 To 5000 correction to J for crack exten 


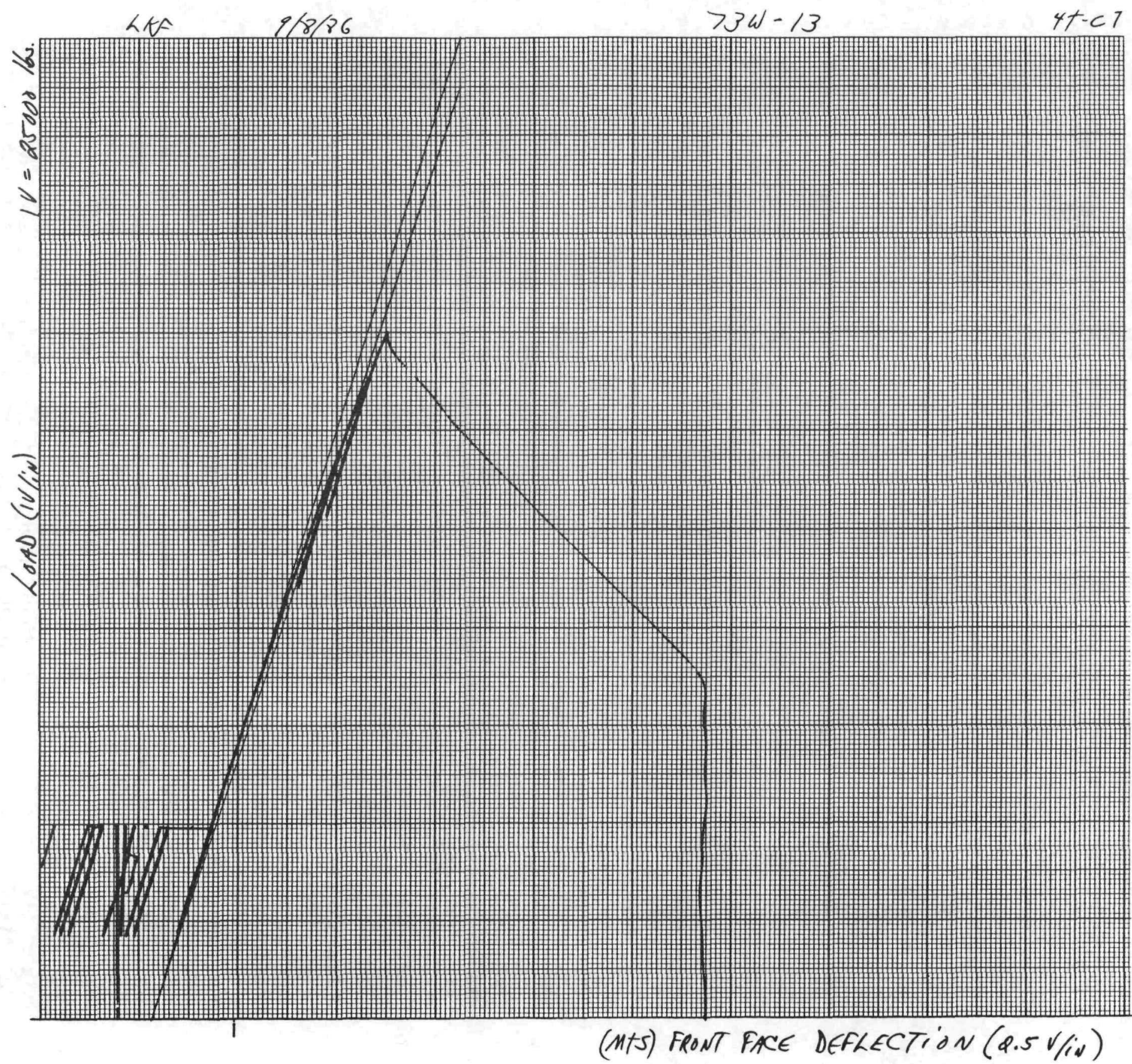


ORNL-DWG 92-11433

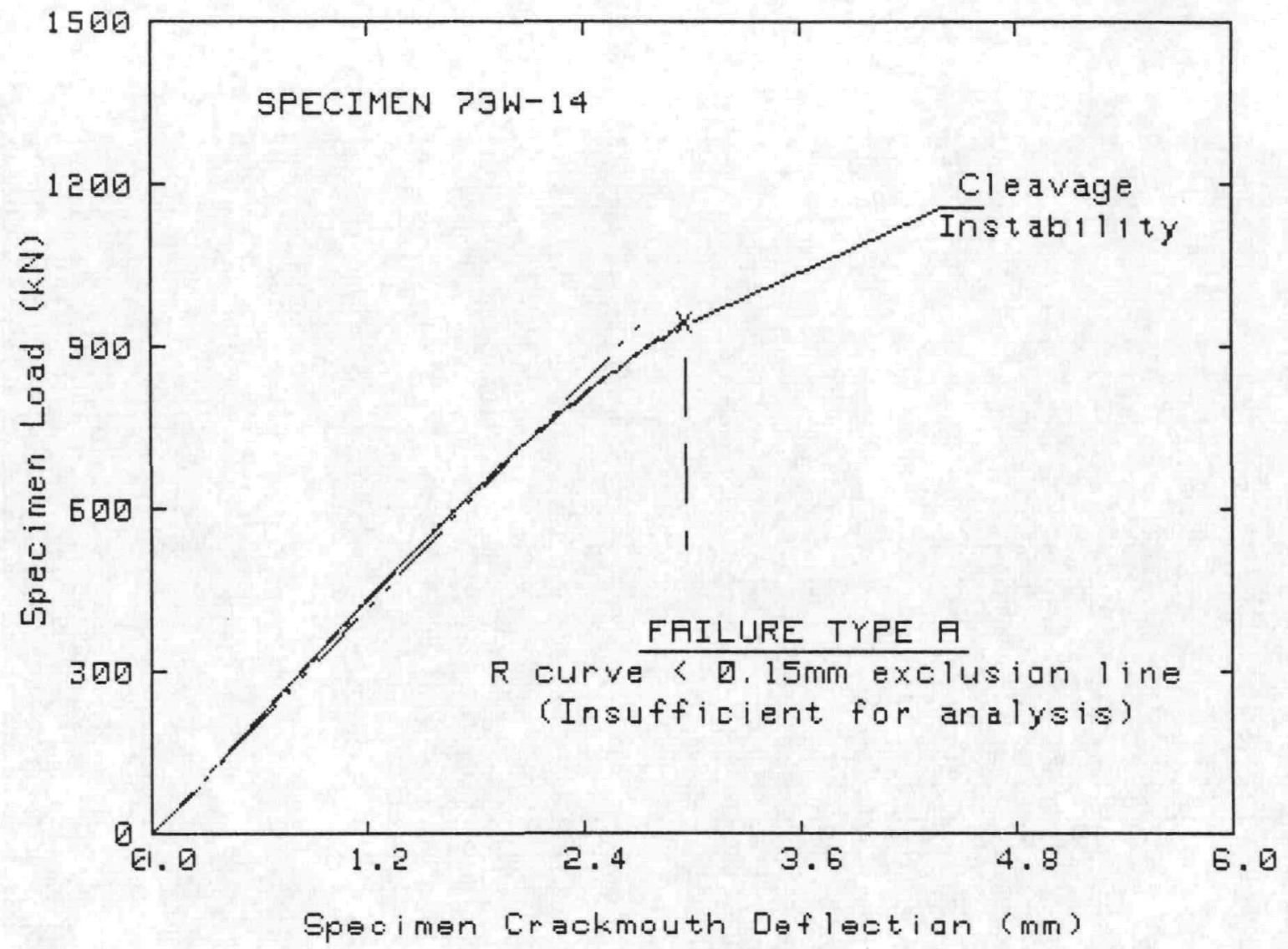

TEST SPECIMEN DATA

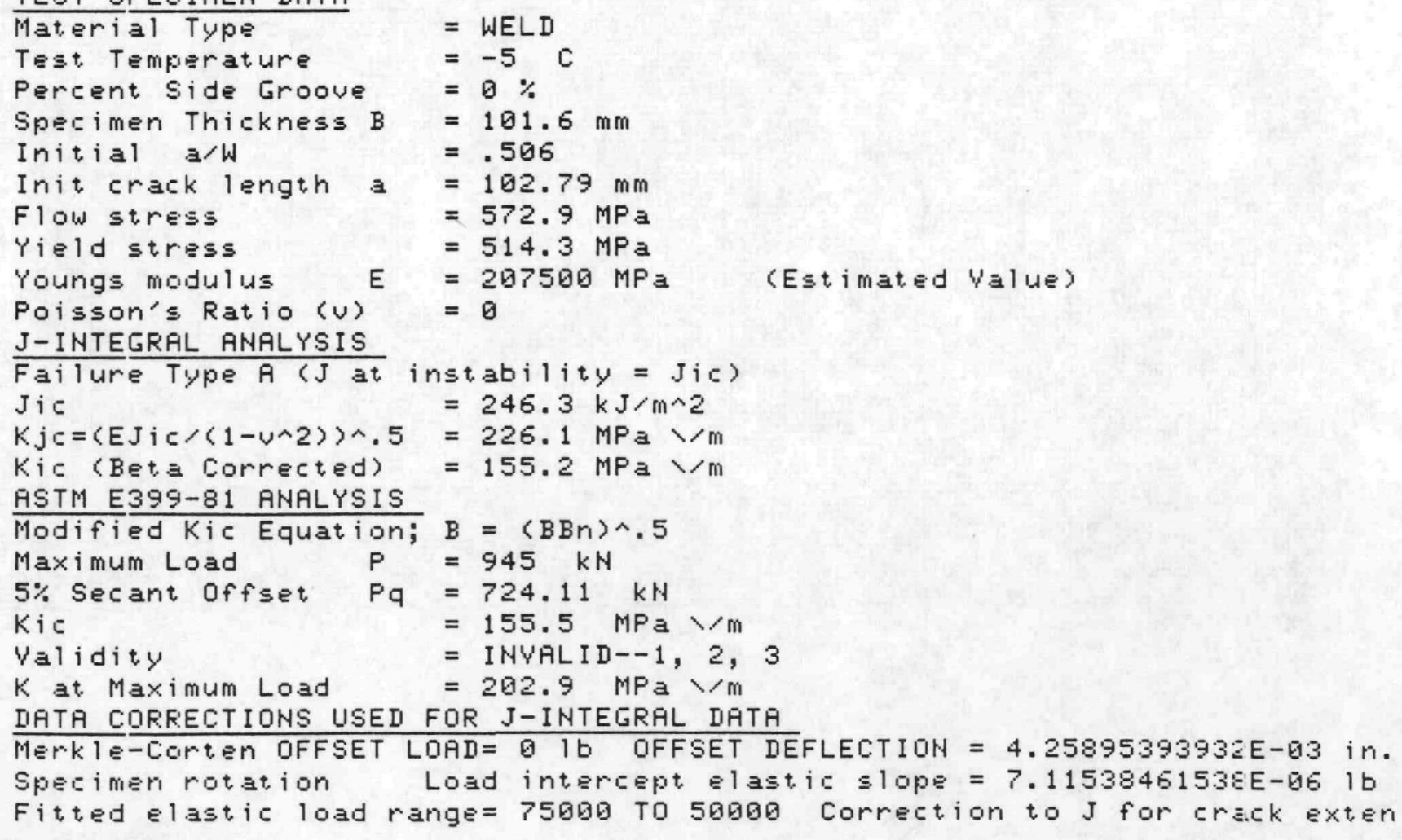


LEF

$9 / 12 / 86$

$73 w-14$

$4 T-<7$

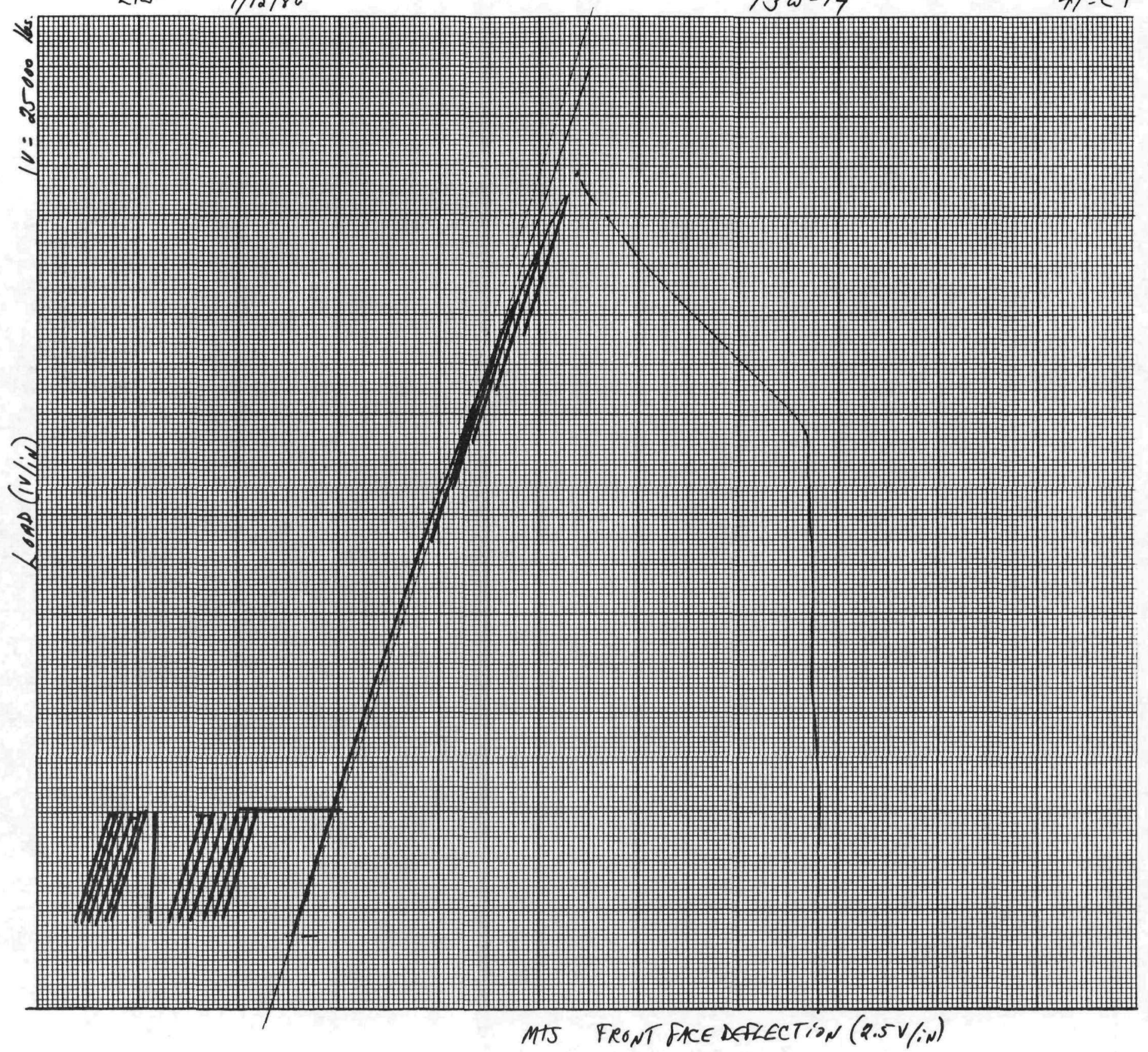




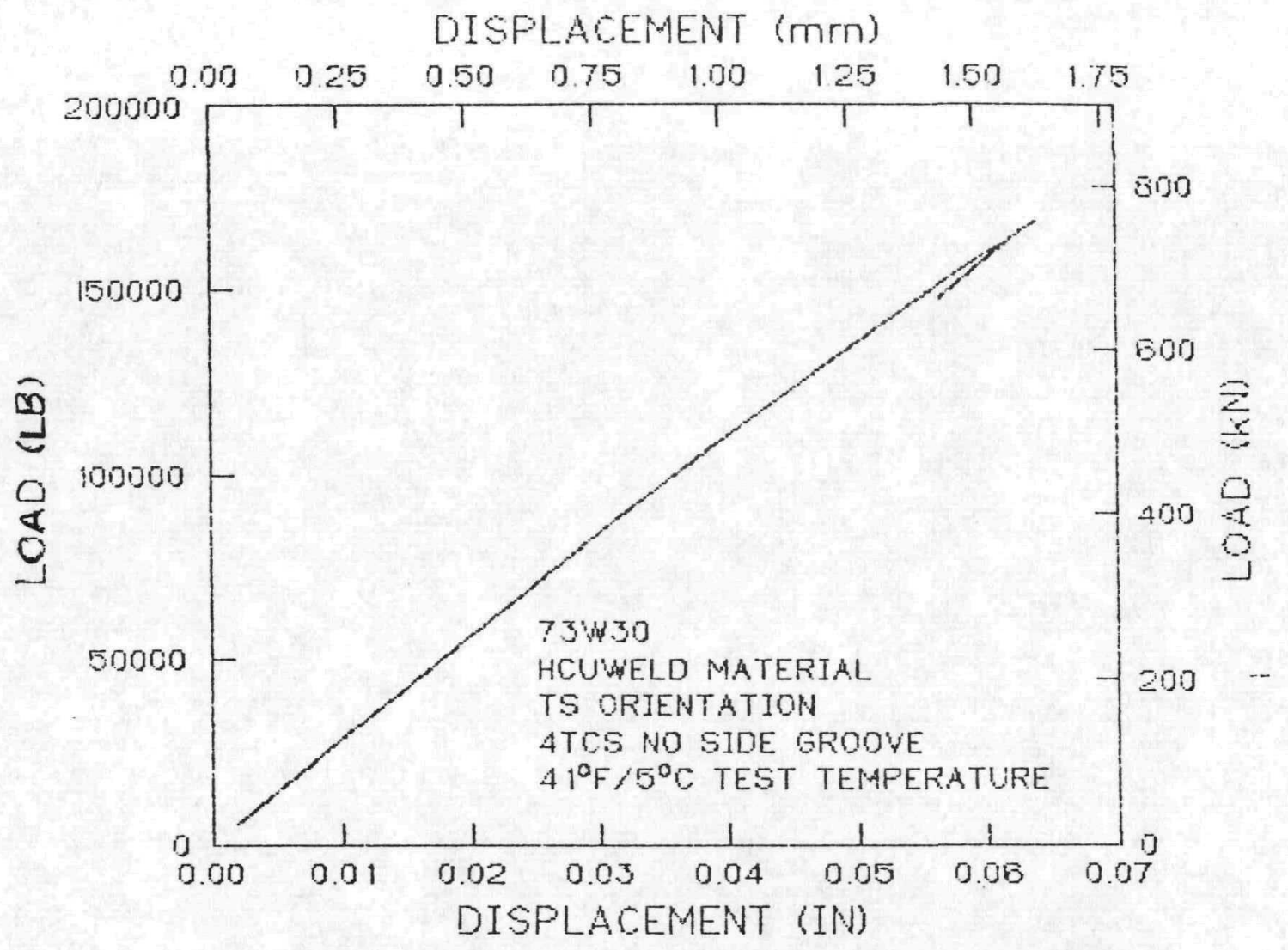

$73 W-30$

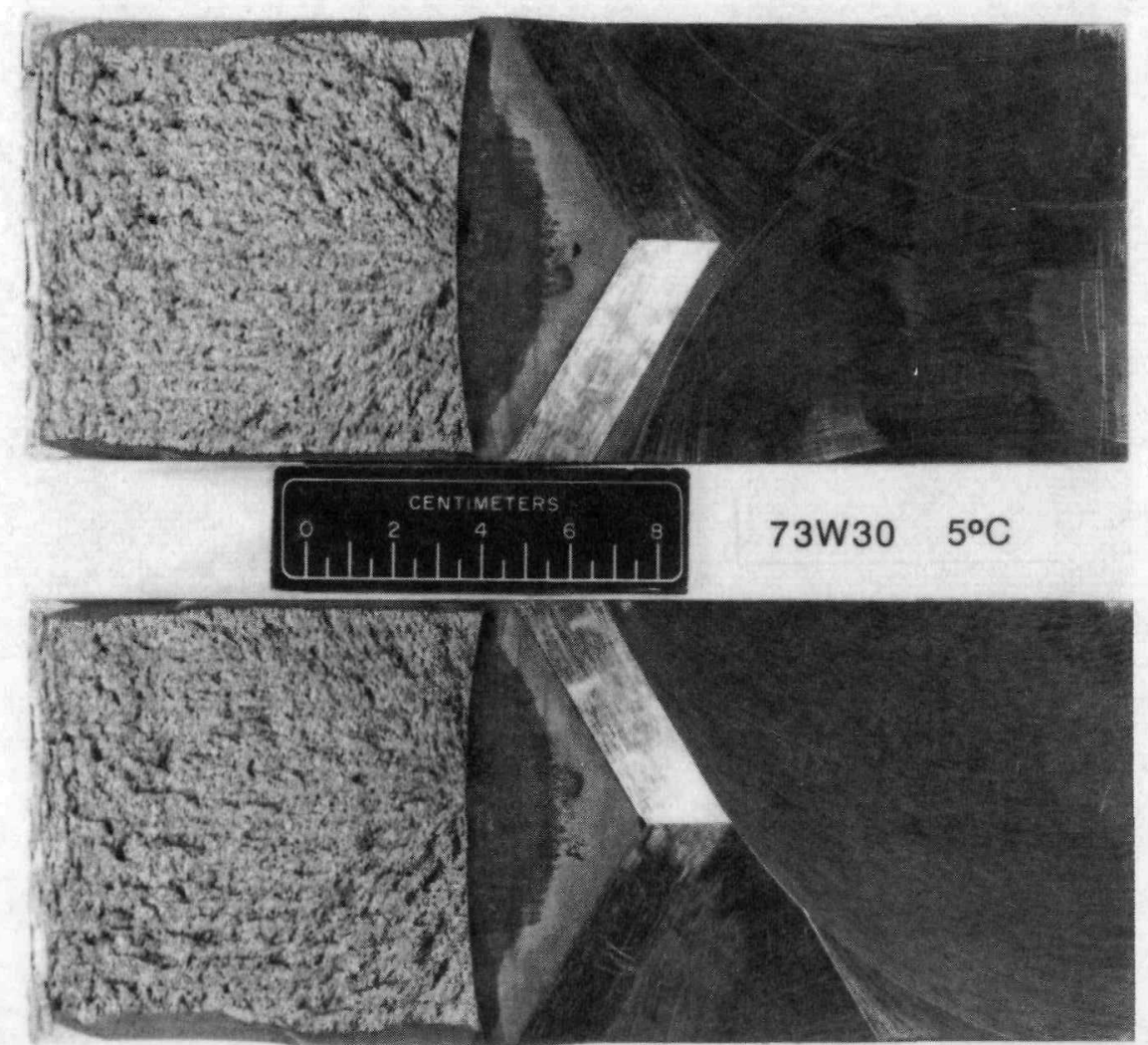


SPECIMEN CLEAVED
147910
657.91
168490
749.46
0.005
0.127
$P Q$ (LB)
$P Q(K N)$
MAX LOAD (LB)
MAX LUAD (KN)
MEASURED FINAL CRACK EXTENSION (IN)
MEASURED FINAL CRACK EXTENSION (MM)
30009 REFERENCE MODULUS (KSI)
206.91 REFERENCE MODULUS (GPA)
134.47 UC KQ (KSI*SQRT (IN))
147.77 UC KO (MPA*SDRT(M))
153.18 UC MAX LOAD K (KSI*SQRT (IN))
168.33 UC MAX LOAD K (MPA*SQRT (M))
$-0.0414$
$-1.05$
\#UC FINAL CRACK EXTENSION (IN)
\#UC FINAL CRACK EXTENSION (MM)
\#UC FINAL CRACK EXTENSION ERROR (PERCENT)

INUALID UC KIC TEST

851.1

149.05

159.81

175.62

122.28

134.37

159.81

175.62

122.28

134.37

831.3

145.57

157.94

173.56

121.49

133.51

157.94

173.56

121.49

133.51

840.1

147.12

158.77

174.48

121.84

133.9

158.77

174.48

121.84

133.9
UC MERKLE-CORTEN

UC MERKLE-CORTEN

UC MERKLE-CORTEN

UC MERKLE-CORTEN

UC MERKLE-CORTEN

UC MERKLE-CORTEN

UC MERKLE-CORTEN

UC MERKLE-CORTEN

UC MERKLE-CORTEN

UC MERKLE-CORTEN
MAX LOAD J-INTEGRAL (IN*LB/IN`2)

MAX LOAD J-JNTEGRAL (KJ/M`2)

MAX LOAD KJ (KSI*SORT (IN))

MAX LOAD KJ (MPA*SQRT (M))

BETA-ADJ MAX LOAD K.J (KSI*SQRT (IN))

BETA-ADJ MAX LOAD KJ (MPA $* S Q R T(M)$ )

FINAL KJ (KSI*SQRT (IN))

FINAL KJ (MPA*SQRT(M))

BETA-ADJ FINAL KJ (KSI*SQRT (IN))

BETA-ADJ FINAL KJ (MPA*SQRT (M))

\section{\# ESTIMATED VALUE FOR FINAL UC CRACK EXTENSION}

UC MERKLE-CORTEN JIC,CEIC,KJIC AND TAVG NOT DEFINED--NOT ENOUGHT DATA FOINTS FOR POWER LAW FIT 


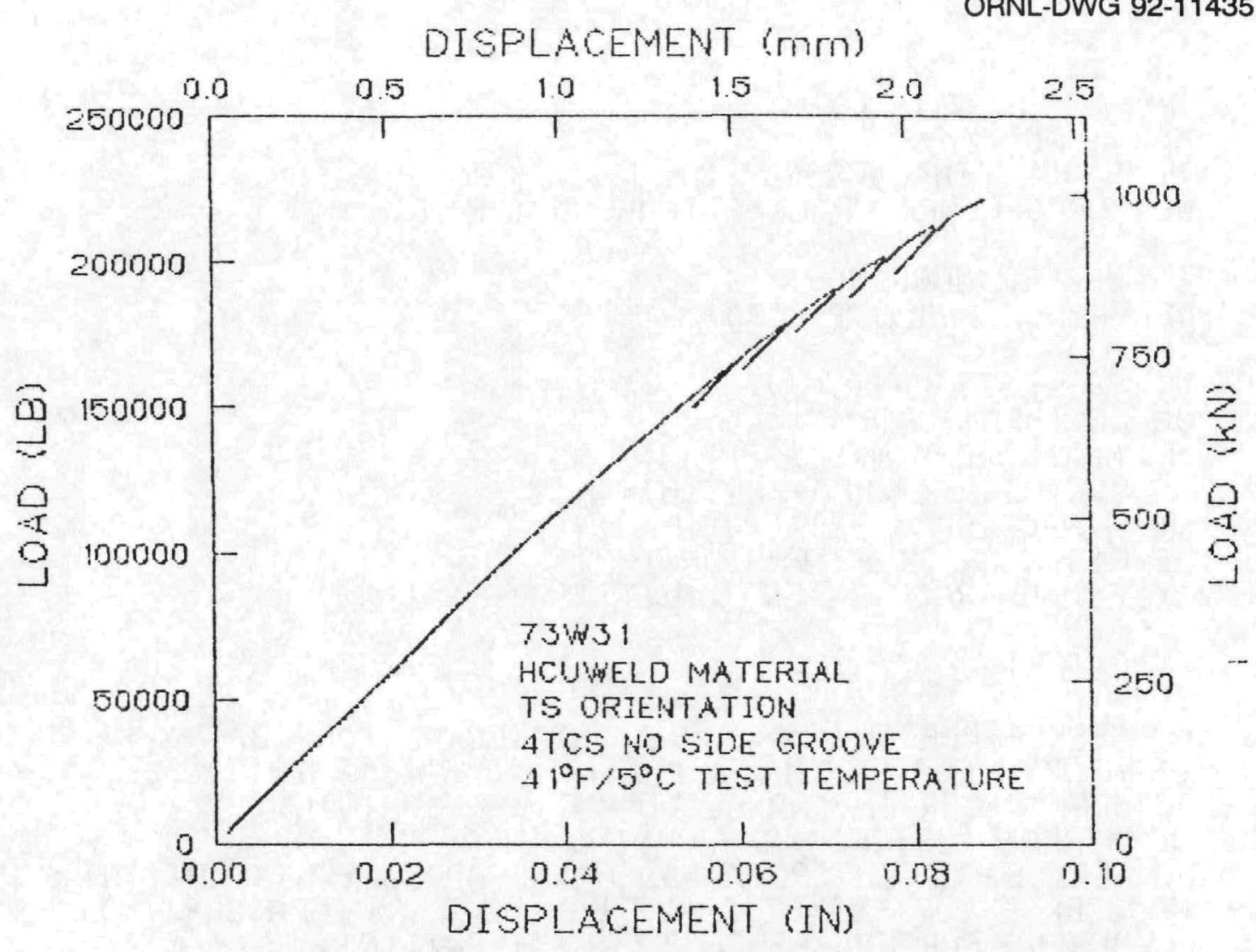

73W-31

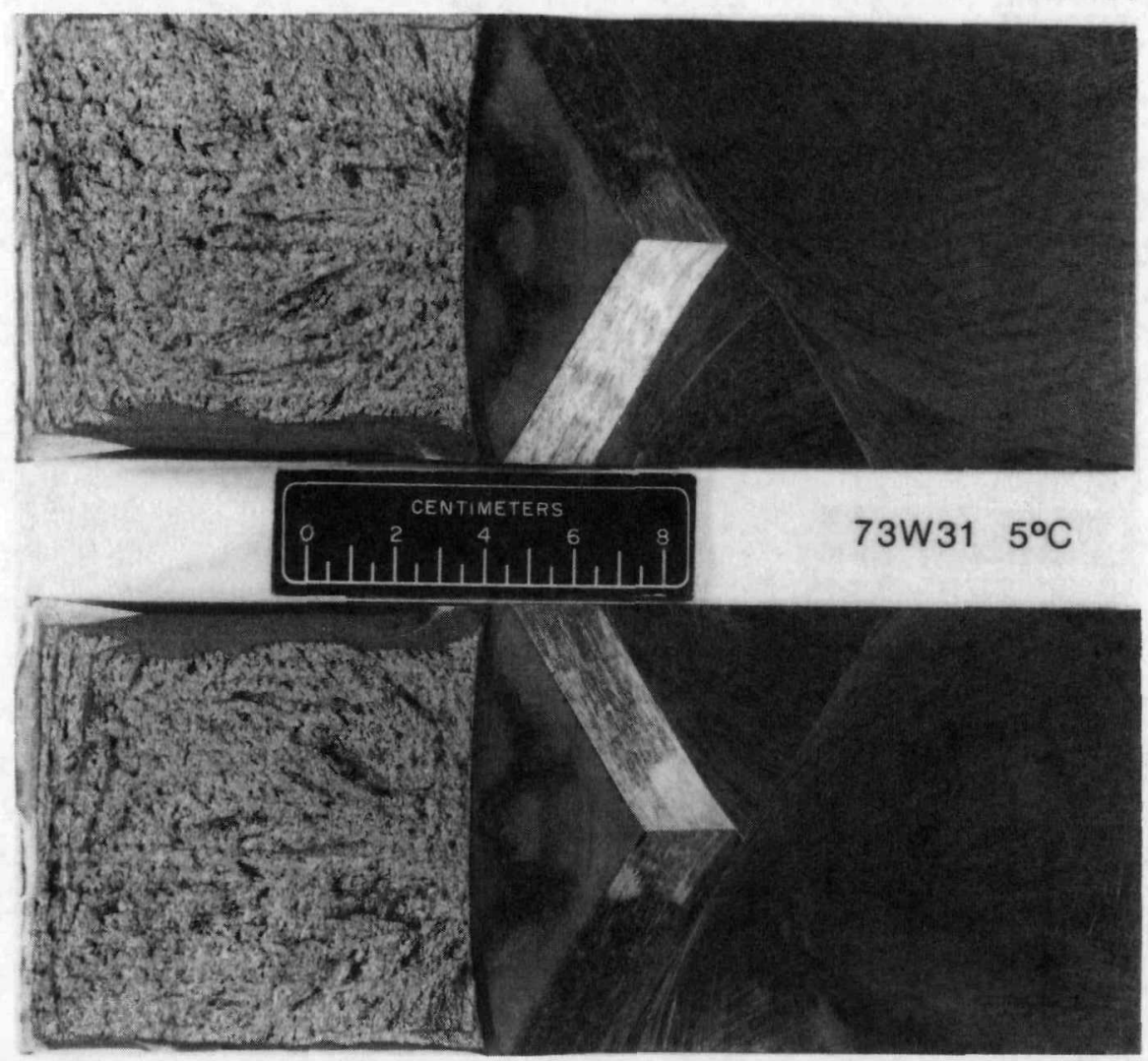


SPECIMEN CLEAVED

$\begin{array}{ll}152800 & P Q(L B) \\ 679.53 & \text { PQ (KN) } \\ 221260 & \text { MAX LOAD (LB) } \\ 984.19 & \text { MAX LOAD (KN) } \\ 0.05 & \text { MEASURED FINAL CRACK EXTENSION (IN) } \\ 1.27 & \text { MEASURED FINAL CRACK EXTENSION (MM) } \\ 30009 & \text { REFERENCE MODULUS (KSI) } \\ 206.91 & \text { REFERENCE MODULUS (GPA) } \\ 135.63 & \text { UC KQ (KSI*SQRT(IN)) } \\ 149.05 & \text { UC KQ (MPA*SORT(M)) } \\ 196.41 & \text { UC MAX LOAD K (KSI*SQRT(IN)) } \\ 215.84 & \text { UC MAX LOAD K (MPA*SQRT(M)) } \\ -0.0472 & \text { \#UC FINAL CRACK EXTENSION (IN) } \\ -1.199 & \text { \#UC FINAL CRACK EXTENSION (MM) } \\ -194.4 & \text { \#UC FINAL CRACK EXTENSION ERROR (PERCENT) }\end{array}$

INUALID UC KIC TEST

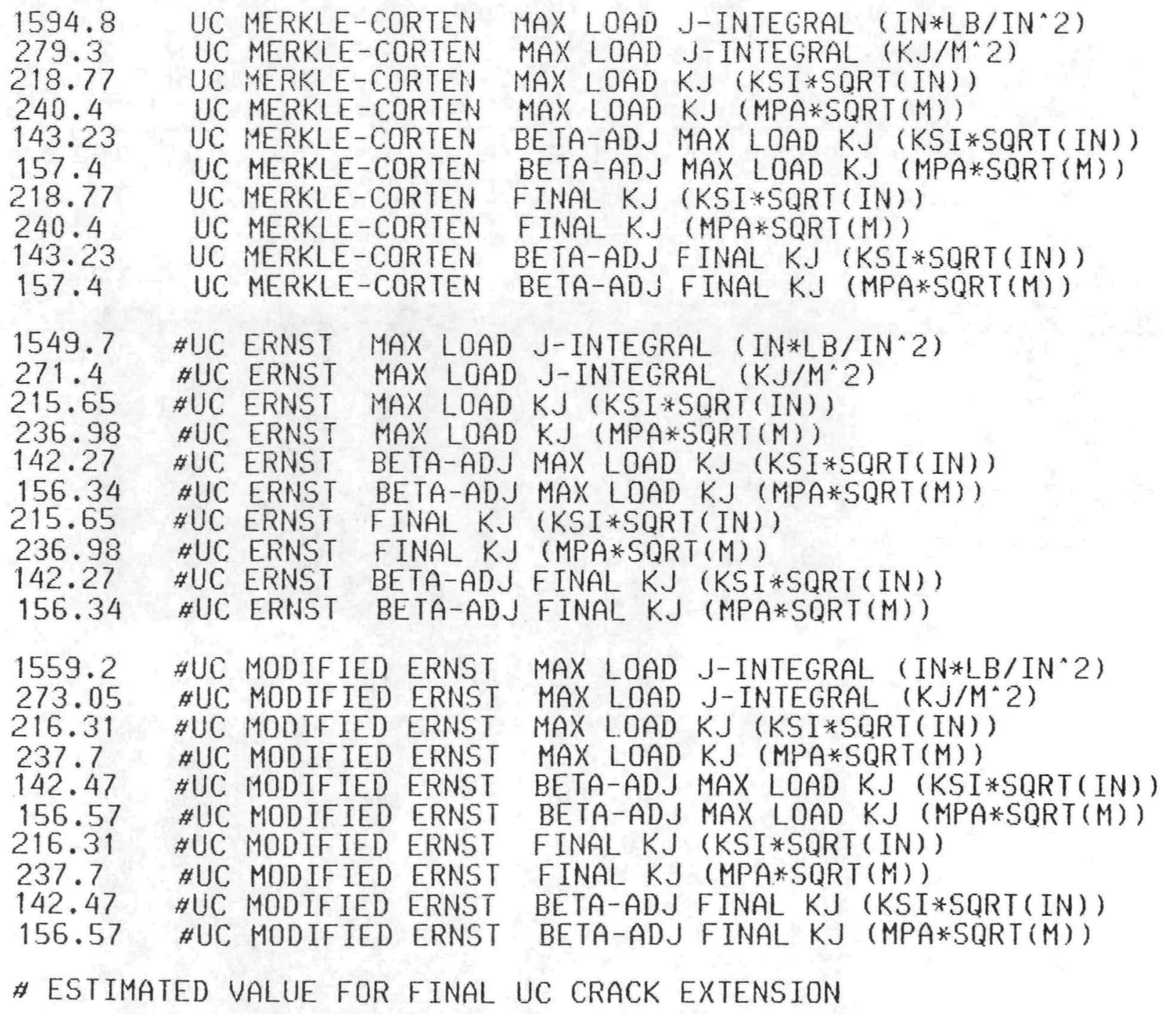

GET POWER LAW FIT TO UC MERKLE-CORTEN TEST RESULTS IN NO 
F-136

ORNL-DWG 92-11436

DISPLACEMENT $(\mathrm{mm})$

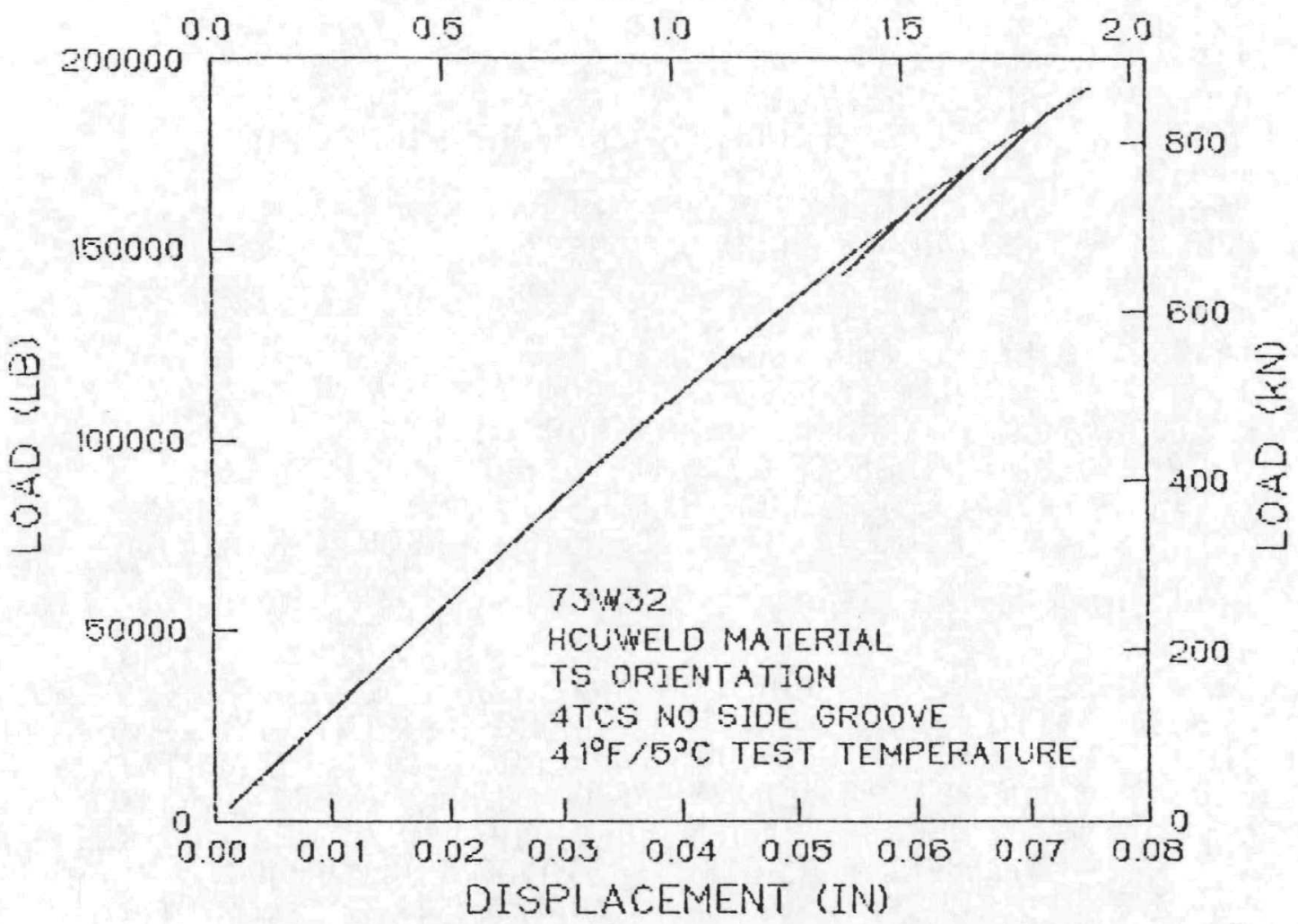

$73 W-32$

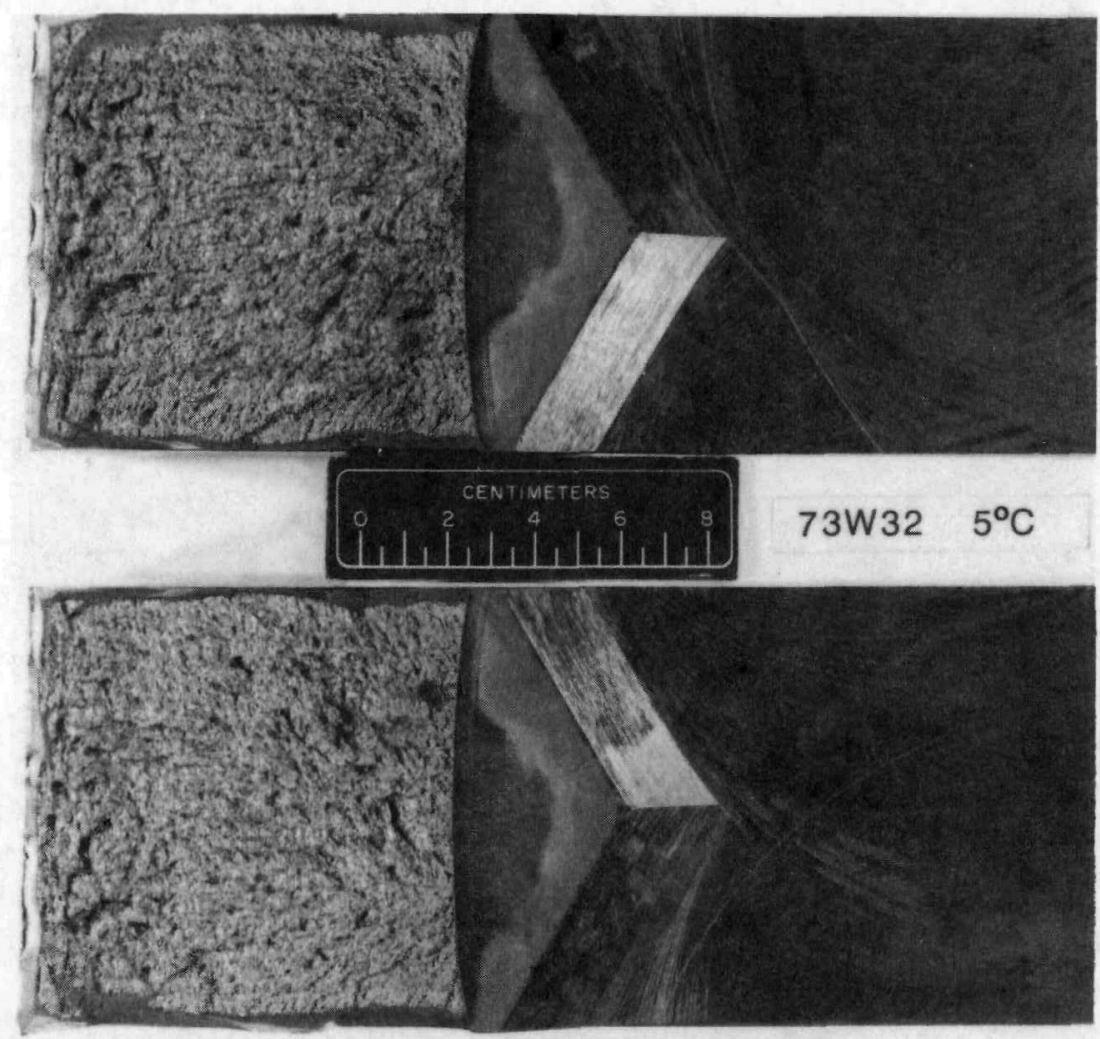




\section{SPECIMEN CLEAVED}
144290
$P Q \quad(L B)$
$64 ! .78$
$P Q(K N)$
192640
856.86
0.005
0.127
MAX LOAD (LB)
MAX LOAD (KN)
MEASURED FINAL CRACK EXTENSION (IN)
MEASURED FINAL CRACK EXTENSION (MM)

\section{9 \\ 206.91 \\ REFERENCE MODLLLUS (KSI) \\ REFERENCE MODULUS (GPA)}
130.65
143.57
UC KQ (KSI*SQRT (IN))
174.44
191.69
$-0.0439$
$-1.116$
UC KQ (MPA*SQRT (M))
UC MAX LOAD K (KSI*SQRT (IN))
UC MAX LOAD K (MPA*SORT $(M))$
\#UC FINAL CRACK EXTENSION (IN)
\#UC FINAL CRACK EXTENSION (MM)
$-978.5$
\#UC FINAL CRACK EXTENSION ERROR (PERCENT)

\section{INVALID UC KIC TEST}

1169

204.72

187.3

205.82

132.87

146.01

187.3

205.82

132.87

146.01

1137

199.12

184.71

202.98

131.94

144.99

184.71

202.98

131.94

144.99

1144.1

200.35

185.29

203.61

132.15

145.22

185.29

203.61

132.15

145.22
UC MERKLE-CORTEN

UC MERKLE-CORTEN

UC MERKLE-CORTEN

UC MERKLE-CORTEN

UC MERKLE-CORTEN

UC MERKLE-CORTEN

UC MERKLE-CORTEN

UC MERKLE-CORTEN

UC MERKLE-CORTEN

UC MERKLE-CORTEN
MAX LOAD J-INTEGRAL (IN*LB/IN“2)

MAX LOAD J-INTEGRAL (KJ/M*2)

MAX LOAD KJ (KSI*SQRT (IN))

MAX LOAD K.J (MPA $* S Q R T(M))$

BETA-ADJ MAX LOAD KJ (KSI*SQRT (IN))

BETA-ADJ MAX LOAD KJ (MPA*SQRT(M))

FINAL KJ (KSI*SQRT (IN))

FINAL KJ (MPA*SQRT $(M)$ )

BETA-ADJ FINAL KJ (KST*SQRT (IN))

BETA-ADJ FINAL KJ (MPA KSQRT (M))
\#UC ERNST
MAX LOAD J-INTEGRAL (IN*LB/IN*2)
\#UC ERNST
\#UC ERNST
MAX LOAD J-INTEGRAL (KJ/M^2)
\#UC ERNST
MAX LOAD KJ (KSI*SQRT ( IN))
\#UC ERNST
MAX LOAD KJ (MPA*SQRT (M))
\#UC ERNST
BETA-ADJ MAX LOAD KJ (KSI*SQRT(IN))
\#UC ERNST
BETA-ADJ MAX LOAD KJ (MPA KQRT (M))
\#UC ERNST
FINAL KJ (KSI*SQRT ( IN))
$\#$ AC ERNST
FINAL KJ (MPA*SQRT (M))
\#UC ERNST
BETA-ADJ FINAL KJ (KSI*SQRT(IN))
BETA-ADJ FINAL K.J (MPA*SQRT $(M)$ )
\#UC MODIFIED ERNST
MAX LOAD J-INTEGRAL (IN*LB/IN`2)
\#UC MODIFIED ERINST
$\# U C$ MODIFIED ERNST
\#UC MODIFIED ERNST
\#UC MODIFIED ERNST
MAX LOAD J-INTEGRAL (KJ/M^2)
MAX LOAD KJ (KSI*SQRT (IN))
MAX LOAD KJ (MPA*SQRT $(M)$ )
\#UC HODIFIED ERNST
\#UC MODIFIED ERNST
BETA-ADJ MAX LOAD KJ (KSI*SQRT (IN))
$\#$ \#C MODIFIED ERNST
BETA-ADJ MAX LOAD KJ (MPA*SQRT(M))
\#UC MODIFIED ERNST
FINAL KJ (KSI*SQRT ( IN))
FINAL KJ (MPA*SQRT $(M))$
\#UC MODIFIED ERNST
BETA-ADJ FINAL KJ (KSI*SQRT(IN))
BETA-ADJ FINAL KJ (MPA*SQRT (M))

* ESTIMATED VALUE FOR FINAL UC CRACK EXTENSION

GET POWER LAW FIT TO UC MERKLE-CORTEN TEST RESULTS NO NO 


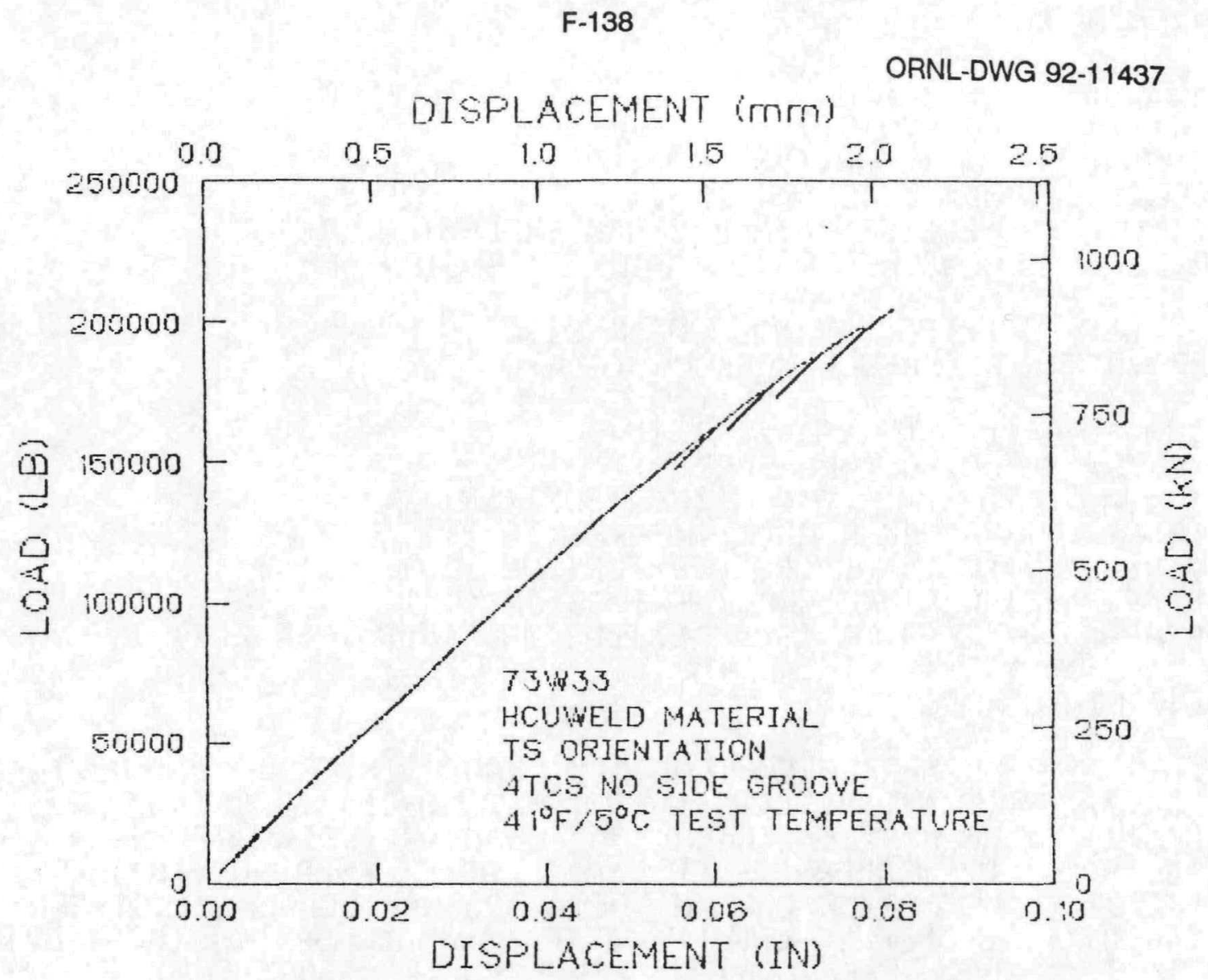

73W-33

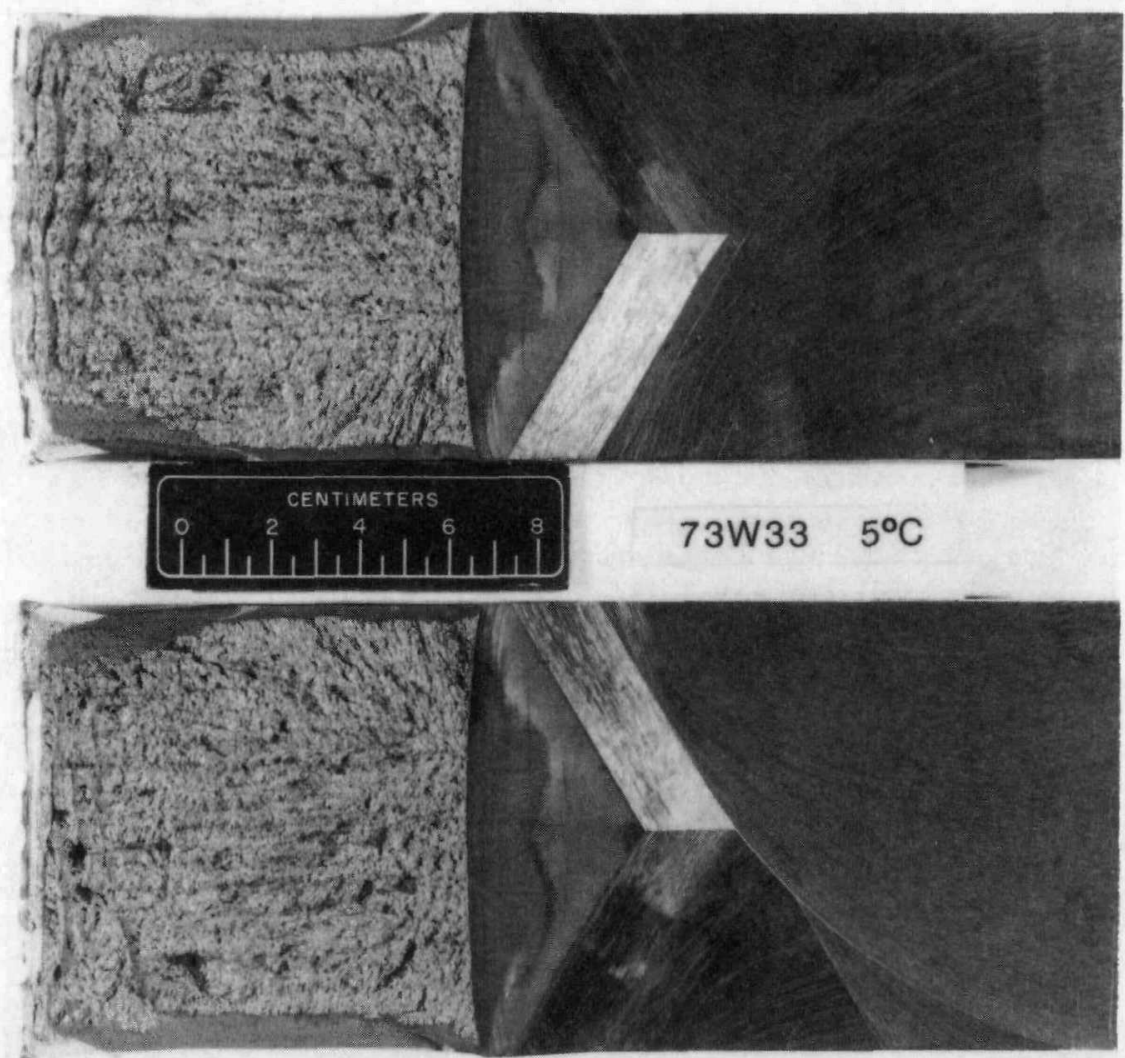




\author{
$148940 \quad P Q(L B)$ \\ $662.49 \quad P Q(K N)$ \\ 204320 MAX LOAD (LB) \\ 908.79 MAX LOAD (KN) \\ 0.005 MEASURED FINAL CRACK EXTENSION (IN) \\ 0.127 MEASURED FINAL CRACK EXTENSION (MM) \\ $\begin{array}{ll}30009 & \text { REFERENCE MODULUS (KSI) } \\ 206.91 & \text { REFERENCE MODULUS (GPA) }\end{array}$ \\ 133.79 UC KO (KSI $\$ S Q R T(I N))$ \\ 147.03 UC KO (MPA*SQRT(M)) \\ 183.54 UC MAX LOAD K (KSI*SQRT(IN)) \\ 201.69 UC MAX LOAD K (MPA*SQRT (M)) \\ $-0.0407 \quad \#$ UC FINAL CRACK EXTENSION (IN) \\ -1.035 \#UC FINAL CRACK EXTENSION (MM) \\ -914.8 \#UC FINAL CRACK EXTENSION ERROR (PERCENT)
}

INUALID UC KIC TEST

1345.7

235.67

200.96

220.83

137.56

151.17

200.96

220.83

137.56

151.17

1305.4

228.61

197.92

217.5

136.55

150.05

197.92

217.5

136.55

150.05

1314.5

230.2

198.61

218.25

136.78

150.31

198.61

218.25

136.78

150.31
UC MERKLE-CORTEN

UC MERKLE-CORTEN

UC MERKLE-CORTEN

UC MERKLE-CORTEN

UC MERKLE-CORTEN

UC MERKLE-CORTEN

UC MERKLE-CORTEN

UC MERKLE-CORTEN

UC MERKLE-CORTEN

UC MERKLE-CORTEN
MAX LOAD J-INTEGRAL (IN*LB/IN`2)

MAX LDAD J-INTEGRAL (K.J/M`2)

MAX LOAD KJ (KSI*SORT(IN))

MAX LOAD KJ (MPA*SORT (M))

BETA-ADJ MAX LOAD KJ (KSI*SQRT (IN))

BETA-ADJ MAX LOAD KJJ (MPA*SDRT(M))

FINAL K.J (KSI*SORT (IN))

FINAL KJ (MPA*SQRT(M))

BETA-ADJ FINAL KJ (KSI*SQRT (IN))

BETA-ADJ FINAL KJ (MPA*SQRT (M))
\#UC ERNST
AUC ERINST
\#UC ERNST
피C ERNST
\#UC ERNST
MAX LOAD J-INTEGRAL (IN*LB/IN`2)
MAX LOAD J-INTEGRAL (KJ/M^2)
MAX LOAD KJ (KSI*SQRT (IN))
MAX LOAD KJ (MPA*SQRT (M))
\#UC ERNST
BETA-ADJ MAX LOAD KJ (KSI*SQRT (IN))
\#UC ERNST
BETA-ADJ MAX LOAD KJ (MPA*SQRT(M))
\#UC ERNST
FINAL KJ (KSI*SORT (IN))
\#UC ERNST
FINAL KJ (MPA KSQRT (M))
\#UC ERNST
BETA-ADJ FINAL KJ (KSI*SQRT (IN))
BETA-ADJ FINAL KJ (MPA $* S Q R T(M))$
\#UC MODIFIED ERNST
\#UC MODIFIED ERNST
\#UC MODIFIED ERNST
$\#$ AC MODIFIED ERNST
\#UC MODIFIED ERNST
\#UC MODIFIED ERNST
\#UC MODIFIED ERNST
\#UC MODIFIED ERNST
AUC MODIFIED ERNST
\#UC MODIFIED ERNST
MAX LOAD J-INTEGRAL (IN*LB/IN`2)
MAX LOAD J-INTEGRAL (KJ/M`2)
MAX LOAD KJ (KSI*SQRT (IN))
MAX LOAD KJ (MPA*SQRT (M))
BETA-ADJ MAX LDAD KJ (KSI*SQRT(IN))
BETA-ADJ MAX LOAD KJ (MPA*SQRT (M))
FINAL KJ (KSI*SQRT(IN))
FINAL KJ (MPA*SQRT(M))
BETA-ADJ FINAL KJ (KSI*SQRT (IN))
BETA-ADJ FINAL KJ (MPA*SQRT (M))

\# ESTIMATED VALUE FOR FINAL UC CRACK EXTENSION

GET POWER LAW FIT TO UC MERKLE-CORTEN TEST RESULTS A NO 
Specimen Crackmauth Deflectian (in.)

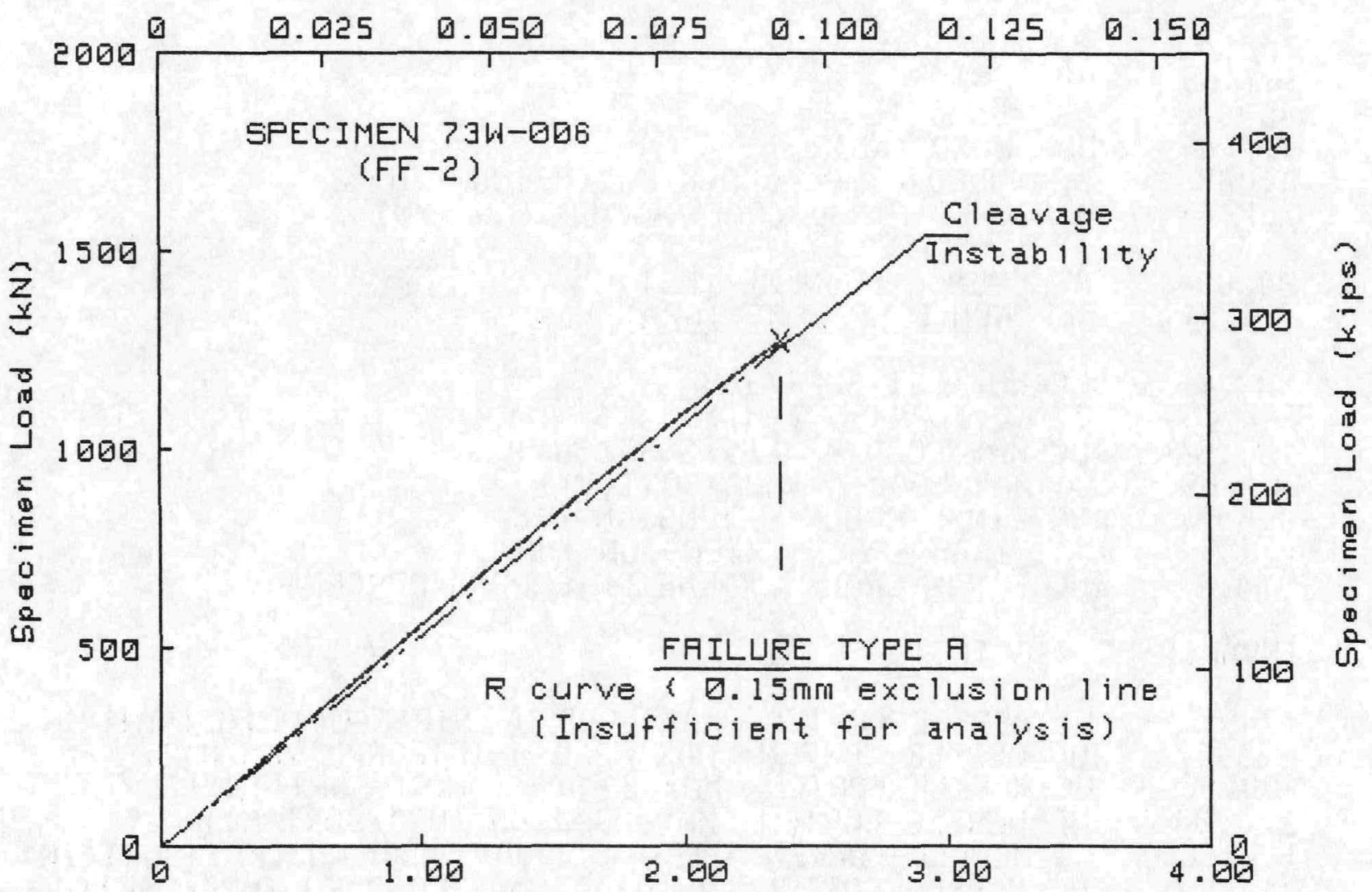

Specimen Crackmouth Deflection ( $m m$ )

TEST SPECIMEN DATA

Material Type
Test Temperature
Percent Side Grooue
Specimen Thickness B
Initial a/W
Init crack length a
Flow strength
Yield strength
Youngs modulus E
Poisson's Ratio ( $U$ )
J-INTEGRAL ANALYSIS

$\mathrm{Jc}$

$K j c=\left\langle E J c /\left(1-w^{2}\right\rangle\right) 1 / 2$

Ke (Beta Corrected)

ASTM E399-81 ANALYSIS

Modified Kic Equation;

Maximum Load

$5 \%$ Secant offset

$\mathrm{Kq}$

Validity

$K$ at Maximum Load

$$
\begin{aligned}
\mathrm{P} & =1276.09 \mathrm{kN} \\
\mathrm{Pq} & =1276.09 \mathrm{kN} \\
& =151.4 \mathrm{MPa} \sqrt{\mathrm{m}} \\
& =I \mathrm{NYRLI} \mathrm{D}--1,2 \\
& =151.4 \mathrm{MPa}{ }^{2}
\end{aligned}
$$

DATA CORRECTIONS USED FOR J-INTEGRAL DATA

Merkle-Corten correction

Correction to J for crack extension (Deformation-J)

OFFSET LOAD $=0 \mathrm{kN}$ DFFSET DEFLECTION $=.006 \mathrm{~mm}$

Fitted elastic load range $=250 \mathrm{kN}$ TO $500 \mathrm{kN}$

Elastic slope $=5.595 E+02 \mathrm{kN} / \mathrm{mm}$ 


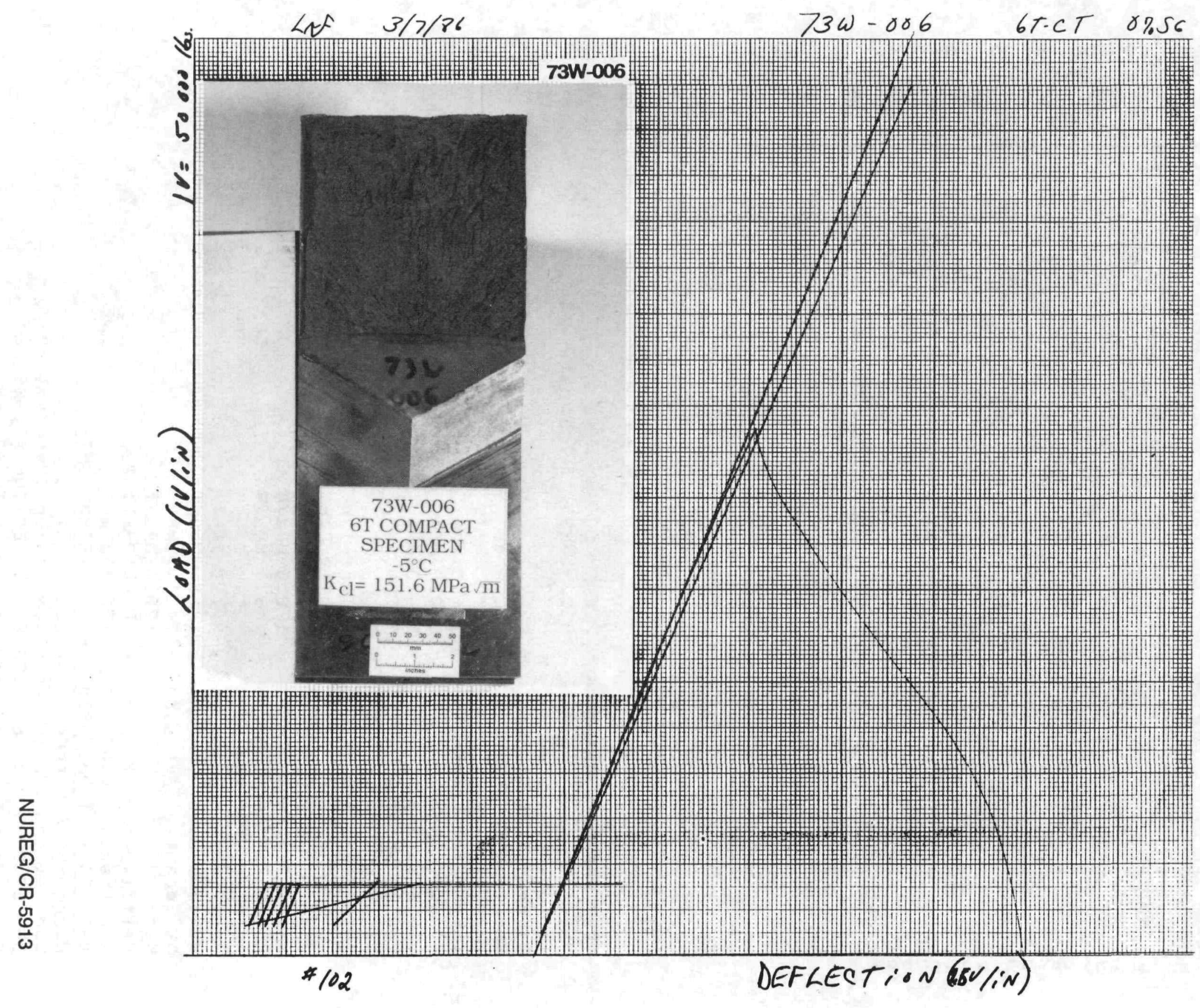

$\stackrel{T}{\vec{E}}$ 
Specimen Crackmouth Deflection (in.)

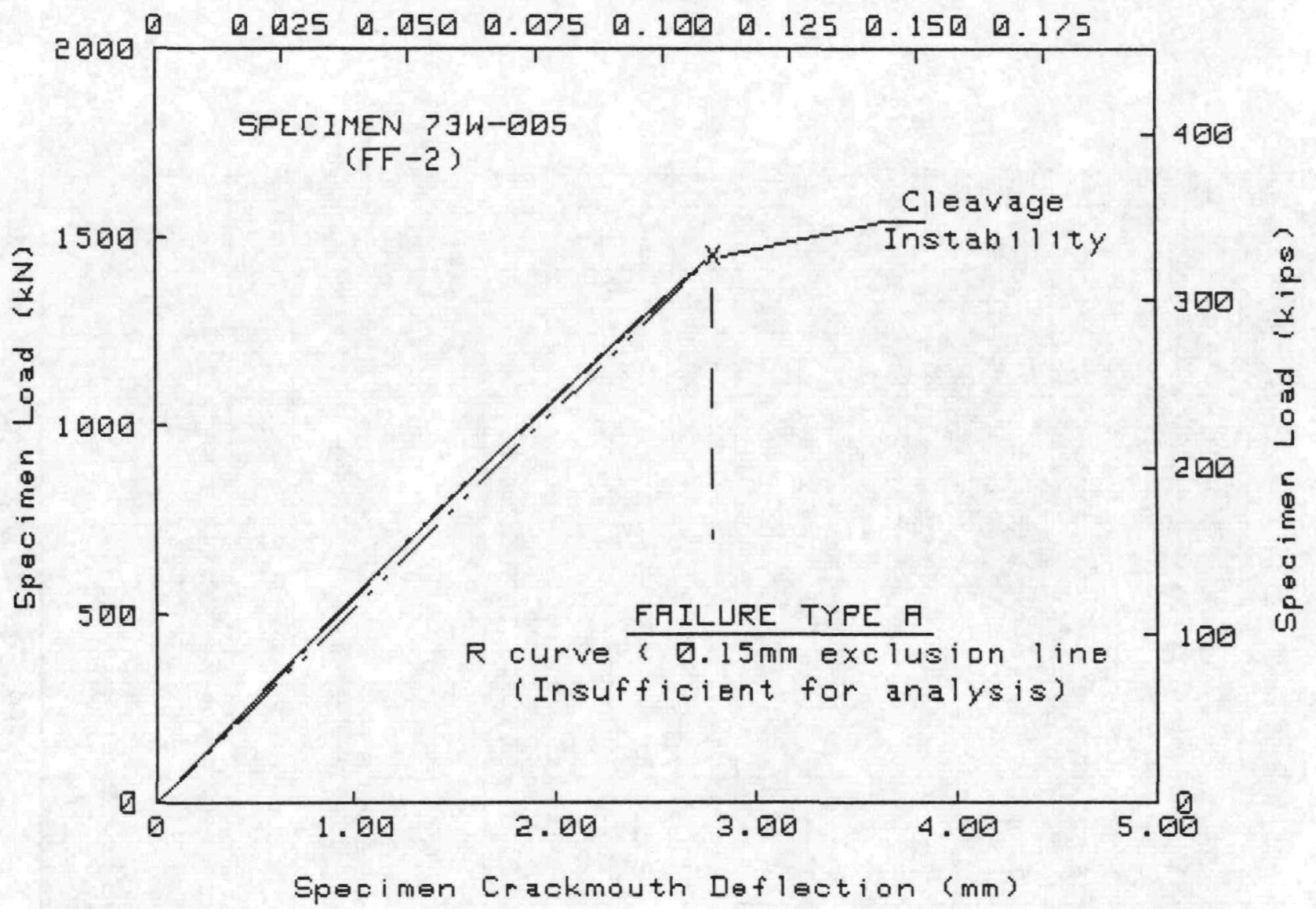

TEST SPECIMEN DATA

Material Type
Test Temperature
Percent Side Groove
Specimen Thickness B
Initial a/w
Init crack length a
Flow strength
Yield strength
Youngs modulus
Poisson's Ratio ( $E$ )
J-INTEGRAL ANALYSIS

= A533-B HSST 5th Irrad. Weld

$=-5^{\circ} \mathrm{C}$

$=0 \%$

$=152.4 \mathrm{~mm}$

$=.512$

$=156.01 \mathrm{~mm}$

$=572.9 \mathrm{MPa}$

$=514.3 \mathrm{MPa}$

$=207500 \mathrm{MPa}$

$=0$

J-INTEGRAL ANALYSIS

[ Failure Type $A$ ( $J$ at instability = Jc)]

$\mathrm{Jc}$

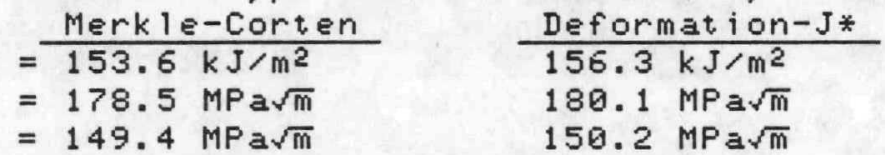

$K j c=\left\langle E J c /\left(1-v^{2}\right\rangle\right) 1 / 2$

Ke (Beta Corrected)

$=149.4 \mathrm{MPa} \sqrt{\mathrm{m}}$

(Est imated Value)

ASTM E399-81 ANALYSIS

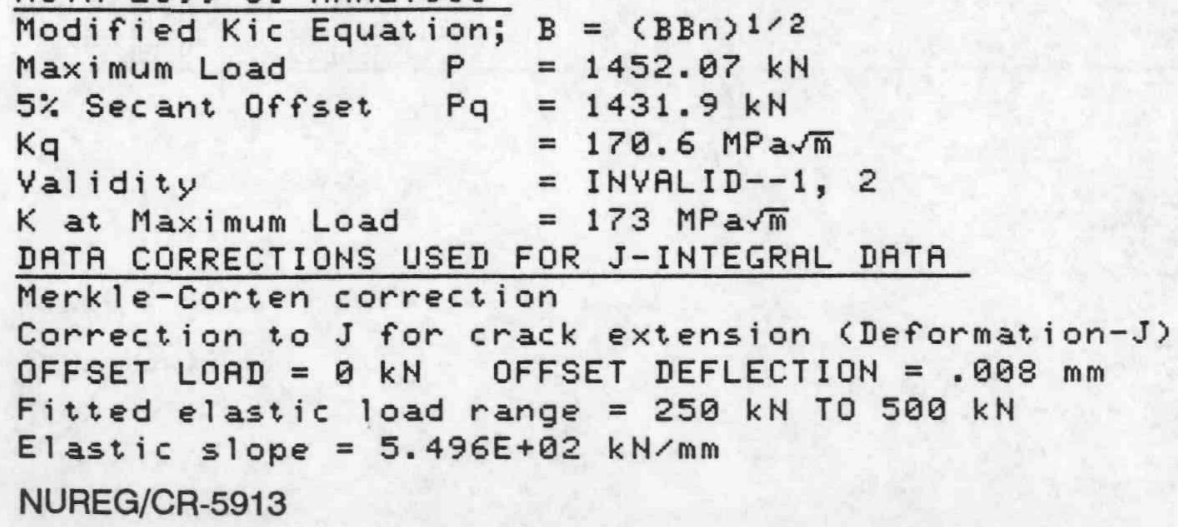




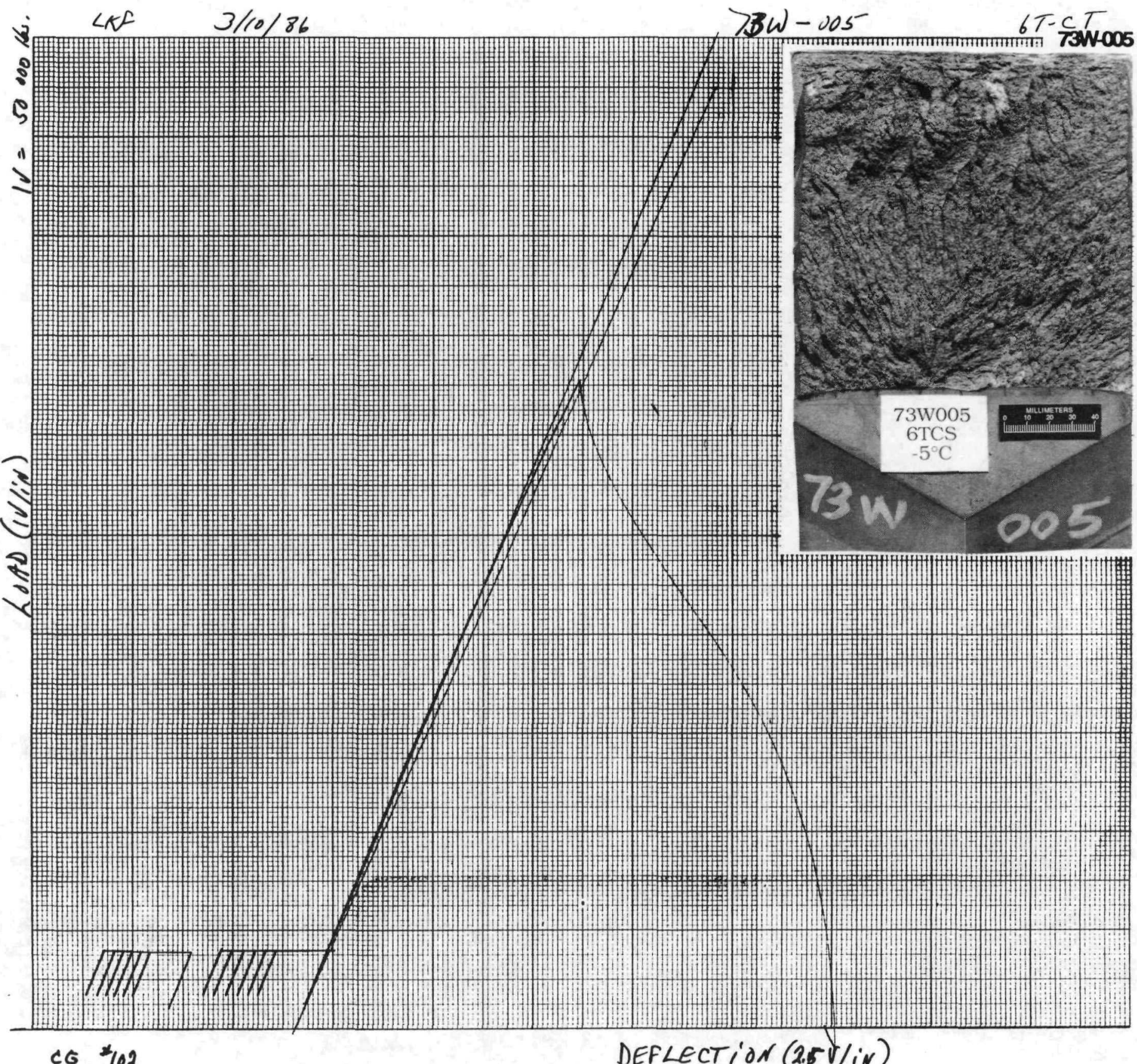


Specimen Crackmauth Deflection (in.)

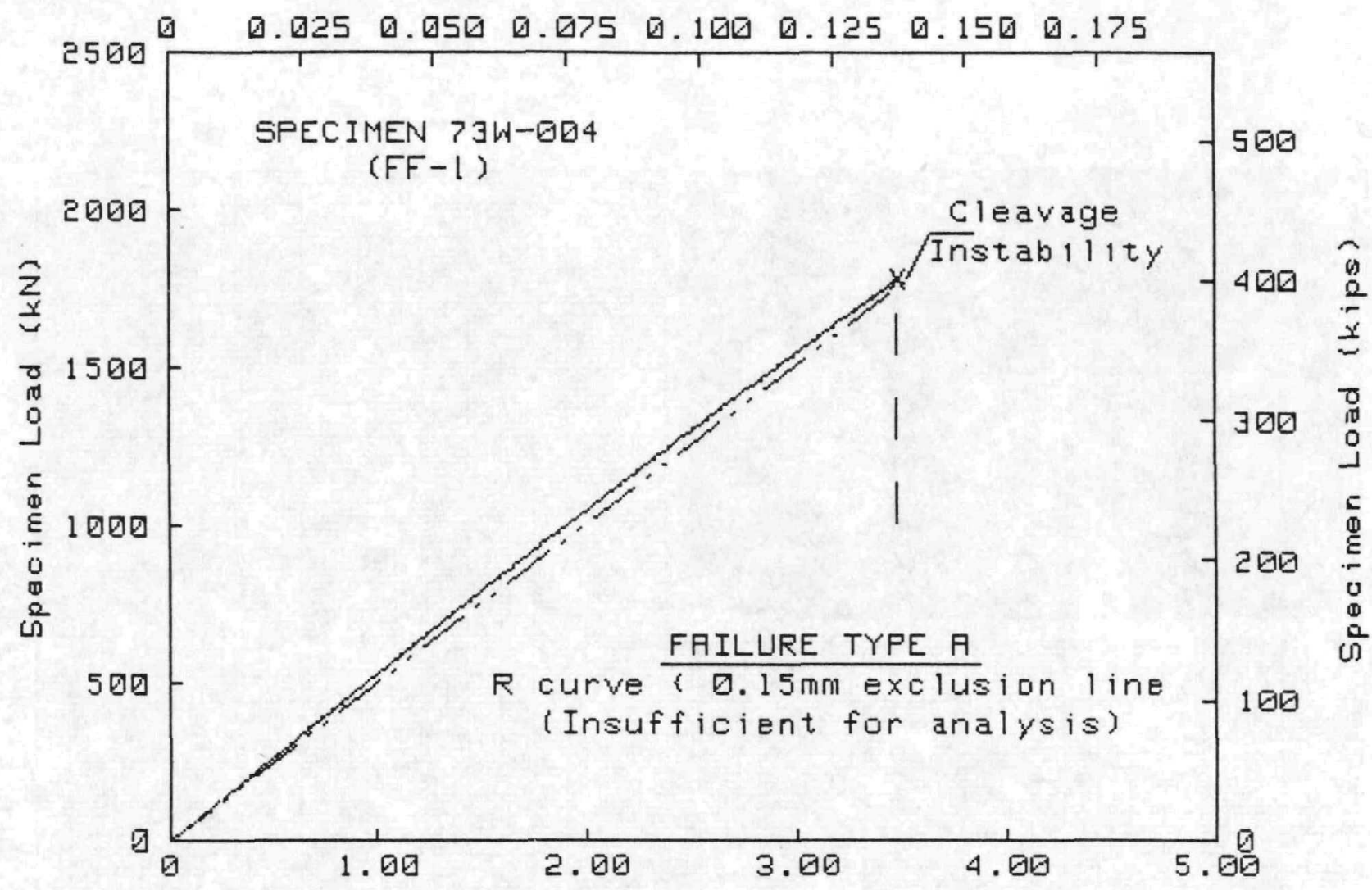

Specimen Crackmouth Deflection ( $m$ )

TEST SPECIMEN DATA

Material Type
Test Temperature
Percent Side Groove
Specimen Thickness B
Initial a/W
Init crack length a
Flow strength
Yield strength
Youngs modulus
Poisson's Ratio (
J-INTEGRAL ANALYSIS

$\mathrm{JC}$

$K j c=\left\langle E J c /\left(1-v^{2}\right\rangle\right) 1 / 2$

$\mathrm{Kc}$ (Beta Corrected)

ASTM E399-81 ANALYSIS

Modified Kic Equation;

Maximum Load

$5 \%$ secant offset

$\mathrm{Kq}$

Validity

$K$ at Maximum Load

$$
\begin{aligned}
\mathrm{P} & =1779.25 \mathrm{kN} \\
\mathrm{PQ} & =1779.25 \mathrm{kN} \\
& =175.2 \mathrm{MPa} \sqrt{\mathrm{m}} \\
& =I \mathrm{NVALII}-1,{ }^{2} \\
& =175.2 \mathrm{MPa} \sqrt{\mathrm{m}}
\end{aligned}
$$

$=-5^{\circ} \mathrm{C}$

$=0 \%$

$=203.2 \mathrm{~mm}$

$=.582$

$=236.61 \mathrm{~mm}$

$=572.9 \mathrm{MPa}$

$=514.3 \mathrm{MPa}$

= $207500 \mathrm{MPa} \quad$ (Estimated Value)

$=0$

Failure Type $A$ ( $J$ at instability = Je) ]

$=\frac{\text { Merkle-Corten }}{150.8 \mathrm{~kJ} / \mathrm{m}^{2}}$

$=176.9 \mathrm{MPa} \sqrt{\mathrm{m}}$

$=155.9 \mathrm{MPa} \sqrt{\mathrm{m}}$ $\frac{\text { Deformation }-\mathrm{J} *}{156.6 \mathrm{~kJ} / \mathrm{m}^{2}}$

$180.2 \mathrm{MPa} \sqrt{\mathrm{m}}$

$158 \mathrm{MP} a \sqrt{\mathrm{m}}$

DATA CORRECTIONS USED FOR J-INTEGRAL DATA

Merkle-Corten correction

Correction to J for crack extension (Deformation-J)

OFFSET LOAD $=0 \mathrm{kN}$ OFFSET DEFLECTION $=.011 \mathrm{~mm}$

Fitted elastic load range $=250 \mathrm{kN}$ TO $500 \mathrm{kN}$

Elastic slope $=5.308 \mathrm{E}+02 \mathrm{kN} / \mathrm{mm}$ 


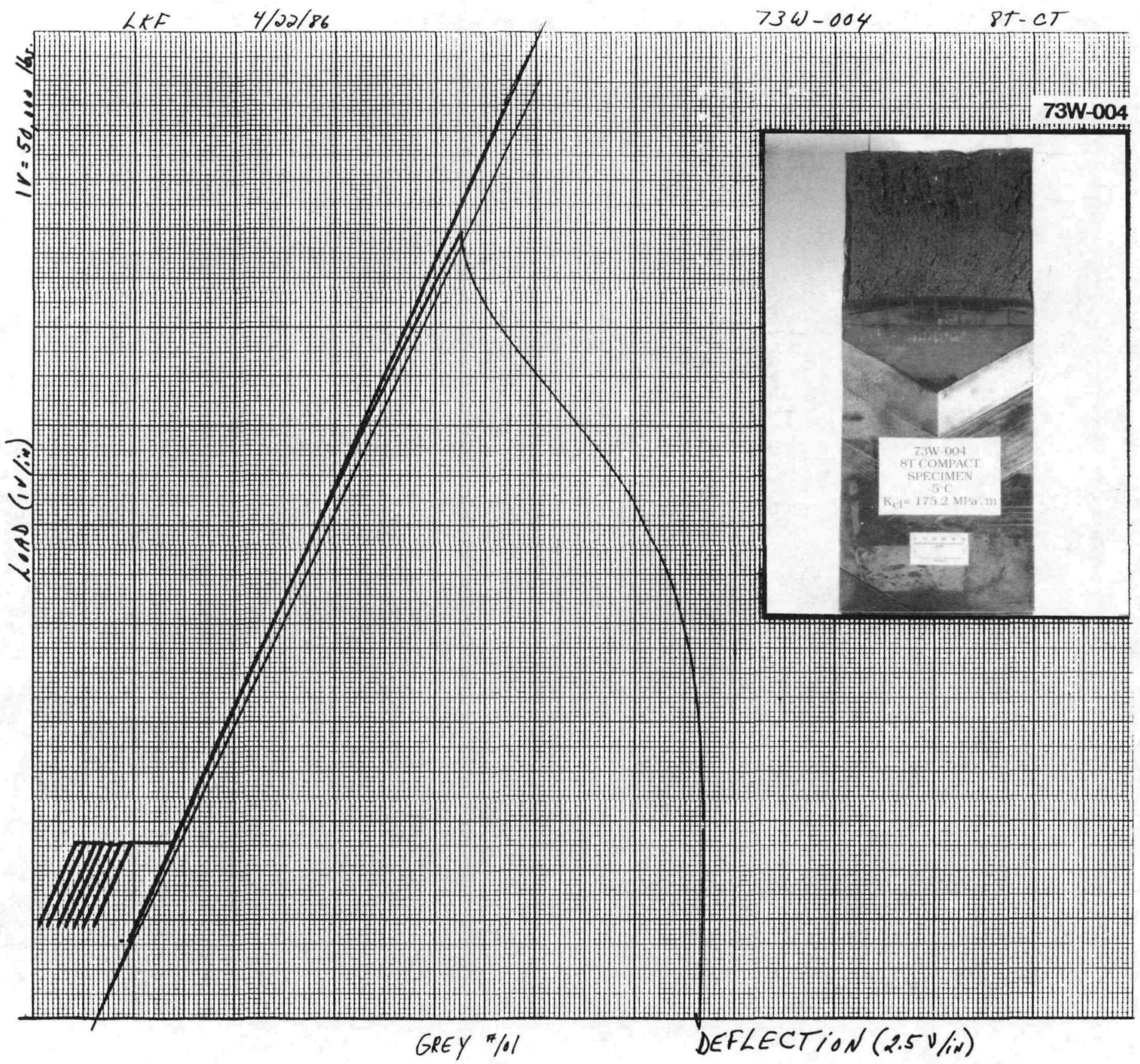




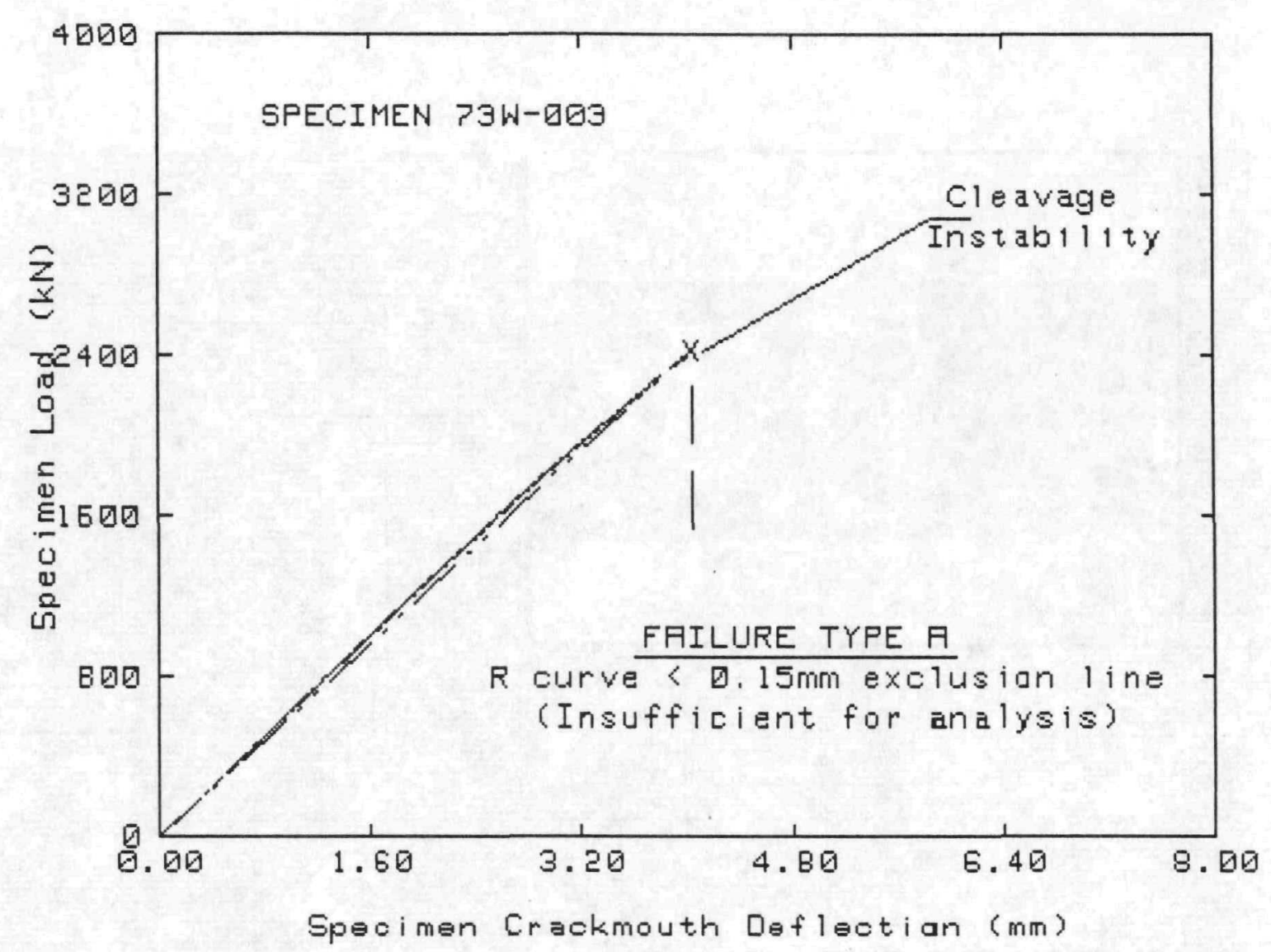

TEST SPECIMEN DRTA

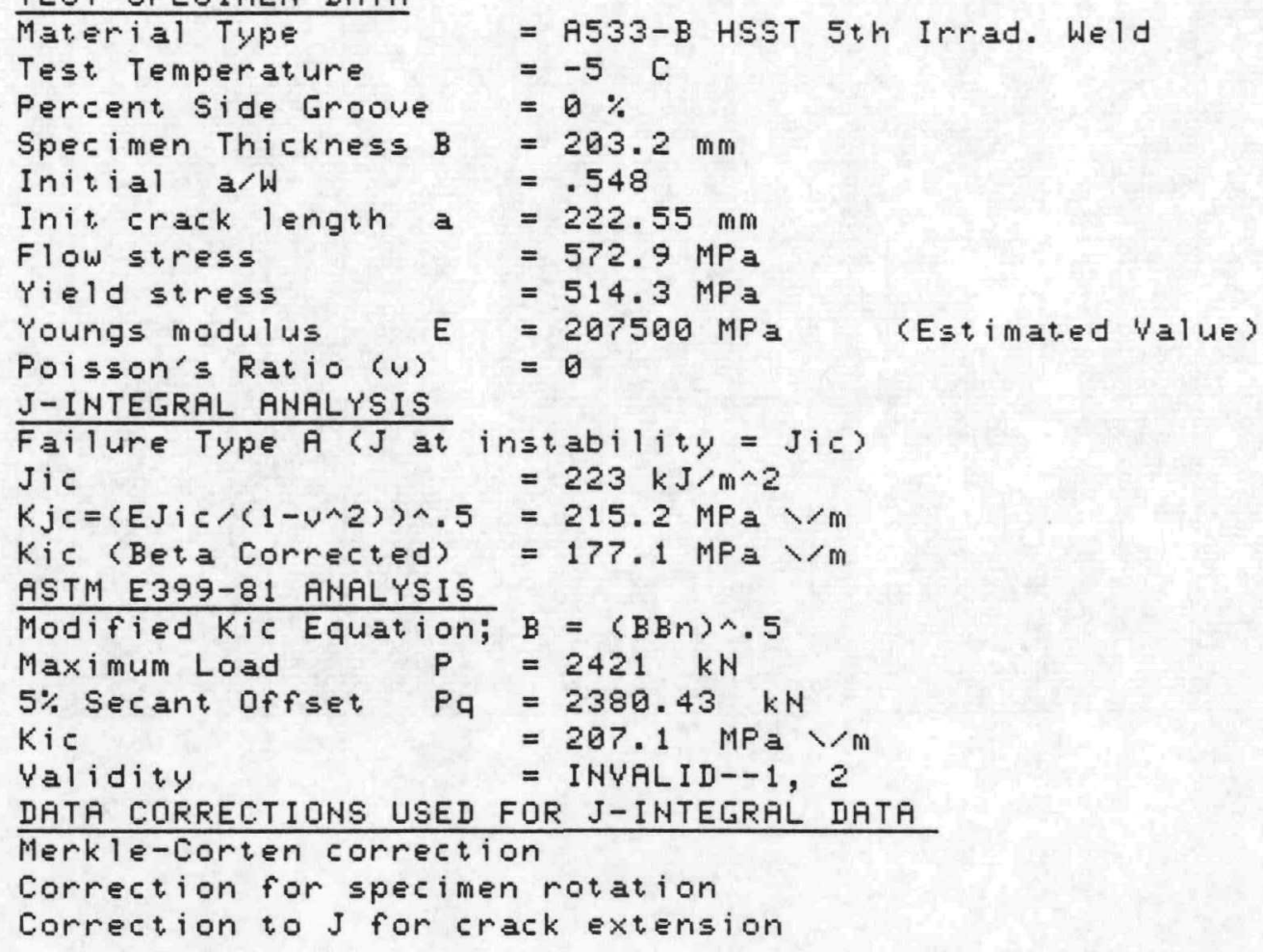


$\angle K F$

$4 / 1186$

$23 w-003$ $B T-C T$ $0 \%$

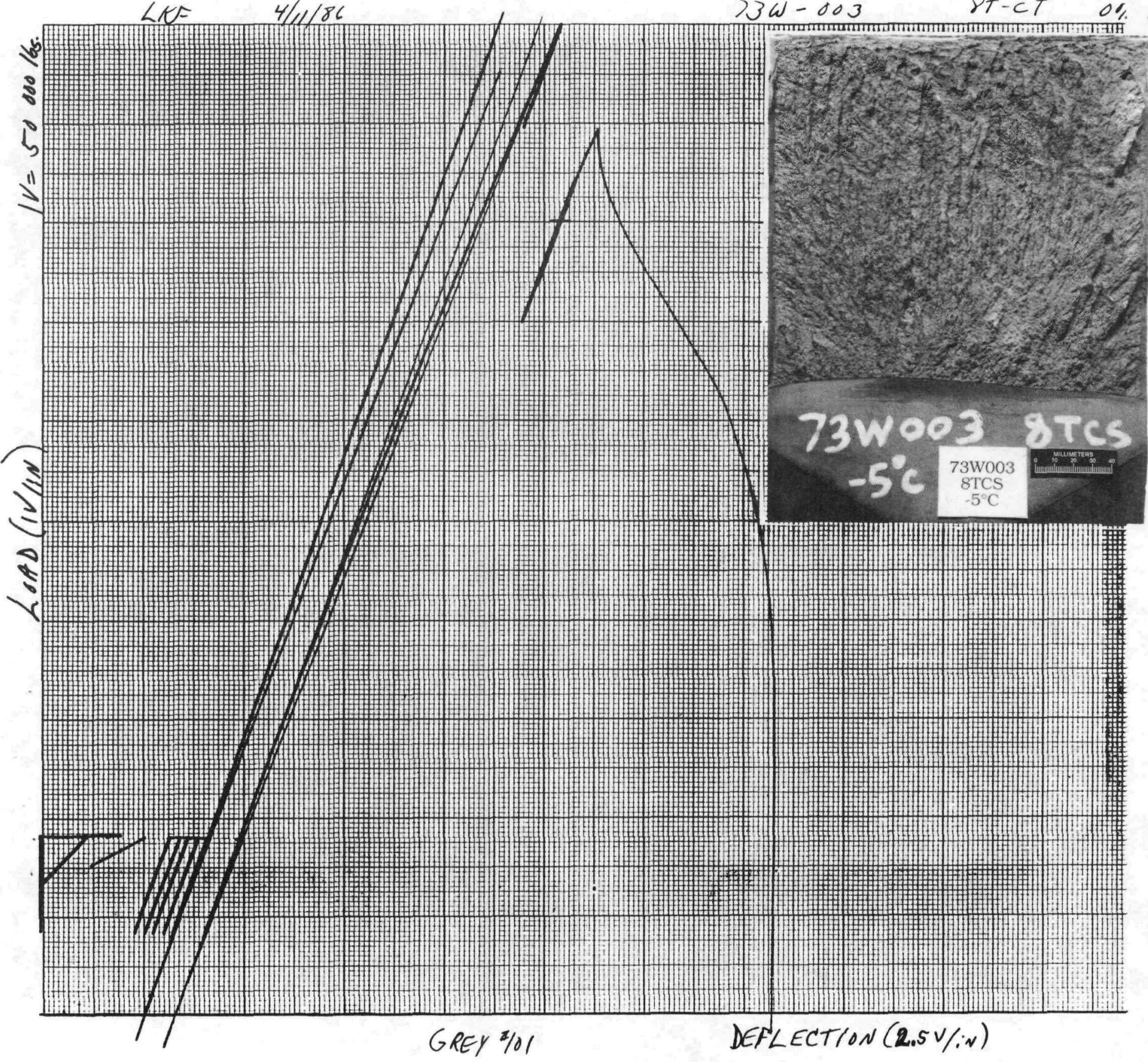


73W-001
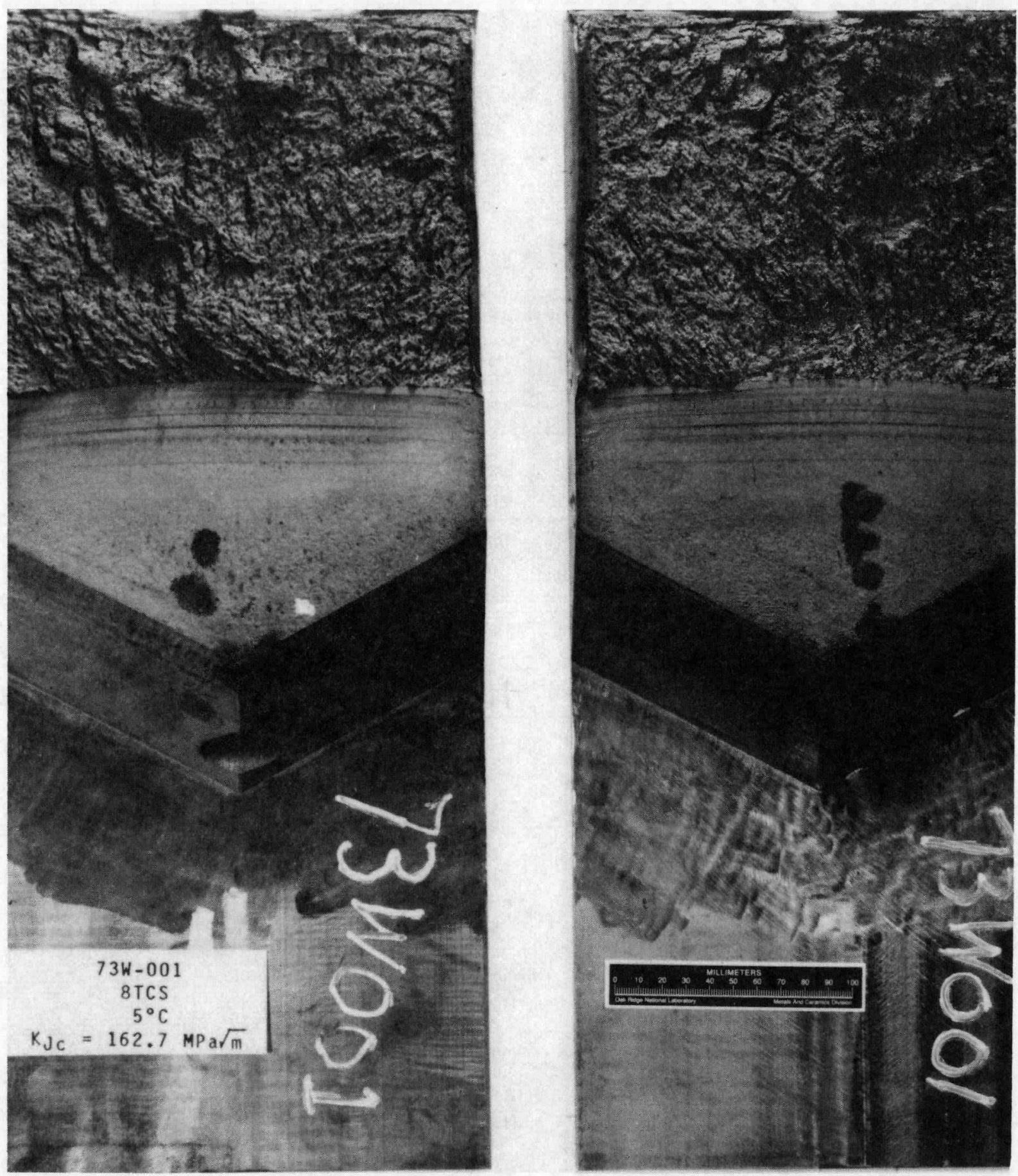


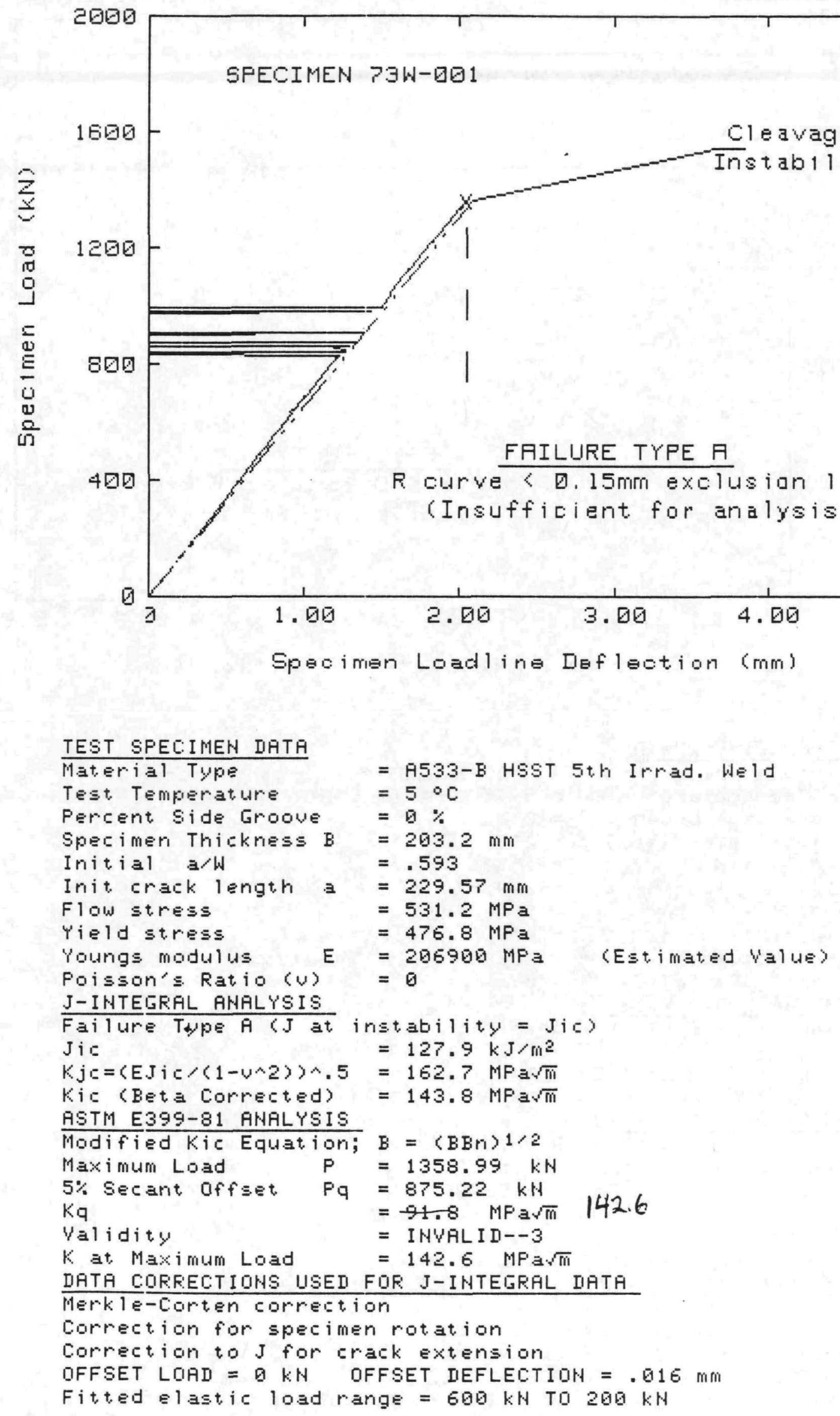




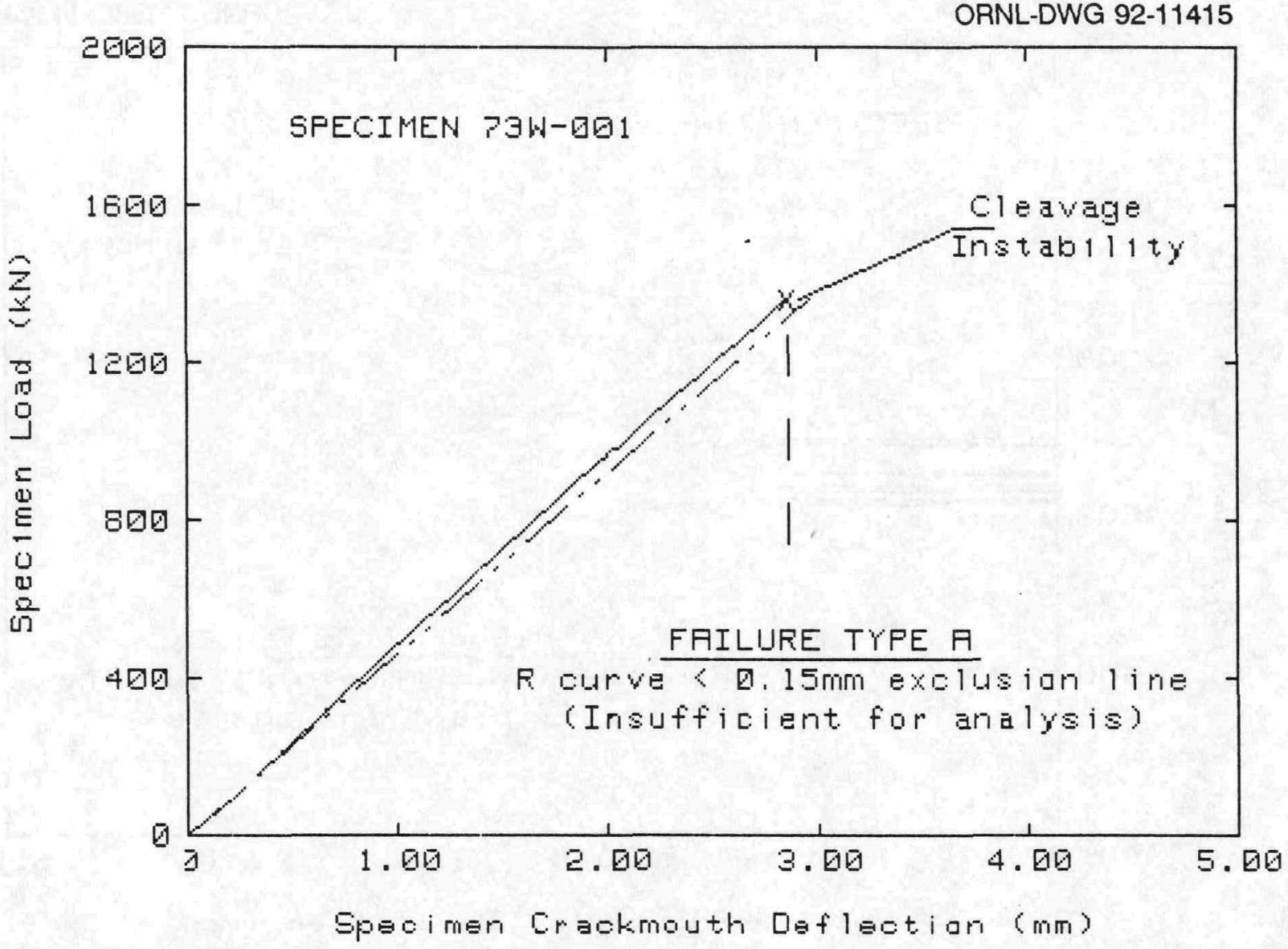

TEST SPELIMEN IATH

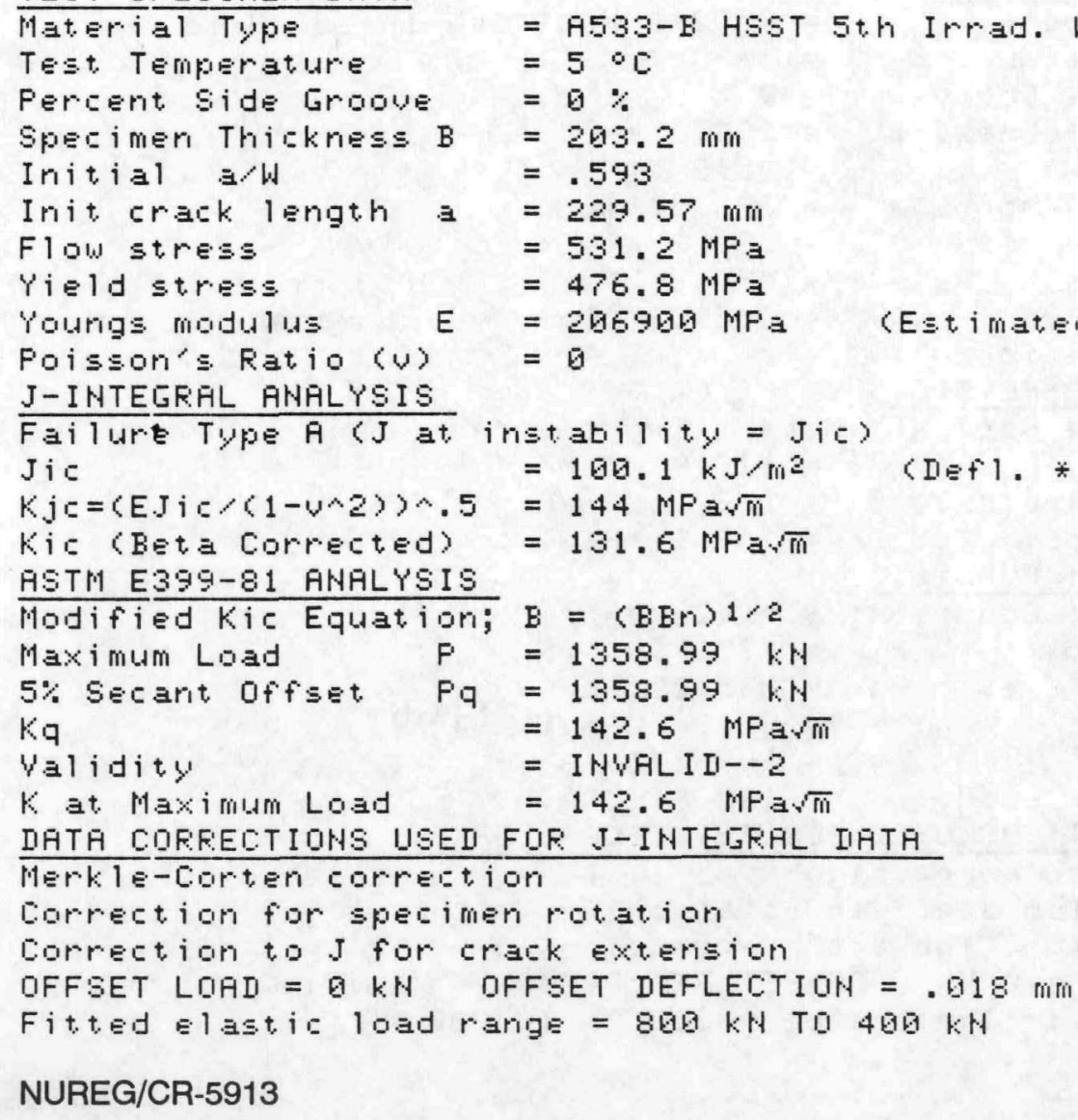




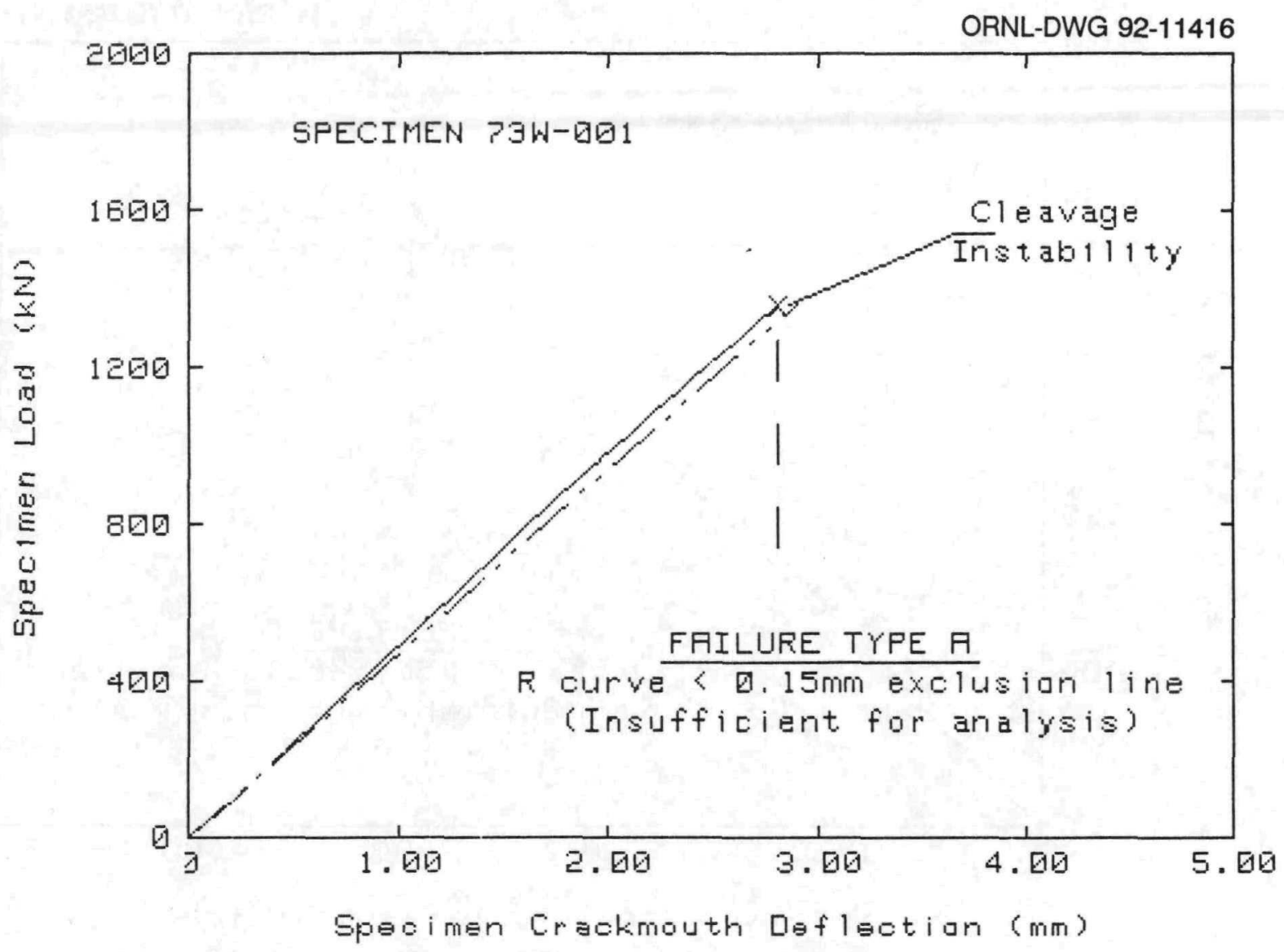

\section{TEST SPELIMEN DATH}

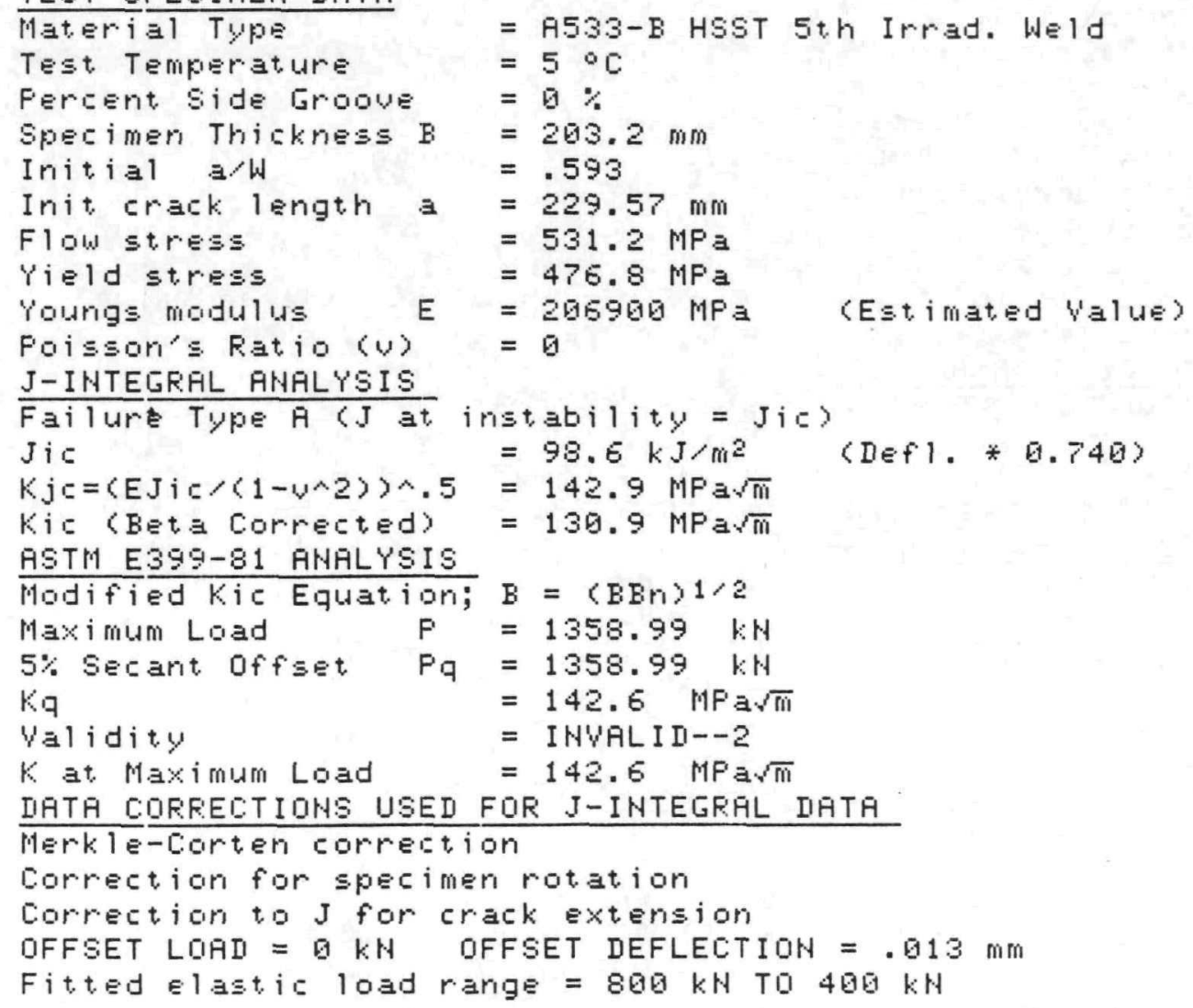




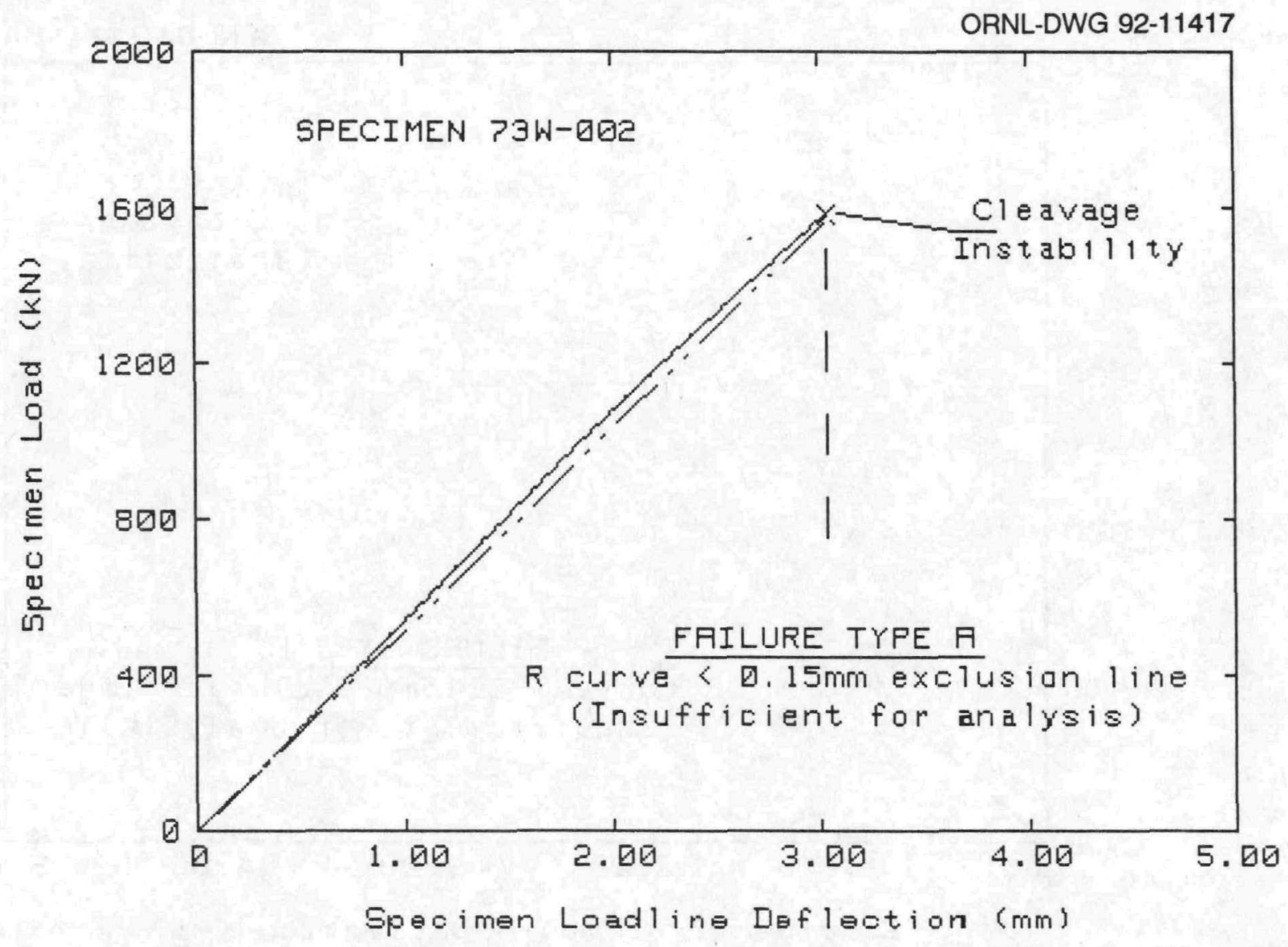

TEST SPECIMEN IATA

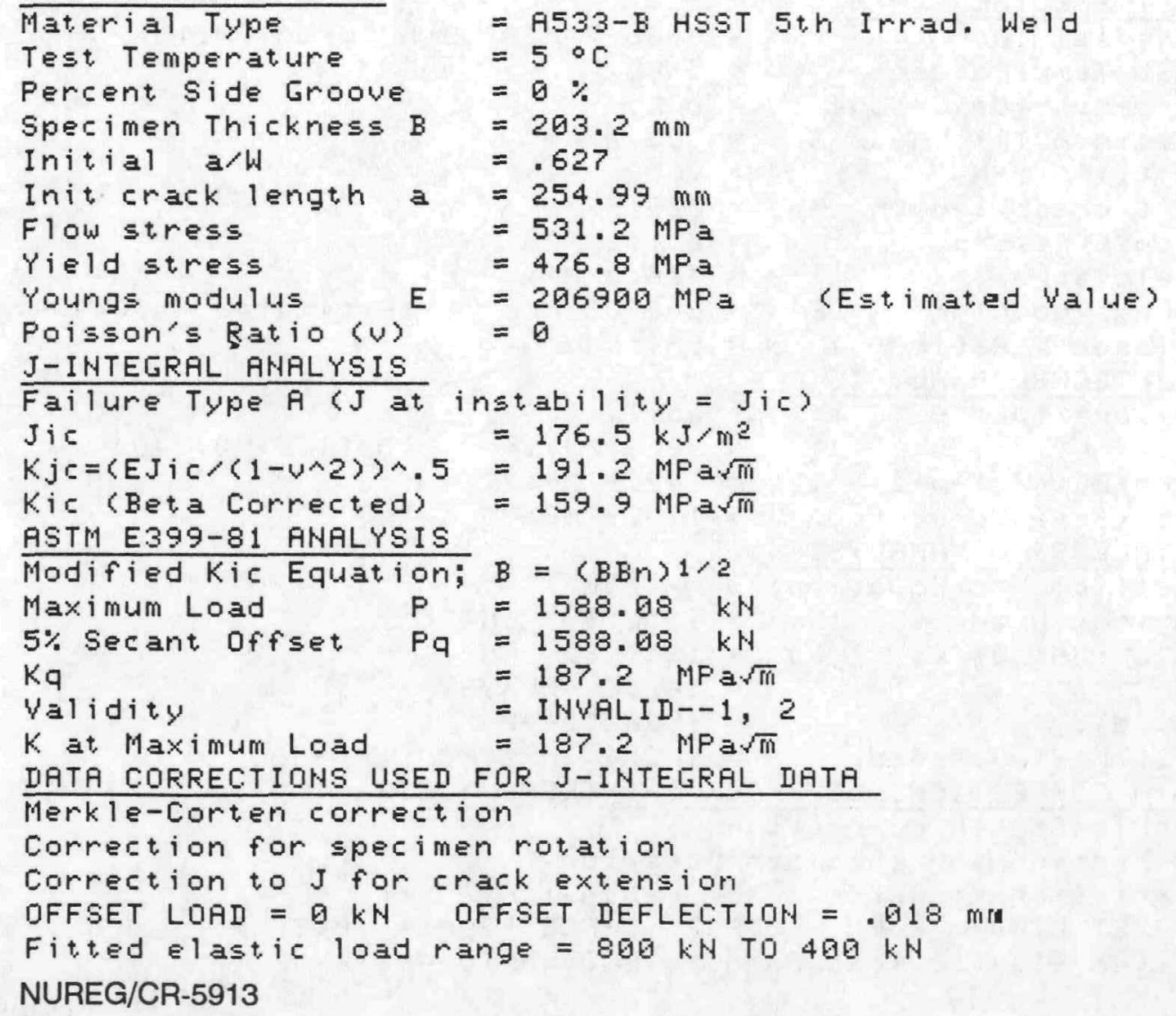




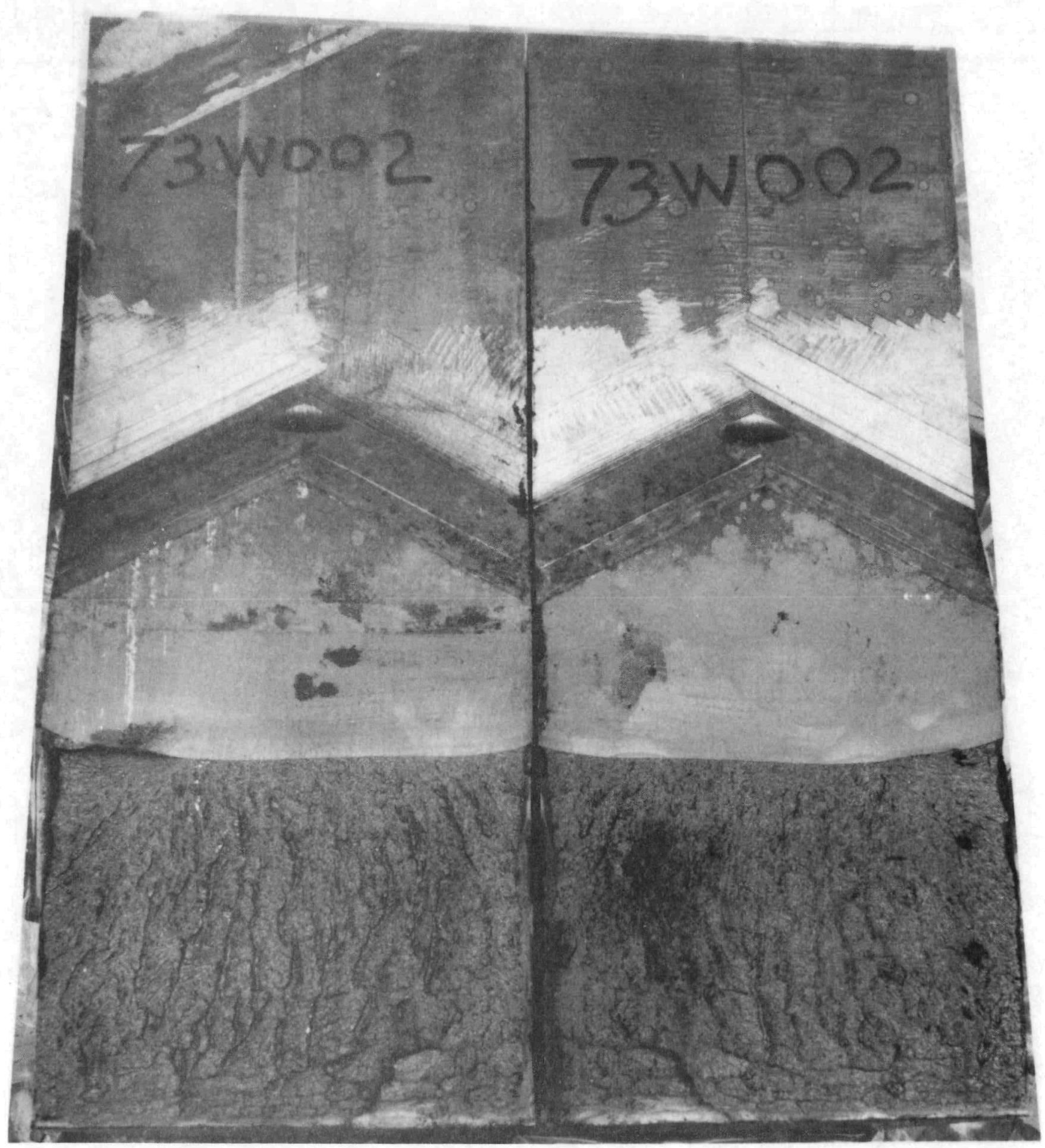


ORNL-DWG 92-11418

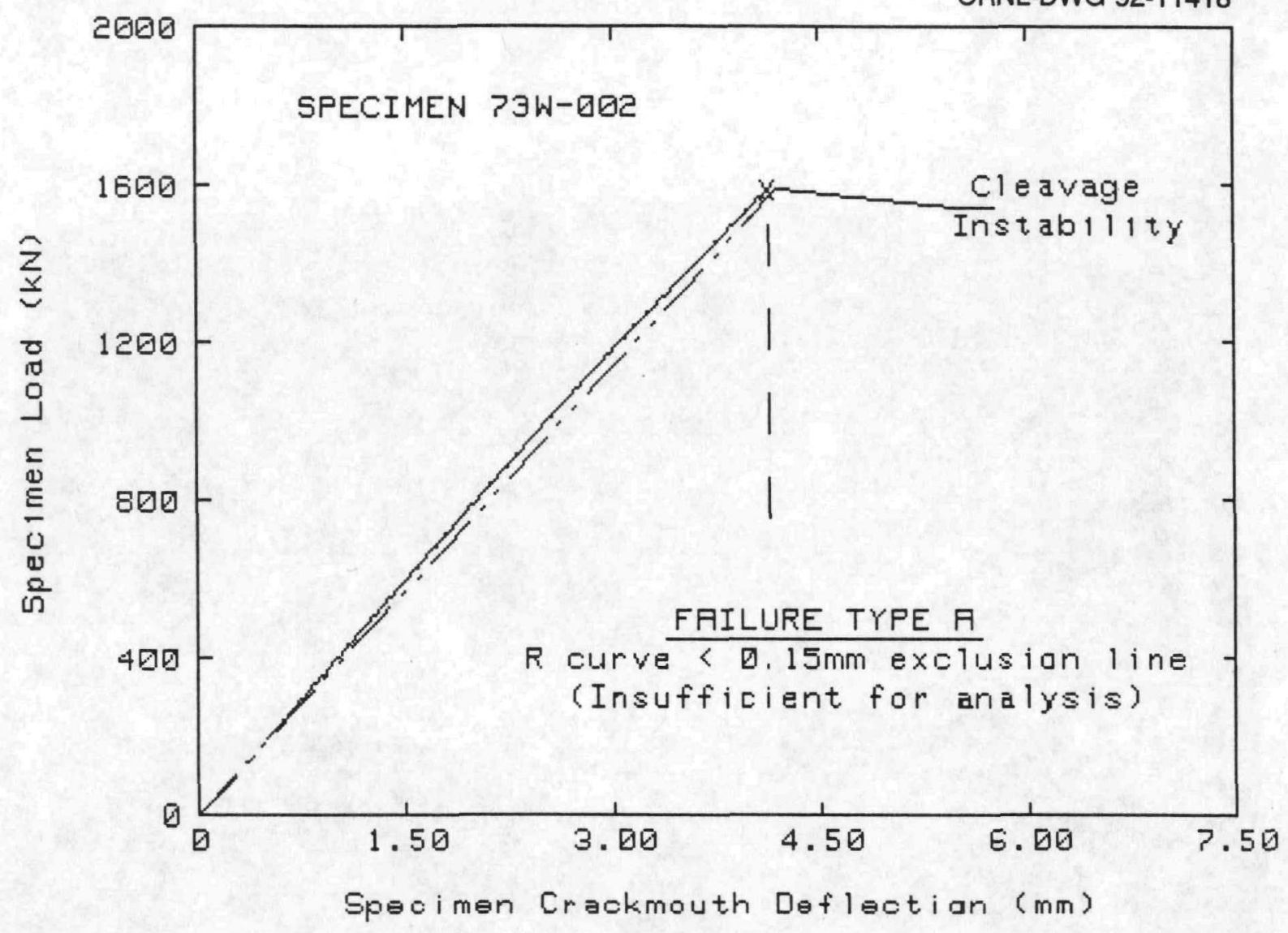

TEST SPECIMEN DATA

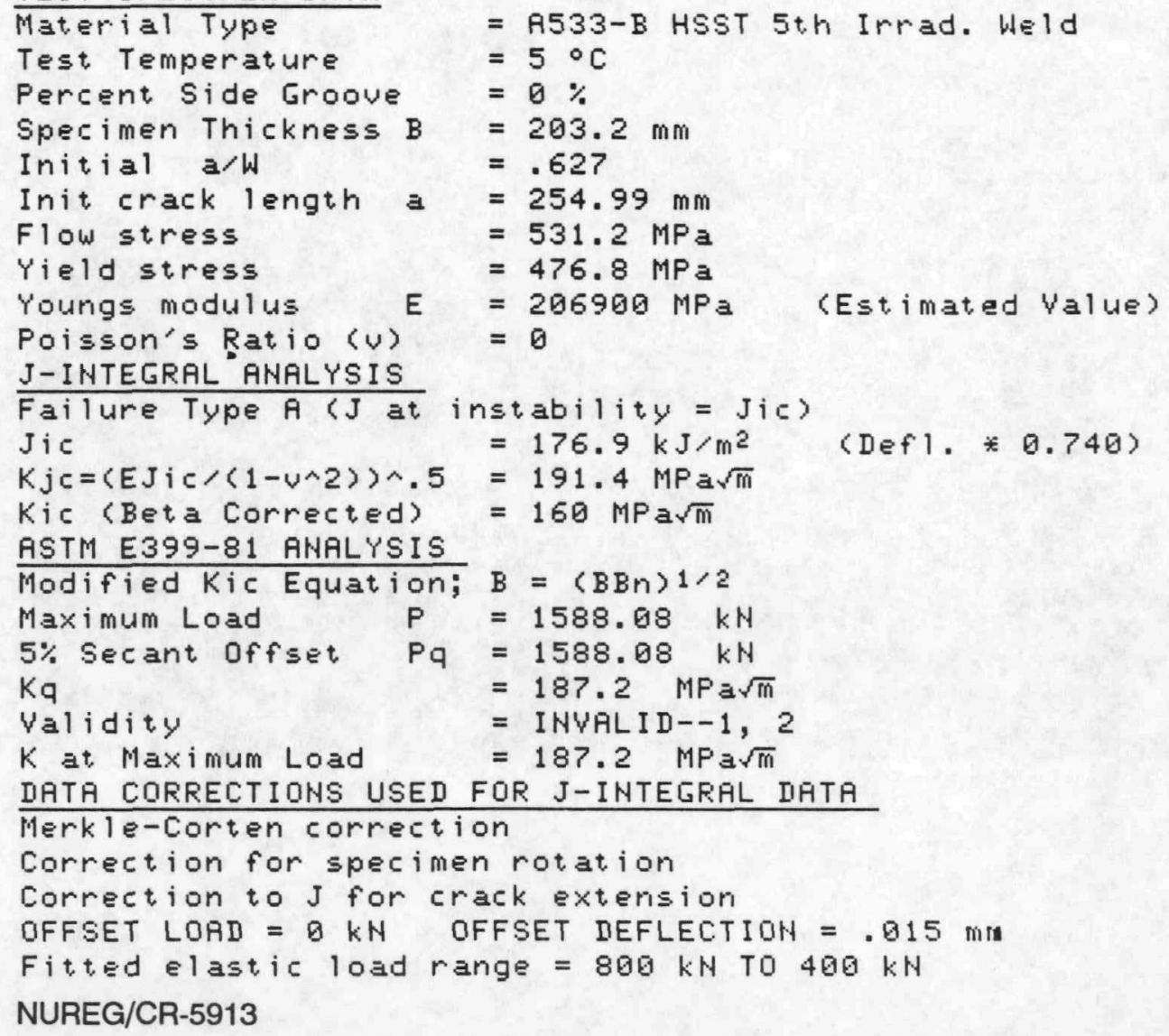




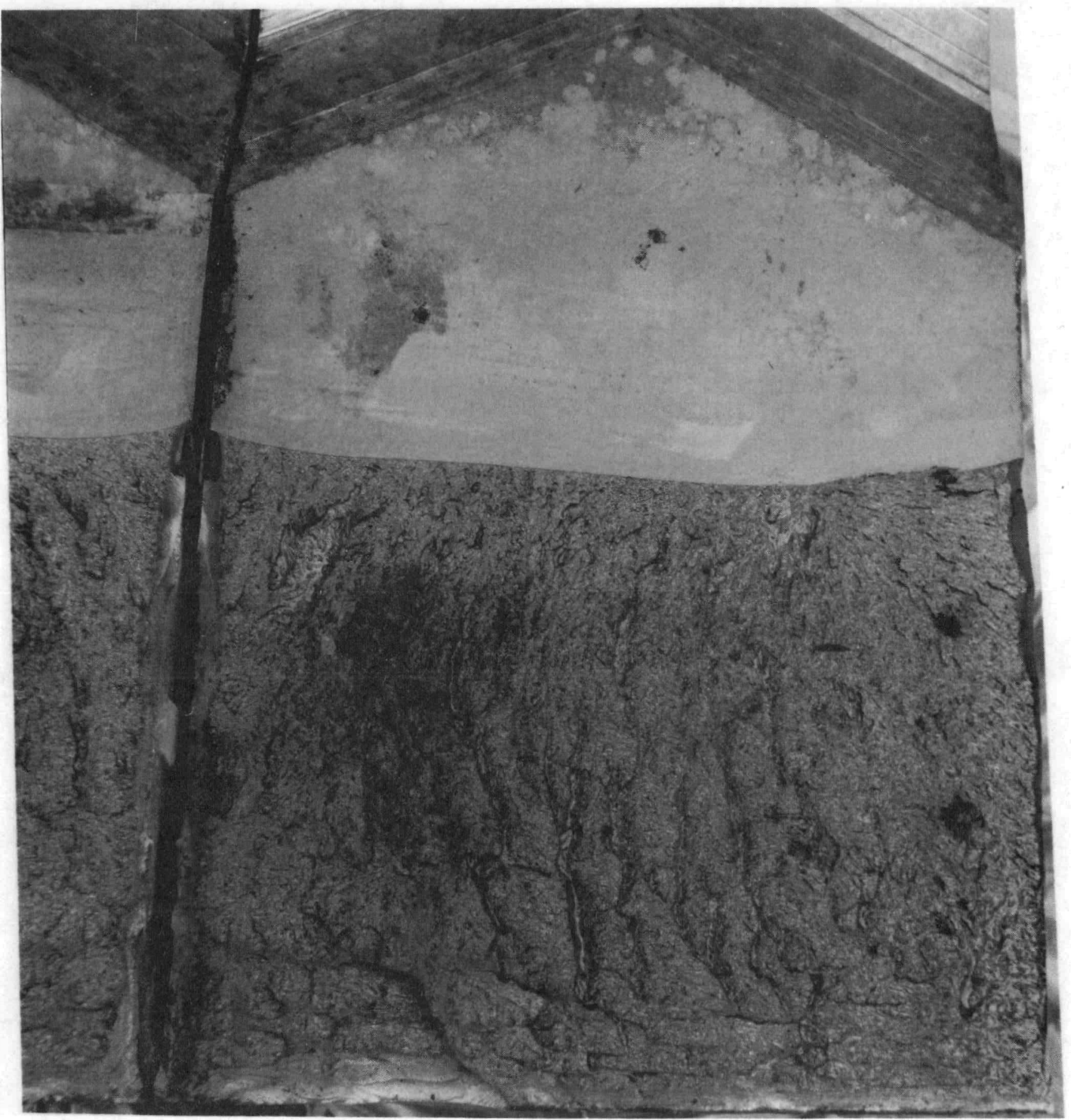

NUREG/CR-5913 

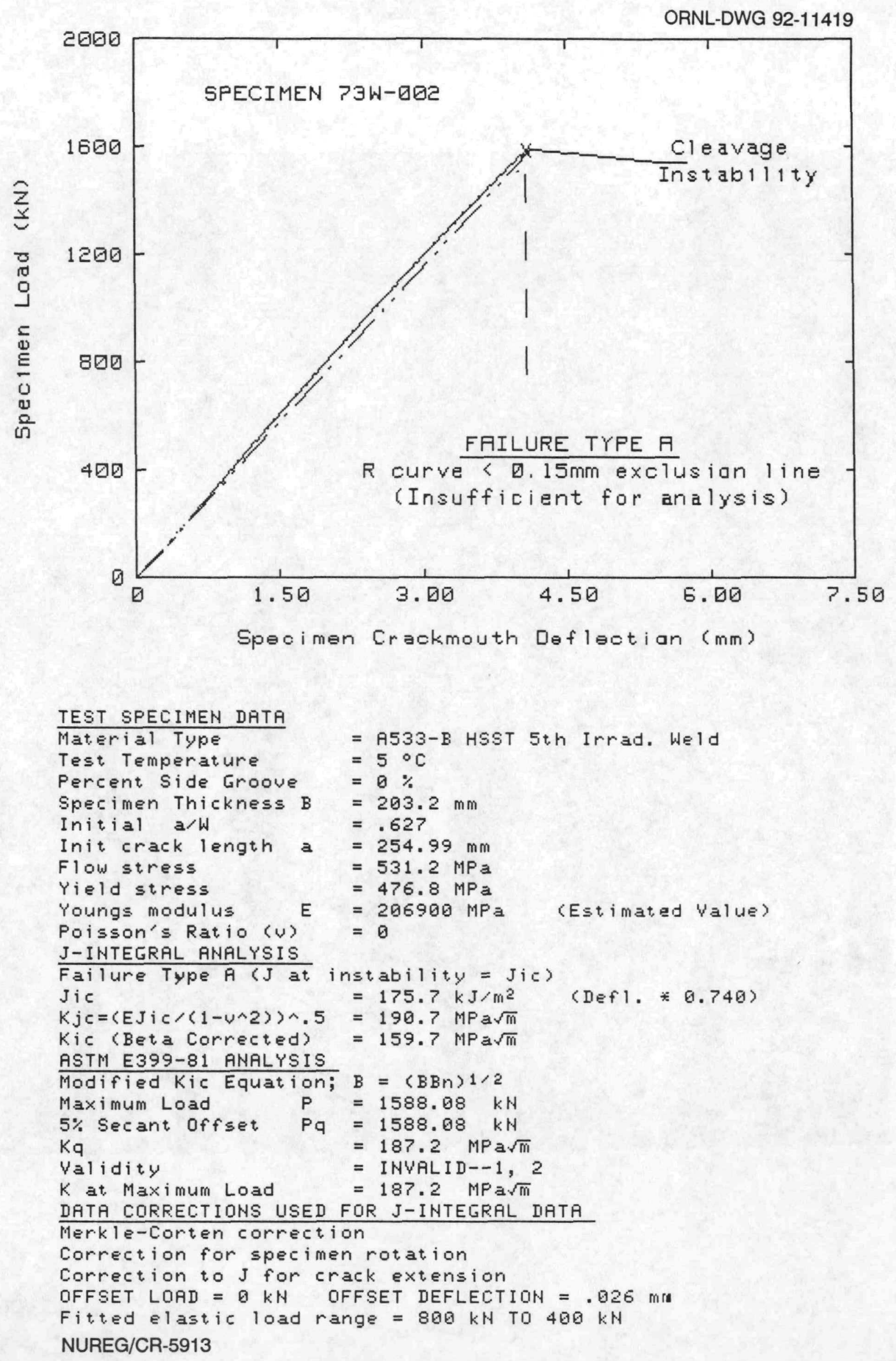
YP6459A

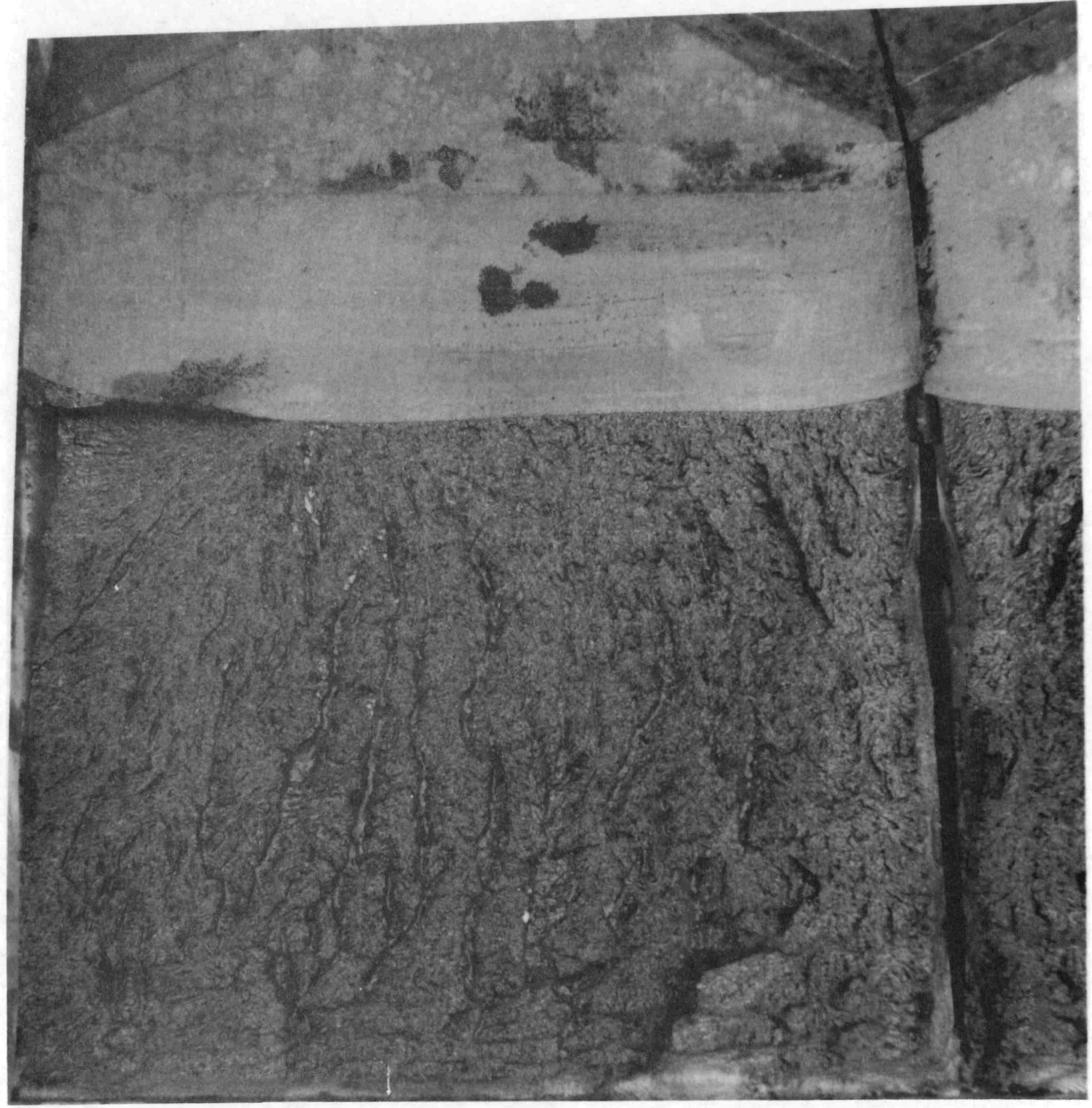




\section{Load-Displacement Curves and Photographs of the Fracture Toughness Specimens From the Irradiated $73 \mathrm{~W}$ Weld $(0.31 \mathrm{wt} \% \mathrm{Cu})$}


SPEC IMEN ID $=73 W 158$

MATERIAL TYPE $=$ HCUWELD

TEST TEMPERATURE $=-75$ DEG $C$

THICKNESS $=25.5 \mathrm{~mm}$

SIDE GRODUES = $0 \%$

INITIAL CRACK LENGTH $=27.3 \mathrm{~mm}$

MEASURED DUCTILE DELTA-A $=.05 \mathrm{~mm}$

$a / W\rangle i=.538$

$Y$ IELD STRENGTH $=621 \mathrm{MPa}$

FLOW STRENGTH $=672 \mathrm{MPa}$

ESTIMATED YOUNG'S MODULUS= $211 \mathrm{GPa}$

SPECIMEN CLEAUED AT KIC= $44.3 \mathrm{MPa} S Q R[\mathrm{~m}]$ (UALID ASTM E399)

UALUES AT MAXIMUM LDAD

J-INTEGRAL $=10.4 \mathrm{~kJ} / \mathrm{m}^{\wedge} 2$

$\mathrm{KJc}=46.9 \mathrm{MPa} \operatorname{SQR}[\mathrm{m}]$

$K$-beta-c $=45.5 \mathrm{MPa} \operatorname{SQR}[\mathrm{m}]$
UALUES AT CLEAUAGE LOAD

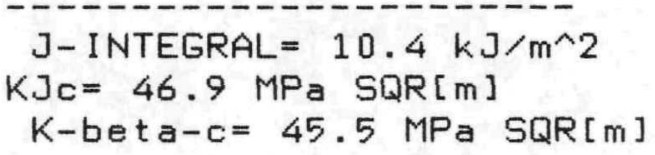


F-160

ORNL-DWG 92-11364

73W158 -75 DEGREES C

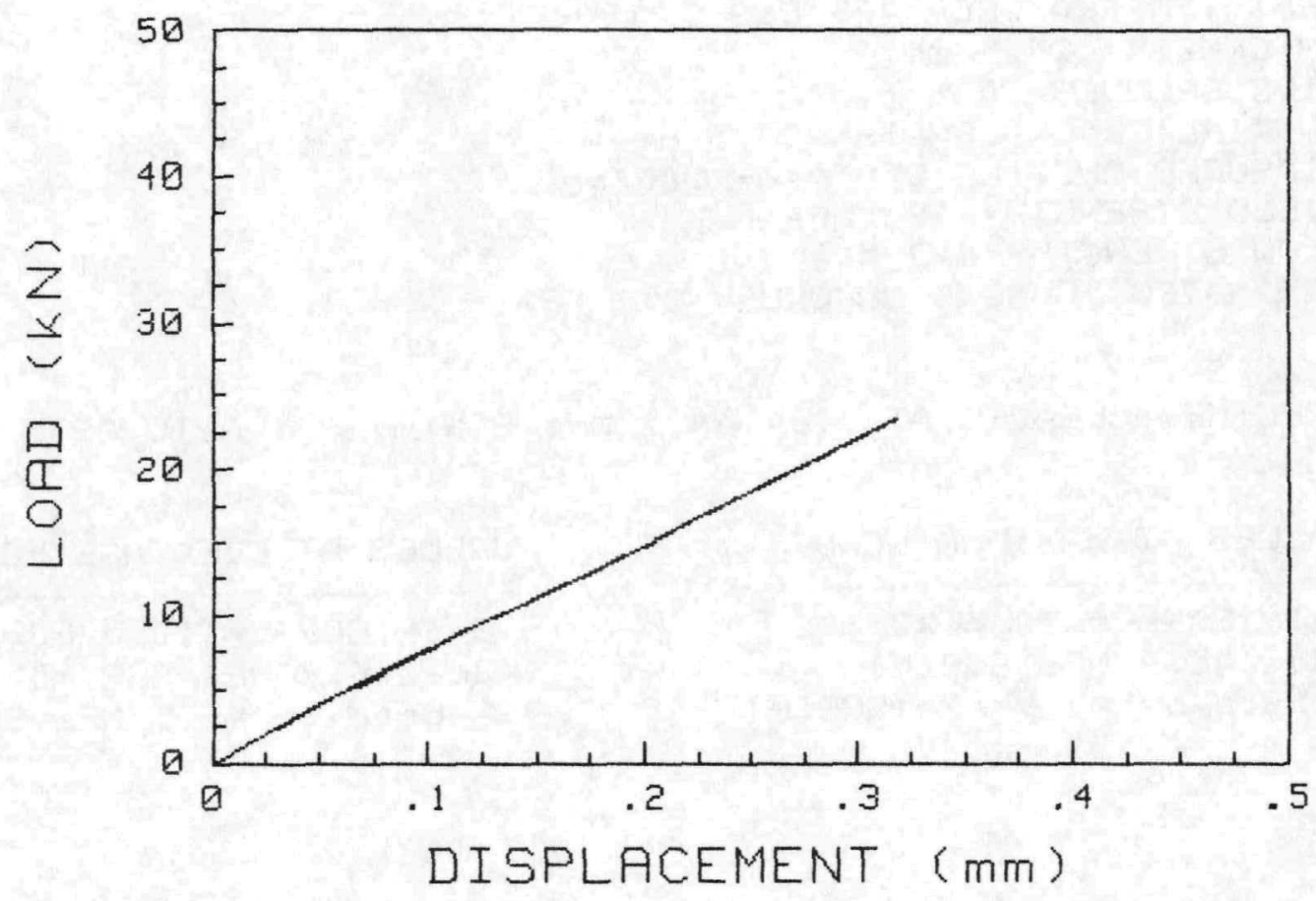


SPECIMEN ID $=73 W 160$

MATERIAL TYPE = HCUWELD

TEST TEMPERATURE $=-25$ DEG $C$

THICKNESS $=25.5 \mathrm{~mm}$

SIDE GRDOUES $=0 \%$

INITIAL CRACK LENGTH $=27.8 \mathrm{~mm}$

MEASURED DUCTILE DELTA-A $=.05 \mathrm{~mm}$

$a / W) i=.546$

YIELD STRENGTH $=621 \mathrm{MPa}$

FLOW STRENGTH $=672 \mathrm{MPa}$

ESTIMATED YOUNG'S MODULUS $=209 \mathrm{GPa}$

SPECIMEN CLEAUED AT KIc= $57.3 \mathrm{MPa}$ SQR $[\mathrm{m}]$ (UALID ASTM E399)

UALUES AT MAXIMUM LOAD

J-INTEGRAL $=19 \mathrm{~kJ} / \mathrm{m}^{\wedge} 2$

$\mathrm{KJ} c=62.9 \mathrm{MPa} \operatorname{SQR}[\mathrm{m}]$

$K$-beta-c $=58.2 \mathrm{MPa} \operatorname{SQR}[\mathrm{m}]$
UALUES AT CLEAUAGE LDAD

$J-I N T E G R A L=19 \mathrm{~kJ} / \mathrm{m}^{\wedge} 2$

$\mathrm{KJ} c=62.9 \mathrm{MPa} \operatorname{SQR}[\mathrm{m}]$

$K$-bet $a-c=58.2 \mathrm{MPa} \operatorname{SQR}[\mathrm{m}]$ 
F-162

ORNL-DWG 92-11365

73W160 -25 DEGREES C

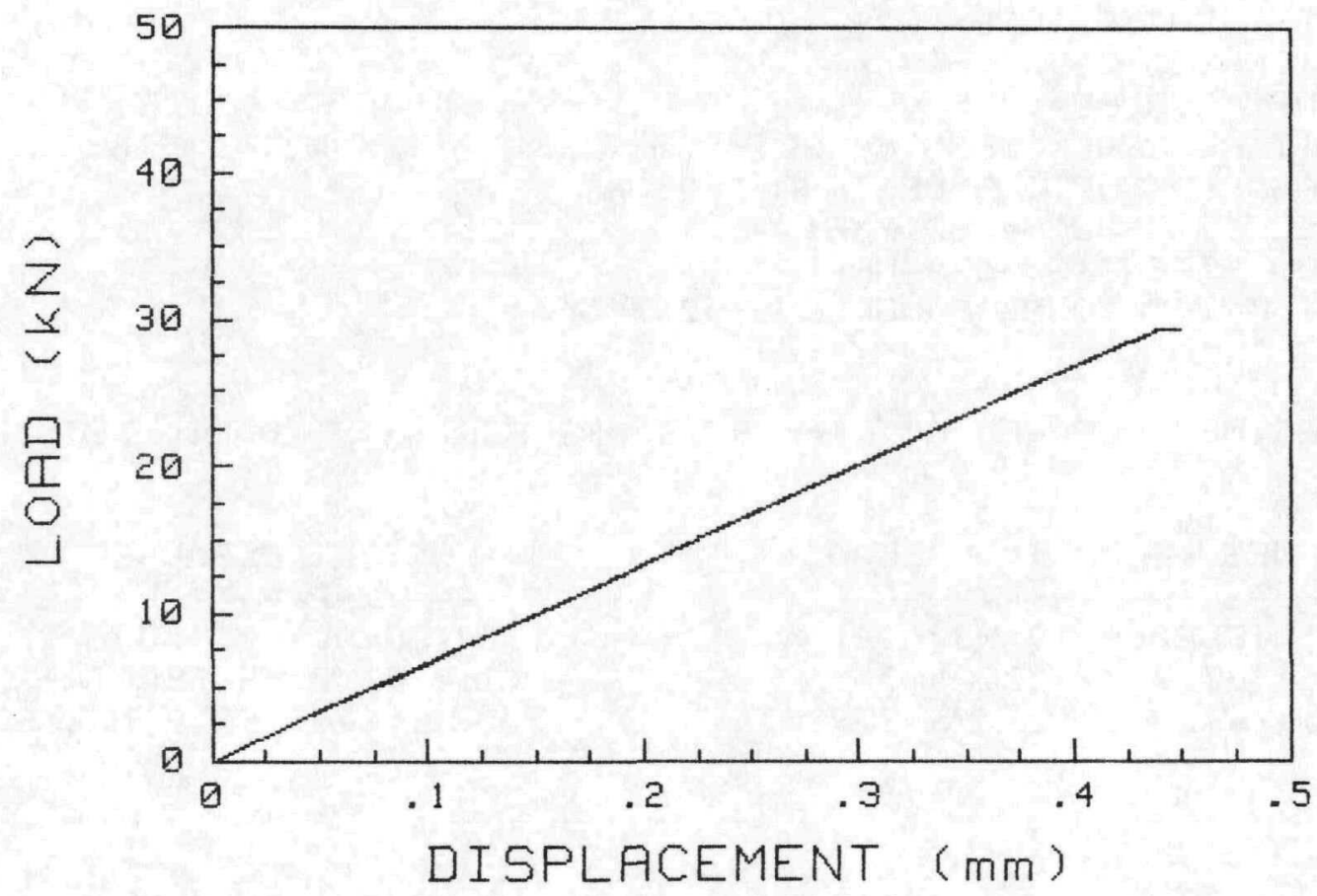


Specimen Loadline Deflection (in.)

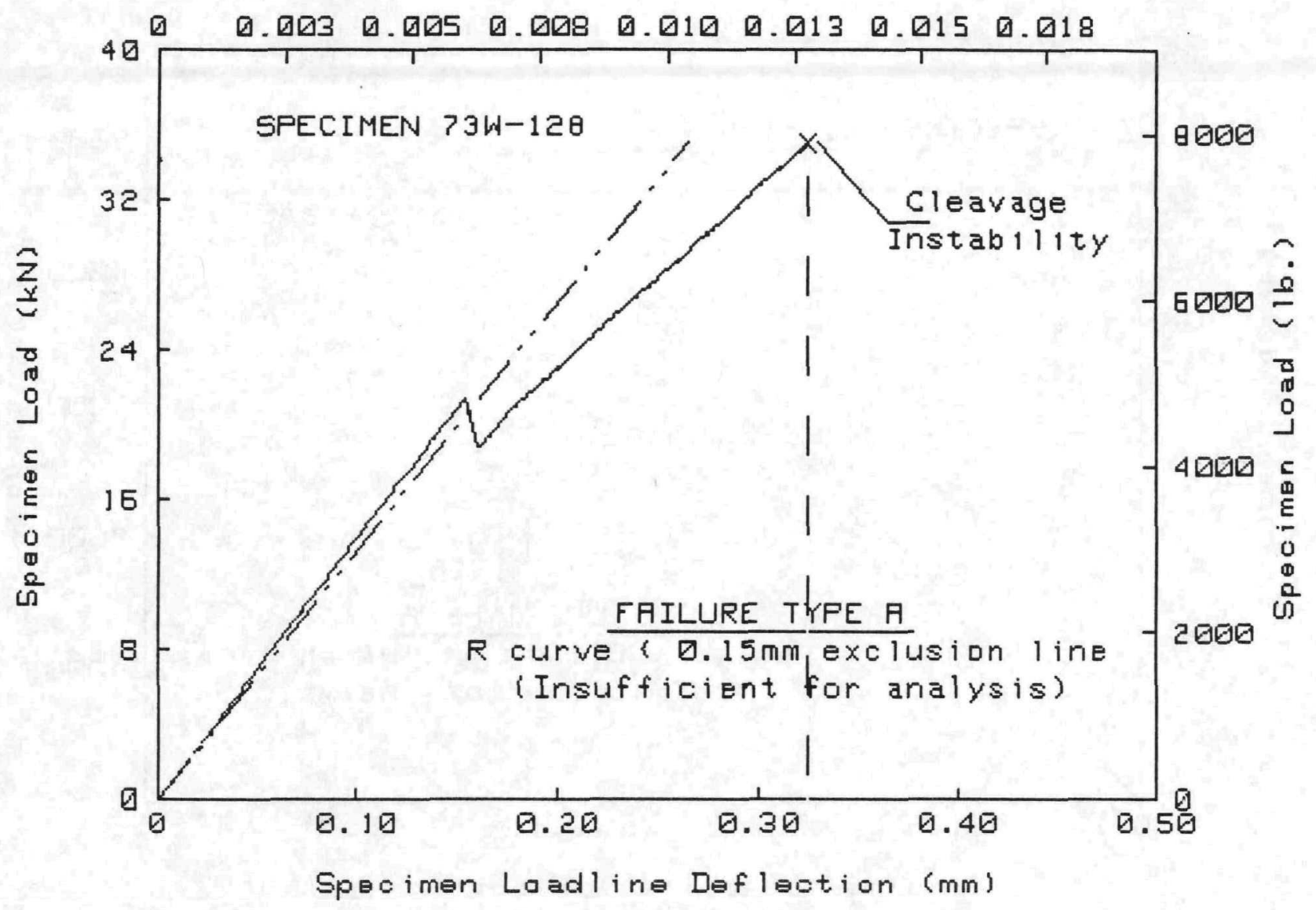

TEST SPECIMEN DATA

Material Type
Test Temperature
Percent Side Groove
Specimen Thickness B
Initial a/W
Init crack length a
Flow stress
Yield stress
Youngs modulus
Poisson's Ratio (U)
J-INTEGRAL RNRLYSIS

Je

$K j c=\left\langle E J c /\left(1-v^{2}\right\rangle\right\rangle \wedge .5$

Ke (Beta Corrected)

ASTM E399-81 ANALYSIS

Modified Kic Equation; $B=\langle B B n\rangle 1 / 2$

Maximum Load $P=35.07 \mathrm{kN}$

$5 \%$ Secant offset $\mathrm{Pq}=21.36 \mathrm{kN}$

$\mathrm{Kq}$

= A533-B Weld (I)

$=-25^{\circ} \mathrm{C}$

$=0 \%$

$=25.4 \mathrm{~mm}$

$=.536$

$=27.24 \mathrm{~mm}$

$=722.8 \mathrm{MPa}$

$=675 \mathrm{MPa}$

$=208600 \mathrm{MPa}$ (Estimated Valwe)

$=0$

[ Failure Type $A\langle J$ at instability $=J c\rangle$ ]

$\begin{array}{lll}=\frac{\text { Merkle-Corten }}{23.4 \mathrm{~kJ} / \mathrm{m}^{2}} & & \text { Deformation-J* } \\ =69.8 \mathrm{MPa} \sqrt{\mathrm{m}} & & 28 \mathrm{~kJ} / \mathrm{m}^{2} \\ =64.3 \mathrm{MPa} \sqrt{\mathrm{m}} & & 76.5 \mathrm{MPa} \sqrt{\mathrm{m}} \\ =68.8 \mathrm{MPa} \sqrt{\mathrm{m}}\end{array}$

Validity

$=40.5 \mathrm{MPa} \sqrt{\mathrm{m}}$

$=$ INVALID --3

$\mathrm{K}$ at Maximum Load $=66.4 \mathrm{MPa} \sqrt{\mathrm{m}}$

DATA CORRECTIONS USED FOR J-INTEGRAL DATA

Merkle-Corten correction

Correction to J for crack extension (Deformation-J)

OFFSET LOAD $=0 \mathrm{kN}$ OFFSET DEFLECTION $=.003 \mathrm{~mm}$

Fitted elastic load range $=5 \mathrm{kN}$ TO $15 \mathrm{kN}$

Elastic slope $=1.399 E+02 \mathrm{kN} / \mathrm{mm}$ 
Specimen Loadline Deflection. (in.)

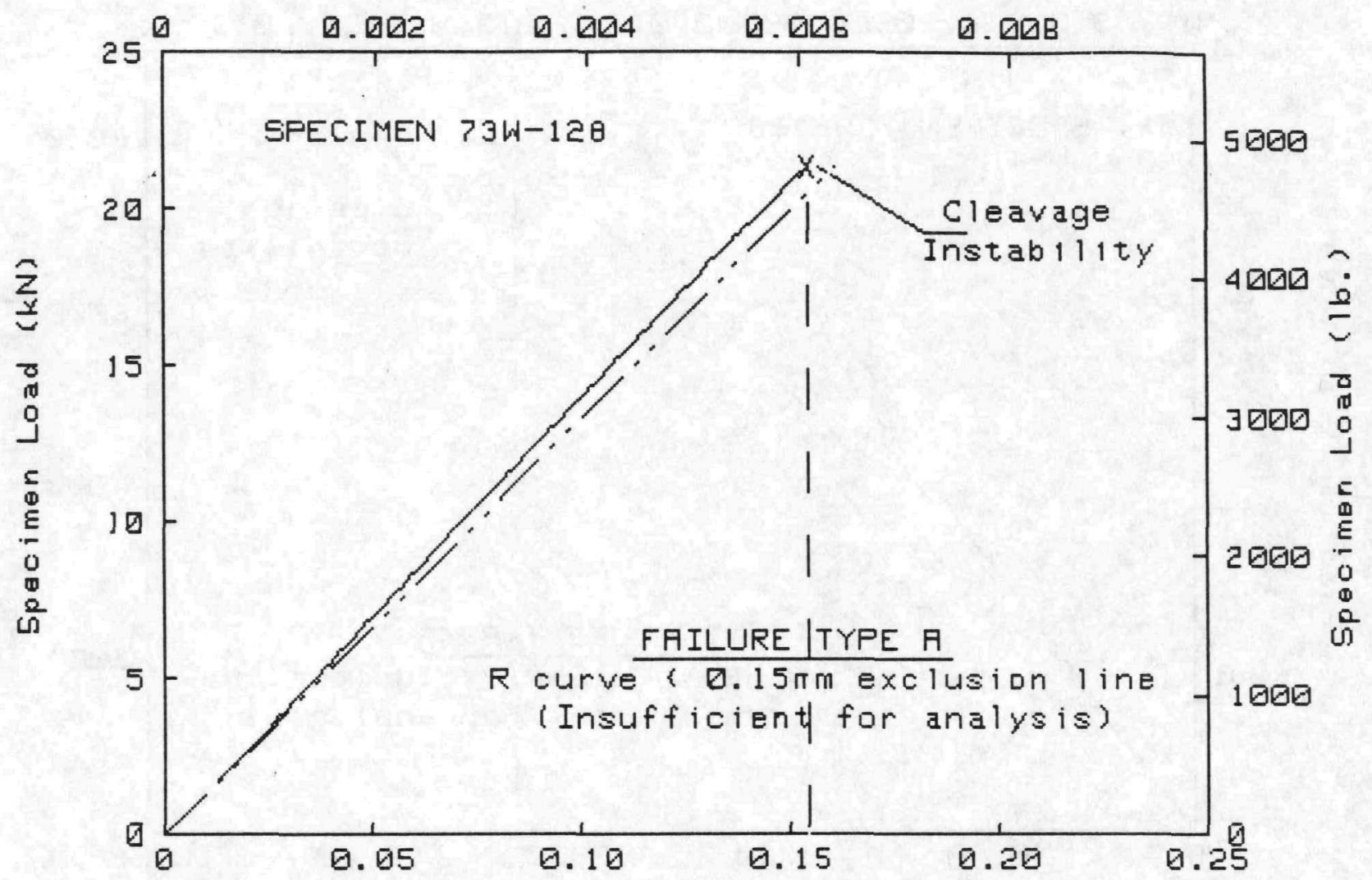

Spooimen Laadine Deflection (mm)

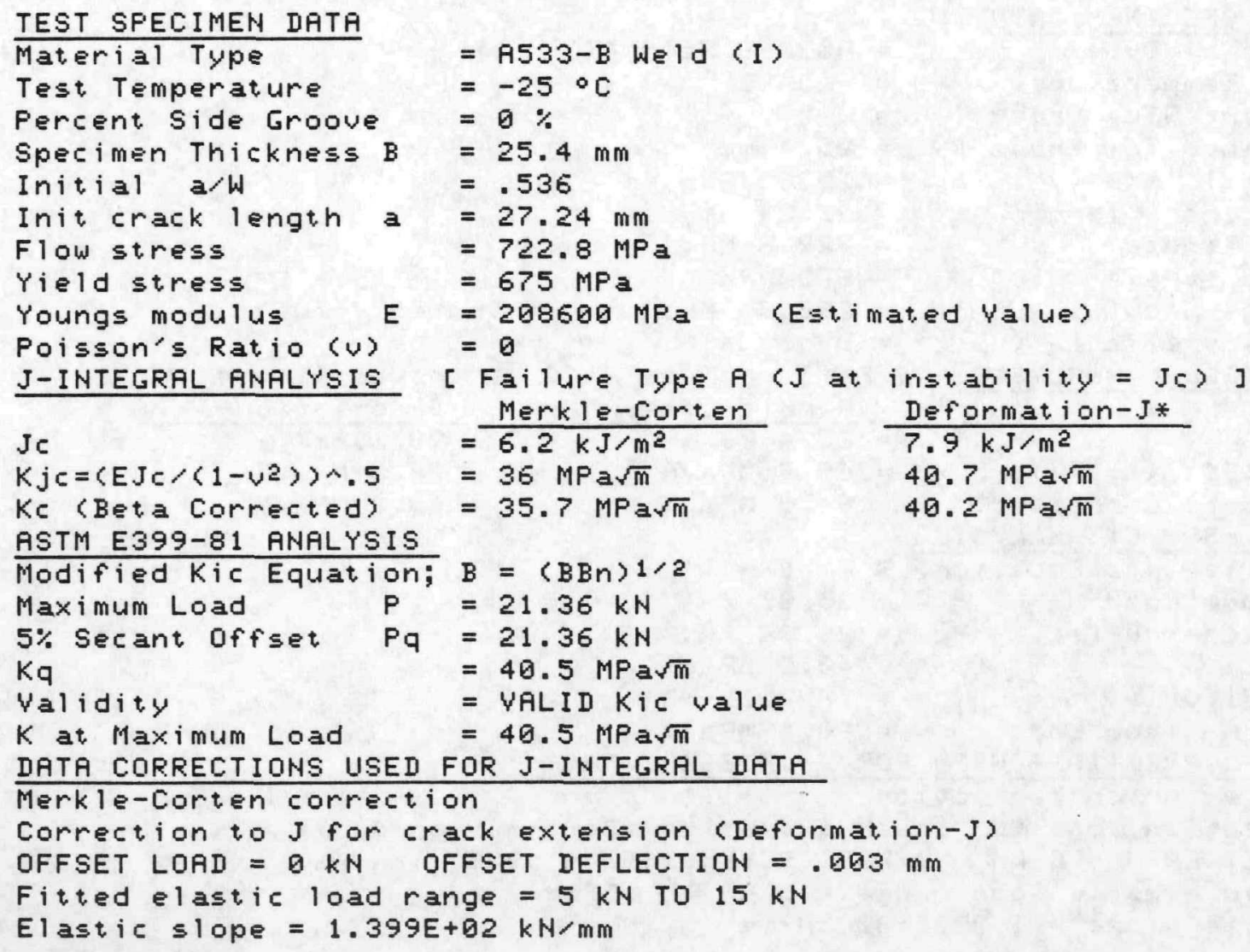


Specimen Crackmouth Def lection (in.)

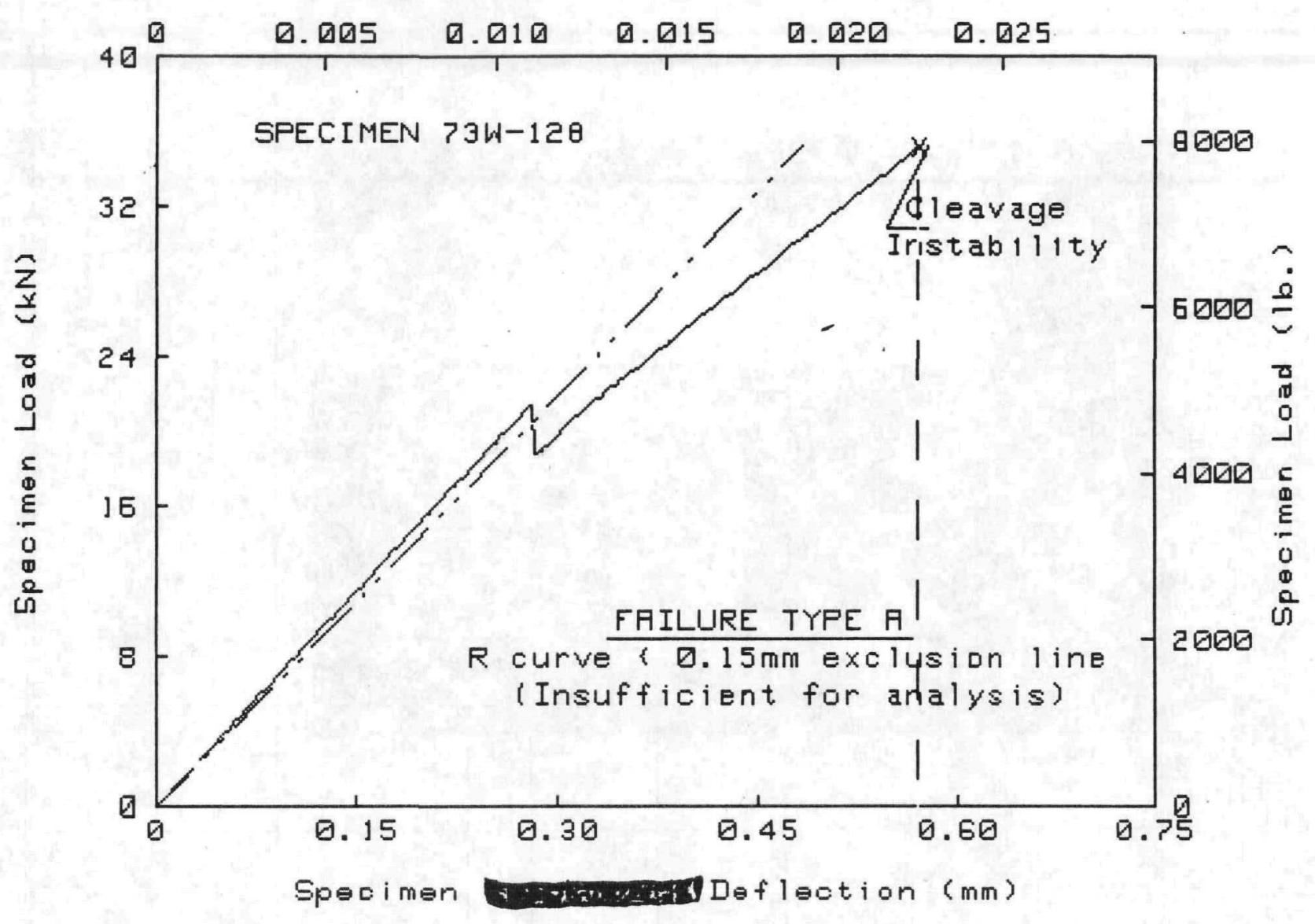

TEST SPECIMEN DATA

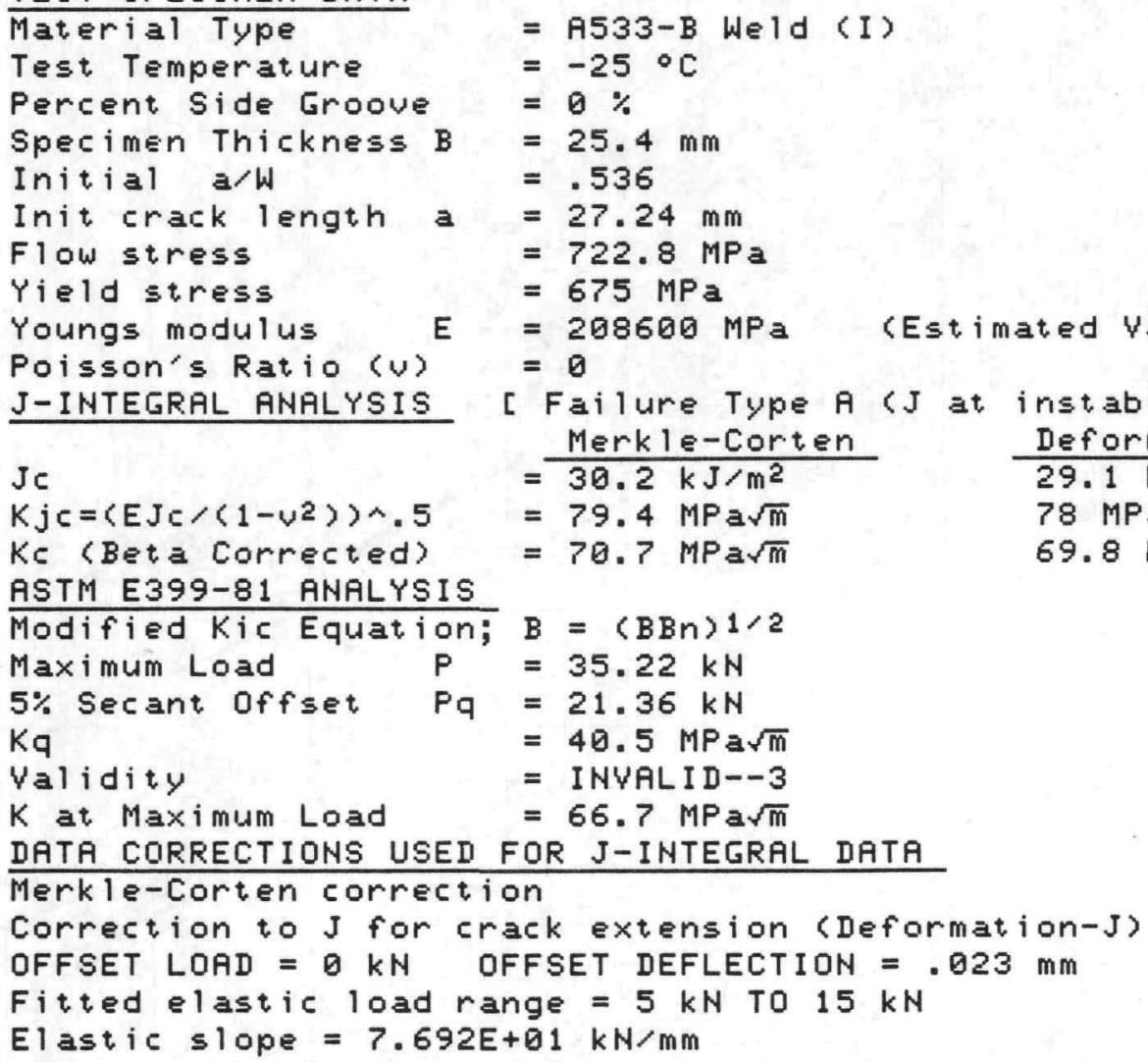




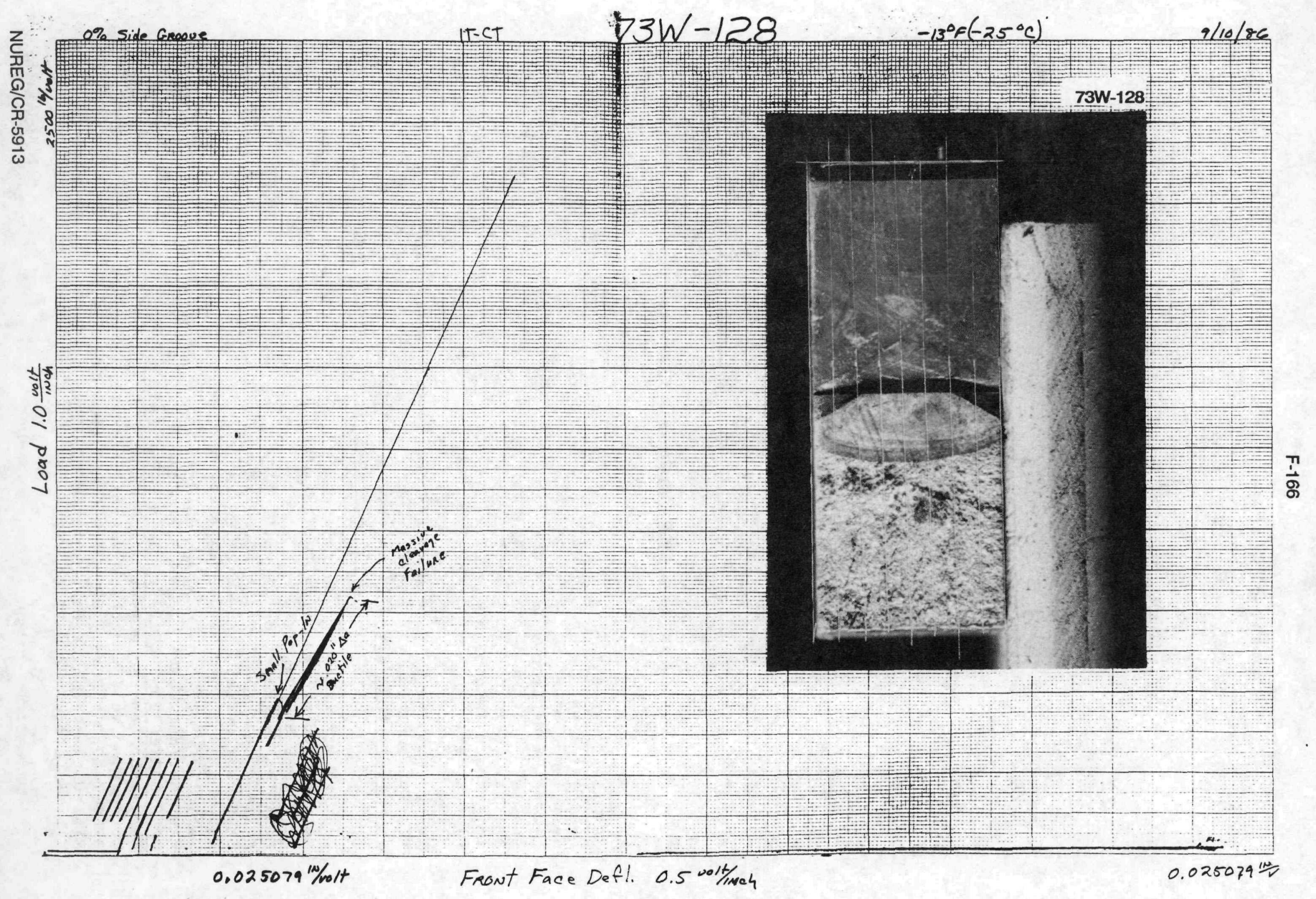


Specimen Crackmouth Deflectian (in.)

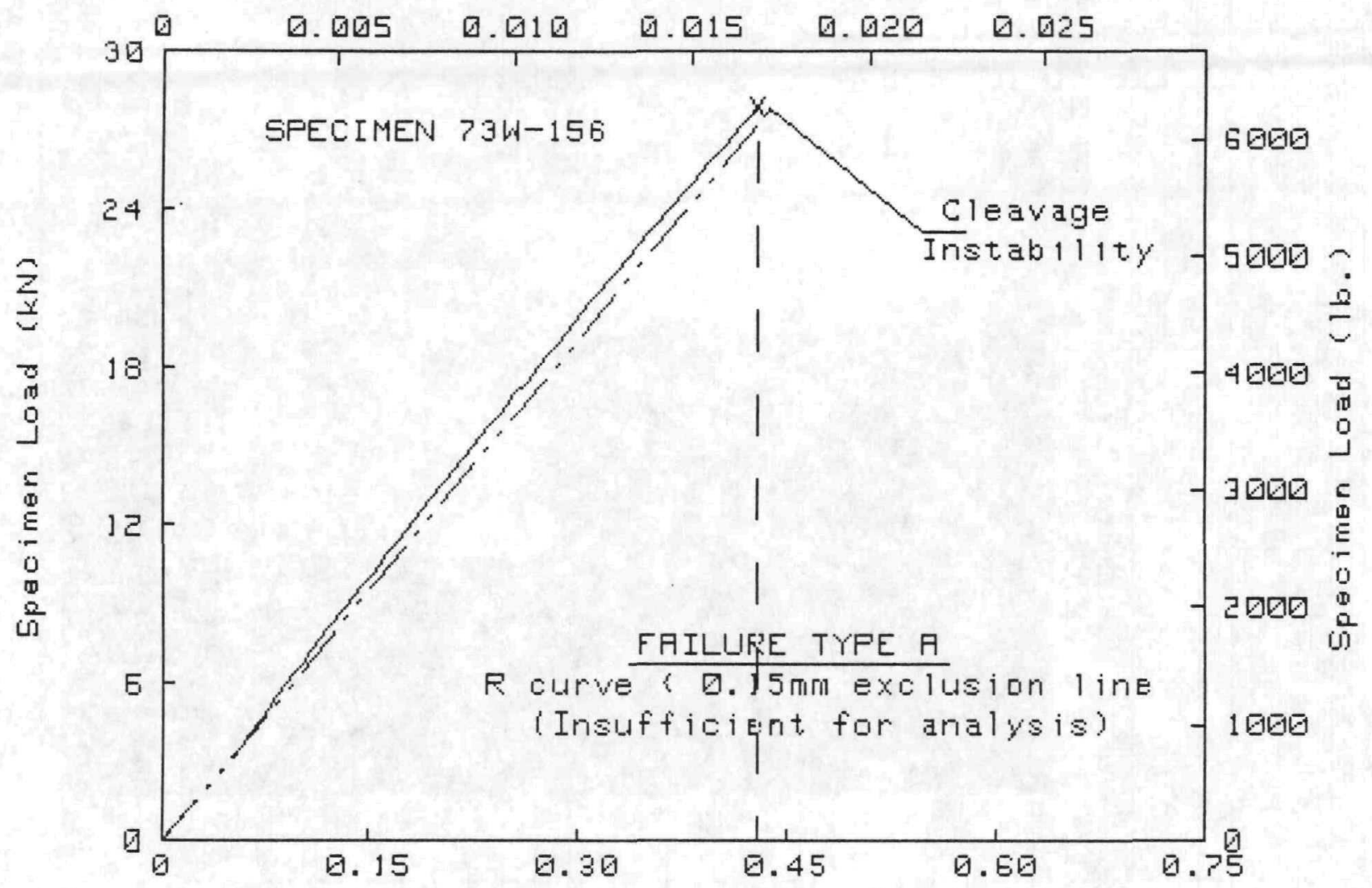

Specimen Crackmouth Defleotion (mm)

TEST SFECIMEN DATA

Material Type
Test Temperature
Percent Side Groove
Specimen Thickness B
Initial a/w
Init crack length a
Flow stress
Yield stress
Youngs modulus
Poisson's Ratio (U)
J-INTEGRAL ANALYSIS

Jc

$K j c=\left\langle E J c /\left(1-v^{2}\right\rangle\right) \wedge .5$

$\mathrm{Kc}$ (Beta Corrected)

ASTM E399-81 ANALYSIS

Modified Kic Equation; $B=\left(B B_{n}\right) 1 / 2$

Maximum Load $P=27.87 \mathrm{kN}$

5\% Secant offset $\mathrm{Pq}=27.87 \mathrm{kN}$

$\mathrm{Kq}$

Validity

$K$ at Maximum Load

$=61.5 \mathrm{MPa} \sqrt{\mathrm{m}}$

= VALID Kic value

$=61.5 \mathrm{MPa} \sqrt{\mathrm{m}}$

$=$ A533-B Weld (I)

$=-25^{\circ} \mathrm{C}$

$=0 \%$

$=722.8 \mathrm{MPa}$

$=675 \mathrm{MPa}$

$=208600 \mathrm{MPa} \quad$ (Estimated Value)

$=0$

Failure Type A (J at, instability = Jc) J

$=\frac{\text { Merkle-Corten }}{18.4 \mathrm{~kJ} / \mathrm{m}^{2}} \quad \frac{\text { Deformation-J* }}{18.9 \mathrm{~kJ} / \mathrm{m}^{2}}$

$=61.9 \mathrm{MPa} \sqrt{\mathrm{m}}$

$=58.5 \mathrm{MPa} \sqrt{\mathrm{m}}$

$62.8 \mathrm{MPa} \sqrt{\mathrm{m}}$

$59.1 \mathrm{MPa} \sqrt{\mathrm{m}}$

DATA CORRECTIONS USEI FOR J-INTEGRAL IATA

Merkle-Corten correction

Correction to J for crack extension (Deformation-J)

OFFSET LOAD $=0 \mathrm{kN}$ OFFSET DEFLECTION $=.019 \mathrm{~mm}$

Fitted elastic load range $=5 \mathrm{kN}$ To $15 \mathrm{kN}$

Elastic slope $=6.689 \mathrm{E}+01 \mathrm{kN} / \mathrm{mm}$ 


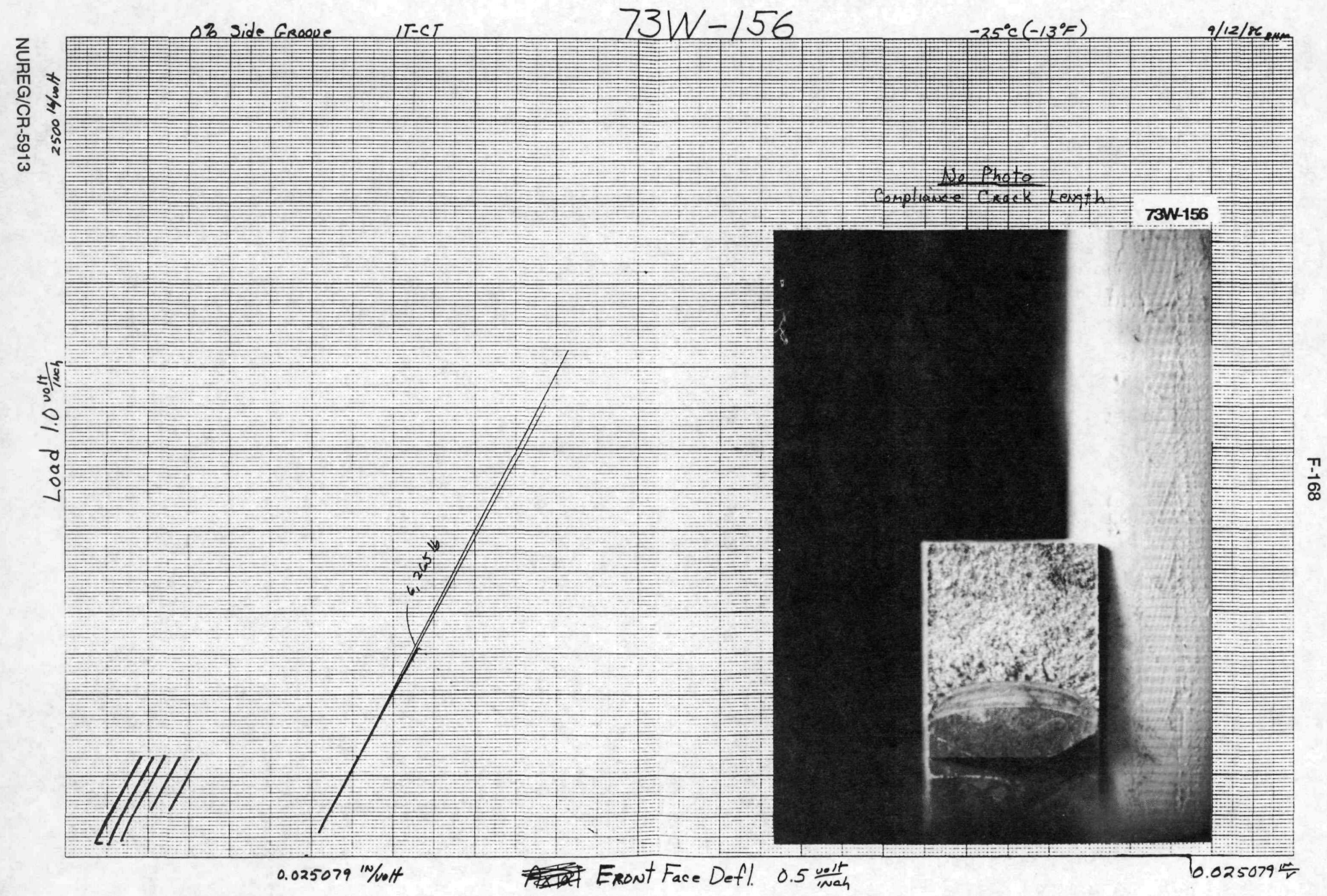


SPECIMEN ID $=73 W 130$

MATERIAL TYPE = HCUWELD

TEST TEMPERATURE $=25$ DEG C

THICKNESS $=25.5 \mathrm{~mm}$

SIDE GROOUES $=0 \%$

INITIAL CRACK LENGTH $=27.4 \mathrm{~mm}$

$a / W) i=.539$

MEASURED DUCTILE DELTA-A $=.05 \mathrm{~mm}$

YIELD STRENGTH $=621 \mathrm{MPa}$

FLOW STRENGTH $=672 \mathrm{MPa}$

ESTIMATED YOUNG'S MDDULUS $=206$ GPa

SPECIMEN CLEAUED AT KQ $=63.8 \mathrm{MPa} S Q R[\mathrm{~m}]$ (NDT UALID KIc)

UALUES AT MAXIMUM LDAD

- - - - -

J-INTEGRAL $=25.4 \mathrm{~kJ} / \mathrm{m}^{\wedge} 2$

$\mathrm{KJ} c=72.3 \mathrm{MPa} S \mathrm{SQ}[\mathrm{m}]$

$K-b e t a-c=64.6 \mathrm{MPa} \operatorname{SQR}[\mathrm{m}]$
Walues at cleavage load

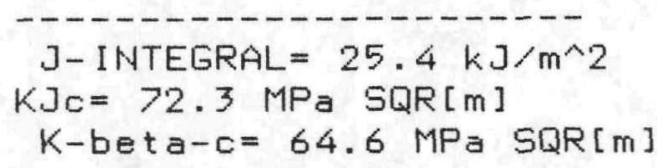


F-170

ORNL-DWG 92-11370

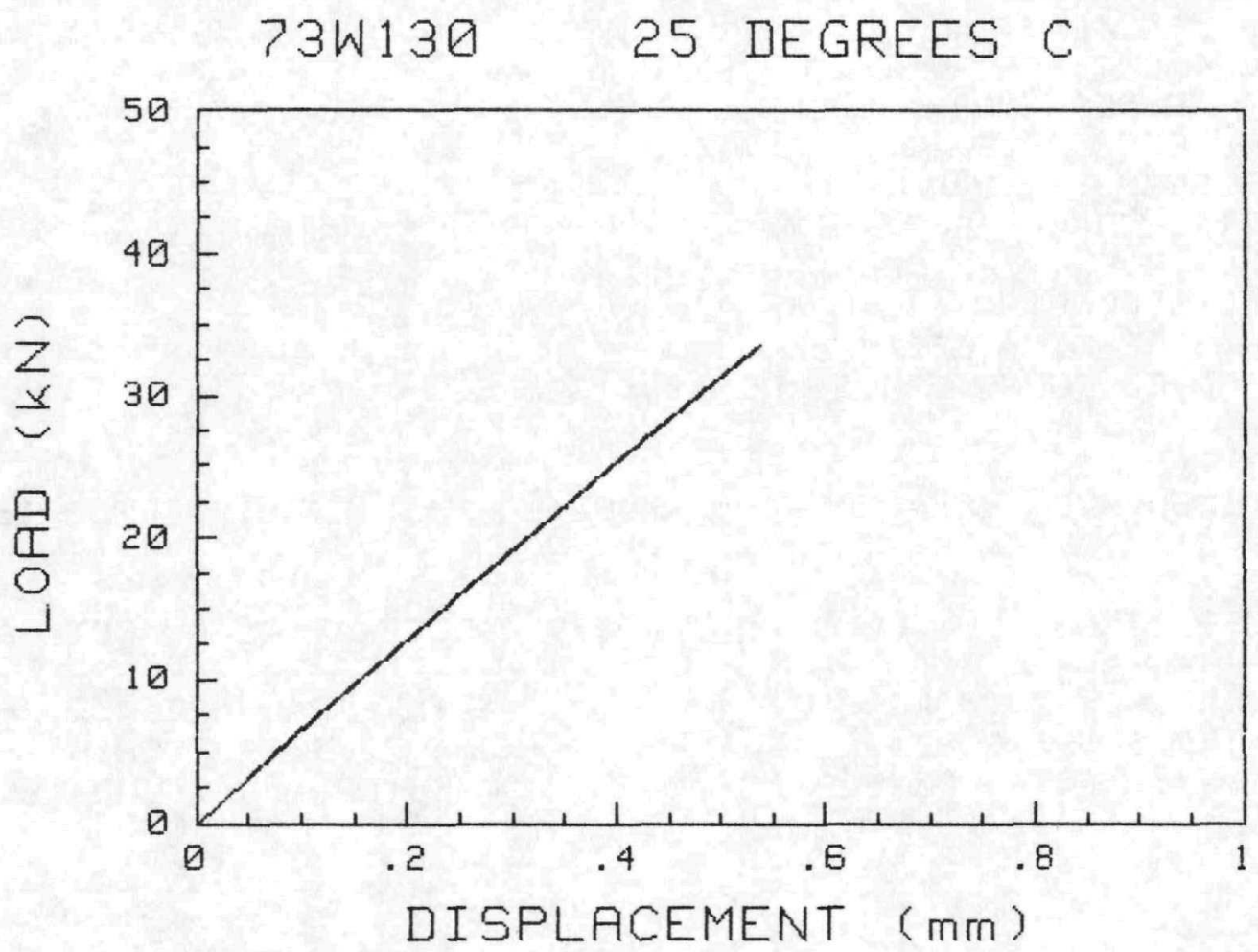


Specimen Loadline Deflection (in.)

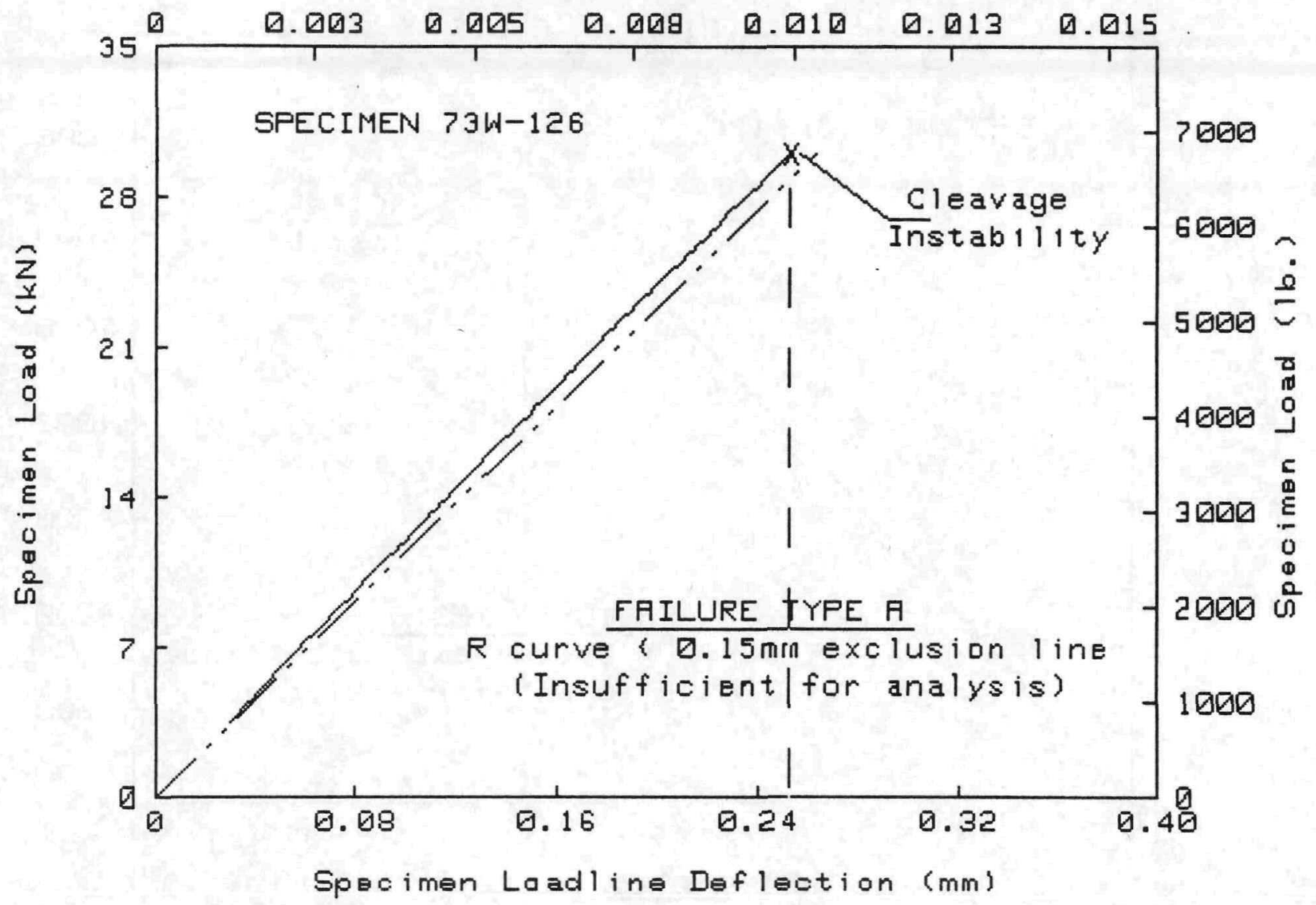

TEST SPECIMEN DATA

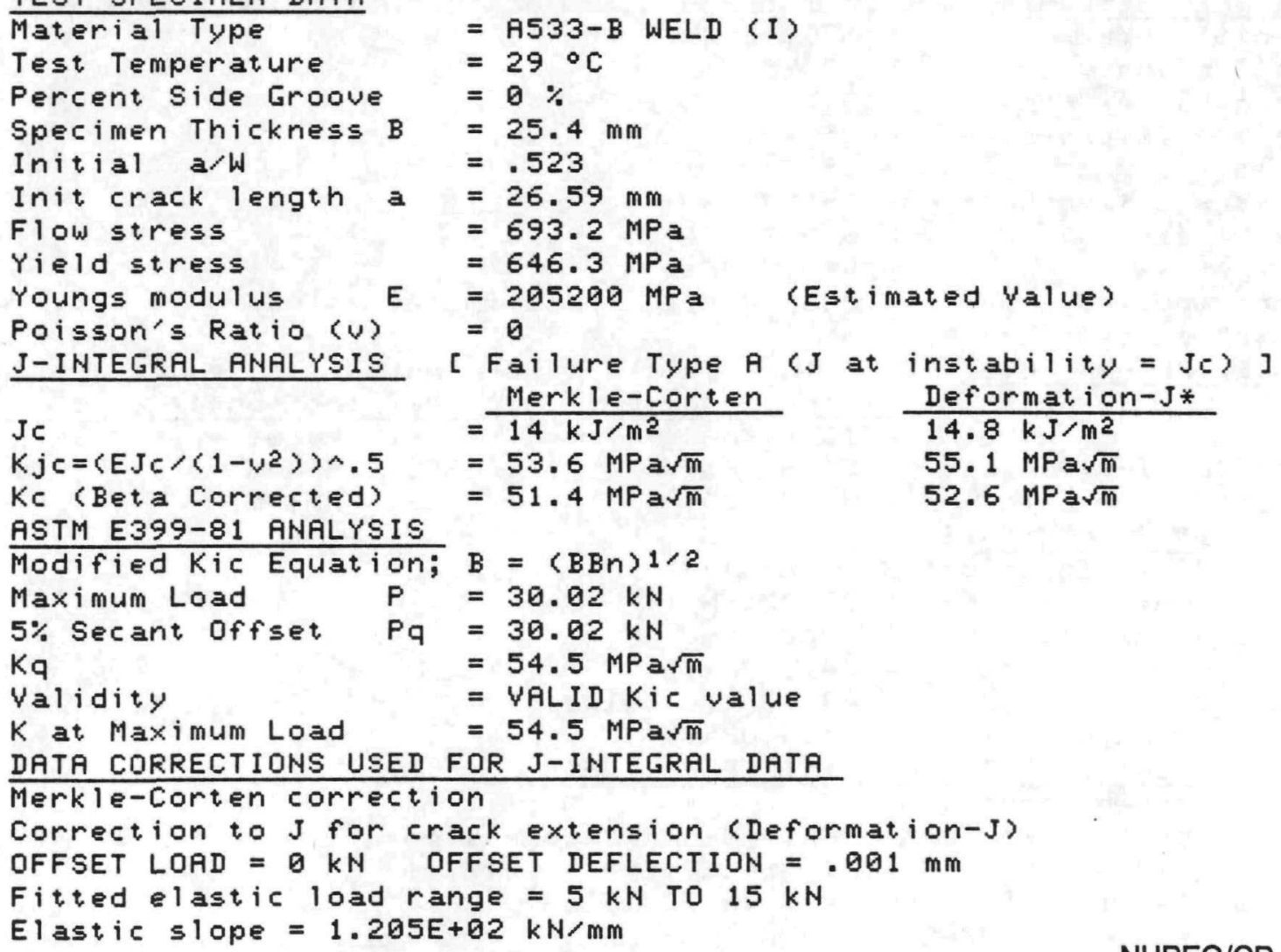


Specimen Crackmouth Deflection (in.)

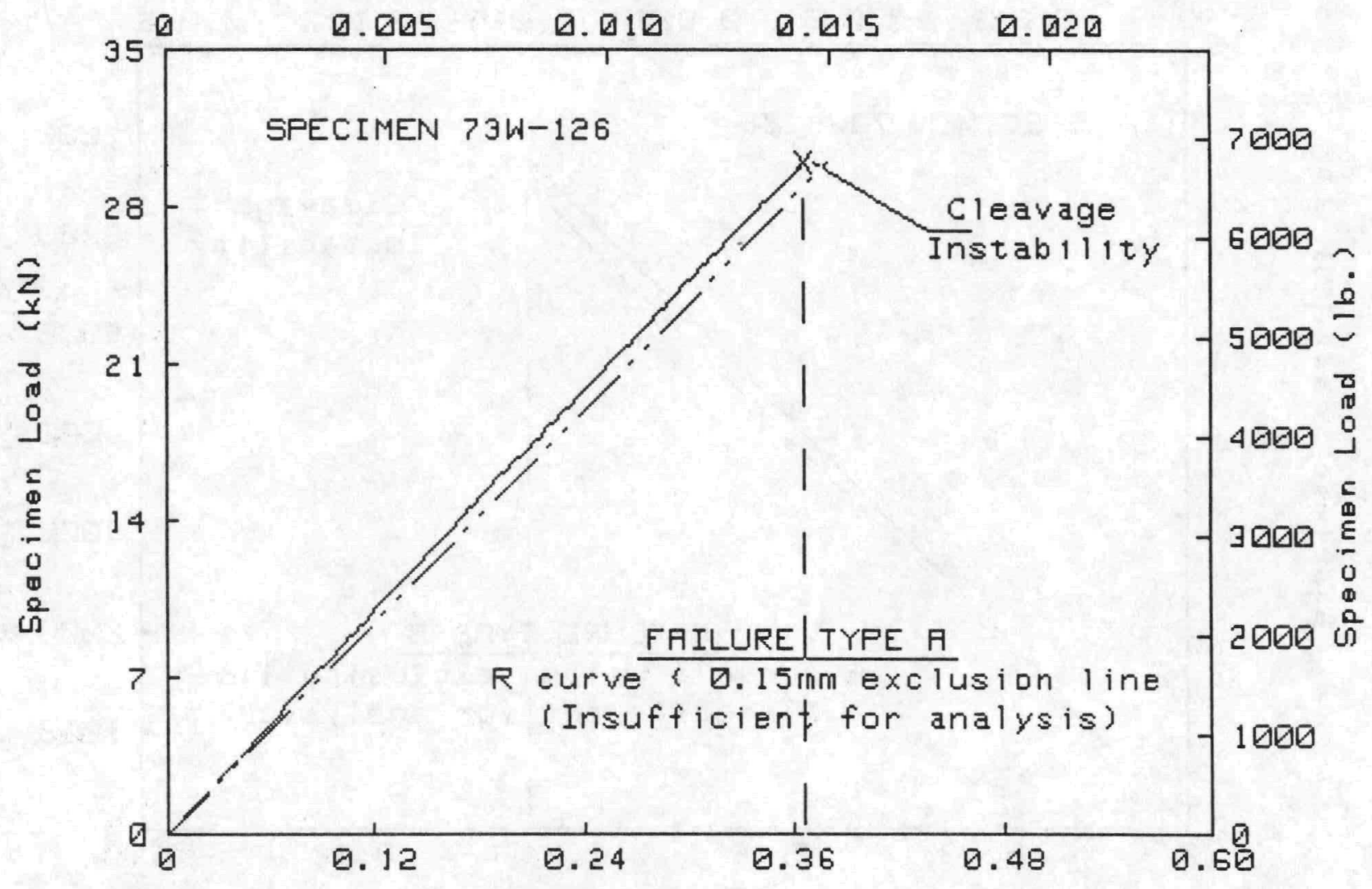

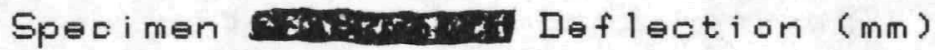

TEST SPECIMEN DATA

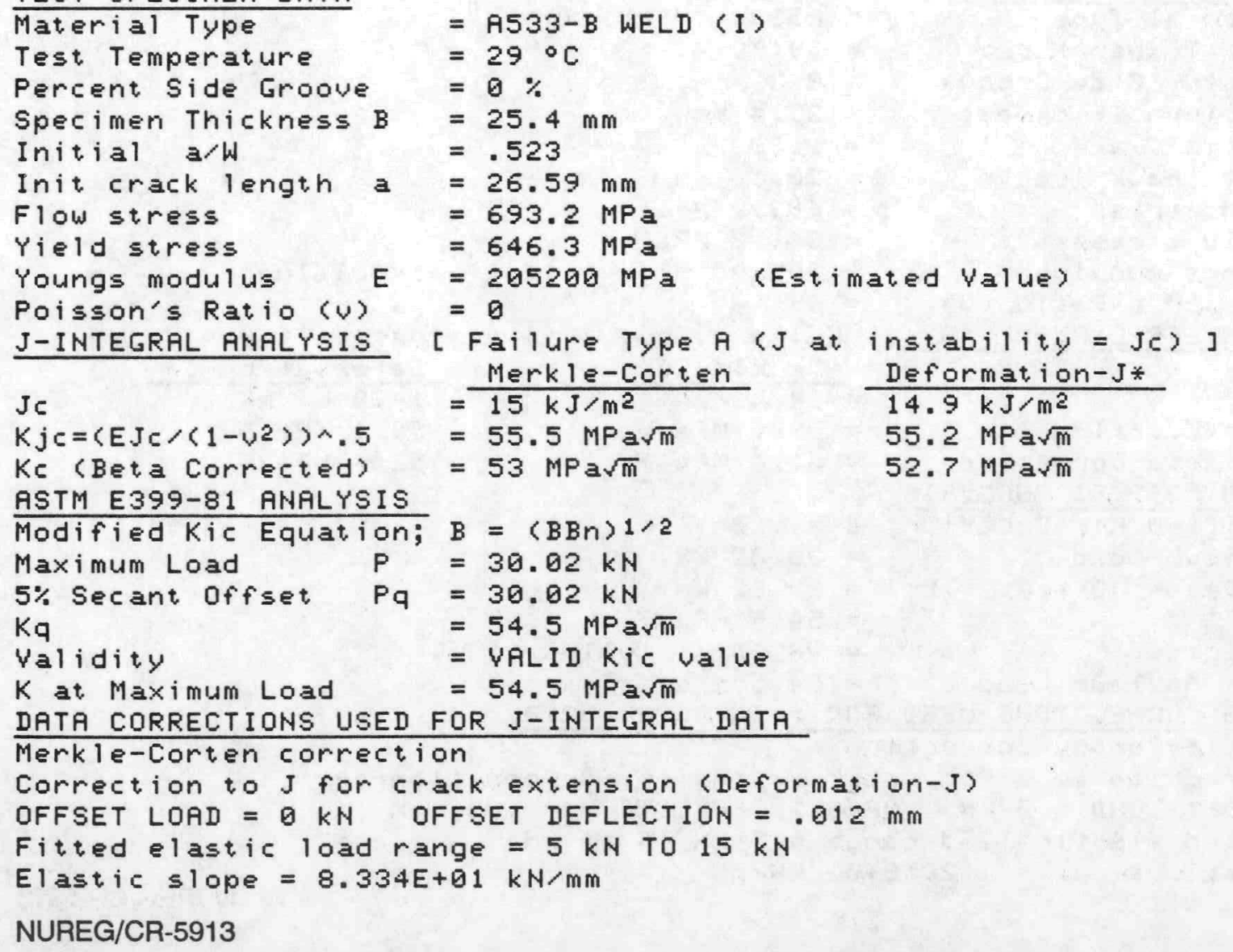




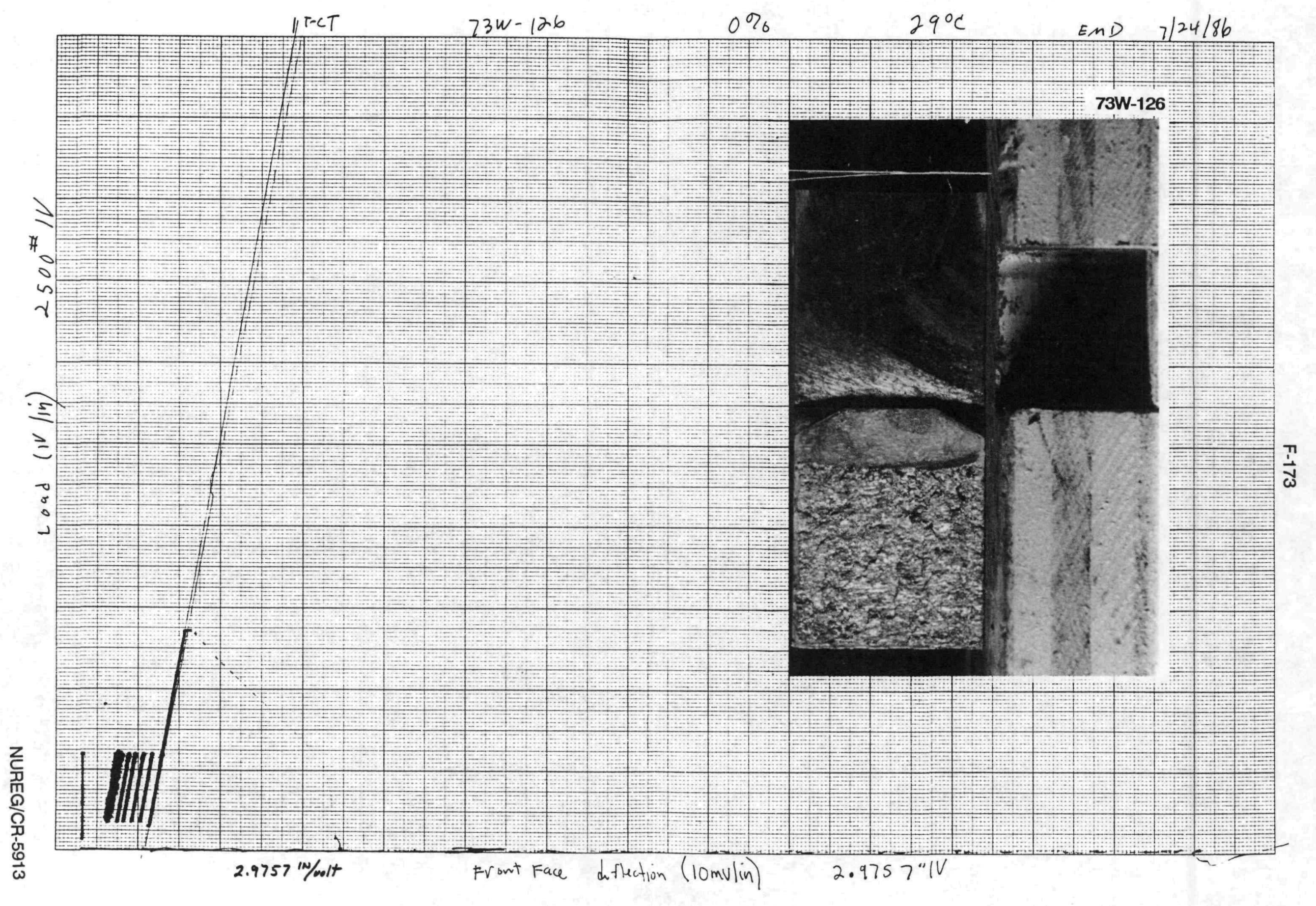


Specimen Loadline Deflection (in.)
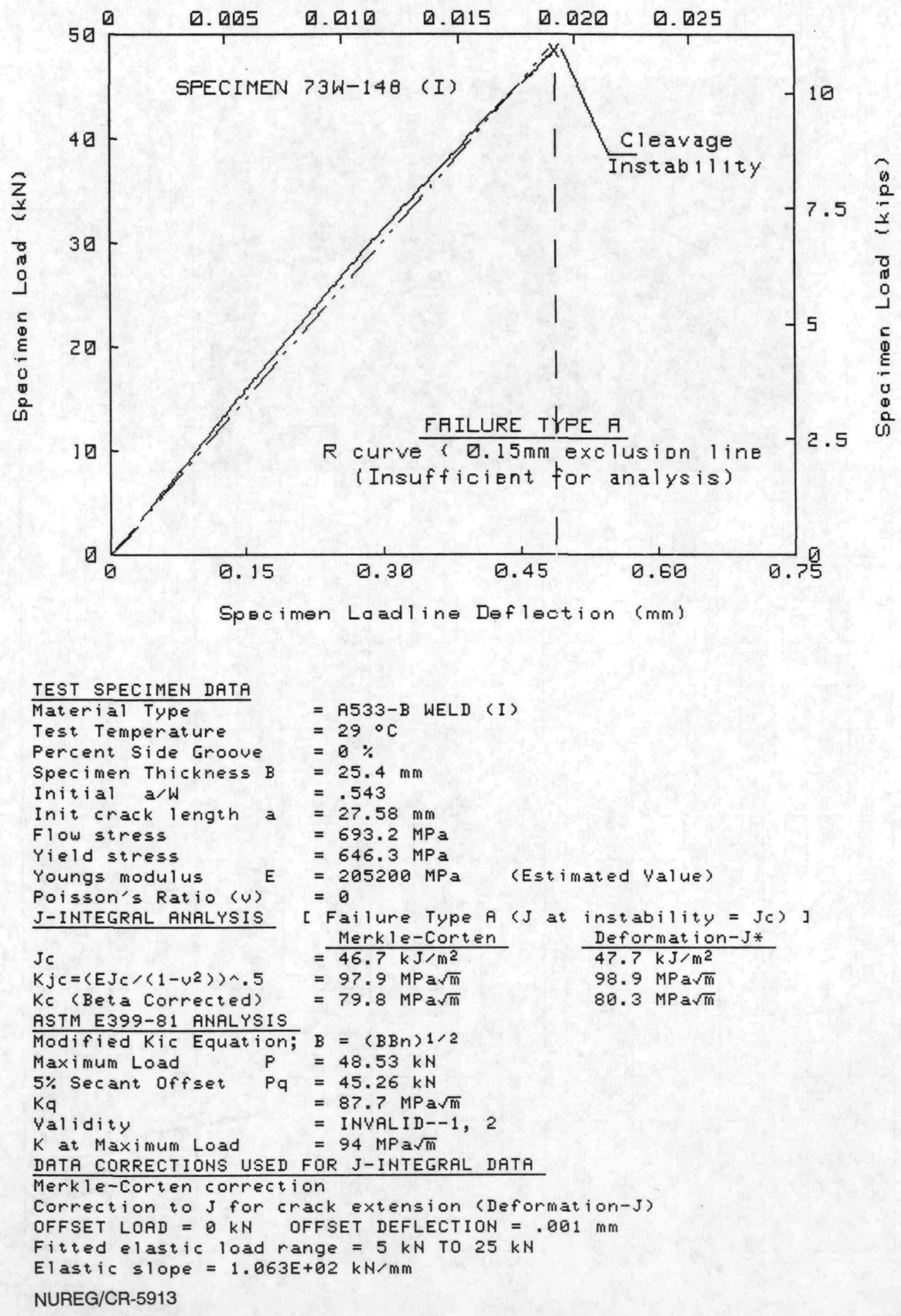
Specimen Crackmouth Deflection (in.)

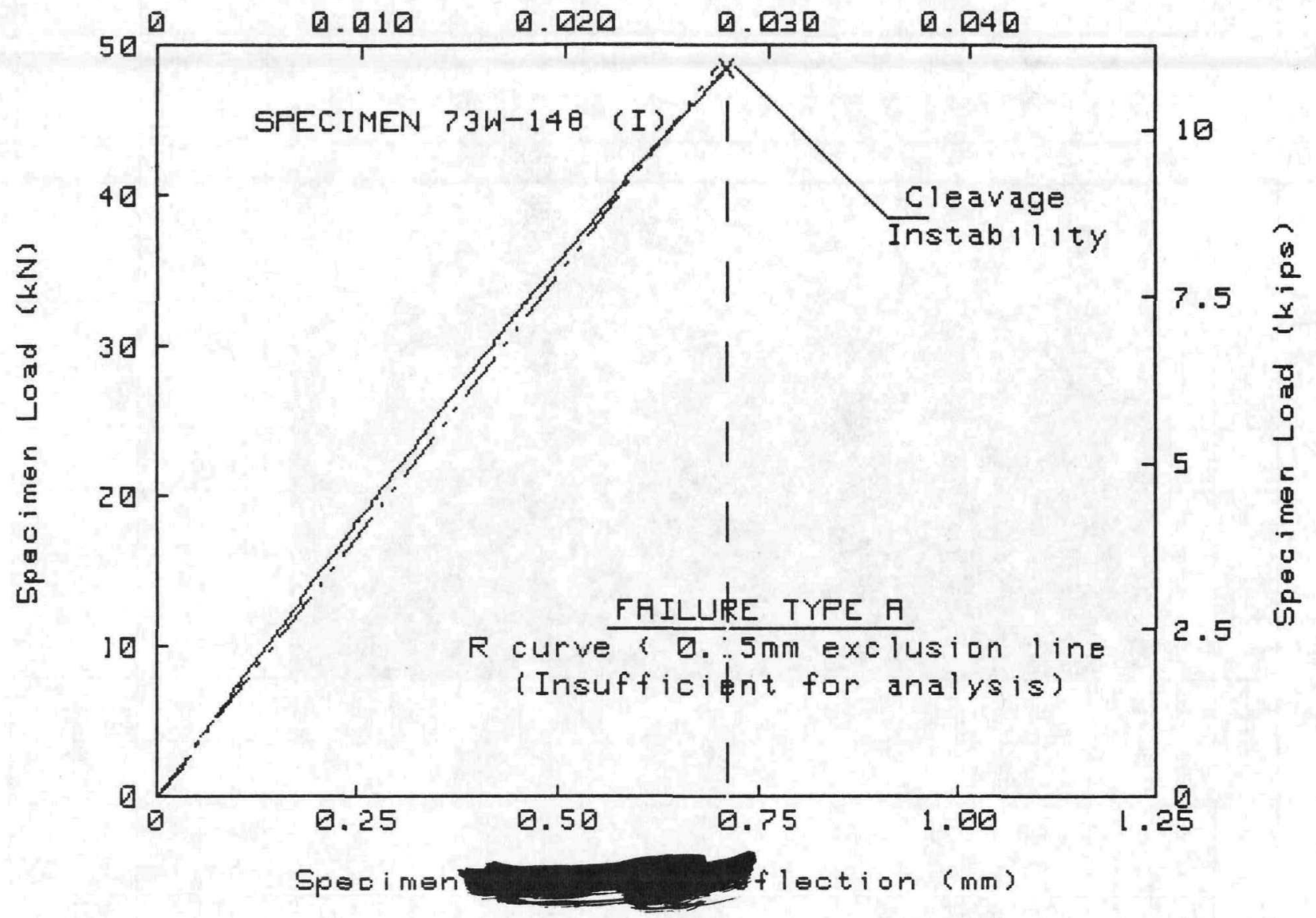

TEST SPECIMEN DATA

Material Type
Test Temperature
Percent Side Groove
Specimen Thickness B
Initial a/W
Init crack length a
Flow stress
Yield stress
Youngs modulus
Poisson's Ratio (U)
J-INTEGRAL ANALYSIS

$=A 533-B$ WELD (I)

$=29^{\circ} \mathrm{C}$

$=0 \%$

$=25.4 \mathrm{~mm}$

$=.543$

$=27.58 \mathrm{~mm}$

$=693.2 \mathrm{MPa}$

$=646.3 \mathrm{MPa}$

$=205200 \mathrm{MPa}$

(Estimated Value)

$=0$

J-INTEGRAL ANALYSIS

$\mathrm{Jc}$

[ Failure Type $A$ ( $J$ at instability = Jc) ]

$\mathrm{Kj} c=\left\langle\mathrm{E} . \mathrm{J} c /\left(1-v^{2}\right\rangle\right) \wedge .5$

$=\frac{\text { Merkle-Corten }}{50.4 \mathrm{~kJ} / \mathrm{m}^{2}}$

Deformation-J*

$\mathrm{Kc}$ (Beta Corrected)

$=101.7 \mathrm{MPa} \sqrt{\mathrm{m}}$

$99.4 \mathrm{MPa} \sqrt{\mathrm{m}}$

$=81.6 \mathrm{MPa} \sqrt{\mathrm{m}}$

$80.5 \mathrm{MPa} \sqrt{\mathrm{m}}$

ASTM E399-81 ANALYSIS

Modified Kic Equation; $B=(B B n) 1 / 2$

Maximum Load $P=48.53 \mathrm{kN}$

$5 \%$ Secant offset $\mathrm{Pq}=44.3 \mathrm{kN}$

$\mathrm{Kq}$

$=85.8 \mathrm{MPa} r \mathrm{~m}$

Validity

$K$ at Maximum Load

= INVALID--1, 2

$=94 \mathrm{MPa} \sqrt{\mathrm{m}}$

DATA CORRECTIONS USED FOR J-INTEGRAL DATA

Merkle-Corten correction

Correction to J for crack extension (Deformation-J)

OFFSET LOAD $=0 \mathrm{kN}$ OFFSET DEFLECTION $=.011 \mathrm{~mm}$

Fitted elastic load range $=5 \mathrm{kN}$ To $25 \mathrm{kN}$

Elastic slope $=7.310 E+01 \mathrm{kN} / \mathrm{mm}$ 


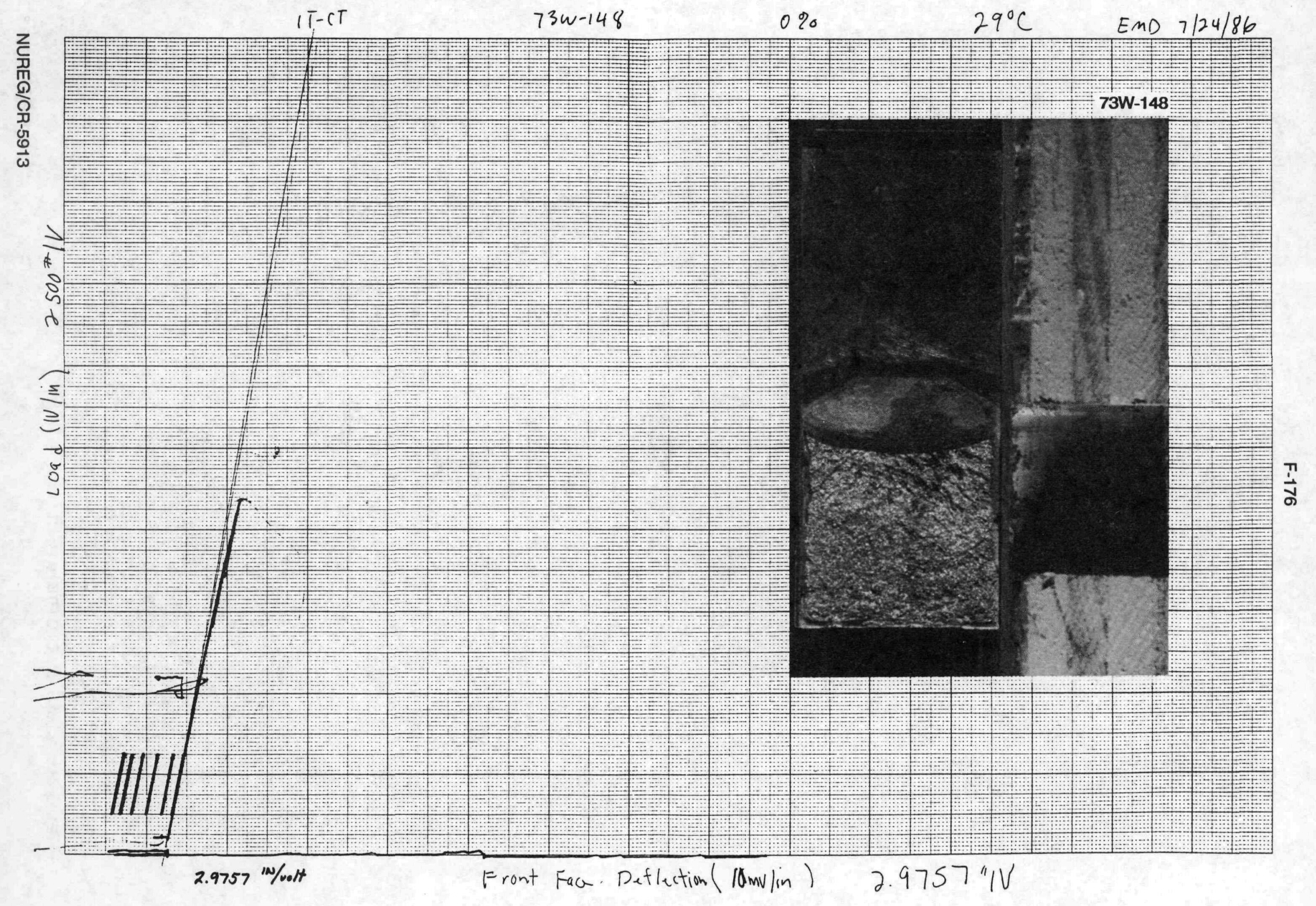


Specimen Loadline Deflection (in.)

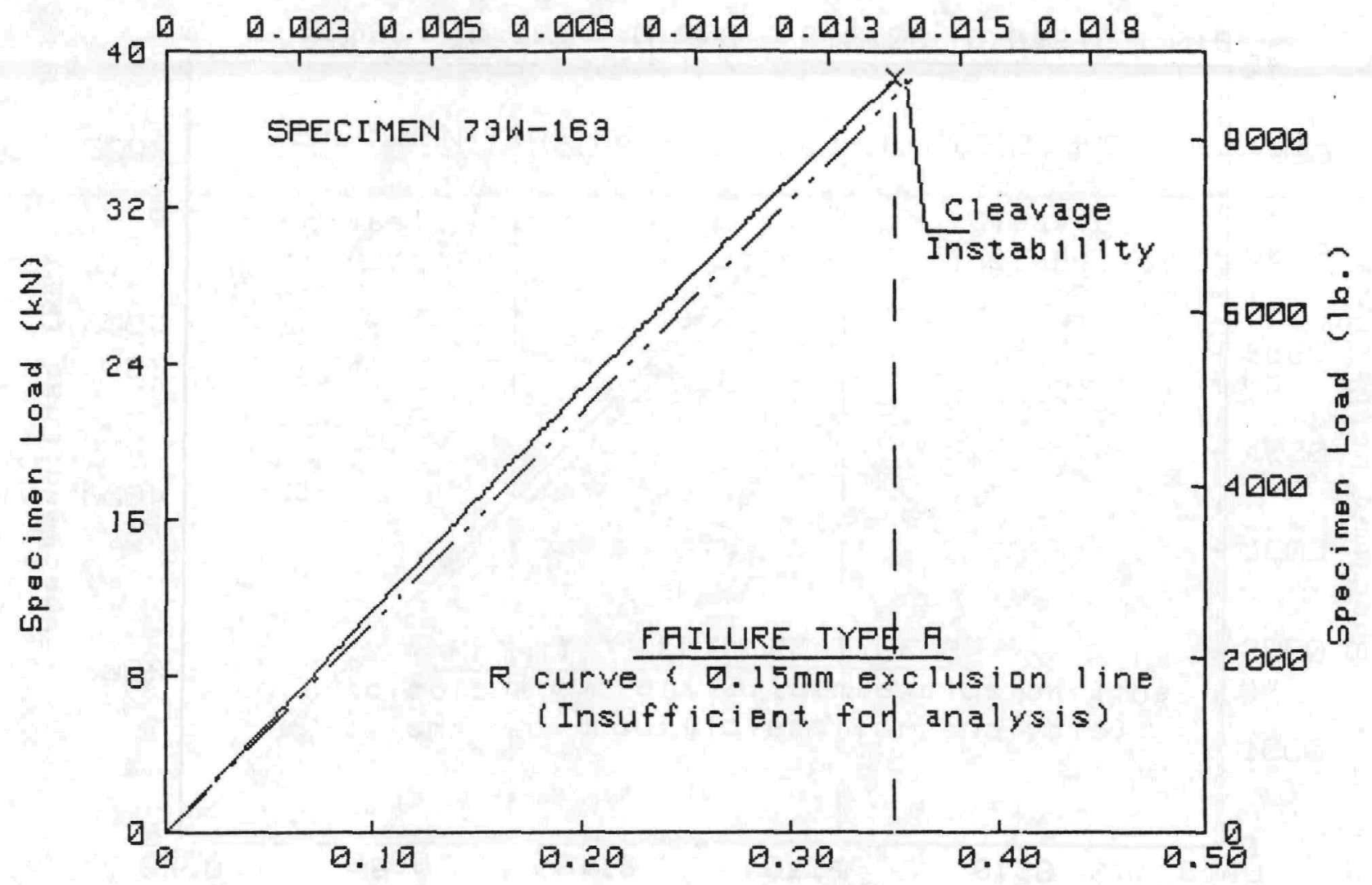

Specimen Laadiine Deflection (mm)

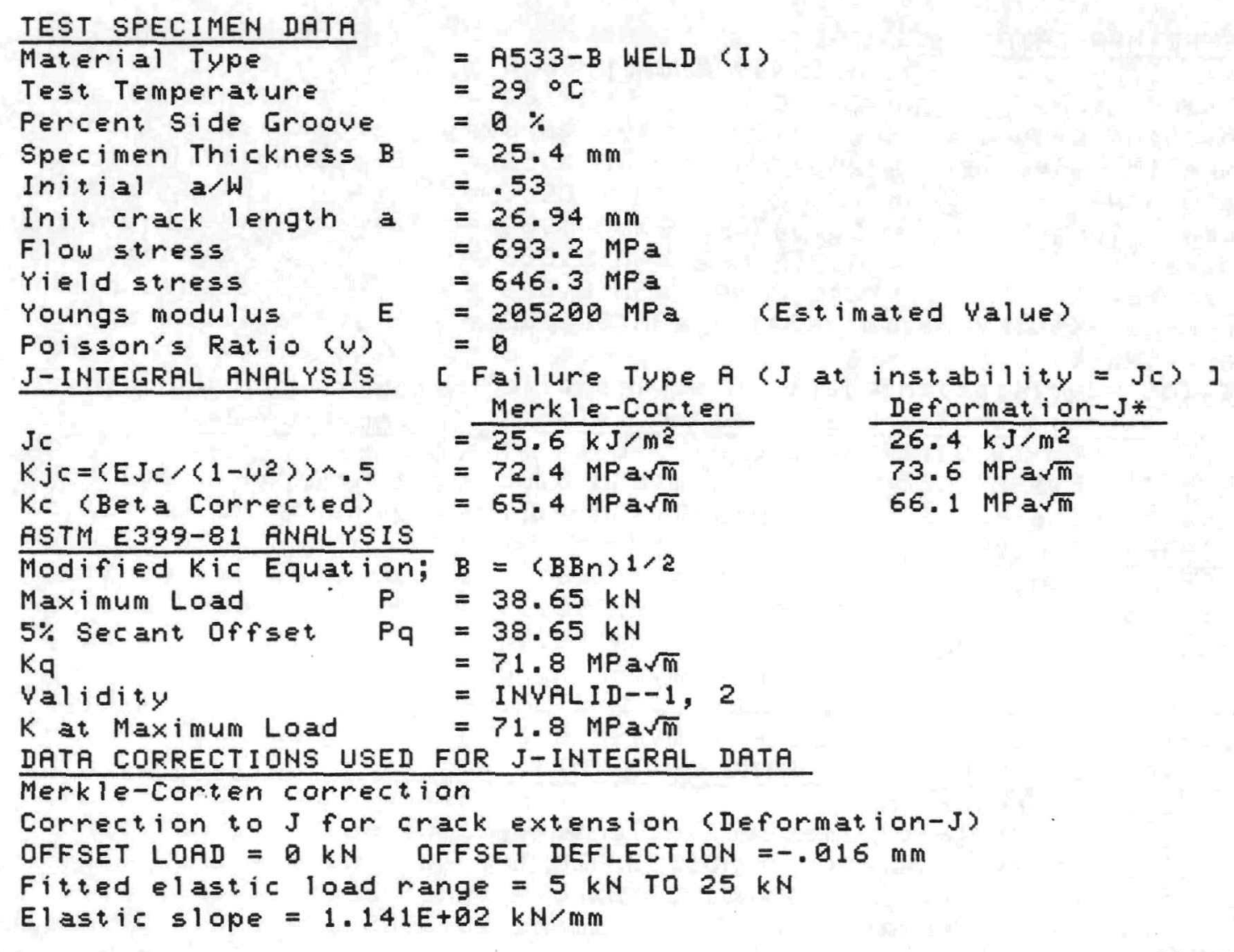


Specimen Crackmouth Deflection (in.)

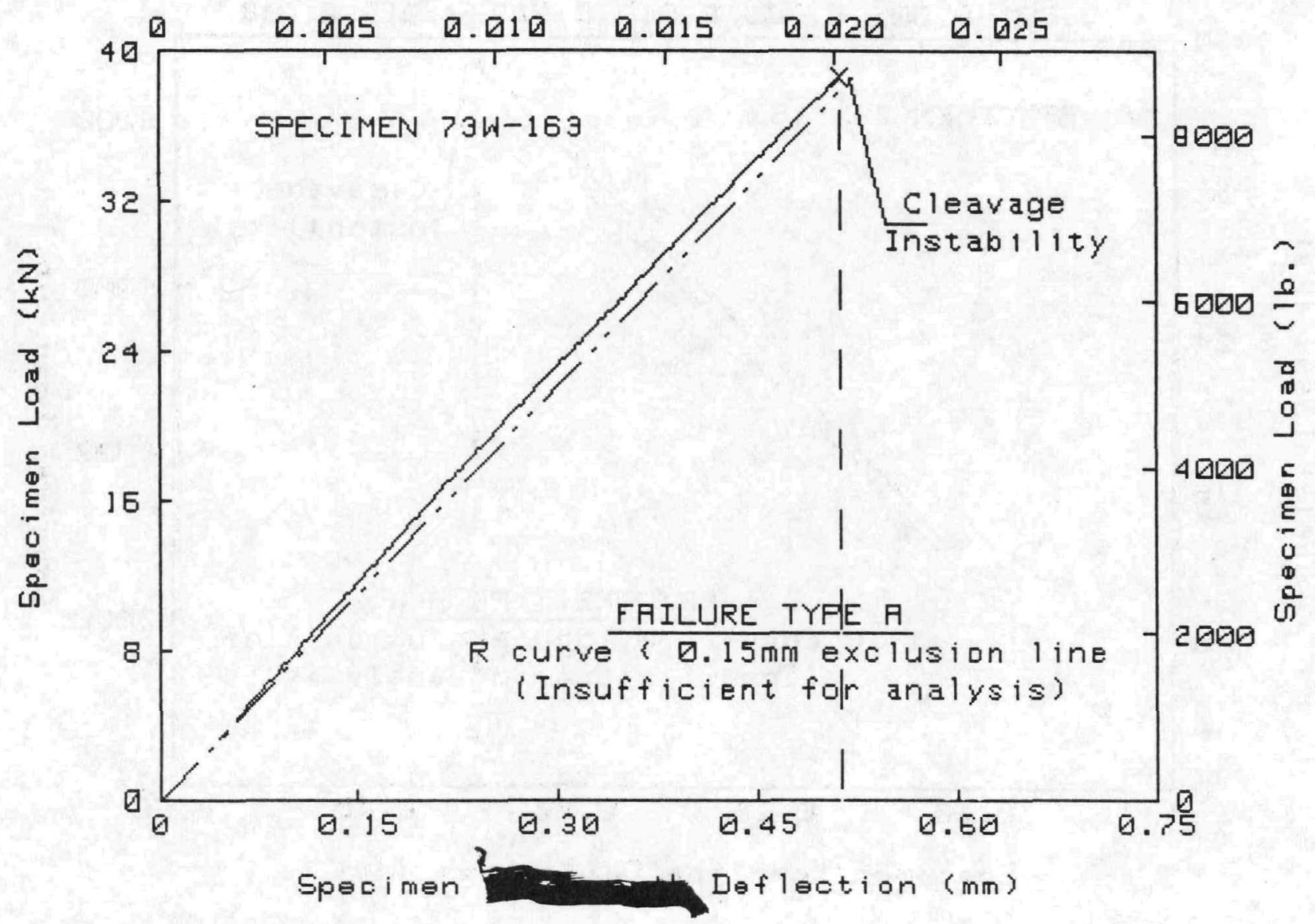

TEST SPECIMEN DATA

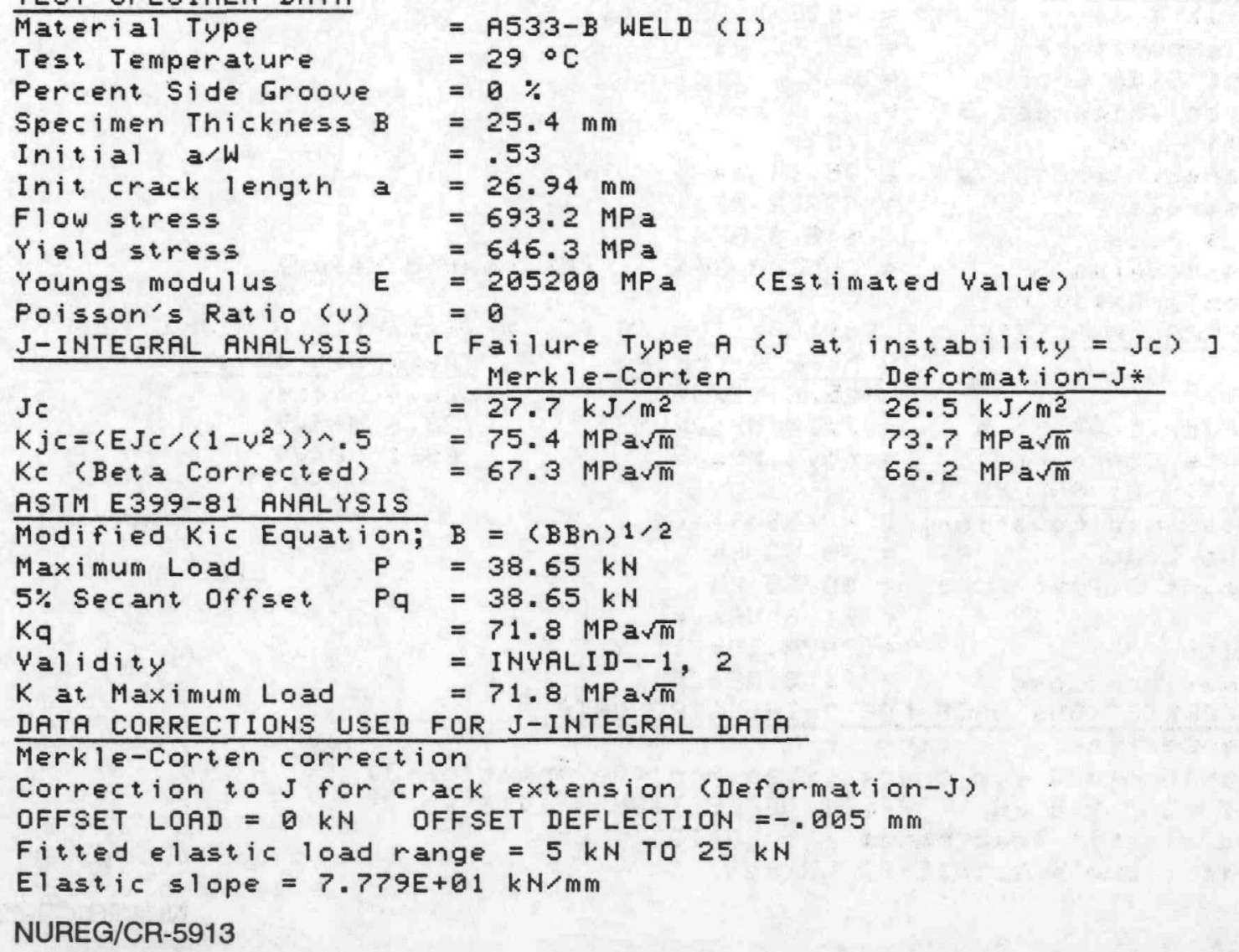




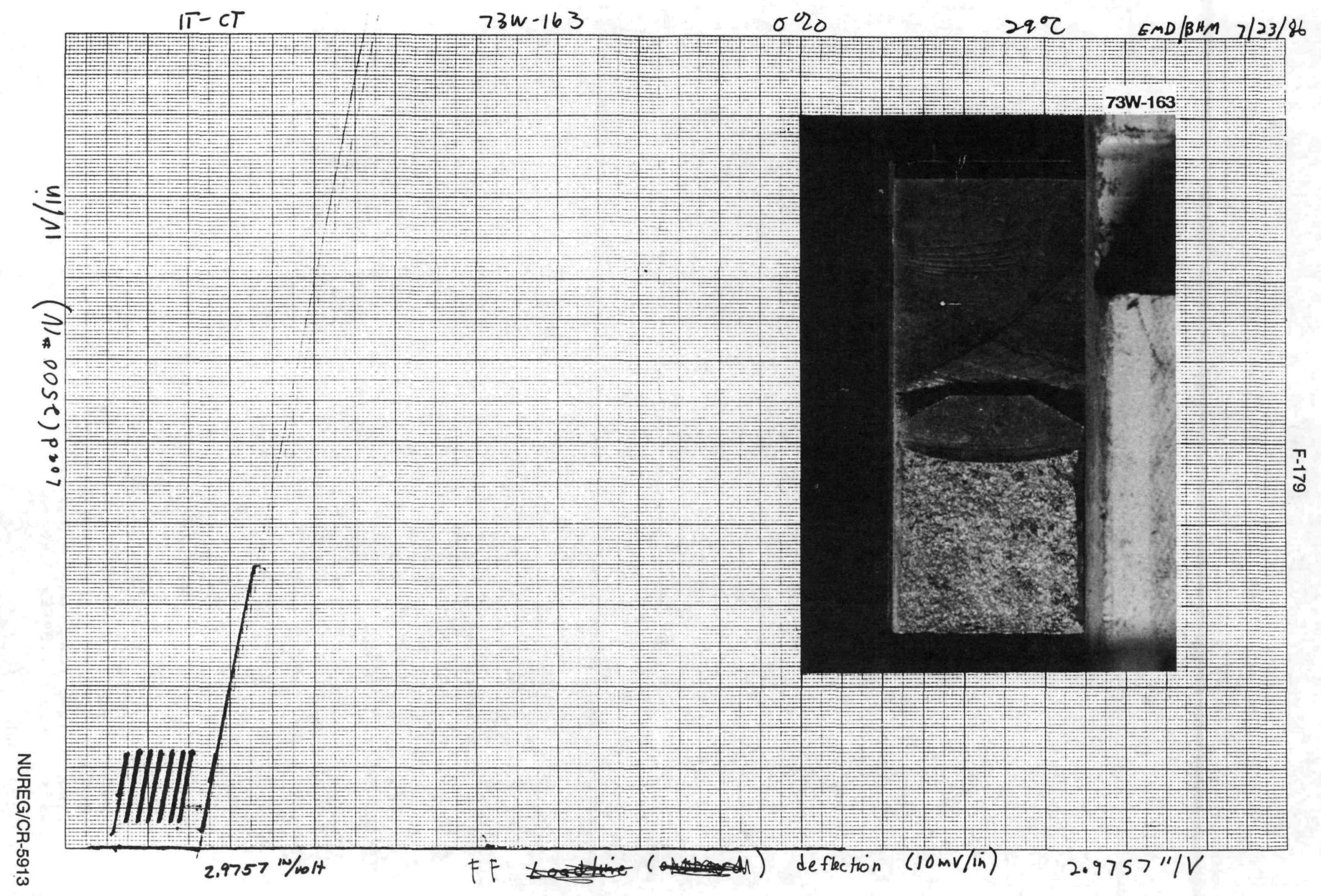


Specimen Loadline Deflection (in.)

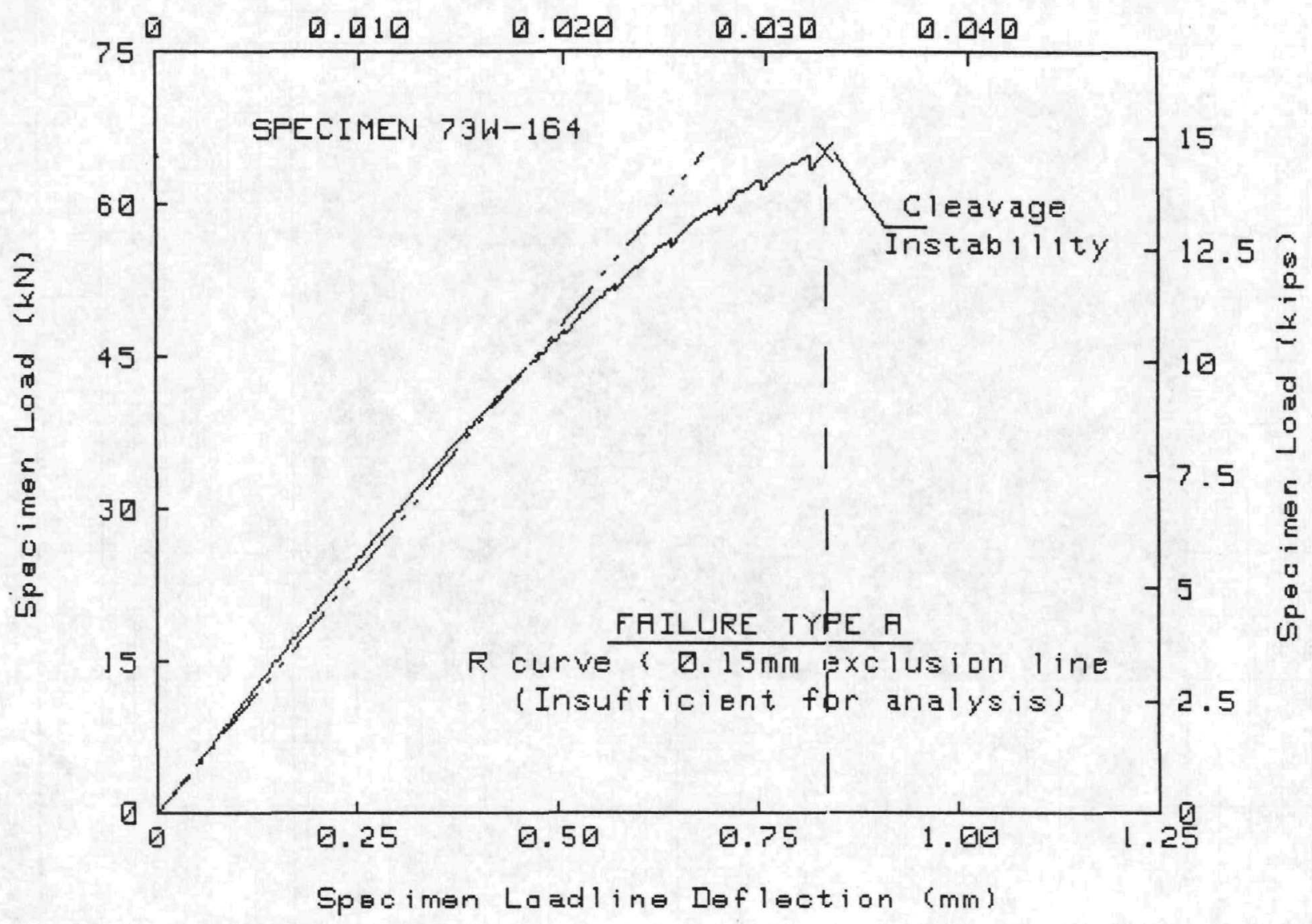

TEST SPECIMEN DATH

Material Type
Test Temperature
Percent Side Groove
Specimen Thickness B
Initial a/W
Init crack length a
Flow stress
Yield stress
Youngs modulus
Poisson's Ratio (U)
J-INTEGRAL RNALYSIS

$=A 533-B$ Weld (I)

$=50 \circ \mathrm{C}$

$=0 \%$

$=25.4 \mathrm{~mm}$

$=.547$

$=27.8 \mathrm{~mm}$

$=633.1 \mathrm{MPa}$

$=636.6 \mathrm{MPa}$

$=204400 \mathrm{MPa}$

$=0$

(Estimated Value)

[ Failure Type A ( $J$ at instability = Jc) ]

$\mathrm{Jc}$

$=\frac{\text { Merkle-Corten }}{120.6 \mathrm{~kJ} / \mathrm{m}^{2}}$

$\frac{\text { Deformat i on }-\mathrm{J} \text { * }}{120 \mathrm{~kJ} / \mathrm{m}^{2}}$

$K j c=\left\langle E J c /\left(1-v^{2}\right)\right\rangle \wedge .5$

Kc (Beta Corrected)

$=157$ MPa $\sqrt{m}$

$=101.4 \mathrm{MPa} \sqrt{\mathrm{m}}$ $156.6 \mathrm{MPa} \sqrt{\mathrm{m}}$ $101.3 \mathrm{MPa} \sqrt{\mathrm{m}}$

ASTM E399-81 ANALYSIS

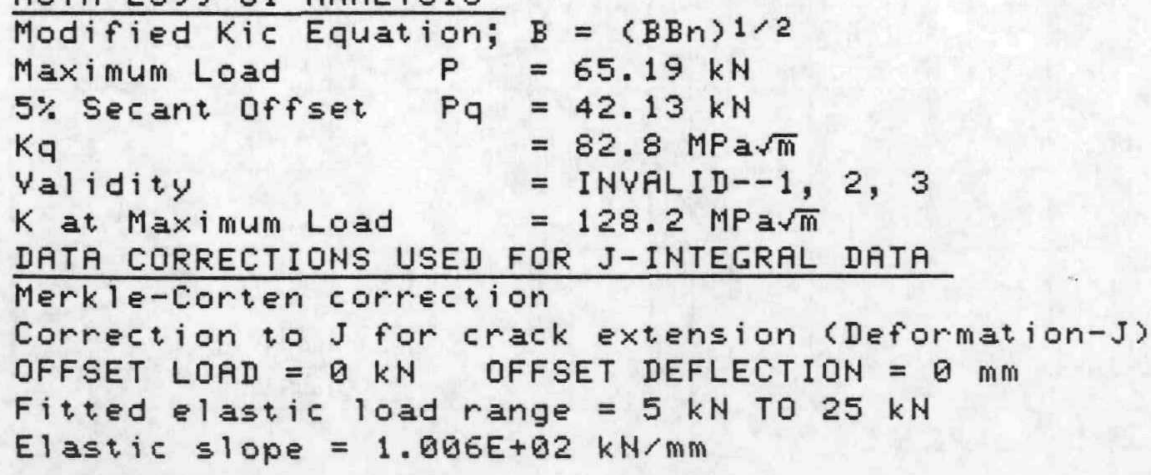


Specimen Crackmouth Deflection (in.)

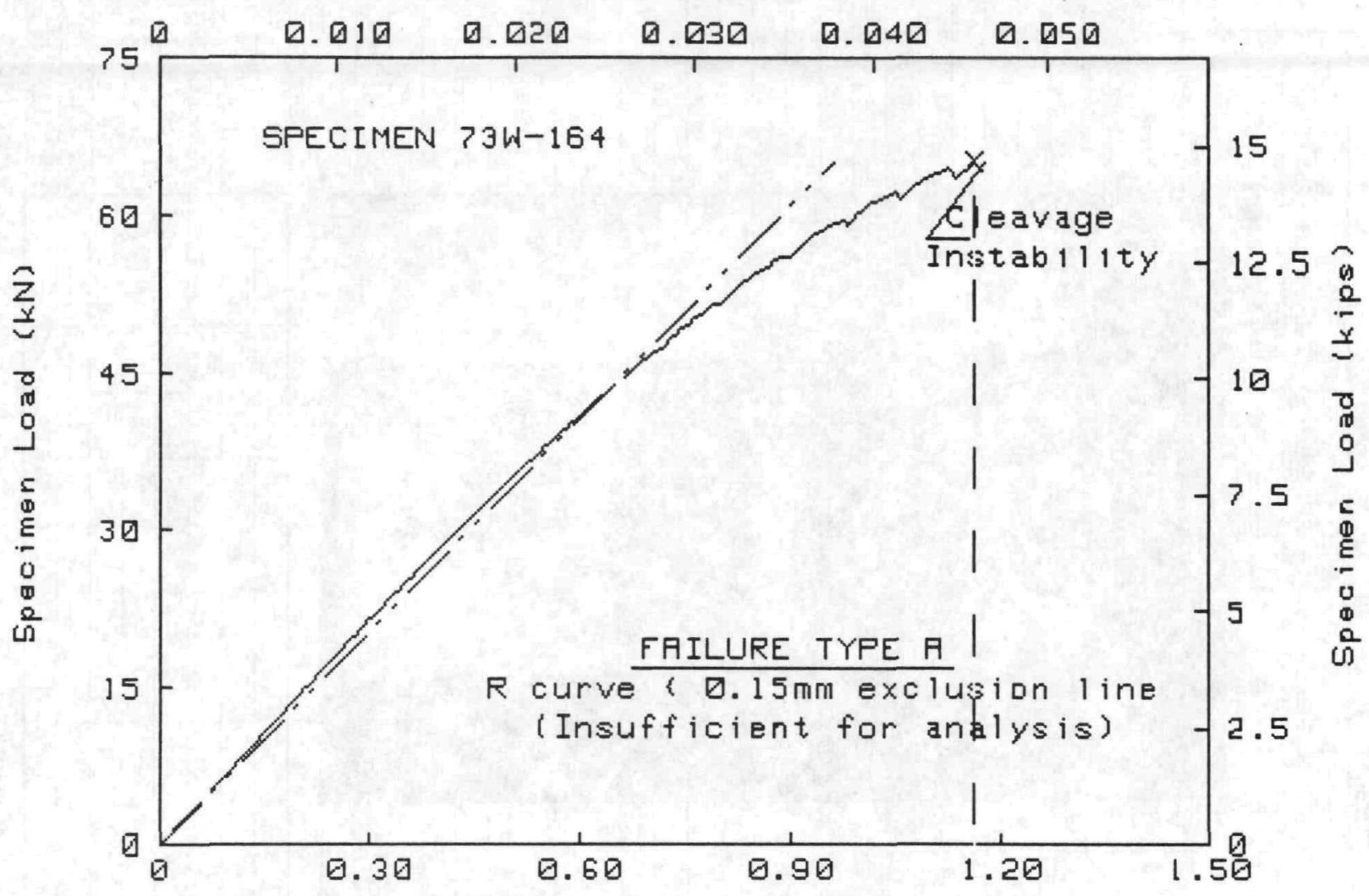

Specimen Crackmouth Deflection ( $\mathrm{mm}$ )

TEST SPECIMEN DATA

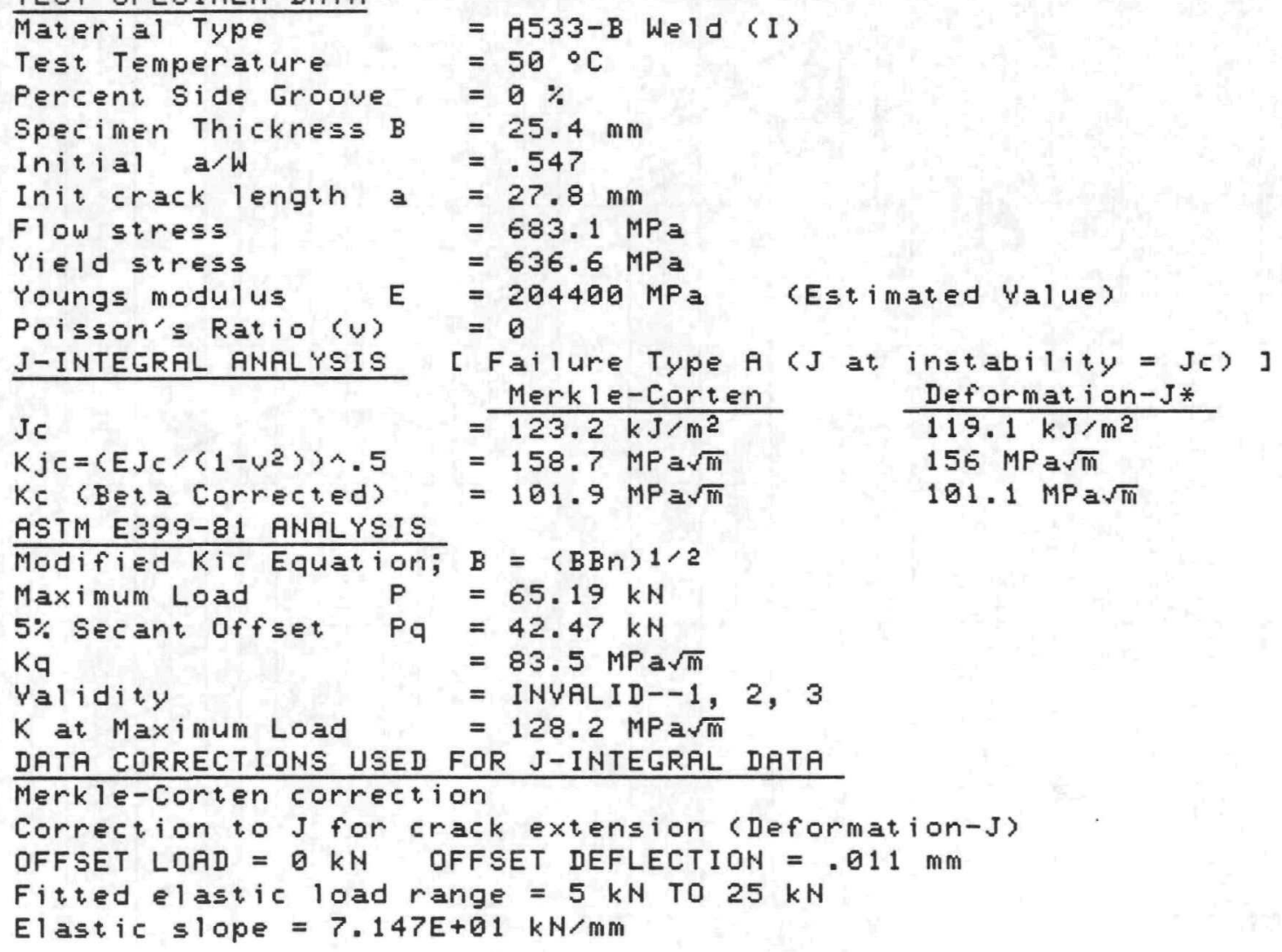




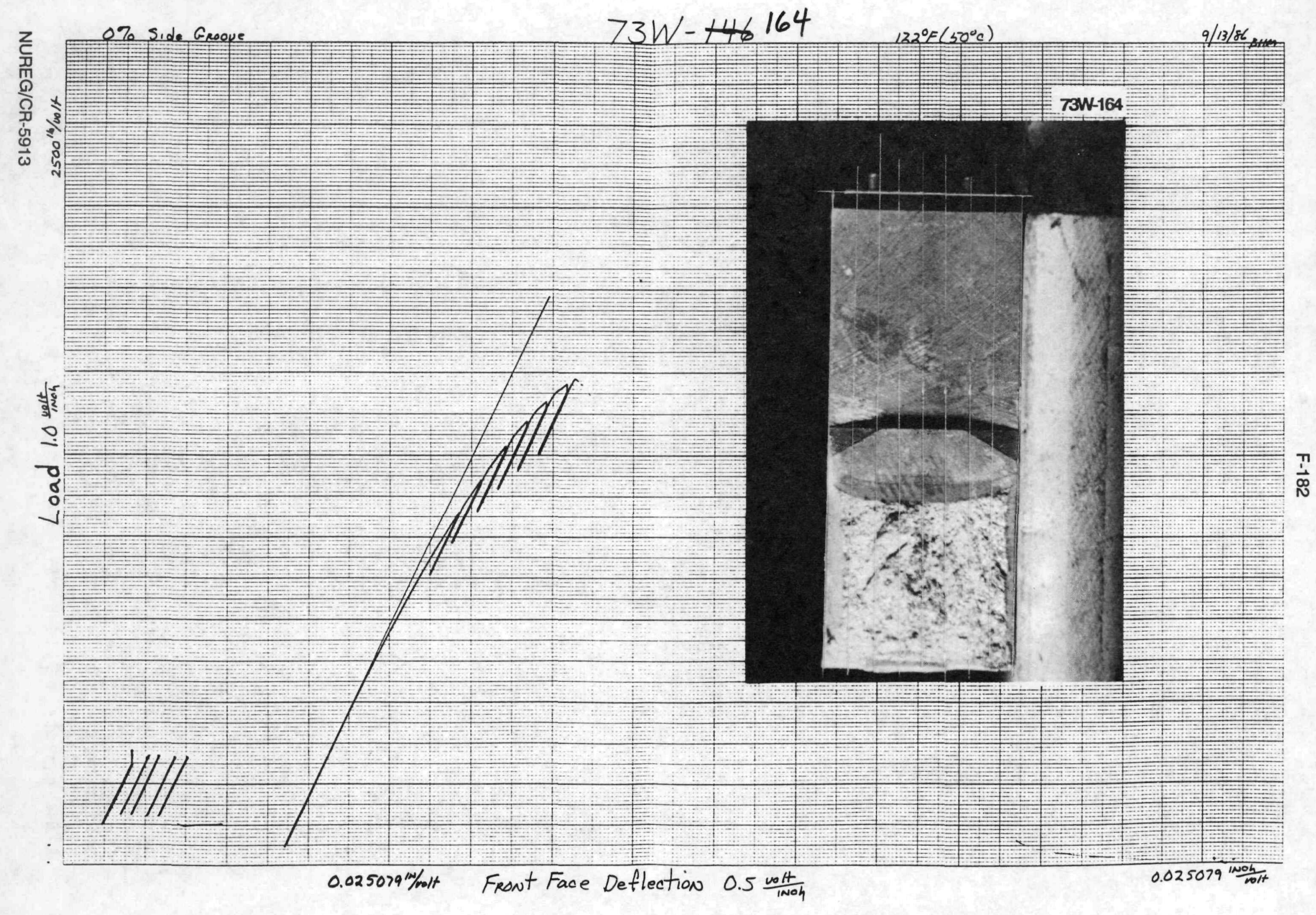




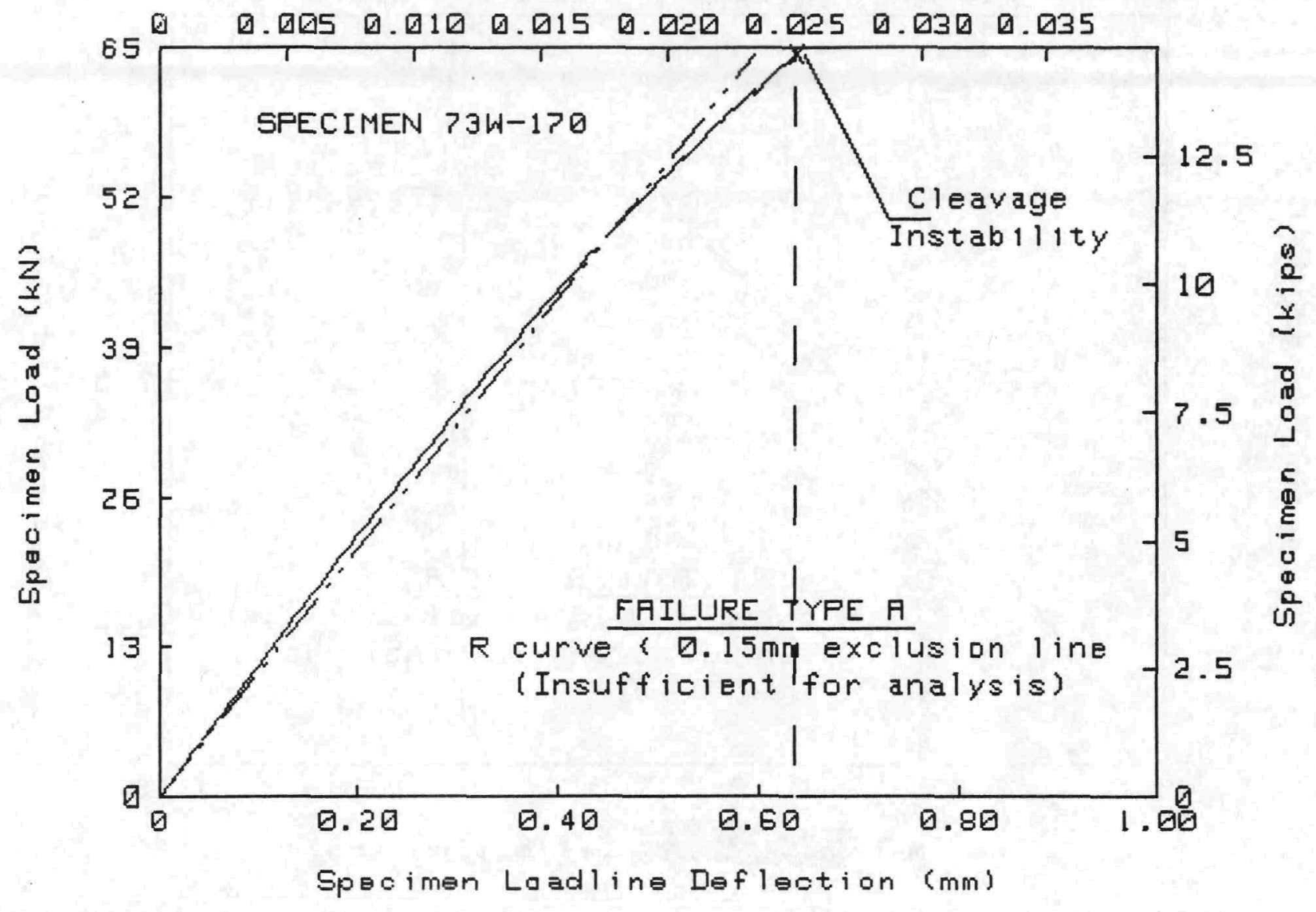

TEST SFECIMEN DATA

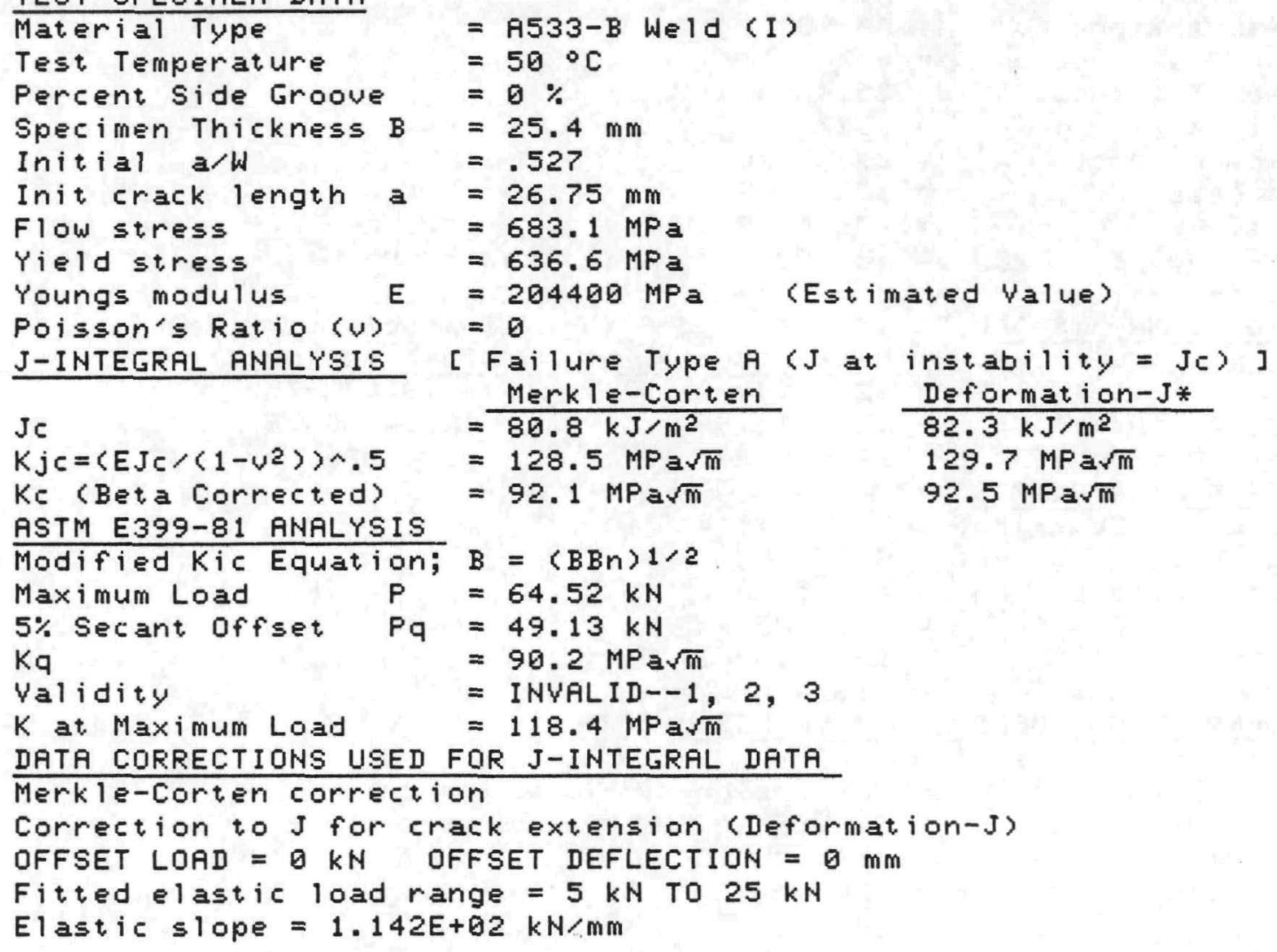




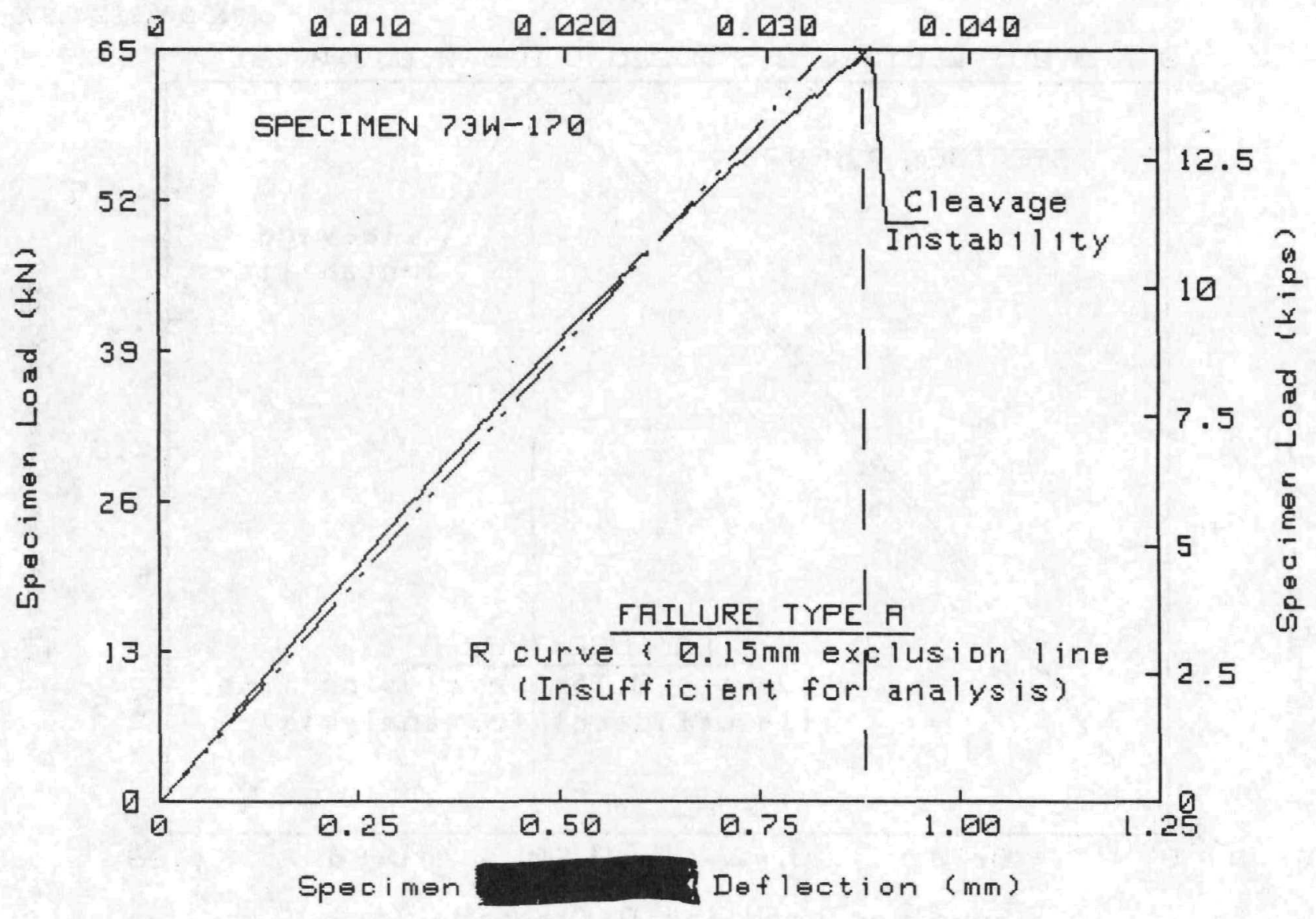

TEST SPECIMEN DATA

Material Type
Test Temperature
Percent Side Groove
Specimen Thickness B
Initial a/W
Init crack length a
Flow stress
Yield stress
Youngs modulus
Poisson's Ratio (
J-INTEGRA RNALYS

$=$ A533-B Weld (I)

$=50^{\circ} \mathrm{C}$

$=0 \%$

$=25.4 \mathrm{~mm}$

$=.527$

$=26.75 \mathrm{~mm}$

$=683.1 \mathrm{MPa}$

$=636.6 \mathrm{MPa}$

$=204400 \mathrm{MPa}$ (Estimated Value)

I-INTEGRAL ANALYSIS

$\mathrm{Jc}$ $=0$

[ Failure Type $A$ ( $J$ at instability = JC) ]

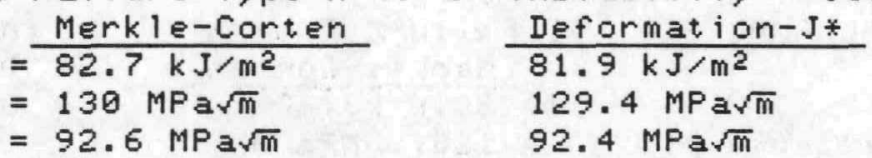

$K j c=\left\langle E J c /\left(1-v^{2}\right\rangle\right) \wedge .5=130 \mathrm{MPa} \sqrt{m}$

$92.4 \mathrm{MPa} \sqrt{\mathrm{m}}$

ASTM E399-81 ANALYSIS

Modified Kic Equation; $B=(B B n) 1 / 2$

Maximum Load $P=64.52 \mathrm{kN}$

$5 \%$ Secant offset. $\mathrm{Pq}=49.55 \mathrm{kN}$

$\mathrm{Kq} \quad=90.9 \mathrm{MPa} \sqrt{\mathrm{m}}$

Validity

$K$ at Maximum Load

= INYALID--1, 2, 3

DATA CORRECTIDNS USED FOR J-INTEGRAL DATA

Merkle-Corten correction

Correction to J for crack extension (Deformation-J)

OFFSET LOAD $=0 \mathrm{kN}$ OFFSET DEFLECTION $=.098 \mathrm{~mm}$

Fitted elastic load range $=5 \mathrm{kN}$ TO $25 \mathrm{kN}$

Elastic slope $=8.184 E+01 \mathrm{kN} / \mathrm{mm}$

NUREG/CR-5913 


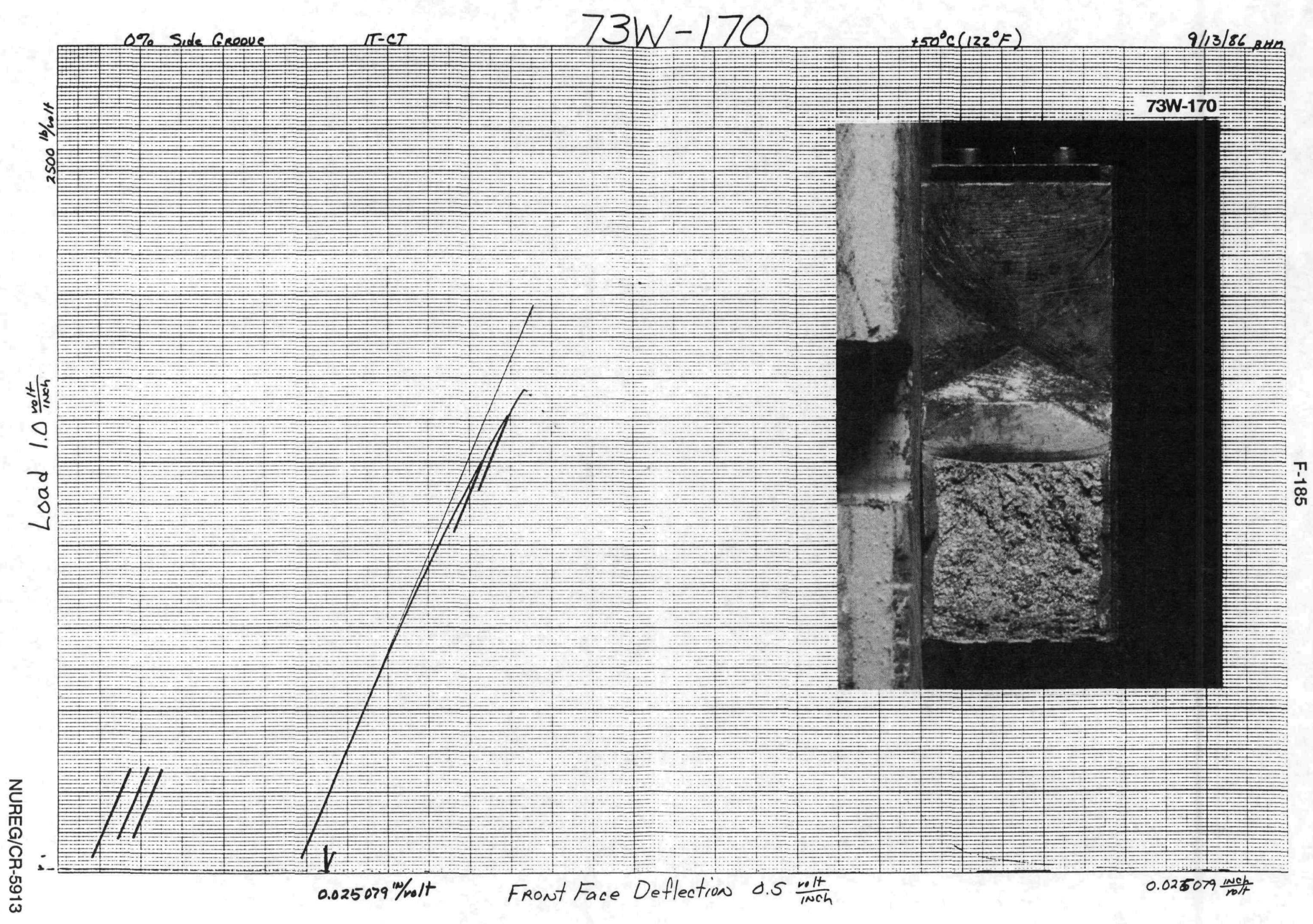


SPECIMEN ID $=73 W 139$

MATERIAL TYPE = HCUWELD

TEST TEMPERATURE $=75$ DEG $C$

THICKNESS $=25.4 \mathrm{~mm}$

SIDE GROOUES = $0 \%$

INITIAL CRACK LENGTH $=27.2 \mathrm{~mm}$

$a /$ (W) $i=.535$

MEASURED DULT ILE DELTA-A $=.05 \mathrm{~mm}$

YIELD STRENGTH $=621 \mathrm{MPa}$

FLOW STRENGTH $=672 \mathrm{MPa}$

ESTIMATED YOUNG'S MODULUS $=203 \mathrm{GPa}$

SPECIMEN CLEAUED AT KQ $=126.9 \mathrm{MPa} S Q R[\mathrm{~m}]$ (NOT UALID KIC)

UALUES AT MAXIMUM LOAD

J-INTEGRAL $=125.3 \mathrm{~kJ} / \mathrm{m}^{\wedge} 2$

$K J c=159.4 \mathrm{MPa} S Q R[\mathrm{~m}]$

$K$-beta-c $=100.7 \mathrm{MPa} S Q R[\mathrm{~m}]$

\author{
UALUES aT CLEAUAGE LDAD \\ J-INTEGRAL $=125 \mathrm{~kJ} / \mathrm{m}^{\wedge} 2$ \\ $K J c=159.3 \mathrm{MPa} S Q R[\mathrm{~m}]$ \\ K-beta-c $=100.7 \mathrm{MPa} S Q R[\mathrm{~m}]$
}


F-187

ORNL-DWG 92-11381

73W139 75 DEGREES C

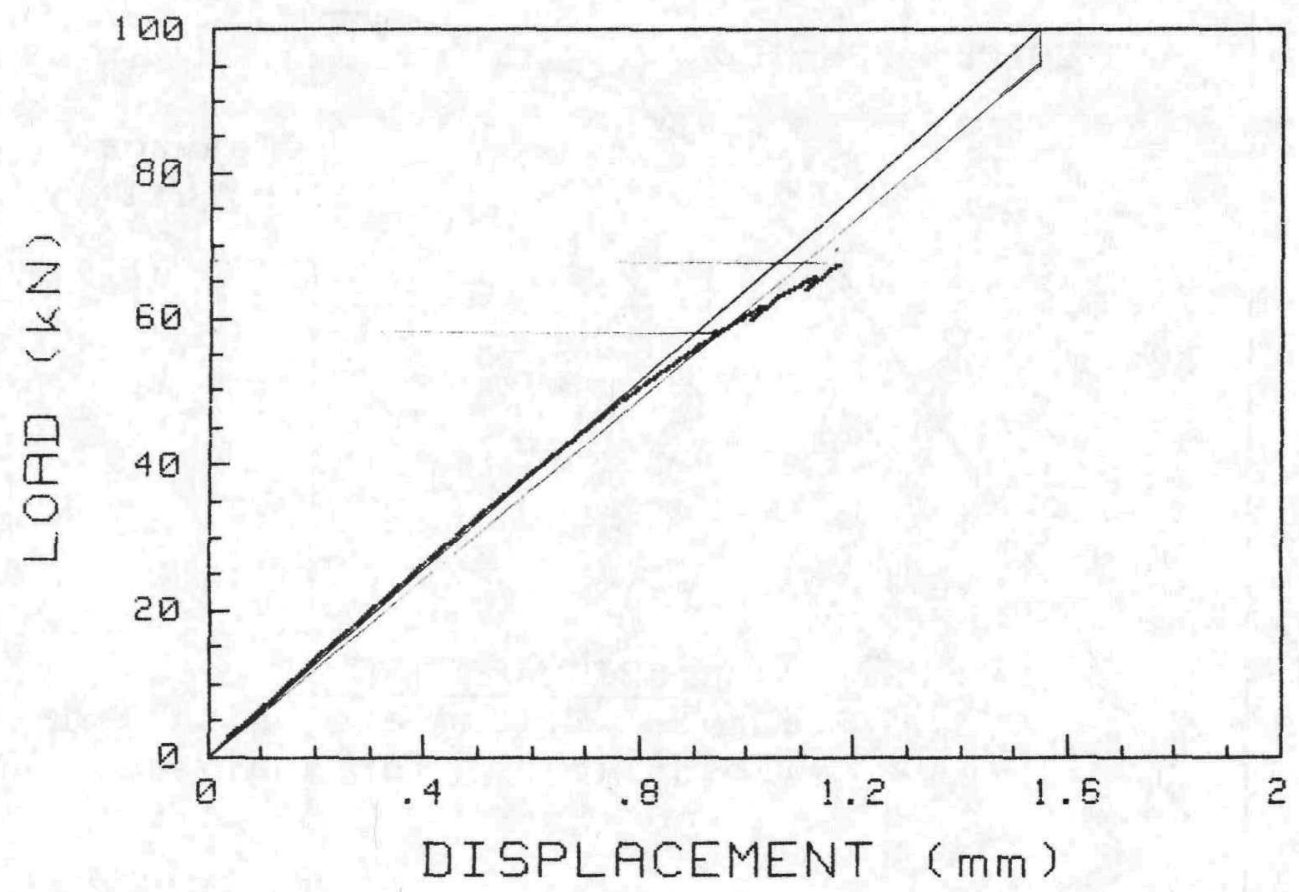


Specimen Loadline Deflection (in.)

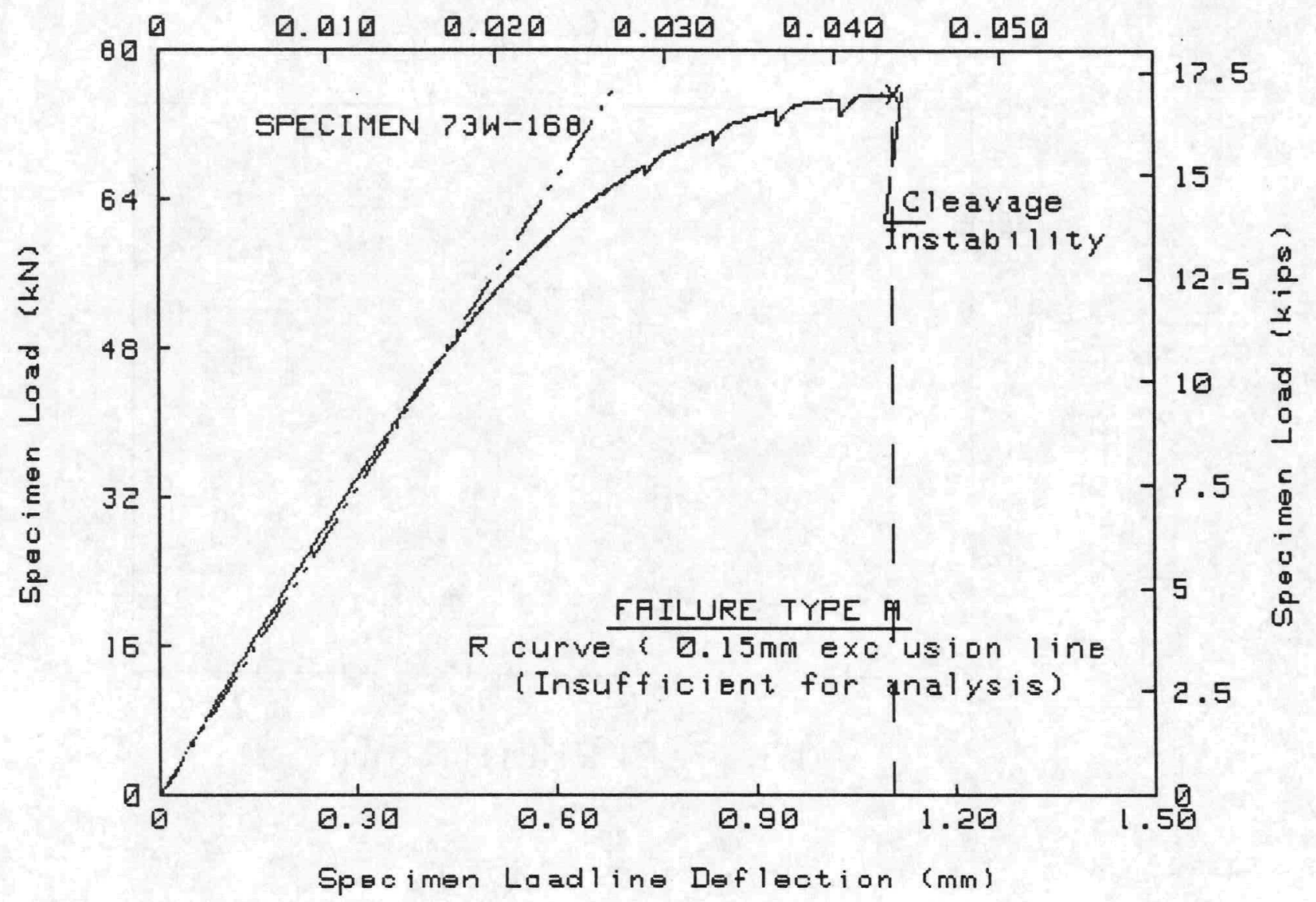

TEST SPECIMEN DATA

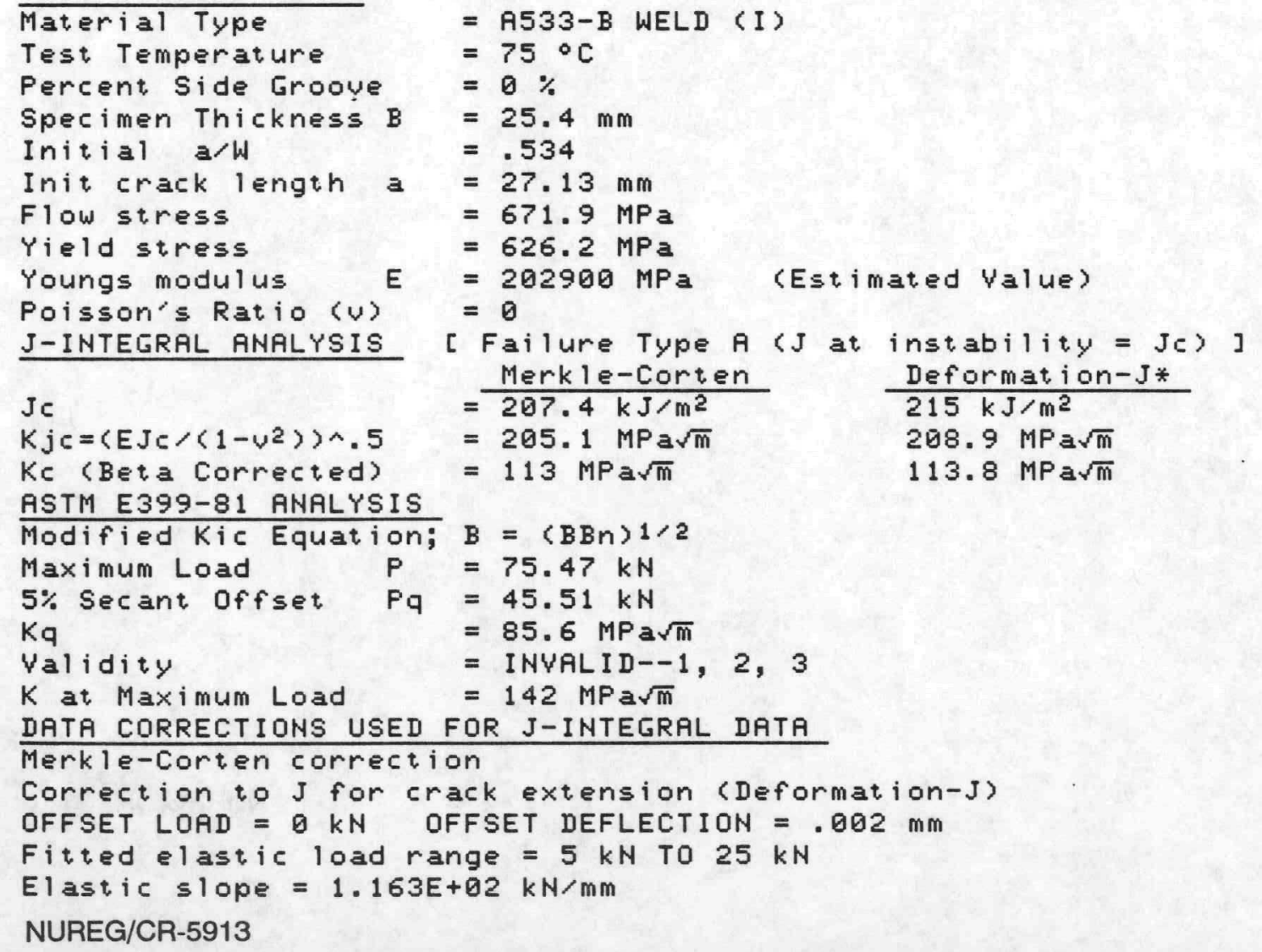


Specimen Crackmouth Deflection (in.)

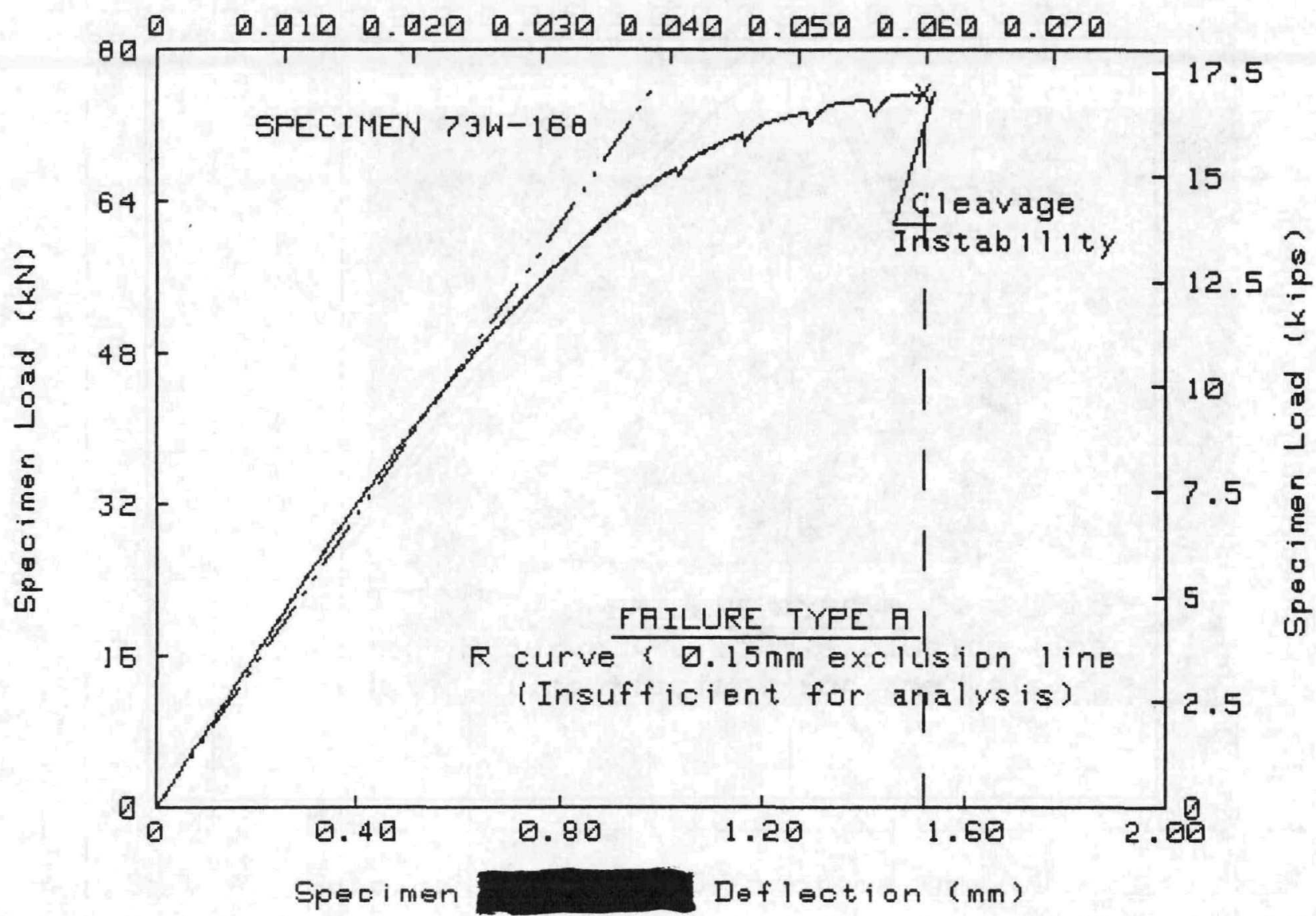

TEST SPECIMEN DATA

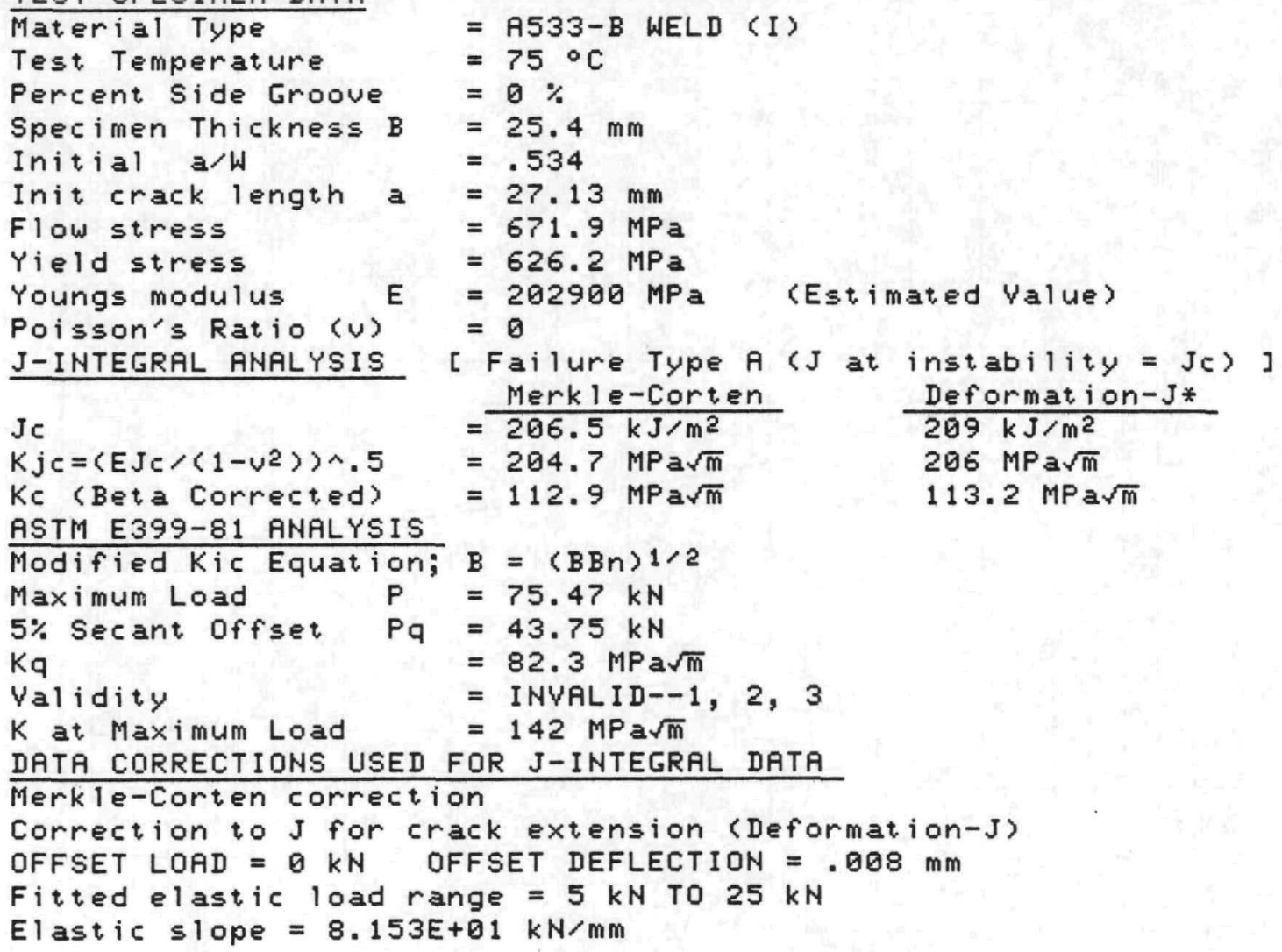




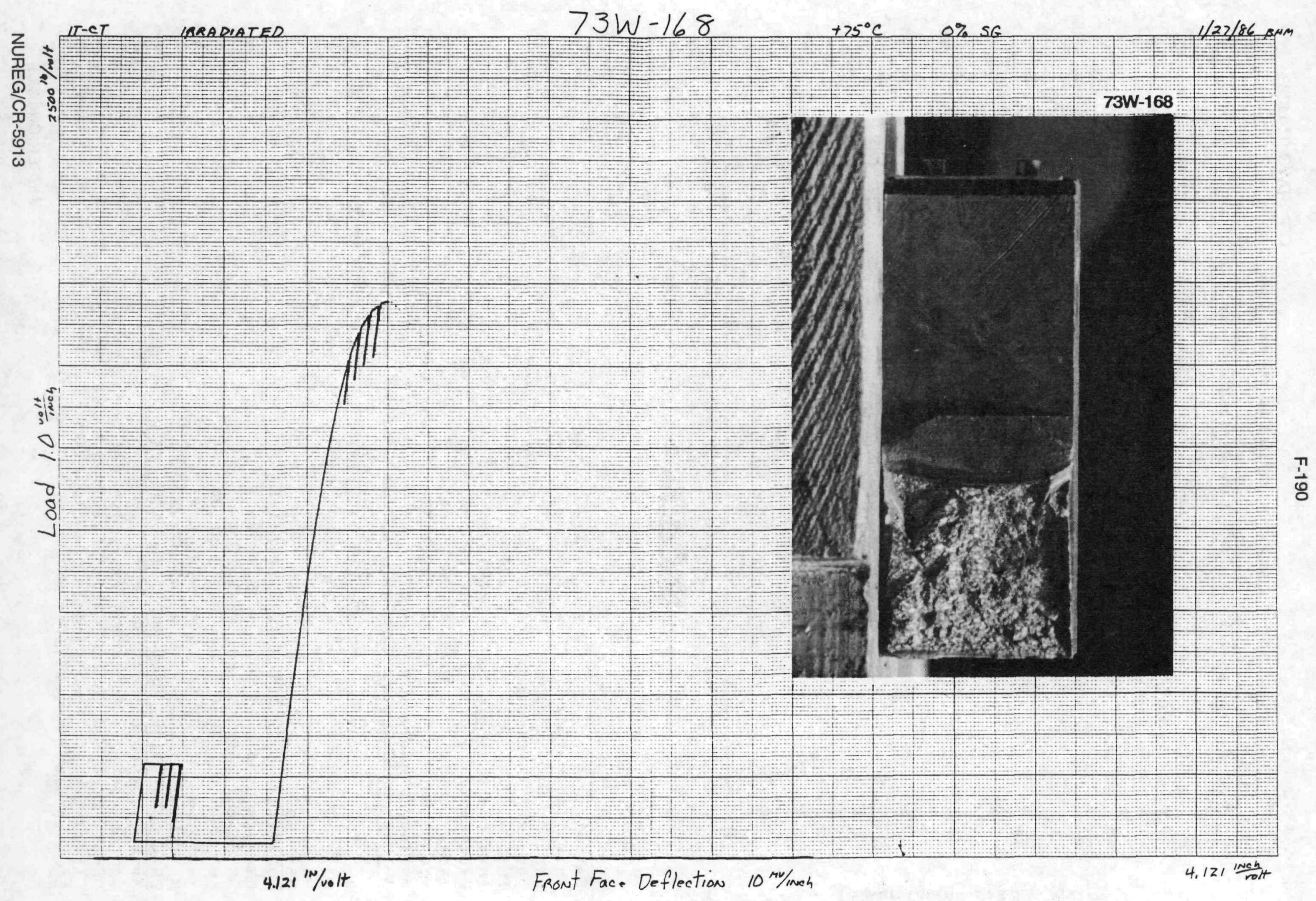


Specimen Loadline Deflection (in.)
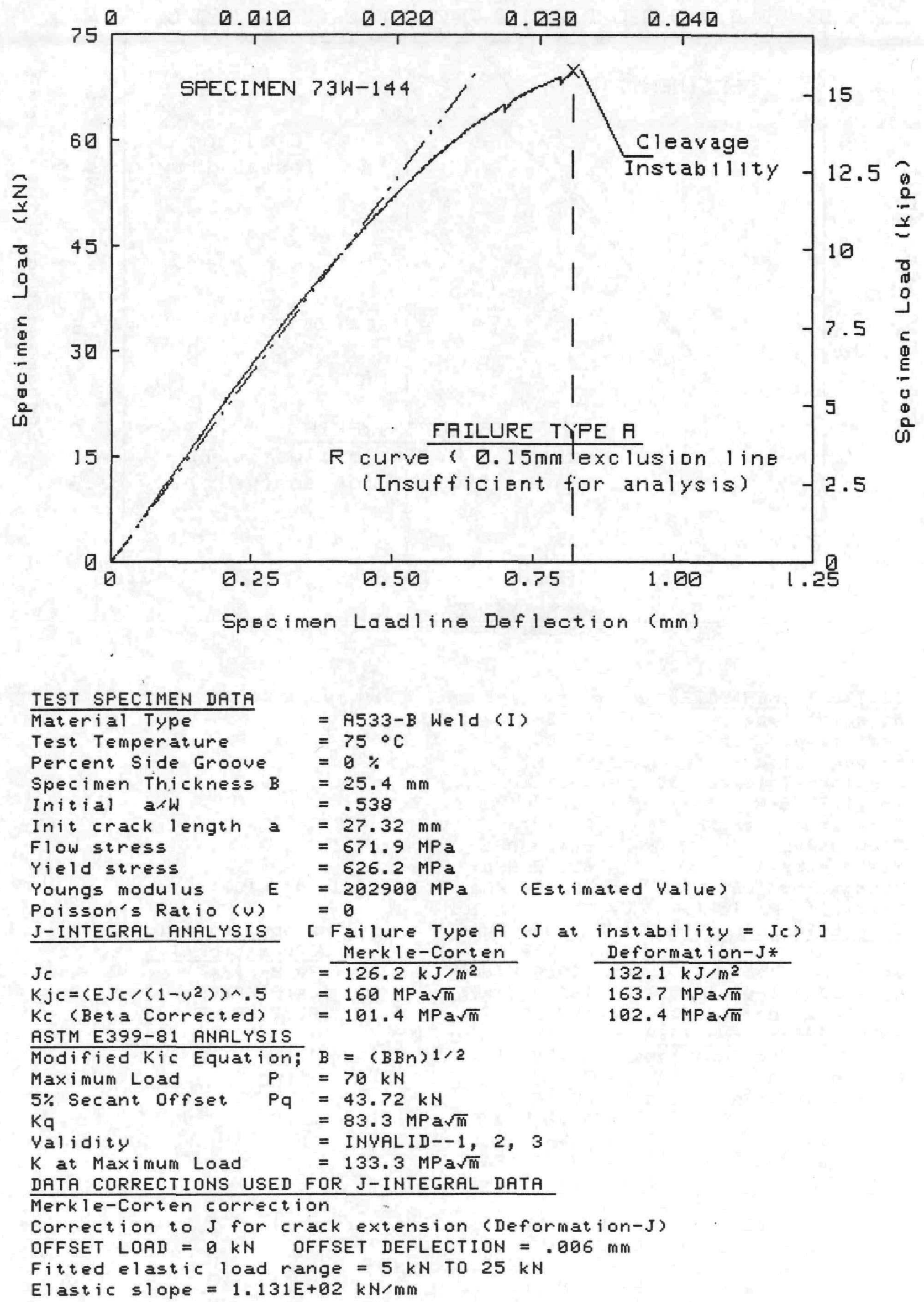
Specimen Crackmouth Deflection (in.)

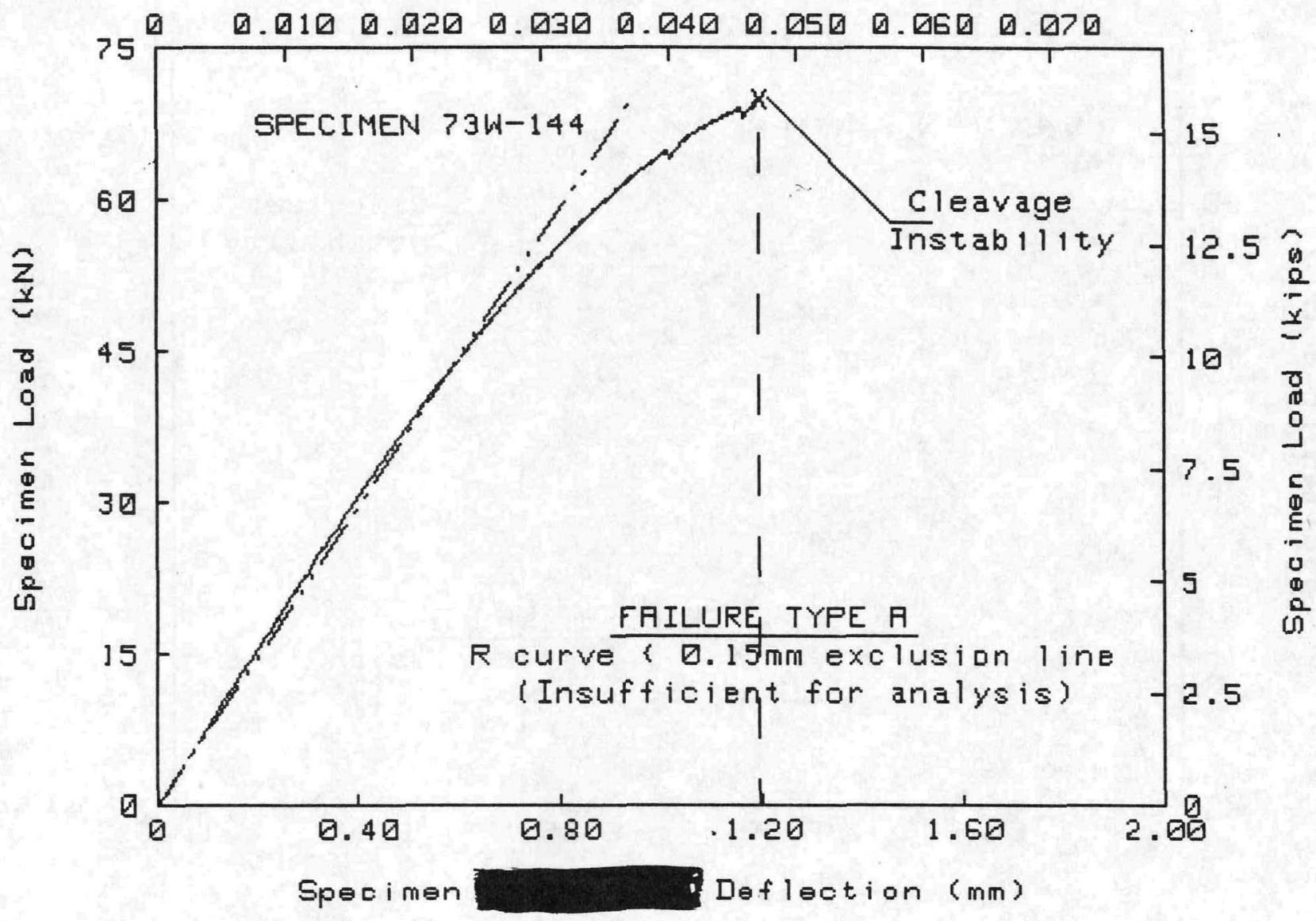

TEST SPECIMEN DATA

Material Type
Test Temperature
Percent Side Groove
Specimen Thickness B
Initial a/W
Init crack length a
Flow stress
Yield stress
Youngs modulus
Poisson's Ratio (U)
J-INTEGRAL ANALYSIS

$=$ A533-B Weld (I)

$=75 \circ \mathrm{C}$

$=0 \%$

$=25.4 \mathrm{~mm}$

$=.534$

$=27.13 \mathrm{~mm}$

$=671.9 \mathrm{MPa}$

$=626.2 \mathrm{MPa}$

$=202900 \mathrm{MPa}$ (Estimated Valwe)

Poisson's Ratio ( $v$ )

$=0$

$\mathrm{JC}$

[ Failure Type A ( $J$ at instability = JE) ]

$=\frac{\text { Merkle-Corten }}{135.6 \mathrm{~kJ} / \mathrm{m}^{2}}$
$=165.9 \mathrm{MPa} \sqrt{\mathrm{m}}$ Deformation $-\mathrm{J} *$ $164.8 \mathrm{MPa} \sqrt{\mathrm{m}}$

$K j c=\left\langle E J C<\left(1-v^{2}\right\rangle\right) \wedge .5$

$=103 \mathrm{MPa} \sqrt{\mathrm{m}}$ $102.7 \mathrm{MP} a \sqrt{\mathrm{m}}$

ASTM E399-81 ANALYSIS
Modified Kic Equation; $B=(B B n) 1 / 2$
Maximum Load $P=70 \mathrm{kN}$
5\% Secant offset $P q=43.16 \mathrm{kN}$
$\mathrm{Kq} \quad=81.2 \mathrm{MPa} \sqrt{\mathrm{m}}$
Validity
$K$ at Maximum Load
$=$ INVALID--1, 2, 3
$=131.7 \mathrm{MPa} \sqrt{\mathrm{m}}$

DATA CORRECTIONS USED FOR J-INTEGRAL DATA

Merkle-corten correction

Correction to J for erack extension (Deformation-J)

OFFSET LOAD $=0 \mathrm{kN}$ OFFSET DEFLECTION $=.014 \mathrm{~mm}$

Fitted elastic load range $=5 \mathrm{kN}$ TO $25 \mathrm{kN}$

Elastic slope $=7.778 E+01 \mathrm{kN} / \mathrm{mm}$

NUREG/CR-5913 


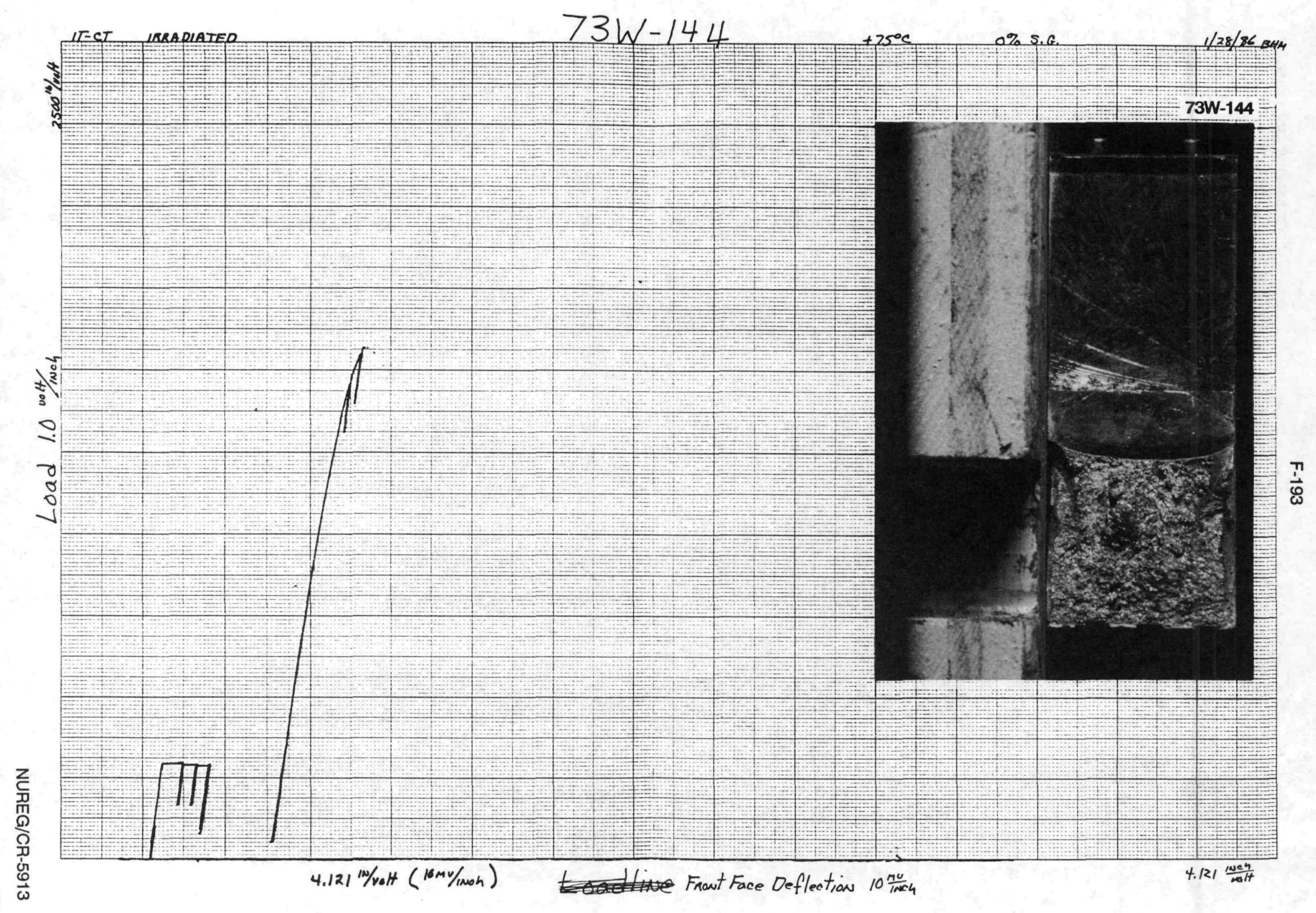


Specimen Loadline Deflection (in.)

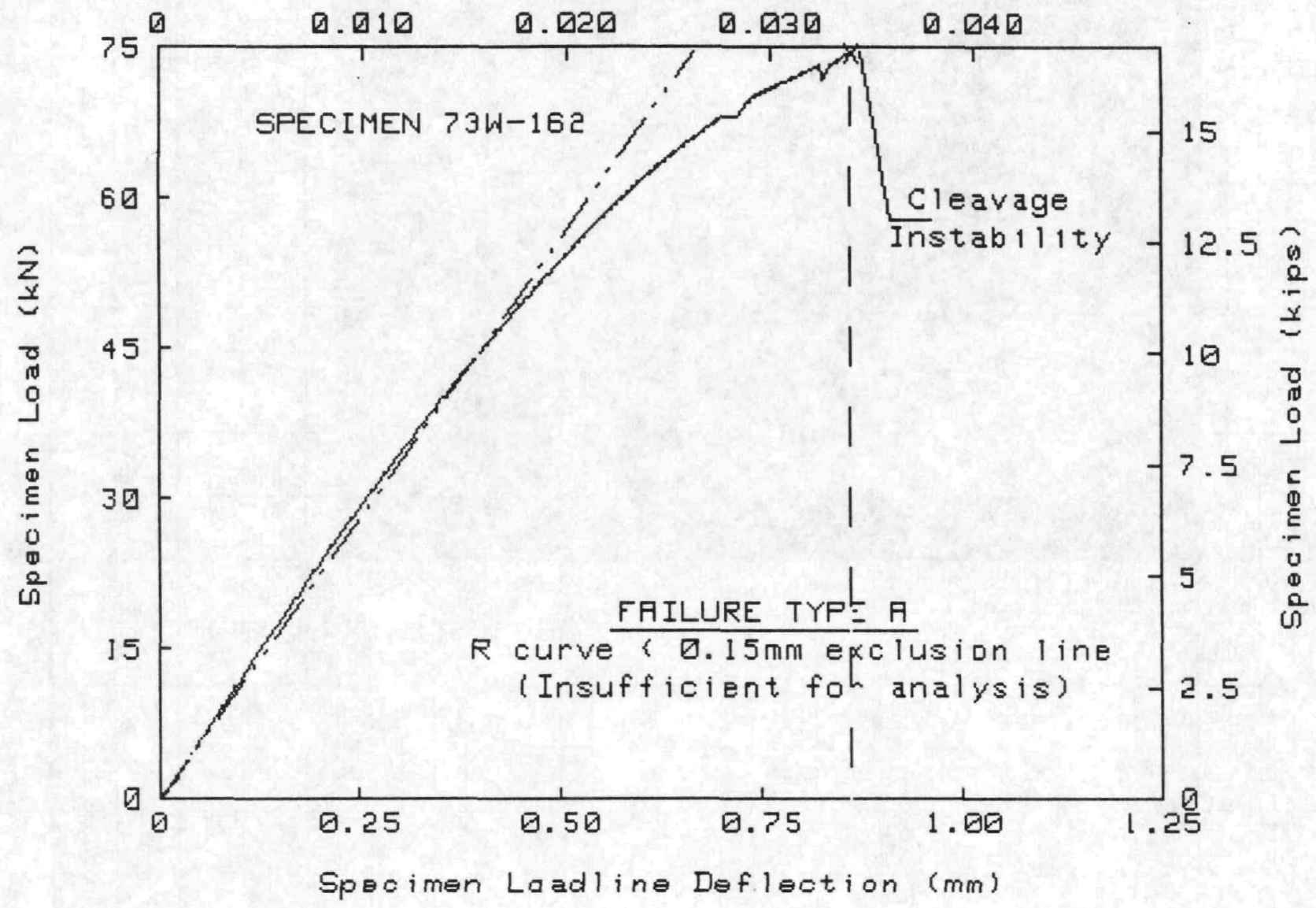

TEST SPECIMEN DATA

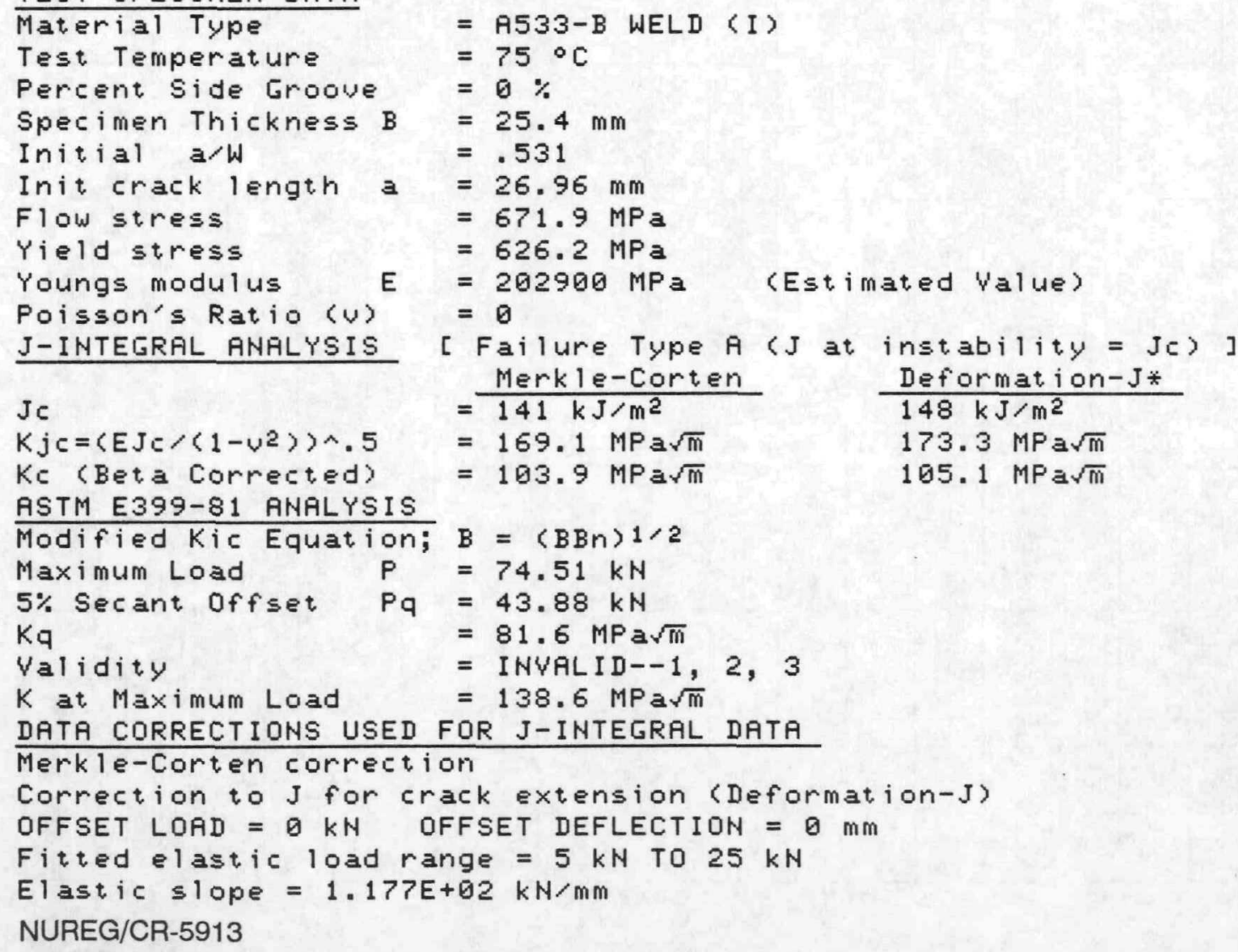


Specimen Crackmouth Deflection (in.)

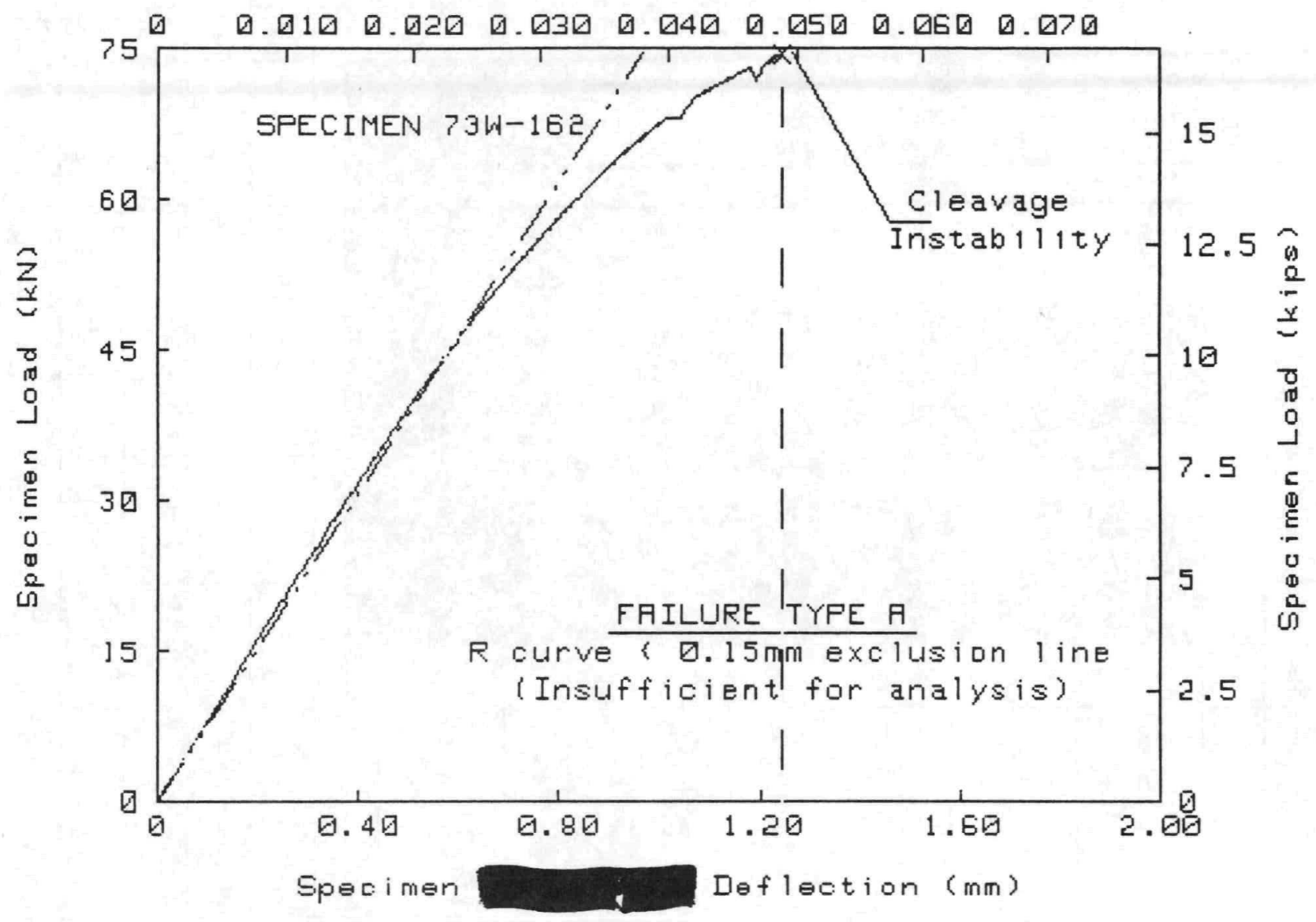

TEST SFECIMEN DATA

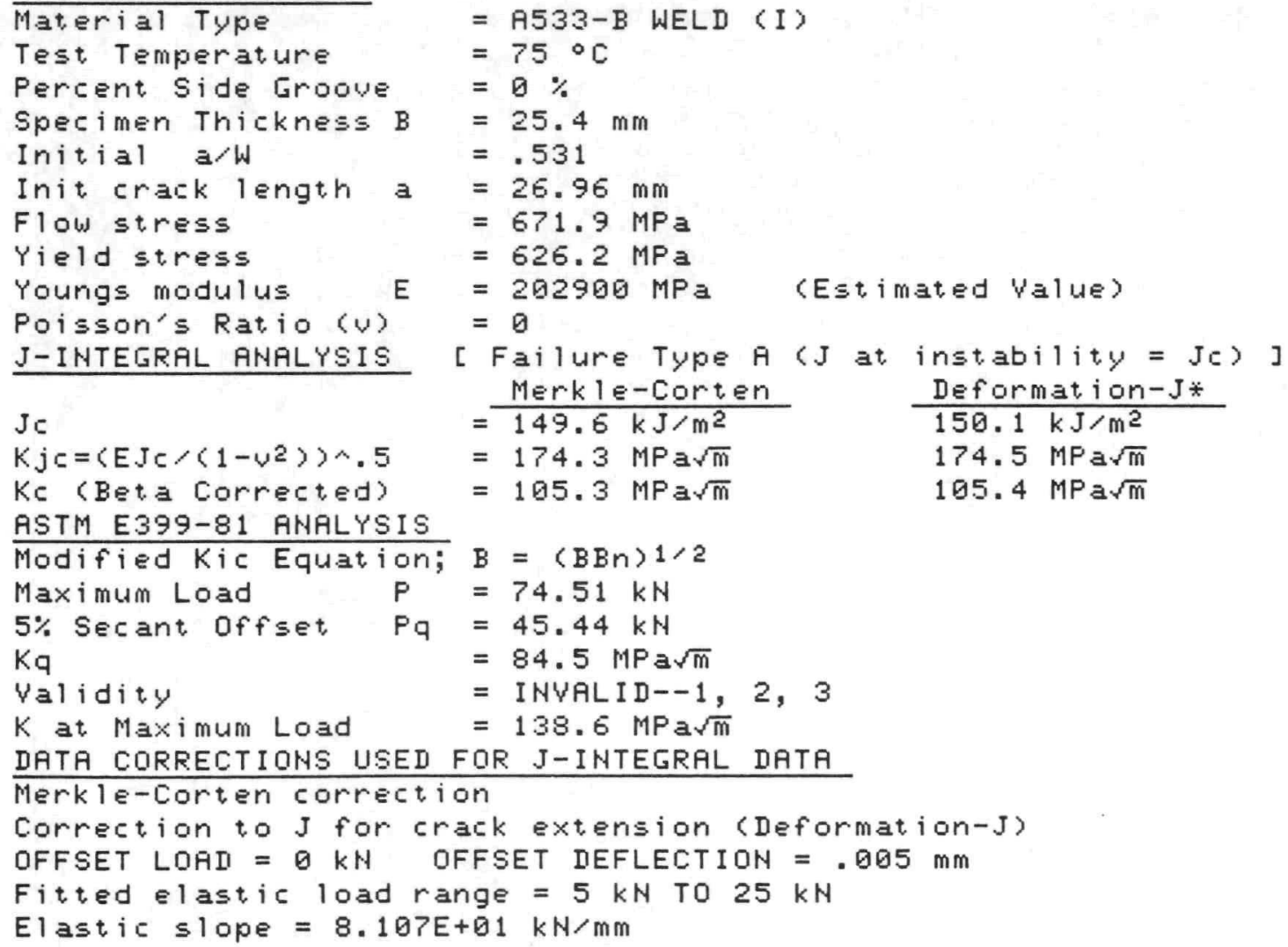




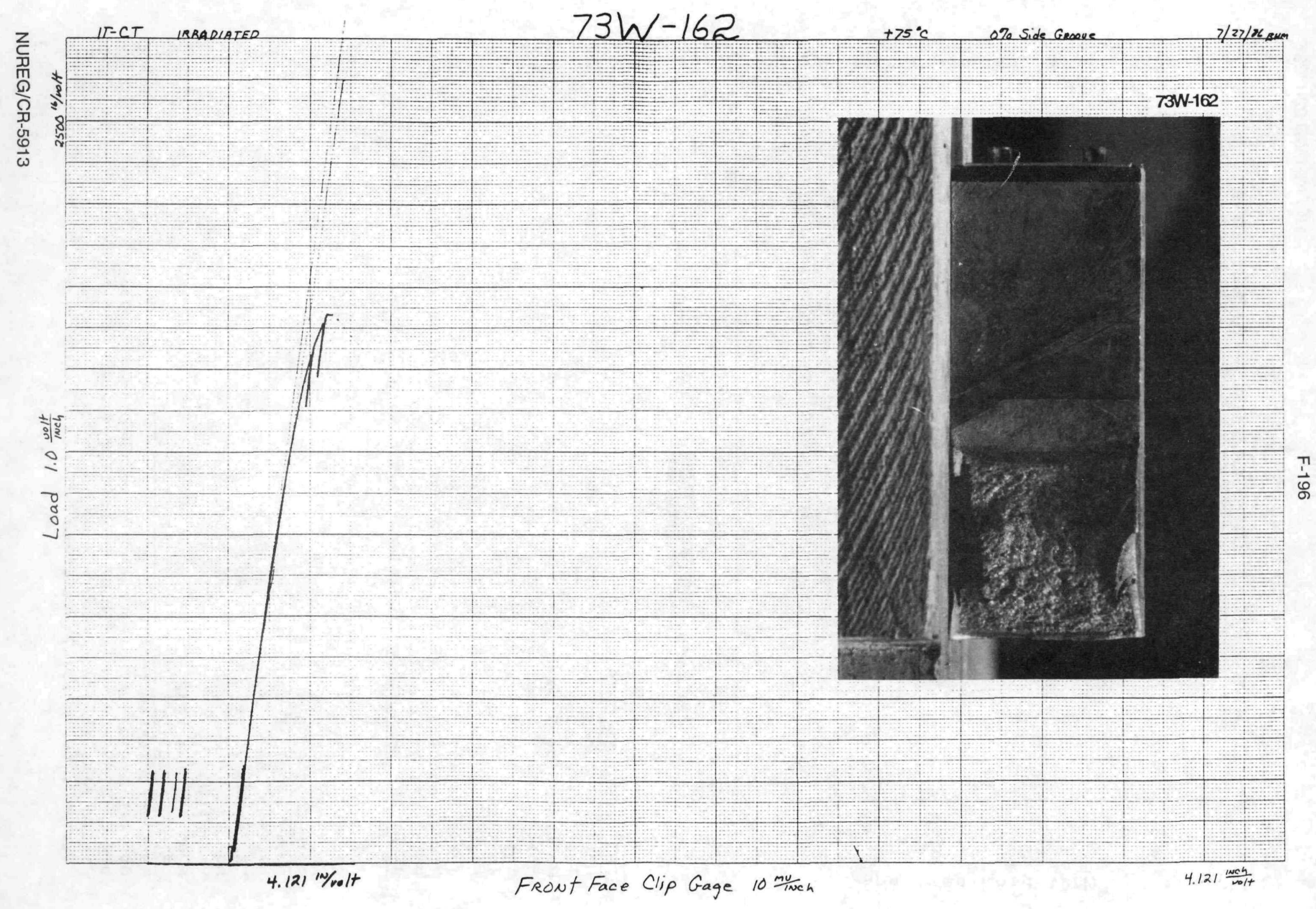




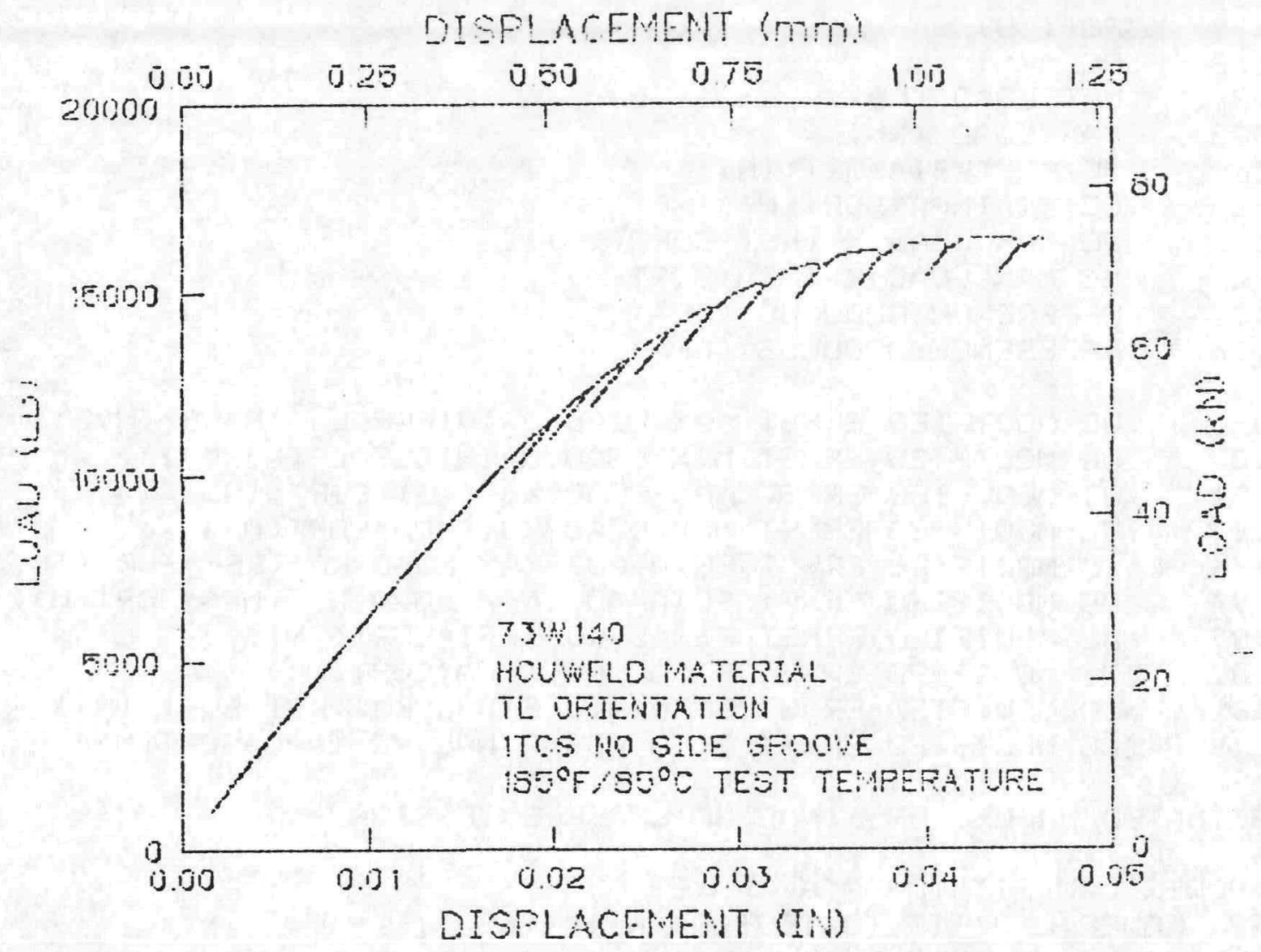

$73 W-140$

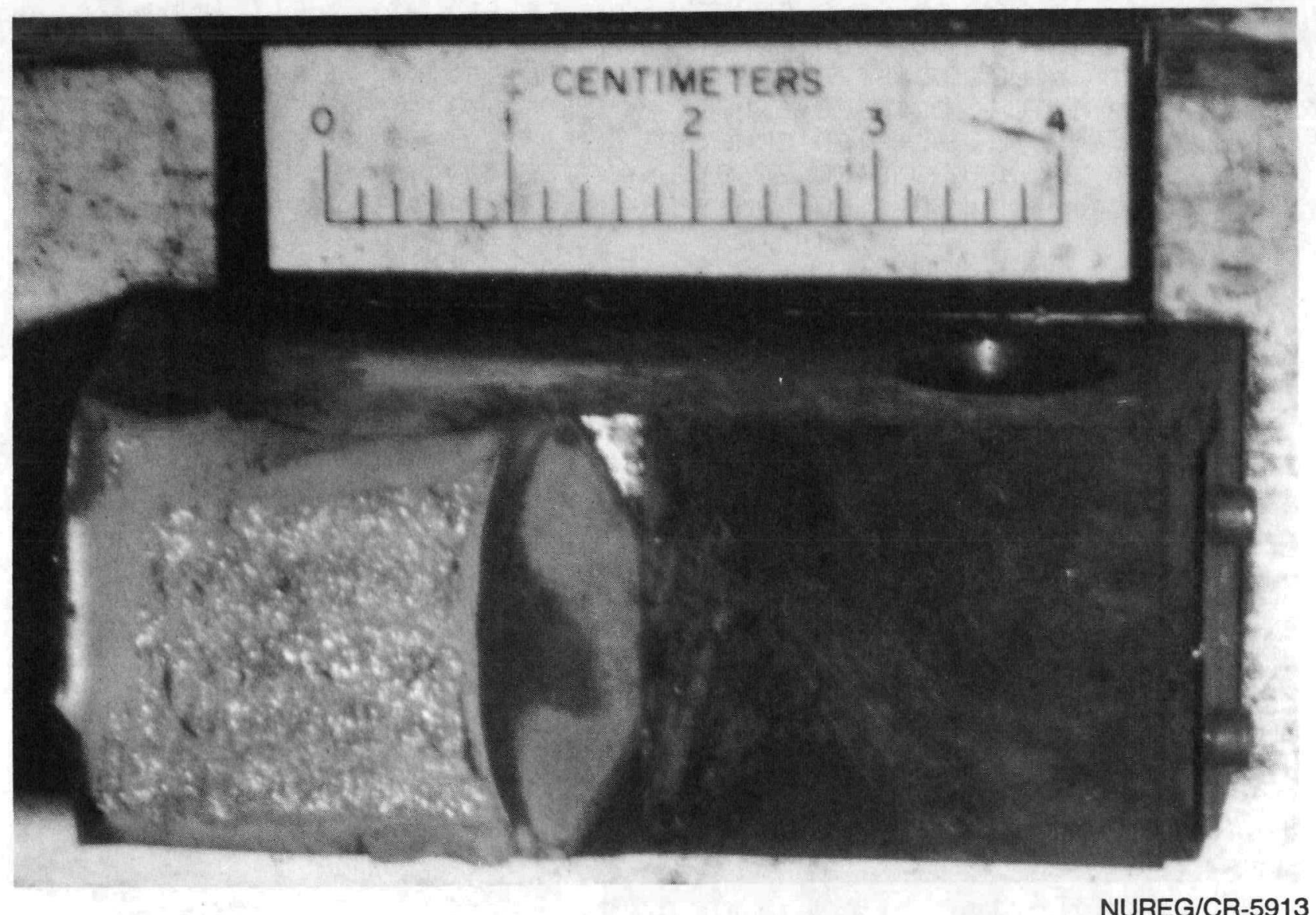




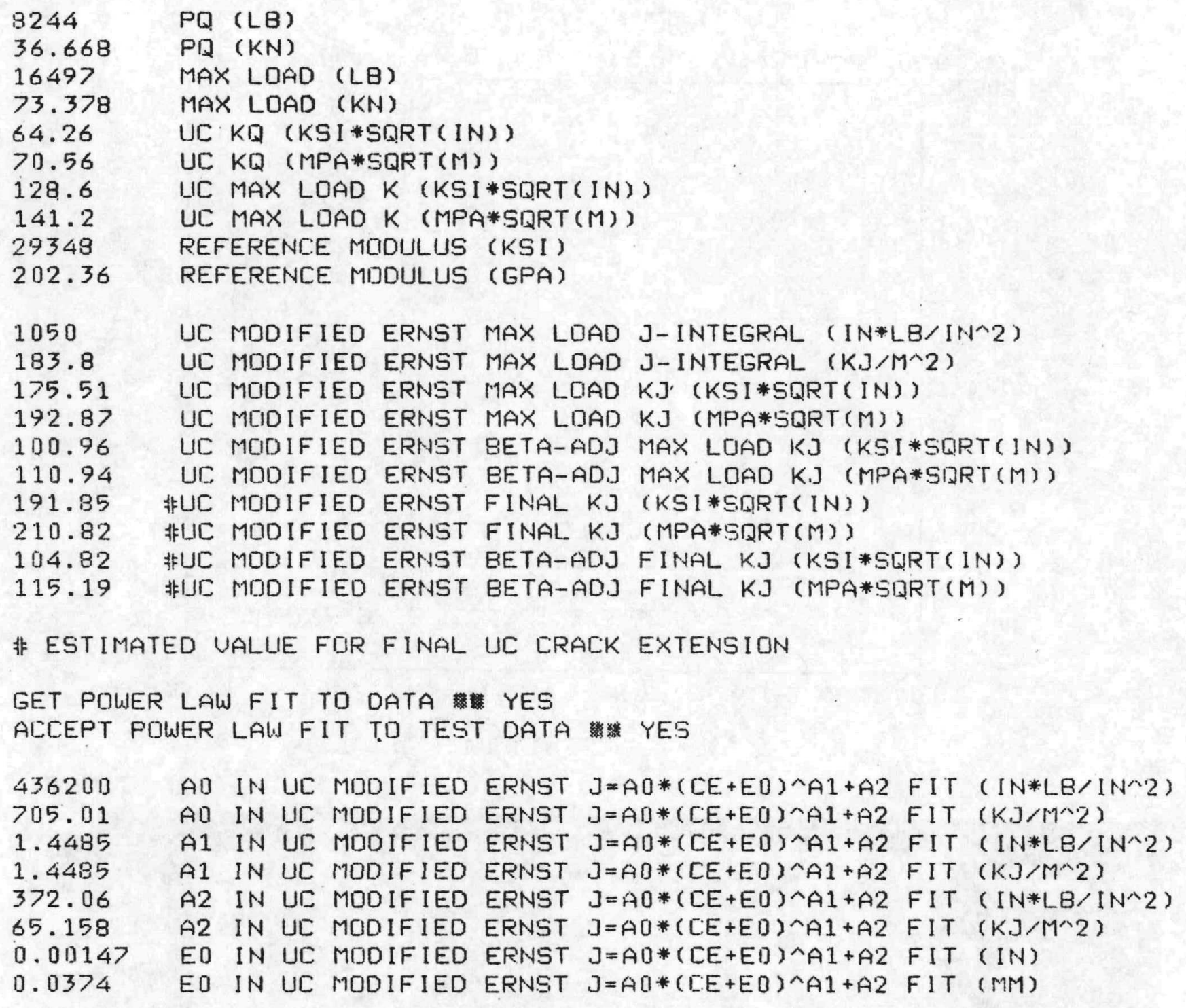


ORNL-DWG 92-11389
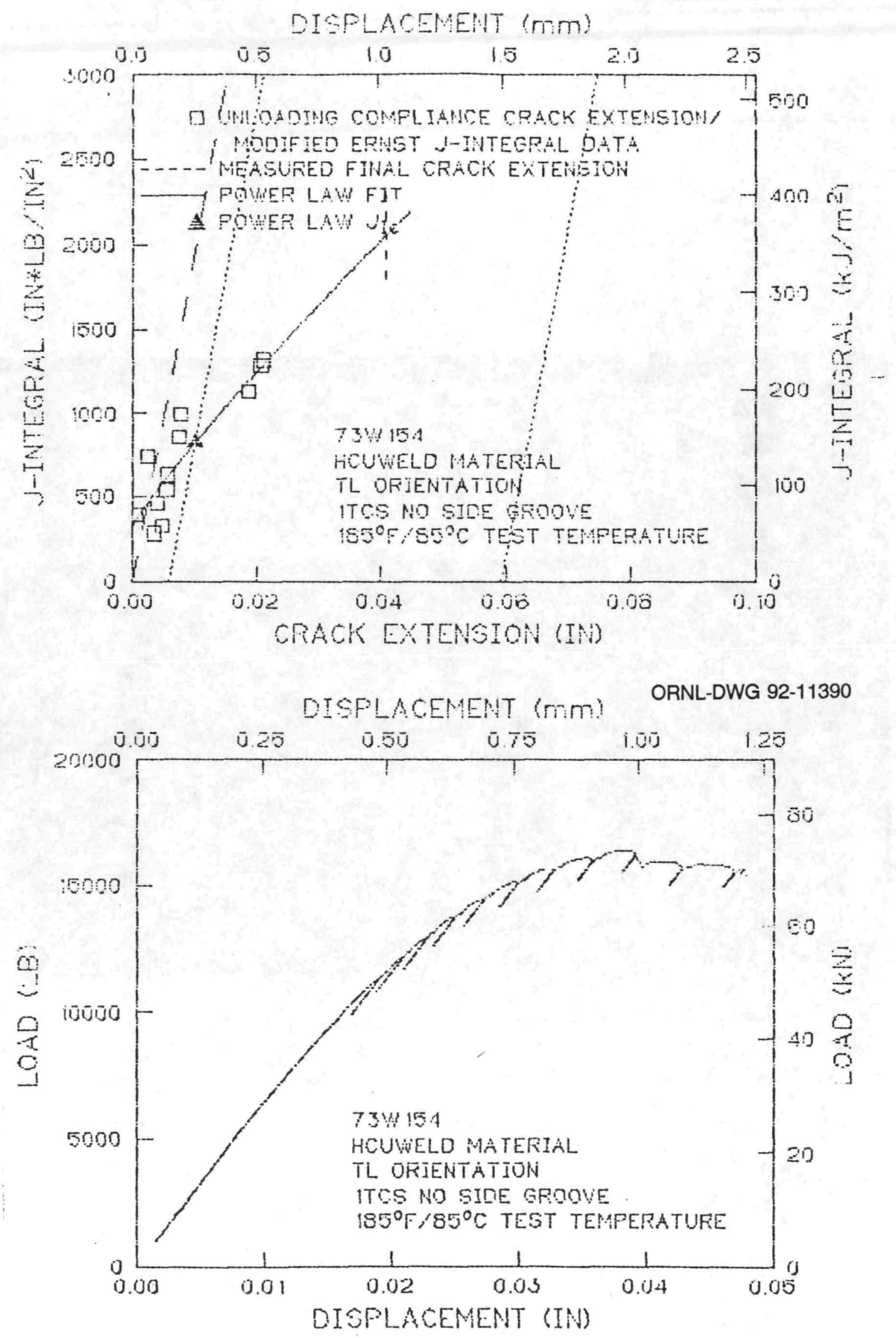


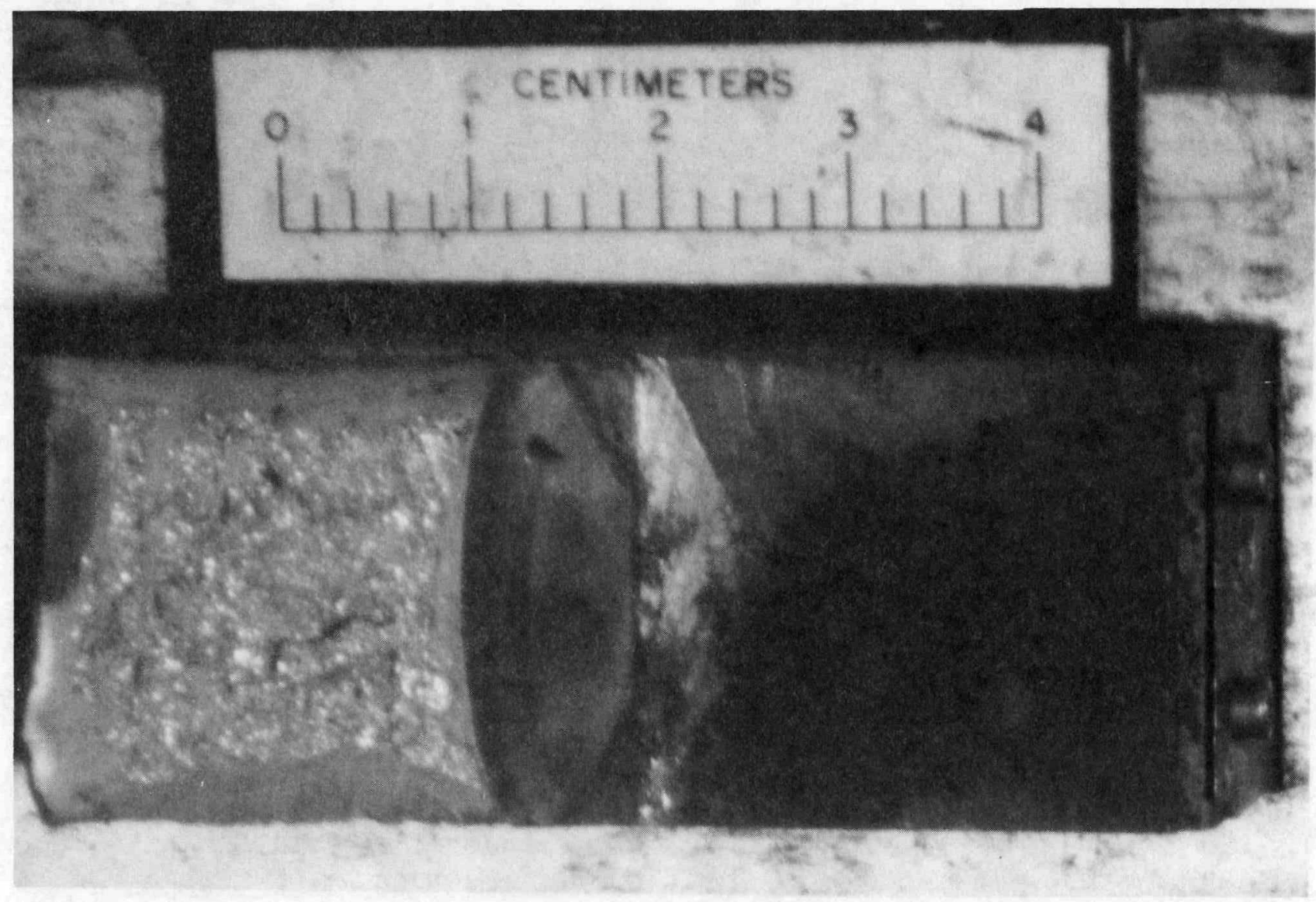




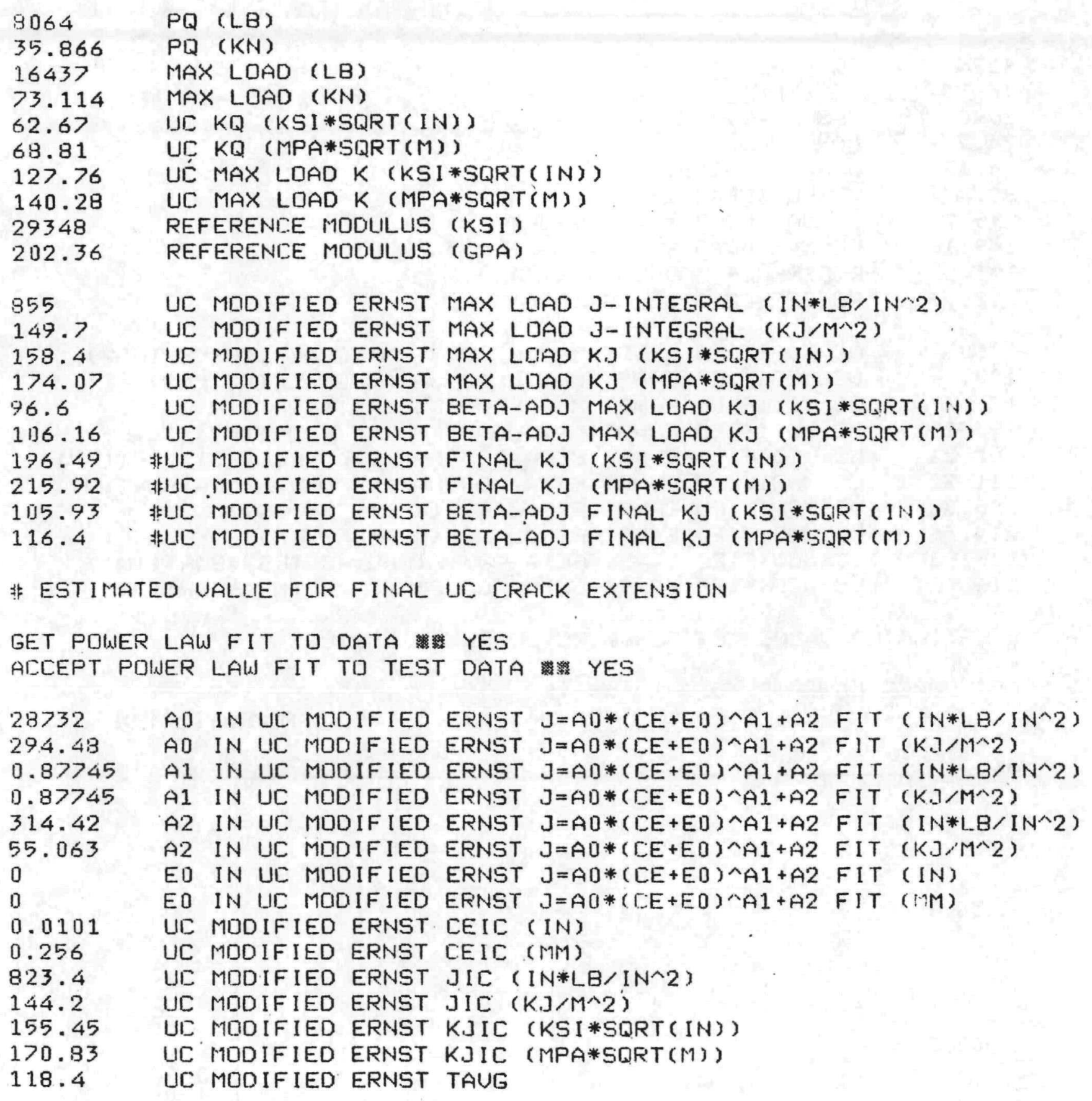




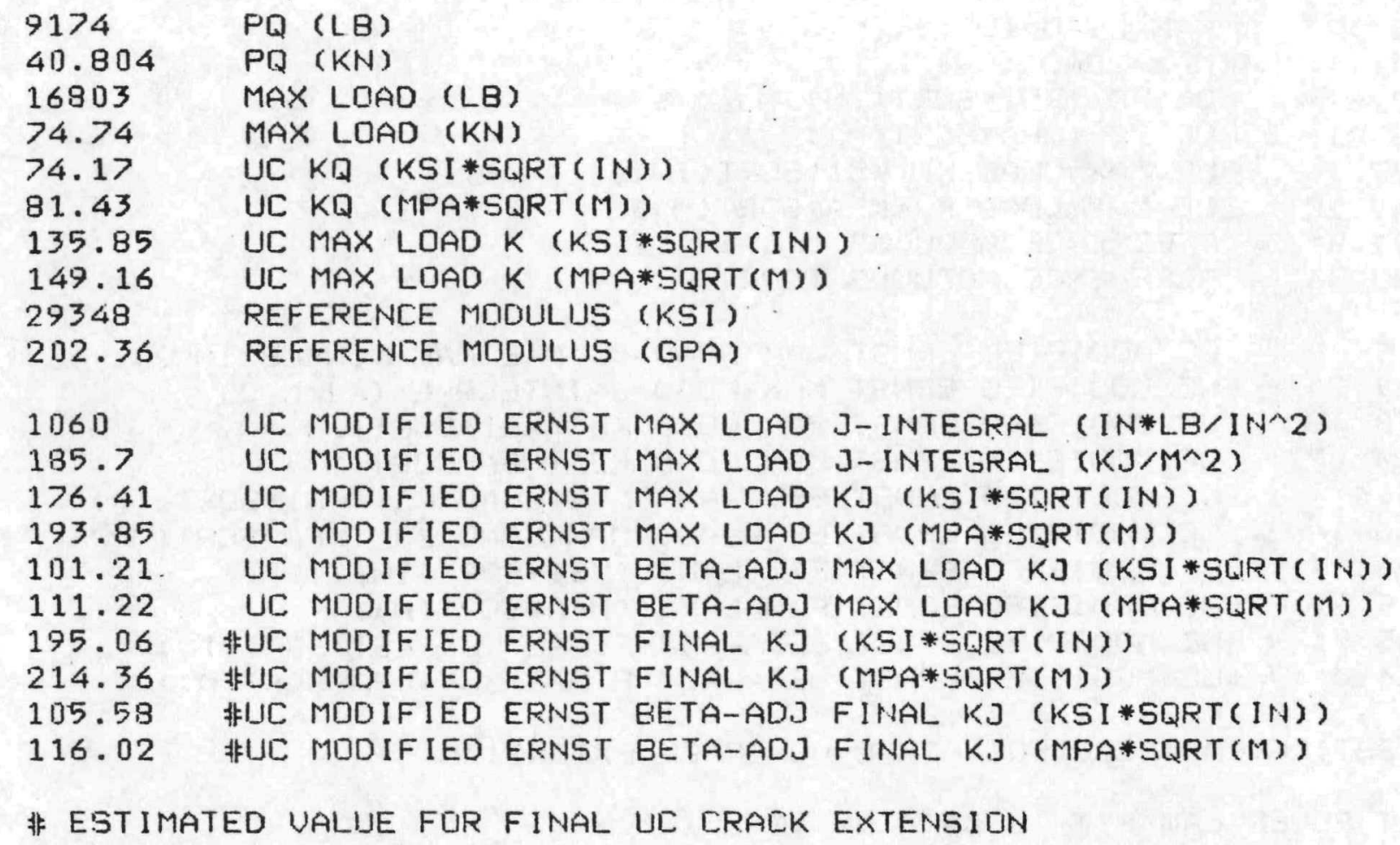

GET POWER LAW FIT TO DATA YES

DISPLACEMENT ( $\mathrm{mim}) \quad$ ORNL-DWG 92-11391

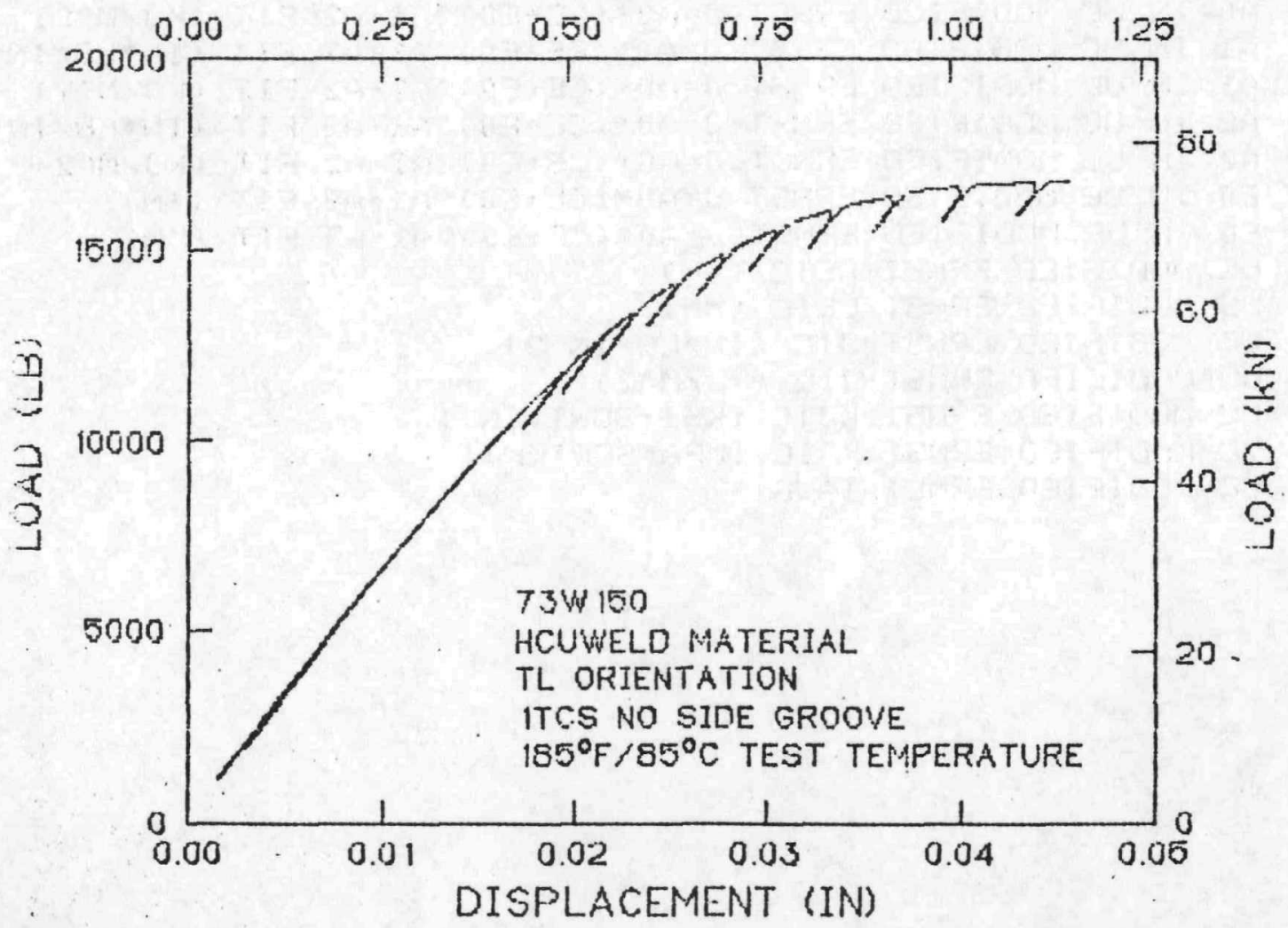




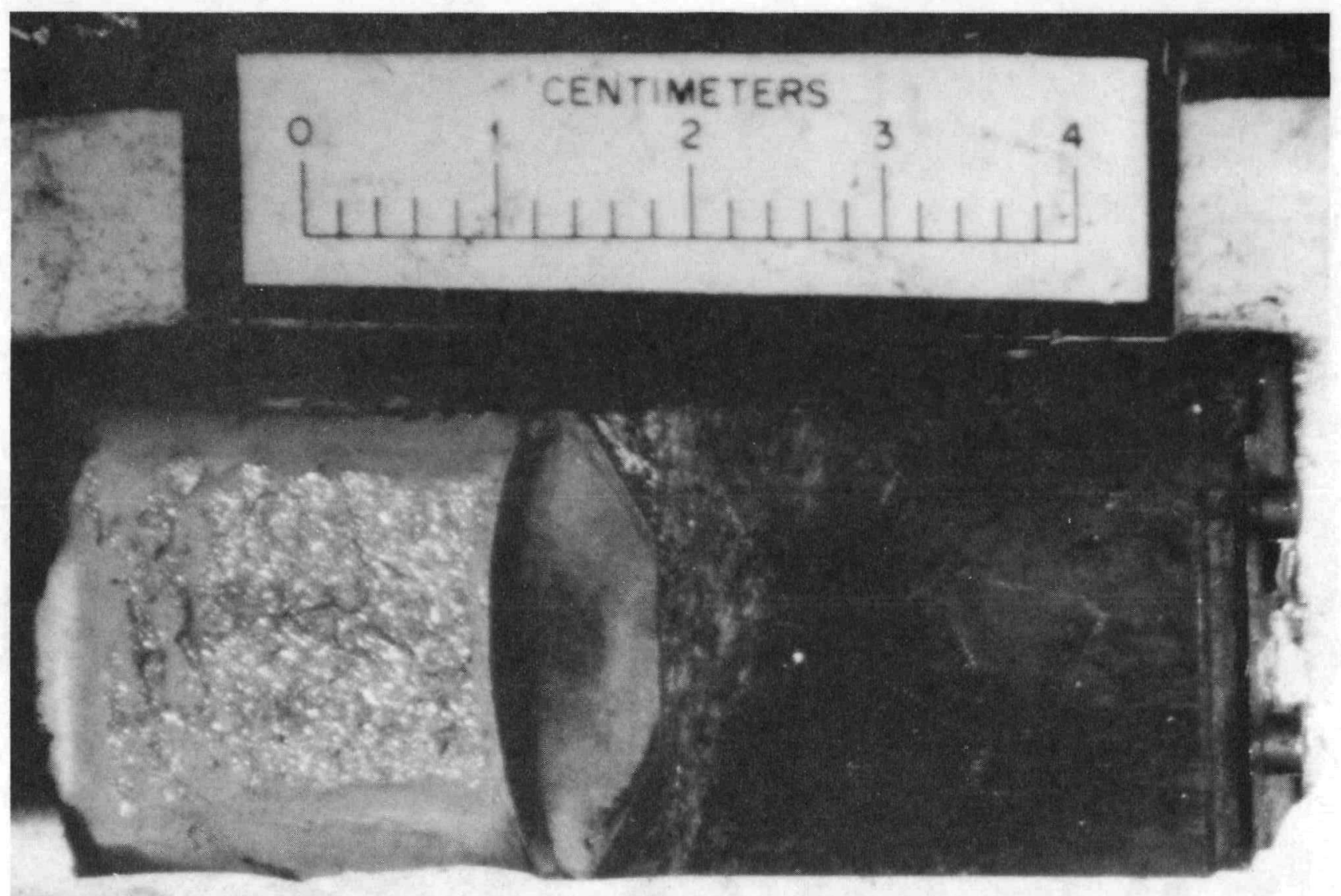




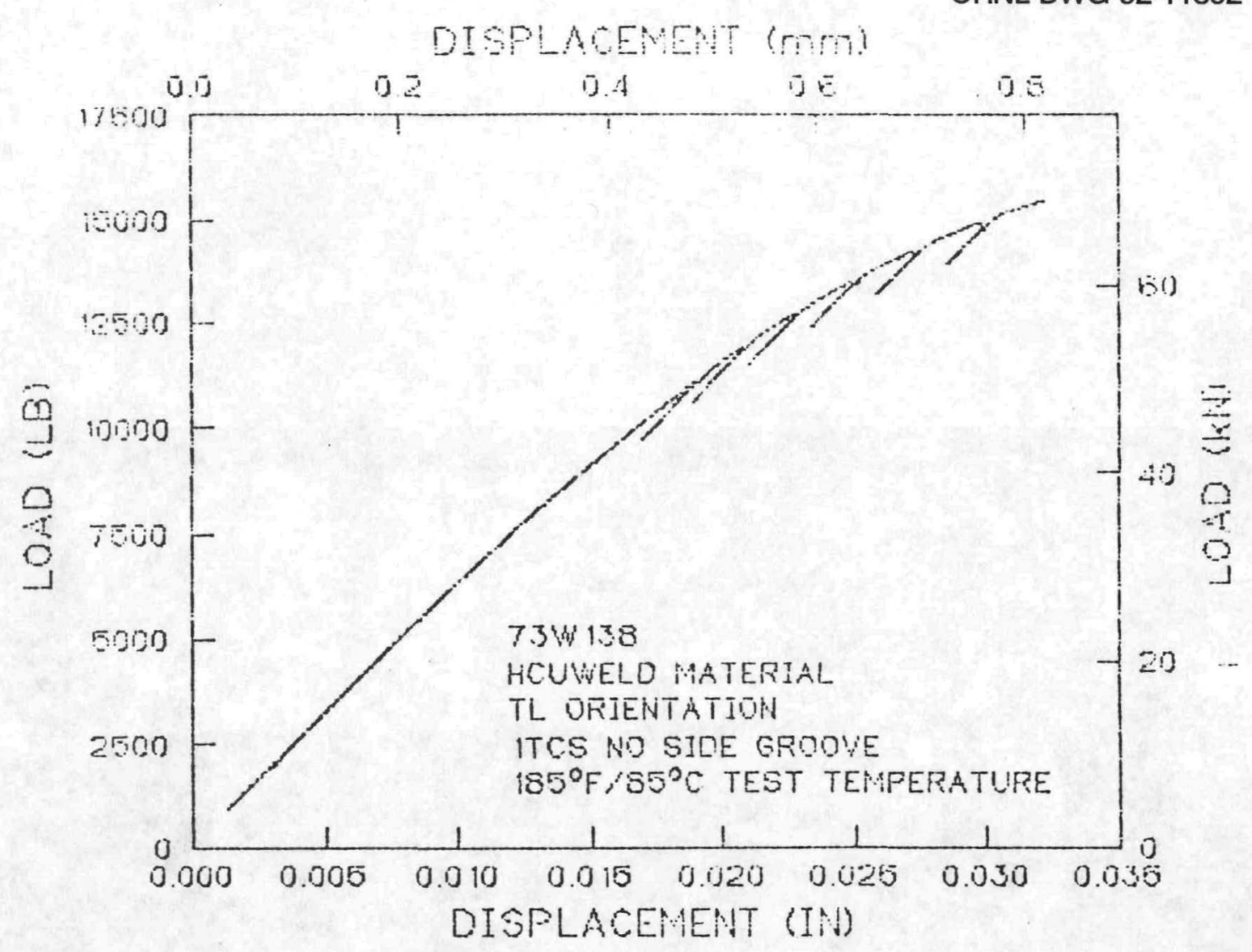

73W-138

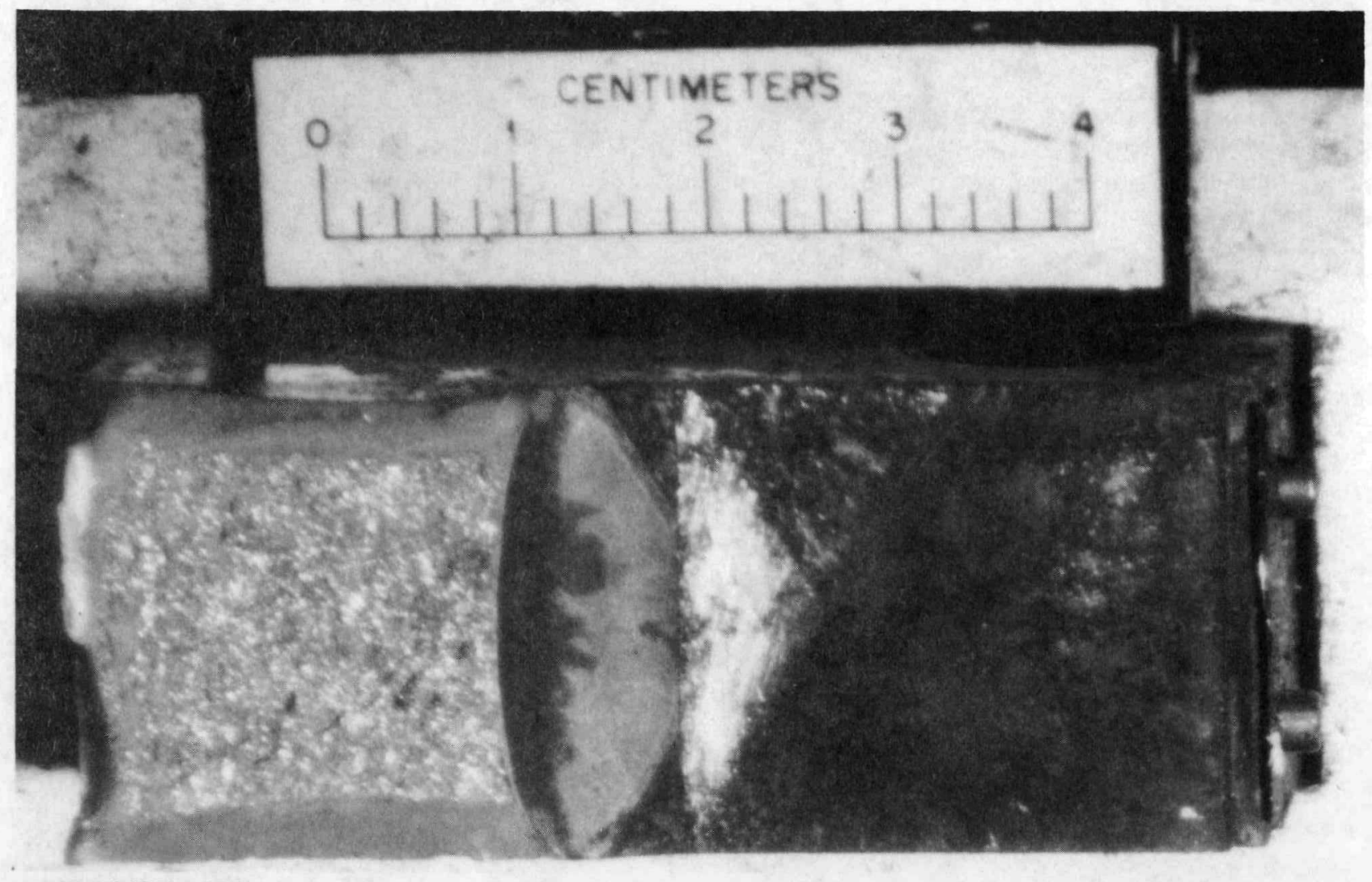




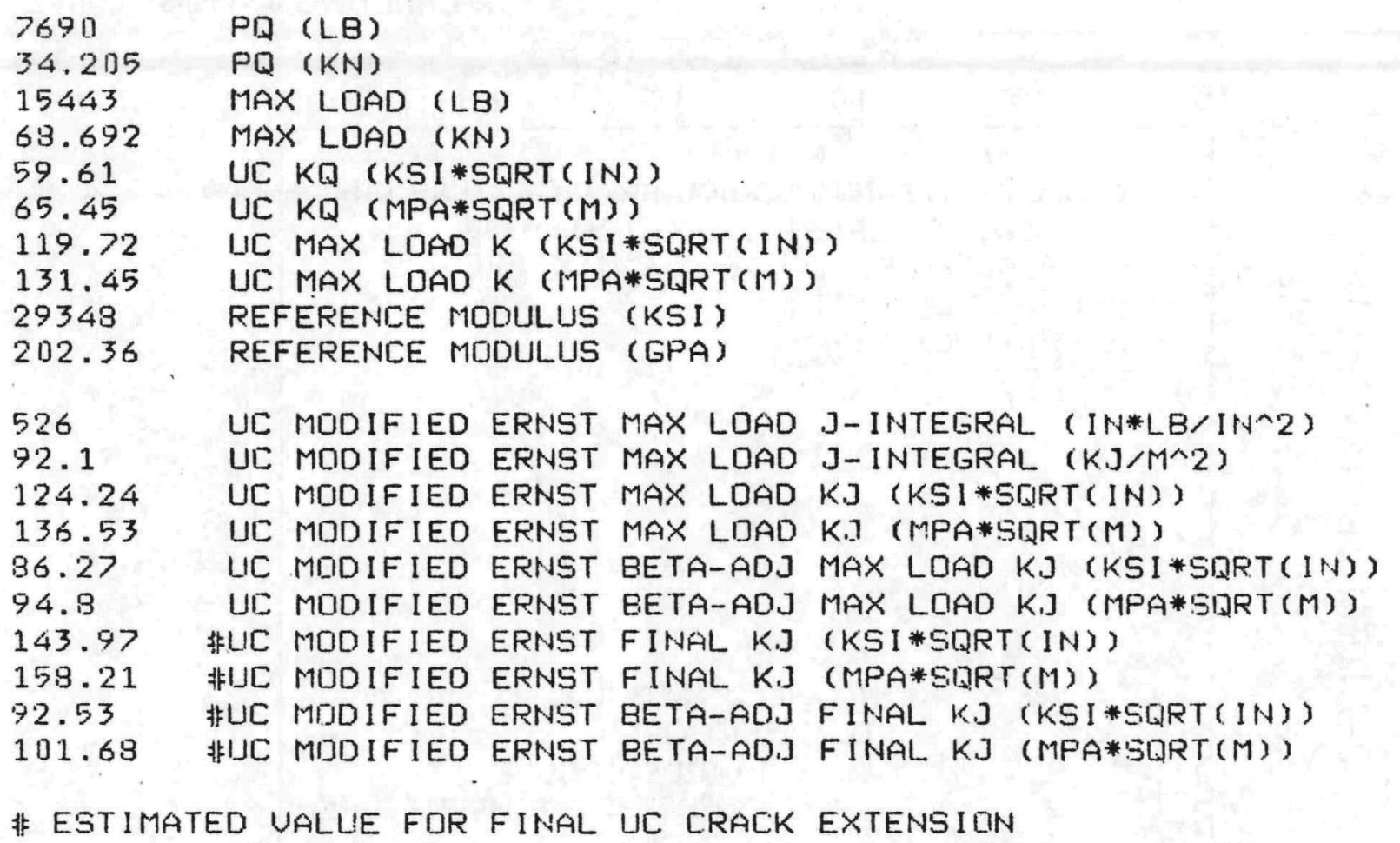

GET POWER LAW FIT TO DATA 絽 NO

UL MIDIFIED ERNST JIC, CEIC,KJIC AND TAUG NDT DEFINED--PDWER LAW FIT NDT REDLESTED BY TECHNICIAN 

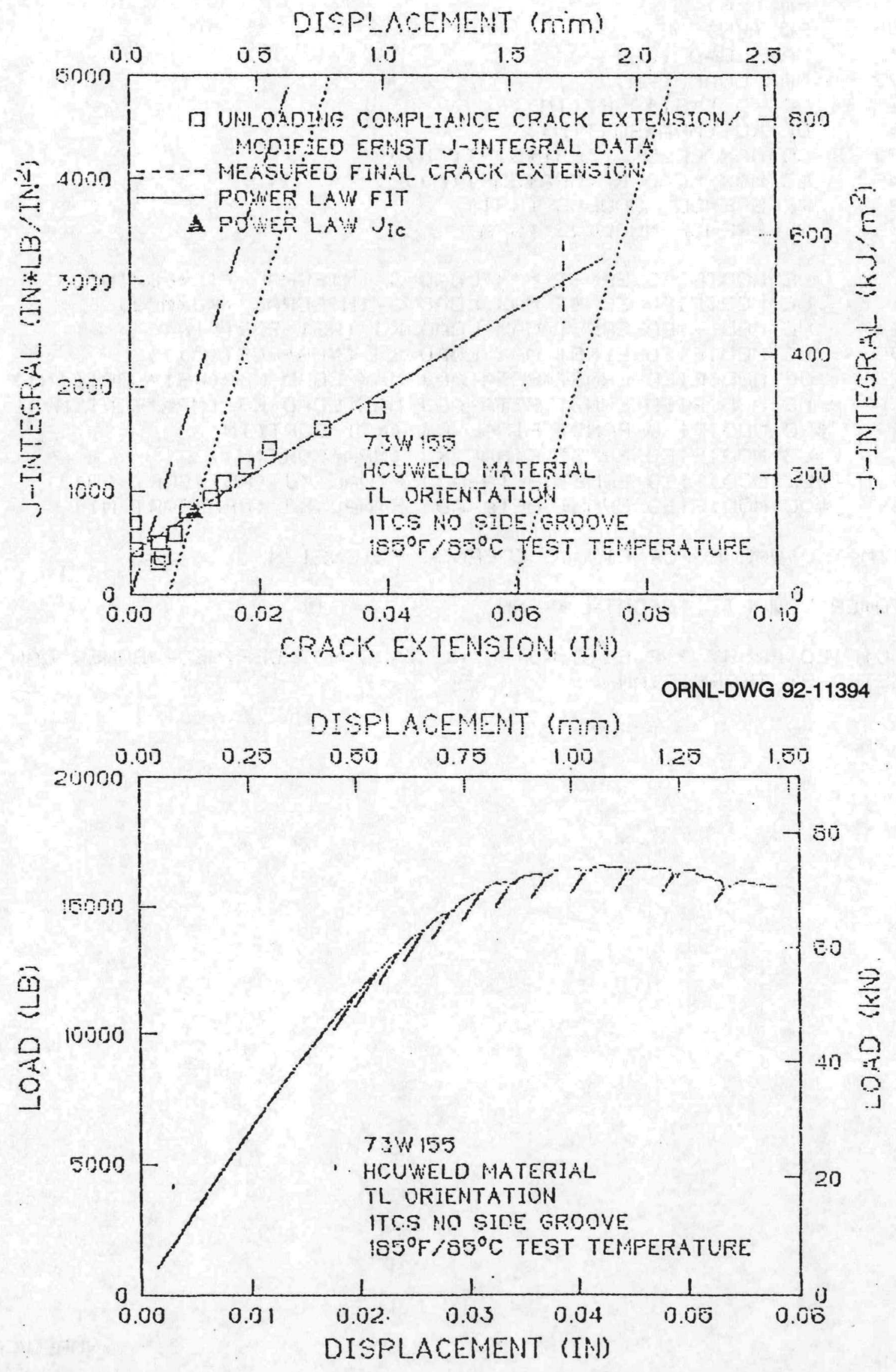


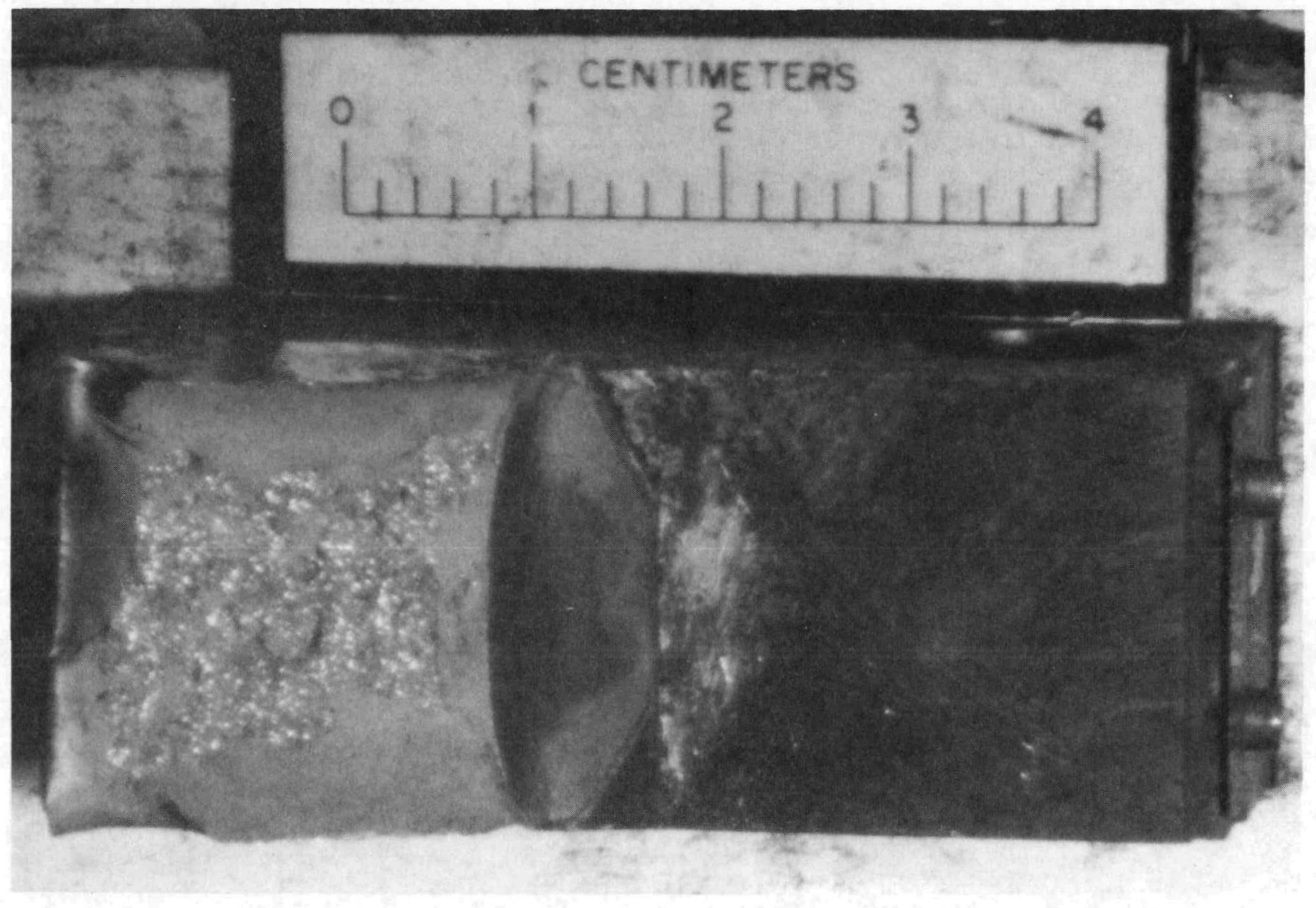




\section{F-208}

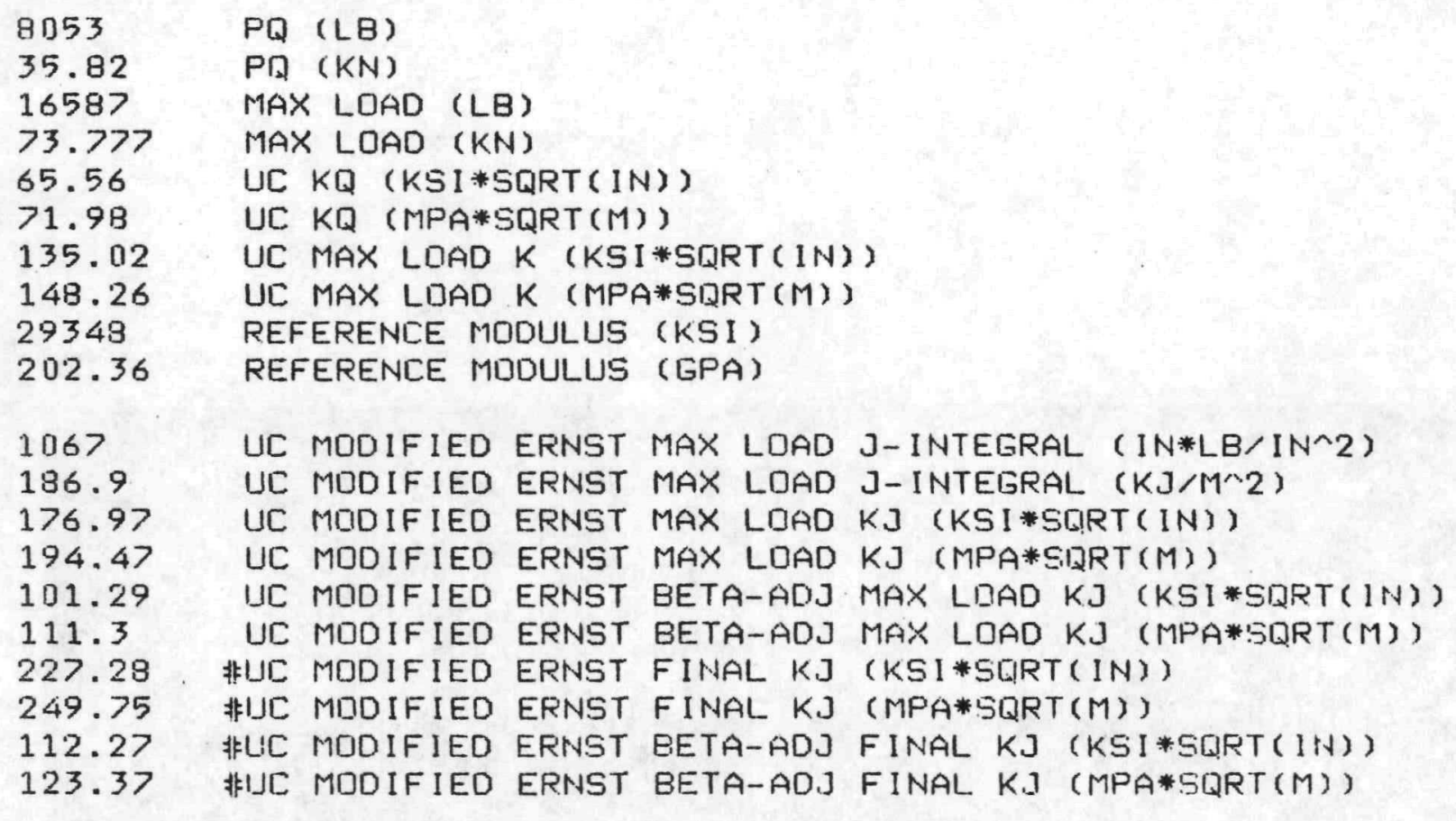

\# ESTIMATED UALLIE FOR FINAL UIC CRACK EXTENSION

GET PDWER LAW FIT TO DATA 繰 YES

ACLEPT POWER LAW FIT TD TEST DATA

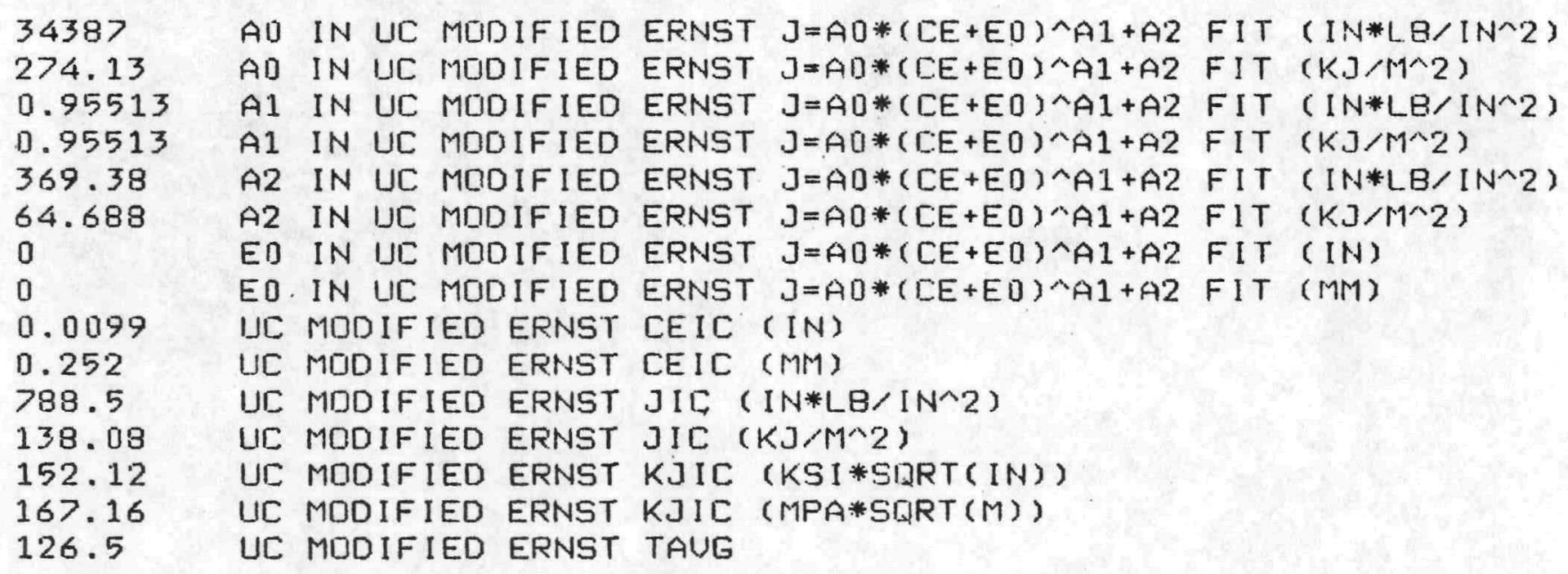


DISPLACEMENT (mim)

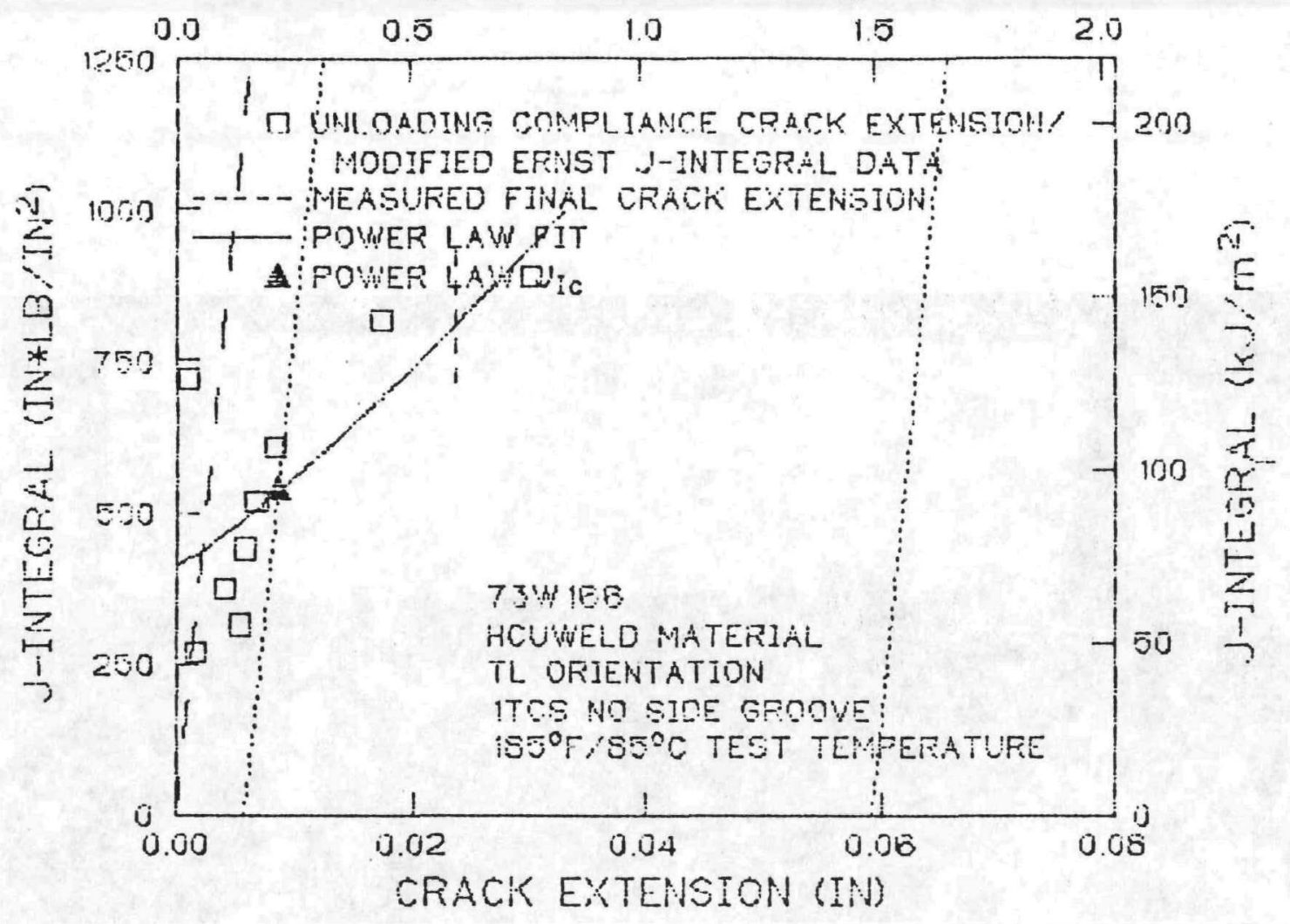

ORNL-DWG 92-11395

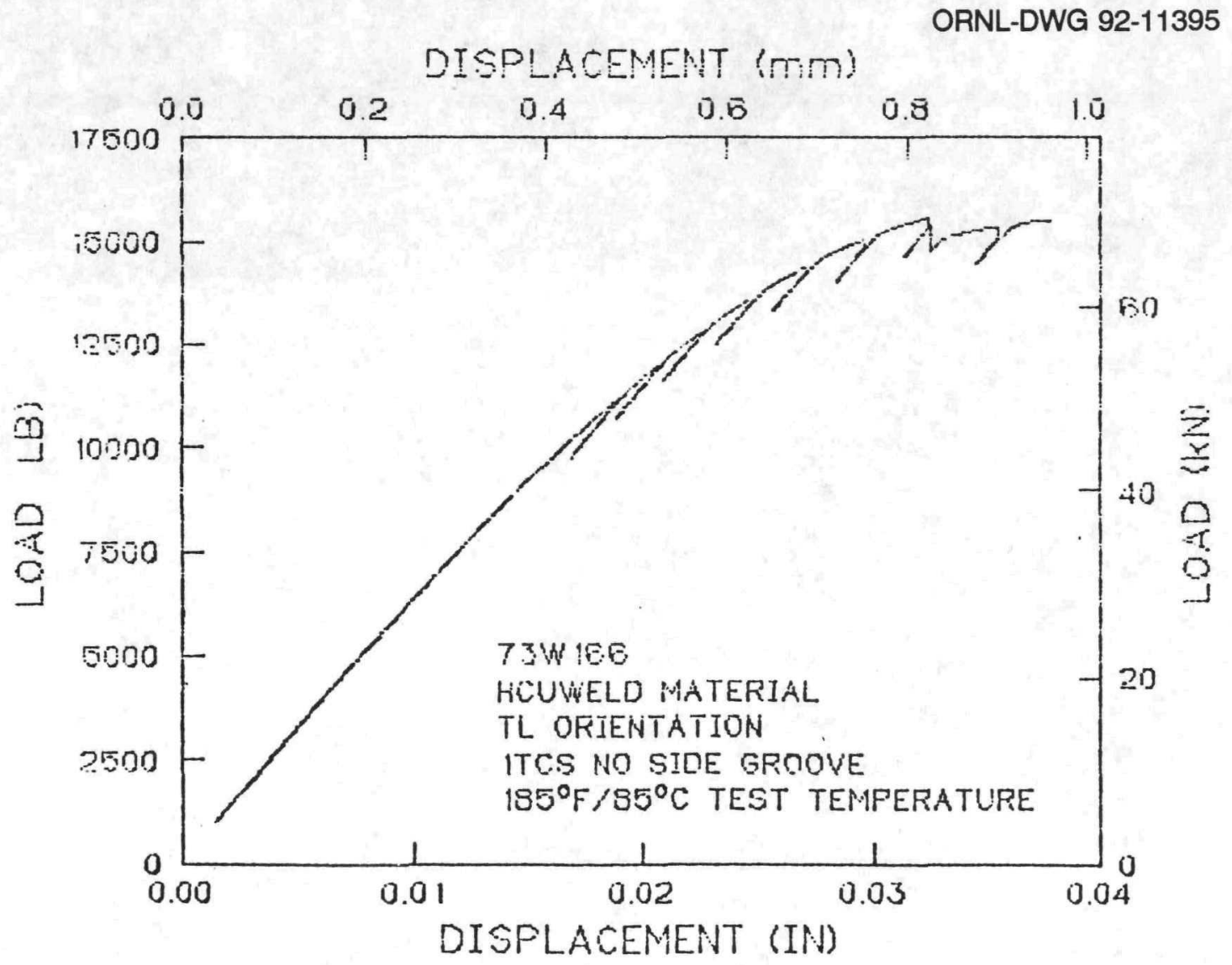


F-210

$73 W-166$

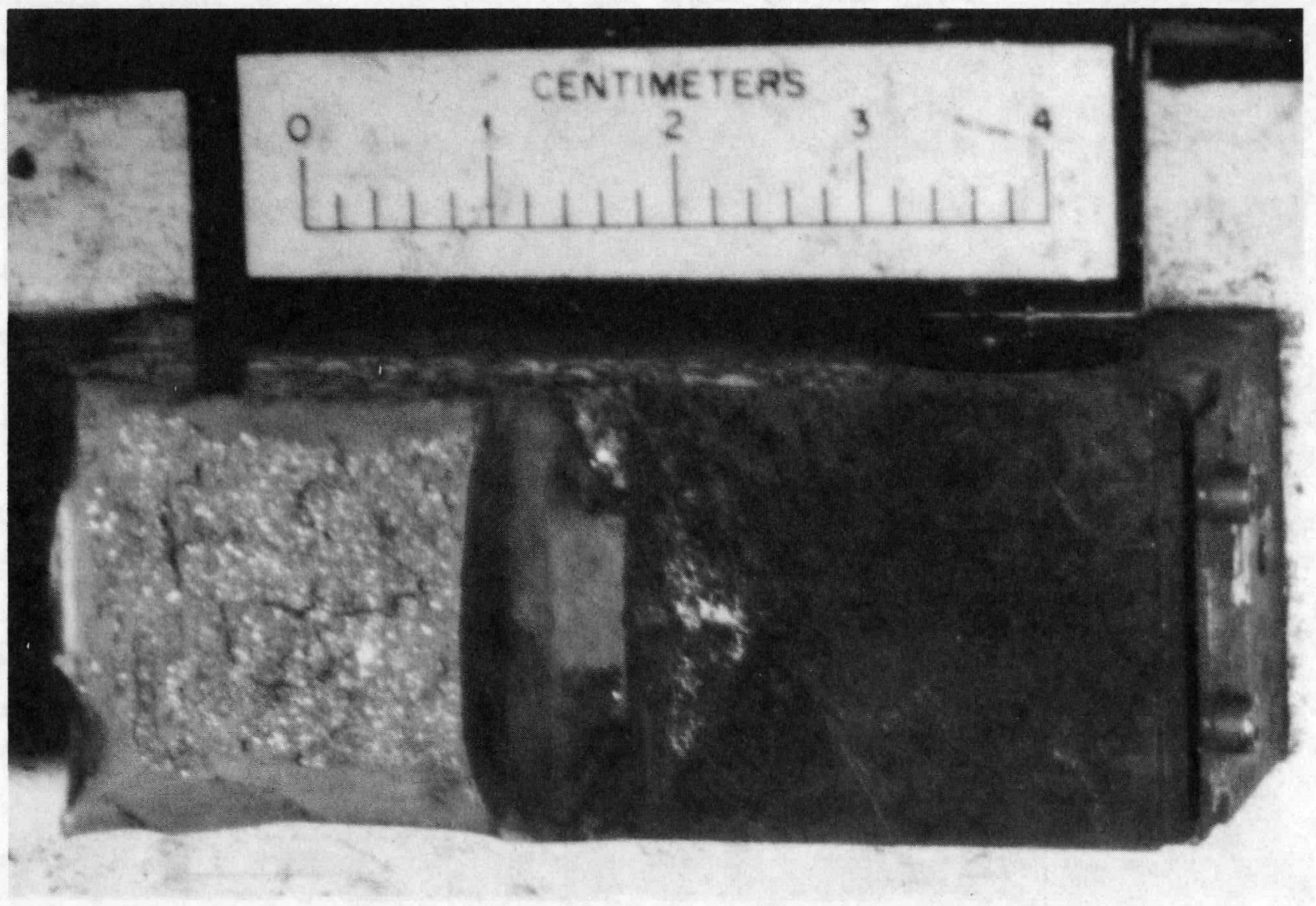




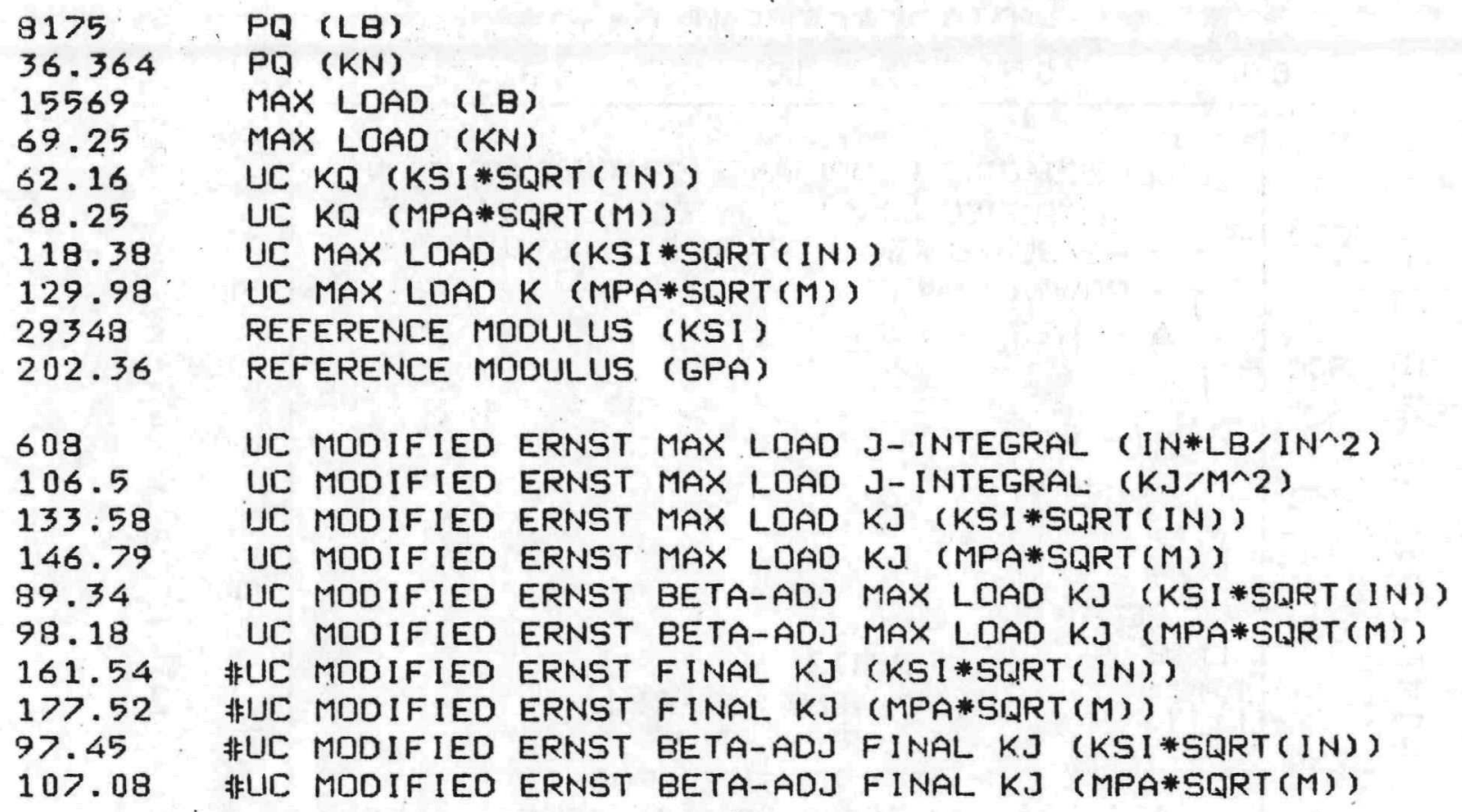

\# ESTIMATED UALLIE FOR FINAL UC CRACK EXTENSION

GET POWER LAW FIT TO DATA Y*: YES

ACCEPT POWER LAW FIT TO TEST DATA YII: YES

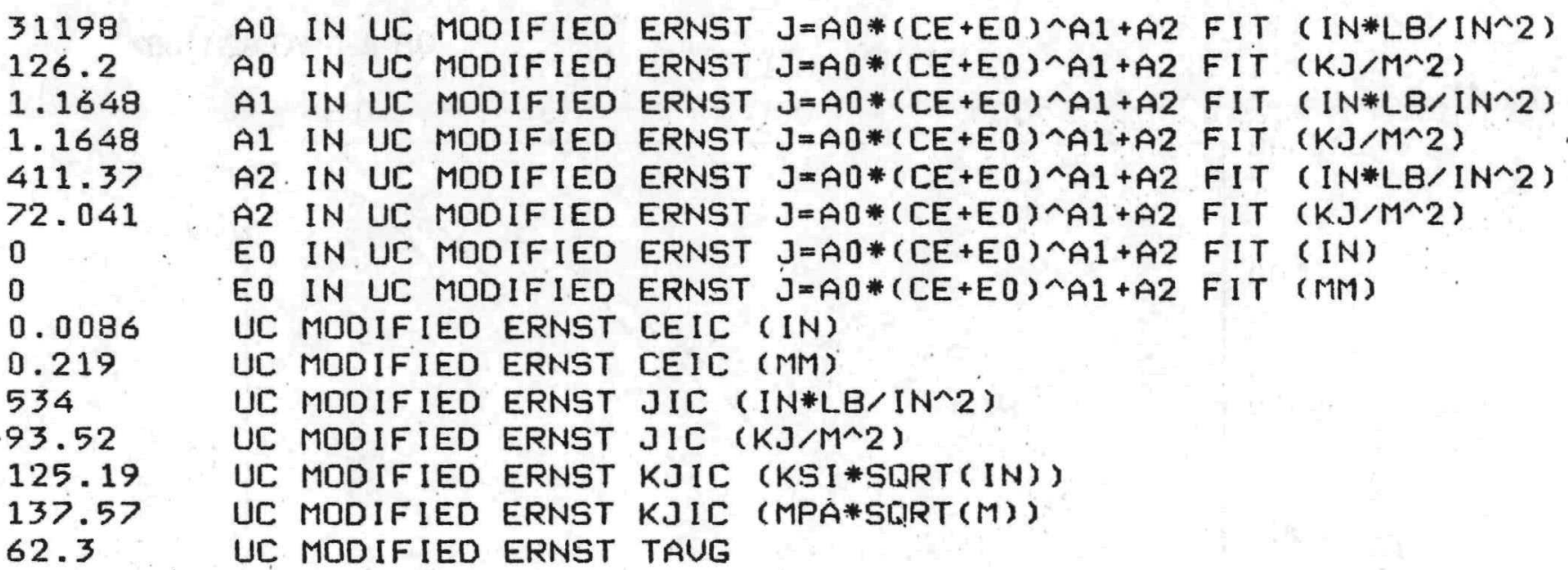


DISPLACEMENT (mm)
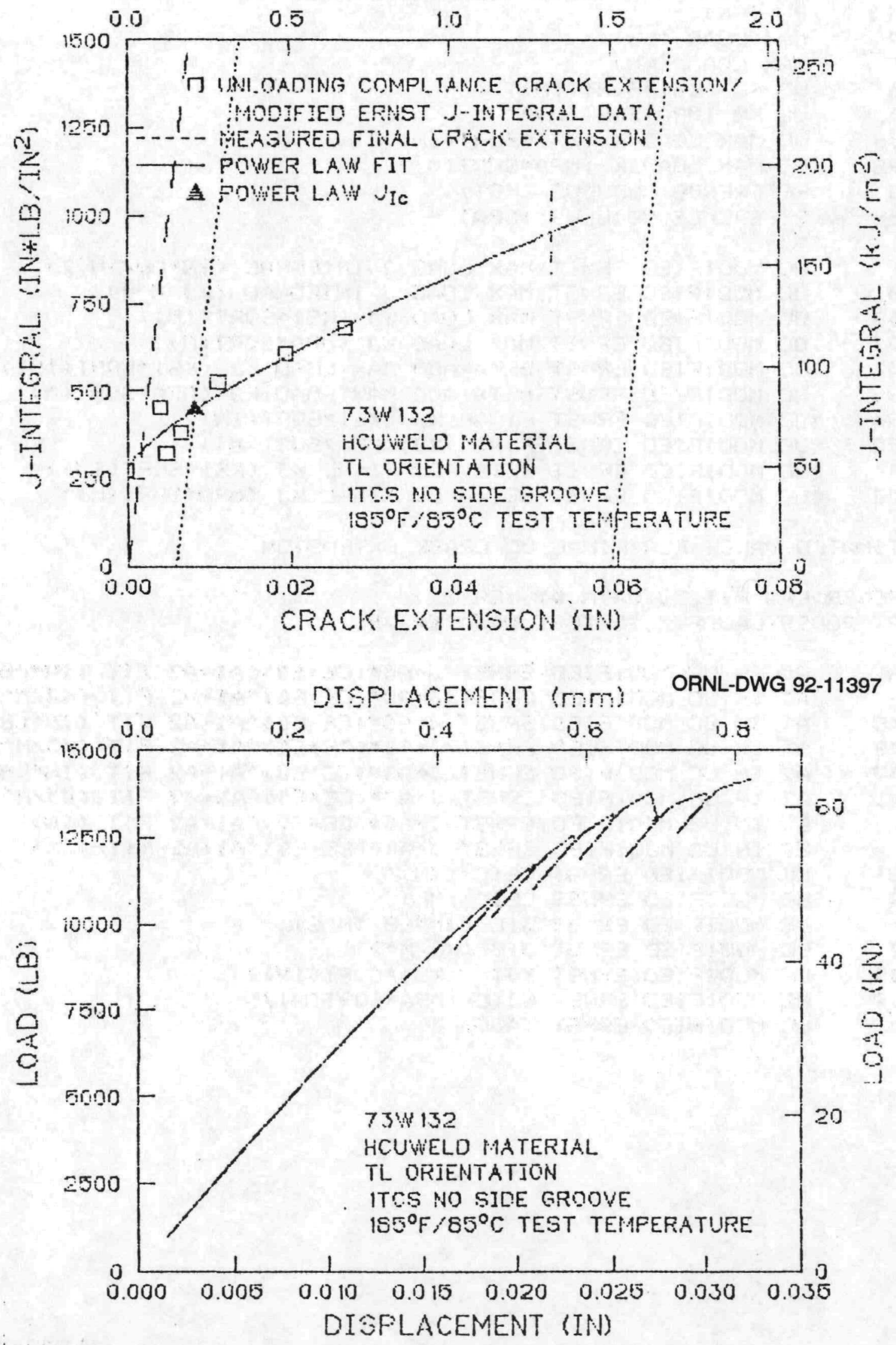
F-213

73W-132

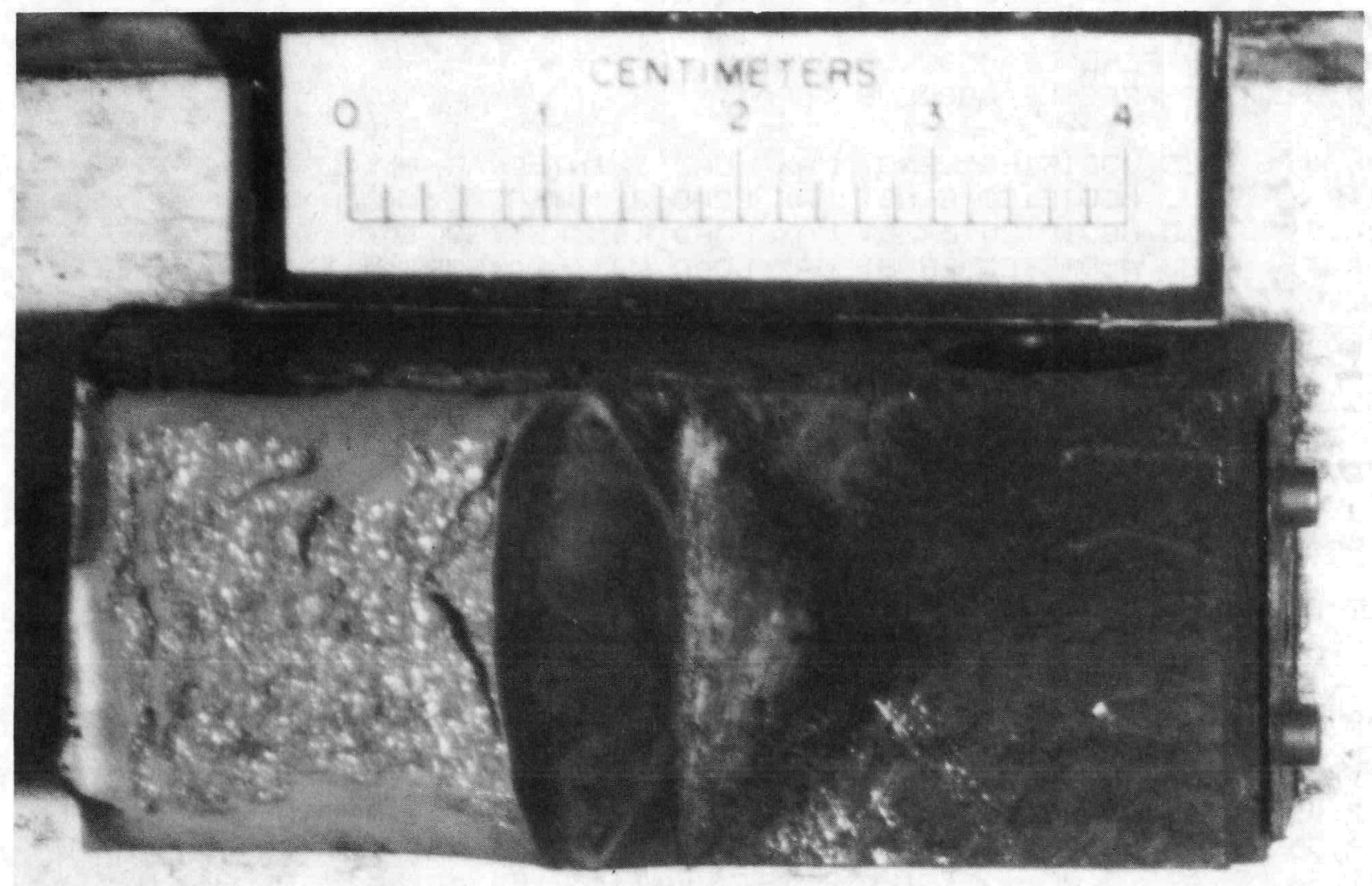


F-214

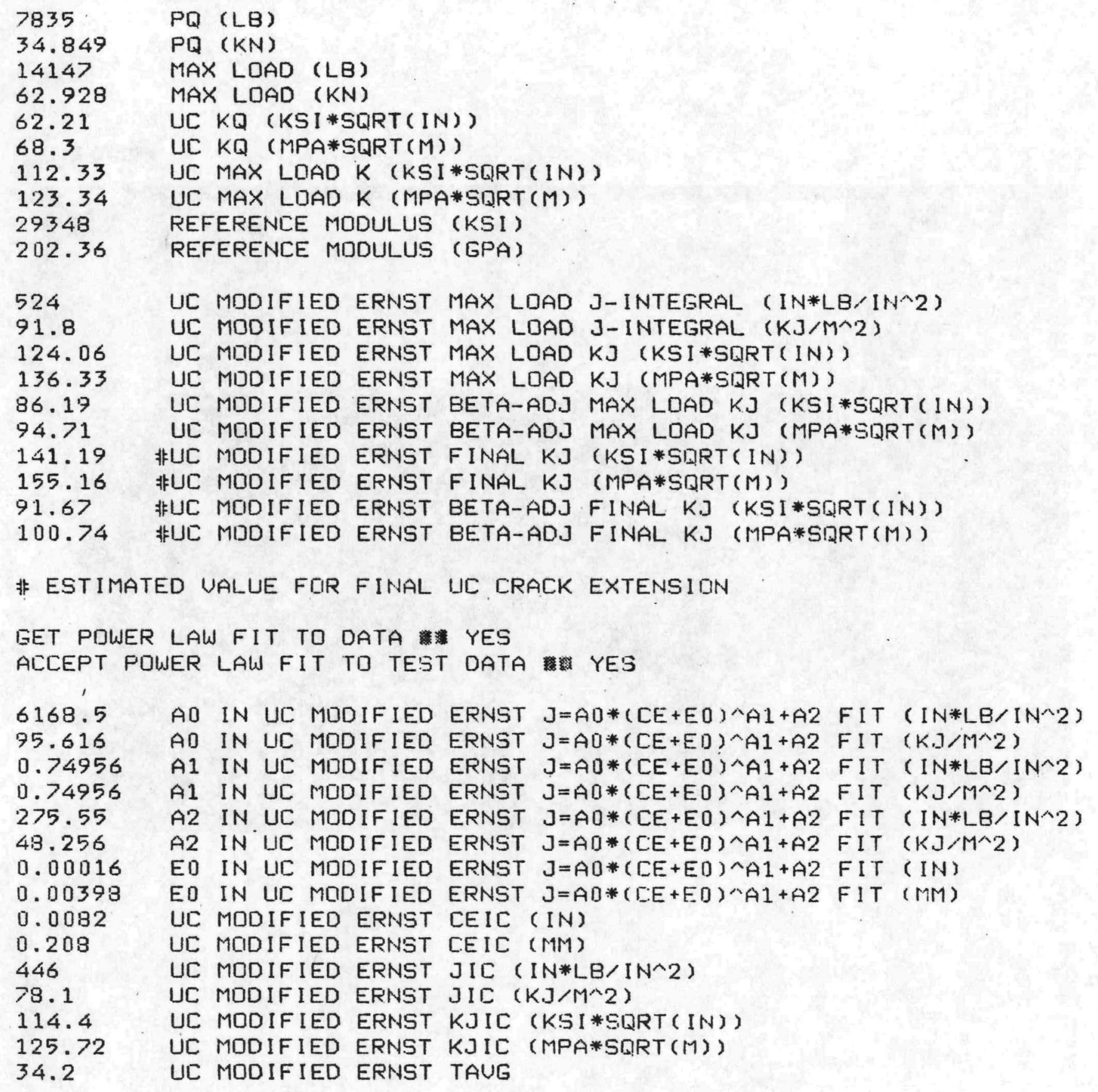




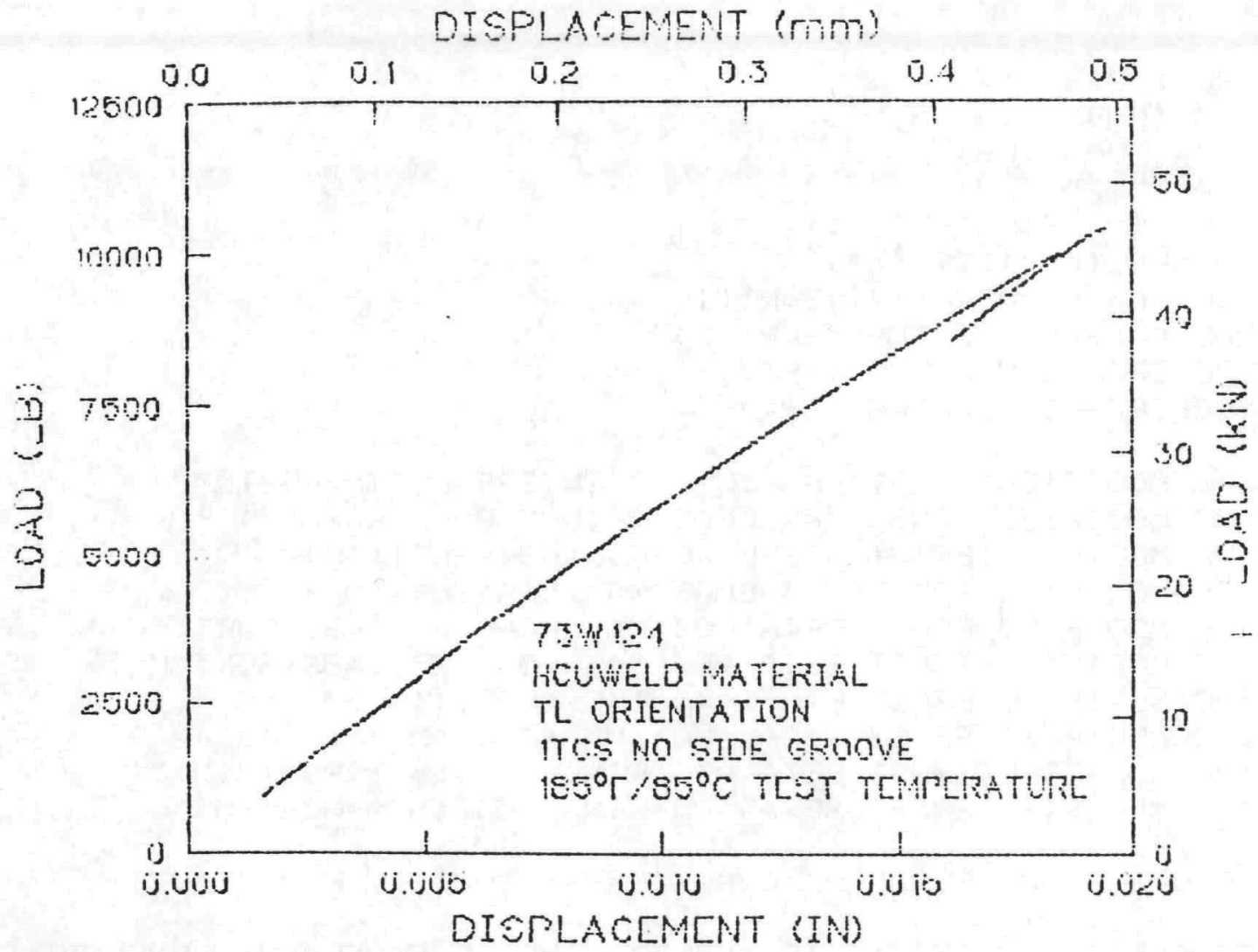

73W-124

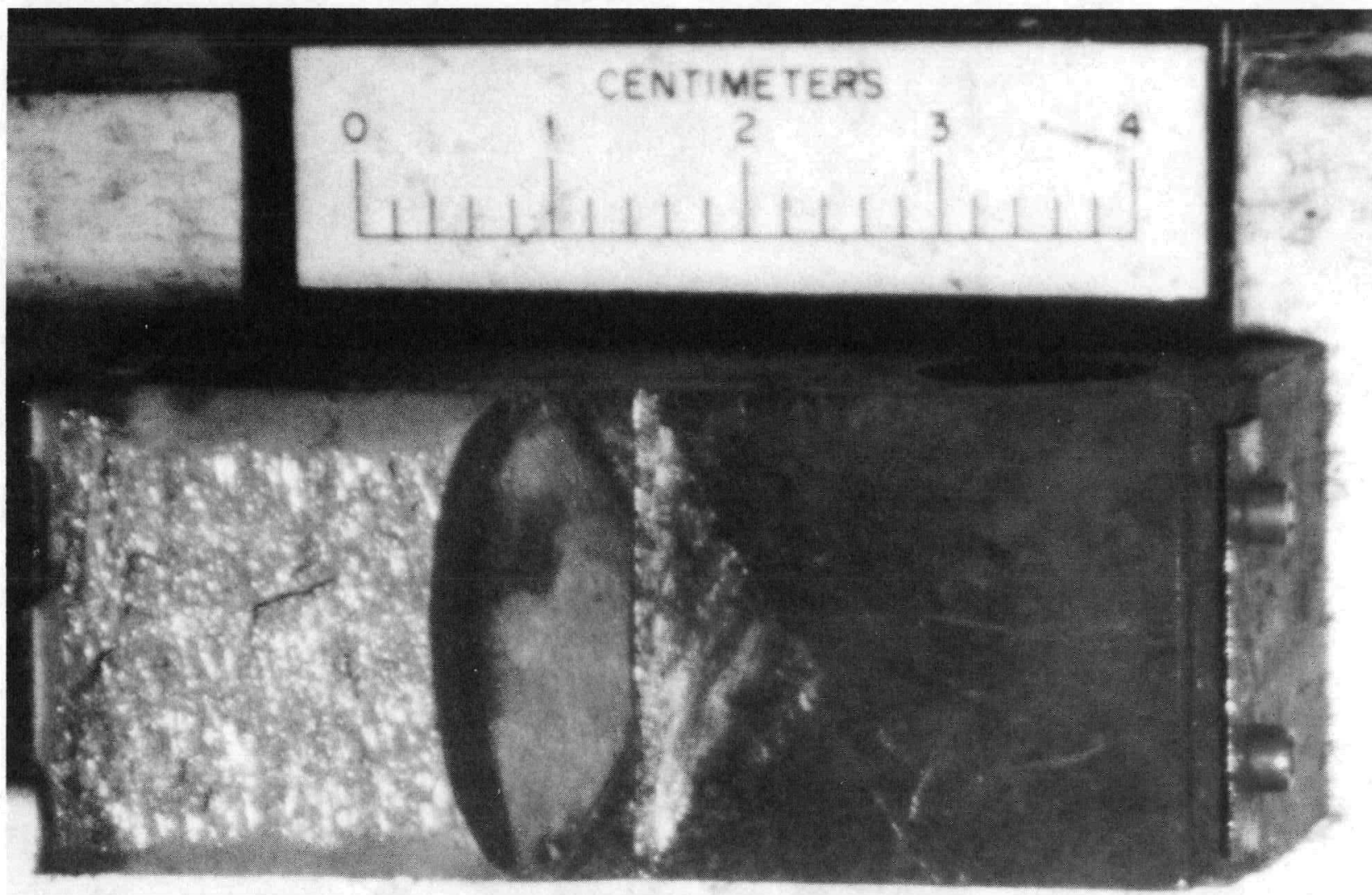




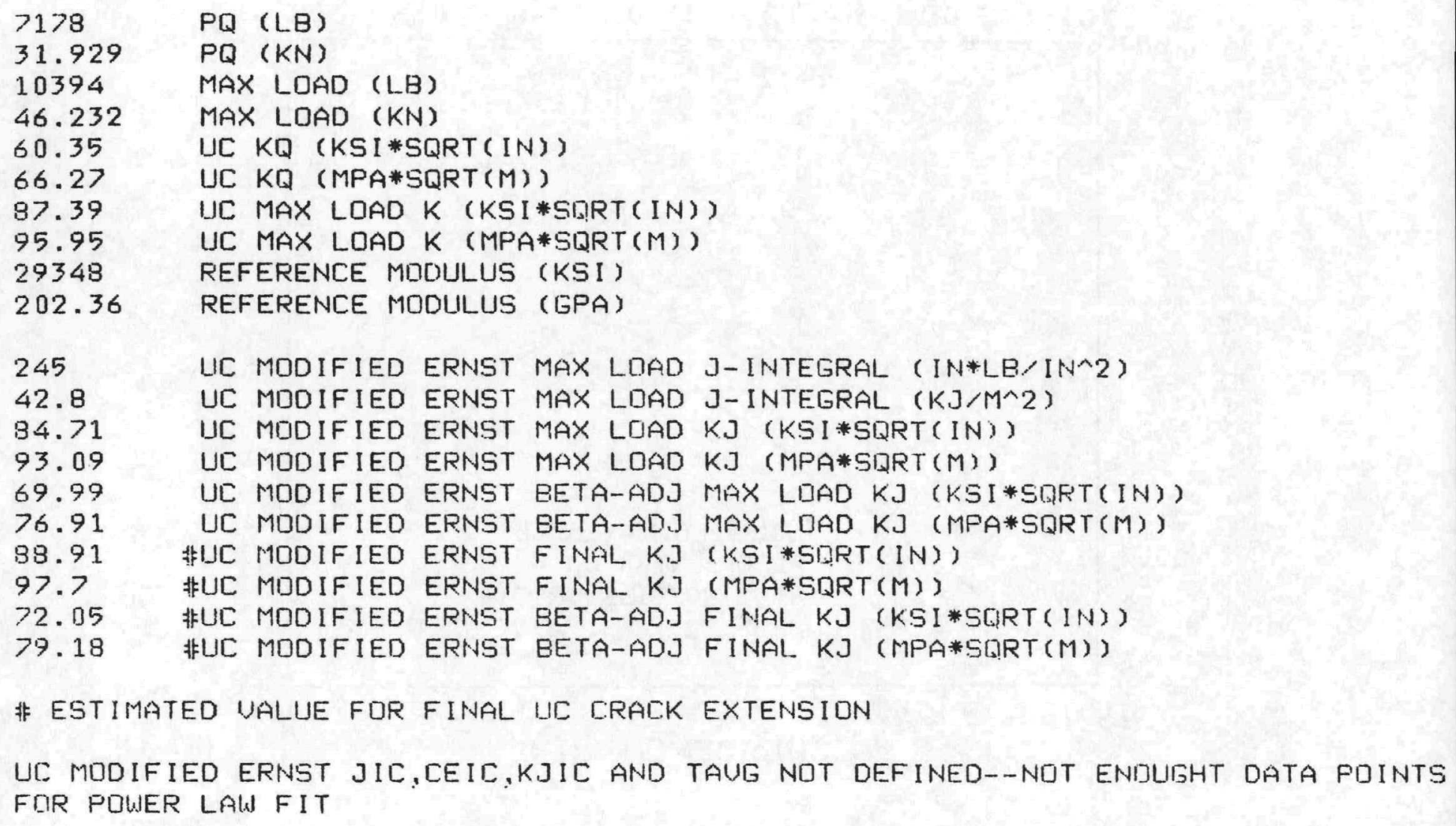




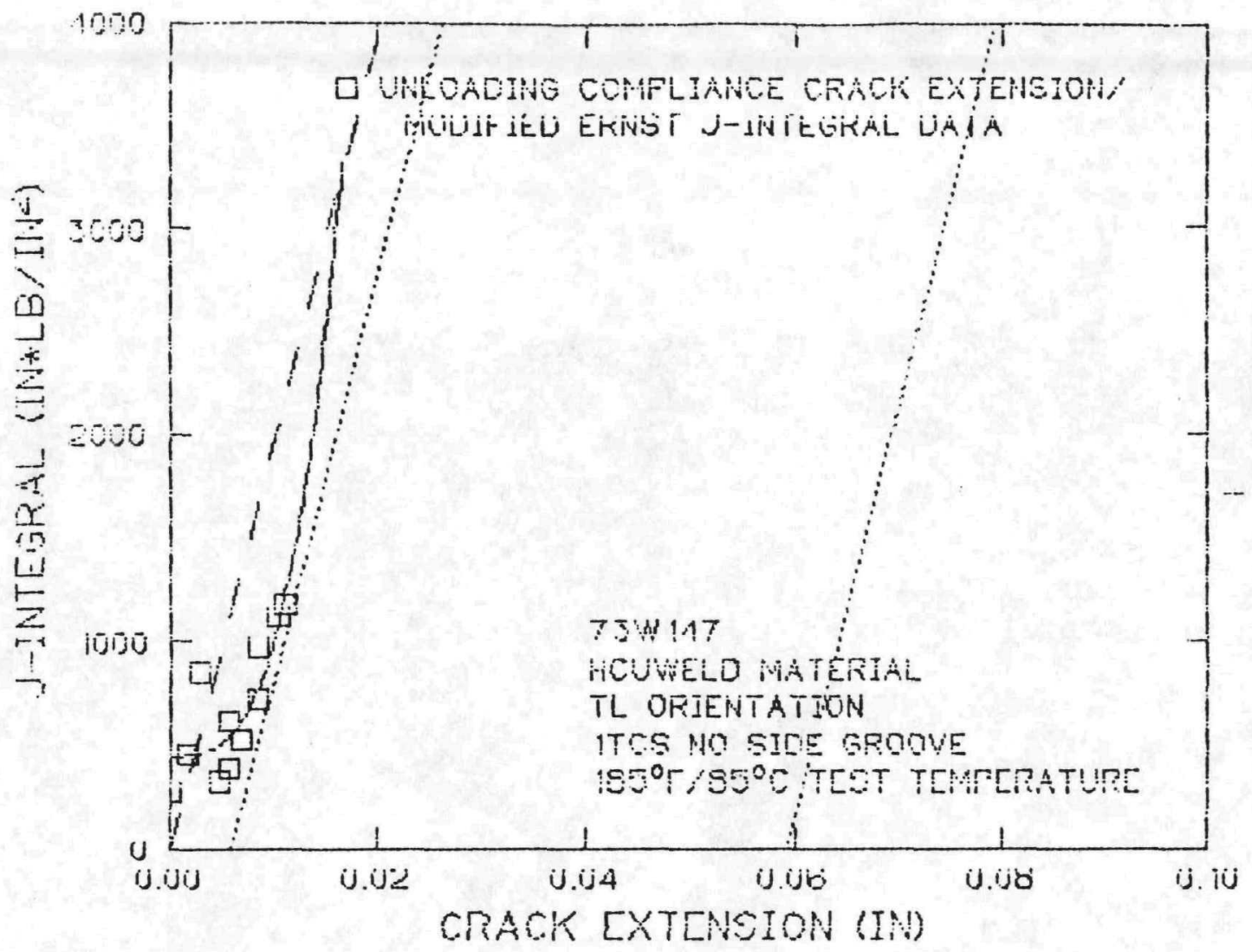

ORNL-DWG 92-11400

DISPLACEMENT (mM)

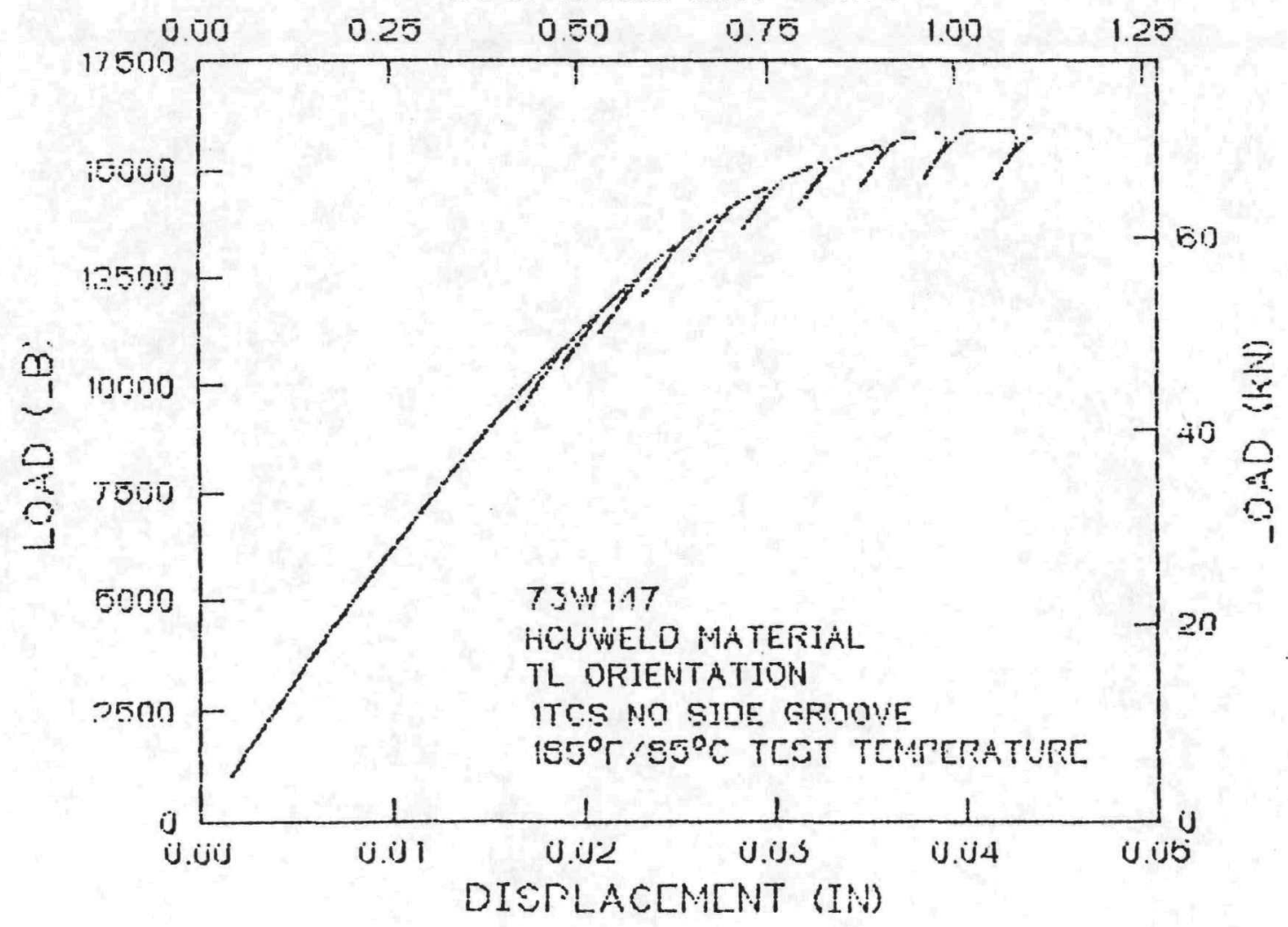


F-218

$73 W-147$

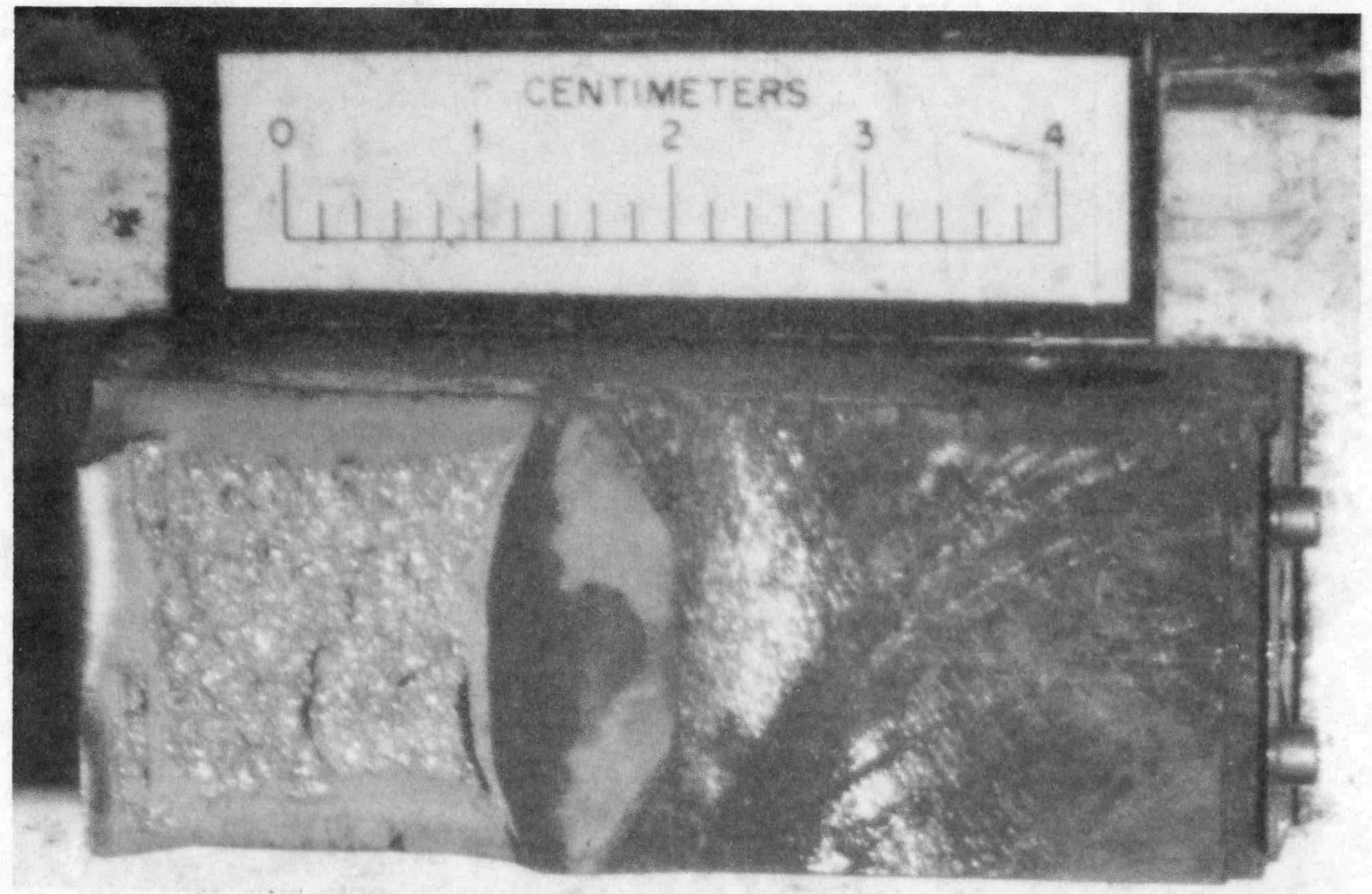

$73 W-147$

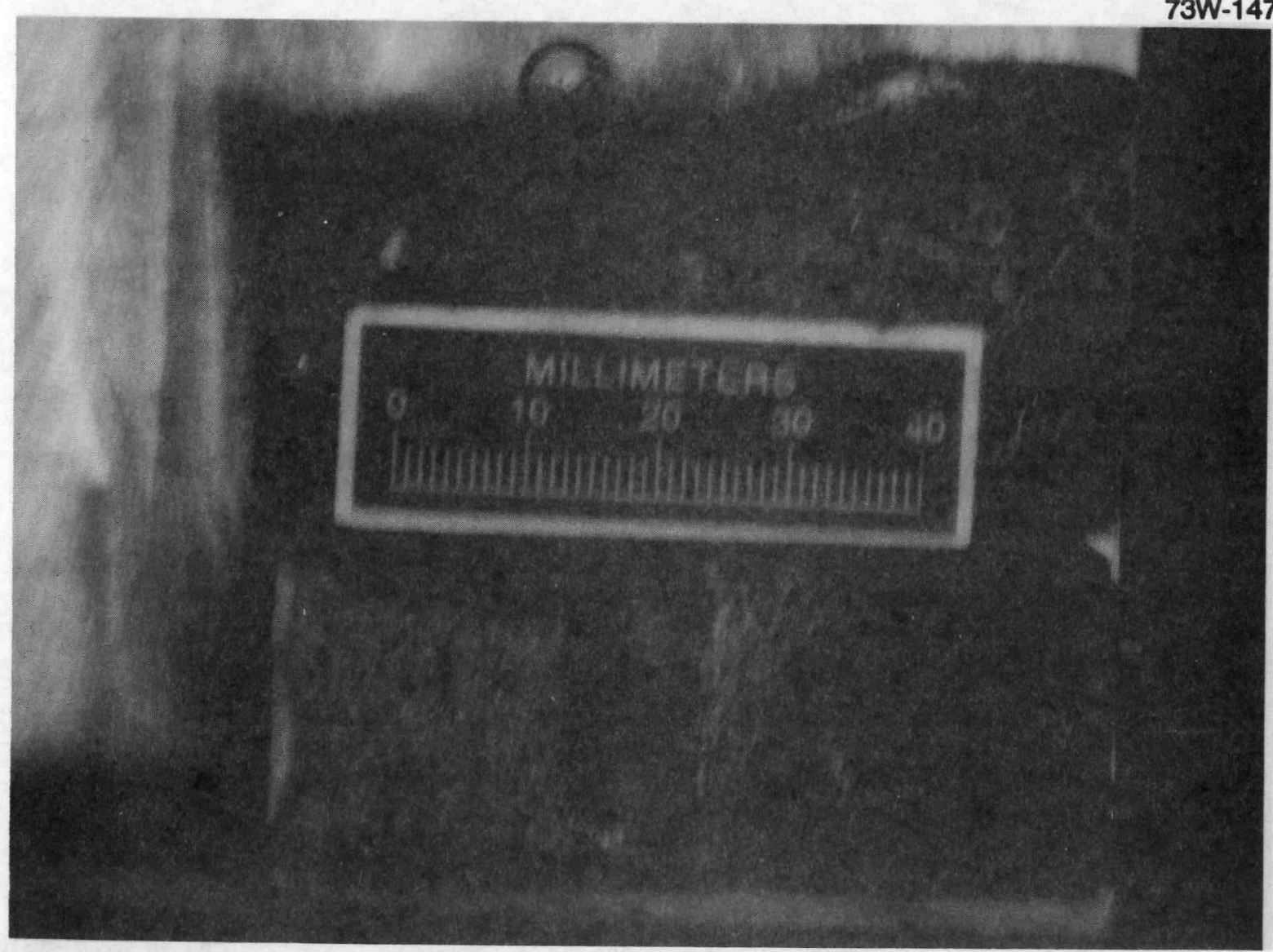

NUREG/CR-5913 
DISPIACEMENT (mm)

ORNL-DWG 92-11401

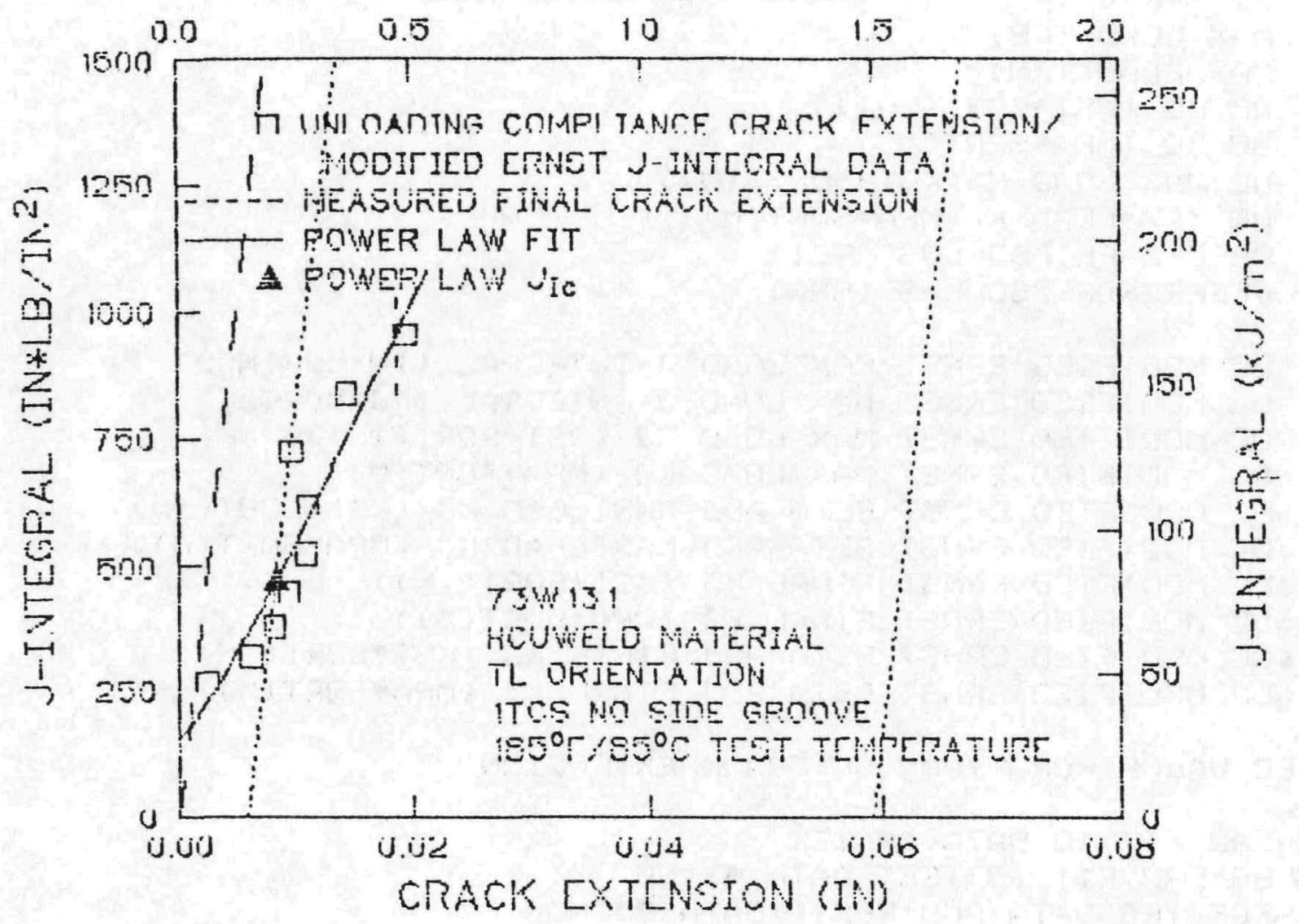

ORNL-DWG 92-11402

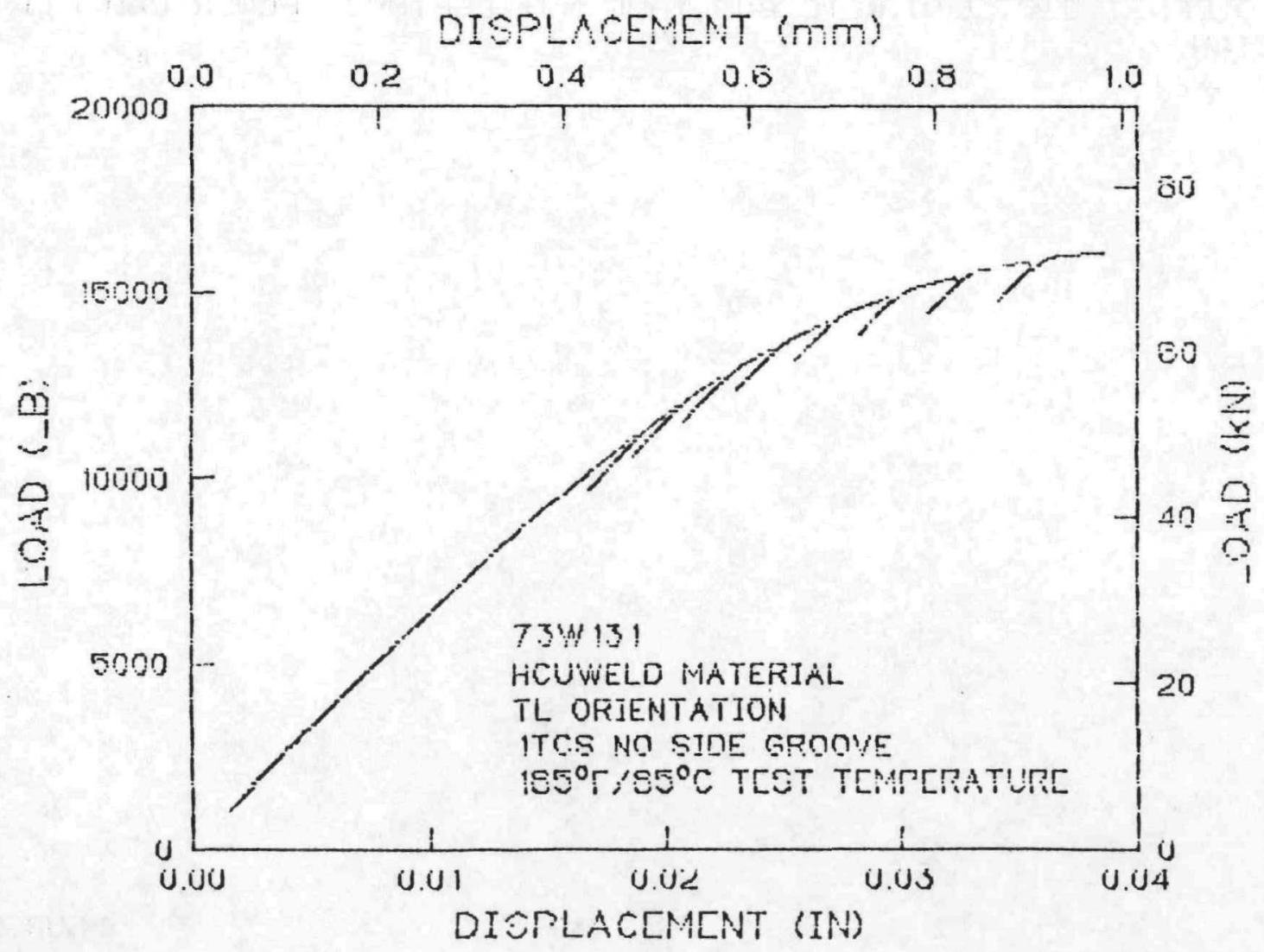




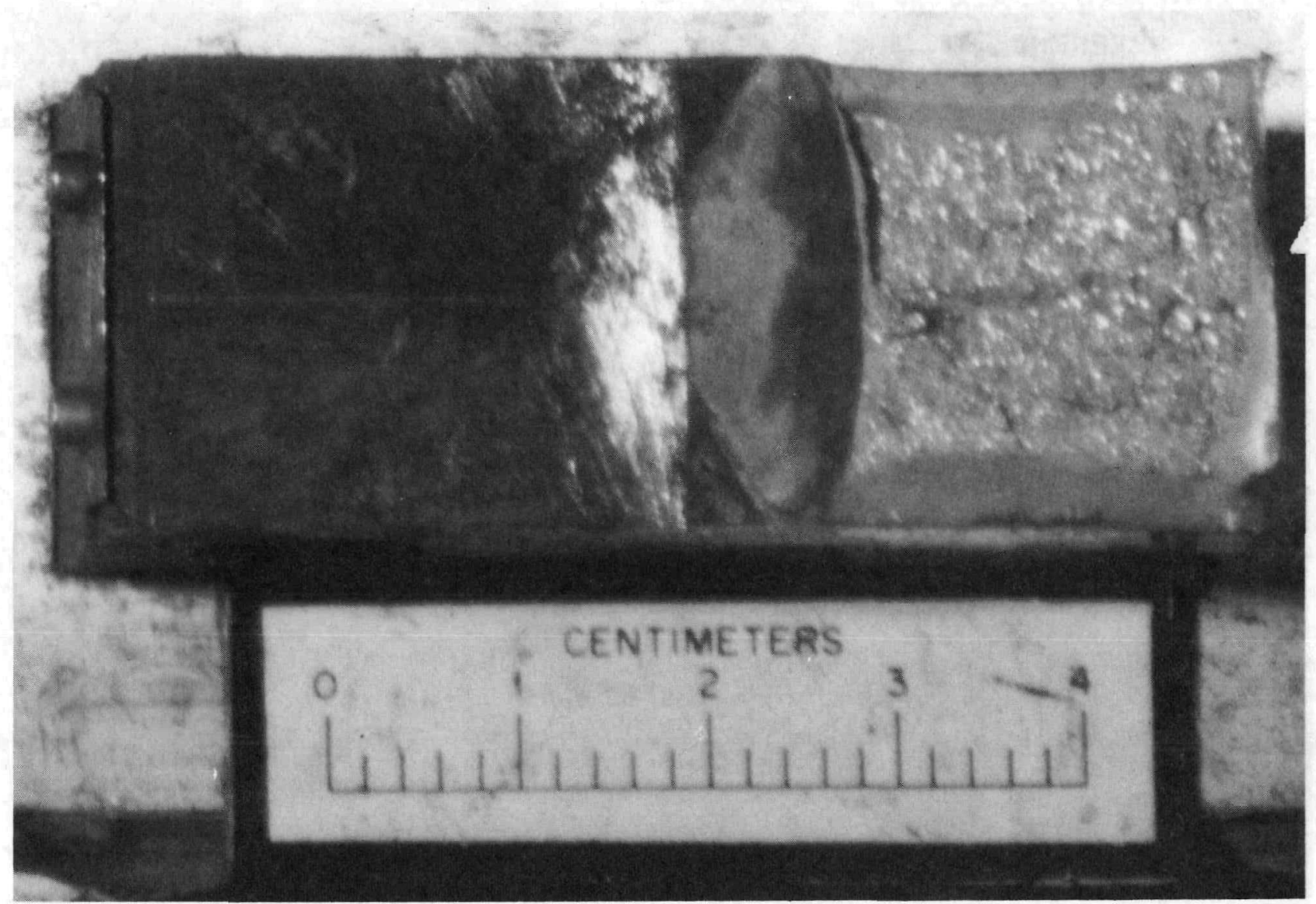




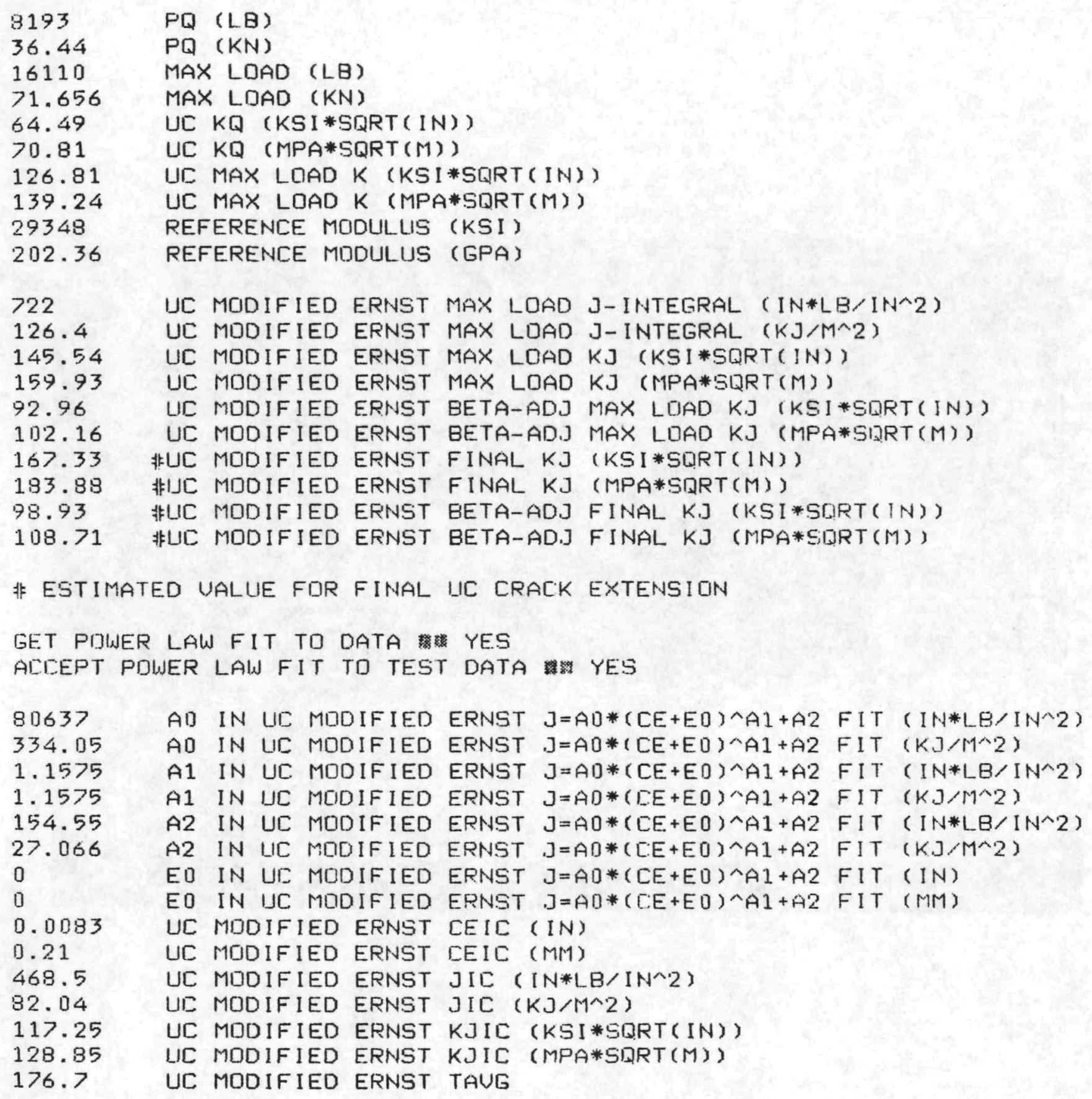



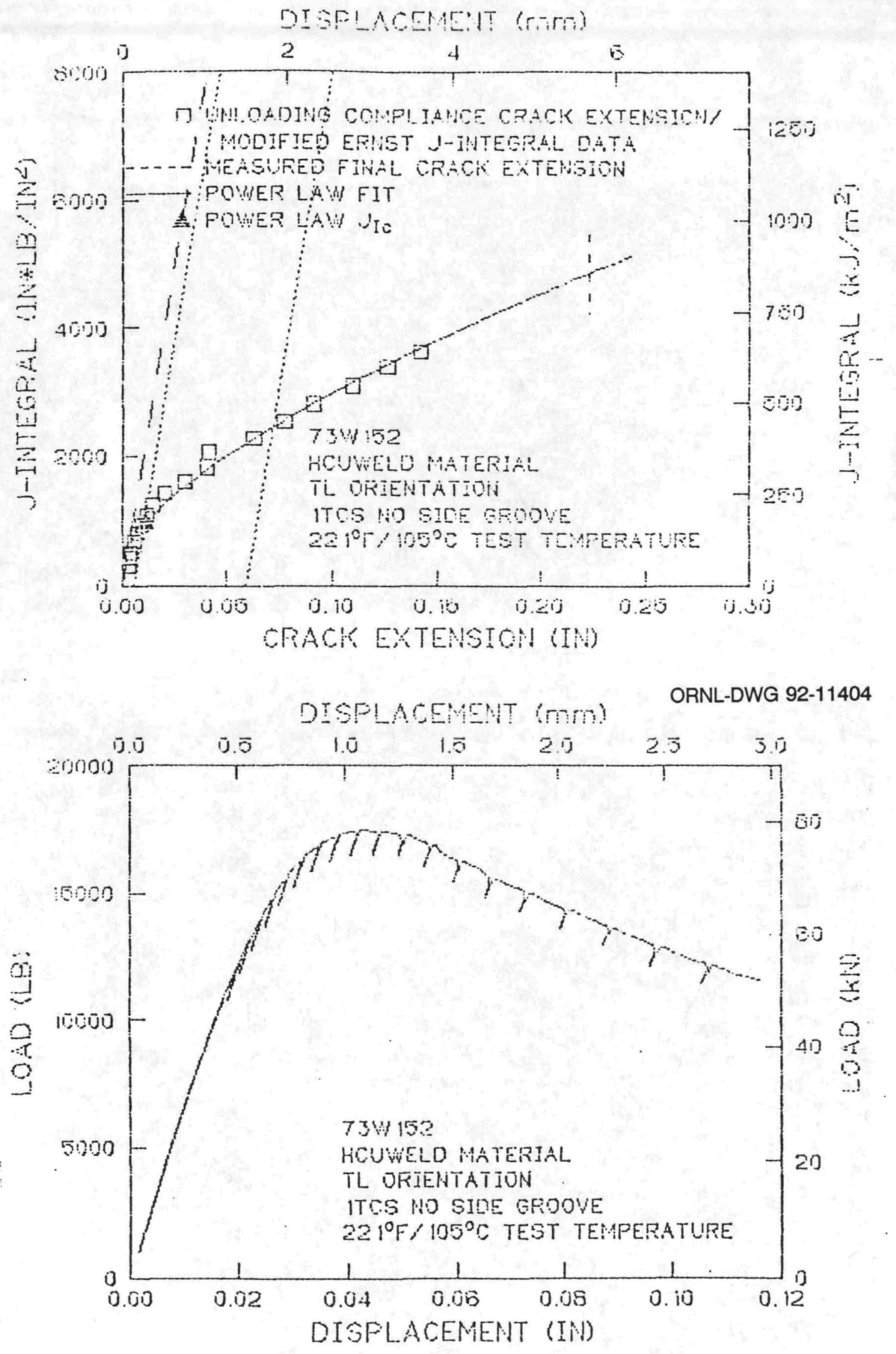
$73 W-152$

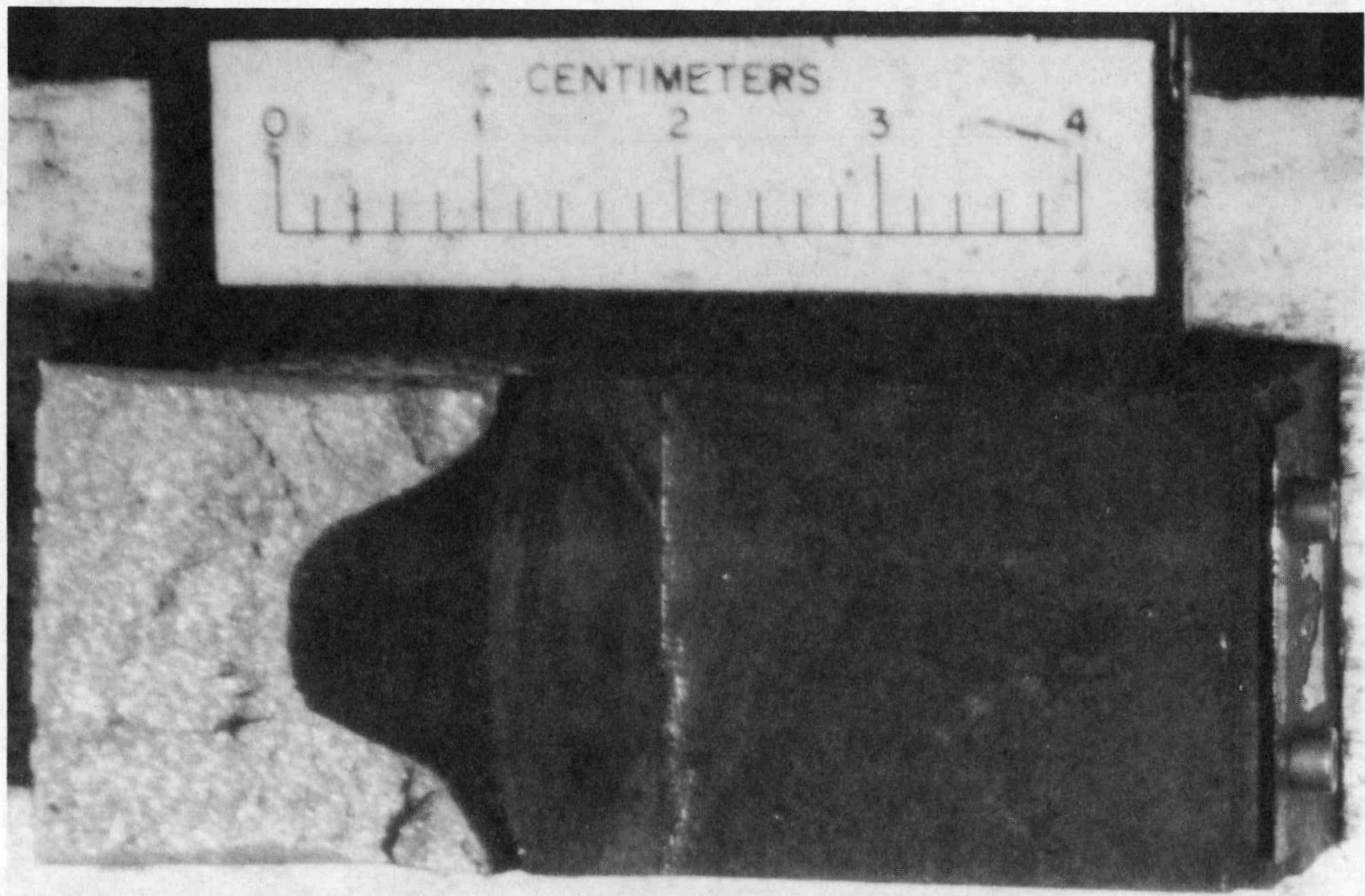

T3W-152

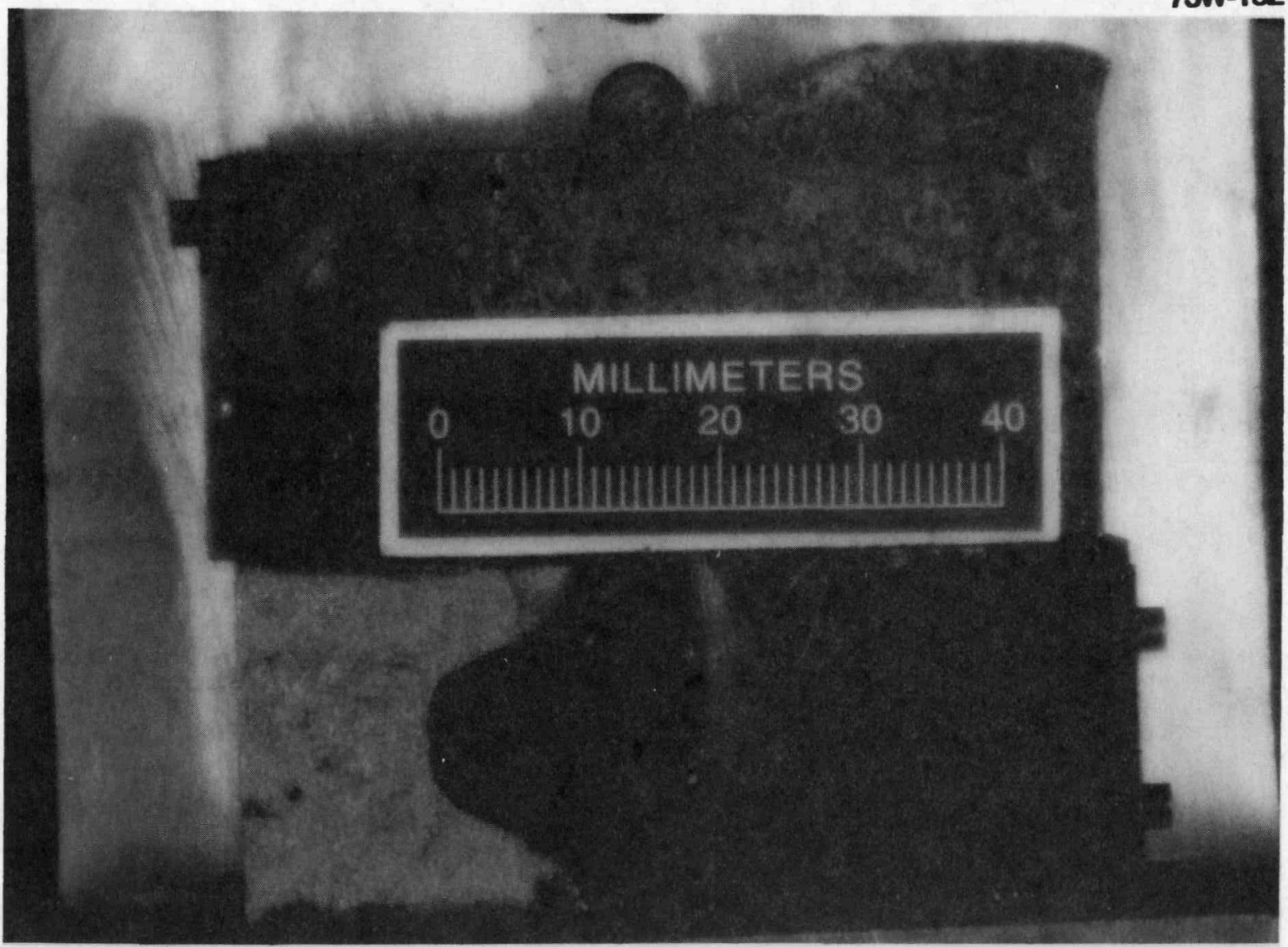




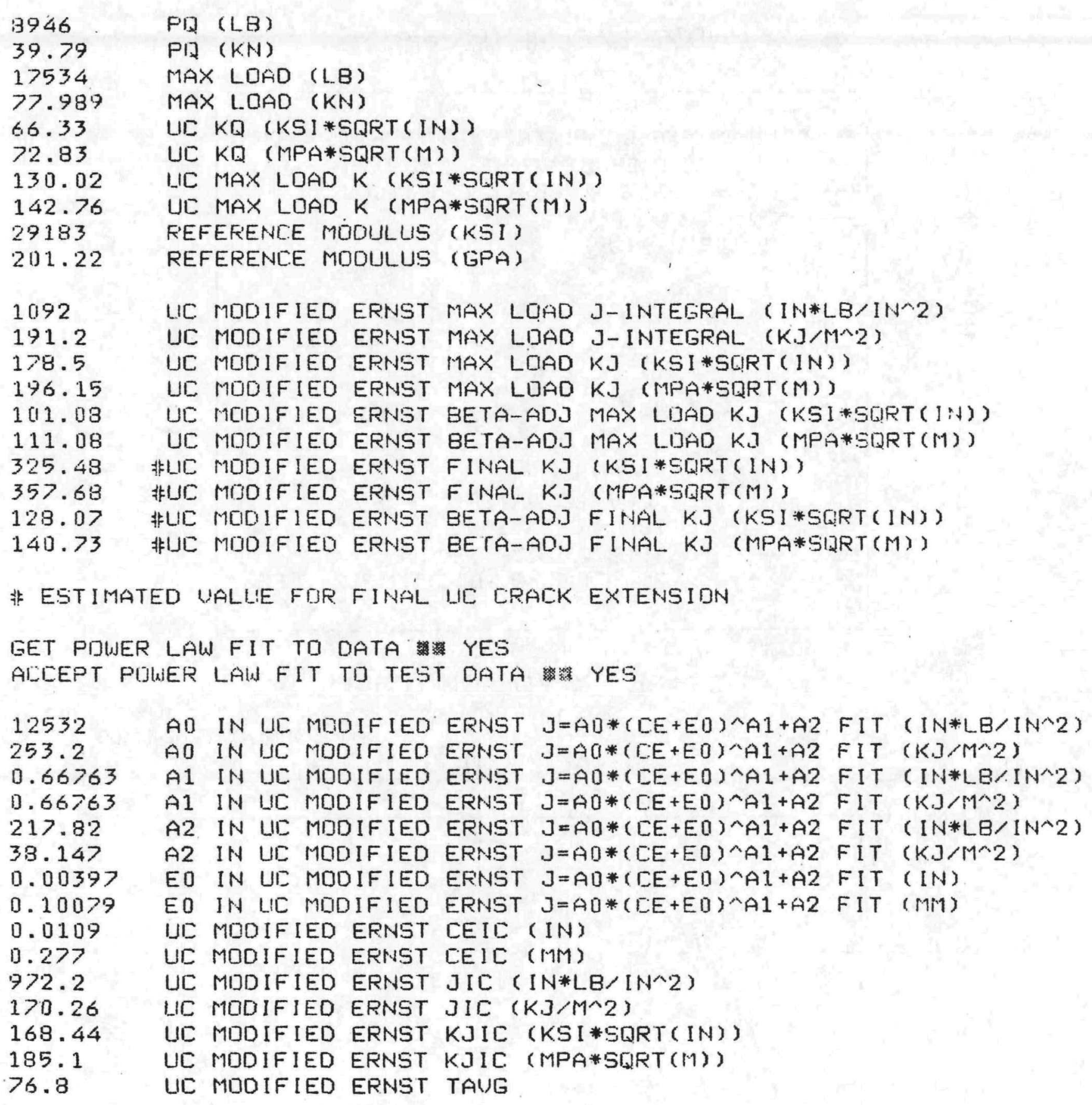


ORNL-DWG 92-11405
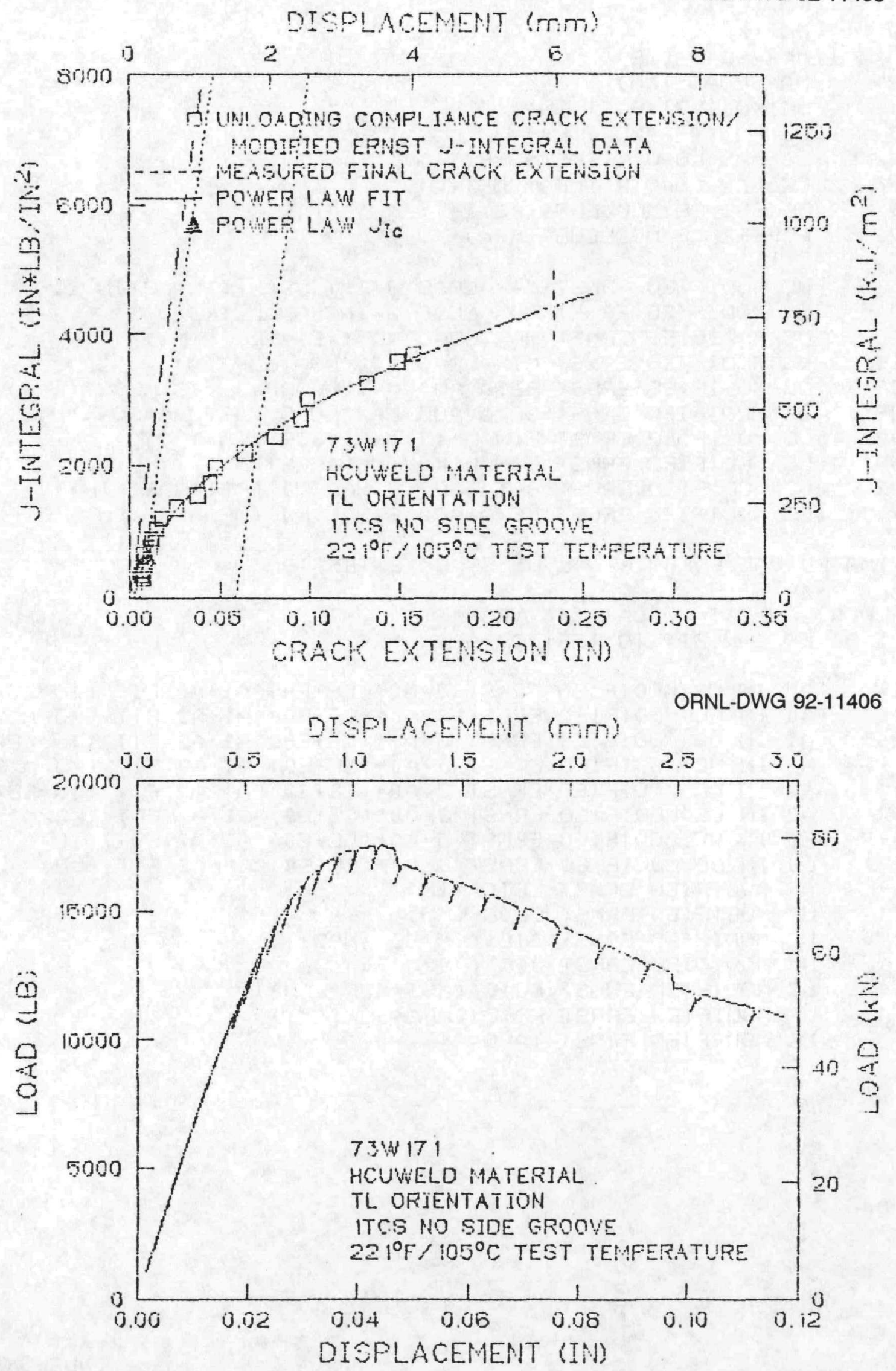


\section{F-227}

73W-171

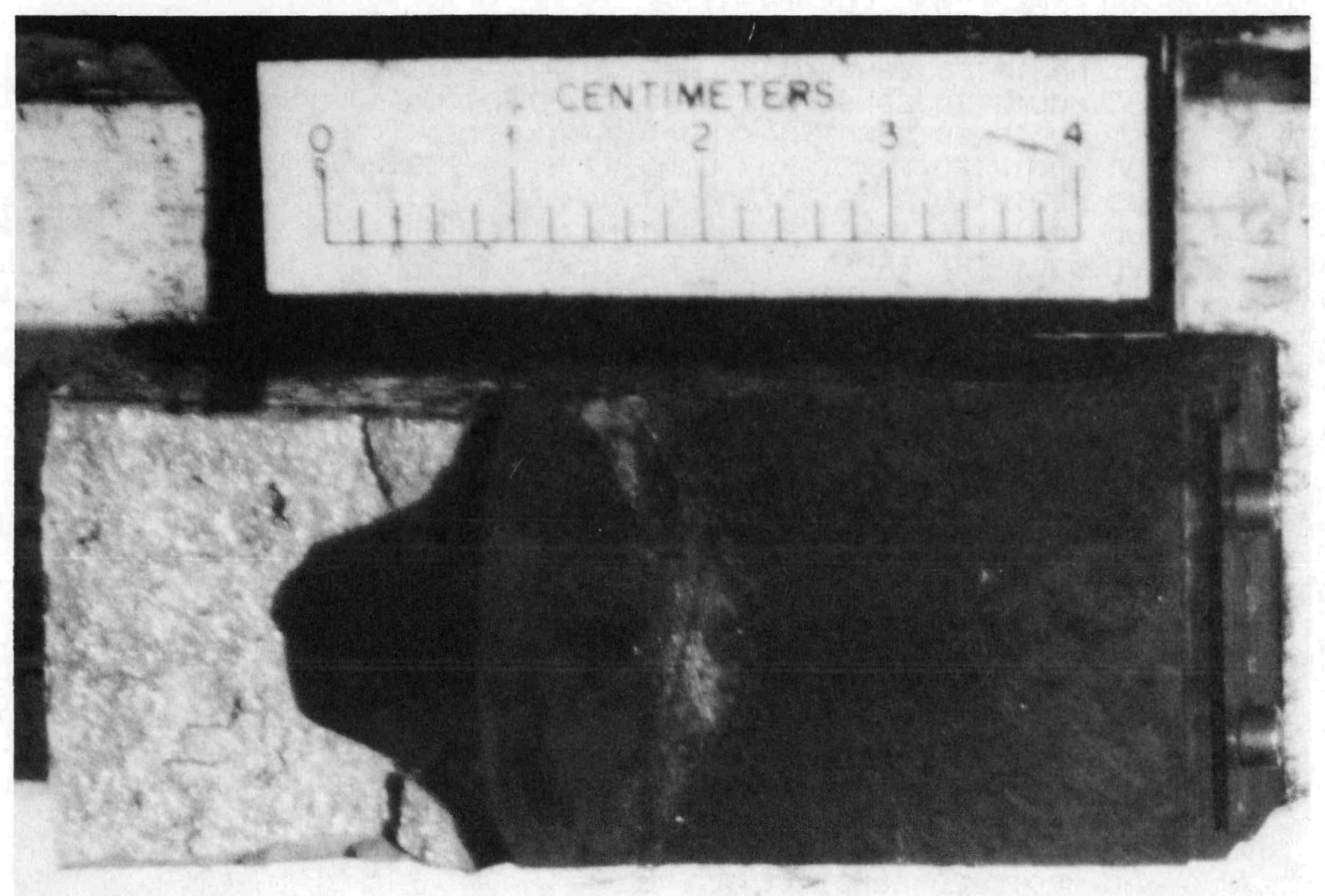




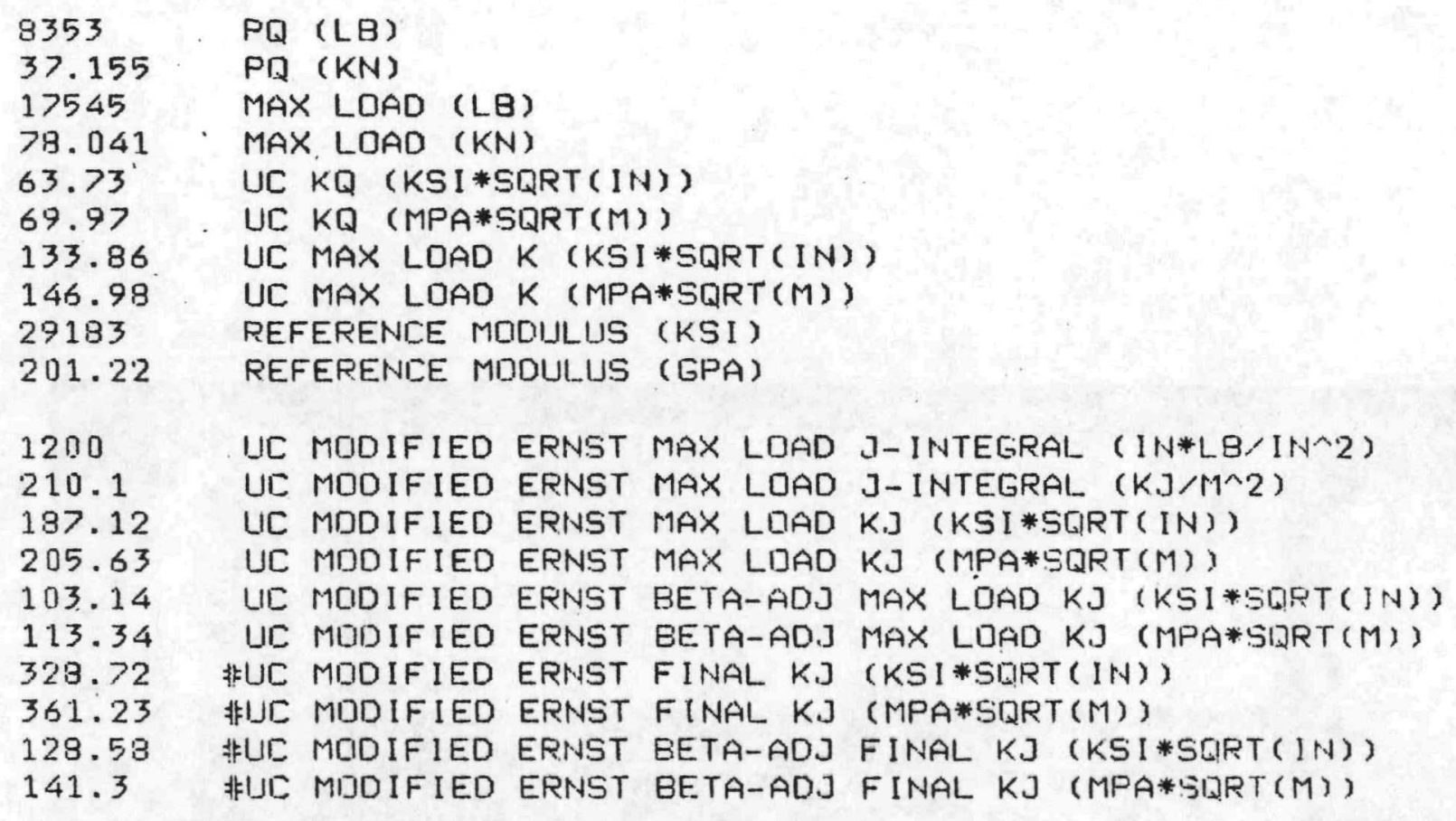

非 ESTIMATED VALUE FUR FINAL UC CRACK EXTENSION

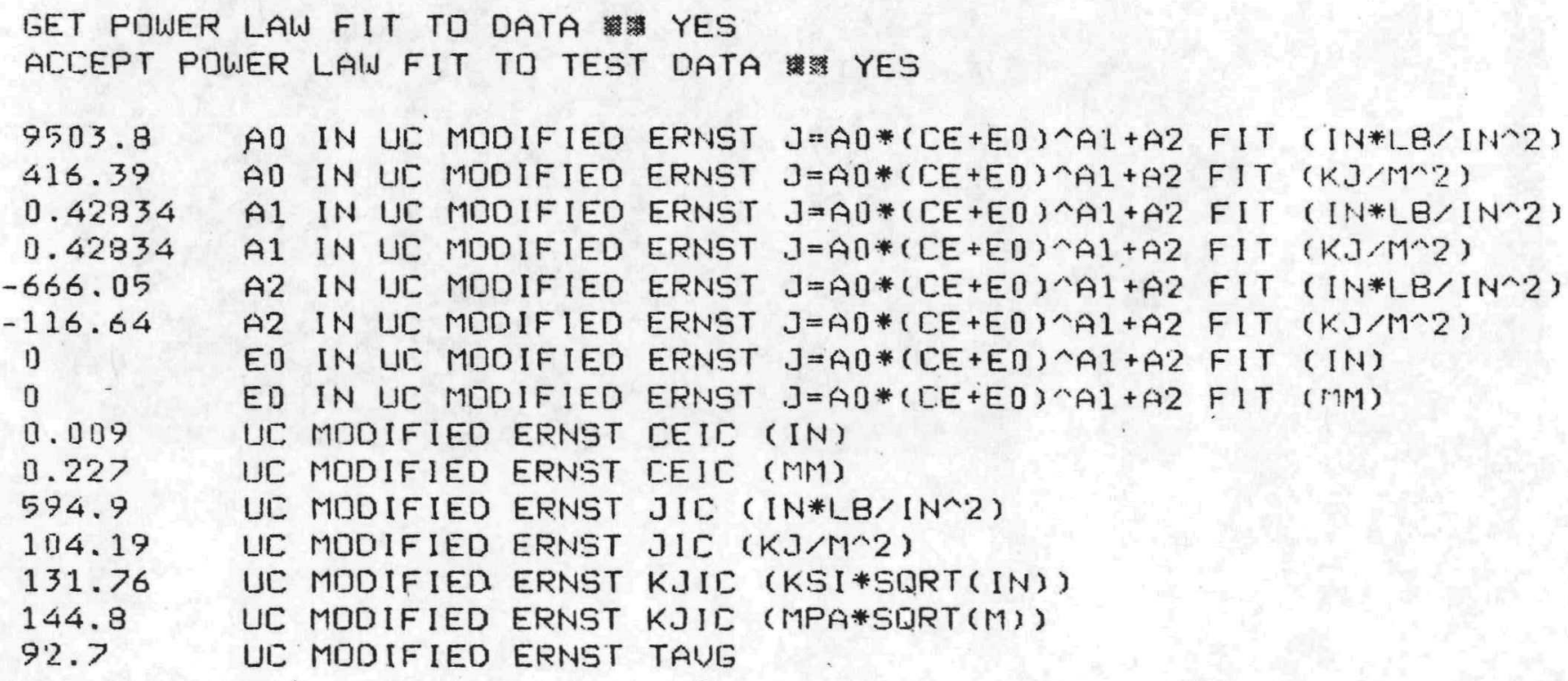




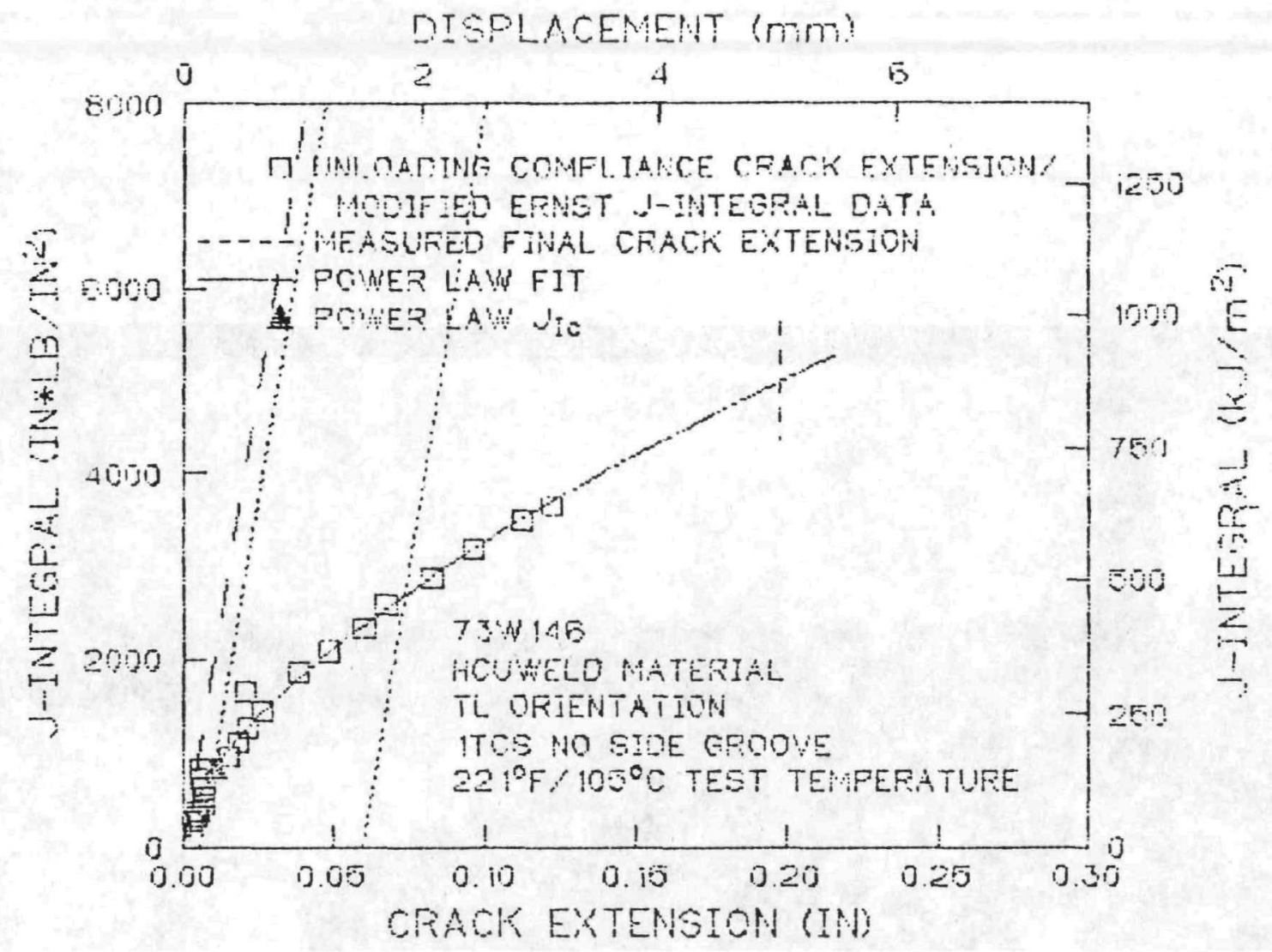

ORNL-DWG 92-11408

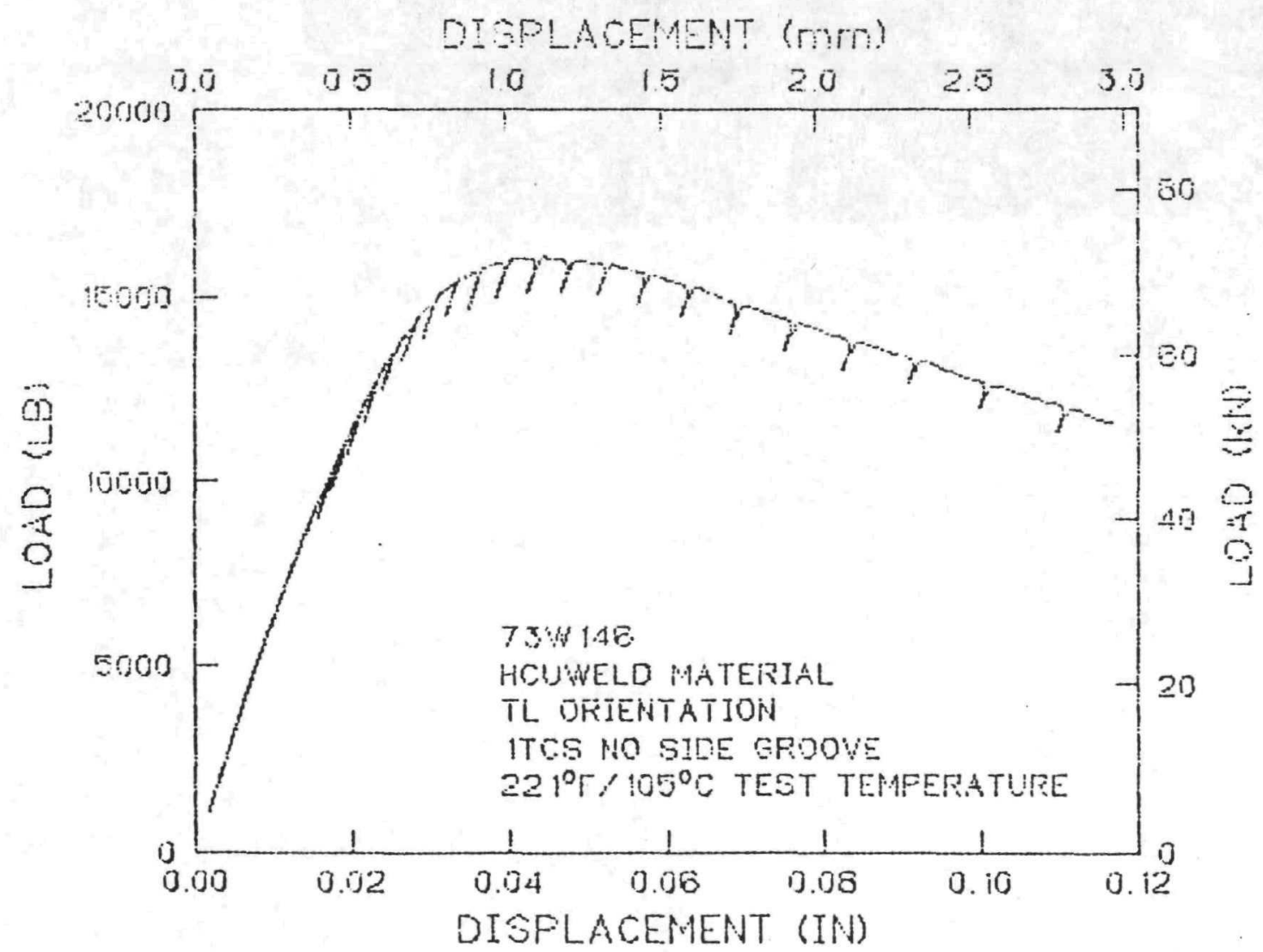


F-230

73W-146

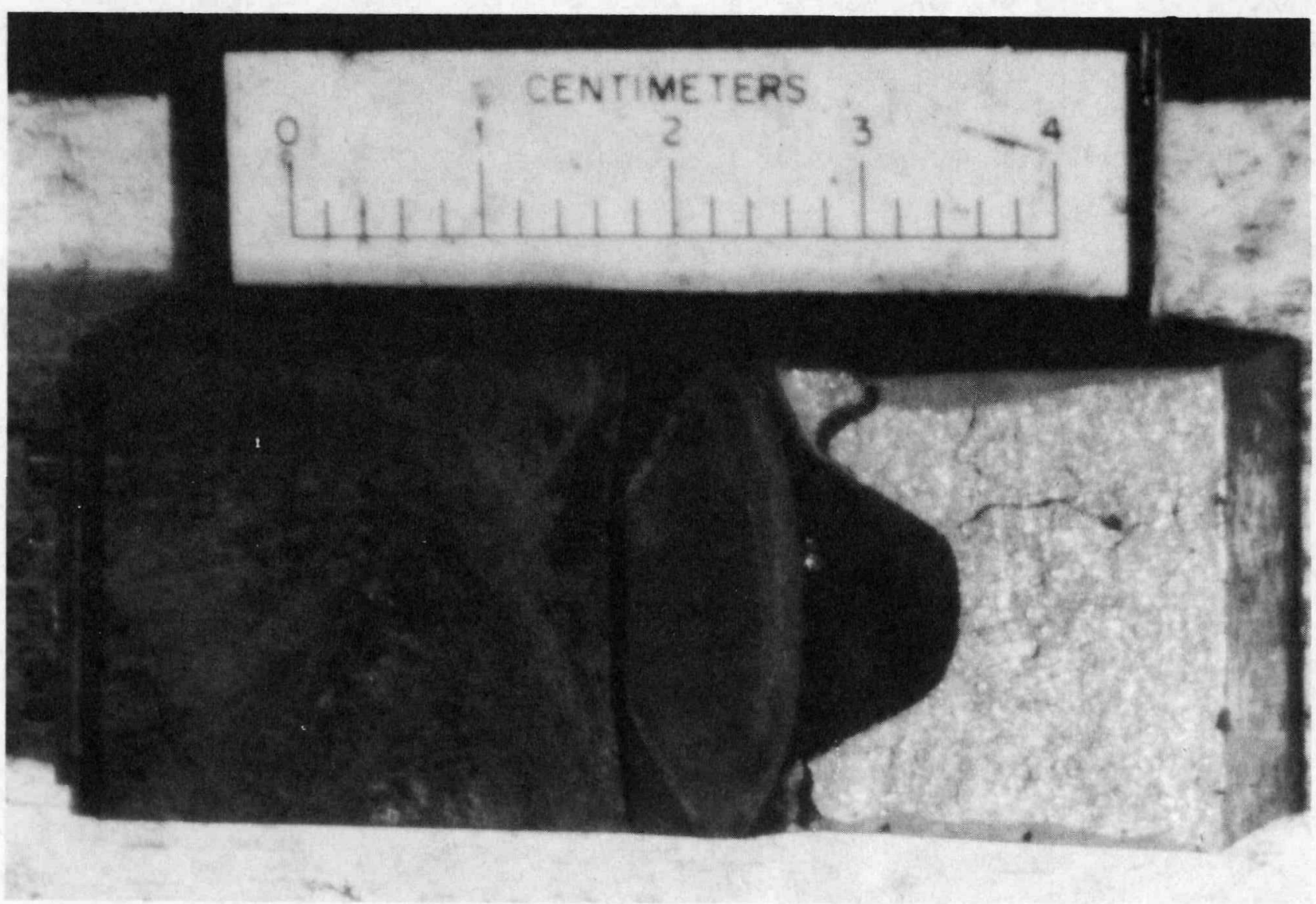

NUREG/CR-5913 


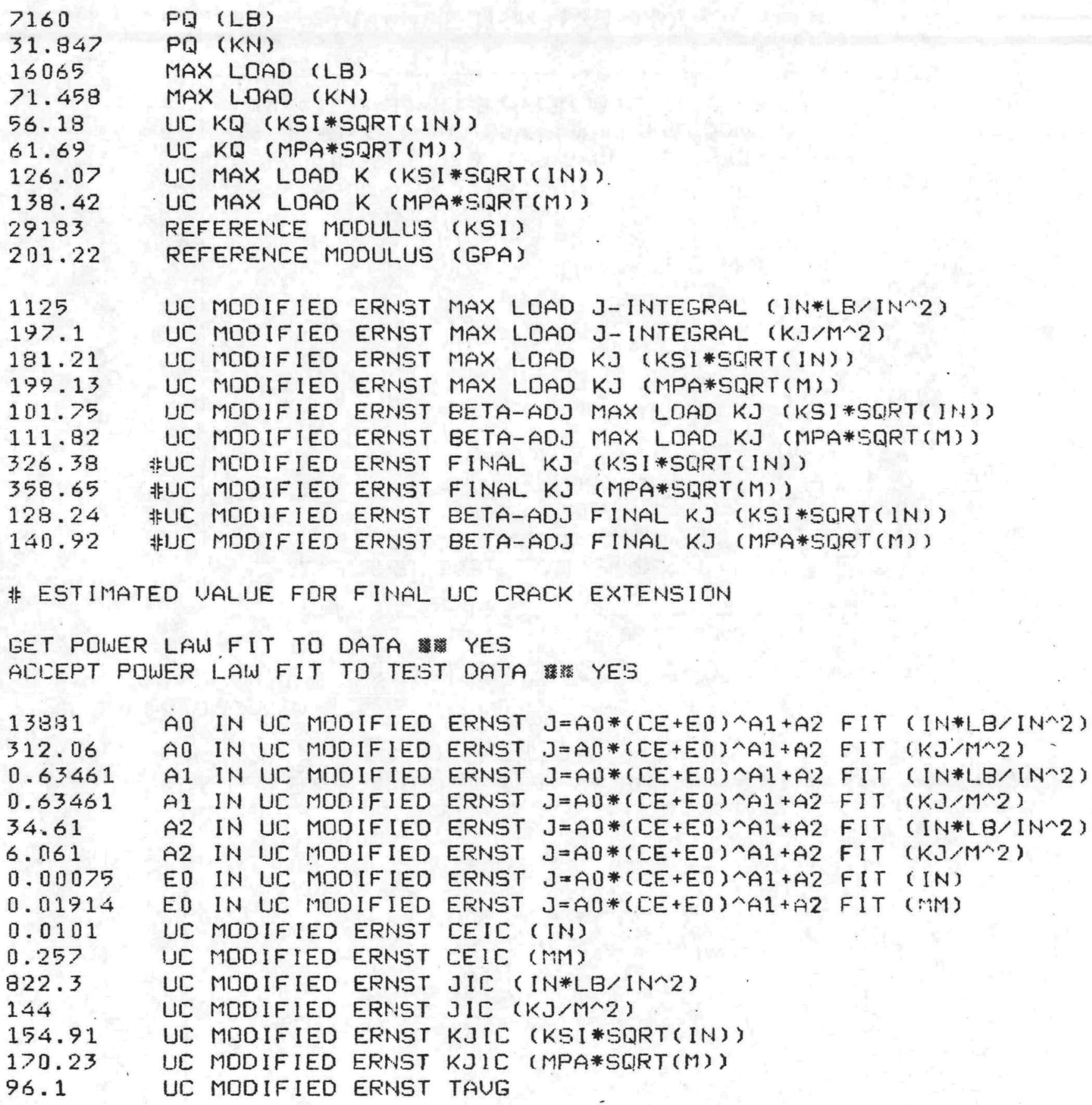




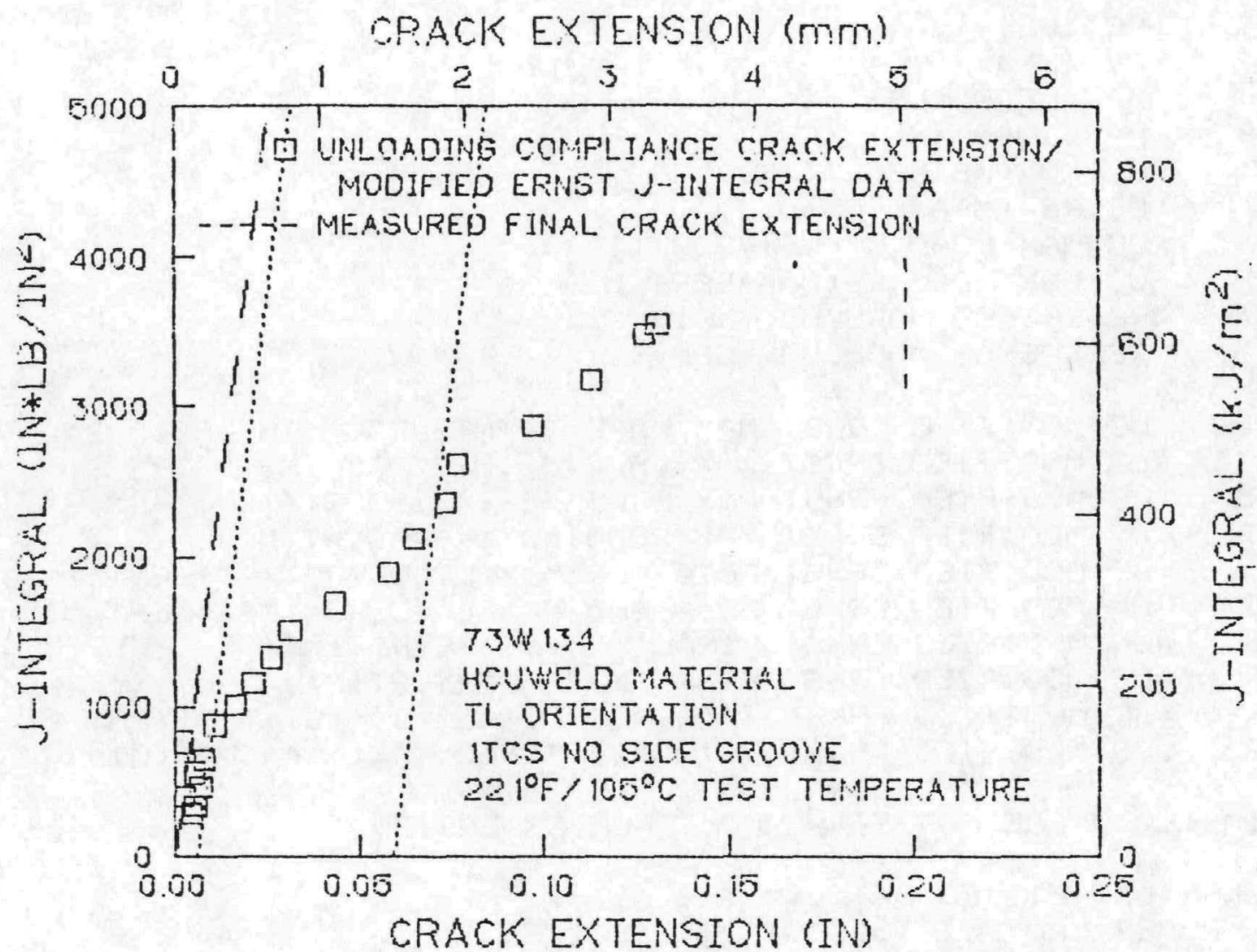

ORNL-DWG 92-11410

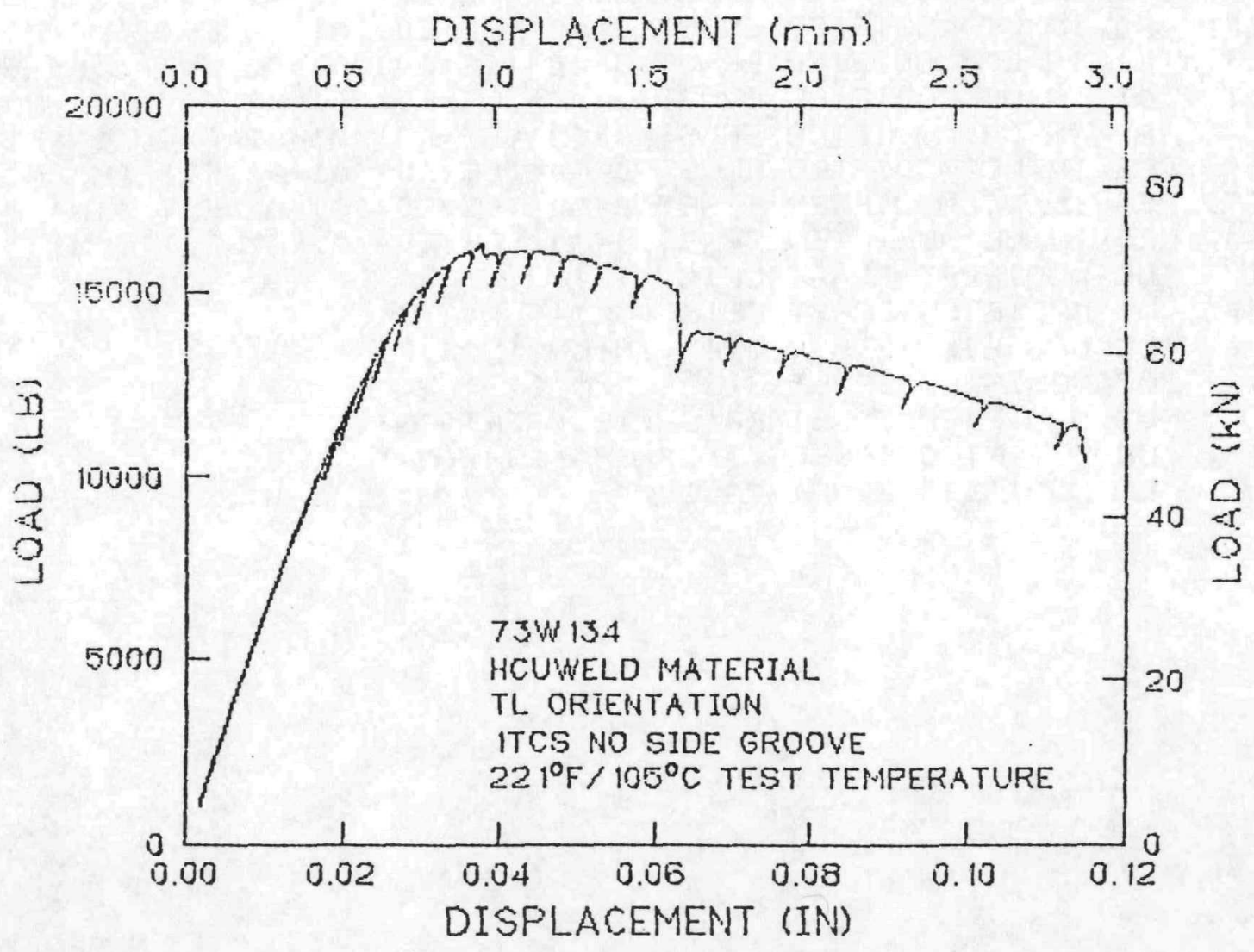


734134

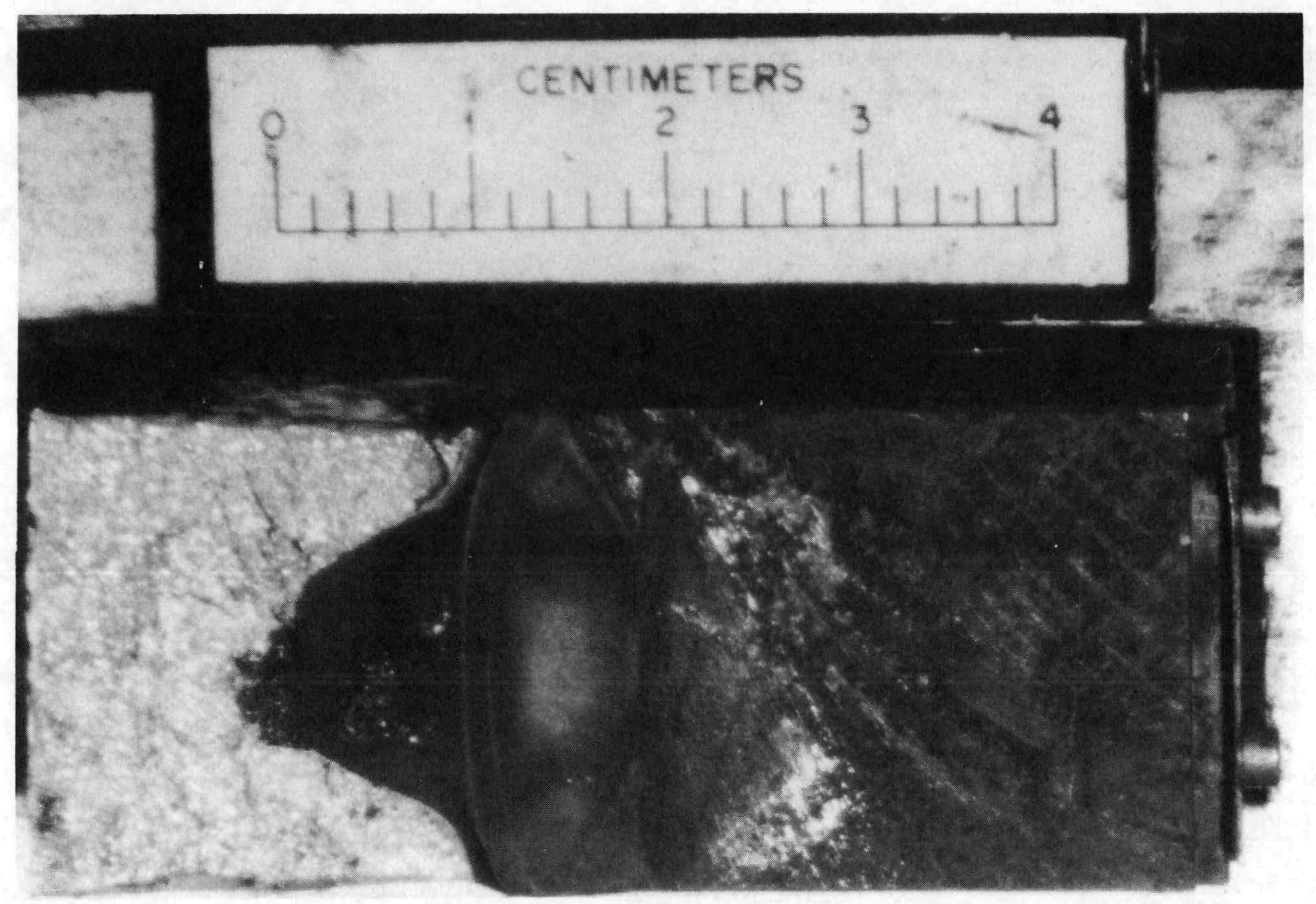



$83>3$
PQ (LB)
37.242
$P Q(K N)$
16263
MAX LOAD (LB)
72.336
MAX LOAD (KN)
0.1984
MEASURED FINAL CRACK EXTENSION (IN)
5.039
MEASURED FINAL CRACK EXTENSION (MM)
29183
201.22

\section{REFERENCE MODULUS (KSI)}
REFERENCE MODULUS (GPA)
66.38
72.95
128.93
141.69
0.131
3.327
34
UC KQ (KSI*SQRT (IN))
UC KQ (MPA*SQRT (M))
UC MAX LOAD K (KSI*SQRT (IN))
UC MAX LOAD K (MPA*SQRT (M))
\#⿰LIC FINAL CRACK EXTENSION (IN)
非C FINAL CRACK EXTENSION (MM)
\#⿰IC FINAL CRACK EXTENSION ERROR (PERCENT)

INUALID UC KIC TEST

1030.5

180.47

173.42

190.57

99.87

109.75

316.12

347.38

126.72

139.26
UC MERKLE-CDRTEN MAX LOAD J-INTEGRAL (IN*LB/IN^2)
UC MERKLE-CORTEN MAX LOAD J-INTEGRAL (KJ/M^2)
UC MERKLE-CORTEN MAX LOAD KJ (KSI*SQRT (IN))
UC MERKLE-CORTEN MAX LDAD KJ (MPA*SQRT (M))
UC MERKLE-CORTEN BETA-ADJ MAX LOAD KJ (KSI*SDRT (IN))
UC MERKLE-CORTEN BETA-ADJ MAX LOAD KJ (MPA*SDRT (M))
UC MERKLE-CORTEN FINAL KJ (KSI*SQRT (IN))
UC MERKLE-CORTEN FINAL KJ (MPA*SQRT(M))
UC MERKLE-CORTEN BETA-ADJ FINAL KJ (KSI*SQRT (IN))
UC MERKLE-CORTEN BETA-ADJ FINAL KJ (MPA*SQRT (M))

1003.9

175.81

171.16

188.09

99.31

109.14

308.6

UC ERNST MAX LOAD J-INTEGRAL (IN*LB/IN^2)

UC ERNST MAX LOAD J-INTEGRAL (KJ/M^2)

UC ERNST MAX LDAD KJ (KSI*SDRT (IN))

UC ERNST MAX LOAD KJ (MPA*SLRT (M)

UC ERNST BETA-ADJ MAX LOAD KJ (KSI*SQRT (IN))

UC ERNST BETA-ADJ MAX LDAD KJ (MPA*SQRT(M))

UC ERNST FINAL KJ (KSI*SIRT (IN))

339.12

125.59

UC ERNST FINAL KJ (MPA*SQRT (M))

UC ERNST BETA-ADJ FINAL KJ (KSI*SQRT(IN))

UC ERNST BETA-ADJ FINAL KJ (MPA*SDRT (M))

1007.8

UC MODIFIED ERNST MAX LOAD. J-INTEGRAL (IN*LB/IN^2)

176.49

171.5

UC MODIFIED ERNST MAX LOAD J-INTEGRAL ( $K J / M \wedge 2)$

UC MODIFIED ERNST MAX LOAD KJ (KSI*SQRT (IN))

188.46

99.4

109.23

322.36

354.24

127.65

140.27

UC MODIFIED ERNST MAX LOAD KJ (MPA*SQRT (M))

UC MODIFIED ERNST BETA-ADJ MAX LOAD KJ (KSI*SQRT (IN))

UC MODIFIED ERNST BETA-ADJ MAX LOAD KJ (MPA*SQRT(M))

UC MODIFIED ERNST FINAL KJ (KSI*SQRT (IN))

UC MODIFIED ERNST FINAL KJ (MPA*SQRT (M))

UC MODIFIED ERNST BETA-ADJ FINAL KJ (KSI*SQRT (IN))

UC MODIFIED ERNST BETA-ADJ FINAL KJ (MPA*SDRT (M))

\# ESTIMATED UALUE FOR FINAL UC CRACK EXTENSION

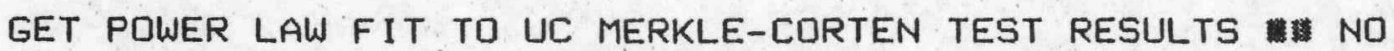

UC MERKLE-CORTEN JIC, CEIC, KJIC AND TAUG NOT DEFINED--POWER LAW FIT DECLINED BY TECHNICIAN

NUREG/CR-5913 
SPECIMEN ID $=7313136$

TEST TEMPERATURE $=125$ DEG C

THICKNESS $=25.5 \mathrm{~mm}$

SIDE GRODUES $=0 \%$

INITIAL CRACK LENGTH $=27.2 \mathrm{~mm}$

MEASURED DUCTILE DELTA-A $=1.98$

YIELD STRENGTH $=621 \mathrm{MPa}$

FLDW STRENGTH $=672 \mathrm{MPa}$

ESTIMATED YOUNG'S MODULUS $=200$ GPa

SPECIMEN DID NOT CLEAUE, J-MAX LDAD $=245.7 \mathrm{~kJ} / \mathrm{m}^{\wedge} 2$

$K J-M A X L D A D=221.7 \mathrm{MPa} \operatorname{SQR}[\mathrm{m}]$

POWER LAW PROCEDURE

JI $=-1000 \mathrm{~kJ} / \mathrm{m}^{\wedge} 2$

$K-J I c=0 \quad M P a S Q R[m]$

PDWER $=0$

COEFFICIENT $=0 \mathrm{~kJ} / \mathrm{m}^{\wedge} 2$

$T(A \cup E R A G E)=D$

JI-R CURUE CALCULATIONS

J $($ 回J $/ T=8.8)=0 \mathrm{~kJ} / \mathrm{m}^{\wedge} 2$

POWER $=0$

$J-M A X$ ALLDWED $=790.2 \mathrm{~kJ} / \mathrm{m}^{\wedge} 2$

RCURUE INUALID, 23
ASTM LINEAR PROCEDURE

$J I c=0 \quad \mathrm{~kJ} / \mathrm{m}^{\wedge} 2$
$\mathrm{~K}-J I \mathrm{I}=0 \mathrm{MPa}$ SQR $[\mathrm{m}]$
SLOPE $=0 \mathrm{~kJ} / \mathrm{m}^{\wedge} 3$
INTERCEPT $=0 \mathrm{~kJ} / \mathrm{m}^{\wedge} 2$
$T$ (ASTM) $=0$
INUALID, 16

COEFF ICIENT $=0 \mathrm{~kJ} / \mathrm{m}^{\wedge} 2$

DELTA-A MAX ALLOWED $=2.35 \mathrm{~mm}$ 
F-236

ORNL-DWG 92-11411

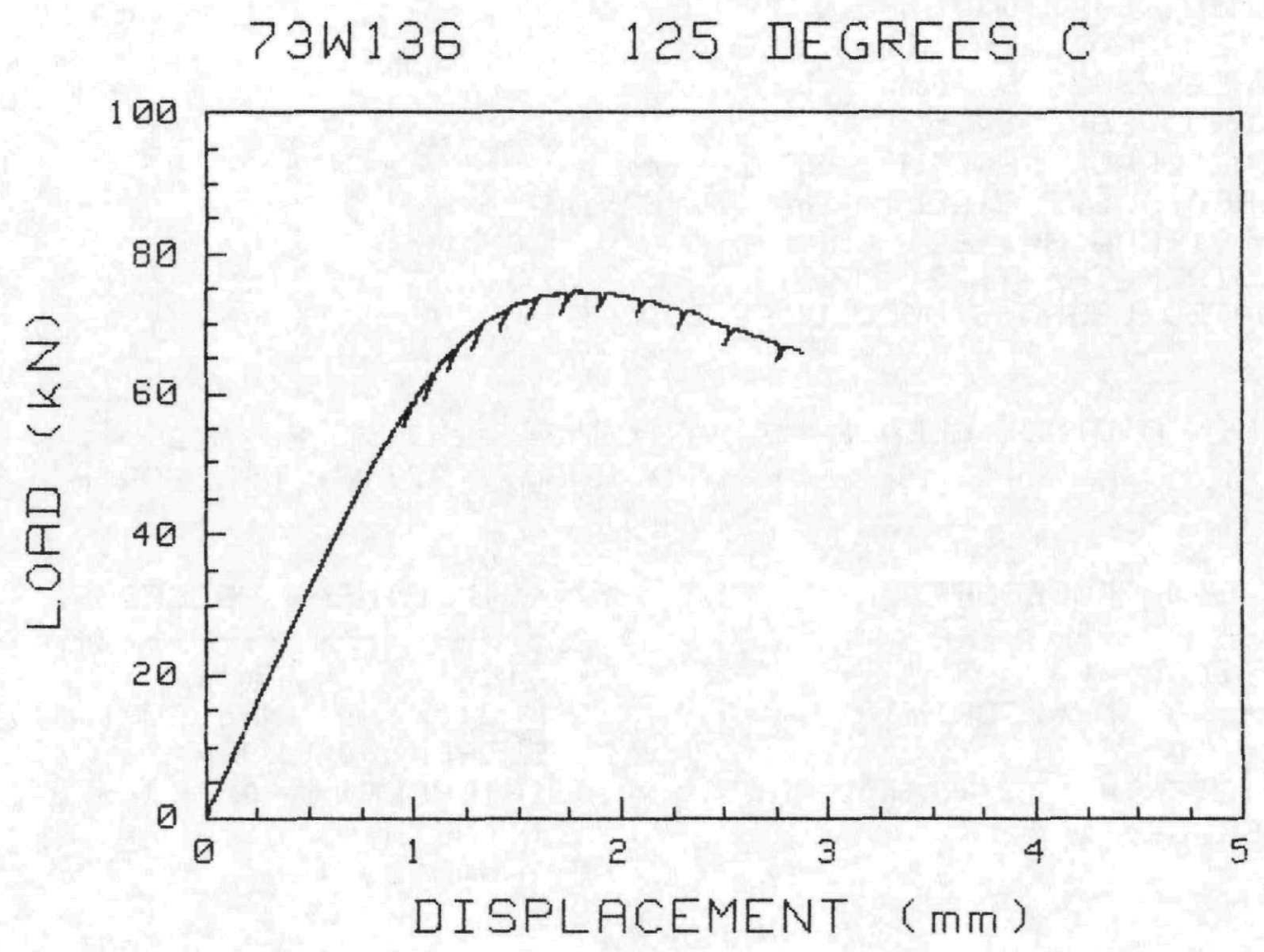


Specimen Lajdline Deflection (in.)

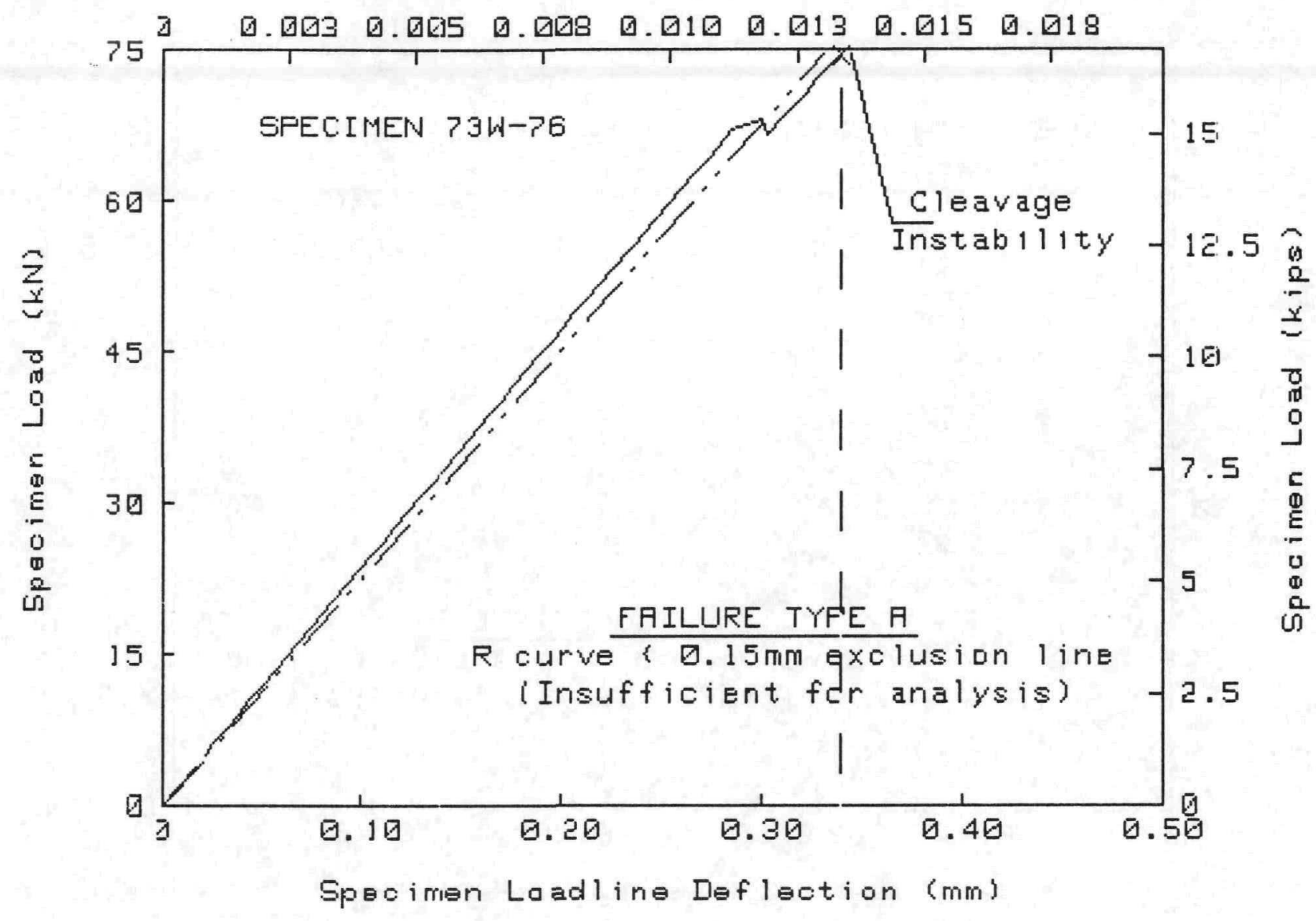

\section{TEST SPECIMEN IATA}

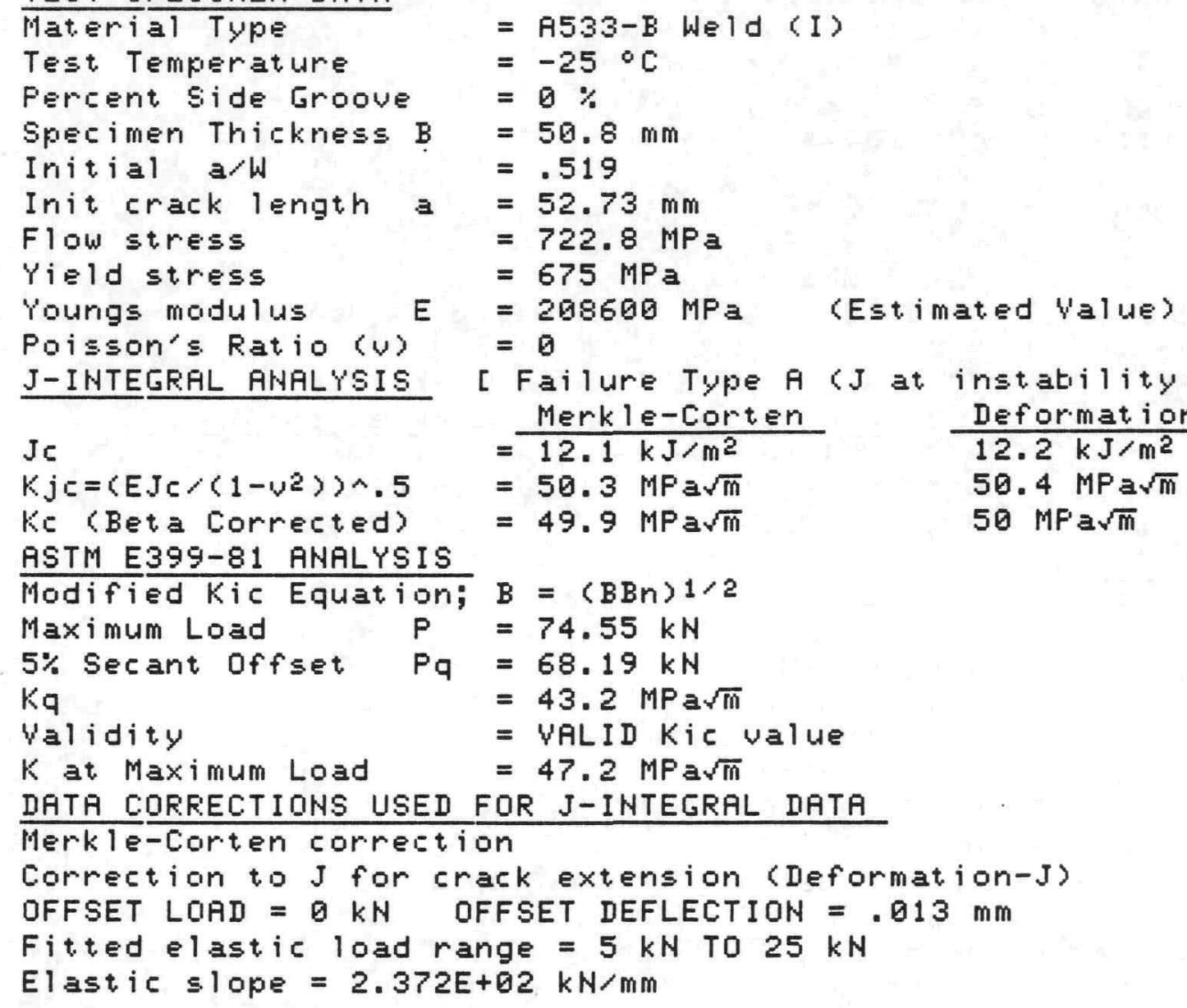


Specimen Loadline Deflectian (in.)

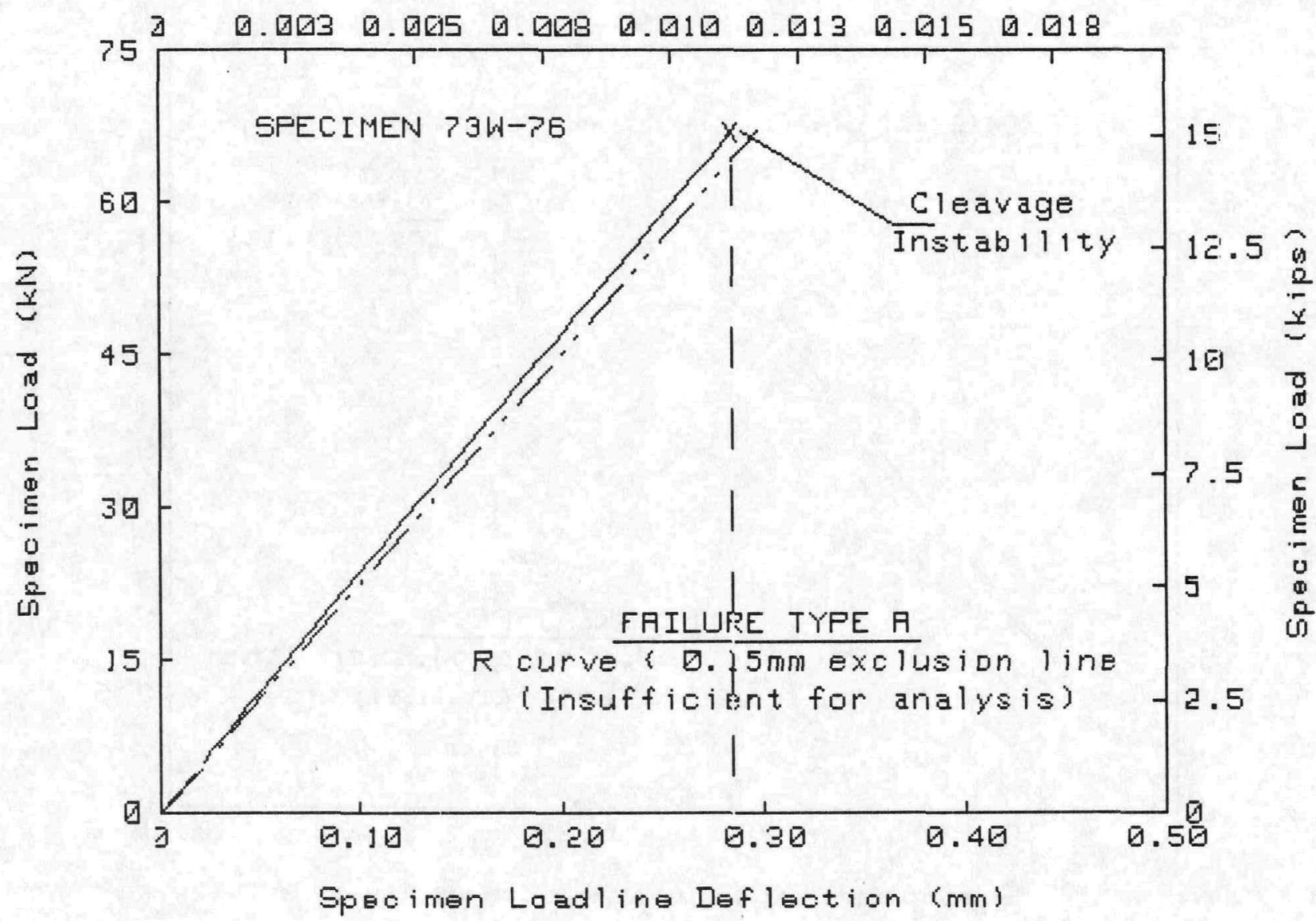

TEST SPEC:IMEN IATA

Material Type
Test Temperature
Percent Side Groove
Specimen Thickness B
Initial a/W
Init crack length a
Flow stress
Yield stress
Youngs modulus
Poisson's. Ratio (U)
J-INTEGRAL ANALYSIS

$=A 533-B$ Weld (I)

$=-25 \circ \mathrm{C}$

$=0 \%$

$=50.8 \mathrm{~mm}$

$=.519$

$=52.73 \mathrm{~mm}$

$=722.8 \mathrm{MPa}$

$=675 \mathrm{MPa}$

$=208600 \mathrm{MPa}$ (Estimated Yalue)

J-INTEGRAL RNALYSIS

\section{$\mathrm{Jc}$}

$K j c=\left(E J c /\left(1-v^{2}\right)\right\rangle \wedge .5$

$\mathrm{Ke}$ (Beta Corrected)

ASTM E399-81 ANALYSIS

Modified Kic Equation;

$5 \%$ Secant offset

$\mathrm{Kq}$

Validity

$K$ at Maximum Load

$$
\begin{aligned}
\mathrm{P} & =67.01 \mathrm{kN} \\
\mathrm{Pq} & =67.01 \mathrm{kN} \\
& =42.4 \mathrm{MParm} \\
& =\text { VALID Kic value }
\end{aligned}
$$$$
=42.4 \mathrm{MPa} \sqrt{\mathrm{m}}
$$

$=42.5 \mathrm{MPa} \sqrt{\mathrm{m}}$

$=42.3 \mathrm{MPa} \sqrt{\mathrm{m}}$

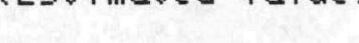

DATA CORRECTIONS USED FOR J-INTEGRAL DATA

Merkle-Carten correction

Correction to J for crack extension (Deformation-J)

OFFSET LOAD $=0 \mathrm{kN}$ OFFSET DEFLECTION $=.013 \mathrm{~mm}$

Fitted elastic load range $=5 \mathrm{kN}$ To $25 \mathrm{kN}$

Elastic slope $=2.372 \mathrm{E}+02 \mathrm{kN} / \mathrm{mm}$ 
Specimen Crackmauth Deflection (in.)

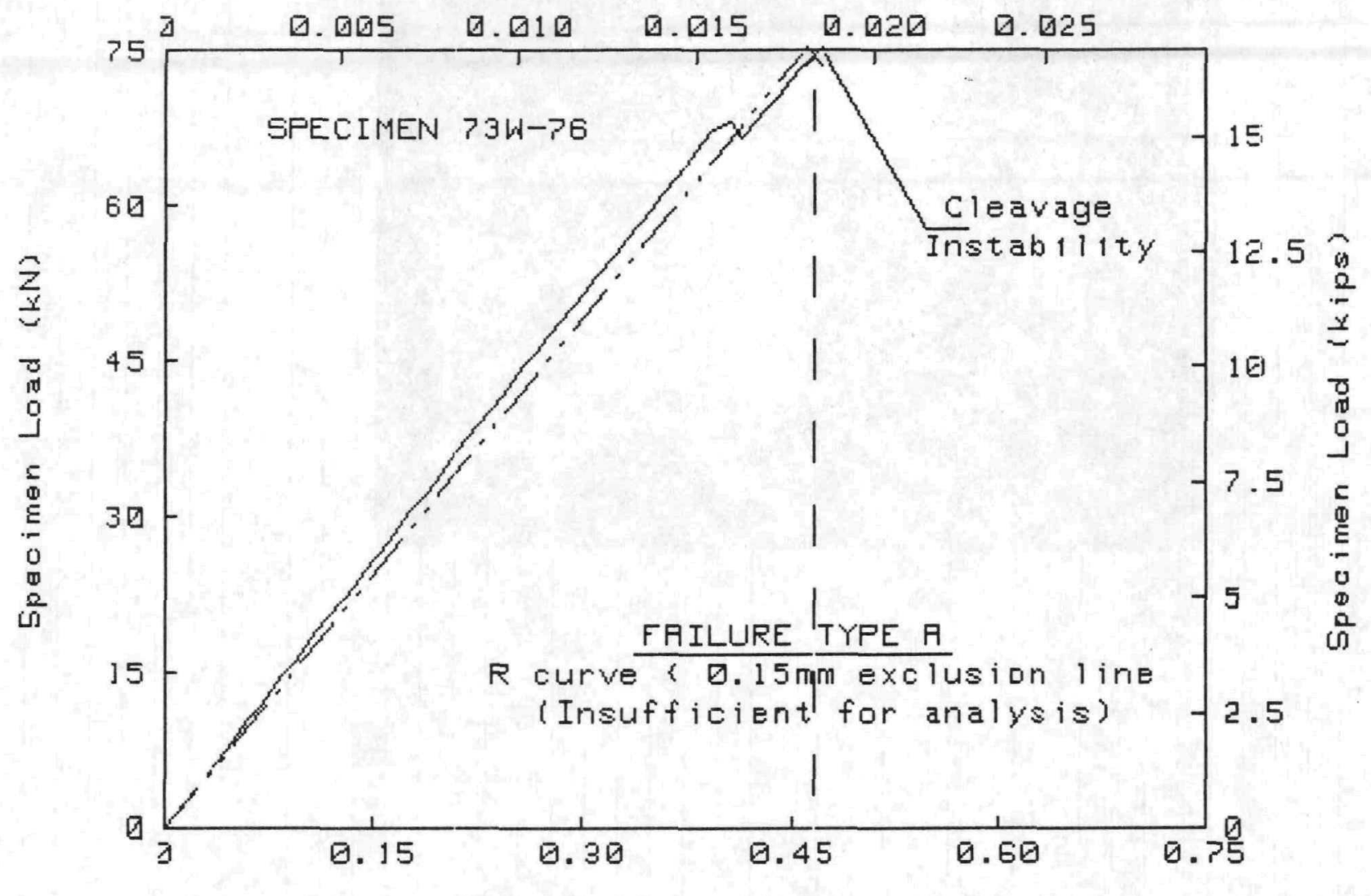

Specimen Crackmouth Deflection ( $\mathrm{mm}$ )

TEST SPECIMEH DATA

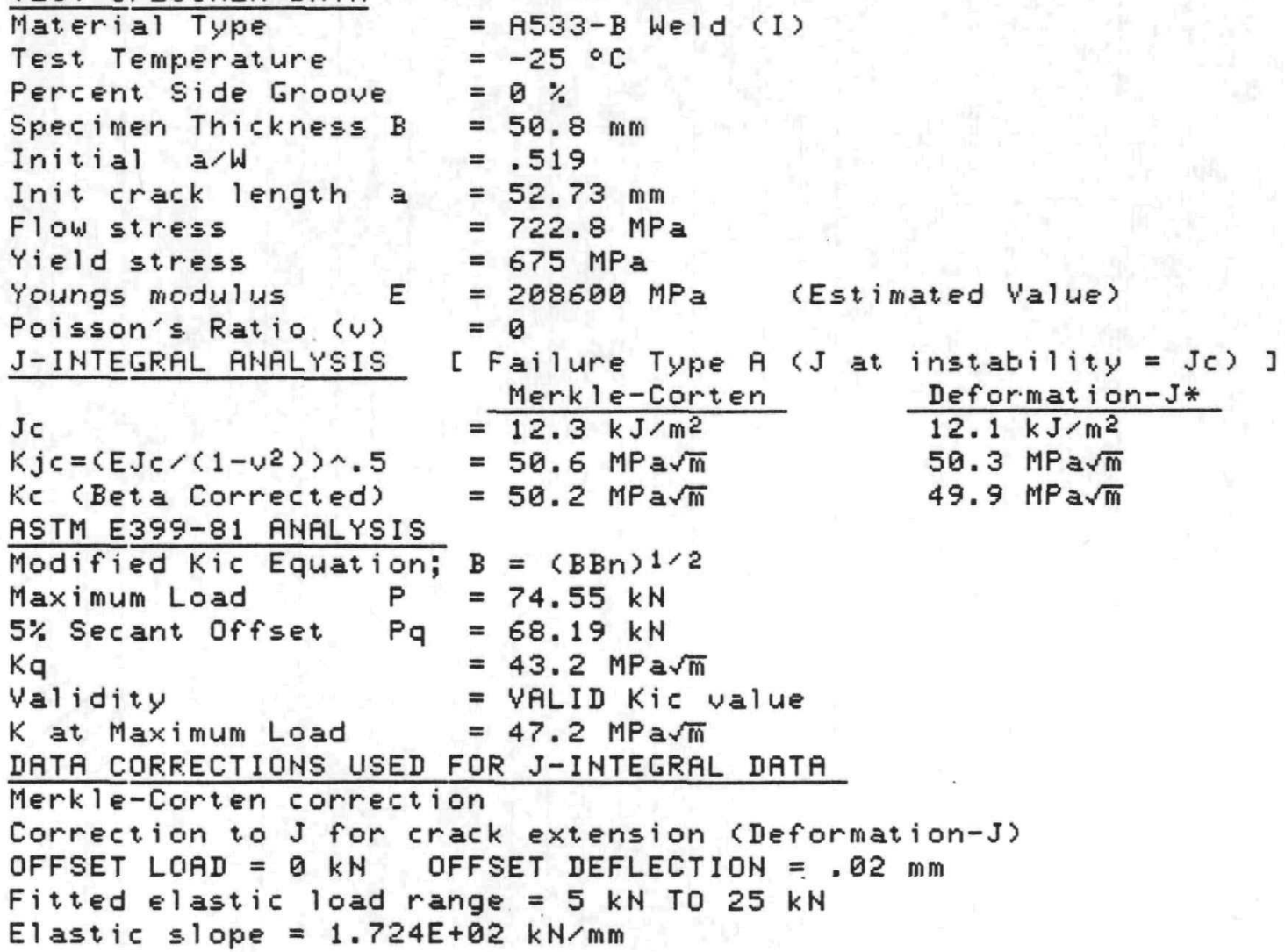




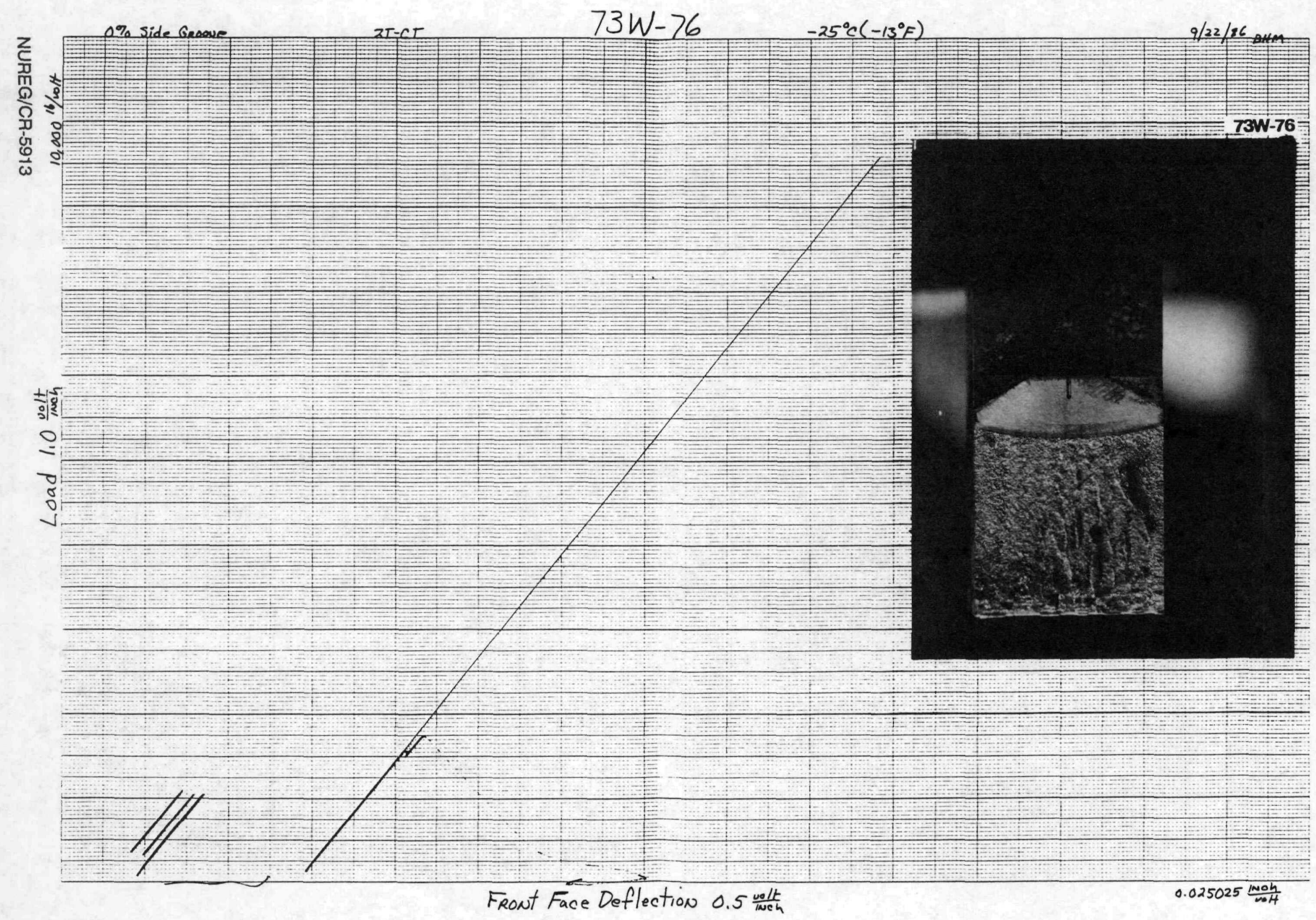




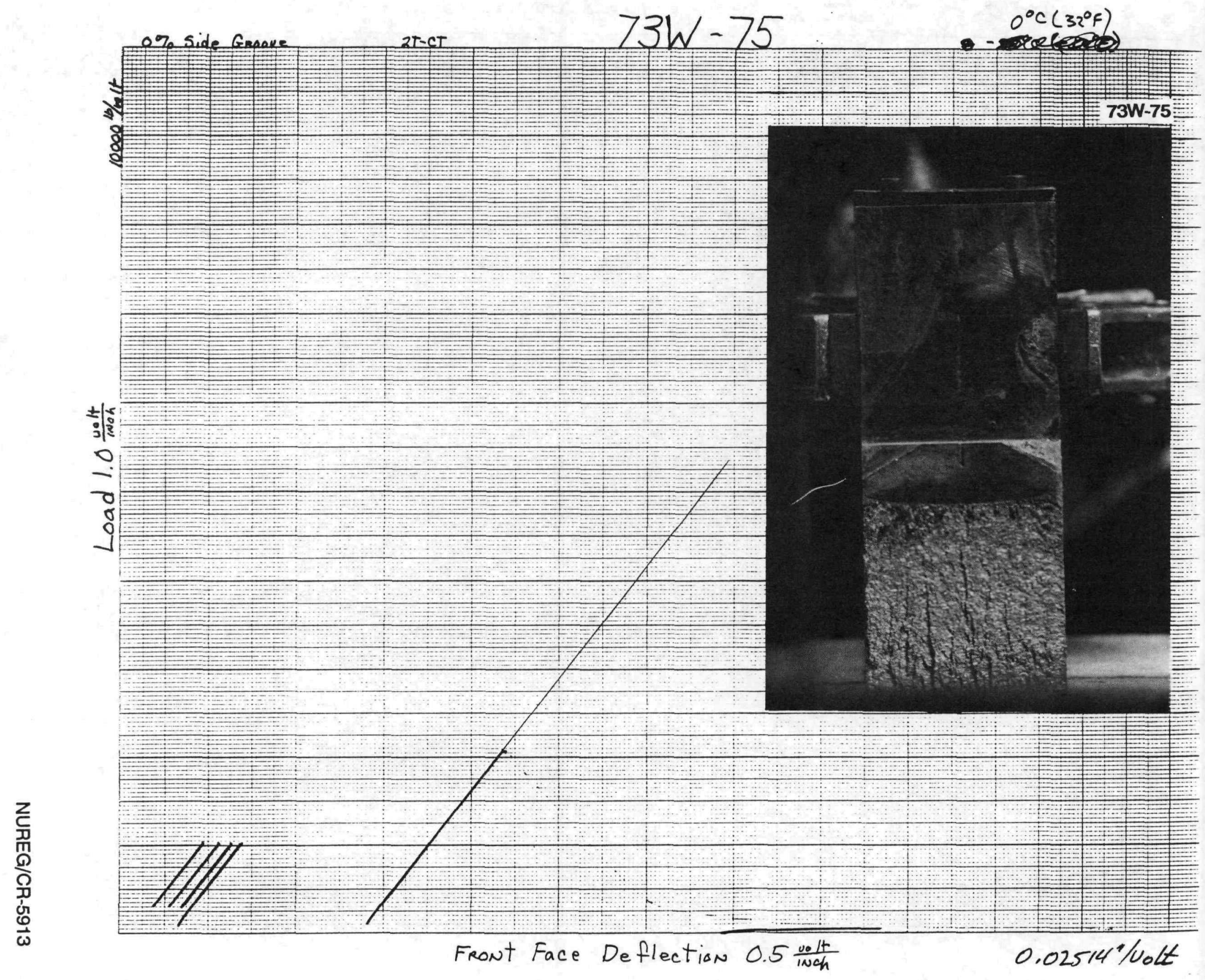




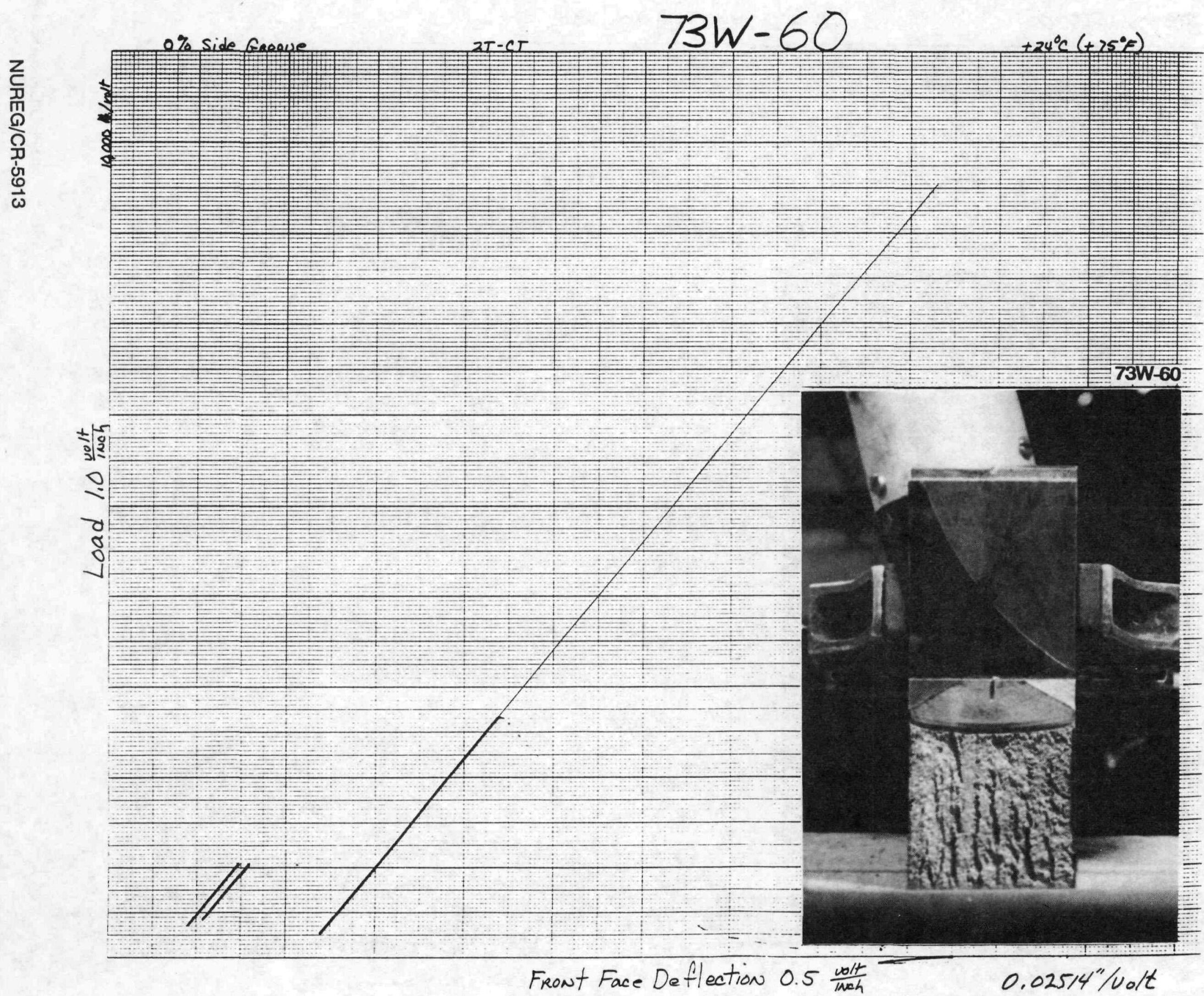


Specimen Loadline Deflection (in.)

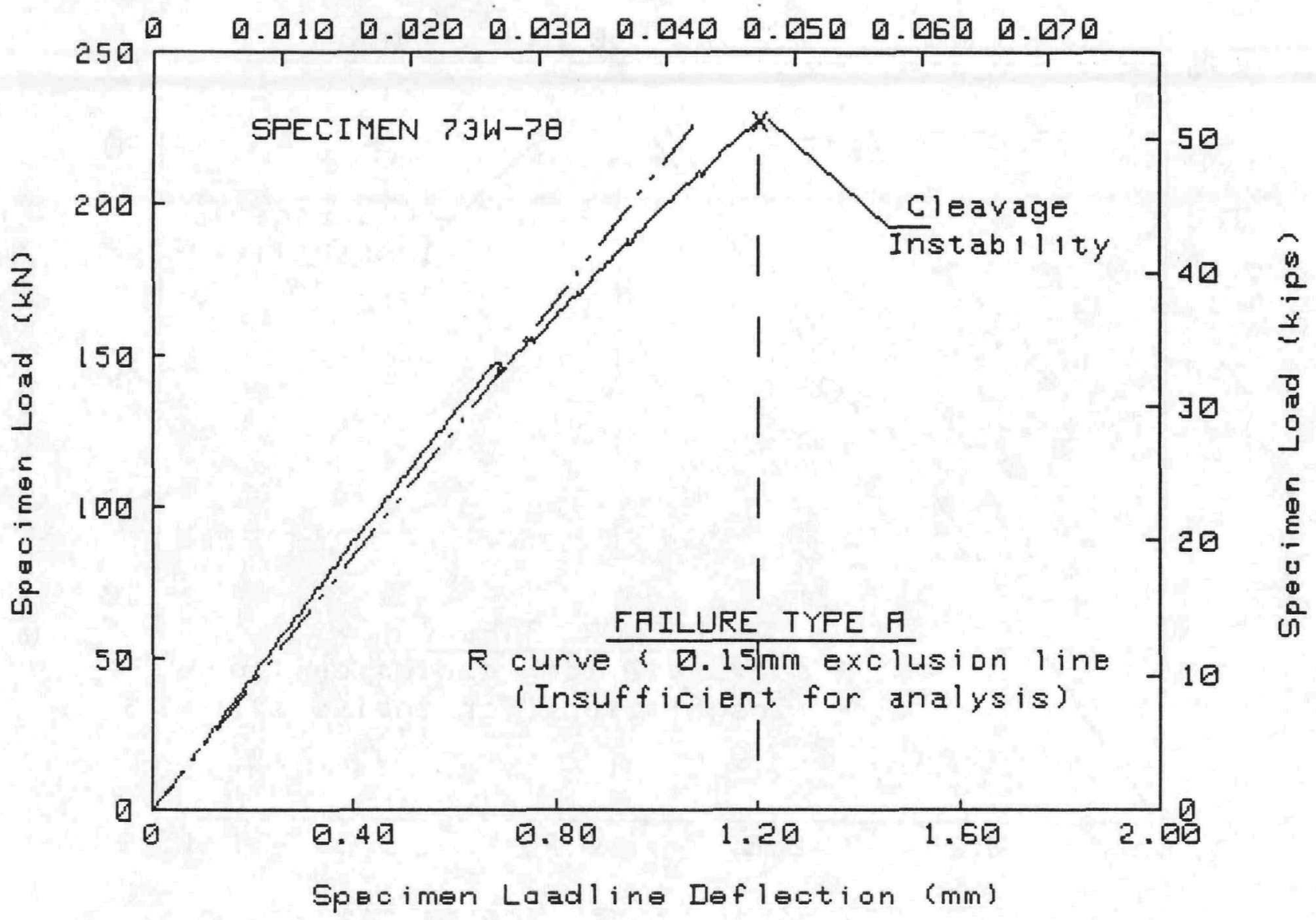

TEST SFECIMEN DATA

Material Type
Test Temperature
Percent Side Groove
Specimen Thickness B
Initial a/W
Init erack length a
Flow stress
Yield stress
Youngs modulus E
Poisson's Ratio (U)
J-INTEGRAL ANALYSIS

Je

$K j c=\left\langle E J c /\left(1-v^{2}\right\rangle\right) \wedge .5$

Kc (Beta Corrected)

ASTM E399-81 ANALYSIS

Modified Kic Equation;

Maximum Load

$5 \%$ Secant offset

$\mathrm{Kq}$

Validity

$K$ at Maximum Load
= A533-B Weld (I)

$=50^{\circ} \mathrm{C}$

$=0 \%$

$=50.8 \mathrm{~mm}$

$=.523$

$=53.15 \mathrm{~mm}$

$=683.1 \mathrm{MPa}$

$=636.6 \mathrm{MPa}$

$=204400 \mathrm{MPa}$ (Estimated Value)

$=0$

[ Failure Type $A$ ( $J$ at instability = J $c$ ) ]

$=\frac{\text { Merk le-Corten }}{136.1 \mathrm{~kJ} / \mathrm{m}^{2}}$

$=166.8 \mathrm{MPa} \sqrt{\mathrm{m}}$

$=124.5 \mathrm{MPa} \sqrt{\mathrm{m}}$
Deformat ion-J*

$165.5 \mathrm{MPa} \sqrt{\mathrm{m}}$

$124 \mathrm{MPa} \sqrt{\mathrm{m}}$

DATA CORRECTIONS USED FOR J-INTEGRAL DATA

Merkle-Corten correction

Correction to J for crack extension (Deformation-J)

OFFSET LOAD $=0 \mathrm{kN}$ OFFSET DEFLECTION $=-.021 \mathrm{~mm}$

Fitted elastic load range $=20 \mathrm{kN}$ TO $110 \mathrm{kN}$

Elastic slope $=2.229 E+02 \mathrm{kN} / \mathrm{mm}$ 
Specimen Laadline Deflection (in.)

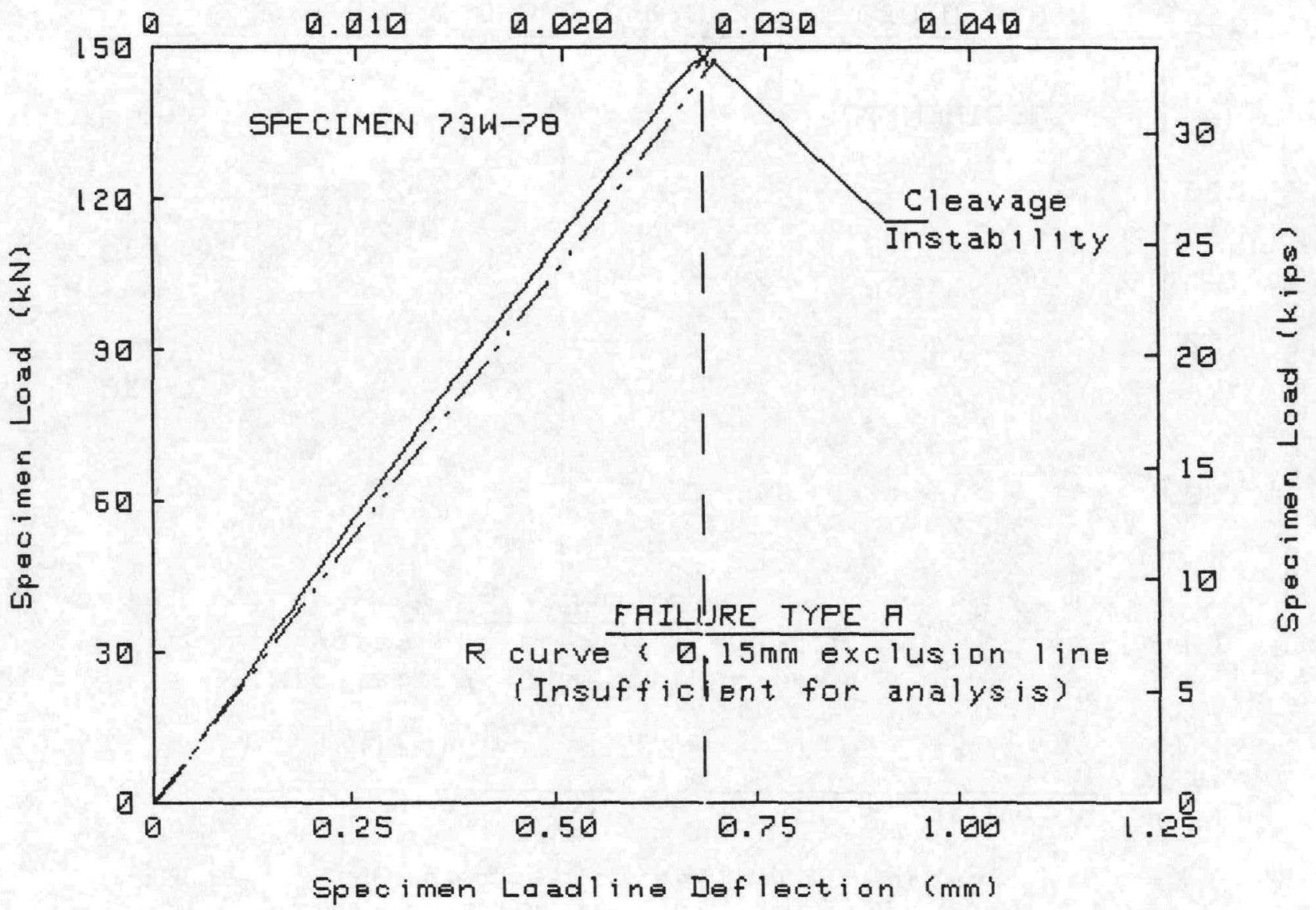

TEST SPECIMEN DATA

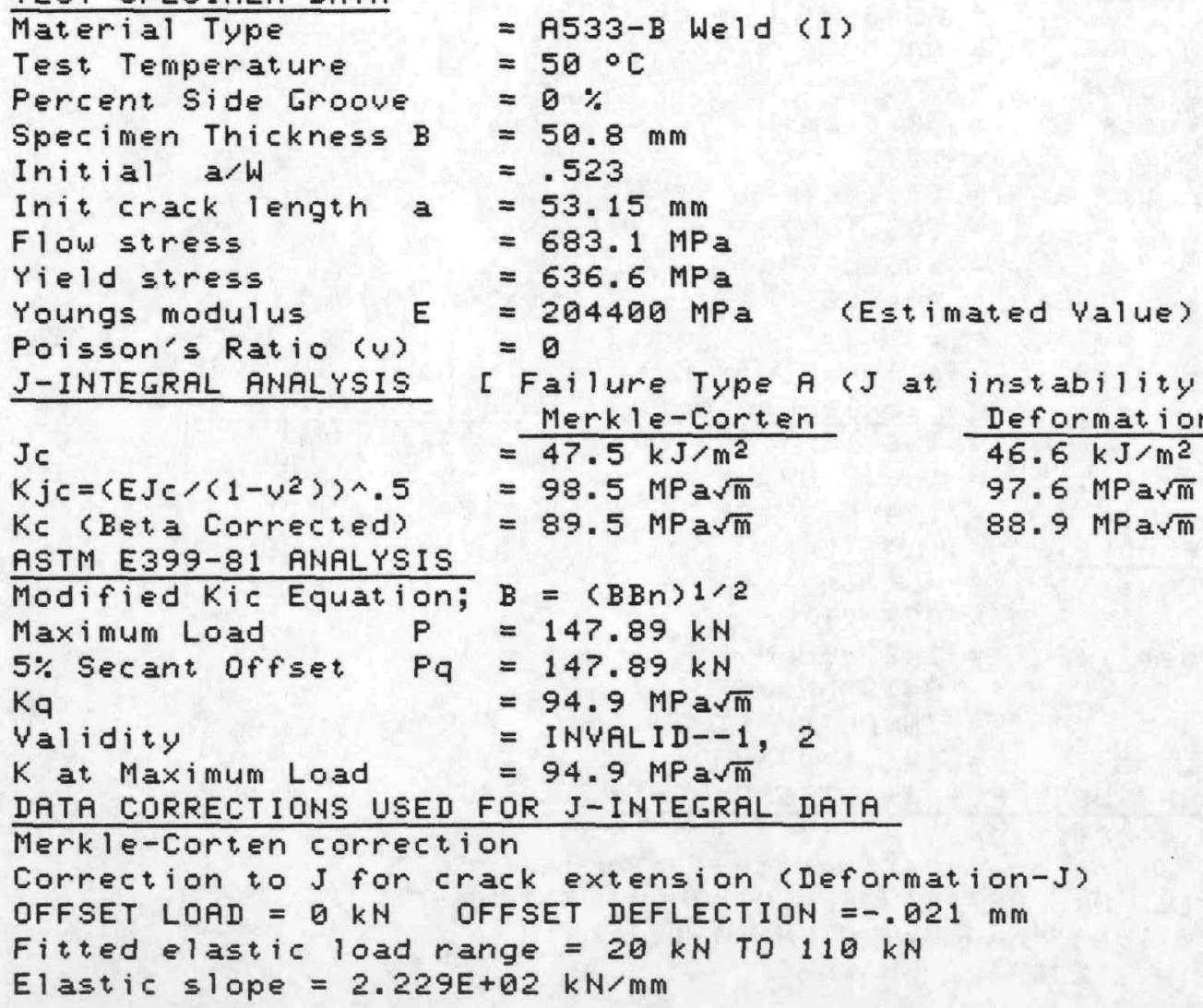


Specimen Crackmouth Deflection (in.)

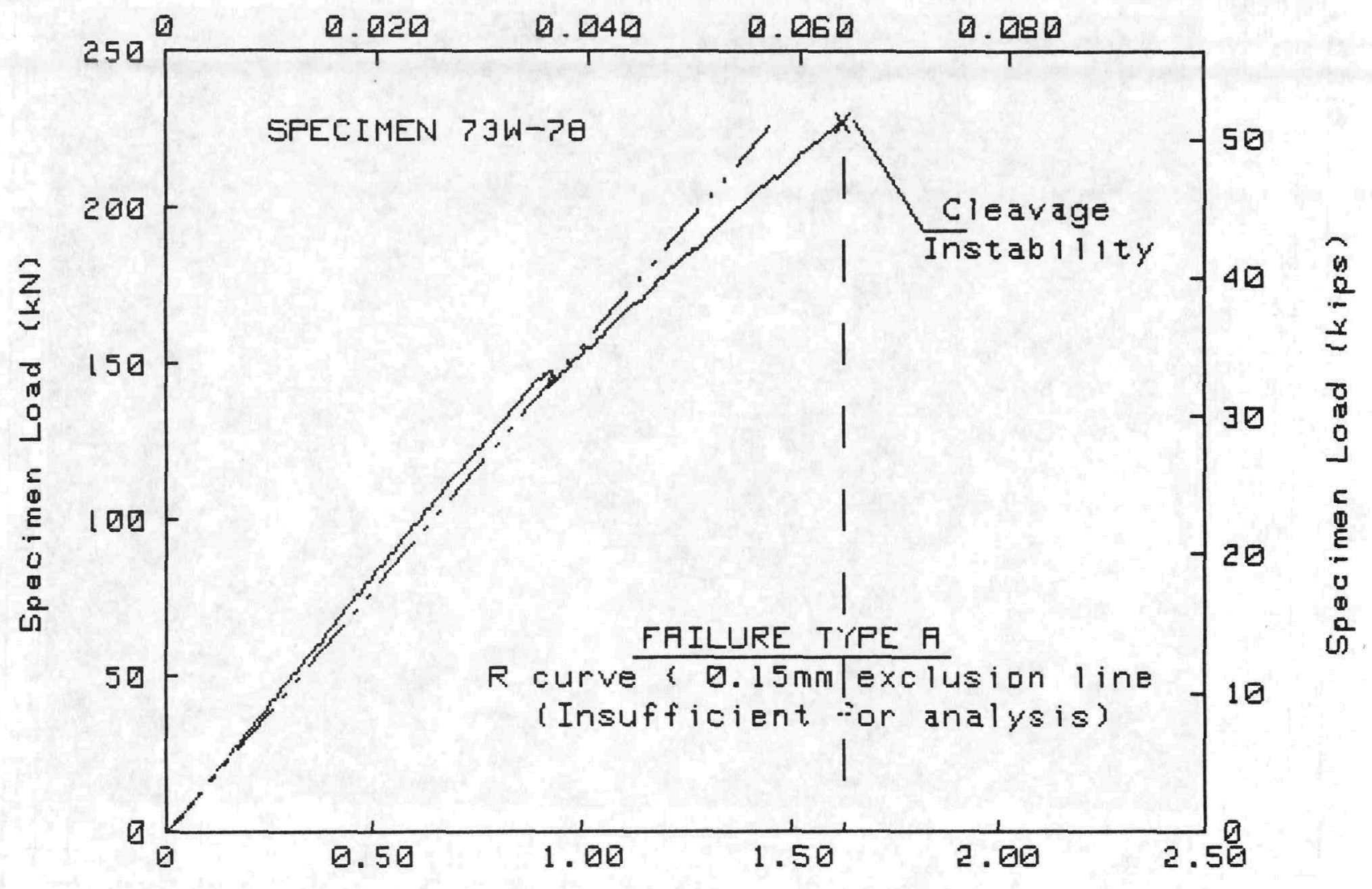

Specimen Crackmouth Deflection (mm)

TEST SFECIMEN DATH

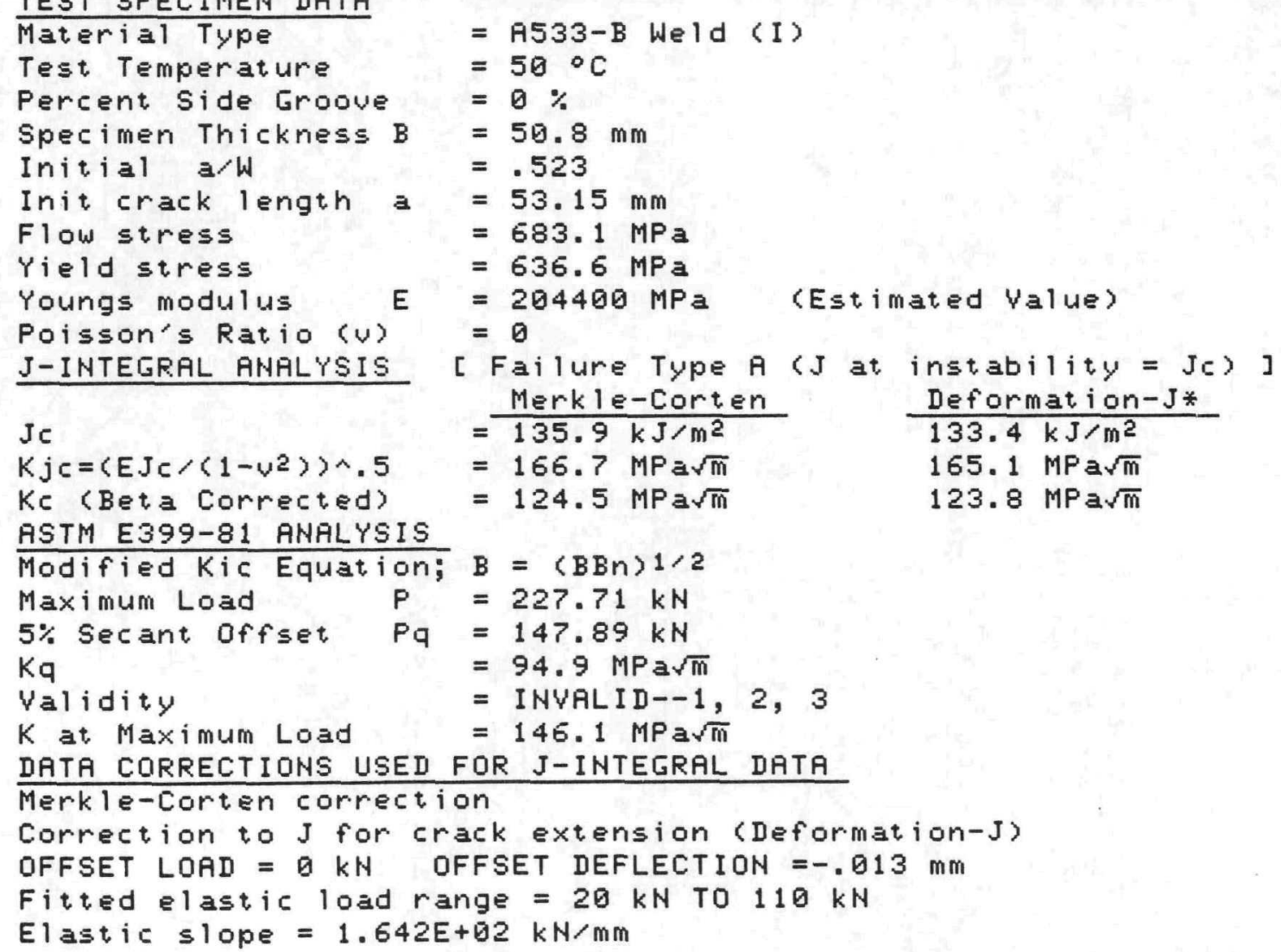




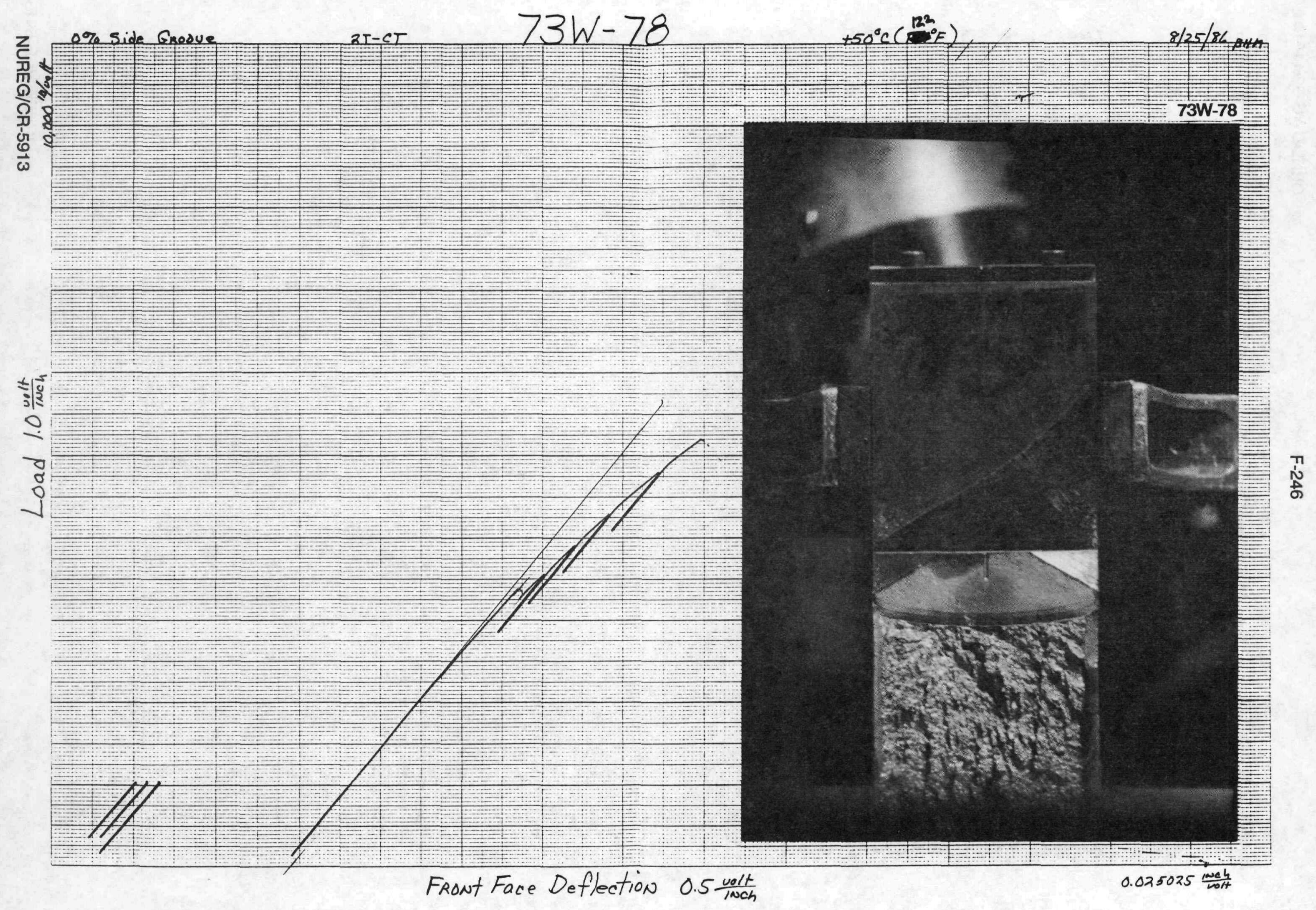




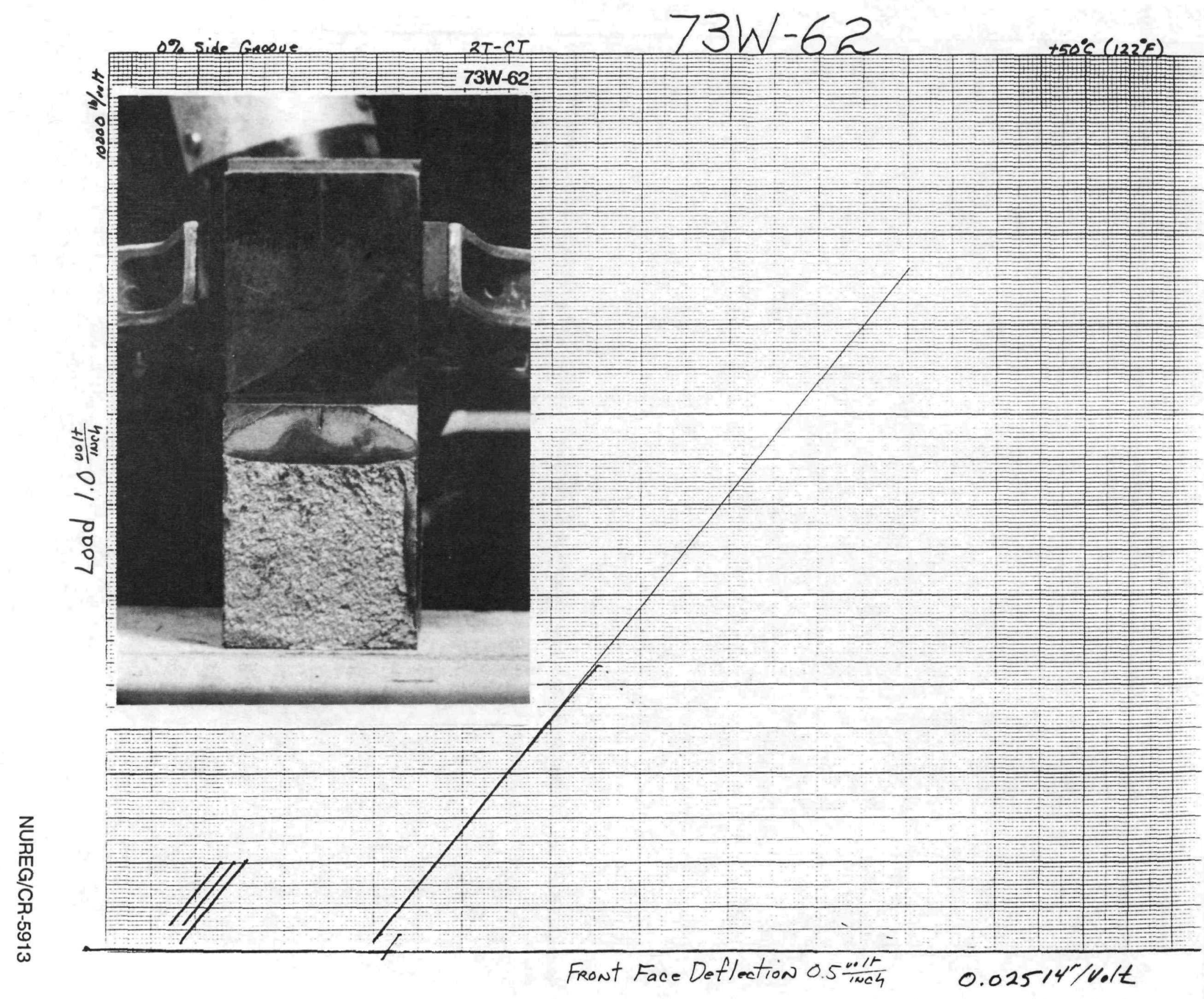




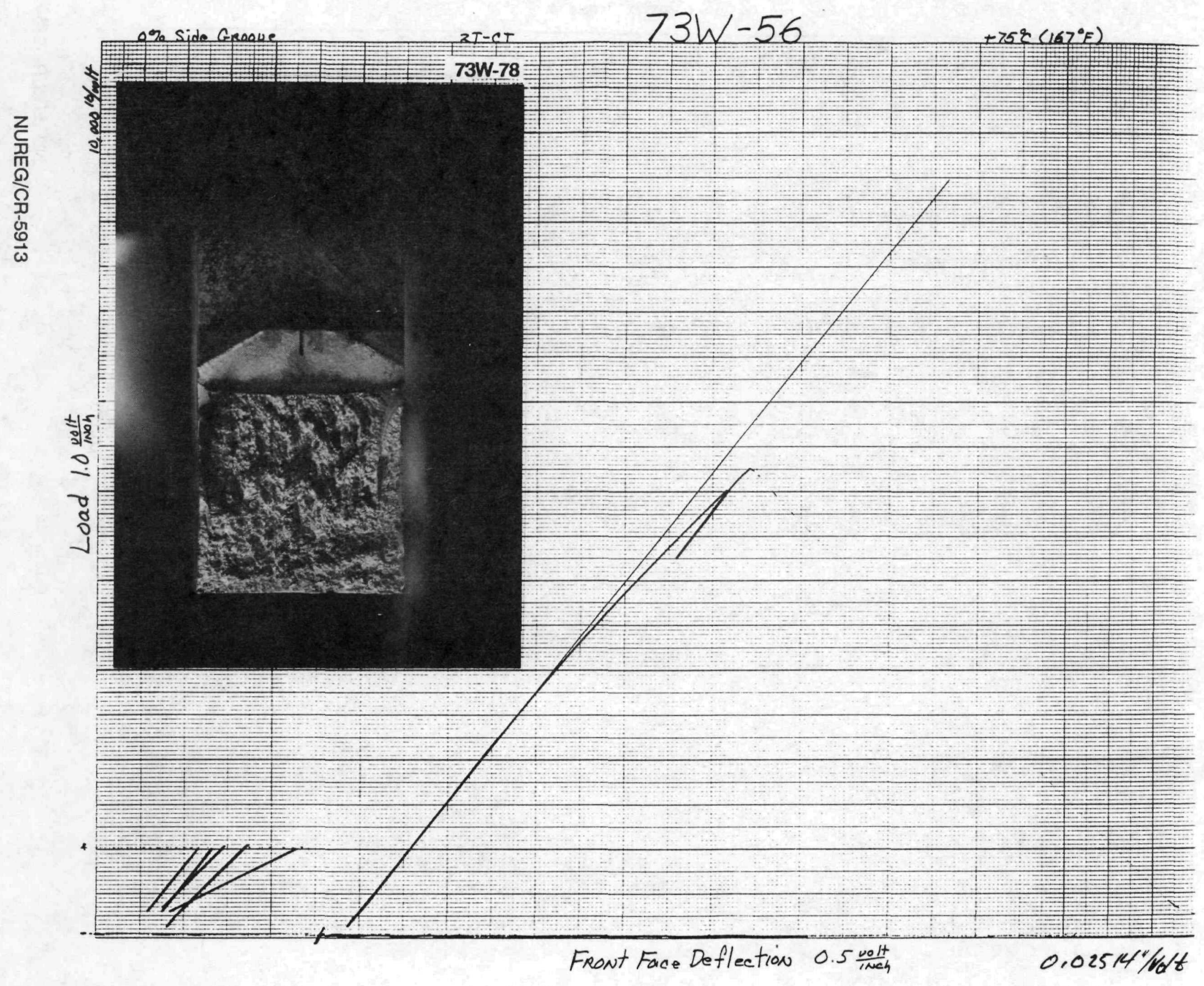




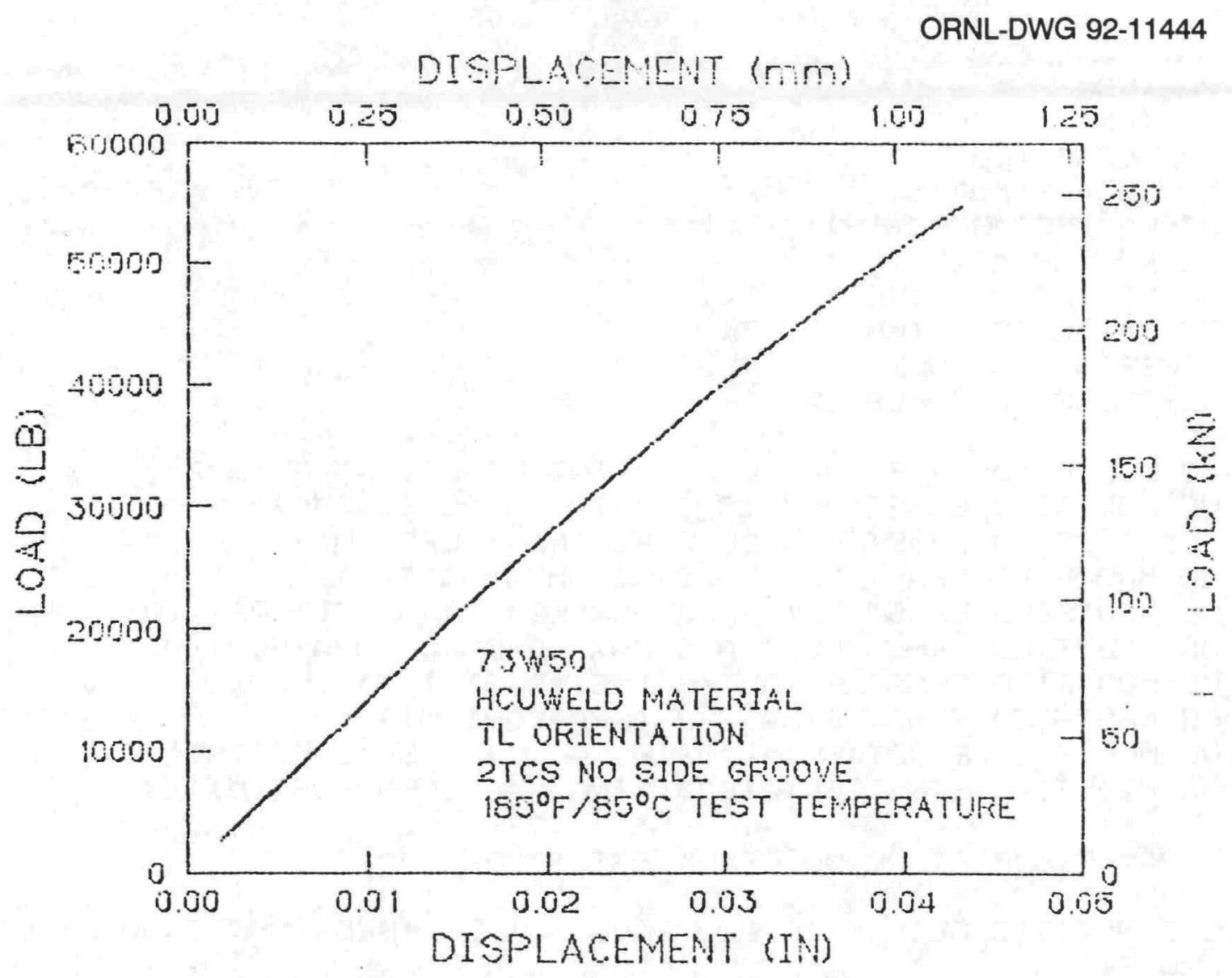

$73 W-50$

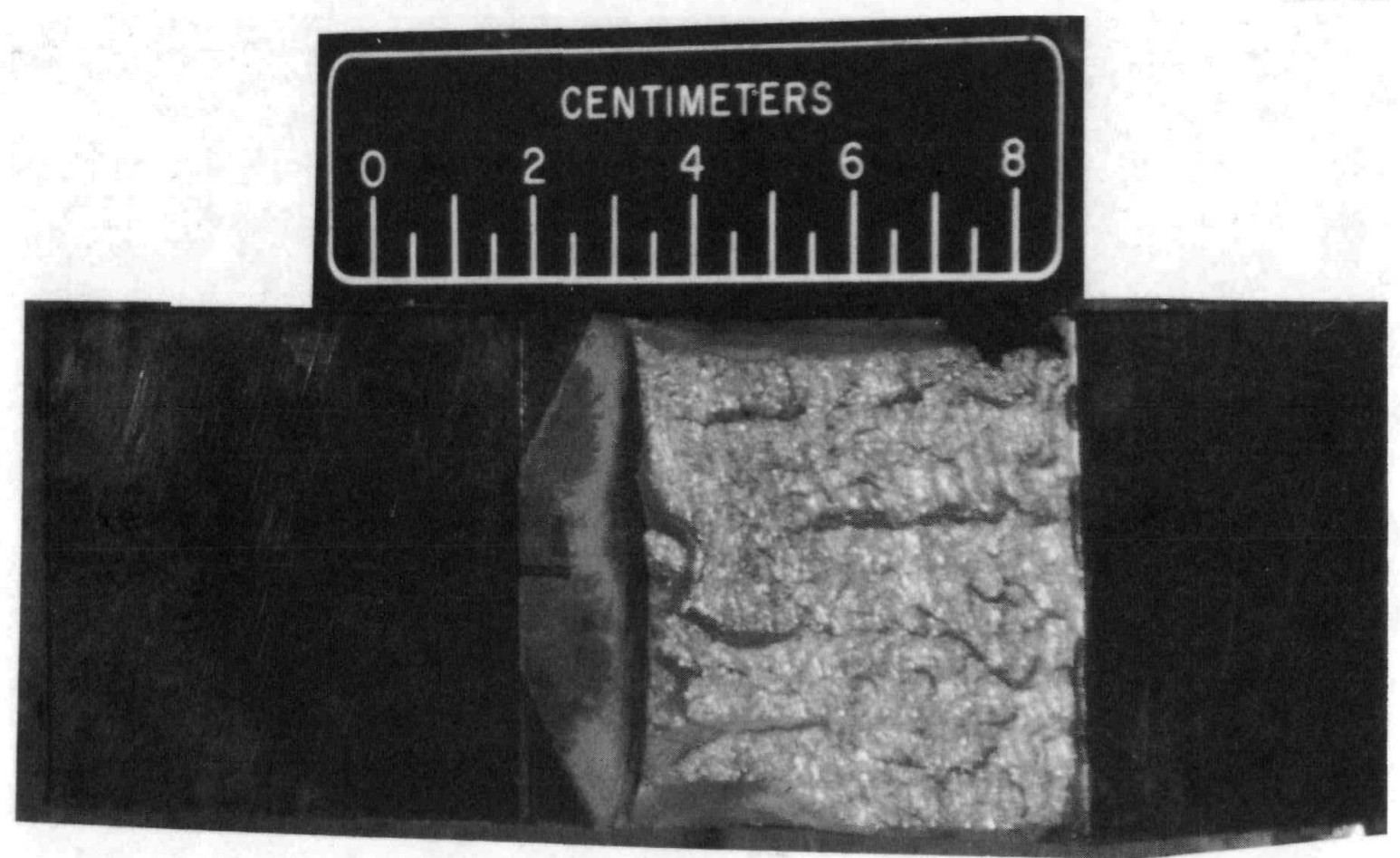




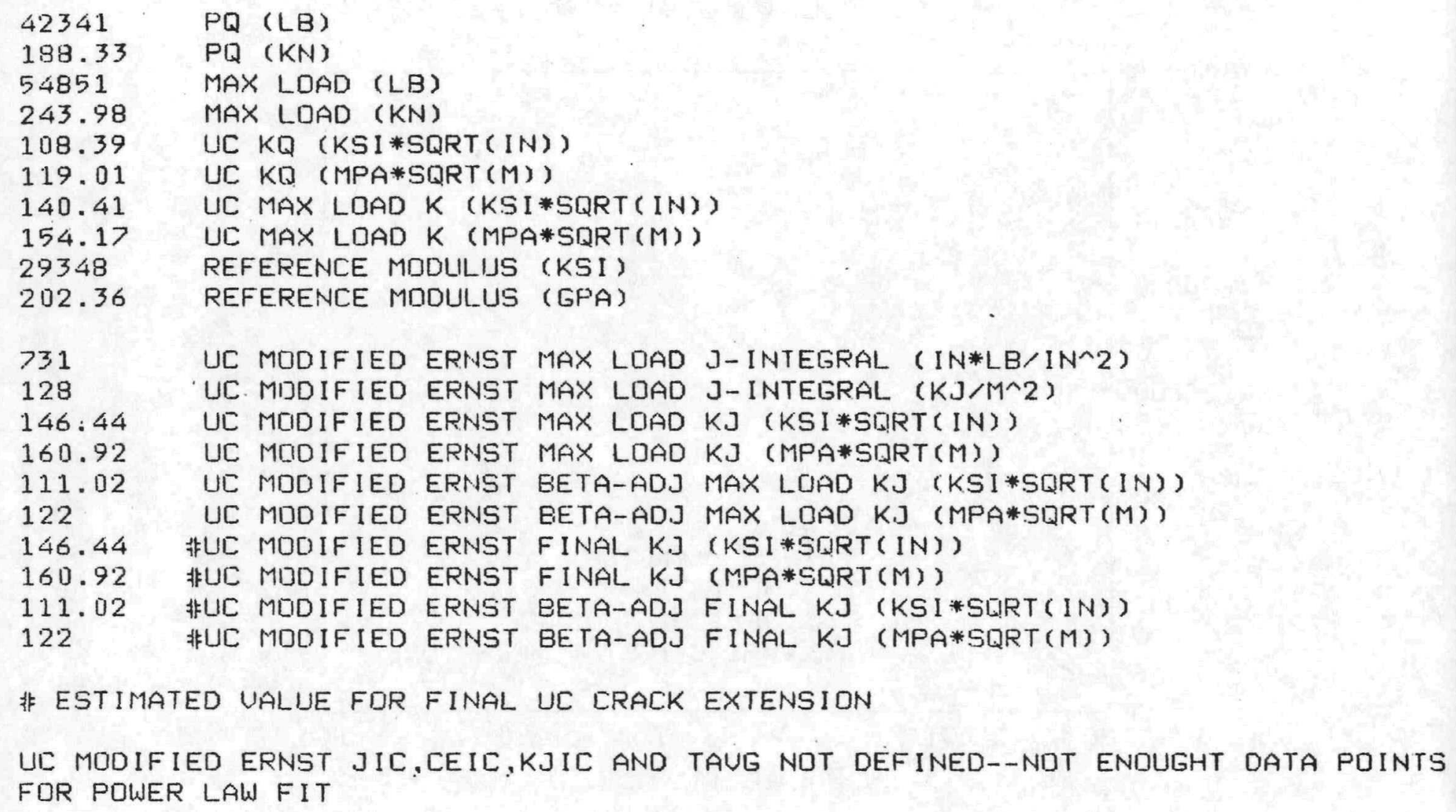


DISPLACEMEMT (MIM!
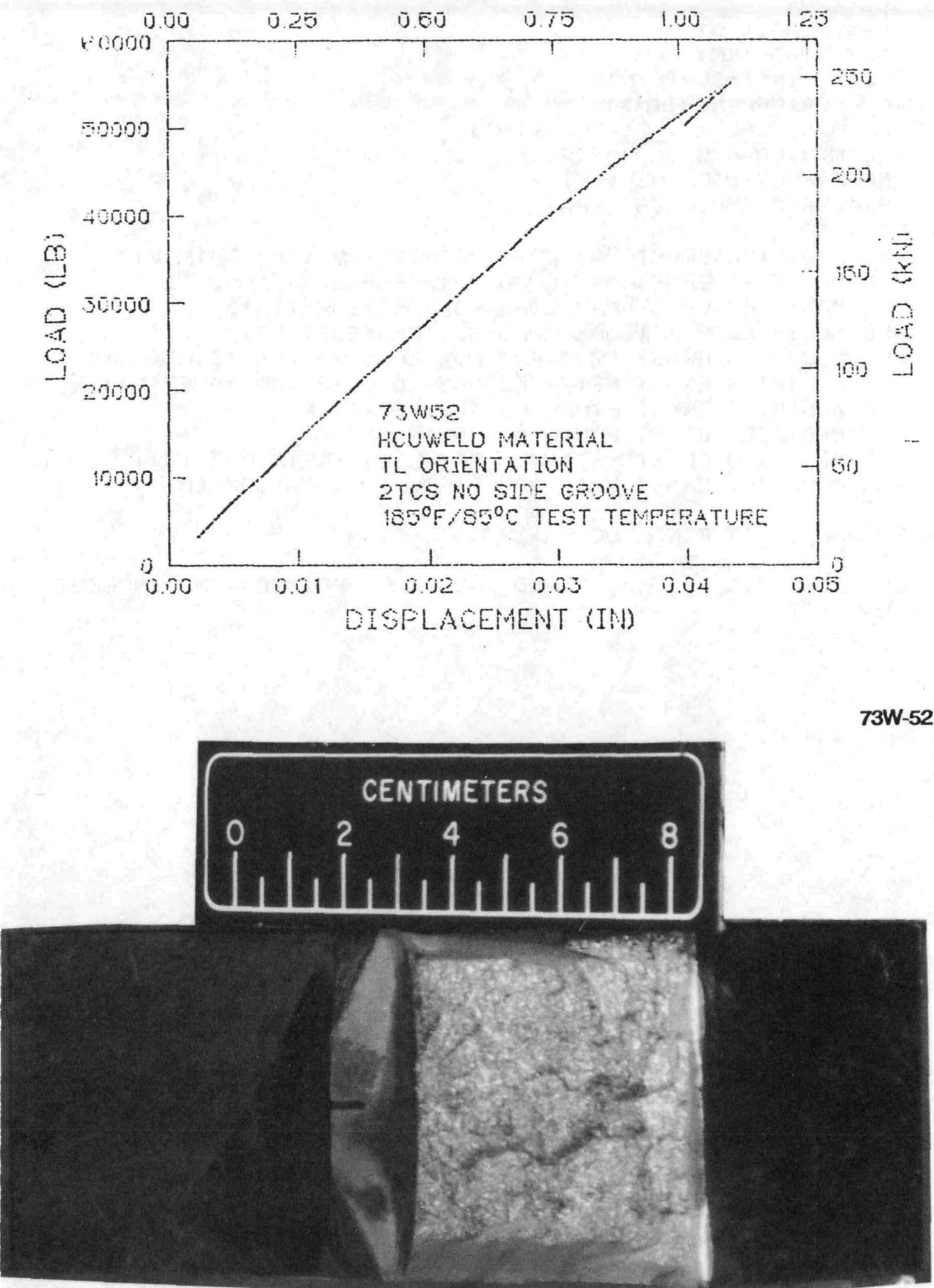


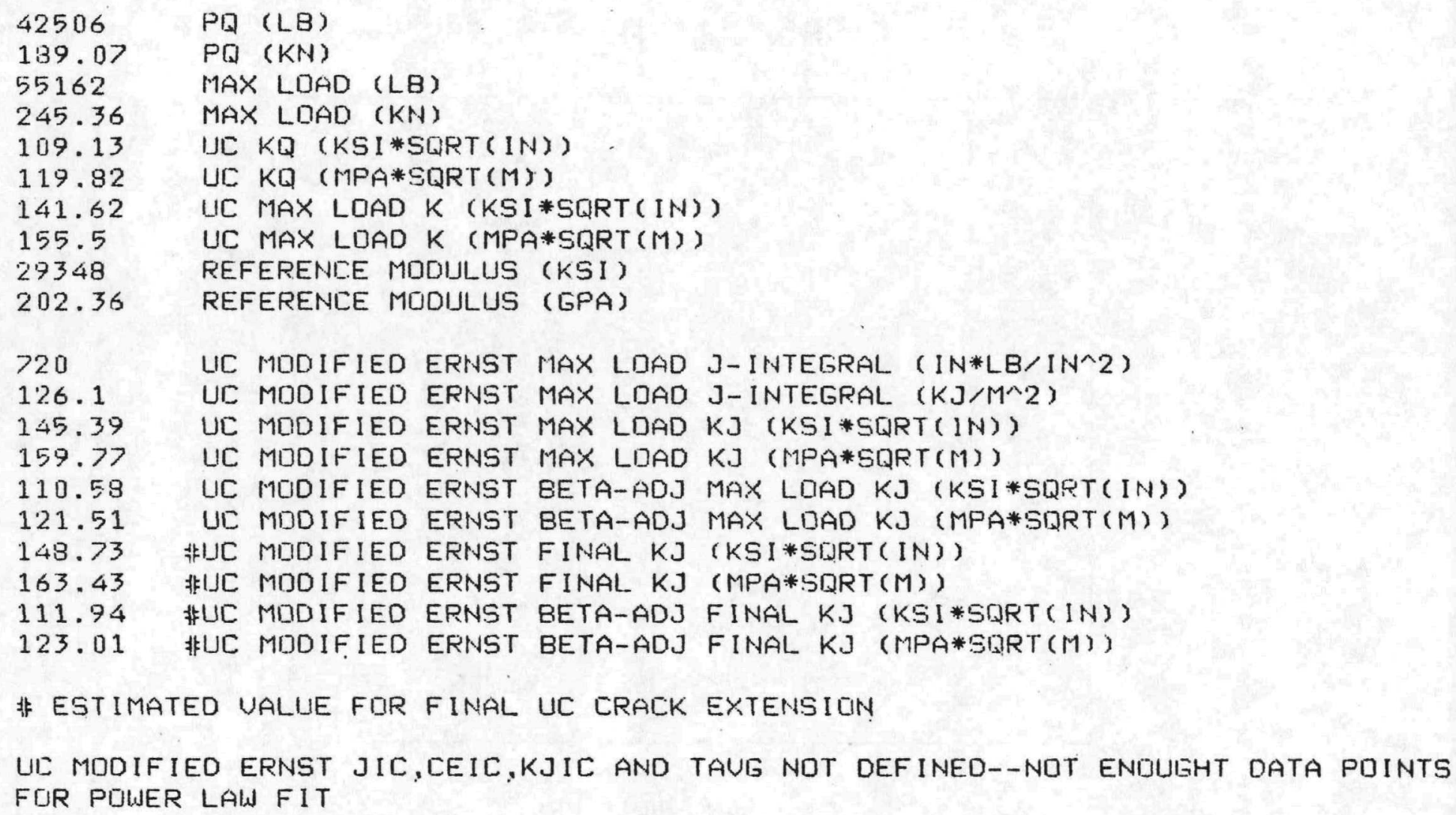




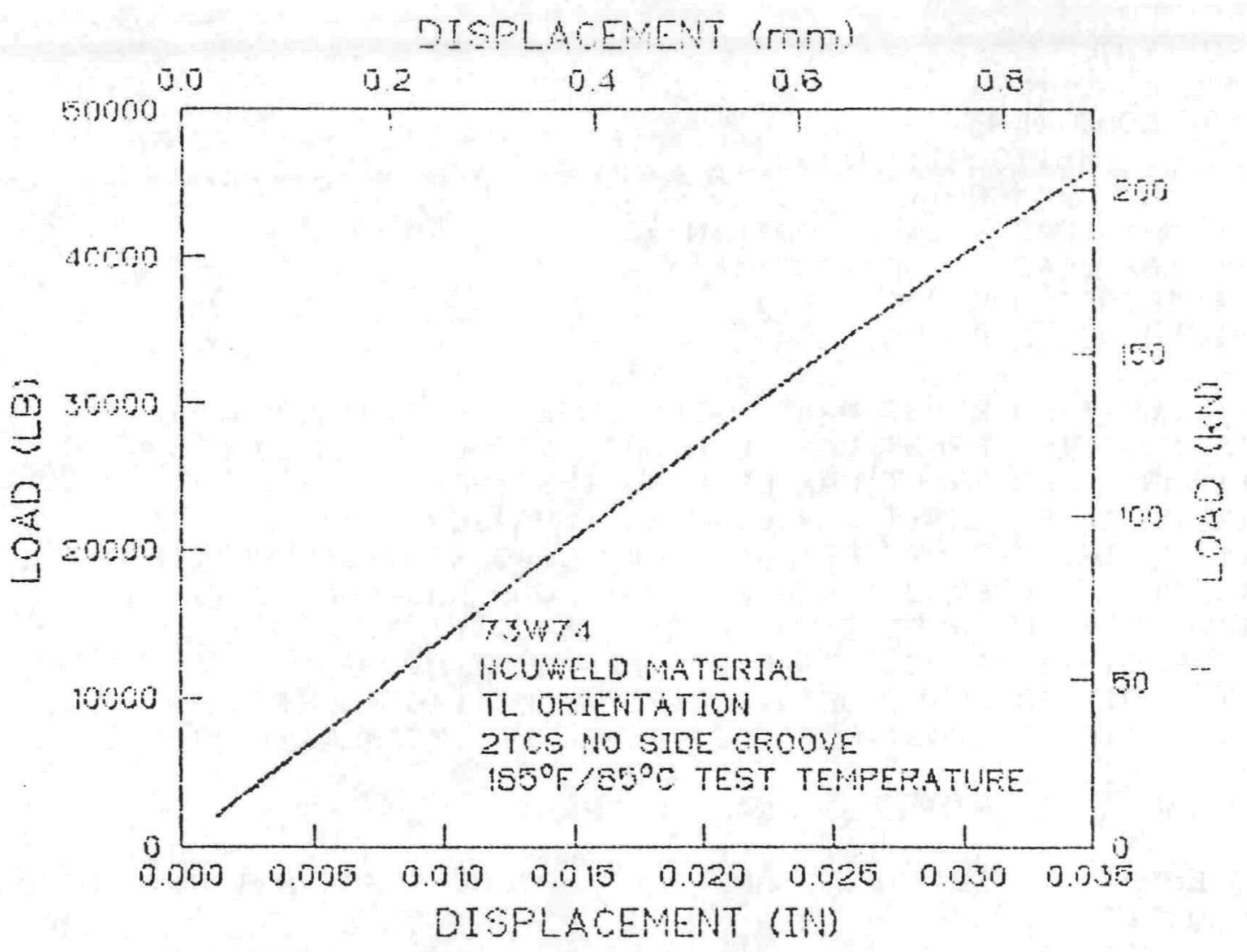

73W-74
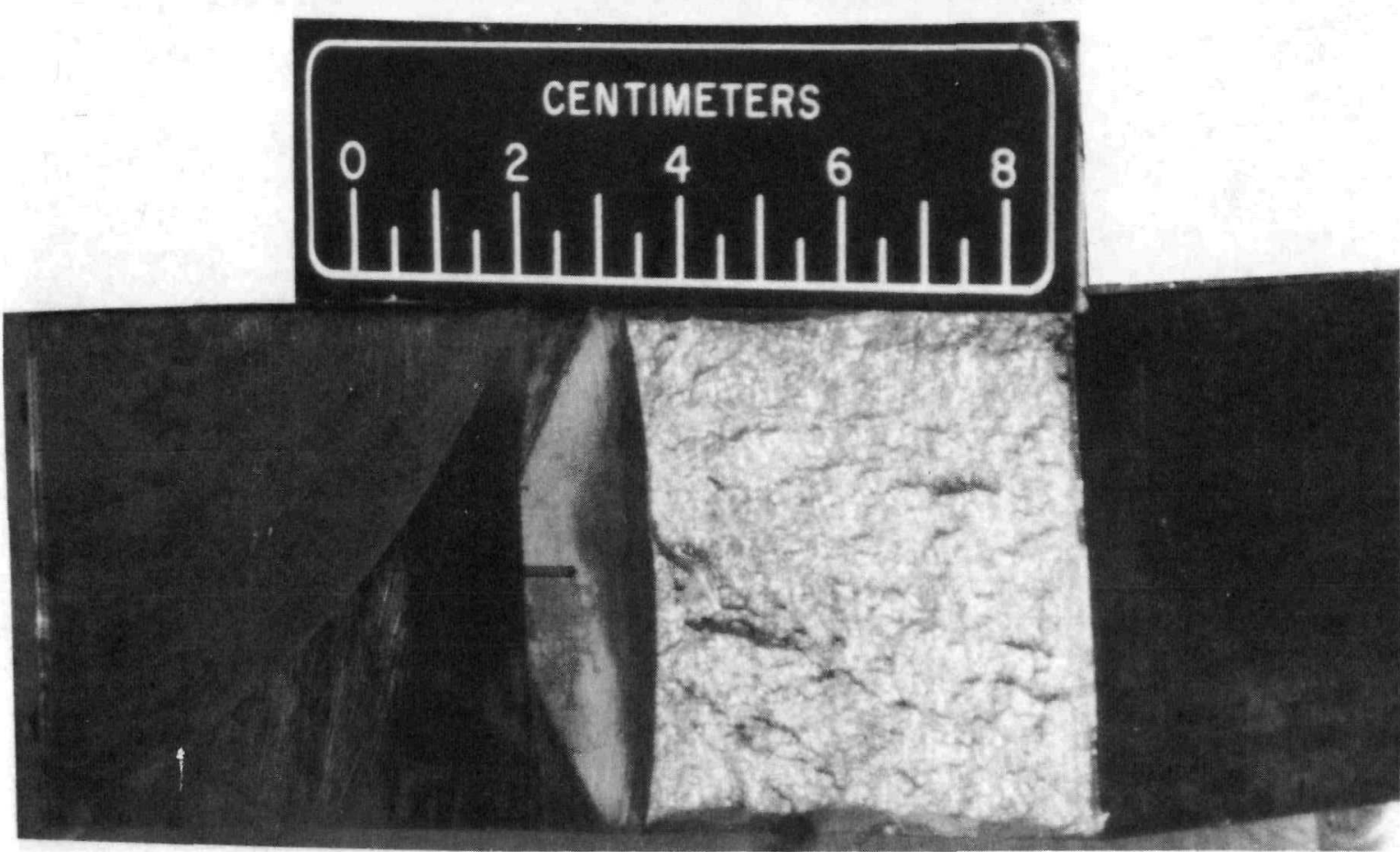


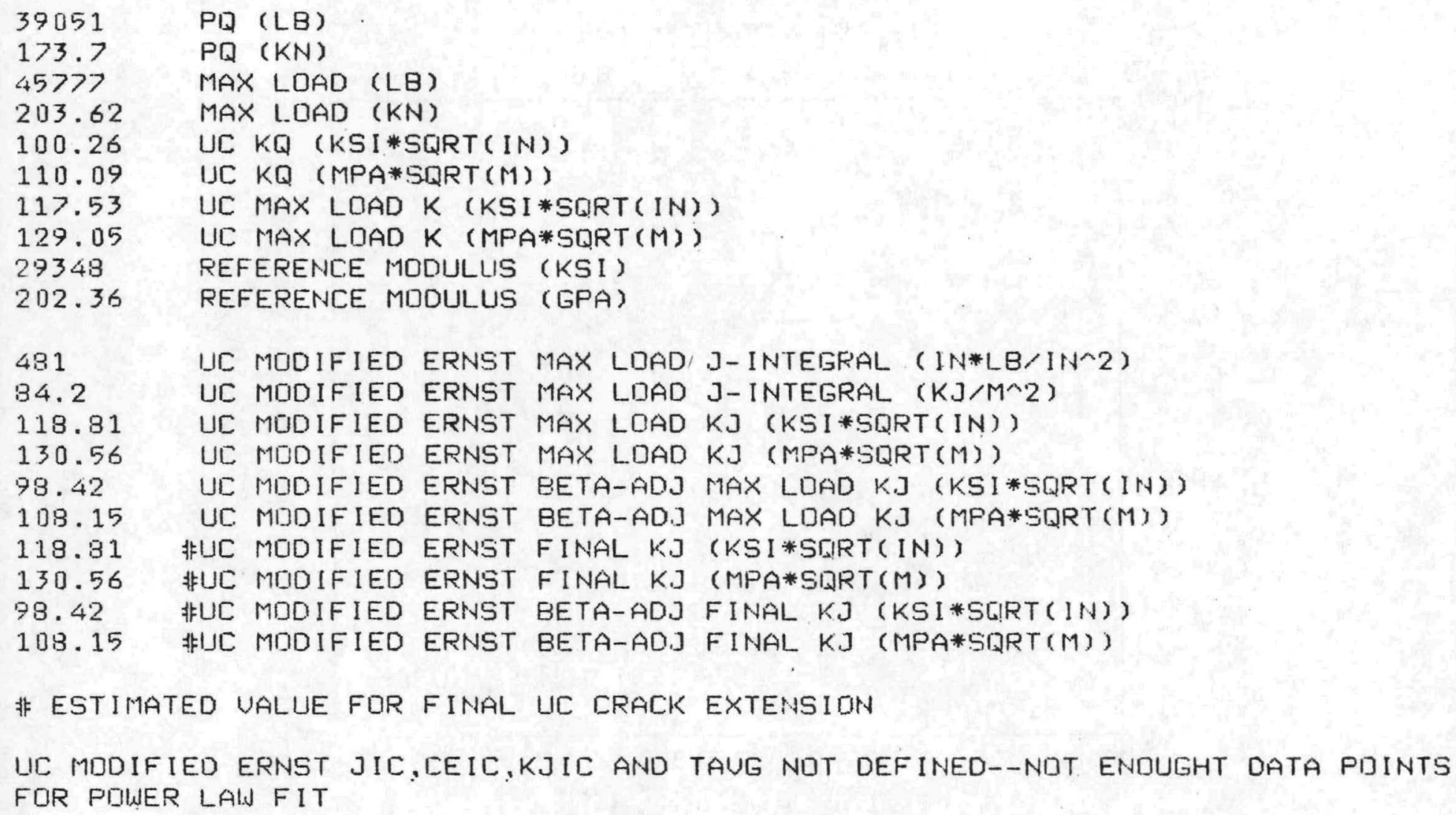




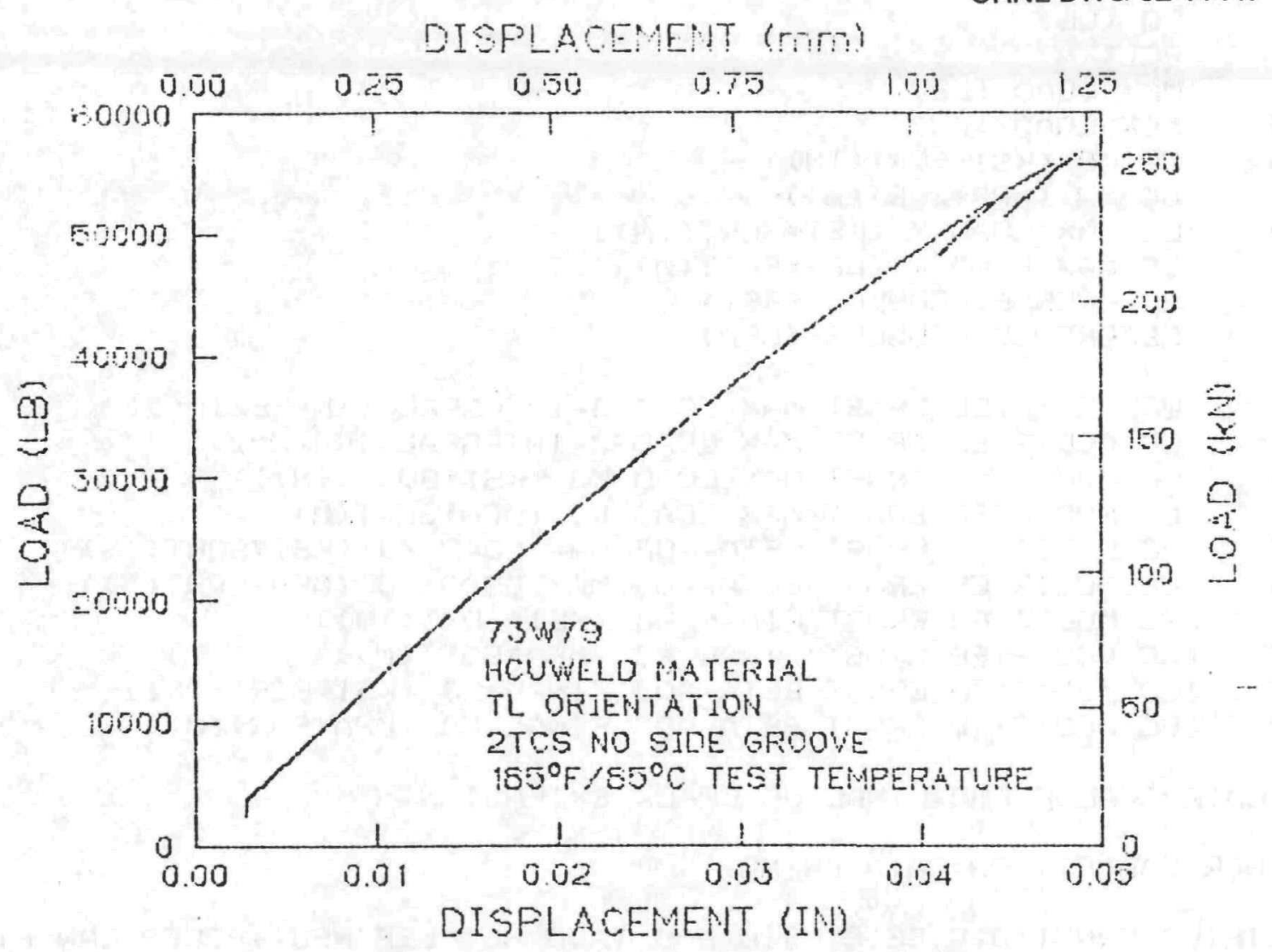

73W-79

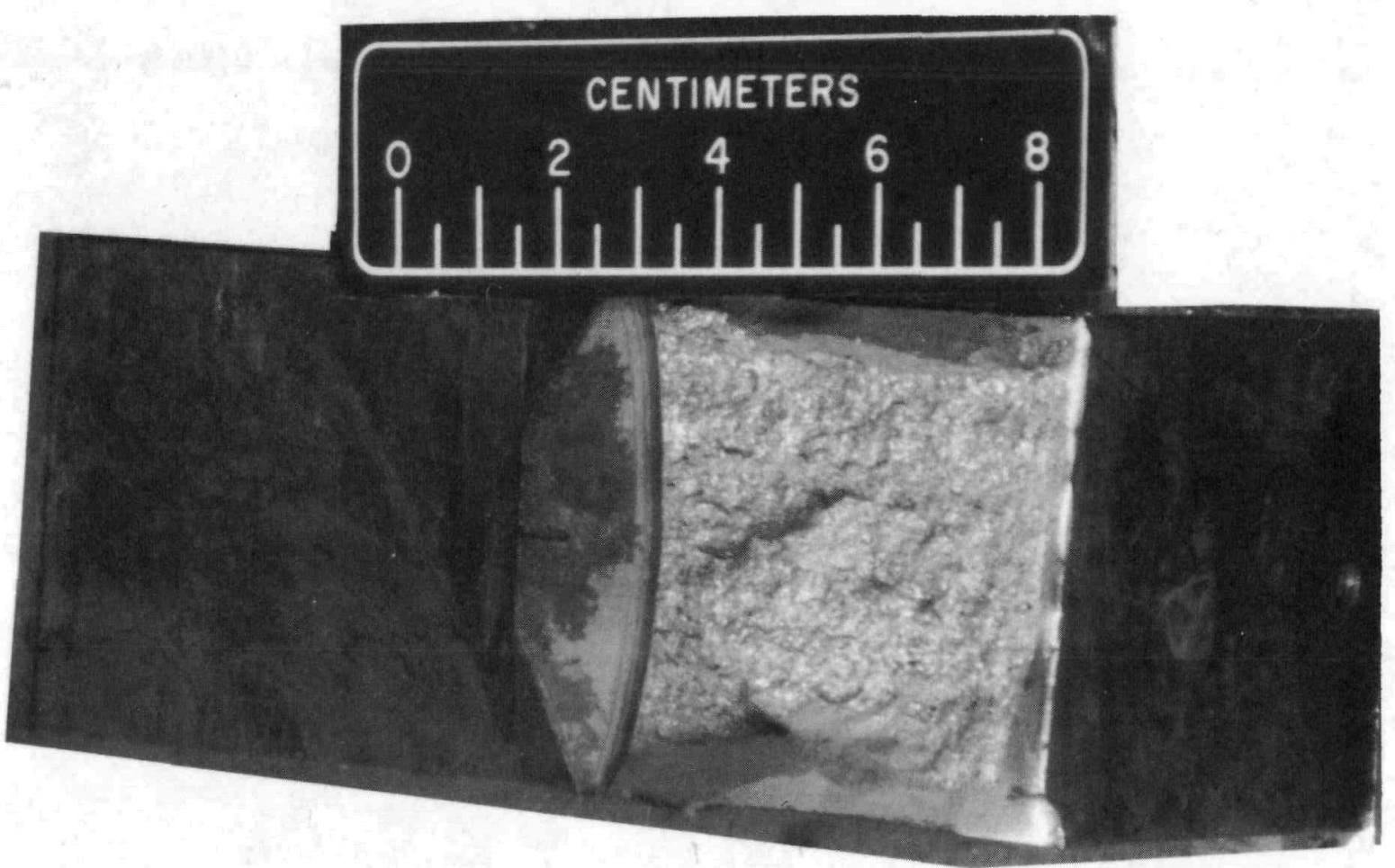




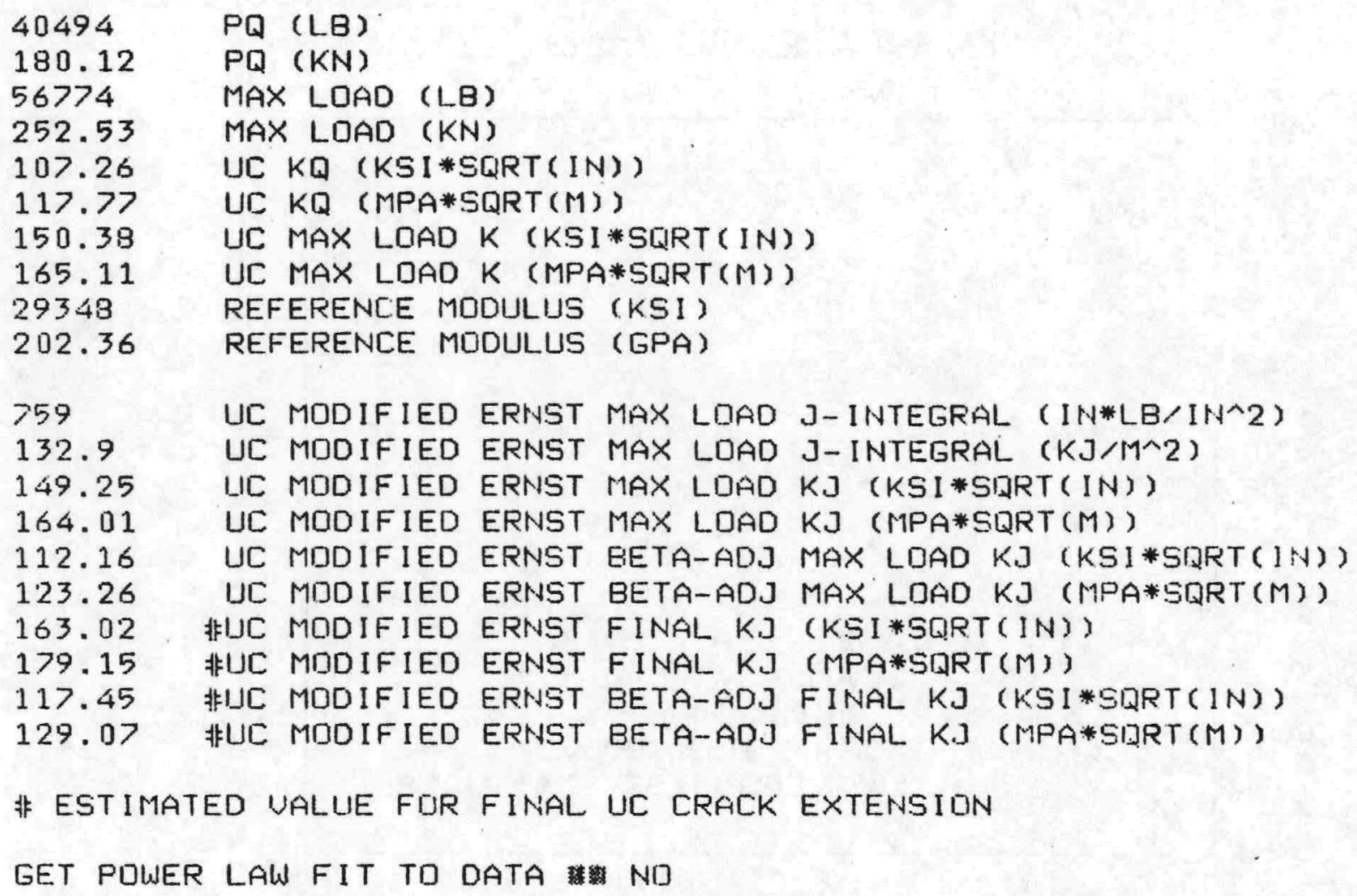


Specimen Loadline Deflection (in.)

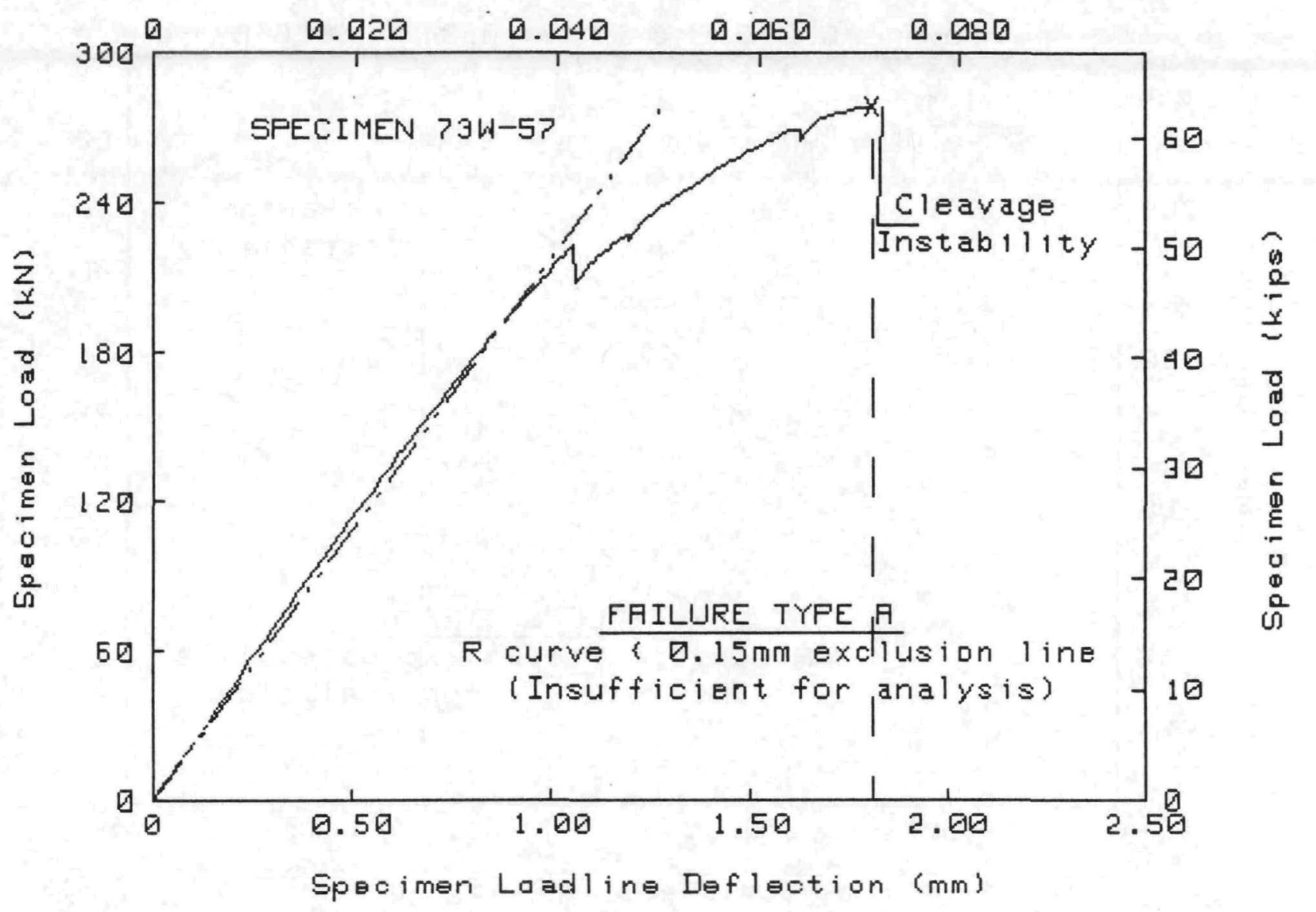

TEST SPECIMEN DATA

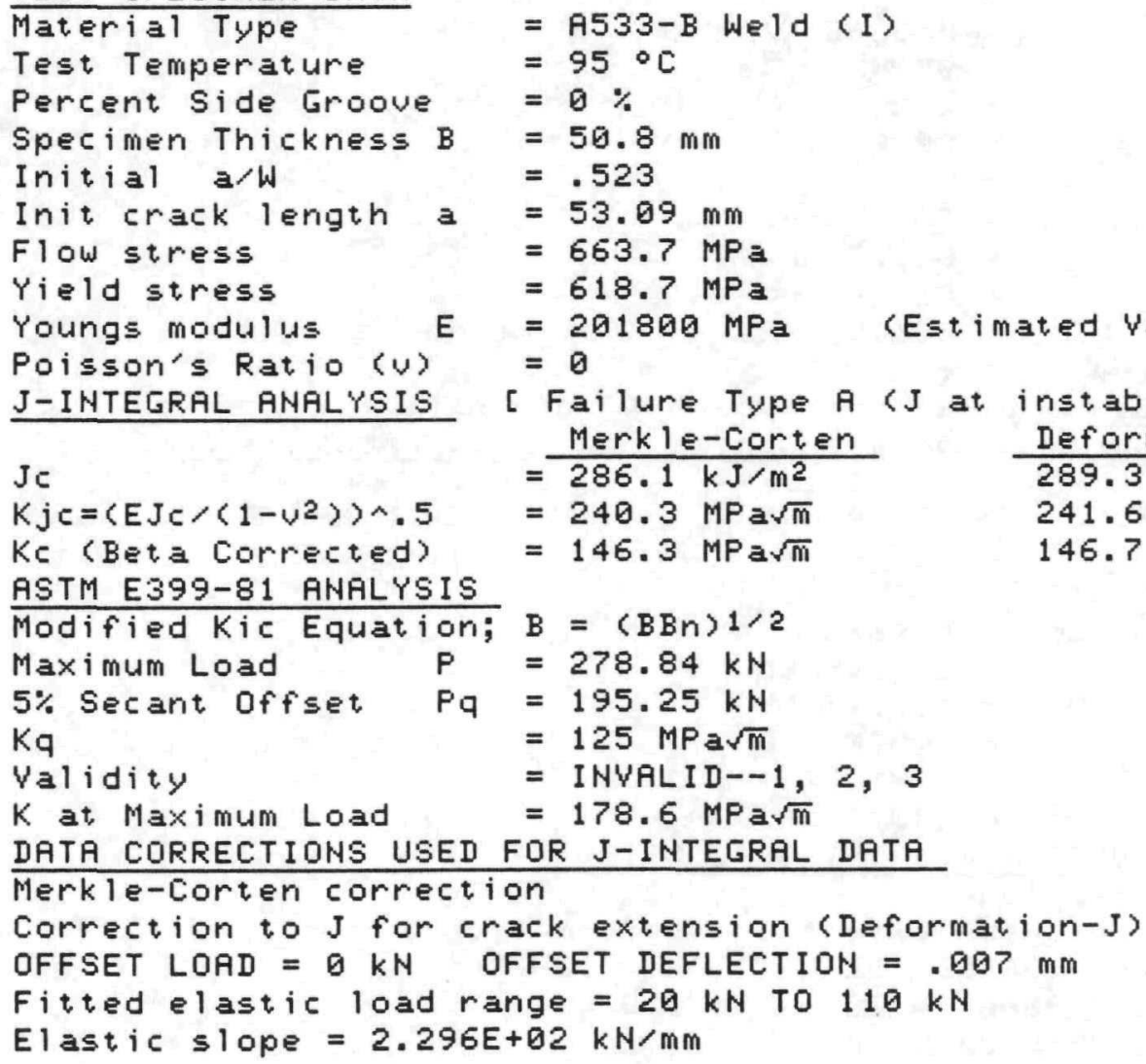


Specimen Loadline Deflection (in.)

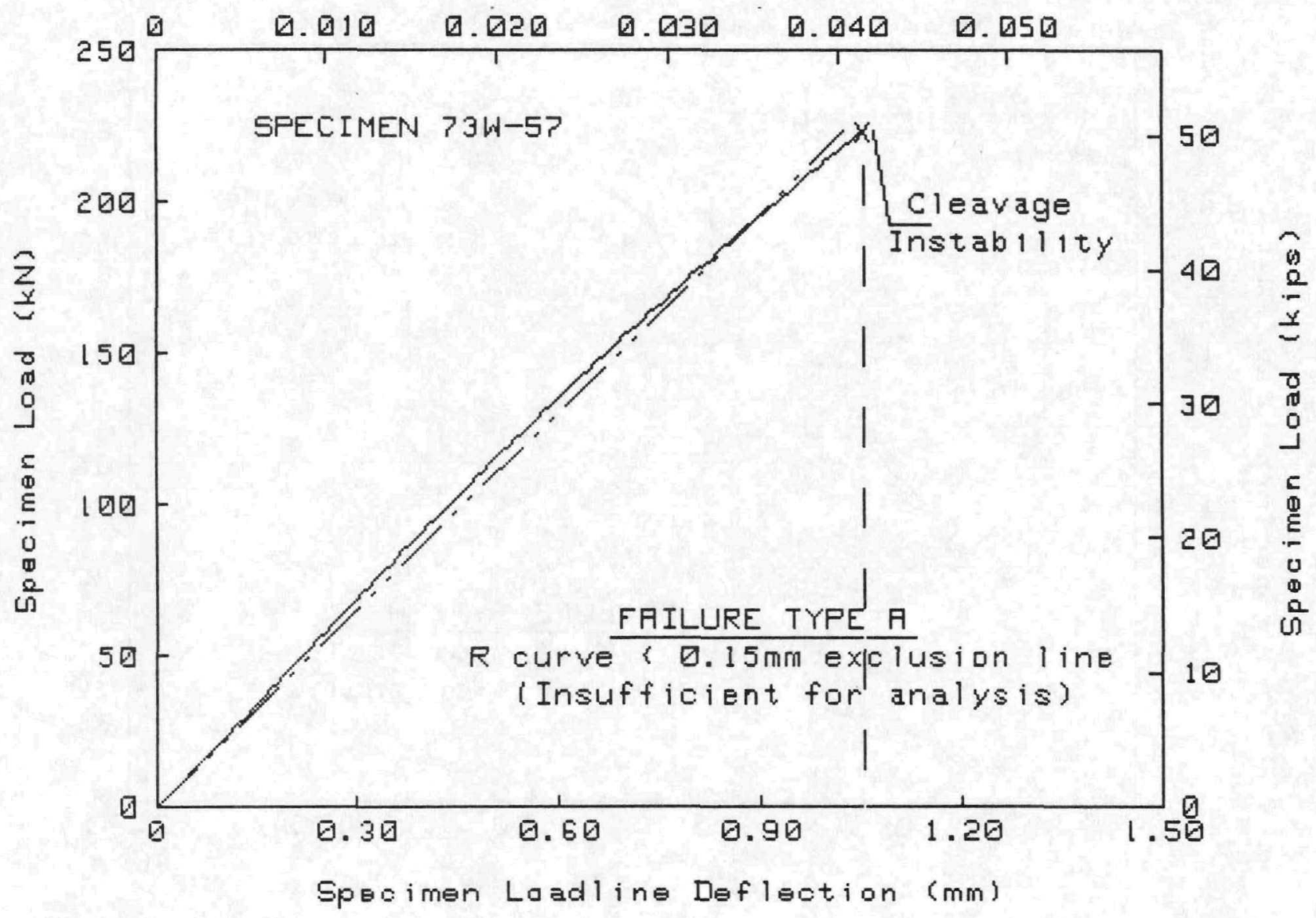

TEST SPECIMEN DATA

Material Type
Test Temperature
Percent Side Groove
Specimen Thickness B
Initial a/W
Init crack length a
Flow stress
Yield stress
Youngs modulus
PoisSon's Ratio (U)
J-INTEGRAL ANALYSIS

$\mathrm{Jc}$

$K j c=\left\langle E J c<\left(1-v^{2}\right\rangle\right) \wedge .5$

Ke (Beta Corrected)

ASTM E399-81 ANALYSIS

Modified Kic Equation;

Maximum Load

$5 \%$ Secant offset

$\mathrm{Kq}$

Yal idity

$K$ at Maximum Load

$$
\begin{aligned}
\mathrm{P} & =223.49 \mathrm{kN} \\
\mathrm{Pq} & =195.25 \mathrm{kN} \\
& =125 \mathrm{MPa} \sqrt{\mathrm{m}} \\
& =I N Y A L I \mathrm{D}--1,2,3 \\
& =143.1 \mathrm{MPa} \sqrt{\mathrm{m}}
\end{aligned}
$$$$
\text { = A533-B Weld (I) }
$$$$
=95^{\circ} \mathrm{C}
$$$$
=0 \%
$$$$
=50.8 \mathrm{~mm}
$$$$
=.523
$$$$
=53.09 \mathrm{~mm}
$$$$
=663.7 \mathrm{MPa}
$$$$
=618.7 \mathrm{MPa}
$$$$
=201800 \mathrm{MPa} \text { (Estimated Value) }
$$$$
=\frac{\text { Merkle-Corten }}{112.8 \mathrm{~kJ} / \mathrm{m}^{2}}
$$$$
=150.9 \mathrm{MPa} \sqrt{\mathrm{m}}
$$$$
=116.4 \mathrm{MPa} \sqrt{\mathrm{m}}
$$

Etimated value)

DRTA CORRECTIONS USED FOR J-INTEGRAL DATA

Merkle-Corten correction

Correction to J for crack extension (Deformation-J)

OFFSET LOAD $=0 \mathrm{kN}$ OFFSET DEFLECTION $=.007 \mathrm{~mm}$

Fitied elastic load range $=20 \mathrm{kN}$ TO $110 \mathrm{kN}$

Elastic slope $=2.296 \mathrm{E}+02 \mathrm{kN} / \mathrm{mm}$ 
Specimen Crackmouth Deflection (in.)

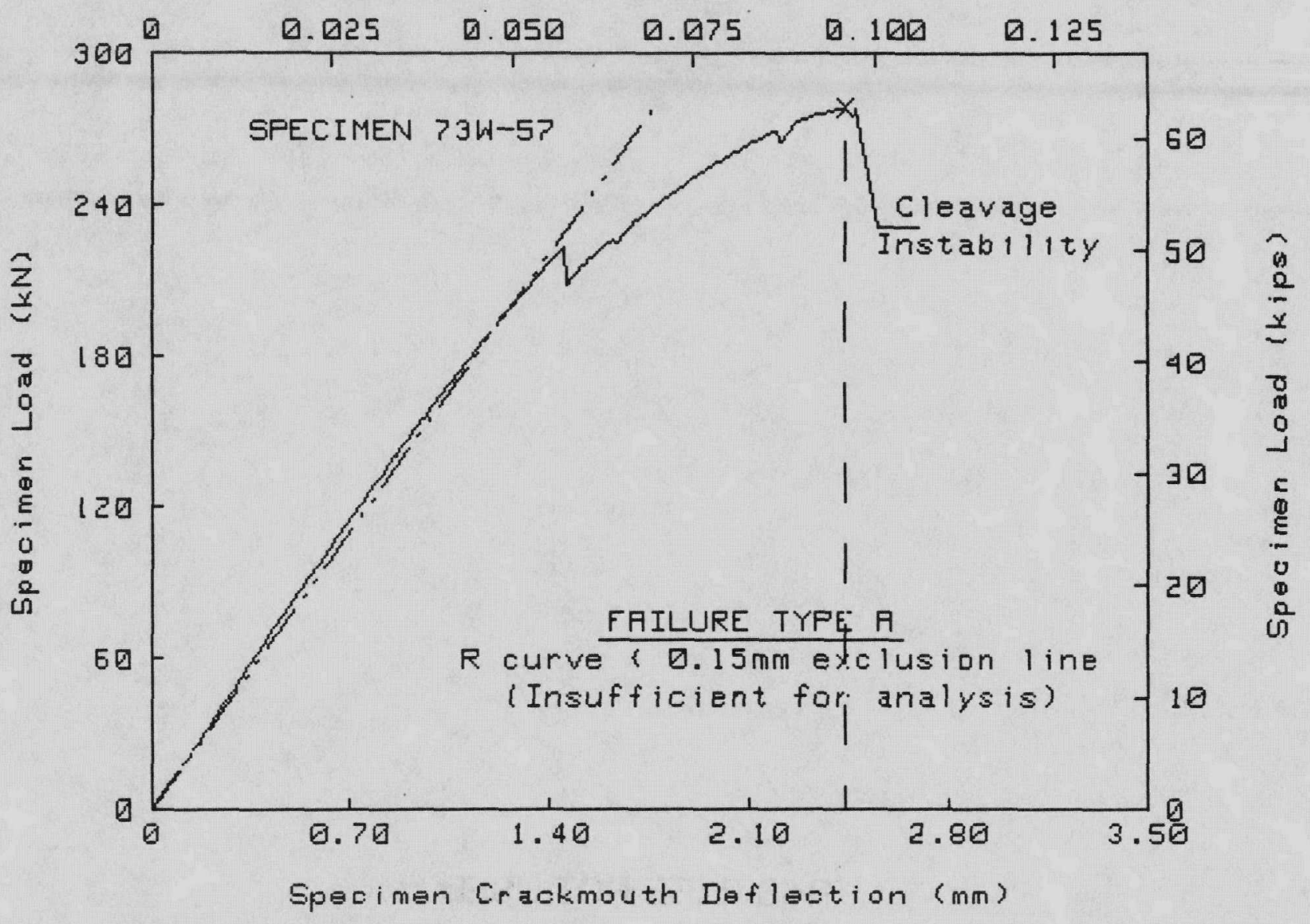

TEST SPECIMEN DATA

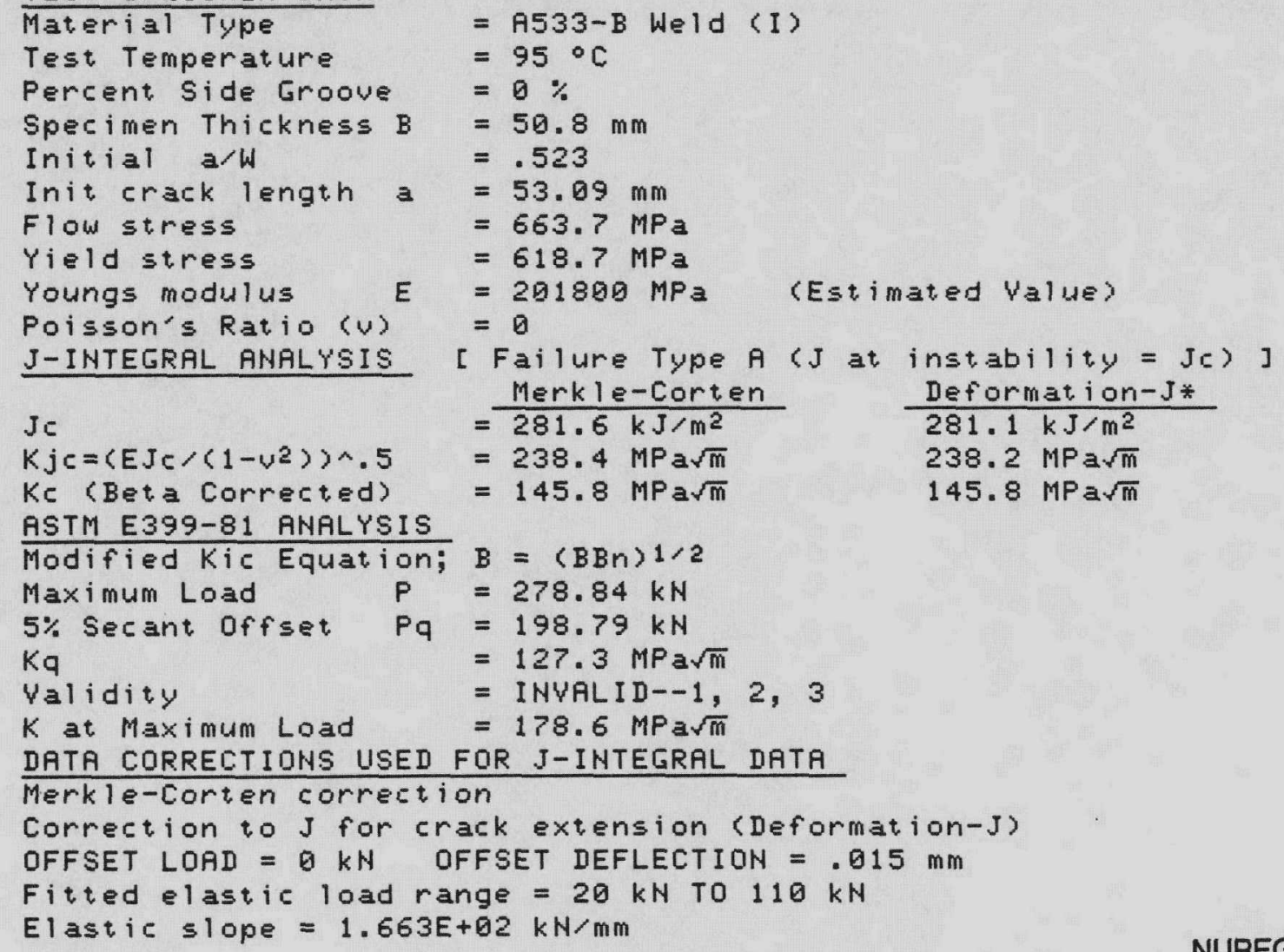



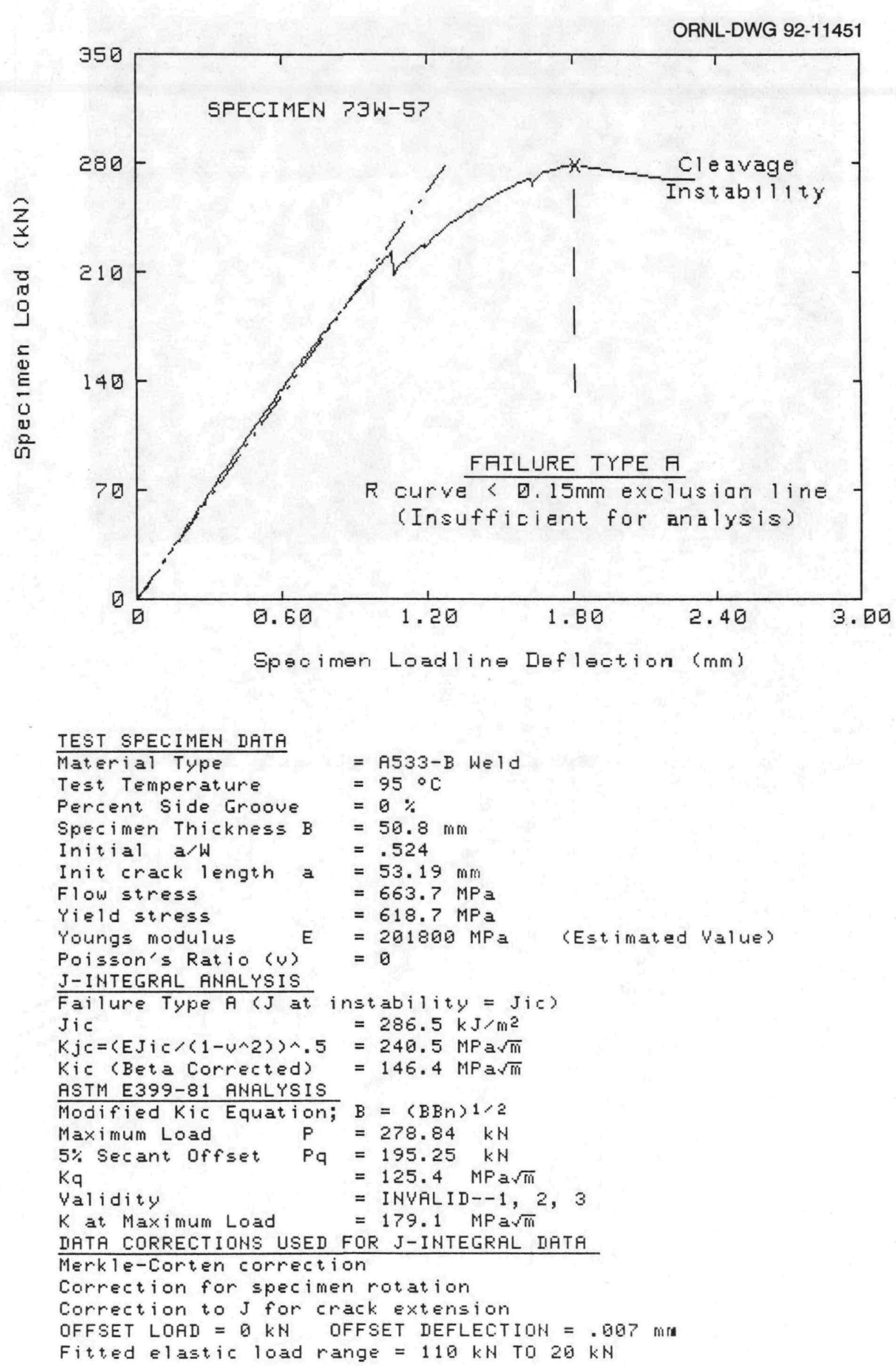


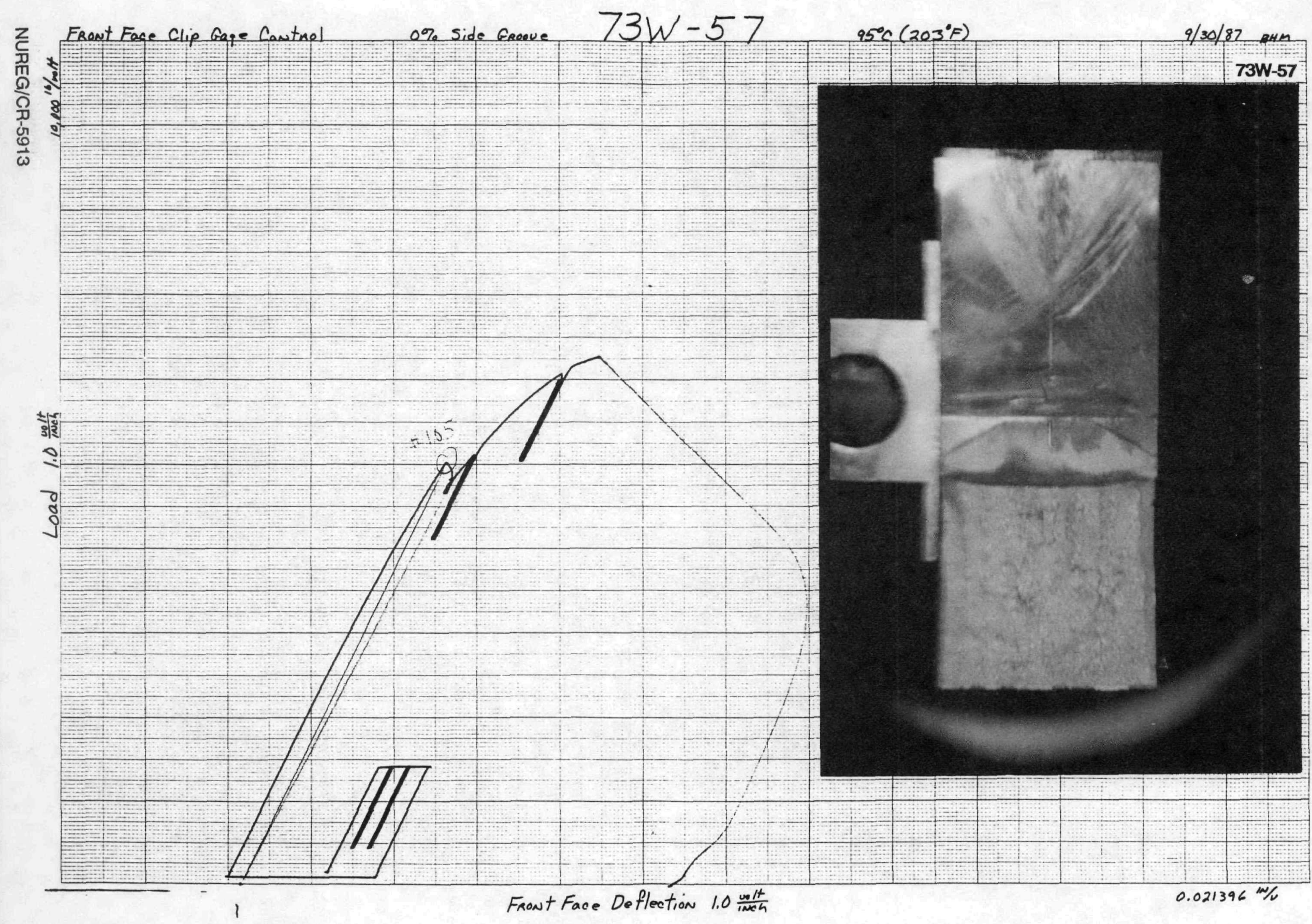


Specimen Loadline Deflection (in.)

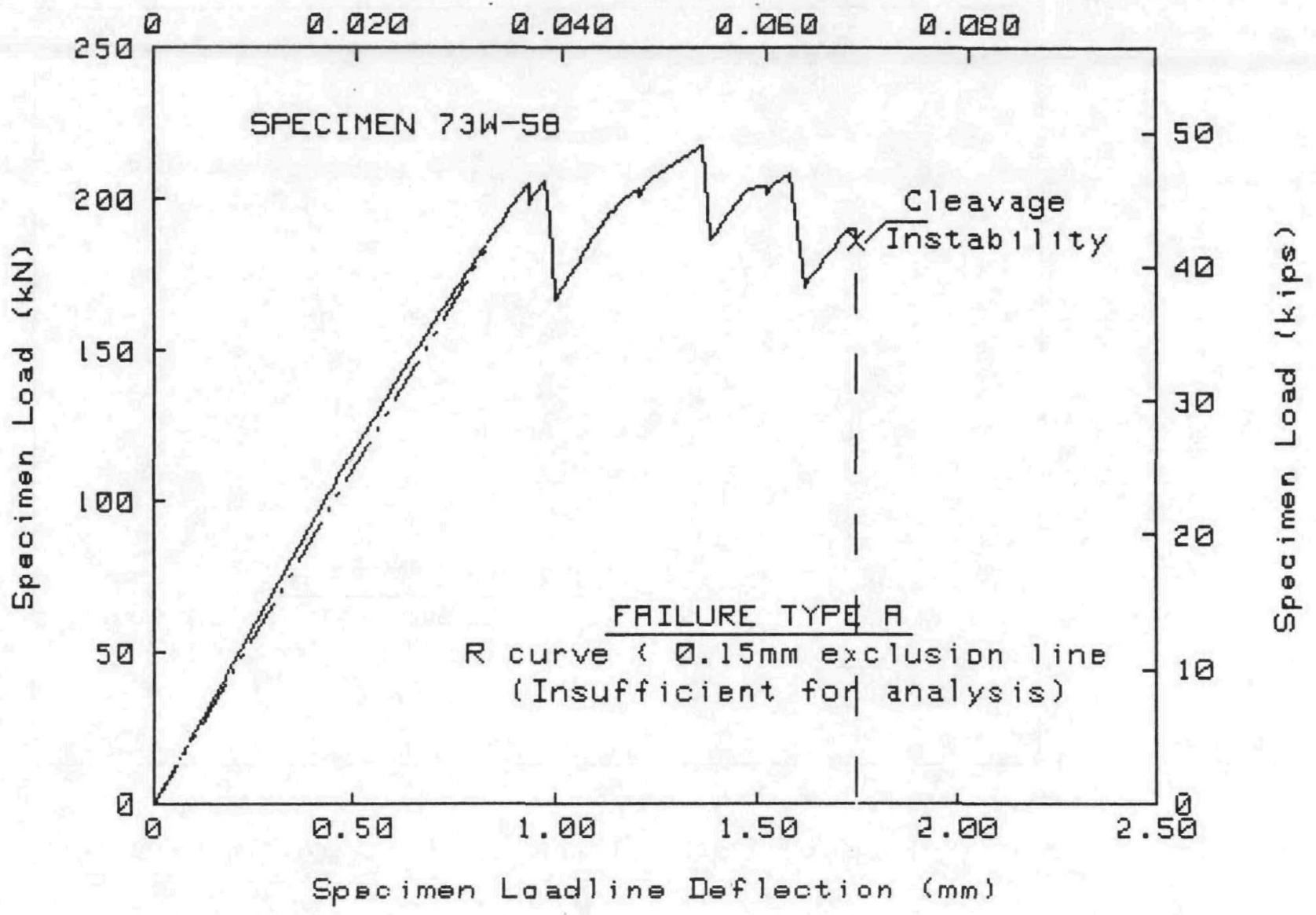

TEST SPECIMEN IATA

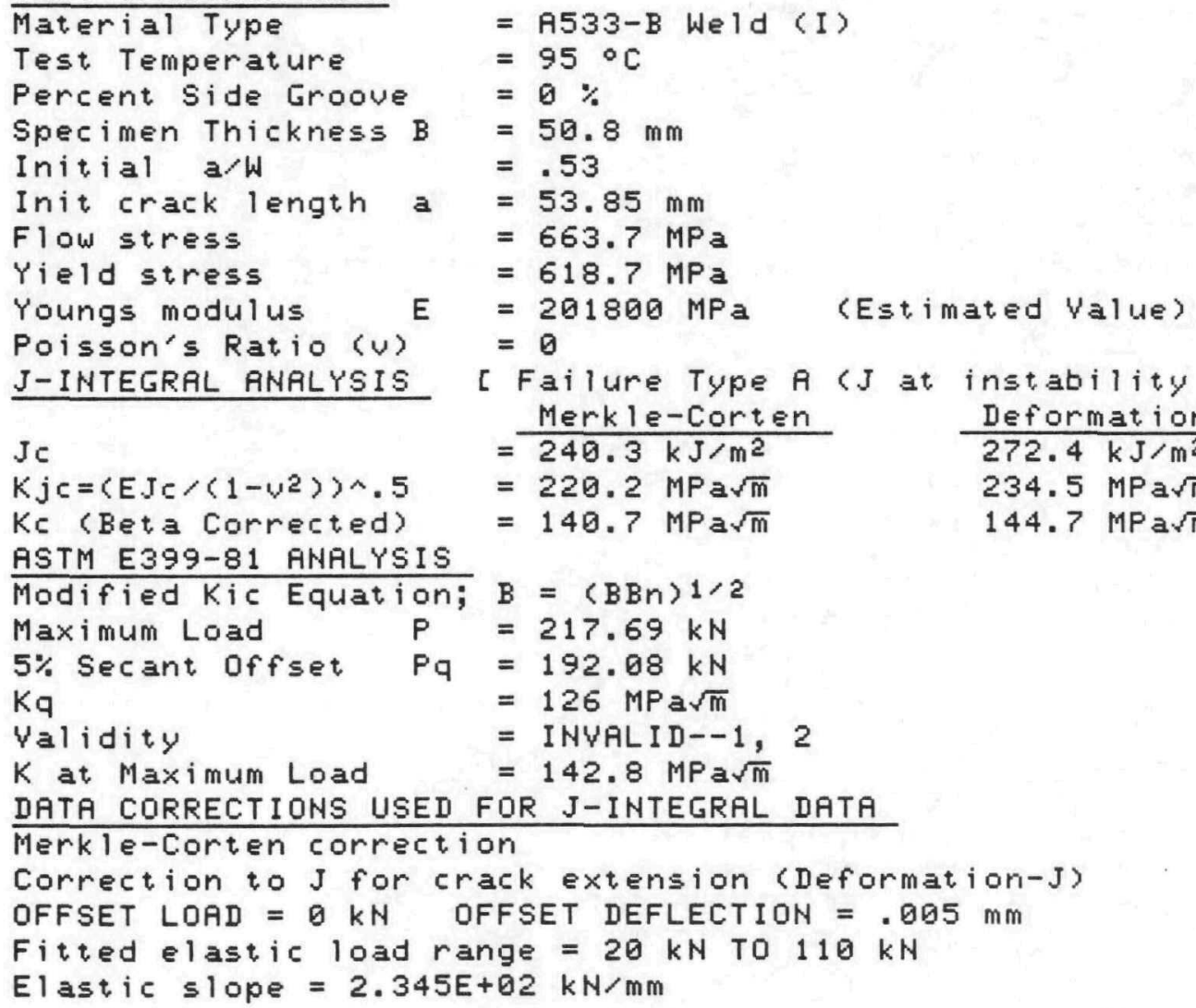




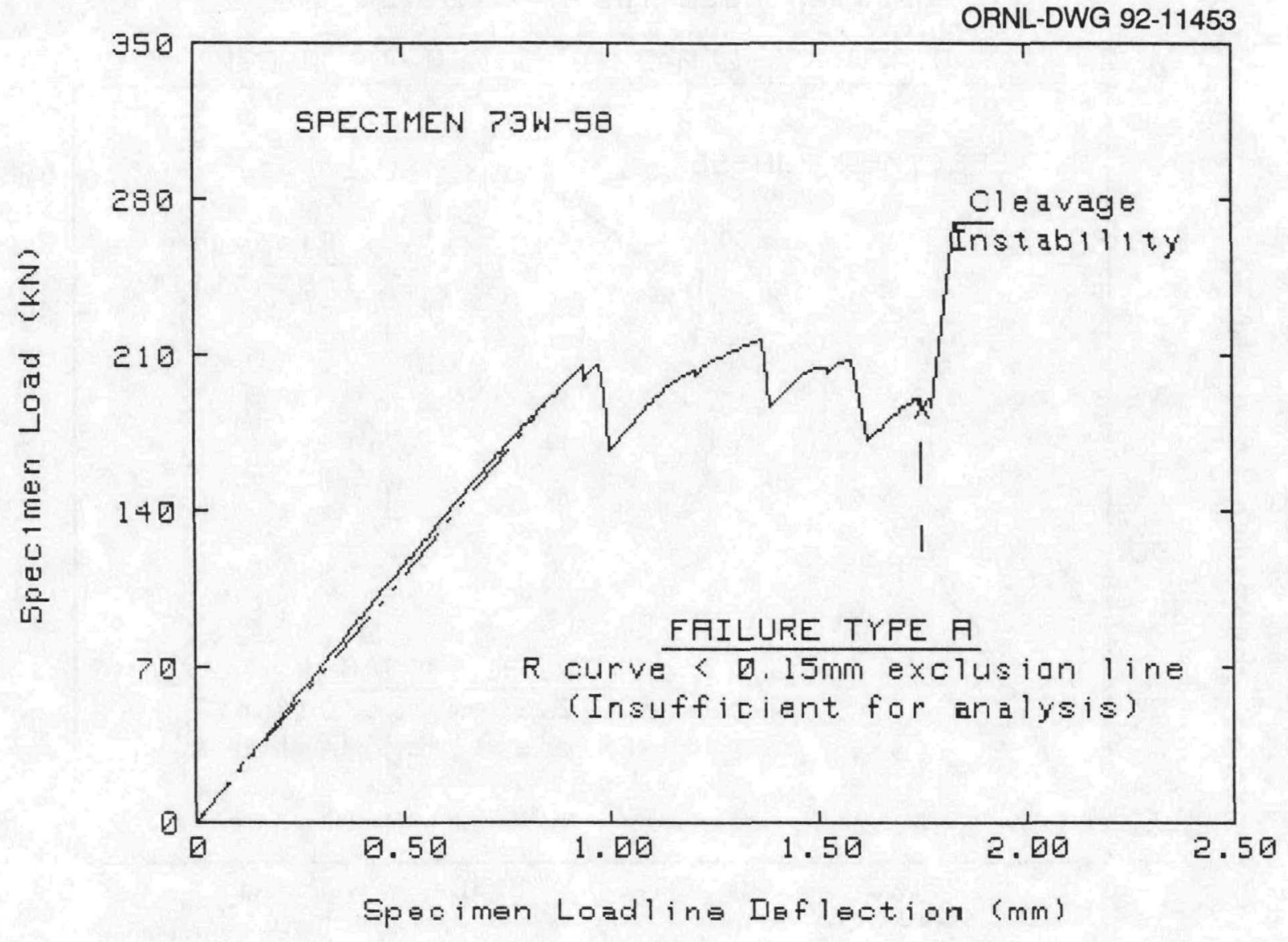

TEST SPECIMEN DATH

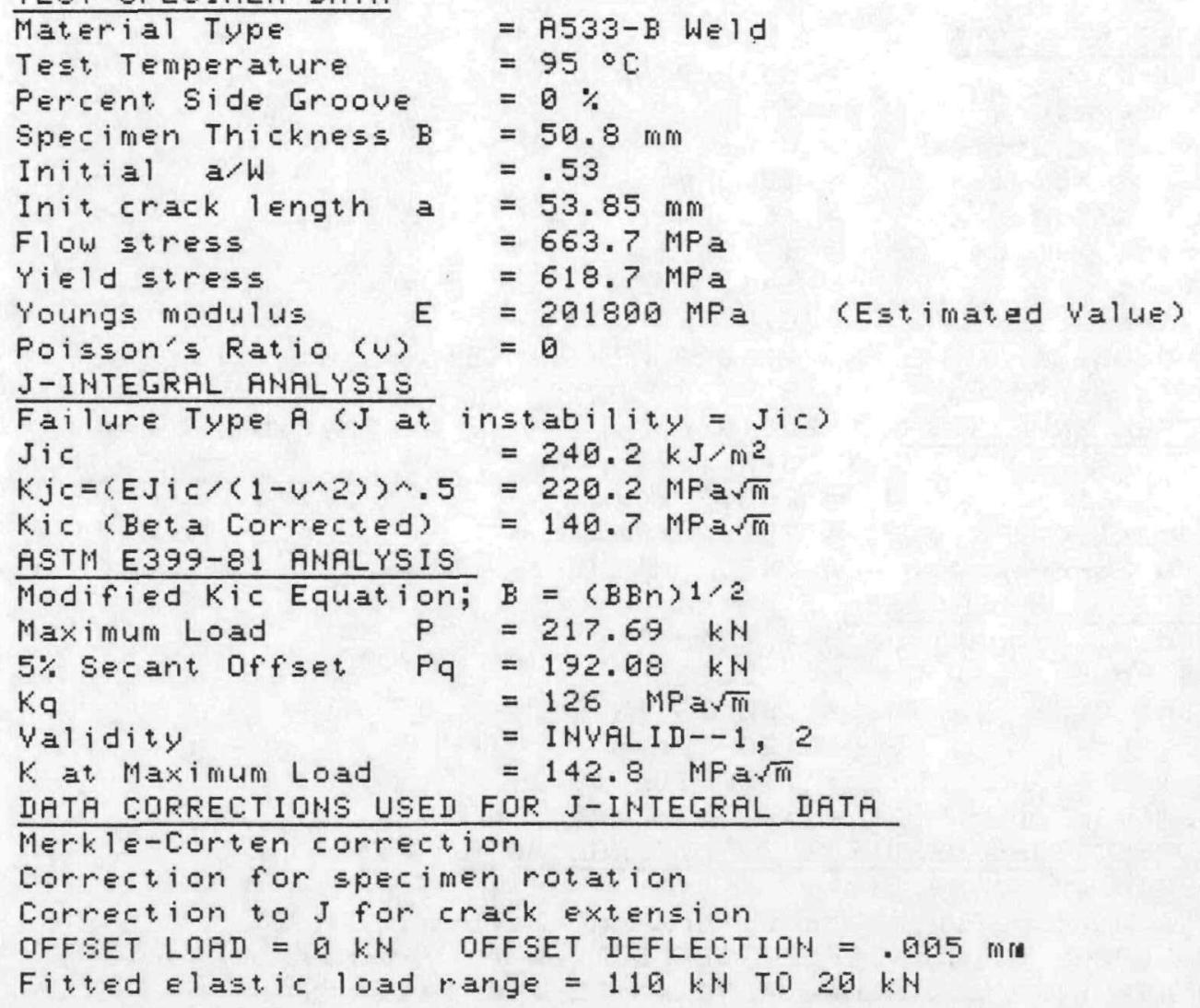


Specimen Laadline Leflectian (in.)

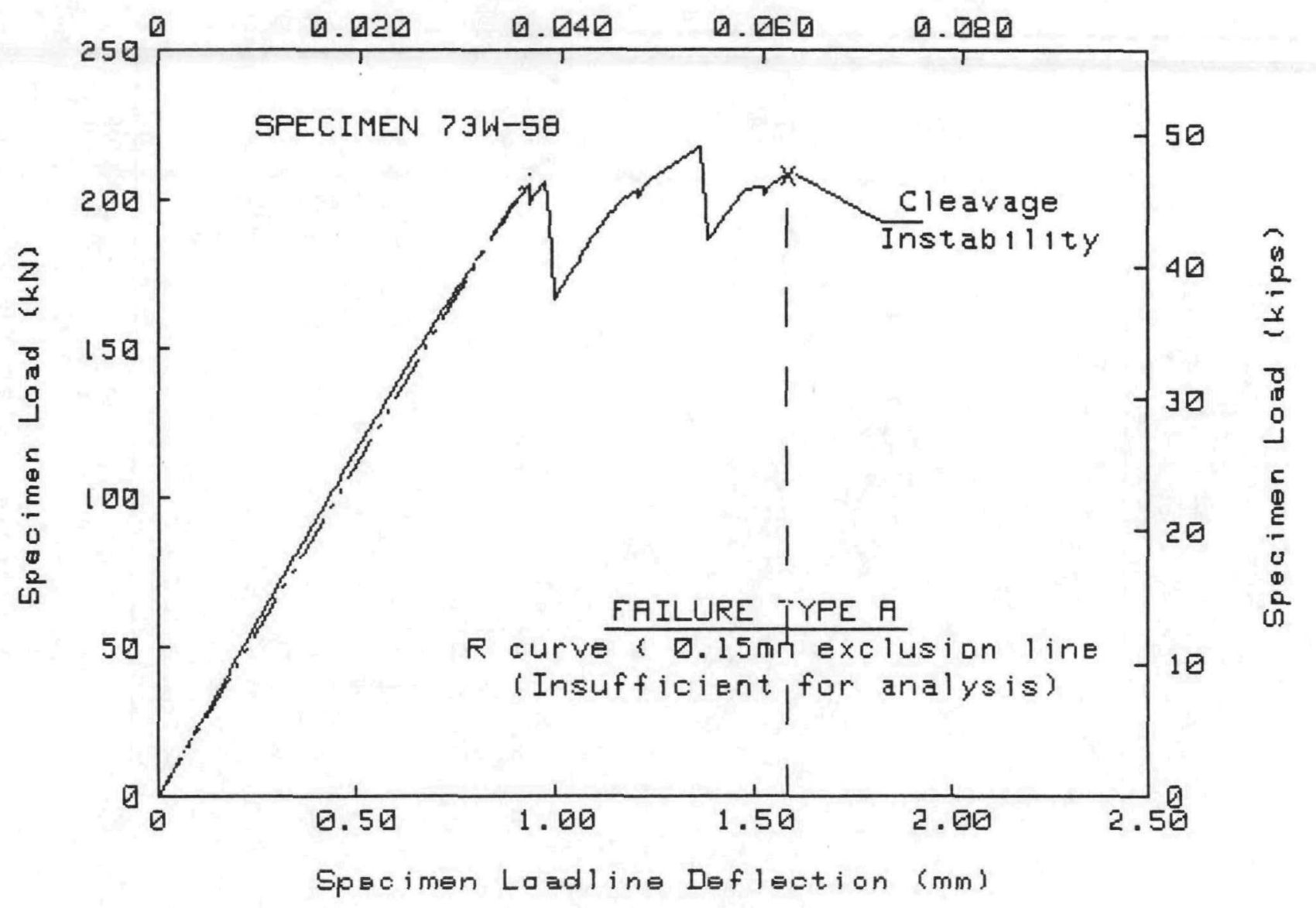

TEST SPECIMEN DATA

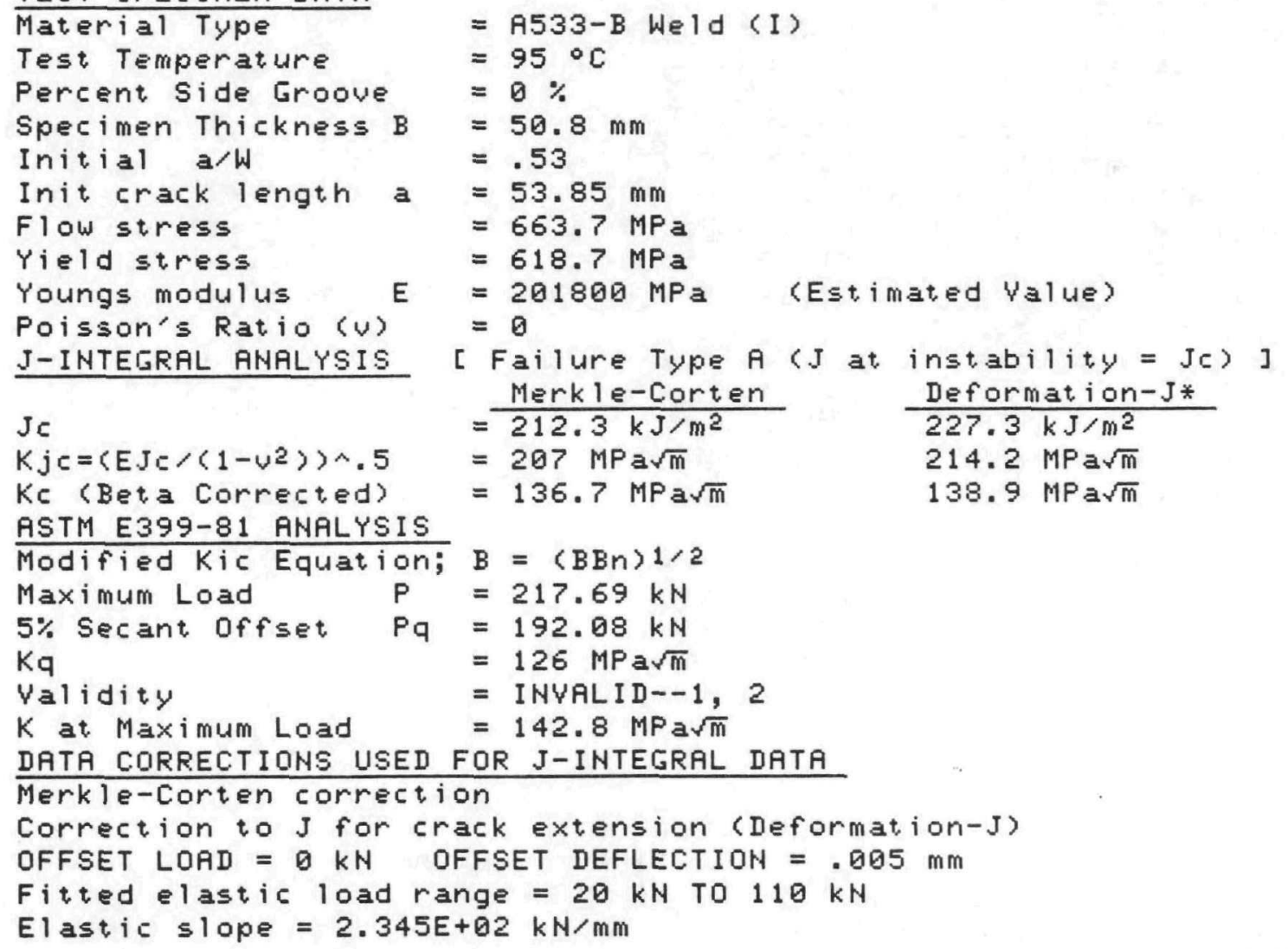


Specimen Loadline Deflection (in.)

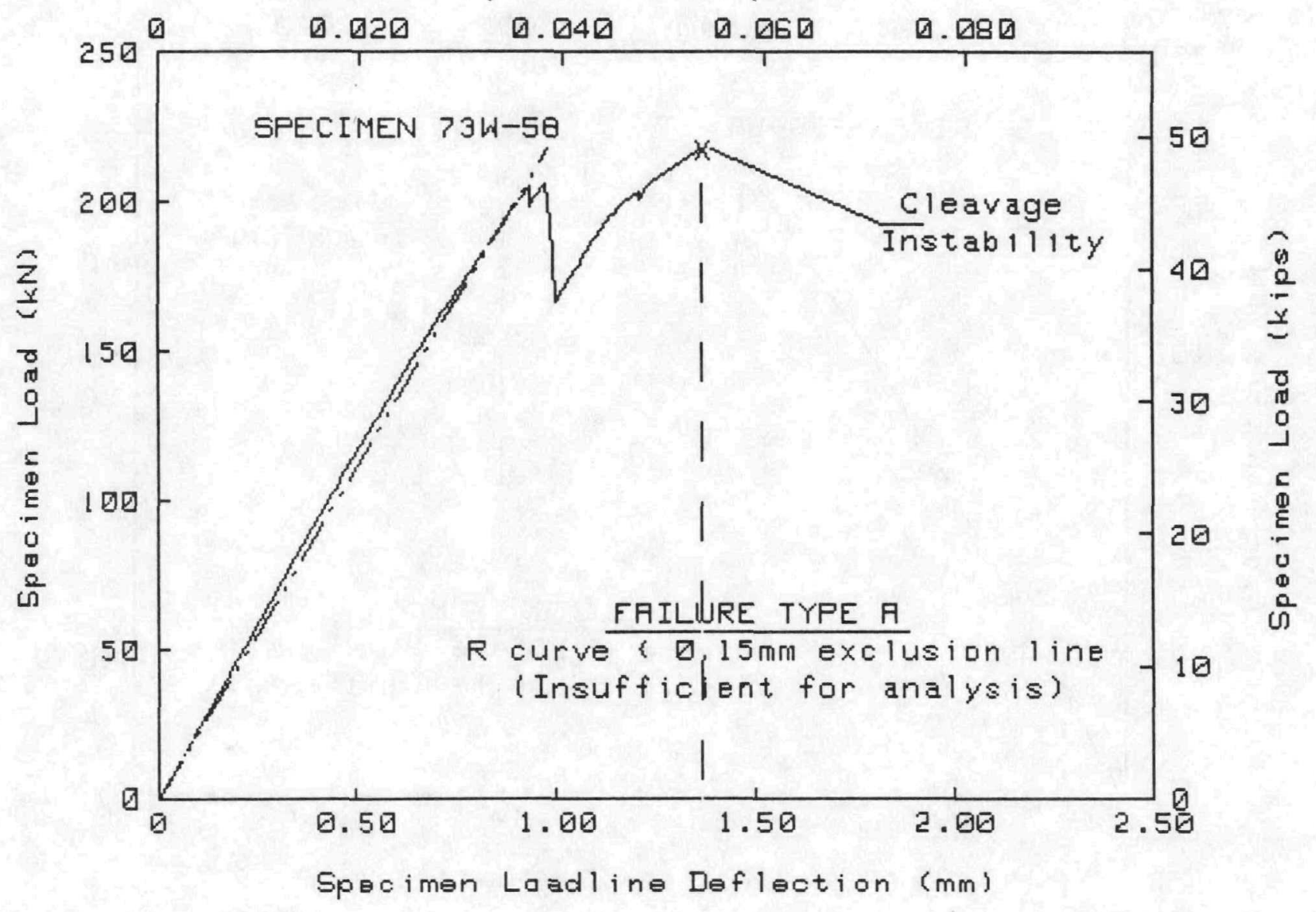

TEST SPECIMEN DATA

Material Type
Test Temperature
Percent Side Groove
Specimen Thickness B
Initial a/W
Init crack length a
Flow stress
Yield stress
Youngs modulus
Poisson's Ratio (U)
J-INTEGRAL ANALYSIS

$=A 533-B$ Weld (I)

$=95^{\circ} \mathrm{C}$

$=0 \%$

$=50.8 \mathrm{~mm}$

$=.53$

$=53.85 \mathrm{~mm}$

$=663.7 \mathrm{MPa}$

$=618.7 \mathrm{MPa}$

$=201800 \mathrm{MPa}$

$=0$

J-INTEGRAL ANALYSIS

$\mathrm{Jc}$

$K j c=\left\langle E J c /\left\langle 1-v^{2}\right\rangle\right\rangle \wedge .5$

Kc (Beta Corrected)

[ Failure Type A

$\begin{aligned}= & \text { Merkle-Corten } \\ = & 172 \mathrm{~kJ} / \mathrm{m}^{2} \\ = & 136.3 \mathrm{MPa} \sqrt{\mathrm{mPa}} \\ = & 13 \mathrm{~m}\end{aligned}$

(Est imated Value)

ASTM E399-81 ANALYSIS

Modified Kic Equation; $B=\langle B B n\rangle 1 / 2$

Maximum Load $P=217.69 \mathrm{kN}$

$5 \%$ Secant offset $\mathrm{Pq}=192.08 \mathrm{kN}$

$\mathrm{Kq}$

$=126 \mathrm{MPa} \sqrt{\mathrm{m}}$

Validity

$K$ at Maximum Load

= INYALID--1, 2, 3

$=142.8 \mathrm{MPa} \sqrt{\mathrm{m}}$

DATA CORRECTIONS USED FOR J-INTEGRAL DATA

Merkle-Corten correction

Correction to J for crack extension (Deformation-J)

OFFSET LOAD $=0 \mathrm{kN}$ OFFSET DEFLECTION $=.005 \mathrm{~mm}$

Fitted elastic load range $=20 \mathrm{kN}$ TO $110 \mathrm{kN}$

Elastic slope $=2.345 E+02 \mathrm{kN} / \mathrm{mm}$

NUREG/CR-5913 
Specimen Loadline Deflection (in.)

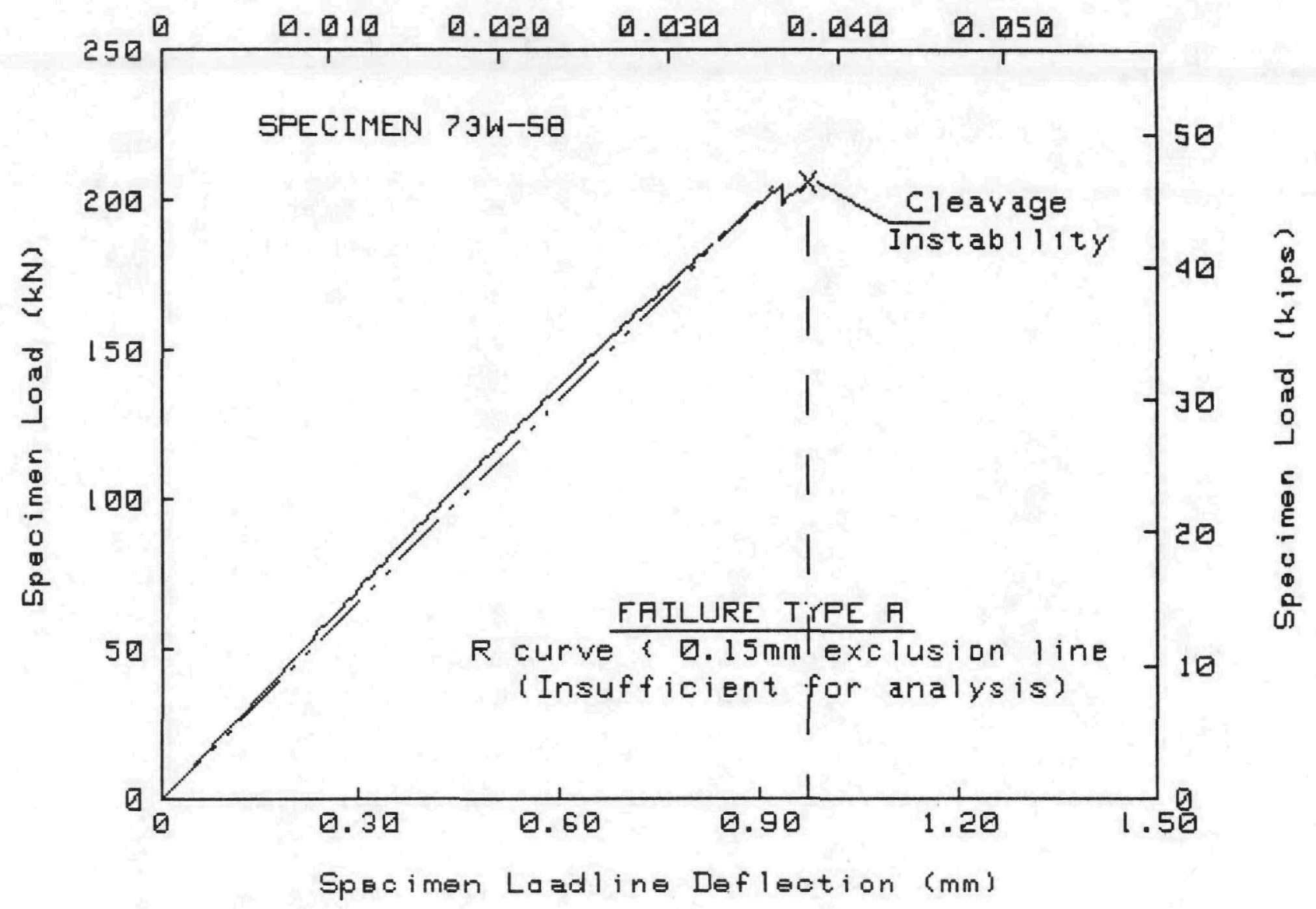

\section{TEST SPECIMEN DATA}

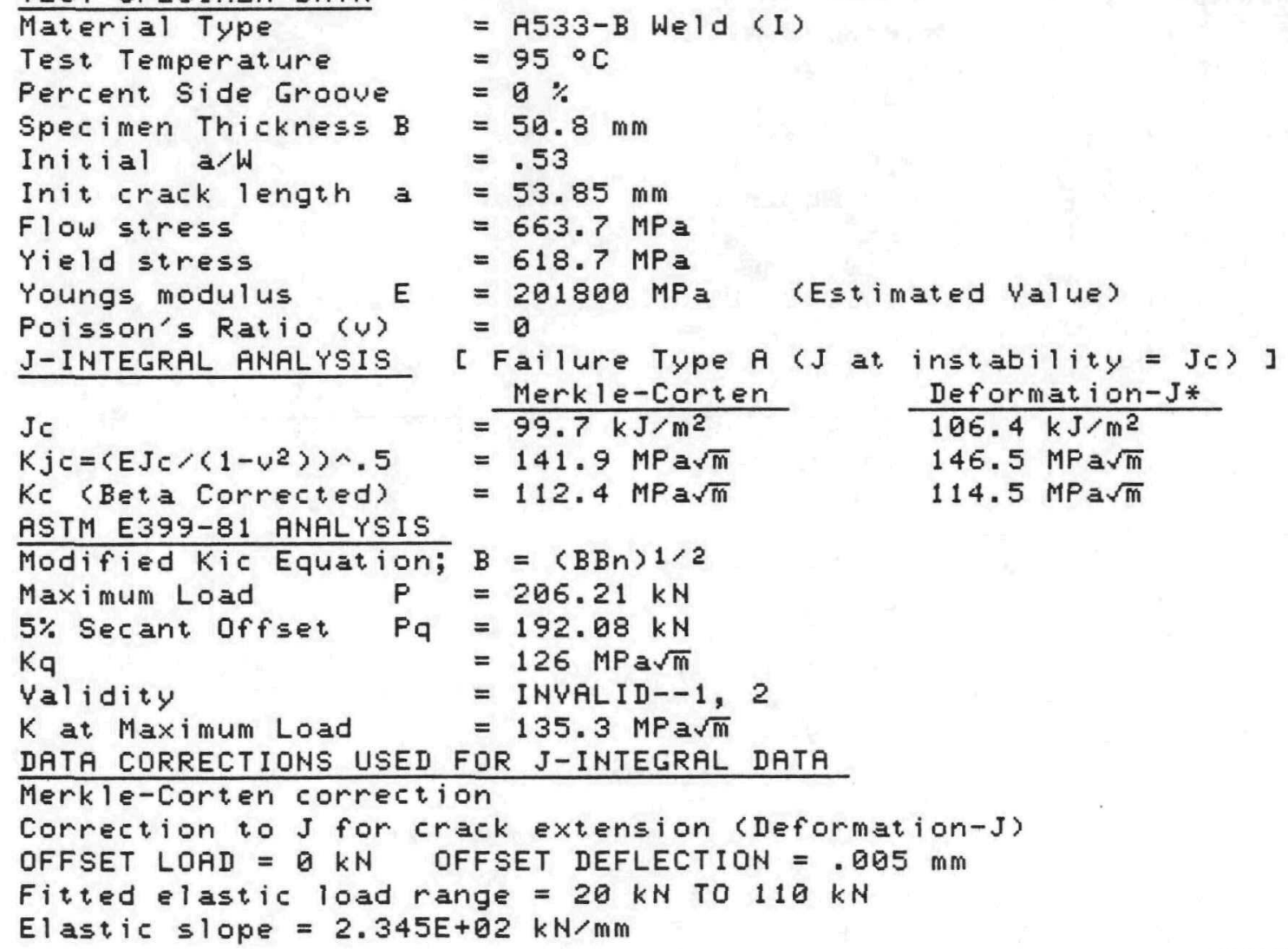


Specimen Loadline Deflection (in.)

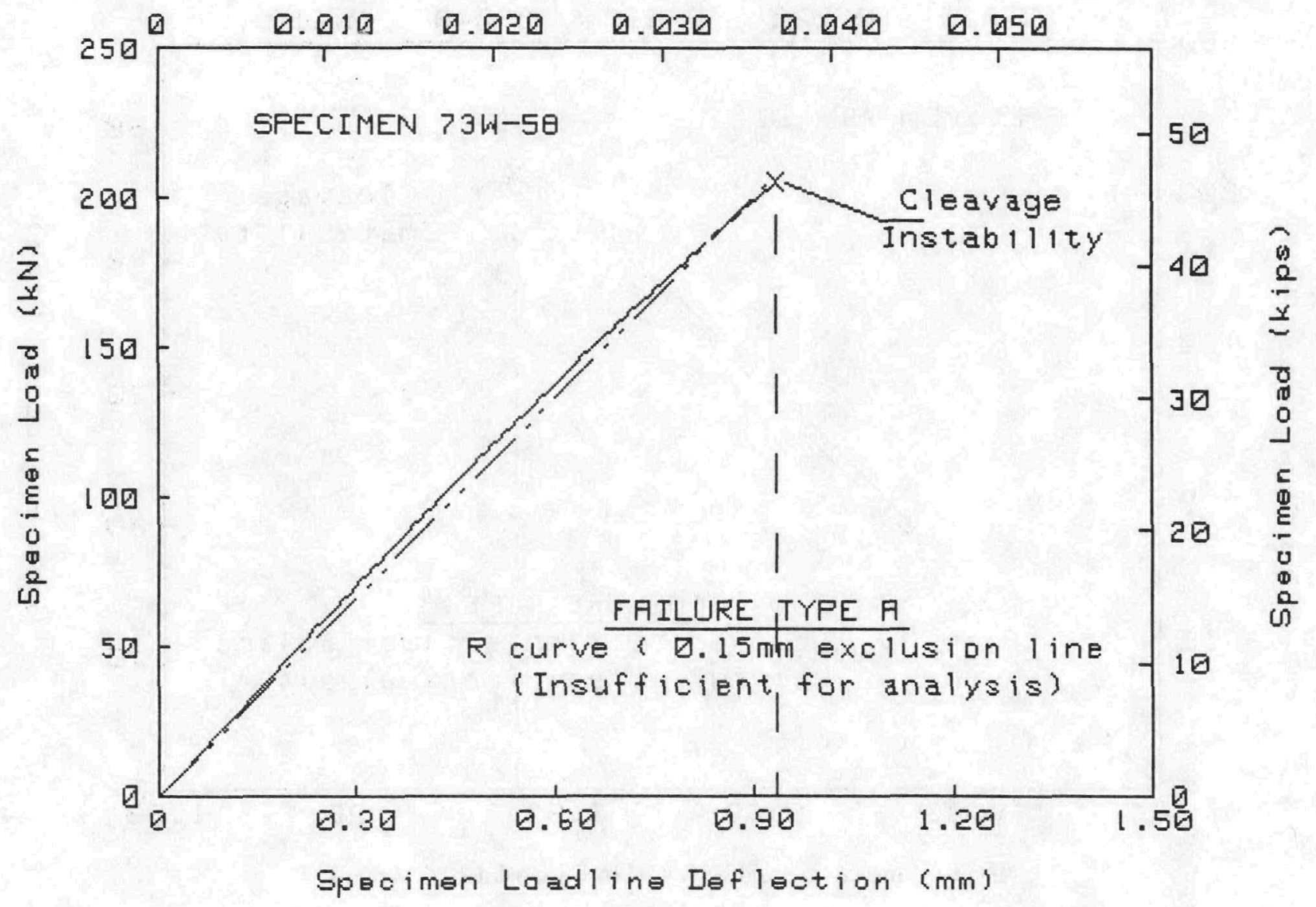

TEST SPECIMEN DATA

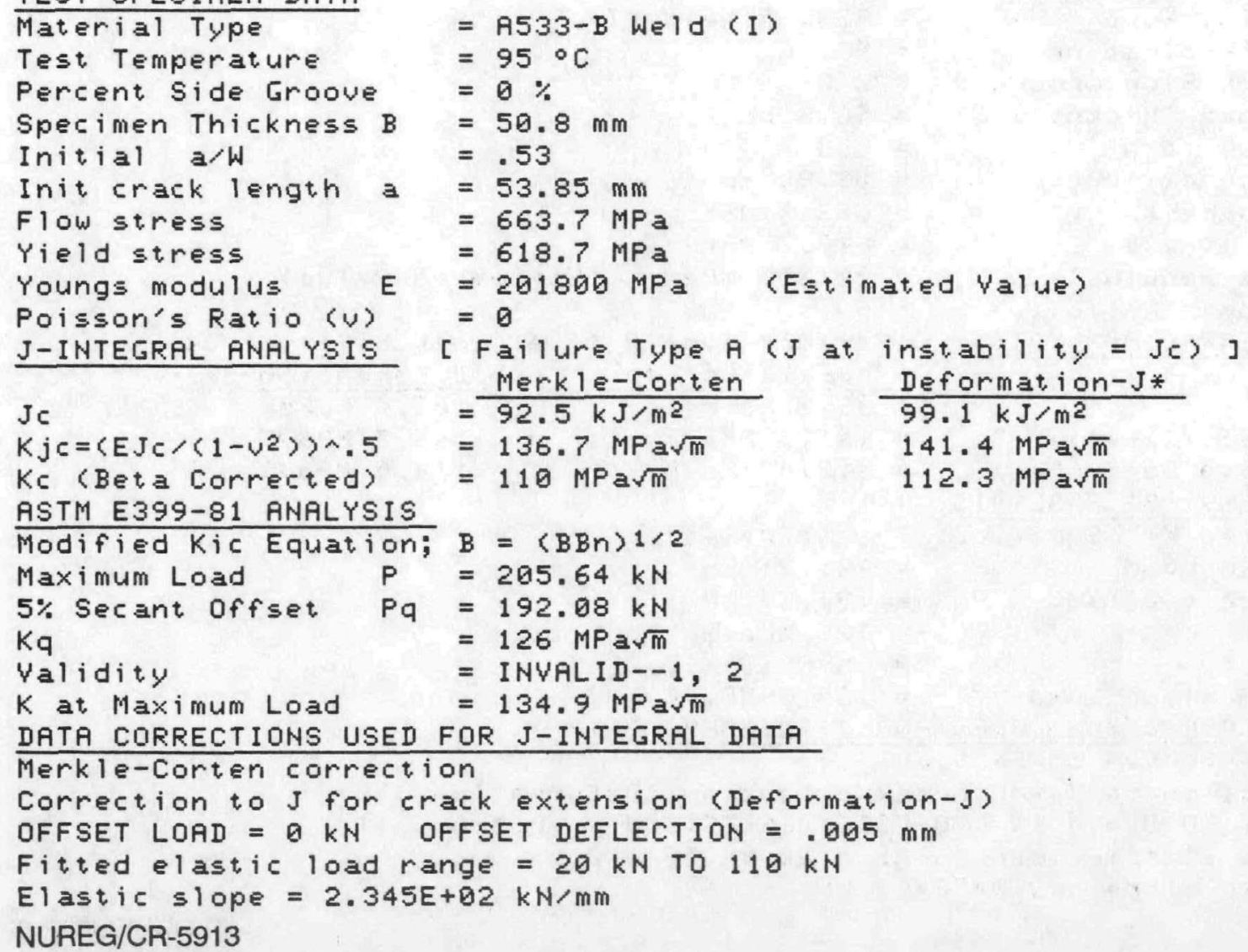


Specimen Crackmouth Deflection 〔in.)

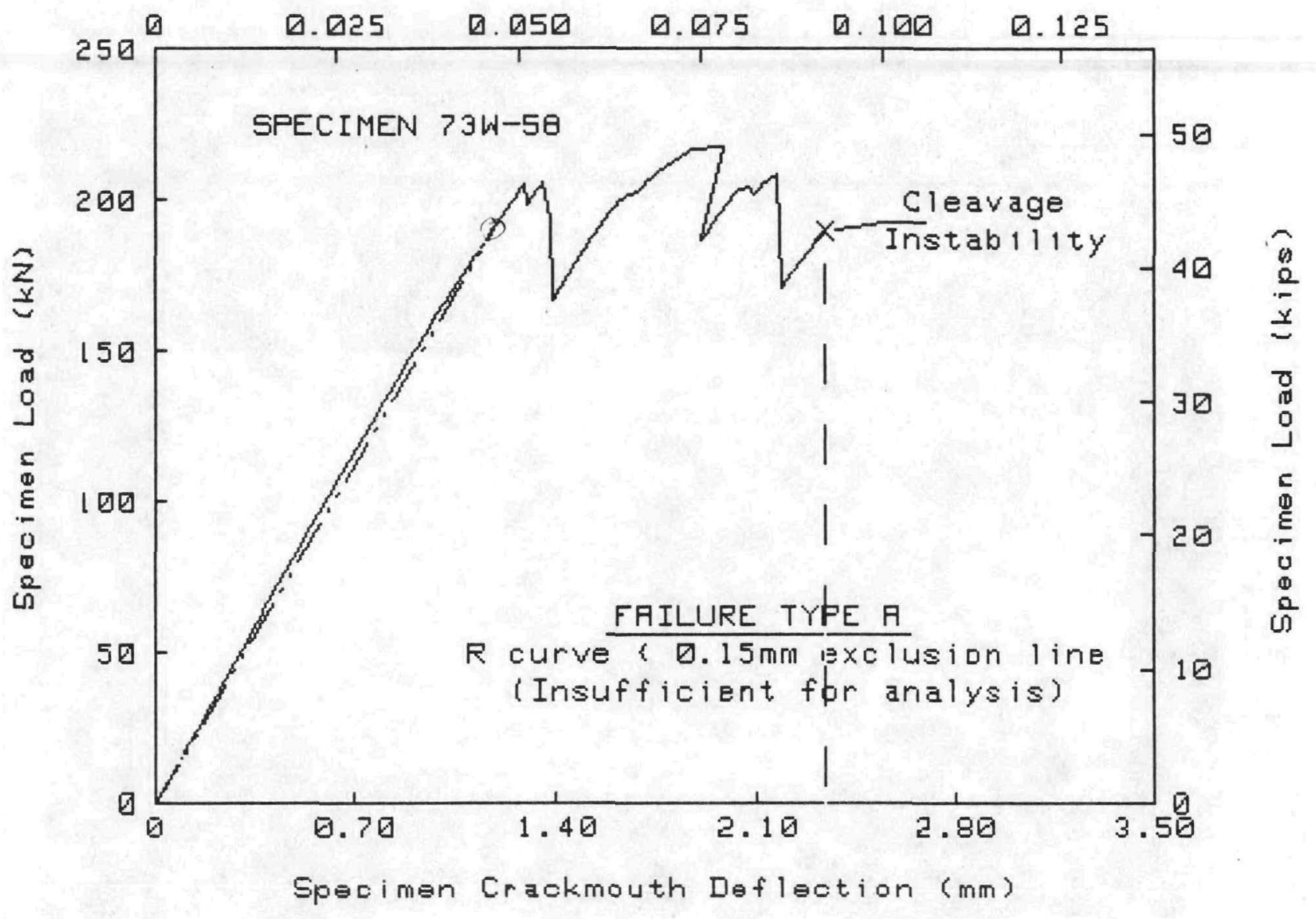

TEST SPECIMEN DATA

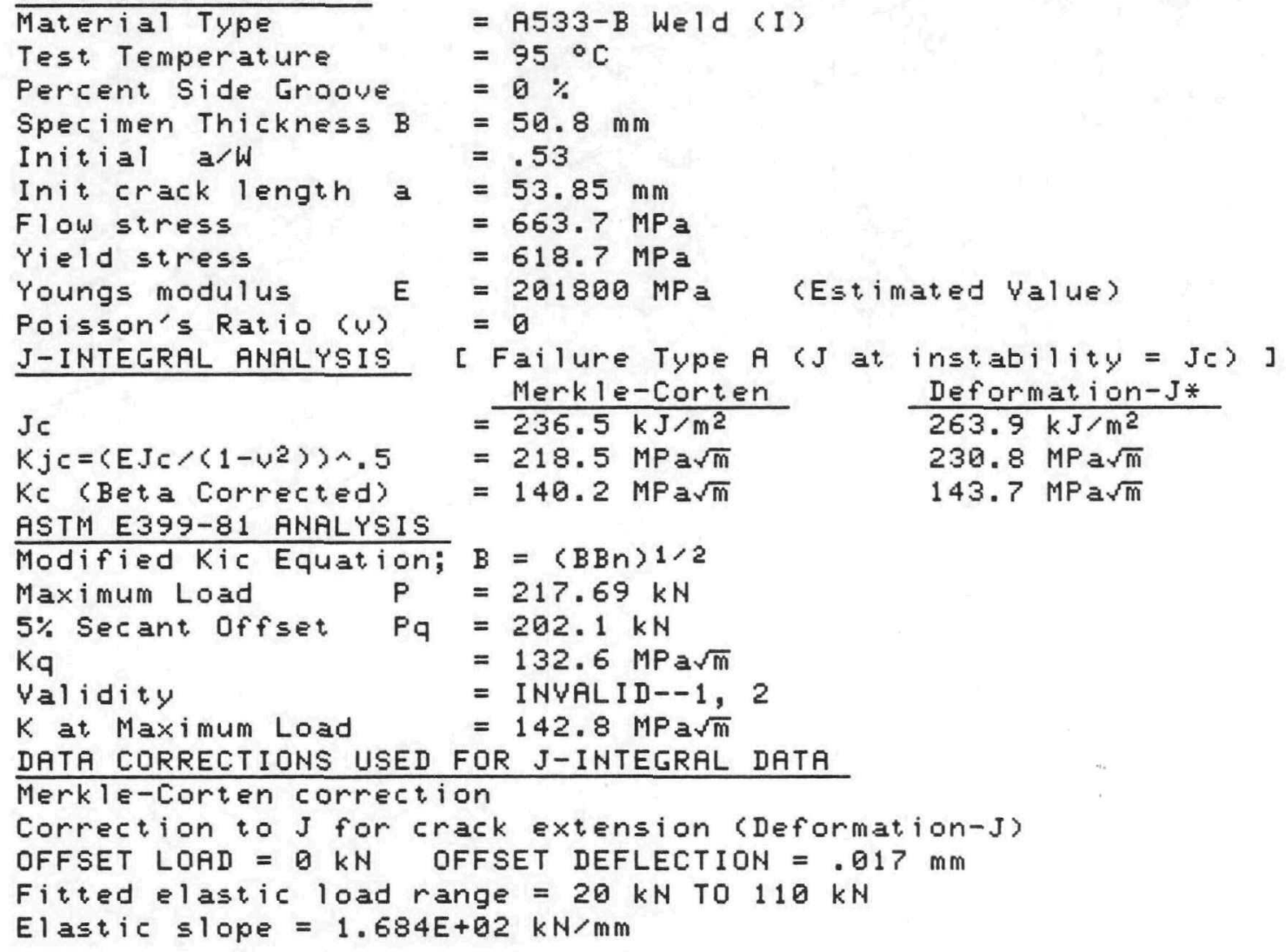




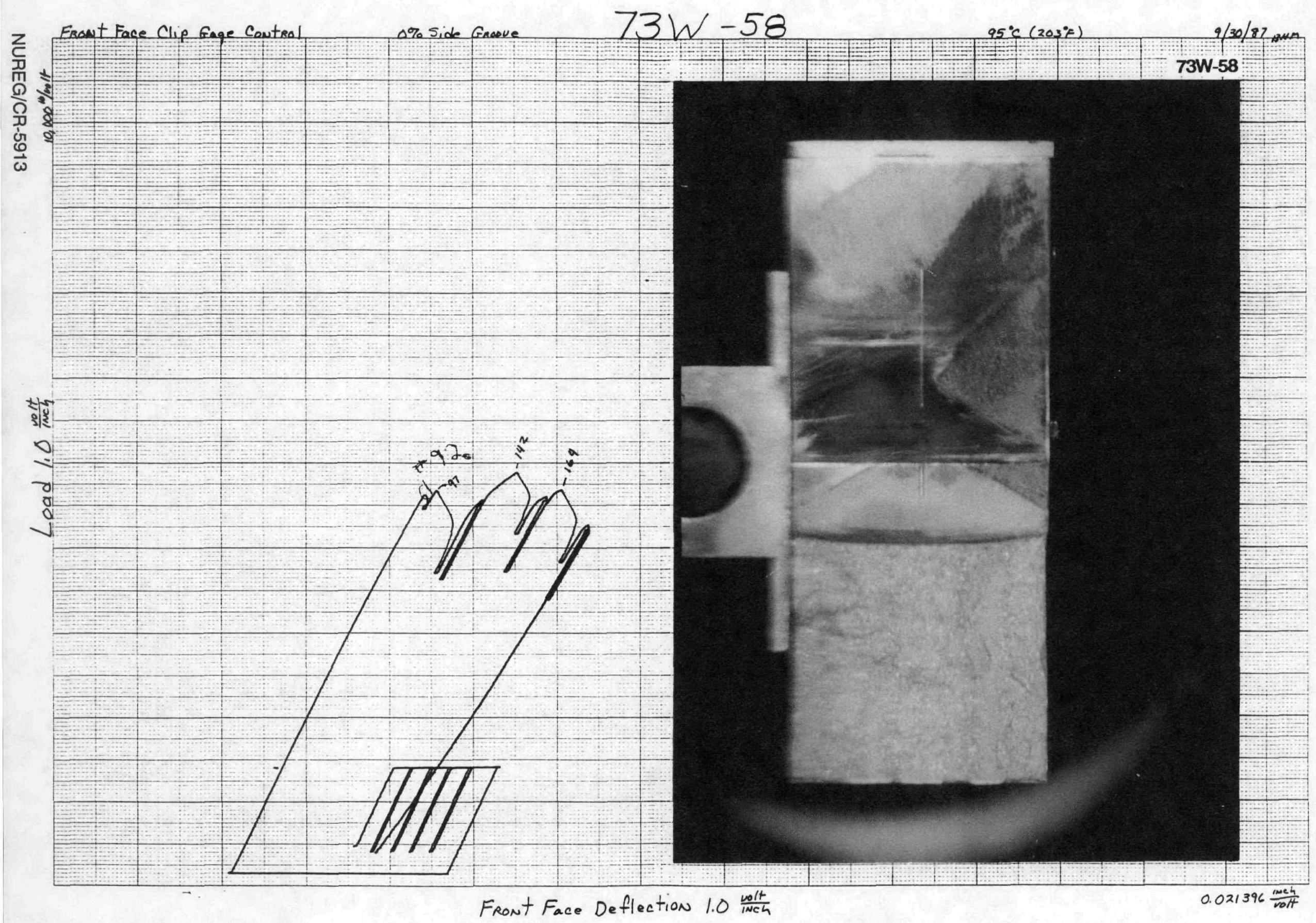


ORNL-DWG 92-11458

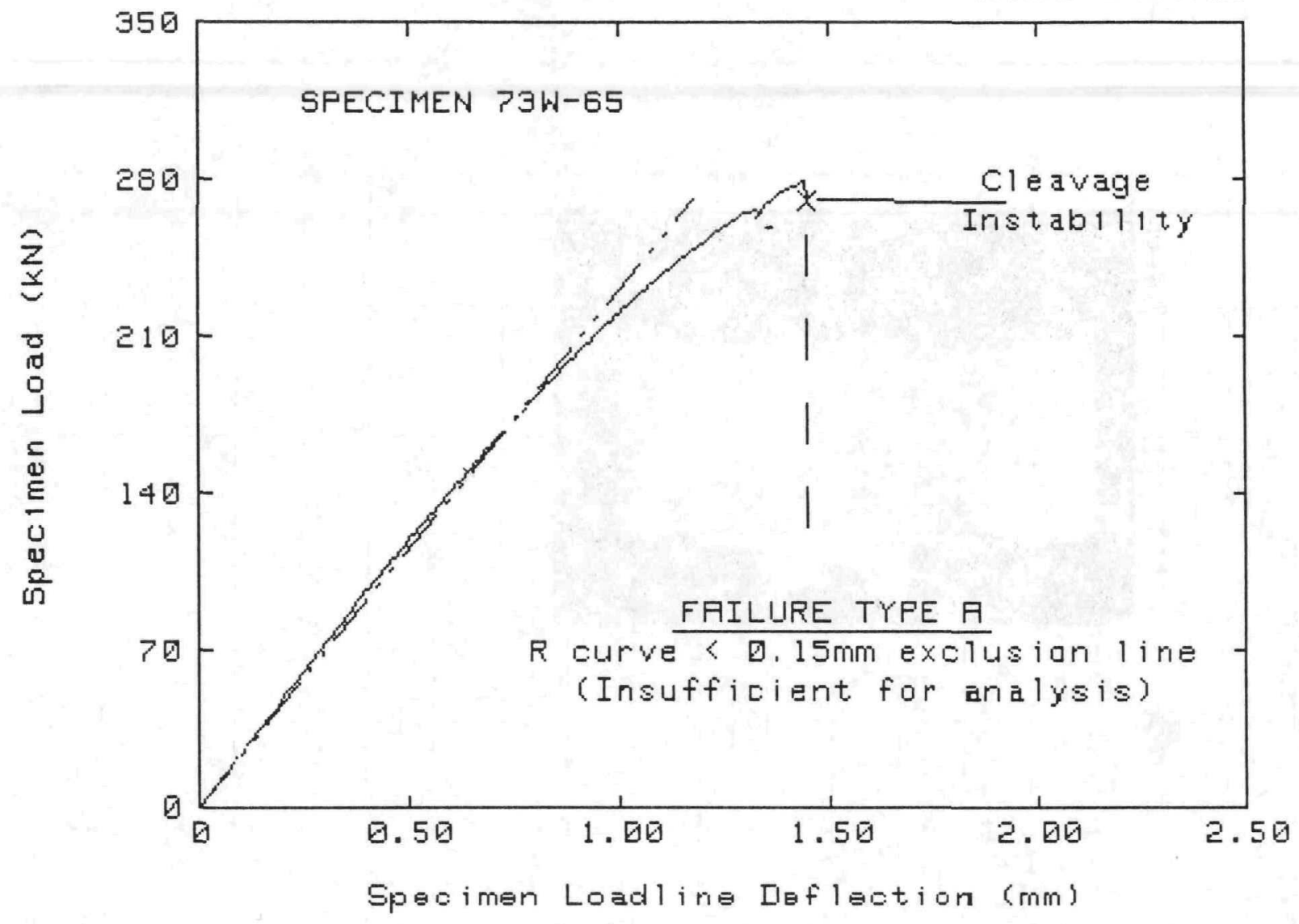

TEST SPECIMEN DATA

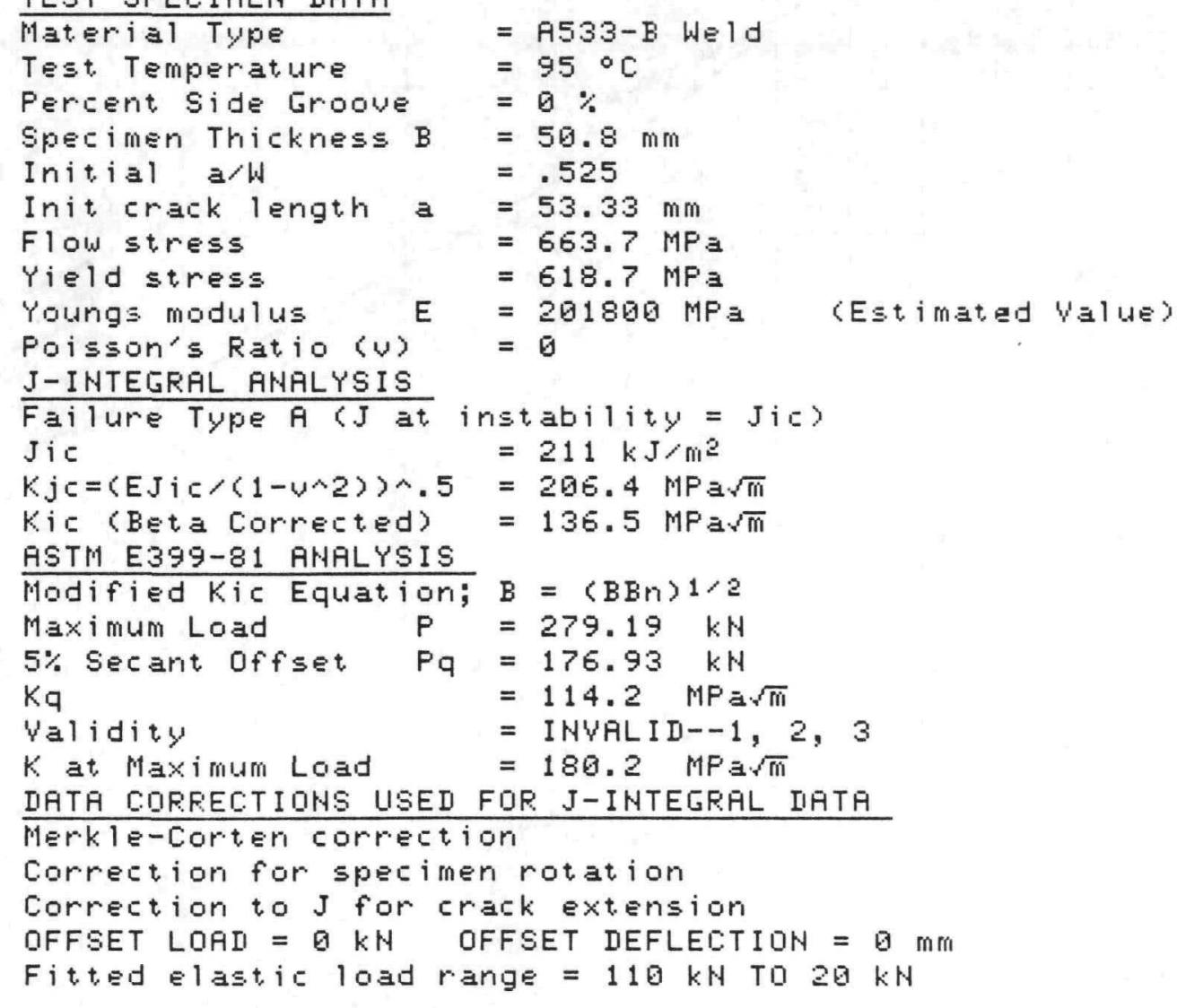




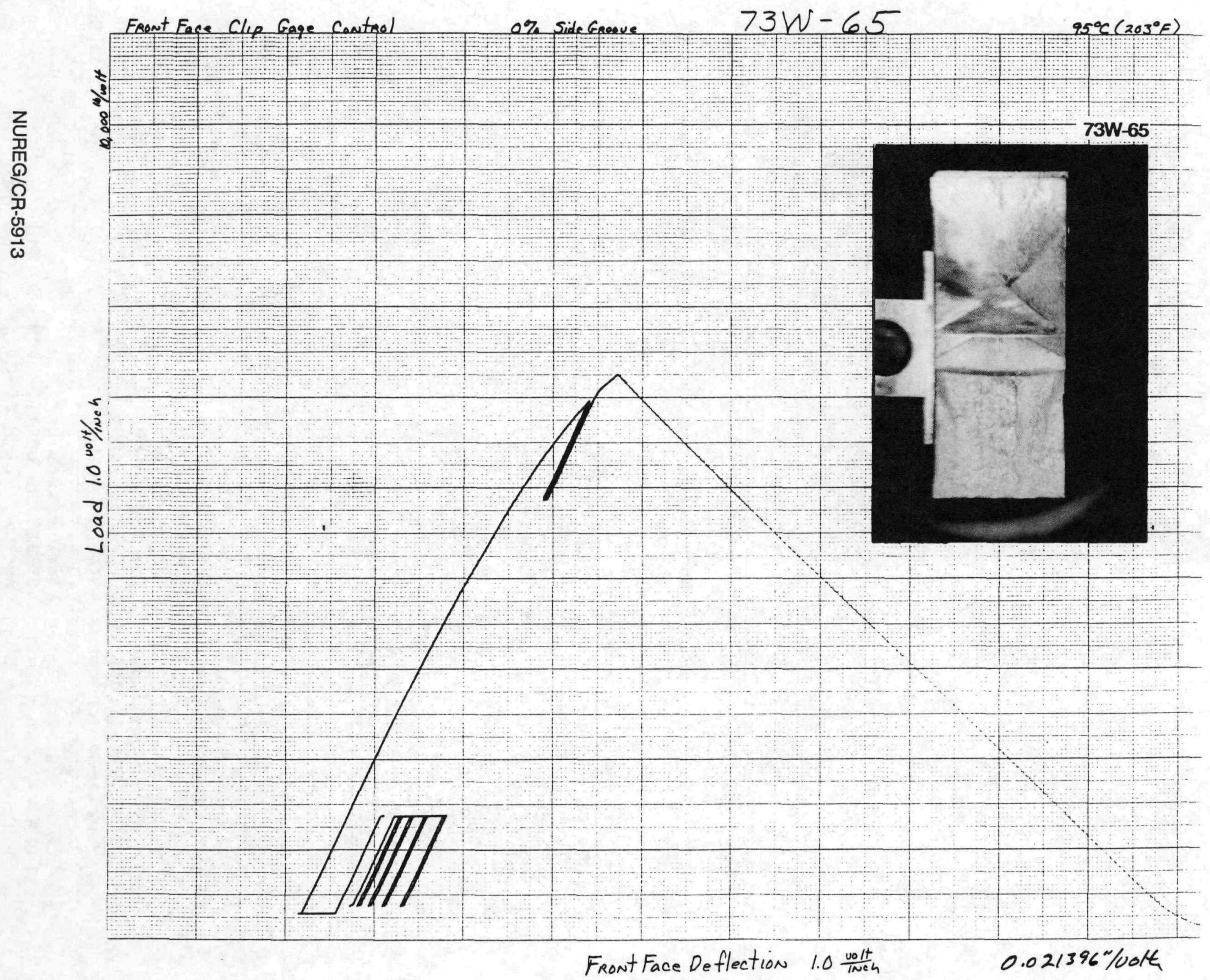




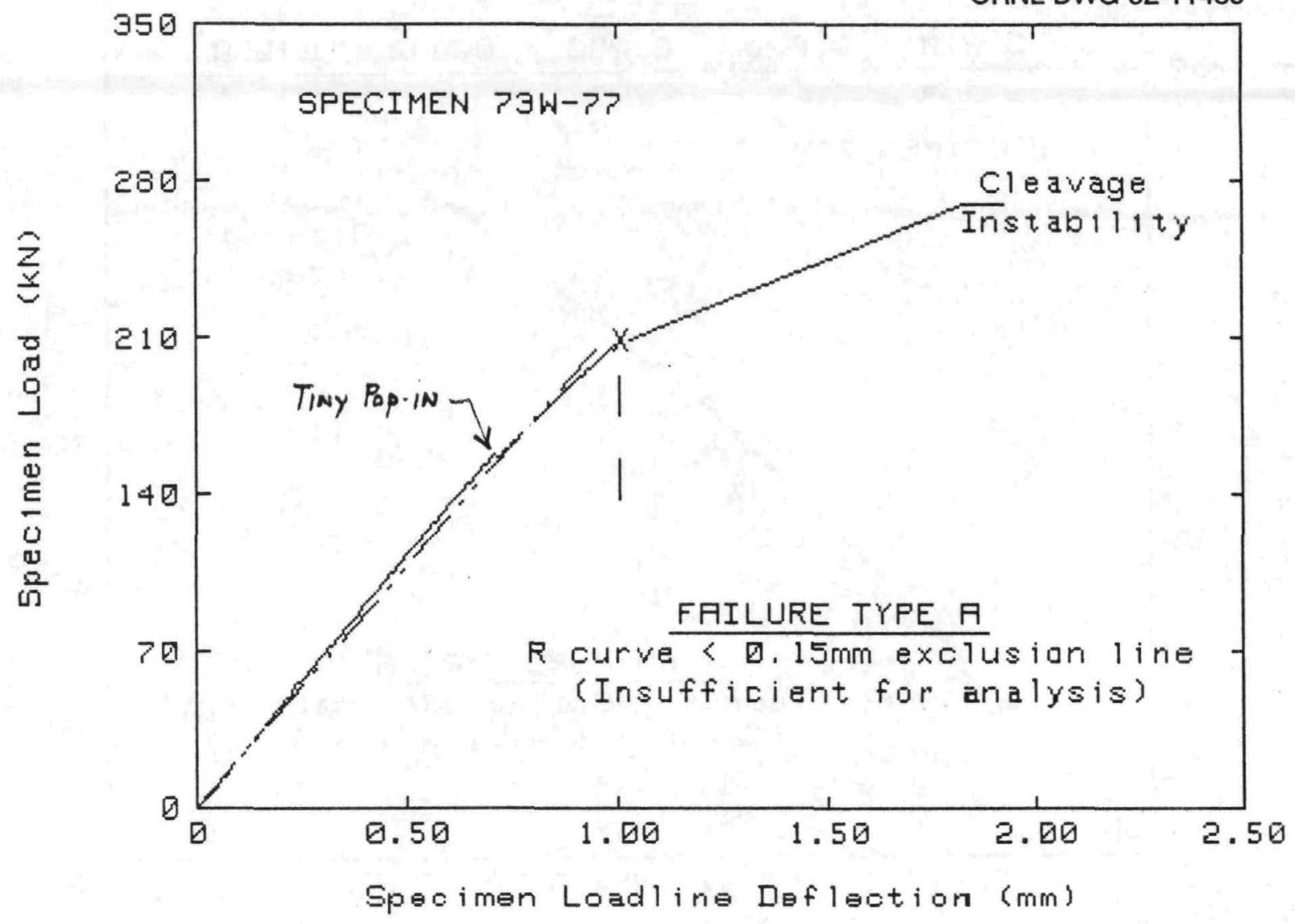

TEST SPECIMEN DATA

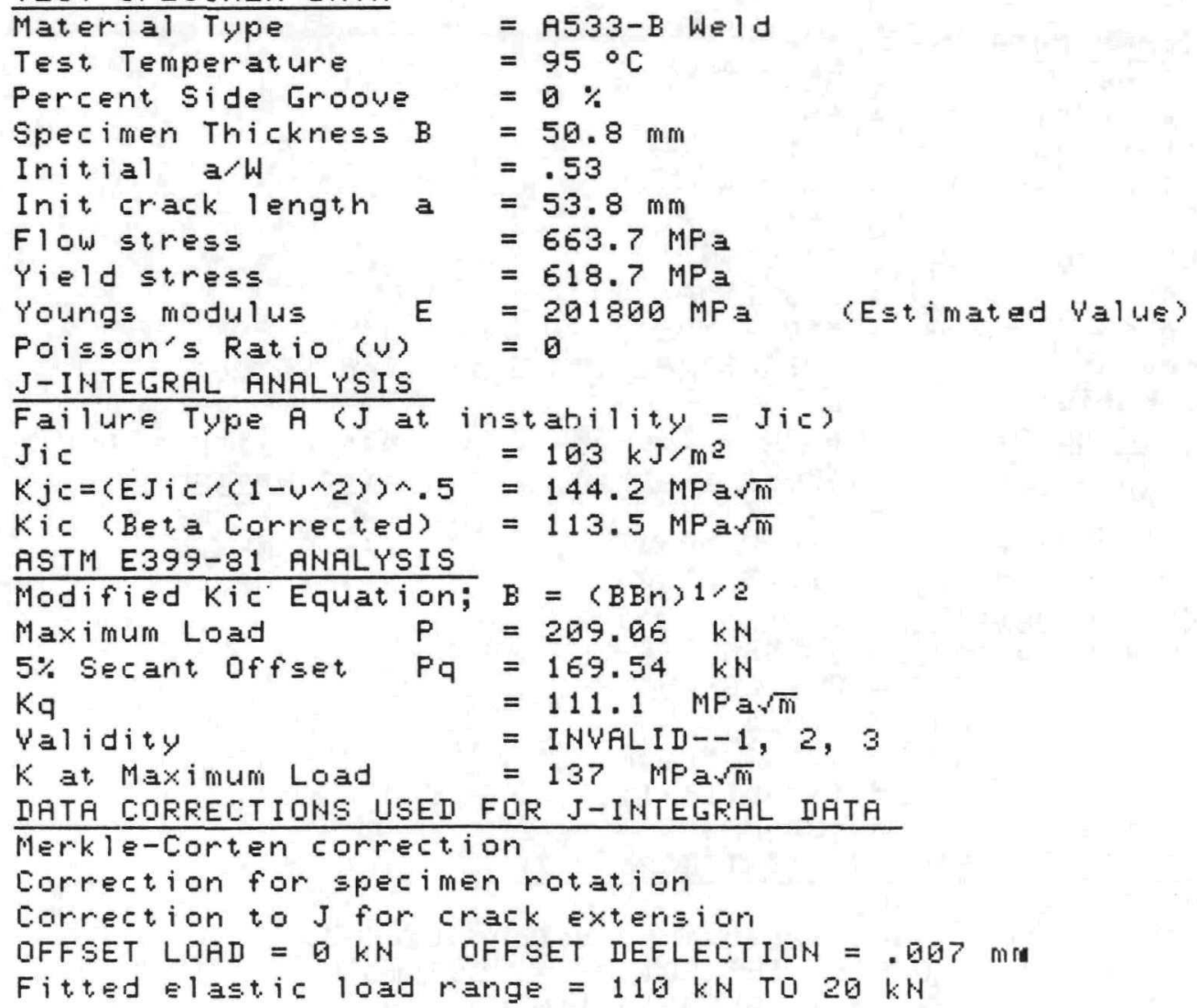


Specimen Loadline Deflection (in.)

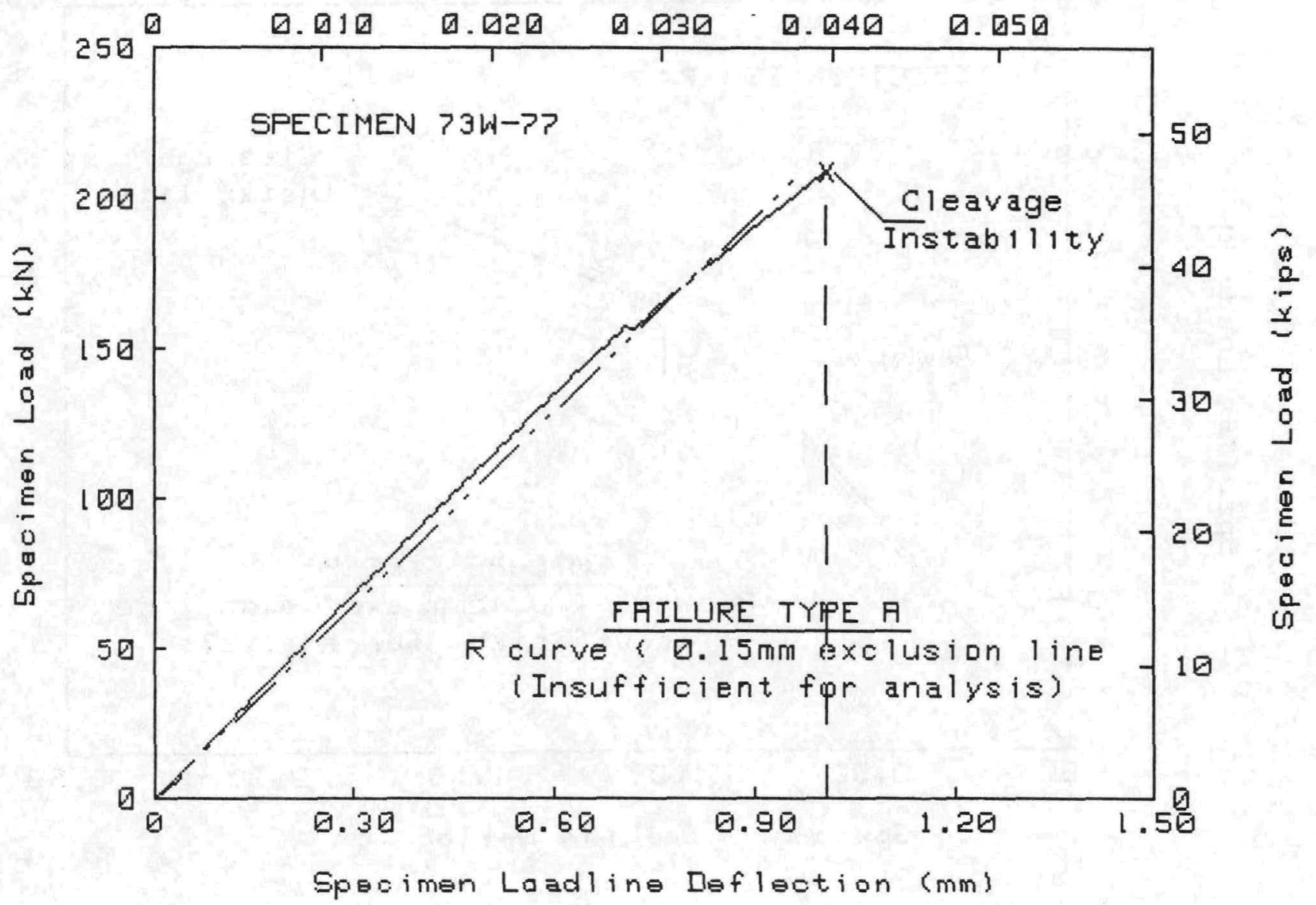

TEST SPECIMEN DATA

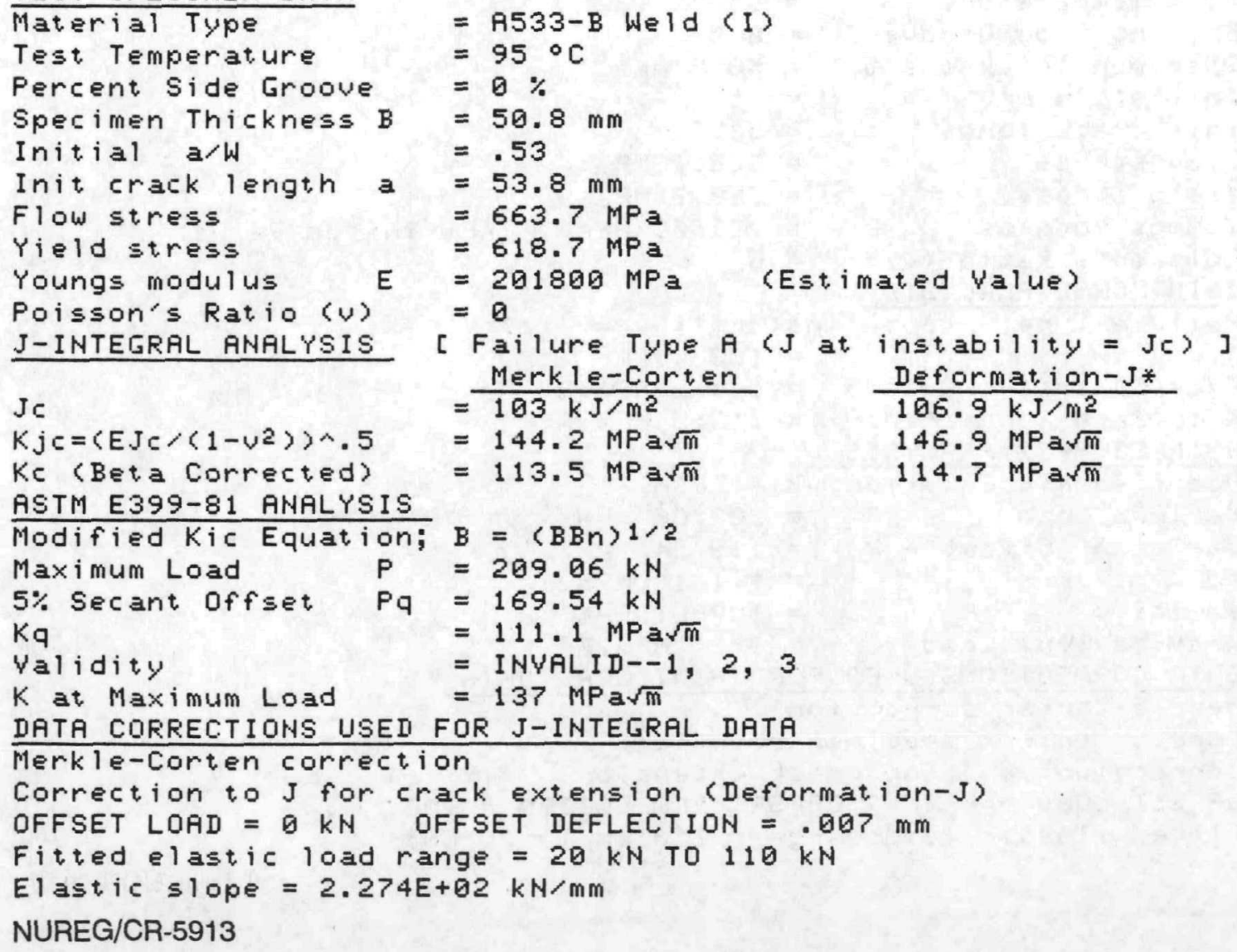


Specimen Loadline Deflectian (in.)

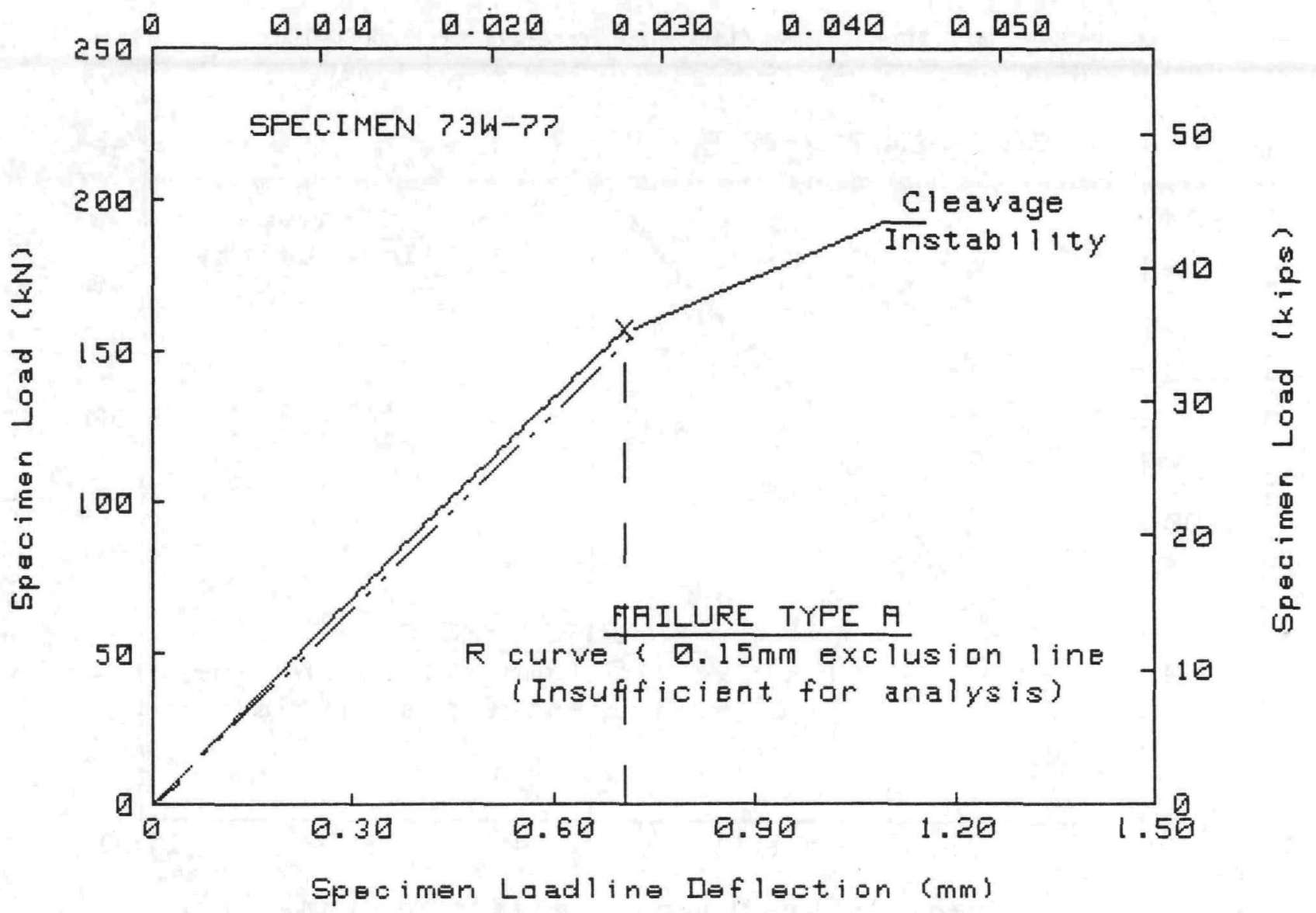

TEST SFECIMEN IATA

Material Type
Test Temperature
Percent Side Groove
Specimen Thickness B
Initial a/W
Init crack length a
Flow stress
Yield stress
Youngs modulus E
Poisson's Ratio 〈U〉
J-INTEGRAL ANALYSIS

$\mathrm{Je}$

$K j c=\left\langle E J c\left\langle\left(1-v^{2}\right\rangle\right\rangle \wedge .5\right.$

Ke (Beta Corrected)

ASTM E399-81 ANALYSIS

Modified Kic Equation; $B=\langle B B n\rangle 1 / 2$

Maximum Load $P=157.51 \mathrm{kN}$

$5 \%$ Secant offset $\mathrm{Pq}=157.51 \mathrm{kN}$

$\mathrm{Kq}$

Validity

$K$ at Maximum Load

$=103.2 \mathrm{MPa} \sqrt{\mathrm{m}}$

= INVALID--1, 2

$=103.2 \mathrm{MPa} \sqrt{\mathrm{m}}$

$=$ A533-B Weld (I)

$=95^{\circ} \mathrm{C}$

$=0 \%$

$=50.8 \mathrm{~mm}$

$=.53$

$=663.7 \mathrm{MPa}$

$=618.7 \mathrm{MPa}$

$=201800 \mathrm{MPa}$ 〈Estimated Value〉

\begin{tabular}{|c|c|}
\hline $\begin{aligned} & \text { Merk le-Corten } \\
= & 52.2 \mathrm{~kJ} / \mathrm{m}^{2} \\
= & 102.6 \mathrm{MPa} \sqrt{\mathrm{m}} \\
= & 91.5 \mathrm{MPa} \sqrt{\mathrm{m}}\end{aligned}$ & $\begin{array}{l}\text { Deformation-J* } \\
54.4 \mathrm{~kJ} / \mathrm{m}^{2} \\
104.8 \mathrm{MPa} \sqrt{\mathrm{m}} \\
92.8 \mathrm{MPa} \sqrt{\mathrm{m}}\end{array}$ \\
\hline
\end{tabular}

FOR J-INTEGRAL DATA

Merkle-Corten correction

Correction to J for crack extension (Deformation-J)

OFFSET LOAD $=0 \mathrm{kN}$ OFFSET DEFLECTION $=.007 \mathrm{~mm}$

Fitted elastic load range $=20 \mathrm{kN}$ T0 $110 \mathrm{kN}$

Elastic slope $=2.274 E+02 \mathrm{kN} / \mathrm{mm}$ 
Specimen Crackmouth Deflection (in.)

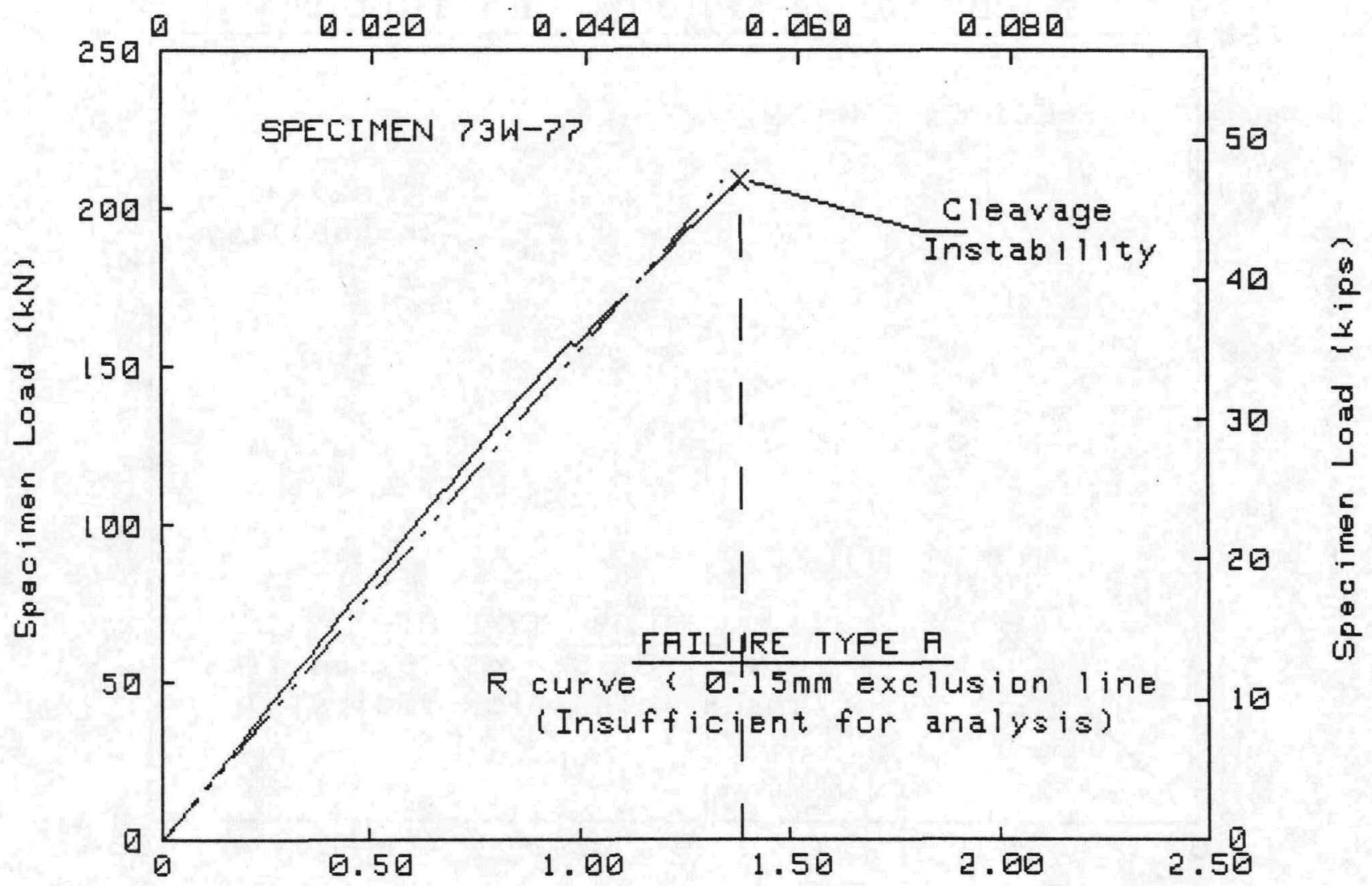

Specimen Crackmouth Defleotion ( $m m$ )

TEST SPECIMEN DATA

Material Type
Test Temperature
Percent Side Groove
Specimen Thickness B
Initial a/W
Init crack length a
Flow stress
Yield stress
Youngs modulus
Poisson's Ratio (U)
J-INTEGRAL ANALYSIS

$=$ A533-B Weld (I)

$=95^{\circ} \mathrm{C}$

$=0 \%$

$=50.8 \mathrm{~mm}$

$=.53$

$=53.8 \mathrm{~mm}$

$=663.7 \mathrm{MPa}$

$=618.7 \mathrm{MPa}$

$=201800 \mathrm{MPa}$

$=0$

J-INTEGRAL ANALYSIS

$\mathrm{Jc}$

$K j c=\left\langle E J c /\left(1-v^{2}\right\rangle\right) \wedge .5$

Ke (Beta Corrected)

[ Failure Type A ( $J$ at instability = Jc) ]

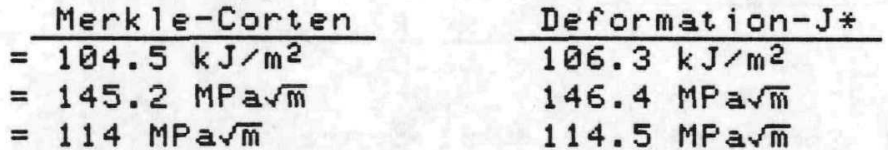

ASTM E399-81 ANALYSIS

Modified Kic Equation; $B=\langle B B n\rangle 1 / 2$

Maximum Load $P=209.06 \mathrm{kN}$

$5 \%$ Secant offset $\mathrm{Pq}=173.14 \mathrm{kN}$

$\mathrm{Kq}$

$=113.4 \mathrm{MPa} \sqrt{\mathrm{m}}$

= INVALID--1, 2, 3

Validity

$K$ at Maximum Load

$=137 \mathrm{MF} a \sqrt{\mathrm{m}}$

DATA CORRECTIONS USED FOR J-INTEGRAL DATA

Merkle-Corten correction

Correction to J for crack extension (Deformation-J)

OFFSET LOAD $=0 \mathrm{kN}$ OFFSET DEFLECTION $=.012 \mathrm{~mm}$

Fitted elastic load range $=20 \mathrm{kN}$ TO $110 \mathrm{kN}$

Elastic slope $=1.643 E+02 \mathrm{kN} / \mathrm{mm}$

NUREG/CR-5913 


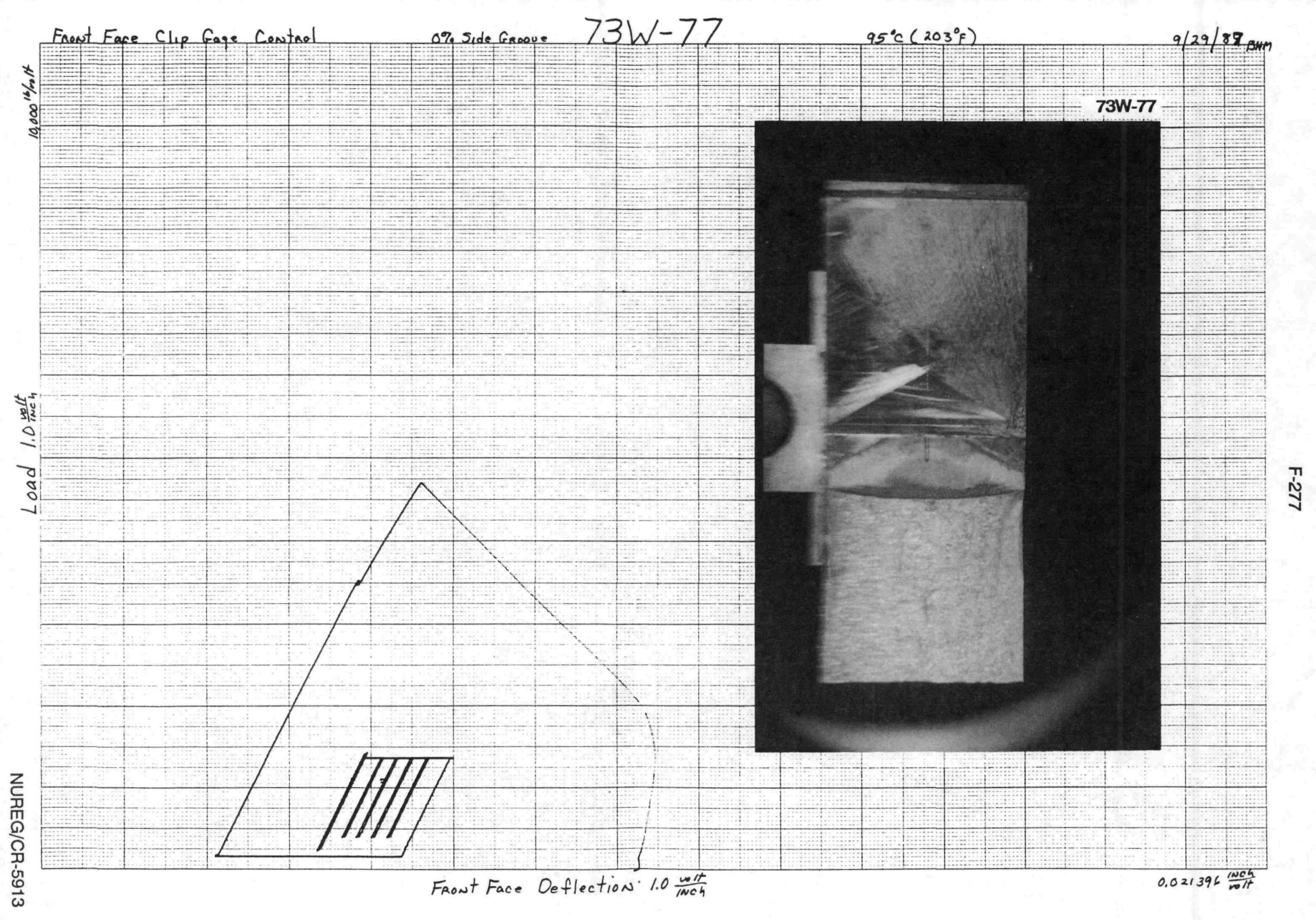


ORNL-DWG 92-11463

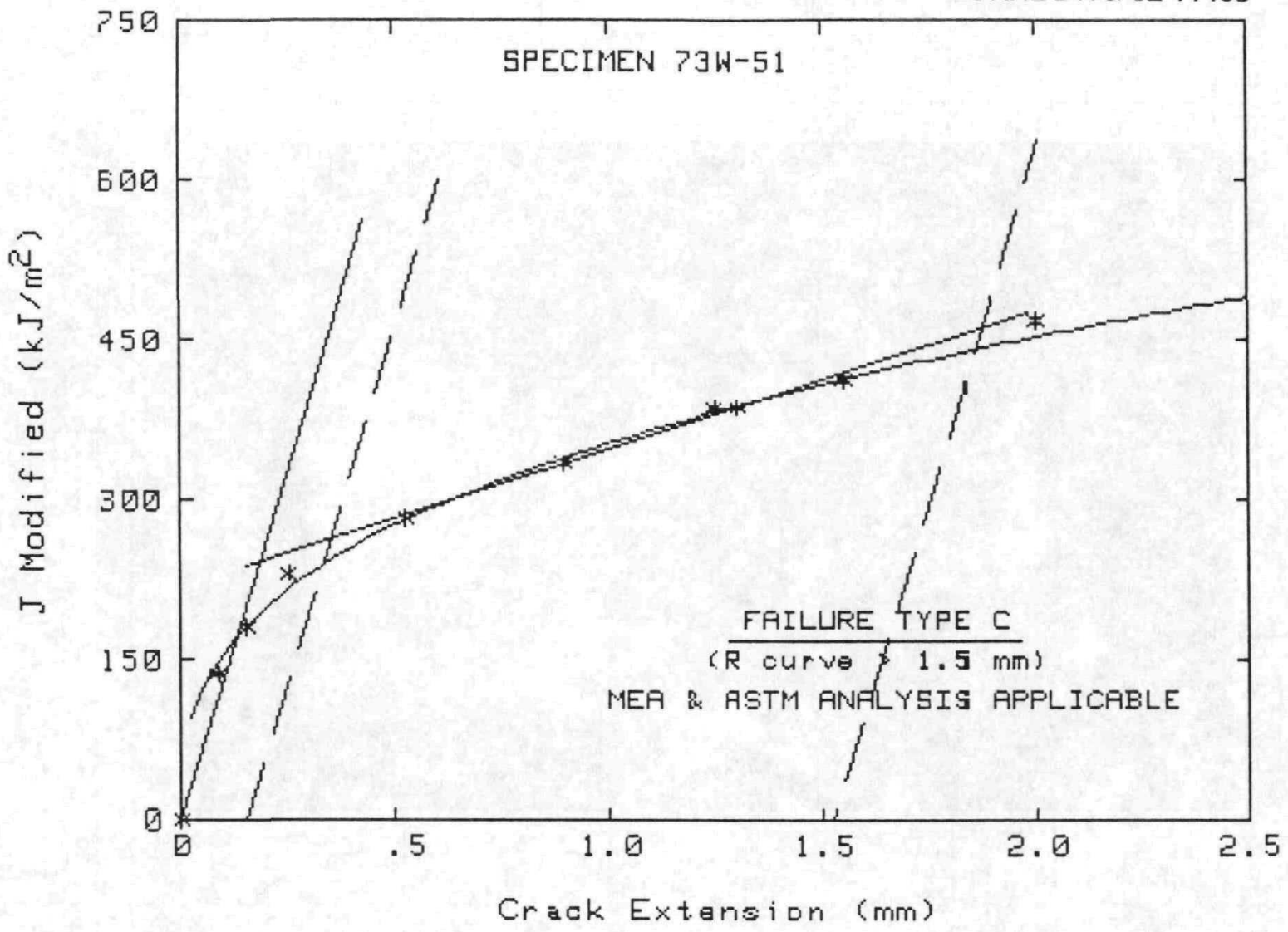

\section{TEST SPECIMEN IATA}

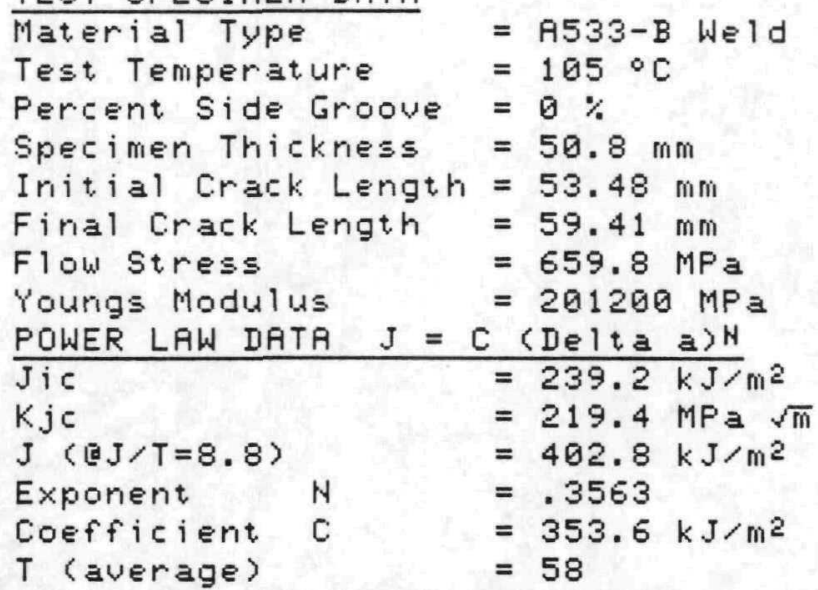

$\begin{array}{ll}\text { LEAST SQUARE LIHEAR LINE (ASTM) J } & =M \text { (DElta } a \text { ) }+B \\ \mathrm{Jic} & =241.2 \mathrm{~kJ} / \mathrm{m}^{2}\end{array}$

Kje

Slope $M=130230.1 \mathrm{~kJ} / \mathrm{m}^{3}$

Intercept B $\quad=217.4 \mathrm{~kJ} / \mathrm{m}^{2}$

$T$ (ASTM) $\quad=60$

Validity (Jic) = INVALID--c (1.83 Us .88)

Validity (R-curve) = INVALID--3 (1.83 Us . . 2 )

$\mathrm{J}$ maximum allowed $=1586.4 \mathrm{~kJ} / \mathrm{m}^{2}$ (Jmax=Bnet.*Flow stress/29)

Delta a max. allowed $=4.81 \mathrm{~mm}$ (Delta a max $=0.1$ *bo)

Final Delta a $\quad=4.1 \mathrm{~mm}$

Poisson's Ratio (u) $=0$

Points Left $=0 \quad$ Points Right $=0$ 


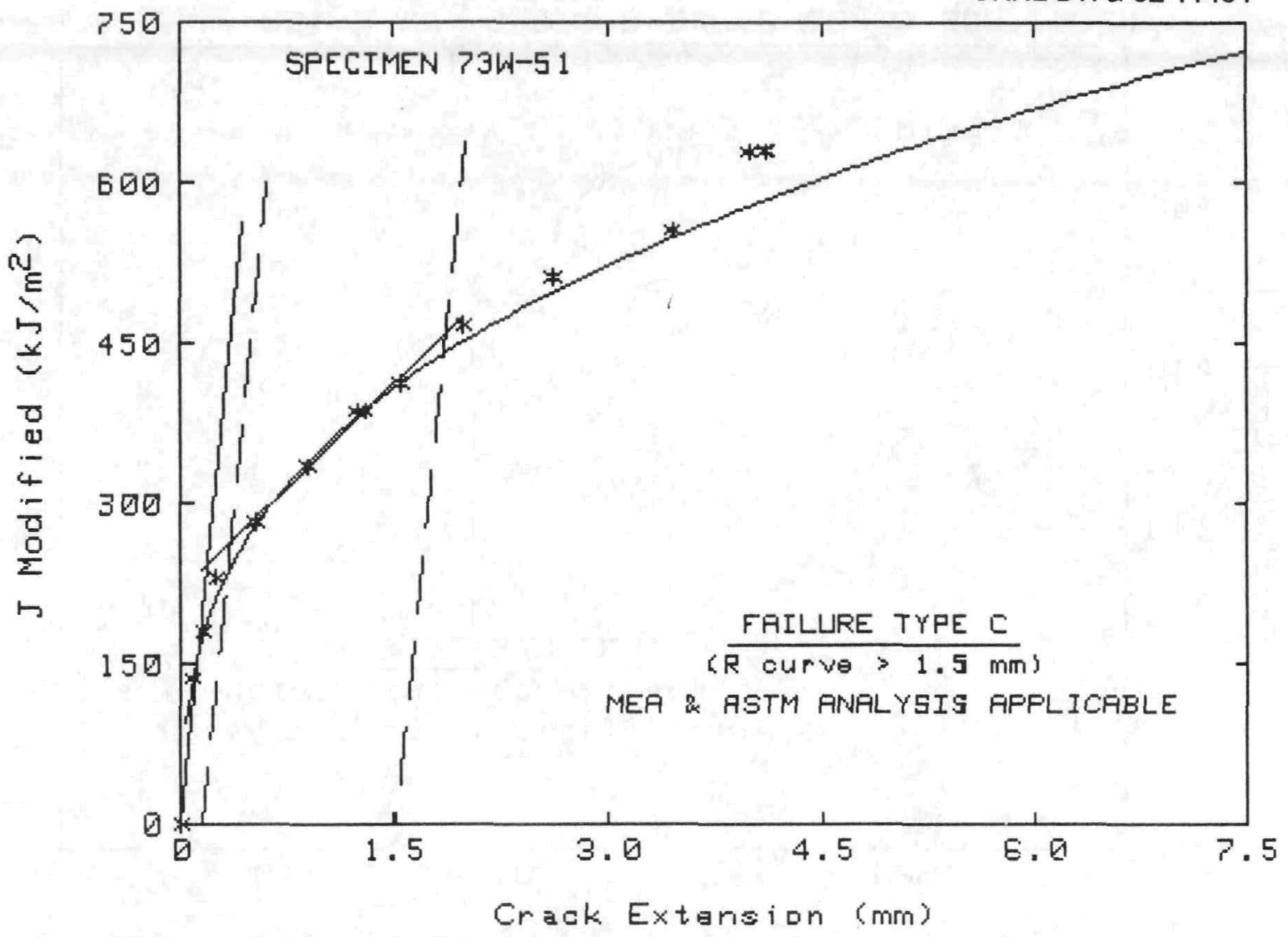

TEST SPECIMEN DATA

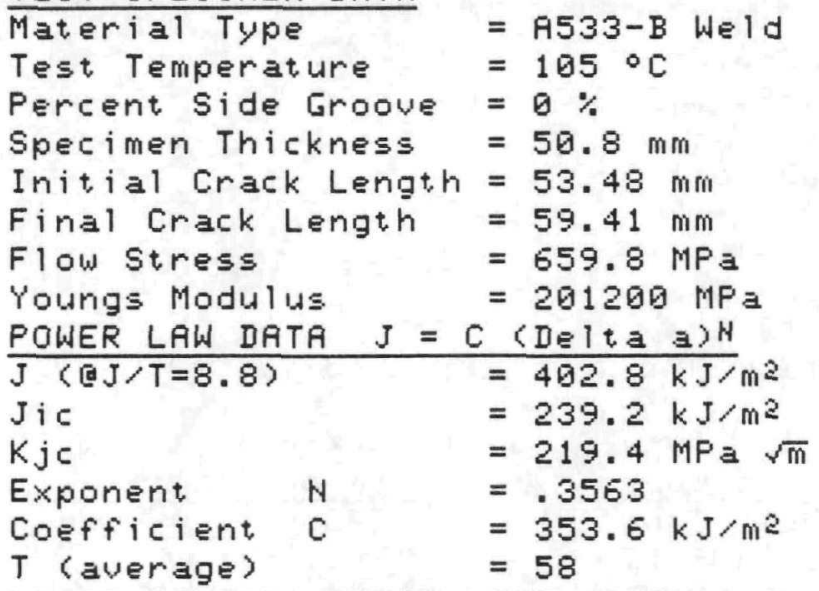

Init $a / W=.526$

Final $a / W=.584$

(Estimated Value)

$\begin{array}{ll}\text { LEAST SQUARE LINEAR LINE (ASTM) J } & \mathrm{J} \text { (DEIta } \mathrm{a}\rangle+\mathrm{B} \\ \mathrm{Jic} & =241.2 \mathrm{~kJ} / \mathrm{m}^{2}\end{array}$

$\mathrm{K} j \mathrm{C}$

Slope M

Intercept B

$T$ (ASTM)

$=220.3 \mathrm{MPa} \sqrt{\mathrm{m}}$

$=130230.1 \mathrm{~kJ} / \mathrm{m}^{3}$

Validity (Jic) = INVALID--C (1.83 vs.88)

Validity (R-curve) = INVALID--3 (1.83 vs .72)

J maximum allowed

$=1586.4 \mathrm{~kJ} / \mathrm{m}^{2}$

(Jmax=Bnet.Flow stress/20)

Delta a max. allowed $=4.81 \mathrm{~mm}$

(Delta a max $=0.1 *$ bo $)$

Final Delta a $=4.1 \mathrm{~mm}$

Poisson's Ratio $\langle u\rangle=0$

Points Left $=0 \quad$ Points Right $=0$ 
Specimen Loadline Deflection (in.)

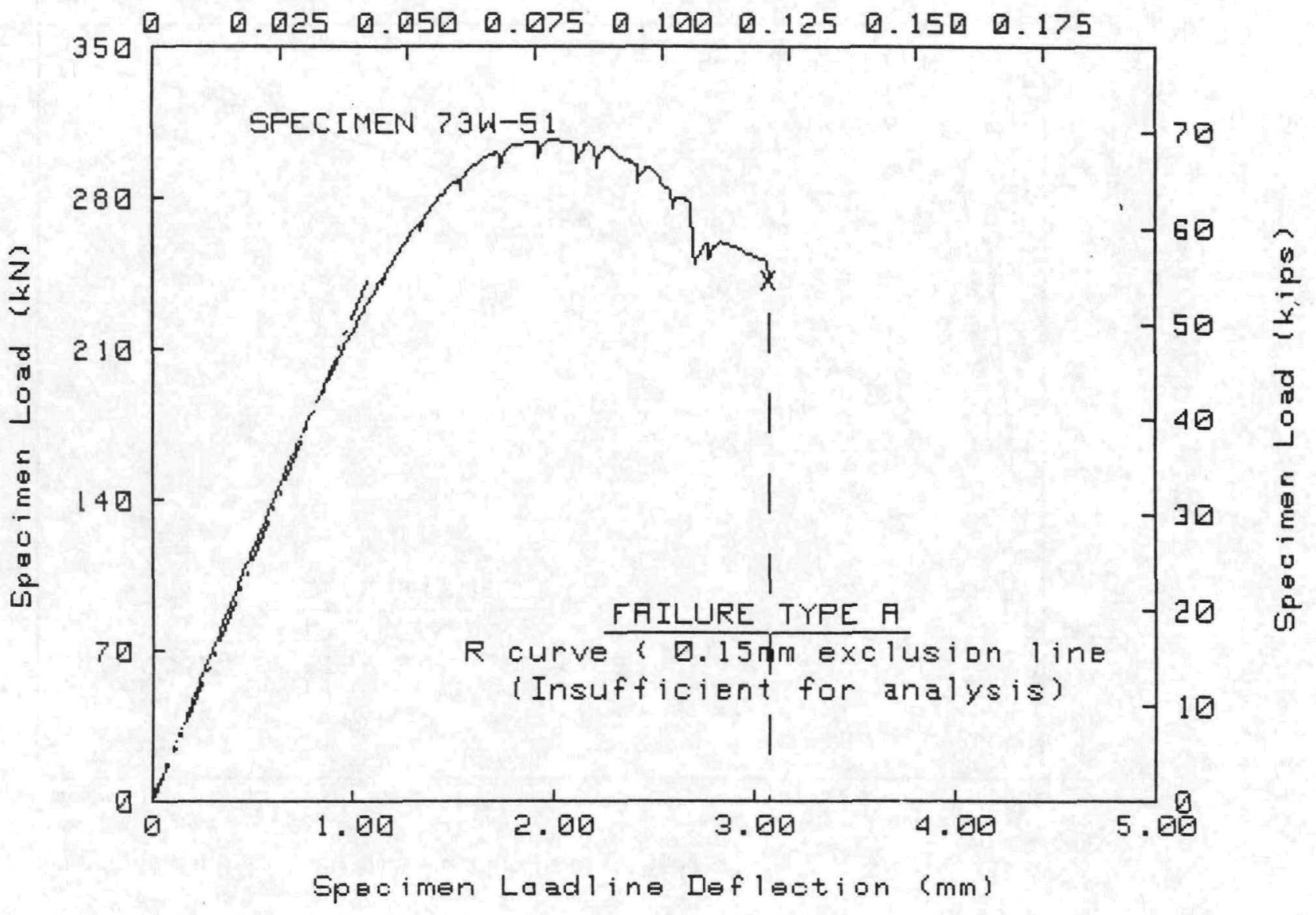

TEST SPECIMEN DATA

Material Type
Test Temperature
Percent Side Groove
Specimen Thickness B
Initial a/W
Init crack length a
Flow stress
Yield stress
Youngs modulus E
Poisson's Ratio (U)
J-INTEGRAL ANALYSIS

$\mathrm{Jc}$

$K j c=\left\langle E J c\left\langle\left(1-u^{2}\right\rangle\right\rangle \wedge .5\right.$

KC (Beta Corrected)

ASTM E399-81 RNALYSIS

Modified Kic Equation;

Maximum Load

$5 \%$ Secant offset.

$\mathrm{Kq}$

Validity

$K$ at Maximum Load

$P=307.85 \mathrm{kN}$

$\mathrm{Pq}=187.86 \mathrm{kN}$

$=121.8 \mathrm{MPa} \sqrt{\mathrm{m}}$

$=$ INYALID--1, 2, 3

$=199.6 \mathrm{MPa} \sqrt{\mathrm{m}}$
$=A 533-B$ Weld (I)

$=105^{\circ} \mathrm{C}$

$=0 \%$

$=50.8 \mathrm{~mm}$

$=.526$

$=53.48 \mathrm{~mm}$

$=659.8 \mathrm{MPa}$

$=615.2 \mathrm{MPa}$

$=201200 \mathrm{MPa}$ (Estimated Value)

$$
=0
$$

[ Failure Type A ( $J$ at instability = JC) ]

$=\frac{\text { Merkle-Corten }}{640.1 \mathrm{~kJ} / \mathrm{m}^{2}}$

$=358.9 \mathrm{MPa} \sqrt{\mathrm{m}}$

$=172.3 \mathrm{MPa} \sqrt{\mathrm{m}}$ $\frac{\text { Deformation }-\mathrm{J} *}{723.6 \mathrm{~kJ} / \mathrm{m}^{2}}$

$381.6 \mathrm{MPa} \sqrt{\mathrm{m}}$

$176.5 \mathrm{MPa} \sqrt{\mathrm{m}}$

DATA CORRECTIONS USED FOR J-INTEGRAL DATA

Merkle-Corten correction

Correction to J for crack extension (Deformation-J)

OFFSET LDAD $=0 \mathrm{kN}$ OFFSET DEFLECTIDN $=.003 \mathrm{~mm}$

Fitted elastic load range $=20 \mathrm{kN} \mathrm{T0} 110 \mathrm{kN}$

Elastic slope $=2.380 E+02 \mathrm{kN} / \mathrm{mm}$ 
Specimen Crackmouth Deflection (in.)

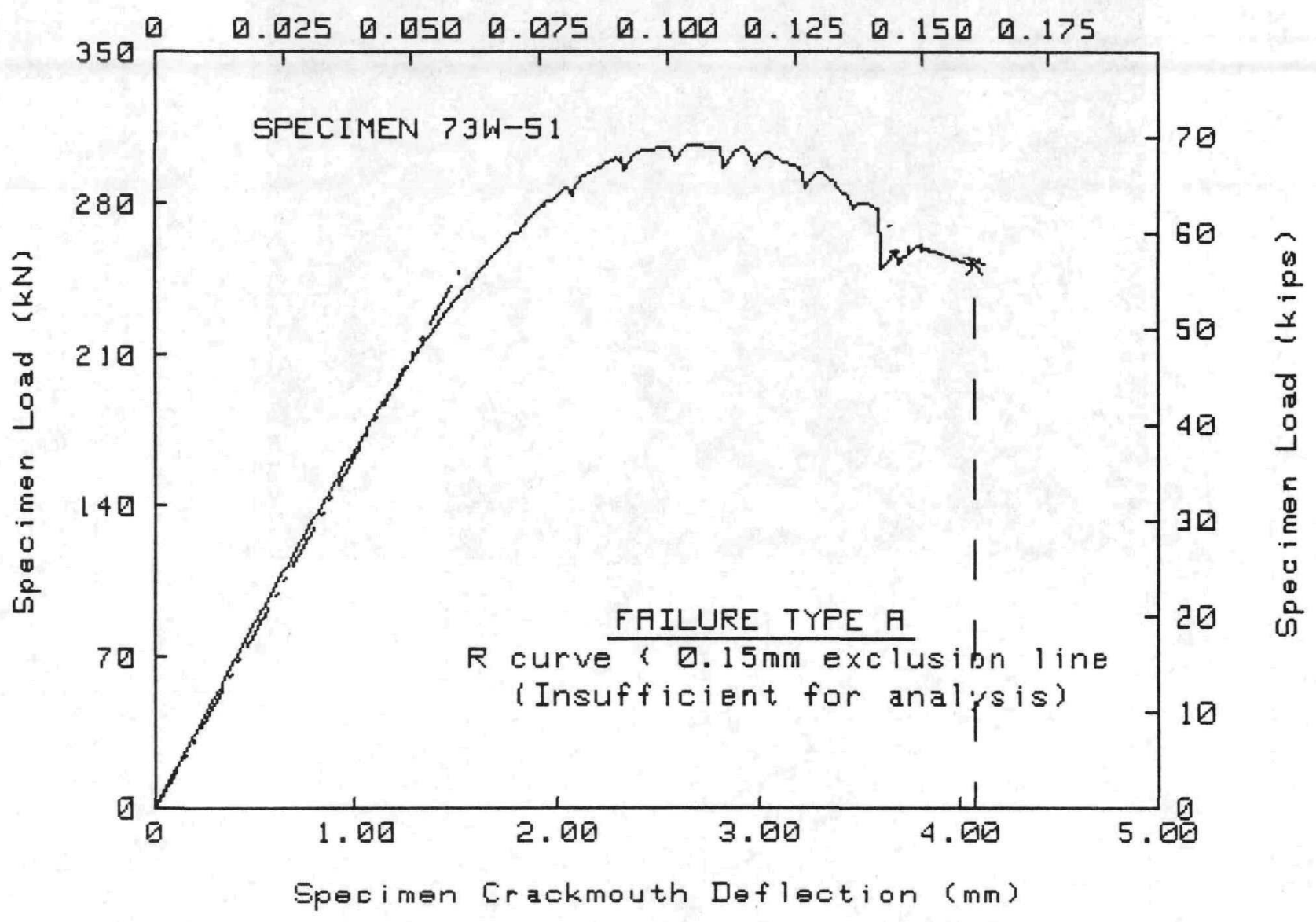

TEST SPECIMEN DATA

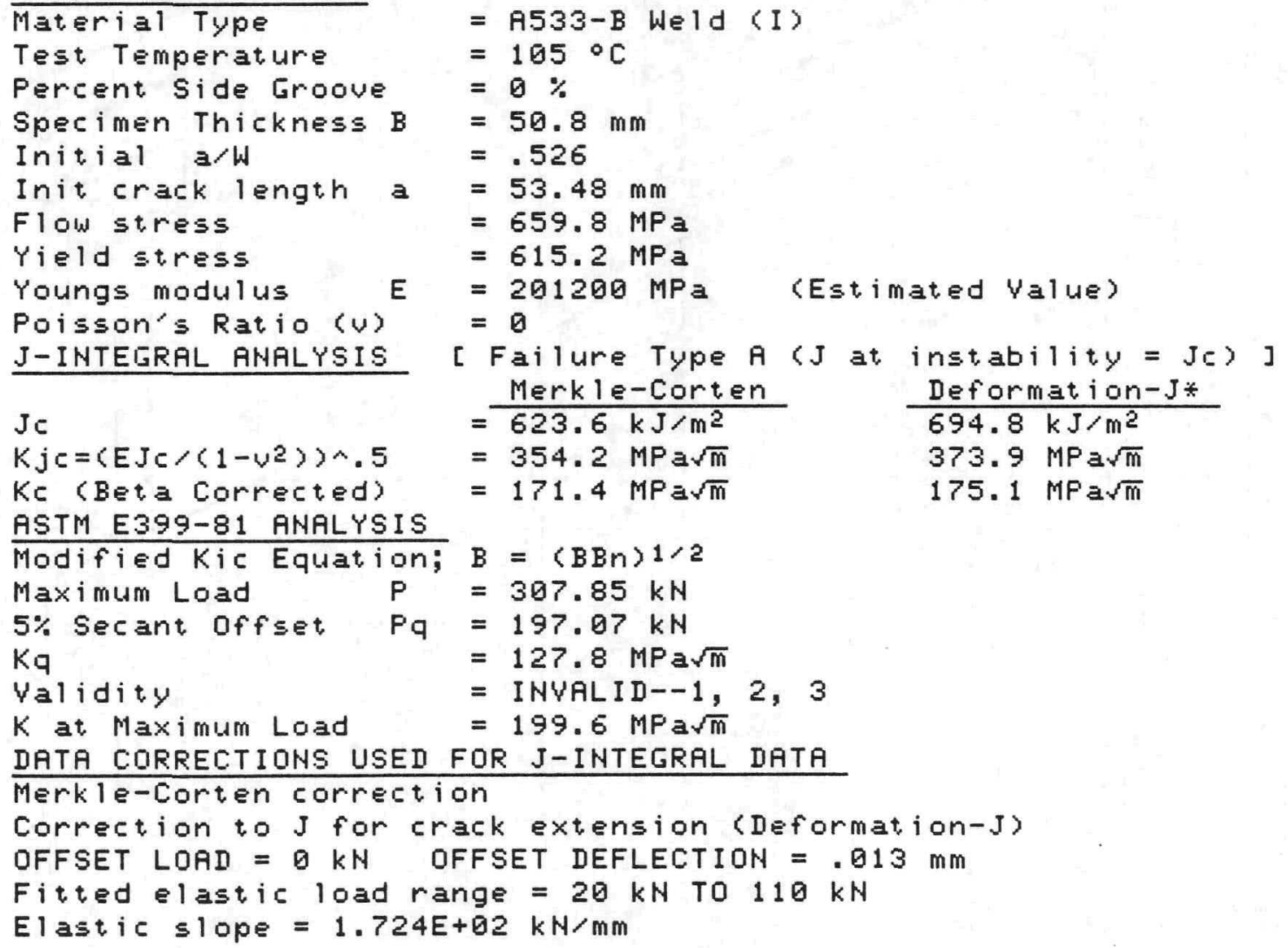




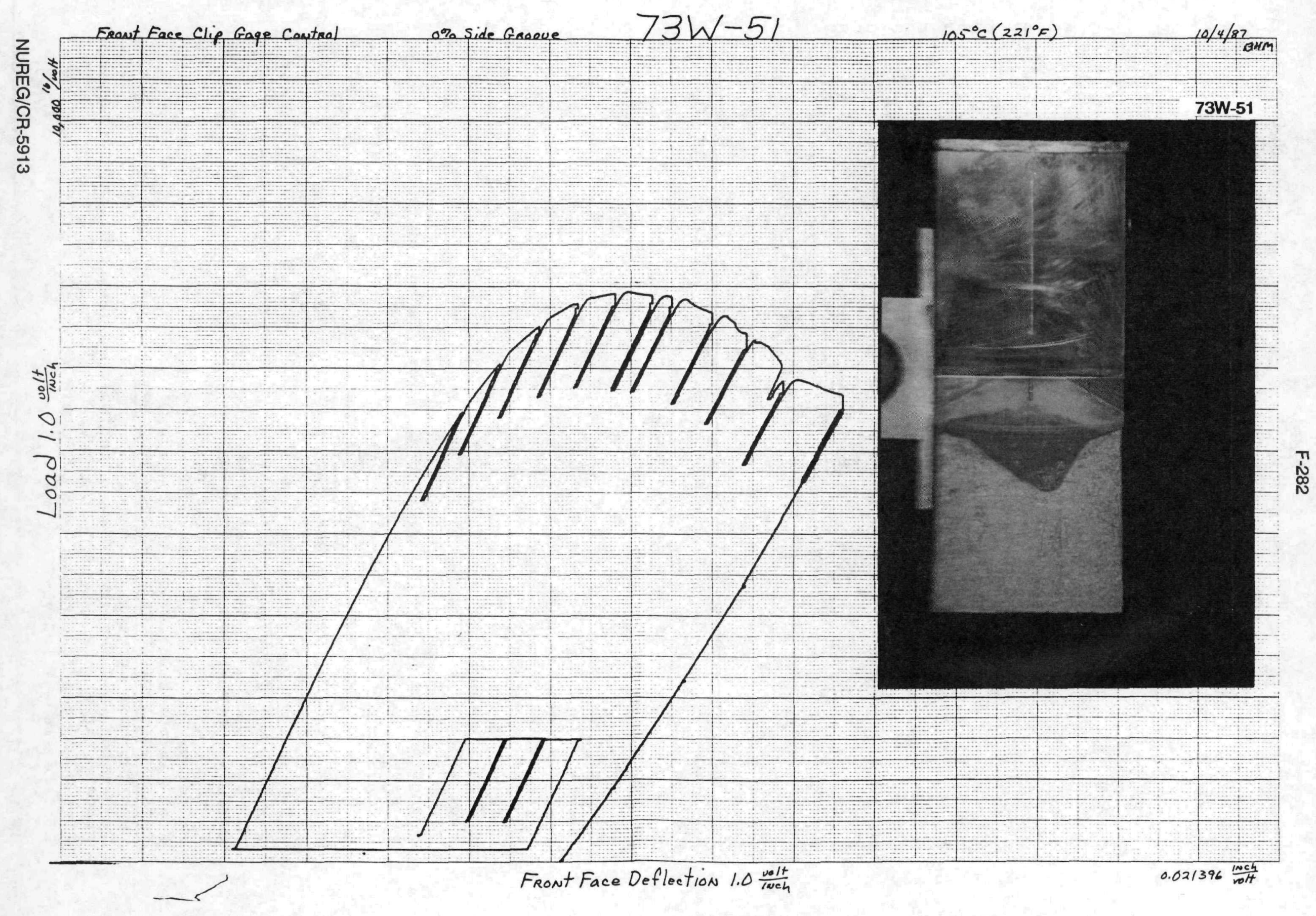




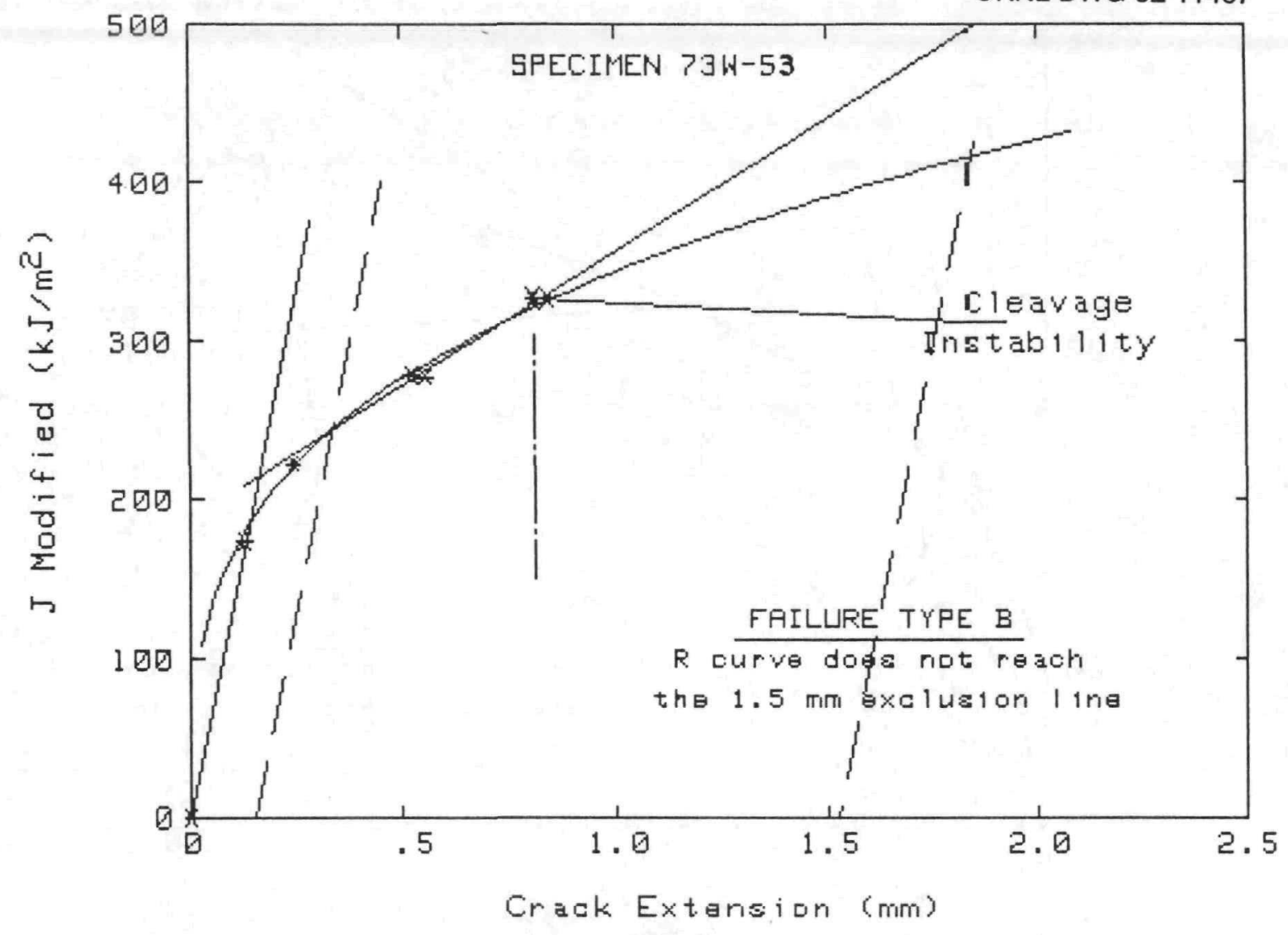

TEST SPECIMEN IATA

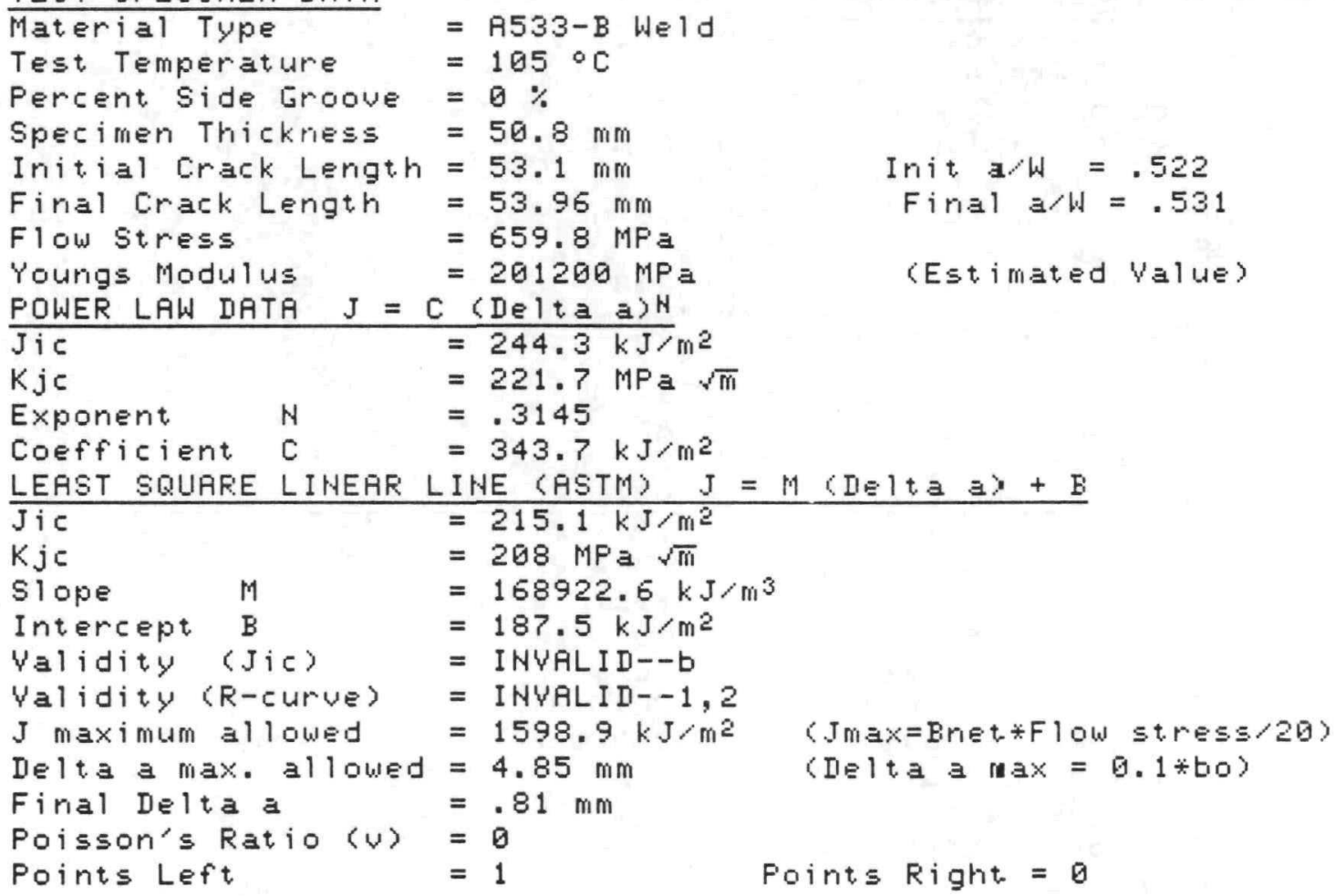


ORNL-DWG 92-11468

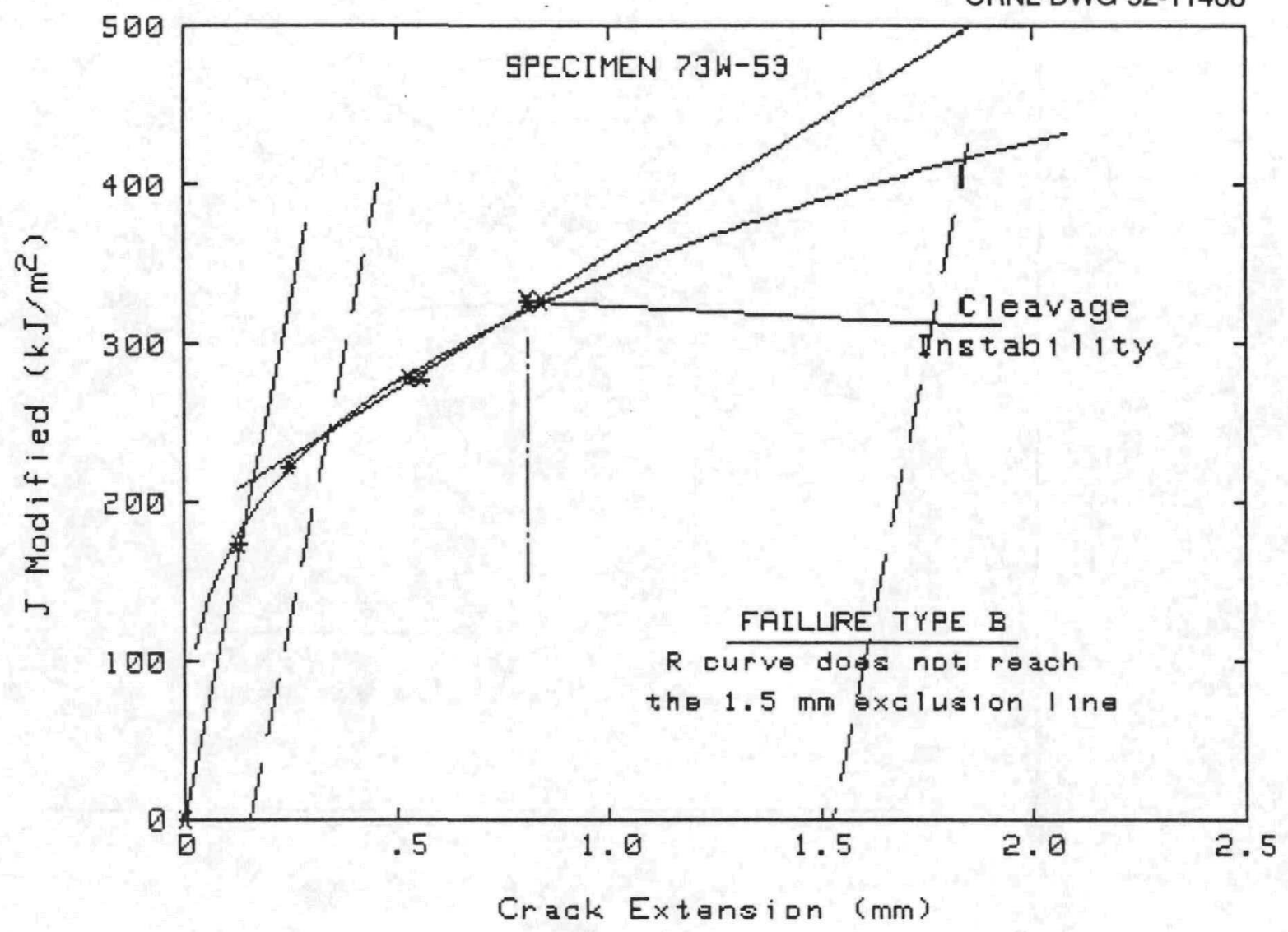

TEST SPECIMEN DATA

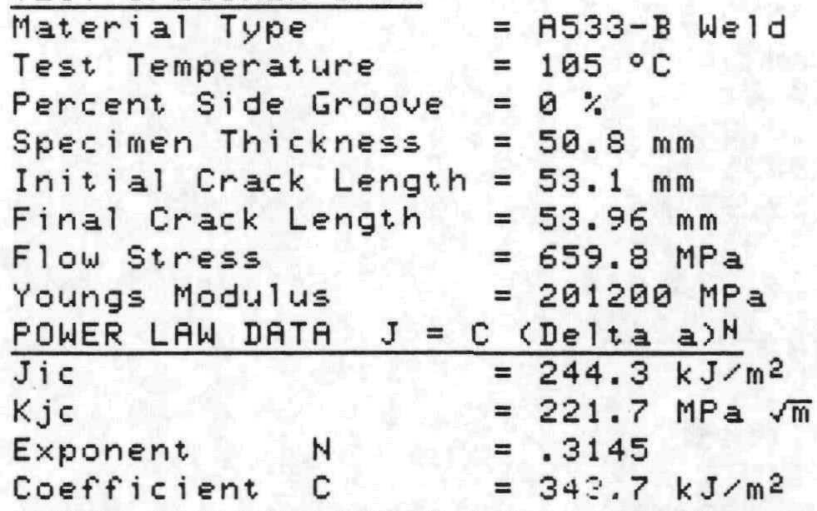

$\begin{array}{ll}\text { LEAST SQUARE LINERR LINE (ASTM) J } & =M\langle\mathrm{DE} I \mathrm{ta} a\rangle+\mathrm{B} \\ \mathrm{Jic} & =215.1 \mathrm{~kJ} / \mathrm{m}^{2}\end{array}$

$\mathrm{Kje}$

Slope M

Intercept $B$

Validity (Jic)

Validity (R-curve)

J maximum allowed

$=208 \mathrm{MPa} \sqrt{\mathrm{m}}$

$=168922.6 \mathrm{~kJ} / \mathrm{m}^{3}$

$=1.5 \mathrm{~kJ} / \mathrm{m}^{2}$

$=\quad$ LID $--b$

$=: \quad H L I D--1,2$

Delta a max. allowed $=4.85 \mathrm{~mm}$

Init $a / W=.522$

Final $a / W=.531$

(Estimated Value)

Final Delta a

$=.81 \mathrm{~mm}$

Poisson's Ratio $u\rangle=0$

Points Left $\quad=1$

( $\mathrm{Jmax}=\mathrm{Bnet} * \mathrm{~F}$ low stress/20)

(DE 1 ta a $\max =0.1 *$ bo $)$

$=1$

Points Right $=0$ 
Specimen Loadine Deflection (in.)

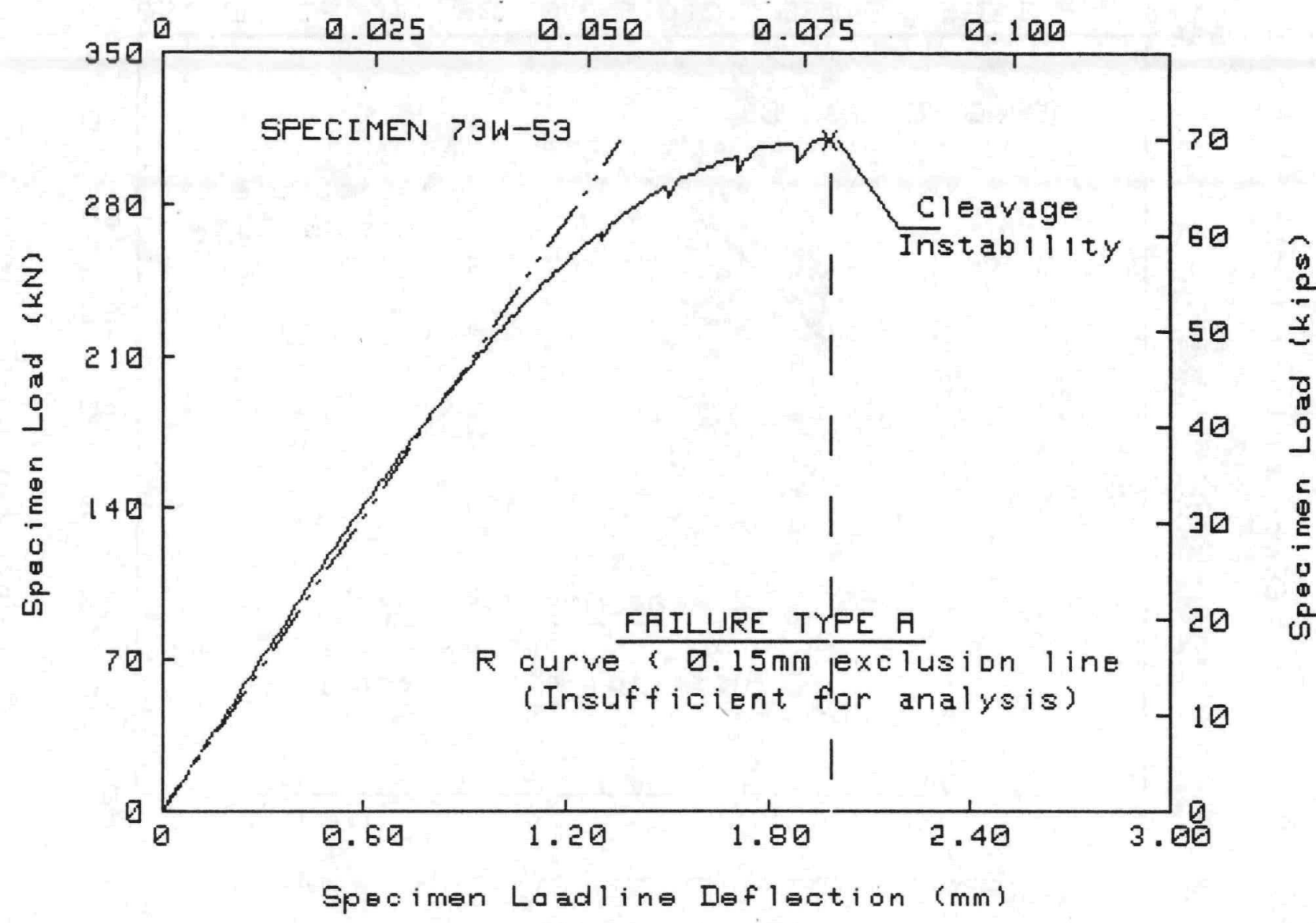

TEST SFECIMEN DATA

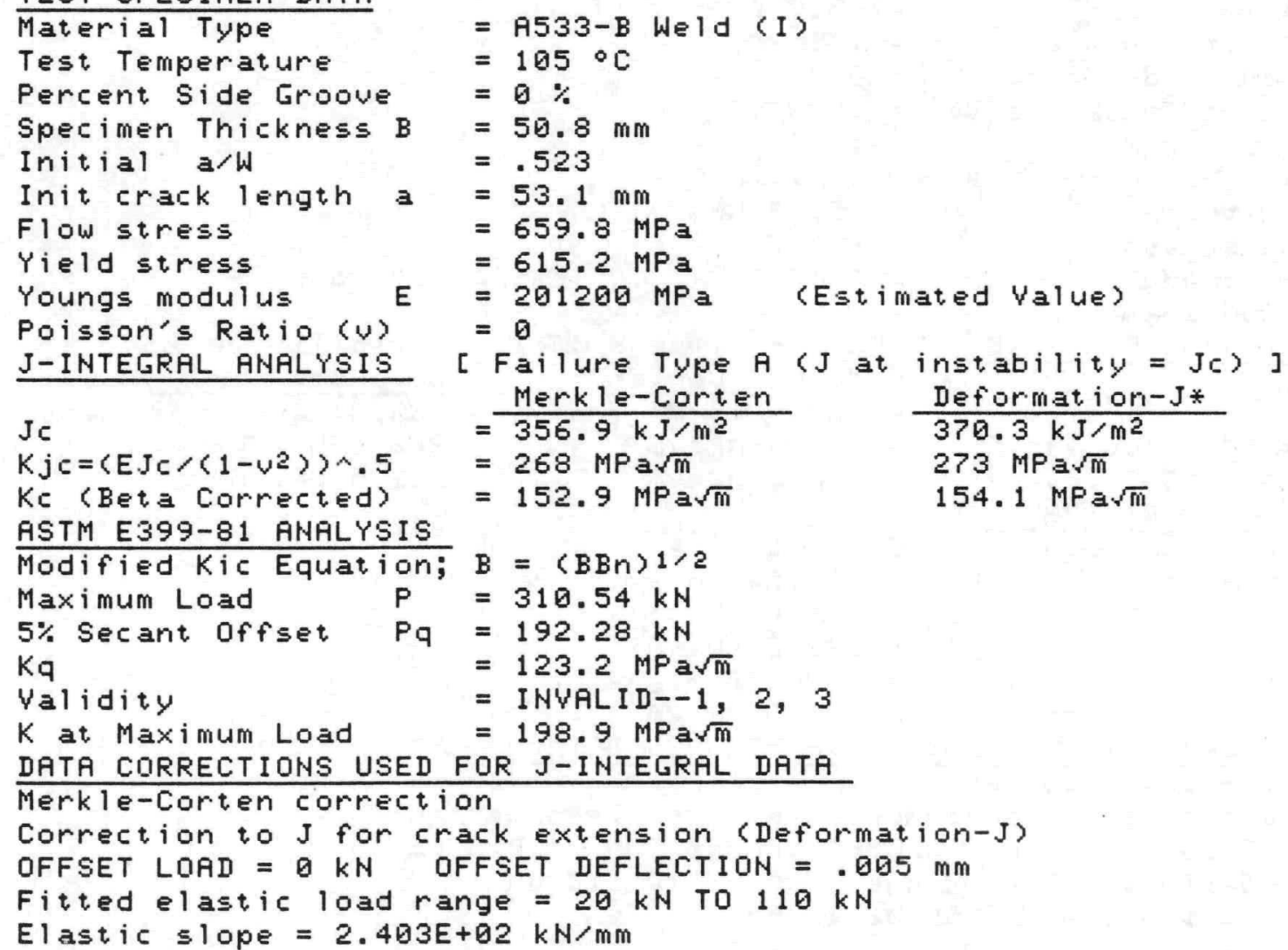


Specimen Crackmouth Deflection (in.)

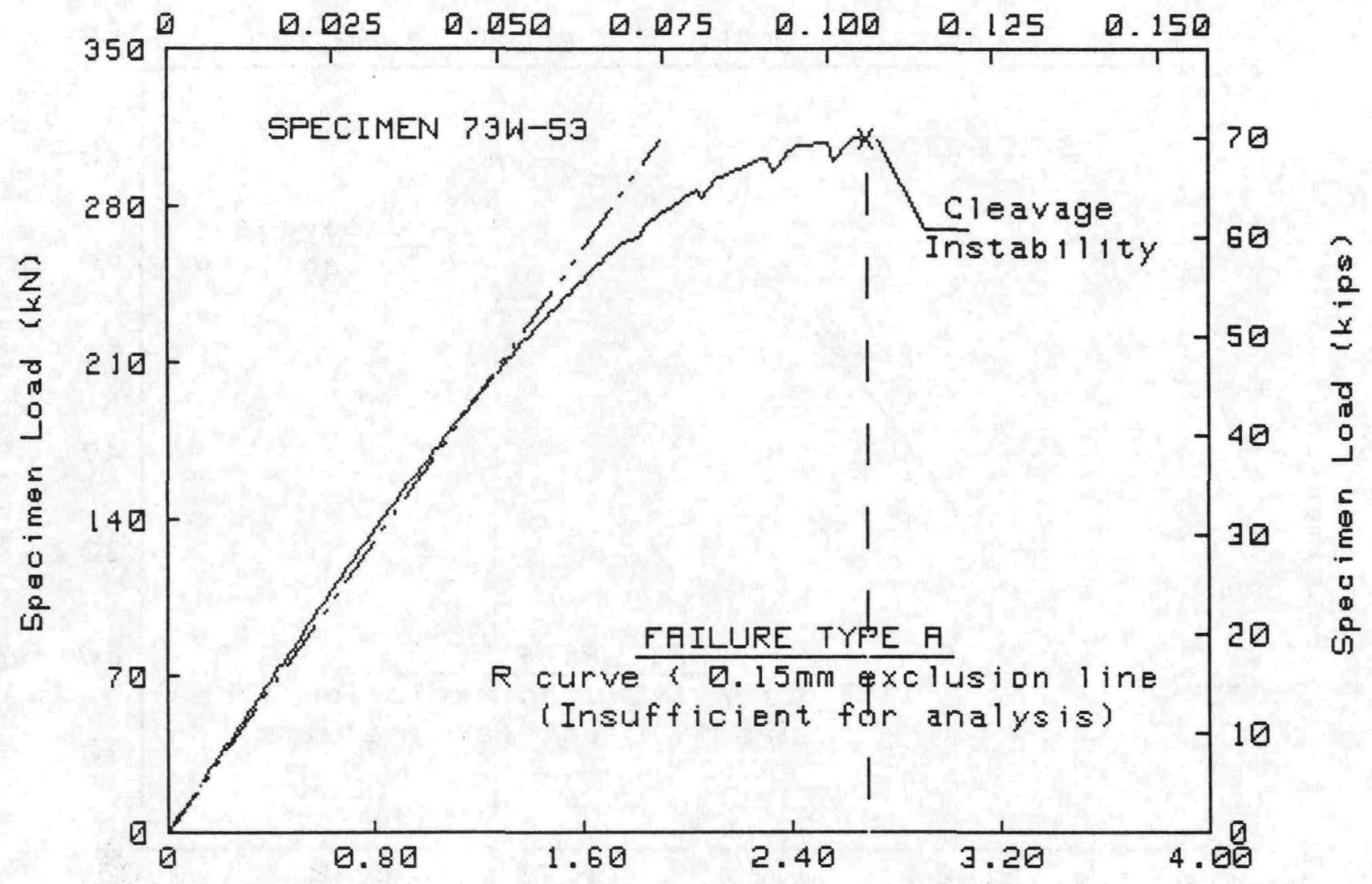

Specimen Crackmouth Deflection ( $m$ )

TEST SPECIMEN DATA

Material Type
Test Temperature
Percent Side Groove
Specimen Thickness B
Initial a/W
Init crack length a
Flow stress
Yield stress
Youngs modulus
Poisson's Ratio ( $)$
J-INTEGRAL ANALYSIS

$\mathrm{Jc}$

$K j c=\left\langle E J c\left\langle\left\langle 1-v^{2}\right\rangle\right\rangle \wedge .5\right.$

Ke (Beta Corrected)

ASTM E399-81 ANALYSIS

Modified Kic Equation; $B=\langle B B n\rangle 1 / 2$

Maximum Load $P=310.54 \mathrm{kN}$

$5 \%$ Secant offset Pq $=196.01 \mathrm{kN}$

$\mathrm{Kq}$

Validity

$K$ at. Maximum Load

$=125.6 \mathrm{MPa} \sqrt{\mathrm{m}}$

= INVALID--1, 2, 3

$=198.9 \mathrm{MPa} \sqrt{\mathrm{m}}$ $=105^{\circ} \mathrm{C}$

$=0 \%$

$=50.8 \mathrm{~mm}$

$=.523$

$=53.1 \mathrm{~mm}$

$=659.8 \mathrm{MPa}$

$=615.2 \mathrm{MPa}$

$=201200 \mathrm{MPa}$ (Estimated Value)

$=0$

$=\frac{\text { Merkle-Corten }}{355.4 \mathrm{~kJ} / \mathrm{m}^{2}}$

$=267.4 \mathrm{MPa} \sqrt{\mathrm{m}}$

$=152.8 \mathrm{MPa} \sqrt{\mathrm{m}}$
(I)

Cstimated Value

( $J$ at instability $=J c$ ) $J$

$\frac{\text { Deformation }-\mathrm{J} *}{363.3 \mathrm{~kJ} / \mathrm{m}^{2}}$

$270.4 \mathrm{MPa} \sqrt{\mathrm{m}}$

$153.5 \mathrm{MPa} \sqrt{\mathrm{m}}$

DATA CORRECTIONS USED FOR J-INTEGRAL DATA

Merkle-Corten correction

Correction to J for crack extension (Deformation-J)

OFFSET LOAD $=0 \mathrm{kN}$ OFFSET DEFLECTION $=.015 \mathrm{~mm}$

Fitted elastic load range $=20 \mathrm{kN}$ TO $110 \mathrm{kN}$

Elastic slope $=1.725 E+02 \mathrm{kN} / \mathrm{mm}$

NUREG/CR-5913 


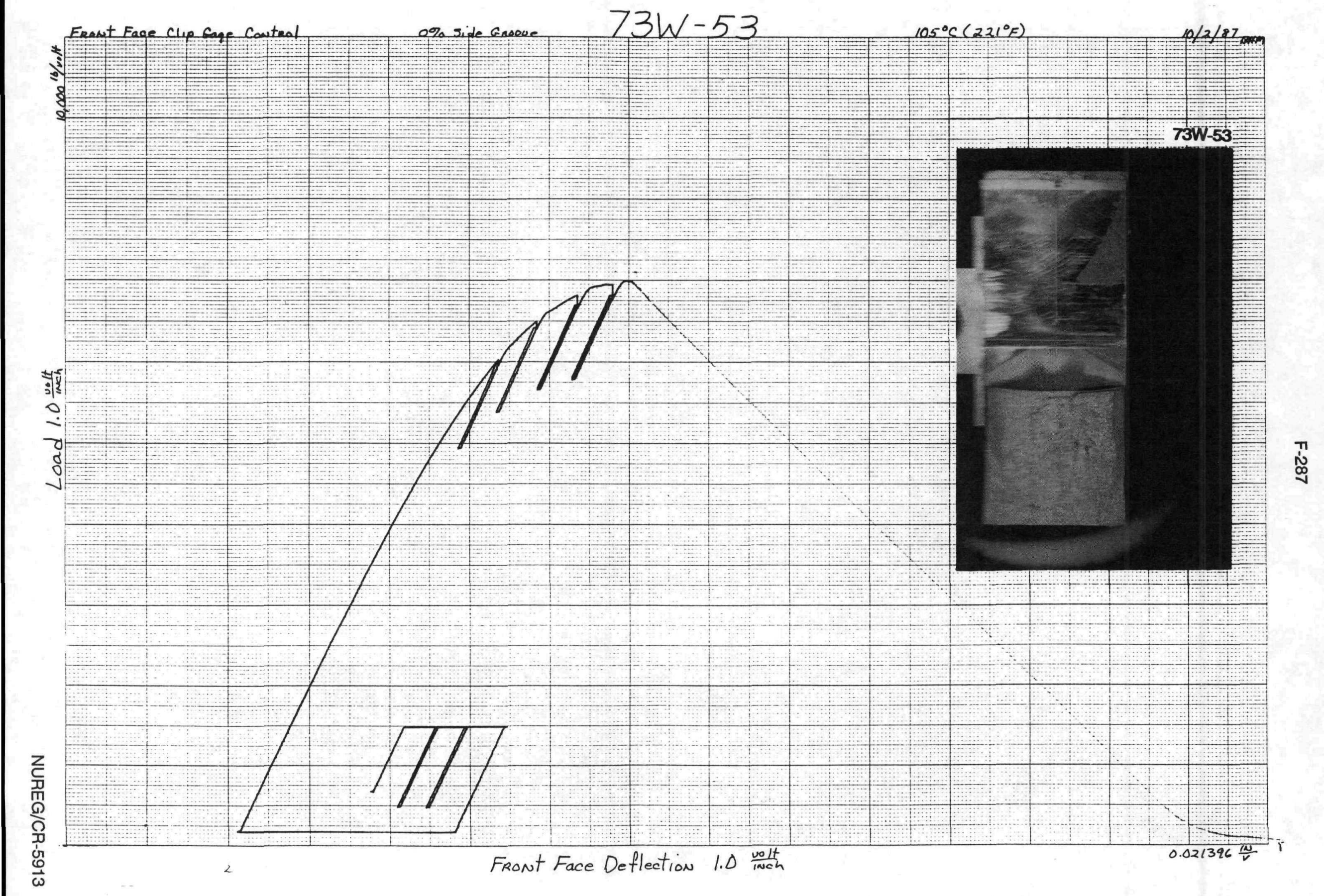




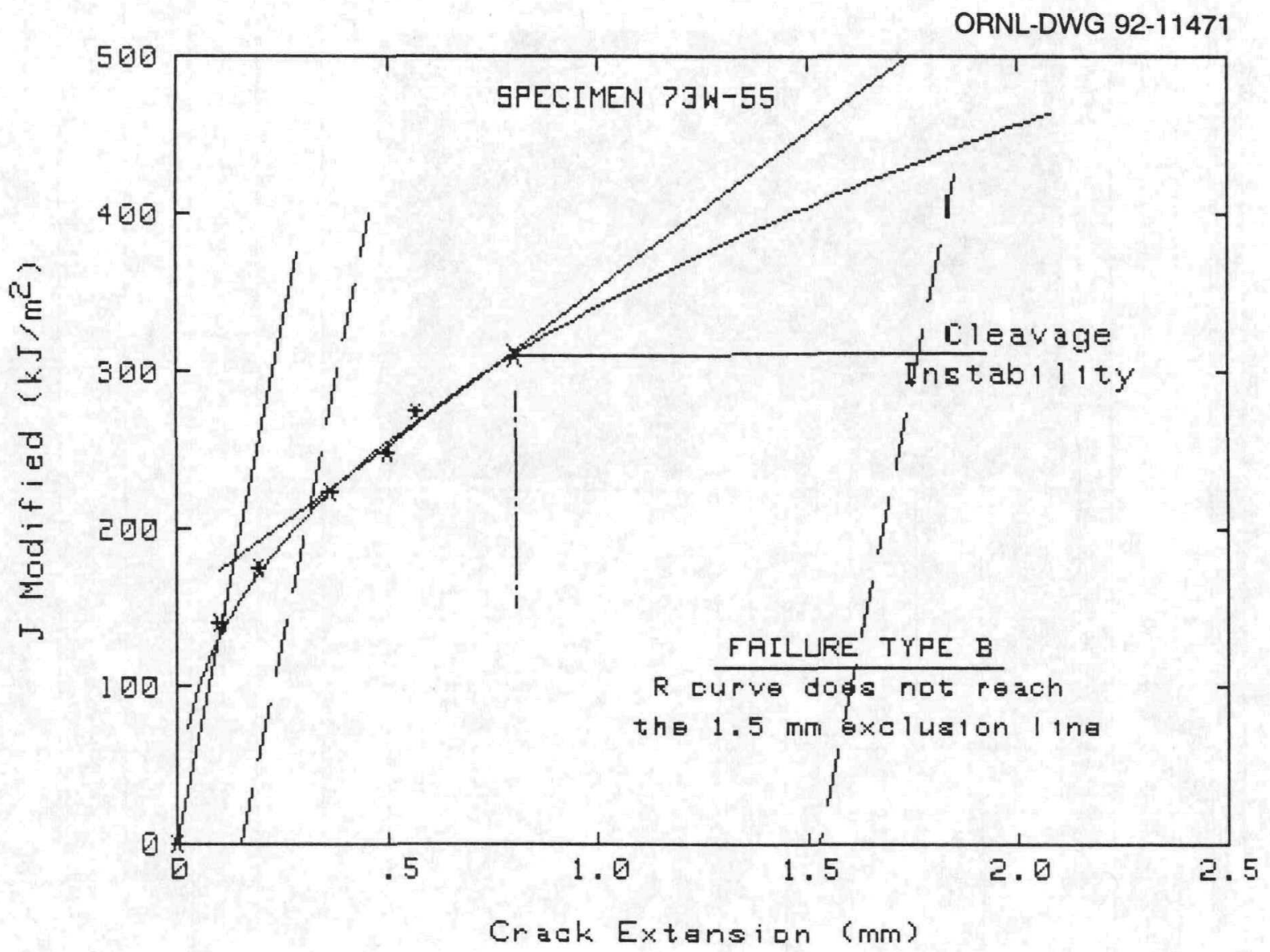

\section{TEST SPECIMEN IATA}

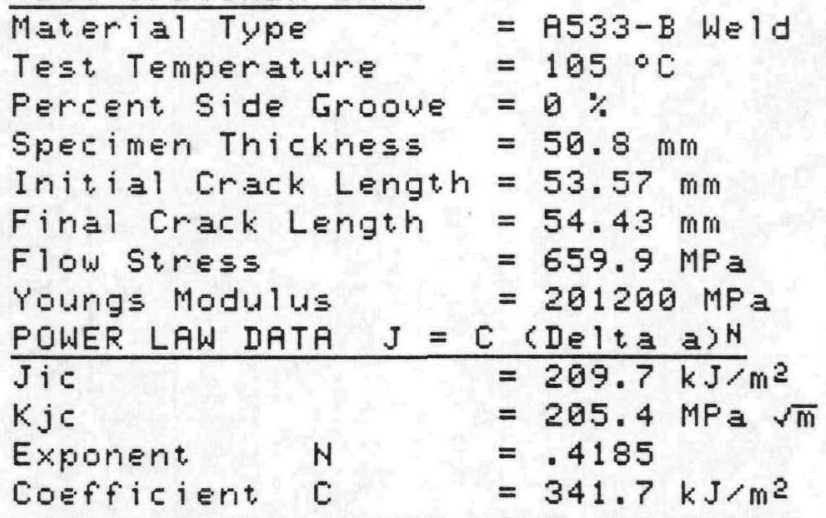

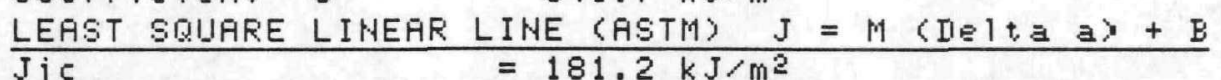

$\mathrm{Kj} \mathrm{c}$

$=153.8 \mathrm{~kJ} / \mathrm{m}^{2}$

Validity (Jic) = INVALID--b

Validity (R-curve) = INVALID--1, 2

$\mathrm{J}$ maximum allowed $=1583.6 \mathrm{~kJ} / \mathrm{m}^{2}$

Delta a max. allowed $=4.8 \mathrm{~mm}$ (Delta a max $=0.1 * \mathrm{bo}$ )

Final Delta a $=.8 \mathrm{~mm}$

Poisson's Ratio $(u)=\theta$

Points Left $=0$

Init $a / w=.527$

Final $a / h=.535$

(Est, inated Value) 


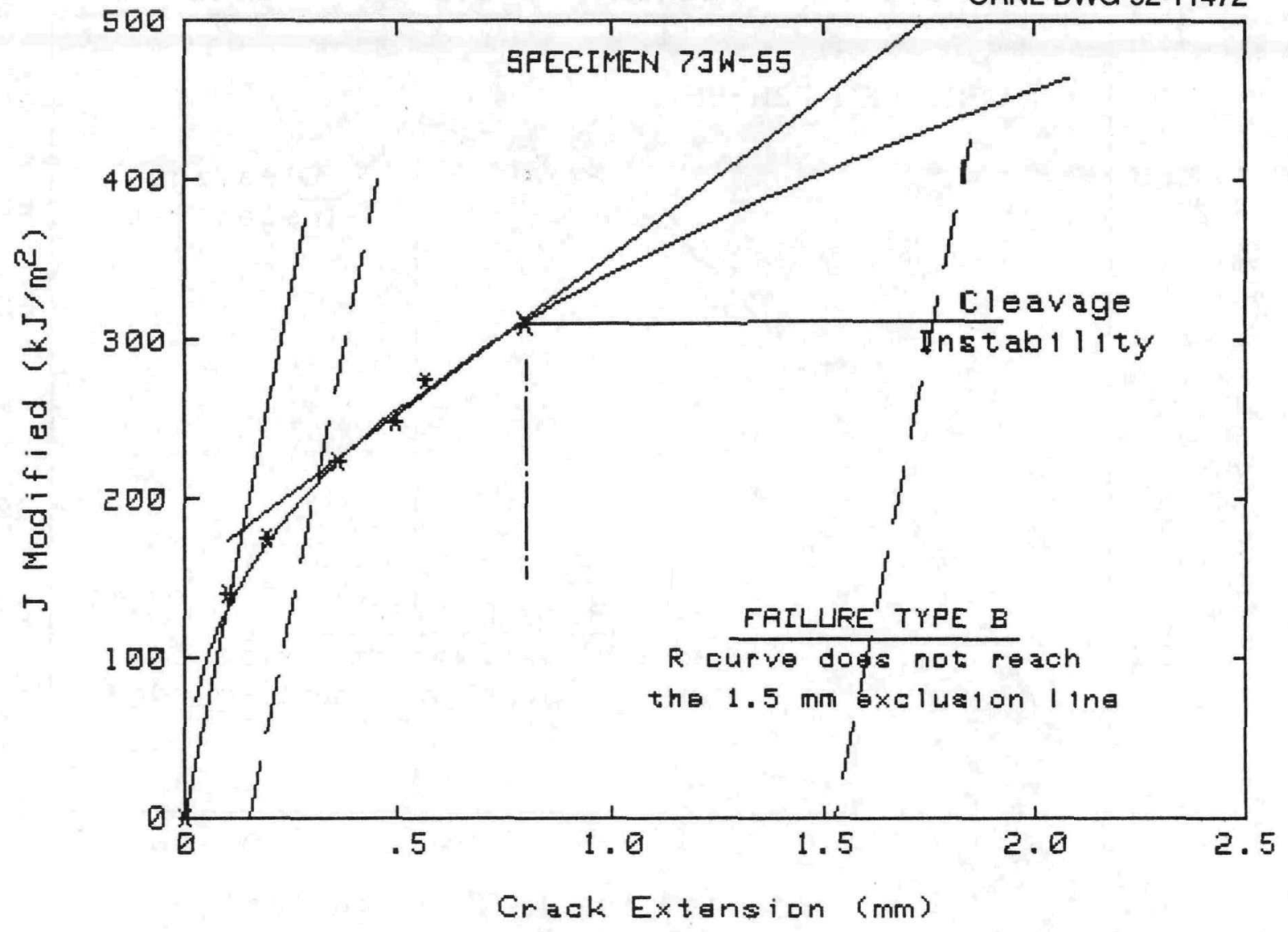

TEST SPECIMEN DATA

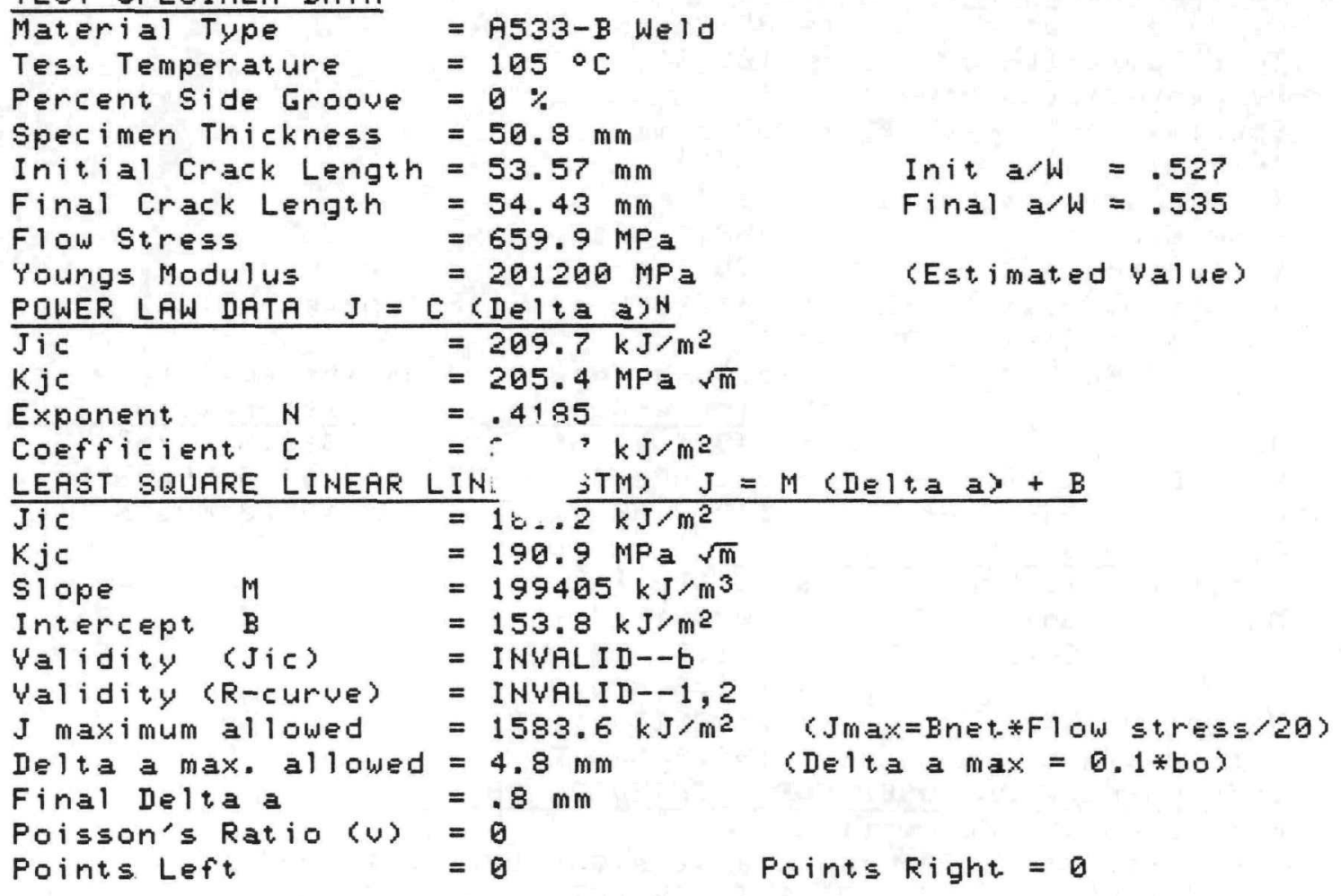


Specimen Loadline Deflection (in.)

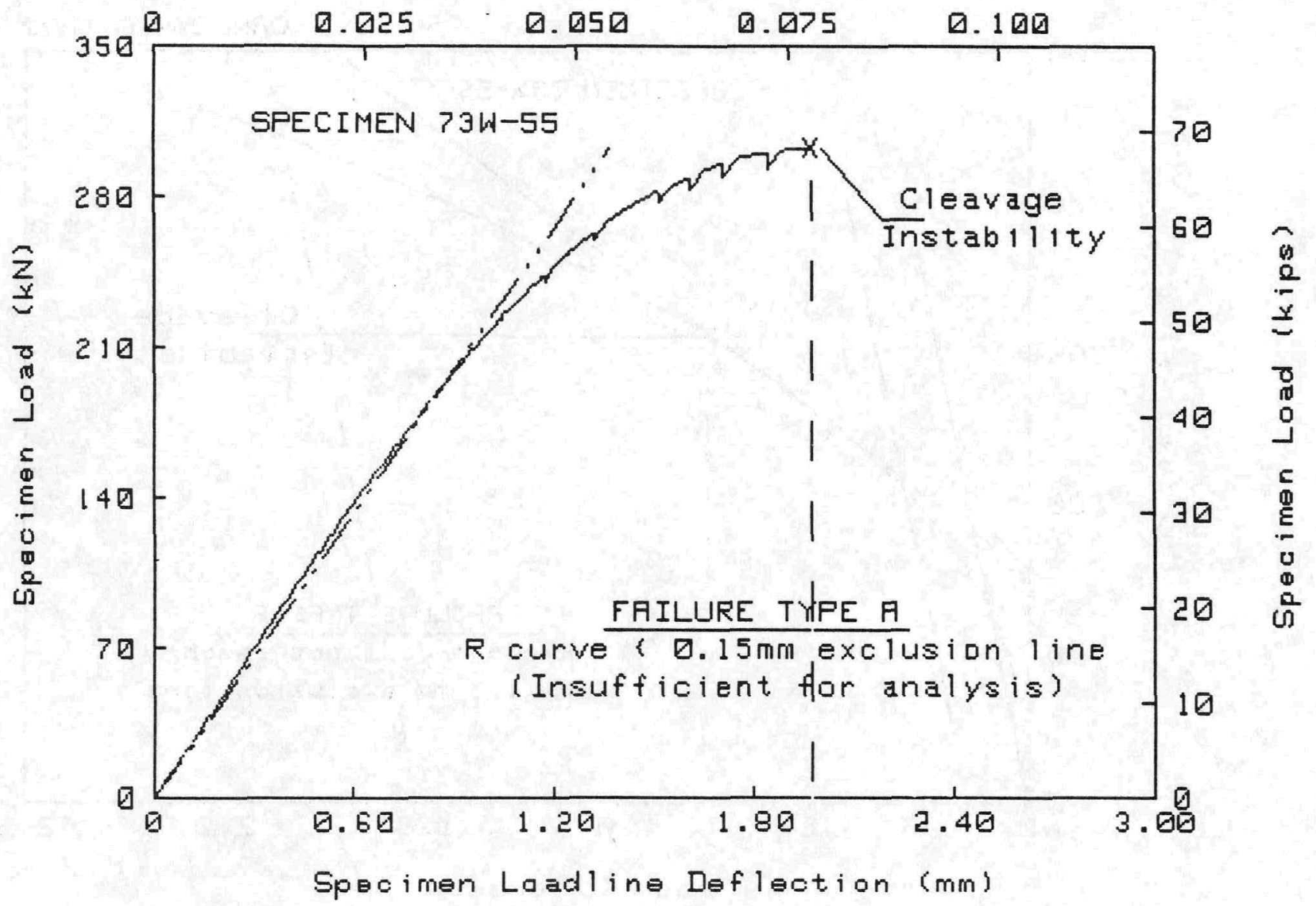

TEST SPECIMEN DATA

Material Type
Test Temperature
Percent Side Groove
Specimen Thickness B
Initial a/W
Init crack length a
Flow stress
Yield stress
Youngs modulus
Poisson's Ratio (U)
J-INTEGRAL ANALYSIS

$=A 533-B$ Weld (I)

$=105^{\circ} \mathrm{C}$

$=0 \%$

$=50.8 \mathrm{~mm}$

$=.527$

$=53.57 \mathrm{~mm}$

$=659.8 \mathrm{MPa}$

$=615.2 \mathrm{MPa}$

$=201200 \mathrm{MPa}$

$=0$

J-INTEGRAL ANALYSIS

$\mathrm{JC}$

[ Failure Type A ( $J$ at instability = J $c$ ) ]

$=\frac{\text { Merkle-Corten }}{349.1 \mathrm{~kJ} / \mathrm{m}^{2}}$

$=265 \mathrm{MParm}$

$K j c=\left\langle E J c /\left(1-v^{2}\right\rangle\right\rangle \wedge .5$

$=152.2 \mathrm{MPa} \sqrt{\mathrm{m}}$ $\frac{\text { Deformation }-\mathrm{J} *}{360.8 \mathrm{~kJ} / \mathrm{m}^{2}}$ $269.5 \mathrm{MPa} \sqrt{\mathrm{m}}$ $153.3 \mathrm{MPa} \sqrt{\mathrm{m}}$

Ke (Beta Corrected)
ASTM E399-81 AHALYSIS

Modified Kic Equation;

$B=(B B \cap) 1 / 2$

5\% Secant offset.

$\mathrm{Kq}$

Validity

$K$ at Maximum Load

$=302.81 \mathrm{kN}$

$\mathrm{Pq}=186.95 \mathrm{kN}$

$=121.6 \mathrm{MPa} \sqrt{\mathrm{m}}$

= INYALID--1, 2, 3

$=196.9 \mathrm{MPa} \sqrt{\mathrm{m}}$

DATA CORRECTIONS USED FOR J-INTEGRAL DATA

Merkle-Corten correction

Correction to J for crack extension (Deformation-J)

OFFSET LOAD $=0 \mathrm{kN}$ OFFSET DEFLECTION $=.005 \mathrm{~mm}$

Fitied elastic load range $=20 \mathrm{kN}$ To $110 \mathrm{kN}$

Elastic slope $=2.341 \mathrm{E}+02 \mathrm{kN} / \mathrm{mm}$

NUREG/CR-5913 
Specimen Crackmouth Deflection (in.)

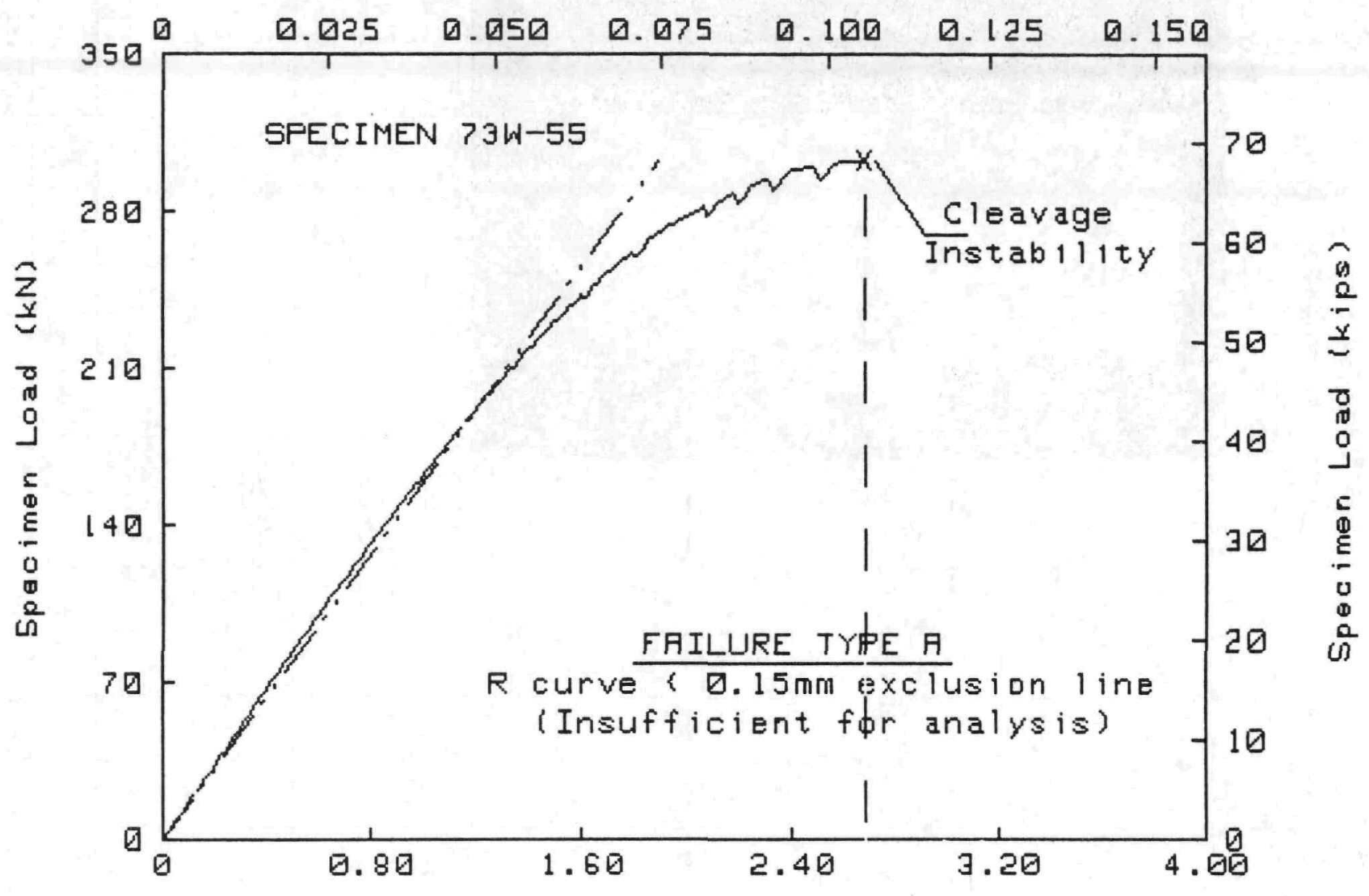

Specimen Crackmouth Deflection ( $m m$ )

TEST SPECIMEN DATA

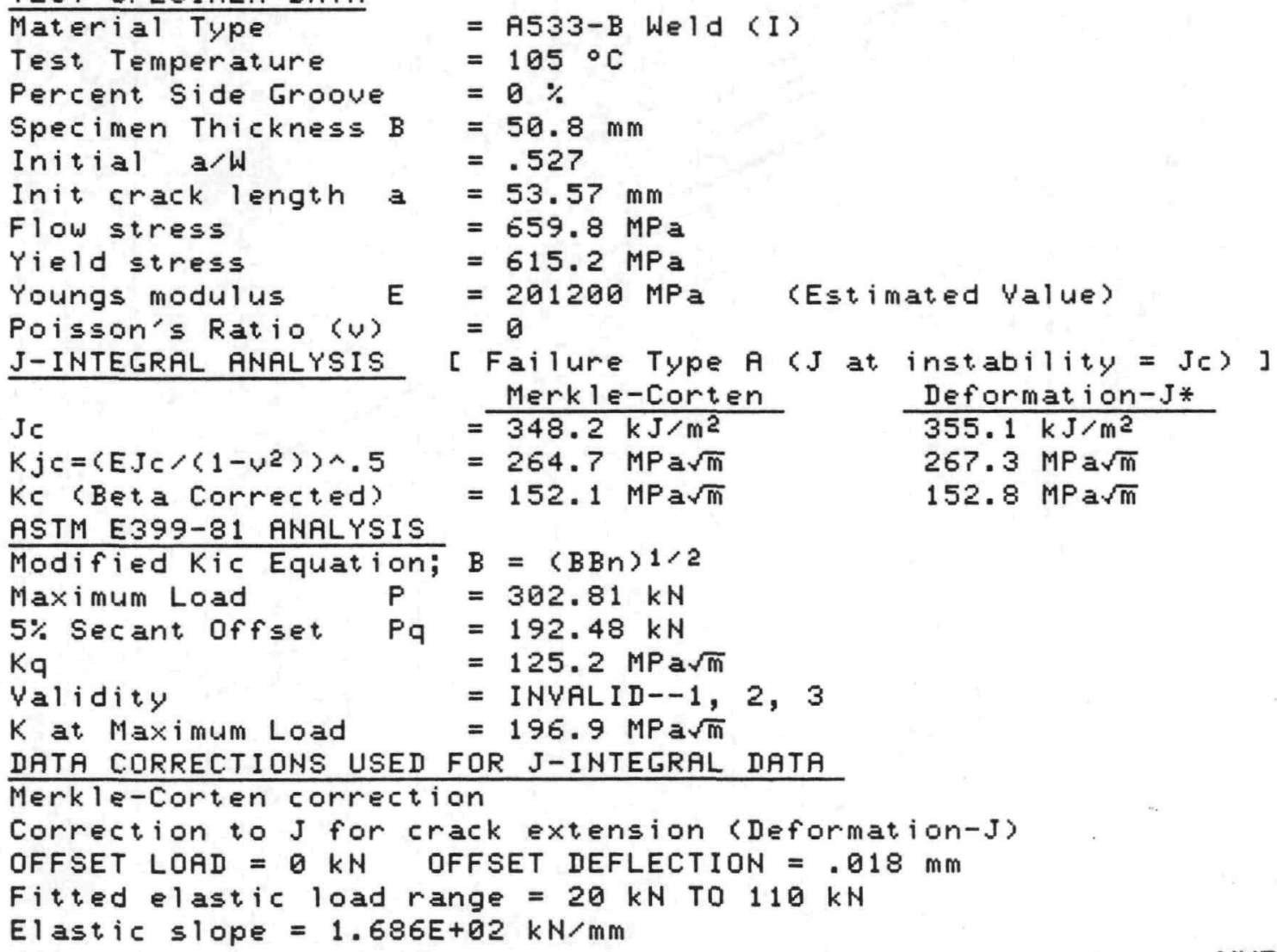




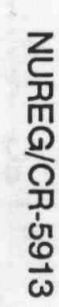

Erout Face Clip Gage Coutrol or side Gropue $\quad 73 W-55$

$105^{\circ} \mathrm{C}\left(231^{\circ} \mathrm{F}\right)$

$10 / 4 / 27$

盈

忿

के
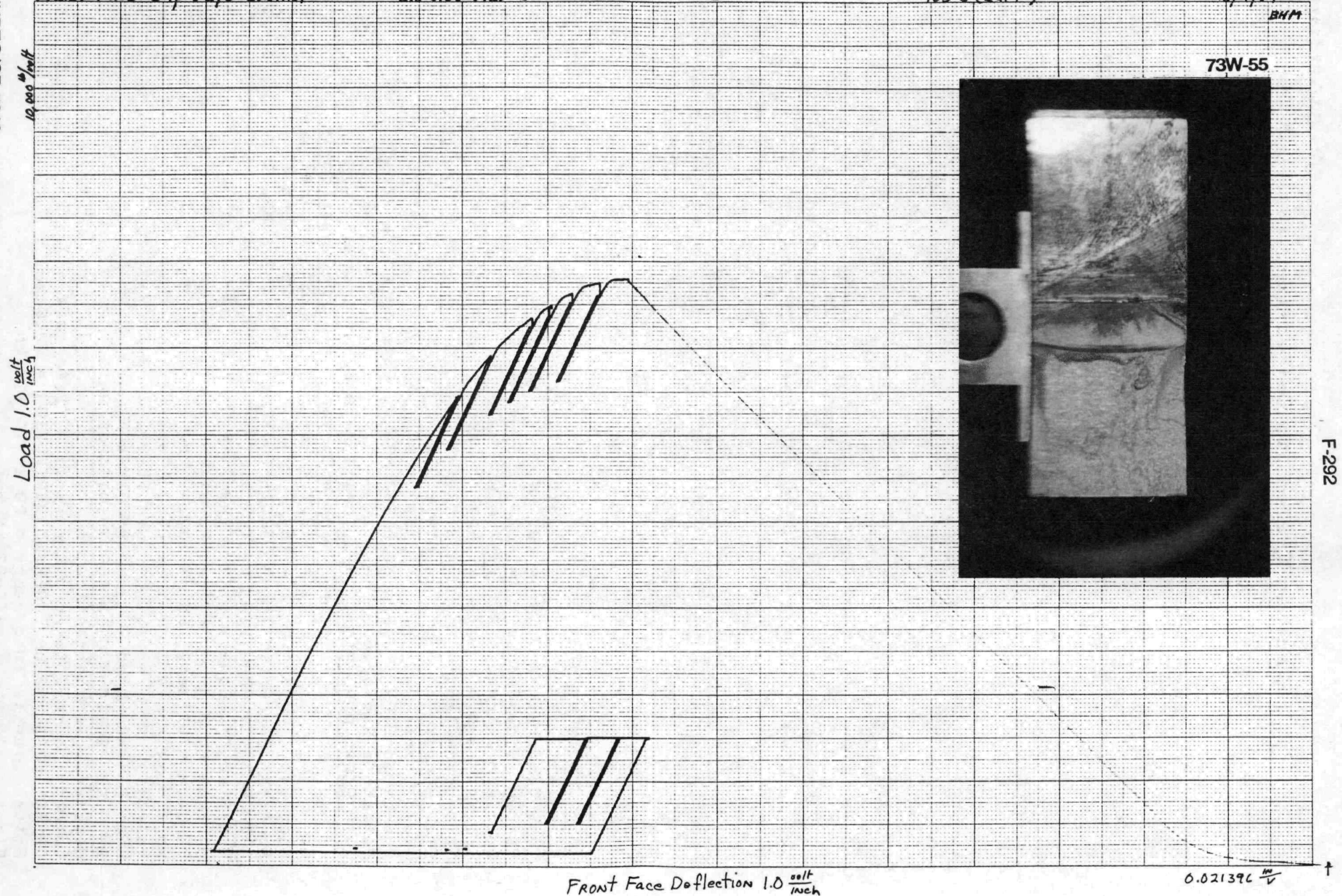


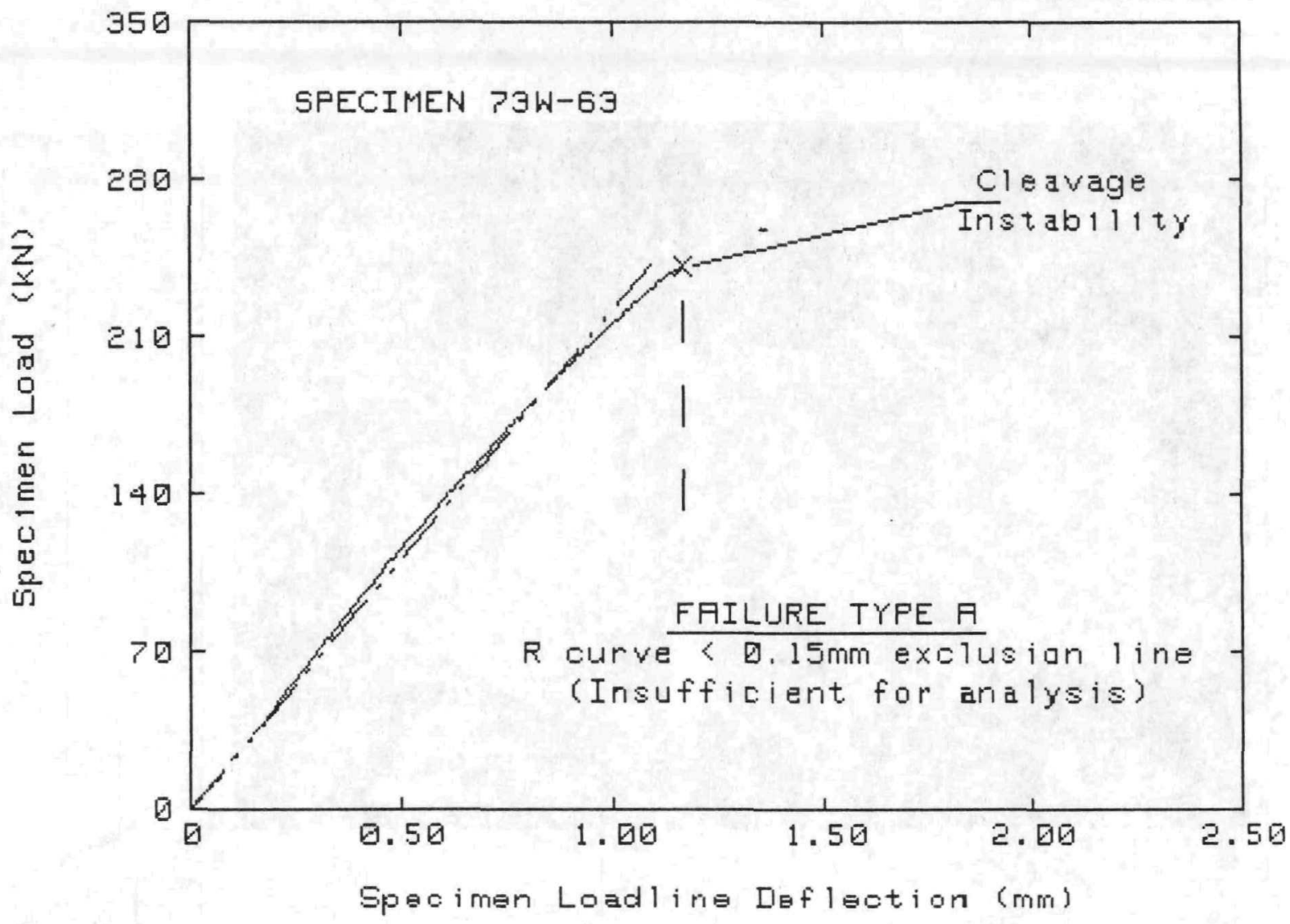

TEST SPECIMEN DATA

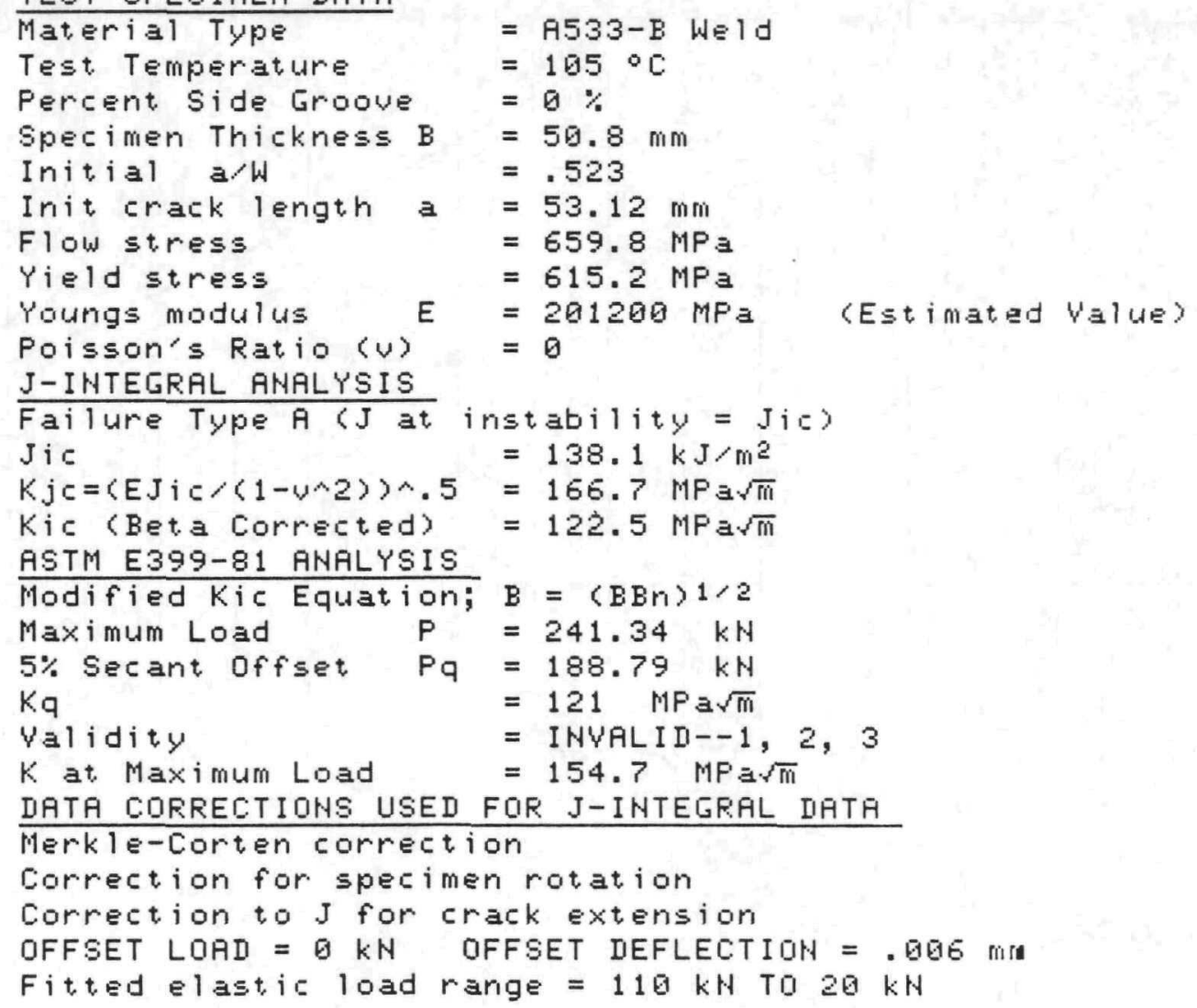




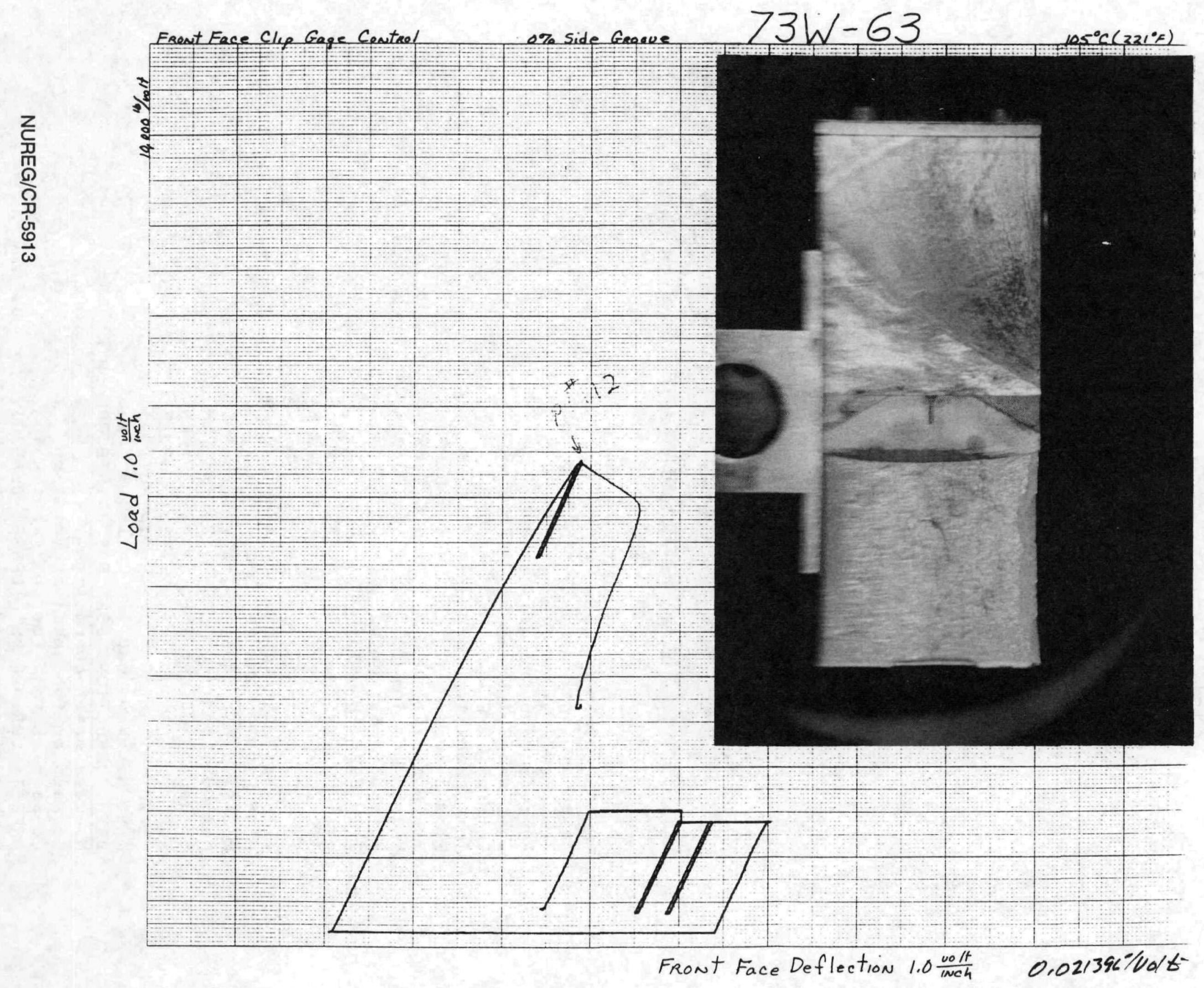


Specimen Loadline Deflection (in.)
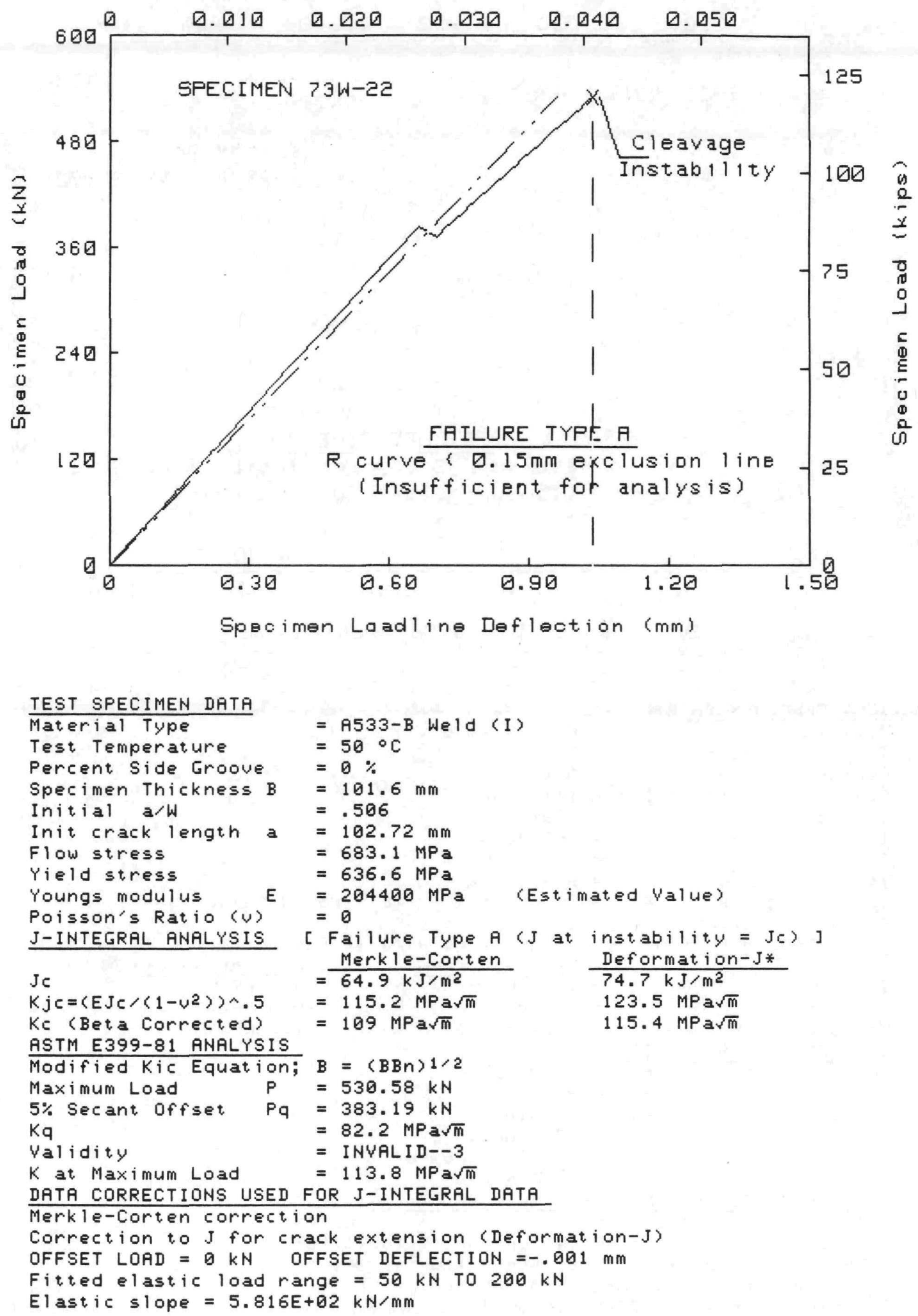
Specimen Loadline Deflection (in.)

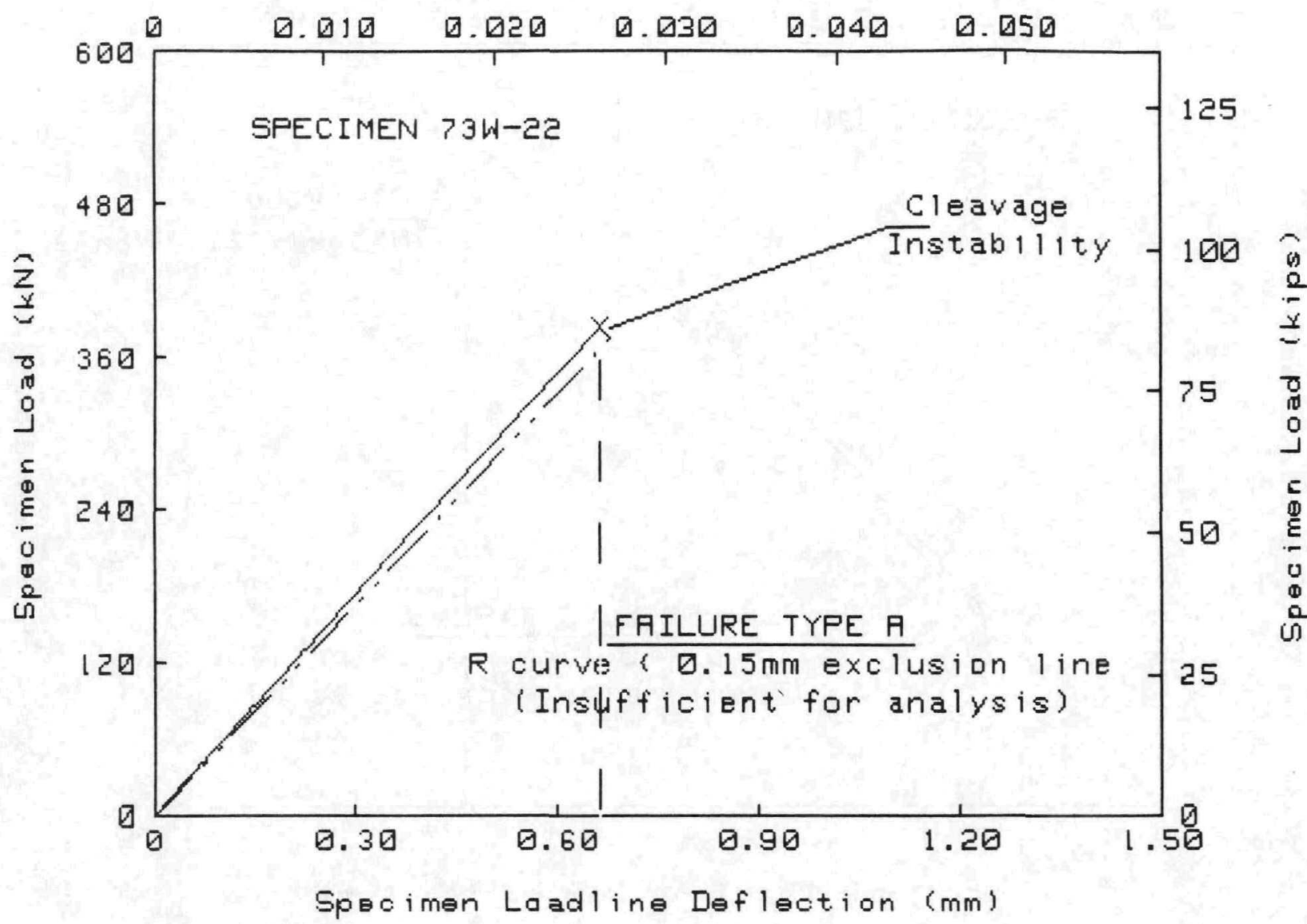

TEST SPECIMEN DATA

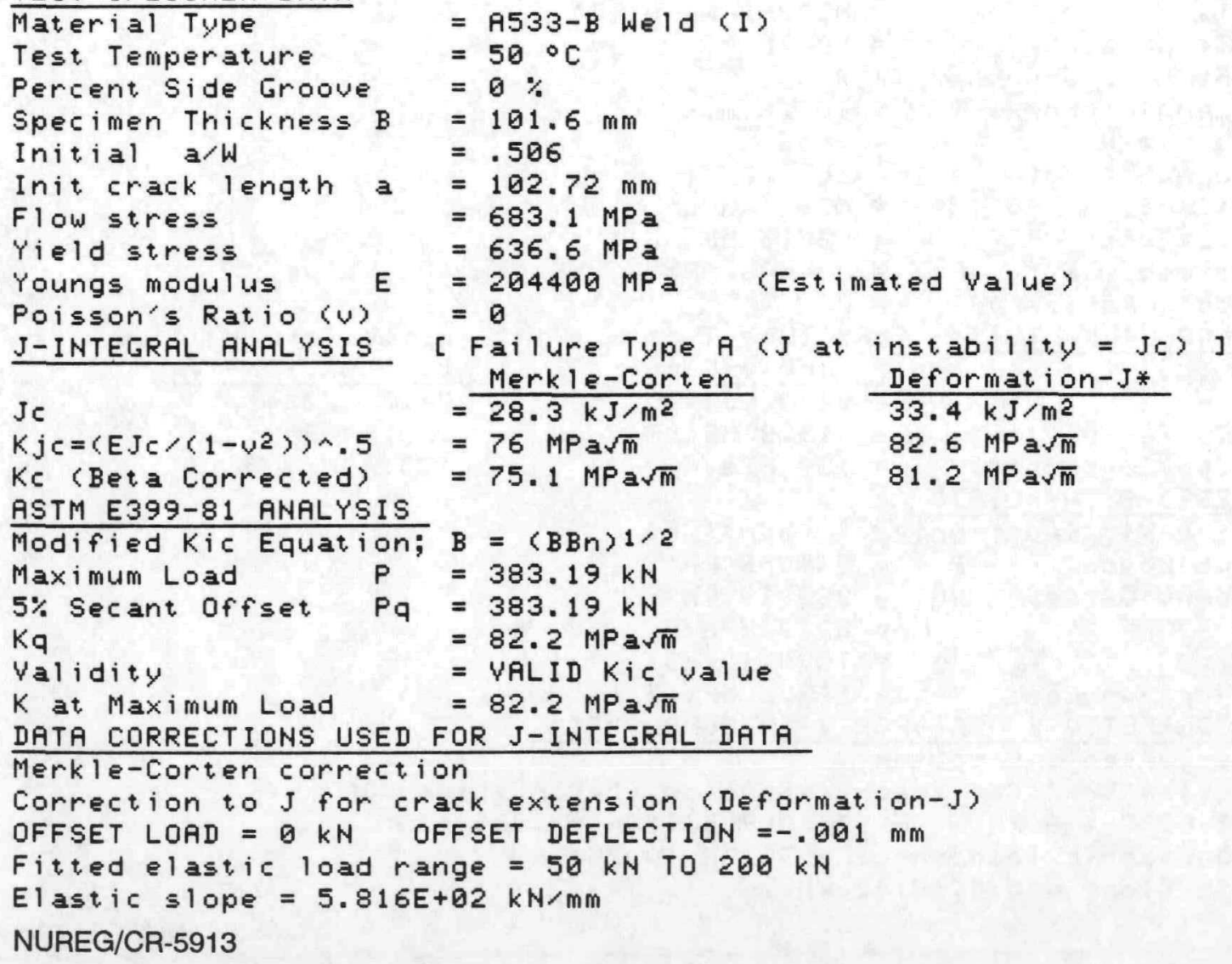


Specimen Crackmauth Deflection (in.)
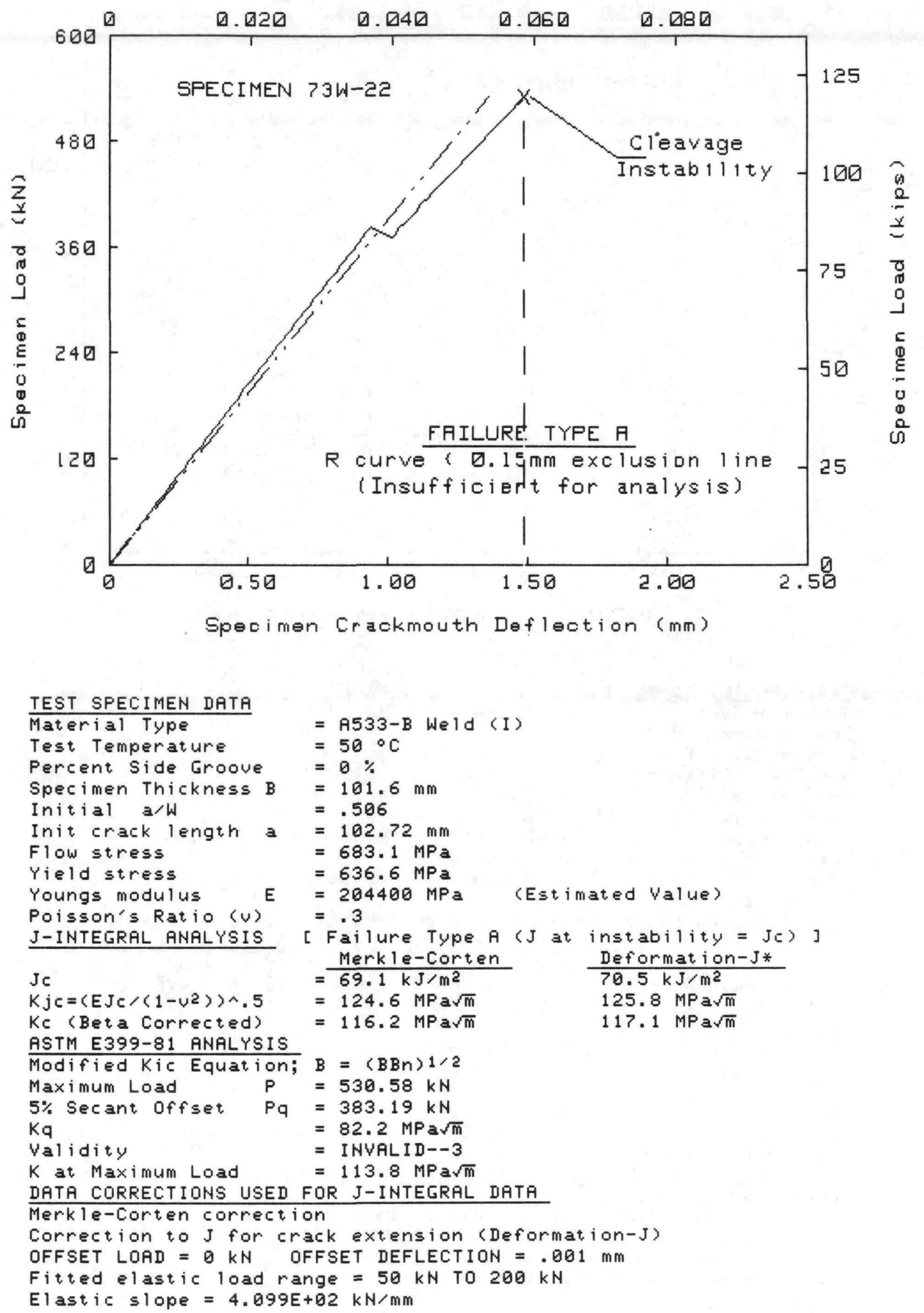
Specimen Crackmouth Deflection (in.)

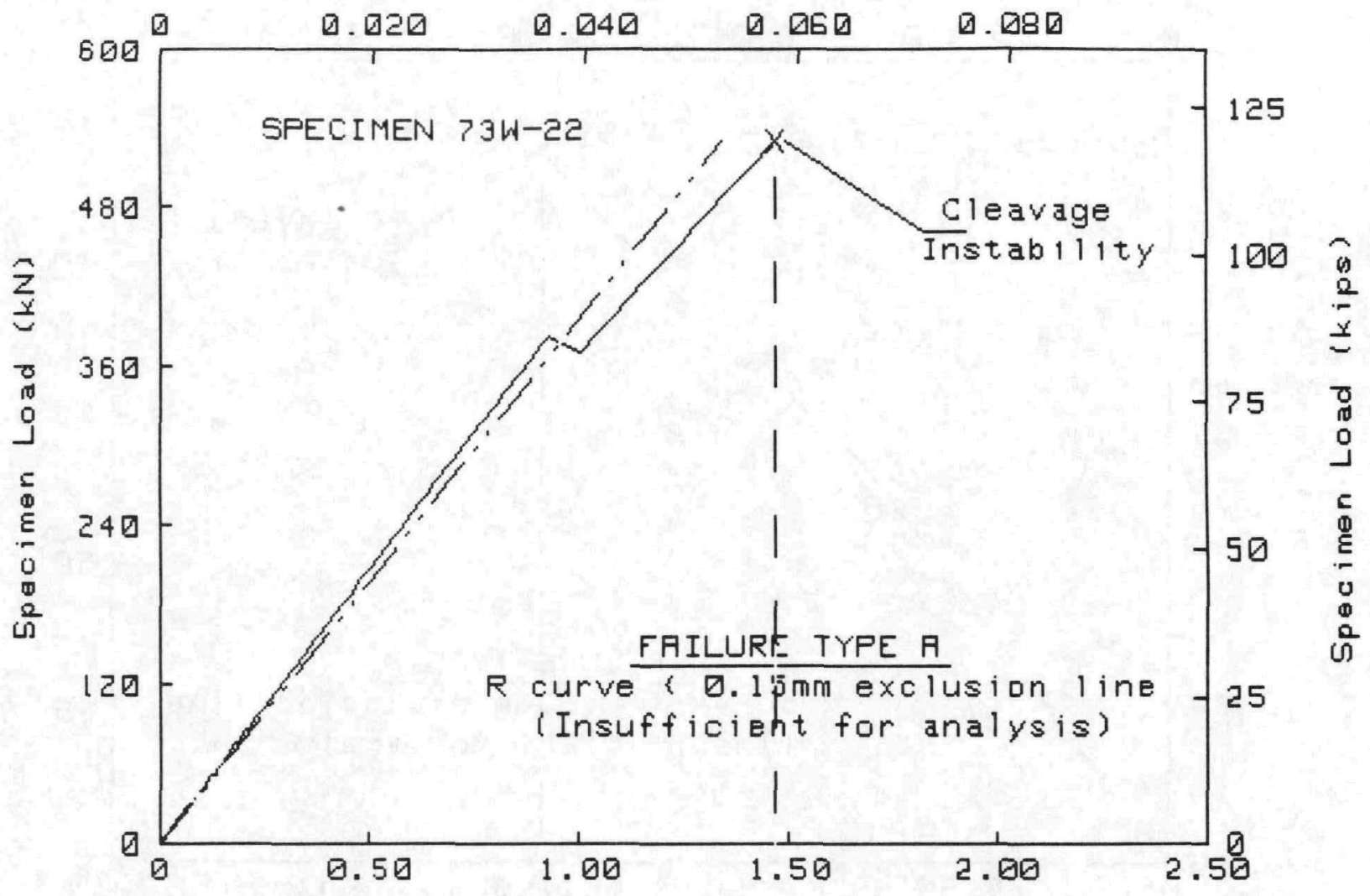

Specimen Crackmouth Deflection ( $m$ )

\section{TEST SPECIMEN DATA}

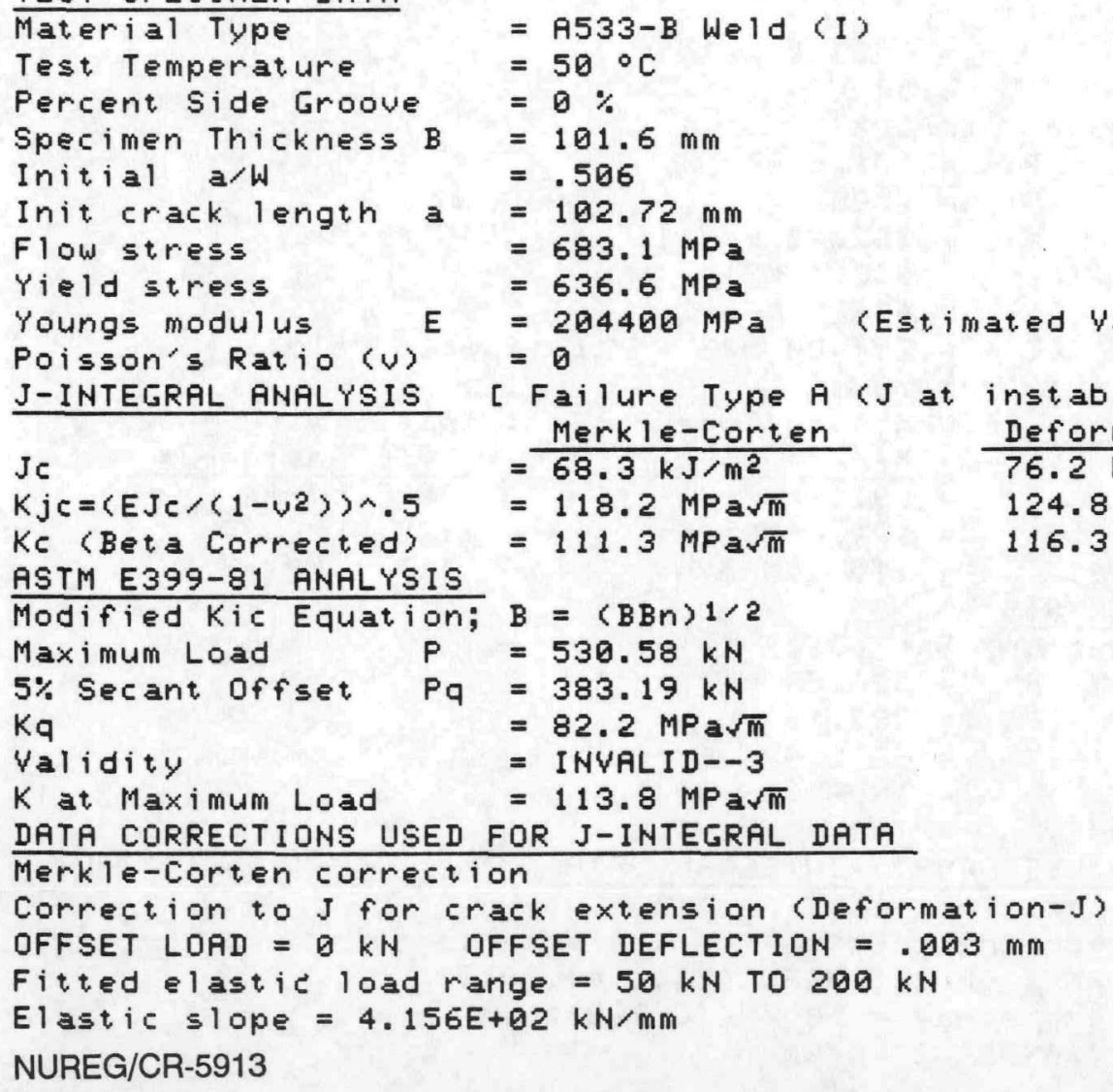




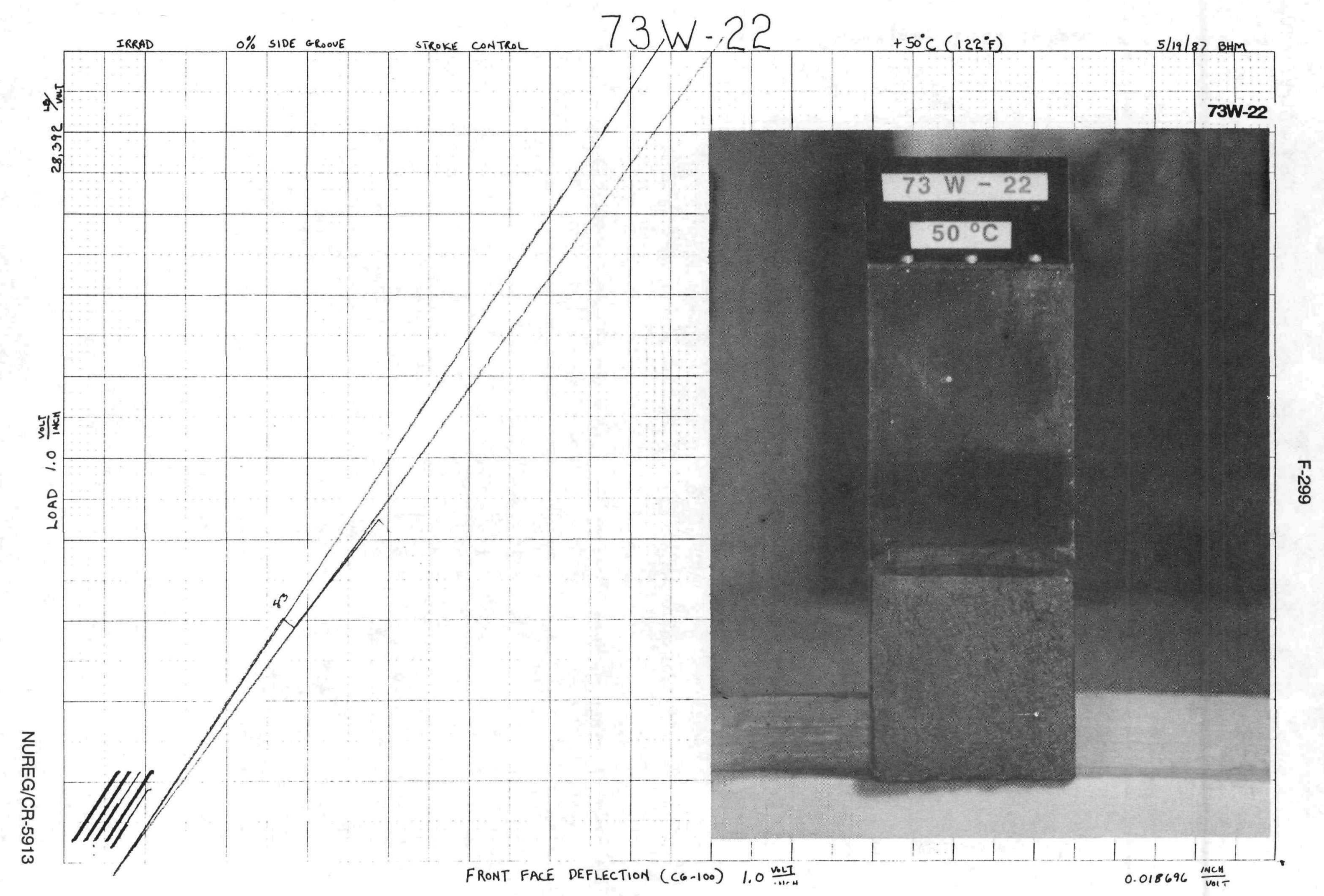


Specimen Laadline Deflectian (in.)

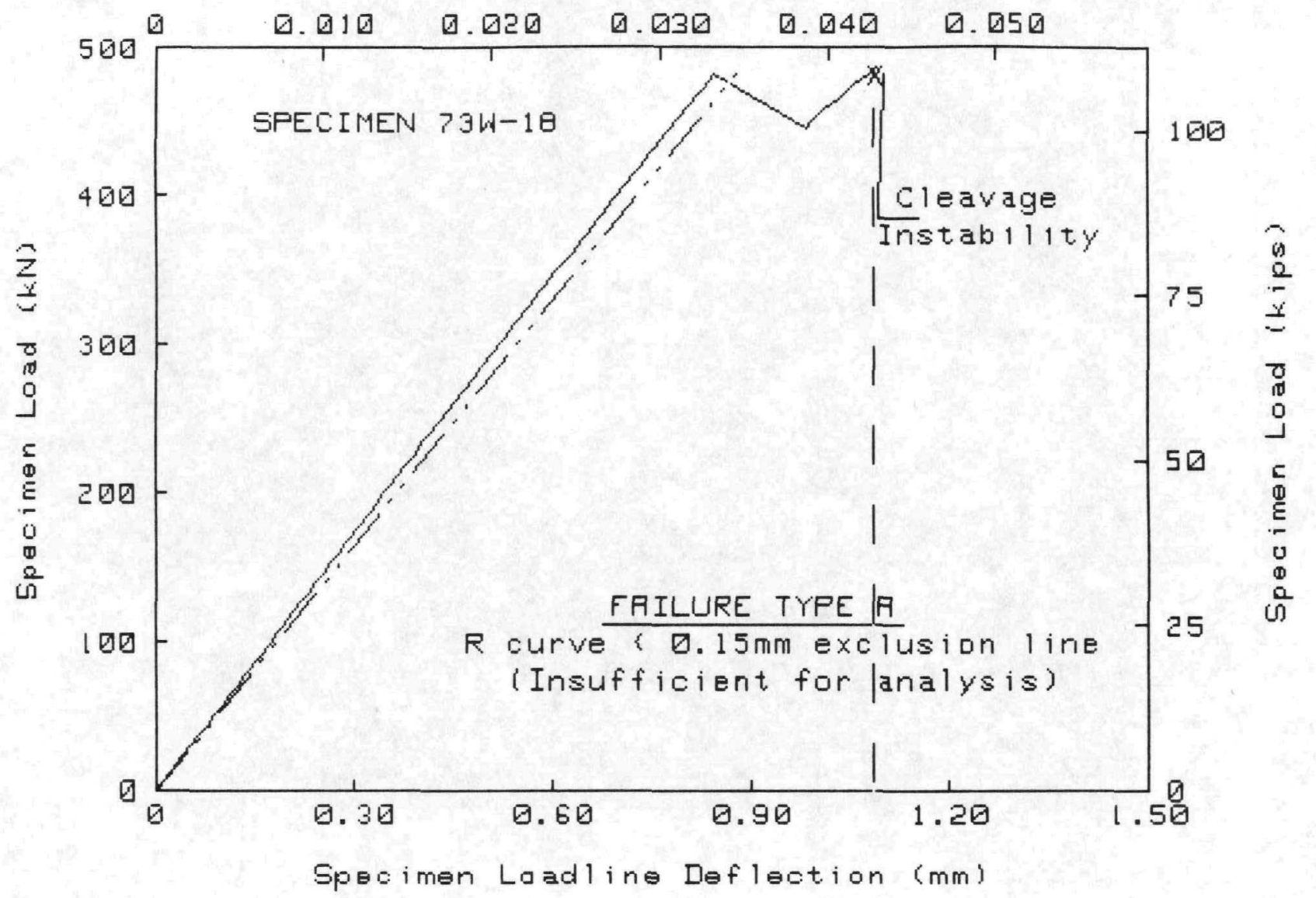

TEST SPECIMEN DATA

Material Type
Test Temperature
Percent Side Groove
Specimen Thickness B
Initial a/W
Init crack length a
Flow stress
Yield stress
Youngs modulus
Poisson's Ratio (U)
J-INTEGRAL ANALYSIS

$=A 533-B$ Weld (I)

$=50^{\circ} \mathrm{C}$

$=0 \%$

$=101.6 \mathrm{~mm}$

$=.505$

$=102.51 \mathrm{~mm}$

$=683.1 \mathrm{MPa}$

$=636.6 \mathrm{MPa}$

$=204400 \mathrm{MPa}$

$=0$

$\mathrm{Jc}$

[ Failure Type A

(Est, imated Value)

$=\frac{\text { Merkle-Corten }}{70.4 \mathrm{~kJ} / \mathrm{m}^{2}}$
$=120 \mathrm{MPa} \sqrt{\mathrm{m}}$
$=112.7 \mathrm{MPa} \sqrt{\mathrm{m}}$

instability $=$ Jc
Deformation-J*

$K j c=\left\langle E J c /\left(1-v^{2}\right\rangle\right\rangle \wedge .5$

$=120 \mathrm{MPa} \sqrt{\mathrm{m}}$

$79.1 \mathrm{~kJ} / \mathrm{m}^{2}$

$127.2 \mathrm{MPa} \sqrt{\mathrm{m}}$

KC (Beta Corrected)
Modified Kic Equation; $B=\langle B B n\rangle 1 / 2$
Maximum Load $P=487.26 \mathrm{kN}$
5\% Secant offset $\mathrm{Fq}=481.96 \mathrm{kN}$
$\mathrm{Kq}$
Validity
$K$ at Maximum Load
$=103.1 \mathrm{MPa} \sqrt{\mathrm{m}}$
= VALID Kic value
$=104.2 \mathrm{MPa} \sqrt{\mathrm{m}}$

DATA CORRECTIONS USED FOR J-INTEGRAL DATA

Merkle-Corten correction

Correction to J for crack extension (Deformation-J)

OFFSET LOAD $=0 \mathrm{kN}$ OFFSET DEFLECTION $=-.001 \mathrm{~mm}$

Fitted elastic load range $=50 \mathrm{kN}$ To $200 \mathrm{kN}$

Elastic slope $=5.786 \mathrm{E}+02 \mathrm{kN} / \mathrm{mm}$

NUREG/CR-5913 
Specimen Loadline Deflection bin.)

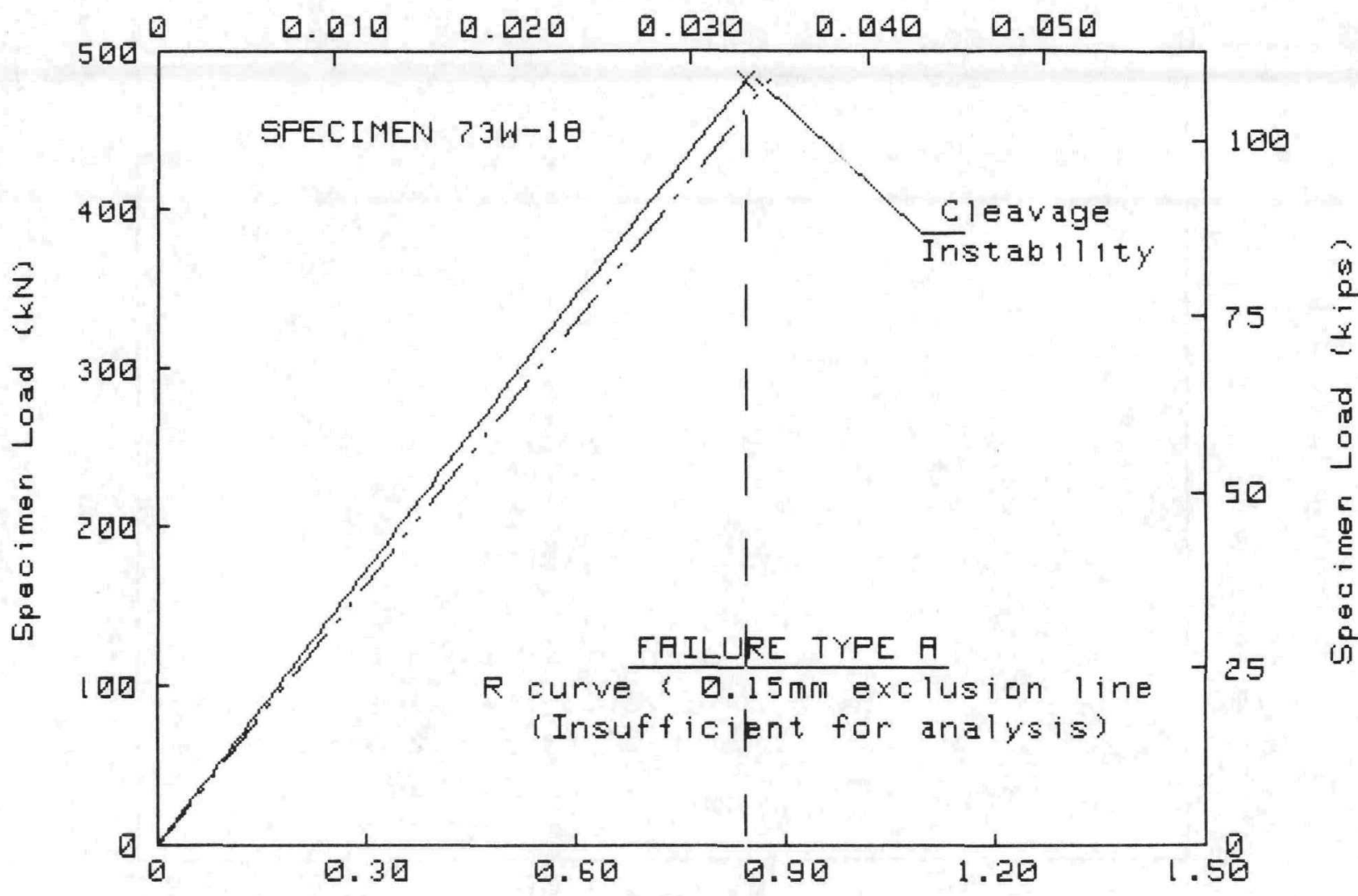

Specimen Laadine Deflection (mm)

TEST SPECIMEN DATA

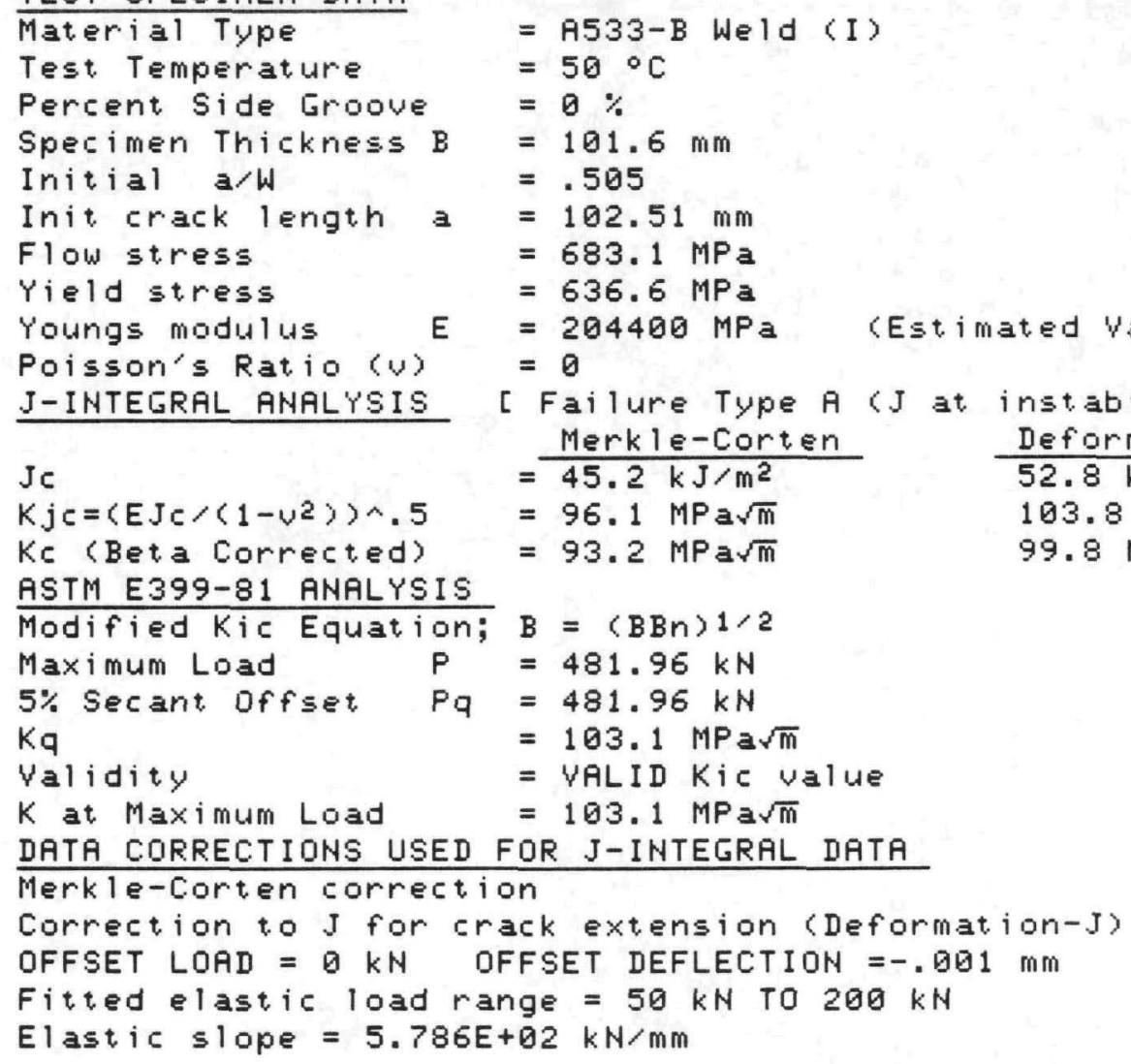


Specimen Crackmauth Deflectian (in.)

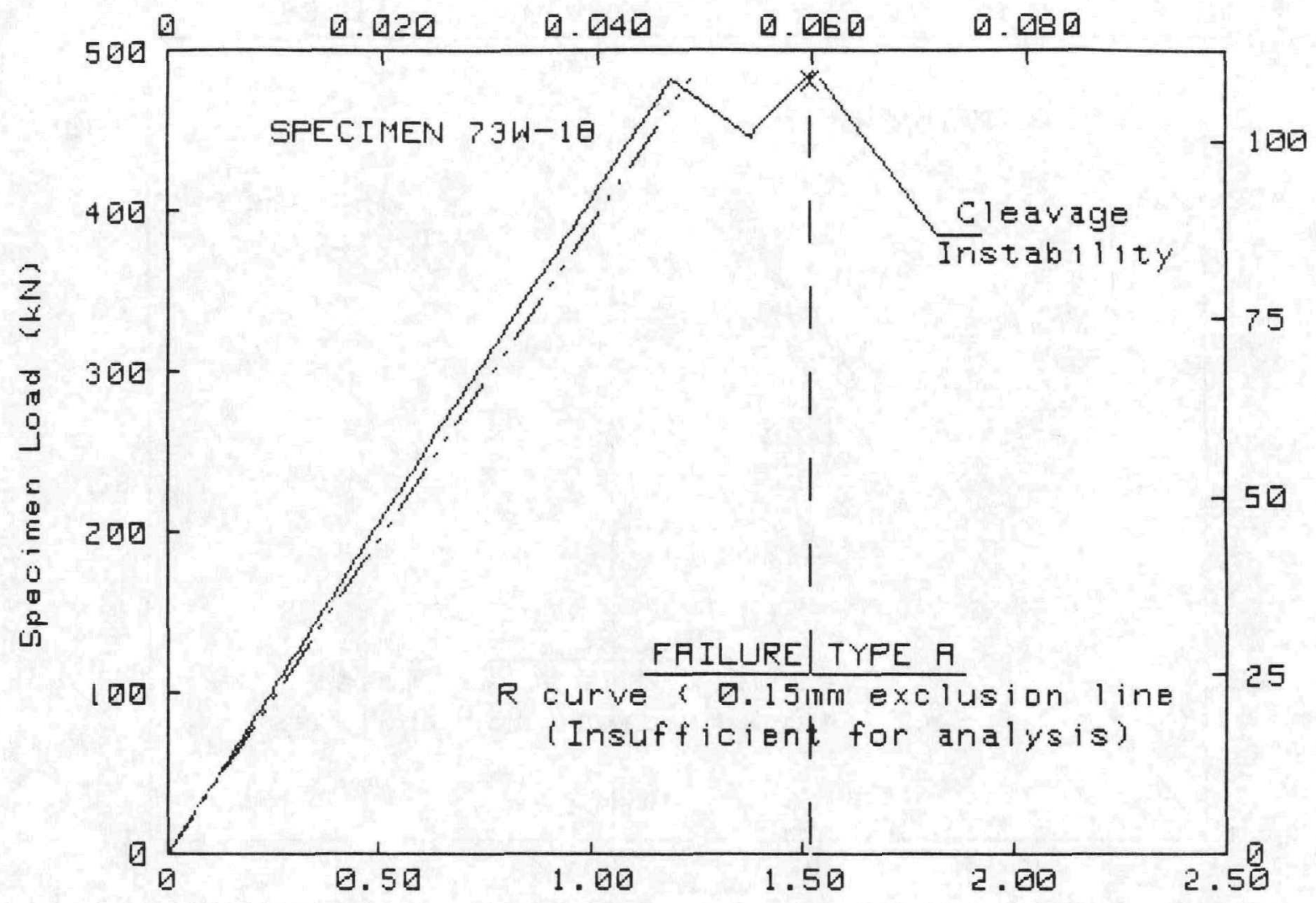

Specimen Cirackmouth Defleotion (mm)

\section{TEST SPECIMEN DATA}

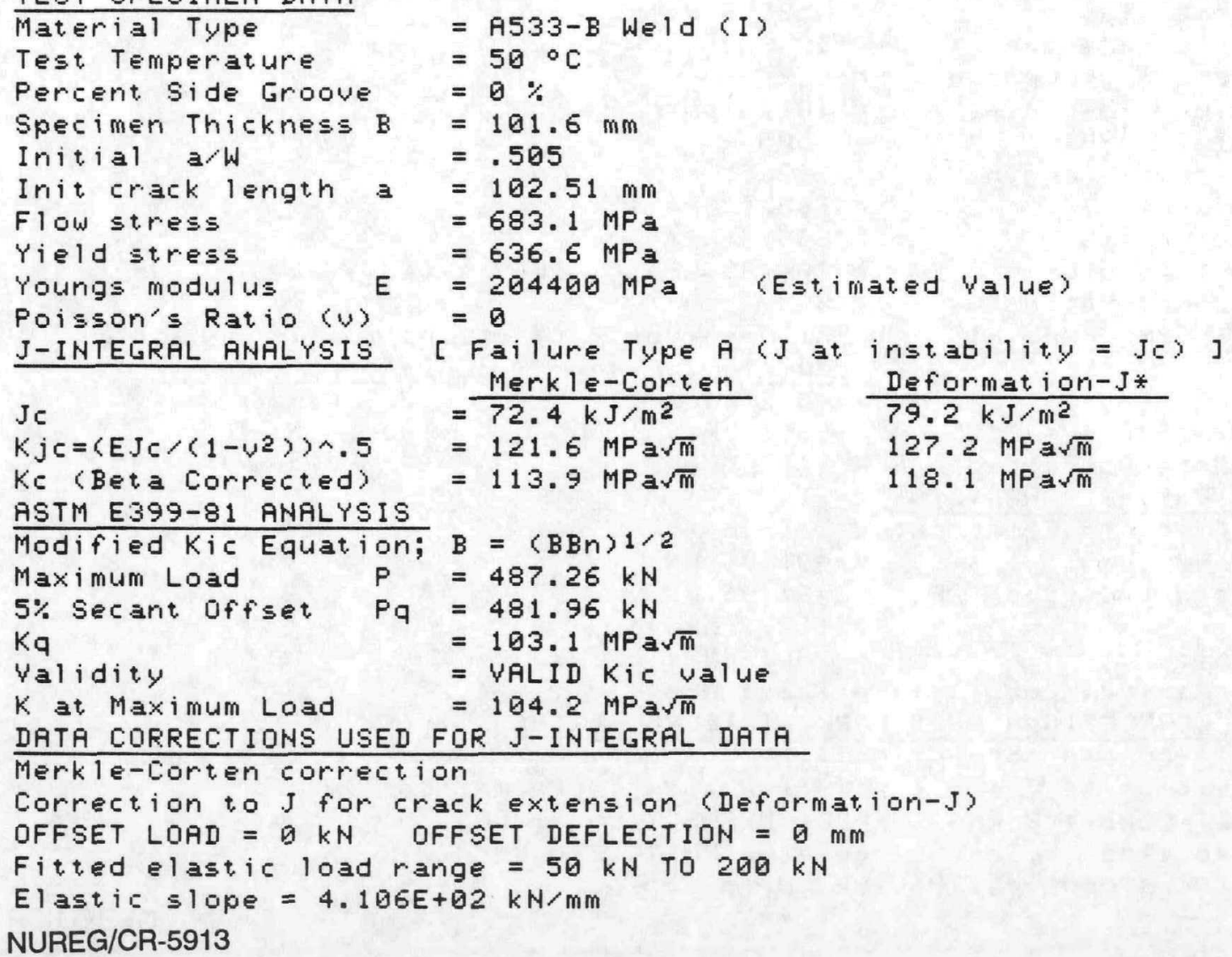


Specimen Crackmouth Deflection (in.)
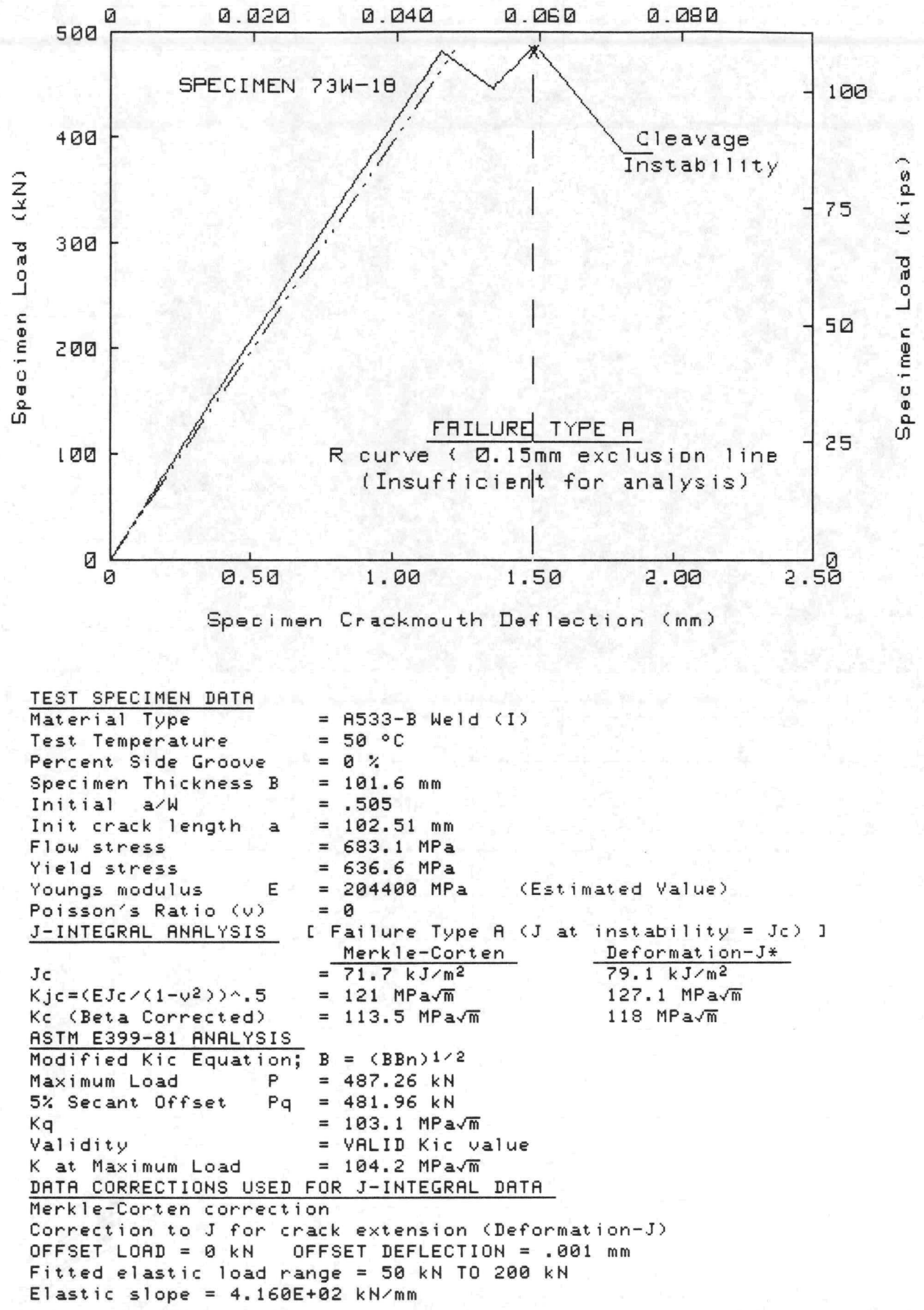


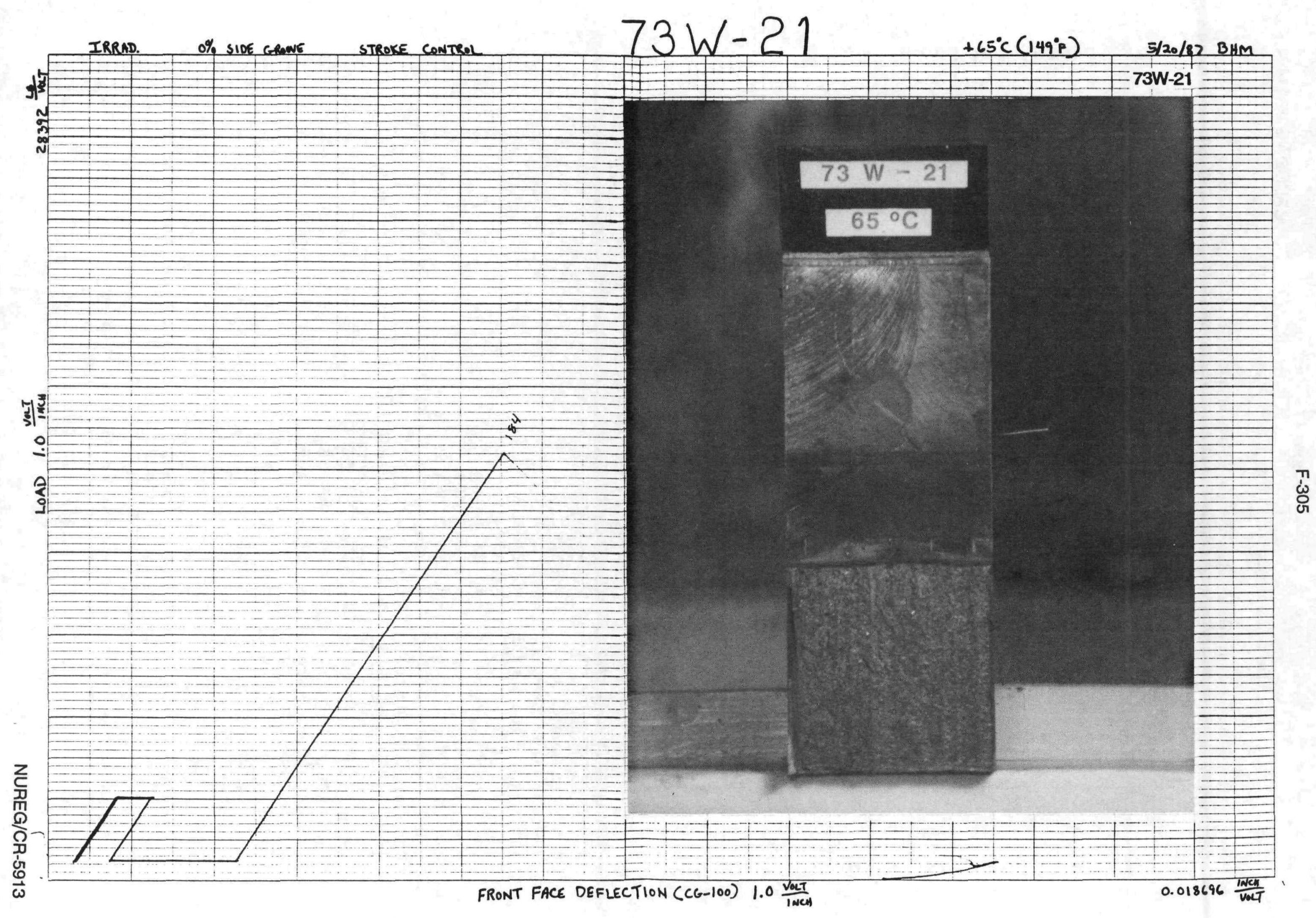


Specimen Loadline Deflection (in.)

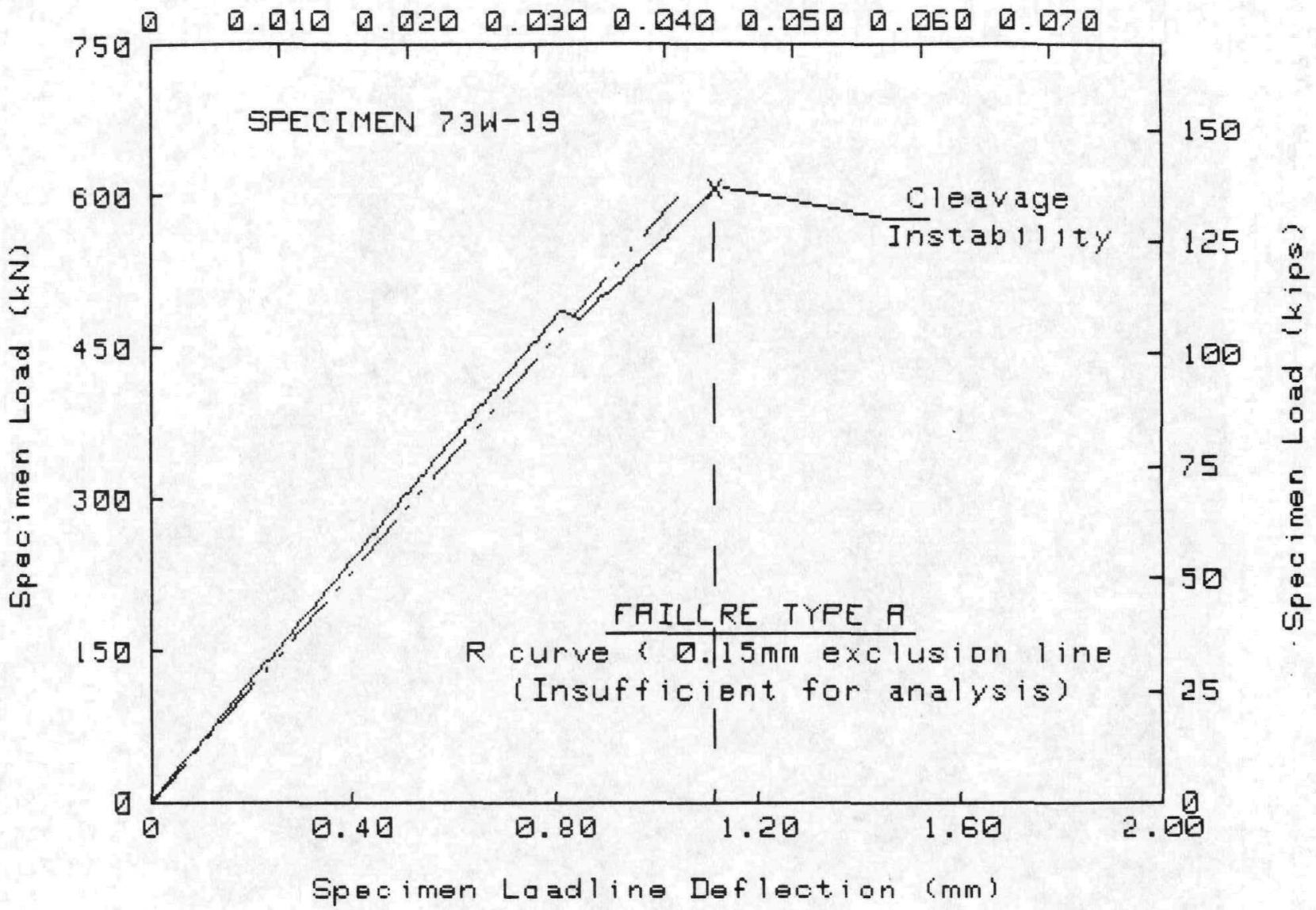

TEST SPECIMEN DATA

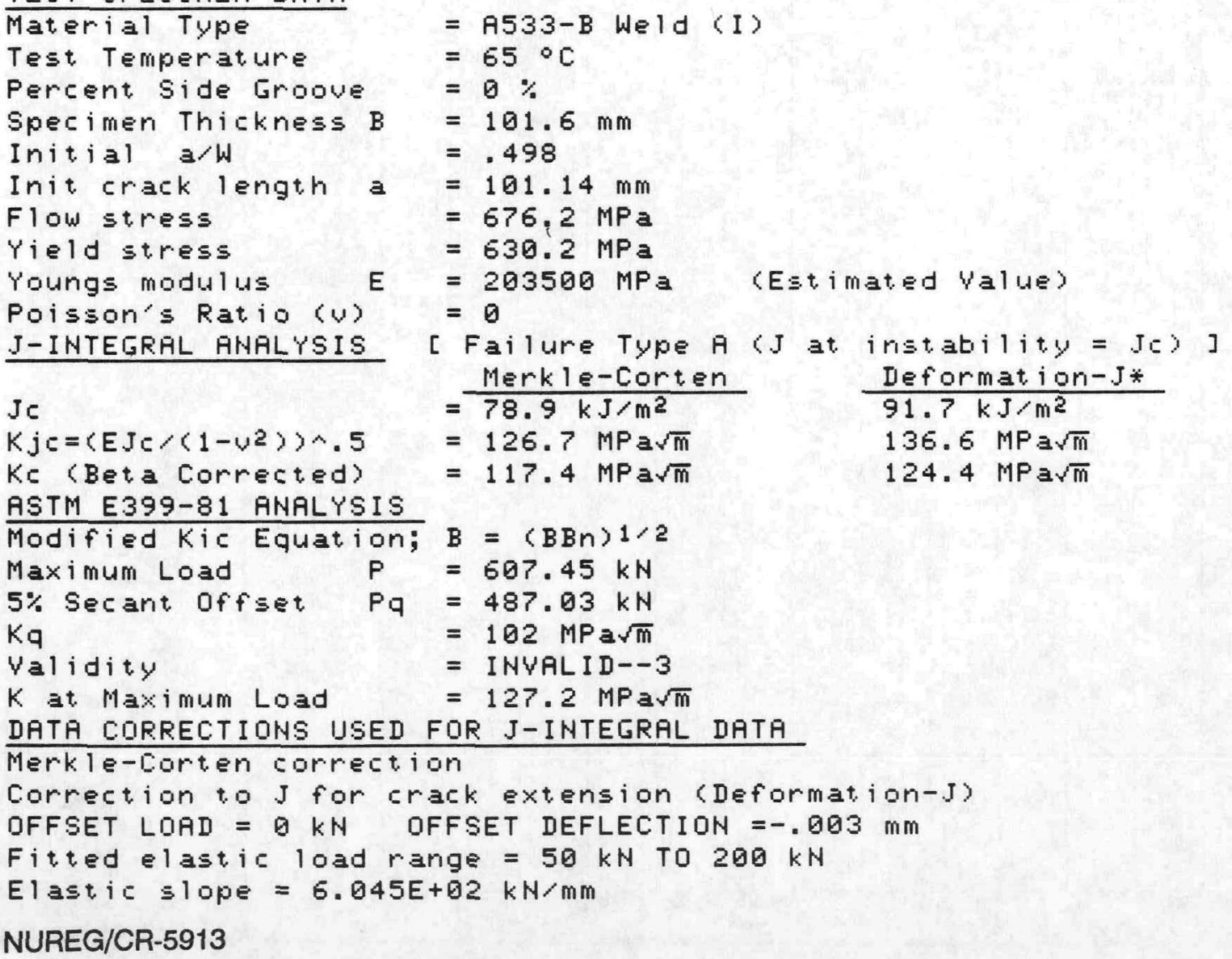


Specimen Loadline Deflection (in.)

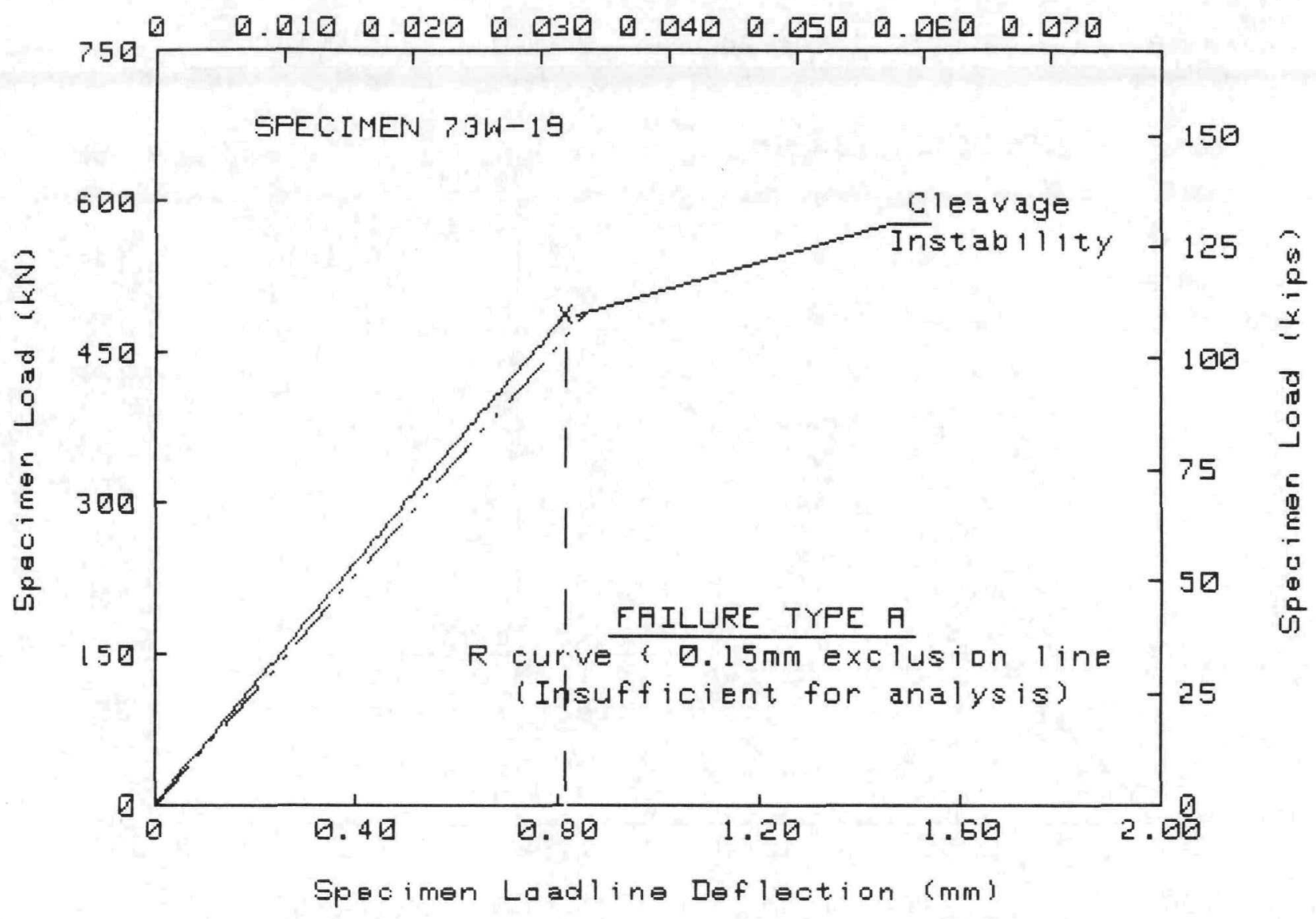

TEST SPECIMEN DATA

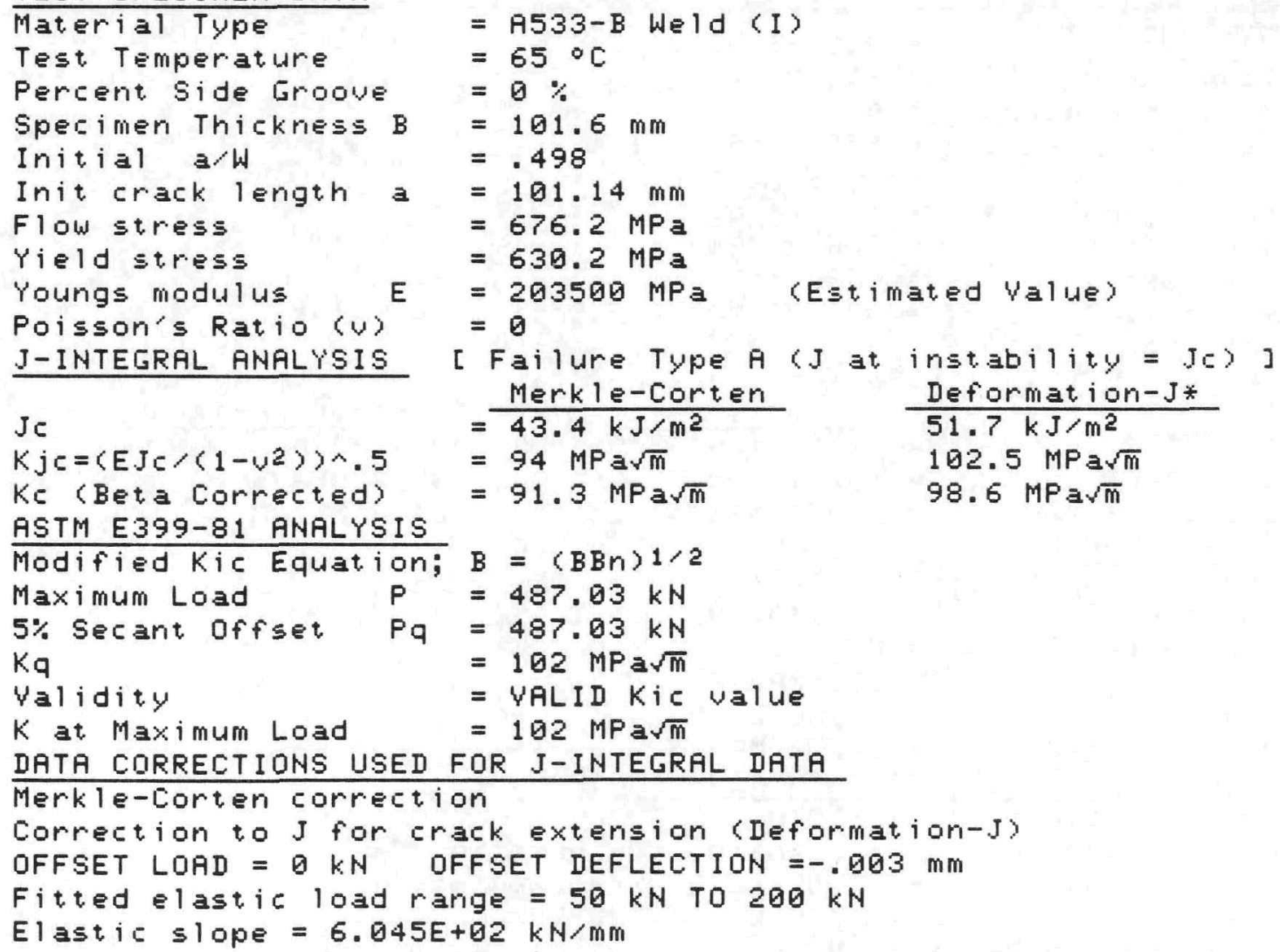


Gpecimen Crackmauth Deflectian (in.)

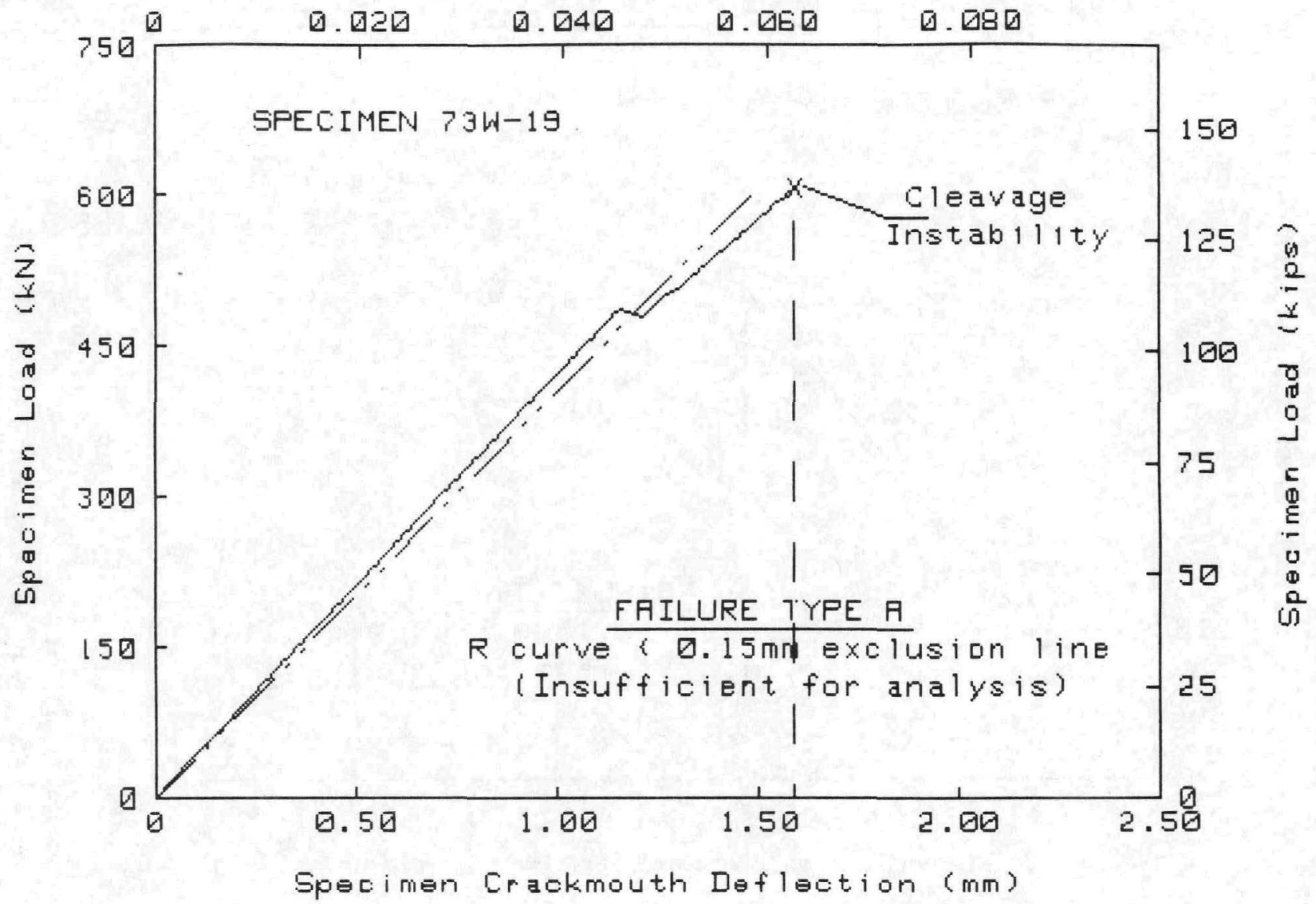

TEST SPECIMEN IATA

Material Type
Test Temperature
Percent Side Groove
Specimen Thickness B
Initial a/W
Init crack length a
Flow stress
Yield stress
Youngs modulus
Poisson S Ratio (U)
J-INTEGRAL ANRLYSIS

= A533-B Weld (I)

$=65 \circ \mathrm{C}$

$=0 \%$

$=101.6 \mathrm{~mm}$

$=.498$

$=101.14 \mathrm{~mm}$

$=676.2 \mathrm{MPa}$

$=630.2 \mathrm{MPa}$

$=203500 \mathrm{MPa}$ (Estimated Value)

I-INTEIRAL ANALYSIS

$\mathrm{Je}$ $=0$

[ Failure Type $A$ (J at instability = Je) ]

$K j c=\left\langle E J c /\left(1-v^{2}\right)\right\rangle \wedge .5$ $=\frac{\text { Merkle-Corten }}{83.2 \mathrm{~kJ} / \mathrm{m}^{2}}$ Deformation-J*

$=130.1 \mathrm{MPa} \sqrt{\mathrm{m}}$ $92.4 \mathrm{~kJ} / \mathrm{m}^{2}$

$=119.9 \mathrm{MPa} \sqrt{\mathrm{m}}$ $137.2 \mathrm{MPa}$ m $124.8 \mathrm{MPa} \sqrt{\mathrm{m}}$

Ke (Bet,a Corrected)
ASTM E399-81 ANALYSIS

Modified Kic Equation; $B=(B B n) 1 / 2$

Maximum Load $P=607.45 \mathrm{kN}$

$5 \%$ Secant offset $\mathrm{Pq}=487.03 \mathrm{kN}$

$\mathrm{Kq}$

Validity

$=102 \mathrm{MPa} \sqrt{\mathrm{m}}$

$=$ INUALID --3

$K$ at Maximum Load

$=127.2 \mathrm{MPa} \sqrt{\mathrm{m}}$

DATA CORRECTIONS USED FOR J-INTEGRAL DATA

Merkle-Corten correction

Correction to J for crack extension (Deformation-J)

OFFSET LOAD $=0 \mathrm{kN}$ OFFSET DEFLECTION $=.001 \mathrm{~mm}$

Fitted elastic load range $=50 \mathrm{kN}$ TO $200 \mathrm{kN}$

Elastic slope $=4.247 \mathrm{E}+02 \mathrm{kN} / \mathrm{mm}$ 
Specimen Crackmouth Deflection (in.)

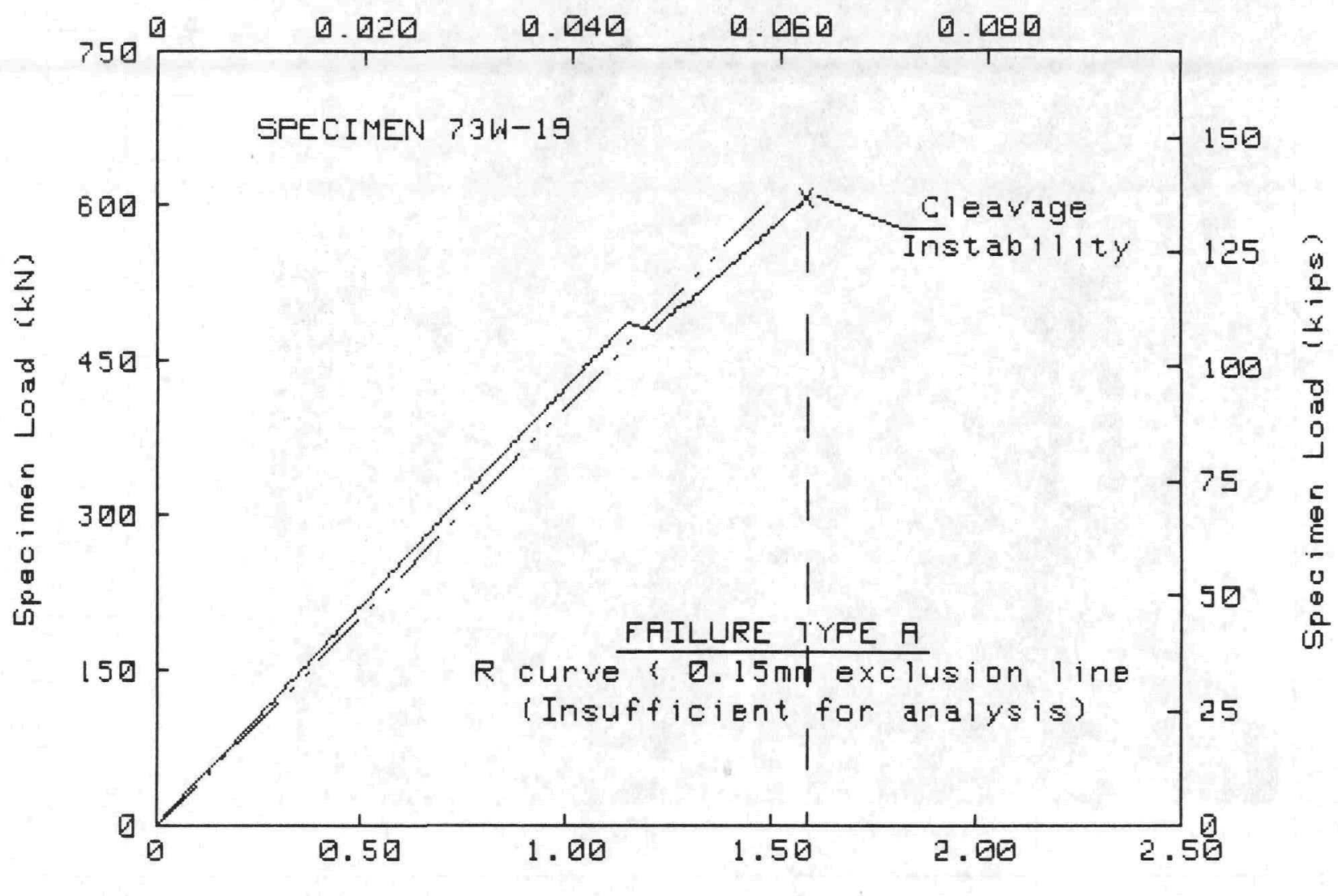

Specimen Crackmouth Defleotion ( $\mathrm{mm}$ )

\section{TEST SPECIMEN IATA}

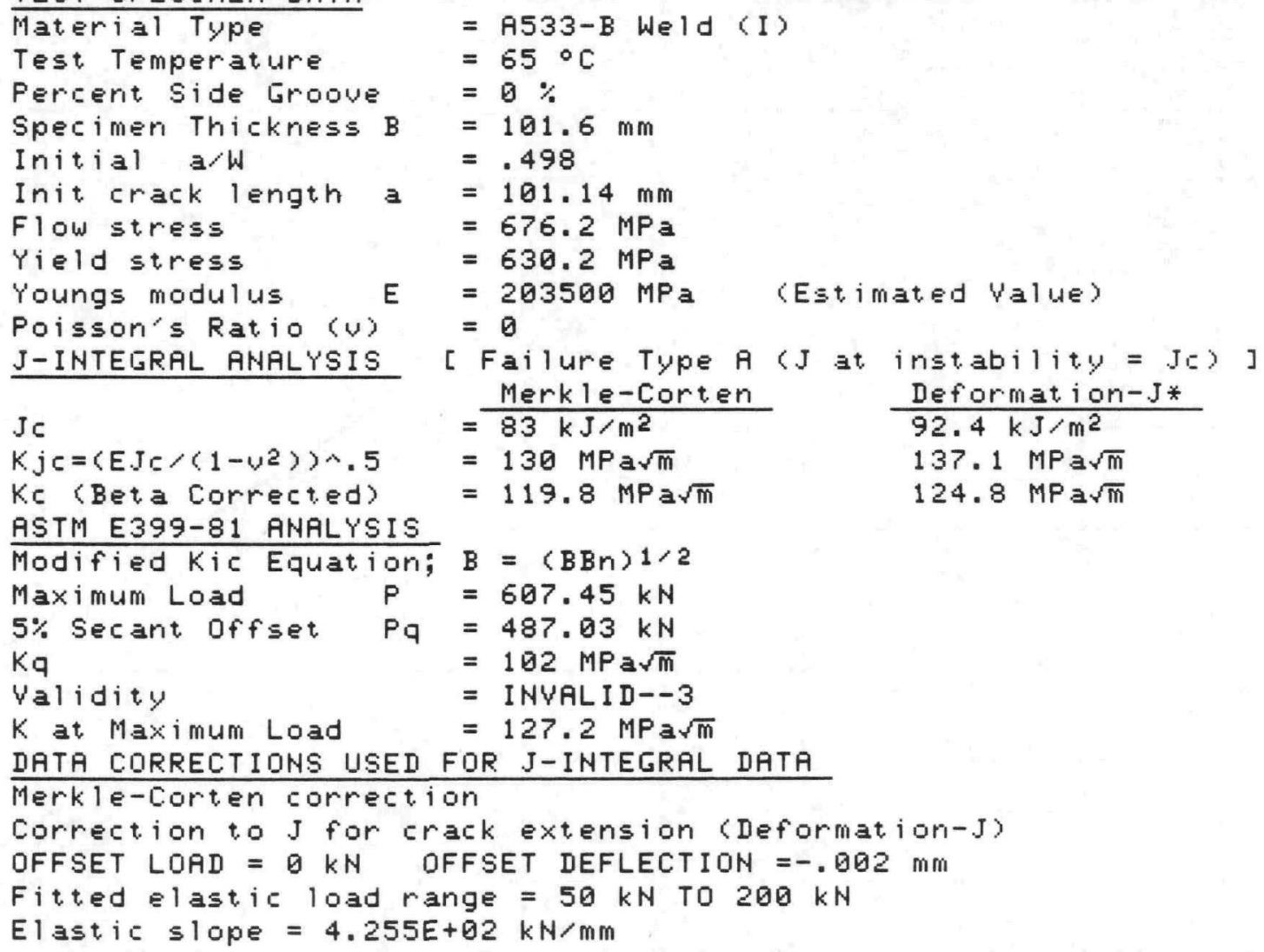




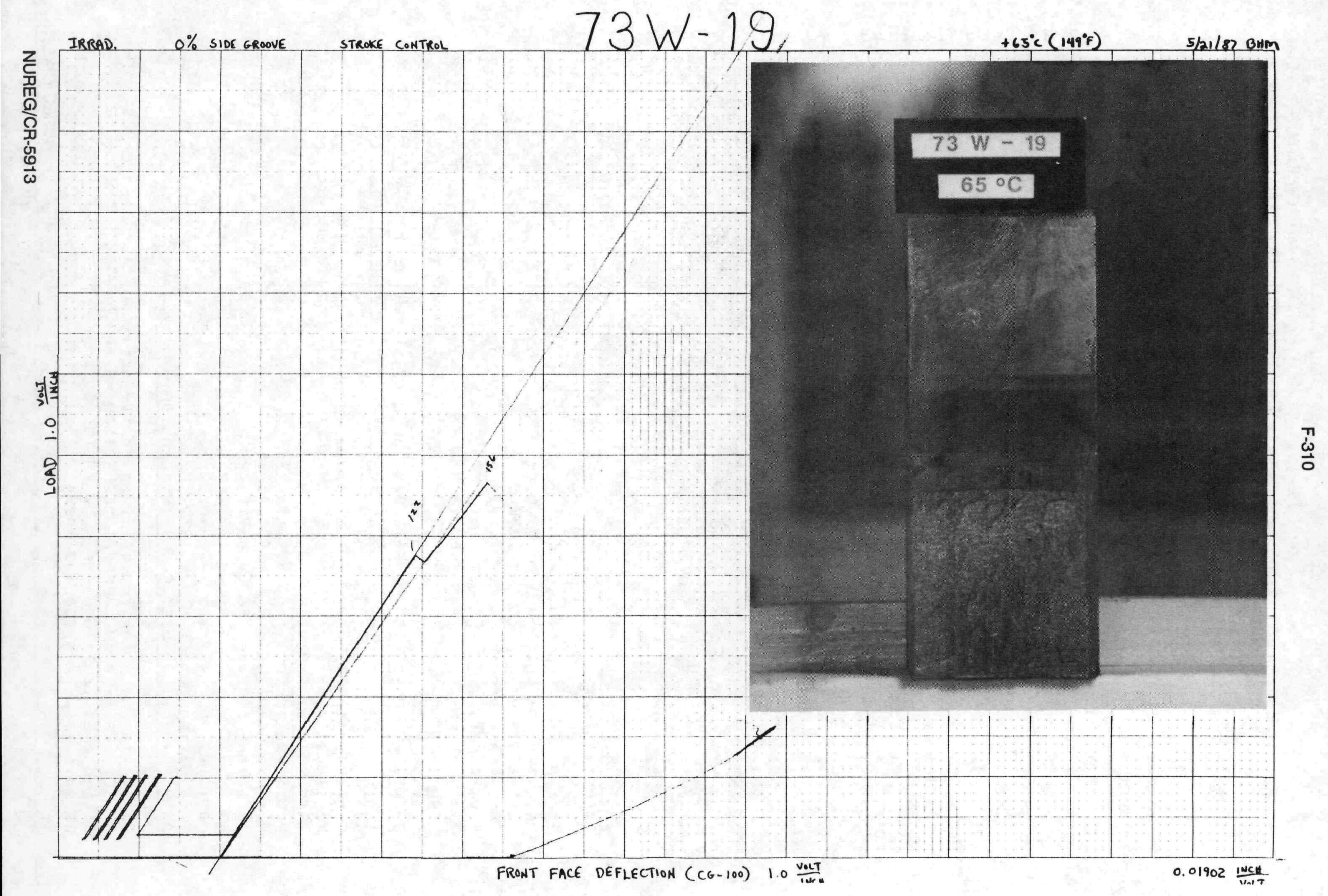




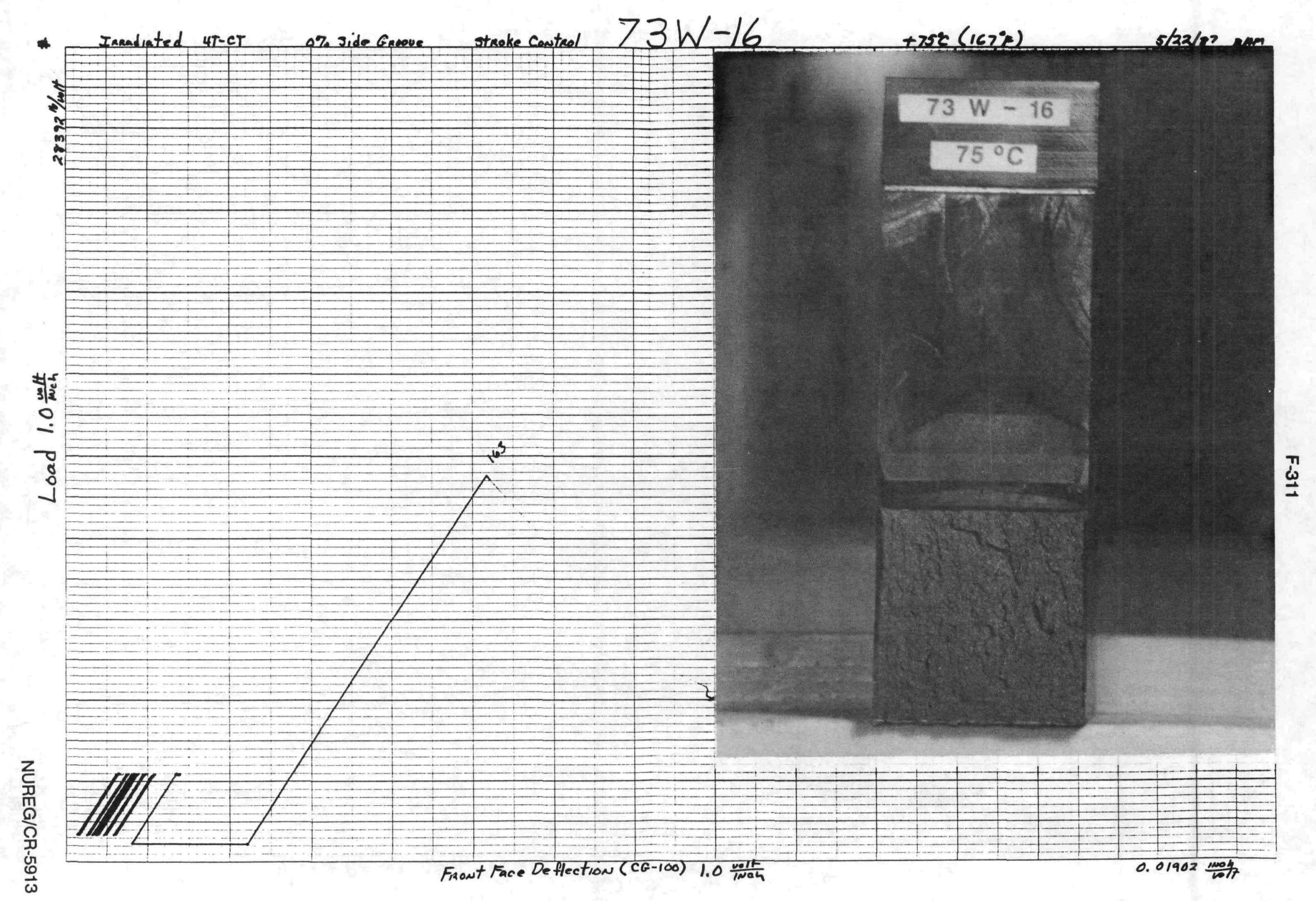


Irradiated $4 T-C T \quad 0 \%$ sideGropue stroke coutrol $73 W-12$

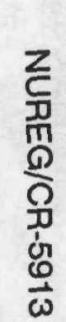

总

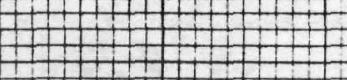

+

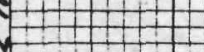

.

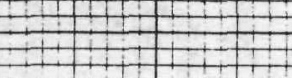

\begin{tabular}{|c|c|c|}
\hline \\
\hline \\
\hline
\end{tabular}

$+1$
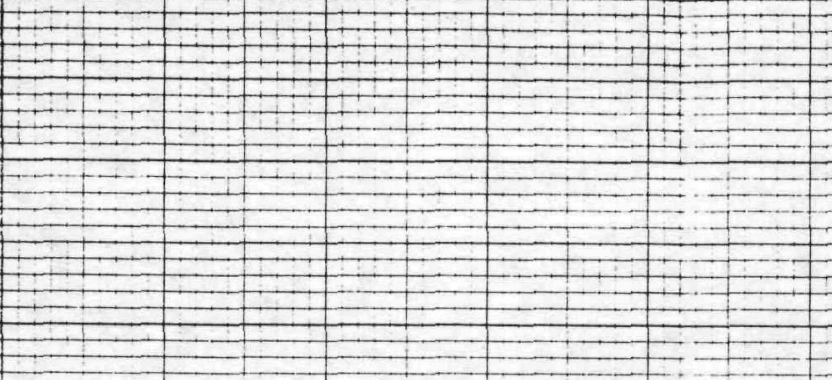

$25^{\circ} \mathrm{C}\left(185^{\circ} \mathrm{F}\right)$

$5 / 22 / 82$ and
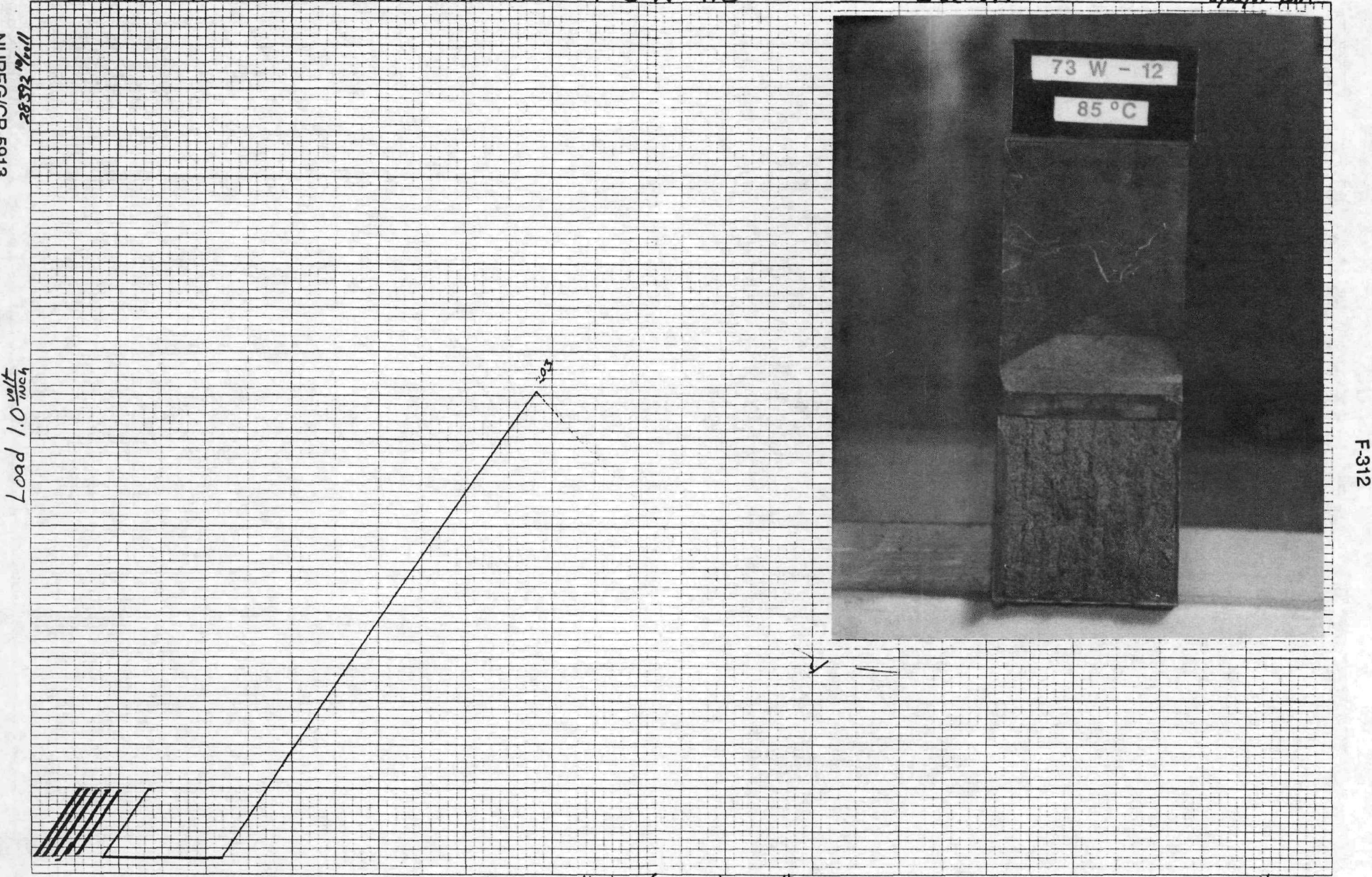

Front Face De flection (GG--100) $1.0 \frac{\text { vell }}{\text { inch }}$ $0.01902 \frac{\mathrm{NeCh}}{\mathrm{NOI}}$ 


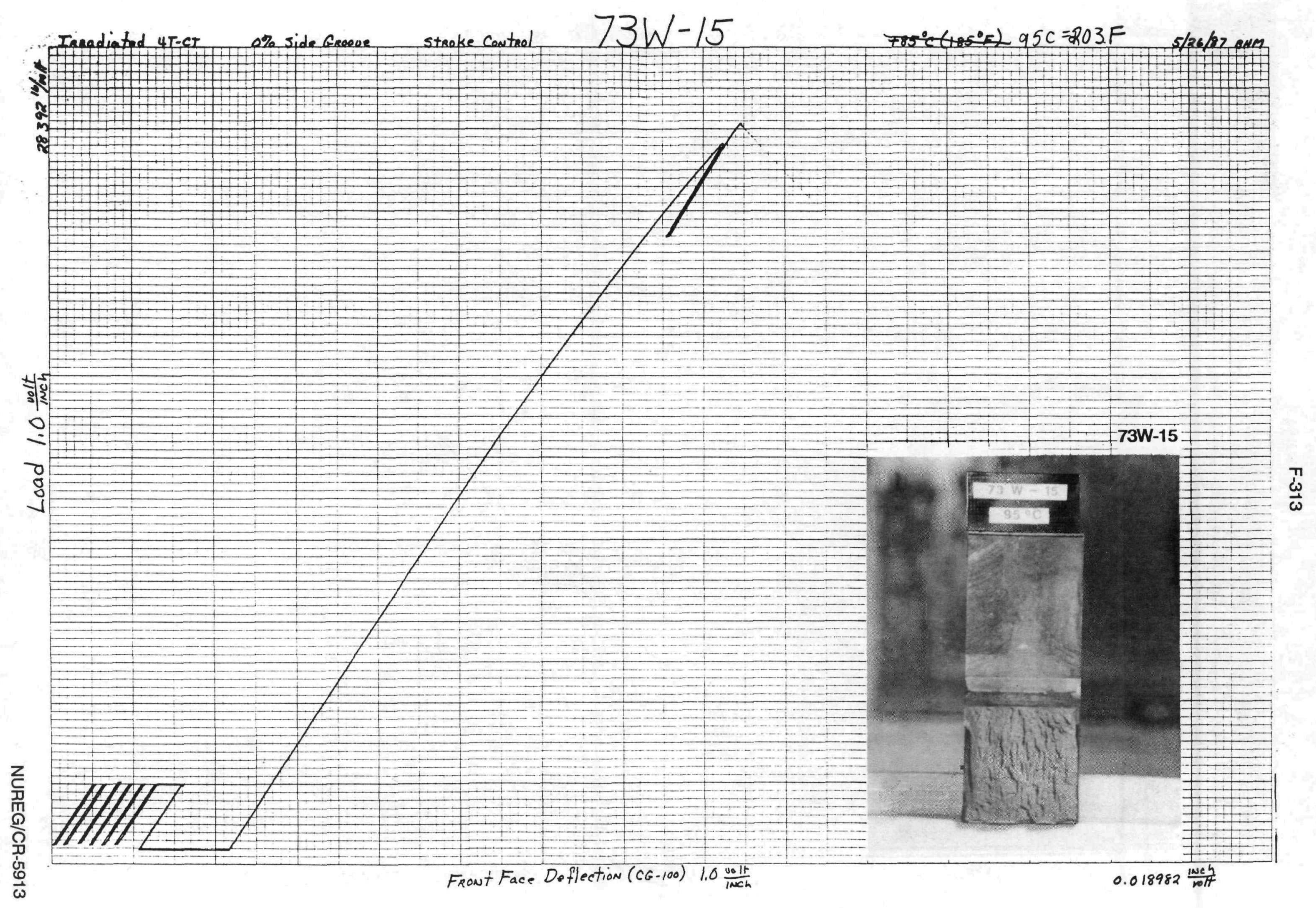




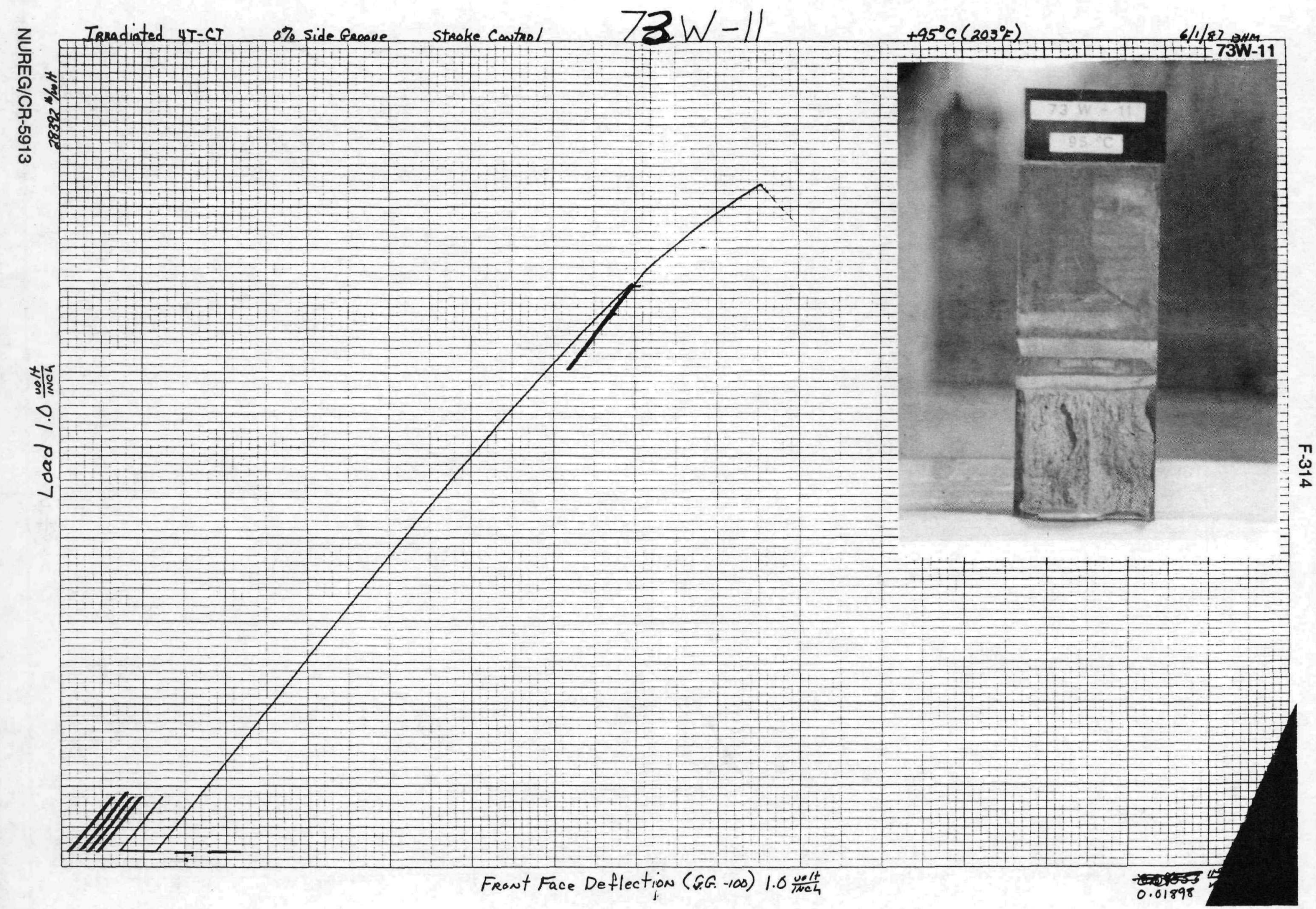


NUREG/CR-5913

Vol. 2

ORNL/TM-12156/N2

Limited

Distr.

\section{INTERNAL DISTRIBUTION}

1. D. J. Alexander

2. B. R. Bass

3-4. K. O. Bowman

5-20. W. R. Corwin

21. D. F. Craig

22. T. L. Dickson

23-24. F. M. Haggag

25. S. K. Iskander

26-28. D. E. McCabe

29. J. G. Merkle

30-34. R. K. Nanstad

35. W. E. Pennell
36. C. E. Pugh

37. D. K. Shum

38. G. M. Slaughter

39. R. E. Stoller

40. R. L. Swain

41. T. J. Theiss

42. ORNL Patent Section

43. Central Research Library

44. Document Reference Section

45-46. Laboratory Records Department

47. Laboratory Records (RC)

48-50. M\&C Records Office

\section{EXTERNAL DISTRIBUTION}

51-52. MATERIALS ENGINEERING ASSOCIATES, 9700B Martin Luther King, Jr., Highway, Lanham, MD 20706

B. H. Menke (2)

53-55. NRC, RES/Division of Engineering, Washington, DC 20555

A. Hiser (MS NS 217C)

M. E. Mayfield (MS NS 217C)

C. Z. Serpan (MS NS 217C)

56-58. NRC, NRR/Division of Engineering Technology, MS 7D4, Washington, DC 20555

B. J. Elliot

J. R. Strosnider

K. R. Wichman

59. DOE OAK RIDGE FIELD OFFICE, P.O. Box 2001, Oak Ridge, TN 37831-6269

Office of Deputy Assistant Manager for Energy Research and Development

60-61. DOE, OFFICE OF SCIENTIFIC AND TECHNICAL INFORMATION, P.O. Box 62,

Oak Ridge, TN 37831 